The Effects of Diaphragm Flexibility on the Seismic Response of One-Storey Steel Buildings

by

Pedram Mortazavi

A thesis submitted to the Faculty of Graduate and Postdoctoral Affairs in partial fulfillment of the requirements for the degree of

\author{
Master of Applied Science \\ in
}

Civil Engineering

Ottawa-Carleton Institute for Civil Engineering

Department of Civil Engineering

Carleton University

Ottawa, Ontario, Canada

(C) 2014, Pedram Mortazavi 


\title{
Effects of Diaphragm Flexibility on the Seismic Response of Single-Storey Steel Buildings
}

\begin{abstract}
The response of single-storey buildings to earthquakes is strongly influenced by the flexibility of the diaphragm. Flexibility increases the period of the building, magnifies the ductility demand on the lateral load resisting system (LLRS), and changes the manner in which the inertia forces are distributed along the length of the diaphragm. These effects are influenced by the manner in which the LLRS responds. In particular, pinched hysteretic behaviour of steel braces can lead to a further increase in the ductility demand on the LLRS.

An analytical study is carried out to examine the various influences of diaphragm flexibility. The study improves on previously suggested methods for finding an appropriate force reduction factor for the seismic design of LLRS. The magnification in bending moment and shear force is investigated. Finally, the effects of the nonlinearity of the diaphragm on the response of the system and the design approaches are studied.
\end{abstract}




\section{Acknowledgements}

First and foremost, I would like to thank my research supervisors, Professor Jagmohan L. Humar and Professor Heng-Aik Khoo without whose support, guidance and financial aid, this study would not have been accomplished. They spent tremendous amount of time and effort instructing me how to write scholarly articles, conduct literature review, and carry out research. I would also like to thank the members of the examination board for taking the time to review my thesis and for their useful suggestions.

I would like to express my appreciation to Professor Jagmohan Humar, Professor Robert Tremblay, Professor Dimitrios G. Lignos and the editors of the journals "Journal of Structural Engineers, ASCE" and "Canadian Journal of Civil Engineers" for granting me permission to reuse their published material in this study.

Special thanks are owed to the Department of Civil and Environmental Engineering all its staff, faculty members, and fellow graduate students. In particular, I like to express my gratitude to Mrs. Payal Chadha, our graduate administrator who tirelessly helps graduate students in their student life.

Last but not least, I would like to thank my family, particularly my parents and siblings for their financial help and for believing in me and encouraging me through all stages of life. Without my family's help and moral support this study would not have been possible. 


\section{Table of Contents}

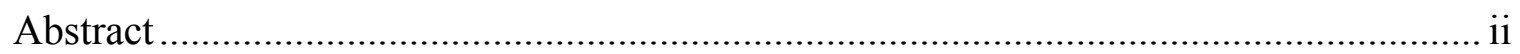

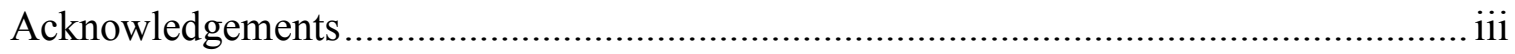

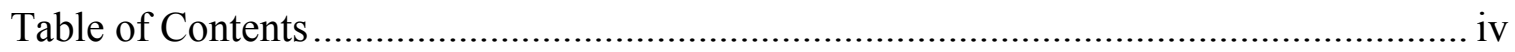

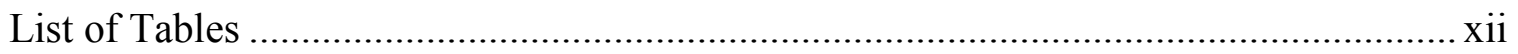

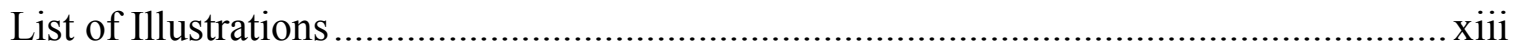

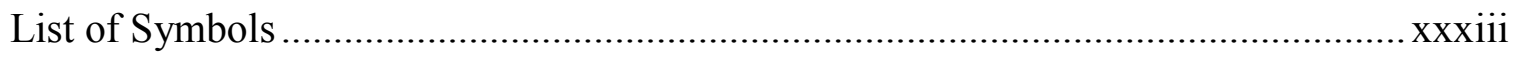

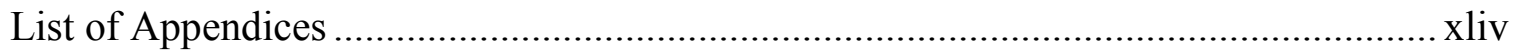

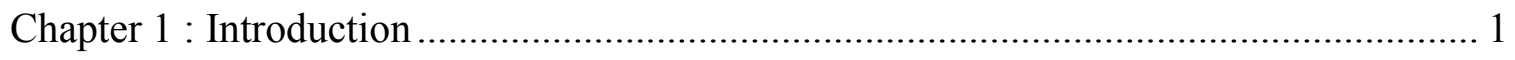

$1.1 \quad$ Diaphragm as a Structural Member ............................................................. 1

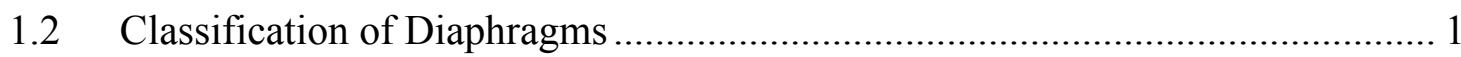

1.2.1 FEMA 356 Methods …………………………................................. 2

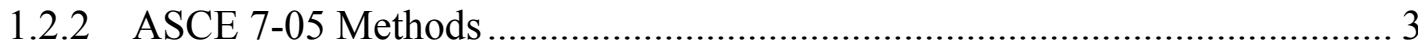

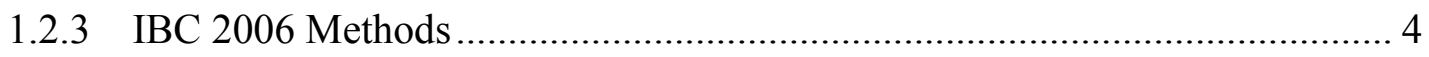

1.3 The Influence of Diaphragm Flexibility on the Response of the Structure ......... 4

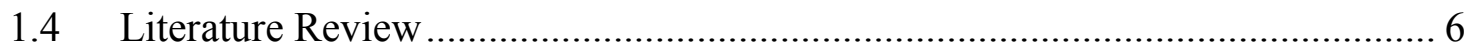

1.4.1 Research on Systems with Flexible Diaphragms ......................................... 7

1.4.2 Previous Research on the Response of Frames with Concentric Braces ...... 24

1.4.3 Previous Research on the Nonlinear Response of Steel Deck Diaphragms . 27 
1.5 Importance of Studying Single-Storey Buildings with Flexible Diaphragms.... 33

1.6 Research Objectives ............................................................................ 34

Chapter 2 : Analytical Model of Buildings with Flexible Diaphragm............................. 37

2.1 Introduction and Chapter Outline............................................................ 37

2.2 One-Storey Buildings with Flexible Diaphragms ...................................... 38

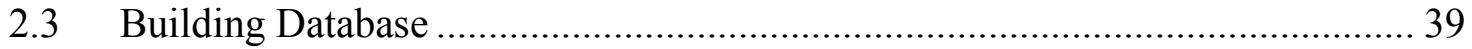

2.3.1 Considerations in Selecting the Buildings ............................................... 40

2.3.2 Design Assumptions ......................................................................... 41

2.3.3 Properties of the Analytical Model ...................................................... 43

2.4 Ground Motion Time Histories ................................................................ 48

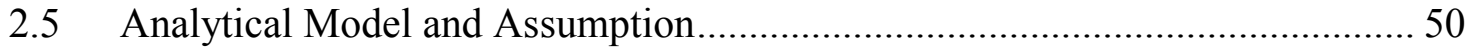

2.5.1 General Analytical Model ....................................................................... 50

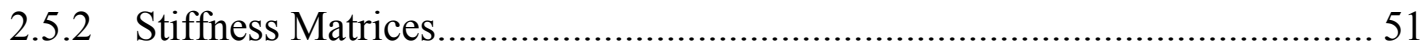

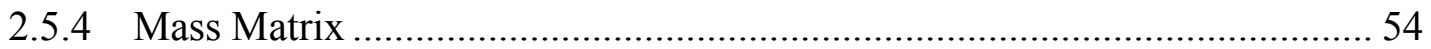

2.5.5 Condensed Stiffness Matrix and Vibration Equations............................... 54

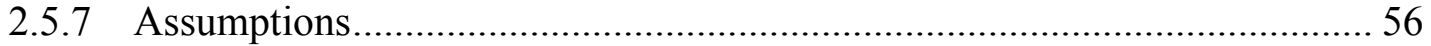

Chapter 3 : Ductility Demand on Lateral Load Resisting System................................. 58

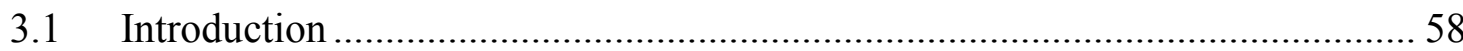

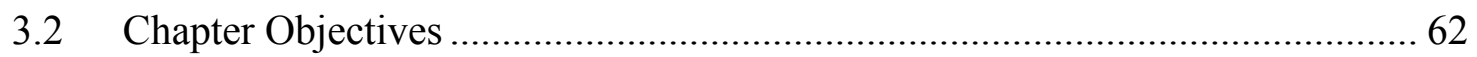

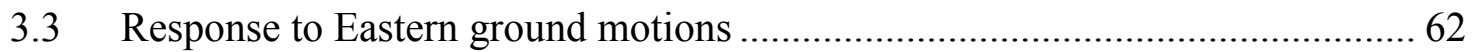


3.3.1 Analytical Model and Assumptions. 62

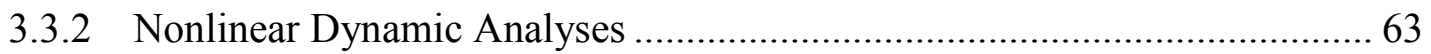

3.4 Development of Relationship between $\kappa$ and the Drift Ratio ........................... 64

3.5 Comparison between the Revised Equation and Tremblay's Method................ 72

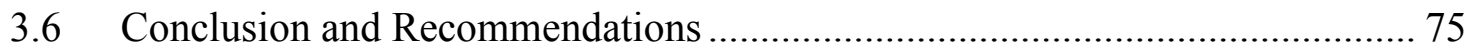

Chapter 4 : Effect of Post-Yield Hardening on Brace Ductility Demand........................ 77

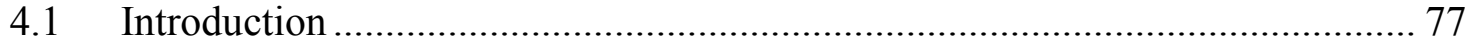

4.2 Analytical Model and Assumptions ....................................................... 78

4.4 Displacement Time Histories for LLRS …........................................... 79

4.6 Development of Relationship between $\kappa$ and the Drift Ratio ......................... 93

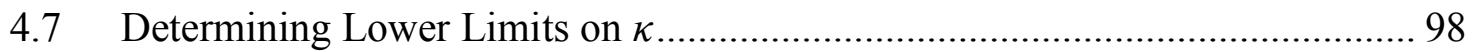

4.8 Conclusions and Recommendations........................................................ 101

Chapter 5 : The Effects of Diaphragm Flexibility on the Distribution of Shear Forces and

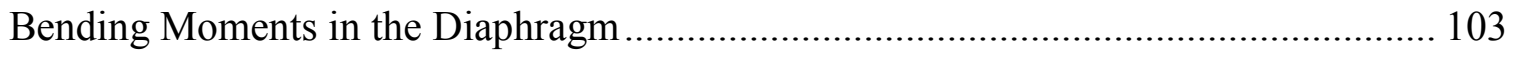

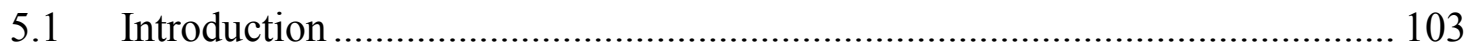

5.1.1 Dynamic Response of Rigid Diaphragms .......................................... 103

5.1.2 Dynamic Response of Flexible Diaphragms......................................... 104

5.1.3 The Importance of Assessing the Magnification of Internal Forces........... 108

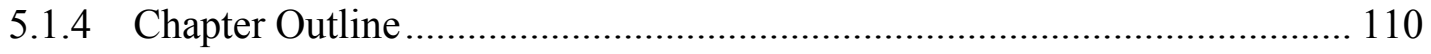

5.2 Analytical Model and Assumptions ......................................................... 111 
5.4 The Distribution of Inertia Forces in Building Diaphragms

5.5 The Relation between Ratio Values and Buildings Characteristics

120

5.6 Development of Relationships between Building Characteristics and the Ratio

Values 125

5.7 Conclusion and Recommendations . 160

5.8 Design Procedure for Diaphragms in which the Diaphragm Remains Linearly Elastic while the LLRS May Yield 162

Chapter 6 : Effect of Hysteretic Behaviour on the Seismic Response of Single-Storey Buildings 166

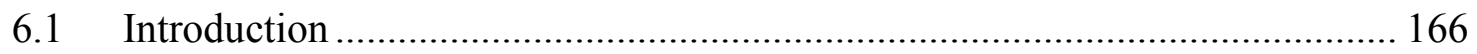

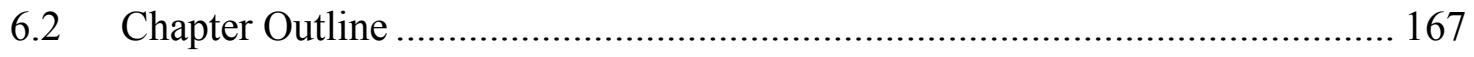

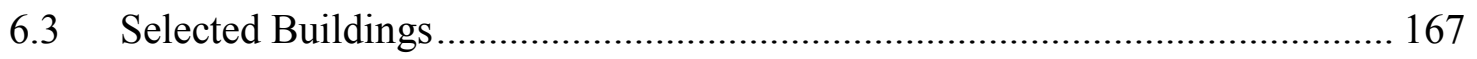

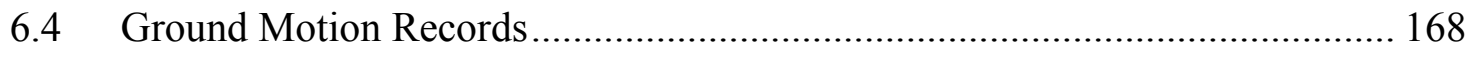

6.5 Assumptions and Analytical Model ...................................................... 169

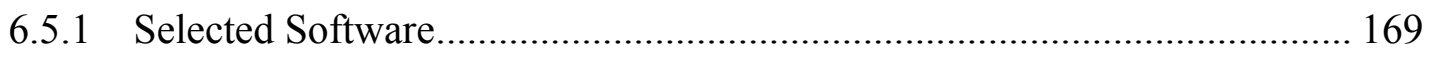

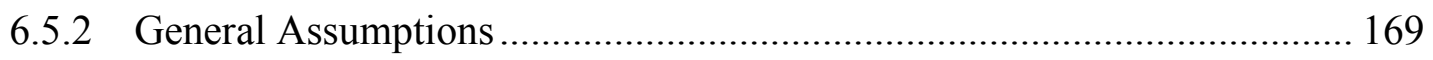

6.6 The OpenSees Model ....................................................................... 170

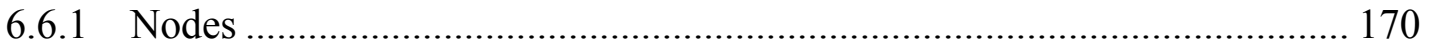

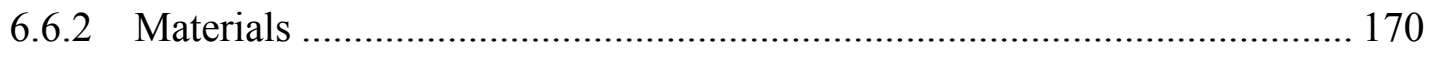

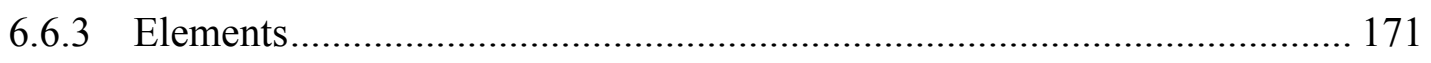


6.6.5 Dynamic Analysis

6.7 Modelling of Pinching Behaviour in OpenSees .......................................... 173

6.7.1 Pinching Behaviour in HSS Concentric Braces.................................... 173

6.7.2 Implementing the Behaviour in OpenSees Platform ............................. 174

6.7.3 Final Pinching Model in OpenSees..................................................... 182

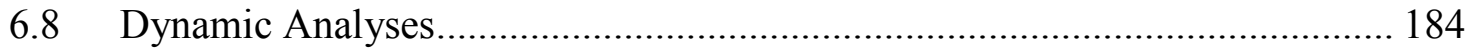

6.9 Results of the Dynamic Analyses ....................................................... 185

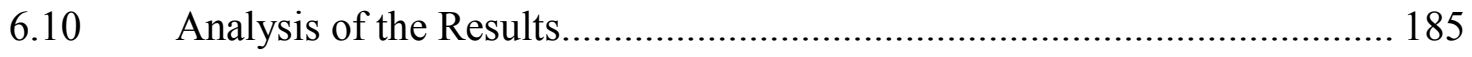

Conclusions and Recommendations ................................................... 194

Chapter 7 : Seismic Response of One-Storey Buildings with Nonlinear Flexible

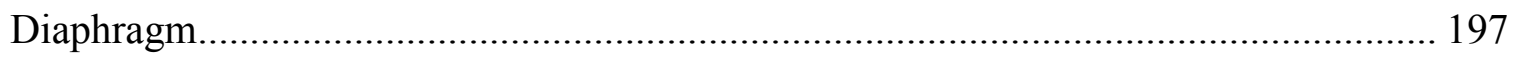

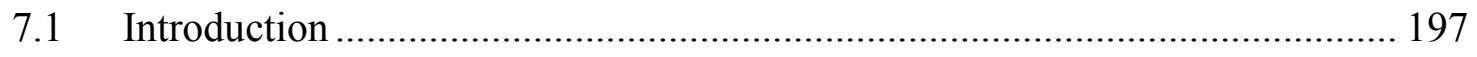

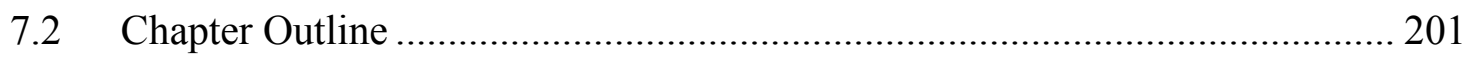

7.3 In-Plane Shear Behaviour of the Diaphragm ............................................ 202

7.3.1 Importance of Connections in Steel Deck Diaphragms ........................... 203

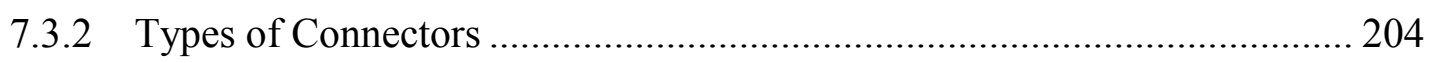

7.3.3 Mechanism of Deformation in a Flexible Diaphragm Subjected to

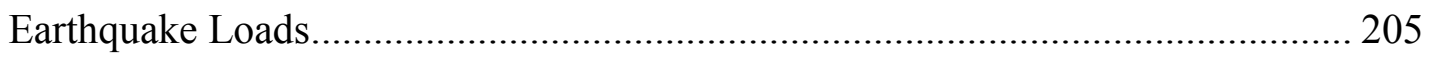

7.3.4 Modelling of Shear Behaviour........................................................ 208 


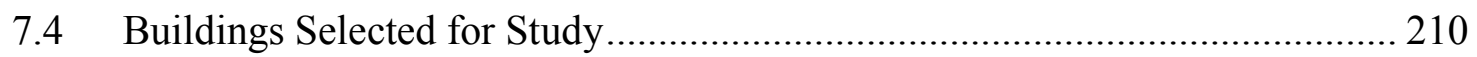

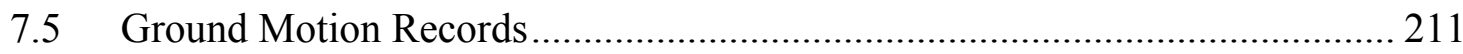

7.6 Assumptions and Analytical Model .............................................................. 211

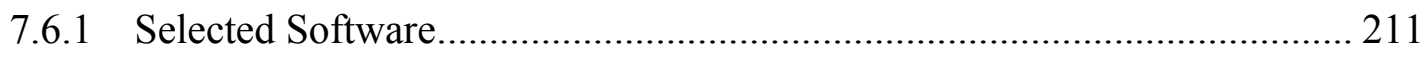

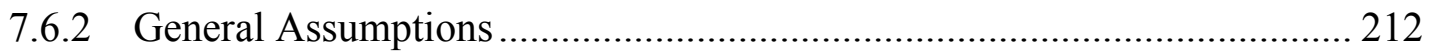

7.6.3 Force - Deformation Behaviour in Shear for Steel Deck ………................ 213

7.6.4 Selected Model and Implementation of the Model in OpenSees Platform. 215

7.6.5 Hysteretic Model Calibration............................................................... 222

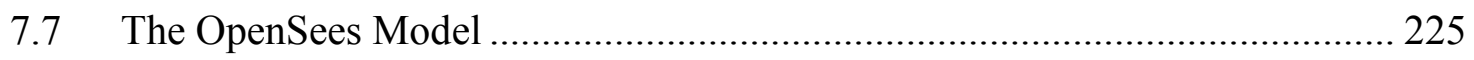

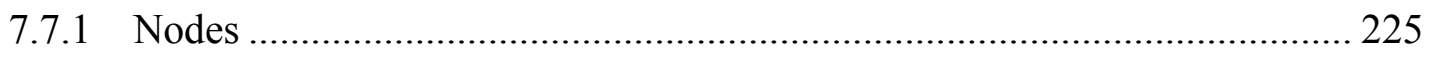

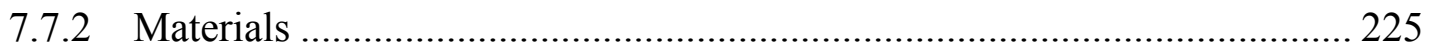

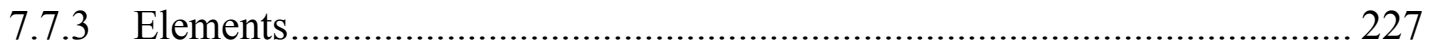

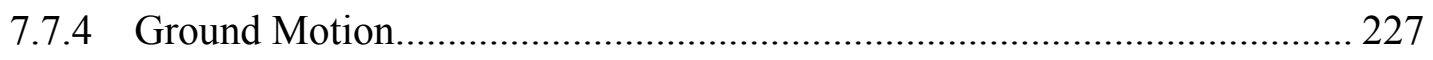

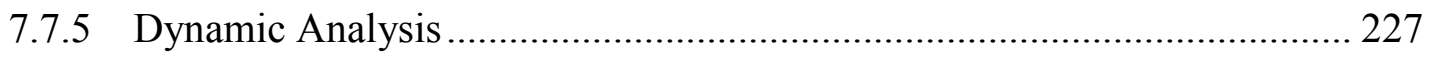

7.8 Hysteretic Response of the Example Building and the Behaviour in Shear .... 228

7.9 Diaphragm as a the Energy Dissipating Element ............................................ 230

7.9.1 Dynamic Analyses Results ................................................................ 230

7.9.2 Force Magnification Factors .................................................................. 232

7.9.3 Development of an Equation for Predicting the $\mathrm{R}_{\mathrm{d}}$ Factor ........................ 236

7.9.4 Residual Displacements .......................................................................... 239 
7.10 Diaphragm Design Force Factors for Different Performance Levels 242

7.10.1 Dynamic Analyses Results 244

7.10.2 Relation between Design Force Factors, $\left(\Phi_{e}\right.$ and $\left.\Phi_{d}\right)$ and Building

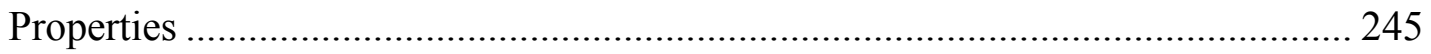

7.10.3 Nonlinearity along the Length of the Diaphragm ................................ 250

7.10.4 Development of the Equation Relating Diaphragm Design Force Factors to the Ductility Demand on the Brace.................................................................... 252

7.10.5 Internal Force Magnification Factors................................................. 253

7.10.6 The Effect of Diaphragm Nonlinearity on the Ductility Demand in the Brace 260

7.11 Selection of the Final Force Reduction Factor ...................................... 263

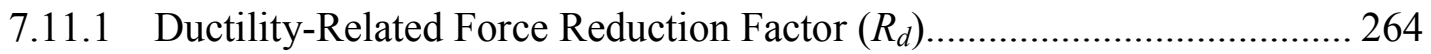

7.11.2 Over-Strength Related Force Reduction Factor...................................... 264

7.11.3 Selection of Design Force Reduction Factor .......................................... 265

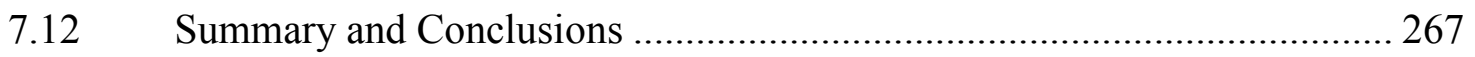

Chapter 8 : Summary, Conclusions and Recommendations ..................................... 271

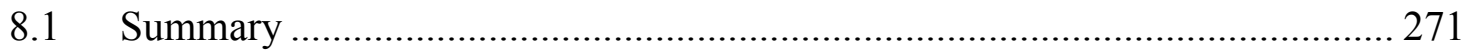

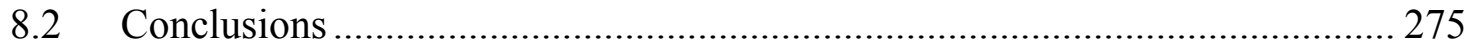

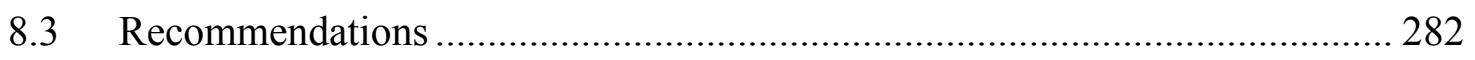

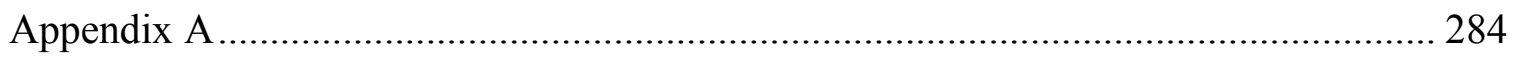


Appendix B

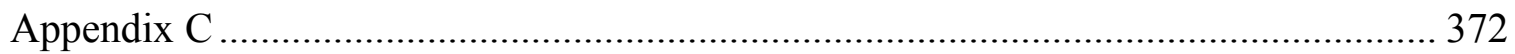

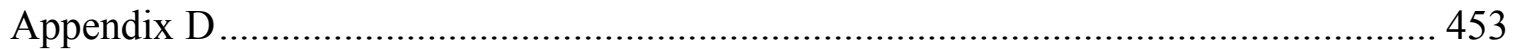

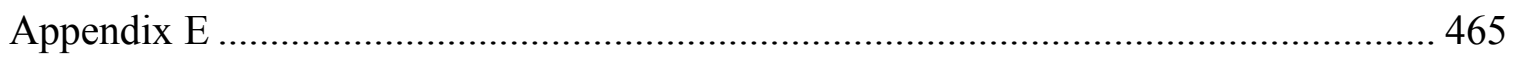

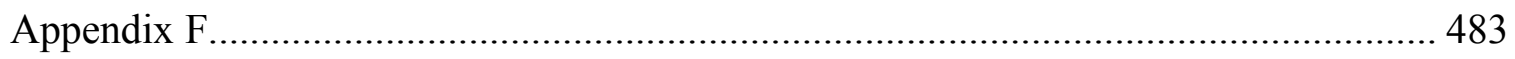

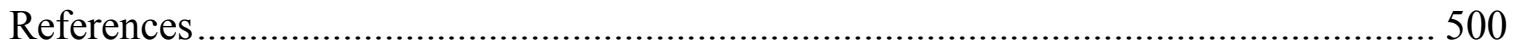




\section{List of Tables}

Table 2.1 - Design sites (taken form Tremblay and Stiemer 1996, with the authors'

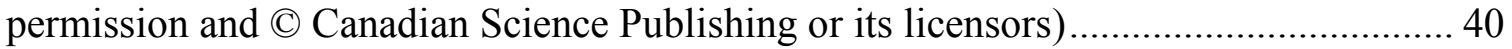
Table 2.2 - Properties of the buildings (taken form Tremblay and Stiemer 1996, with the authors' permission and (C) Canadian Science Publishing or its licensors) 43

Table 2.3 - Properties of the buildings (taken form Tremblay and Stiemer 1996, with the authors' permission and (C) Canadian Science Publishing or its licensors) ..................... 46

Table 3.1 - The lower limits on $\kappa$ without post-yield hardening ................................. 70

Table 4.1 - Lower limits on $\kappa$ for different cases......................................................... 94

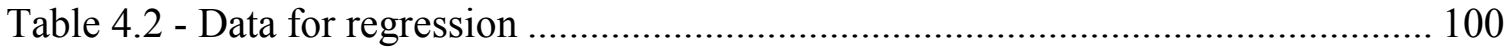

Table 5.1 - M.F and V.F values for different ductility demands ............................... 157

Table 7.1 - Mean values for diaphragm design force factor ................................... 250 


\section{List of Illustrations}

Figure 1.1- One-storey building with flexible diaphragm ............................................. 5

Figure 1.2 - Diaphragm lateral deformations under lateral loads, definitions of $\Delta \boldsymbol{B}$ and $\Delta \boldsymbol{D}$

Figure 2.1 - Schematic illustration of the one-storey buildings designed by Tremblay and Stiemer (1996) 41

Figure 2.2 - Uniform hazard spectrum for Montreal and spectra of generated ground motions. 49

Figure 2.3 - Uniform hazard spectrum for Vancouver and spectra of generated ground motions 49

Figure 2.4 - The simple model for representing one-storey buildings with flexible diaphragms 51

Figure 3.1 - Maximum horizontal deformation of diaphragm $\left(\Delta_{\mathrm{D}}\right)$ and average interstorey $\operatorname{drift}\left(\Delta_{\mathrm{B}}\right)$ 60

Figure 3.2 - Variation of $\kappa$ with drift ratio corresponding to the response of buildings to ground motions compatible with the UHS for Vancouver; target ductility 2 and elastoplastic behaviour for LLRS $(\alpha=0.00)$ 65

Figure 3.3 - Variation of $\kappa$ with drift ratio corresponding to the response of buildings to ground motions compatible with the UHS for Vancouver; target ductility 3 and elastoplastic behaviour for LLRS $(\alpha=0.00)$. 66

Figure 3.4 - Variation of $\kappa$ with drift ratio corresponding to the response of buildings to ground motions compatible with the UHS for Vancouver; target ductility 4 and elastoplastic behaviour for LLRS $(\alpha=0.00)$. 66 
Figure 3.5 - Variation of $\kappa$ with drift ratio corresponding to the response of buildings to ground motions compatible with the UHS for Montreal; target ductility 2 and elastoplastic behaviour for LLRS $(\alpha=0.00)$

Figure 3.6 - Variation of $\kappa$ with drift ratio corresponding to the response of buildings to ground motions compatible with the UHS for Montreal; target ductility 3 and elastoplastic behaviour for LLRS $(\alpha=0.00)$ 67

Figure 3.7 - Variation of $\kappa$ with drift ratio corresponding to the response of buildings to ground motions compatible with the UHS for Montreal; target ductility 4 and elastoplastic behaviour for LLRS $(\alpha=0.00)$. 68

Figure 3.8 - Variation of $\kappa$ with drift ratio for target ductilities 2, 3 and 4 for buildings subjected to ground motions compatible with the UHS for Vancouver, elasto-plastic behaviour for LLRS ( $\alpha=0.00$ ) Humar and Popovski (2013) (with the authors' permission and (C) Canadian Science Publishing or its licensors)

Figure 3.9 - Variation of $\kappa$ with drift ratio for target ductilities 2, 3 and 4 for buildings subjected to ground motions compatible with the UHS for Montreal, elasto-plastic behaviour for LLRS $(\alpha=0.00)$ 70

Figure 3.10 - Variation of $\kappa$ with drift ratio for target ductilities 2, 3 and 4 for buildings subjected to ground motions compatible with the UHS for Vancouver and Montreal, elasto-plastic behaviour for LLRS $(\alpha=0.00)$ 71

Figure 3.11 - Comparison between Equation 3.8 and Equation 3.7 for target ductility of 2 when the buildings are subjected to ground motions compatible with the UHS for Montreal. 72 
Figure 3.12 - Comparison between Equation 3.8 and Equation 3.7 for target ductility of 3 when the buildings are subjected to ground motions compatible with the UHS for Montreal 73

Figure 3.13 - Comparison between Equation 3.8 and Equation 3.7 for target ductility of 2 when the buildings are subjected to ground motions compatible with the UHS for Montreal 73

Figure 3.14 - Comparison between Equation 3.8 and Equation 3.7 for target ductility of 2 when the buildings are subjected to ground motions compatible with the UHS for Vancouver 74

Figure 3.15 - Comparison between Equation 3.8 and Equation 3.7 for target ductility of 3 when the buildings are subjected to ground motions compatible with the UHS for Vancouver 74

Figure 3.16 - Comparison between Equation 3.8 and Equation 3.7 for target ductility of 4 when the buildings are subjected to ground motions compatible with the UHS for Vancouver 75

Figure 4.1 - Displacement time history for Building SH1 subjected to M6C38 ground motion parallel to the short side for $\alpha=0.00$. 81

Figure 4.2 - Displacement time history for Building SH1 subjected to M6C38 ground motion parallel to the short side for $\alpha=0.02$ 81

Figure 4.3 - Displacement time history for Building SH1 subjected to M6C38 ground motion parallel to the short side for $\alpha=0.05$ 82 Figure 4.4 - Displacement time history for Building SH1 subjected to M6C38 ground motion parallel to the short side for $\alpha=0.10$. 82 
Figure 4.5 - Displacement time history for Building SH1 subjected to M6C38 ground motion parallel to the long side for $\alpha=0.00$ 83

Figure 4.6 - Displacement time history for Building SH1 subjected to M6C38 ground motion parallel to the long side for $\alpha=0.02$ 83

Figure 4.7 - Displacement time history for Building SH1 subjected to M6C38 ground motion parallel to the long side for $\alpha=0.05$ 84 Figure 4.8 - Displacement time history for Building SH1 subjected to M6C38 ground motion parallel to the long side for $\alpha=0.10$ 84 Figure 4.9 - Displacement time history for Building MH1 subjected to M6C38 ground motion parallel to the short side for $\alpha=0.00$. 85 Figure 4.10 - Displacement time history for Building MH1 subjected to M6C38 ground

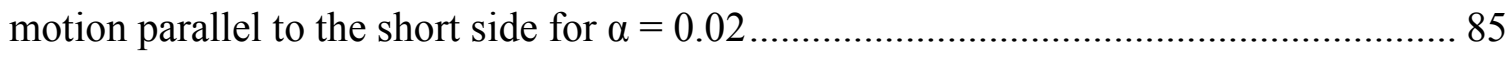

Figure 4.11 - Displacement time history for Building MH1 subjected to M6C38 ground motion parallel to the short side for $\alpha=0.05$ 86

Figure 4.12 - Displacement time history for Building MH1 subjected to M6C38 ground motion parallel to the short side for $\alpha=0.10$. 86 Figure 4.13 - Displacement time history for Building MH1 subjected to M6C38 ground motion parallel to the long side for $\alpha=0.00$ 87 Figure 4.14 - Displacement time history for Building MH1 subjected to M6C38 ground motion parallel to the long side for $\alpha=0.02$ 87 Figure 4.15 - Displacement time history for Building MH1 subjected to M6C38 ground motion parallel to the long side for $\alpha=0.05$ 88 
Figure 4.16 - Displacement time history for Building MH1 subjected to M6C38 ground

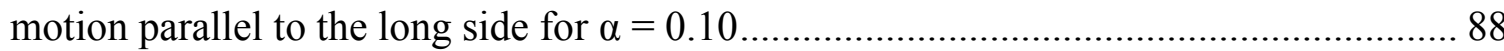

Figure 4.17 - Displacement time history for Building LH1 subjected to M6C38 ground motion parallel to the short side for $\alpha=0.00$. 89

Figure 4.18 - Displacement time history for Building LH1 subjected to M6C38 ground motion parallel to the short side for $\alpha=0.02$. 89 Figure 4.19 - Displacement time history for Building LH1 subjected to M6C38 ground motion parallel to the short side for $\alpha=0.05$. 90 Figure 4.20 - Displacement time history for Building LH1 subjected to M6C38 ground motion parallel to the short side for $\alpha=0.10$. 90 Figure 4.21 - Displacement time history for Building LH1 subjected to M6C38 ground motion parallel to the long side for $\alpha=0.00$ 91

Figure 4.22 - Displacement time history for Building LH1 subjected to M6C38 ground motion parallel to the long side for $\alpha=0.02$ 91 Figure 4.23 - Displacement time history for Building LH1 subjected to M6C38 ground motion parallel to the long side for $\alpha=0.05$ 92 Figure 4.24 - Displacement time history for Building LH1 subjected to M6C38 ground motion parallel to the long side for $\alpha=0.10$ 92 Figure 4.25 - Suggested equation for $\kappa$ and the obtained lower limits on $\kappa, \alpha=0.02$, West 95 Figure 4.26 - Suggested equation for $\kappa$ and the obtained lower limits on $\kappa, \alpha=0.05$, West 96 
Figure 4.27 - Suggested equation for $\kappa$ and the obtained lower limits on $\kappa, \alpha=0.10$, West 96

Figure 4.28 - Suggested equation for $\kappa$ and the obtained lower limits on $\kappa, \alpha=0.02$, East

Figure 4.29 - Suggested equation for $\kappa$ and the obtained lower limits on $\kappa, \alpha=0.05$, East

Figure 4.30 - Suggested equation for $\kappa$ and the obtained lower limits on $\kappa, \alpha=0.10$, East 98

Figure 5.1 - First seven modes of the beam-spring (diaphragm) model 105

Figure 5.2 - Bending moment distribution in building SL1 when subjected to M6C1 ground motion, $\mathrm{Ry}=3, \alpha=0.00$ - in both the flexible case (blue line) and the rigid case (red line). 114

Figure 5.3 - Bending moment distribution in building ML1 when subjected to M6C1 ground motion, $\mathrm{Ry}=3, \alpha=0.00$ - in both the flexible case (blue line) and the rigid case (red line) 115

Figure 5.4 - Bending moment distribution in building LH1 when subjected to M6C1 ground motion, Ry $=3, \alpha=0.00$ - in both the flexible case (blue line) and the rigid case (red line) 115

Figure 5.5 - Shear distribution in building SL1 when subjected to M6C1 ground motion, $\mathrm{Ry}=3, \alpha=0.00$ - in both the flexible case (blue line) and the rigid case (red line) ........ 116 Figure 5.6 - Shear distribution in building ML1 when subjected to M6C1 ground motion, $\mathrm{Ry}=3, \alpha=0.00$ - in both the flexible case (blue line) and the rigid case (red line) ........ 116 
Figure 5.7 - Shear distribution in building LH1 when subjected to M6C1 ground motion, $\mathrm{Ry}=3, \alpha=0.00$ - in both the flexible case (blue line) and the rigid case (red line) ........ 117

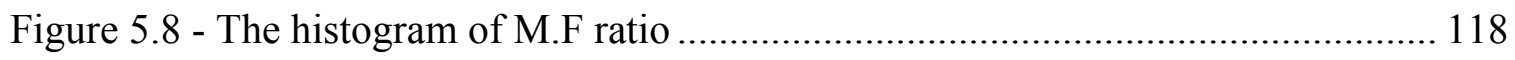

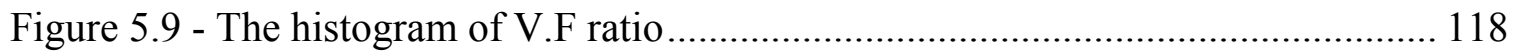

Figure 5.10 - The histogram of M.U ratio ............................................................. 119

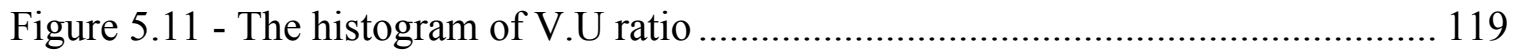

Figure 5.12 - Internal forces along the diaphragm decomposed into the first, third and fifth modes for building ML1 when subjected to M6C1 ground motion with no scale

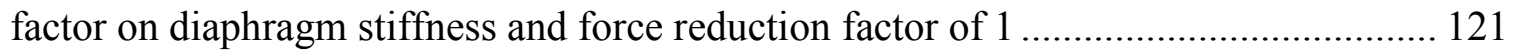

Figure 5.13 - Internal forces along the diaphragm decomposed into the first, third and fifth modes for building ML1 when subjected to M6C1 ground motion with no scale factor on diaphragm stiffness and force reduction factor of 2 122

Figure 5.14 - Internal forces along the diaphragm decomposed into the first, third and fifth modes for building ML1 when subjected to M6C1 ground motion with no scale factor on diaphragm stiffness and force reduction factor of 3 122

Figure 5.15 - Internal forces along the diaphragm decomposed into the first, third and fifth modes for building ML1 when subjected to M6C1 ground motion with the scale factor of 200 on diaphragm stiffness (rigid case) and force reduction factor of 1 123 Figure 5.16 - Internal forces along the diaphragm decomposed into the first, third and fifth modes for building ML1 when subjected to M6C1 ground motion with the scale factor of 200 on diaphragm stiffness (rigid case) and force reduction factor of $2 \ldots \ldots \ldots . .123$ 
Figure 5.17 - Internal forces along the diaphragm decomposed into the first, third and fifth modes for building ML1 when subjected to M6C1 ground motion with the scale factor of 200 on diaphragm stiffness (rigid case) and force reduction factor of 3 124

Figure 5.18 - M.F values mapped with respect to target ductility demands and the suggested equation for M.F for data corresponding to the western compatible spectrum and no post-yield stiffness 127 Figure 5.19 - V.F values mapped with respect to target ductility demands and the suggested equation for V.F for data corresponding to the western compatible spectrum and no post-yield stiffness 127

Figure 5.20 - M.U values mapped with respect to target ductility demands and the suggested equation for M.U for data corresponding to the western compatible spectrum and no post-yield stiffness 128 Figure 5.21 - V.U values mapped with respect to target ductility demands and the suggested equation for V.U for data corresponding to the western compatible spectrum and no post-yield stiffness 128 Figure 5.22 - M.F values mapped with respect to target ductility demands and the suggested equation for M.F for data corresponding to the eastern compatible spectrum and no post-yield stiffness 129 Figure 5.23 - V.F values mapped with respect to target ductility demands and the suggested equation for V.F for data corresponding to the eastern compatible spectrum and no post-yield stiffness 129 
Figure 5.24 - M.U values mapped with respect to target ductility demands and the suggested equation for M.U for data corresponding to the eastern compatible spectrum and no post-yield stiffness 130 Figure 5.25 - V.U values mapped with respect to target ductility demands and the suggested equation for V.U for data corresponding to the eastern compatible spectrum and no post-yield stiffness 130 Figure 5.26 - M.F values mapped with respect to target ductility demands and the suggested equation for M.F for data corresponding to the western compatible spectrum and post-yield stiffness of 0.02 131

Figure 5.27 - V.F values mapped with respect to target ductility demands and the suggested equation for V.F for data corresponding to the western compatible spectrum and post-yield stiffness of 0.02 131

Figure 5.28 - M.U values mapped with respect to target ductility demands and the suggested equation for M.U for data corresponding to the western compatible spectrum and post-yield stiffness of 0.02 132 Figure 5.29 - V.U values mapped with respect to target ductility demands and the suggested equation for V.U for data corresponding to the western compatible spectrum and post-yield stiffness of 0.02 132

Figure 5.30 - M.F values mapped with respect to target ductility demands and the suggested equation for M.F for data corresponding to the eastern compatible spectrum and post-yield stiffness of 0.02 133 
Figure 5.31 - V.F values mapped with respect to target ductility demands and the suggested equation for V.F for data corresponding to the eastern compatible spectrum and post-yield stiffness of 0.02 133

Figure 5.32 - M.U values mapped with respect to target ductility demands and the suggested equation for M.U for data corresponding to the eastern compatible spectrum and post-yield stiffness of 0.02 134

Figure 5.33 - V.U values mapped with respect to target ductility demands and the suggested equation for V.U for data corresponding to the eastern compatible spectrum and post-yield stiffness of 0.02 134

Figure 5.34 - M.F values mapped with respect to target ductility demands and the suggested equation for M.F for data corresponding to the western compatible spectrum and post-yield stiffness of 0.05 135

Figure 5.35 - V.F values mapped with respect to target ductility demands and the suggested equation for V.F for data corresponding to the western compatible spectrum and post-yield stiffness of 0.05 135

Figure 5.36 - M.U values mapped with respect to target ductility demands and the suggested equation for M.U for data corresponding to the western compatible spectrum and post-yield stiffness of 0.05 136

Figure 5.37 - V.U values mapped with respect to target ductility demands and the suggested equation for V.U for data corresponding to the western compatible spectrum and post-yield stiffness of 0.05 136 
Figure 5.38 - M.F values mapped with respect to target ductility demands and the suggested equation for M.F for data corresponding to the eastern compatible spectrum and post-yield stiffness of 0.05

Figure 5.39 - V.F values mapped with respect to target ductility demands and the suggested equation for V.F for data corresponding to the eastern compatible spectrum and post-yield stiffness of 0.05 137

Figure 5.40 - M.U values mapped with respect to target ductility demands and the suggested equation for M.U for data corresponding to the eastern compatible spectrum and post-yield stiffness of 0.05 138

Figure 5.41 - V.U values mapped with respect to target ductility demands and the suggested equation for V.U for data corresponding to the eastern compatible spectrum and post-yield stiffness of 0.05 138

Figure 5.42 - M.F values mapped with respect to target ductility demands and the suggested equation for M.F for data corresponding to the western compatible spectrum and post-yield stiffness of 0.10 139 Figure 5.43 - V.F values mapped with respect to target ductility demands and the suggested equation for V.F for data corresponding to the western compatible spectrum and post-yield stiffness of 0.10 139

Figure 5.44 - M.U values mapped with respect to target ductility demands and the suggested equation for M.U for data corresponding to the western compatible spectrum and post-yield stiffness of 0.10 140 
Figure 5.45 - V.U values mapped with respect to target ductility demands and the suggested equation for V.U for data corresponding to the western compatible spectrum and post-yield stiffness of 0.10 140

Figure 5.46 - M.F values mapped with respect to target ductility demands and the suggested equation for M.F for data corresponding to the eastern compatible spectrum and post-yield stiffness of 0.10 141

Figure 5.47 - V.F values mapped with respect to target ductility demands and the suggested equation for V.F for data corresponding to the eastern compatible spectrum and post-yield stiffness of 0.10 141

Figure 5.48 - M.U values mapped with respect to target ductility demands and the suggested equation for M.U for data corresponding to the eastern compatible spectrum and post-yield stiffness of 0.10 142

Figure 5.49 - V.U values mapped with respect to target ductility demands and the suggested equation for V.U for data corresponding to the eastern compatible spectrum and post-yield stiffness of 0.10 142

Figure 5.50 - M.F values mapped with respect to target ductility demands and the suggested equation for M.F for data corresponding to both spectra and post-yield stiffness of 0.00 147

Figure 5.51 - V.F values mapped with respect to target ductility demands and the suggested equation for V.F for data corresponding to both spectra and post-yield stiffness of 0.00 147 
Figure 5.52 - M.U values mapped with respect to target ductility demands and the suggested equation for M.U for data corresponding to both spectra and post-yield stiffness of 0.00 148

Figure 5.53 - V.U values mapped with respect to target ductility demands and the suggested equation for V.U for data corresponding to both spectra and post-yield stiffness of 0.00 148

Figure 5.54 - M.F values mapped with respect to target ductility demands and the suggested equation for M.F for data corresponding to both spectra and post-yield stiffness of 0.02 149

Figure 5.55 - V.F values mapped with respect to target ductility demands and the suggested equation for V.F for data corresponding to both spectra and post-yield stiffness of 0.02 149

Figure 5.56 - M.U values mapped with respect to target ductility demands and the suggested equation for M.U for data corresponding to both spectra and post-yield stiffness of 0.02 . 150

Figure 5.57 - V.U values mapped with respect to target ductility demands and the suggested equation for V.U for data corresponding to both spectra and post-yield stiffness of 0.02 150

Figure 5.58 - M.F values mapped with respect to target ductility demands and the suggested equation for M.F for data corresponding to both spectra and post-yield stiffness of 0.05 151 
Figure 5.59 - V.F values mapped with respect to target ductility demands and the suggested equation for V.F for data corresponding to both spectra and post-yield stiffness of 0.05 151

Figure 5.60 - M.U values mapped with respect to target ductility demands and the suggested equation for M.U for data corresponding to both spectra and post-yield stiffness of 0.05 152

Figure 5.61 - V.U values mapped with respect to target ductility demands and the suggested equation for V.U for data corresponding to both spectra and post-yield stiffness of 0.05 152

Figure 5.62 - M.F values mapped with respect to target ductility demands and the suggested equation for M.F for data corresponding to both spectra and post-yield stiffness of 0.10 153

Figure 5.63 - V.F values mapped with respect to target ductility demands and the suggested equation for V.F for data corresponding to both spectra and post-yield stiffness of 0.10 153

Figure 5.64 - M.U values mapped with respect to target ductility demands and the suggested equation for M.U for data corresponding to both spectra and post-yield stiffness of 0.10 154

Figure 5.65 - V.U values mapped with respect to target ductility demands and the suggested equation for V.U for data corresponding to both spectra and post-yield stiffness of 0.10 154

Figure 5.66 - The final design expression for M.F magnification factor vs. the original M.F equation 159 
Figure 5.67 - The final design expression for V.F magnification factor vs. the original V.F equation. 159

Figure 6.1 - Schematic illustration of the analytical model with 7 nodes 171

Figure 6.2 - The hysteretic response of concentric braces subjected to lateral loads, from Tremblay et al. (2003) with the authors' permission and, with permission from ASCE 173 Figure 6.3 - The hysteretic response of a single element modelled in OpenSees with Clough model. 175

Figure 6.4 - Hysteretic response of an element with Clough hysteretic behaviour and pinching factors PinchX and PinchY equal to 0.8 and 0.2 , respectively...... 176

Figure 6.5 - Comparison between Clough model with pinching and Clough model without pinching 177

Figure 6.6 - Schematic illustration of the actual behaviour and the behaviour in the model before stiffening of the tensile brace 179

Figure 6.7 - Pinching hysteretic behaviour of LLRS with high slenderness 183

Figure 6.8 - Pinching hysteretic behaviour of LLRS with intermediate slenderness ..... 183 Figure 6.9 - Comparison between the maximum ductility demands on the LLRS considering different material behaviour 186

Figure 6.10 - Comparison between mid-span bending moment magnification ratios with respect to FEMA parabolic distribution of internal forces for different models to represent the hysteretic behaviour of the LLRS 189 Figure 6.11 - Comparison between mid-span bending moment magnification ratios with respect to uniform distribution of internal forces for different models to represent the hysteretic behaviour of the LLRS 189 
Figure 6.12 - Comparison between quarter span shear force magnification ratios with respect to FEMA parabolic distribution of internal forces for different models to represent the hysteretic behaviour of the LLRS 190

Figure 6.13 - Comparison between quarter span shear force magnification ratios with respect to uniform distribution of internal forces for different models to represent the hysteretic behaviour of the LLRS 190

Figure 6.14 - Comparison of the hysteretic responses of the LLRS when represented by 3 different models - Building LH1 subjected to ground motion M6C2 parallel to the short side 192

Figure 6.15 - Comparison of the hysteretic responses of the LLRS when represented by 3 different models - Building LH1 subjected to ground motion M6C1 parallel to the short side 192

Figure 6.16 - Comparison of the hysteretic responses of the LLRS when represented by 3 different models - Building LH1 subjected to ground motion M6C2 parallel to the long side 193

Figure 7.1 - Different connection types in steel deck diaphragms (Taken from Rogers and Tremblay 2003 with the authors' permission and, with permission from ASCE) 205 Figure 7.2 - Load deformation curves for different deck to deck and deck to frame fastener types, (a) welded deck to deck and deck to frame fastener - (b) welded deck to deck and deck to frame fasteners (c) screwed deck to deck and welded deck to frame fasteners - (d) screwed deck to deck and weld with washers deck to frame fasteners (taken from Essa et al. 2003 with the authors' permission and, with permission from ASCE) 209 
Figure 7.3 - Experimental force - deformation hysteretic curve in shear for the specimen with screwed deck to deck and welded deck to frame fasteners (taken from Essa et al. 2003 with the authors' permission and, with permission from ASCE) 215

Figure 7.4 - Backbone curve for the modified IMK deterioration model with peakoriented hysteretic response (taken from Lignos and Krawinkler 2011 with the authors' permission and, with permission from ASCE) 217 Figure 7.5 - Illustration of the modified IMK deterioration model with peak-oriented response (taken from OpenSees command manual) (Lignos and Krawinkler 2012 with the authors' permission) 218 Figure 7.6 - Illustration of the modified IMK deterioration model with pinched response (taken from OpenSees command manual) (Lignos and Krawinkler 2012 with the authors' permission) 219

Figure 7.7 - The calibration of the analytical model based on the experimental results on the diaphragm specimen with screwed and weld sidelap and deck to frame fasteners (Essa et al 2003) - (a) The response of the calibrated element under displacement controlled analysis (b) The experimental hysteretic curve (Essa et al 2003 with permission from ASCE) (c) The superimposed curves for assessing the calibration effectiveness .......... 223

Figure 7.8 - Schematic illustration of the analytical model with 7 nodes....................... 225 Figure 7.9 - Hysteretic shear behaviour in building SH1 subjected to M6C2 - (a) $\mu=2.0$, (b) $\mu=1.5$, and (c) $R_{d}=1.5$ 228

Figure 7.10 - Final shear hysteretic behaviour of the steel deck diaphragms 229

Figure 7.11 - The frequency of occurrence for the ductility demand on the diaphragm 232 Figure 7.12 - M.F magnification factors vs. the ductility demand in the diaphragm ..... 233 
Figure 7.13 - V.F magnification factors vs. the ductility demand in the diaphragm ...... 234 Figure 7.14 - M.U magnification factors vs. the ductility demand in the diaphragm..... 234 Figure 7.15 - V.U magnification factors vs. the ductility demand in the diaphragm ..... 235 Figure 7.16 - Force reduction factors vs. drift ratio values for target ductility of $1.5 \ldots 237$ Figure 7.17 - Force reduction factors vs. drift ratio values for target ductility of $2.0 \ldots .237$ Figure 7.18 - Force reduction factor data vs. the drift ratio and suggested equations .... 238 Figure 7.19 - The brace displacement time history in building SH1 subjected to M6C2 with the brace as the energy dissipating member and the target ductility of $3.0 \ldots \ldots \ldots . .240$ Figure 7.20 - Displacement time-histories at different locations along the length of the diaphragm in building SH1 with brace as the energy dissipating member and the target ductility of 3.0 240

Figure 7.21 - The brace displacement time history in building SH1 subjected to M6C2 with the diaphragm as the energy dissipating member and the target ductility of $1.5 \ldots 241$ Figure 7.22 - Displacement time-histories at different locations along the length of the diaphragm in building SH1 with the diaphragm as the energy dissipating member and the target ductility of 1.5 241

Figure 7.23 - Design force factor for elastic behaviour $\left(\Phi_{e}\right)$ vs. drift ratio for ductility of 1.0 246

Figure 7.24 - Design force factor for elastic behaviour $\left(\Phi_{e}\right)$ vs. drift ratio for ductility of 2.0 246

Figure 7.25 - Design force factor for elastic behaviour $\left(\Phi_{e}\right)$ vs. drift ratio for ductility of 3.0 247 
Figure 7.26 - Design force factor for elastic behaviour $\left(\Phi_{e}\right)$ vs. drift ratio for ductility of 4.0 . 247

Figure 7.27 - Design force factor for elastic behaviour $\left(\Phi_{d}\right)$ vs. drift ratio for ductility of 1.0 248

Figure 7.28 - Design force factor for elastic behaviour $\left(\Phi_{d}\right)$ vs. drift ratio for ductility of 2.0 248

Figure 7.29 - Design force factor for elastic behaviour $\left(\Phi_{d}\right)$ vs. drift ratio for ductility of 3.0 249

Figure 7.30 - Design force factor for elastic behaviour $\left(\Phi_{d}\right)$ vs. drift ratio for ductility of 4.0. 249

Figure $7.31-\Phi_{e}$ factor vs. the ductility demand on the brace when the diaphragm remains elastic and the suggested empirical equation 252

Figure $7.32-\Phi_{d}$ factor vs. the ductility demand on the brace when the ductility demand in the diaphragm is limited to 1.5 and the suggested empirical equation 253

Figure 7.33 - Internal force magnification M.F vs. the ductility demand on the diaphragm with the suggested equation $(\mu=2.0)$....... 254 Figure 7.34 - Internal force magnification V.F vs. the ductility demand on the diaphragm with the suggested equation $(\mu=2.0)$...... 255 Figure 7.35 - Internal force magnification M.U vs. the ductility demand on the diaphragm with the suggested equation $(\mu=2.0)$ 255 Figure 7.36 - Internal force magnification V.U vs. the ductility demand on the diaphragm with the suggested equation $(\mu=2.0)$. 256 
Figure 7.37 - Internal force magnification M.F vs. the ductility demand on the diaphragm

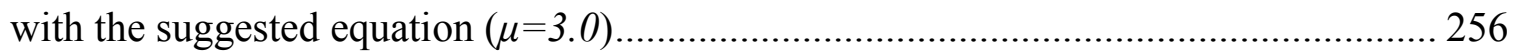

Figure 7.38 - Internal force magnification V.F vs. the ductility demand on the diaphragm

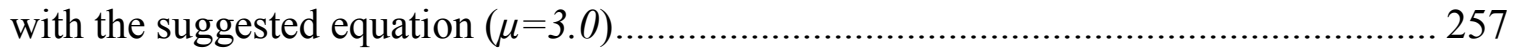
Figure 7.39 - Internal force magnification M.U vs. the ductility demand on the diaphragm

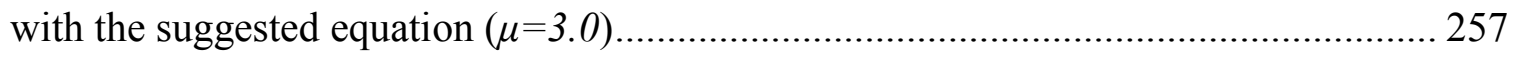
Figure 7.40 - Internal force magnification V.U vs. the ductility demand on the diaphragm

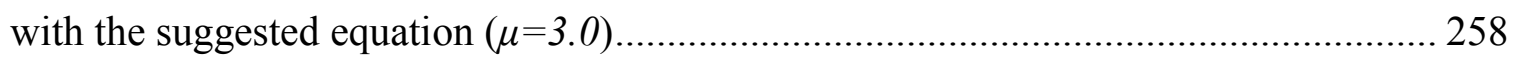
Figure 7.41 - Internal force magnification M.F vs. the ductility demand on the diaphragm

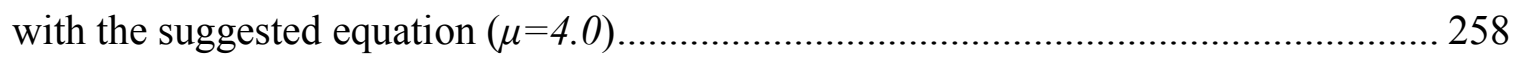
Figure 7.42 - Internal force magnification V.F vs. the ductility demand on the diaphragm with the suggested equation $(\mu=4.0)$ 259

Figure 7.43 - Internal force magnification M.U vs. the ductility demand on the diaphragm with the suggested equation $(\mu=4.0)$ 259

Figure 7.44 - Internal force magnification V.U vs. the ductility demand on the diaphragm with the suggested equation $(\mu=4.0)$....... 260

Figure 7.45 - The reduction in the ductility demand on the brace by allowing limited nonlinearity $\left(\mu_{\mathrm{d}} \leq 1.5\right)$ in the roof diaphragm, target ductility of 2.00 262 Figure 7.46 - The reduction in the ductility demand on the brace by allowing limited nonlinearity $\left(\mu_{\mathrm{d}} \leq 1.5\right)$ in the roof diaphragm, target ductility of 3.00 262 Figure 7.47 - The reduction in the ductility demand on the brace by allowing limited nonlinearity $\left(\mu_{\mathrm{d}} \leq 1.5\right)$ in the roof diaphragm, target ductility of 4.00 263 


\section{List of Symbols}

a The acceleration ratio, ratio of the peak horizontal acceleration to the gravity acceleration

$a^{*} \quad$ The length of the diaphragm over which stronger and stiffer panels and connections are used

A Brace cross sectional area

$A_{e} \quad$ Effective shear area of the diaphragm

$A_{\text {pinch }}$ The ratio of the reloading stiffness to the previous loading or reloading stiffness in the definition of the modified IMK deterioration model with pinched hysteretic response

$B \quad$ Width of the diaphragm (the dimension of the building parallel to the direction of the force)

$c \quad$ empirical parameter in the definition of the modified IMK deterioration model with peak-oriented hysteretic response, usually taken as 1.0

$C_{r} \quad$ The compressive capacity of the brace dictated usually by buckling

$\mathrm{D}_{\mathrm{s}} \quad$ The length of the bracing bents

E Modulus of elasticity

EI Flexural rigidity of the diaphragm 
$E_{i} \quad$ hysteretic energy dissipation in excursion $i$ in the definition of the modified IMK deterioration model with peak-oriented hysteretic response

$E_{t} \quad$ The reference hysteretic energy capacity in the definition of the modified IMK deterioration model with peak-oriented hysteretic response

$f_{d} \quad$ The inertia load intensity at a distance $\mathrm{x}$ from the centre line of the diaphragm (FEMA parabolic distribution)

$f(t) \quad$ External force expressed as function of time

$\mathrm{F}_{d} \quad$ The total inertia force on the diaphragm

$F_{d} \quad$ Diaphragm design force

$F_{e} \quad$ Force associated with buckling (Euler buckling load)

$F_{y} \quad$ Yield strength of the brace material

$F_{p}{ }^{+} \quad$ The ratio of the force at which reloading begins to the force corresponding to the maximum historic deformation demand at positive values in the definition of the modified IMK deterioration model with pinched hysteretic response

$F_{p}^{-} \quad$ The ratio of the force at which reloading begins to the force corresponding to the maximum historic deformation demand at negative values in the definition of the modified IMK deterioration model with pinched hysteretic response

F Vector representing the spatial distribution of the seismic load

$g \quad$ Shear deformation parameter 
$G \quad$ The shear modulus of the material used for the diaphragm

G' The effective shear stiffness of the diaphragm

I Cross-sectional moment of inertia

k Stiffness matrix of an individual beam element

$K \quad$ Effective length factor

$K_{b} \quad$ Stiffness of the springs at two ends representing the braces

$K_{D} \quad$ Stiffness of the springs representing the diaphragm

K The condensed stiffness matrix removing the rotational degrees of freedom

$\mathbf{K}_{\boldsymbol{A} \boldsymbol{A}}$ Portion of the global stiffness matrix associated with translational degrees of freedom

$\mathbf{K}_{A B}$ Portion of the global stiffness matrix associated with the effect of rotational degrees of freedom on the translational degrees of freedom

$\mathbf{K}_{\boldsymbol{B A}}$ Portion of the global stiffness matrix associated with the effect of translational degrees of freedom on the rotational degrees of freedom

$\mathbf{K}_{B B}$ Portion of the global stiffness matrix associated with rotational degrees of freedom

$\mathbf{K}_{\mathrm{s}} \quad$ The global stiffness matrix obtained by assembling the stiffness matrices of individual beam elements and the two springs at ends 
$l \quad$ The length of the inter-connected beam elements in the deep beam representation of diaphragm

$L \quad$ Length of the deep beam model representing the diaphragm; the dimension of the building perpendicular to the direction of the seismic force

$L_{d} \quad$ The unsupported length (span) of the diaphragm

$m \quad$ The mass per unit length of the diaphragm

M The total mass assigned to the level of the diaphragm

$M_{c} \quad$ The capping strength in the definition of the modified IMK deterioration model with peak-oriented hysteretic response

$M_{d} \quad$ The bending moment for which beam to column connections must be designed

$M_{n} \quad$ The mass associated with the nth mode

$M_{p} \quad$ The beam plastic moment capacity

$M_{r} \quad$ The residual moment in the definition of the modified IMK deterioration model with peak-oriented hysteretic response

$M_{y} \quad$ The effective yield moment in the definition of the modified IMK deterioration model with peak-oriented hysteretic response

M The mass matrix of the system

$\boldsymbol{M}_{\boldsymbol{b}} \quad$ The moment capacity of the beams

$\boldsymbol{M}_{\boldsymbol{c}} \quad$ The moment capacity of the columns 
$\mathbf{M}_{A A} \quad$ The lumped mass matrix of the analytical model

M.F The ratio of actual mid span moment to the mid span moment calculated for the case in which the total seismic load is distributed according the FEMA 356 parabolic distribution

M.U The ratio of actual mid span moment to the mid span moment calculated for the case in which the total seismic load is distributed uniformly

$n \quad$ parameter used in the computation of $C_{r} ; 1.34$ for HSS

$N \quad$ Number of elements in the deep beam

PinchY Pinch factor for the vertical axis

PinchX Pinch factor for the horizontal axis

P.F The participation factor for mode $n$, which is a measure of the level of the contribution from mode $n$ to the total response of the system

PHA Peak horizontal acceleration

PHV Peak horizontal velocity

$\boldsymbol{r} \quad$ Drift ratio in the one-storey building defined as the ratio of the maximum deformation along the length of the diaphragm to the average storey drift of the lateral load resisting system

$r \quad$ radius of gyration 
r The vector of rigid body displacements produced by a unit displacement at the supports.

$R_{d} \quad$ Ductility-related force reduction factor for the design of the diaphragm

$\mathrm{R}_{\mathrm{o}} \quad$ Overstrength-related force reduction factor

$R \quad$ A factor for the seismic design of beam to column connections

$R_{s h} \quad$ Over strength owing to strain hardening in the material

$R_{\text {size }} \quad$ The over strength caused by restrictions in the selection of members and rounding up of the sizes and dimensions in the design

$R_{\text {mech }} \quad$ The over strength resulting from mobilization of the full capacity of the structure until a collapse mechanism is formed.

$R_{y} \quad$ Ductility related force reduction factor for the seismic design of the LLRS (braces)

$R_{\text {yield }} \quad$ The ratio of the actual yield strength to the minimum specified yield strength

$R \mu \quad$ The reduction in the ductility demand on the brace by allowing the diaphragm to behave nonlinearly

$R_{\phi} \quad$ A factor accounting for the difference between the nominal resistance and the factored resistance.

$S(T) \quad$ The spectral acceleration for the period $T$

$T \quad$ Fundamental period of the beam-spring model 
$\operatorname{Tr} \quad$ The tensile capacity of the brace in tension

$u_{d} \quad$ Diaphragm deformation at mid-span under a uniformly distributed lateral load

$u_{b} \quad$ Brace displacement

u Vector of displacements along the translational degrees of freedom

$\ddot{\mathbf{u}}_{\mathrm{g}} \quad$ Vector of ground accelerations

$v \quad$ The velocity ratio, peak horizontal velocity divided by $1 \mathrm{~m} / \mathrm{s}$

$V_{e} \quad$ Elastic base shear based on the uniform hazard spectrum for the site

$V_{f} \quad$ Base shear induced on one-storey buildings

$V_{y} \quad$ The yield base shear

$V_{w e} \quad$ Maximum force in the walls based on a linear analysis

$V_{w e} \quad$ Yield force of the walls

V.F The ratio of actual quarter span shear to the quarter span shear calculated for the case in which the total seismic load is distributed according the FEMA 356 parabolic distribution

V.U The ratio of actual quarter span shear to the quarter span shear calculated for the case in which the total seismic load is distributed uniformly

$w \quad$ Uniformly distributed lateral load applied along the length of the diaphragm

W Total seismic weight 
$Z_{a} \quad$ Zone number corresponding to the acceleration ratio

$Z_{v} \quad$ Zone number corresponding to the velocity ratio

$y_{n} \quad$ Displacement component in the nth mode of vibration

$\dot{y}_{n} \quad$ Velocity component in the nth mode of vibration

$\ddot{y_{n}} \quad$ Acceleration component in the nth mode of vibration

$\alpha \quad$ Post-yield stiffness ratio for the springs representing the braces

$\alpha \quad$ A term accounting for the stiffness of the LLRS when the stiffness of the LLRS is expressed as $K_{B}=\beta E I / L^{3}$

$\beta \quad$ A parameter that defines the stiffness of the springs representing the braces as a factor of $E I / L^{3}$

$\beta_{i} \quad$ The energy-based deterioration parameter in the definition of the modified IMK deterioration model with peak-oriented hysteretic response

$\beta_{c} \quad$ The ratio of the capacity of the column to the capacity of the beams

$\gamma_{w} \quad$ Nominal over-strength ratio

$\delta_{r} \quad$ Maximum roof displacement determined from linear analysis

$\delta_{w} \quad$ Maximum wall displacement determined from linear analysis

$\delta_{d}(T)$ Spectral displacement based on the period of the system

$\Delta_{e} \quad$ Total elastic displacement of the combined spring-beam system 
$\Delta_{i} \quad$ Total displacement of the combined spring-beam system with nonlinear springs

$\Delta_{B} \quad$ Average storey drift of the lateral load resisting system

$\Delta_{D} \quad$ Maximum deformation along the length of the diaphragm

$\Delta_{w} \quad$ Total inelastic drift of the wall

$\Delta_{w i} \quad$ Inelastic displacement of the wall

$\Delta_{w y} \quad$ Yield displacement of the wall

$\theta_{p} \quad$ The pre capping plastic deformation for monotonic loading, in other words the difference between the yield deformation and deformation at maximum moment, in the definition of the modified IMK deterioration model with peak-oriented hysteretic response

$\theta_{p c} \quad$ The post-capping plastic deformation (difference between deformation at maximum moment and deformation at complete loss of strength) in the definition of the modified IMK deterioration model with peak-oriented hysteretic response

$\theta_{u} \quad$ The ultimate deformation capacity in the definition of the modified IMK deterioration model with peak-oriented hysteretic response

$\theta_{y} \quad$ The yield deformation in the definition of the modified IMK deterioration model with peak-oriented hysteretic response

$\kappa \quad$ The ratio of the force reduction factor for the brace to the maximum ductility demand on the brace 
$\lambda \quad$ A parameter $=\sqrt{F_{y} / F_{e}}$ defined for determining the compressive resistance of members

$\Lambda$ The reference cumulative rotation capacity in the definition of the modified IMK deterioration model with peak-oriented hysteretic response

$\mu \quad$ Ductility demand on the lateral load resisting system

$\mu_{d} \quad$ The ductility demand on the diaphragm steel panels

$\zeta_{n} \quad$ Damping ratio in mode $\mathrm{n}$

$\Sigma E_{j} \quad$ cumulative energy dissipation in previous excursions in the definition of the modified IMK deterioration model with peak-oriented hysteretic response

$\Phi \quad$ Resistance factor for steel

$\Phi_{e} \quad$ Diaphragm design force factor to ensure elastic behaviour in the diaphragm, defined as the ratio of the diaphragm yield force to the brace yield force

$\Phi_{d} \quad$ Diaphragm design force factor to ensure that the ductility demand on the diaphragm would be limited to 1.5 , defined as the ratio of the diaphragm yield force to the brace yield force

$\Phi_{n}^{T} \quad n$th mode shape

$\omega \quad$ Fundamental frequency of the beam-spring model as a combined structural system

$\omega_{b} \quad$ Frequency of the springs supporting a rigid diaphragm 
$\omega_{d l} \quad$ First mode frequency of the diaphragm resting on rigid supports

$\omega_{d n} \quad$ Nth mode frequency of the diaphragm resting on rigid supports

$\omega_{n} \quad$ Frequency of the nth mode 


\section{List of Appendices}

Appendix A.............................................................284

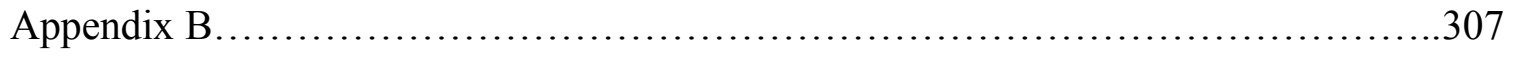

Appendix C................................................................. 372

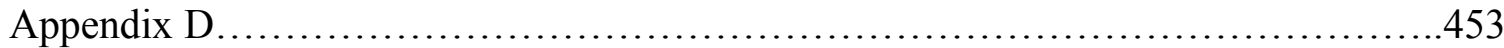

Appendix E............................................................465

Appendix F.......................................................... 483 


\section{Chapter 1 : Introduction}

\subsection{Diaphragm as a Structural Member}

The roofing system, also referred to as a diaphragm, is an important component of the structure of a building. The diaphragm resists the gravity loads imposed on the building through its stiffness in the direction perpendicular to the roof plane. The other function of the diaphragm is the distribution of lateral forces among the elements of the lateral load resisting system (LLRS) of the building. Extreme events such as wind, earthquake, and blast can give rise to the imposition of significant lateral loads on the LLRS. For the distribution of such lateral loads the roofing system relies on its in-plane stiffness. Thus the in-plane stiffness of the roof diaphragm relative to the stiffness of the LLRS greatly influences the response of the structure to the lateral loads.

\subsection{Classification of Diaphragms}

In multi-storey buildings, where the gravity loads are fairly large, the diaphragm usually consists of a reinforced concrete slab or a steel deck with structural concrete topping. The in-plane stiffness of such a diaphragm is quite large. It, therefore, acts as a rigid body in its plane and is classified as a rigid diaphragm. On the other hand, in low-rise buildings, particularly in one-storey buildings with large footprints, the gravity load on the roof is limited. In such cases, the roofing system normally consists of un-topped steel deck panels or wood structural panels or other structural systems with low in-plane stiffness. In such cases the behaviour of the diaphragm is no longer similar to that of a rigid body; consequently the diaphragm is categorized as a flexible diaphragm. Several codes and 
guidelines also recognize a third group of diaphragms that are referred to as stiff diaphragms. Such diaphragms are neither rigid, nor flexible and their behaviour is somewhere in between the behaviour of rigid and flexible diaphragms.

For choosing an appropriate method for the design of a diaphragm, one must first classify the diaphragm into one of the three mentioned categories. Obviously, the classification of the diaphragm primarily depends on the in-plane stiffness of the diaphragm relative to that of the lateral load resisting system that transfers the lateral loads to the foundation. Design guidelines and codes have recommended several methods, both prescriptive and calculation based, to assist in such classifications. Some of these methods are outlined in the following paragraphs.

\subsubsection{FEMA 356 Methods}

According to the provisions of FEMA 356 of The Federal Emergency Management Agency of United States (FEMA 2000), a diaphragm should be classified as flexible when the maximum horizontal deformation of the diaphragm along its length produced by a uniformly distributed static lateral load is more than twice the average inter-storey drift of the lateral load resisting system elements of the storey that is immediately below the diaphragm. FEMA 356 further recommends that a diaphragm may be considered as rigid if the maximum diaphragm deformation along its length is equal to or less than half the average inter-storey drifts of the storey below the diaphragm. Diaphragms, which fall into neither of the categories, are classified as stiff diaphragms, and the design of such diaphragms should be based on a method that takes into account both the in-plane stiffness of the diaphragm and the stiffness of the lateral load resisting system. 


\subsubsection{ASCE 7-05 Methods}

The Design Load Standard of the American Society of Civil Engineering ASCE 7-05 (ASCE 2005) recommends both prescriptive and calculation based methods for the classification of the diaphragms.

Diaphragm systems that consist of un-topped steel decking or wood structural panels can be idealized and designed as flexible diaphragms when the vertical lateral load-resisting elements are steel or composite steel and concrete braced frames, or concrete, masonry, steel or composite shear walls. In such situations, the diaphragm easily meets the flexibility criteria of FEMA 356, since the diaphragm is almost always more flexible than the lateral load resisting system. Furthermore, the ASCE standard permits diaphragms consisting of wood structural panels or un-topped steel deck in one- and two-storey family residential buildings of light-frame construction to be treated as flexible diaphragms. One can reason that such diaphragms may not always meet the deflection criteria of FEMA 356. However, the ASCE recommendation is supported by some recent studies which showed that for regular light-framed wood diaphragm buildings a force distribution based on flexible diaphragm assumption leads to better performance. The ASCE 7-05 standard also states that diaphragms consisting of either concrete slabs or metal decks with concrete fillings that have span-to-depth ratios equal or less than 3 can be idealized as rigid provided that the structure has no irregularities in its horizontal plane.

As an alternative to the prescriptive methods outline in the preceding paragraph, or when the structure does not satisfy the conditions associated with the prescriptive method, a 
computational method may be used for the classification of the diaphragms. According to this method, if the maximum in-plane deflection of the diaphragm under lateral loads is more than twice the average story drift of the vertical elements of the seismic forceresisting system (SFRS) in the story below the diaphragm, the diaphragm can be idealized as flexible.

\subsubsection{IBC 2006 Methods}

The 2006 International Building Code (ICC 2006) has generally adopted the same provisions as ASCE 7-05 with some minor modifications to the prescriptive requirements regarding buildings of light-frame construction. Based on IBC 2006 provisions, any diaphragm that is not flexible may be treated as rigid. On the other hand, both ASCE 7-05 and FEMA 356 have characterized rigid diaphragms quite specifically and these characteristics must be satisfied by a diaphragm in order to be classified as rigid. FEMA and ASCE 7-05 further specify that when the requirements related to flexible conditions as well as those related to rigid conditions are not met by a diaphragm, the diaphragm should be classified as stiff and its stiffness considered in the analysis.

\subsection{The Influence of Diaphragm Flexibility on the Response of the Structure}

A large number of studies have been carried out on buildings with flexible diaphragms, particularly on one-storey buildings with flexible diaphragms. Usually, in one-storey buildings, the roof system consists of un-topped steel deck or structural wood panels or other roofing systems with low in-plane stiffness. The lateral load resisting system (LLRS) can consist of concentric steel braces, eccentric steel braces, reinforced shear walls, masonry shear walls or steel plate shear walls. The in-plane stiffness of the 
diaphragm is thus quite small relative to that of the lateral load resisting system. Hence, the diaphragm in one-storey buildings with such structural systems usually belongs to the category of a flexible diaphragm. Figure 1.1 illustrates a one-storey building with flexible diaphragm.

Different combinations of structural systems (i.e. steel deck as the diaphragm with concentric braces as LLRS, steel deck as the diaphragm supported by masonry shear walls as the LLRS and so on) have been the subject of previous studies and they have all come to the same conclusion. The results of all such studies suggest that the flexibility of the diaphragm alters the response of the building to lateral loads, especially that produced

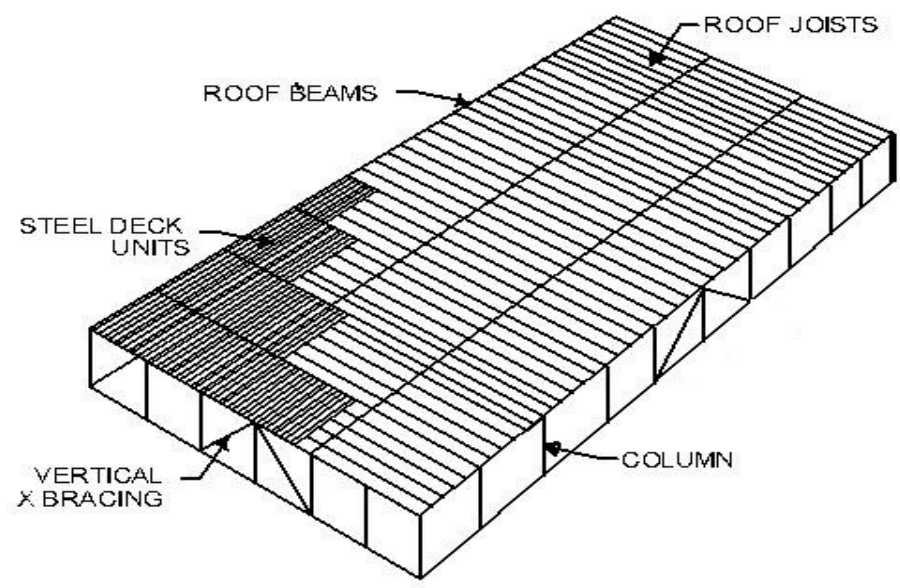

Figure 1.1- One-storey building with flexible diaphragm 
by seismic ground motions. Diaphragm flexibility also changes the dynamic characteristics of the system. Some of the more important influences of diaphragm flexibility identified in the previous studies are as follows:

- The dynamic characteristic of a building with flexible diaphragm, and consequently its response to seismic ground motion, is different from that of a building with rigid diaphragm.

- The flexibility of the diaphragm lengthens the period of the building.

- The flexibility also changes the manner in which the inertia forces are distributed along the length of the diaphragm.

- The flexibility of the diaphragm, gives rise to a significant increase in the ductility demand on the lateral load resisting elements.

- The variation in the distribution of inertia forces, leads to a change in the distribution of shear forces and bending moments in the diaphragm. The mid-span bending moment and the quarter-span shear force both increase significantly.

The effects of diaphragm flexibility on the response of the system, as reported in the previous studies, are discussed in more detail in the following section.

\subsection{Literature Review}

The diaphragm flexibility and its effects on the response of the structure to seismic ground motions has been the subject of many studies. A selected number of previous research papers, particularly those with emphasis on the response of one-storey buildings with flexible diaphragms consisting of steel decks, are briefly reviewed in the following 
paragraphs. In addition, some papers regarding the nonlinear cyclic behaviour of concentric bracing members and the cyclic response of steel deck diaphragm are also reviewed. The referenced papers provide a good understanding of the behaviour of the structural system as well as that of its components.

\subsubsection{Research on Systems with Flexible Diaphragms}

In their paper, Jain and Jennings (1985) presented an analytical method for the dynamic analysis of one- and two-storey buildings with flexible diaphragms. In this method, the floor and roof diaphragms were modeled as flexural beams and the end walls were modeled as shear beams. The authors provided an example to illustrate the use of their analytical method. In that example, the Arvin high school administration building in California was analyzed. The authors pointed out that while the suggested method could be suitable for low-rise buildings it might be less effective for multi-storey buildings.

Button et al. (1984) carried out a study to investigate the influence of diaphragm flexibility on the seismic response of buildings. They presented a technique for modelling the flexibility of the diaphragm. They pointed out that just as the in-plane displacement of a rigid diaphragm can be expressed by three degrees of freedom at one master node, the displacement of a flexible diaphragm can be described by three degrees of freedom at each of a series of master nodes which are linked by floor beams representing the diaphragm stiffness. The authors studied three hypothetical structures, both with and without diaphragm flexibility, using their suggested method. The results suggested that the flexibility of the diaphragm could have significant effect on the response of buildings with a number of different forms that are widely used these days. The authors showed 
that neglecting the floor flexibility could lead to results that were potentially in error on the non-conservative side.

In a study carried out by Kunnath et al. (1991), the seismic response of RC buildings with inelastic floor diaphragms was examined. The authors showed that while in most simple buildings the rigid floor assumption could be useful; for certain types of buildings, such as long and narrow buildings and buildings with horizontal and vertical offsets, the flexibility of the diaphragm could not be disregarded. In their research, they studied the seismic response of a set of narrow rectangular buildings with end walls when subjected to El Centro 1940 earthquake, accounting for diaphragm flexibility and diaphragm nonlinearity through stiffness degradation models. The authors developed a modeling scheme for nonlinear response of RC buildings which accounted for the inelastic in-plane deformations of slab systems. The results obtained from the study suggested that the inplane inelastic deflection of floor slabs imposed a much more significant ductility demand and strength demand on the interior frames than that predicted from elastic behaviour of the floor slab or a rigid floor assumption. Such effects could lead to the failure of vertical load resisting elements and eventually the entire structural system. The study clearly showed that a distinct collapse mechanism, due to diaphragm yielding, existed for the class of buildings discussed by the authors.

In their 1996 paper, Tena-Colunga and Abrams (1996) presented a study in which they investigated the seismic response of buildings with flexible diaphragms. In that analytical study, three existing instrumented buildings: a two-storey building with unreinforced masonry walls, a two-storey timber building with reinforced clay-units masonry shear walls and an eight-storey building with unreinforced masonry clay-units and reinforced 
concrete shear walls were subjected to the 1989 Loma Prieta ground motion. The results were then compared with the recorded results from the accelerometers in the buildings. In addition, the responses of analogous hypothetical buildings with rigid diaphragms were computed and compared with the measured responses in order to illustrate the effect of the flexibility of the diaphragm. Such comparison led to the following observations:

With an increase in the diaphragm flexibility, the diaphragm and shear wall accelerations could also increase. Moreover, in buildings having flexible diaphragms, the flexible shear walls could accelerate at higher accelerations than stiffer shear walls. Further the design criteria for rigid diaphragms would not necessarily be conservative for flexible diaphragms. Also, the flexibility of the diaphragm was found to lengthen the period of the system.

Dolce et al. (1994) carried out a study to investigate the response of structures with flexible inelastic diaphragms. They investigated the nonlinear dynamic response of a large number of simple symmetric structures. From their results, the authors had the following observations:

The flexibility of the diaphragm could significantly affect the behaviour of the system in cases where the stiffness distribution among the lateral resisting elements was not uniform. In addition, it was found that while the rigid floor assumption for design could be conservative for stiffer elements, it would not be as appropriate for less stiff elements and for the diaphragm floor itself. The flexible diaphragm hypothesis seemed to be more suitable for the design of both less stiff lateral resisting elements and the diaphragm but produced substantial ductility demand in the stiffer elements. A design based on the 
envelope of the stresses obtained from the two assumptions led to reasonable values of ductility demand in vertical resisting elements, but would not reduce the floor diaphragm ductility demand. The study suggested that the usual rigid diaphragm assumption could be more conservative with regards to overall safety as long as the diaphragm was provided with the maximum elastic strength and the less stiff vertical resisting elements were highly ductile or had more strength than required. It was recommended that the seismic code provisions gave more emphasis to floor diaphragms in terms of both strength design and detailing.

Tremblay and Stiemer (1996) carried out a study to investigate the seismic behaviour of single-storey steel structures with flexible diaphragms. They examined the nonlinear response of 36 rectangular single-storey steel buildings when subjected to historical earthquakes. The buildings were designed according to the provisions of the National Building Code of Canada (NBCC). The braces were designed to carry the base shear calculated from NBCC equations, while a capacity design procedure was adopted for designing the diaphragm.

The authors considered 6 sites (two for each of the three main seismic regions defined in NBCC). For each site, 6 buildings were considered: small $(15 \times 30 \times 5.4 \mathrm{~m})$, medium $(30 \times 60 \times 6.6 \mathrm{~m})$ and large $(60 \times 120 \times 9 \mathrm{~m})$. For each building size two alternative roofing systems were considered: a heavy roofing system, and a light roofing system. The response of each building was investigated for the earthquake acting in each of two principal directions, which effectively meant that 72 one-storey buildings were analysed. The LLRS for the buildings consisted of a pair of diagonal tension and compression 
tubular bracing members located on each exterior wall. The length of bracing bents was 7.5, 10 and 15 meters for small, medium and large buildings, respectively.

The authors modeled the one-storey building with flexible diaphragm as a deep girder (representing the diaphragm) supported on two springs (representing the bracing system). They calibrated the spring stiffness $\left(K_{b}\right)$ as well as the beam properties (shear stiffness $G^{\prime}$, flexural rigidity $E I$, and mass $m$ ) to match the values obtained from the geometric dimensions and the material properties of the components. The force-displacement relationship for the lateral load resisting system (LLRS) was assumed to be elasto-plastic while a linear elastic behaviour was considered for the diaphragm. After conducting nonlinear dynamic time history analyses for all 72 buildings, the authors made the following observations:

The period of the building with flexible diaphragm was longer than that of the analogous buildings with perfectly rigid diaphragm. This implied that the expressions to be used for determining the period of the building had to take into account the in-plane flexibility of the roof diaphragm. For all buildings the dominant mode was the first mode. It was seen that for each building the peak storey drift was less than $2 \%$ of the height of the buildings, which is the NBCC requirement for the maximum storey drift. The in-plane deformations of the diaphragms obtained from dynamic analysis exceeded the static deformations in all cases. There was a significant increase in the ductility demand on the LLRS. This meant that smaller force modification factors had to be used in such structures to lower the ductility demand in the LLRS. The authors indicated that while the distribution pattern of moments in dynamic analyses was similar to that of the static case (parabolic), the maximum value was amplified by a factor between 1.25 and 2.3. Also, 
the distribution of shear forces along the length of the diaphragm was not linear and the shear at quarter span was larger than that produced by a uniform distribution of forces. Evidently, the forces and deformations obtained from static analysis had to be modified for dynamic effects and the flexibility of the diaphragm.

An experimental study on the behaviour of low-rise building with flexible roof diaphragms was performed by Tremblay et al. (2000). A first order model with artificial mass-simulation (Moncarz and Krawinkler 1981) of the prototype building was designed and built and subjected to shake table test. The prototype building was designed based on NBCC provisions (Tremblay et al. 2000)

The results from the shake table test confirmed the findings of the previous analytical studies. The measured shear force matched that found by nonlinear dynamic analyses. It was also noted that the high strain rate effects caused an increase in the brace resisting yield force and therefore caused higher forces in the roof diaphragm. In addition, strength eccentricity was observed to have a significant effect on the ductility of the bracing elements. The authors recommended further numerical study, calibrated against this test data, to better understand the behaviour of such structures.

A study on low rise masonry buildings with flexible diaphragms was carried out by Kim and White (2004a). They proposed a linear static methodology applicable to buildings with flexible diaphragms. Their method was based on the assumption that the stiffness of the walls was much greater than that of the flexible diaphragm and that the response of flexible diaphragms within a structure were independent of one another. Based on these assumptions, the buildings could be analyzed as a combination of separate sub-assembly 
models for each of the diaphragms. One could then additively combine the results from separate models. The authors observed that the calculated lateral forces were in fair agreement with the results obtained from time history analyses.

Kim and White also concluded that low-rise shear wall buildings with flexible diaphragms could not be idealized as SDOF systems and should be studied as MDOF systems that took into account both the in-plane and out of plane walls. In addition, they recommended their simplified procedure as a useful method that could accurately predict the response of buildings with flexible diaphragms. The authors further pointed out that the walls should be designed and investigated as force-controlled elements in their plane and as deformation-controlled elements in the direction perpendicular to their in-plane direction (Kim and White 2004a)

In their 2004 paper, Adebar et al. (2004) showed that there were two differences between the behaviour of typical tilt-up frames and typical cast-in-place frames. Not only was the seismic detailing in tilt-up frames much less stringent, but also tilt-up frames were usually associated with flexible diaphragms which would cause the tilt-up walls to be subjected to much larger drift demands than what a linear analysis might suggest.

Using data from previous experimental studies on both steel deck diaphragms and tilt-up frames, Adebar et al. (2004) carried out a series of linear and nonlinear dynamic analyses on single-storey buildings consisting of tilt-up frames and steel deck diaphragms. They assumed linear elastic behaviour for the steel deck diaphragm in both cases. The results suggested significant increase in the ductility demands on the tilt-up frames when 
responding in the nonlinear range. The authors recommended a simplified equation for estimating the inelastic drift of tilt-up walls:

$$
\Delta_{w}=\delta_{r}-\left(\delta_{r}-\delta_{w}\right) \frac{V_{w y}}{V_{w e}}
$$

in which $\Delta_{w}$ is the total inelastic drift of the wall, $\delta_{r}$ and $\delta_{w}$ are the maximum roof and wall displacement, respectively, determined from a linear analysis, $V_{w y}$ is the yield strength of the walls and $V_{w e}$ is the maximum force in the walls based on a linear analysis. The authors pointed out that the total drift of the tilt-up walls responding in the inelastic range could be broken into two portions: the elastic part equal to yield displacement $\Delta_{w y}$ and the inelastic part $\Delta_{w i}$ where:

$$
\begin{aligned}
& \Delta_{w y}=\delta_{w} \frac{V_{w y}}{V_{w e}} \\
& \Delta_{w i}=\delta_{d}(T)\left(1-\frac{\gamma_{w}}{R_{y}}\right)
\end{aligned}
$$

In Equations 1.2 and $1.3 \delta_{d}(T)$ is the spectral displacement based at the period of the system $T, R_{y}$ is the force reduction factor and $\gamma_{w}$ is the nominal over-strength ratio.

Adebar et al suggested that the period of the building could be determined by using the following equation given by FEMA 356 (FEMA 2000).

$$
T=\sqrt{0.1 \delta_{w}+0.078 \delta_{r}}
$$


The authors recommended that it would be useful to classify the tilt up frames into three groups: (1) short roof diaphragm spans in which the frames do not need to be designed for significant inelastic rotations, (2) long roof diaphragm spans in which the frames must be designed to remain elastic, and (3) intermediate roof diaphragm spans where a proper combination of strength and ductility must be provided depending on the dimension of the roof (Adebar et al. 2004).

Kim and White (2004b) carried out a study that focused on masonry buildings with flexible diaphragms. Their study was related to the analysis of the dynamic response of two single-storey single-diaphragm half-scale reinforced masonry buildings that were earlier tested at the University of Texas Austin and the United States Army Construction Engineering (CERL). The tests were designed to produce elastic response, inelastic response with moderate damage, and inelastic response with extensive damage. One objective of Kim and White's study was to define proper structural properties for predicting the dynamic response. In their study the authors used a three dimensional model, with a limited number of degrees of freedom that took into account three idealized key modes of diaphragm displacement: an out-of-plane bending mode in the N-S direction, an out-of-plane bending mode in the E-W direction and a shear raking mode (Kim and White 2004b). They assumed that in terms of bending, the diaphragm behaved as a horizontal plate girder in which the boundary members or chords acted as the flanges and the diaphragm functioned as the web. Both in-plane and out-of-plane walls were modeled. Only the E-S ground excitation (short side) was considered in the study since the response of the system in that direction was much more significant than that in the other direction. The authors adopted a model calibration method to determine the 
structural properties. Based on various experimental tests, they determined the properties of the system and members. The structure that was tested at CERL consisted of a flexible wood diaphragm supported by four masonry walls. Using the simplified MDOF modeling the authors modeled the structure that was tested at CERL and applied the E-W ground motion to their analytical model. The analytical responses were compared with the recorded experimental results. Satisfactory match was found between the acceleration and displacement time histories recorded in the experiment and those computed analytically.

The observations of Kim and White can be summarized as follows:

When the ground motion was increased it was the out-of-plane wall that was damaged first. When the out-of-plane wall reached certain degradation in stiffness and strength, the diaphragm response became increasingly nonlinear and larger lateral loads were distributed to the in plane walls. Generally, the flexibility of the diaphragm significantly influenced the response of the out-of-plane walls and the level of flexibility was in direct correlation with the drift ratio in the out-of-plane walls. The authors suggested that the response of the buildings could be improved by strengthening the out-of-plane shear walls (using FRP, pre-stressing or other techniques). As an alternative, the response of the out-of-plane walls could be improved by stiffening the diaphragm.

In 2012, an experimental research was carried out by Massarelli et al. (2012) to assess the seismic characteristics of steel deck diaphragms. The authors also investigated the practicality and effectiveness of retrofitting and repair strategies for the steel deck roof diaphragms. They conducted dynamic tests on nine diaphragm specimens composed of 
corrugated deck panels with nail/screw, weld/button-punch or weld/screw connections. The specimens were supported on open web steel joists spaced at $1.75 \mathrm{~m}$ or $1.828 \mathrm{~m}$. The authors concluded that the ultimate failure was confined to the deck-to-frame and sidelap connections. Also, the steel deck institute (SDI) method overestimated the shear stiffness at high amplitudes of shaking. For $0.76 \mathrm{~mm}$ and $0.91 \mathrm{~mm}$ nail $/ \mathrm{screw}$ specimens a ductile pinched hysteretic behaviour was observed but the weld/button-punch and the thicker nail/screw diaphragms did not display as much energy dissipation.

For the purpose of assessing the retrofitting performance, Massarelli et al. constructed a replica of one of the specimens which was retrofitted prior to the test. The arc-spot weld fasteners were reinforced with Hilti nails placed in every flute at panel ends and at every second flute along joist lines. The button-punch sidelaps were strengthened with $1.21 \mathrm{~mm}$ thick strip screws connected below sidelaps. Using the SDI modified method; they determined the strength and stiffness of the retrofitted diaphragm. Both values increased substantially. The retrofitted specimen was observed to have an improved hysteretic response as well. The authors pointed out that although the retrofitted specimen failed in the same manner as the other specimens, the use of additional nails and screw connected sidelap reinforcement strips could be considered as an effective retrofit scheme to increase the shear strength and stiffness of an existing arc-spot weld and button-punch connected diaphragm (Massarelli et al. 2012).

Humar and Popovski (2012) conducted an analytical study in 2012 to assess the seismic response of single-storey buildings with flexible diaphragms. They studied the response of 33 buildings of the 36 one-storey buildings that were designed by Tremblay and Stiemer (1996). The authors modeled the buildings as a deep beam (modulus of elasticity 
$E$, cross-sectional moment of inertia $I$, and shear stiffness $G^{\prime}$ ) supported by two springs (each of stiffness $K_{b}$ ). The deep beam represented the diaphragm while the springs modeled the lateral load resisting system (LLRS). The diaphragm beam was divided into 20 elements and the mass was lumped at the interconnecting nodes.

The authors determined the ratio of the maximum diaphragm displacement to story drift in each building to verify the FEMA 356 classification criteria for the flexibility of the diaphragm. It was observed that when the load was acting parallel to the long side of the building, for most buildings the ratio lay between 1 and 2, so that the diaphragm would be classified as stiff according to FEMA356. On the other hand, when the load was acting parallel to the short side of the buildings, all buildings exhibited a displacement ratio much greater than 2 , so that the diaphragms would be classified as flexible.

The authors reasoned that the beam representing the diaphragm and the springs representing the bracing could be considered as two springs in series. Based on this analogy, they suggested the following equation for determining the fundamental period of the building:

$$
\omega=\frac{\omega_{\mathbf{b}} \omega_{d 1}}{\sqrt{\omega_{b}^{2}+\omega_{d 1}^{2}}}
$$

In Equation $1.5 \omega$ is the frequency of the combined system, $\omega_{b}$ is the frequency of the system when the diaphragm is rigid and $\omega_{d 1}$ is the first mode frequency of the diaphragm (beam) on rigid supports. The nth mode frequency of the diaphragm on rigid supports can be obtained from the following equation: 
$\omega_{d n}=(n \pi)^{2} \sqrt{\frac{E I}{m L^{4}}} \sqrt{\frac{1}{1+\left(E I / B G^{\prime}\right)(n \pi / L)^{2}}}$

where $L$ is the span of the diaphragm and $B$ its width. When the shear deformations are much larger than flexural deformations, equation 1.6 reduces to:

$\omega_{d n}=(n \pi) \sqrt{\frac{B G^{\prime}}{m L^{2}}}$

When the diaphragm is very stiff in comparison to the LLRS and may be taken as rigid, the fundamental frequency is given by

$\omega_{b}=\sqrt{\frac{2 K_{b}}{m L}}$

It was observed that the flexibility of the diaphragm lengthened the period of the building.

The authors pointed out that the distribution of inertia forces along the length of a flexible diaphragm was not uniform, as would be the case for a rigid diaphragm, but followed a pattern that was close to a parabola. They showed that the FEMA 356 suggestion for the distribution of inertia forces gave a fair approximation of the real distribution. The FEMA 356 suggestion is as follows:

$f_{d}=\frac{1.5 F_{d}}{L_{d}}\left[1-\left(\frac{2 x}{L_{d}}\right)^{2}\right]$ 
where $\mathrm{F}_{d}$ is the total inertial load on the flexible diaphragm, $x$ is the distance from the center line of the diaphragm and $L_{d}$ is the distance between lateral support points for the diaphragm.

The authors analysed the response of a one-storey building with flexible diaphragm to ElCentro ground motion. In their study they assumed that both the LLRS and the diaphragm remained elastic. Based on their analyses, the authors came to the following conclusions:

In a building with flexible diaphragm, shear deformations make a major contribution to the total deformation of the system. The flexibility of the diaphragm increases the period of the system. Since the inertia forces follow a parabolic rather than a uniform distribution, the mid-span moment may be larger, even though the total base shear might be smaller due to elongation of the period.

In their 2012 paper, Trudel-Languedoc et al. (2012) carried out a parametric study to investigate the influence of the flexibility of the diaphragm on the dynamic seismic response and key seismic design parameters of single story buildings with steel deck roof diaphragms. Both elastic and inelastic responses were studied. The authors used modal response spectrum analysis and linear time history analysis for obtaining the elastic response of the buildings and a nonlinear time history analysis for the inelastic response of the buildings. The buildings were designed according to the Canadian code provision for a base shear $V_{f}$ given by.

$$
V_{f}=\frac{S(T) W}{R_{d} R_{o}}
$$


in which $W$ is the total seismic weight, $R_{d}$ and $R_{o}$ are respectively the ductility and over strength related force modification factors, and $S(T)$ is the design spectral value for the period, given by:

$T=2 \pi \sqrt{\frac{W\left(\Delta_{B}+0.76 \Delta_{D}\right)}{g V}}$

In Equation $1.11 \Delta_{B}$ is the average lateral displacement of the storey (brace displacement) and $\Delta_{D}$ is the maximum horizontal deflection of the diaphragm. Figure 1.2 illustrates the lateral displacements $\Delta_{B}$ and $\Delta_{D}$.

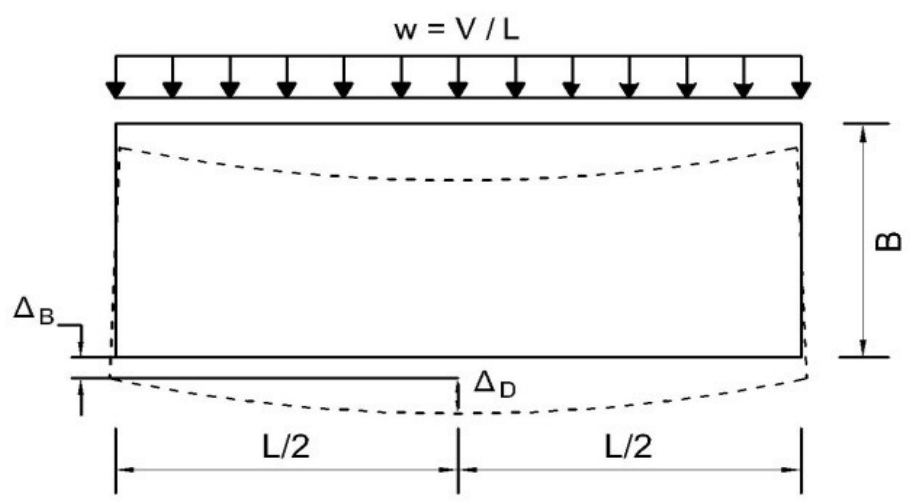

Figure 1.2 - Diaphragm lateral deformations under lateral loads, definitions of $\Delta_{B}$ and $\Delta_{D}$

In their three analysis methods (Modal Response Spectrum Analysis, Linear Time History Analysis and Nonlinear Time History Analysis), Trudel-Languedoc et al., investigated the magnitude of the lateral deflections of the building as well as the distribution of bending moment and shear force along the length of the diaphragm. The 
authors also compared the bending moment and shear force distributions with those obtained by applying the total seismic load as a uniform force along the length of the diaphragm. They also compared the diaphragm deflection with the one determined according to NBCC design provisions.

The modal response analysis and linear time history analysis showed that the values obtained for mid-span bending moments as well as quarter-span shear forces were larger than those determined for a uniform distribution of the total seismic load. Also, the diaphragm deflections were larger. The only difference between modal analysis and linear time history analysis was that in the latter, the period of the system had a more pronounced effect on the level of amplification. In the nonlinear time history analysis, a pinched hysteretic behaviour was assumed for the braces. The yield base shear $V_{y}$ was assumed to be equal to $1.2 \times 1.3 V_{f}=1.56 V_{f}$ and the reduced lateral strength near zero deformation was set equal to $0.3 V_{f}$ which is typical for intermediate brace slenderness (Trudel-Languedoc et al. 2012). The maximum story shear was set equal to $1.25 \mathrm{Vy}$ which was reached at a ductility of 4 (Based on NBCC upper limit on $R_{d} R_{o}$ ). The authors used force modification factor of 2 and 4 (The limits on $R_{d} R_{o}$ ), to account for the effect of $R$. Nonlinear time history analysis showed that the quarter-span shears and mid-span moments as well as the brace ductility demand increased with the flexibility of the diaphragm and the reduction of the design force. Moreover, for higher periods greater bending moments and shears were obtained. On the other hand, greater ductility demand on lateral load resisting system was observed for shorter periods.

In a paper, Humar and Popovski (2013) presented the results of a study that was an extension of their previous study on buildings with flexible diaphragm. This new study 
investigated the inelastic seismic response of single-storey buildings with flexible diaphragms. It was assumed that the inelasticity was confined to the bracing system with an elasto-plastic or bilinear force-displacement relationship. The diaphragm was assumed to remain elastic. The authors subjected 65 of the 72 buildings designed by Tremblay and Stiemer (Tremblay and Stiemer 1996) to 5 synthetic ground motions generated by Atkinson (2009). The selected motions were compatible with the uniform hazard spectrum for Vancouver. The authors performed nonlinear time history analyses on the buildings. The analytical model used was identical to that employed in the previous study referred to above (Humar and Popovski 2012). Based on the results of their nonlinear time history analyses, the authors made the following observations:

The nonlinearity of the LLRS increased the contribution of higher modes, so that the FEMA 356 recommendation for finding the inertia load distribution was no longer as accurate. Consequently, the use of FEMA 356 loading for obtaining moment and shears along the length of the diaphragm could lead to significant underestimation of the true values of these parameters. The authors noted that the shear force at the quarter span and the bending moment at the mid-span were much greater than the corresponding values for a rigid diaphragm. They suggested that further research was required for finding the level of this magnification.

The ductility demand on the LLRS was increased significantly. This was because the contribution of higher modes was more significant and because the displacement produced at the bracings by the higher modes was much larger. It was shown that increase in ductility demand was correlated to the increase in drift ratio, which is a measure of the flexibility of the diaphragm. The increase in ductility was more 
pronounced for systems with low post-yield stiffness. If the ductility demand was to be limited to a reasonable value, a reduced force modification factor would have to be used. The authors suggested the following equations for the adjustment of the force modification factor so as to limit the ductility demand on the LLRS to a target value:

$R_{y}=\kappa \mu$

$\kappa=-0.5 r+1.5$

$1 \geq \kappa \geq 0.64$ for $\mu=2$

$1 \geq \kappa \geq 0.50$ for $\mu=3$

$1 \geq \kappa \geq 0.40$ for $\mu=4$

where $\boldsymbol{r}$ is the drift ratio, $\mu$ is the ductility demand and $R_{y}$ is the force modification factor. The $\kappa$ value is thus a function of the drift ratio.

The authors suggested further studies involving different structural systems and using different target UHS to confirm the validity of the force modification factors obtained by their method (Humar and Popovski 2013).

\subsubsection{Previous Research on the Response of Frames with Concentric Braces}

In an experimental study Tremblay et al. (2003), examined the seismic response of concentrically braced steel frames made with rectangular hollow bracing members. Rectangular hollow sections (RHSS) are known to be quite efficient in resisting compression and seismic load. Moreover, these sections satisfy fairly well the code requirements related to ductile behaviour. In the experimental test, a total of 24 full- 
scaled steel frames, both with single bracing and X-bracing configuration, were tested in order to compare the behaviour of the two configurations. Two displacement sequences $\mathrm{H} 1$ and $\mathrm{H} 2$ were applied to the specimens. Sequence $\mathrm{H} 1$ was a symmetrical displacement series of increasing amplitude. $\mathrm{H} 2$ displacement time history was developed based on the dynamic analysis of a two story building subjected to several ground motions (Archambault et al. 1995). The story shears as well as the story drifts were recorded for both displacement time histories (Tremblay et al. 2003).

Measurements on a single brace showed strength degradation in compression with the number of cycles and the amplitude of deformation. Tension resistance also decreased with the number of cycles because of permanent brace elongation. The combination of the two behaviours resulted in a symmetrical behaviour that showed progressive reduction in the peak story shear with a pinching response that developed over the cycles. The response of X-braces was quite similar to this combination. The authors also studied the effective length and the out of plane deformations of the braces as well as the fracture life of the bracing members.

The authors reached the conclusion that in all cases the braces underwent several cycles of out of plane inelastic buckling and yielding in tension which led to permanent elongation of the brace. In addition, for X-bracing members, the effective brace slenderness obtained with the assumption that the tensile member provided full support at mid-length was found to give satisfactory results. It was observed that local buckling and fracture of the RHS braces occurred at lower ductility level for X-braces than for similar single braces. 
The authors recommended further tests on larger scale specimens to validate the findings of their study.

The experimental results from Tremblay et al. (2003) as well as the results from Archambault et al. (1995) can be used in analytical studies to model the behaviour of the braces. Certain behaviours such as pinching and strength and stiffness degradation can be complicated and software such as OpenSees and Drain-2DX would need details from the experimental studies. Therefore, such experimental data can be quite useful for the purpose of modelling the behaviour of brace members.

In their 2005 paper, Lee and Bruneau (2005) reviewed the experimental data for energy dissipation of compression members in concentrically braced frames. Their review included several previous experimental studies, such as that by Black et al. (1980), which investigated the extent of hysteretic energy dissipation in compressive bracing members as well as the compressive strength degradation in bracing members of a variety of section shapes.

Lee and Bruneau pointed out that, bracing members with tubular cross-section suffered less degradation in compressive strength and energy dissipation. It was observed that under cyclic loading, there was a significant drop in the energy dissipation in compression and in compressive strength of braces with slenderness above 80, which confirmed the validity of the specified limits on the slenderness of braces in seismic provisions. 


\subsubsection{Previous Research on the Nonlinear Response of Steel Deck Diaphragms}

In their paper, Tremblay et al. (2002) studied the possibility of allowing the roof diaphragm panels and their connections to act as elements dissipating energy through inelastic deformations during seismic ground motions. In low-rise buildings it is the braces that usually function as energy dissipating elements. However, for the other elements (diaphragm, foundation etc.) to stay elastic during the action of lateral loads, a capacity design procedure must be adopted. Generally, the ductility provisions for braces will lead to oversizing of the bracing members. Consequently, larger seismic forces would be resisted by the braces which in turn would cause greater forces to be imposed on the diaphragm. This would result in much thicker diaphragm (capacity design) and an uneconomical design.

To assess the plausibility of diaphragm acting as an energy dissipating element, the authors designed one example building using 3 approaches: (1) Elastic roof and inelastic energy dissipating braces (weak braces with $\mathrm{Rd}=2$ ). (2) Elastic roof and inelastic energy dissipating braces (weak braces with $\mathrm{Rd}=3$ ). (3) Inelastic energy dissipating roof and elastic braces (weak diaphragm with $\mathrm{Rd}=3$ ). It was pointed out that the weak diaphragm design was the most economical design (Tremblay et al. 2002). Nonlinear dynamic analyses were performed on all three buildings to determine their response to the same recorded ground motion. The authors modeled the diaphragm as a mesh using truss elements with degrading stiffness hysteretic response. The obtained results showed that for cases (1) and (2) where the energy was dissipated in the braces, large part of the displacement was caused by brace yielding. Moreover, after the ground motion had ceased the braces were left with large residual displacement. In case (3), however, it was 
observed that the inelastic response occurred only in the diaphragm and therefore the braces remained intact and able to carry post-earthquake loads. The authors showed that allowing the inelastic response to take place in the diaphragm could be beneficial as long as the diaphragm did not lose its shear resistance during ground motion and there was no extensive damage in the roof deck diaphragm. The authors pointed out that such distresses could be avoided by choosing an appropriate value for $R_{d}$. In addition to their analytical study, Tremblay et al. (2002) also carried out an experimental research to investigate the hysteretic behaviour of the diaphragm and presented the results in the same paper. The authors pointed out that the cyclic behaviour of diaphragm was mostly dependent on the type of fasteners. They selected for their test specimens the type of connections that exhibited the ability to carry load in the nonlinear range. It was observed that the specimens with weld plus washer frame connections possessed the most promising characteristics in hysteretic behaviour. Diaphragm with screwed side laps and nailed frame fasteners also performed well.

Tremblay et al. came to the conclusion that cost-effective diaphragms that show good ductility under the design earthquake do exist. They recommended values for $R_{d}$ for diaphragms acting as energy dissipating members but observed that further experimental and analytical studies were needed.

Through an experimental test program, Rogers and Tremblay (2003a, b) investigated the inelastic response of steel deck roofing systems to earthquake excitations and presented their findings in two study papers. The authors based their studies on the fact that the capacity of the roof deck diaphragms as well as their in-plane shear stiffness and therefore their capability for energy dissipation is mainly dependent on the performance 
of the connections. The authors reasoned that since the in-plane shear behaviour of the diaphragm was mainly dependent on the performance of its fasteners, the hysteretic behaviour of a single fastener as a single energy dissipating member could provide beneficial information about the in-plane behaviour of steel deck diaphragms as a whole. In one paper, Rogers and Tremblay presented the response of side lap fasteners (2003a) and in a companion paper they presented the response of frame fasteners (2003b).

For studying the response of side lap fasteners, the authors used 5 displacement protocols for the connection specimens. Monotonic, quasistatic, $0.5 \mathrm{~Hz}$ cyclic, $3 \mathrm{~Hz}$ cyclic and simulated earthquake displacement time histories were used. A total of 45 connections were tested, consisting of screwed $(10-14 \times 7 / 8 \mathrm{in})$, button-punched $(10 \mathrm{~mm}$ nominal diameter) and welded (35 mm length using E60-10 electrode for 2-3 s at $200 \mathrm{~A}$ ) connections. In their research program, the authors included the most commonly used deck profile in North America which is either $38 \mathrm{~mm}$ or $76 \mathrm{~mm}$ in depth $\times 914 \mathrm{~mm}$ in width. These sections require button-punched or welded side lap connections. A nestable version of the $38 \mathrm{~mm}$ deck section, which allows using screwed side lap connection, was also used. The specimens were composed of two adjoining $300 \mathrm{~mm}$ long deck sections connected together by two connectors. The authors presented the hysteretic response as well as the energy absorptions of 45 screwed, button punched and welded side lap connections, for different steel types.

The results suggested that the ultimate capacity of the connection was dependent on the type of the connection, where the welded connections offered the highest capacity and the button-punched the lowest. It was observed that the peak load reached during monotonic tests and other displacement protocols were consistent for different side lap connectors. 
However, a degradation of the capacity was observed with increasing number of cycles for given amplitudes. Moreover, a significant pinching behaviour was observed for screwed and button-punched connections. The authors pointed out that except for welds, the ultimate capacity of the connection could be predicted by using existing design methods. Welded connections performed well with limited fractures in the weldment and plastic deformations of the nearby sheet steel. Such behaviour can be quite beneficial for ductile behaviour and energy dissipation. To ensure a ductile behaviour and avoid further fracture in the weld, the authors recommended appropriate quality controls when welds are used as the connectors. Screwed connections showed satisfying capability for carrying loads at low displacements. However, as the amplitude and the number of cycles increased a significant decrease in the capacity, caused by damaging of the sheet material around the screws and the tilting of the screws, was observed. Button punch side lap connections were the most promising for low shear displacements.

In general, all types of side lap fasteners were able to dissipate some amount of energy through inelastic deformations. Among them, weld connections had the best performance since they were able to carry higher loads under larger inelastic deformations and did not exhibit extreme pinching behaviour. As displacements increased, energy dissipation degradation was observed. Such behaviour is expected for screwed connections because of their tendency to tilt under shear and show pinching behaviour. In the end, the authors recommended further experimental tests in which the put-of-plane distortion of the diaphragm steel decks is taken into account as well as analytical study to assess the ductility demand on the steel deck roofs. 
In their companion paper on the performance of deck to frame fasteners, Rogers and Tremblay (2003b), presented the results of their experimental program on different types of frame fasteners. As the steel deck diaphragm depends on deck to frame fasteners in order to transfer the seismic force to the underlying frame, the authors reasoned that the nonlinear performance of these connections was of great importance to assess the overall inelastic behaviour of the roofing system.

In the aforementioned experimental test program, a total of 144 tests (41 screw, 71 powder-actuator, and 26 weld fasteners) were carried out using the same displacement protocols defined in the companion paper by Rogers and Tremblay (2003b).

It was observed that welded connections, although having larger ultimate capacity, would fail under small displacements. The authors pointed out that the connection capacity prediction using current guidelines was conservative regardless of the type of the fastener. However, the uncertainty in predicting the capacity of the welds was higher due to differences in the quality of the weld. It was observed that nail and screw fasteners were able to carry load under inelastic deformations. This was mainly due to the bearing of the sheet steel. However, sheet steel bearing behaviour often shows significant pinching behaviour. For displacements greater than $5 \mathrm{~mm}$, the capacity can drop to a point at which nail and screw connections are no longer adequate for energy dissipation. Using nail and screw connectors with thin flanges for beams and joists could lead to the failure of the system as nails and screws tend to pull out when tilted under earthquake loads. It was observed that when welds with washers were used, the connections were able to show ductile behaviour and energy dissipation comparable with those by screws 
and powder actuators. The authors recommended further full scale experimental tests on steel deck roofing system as well as analytical studies to confirm their findings.

In an experimental study on the cyclic behaviour of flexible diaphragms Essa et al. (2003) carried out monotonic and reversed quasi static tests on cold-formed corrugated steel decks with different types of fasteners. The program was the second phase of the aforementioned study by Rogers and Tremblay (2003a, b). The purpose of the study was to investigate whether the diaphragm could act as the main source of energy absorption through inelastic deformations during an earthquake. Such role would depend on both the diaphragm properties and the type of fasteners used for the connections. Diaphragm assemblies with 0.76 and $0.91 \mathrm{~mm}$ thickness with nine combinations of deck to frame and deck to deck (side lap) fasteners were tested in a full scale experimental program. For each case, monotonic and quasi static cyclic tests were carried out.

The monotonic test showed that the Steel Deck Institute (SDI) (1981) method gave fair predictions for the strength and the stiffness of the diaphragms provided appropriate fastener properties were used. It was observed that the results from monotonic tests generally overestimated the strength that could be mobilized in the inelastic range. The results of the test indicated that diaphragms with welded deck-to-frame connections without washers had limited ductility. On the contrary, diaphragms with B-deck profile sheets, screwed for side lap fasteners, and either nailed or welded-with-washer for deckto-frame connections exhibited much greater ductility and had the ability to maintain their capacity in the inelastic range. The best behaviour was observed for the case in which $0.91 \mathrm{~mm}$ thick steel sheets were screwed to each other (side lap) and nailed to the frame (deck-to-frame connection). The authors pointed out that the assumption of 5\% 
viscous damping for the dynamic analysis of steel deck diaphragm gave results that matched the cyclic test results. The authors suggested that additional tests under similar and different conditions be performed to extend the database and verify the results of their study (Essa et al. 2003). The findings of this study can be applied to code provisions as well to analytical studies wherever a nonlinear behaviour in the steel deck diaphragms is likely.

\subsection{Importance of Studying Single-Storey Buildings with Flexible Diaphragms}

As stated earlier, the roofing system in one-storey buildings, particularly in large foot print buildings used for commercial, recreational, or educational purposes, generally consists of un-topped steel deck or structural wood panels or other roofing systems with low in-plane stiffness. The diaphragm of such structures can be classified as flexible, and the flexibility of the diaphragm affects their response to earthquake motion as confirmed by both analytical and experimental research.

A large number of buildings in North America, including in Canada, are one-storey buildings with large footprints. As stated above, such buildings are often used for industrial, recreational, commercial or institutional centers and are of high importance. It is therefore important that the response of such buildings to earthquake motion and the effect of diaphragm flexibility on such response are studied in detail.

While previous studies have shed light on many of the issues related to flexible diaphragms, there are still many unanswered questions. The questions that need to be studied include the following: 
1. Development or validation of simplified methods to compute the fundamental period of single-storey buildings with flexible diaphragms.

2. Development of new procedures and validation of existing procedure for modifying the force reduction factors to be applied to brace design shears so as to achieve specified target ductility.

3. Development of methods to account for diaphragm flexibility in determining the bending moments and shears in the diaphragm.

4. Effect of pinching in hysteretic behaviour of braced frames on the ductlity demand and bending moment and shear force distributions.

5. The effects of a nonlinear behaviour of the diaphragm in buildings with flexible diaphragms.

\subsection{Research Objectives}

The objectives of the current study are to investigate the following:

1. The effect of diaphragm flexibility on the ductility demand on the LLRS.

2. The impact of post-yield hardening in LLRS on the response of the system.

3. The distribution of shear forces and bending moments along the length of the diaphragm when the system is subjected to ground motions.

4. The effect of pinching behaviour in LLRS on the total response and behaviour of the system.

5. The consequence of nonlinear behaviour in the diaphragm system.

6. The concept of diaphragm acting as the main energy dissipating member during earthquakes. 


\subsection{Layout of the Thesis}

The current study focuses on the response of one-storey buildings with un-topped steel deck diaphragms, supported on concentric braced frames to seismic ground motions.

In chapter 2 the building database used in the study as well as the generated ground motions are introduced. Mathematical assumptions, analytical model and further assumptions in the study are outlined and discussed in detail.

In Chapter 3 analytical studies are carried out to validate the methods previously suggested for determining the ductility demand on the lateral load resisting system in cases where the nonlinearity of the system is confined to lateral load resisting system. The force-displacement relationship for the LLRS is assumed to be elasto-plastic. The diaphragm is assumed to remain elastic. Specifically, the previous study by Humar and Popovski $(2012,2013)$ is extended. Analyses are carried out for a wider range of spectral shapes including the UHS typical of the eastern region of Canada.

Chapter 4 deals with the effect of post-yield hardening in lateral load resisting system elements on the ductility demand on the lateral load resisting system.

In chapter 5 the issue related to the distribution of shear forces and bending moments along the length of the diaphragm is addressed. In this part of the study, the diaphragm is again assumed to remain elastic, but both elasto-plastic and bilinear force-displacement relations are considered for the braced frames. It is recognized that braced frames of steel with concentric braces often exhibit the effect of pinching in their hysteretic behaviour. 
In chapter 6 , the effects of pinching on both the ductility demand on lateral load resisting system and the distribution of shear forces and bending moments are investigated and discussed.

Chapter 7 investigates the effects of allowing the diaphragm system to deform into the nonlinear range. Recently, some design guidelines have permitted the diaphragm to behave nonlinearly during rare events. The effect of such behaviour on the total response of the system is studied. Also, the concept of "diaphragm as the energy dissipating member" or in other words, "weak diaphragm-strong brace" is examined.

In chapter 8 the study and its objectives is summarized. Important conclusions along with recommendations for future studies are presented.

The results obtained and the conclusions reached in the present study are based mainly on the seismic time history analyses of building structures consisting of a steel deck diaphragm and a lateral load resisting system (LLRS) of concentric steel braces. However most of the results are equally applicable to buildings in which a different type of LLRS is used. 


\section{Chapter 2 : Analytical Model of Buildings with Flexible Diaphragm}

\subsection{Introduction and Chapter Outline}

The current research is focussed on an analytical study of the seismic response of onestorey buildings with flexible steel deck roof diaphragms, supported on concentric braced frames. The determination of the response of a structure to seismic ground motions in cases when the structure is expected to yield can be quite complicated. Moreover, as shown by previous studies additional complication is introduced when the building has one or more flexible floor diaphragms.

In order to obtain a reasonable assessment of the seismic response of one-storey buildings with flexible diaphragms, one must first form a basic understanding of the general behaviour and characteristics of such systems. For this purpose, a brief description of such buildings and the manner in which they respond to seismic ground motions is presented under Section 2.2.

The present study carries out the response analysis of a set of single-storey buildings with flexible diaphragms that are representative of such buildings designed and built at locations in Canada. The set of buildings selected for this purpose is introduced in Section 2.3.

The determination of time history of the seismic response of the buildings requires the specification of suitable ground motion histories. Ideally, these ground motions must be compatible with the Uniform Hazard Spectra for selected locations in Canada. The ground motions that are used in this study are presented in Section 2.4. 
Obviously, an important aspect of any analytical study is the simulation of the system through modelling and recognition of the assumptions made in such modelling. The analytical model as well as the assumptions made in developing are presented in Section 2.5.

\subsection{One-Storey Buildings with Flexible Diaphragms}

One-storey buildings with large footprints are extensively used throughout the world, particularly in Canada. The occupancy importance of such buildings is often in the category of high importance. The low level of gravity loads on the roof makes it unnecessary and uneconomical to provide rigid deck roofing systems, such as concrete slabs. In practice, therefore, the roofing system in one-storey buildings usually consists of elements with low in-plane stiffness, such as un-topped steel deck diaphragms or structural wood panels. The roof diaphragm panels are usually supported by open-web steel joists while the joists themselves sit on framing of structural steel. Several types of connections can be used for the connection between the deck panels and the connections between the panels and the frame girders.

The lateral load resisting system in such buildings can be comprised of steel braced frames with eccentric or concentric braces, concrete shear walls, masonry shear walls or steel plate shear walls. Since, the lateral stiffness of all of these systems is much greater than the in-plane stiffness of aforementioned roofing systems; the roofing behaves as a flexible diaphragm. The rigid floor assumption is thus no longer valid and the response analysis must account for the flexibility of the diaphragm. Previous studies have confirmed this conclusion. 
The types of buildings being studied are not subjected to large gravity loads, their mass is therefore low and the base shear due to earthquake may be relatively small. In addition, because of the flexibility of the diaphragm the fundamental period of the building becomes longer, resulting in a further reduction in the base shear. However, the flexibility of the diaphragm alters the response of the structure to seismic ground motion leading to non-uniform distribution of the inertia forces. These forces now become concentrated near the mid-span of the diaphragm producing larger bending moments and in-span shears. Furthermore, the diaphragm flexibility increases the displacement of the lateral load resisting system and the ductility demand placed on it.

Since there are many challenges and problems related to the seismic behaviour of onestorey buildings with flexible diaphragms, it is important to investigate the nature of their response to earthquake forces.

\subsection{Building Database}

The building samples used for the current study are from a set of one-storey buildings which were originally designed by Tremblay and Stiemer and presented in their 1996 paper (Tremblay and Stiemer 1996). The buildings in the set were designed according to the provisions of the 1995 National Building Code of Canada (NBCC). The buildings had metal roof deck diaphragms of different sizes, weight, and stiffness and were designed for six different locations. The selected buildings as well as the assumptions and details relevant to current study are discussed in detail in the following paragraphs. 


\subsubsection{Considerations in Selecting the Buildings}

For each of the six locations presented in Table 2.1, six buildings were designed. The database included 3 building sizes: small $(15 \times 30 \times 5.4 \mathrm{~m})$, medium $(30 \times 60 \times 6.6 \mathrm{~m})$, and large $(60 \times 120 \times 9.0 \mathrm{~m})$. All buildings had the length-to-width ratio of 2 in the plan. For each building size, two types of roofing systems were considered: a heavy roofing system and a light one. The total dead loads for the heavy system and the light system were 2.21 and $1.0 \mathrm{kPa}$, respectively. The total number of buildings was 36 . Figure 2.1 is a schematic diagram of the buildings.

Table 2.1 - Design sites (taken form Tremblay and Stiemer 1996, with the authors' permission and (C) Canadian Science Publishing or its licensors)

\begin{tabular}{lccccc}
\hline Site & & & & $\begin{array}{c}\text { Roof Snow } \\
\text { Load (kPa) }\end{array}$ \\
\hline 1. Victoria, B.C & $v$ & $a / v$ & $Z_{a}$ & $Z_{v}$ & 1 \\
2. Vancouver, B.C & 0.26 & 1.08 & 5 & 5 & 1.48 \\
3. Ottawa, Ont. & 0.21 & 1 & 4 & 4 & 2.16 \\
4. Quebec, Que. & 0.1 & 2.04 & 4 & 2 & 3.14 \\
5. Prince Rupert, B.C. & 0.14 & 1.36 & 4 & 3 & 3.2 \\
6. Whitehorse, Yukon Territory & 0.21 & 0.52 & 3 & 4 & 1.46 \\
\hline
\end{tabular}

In Table 2.1, the acceleration ratio $a$, is the ratio of the peak horizontal acceleration (PHA) to the gravity acceleration, the velocity ratio $v$, is the peak horizontal velocity (PHV) divided by $1 \mathrm{~m} / \mathrm{s}$. Values $Z_{a}$ and $Z_{v}$ are the Zone numbers that correspond to $a$ and $v$, respectively. 


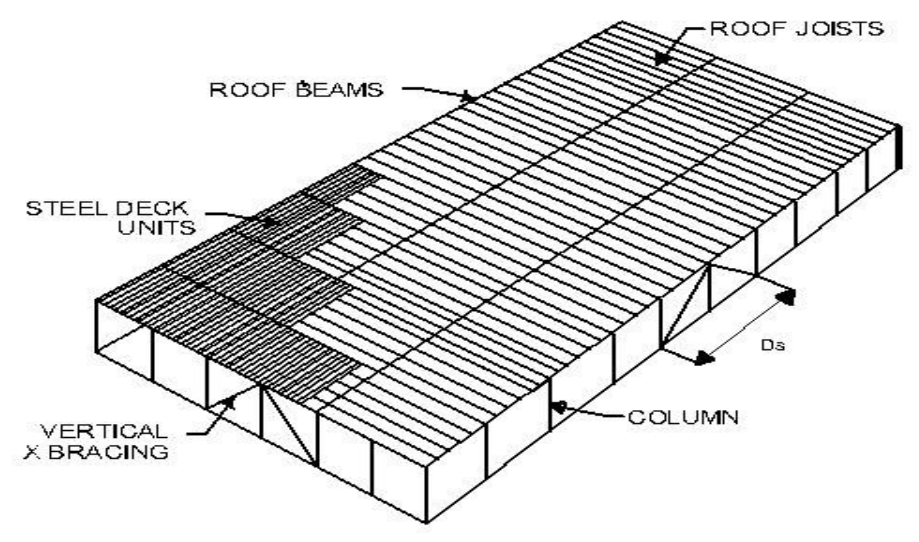

Figure 2.1 - Schematic illustration of the one-storey buildings designed by Tremblay and Stiemer (1996)

All buildings were analyzed for the earthquake acting along each of the two principal directions. Since the structural parameters were different in the two directions of the same building, in effect 72 buildings were analysed. Each building is identified by a 3-digit name. The first digit represents the size of the building ( $\mathrm{S}$ for small, $\mathrm{M}$ for medium and $\mathrm{L}$ for large), the second digit shows the roofing system of the building ( $\mathrm{L}$ for light and $\mathrm{H}$ for Heavy) and the last digit represents the site number given in Table 2.1. For instance, building ML2 is a medium sized building with light roofing system, designed for site class 2 .

\subsubsection{Design Assumptions}

Each building has a pair of diagonal tension- and compression-acting tubular braces on its exterior walls (Figure 2.1). The length of the bracing bents (Ds) was 7.5, 10 and 15 
meters for the small, medium and large buildings respectively. The diaphragm roofing system was supported on open web steel. The joists themselves were supported by the perimeter girders.

As mentioned earlier, the design was based on NBCC provisions. The bracing members were designed based on prescribed seismic loads. A capacity design approach was used for designing the diaphragm roofing system. P-delta effects were neglected as they did not affect the results that much. The buildings were designed so that the maximum inelastic drift, defined as the deformation of the diaphragm mid-point under loads corresponding to base-shear, times the force modification factor, remained within the code-prescribed limits.

First, the deck thickness and the spacing of joists were selected based on the gravity loads. Next, the deck assembly was checked for in-plane shear strength and stiffness (G'). The ultimate thicknesses of the diaphragms were different for different building sizes and were $0.76,0.91$ or $1.22 \mathrm{~mm}$. Arc spot welding ( $16 \mathrm{~mm}$ welds) was assumed for fastening the decks to steel framework. For the sidelap connections, number 10 screws were assumed for $0.76 \mathrm{~mm}$ thick panels, whereas spot welds were assumed for $0.91 \mathrm{~mm}$ decks and thicker ones. For some of the medium and large buildings, the connections as well as the thickness of the decks varied through the length of the roof to make better use of the material. Thus, a stronger and stiffer diaphragm was assumed over a length $\mathrm{a}^{*}$ starting from each short wall of the structure. 


\subsubsection{Properties of the Analytical Model}

Tremblay and Stiemer modelled the diaphragm by a deep beam which was supported on two truss elements representing the braces. Naturally in such a model, the seismic forces acting in the plane of the diaphragm can be represented by forces acting perpendicular to the length of the deep beam. The authors worked out the equivalent properties of this model such as G' (Effective Shear Stiffness), I (Moment of Inertia) and $K_{b}$ (The Stiffness of the Truss Members) for each building so that the response of the beam and the truss members matched the response of the diaphragm in its plane and the response of the braces in the horizontal direction, respectively.

The details of the buildings and the equivalent beam-spring properties of the buildings presented by Tremblay and Stiemer (1996) are shown in Tables 2.2 and 2.3 and in Tables A.1 and A.2.

Table 2.2 - Properties of the buildings (taken form Tremblay and Stiemer 1996, with the authors' permission and (C) Canadian Science Publishing or its licensors)

\begin{tabular}{|c|c|c|c|c|c|c|c|c|c|}
\hline \multirow[b]{4}{*}{ Building } & \multirow{4}{*}{$\begin{array}{c}\mathrm{Kb} \\
(\mathrm{kN} / \mathrm{mm}) \\
\end{array}$} & \multicolumn{5}{|c|}{ Steel roof deck diaphragm } & & & \\
\hline & & \multirow{3}{*}{$\begin{array}{c}\text { Joist } \\
\text { spacing } \\
(\mathrm{mm})\end{array}$} & \multicolumn{3}{|c|}{ Type } & \multirow{3}{*}{$\begin{array}{c}\mathrm{a}^{*} \\
(\mathrm{~mm})\end{array}$} & \multirow{2}{*}{\multicolumn{3}{|c|}{$\begin{array}{l}\text { Loads applied parallel to short } \\
\text { walls }\end{array}$}} \\
\hline & & & & $\begin{array}{l}\text { No. of } \\
\text { frame } \\
\text { fasteners }\end{array}$ & $\begin{array}{l}\text { No. of } \\
\text { stich } \\
\text { fasteners }\end{array}$ & & & & \\
\hline & & & $(\mathrm{mm})$ & width & span & & $(\mathrm{kN} / \mathrm{mm})$ & $\left(\mathrm{x} 10^{\wedge} 9 \mathrm{~mm} 4\right)$ & \\
\hline SL1 & 30.7 & 2100 & 0.76 & 4 & 3 & - & 2.41 & 556 & 0.447 \\
\hline SL2 & 30.7 & 2100 & 0.76 & 4 & 3 & - & 2.41 & 561 & 0.499 \\
\hline SL3 & 30.7 & 2000 & 0.76 & 4 & 3 & - & 2.32 & 663 & 0.537 \\
\hline SL4 & 30.7 & 1800 & 0.76 & 4 & 2 & - & 2.10 & 853 & 0.603 \\
\hline SL5 & 30.7 & 1800 & 0.76 & 4 & 2 & - & 2.10 & 853 & 0.604 \\
\hline SL6 & 30.7 & 2100 & 0.76 & 4 & 3 & - & 2.41 & 561 & 0.500 \\
\hline
\end{tabular}




\begin{tabular}{|c|c|c|c|c|c|c|c|c|c|}
\hline SH1 & 35.4 & 2000 & 0.76 & 4 & 6 & - & 2.41 & 663 & 0.663 \\
\hline $\mathrm{SH} 2$ & 35.4 & 1800 & 0.76 & 4 & 4 & - & 2.17 & 766 & 0.708 \\
\hline SH3 & 30.7 & 1800 & 0.76 & 4 & 2 & - & 2.10 & 853 & 0.747 \\
\hline SH4 & 35.4 & 1600 & 0.76 & 4 & 3 & - & 1.95 & 977 & 0.798 \\
\hline SH5 & 30.7 & 1600 & 0.76 & 4 & 4 & - & 1.97 & 977 & 0.802 \\
\hline SH6 & 30.7 & 1800 & 0.76 & 4 & 2 & - & 2.10 & 766 & 0.724 \\
\hline \multirow[t]{2}{*}{ ML1 } & 48.6 & 2100 & 0.76 & 5 & 5 & 9000 & 3.26 & 2223 & 0.881 \\
\hline & & & 0.76 & 4 & 3 & & 2.41 & & \\
\hline \multirow[t]{2}{*}{ ML2 } & 48.6 & 2100 & 0.76 & 4 & 6 & 6000 & 2.50 & 2246 & 0.978 \\
\hline & & & 0.76 & 4 & 3 & & 2.41 & & \\
\hline ML3 & 40.2 & 2000 & 0.76 & 4 & 3 & - & 2.32 & 2650 & 1.068 \\
\hline \multirow[t]{2}{*}{ ML4 } & 48.6 & 1800 & 0.76 & 5 & 4 & 9000 & 2.87 & 3411 & 1.079 \\
\hline & & & 0.76 & 4 & 3 & & 2.32 & & \\
\hline \multirow[t]{2}{*}{ ML5 } & 48.6 & 1800 & 0.76 & 5 & 5 & 9000 & 2.90 & 3411 & 1.099 \\
\hline & & & 0.76 & 4 & 3 & & 2.14 & & \\
\hline \multirow[t]{2}{*}{ ML6 } & 40.2 & 2100 & 0.76 & 4 & 4 & 3000 & 2.45 & 2246 & 0.911 \\
\hline & & & 0.76 & 4 & 3 & & 2.41 & & \\
\hline \multirow[t]{2}{*}{ MH1 } & 74.9 & 2000 & 0.91 & 5 & 6 & 15000 & 5.11 & 2651 & 0.962 \\
\hline & & & 0.91 & 4 & 3 & & 3.76 & & \\
\hline \multirow[t]{2}{*}{ MH2 } & 57.2 & 1800 & 0.91 & 5 & 4 & 12000 & 4.61 & 3065 & 1.054 \\
\hline & & & 0.91 & 4 & 2 & & 3.39 & & \\
\hline \multirow[t]{2}{*}{ MH3 } & 48.6 & 1800 & 0.76 & 5 & 5 & 12000 & 2.90 & 3411 & 1.317 \\
\hline & & & 0.76 & 4 & 3 & & 2.32 & & \\
\hline MH4 & 74.9 & 1600 & 0.91 & 5 & 4 & - & 4.20 & 3906 & 1.110 \\
\hline \multirow[t]{2}{*}{ MH5 } & 57.2 & 1600 & 0.91 & 5 & 5 & 12000 & 4.24 & 3906 & 1.174 \\
\hline & & & 0.91 & 4 & 2 & & 3.09 & & \\
\hline \multirow[t]{2}{*}{ MH6 } & 48.6 & 1800 & 0.76 & 7 & 6 & 12000 & 8.27 & 3065 & 1.045 \\
\hline & & & 0.76 & 4 & 3 & & 3.14 & & \\
\hline \multirow[t]{2}{*}{ LL1 } & 107 & 2100 & 0.91 & 7 & 6 & 30000 & 13.90 & 8982 & 1.094 \\
\hline & & & 0.91 & 5 & 3 & & 5.07 & & \\
\hline \multirow[t]{2}{*}{ LL2 } & 107 & 2100 & 0.91 & 7 & 5 & 30000 & 13.50 & 8982 & 1.190 \\
\hline & & & 0.91 & 4 & 3 & & 3.90 & & \\
\hline \multirow[t]{2}{*}{ LL3 } & 84.7 & 2000 & 0.76 & 7 & 6 & 18000 & 8.65 & 10600 & 1.499 \\
\hline & & & 0.76 & 5 & 3 & & 3.75 & & \\
\hline \multirow[t]{2}{*}{ LL4 } & 107 & 1800 & 0.91 & 7 & 5 & 36000 & 12.80 & 13640 & 1.231 \\
\hline & & & 0.91 & 5 & 2 & & 4.43 & & \\
\hline \multirow[t]{2}{*}{ LL5 } & 107 & 1800 & 0.91 & 7 & 6 & 24000 & 13.00 & 13640 & 1.428 \\
\hline & & & 0.91 & 4 & 4 & & 3.51 & & \\
\hline
\end{tabular}




\begin{tabular}{cccccccccc} 
LL6 & 68.6 & 2100 & 0.91 & 4 & 7 & 12000 & 4.08 & 8982 & 1.631 \\
& & & 0.91 & 4 & 3 & & 3.90 & & \\
\hline LH1 & 154 & 1500 & 1.22 & 7 & 8 & - & 20.50 & 17900 & 1.120 \\
\hline LH2 & 130 & 2000 & 1.22 & 7 & 8 & 36000 & 22.90 & 13900 & 1.217 \\
& & & 1.22 & 5 & 3 & & 8.80 & & \\
\hline LH3 & 107 & 2000 & 0.91 & 7 & 6 & 36000 & 13.00 & 13640 & 1.508 \\
& & & 0.91 & 5 & 3 & & 4.90 & & \\
\hline LH4 & 130 & 1600 & 1.22 & 7 & 7 & - & 20.80 & 15620 & 1.274 \\
\hline LH5 & 127 & 1500 & 1.22 & 7 & 8 & 30000 & 20.50 & 15620 & 1.436 \\
& & & 1.22 & 4 & 4 & & 5.72 & & \\
\hline LH6 & 107 & 1600 & 0.91 & 7 & 7 & 30000 & 12.40 & 12260 & 1.589 \\
& & & 0.91 & 5 & 2 & & 4.07 & & \\
\hline
\end{tabular}


Table 2.3 - Properties of the buildings (taken form Tremblay and Stiemer 1996, with the authors' permission and (C) Canadian Science Publishing or its licensors)

\begin{tabular}{|c|c|c|c|c|c|c|c|c|c|}
\hline \multirow[b]{4}{*}{ Building } & \multirow{4}{*}{$\begin{array}{c}\mathrm{Kb} \\
(\mathrm{kN} / \mathrm{mm}) \\
\end{array}$} & \multicolumn{5}{|c|}{ Steel roof deck diaphragm } & \multirow{3}{*}{\multicolumn{3}{|c|}{$\begin{array}{l}\text { Loads applied parallel to long } \\
\text { walls }\end{array}$}} \\
\hline & & \multirow{3}{*}{$\begin{array}{c}\text { Joist } \\
\text { spacing } \\
(\mathrm{mm})\end{array}$} & \multicolumn{3}{|c|}{ Type } & \multirow{3}{*}{$\begin{array}{c}\mathrm{a}^{*} \\
(\mathrm{~mm})\end{array}$} & & & \\
\hline & & & \multirow{2}{*}{$\begin{array}{c}\text { Steel } \\
\text { thickness } \\
(\mathrm{mm})\end{array}$} & \multirow{2}{*}{$\begin{array}{l}\text { No. of } \\
\text { frame } \\
\text { fasteners } \\
\text { per } \\
\text { panel } \\
\text { width }\end{array}$} & \multirow{2}{*}{$\begin{array}{c}\text { No. of } \\
\text { stich } \\
\text { fasteners } \\
\text { within } \\
\text { joist } \\
\text { span }\end{array}$} & & & & \\
\hline & & & & & & & $\begin{array}{c}\mathrm{G}^{\prime} \\
(\mathrm{kN} / \mathrm{mm})\end{array}$ & $\begin{array}{c}\mathrm{I} \\
\left(\mathrm{x} 10^{\wedge} 9 \mathrm{~mm} 4\right) \\
\end{array}$ & $\mathrm{T}(\mathrm{s})$ \\
\hline SL1 & 30.7 & 2100 & 0.76 & 4 & 3 & - & 2.41 & 1526 & 0.284 \\
\hline SL2 & 30.7 & 2100 & 0.76 & 4 & 3 & - & 2.41 & 1526 & 0.297 \\
\hline SL3 & 30.7 & 2000 & 0.76 & 4 & 3 & - & 2.32 & 1526 & 0.319 \\
\hline SL4 & 30.7 & 1800 & 0.76 & 4 & 2 & - & 2.10 & 1526 & 0.354 \\
\hline SL5 & 30.7 & 1800 & 0.76 & 4 & 2 & - & 2.10 & 1526 & 0.355 \\
\hline SL6 & 30.7 & 2100 & 0.76 & 4 & 3 & - & 2.41 & 1526 & 0.298 \\
\hline SH1 & 35.4 & 2000 & 0.76 & 4 & 6 & - & 2.40 & 1526 & 0.388 \\
\hline $\mathrm{SH} 2$ & 35.4 & 1800 & 0.76 & 4 & 4 & - & 2.17 & 1526 & 0.410 \\
\hline $\mathrm{SH} 3$ & 30.7 & 1800 & 0.76 & 4 & 2 & - & 2.10 & 1526 & 0.439 \\
\hline SH4 & 35.4 & 1600 & 0.76 & 4 & 3 & - & 1.95 & 1526 & 0.457 \\
\hline SH5 & 30.7 & 1600 & 0.76 & 4 & 4 & - & 1.97 & 1526 & 0.468 \\
\hline SH6 & 30.7 & 1800 & 0.76 & 4 & 2 & - & 2.10 & 1526 & 0.425 \\
\hline \multirow[t]{2}{*}{ ML1 } & 48.6 & 2100 & 0.76 & 5 & 5 & 9000 & 2.67 & 6102 & 0.506 \\
\hline & & & 0.76 & 4 & 3 & & & & \\
\hline \multirow[t]{2}{*}{ ML2 } & 48.6 & 2100 & 0.76 & 4 & 6 & 6000 & 2.43 & 6102 & 0.545 \\
\hline & & & 0.76 & 4 & 3 & & & & \\
\hline ML3 & 40.2 & 2000 & 0.76 & 4 & 3 & - & 2.32 & 6102 & 0.607 \\
\hline \multirow[t]{2}{*}{ ML4 } & 48.6 & 1800 & 0.76 & 5 & 4 & 9000 & 2.49 & 6102 & 0.619 \\
\hline & & & 0.76 & 4 & 3 & & & & \\
\hline \multirow[t]{2}{*}{ ML5 } & 48.6 & 1800 & 0.76 & 5 & 5 & 9000 & 2.37 & 6102 & 0.631 \\
\hline & & & 0.76 & 4 & 3 & & & & \\
\hline \multirow[t]{2}{*}{ ML6 } & 40.2 & 2100 & 0.76 & 4 & 4 & 3000 & 2.41 & 6102 & 0.565 \\
\hline & & & 0.76 & 4 & 3 & & & & \\
\hline \multirow[t]{2}{*}{ MH1 } & 74.9 & 2000 & 0.91 & 5 & 6 & 15000 & 4.44 & 6102 & 0.558 \\
\hline & & & 0.91 & 4 & 3 & & & & \\
\hline \multirow[t]{2}{*}{$\mathrm{MH} 2$} & 57.2 & 1800 & 0.91 & 5 & 4 & 12000 & 3.88 & 6102 & 0.626 \\
\hline & & & 0.91 & 4 & 2 & & & & \\
\hline \multirow[t]{2}{*}{ MH3 } & 48.6 & 1800 & 0.76 & 5 & 5 & 12000 & 2.55 & 6102 & 0.762 \\
\hline & & & 0.76 & 4 & 3 & & & & \\
\hline
\end{tabular}




\begin{tabular}{|c|c|c|c|c|c|c|c|c|c|}
\hline MH4 & 74.9 & 1600 & 0.91 & 5 & 4 & - & 4.20 & 6102 & 0.628 \\
\hline \multirow[t]{2}{*}{ MH5 } & 57.2 & 1600 & 0.91 & 5 & 5 & 12000 & 3.55 & 6102 & 0.696 \\
\hline & & & 0.91 & 4 & 2 & & & & \\
\hline \multirow[t]{2}{*}{ MH6 } & 48.6 & 1800 & 0.76 & 7 & 6 & 12000 & 4.59 & 6102 & 0.619 \\
\hline & & & 0.76 & 4 & 3 & & & & \\
\hline \multirow[t]{2}{*}{ LL1 } & 107 & 2100 & 0.91 & 7 & 6 & 30000 & 9.50 & 24400 & 0.601 \\
\hline & & & 0.91 & 5 & 3 & & & & \\
\hline \multirow[t]{2}{*}{ LL2 } & 107 & 2100 & 0.91 & 7 & 5 & 30000 & 8.74 & 24400 & 0.646 \\
\hline & & & 0.91 & 4 & 3 & & & & \\
\hline \multirow[t]{2}{*}{ LL3 } & 84.7 & 2000 & 0.76 & 7 & 6 & 18000 & 5.22 & 24400 & 0.827 \\
\hline & & & 0.76 & 5 & 3 & & & & \\
\hline \multirow[t]{2}{*}{ LL4 } & 107 & 1800 & 0.91 & 7 & 5 & 36000 & 9.43 & 24400 & 0.720 \\
\hline & & & 0.91 & 5 & 2 & & & & \\
\hline \multirow[t]{2}{*}{ LL5 } & 107 & 1800 & 0.91 & 7 & 6 & 24000 & 7.31 & 24400 & 0.775 \\
\hline & & & 0.91 & 4 & 4 & & & & \\
\hline \multirow[t]{2}{*}{ LL6 } & 68.6 & 2100 & 0.91 & 4 & 7 & 12000 & 3.94 & 24400 & 0.884 \\
\hline & & & 0.91 & 4 & 3 & & & & \\
\hline LH1 & 154 & 1500 & 1.22 & 7 & 8 & - & 20.50 & 35570 & 0.640 \\
\hline \multirow[t]{2}{*}{ LH2 } & 130 & 2000 & 1.22 & 7 & 8 & 36000 & 17.30 & 35570 & 0.710 \\
\hline & & & 1.22 & 5 & 3 & & & & \\
\hline \multirow[t]{2}{*}{ LH3 } & 107 & 2000 & 0.91 & 7 & 6 & 36000 & 9.76 & 28800 & 0.882 \\
\hline & & & 0.91 & 5 & 3 & & & & \\
\hline LH4 & 130 & 1600 & 1.22 & 7 & 7 & - & 20.80 & 35570 & 0.739 \\
\hline \multirow[t]{2}{*}{ LH5 } & 127 & 1500 & 1.22 & 7 & 8 & 30000 & 13.10 & 35570 & 0.821 \\
\hline & & & 1.22 & 4 & 4 & & & & \\
\hline \multirow[t]{2}{*}{ LH6 } & 107 & 1600 & 0.91 & 7 & 7 & 30000 & 8.24 & 24400 & 0.896 \\
\hline & & & 0.91 & 5 & 2 & & & & \\
\hline
\end{tabular}

For the current study, 65 out of the 72 buildings have been selected. Of the selected buildings 33 are from Table 2.2. The properties listed in that table are to be used when the lateral loads act in a direction parallel to the short side of the building. The remaining 32 buildings are selected from Table 2.3 in which the listed properties are based on the assumption that loads are applied in a direction parallel to the long side of the building. 
Clearly, the span of the deep beam (diaphragm) in each case is equal to the plan dimension of the diaphragm in a direction perpendicular to the applied loads.

Buildings SL2, SL5, SL6 from Table 2.2 and SL2, SL5, SL6 and SH6 from Table 2.3 were excluded for the current study.

\subsection{Ground Motion Time Histories}

For conducting nonlinear dynamic analyses, a set of ground motion time histories is needed. While historical time histories may be used for the purpose, such time histories may not always be available or, in some cases, they may not provide a good representation of the seismic characteristics of the region under study. In such cases, the use of generated time histories can be quite advantageous.

Atkinson (2009) has carried out a study to generate ground motion time histories compatible with eastern (Montreal) spectrum and western (Vancouver) spectrum. The stochastic finite fault method was used for the generation of these ground motions. A series of time histories for a range of magnitudes, distances and site conditions were generated. With appropriate scaling such time histories could provide a response spectrum matching the UHS for a specified site.

As examples, records M6C1, M6C2, M6C26, M6C31 and M6C 38 with scaling factors of $0.78,0.87,1.19,0.99$ and 1.43 , respectively, would provide a match for the Vancouver UHS. Records E6C1, E6C13, E6C15, E6C18 and E6C42 with scaling factors 0.55, 0.74, 0.56, 0.61 and 1.01, respectively, were appropriate for matching the UHS for Montreal. 
The uniform hazard spectra for Montreal and Vancouver along with the spectra for the matching records are shown in Figures 1.3 and 1.4.

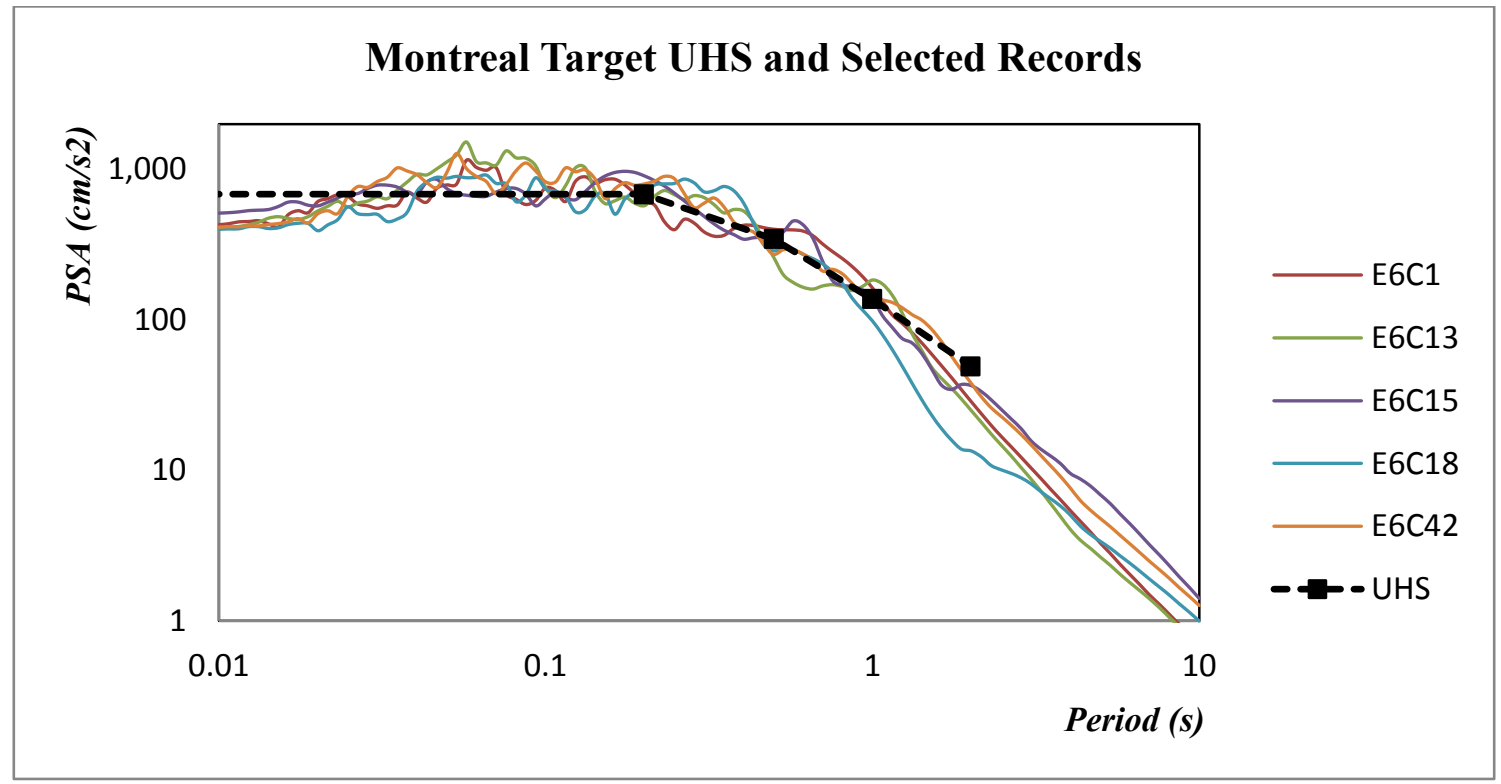

Figure 2.2 - Uniform hazard spectrum for Montreal and spectra of generated ground motions

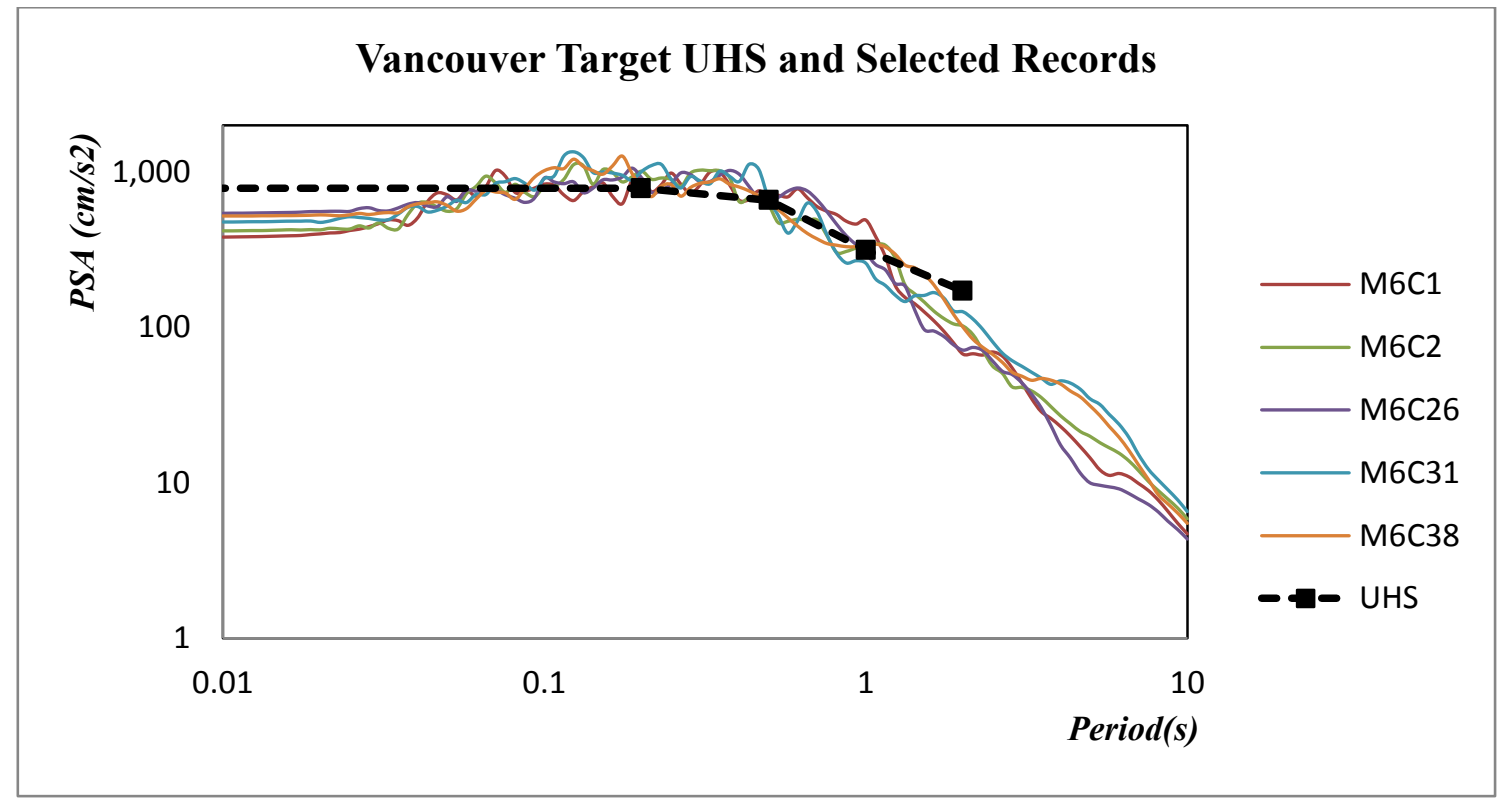

Figure 2.3 - Uniform hazard spectrum for Vancouver and spectra of generated ground motions 
Atkinson time history records have been widely used in research studies. For instance, Humar and Popovski (2013) have used the scaled western records in their study of flexible diaphragms. The advantage of using generated records is that the user has a wider selection of compatible ground motion histories and is not limited to historical ground motions.

\subsection{Analytical Model and Assumption}

In order to investigate the impact of the flexibility of the diaphragm on the response of the system, a simple model must be used to characterize the building. The response of such a model to lateral loads including seismic loads should match the response of the building fairly well and the model should represent the important characteristics of the system. The model used in this study is described in the following paragraphs.

\subsubsection{General Analytical Model}

A one-storey building with flexible diaphragm is represented here by a deep flexural beam supported on two springs. Previous studies have suggested that shear deformations play an important role in the behaviour of the diaphragm. Therefore, shear deformations in the deep beam are taken into account in the model properties. For steel deck diaphragms and wood panels, the bending moment resistance of the beam is provided entirely by the chords located at the boundaries of the diaphragm. Shear is mostly resisted by the web of the beam (diaphragm). The lateral load resisting system (LLRS) that resists the loads arising from wind and earthquake and transfers them to the foundations is modelled by spring supports at the two ends of the beam. 
A simplified example of the model used in this study is presented in Figure 2.4, where the diaphragm beam of length of $L$ is divided into 6 one-dimensional elements of equal length $l$, and supported at the two ends by springs of stiffness $K_{b}$.

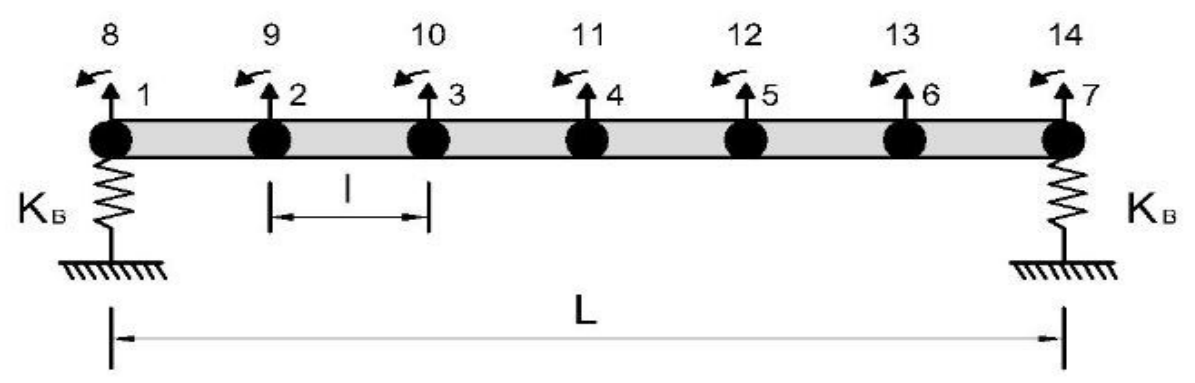

Figure 2.4 - The simple model for representing one-storey buildings with flexible diaphragms

\subsubsection{Stiffness Matrices}

In the model shown in Figure 2.4, the beam-spring system has 14 degrees of freedom. By representing each rotational degree of freedom as $l \theta$, and neglecting shear deformations, the stiffness matrix of an individual element becomes:

$\mathbf{k}=\frac{N^{3} E I}{L^{3}}\left[\begin{array}{cccc}12 & -12 & 6 & 6 \\ -12 & 12 & -6 & -6 \\ 6 & -6 & 4 & 2 \\ 6 & -6 & 2 & 4\end{array}\right]$

in which EI represents the flexural rigidity of the diaphragm and $N$ is the number of elements. Assembling the element matrices at the global level and including the stiffness of spring supports yields the following global matrix: 
$\mathbf{K}_{S}=\frac{N^{3} E I}{L^{3}}\left[\begin{array}{ll}\mathbf{K}_{A A} & \mathbf{K}_{A B} \\ \mathbf{K}_{B A} & \mathbf{K}_{B B}\end{array}\right]$

where subscript A refers to the translational degrees of freedom, and subscript B refers to the rotational degrees of freedom.

$$
\begin{aligned}
\mathbf{K}_{A A} & =\left[\begin{array}{ccccccc}
12+\alpha & -12 & 0 & 0 & 0 & 0 & 0 \\
-12 & 24 & -12 & 0 & 0 & 0 & 0 \\
0 & -12 & 24 & -12 & 0 & 0 & 0 \\
0 & 0 & -12 & 24 & -12 & 0 & 0 \\
0 & 0 & 0 & -12 & 24 & -12 & 0 \\
0 & 0 & 0 & 0 & -12 & 24 & -12 \\
0 & 0 & 0 & 0 & 0 & -12 & 12+\alpha
\end{array}\right] \\
\mathbf{K}_{A B} & =\left[\begin{array}{ccccccc}
6 & 6 & 0 & 0 & 0 & 0 & 0 \\
-6 & 0 & 6 & 0 & 0 & 0 & 0 \\
0 & -6 & 0 & 6 & 0 & 0 & 0 \\
0 & 0 & -6 & 0 & 6 & 0 & 0 \\
0 & 0 & 0 & -6 & 0 & 6 & 0 \\
0 & 0 & 0 & 0 & -6 & 0 & 6 \\
0 & 0 & 0 & 0 & 0 & -6 & -6
\end{array}\right] \\
\mathbf{K}_{B B} & =\left[\begin{array}{lllllll}
4 & 2 & 0 & 0 & 0 & 0 & 0 \\
2 & 8 & 2 & 0 & 0 & 0 & 0 \\
0 & 2 & 8 & 2 & 0 & 0 & 0 \\
0 & 0 & 2 & 8 & 2 & 0 & 0 \\
0 & 0 & 0 & 2 & 8 & 2 & 0 \\
0 & 0 & 0 & 0 & 2 & 8 & 2 \\
0 & 0 & 0 & 0 & 0 & 2 & 4
\end{array}\right]
\end{aligned}
$$

Also

$$
\mathbf{K}_{B A}=\mathbf{K}_{A B}^{T}
$$


The term $\alpha=\beta / N^{3}$ in Equation 2.3 accounts for the stiffness of the LLRS, when the stiffness of the LLRS is expressed as $K_{b}=\beta E I / L^{3}$.

As pointed out earlier, the shear deformations play an important role in the behaviour of the diaphragm and are usually much greater than flexural deformations. A realistic model must, therefore, account for the effect of shear deformations in the diaphragm In order to make the formulation easier, a shear deformation parameter is defined as follows:

$g=\frac{6 E I}{l^{2} G A_{e}}=\frac{6 E I}{l^{2} G^{\prime} B}$

In this expression, $l$ is the length of an individual diaphragm element, $B$ is the width of the diaphragm, $I$ is its moment of inertia, $A_{e}$ is the effective shear area of the diaphragm, $E$ is the modulus of elasticity of the diaphragm material, $G$ is the shear modulus and $G^{\prime}$ ' is the effective shear stiffness of the diaphragm. On taking the shear deformations into account, the stiffness matrix of an individual element becomes

$$
\mathbf{k}=\frac{N^{3} E I}{L^{3}(1+2 g)}\left[\begin{array}{cccc}
12 & -12 & 6 & 6 \\
-12 & 12 & -6 & -6 \\
6 & -6 & 2(2+g) & 2(1-g) \\
6 & -6 & 2(1-g) & 2(2+g)
\end{array}\right]
$$

The global stiffness obtained from an assembly of the element stiffness matrices given by Equation 2.8 is

$$
\mathbf{K}_{S}=\frac{N^{3} E I}{L^{3}(1+2 g)}\left[\begin{array}{ll}
\mathbf{K}_{A A} & \mathbf{K}_{A B} \\
\mathbf{K}_{B A} & \mathbf{K}_{B B}
\end{array}\right]
$$


Sub-matrices $\mathbf{K}_{\mathrm{AA}}, \mathbf{K}_{\mathrm{AB}}$ and $\mathbf{K}_{\mathrm{BA}}$ will not undergo any changes except that in this case $\alpha=\beta(1+2 g) / N^{3}$. Matrix $\mathbf{K}_{\mathrm{BB}}$ is modified as follows:

$$
\mathbf{K}_{B B}=\left[\begin{array}{ccccccc}
2(2+g) & 2(1-g) & 0 & 0 & 0 & 0 & 0 \\
2(1-g) & 4(2+g) & 2(1-g) & 0 & 0 & 0 & 0 \\
0 & 2(1-g) & 4(2+g) & 2(1-g) & 0 & 0 & 0 \\
0 & 0 & 2(1-g) & 4(2+g) & 2(1-g) & 0 & 0 \\
0 & 0 & 0 & 2(1-g) & 4(2+g) & 2(1-g) & 0 \\
0 & 0 & 0 & 0 & 2(1-g) & 4(2+g) & 2(1-g) \\
0 & 0 & 0 & 0 & 0 & 2(1-g) & 2(2+g)
\end{array}\right]
$$

\subsubsection{Mass Matrix}

In the analytical model used in the study, the mass of the diaphragm is lumped along the translational degrees of freedom and the rotational inertias are neglected. For the model shown in Figure 2.2 the resulting mass matrix becomes:

$$
\mathbf{M}_{A A}=\frac{m L}{N}\left[\begin{array}{ccccccc}
0.5 & 0 & 0 & 0 & 0 & 0 & 0 \\
0 & 1 & 0 & 0 & 0 & 0 & 0 \\
0 & 0 & 1 & 0 & 0 & 0 & 0 \\
0 & 0 & 0 & 1 & 0 & 0 & 0 \\
0 & 0 & 0 & 0 & 1 & 0 & 0 \\
0 & 0 & 0 & 0 & 0 & 1 & 0 \\
0 & 0 & 0 & 0 & 0 & 0 & 0.5
\end{array}\right]
$$

In this equation $m$ is the mass per unit length of the diaphragm and the rotational degrees of freedom have been omitted.

\subsubsection{Condensed Stiffness Matrix and Vibration Equations}

A condensed stiffness matrix should be used to remove the rotational degrees of freedom from the stiffness matrix as well. The condensed stiffness matrix is given by 
$\mathbf{K}=\frac{N^{3} E I}{L^{3}(1+2 g)}\left(\mathbf{K}_{A A}-\mathbf{K}_{A B} \mathbf{K}_{B B}^{-1} \mathbf{K}_{A B}\right)$

In other words, in such model, the translational degrees of freedom are the master degrees of freedom and the rotational degrees of freedom are the slave degrees of freedom.

If $\mathbf{u}$ represents the vector of displacements along the translational degrees of freedom, and $\ddot{\mathbf{u}}_{g}$ represents the vector of ground motion acceleration, Equations 2.13 and 2.14 govern the free vibration of the system and the system response to ground excitations, respectively.

$$
\begin{aligned}
& \mathbf{M u ̈}+\mathbf{C} \dot{\mathbf{u}}+\mathbf{K u}=\mathbf{0} \\
& \mathbf{M u ̈}+\mathbf{C} \dot{\mathbf{u}}+\mathbf{K u}=-\mathbf{M u ̈}_{\mathrm{g}}
\end{aligned}
$$

A beam-spring model similar to that shown in Figure 2.4 is used in the current study; the only difference is that instead of 6 beam elements, the diaphragm has been divided into 20 beam elements to improve the accuracy of the analysis. Thus, instead of 7 nodes, the model actually has 21 nodes along the diaphragm length and hence 21 translational degrees of freedom. Consequently, the lumped mass matrix and the condensed stiffness matrix both are of size $21 \times 21$. The system properties and the other definitions are identical to those described in the simplified example model.

In the current study, each building is therefore modeled as a deep beam supported on two spring supports. The properties of the system and the introduced quantities (i.e. $L, B, m$, $K_{b}$ and so on) are then determined for each building in the database. 


\subsubsection{Assumptions}

As pointed out in Chapter 1, the current study investigates different aspects of the behaviour of one-storey buildings with flexible steel deck roof diaphragms. While this may lead to slightly different assumptions in the work described in individual chapters, most of the assumptions in the study are consistent. Some of these assumptions are presented in the following paragraphs:

Since the supports of the model are relatively close, both undergo the same earthquake induced ground motion. Consequently, the unsymmetrical modes of vibration for the beam make negligible contribution to the response of the system. Rayleigh damping is used in the analysis. It is a sound proposition that when proportional Rayleigh damping is being used, the damping matrix should be determined such as to provide selected values of damping ratios in the modes that make the major contribution to the response of the system. Based on this consideration, the first and the third modes have been selected in the derivation of proportional Rayleigh damping matrices.

In the study presented in Chapters 3, 4, 5 and 6 it is assumed that the nonlinearity is confined to the elements of the lateral load resisting system (LLRS) and the diaphragm is assumed to behave in a linear elastic manner. However, in Chapter 7, a nonlinear behaviour is assumed for the diaphragm while the elements of LLRS elements may remain elastic or be strained into the inelastic range. The stress-strain relationship under cycling loading (hysteretic behaviour) of the LLRS is different in each portion of the study. In Chapter 3, elasto-plastic behaviour is assumed for LLRS in investigating the effect of diaphragm flexibility on the ductility demand on the lateral load resisting 
system. In chapter 4, a bi-linear force-displacement relationship is assigned to the LLRS with 3 different post-yield stiffness values of $\alpha=0.02,0.05$ and 0.1 to account for the impact of post-yield stiffness on the ductility demand on the LLRS. In Chapter 5, all four force-displacement relationships are considered for the LLRS $(\alpha=0.00,0.02,0.05$ and 0.1). In Chapter 6, an attempt is made to account for the effects of pinching behaviour in the hysteretic response of LLRS on the total response of the system by assuming a pinching behaviour for the lateral load resisting system. In Chapter 7, a nonlinear forcedisplacement relationship is assigned to the diaphragm in order to investigate the effect of such behaviour on the overall response of the structure. Further assumptions regarding material behaviour are presented in individual chapters. 


\section{Chapter 3 : Ductility Demand on Lateral Load Resisting System}

\subsection{Introduction}

As pointed out earlier, most previous research studies have shown that the flexibility of the diaphragm causes an increase in the ductility demand on the lateral load resisting elements of buildings subjected to earthquake ground motion. In buildings with rigid diaphragms, the ductility demand on the lateral load resisting elements is roughly equal to the force reduction factor that is applied to the elastic base shear to obtain the design shear. In buildings with flexible diaphragms, however, this is no longer the case. The results of inelastic time history analyses of buildings with flexible diaphragms, presented in this chapter, show that a force modification factor of 3 , as an example, may lead to a ductility demand much greater than 3 and in some cases even as high as 20. Evidently, for such buildings the force reduction factor used to scale down the elastic shear must be significantly smaller than the ductility capacity. The present study investigates the ductility demand in buildings with flexible diaphragms with a view to determine appropriate values for the force modification factor. This force reduction factor must lead to reasonable ductility demands which are achievable with current practical detailing of structural members.

Humar and Popovski (2013) have studied the effect of diaphragm flexibility on the ductility demand on lateral load resisting system. They carried out time history analyses on 65 single-storey buildings with flexible steel deck diaphragm designed by Tremblay and Stiemer (1996) for their response to El Centro 1941 ground motion as well as to 5 other ground motions that were compatible with the uniform hazard spectrum (UHS) for 
Vancouver. The spectrum compatible ground motions were generated by Atkinson (2009). The records selected for time history analyses were M6C1, M6C2, M6C26, M6C31 and M6C38 scaled by factors of $0.78,0.87,1.19,0.99$ and 1.43, respectively. Based on the results of their study, Humar and Popovski (2013) suggested an equation relating the force reduction factor to the ductility demand on the lateral load resisting system and the drift ratio $(r)$. The latter was defined as the ratio of the maximum horizontal deformation of the diaphragm along its length to the average inter-story drift of the lateral load resisting elements produced by the action of uniform static lateral load acting along the length of the diaphragm. Equation 3.3 shows the calculation of drift ratio. Maximum horizontal deformations of the diaphragm $\left(\Delta_{\mathrm{D}}\right)$ as well as the average storey drift of the lateral load resisting elements $\left(\Delta_{\mathrm{B}}\right)$ are shown in Figure 3.1. The proposed relationships are as follows:

$$
\begin{aligned}
& R_{y}=\kappa \mu \\
& \kappa=-0.5 r+1.5 \\
& 1 \geq \kappa \geq 0.64 \text { for } \mu=2 \\
& 1 \geq \kappa \geq 0.5 \text { for } \mu=3 \\
& 1 \geq \kappa \geq 0.4 \text { for } \mu=4
\end{aligned}
$$

where $R_{y}$ is the force reduction factor, $\mu$ is the ductility demand and $\boldsymbol{r}$ is the drift ratio defined by

$$
r=\frac{\Delta_{D}}{\Delta_{B}}
$$


Naturally, the upper limit on $\kappa$ values should be 1.0 , since the force modification factor is always less than or equal to the ductility demand. Humar and Popovski (2013) obtained the lower limits on $\kappa$ by mapping the results from their response analysis of the buildings subjected to generated ground motions that were compatible for the uniform hazard spectrum for the western regions, specifically for Vancouver. The validity of these lower limits should be confirmed and the limits revised if necessary for cases where the buildings are subjected to a different set of ground motions.

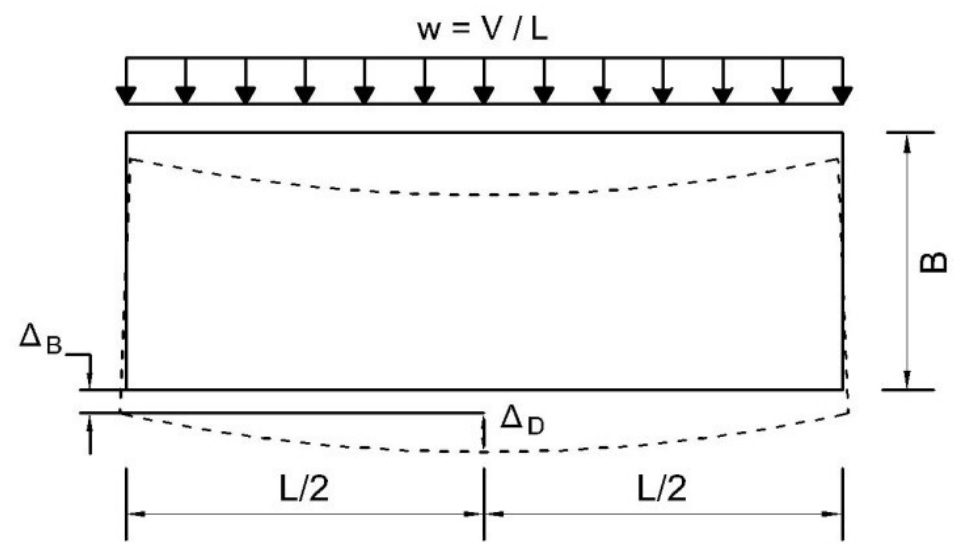

Figure 3.1 - Maximum horizontal deformation of diaphragm $\left(\Delta_{D}\right)$ and average inter-storey drift $\left(\Delta_{B}\right)$

An alternative method for determining the force modification factor has been recommended by Tremblay ${ }^{1}$. The modification factor is again related to the ductility demand on the lateral load resisting system and the drift ratio in the diaphragm defined in

\footnotetext{
${ }^{1}$ Personal communication
} 
Equation 3.3. The procedure through which Tremblay has derived the equation as well as the equation itself is presented in the following paragraphs:

Consider two springs in series. Spring 1 has stiffness $K_{b}$ and represents the brace; Spring 2 has stiffness $K_{D}$ and represents the diaphragm. Assume that the whole system behaves elastically and attracts a force $F$ applied at the end of second spring. The total displacement is given by

$$
\Delta_{e}=\frac{F}{K_{b}}+\frac{F}{K_{D}}=\Delta_{B}+\Delta_{D}
$$

Now suppose we design the system to yield at a force of $F / R_{y}$ and assume also that only Spring 1 is yielding but Spring 2 is still elastic. The displacement is now given by

$$
\Delta_{i}=\frac{F}{R_{y} K_{b}} \mu+\frac{F}{R_{y} K_{D}}=\frac{\mu}{R_{y}} \Delta_{B}+\frac{1}{R_{y}} \Delta_{D}
$$

where $\mu$ is the ductility in brace.

Now assuming that the equal displacement theory is valid, we get

$$
\begin{aligned}
& \Delta_{i}=\Delta_{e} \\
& R_{y}=\frac{\mu+\Delta_{D} / \Delta_{B}}{1+\Delta_{D} / \Delta_{B}}
\end{aligned}
$$

By combining equations (3.6) and (3.3), the equation proposed by Tremblay becomes:

$$
R_{y}=\frac{\mu+r}{1+r}
$$

In equation 3.7, $R_{y}$ is the force reduction factor, $\mu$ is the ductility demand, and $\boldsymbol{r}$ is the drift ratio. 


\subsection{Chapter Objectives}

In this chapter, we investigate whether the relationships based on the results of time history analyses of the selected buildings for ground motions compatible with the UHS for the western region of Canada (Vancouver) are also valid for the eastern region. Specifically, validation is carried out for ground motions that are compatible with the UHS for Montreal. In addition, the limiting values of $\kappa$ given in Equation 3.2 are evaluated by reference to a more comprehensive database of analytical results. Also, the proposed method for calculating the force reduction factor is compared to the method suggested by Tremblay.

\subsection{Response to Eastern ground motions}

The response of one-storey buildings with flexible diaphragms when subjected to eastern compatible ground motions is studied for confirmation of, and if necessary, a revision of the equation suggested by Humar and Popovski (2013)

\subsubsection{Analytical Model and Assumptions}

In order to study the response of one-storey buildings to eastern compatible ground motions, the 65 selected buildings (32 buildings for the case when the load is acting parallel to the long side of the building and 33 buildings for the case when the load is acting parallel to the short side of the system) from the database of buildings designed by Tremblay and Stiemer (1996) are subjected to the generated ground motions that are compatible with the UHS for the eastern region (Atkinson 2009). Records E6C1, E6C13, E6C15, E6C18 and E6C42 scaled by factors of $0.55,0.74,0.56,0.61$ and 1.01, 
respectively, are selected for this purpose. These scaled ground motion time histories match the UHS for Montreal. As described in Section 2.5 of Chapter 2, the buildings are modelled as deep beams (diaphragm) supported on two springs (LLRS). In addition, it is assumed that the nonlinearity in the system is confined to the lateral load resisting system (the springs in the analytical model). Elasto-plastic hysteretic behaviour with no postyield hardening ( $\alpha=0.00)$ is assumed for the lateral load resisting elements, while a linear elastic behaviour is assumed for the diaphragm (the beam in the analytical model). The assumption of elasto-plastic force displacement relationship, as opposed to a bilinear relation exhibiting post-yield hardening, is a conservative assumption since elasto-plastic behaviour is likely to give larger values for the ductility demand. Additional details related to the analytical model and properties of the system are presented in Section 2.5.

\subsubsection{Nonlinear Dynamic Analyses}

In the response analyses, carried out with the help of a MATLAB program developed for the purpose, direct time step integration of the equations of motion is carried out using average acceleration method. The time-step used in the analyses is $0.001 \mathrm{~s}$. Proportional Rayleigh damping of $5 \%$ is assumed for the first and the third mode. For better comparison and in order to confirm that the analyses for buildings subjected to eastern ground motions match the results of those from the studies carried out earlier for western motions by Humar and Popovski (2013), the latter sets of analyses are repeated. Furthermore, the analysis is based on models and assumptions that are identical to those used in Humar and Popovski study. 
Analyses are carried out first to determine the ductility demands on the lateral load resisting system, which has been designed assuming a force modification factor of 3 $\left(R_{y}=3\right)$. This set of analyses helps to confirm that ductility demand can significantly exceed the force modification factor and to establish the need for the present study. Response analyses are then carried out in the reverse order to determine the force reduction factors that will lead to ductility demands of 2, 3 and 4, respectively. This way the $\kappa$ value introduced earlier can be determined as the ratio of the force modification factor $\left(R_{y}\right)$ to the ductility demand $(\mu)$ for each case. This portion of the analytical study requires several iterations for each building model, each earthquake ground motion, and each ductility capacity. The results of the analyses are presented in the tables A.3 to A.22 in Appendix A.

\subsection{Development of Relationship between $\kappa$ and the Drift Ratio}

The values of $\kappa$ for each building reported in Tables A.3 to A.22 are plotted against the corresponding drift ratios in Figures 3.2 to 3.7. These plots represent the results for all 65 building models when subjected to ground motions compatible with eastern and western spectra and the three target ductilities of 2,3 and 4 .

To find an appropriate relationship between $\kappa$ and drift ratio and to validate the equation suggested by Humar and Popovski.(2013), a regression fit is carried out on the data corresponding to the cases in which the ground motion is parallel to the long side of the building (smaller values of $r$ ). In addition, the average value of $\kappa$ obtained from the data corresponding to cases for which the earthquake ground motion is parallel to the short side of the building (larger values of $r$ ), is selected as the lower limit of $\kappa$. Naturally, the 
upper limit on $\kappa$ should be 1 , as suggested by Humar and Popovski, since the force reduction factor is generally smaller than the ductility demand.

The reason for choosing the foregoing approach for finding the relationship between $\kappa$ and the drift ratio is simply because of the manner in which the data are distributed. For smaller values of $r$, there is considerable scatter in the data, whereas for larger values of $r$ the scatter is comparatively less and the $\kappa$ values do not vary much with changing $r$.

The regression lines are also shown in Figures 3.2 to 3.7.

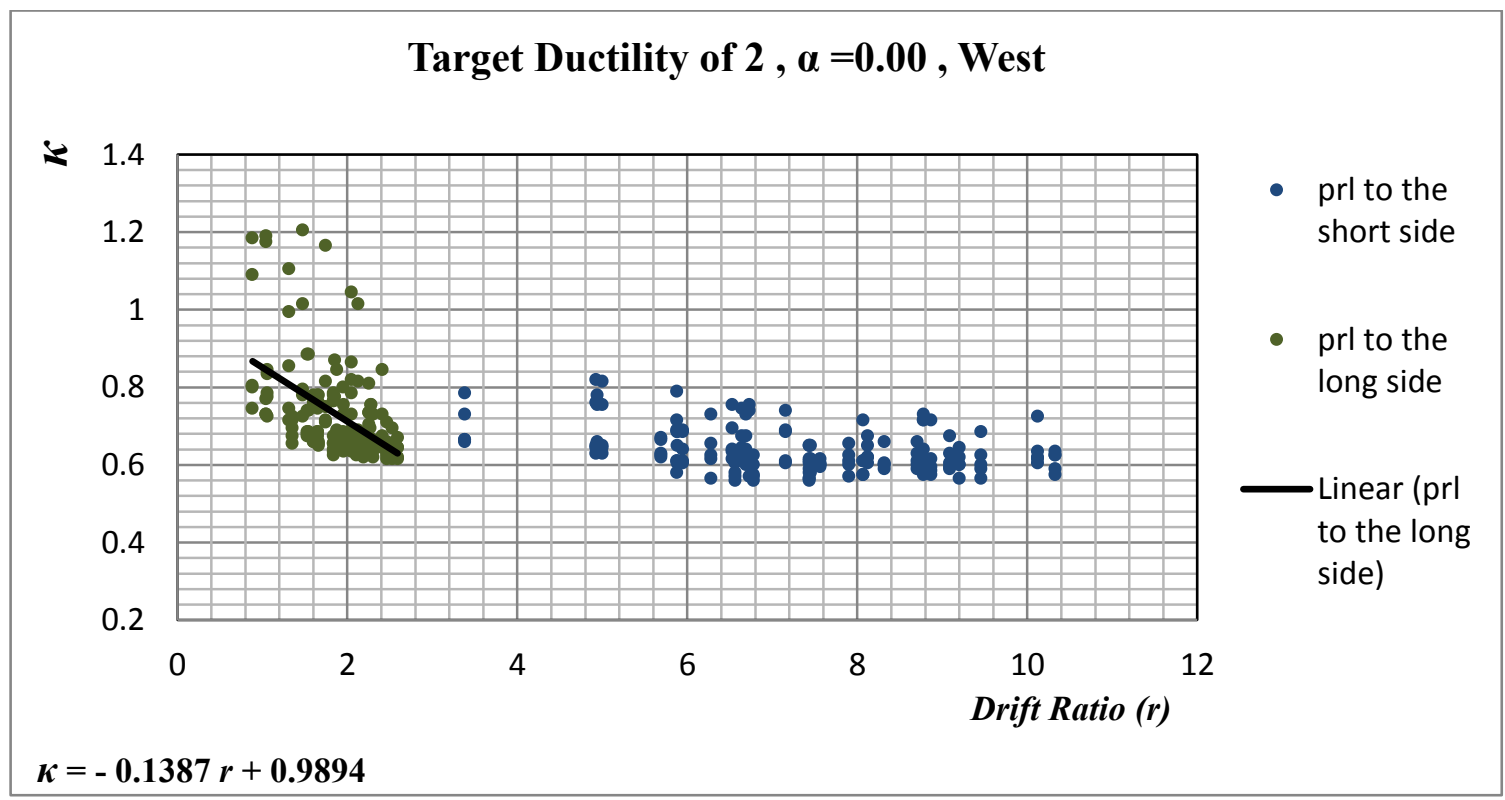

Figure 3.2 - Variation of $\kappa$ with drift ratio corresponding to the response of buildings to ground motions compatible with the UHS for Vancouver; target ductility 2 and elasto-plastic behaviour for $\operatorname{LLRS}(\boldsymbol{\alpha}=\mathbf{0 . 0 0})$ 


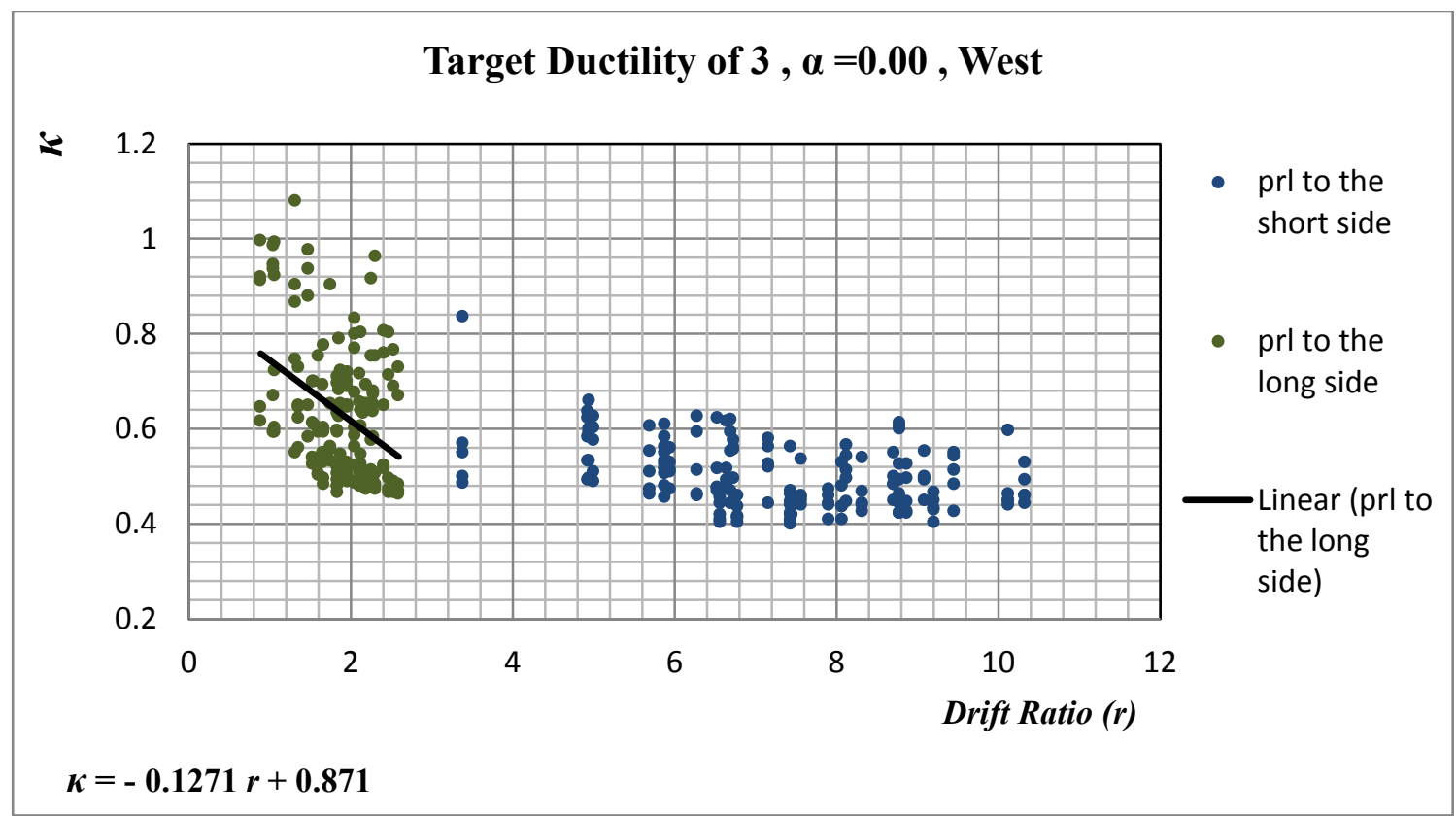

Figure 3.3 - Variation of $\kappa$ with drift ratio corresponding to the response of buildings to ground motions compatible with the UHS for Vancouver; target ductility 3 and elasto-plastic behaviour for $\operatorname{LLRS}(\boldsymbol{\alpha}=\mathbf{0 . 0 0})$

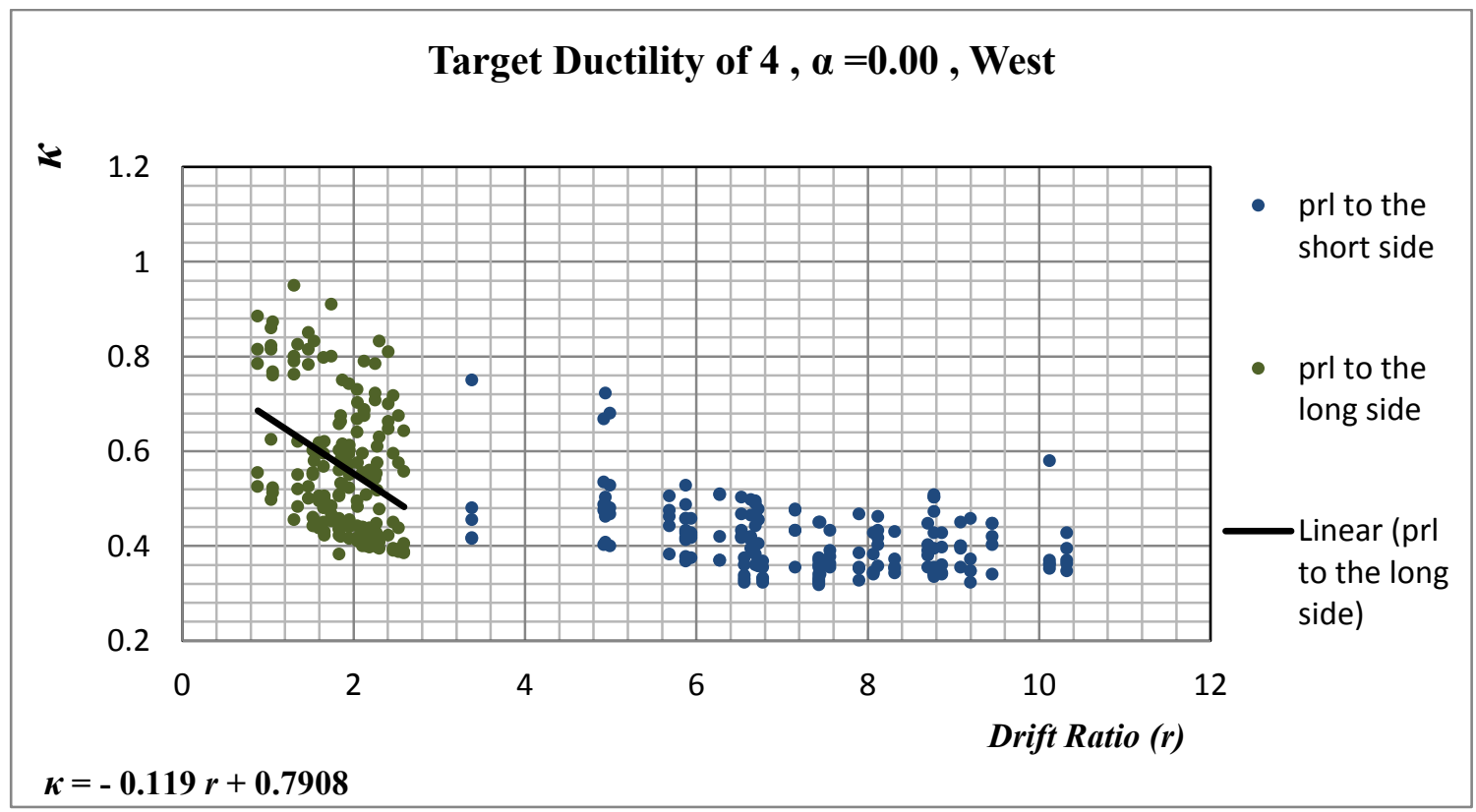

Figure 3.4 - Variation of $\kappa$ with drift ratio corresponding to the response of buildings to ground motions compatible with the UHS for Vancouver; target ductility 4 and elasto-plastic behaviour for LLRS $(\boldsymbol{\alpha}=\mathbf{0 . 0 0})$ 


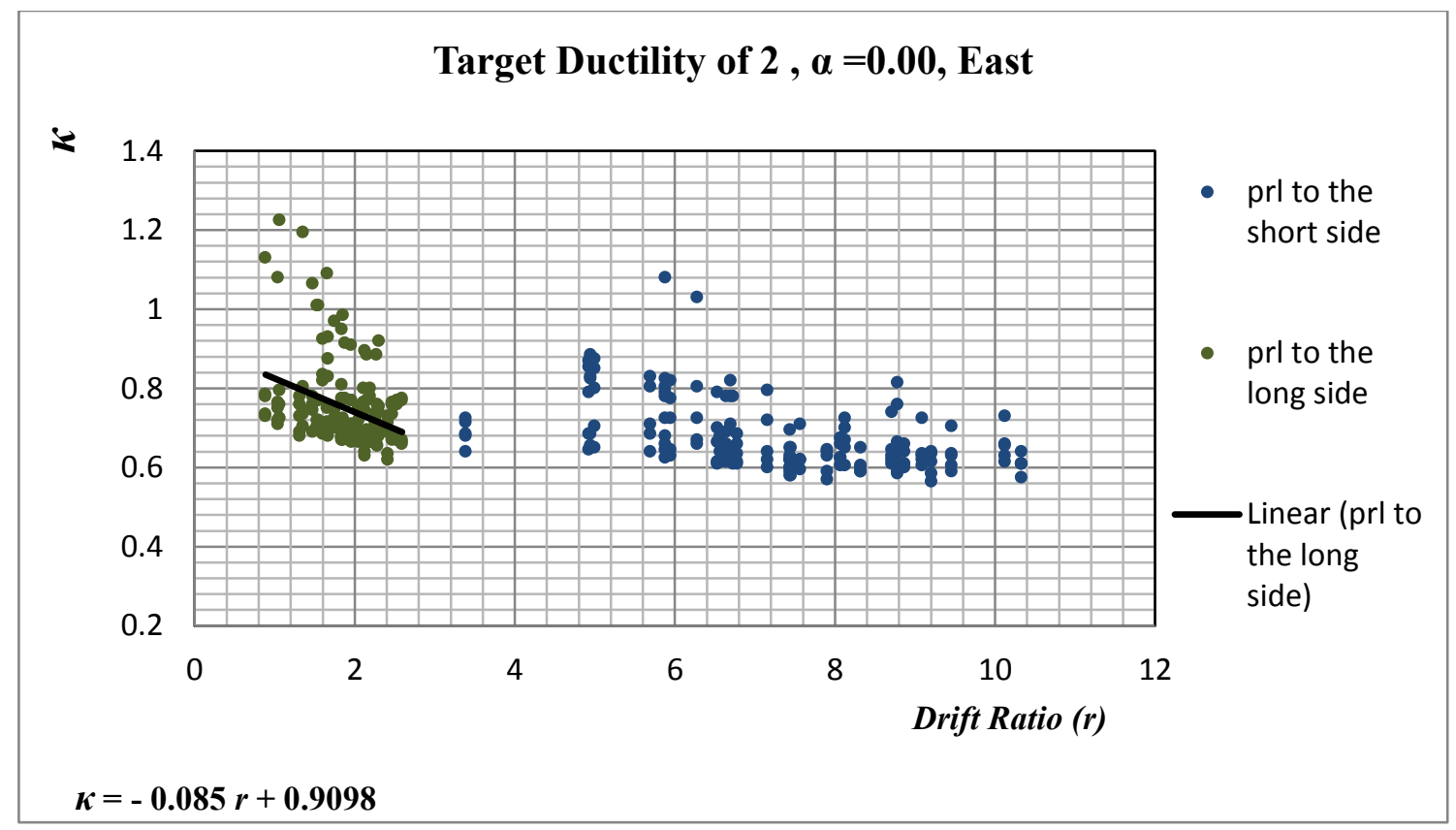

Figure 3.5 - Variation of $\kappa$ with drift ratio corresponding to the response of buildings to ground motions compatible with the UHS for Montreal; target ductility 2 and elasto-plastic behaviour for $\operatorname{LLRS}(\boldsymbol{\alpha}=\mathbf{0 . 0 0})$

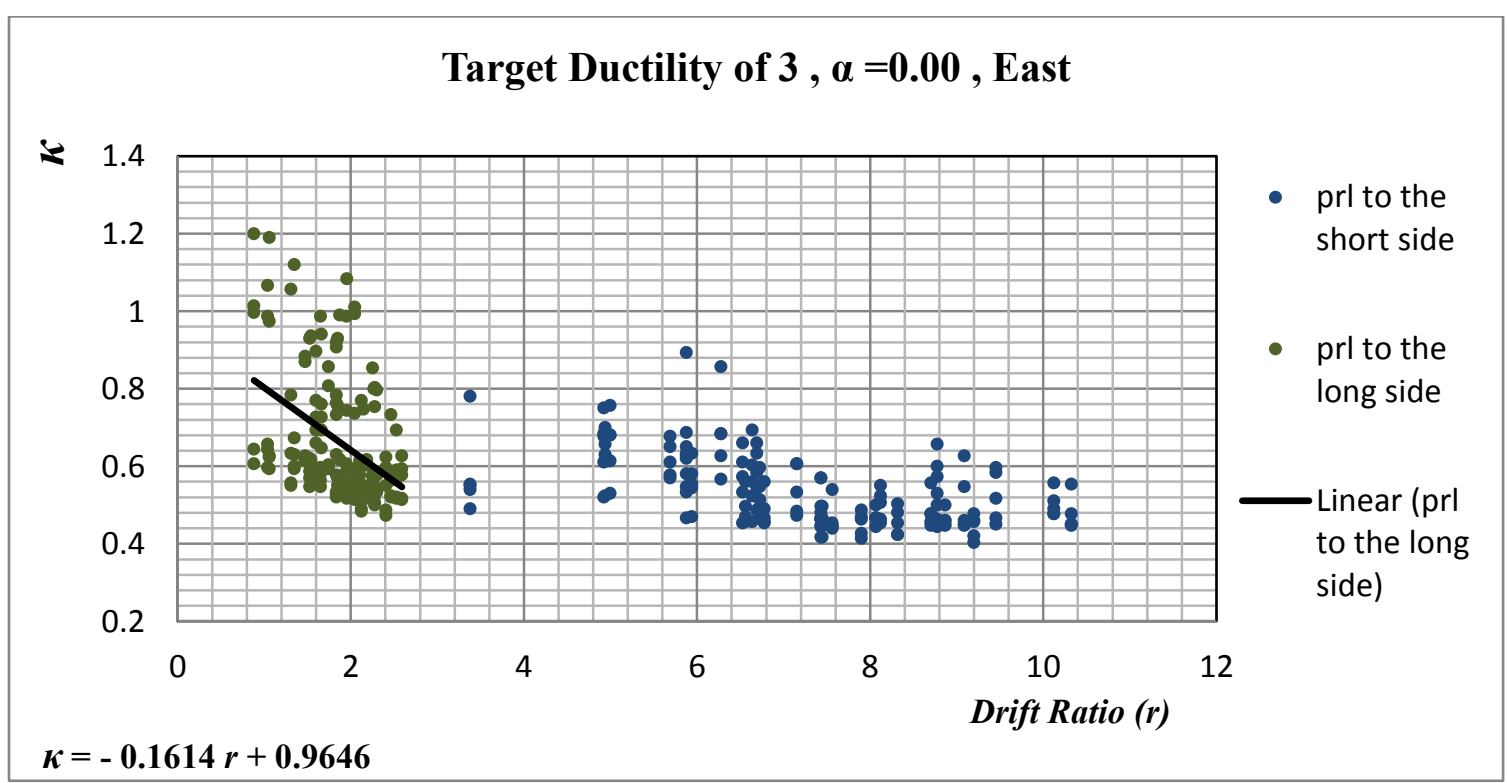

Figure 3.6 - Variation of $\kappa$ with drift ratio corresponding to the response of buildings to ground motions compatible with the UHS for Montreal; target ductility 3 and elasto-plastic behaviour for $\operatorname{LLRS}(\alpha=0.00)$ 


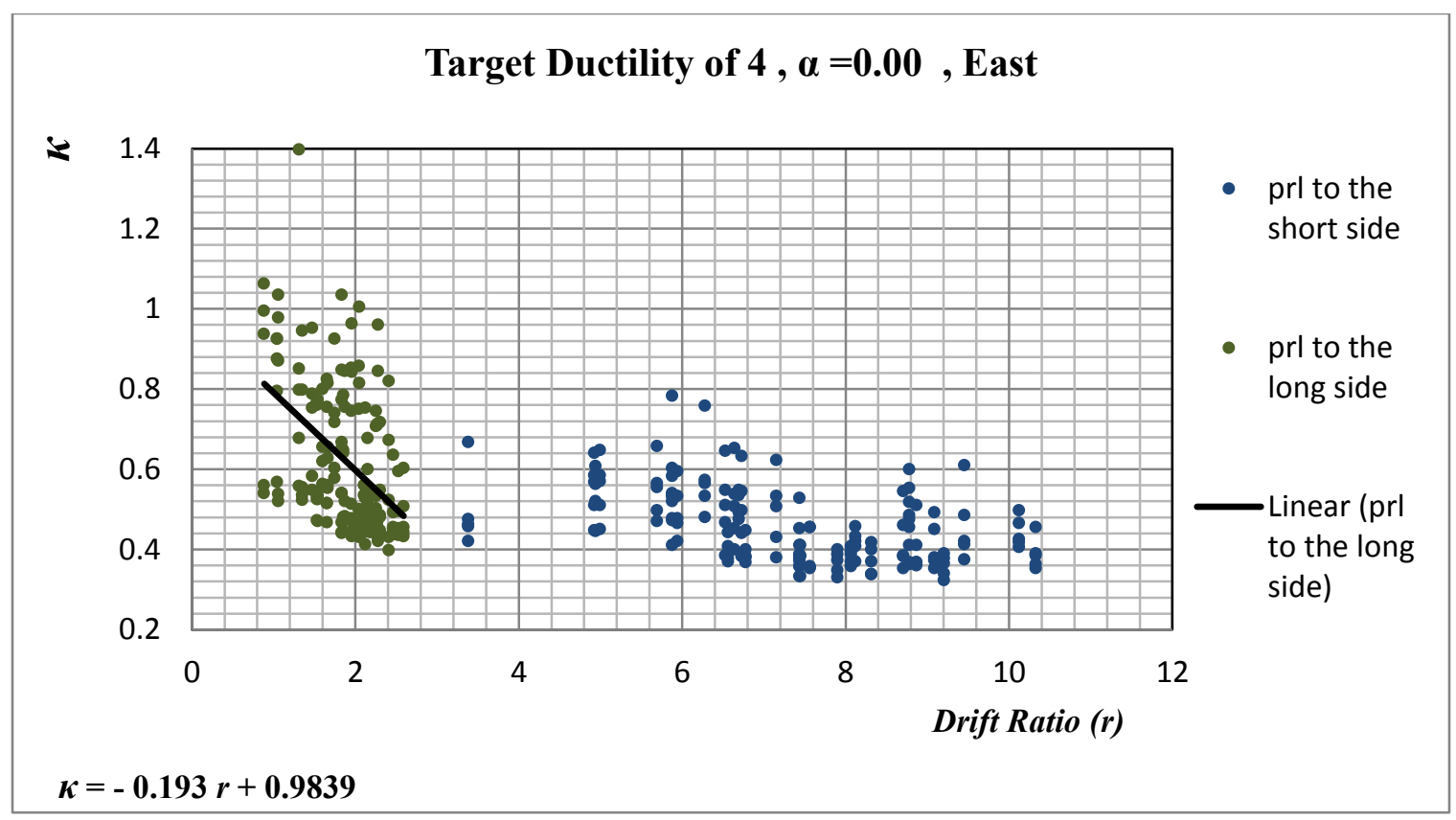

Figure 3.7 - Variation of $\kappa$ with drift ratio corresponding to the response of buildings to ground motions compatible with the UHS for Montreal; target ductility 4 and elasto-plastic behaviour for $\operatorname{LLRS}(\boldsymbol{\alpha}=\mathbf{0 . 0 0})$

It is evident from the plots that the regression fits on the portion of the data corresponding to the case in which the ground motion is applied parallel to the long side of the building (smaller values of $\boldsymbol{r}$ ), needs to be modified and simplified in order to obtain a consistent relationship for all cases. As can be seen from Figures 3.2 to 3.7, the data corresponding to smaller values of drift ratios are denser in the region with values of $\kappa$ ranging between about 0.4 and 0.7 , causing the regression fit to have a smaller slope and a smaller intercept. The equation suggested by Humar and Popovski (2013) provides a more reasonable and more consistent value for $\kappa$, making it more suitable for practical design. However, since there is a difference in the lower limits on $\kappa$ for eastern and western spectra, a modification on the lower limit may prove useful. 
Plots of $\kappa$ versus the drift ratio corresponding to all values of the ductilities, namely 2, 3 and 4, are presented in Figures 3.8 and 3.9 for the western spectrum and eastern spectrum, respectively, and compared with the equation suggested by Humar and Popovski (2013). The lower limits obtained by averaging the data are also shown in the graphs.

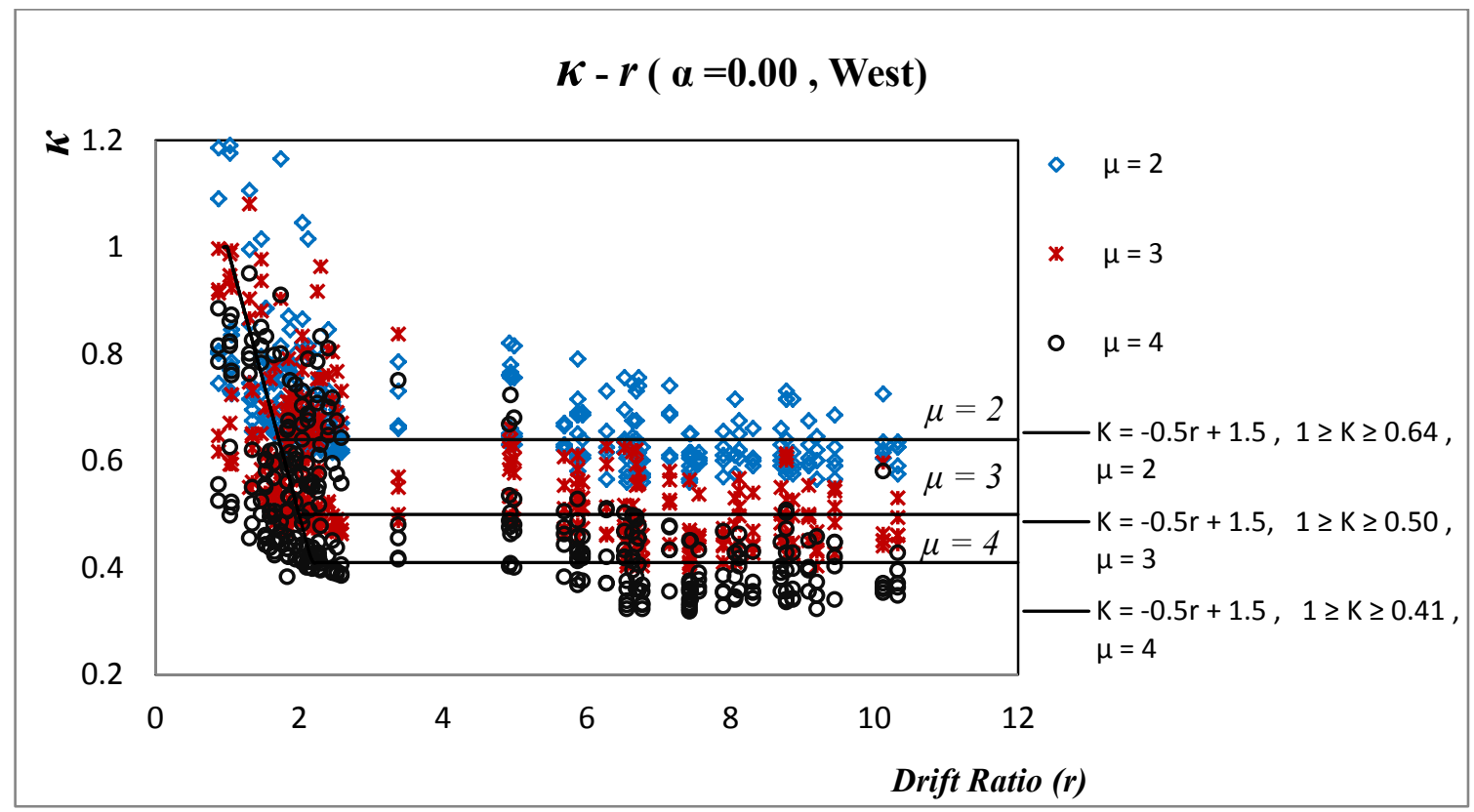

Figure 3.8 - Variation of $\kappa$ with drift ratio for target ductilities 2, 3 and 4 for buildings subjected to ground motions compatible with the UHS for Vancouver, elasto-plastic behaviour for LLRS $(\alpha=0.00)$ Humar and Popovski (2013) (with the authors' permission and C Canadian Science Publishing or its licensors) 


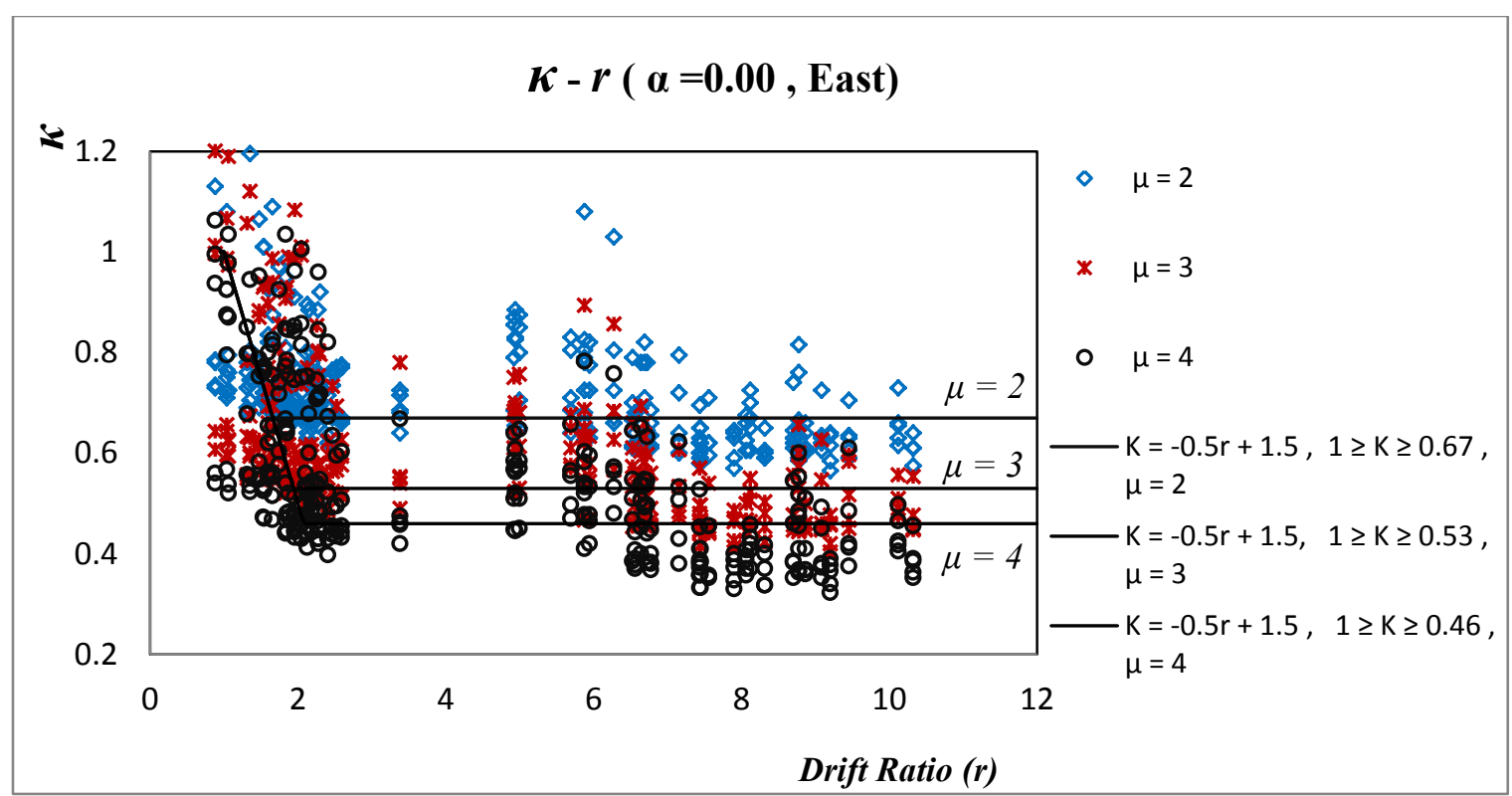

Figure 3.9 - Variation of $\kappa$ with drift ratio for target ductilities 2, 3 and 4 for buildings subjected to ground motions compatible with the UHS for Montreal, elasto-plastic behaviour for LLRS $(\alpha=0.00)$

The lower limits on $\kappa$ values for the various cases (two spectra and three targets of ductility demand) are summarized in Table 3.1:

Table 3.1 - The lower limits on $\kappa$ without post-yield hardening

\begin{tabular}{lccc}
\hline & \multicolumn{3}{c}{ Ductility Targets } \\
\cline { 2 - 4 } & $\mu=2$ & $\mu=3$ & $\mu=4$ \\
\hline Eastern Compatible Ground Motions & 0.67 & 0.53 & 0.46 \\
\hline Western Compatible Ground Motions & 0.64 & 0.50 & 0.41 \\
\hline
\end{tabular}

It will be observed that while the $\kappa$ versus drift ratio relationships in the region of lower values of the drift ratio are similar for the west and the east, the lower limits for the two zones are somewhat different. Since such difference is not large it should be possible to 
develop a single set of relationship for both zones to simplify the design process. In order to obtain consistent lower limits, all the data obtained from all cases, that is data from applying eastern and western ground motions to all 65 buildings when there is no postyield hardening in the LLRS $(\alpha=0.00)$, are compiled into one database and the lower limits are calculated accordingly. The results are presented in one graph in Figure 3.10.

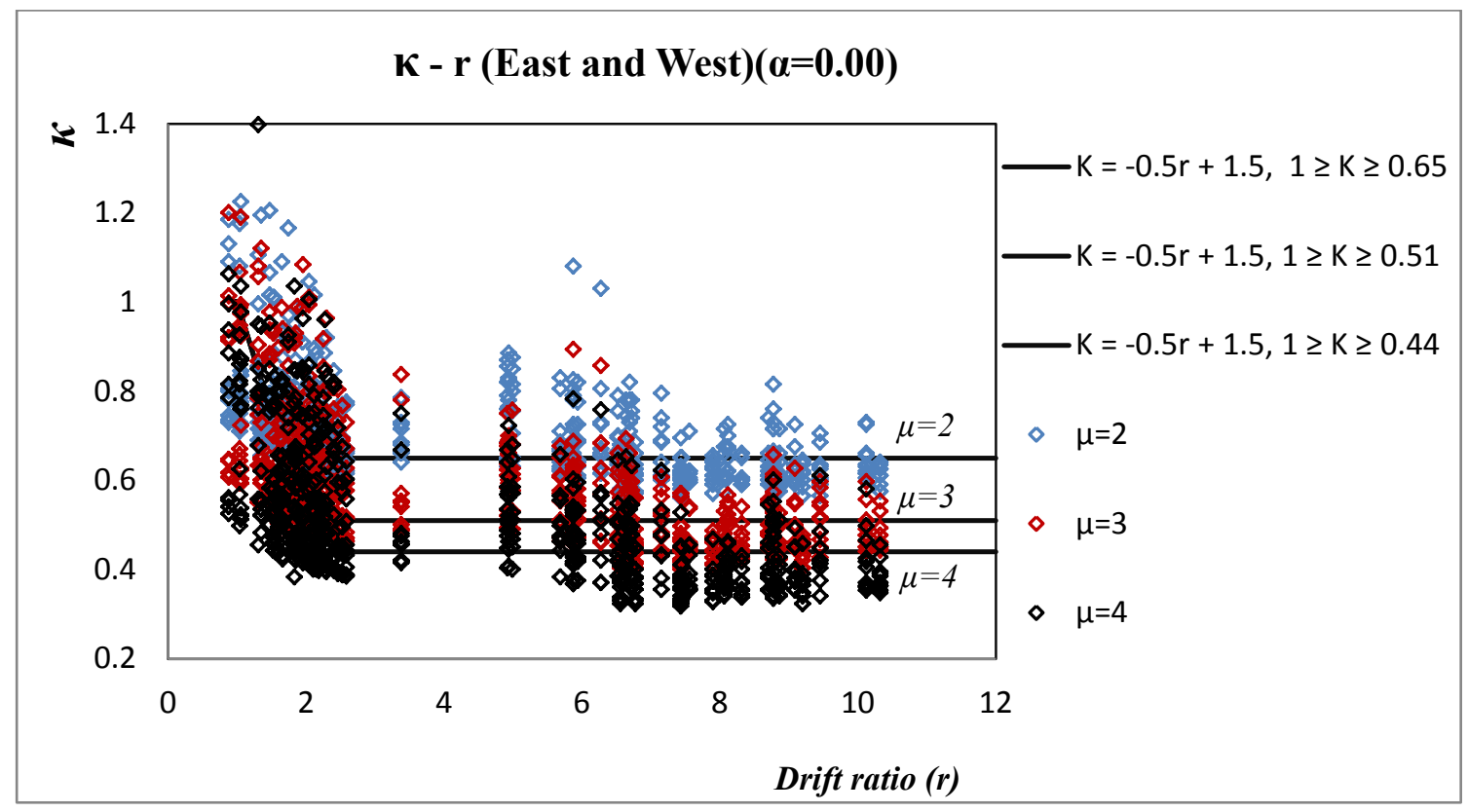

Figure 3.10 - Variation of $\kappa$ with drift ratio for target ductilities 2, 3 and 4 for buildings subjected to ground motions compatible with the UHS for Vancouver and Montreal, elasto-plastic behaviour for LLRS $(\boldsymbol{\alpha}=\mathbf{0 . 0 0})$

Use of the more comprehensive database indicates that Equation 3.2 may be revised as follows. The revised equation is applicable to all zones of Canada. 
$\kappa=-0.5 r+1.5$

$\kappa \geq 0.65$ for $\mu=2$

$\kappa \geq 0.51$ for $\mu=3$

$\kappa \geq 0.44$ for $\mu=4$

\subsection{Comparison between the Revised Equation and Tremblay's Method}

As mentioned earlier, Tremblay has proposed that Equation 3.7 may be used to determine the force reduction factor based on ductility demand and the drift ratio. Figures 3.11 to 3.16 compare the equation proposed by Tremblay and the equation developed in the previous sections with the data obtained from the analyses. Comparisons have been done for each case separately (three values of ductility demand and two spectra) which leads to 6 cases in total.

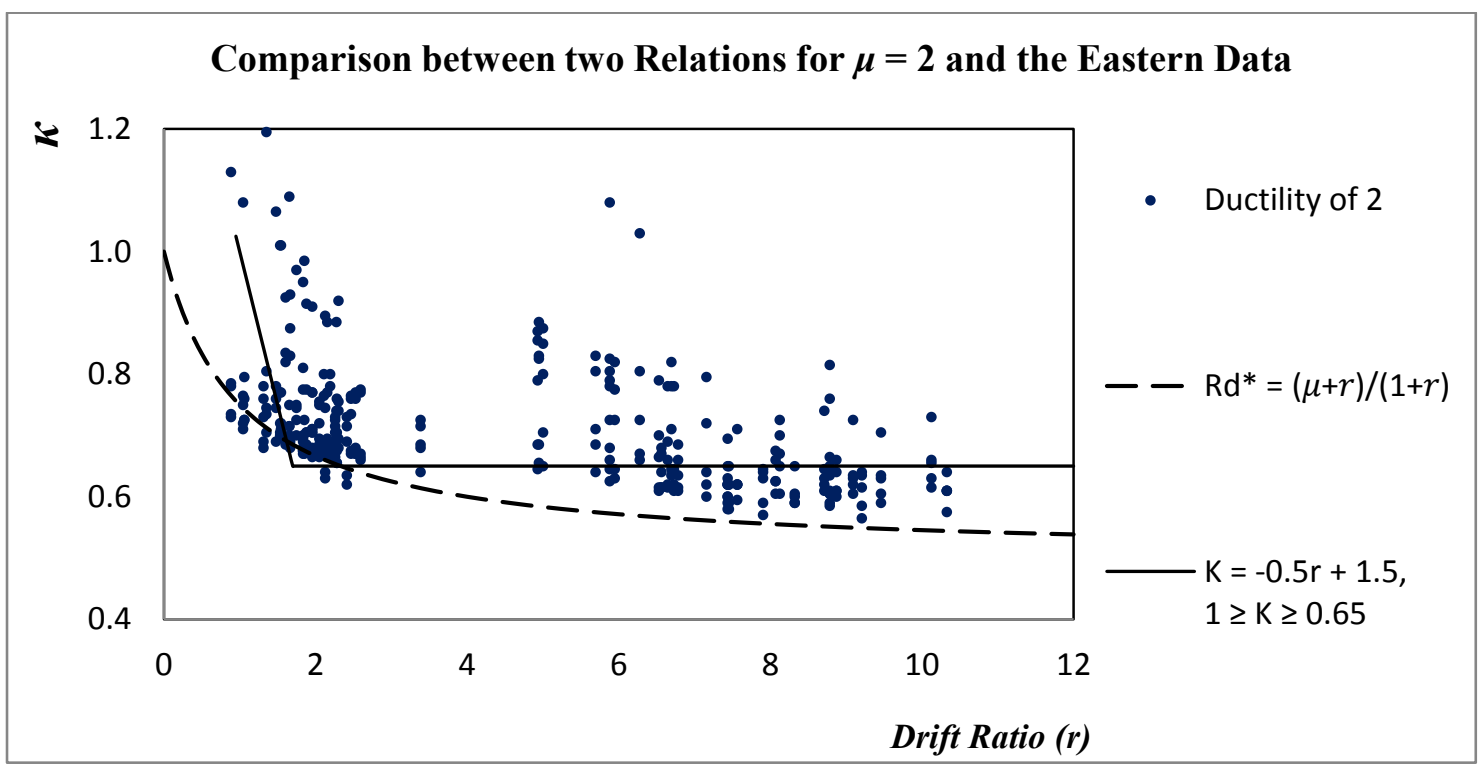

Figure 3.11 - Comparison between Equation 3.8 and Equation 3.7 for target ductility of 2 when the buildings are subjected to ground motions compatible with the UHS for Montreal 


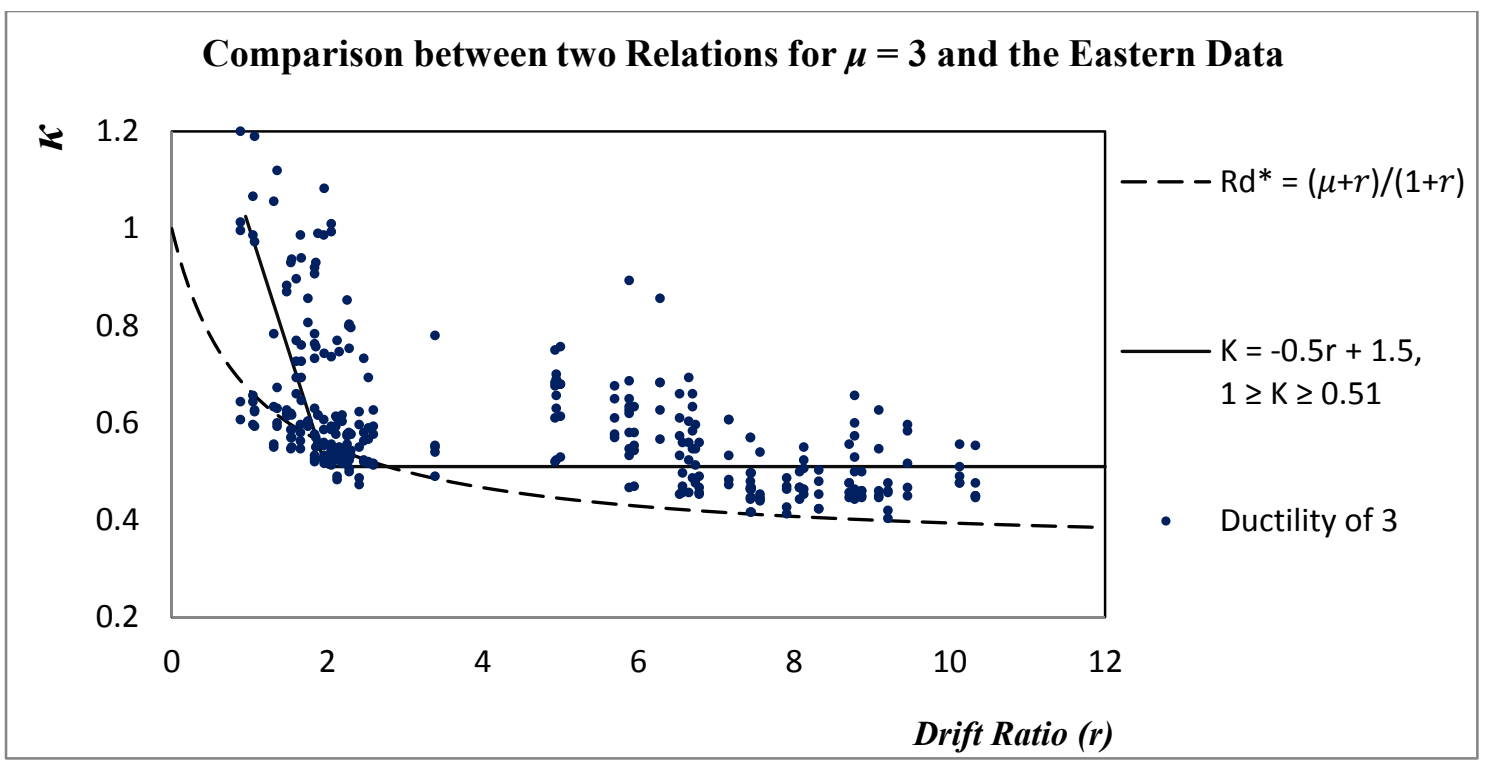

Figure 3.12 - Comparison between Equation 3.8 and Equation 3.7 for target ductility of 3 when the buildings are subjected to ground motions compatible with the UHS for Montreal

\section{Comparison between two Relations for $\mu=4$ and the Eastern Data}

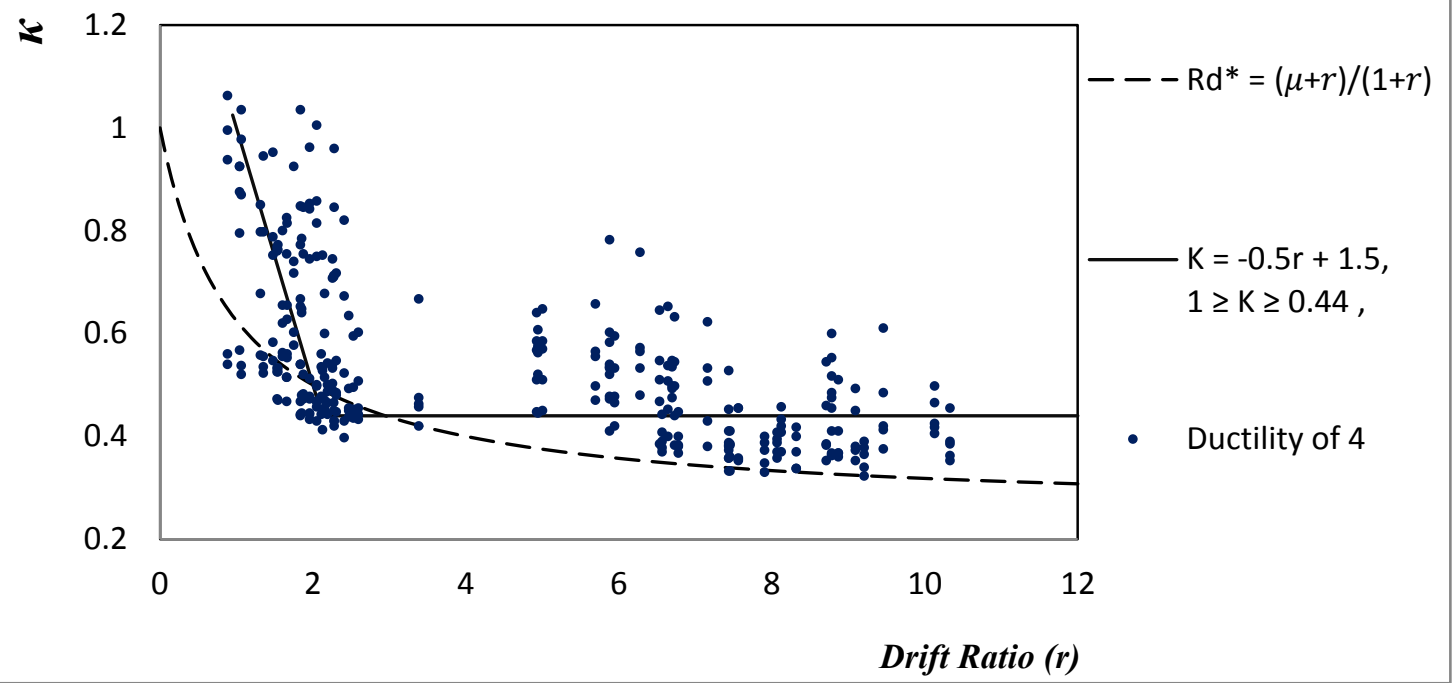

Figure 3.13 - Comparison between Equation 3.8 and Equation 3.7 for target ductility of 2 when the buildings are subjected to ground motions compatible with the UHS for Montreal 


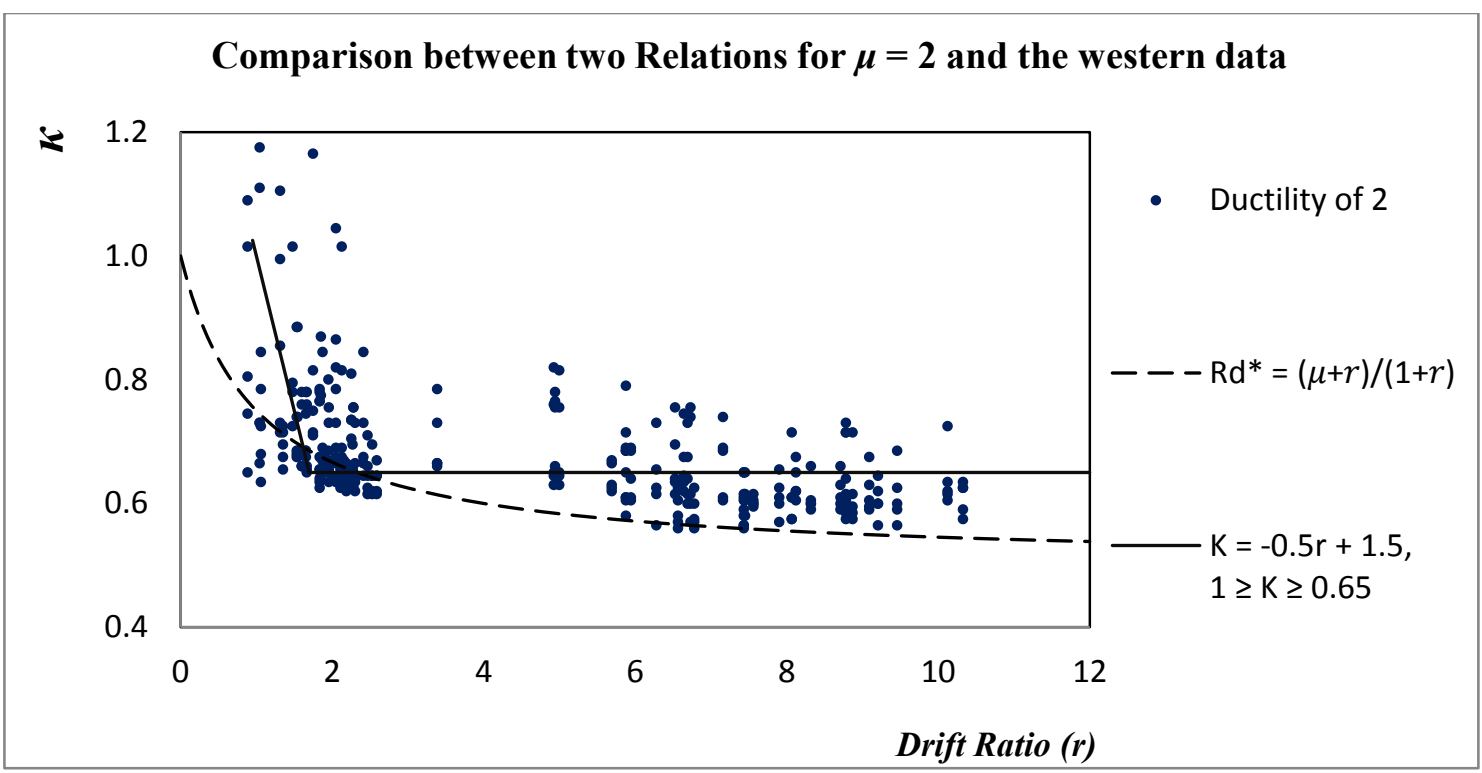

Figure 3.14 - Comparison between Equation 3.8 and Equation 3.7 for target ductility of 2 when the buildings are subjected to ground motions compatible with the UHS for Vancouver

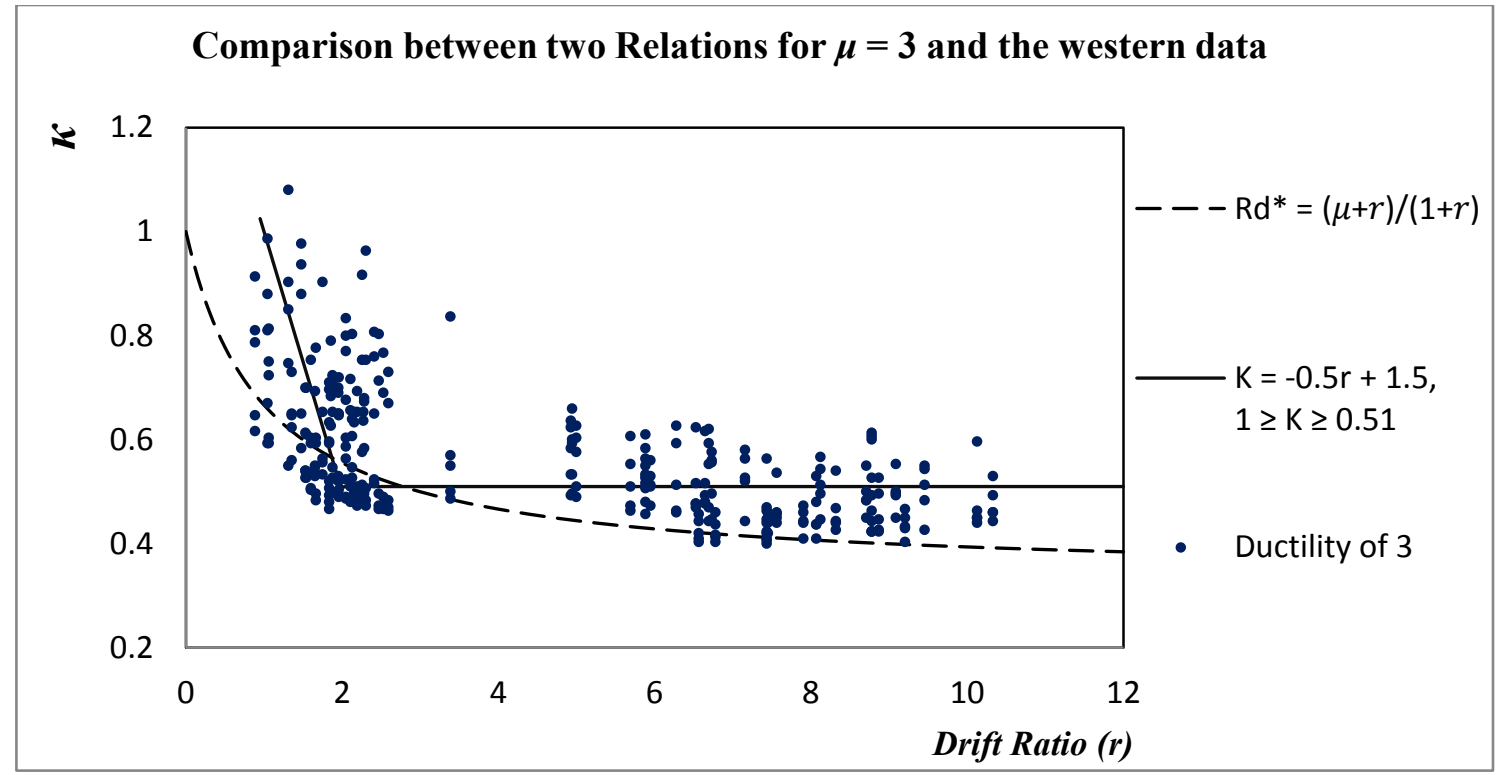

Figure 3.15 - Comparison between Equation 3.8 and Equation 3.7 for target ductility of 3 when the buildings are subjected to ground motions compatible with the UHS for Vancouver 


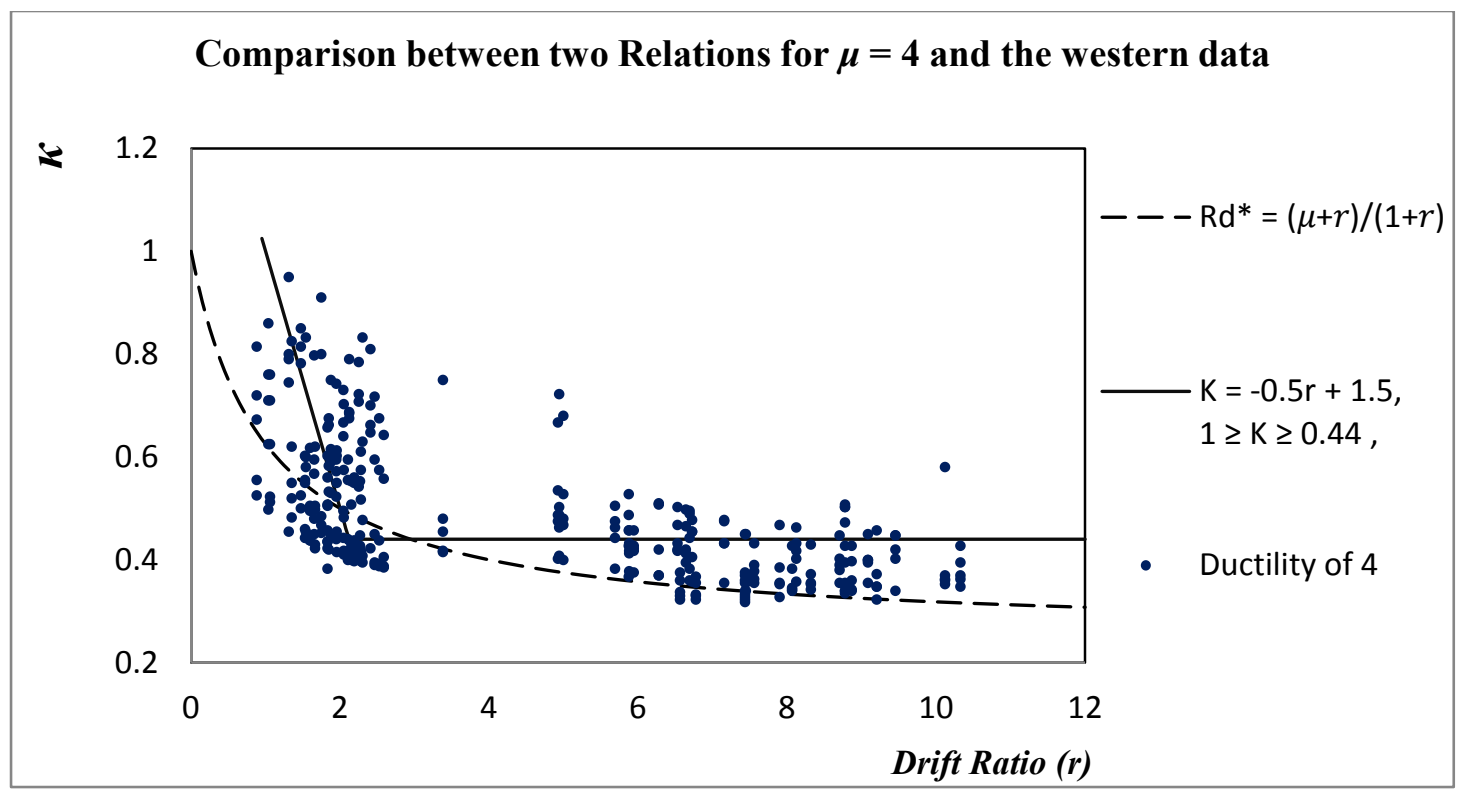

Figure 3.16 - Comparison between Equation 3.8 and Equation 3.7 for target ductility of 4 when the buildings are subjected to ground motions compatible with the UHS for Vancouver

Judging from Figures 3.11 to 3.16, it is clear that Equation 3.7 is quite conservative, and underestimates the force reduction factor values in most cases. The plots show that most data points lie above the curve represented by Equation 3.7, which thus provides lower bound values. In author's view, given the large uncertainties involved in estimating the hazard and in obtaining the nonlinear response, Equation 3.8 is reasonable for use in design.

\subsection{Conclusion and Recommendations}

The results presented in the previous sections lead to the following conclusions:

1. The flexibility of the diaphragm causes a significant increase in the ductility demand on the lateral load resisting system. In many instances such increase is quite large. It is therefore imperative that a smaller force reduction factor be used 
in the design in order to ensure that the ductility demands are achievable with the current detailing techniques.

2. The equation and the method proposed by Humar and Popovski (2013) provide a reasonable method for determining an appropriate force reduction factor for buildings having flexible diaphragms and located within both the western and the eastern regions of Canada.

3. The lower limits on the $\kappa$ value, suggested by Humar and Popovski may be slightly adjusted on the basis of a more comprehensive database of analytical results obtained in this study for both the western and eastern regions. The suggested lower limits are as follows:

- $\quad 0.65$ For the target ductility of 2 .

- $\quad 0.51$ For the target ductility of 3 .

- $\quad 0.44$ For the target ductility of 4 .

4. The relationship between force reduction factor, drift ratio, and ductility demand suggested by Tremblay would generally lead to quite conservative seismic design forces. The equation proposed by Humar and Popovski and slightly modified as a result of this study provides more realistic and economic design. 


\section{Chapter 4 : Effect of Post-Yield Hardening on Brace Ductility Demand}

\subsection{Introduction}

The results of a study on the effect of the flexibility of the diaphragm on the ductility demand on the lateral load resisting system were presented in Chapter 3. As a part of that study, a series of nonlinear dynamic analyses were carried out on selected buildings. It was assumed that the nonlinearity was confined to the lateral load resisting system (LLRS) with an elasto-plastic behaviour and no post-yield stiffness (alpha $=0.00)$. Many materials have significant post-yield hardening effect that could be responsible for sustaining a significant portion of the earthquake energy. When the elements of LLRS are built from such a material, the post-yield hardening would affect the ductility demand on the LLRS and the selection of a proper force reduction factor should take such effect into account. Intuitively, one can make the assertion that post-yield hardening will lead to smaller ductility demands since energy dissipation (area under the force-displacement curve) would be greater. As a result, the use of higher force reduction factors would be justified. The level of decrease in the ductility demand or, in other words, how large this effect can be is a valid subject for study. The fact that no material is perfectly elastoplastic makes it worthwhile to investigate the effect of post-yield hardening on the ductility demand on LLRS and in the selection of force reduction factor in buildings with flexible diaphragms.

The results of a study on the effects of post-yield hardening in the lateral load resisting members on the ductility demand on the LLRS are presented in this chapter. The study includes an investigation of whether the post-yield stiffness in the lateral load resisting 
members will affect the equations developed for determining the force reduction factor. A series of nonlinear dynamic analyses are carried out on the selected buildings with LLRS consisting of material with post-yield hardening for their response to ground motion time histories compatible with the UHS for both the eastern and western regions.

\subsection{Analytical Model and Assumptions}

The 65 selected buildings ( 32 buildings for the case when the load is acting parallel to the long side of the building and 33 buildings for the case when the load is acting parallel to the short side of the system) from the building database designed by Tremblay and Stiemer (1996), introduced earlier, are subjected to the UHS compatible ground motions generated by Atkinson (2009). Records E6C1, E6C13, E6C15, E6C18 and E6C42 scaled by factors of $0.55,0.74,0.56,0.61$ and 1.01 respectively are selected to represent the eastern uniform hazard spectrum (UHS), specifically for Montreal. For ground motions compatible with the UHS for west, specifically for Vancouver, records M6C1, M6C2, M6C26, M6C31 and M6C38 scaled by factors of 0.78, 0.87, 1.19, 0.99 and 1.43, respectively, are used. As described in Section 2.5, the buildings are modelled by deep beams, representing the diaphragm, supported on two springs, representing the LLRS. In addition, it is assumed that the nonlinearity in the system is confined to the lateral load resisting system (the springs in the analytical model). In order to investigate the effects of post-yield hardening in LLRS on the response of the structures, a set of post-yield hardening ratios $(\alpha=0.02, \alpha=0.05$ and $\alpha=0.10)$ are used for the lateral load resisting elements. Linear elastic behaviour is assumed for the diaphragm (the beam in the 
analytical model). Additional details related to the analytical model and properties of the system as well as the ground motions are presented in Section 2.5.

\subsection{Nonlinear Dynamic Analyses}

Using a MATLAB program developed for the purpose, direct time step integration of the equations of motion is carried out using average acceleration method for all 65 buildings subjected to 10 ground motions considering three material behaviours and for three targets of ductility demands. The time-step used in the analyses is $0.001 \mathrm{~s}$. Proportional Rayleigh damping of $5 \%$ is assumed for the first and the third modes.

Analyses are carried out first to determine the ductility demands on the lateral load resisting system, which has been designed assuming a force modification factor of 3 $\left(R_{y}=3\right)$. Response analyses are then carried out in the reverse order to determine the force reduction factors that will lead to ductility demands of 2,3 and 4 , respectively. This way the $\kappa$ value introduced in previous chapter can be determined as the ratio of the force modification factor $R_{y}$ to the ductility demand $\mu$ for each case. This portion of the analytical study requires several iterations for each building model, each earthquake ground motion, and each ductility capacity. The results of the analyses are presented in Tables B. 1 to B.60 in Appendix B.

\subsection{Displacement Time Histories for LLRS}

In order to form a fundamental understanding about how post-yield hardening behaviour in lateral load resisting members can affect the behaviour of the system and particularly the ductility demand on the lateral load resisting system, the displacement time histories 
for three selected buildings of three different sizes are presented. The displacement time histories for buildings SH1, MH1 and LH1 subjected to M6C38 ground motion have been plotted for different values of post-yield hardening $(\alpha=0.00,0.02,0.05$ and 0.10$)$ and are presented in Figures 4.1 to 4.24. Each building has been analyzed twice; once when the earthquake load is acting parallel to the short side of the building and next when the ground motion is acting parallel to the long side of the building. In all cases a force reduction factor of $3\left(R_{y}=3\right)$ is used since the purpose at this stage is just to observe the effects on displacements and the ductility demand.

From Figures 4.1 to 4.24 , it is evident that post-yield hardening generally leads to smaller displacements. There is an inverse relation between the value of $\alpha$ and the maximum displacement in LLRS, so that as $\alpha$ increases, the maximum displacement decreases. Consequently, smaller ductility demands are expected. Naturally, smaller values of ductility demand on the lateral load resisting elements suggest that a larger force reduction factor may be used leading to smaller design earthquake loads. Evidently, it is worthwhile investigating how extensive is the reduction in ductility demand on the lateral load resisting system members, and how much larger a force reduction factor could be justified. 


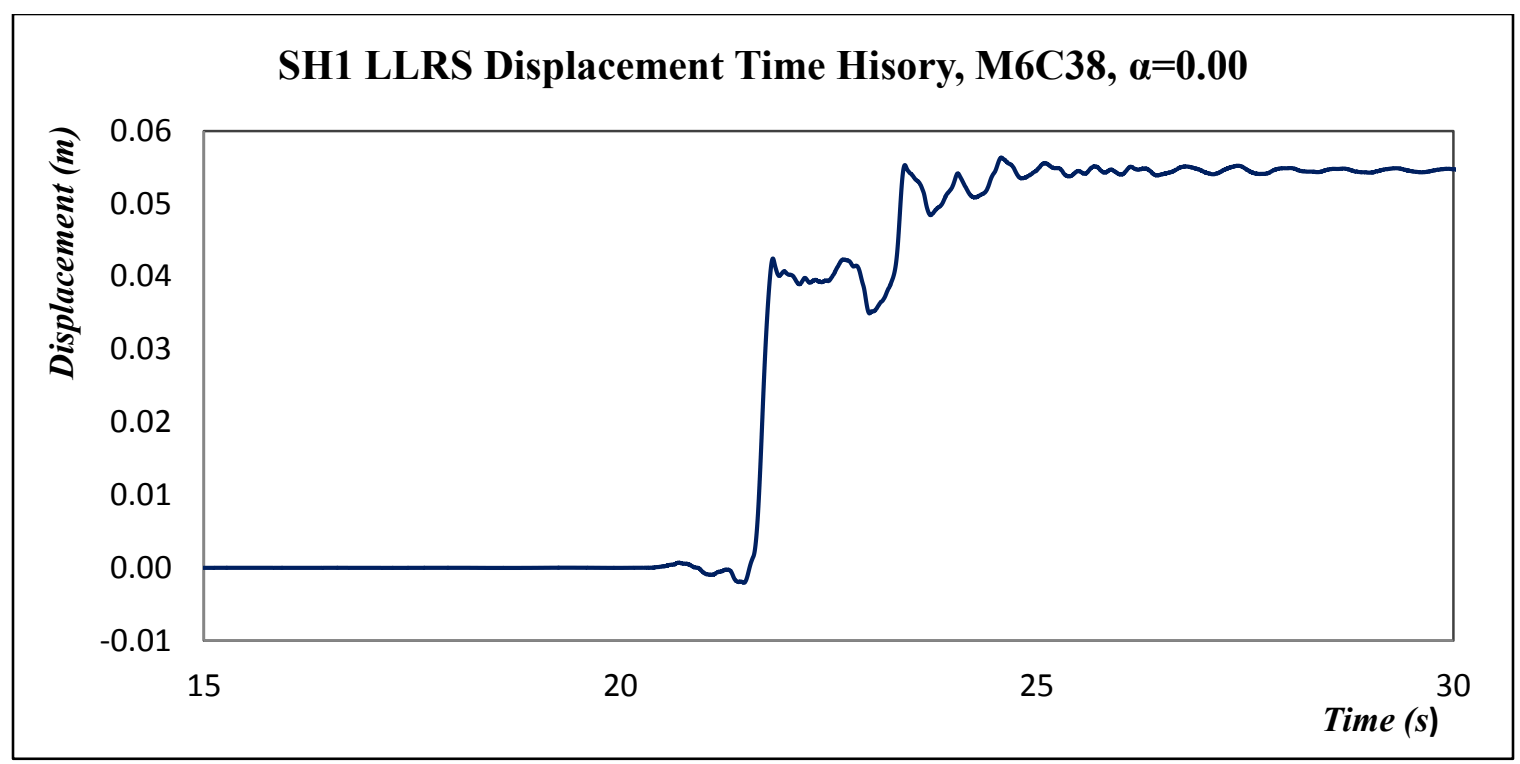

Figure 4.1 - Displacement time history for Building SH1 subjected to M6C38 ground motion parallel to the short side for $\alpha=\mathbf{0 . 0 0}$

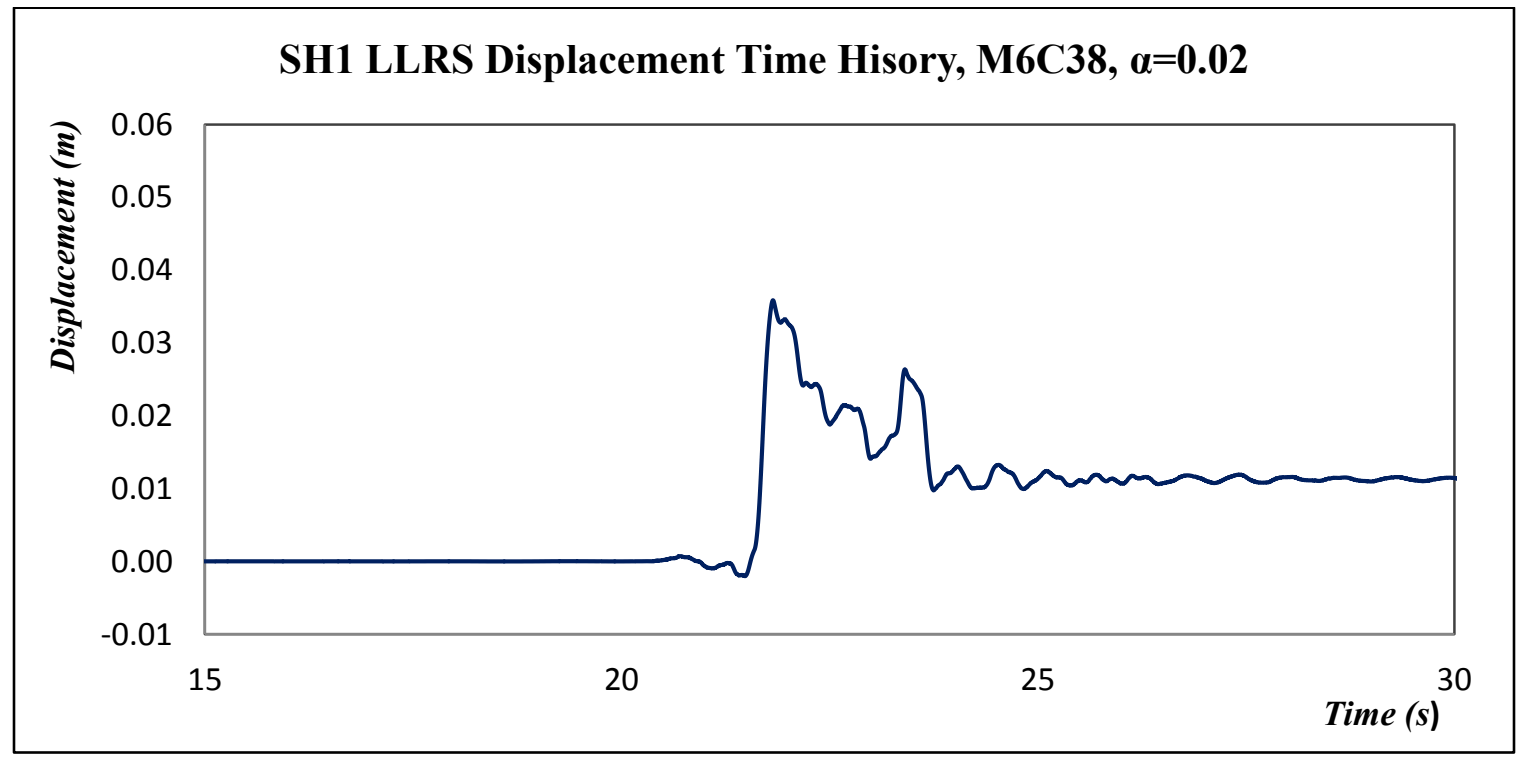

Figure 4.2 - Displacement time history for Building SH1 subjected to M6C38 ground motion parallel to the short side for $\alpha=0.02$ 


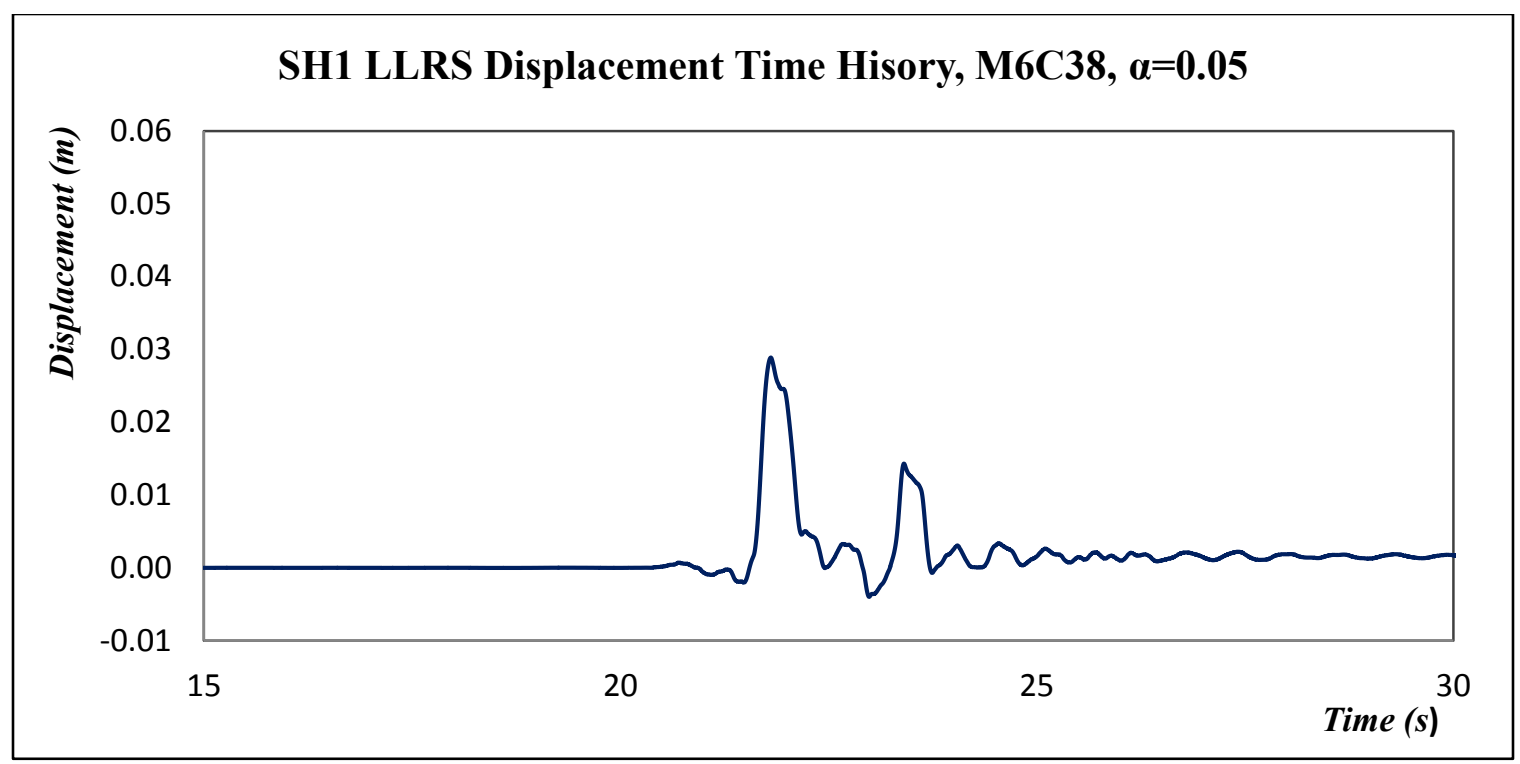

Figure 4.3 - Displacement time history for Building SH1 subjected to M6C38 ground motion parallel to the short side for $\alpha=0.05$

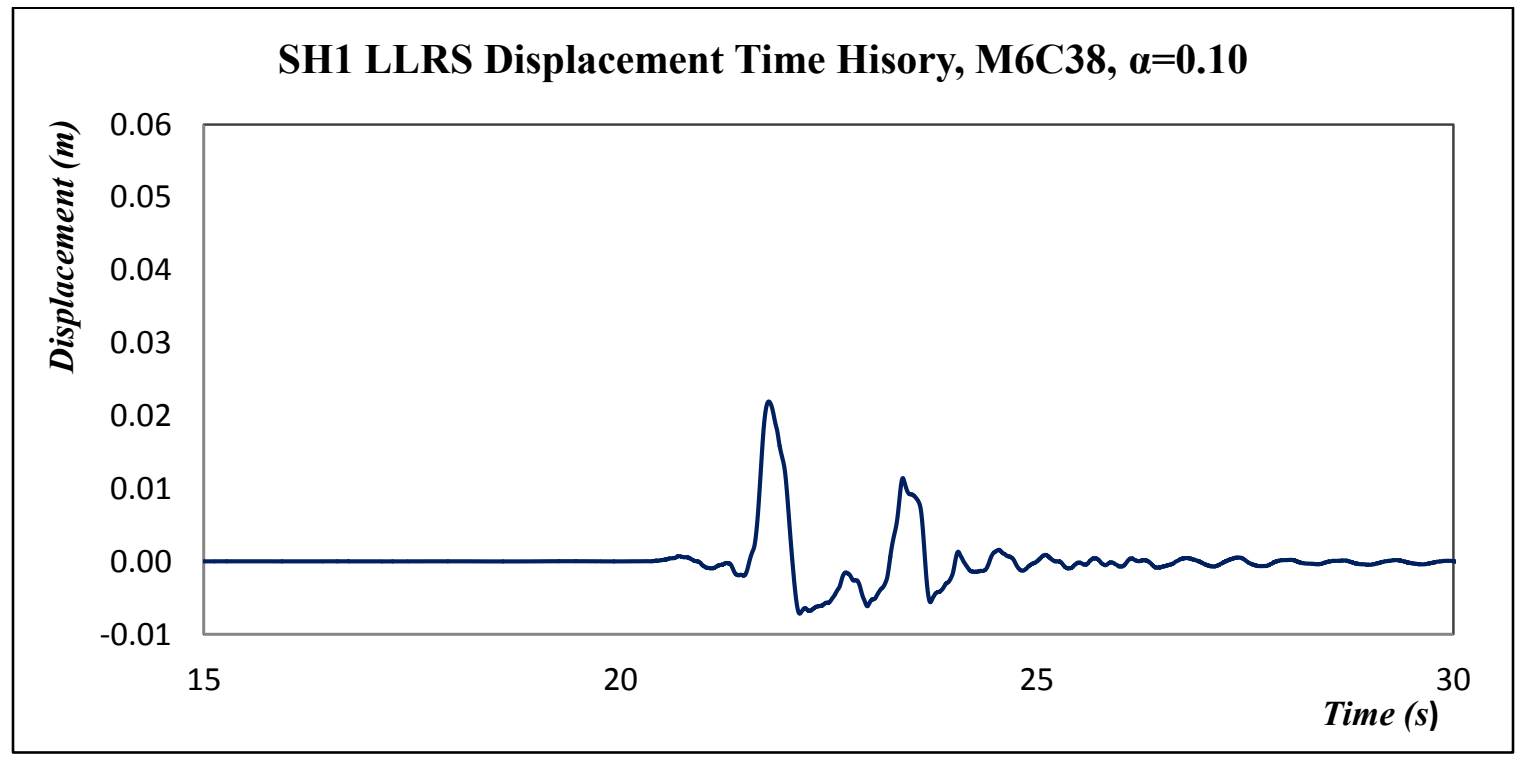

Figure 4.4 - Displacement time history for Building SH1 subjected to M6C38 ground motion parallel to the short side for $\alpha=\mathbf{0 . 1 0}$ 


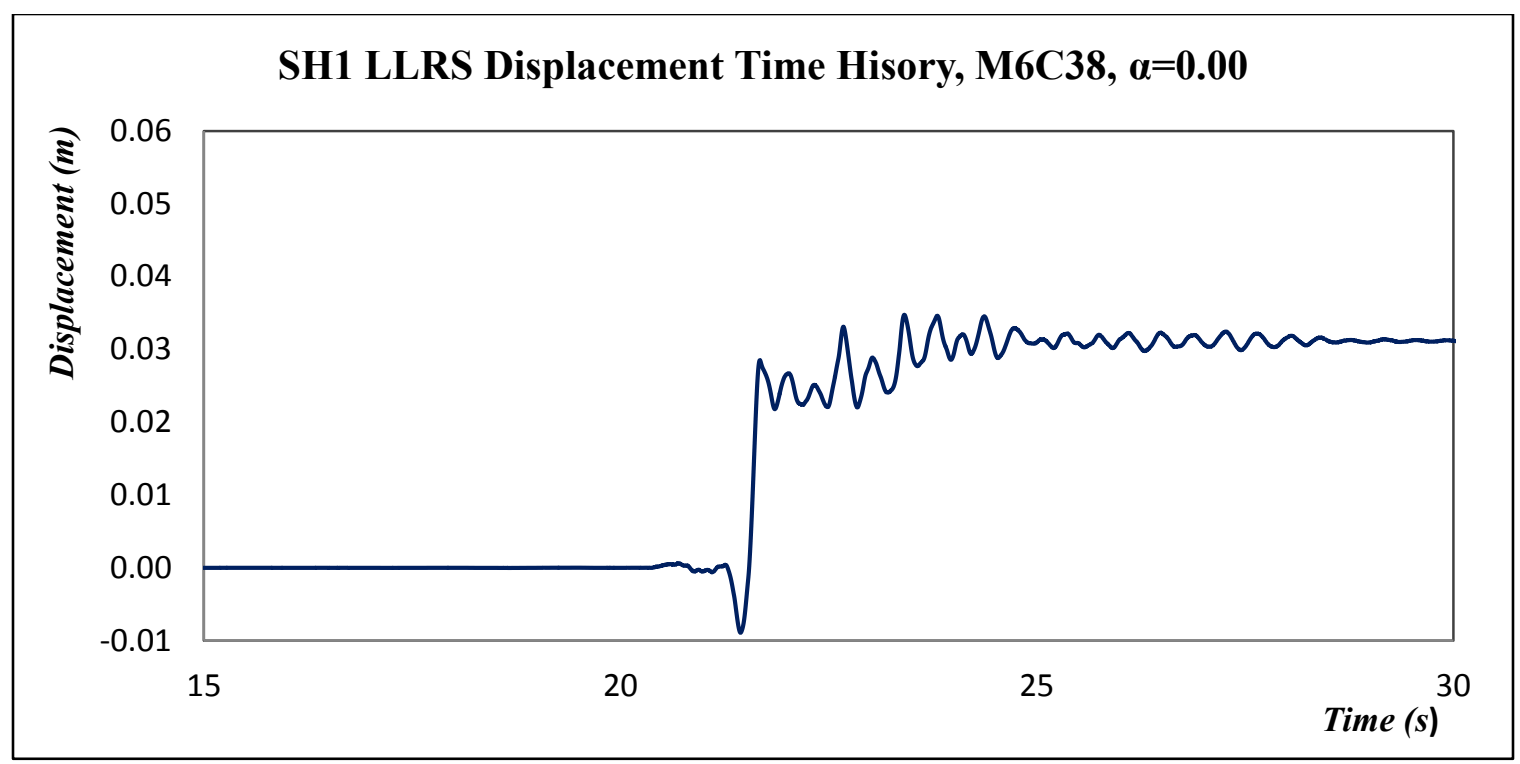

Figure 4.5 - Displacement time history for Building SH1 subjected to M6C38 ground motion parallel to the long side for $\alpha=0.00$

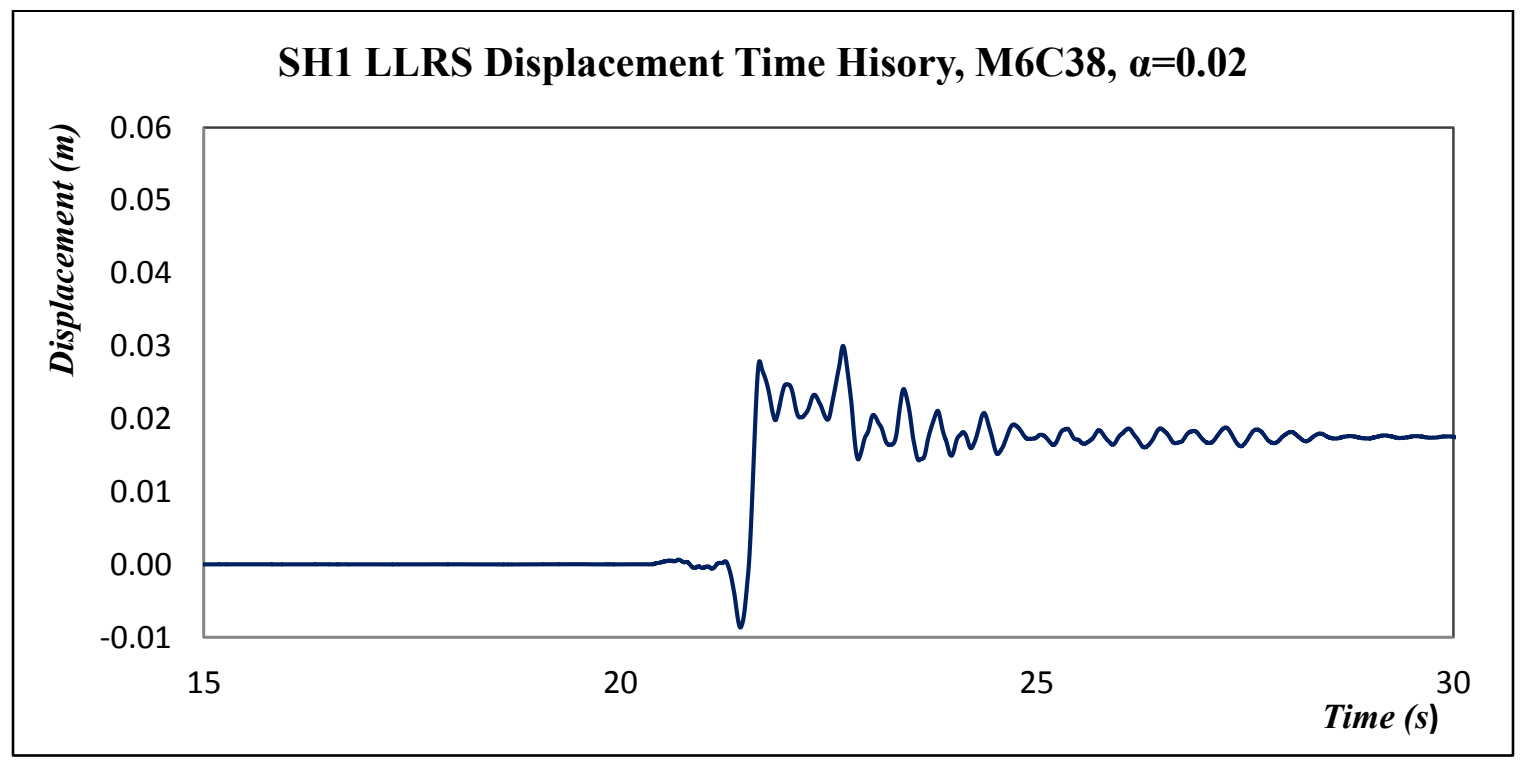

Figure 4.6 - Displacement time history for Building SH1 subjected to M6C38 ground motion parallel to the long side for $\alpha=0.02$ 


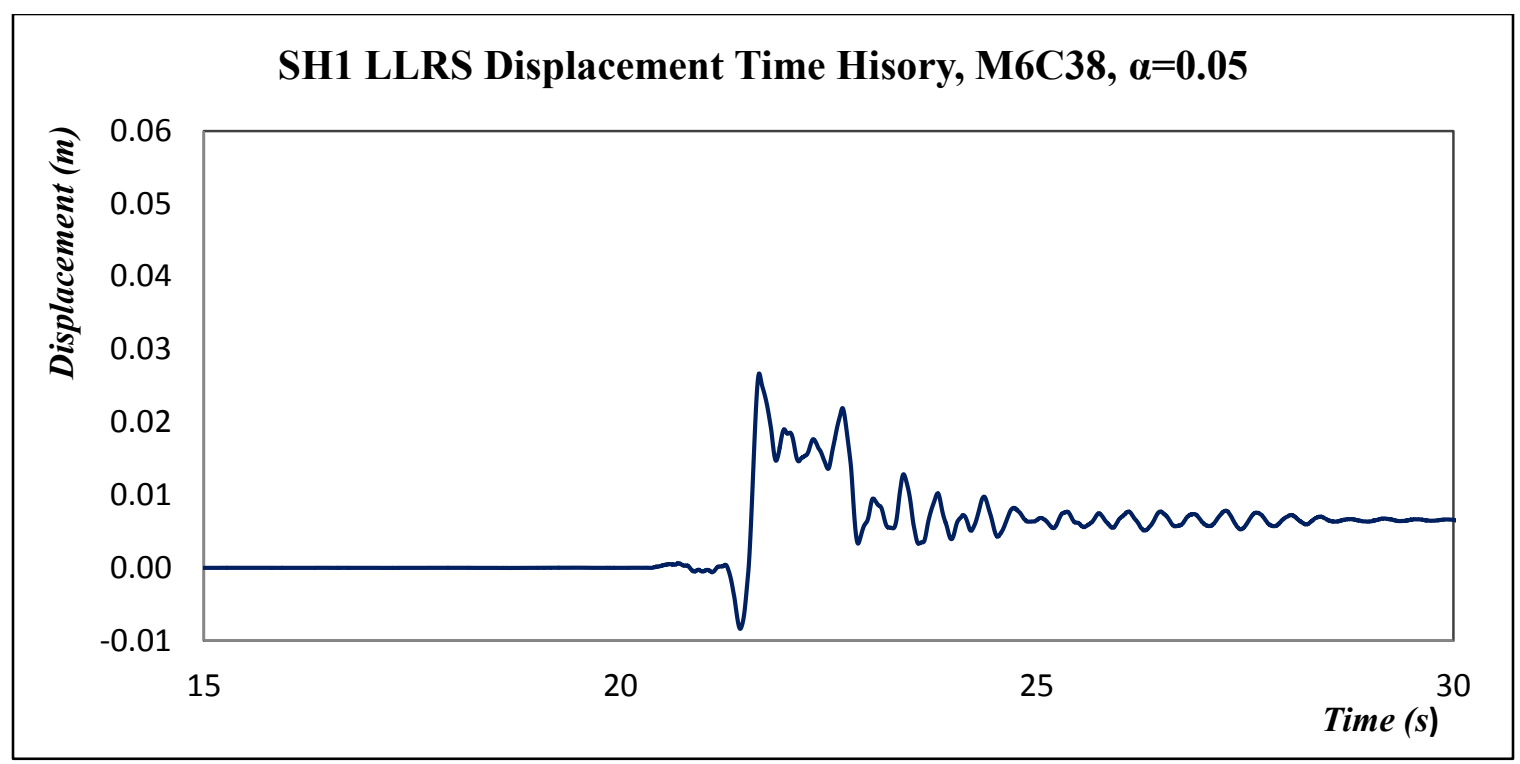

Figure 4.7 - Displacement time history for Building SH1 subjected to M6C38 ground motion parallel to the long side for $\alpha=0.05$

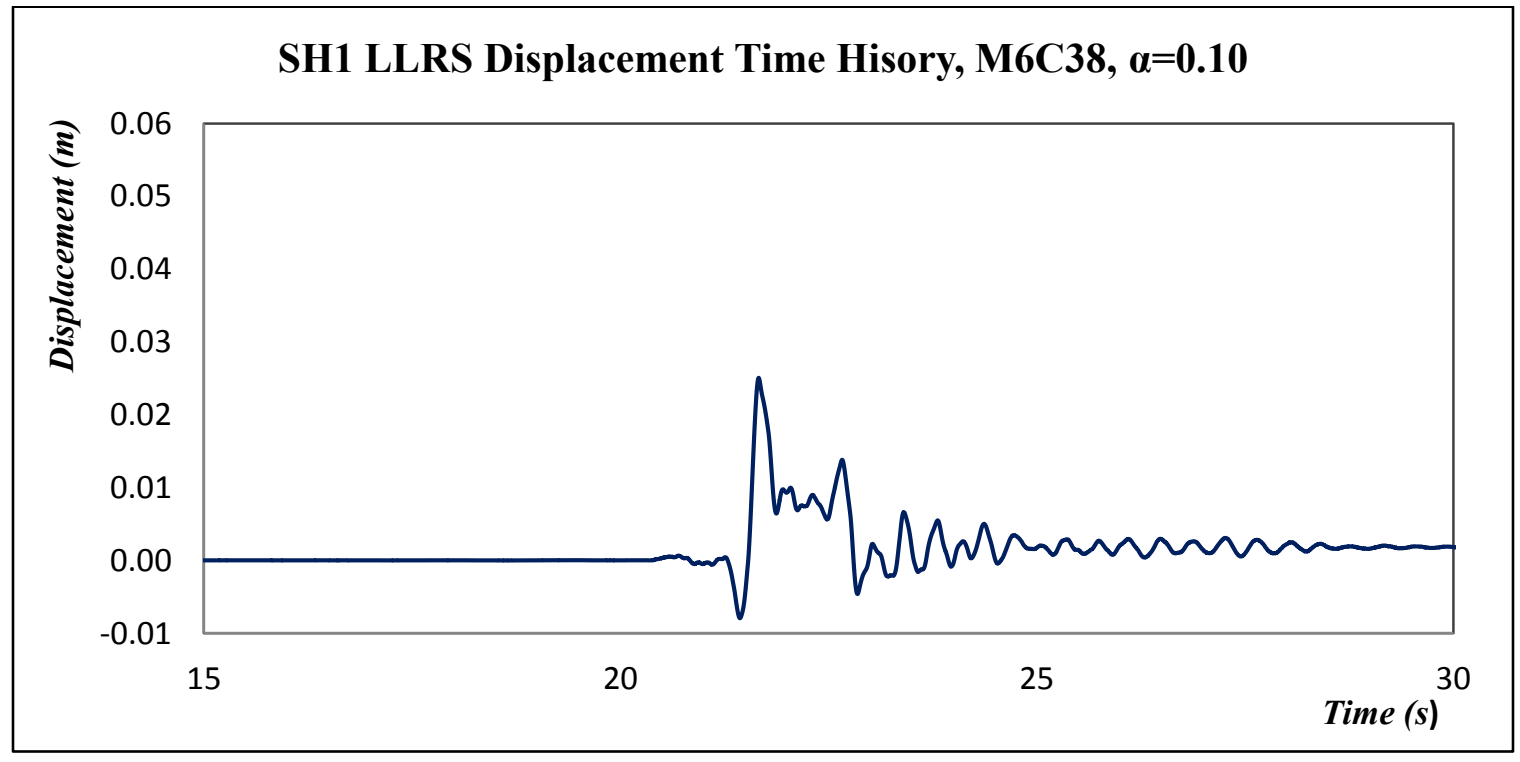

Figure 4.8 - Displacement time history for Building SH1 subjected to M6C38 ground motion parallel to the long side for $\alpha=0.10$ 


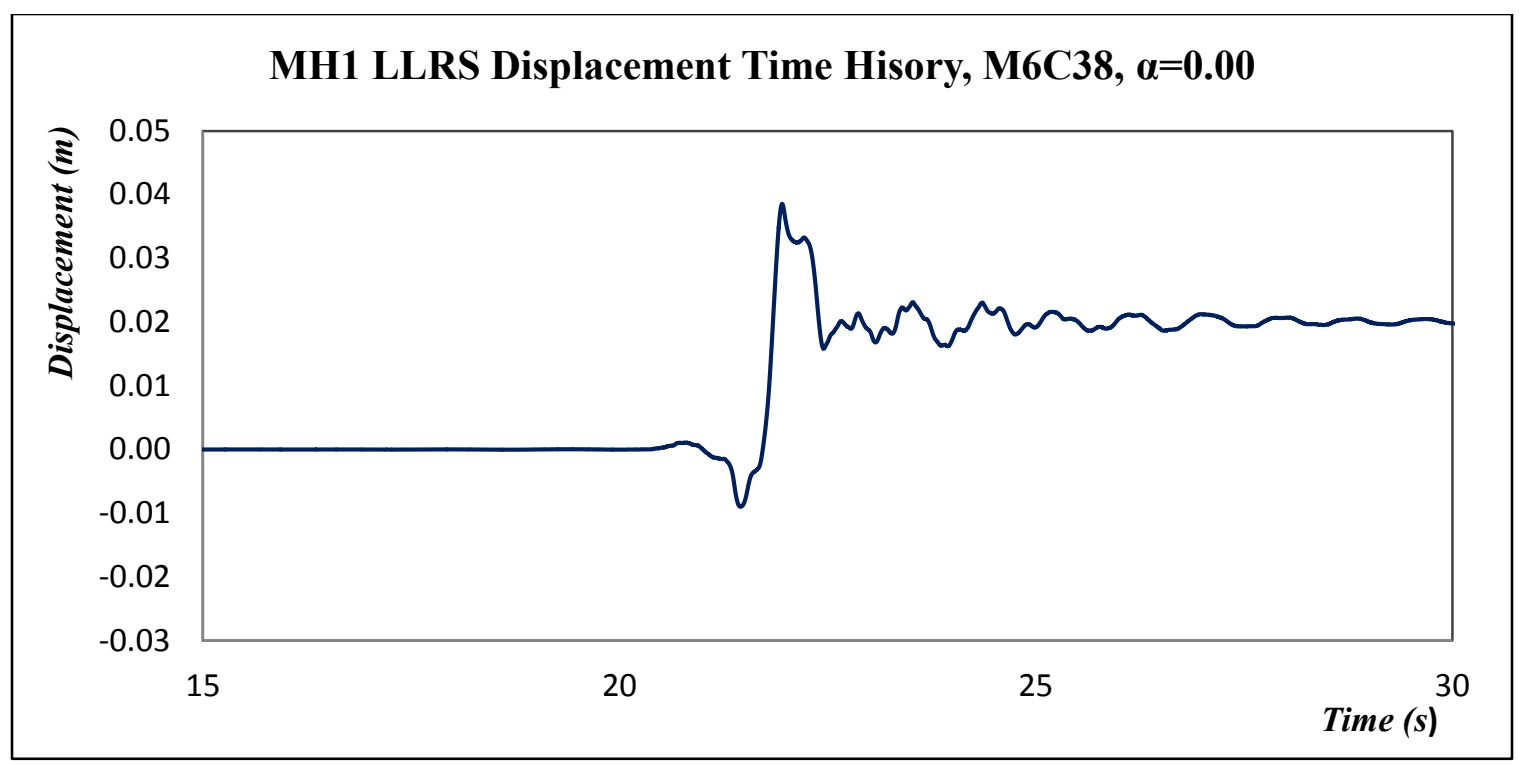

Figure 4.9 - Displacement time history for Building MH1 subjected to M6C38 ground motion parallel to the short side for $\alpha=\mathbf{0 . 0 0}$

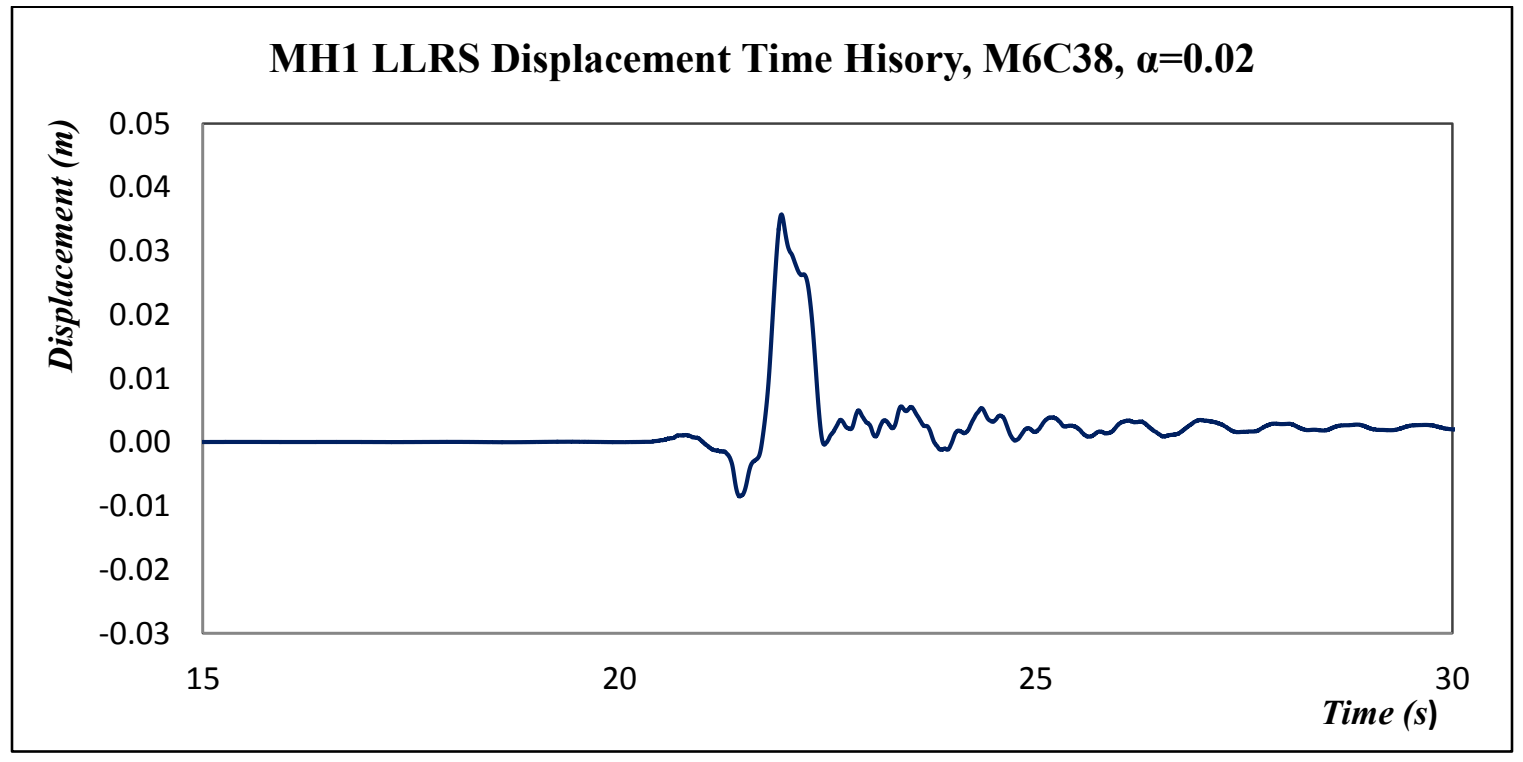

Figure 4.10 - Displacement time history for Building MH1 subjected to M6C38 ground motion parallel to the short side for $\alpha=\mathbf{0 . 0 2}$ 


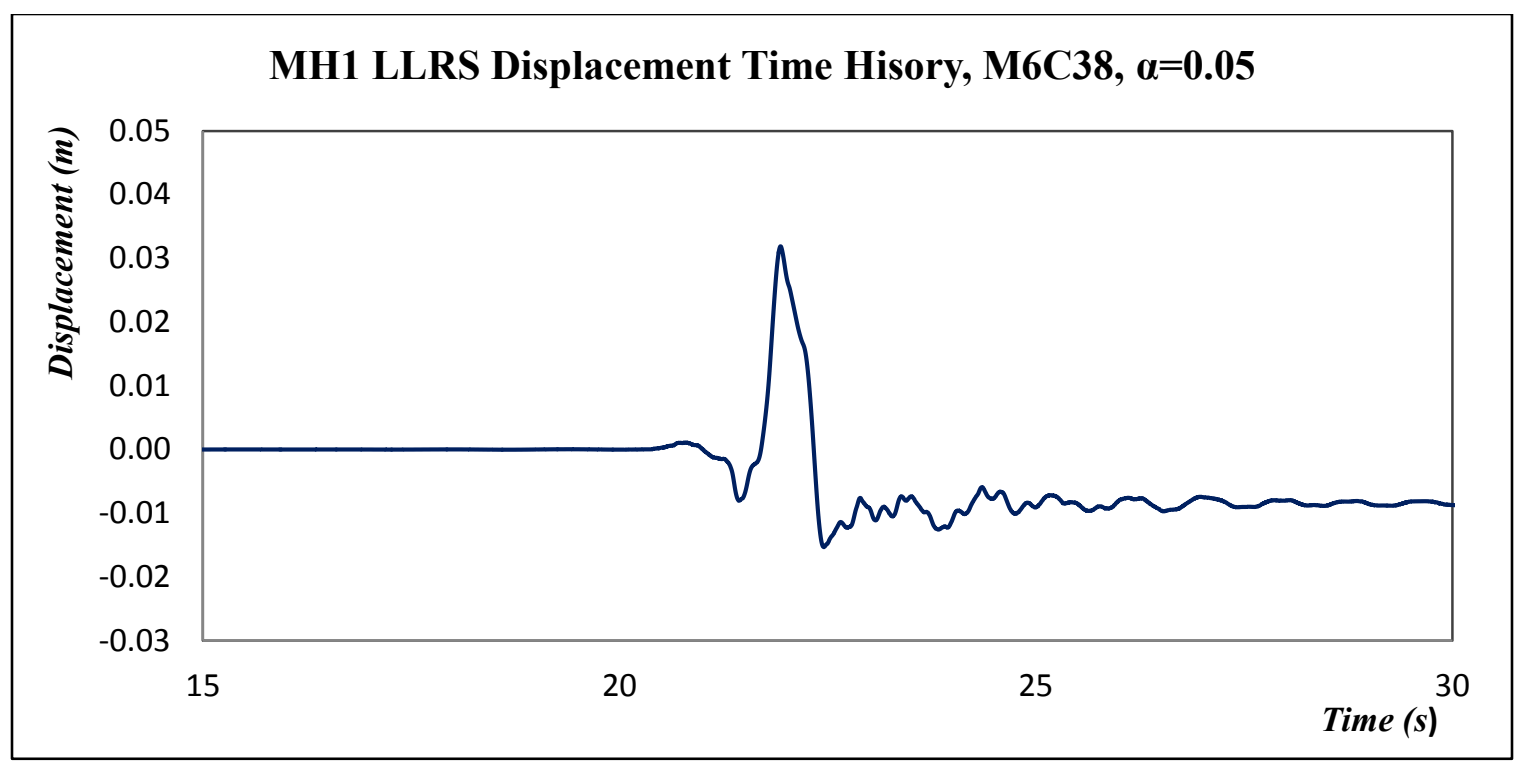

Figure 4.11 - Displacement time history for Building MH1 subjected to M6C38 ground motion parallel to the short side for $\alpha=\mathbf{0 . 0 5}$

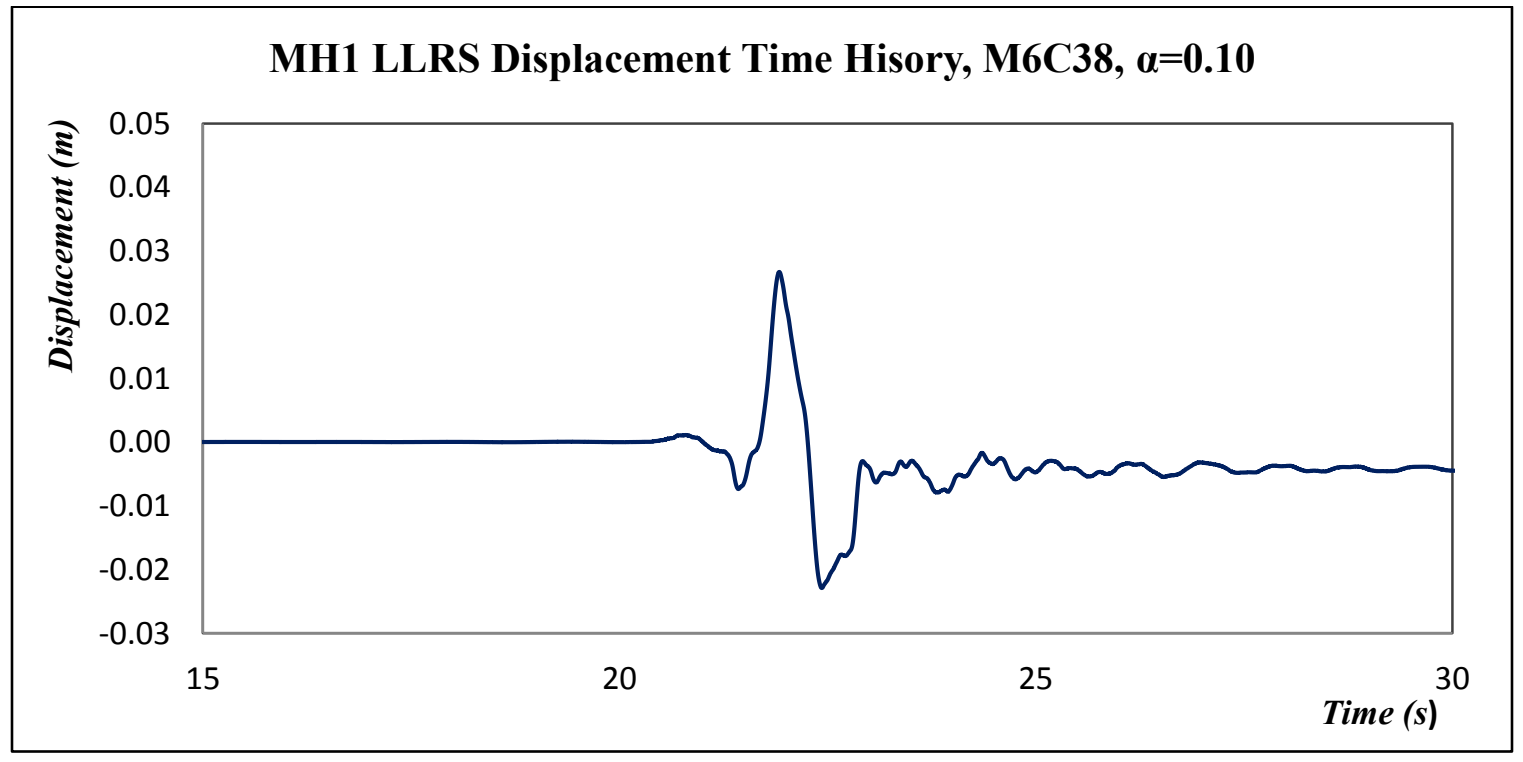

Figure 4.12 - Displacement time history for Building MH1 subjected to M6C38 ground motion parallel to the short side for $\alpha=\mathbf{0 . 1 0}$ 


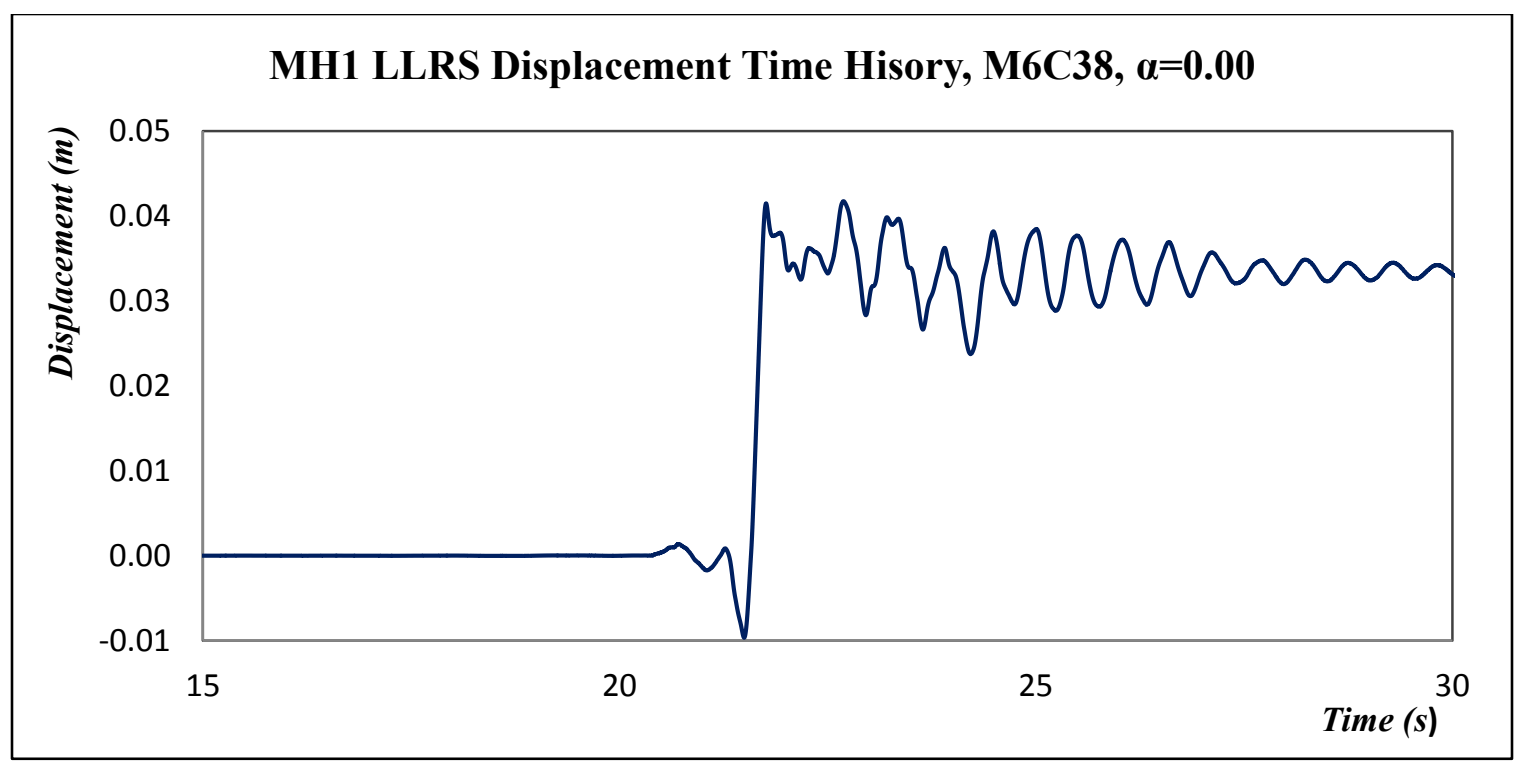

Figure 4.13 - Displacement time history for Building MH1 subjected to M6C38 ground motion parallel to the long side for $\alpha=\mathbf{0 . 0 0}$

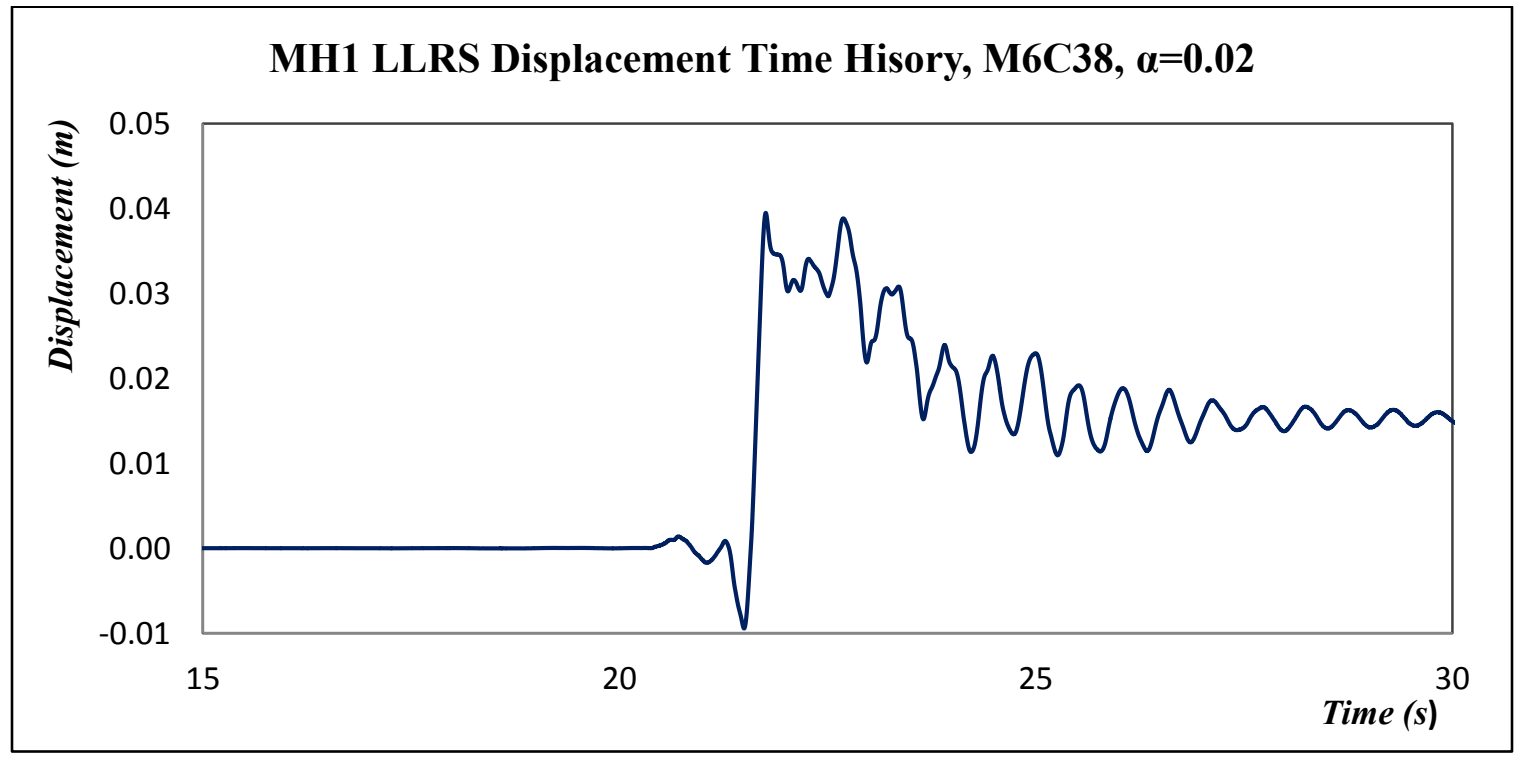

Figure 4.14 - Displacement time history for Building MH1 subjected to M6C38 ground motion parallel to the long side for $\alpha=\mathbf{0 . 0 2}$ 


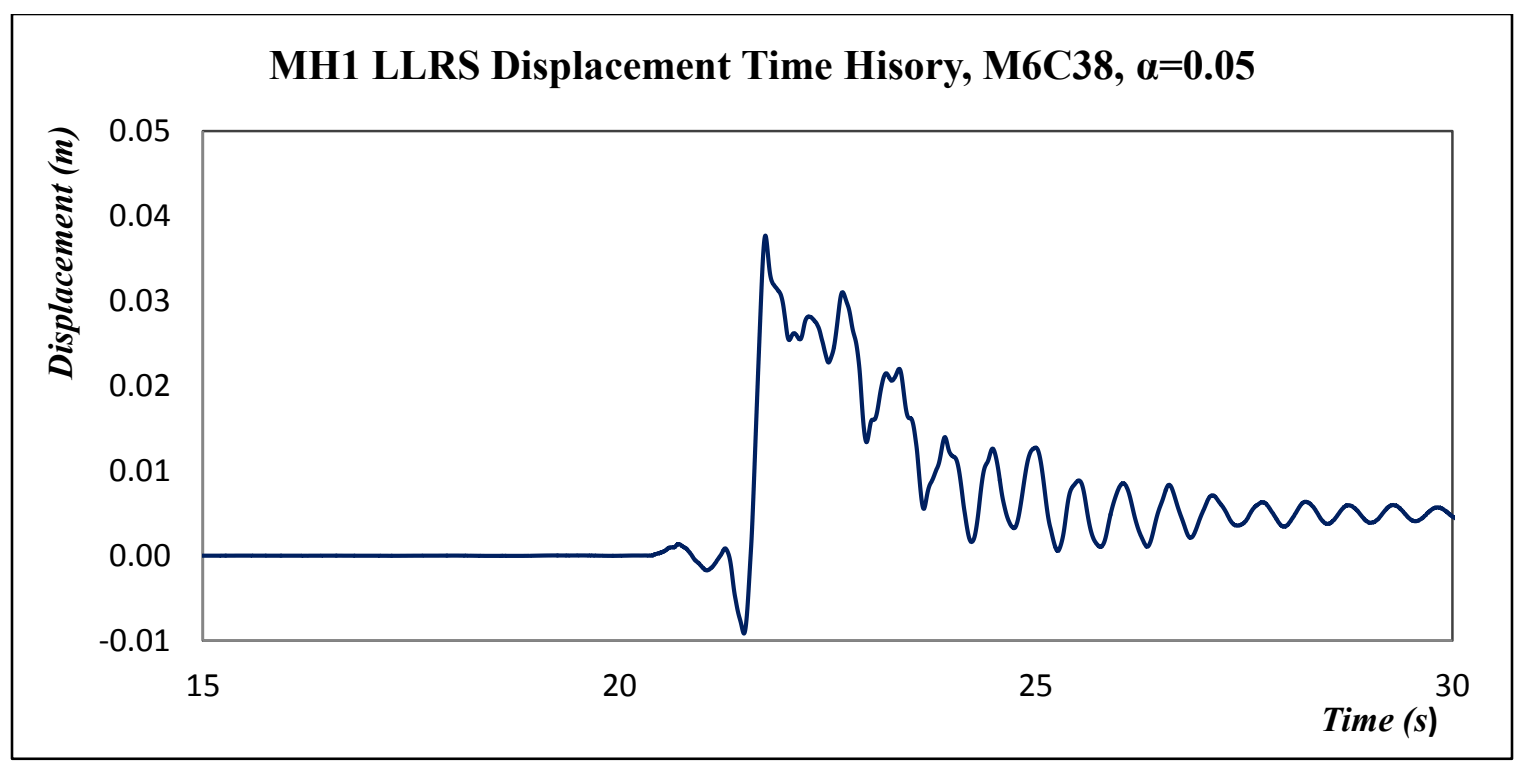

Figure 4.15 - Displacement time history for Building MH1 subjected to M6C38 ground motion parallel to the long side for $\alpha=\mathbf{0 . 0 5}$

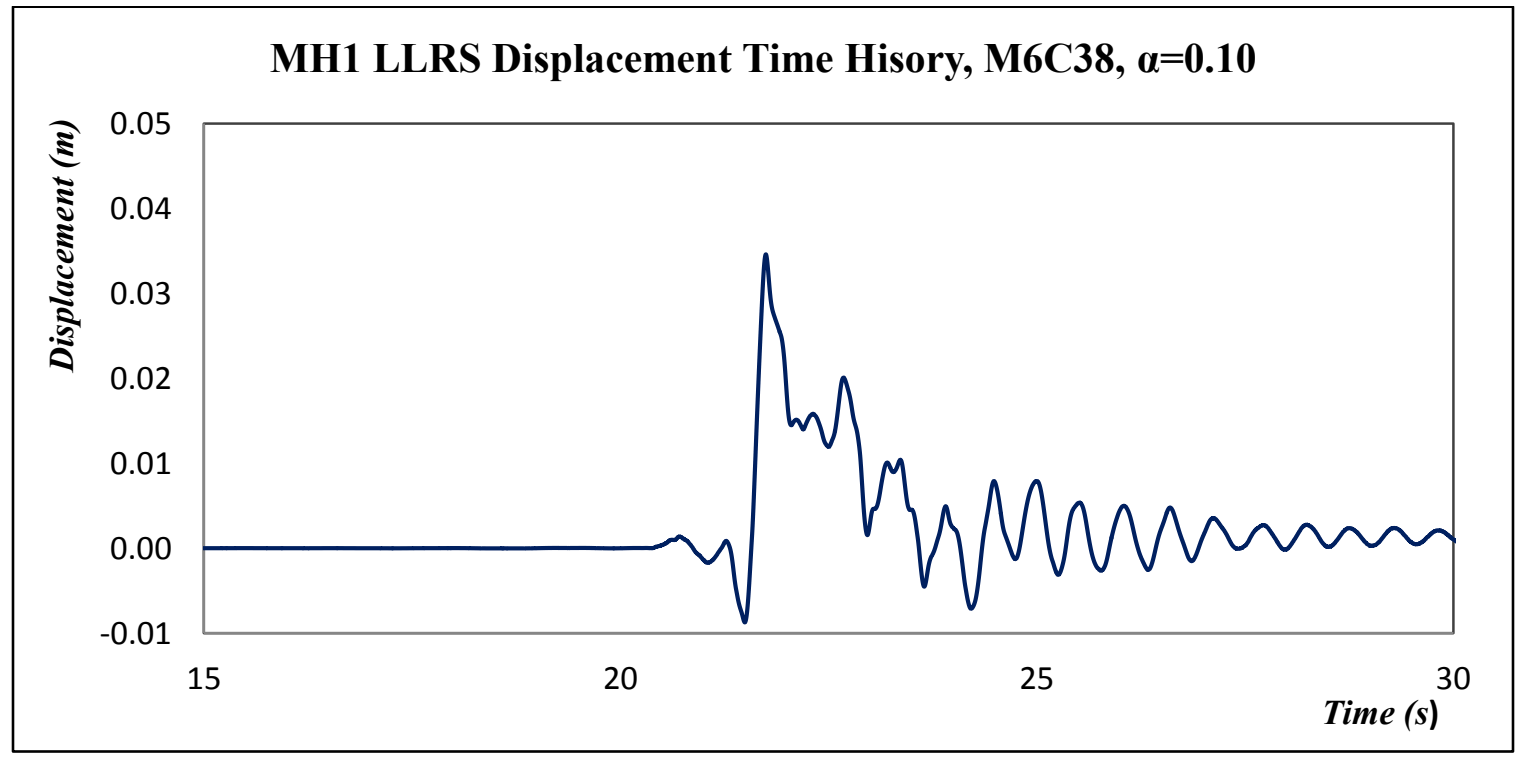

Figure 4.16 - Displacement time history for Building MH1 subjected to M6C38 ground motion parallel to the long side for $\alpha=\mathbf{0 . 1 0}$ 


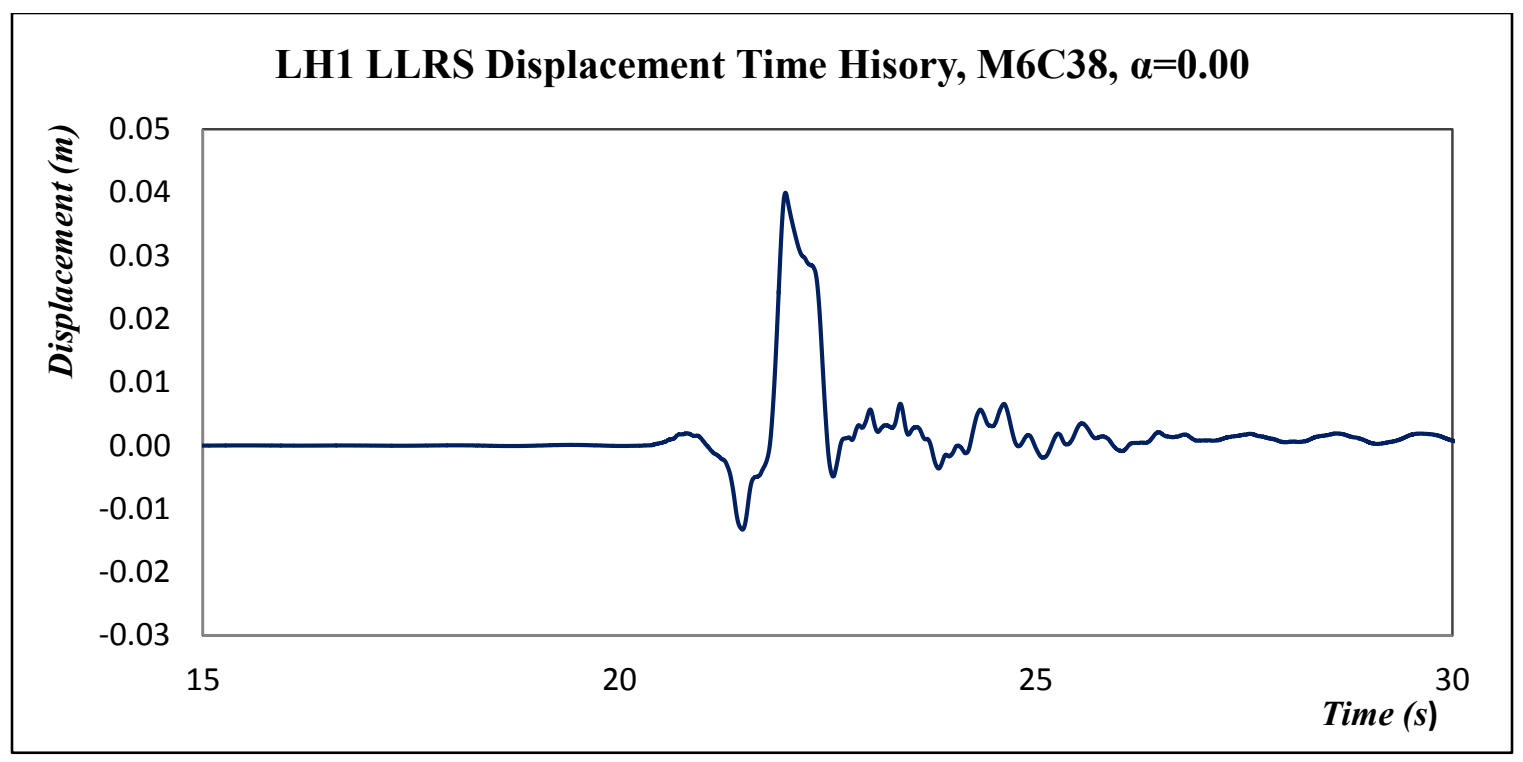

Figure 4.17 - Displacement time history for Building LH1 subjected to M6C38 ground motion parallel to the short side for $\alpha=\mathbf{0 . 0 0}$

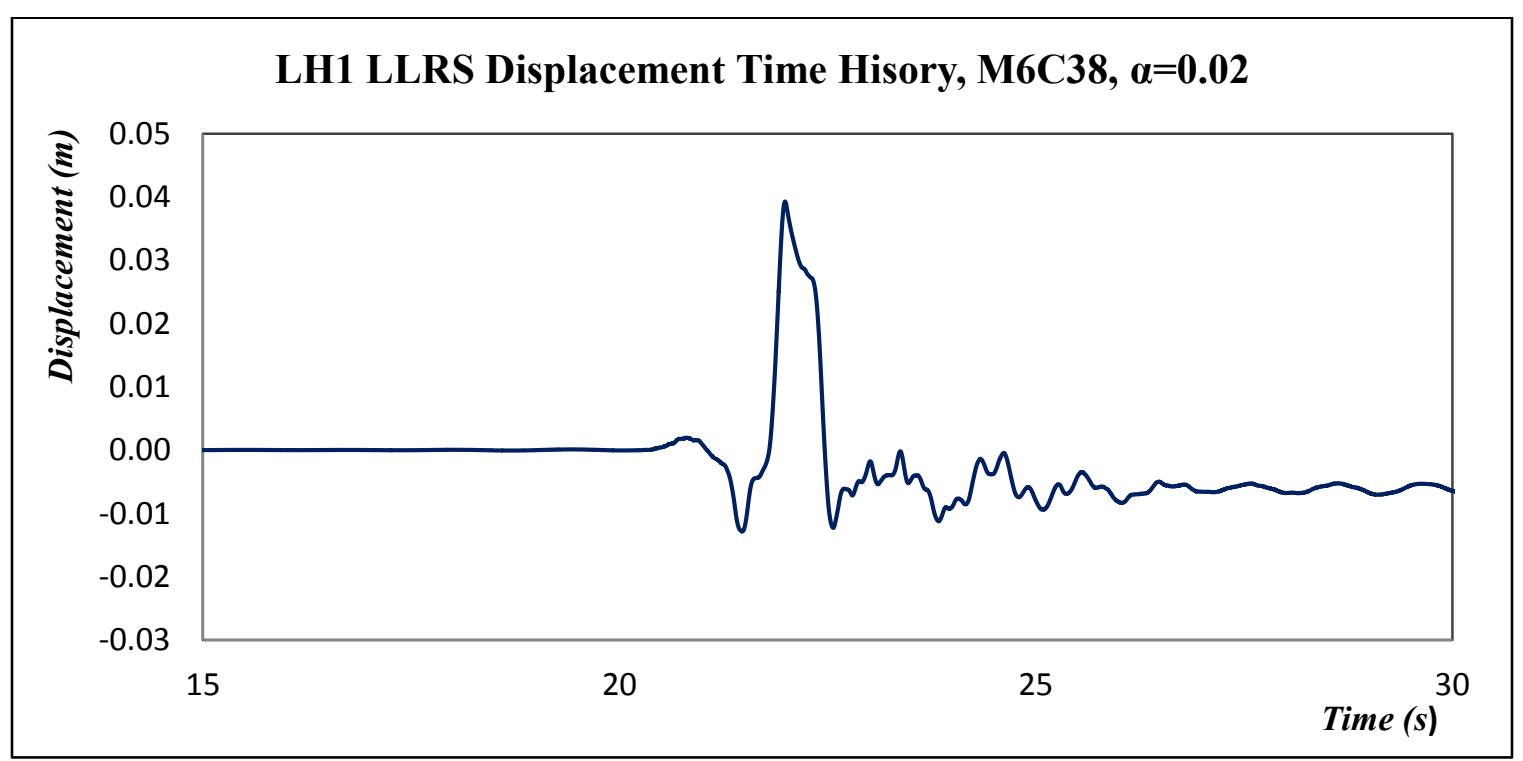

Figure 4.18 - Displacement time history for Building LH1 subjected to M6C38 ground motion parallel to the short side for $\alpha=\mathbf{0 . 0 2}$ 


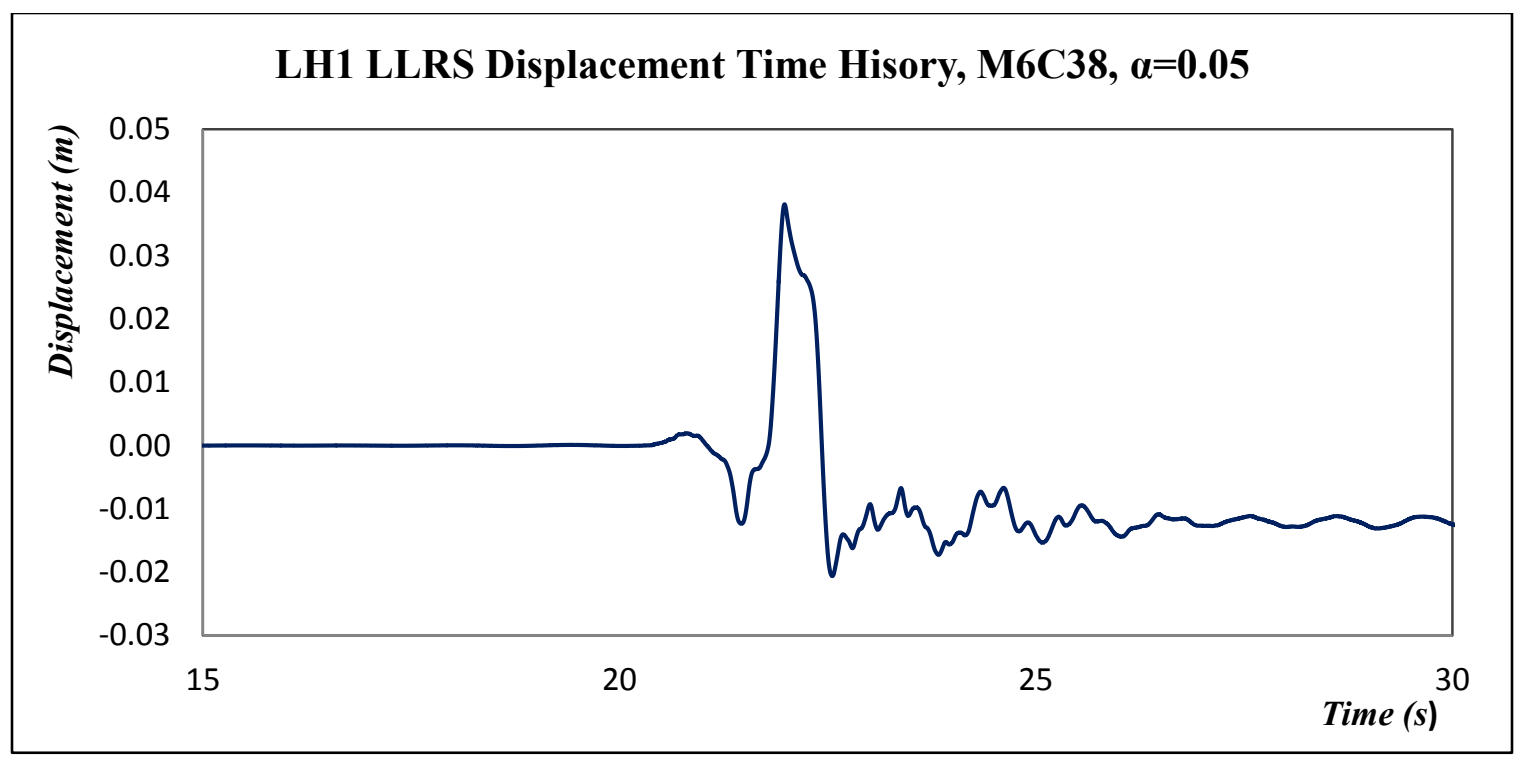

Figure 4.19 - Displacement time history for Building LH1 subjected to M6C38 ground motion parallel to the short side for $\alpha=\mathbf{0 . 0 5}$

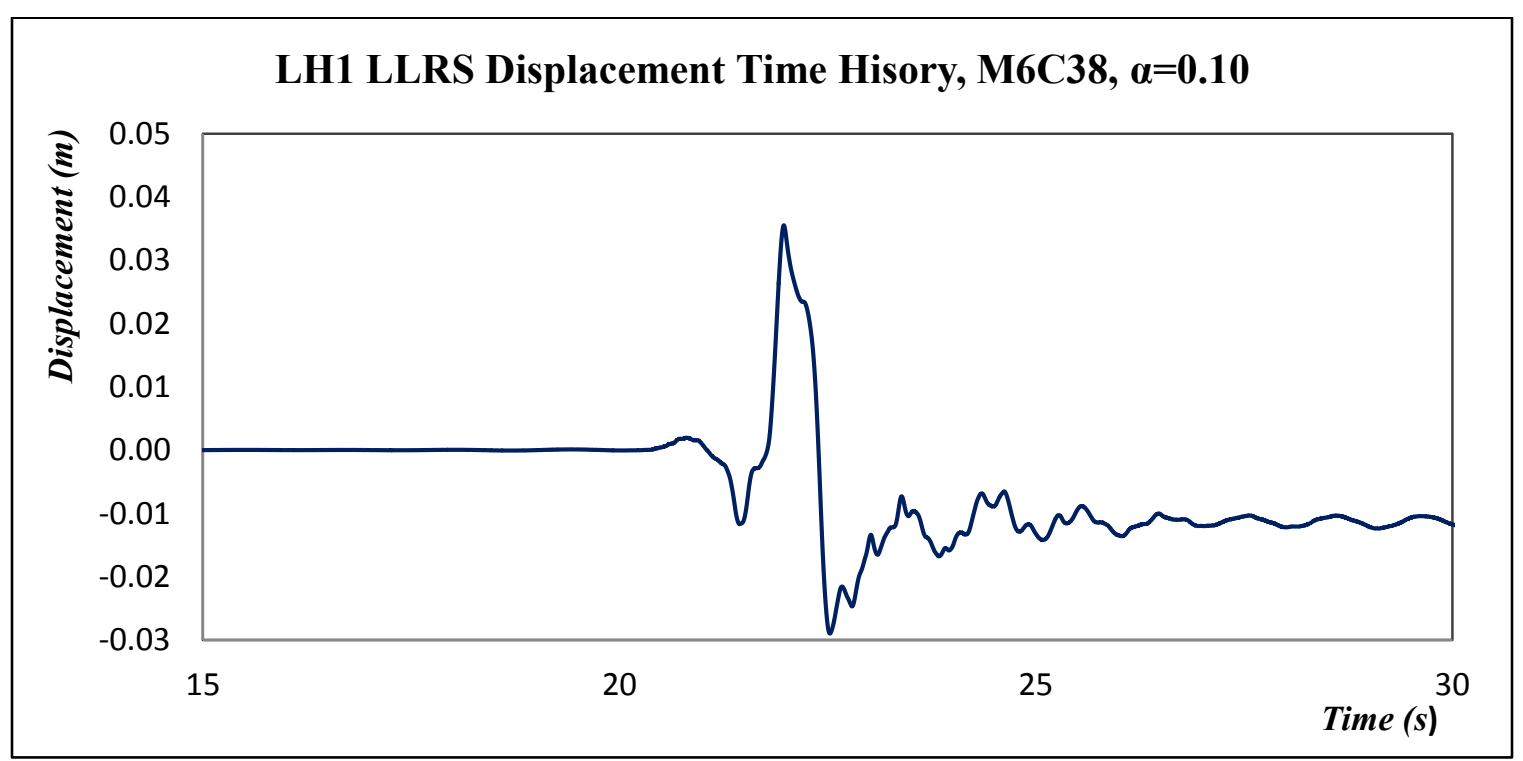

Figure 4.20 - Displacement time history for Building LH1 subjected to M6C38 ground motion parallel to the short side for $\alpha=\mathbf{0 . 1 0}$ 


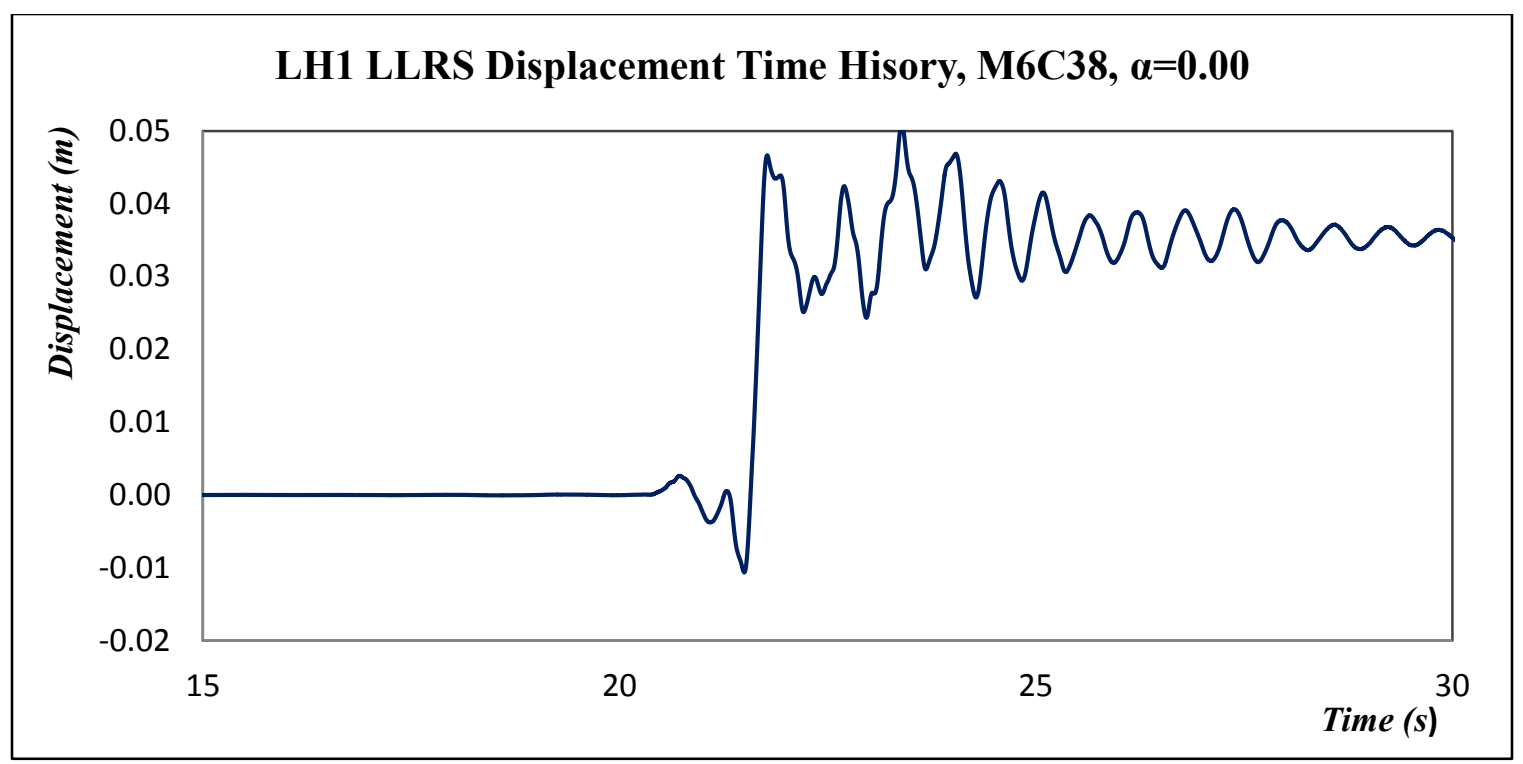

Figure 4.21 - Displacement time history for Building LH1 subjected to M6C38 ground motion parallel to the long side for $\alpha=\mathbf{0 . 0 0}$

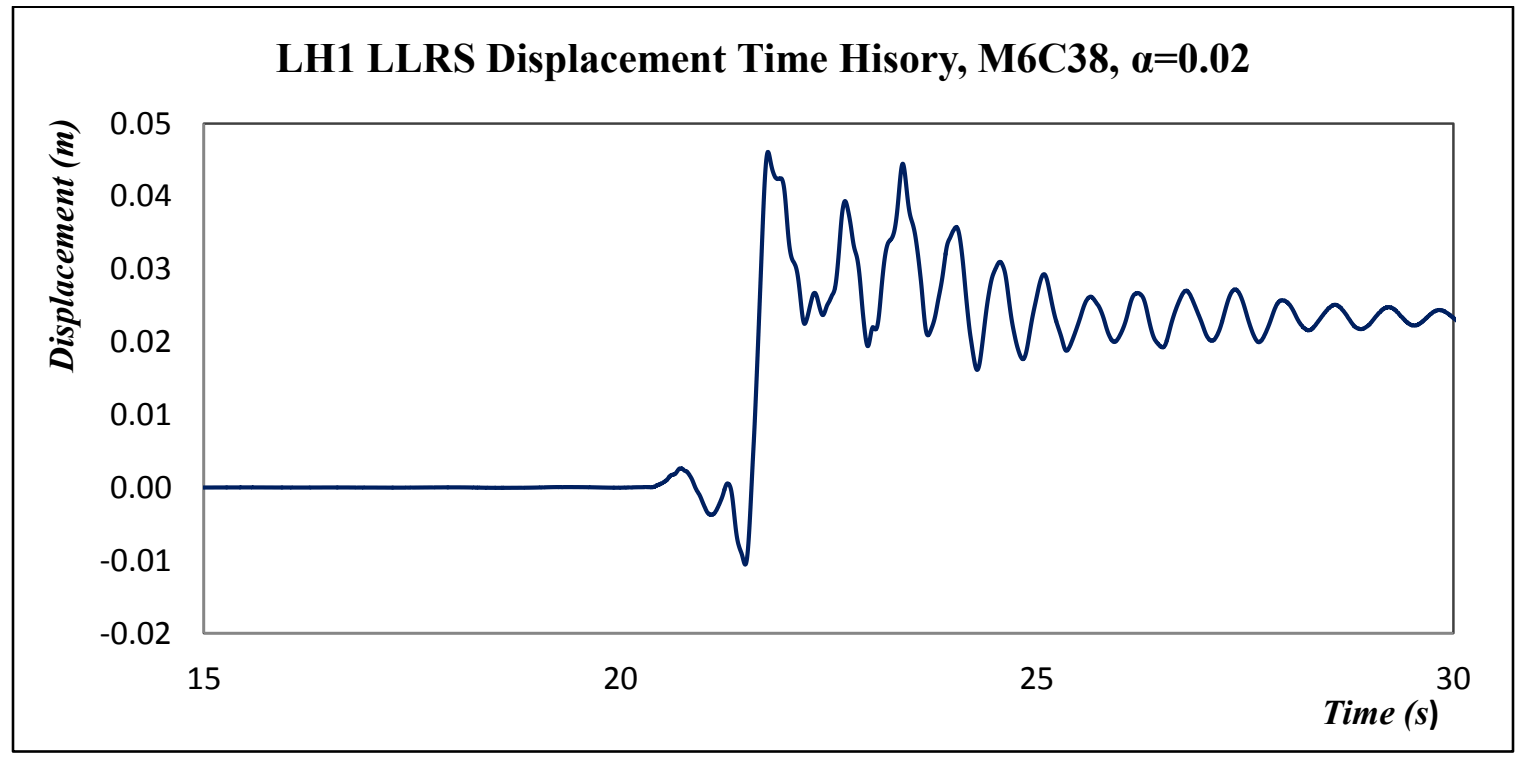

Figure 4.22 - Displacement time history for Building LH1 subjected to M6C38 ground motion parallel to the long side for $\alpha=\mathbf{0 . 0 2}$ 


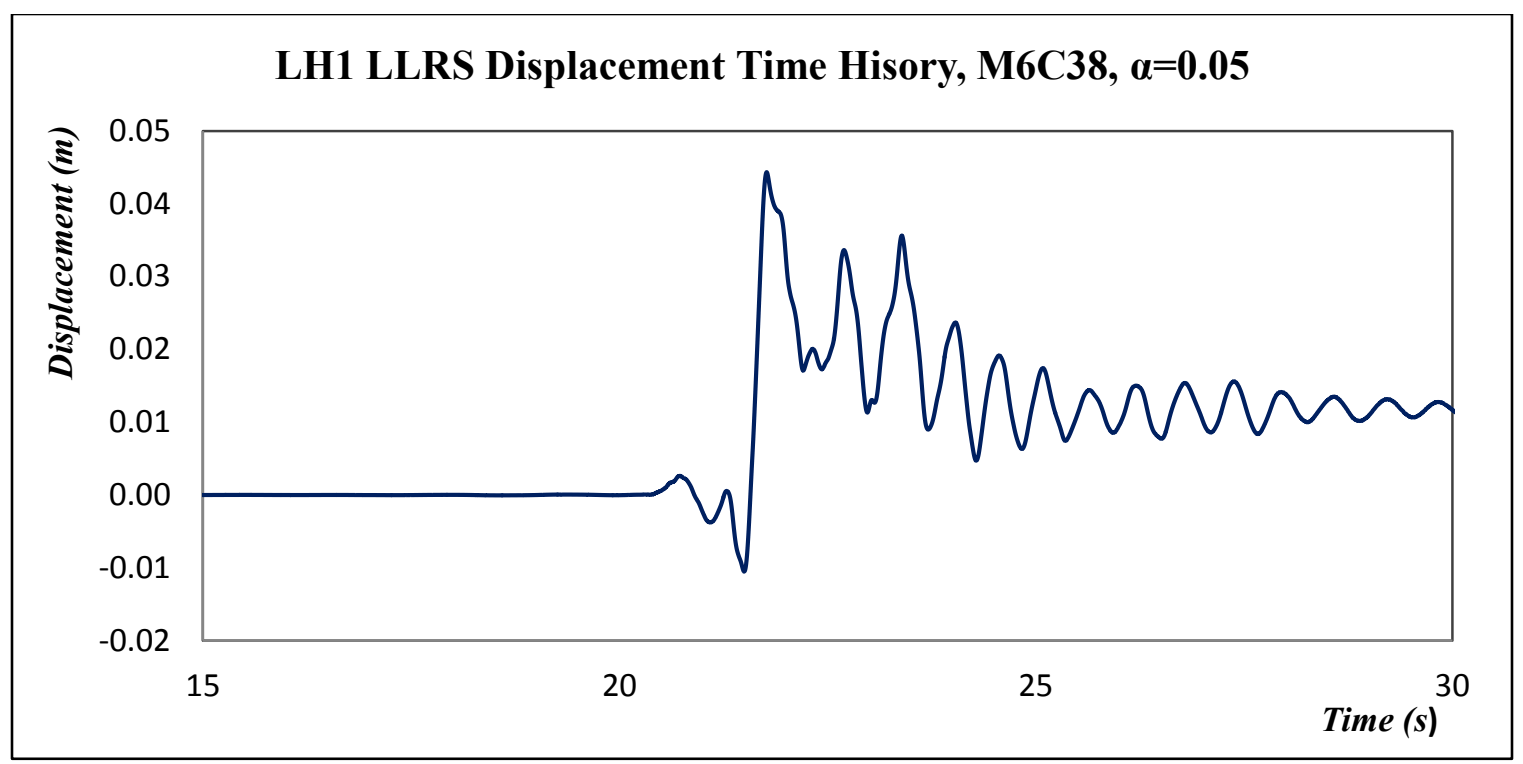

Figure 4.23 - Displacement time history for Building LH1 subjected to M6C38 ground motion parallel to the long side for $\alpha=\mathbf{0 . 0 5}$

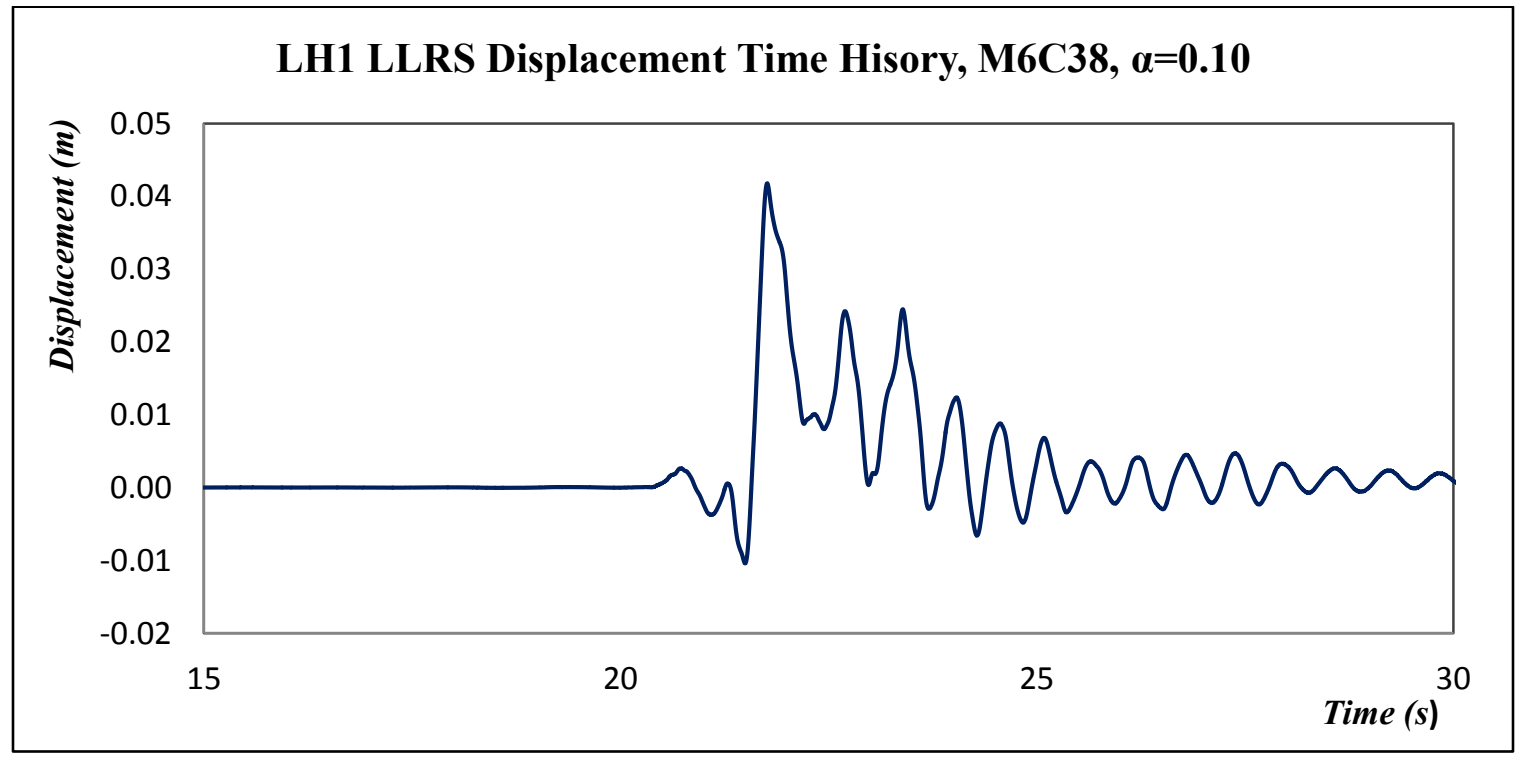

Figure 4.24 - Displacement time history for Building LH1 subjected to M6C38 ground motion parallel to the long side for $\alpha=\mathbf{0 . 1 0}$ 
Figures 4.1 to 4.24 also show that the residual displacement generally decreases as the post-yield stiffness increases. The data presented in Tables B.1 to B.60, confirm that with an increase in $\alpha$, the ductility demand decreases and a larger force reduction factor is justified.

\subsection{Development of Relationship between $\kappa$ and the Drift Ratio}

Values of $\kappa$, the multiplier to be applied to the ductility demand to get the force reduction factor as defined in Section 4.3, for each building are reported in Tables B.1 to B.60 and are plotted against the corresponding drift ratios in Figures B1 to B8. Plots are given separately for each value of the post-yield hardening ratio, from $\alpha=0$ to $\alpha=0.1$. The plots are for all 65 building models subjected to ground motions compatible with the UHS for Vancouver as well as Montreal for each of the three target ductility demand values: 2,3 and 4 .

As described earlier in Chapter 3, to find an appropriate relationship between $\kappa$ and drift ratio a linear regression fit is carried out on the data corresponding to the cases in which the ground motion is parallel to the long side of the building (smaller values of $r$ ). The average value of $\kappa$ obtained from the data corresponding to cases for which the earthquake ground motion is parallel to the short side of the building (larger values of r), is selected as the lower limit of $\kappa$.

The regression lines are also shown in Figures B1 to B8. In each chart, the data corresponding to the three values of target ductility demands and the corresponding $\kappa$ equations are presented. 
The lower limits on $\kappa$ values for the various cases (two spectra and three targets of ductility demand and three $\alpha$ values) are presented in Table 4.1

Table 4.1 - Lower limits on $\boldsymbol{\kappa}$ for different cases

\begin{tabular}{ccccccccc}
\hline & \multicolumn{2}{c}{$\alpha=0.00$} & \multicolumn{2}{c}{$\alpha=0.02$} & $\alpha=0.05$ & \multicolumn{2}{c}{$\alpha=0.10$} \\
\cline { 2 - 9 } $\begin{array}{c}\text { Target Ductility } \\
\text { Demand }(\mu)\end{array}$ & \multicolumn{2}{c}{ Target Spectrum } & \multicolumn{2}{c}{ Target Spectrum } & \multicolumn{2}{c}{ Target Spectrum } & \multicolumn{2}{c}{ Target Spectrum } \\
\cline { 2 - 10 } & East & West & East & West & East & West & East & West \\
\hline 2 & 0.67 & 0.64 & 0.68 & 0.65 & 0.69 & 0.66 & 0.70 & 0.67 \\
3 & 0.53 & 0.50 & 0.54 & 0.51 & 0.56 & 0.53 & 0.58 & 0.55 \\
4 & 0.46 & 0.41 & 0.47 & 0.43 & 0.49 & 0.46 & 0.51 & 0.49 \\
\hline
\end{tabular}

As for the case of $\alpha=0$ described in Chapter 3, it is evident from the plots that the regression fits on the portion of the data corresponding to the case in which the ground motion is applied parallel to the long side of the building (smaller values of $r$ ), needs to be modified and simplified in order to obtain a consistent relationship for all cases. As can be seen from Figures B1 to B8, the data corresponding to smaller values of drift ratios are denser in the region with smaller values of $\kappa$, causing the regression fit to have a smaller slope and a smaller intercept. On the other hand, the equation suggested by Humar and Popovski (2013) presented in the Chapter 3, provides a more reasonable and more consistent value for $\kappa$, making it more suitable for practical design. However, since there are differences in the lower limits on $\kappa$ for various cases, a different approach may prove useful.

In author's view, since the focus of the study is on buildings with flexible diaphragms and generally such buildings exhibit large drift ratios, the lower limits on $\kappa$ which are 
related to the data corresponding to larger values of drift ratio and which will be used to determine the force modification factor for buildings with higher drift ratios, are of importance.. Therefore, a more detailed processing is carried out of the portion of the data corresponding to higher values of $r$ (the range of $2-10)$.

According to the data presented in Table 4.1, the lower limits on $\kappa$ increase with higher values of $\alpha$. Further, ground motions compatible with the UHS for eastern region is generally seen to give larger values for the lower limits on $\kappa$.

For each value of post-yield hardening ratio, plots of $\kappa$ versus the drift ratio corresponding to all values of the ductility demand, namely 2, 3 and 4, are presented in Figures 4.25 to 4.30 for the ground motions compatible to the UHS for Vancouver and Montreal, and compared with the equation suggested by Humar and Popovski (2013). The lower limits obtained by averaging the data are also shown in the graphs.

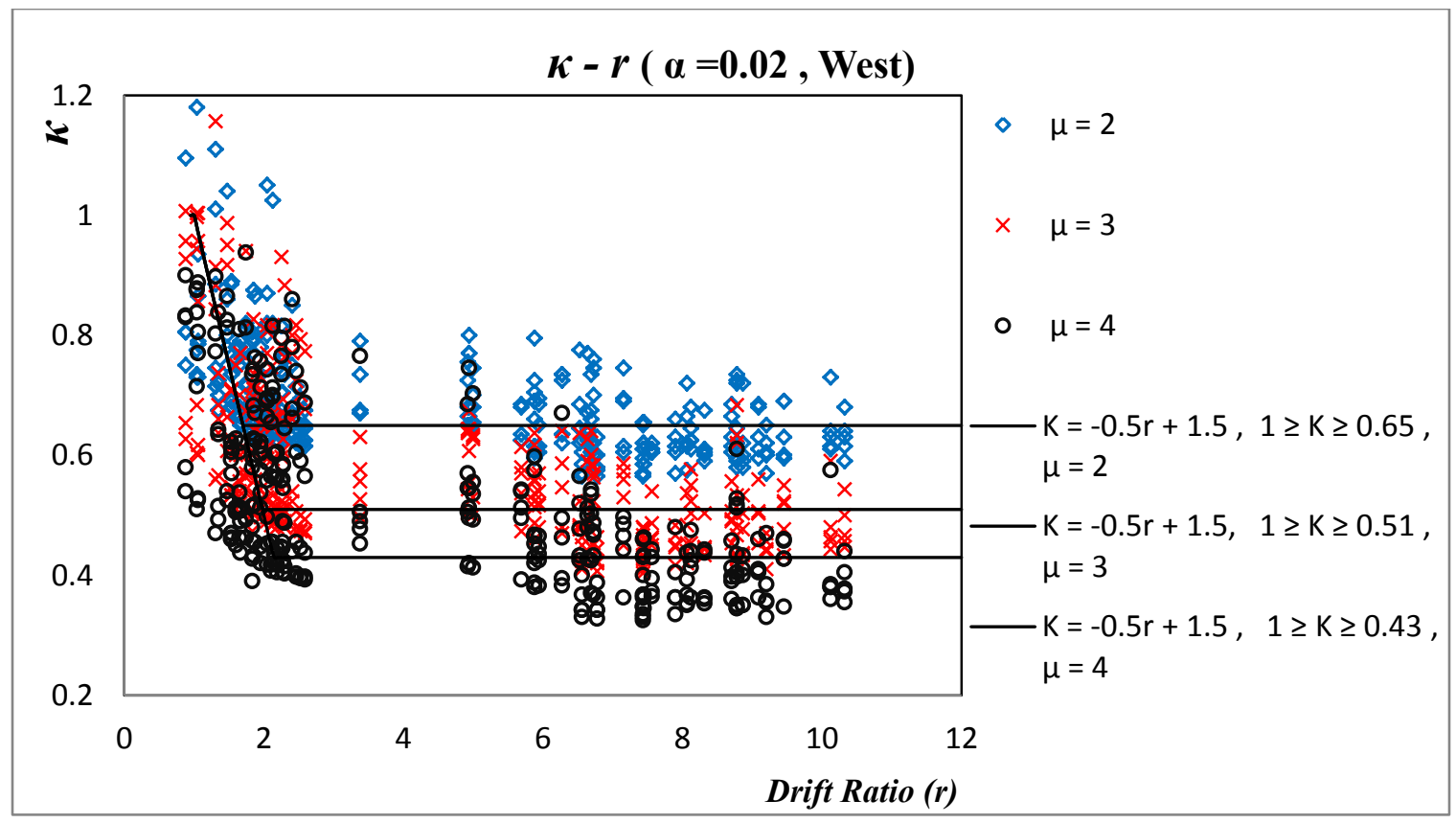

Figure 4.25 - Suggested equation for $\kappa$ and the obtained lower limits on $\kappa, \alpha=0.02$, West 


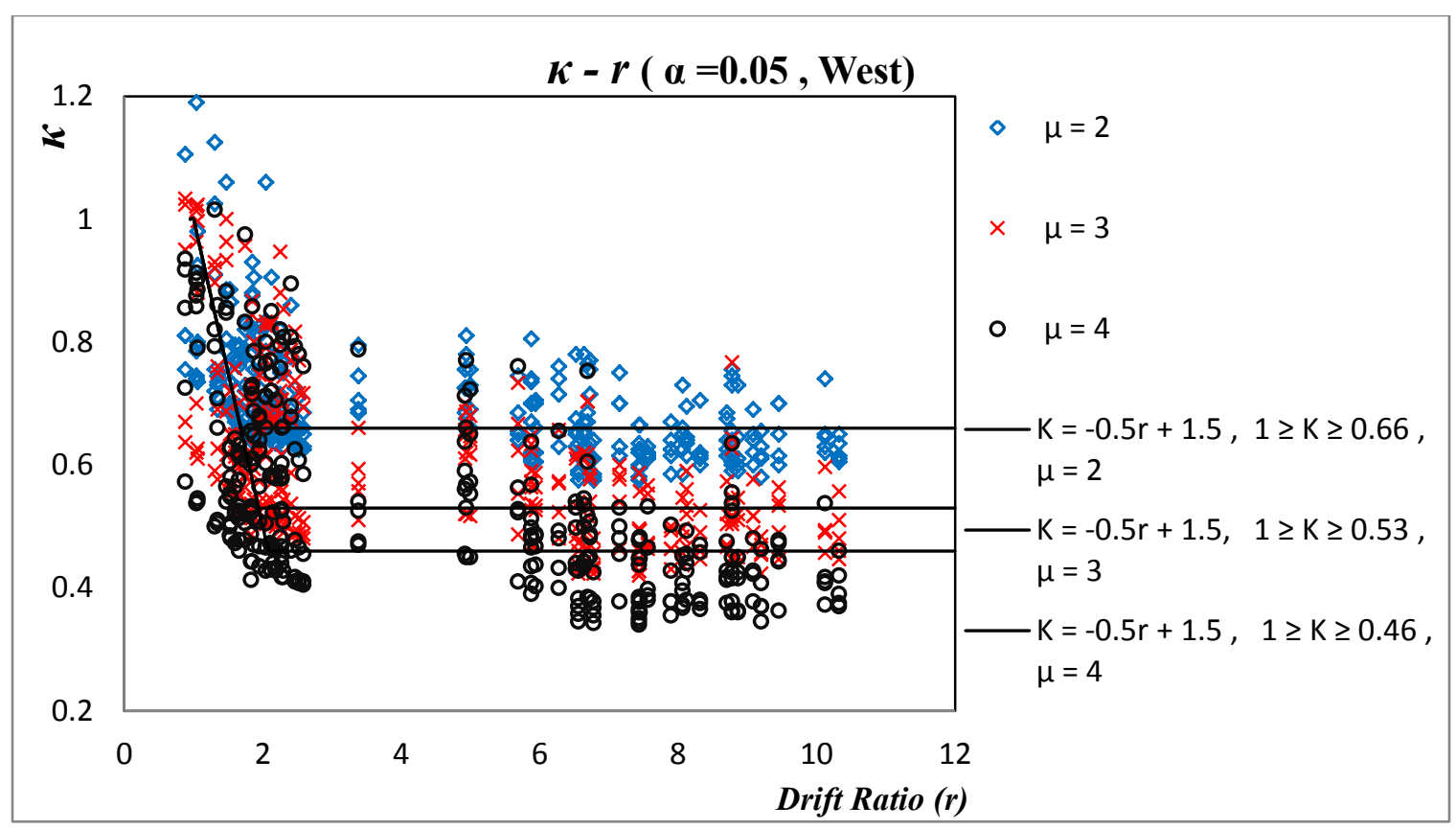

Figure 4.26 - Suggested equation for $\kappa$ and the obtained lower limits on $\kappa, \alpha=0.05$, West

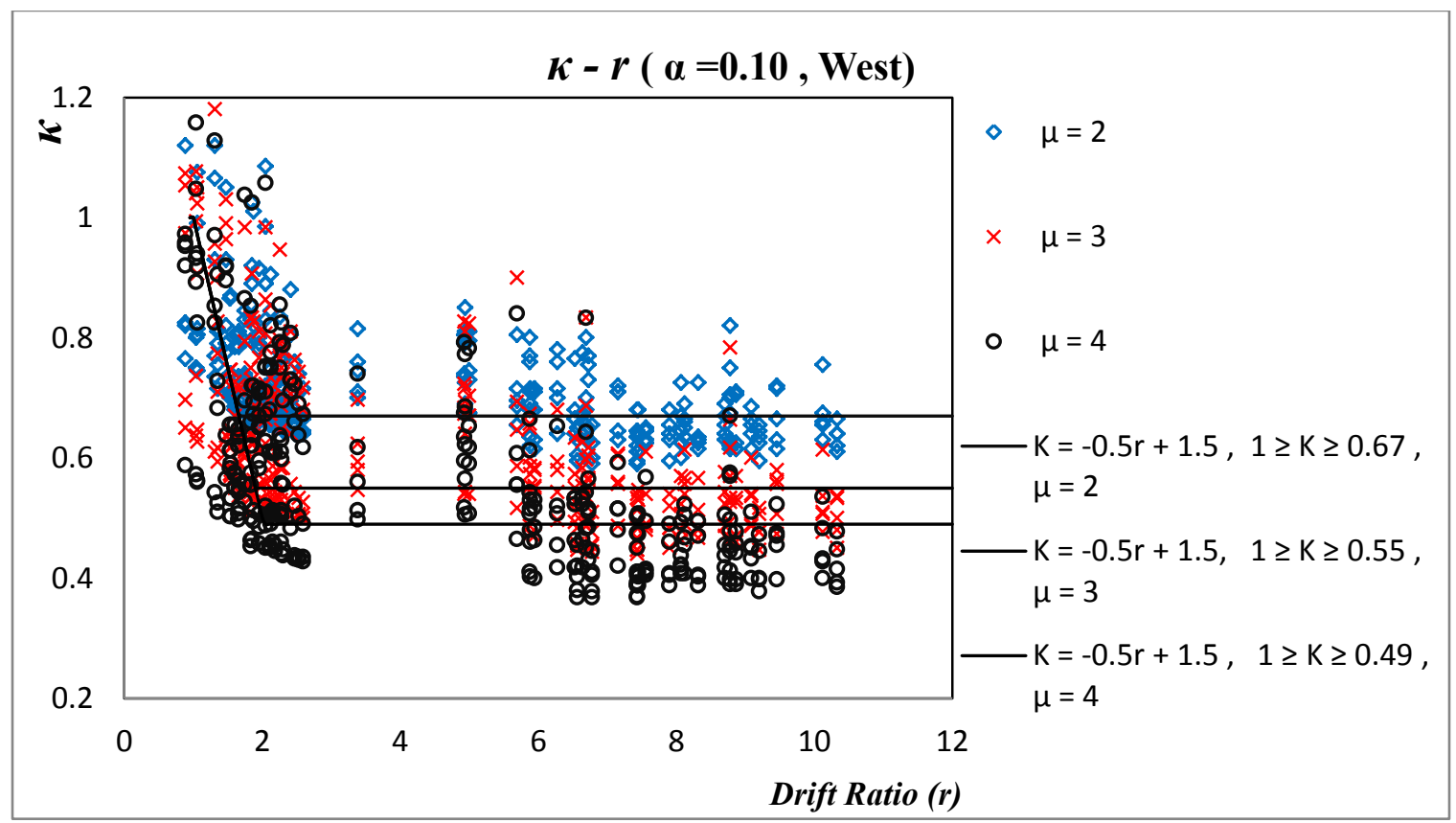

Figure 4.27 - Suggested equation for $\kappa$ and the obtained lower limits on $\kappa, \alpha=0.10$, West 


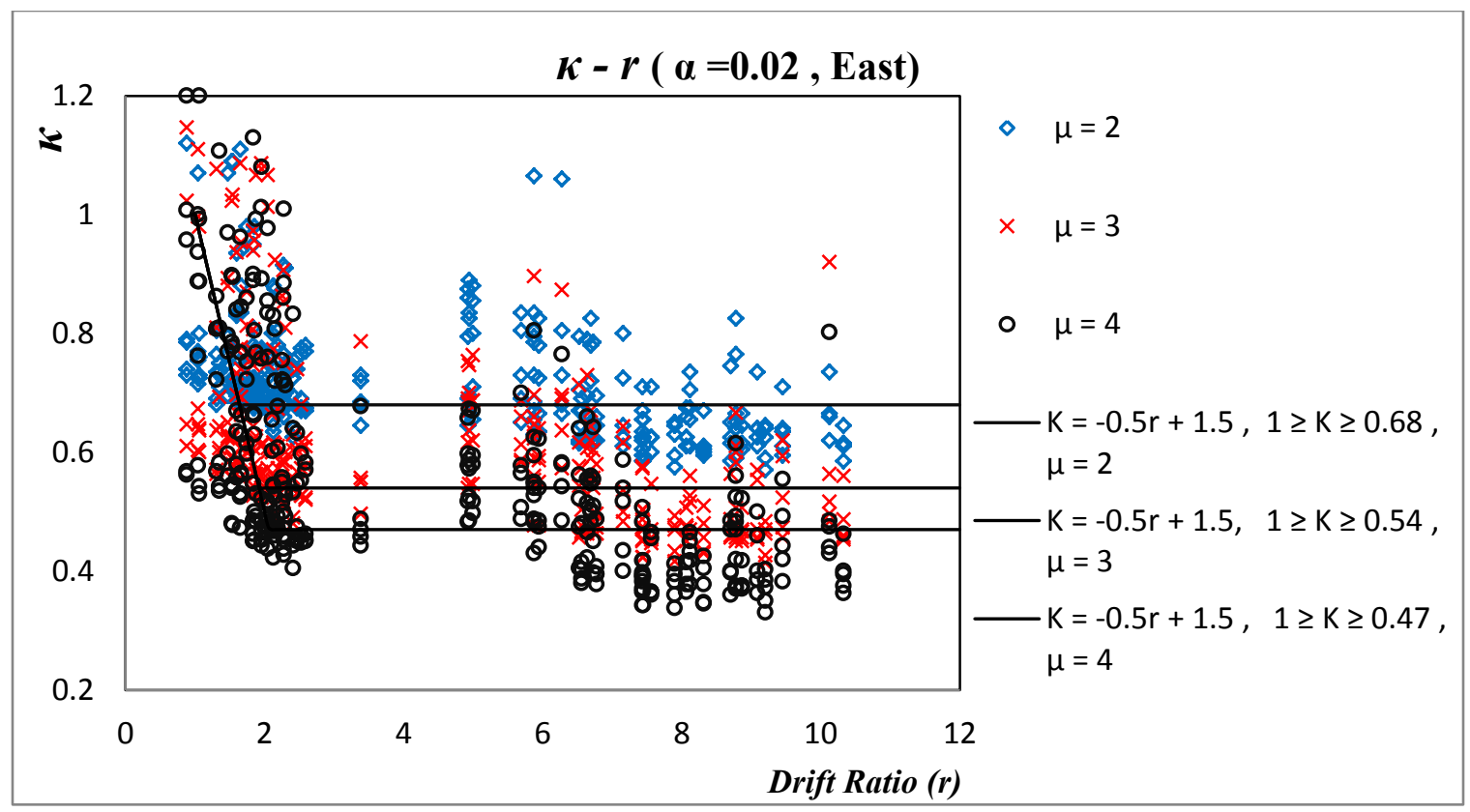

Figure 4.28 - Suggested equation for $\kappa$ and the obtained lower limits on $\kappa, \alpha=0.02$, East

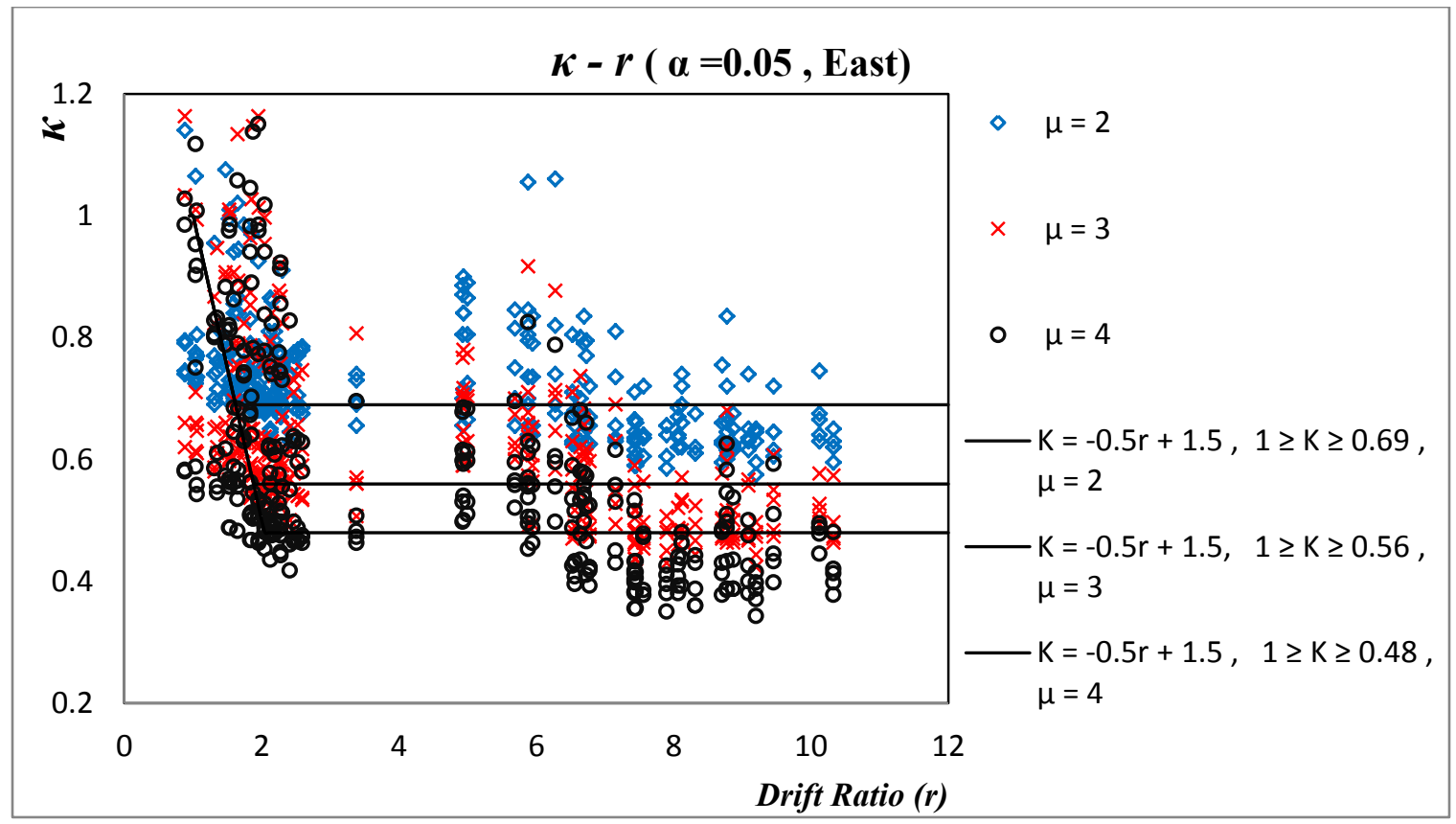

Figure 4.29 - Suggested equation for $\kappa$ and the obtained lower limits on $\kappa, \alpha=0.05$, East 


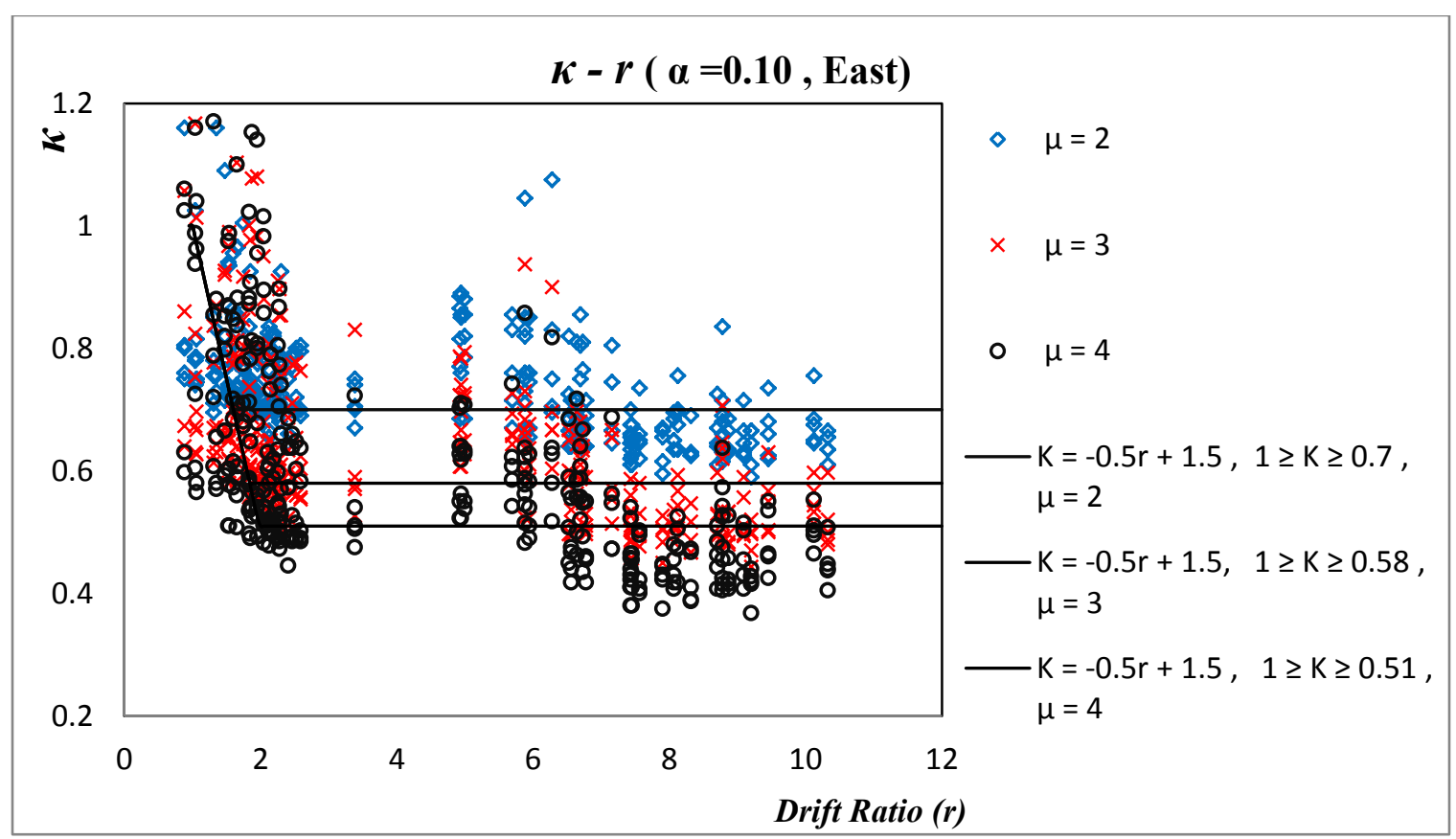

Figure 4.30 - Suggested equation for $\kappa$ and the obtained lower limits on $\kappa, \alpha=0.10$, East

As can be seen in Figures 4.25 to 4.30 , in each case, the equation suggested by Humar and Popovski (2013) provides reasonable prediction for the $\kappa$ values for the lower values of the drift ratio. The lower limits on $\kappa$, however, do change and are different for different cases. Elasto-plastic behaviour $(\alpha=0.00)$, provides the most critical case with the smallest lower limits, while $\alpha=0.10$ provides the least critical case with the largest lower limits. The differences are, however, relatively small.

\subsection{Determining Lower Limits on $\boldsymbol{\kappa}$}

In the following paragraphs three different alternatives are suggested for determining the lower limits on $\kappa$ : 
1. Using the lower limits suggested for $\alpha=0.00$. Since the smallest lower limits were suggested in this case, the designer can use these values with ease. This approach may be a bit conservative. However it gives a consistent method in finding the force reduction factor in buildings with flexible diaphragm for all values of $\alpha$. This method is recommended for buildings in regions with low seismic activity or buildings in which precision is not important (buildings with small base shear or of low level of importance).

2. Finding the lower limits using the Table 4.1. This method is more precise. This method may be justified for buildings in areas of high seismicity or for buildings where earthquake loading is critical.

3. As an alternative to the second method, one can use the equations for the lower limits on $\kappa$ that are presented in the following section. This method is again suggested for buildings with critical base shear (high seismicity area or buildings with high level of importance). While it lacks the consistency that method one holds, it gives a fair precision in determining the lower limits on $\kappa$ values.

The lower limits of $\kappa$ seem to change for different cases. In addition, since they are responsible for determining the force modification factor for buildings with drift ratios in the range of 2 to 10 (buildings with flexible diaphragms according to FEMA 356 provisions), they are of greater importance. Therefore, a multiple regression has been carried out in order to find equations that can predict the lower limits on $\kappa$. The data for different cases are first presented in Table 4.2: 
Table 4.2 - Data for regression

\begin{tabular}{cccc}
\hline $\begin{array}{c}\text { Ductility } \\
\text { Demand }(\mu)\end{array}$ & $\begin{array}{c}\text { Post-Yield } \\
\text { Hardening }(\alpha)\end{array}$ & $\begin{array}{c}\text { Dummy Variable } \\
(d)\end{array}$ & $\begin{array}{c}\text { Lower } \\
\text { Limits }\end{array}$ \\
\hline 2 & 0 & 1 & 0.67 \\
3 & 0 & 1 & 0.53 \\
4 & 0 & 1 & 0.46 \\
2 & 0.02 & 1 & 0.68 \\
3 & 0.02 & 1 & 0.54 \\
4 & 0.02 & 1 & 0.47 \\
2 & 0.05 & 1 & 0.69 \\
3 & 0.05 & 1 & 0.56 \\
4 & 0.05 & 1 & 0.49 \\
2 & 0.1 & 1 & 0.7 \\
3 & 0.1 & 1 & 0.58 \\
4 & 0.1 & 1 & 0.51 \\
2 & 0 & 0 & 0.64 \\
3 & 0 & 0 & 0.5 \\
4 & 0 & 0 & 0.41 \\
2 & 0.02 & 0 & 0.65 \\
3 & 0.02 & 0 & 0.51 \\
4 & 0.02 & 0 & 0.43 \\
2 & 0.05 & 0 & 0.66 \\
3 & 0.05 & 0 & 0.53 \\
4 & 0.05 & 0 & 0.46 \\
2 & 0.1 & 0 & 0.67 \\
3 & 0.1 & 0 & 0.55 \\
4 & 0.1 & 0 & 0.49 \\
\hline
\end{tabular}

The multiple regression calculations are conducted twice. In the first set of calculations, the type of spectrum is not taken into account; in the second set, a distinction is made depending on the type of the spectrum. For this purpose, a dummy variable is defined to account for the type of spectrum as can be seen in the table presenting the data. The 
dummy variable is defined such that for eastern spectrum target, $d=1$ while $d=0$ for any other case.

After the regressions, the following equations are suggested for determining the lower limit on $\kappa$.

When the type of spectrum is not considered

$\kappa=0.84-0.1 \mu+0.47 \alpha$

When the type of spectrum is considered

$\kappa=0.83-0.1 \mu+0.47 \alpha$

$\kappa=0.86-0.1 \mu+0.47 \alpha$

Equation 4.2a, applies for sites in the western region, while Equation 4.2b applies for sites in the eastern region.

\subsection{Conclusions and Recommendations}

The results presented in the previous sections lead to the following conclusions:

1. Post-yield stiffness decreases the ductility demand on the LLRS, relative to the case for zero post-yield stiffness, and consequently increases the force reduction factor. For systems with higher post-yield stiffness, smaller residual displacements are observed.

2. Although there are differences in the results obtained from the analyses on systems with post-yield stiffness to those for buildings with elasto-plastic 
behaviour, the equation suggested by Humar and Popovski (2013) still gives reasonable estimation of the force reduction factor, particularly for smaller values of the drift ratio. Even for larger drift ratio, the $\kappa$ values (the lower limits obtained for the elasto-plastic case could be considered quite adequate for the purpose of design.

3. For determining more refined values for the lower limits on $\kappa$ either the values suggested in Table 4.1 may be used or Equation 4.1 and 4.2 may be used. 


\section{Chapter 5 : The Effects of Diaphragm Flexibility on the Distribution of Shear Forces and Bending Moments in the Diaphragm}

\section{$5.1 \quad$ Introduction}

As pointed out earlier, one of the effects of the flexibility of the diaphragm on the seismic response of one-storey buildings with flexible diaphragm is the magnification of the internal forces arising from earthquake excitation. Recent studies have shown that internal bending moment at mid-span and the shear force at the quarter span increase significantly due to the flexibility of the diaphragm. To comprehend the reason for this behaviour one must first study the dynamic response of both perfectly rigid diaphragms and flexible diaphragms.

\subsubsection{Dynamic Response of Rigid Diaphragms}

When a perfectly rigid diaphragm undergoes vibrations due to identical ground motions applied to its supports, every point along the length of the diaphragm experiences the same displacement. In other words, the diaphragm translates in its plane but does not deform. Uniform displacements along the length of the diaphragm lead to uniform accelerations and consequently to a uniform distribution of inertia forces along the length of the diaphragm. As a result, internal shear forces and bending moments along the length of the diaphragm follow linear and parabolic distributions, respectively. Under such force distribution, the maximum shear force and bending moment values occur at the supports and mid-span, respectively. Although the foregoing is true only for perfectly rigid diaphragms, often in the design of diaphragms that behave close to a rigid diaphragm, it 
is assumed that the inertia forces are distributed uniformly along the length of the diaphragm.

\subsubsection{Dynamic Response of Flexible Diaphragms}

In a flexible diaphragm several vibration modes contribute to the response and the distribution of inertia forces depends on the shape of the predominant mode. To consider this further, the first seven modes of vibration for the analytical model used in this study (beam - spring model) are presented in Figure 5.1.

Consider the modal equation for mode $n$ in a system with proportional damping:

$\ddot{y}_{n}+2 \zeta_{n} \omega_{n} \dot{y}_{n}+\omega_{n}^{2} y_{n}=\frac{\Phi_{n}^{T} \mathbf{F}}{M_{n}} f(t)$

For earthquake excitation

$$
\begin{aligned}
& \ddot{y}_{n}+2 \zeta_{n} \omega_{n} \dot{y}_{n}+\omega_{n}^{2} y_{n}=-\frac{\Phi_{n}^{T} \mathbf{M r}}{M_{n}} \ddot{v}_{g}(t) \\
& P . F=\frac{\Phi_{n}^{T} \mathbf{F}}{M_{n}}
\end{aligned}
$$

or

$$
P . F=\frac{\Phi_{n}^{T} \mathbf{M r}}{M_{n}}
$$




\section{Schematic Mode Shapes}
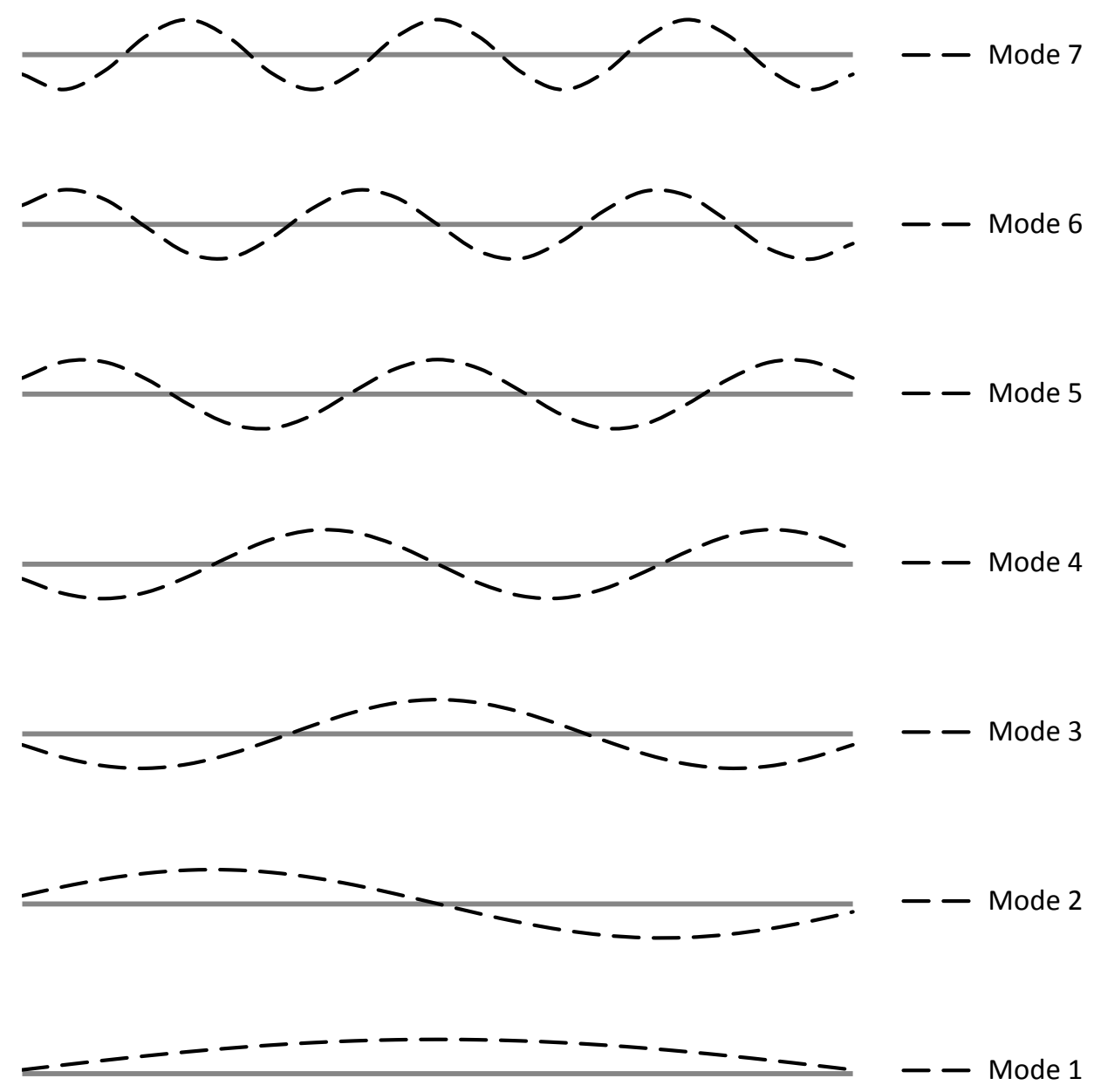

Diaphragm Length

Figure 5.1 - First seven modes of the beam-spring (diaphragm) model

Equations 5.1 and 5.2 represent the response in mode No. $n$ for forced vibration and vibrations due to ground motions, respectively. In these equations, $\ddot{y}_{n}$ is the acceleration in mode $n, \dot{y}_{n}$ is the velocity in mode $n, y_{n}$ is the displacement in mode $n, \omega_{n}$ is the frequency of mode $n, \zeta_{n}$ is the damping ratio for mode $n, \Phi_{n}^{T}$ is the $n$th mode shape, $M_{n}$ is the mass associated with the $n$th mode, $\mathbf{F}$ is a vector representing the spatial 
distribution of the exciting force, $f(t)$ is the variation of the external force with time, $\mathbf{M}$ is the mass matrix of the system, and $\mathbf{r}$ is the vector of rigid body displacements produced by a unit displacement at the supports. The term P.F in Equations 5.3 and 5.4 is the participation factor for mode $n$, which is a measure of the level of the contribution from mode $n$ to the total response of the system. As can be seen from Equations 5.3 and 5.4, the contribution of a mode is dependent on the dot product of the mode shape and the spatial distribution of the exciting force. If the mode shape and the spatial distribution of the external force are orthogonal, the participation factor for that mode would be zero meaning that the contribution of that mode to the response would be zero percent of the total response. On the other hand, if the spatial distribution and the mode shape of a given mode are almost identical vectors, the response of that mode becomes dominant.

In a flexible diaphragm the displacements along the length of the system are no longer uniform. The diaphragm experiences larger displacements near mid span. This suggests that larger accelerations and therefore larger inertia forces will be produced near midspan. In fact, the distribution of inertia forces along the length of the diaphragm follows a distribution similar to that of a parabola. Since the response matches the first mode closely, larger inertia forces would be concentrated near the mid-span which would cause an increase in the bending moment at the mid-span and the shear force at quarter-span. Equation 5.5, which is the equation of a parabola, has therefore been proposed by FEMA 356 (FEMA 2006) for predicting the distribution of inertia forces along the length of the diaphragm.

$$
f_{d}=\frac{1.5 F_{d}}{L_{d}}\left[1-\left(\frac{2 x}{L_{d}}\right)^{2}\right]
$$


where $f_{d}$ is the inertia load intensity at a distance $\mathrm{x}$ from the centre line of the diaphragm, $\mathrm{F}_{d}$ is the total inertia force on the diaphragm, and $L_{d}$ is the unsupported length of the diaphragm.

Although the participation factors for higher modes are always relatively small compared to the first mode, their contributions, especially that of the third mode, is still important in the assessment of the behaviour of the system. When the effects of the third mode are added to the results obtained from the first mode, it becomes evident that around the midspan the inertia forces increase. This leads to even greater values of bending moment near the mid-span. The addition of response results from the third mode to those of the first mode also leads to a decrease of forces near the ends of the span. Although this effect is small, since the magnitude of seismic forces is small near the ends, it still affects the results and further magnifies the shear force at quarter span. This effect can often cause the shear force at the quarter span to even exceed the shear force value at end supports. The bending moment at mid-span and the shear force at quarter span are further magnified by the contributions of modes higher than the third.

In conclusion it can be stated that, the changed spatial distribution of the seismic force in flexible diaphragms and the contribution of higher modes are the main reasons for the magnification in bending moments and shear forces in flexible diaphragms.

It may be noted that for the model considered in this study the distributions of the inertia forces in both rigid and flexible case are symmetrical. Consequently, the participation factor presented in Equation 5.4 is zero for the unsymmetrical even number modes (i.e. second, fourth and so on.) This confirms the assumption that was made earlier in the 
study which stated that even number modes would not be excited for such a system under identical support ground motions.

\subsubsection{The Importance of Assessing the Magnification of Internal Forces}

Another effect of the flexibility of the diaphragm is the elongation of the period of the system. As a consequence of the lengthening of the period, the total base shear in the building becomes smaller. This can be understood by examining the shape of a uniform hazard spectrum (UHS) in which longer periods lead to smaller base shears. However, this reduced shear is accompanied by concentration of inertia forces near mid-span. This results in larger bending moments at mid span and larger shear forces at quarter span that sometimes can exceed the corresponding values obtained from the rigid diaphragm case, with a larger base shear, but with a uniform distribution of inertia forces. Thus, even if a building with flexible diaphragm is treated as one with a rigid diaphragm, and designed for the larger base shear, the design may not be conservative in terms of the internal shear forces and bending moments. Also, the ductility demand on the lateral load resisting system may be underestimated. It is therefore necessary to understand the pattern in which the shear forces and bending moments are distributed along the length of flexible diaphragms, or as an alternative develop methods for predicting the critical shear force (at quarter span) and bending moments (at mid span) in flexible diaphragms based on their actual distribution.

As stated in the previous paragraphs, greater contributions from the higher modes, particularly the third mode, in diaphragms of increasing flexibility supported by a LLRS that is strained into the inelastic range, cause distribution to further depart from being 
parabolic. This suggests that the assumption that shear forces follow a linear distribution and bending moments follow a parabolic distribution becomes more and more inaccurate. If one compares a parabolic distribution of forces to a uniform distribution of forces with the total force being equal for both cases, the shear force is greater at the quarter span and the bending moment at mid-span is larger in the former case. Such changes in the shear forces and bending moments becomes more pronounced as the flexibility increases and the LLRS becomes nonlinear.

The foregoing reasoning makes it evident that while a uniform distribution of the seismic force, linear distribution for shear and a parabolic distribution for bending moment can be used for designing rigid diaphragms or diaphragms with a behaviour quite close to a rigid one, for flexible diaphragms the distribution of inertia forces, shear forces and bending moments can be quite complicated and dependent on the characteristics of the ground motion as well as the characteristics of the building structure including the diaphragm. While limited research on this issue has been reported in the literature, it has always been recognized as one of the problems in the design of buildings with flexible diaphragms. Humar and Popovski (2012) made the observation that applying the total seismic load to the diaphragm with the parabolic distribution suggested by FEMA gives a fair prediction of actual internal bending moments obtained from dynamic analyses. This is generally true if the supporting LLRS remains elastic, but as will be shown in the present study, inelasticity in the LLRS results in further increase in the bending moment at the mid-span and shear force at the quarter span. 


\subsubsection{Chapter Outline}

It will be shown here that with increasing flexibility of diaphragm and nonlinearity in the supporting lateral load resisting system (LLRS), the distribution of inertia forces departs more and more from a parabola, further increasing the inertia forces toward the mid-span and increasing the contribution from the higher modes.

In the current study, an attempt is made to compare the actual internal shear forces and bending moments at quarter-span and mid-span, respectively, to their corresponding values obtained from a uniform distribution of the total seismic loads or from a parabolic distribution of such loads as suggested by FEMA 356. The increase in shear force and bending moment is largest at the quarter-span and mid-span, respectively. Therefore, if we know the extent of magnification in shear force at the quarter-span and in the bending moment at the mid-span, we can use the uniform distribution or the FEMA 356 parabolic distribution for seismic inertia forces and simply magnify the shear force at quarter- and the bending moment at mid-span. In the current study, an attempt is made to develop appropriate magnification factors for predicting the actual shear force at quarter-span and bending moment at mid-span by determining the corresponding values from uniform distribution or FEMA parabolic distribution.

Clearly, the magnification factors referred to above can be different for different buildings with different characteristics. In the present study, an attempt is made to determine the system characteristics which govern the magnification factors. Also, equations are proposed for finding the magnification factor for different cases. 
Toward the end of the chapter a simplified method for designing a one-storey building with elastic flexible diaphragm is suggested. The suggested method is based on previous work by Humar and Popovski $(2012,2013)$

To accomplish the objectives outlined above, a series of nonlinear dynamic analyses are carried out to observe the pattern of shear and moment magnifications. A comprehensive database consisting of 65 buildings, 10 ground motions, several material behaviours and different targets of ductility demand is used to reach sound conclusions regarding the addressed issues.

\subsection{Analytical Model and Assumptions}

The 65 buildings ( 32 buildings for the case when the load is acting parallel to the long side of the building and 33 buildings for the case when the load is acting parallel to the short side of the system) selected from the database of buildings designed by Tremblay and Stiemer (1996), introduced earlier, are analysed for their response to UHS compatible ground motions generated by Atkinson (2009). Records E6C1, E6C13, E6C15, E6C18 and E6C42 scaled by factors of $0.55,0.74,0.56,0.61$ and 1.01 respectively are selected to represent the uniform hazard spectrum (UHS), for Montreal. For ground motions compatible with a typical UHS for west, specifically for Vancouver, records M6C1, M6C2, M6C26, M6C31 and M6C38 scaled by factors of 0.78, 0.87, 1.19, 0.99 and 1.43, respectively, are used. As described in Section 2.5, the buildings are modelled by deep beams, representing the diaphragm, supported on two springs, representing the LLRS. In addition, it is assumed that the nonlinearity in the system is confined to the lateral load resisting system (the springs in the analytical model). In order to take into account the 
effects of post-yield hardening as well as elasto-plastic behaviour in LLRS on the response of the structures, a set of bilinear behaviours $(\alpha=0.00, \alpha=0.02, \alpha=0.05$ and $\alpha$ $=0.10)$ is considered for the lateral load resisting elements, while a linear elastic behaviour is assumed for the diaphragm (the beam in the analytical model). Additional details related to the analytical model and properties of the system as well as the ground motions are presented in Section 2.5.

\subsection{Nonlinear Dynamic Analyses}

Direct time step integration of the equations of motion is carried out with the help of the MATLAB program referred to earlier, using average acceleration method for all 65 buildings subjected to 10 ground motions considering four material behaviours and three target ductility demands. This combination leads to 7800 cases. The time-step used in the analyses is $0.001 \mathrm{~s}$. Proportional Rayleigh damping of $5 \%$ is assumed for the first and the third modes.

In each case, the total seismic load which is twice the total load in each braced frame is calculated. The total seismic load is then distributed either as a uniform load or in the form of a parabola as suggested by FEMA 356. The shear force at quarter span and the bending moment at mid span are determined for each of these distributions. The values so obtained are compared to the corresponding actual values calculated from nonlinear dynamic analyses. The following 4 parameters are used in the study:

- M.F: The ratio of actual mid span moment to the mid span moment calculated for the case in which the total seismic load is distributed according the FEMA 356 parabolic distribution. 
- V.F: The ratio of actual quarter span shear to the quarter span shear calculated for the case in which the total seismic load is distributed according the FEMA 356 parabolic distribution.

- M.U: The ratio of actual mid span moment to the mid span moment calculated for the case in which the total seismic load is distributed uniformly.

- V.U: The ratio of actual quarter span shear to the quarter span shear calculated for the case in which the total seismic load is distributed uniformly.

The results of the analyses which provide the aforementioned ratios are presented in Tables C. 1 to C.80 in Appendix C.

\subsection{The Distribution of Inertia Forces in Building Diaphragms}

In order to form a better understanding of the effect that the flexibility of the diaphragm has on the distribution of shear forces and bending moments along the length of the diaphragm and to confirm the importance of the current study, the response of three example buildings SL1, ML1 and LH1 from the buildings that were originally designed by Tremblay and Stiemer (1996), are analysed first. The ground motion used in the analysis is record $\mathrm{M} 6 \mathrm{C} 1$ generated by Atkinson (2009). For each of the three example buildings the analyses are carried out twice, once with no scale factor on the stiffness of the diaphragm (flexible case) and next, with a scale factor of 200 on the stiffness of the diaphragm (rigid case). Since the intention here is just to compare the distributions of shears and moments between the flexible and the rigid case, $R_{y}=3$ and $\alpha=0.00$ are chosen for all cases to isolate the effect of diaphragm flexibility on the distribution on internal forces. Furthermore, the analyses are carried out for the case when the seismic 
load is applied parallel to the short side of the buildings. The results of the analyses are presented in the following paragraphs.

Figures 5.2 to 5.4 show that for each building the bending moment at mid span for flexible diaphragm exceeds that for the rigid cases even though the total base shear is smaller in the flexible case. For instance, for building ML1, in the flexible case the base shear is $339.2 \mathrm{KN}$ whereas the base shear corresponding to the rigid case is $454.8 \mathrm{KN}$. Even for building LH1, where the base shears are $1955.9 \mathrm{KN}$ and $4101.6 \mathrm{KN}$ for the flexible case and rigid case, respectively, the mid span bending moment in the flexible case exceeds the corresponding value for the rigid case. Similarly, Figures 5.5 to 5.7 suggest that the shear forces at the quarter span are larger for flexible diaphragms as compared to their corresponding values for the rigid cases. Sometimes this shear magnification at quarter span is so large that the quarter-span shear exceeds the shear force at the two ends.

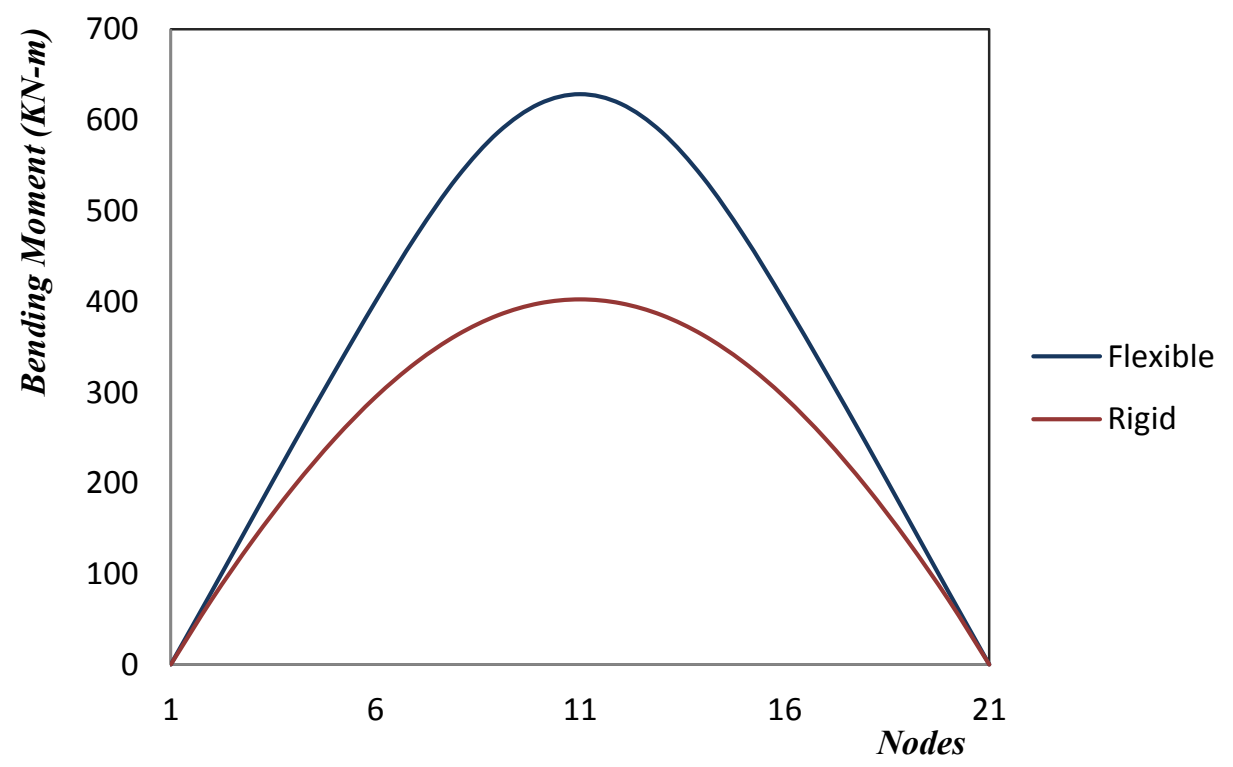

Figure 5.2 - Bending moment distribution in building SL1 when subjected to M6C1 ground motion, $\mathrm{Ry}=3, \alpha=0.00$ - in both the flexible case (blue line) and the rigid case (red line) 


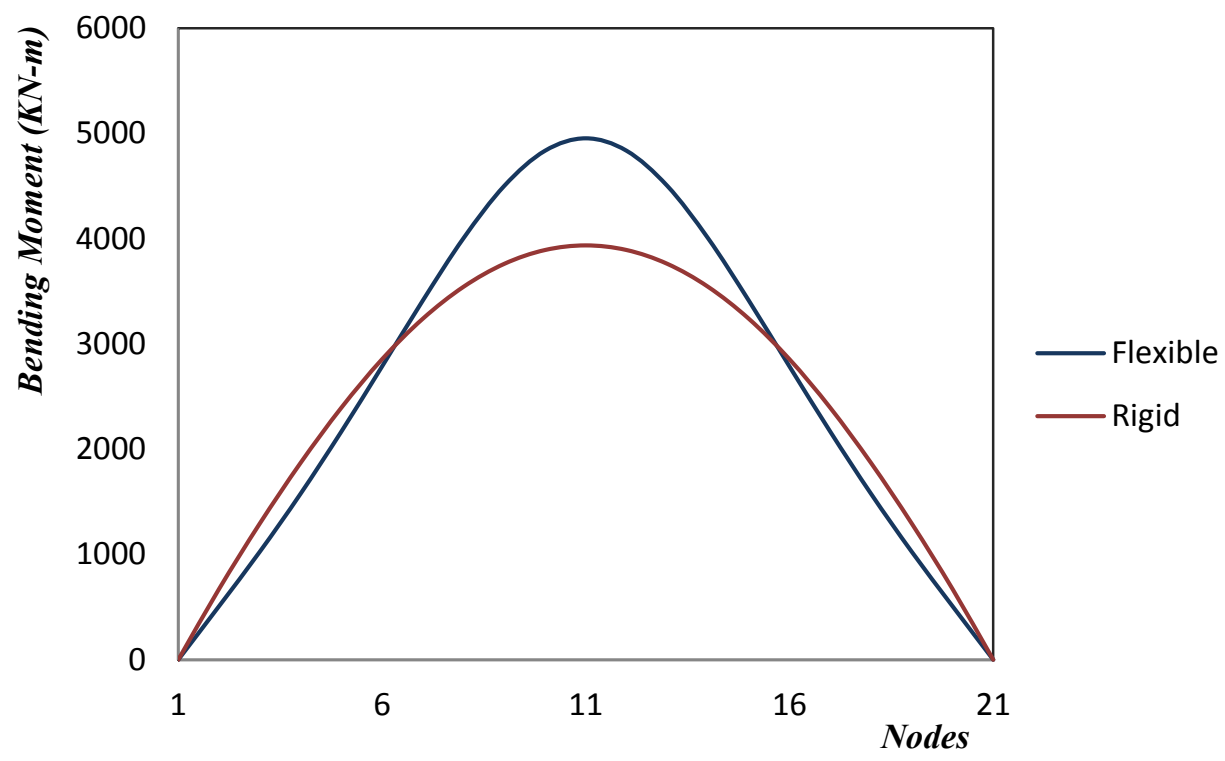

Figure 5.3 - Bending moment distribution in building ML1 when subjected to M6C1 ground motion, $R y=3, \alpha=0.00$ - in both the flexible case (blue line) and the rigid case (red line)

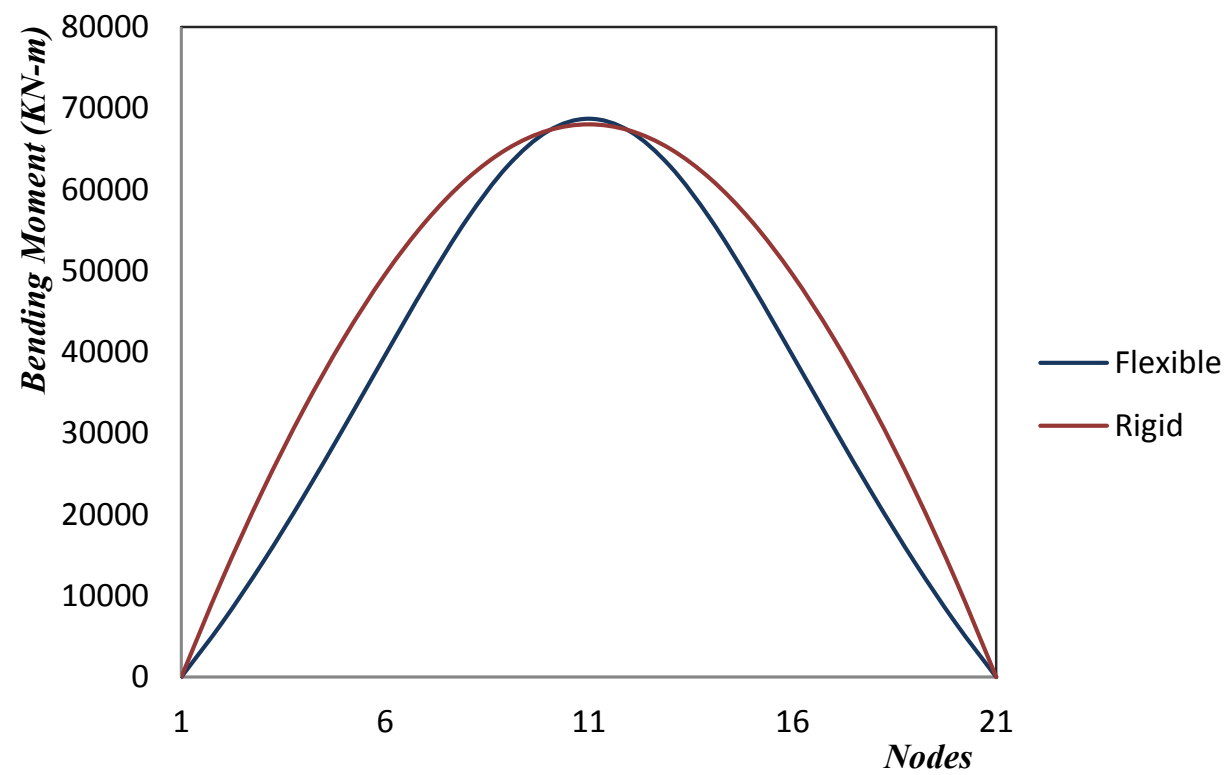

Figure 5.4 - Bending moment distribution in building LH1 when subjected to M6C1 ground motion, $R y=3, \alpha=0.00$ - in both the flexible case (blue line) and the rigid case (red line) 


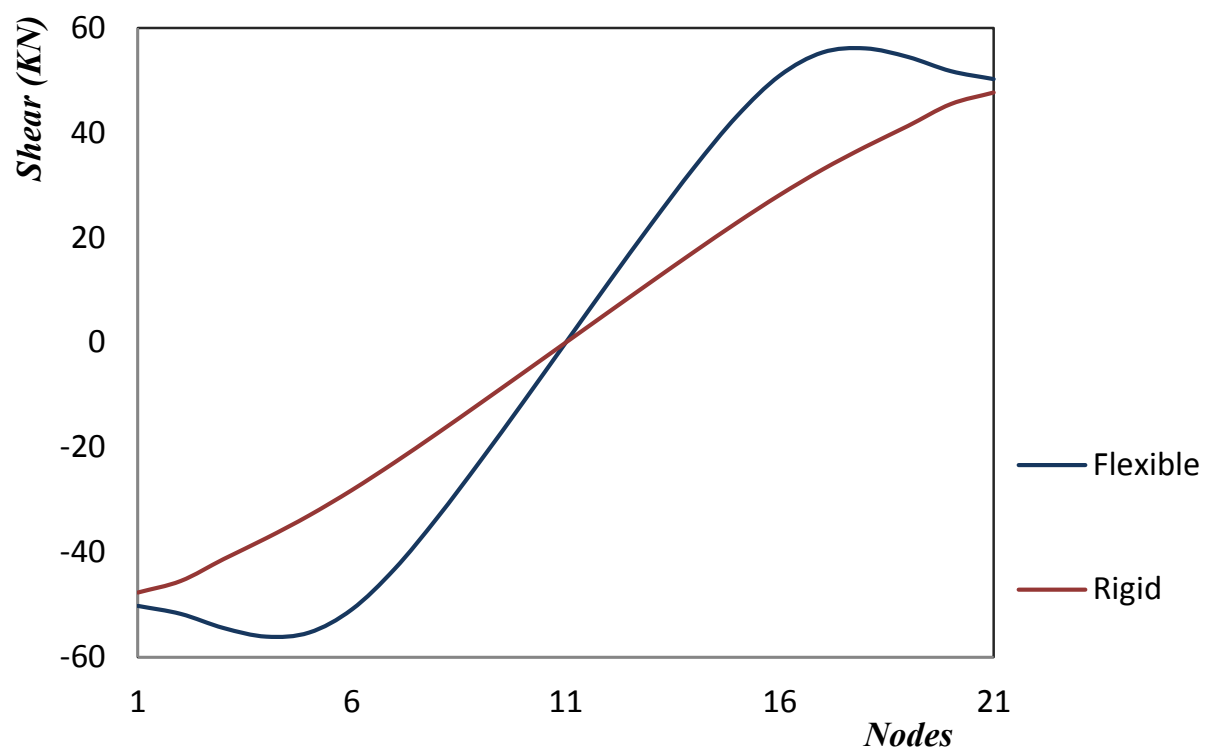

Figure 5.5 - Shear distribution in building SL1 when subjected to M6C1 ground motion, Ry =3, $\alpha=0.00$ - in both the flexible case (blue line) and the rigid case (red line)

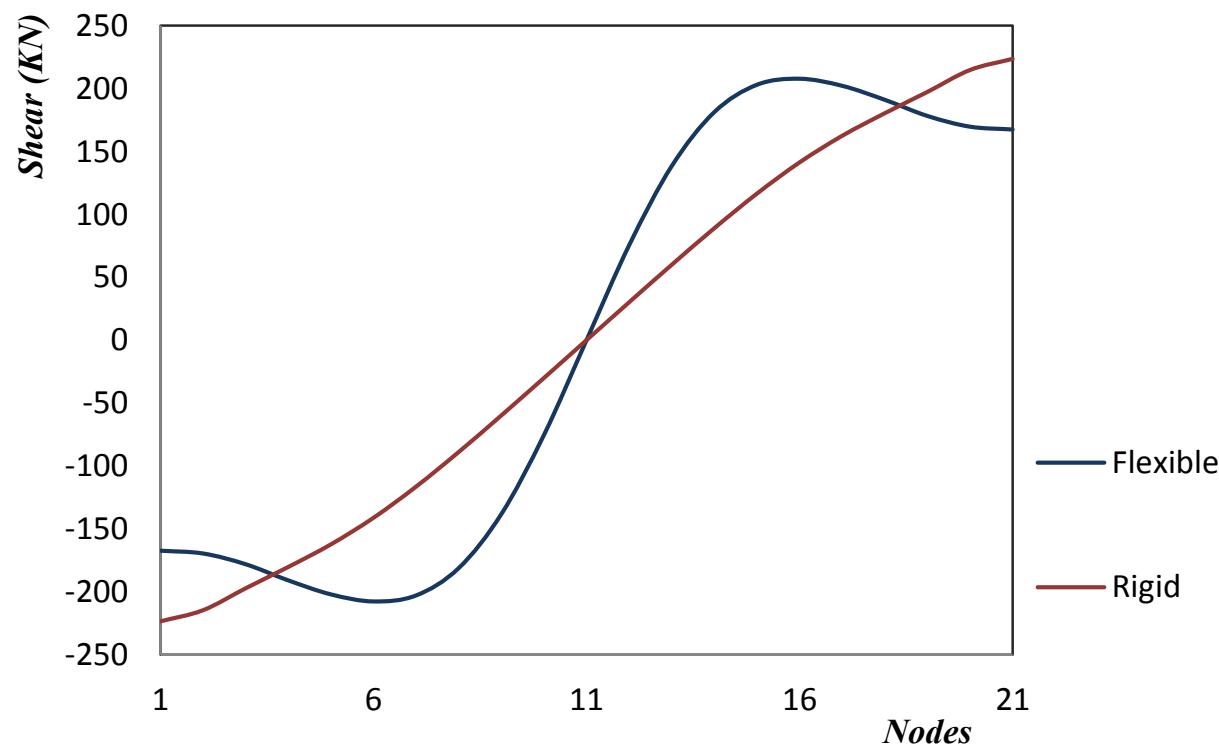

Figure 5.6 - Shear distribution in building ML1 when subjected to M6C1 ground motion, Ry =3, $\alpha=0.00$ - in both the flexible case (blue line) and the rigid case (red line) 


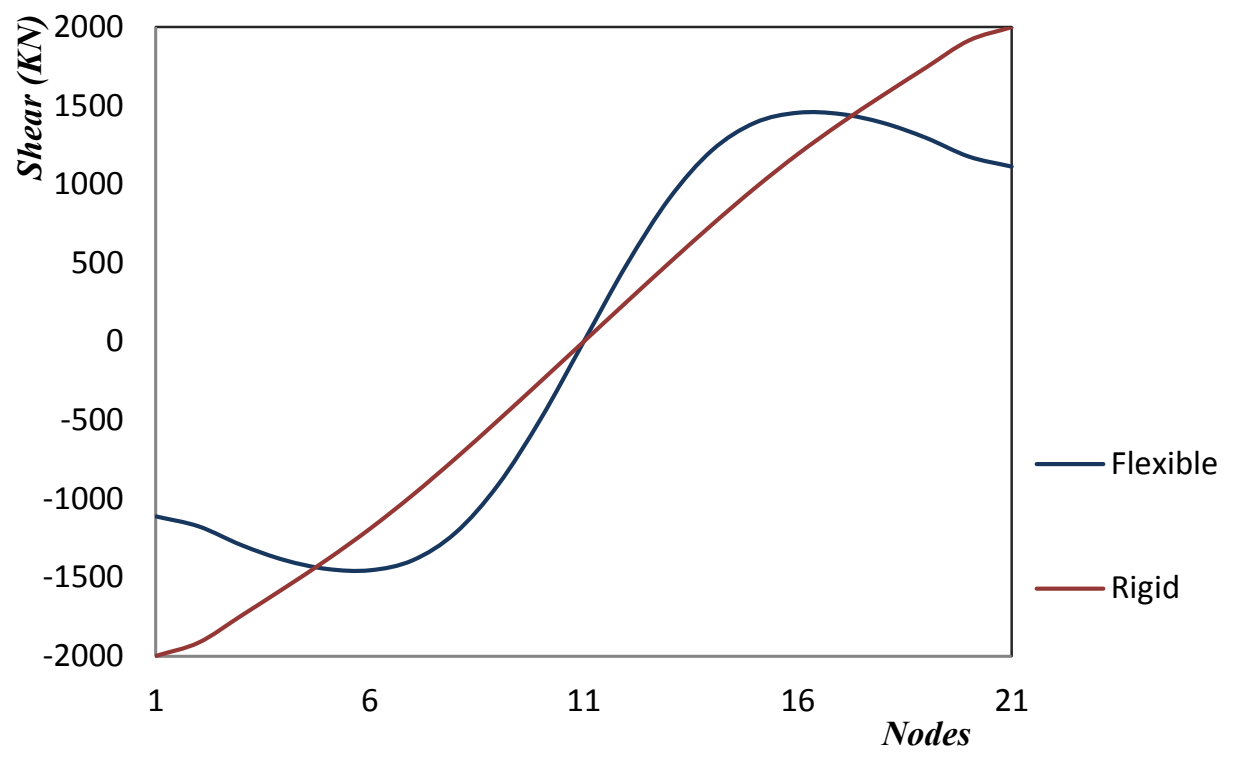

Figure 5.7 - Shear distribution in building LH1 when subjected to M6C1 ground motion, Ry =3, $\alpha=0.00$ - in both the flexible case (blue line) and the rigid case (red line)

The results of the nonlinear analyses for the 7800 cases referred to earlier are presented next in Figures 5.8 to 5.11 in the form of histograms of the defined ratio parameters (M.F, V.F, M.U and V.U) As can be observed from those figures, the ratios are almost always larger than 1. Figures 5.8 and 5.9, which present M.F and V.F, the ratios of the bending moments and shear forces determined from nonlinear dynamic analyses to the bending moments and shear forces obtained from a parabolic distribution (FEMA 356) of seismic forces, show values that are comparatively smaller than the ratios M.U and V.U, presented in Figures 5.10 and 5.11. This is to be expected since the parabolic distribution of FEMA 356 is much closer to the real distribution of forces. Nevertheless, use of an appropriate magnification factor is necessary even if the parabolic distribution of FEMA is used. The ratios of actual bending moments and shear forces to those obtained from a uniform distribution of the total seismic load are significantly larger. It can be seen that 
the value V.U sometimes exceeds 2.0, which means that the actual shear force at the quarter span occasionally is even larger than the shear in the braced frame.

\section{M.F Histogram}

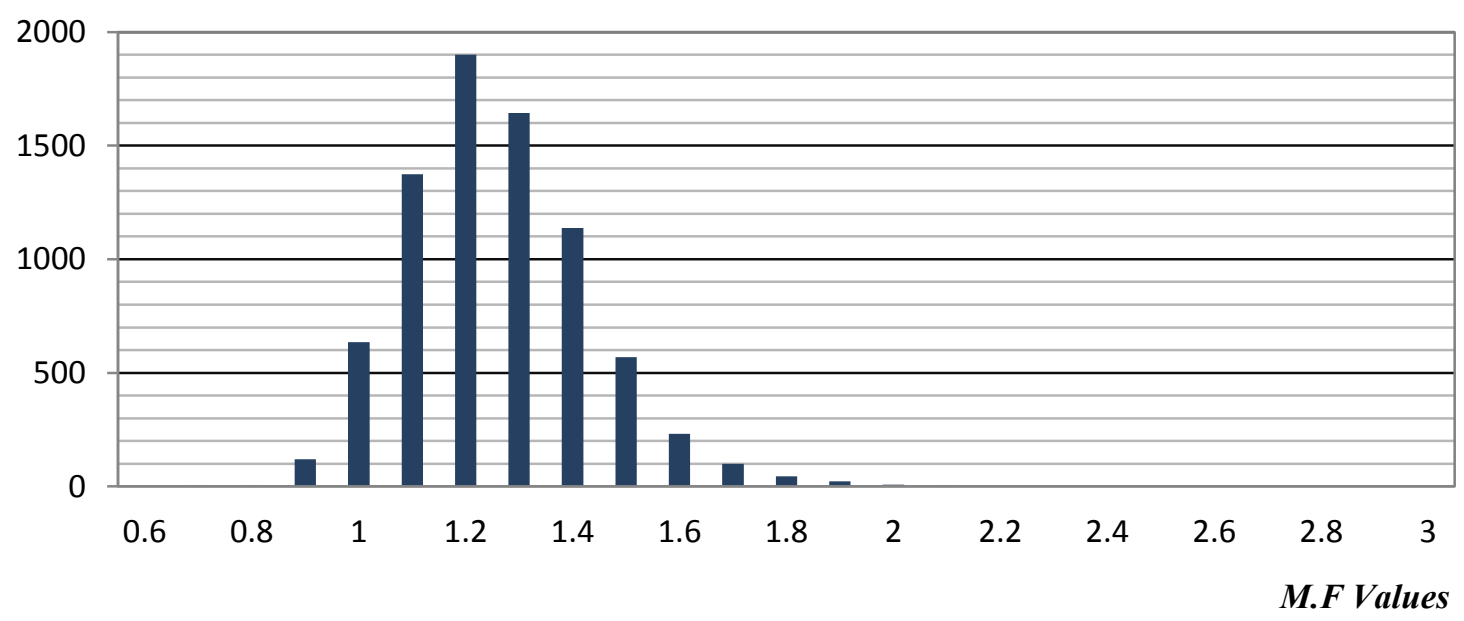

Figure 5.8 - The histogram of M.F ratio

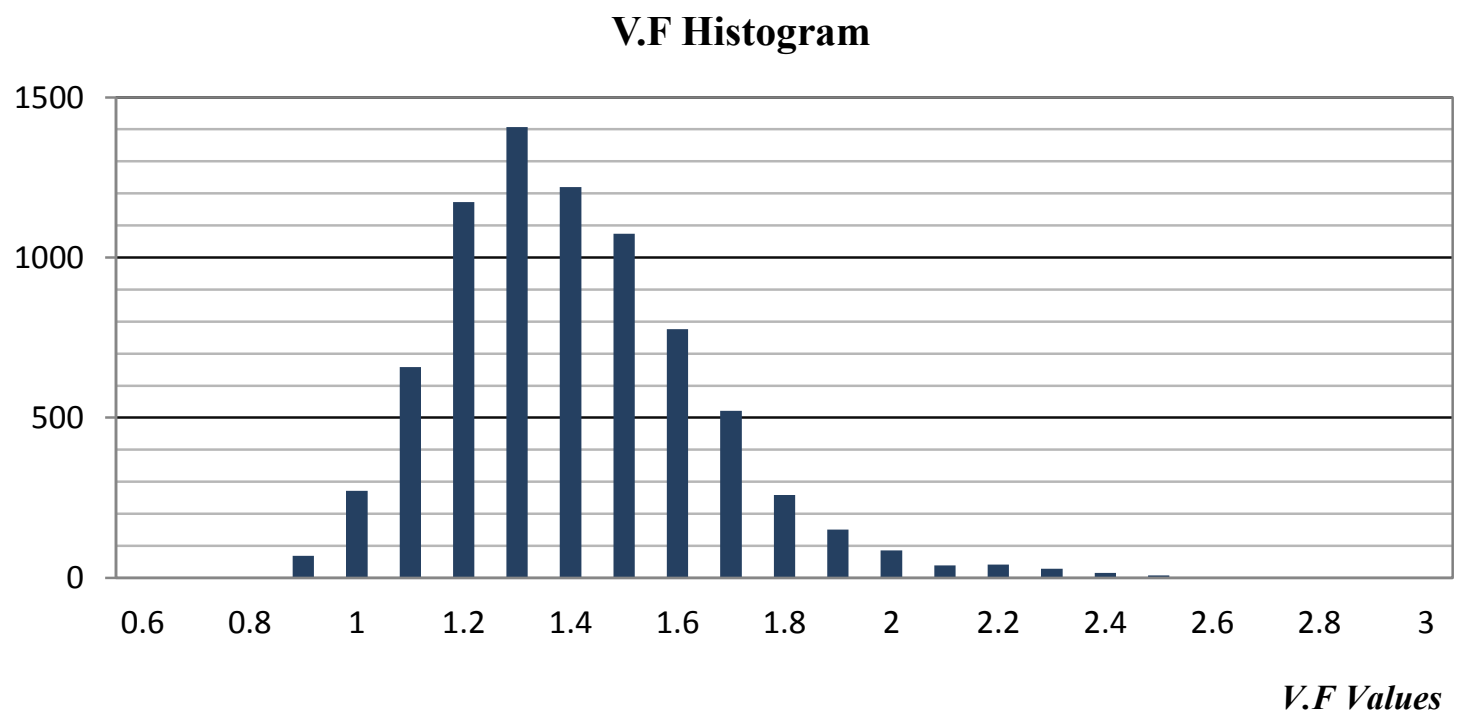

Figure 5.9 - The histogram of V.F ratio 


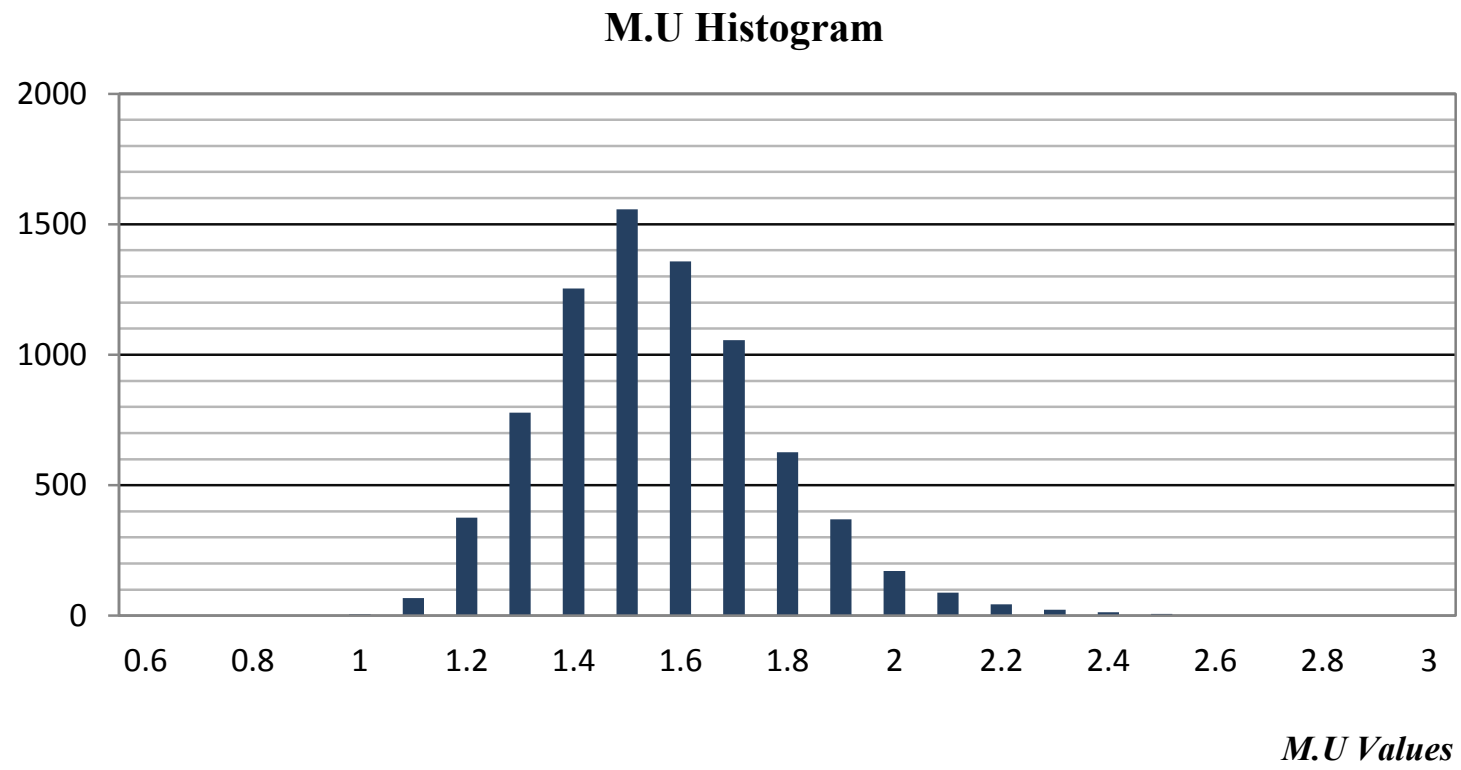

Figure 5.10 - The histogram of M.U ratio

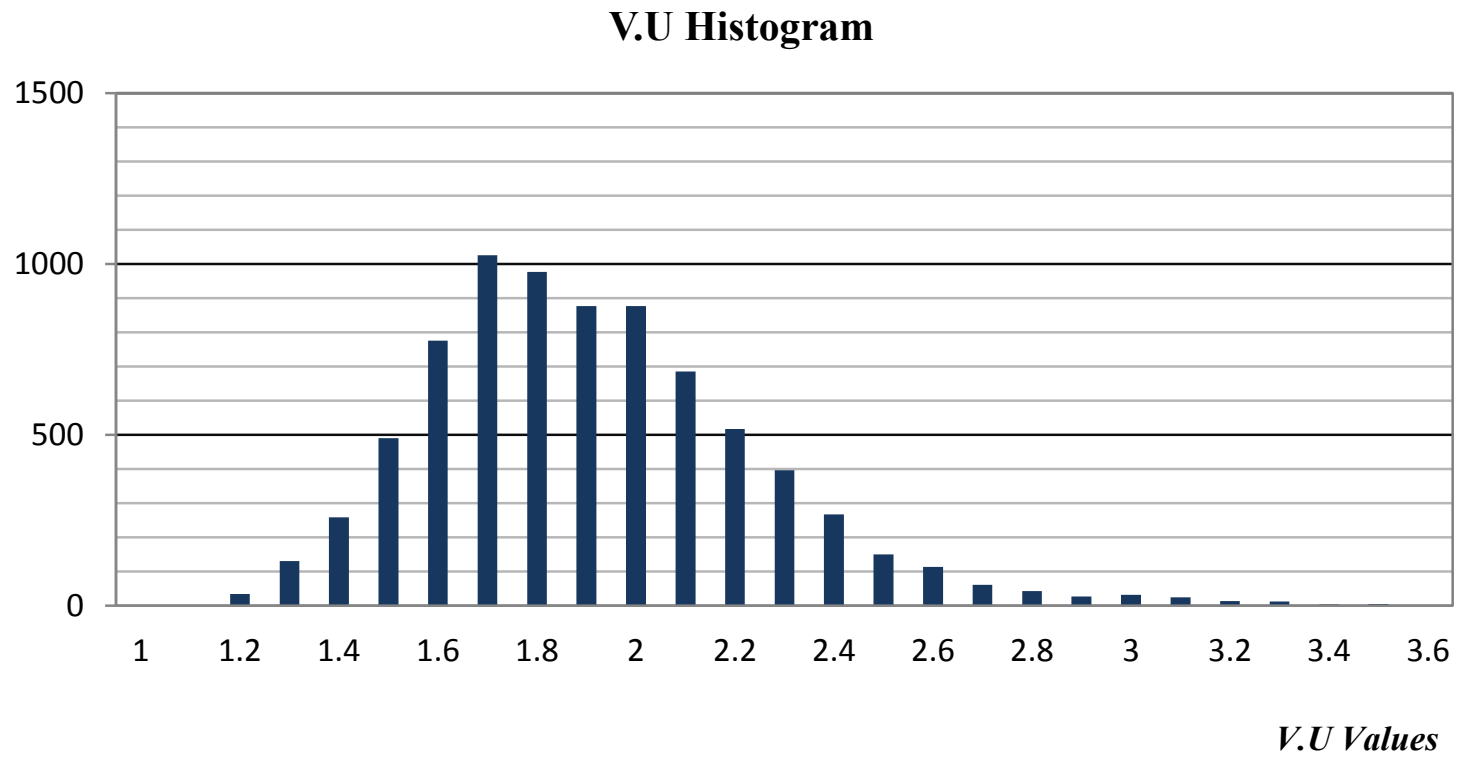

Figure 5.11 - The histogram of V.U ratio 
The foregoing results confirm the validity of the earlier observations and the importance of the current study for developing an appropriate method for estimating the actual shear forces at the quarter span as well as the actual bending moment at the mid-span.

\subsection{The Relation between Ratio Values and Buildings Characteristics}

As pointer out earlier, the main reason for the magnification in shear forces and bending moments is the greater contribution of higher modes. It was observed that the flexibility of the diaphragm attracts greater contribution from higher modes. As a result, in flexible diaphragms, larger forces are concentrated in the mid-span region, which results in larger shear forces at quarter span and larger bending moments at mid-span compared to the corresponding values obtained for a rigid diaphragm. While the flexibility of the diaphragm is the cause of increase in internal bending moments and shears, the magnitude of such increase can be different for buildings with different characteristics. Since this effect can be traced to higher mode-contributions, by finding a relation between building characteristics and higher mode-contributions, a relation between the building characteristics and the increase in shear forces and bending moments can be established.

Through a series of analyses, an attempt is made here to assess the relationship between the level of contributions from higher modes (increase in internal forces) and the building's characteristics. In each case, the internal forces are decomposed into their modal contributions in order to evaluate the relation between buildings characteristics and the level of contribution from higher modes. For illustration, the results of the analyses carried out on building ML1 when subjected to $\mathrm{M} 6 \mathrm{C} 1$ ground motion are 
presented here. In the first set of analyses, the diaphragm stiffness has been scaled by the factor of 200 so that building ML1 acts as a building with rigid diaphragm. Analyses are carried out for three values of force reduction factors: 1, 2 and 3. The same sets of analyses are then repeated, with no scale factor on diaphragm stiffness. Through these results one can confirm the effect of diaphragm flexibility on the level of the contribution of higher modes and also assess the impact that force reduction factor has on the contribution of higher modes.

The results of the analyses are presented in Figures 5.12 to 5.17 .

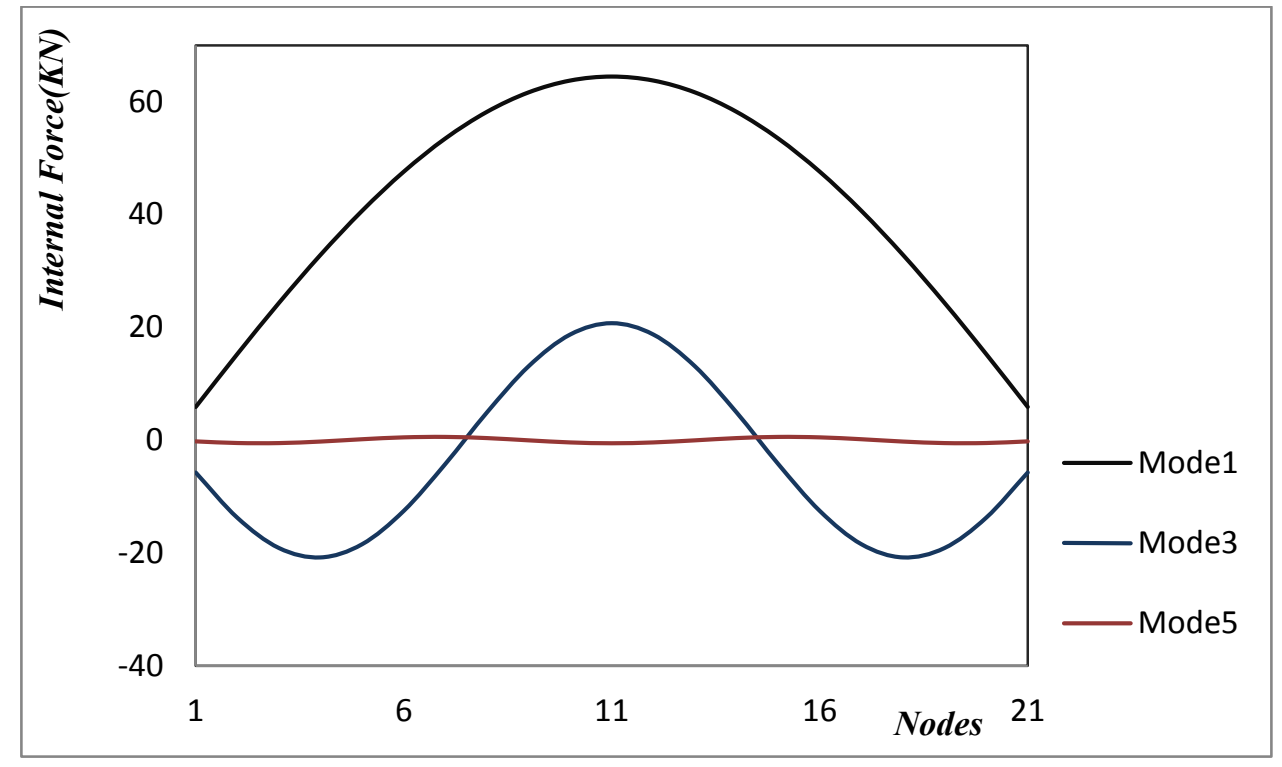

Figure 5.12 - Internal forces along the diaphragm decomposed into the first, third and fifth modes for building ML1 when subjected to M6C1 ground motion with no scale factor on diaphragm stiffness and force reduction factor of 1 


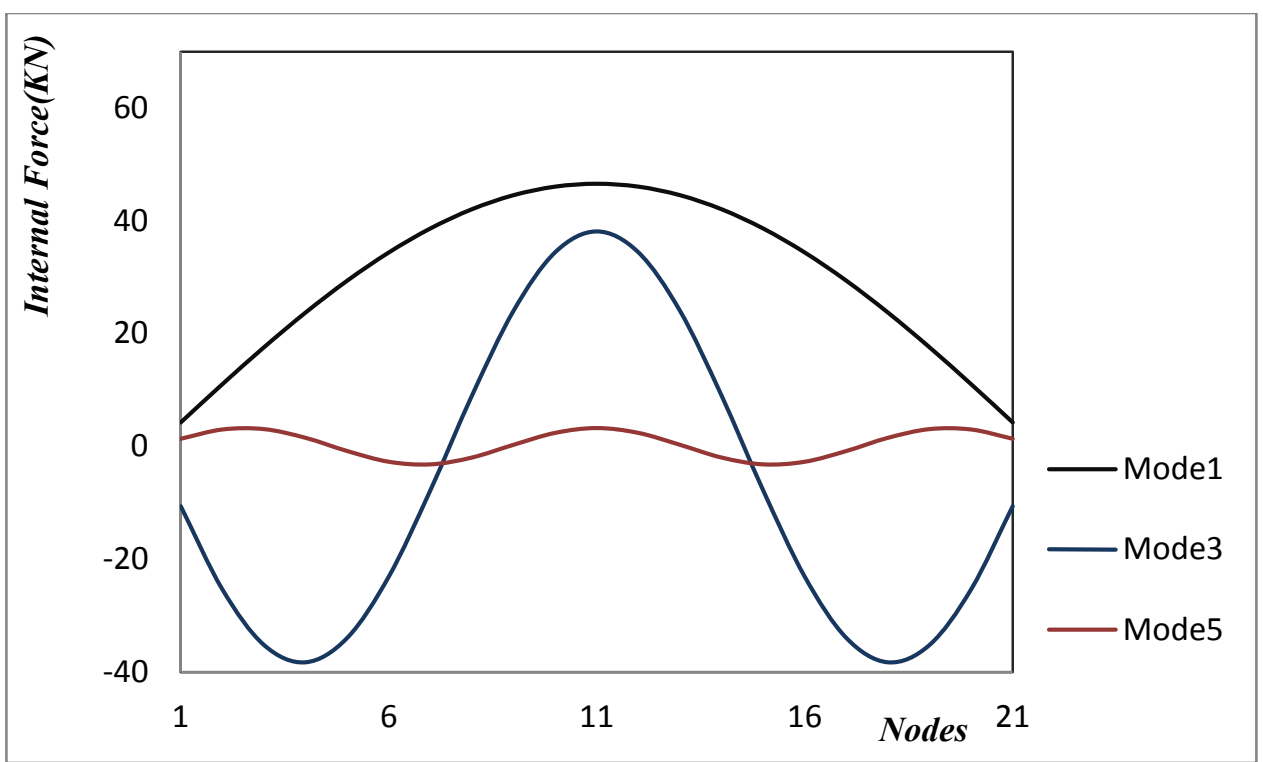

Figure 5.13 - Internal forces along the diaphragm decomposed into the first, third and fifth modes for building ML1 when subjected to M6C1 ground motion with no scale factor on diaphragm stiffness and force reduction factor of 2

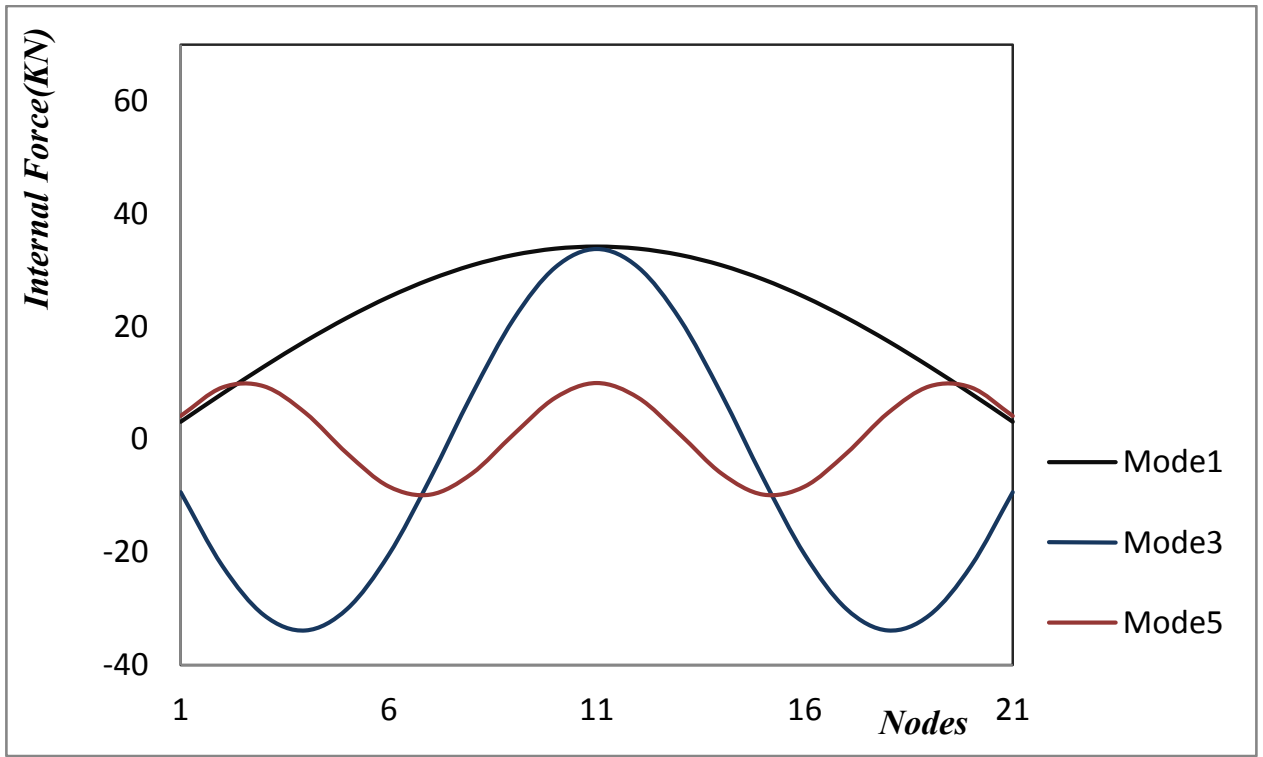

Figure 5.14 - Internal forces along the diaphragm decomposed into the first, third and fifth modes for building ML1 when subjected to M6C1 ground motion with no scale factor on diaphragm stiffness and force reduction factor of 3 


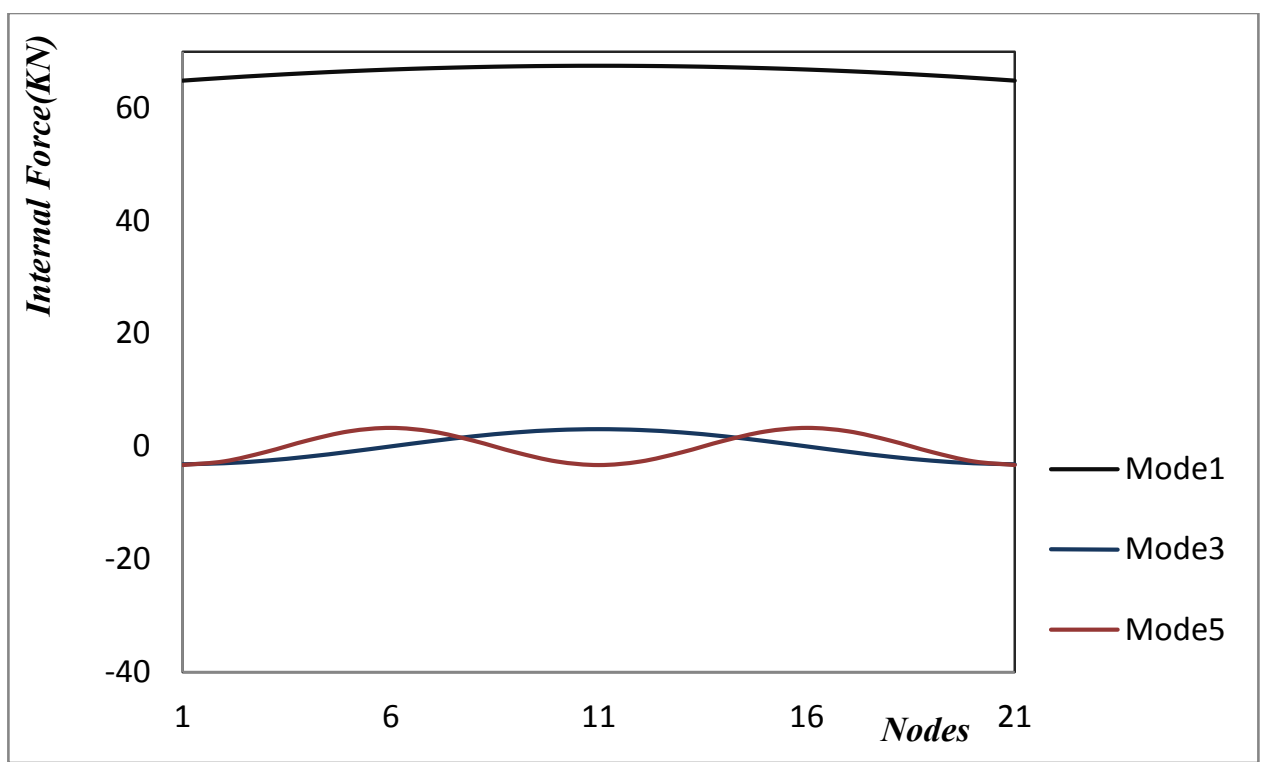

Figure 5.15 - Internal forces along the diaphragm decomposed into the first, third and fifth modes for building ML1 when subjected to M6C1 ground motion with the scale factor of 200 on diaphragm stiffness (rigid case) and force reduction factor of 1

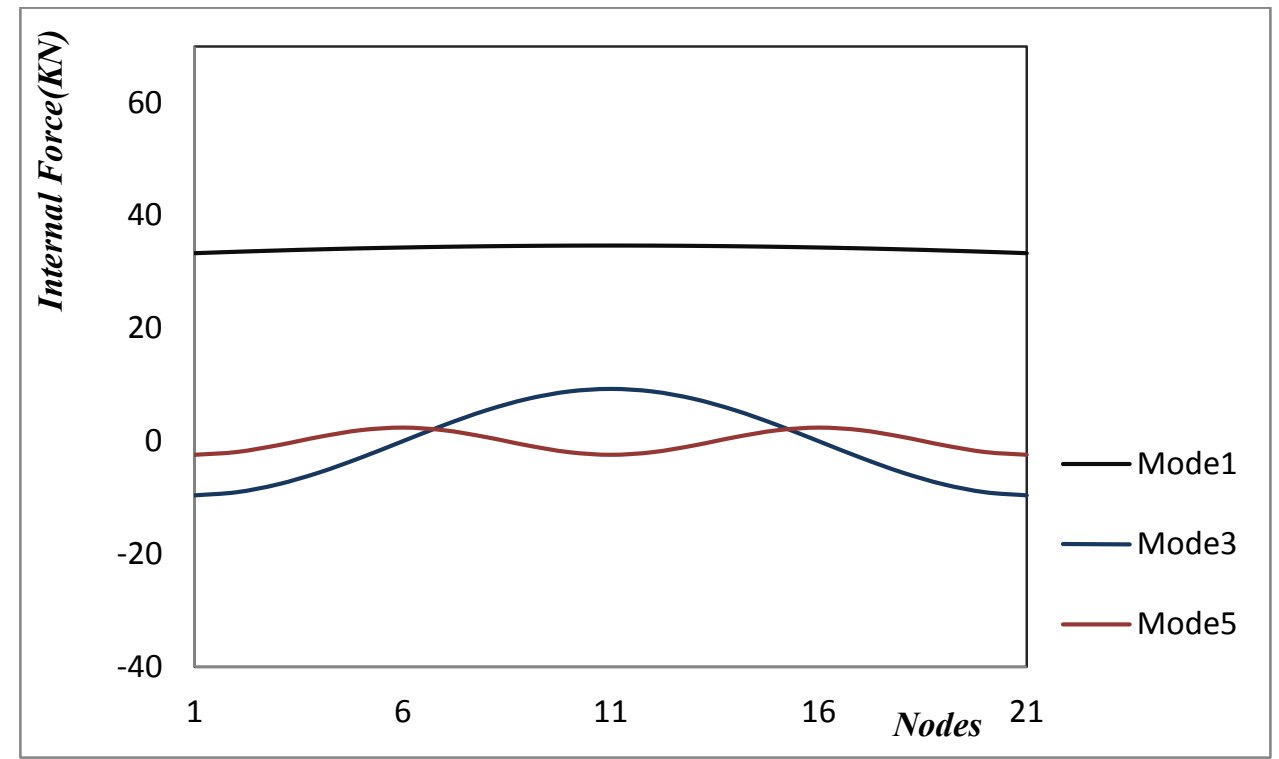

Figure 5.16 - Internal forces along the diaphragm decomposed into the first, third and fifth modes for building ML1 when subjected to $\mathrm{M6C1}$ ground motion with the scale factor of 200 on diaphragm stiffness (rigid case) and force reduction factor of 2 


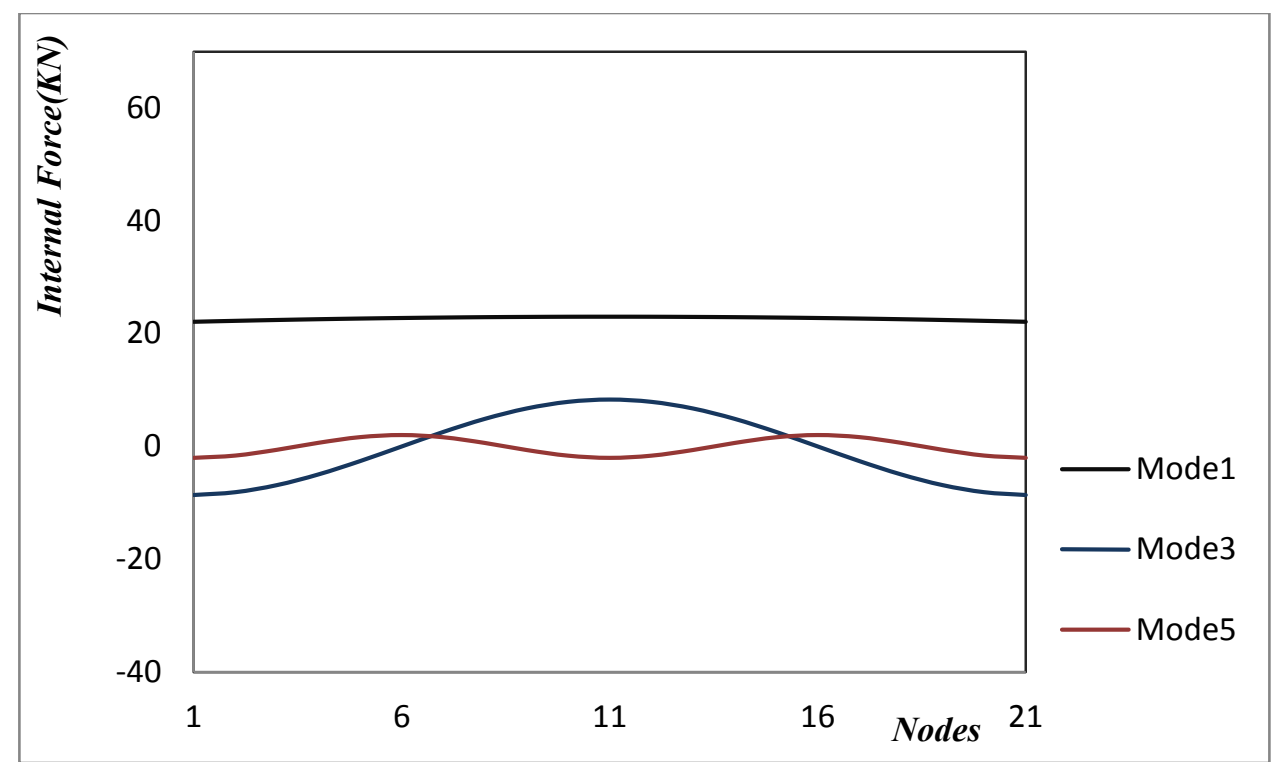

Figure 5.17 - Internal forces along the diaphragm decomposed into the first, third and fifth modes for building ML1 when subjected to M6C1 ground motion with the scale factor of 200 on diaphragm stiffness (rigid case) and force reduction factor of 3

Figures 5.12 to 5.17 confirm the fact that the flexibility of the diaphragm leads to greater contribution from higher modes to the response of the system. It is observed in Figures 5.15 to 5.17 that in the near rigid case the first mode which dominates the response of the system has the shape of a flat straight line and therefore an assumed uniform distribution should provide reasonable estimates of the shears and bending moments in the diaphragm. On the other hand, in the flexible case (with no scale factor on the diaphragm stiffness) it is observed that the contribution of higher modes becomes more significant. This leads to the concentration of seismic inertia forces towards the mid-span, which causes greater shear forces at quarter-span and greater bending moments at mid-span.

There appears to be a direct relation between the nonlinearity of the system and the level of the contribution from higher modes, so that as the system is strained more into the 
inelastic range, the contribution from higher modes to the total response of the system becomes greater. Obviously, the ductility demand as a measure of the nonlinearity of the system must be taken into account in determining the magnifying ratio between the true internal actions and those determined from a uniform distribution of the inertia forces. The results presented in Appendix C confirm such conclusion.

On reviewing the data presented in Tables C.1 to C. 80 in Appendix C, it can be observed that there is also a direct correlation between the ratio values and the period of the system. On the other hand, the results suggest that there is an inverse correlation between the ratio values (shear force and bending moment increase) and the post-yield hardening, so that smaller values of $\alpha$ lead to greater ratio values. In addition, post-yield hardening reduces the correlation between the ductility demand and the level of magnification in internal forces.

\subsection{Development of Relationships between Building Characteristics and the} Ratio Values

Multiple regressions are carried out in order to develop equations that relate M.F, V.F, M.U and V.U values, defined earlier, to the building's characteristics. In these multiple regressions, an attempt has been made to find out which building property or properties have the most dominant correlation with M.F, V.F, M.U and V.U and to suggest equations for the four quantities based on such buildings properties. In the first set of regressions, the selected building characteristics are the force modification factor, the fundamental period of the beam-spring model and the post-yield hardening stiffness. The obtained equations are as follows: 
$M . F=0.97+0.14 R_{y}+0.08 T-1.69 \alpha$

$V . F=0.86+0.20 R_{y}+0.30 T-2.01 \alpha$

$M . U=1.21+0.17 R_{y}+0.11 T-2.12 \alpha$

$V . U=1.19+0.28 R_{y}+0.41 T-2.77 \alpha$

where $R_{y}$ is the force reduction factor and can be a measure of the nonlinearity of the system, $T$ is the fundamental period of the beam spring system and $\alpha$ is the post-yield stiffness ratio.

While Equations 5.6 to 5.9 provide good estimates for the magnification ratios for the bending moments at mid-span and shear forces at quarter span, they seem unnecessarily complicated with several variable involved in the equations. In particular, calculation of the fundamental period of the beam/spring model can be time consuming. Nevertheless, these equations can give a good understanding of how the building characteristics relate to the force magnification ratio values.

To develop a simpler set of relationships that will be appropriate for practical design, and since among all the building characteristics the nonlinearity of the system has the most dominant correlation with the ratio values, a series of regressions are carried out to suggest sets of equations relating the ratio values to the ductility demand of the system, which is a measure of how much the system is strained into the inelastic range. Calculations are carried out for data corresponding to eastern spectrum and western spectrum separately. For each spectrum equations are developed for four values of post yield stiffness $(\alpha=0.00, \alpha=0.02, \alpha=0.05, \alpha=0.10)$. For this purpose, first the ratio values 
(M.F, V.F, M.U and V.U values) for each analysis case are plotted against the ductility demand. A parabolic equation is then fitted through the means of the data points. The equations as well as the plots are presented in Figures 5.18 to 5.49:

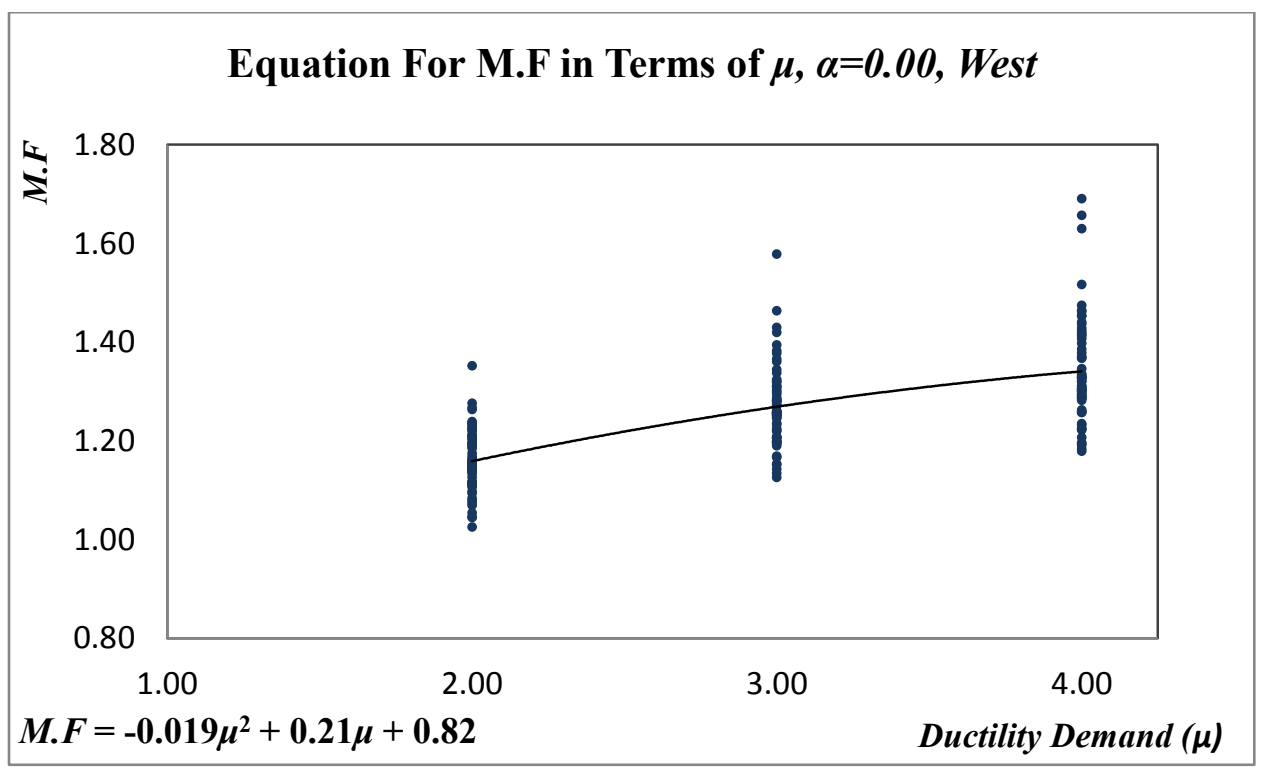

Figure 5.18 - M.F values mapped with respect to target ductility demands and the suggested equation for M.F for data corresponding to the western compatible spectrum and no post-yield stiffness

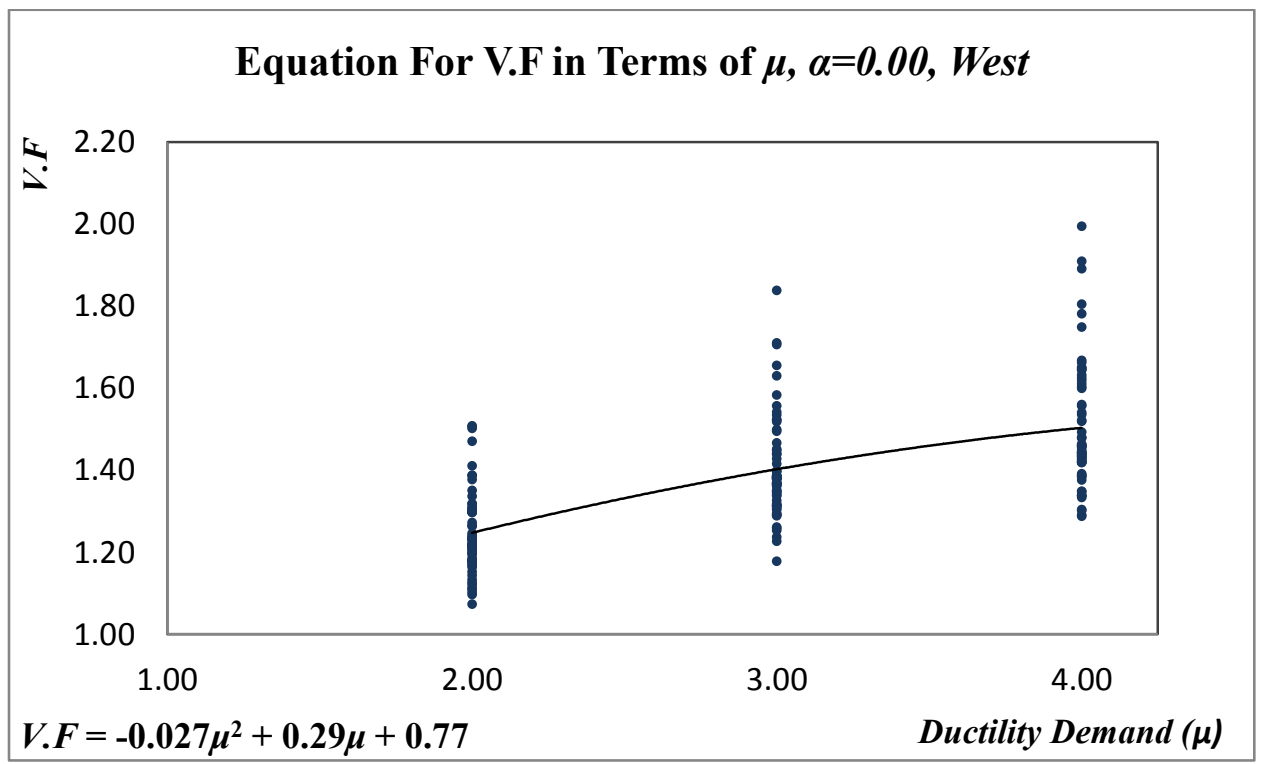

Figure 5.19 - V.F values mapped with respect to target ductility demands and the suggested equation for V.F for data corresponding to the western compatible spectrum and no post-yield stiffness 


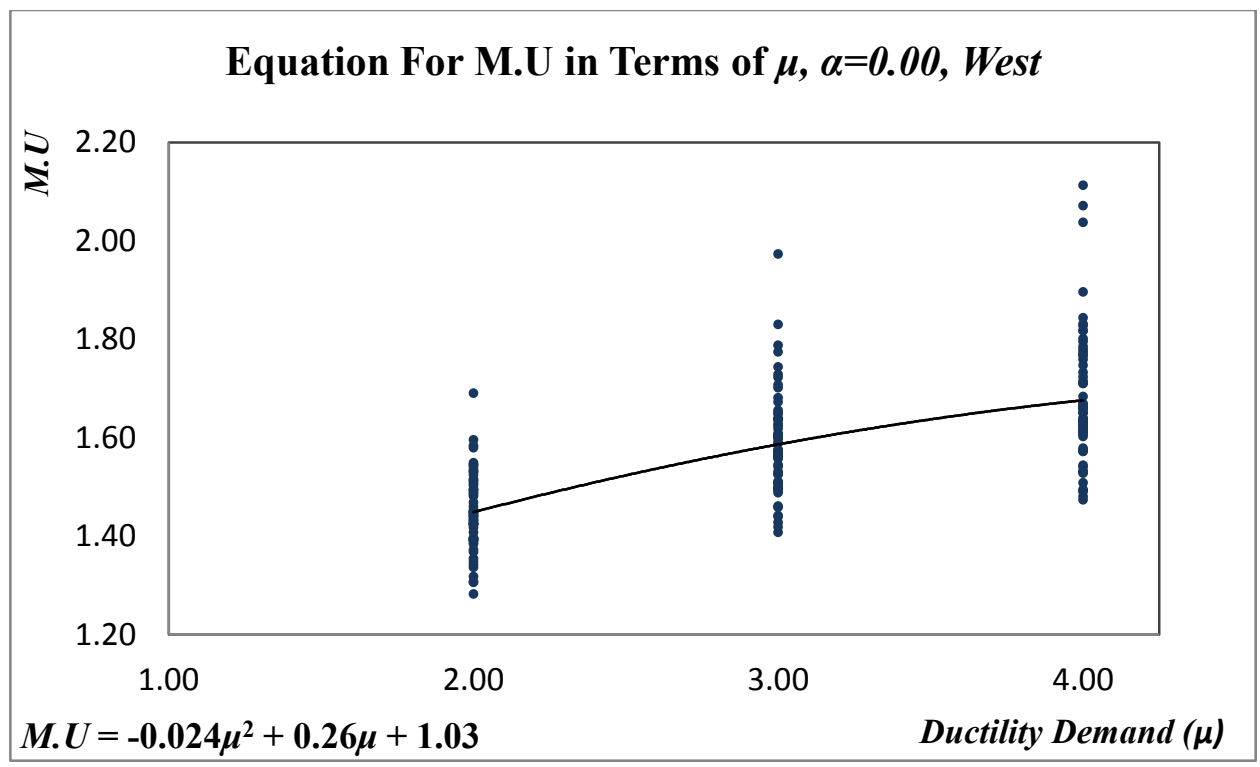

Figure 5.20 - M.U values mapped with respect to target ductility demands and the suggested equation for M.U for data corresponding to the western compatible spectrum and no post-yield stiffness

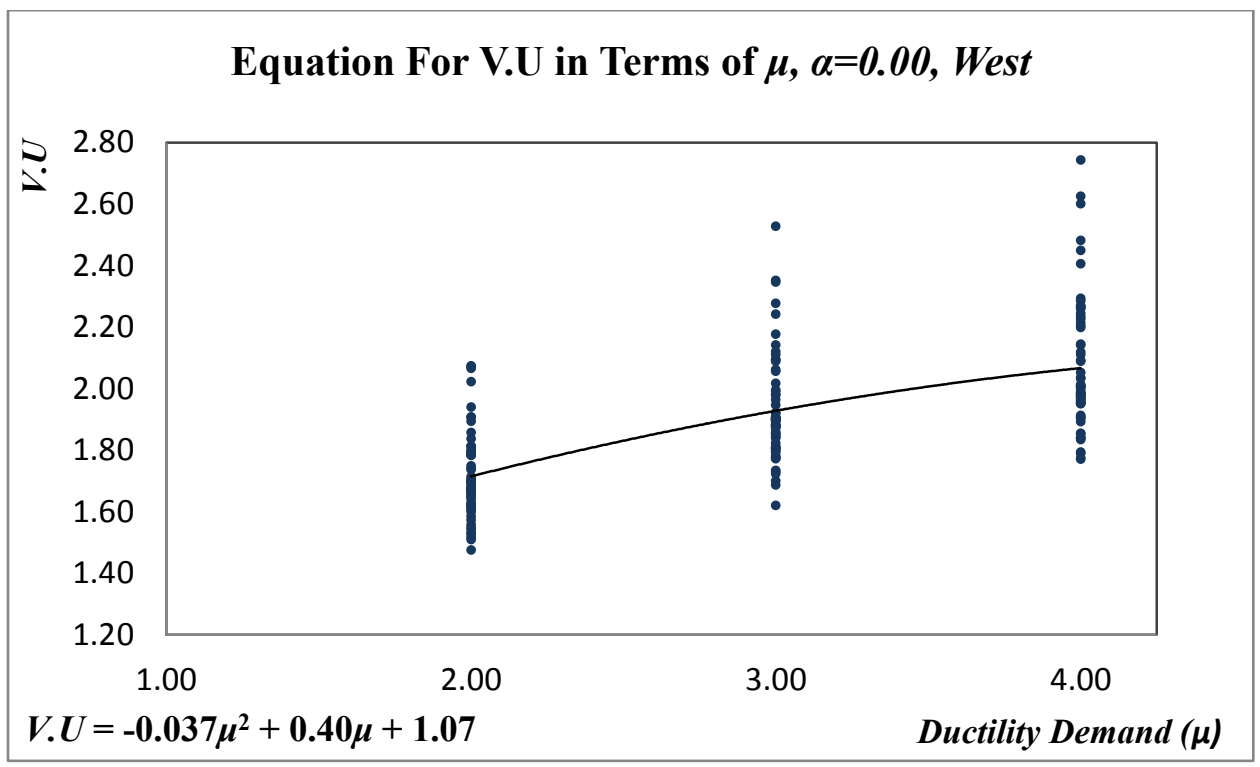

Figure 5.21 - V.U values mapped with respect to target ductility demands and the suggested equation for V.U for data corresponding to the western compatible spectrum and no post-yield stiffness 


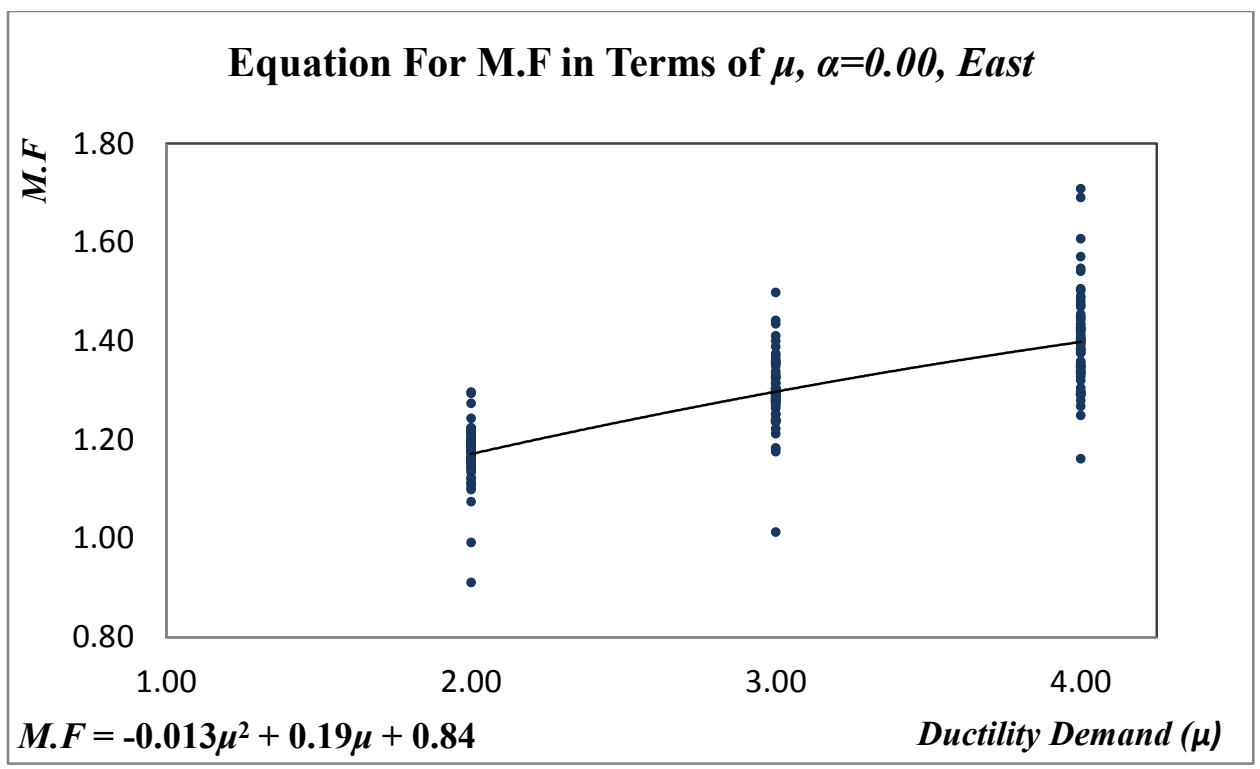

Figure 5.22 - M.F values mapped with respect to target ductility demands and the suggested equation for M.F for data corresponding to the eastern compatible spectrum and no post-yield stiffness

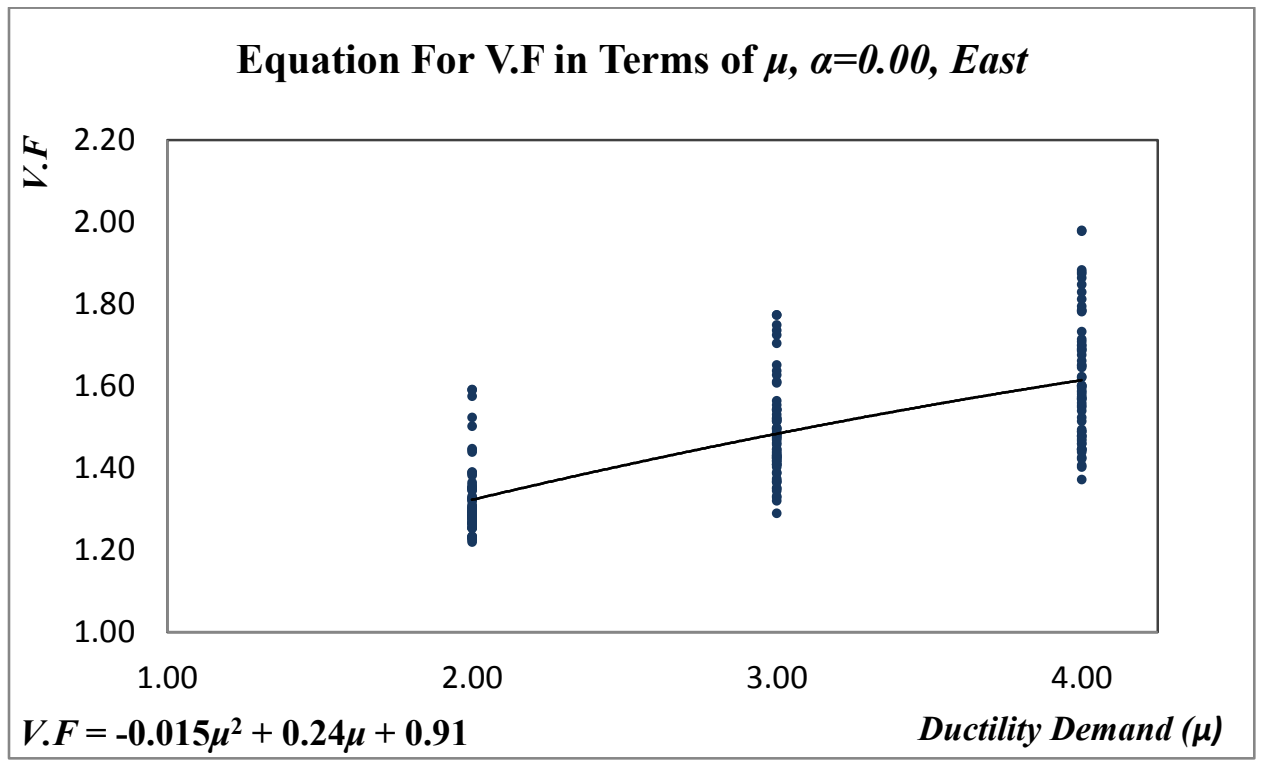

Figure 5.23 - V.F values mapped with respect to target ductility demands and the suggested equation for V.F for data corresponding to the eastern compatible spectrum and no post-yield stiffness 


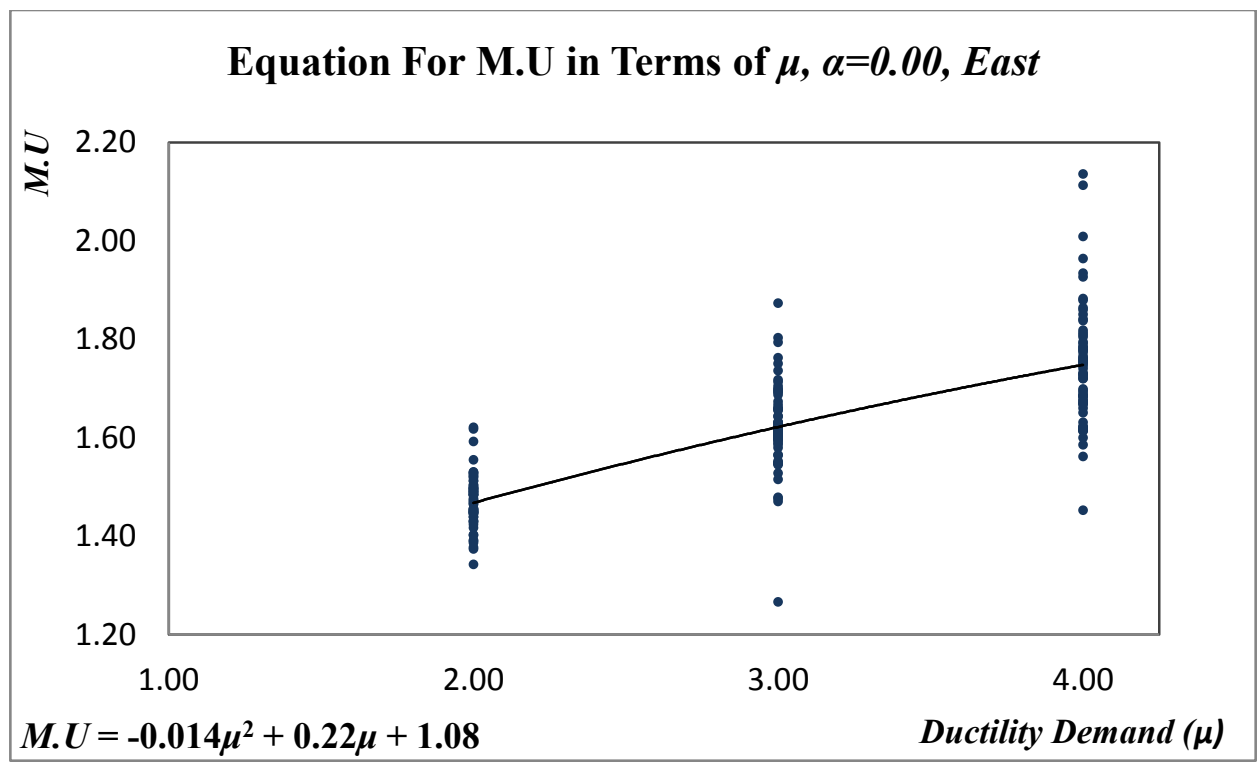

Figure 5.24 - M.U values mapped with respect to target ductility demands and the suggested equation for M.U for data corresponding to the eastern compatible spectrum and no post-yield stiffness

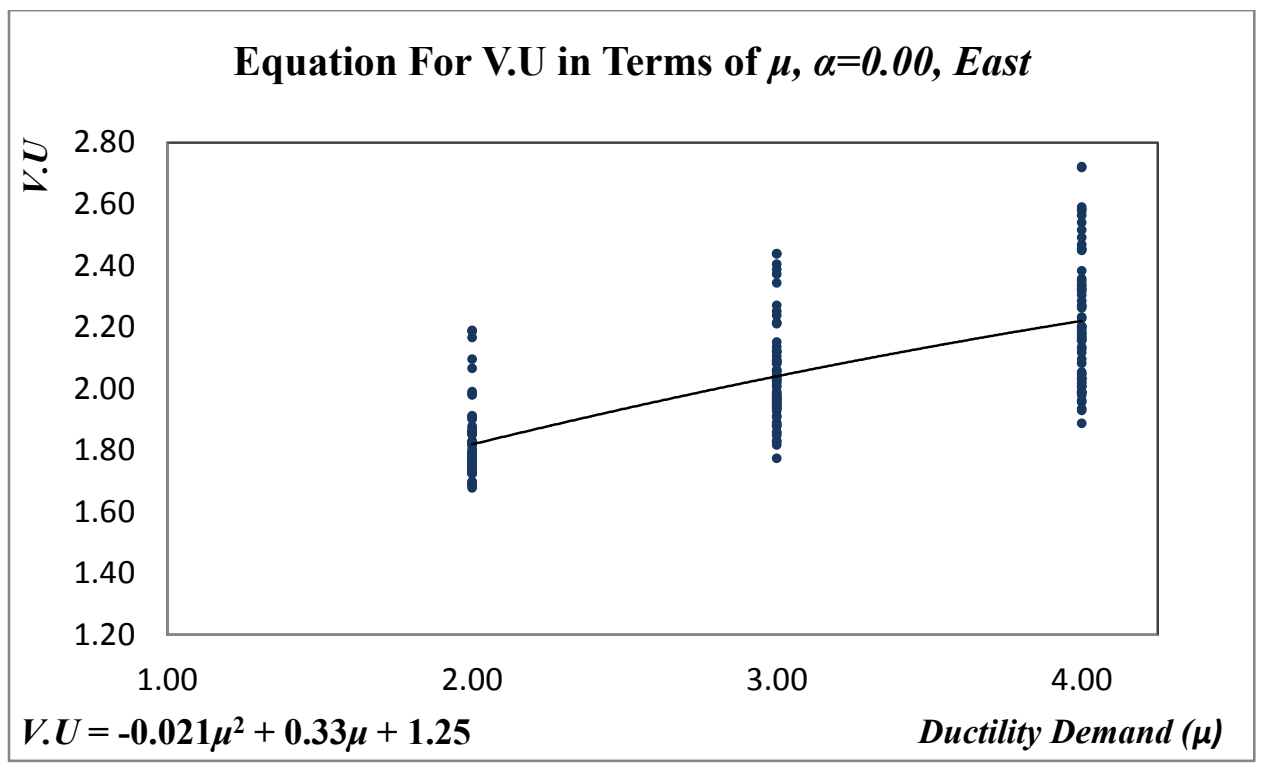

Figure 5.25 - V.U values mapped with respect to target ductility demands and the suggested equation for V.U for data corresponding to the eastern compatible spectrum and no post-yield stiffness 


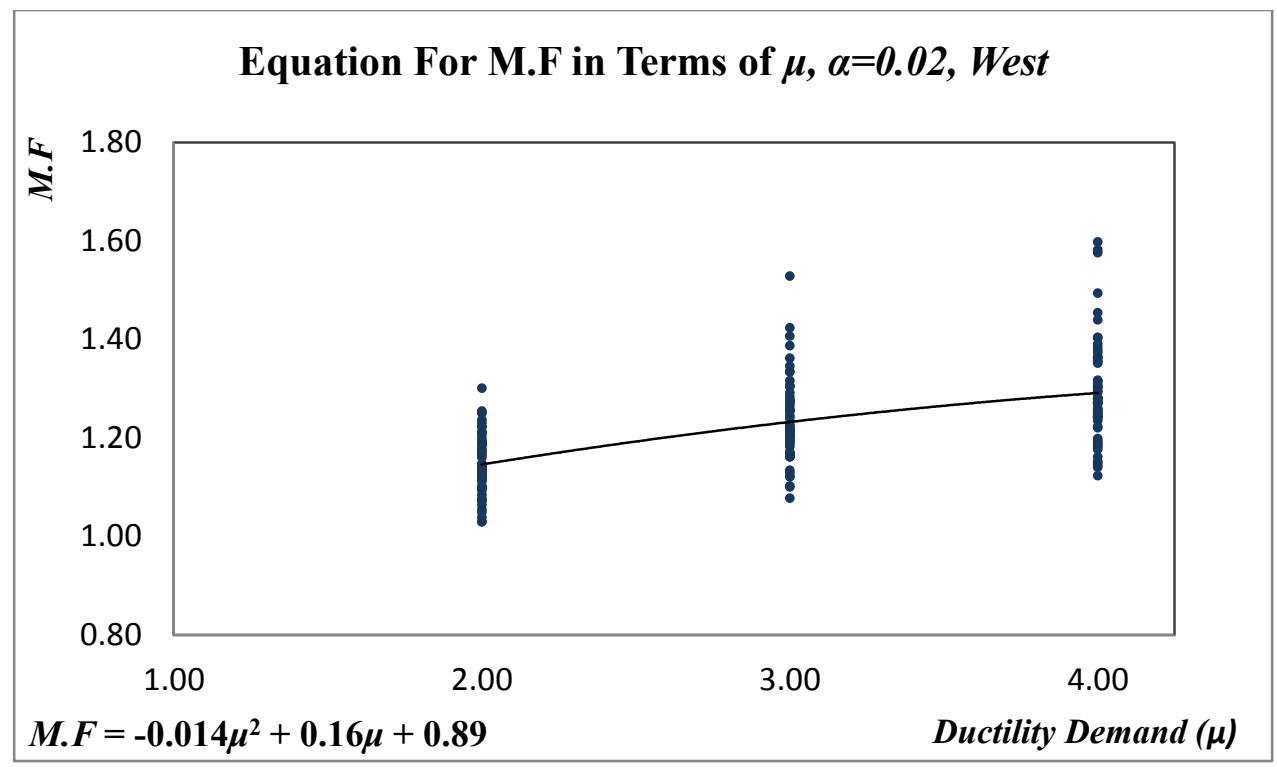

Figure 5.26 - M.F values mapped with respect to target ductility demands and the suggested equation for M.F for data corresponding to the western compatible spectrum and post-yield stiffness of 0.02

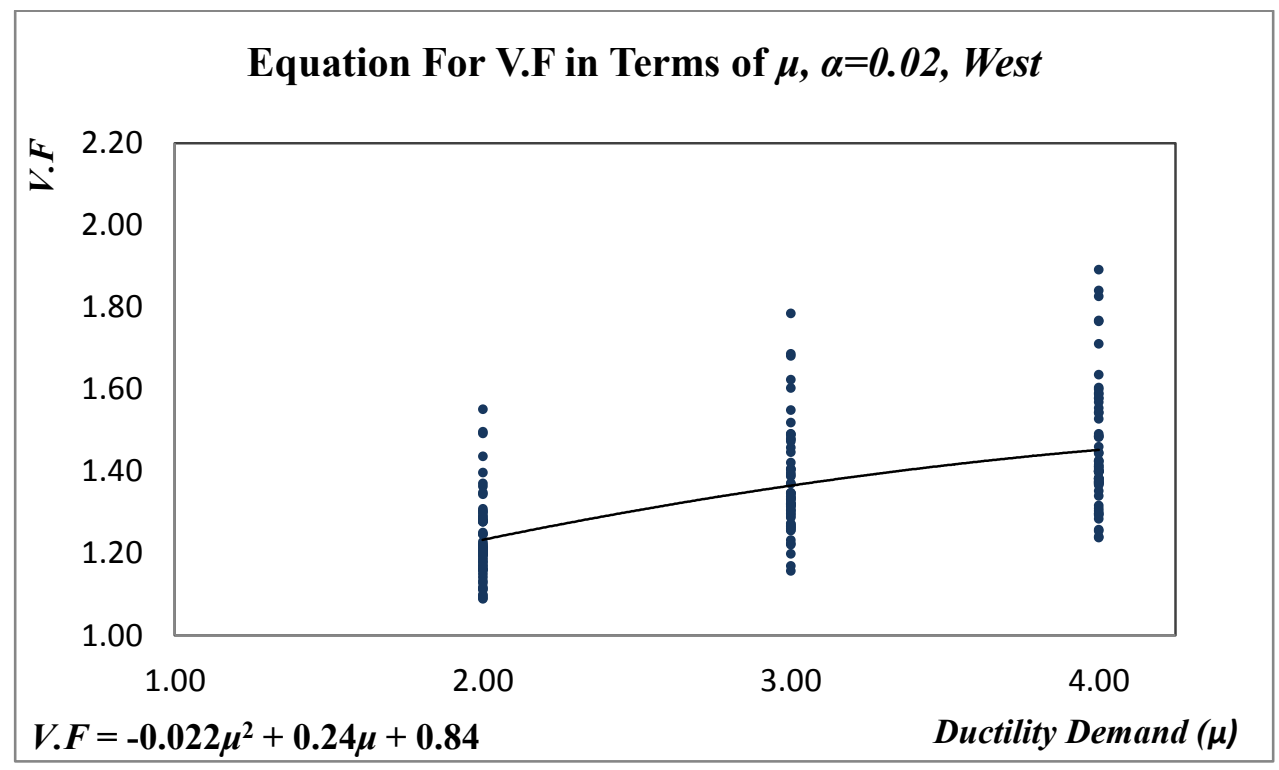

Figure 5.27 - V.F values mapped with respect to target ductility demands and the suggested equation for $V$.F for data corresponding to the western compatible spectrum and post-yield stiffness of 0.02 


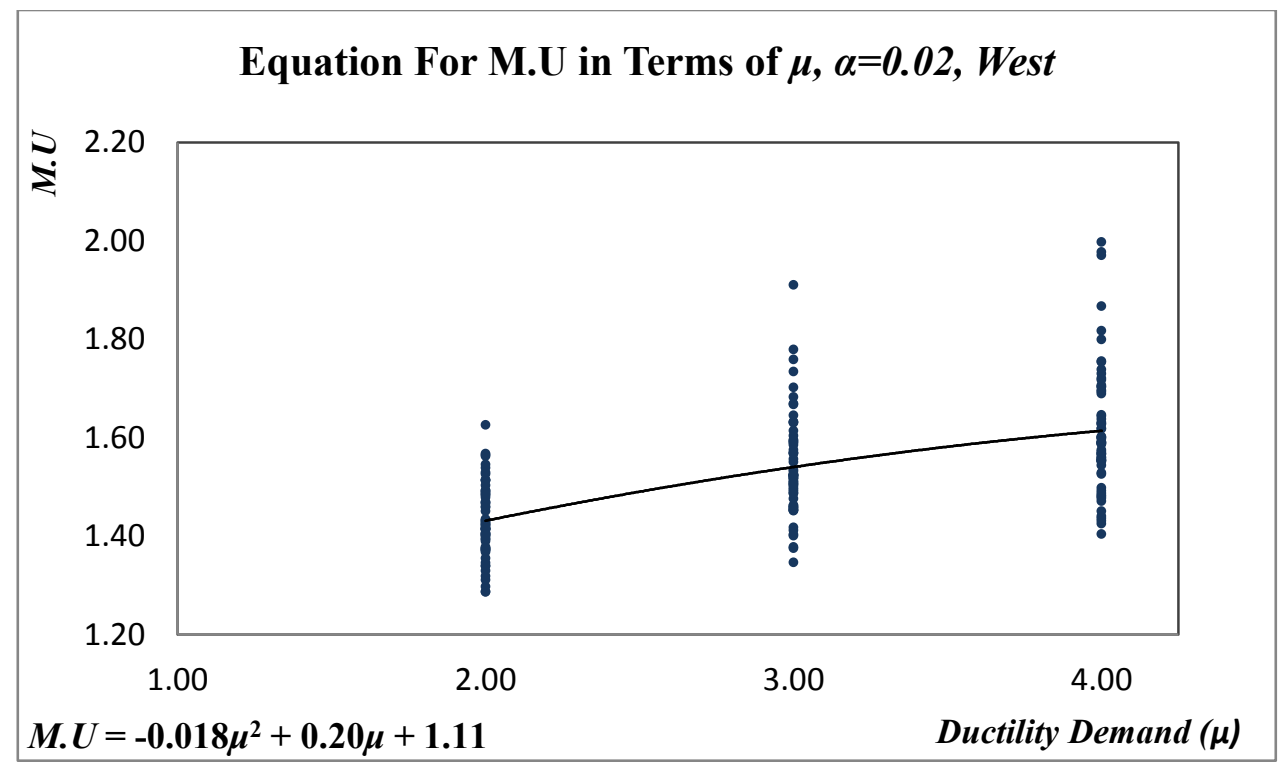

Figure 5.28 - M.U values mapped with respect to target ductility demands and the suggested equation for M.U for data corresponding to the western compatible spectrum and post-yield stiffness of 0.02

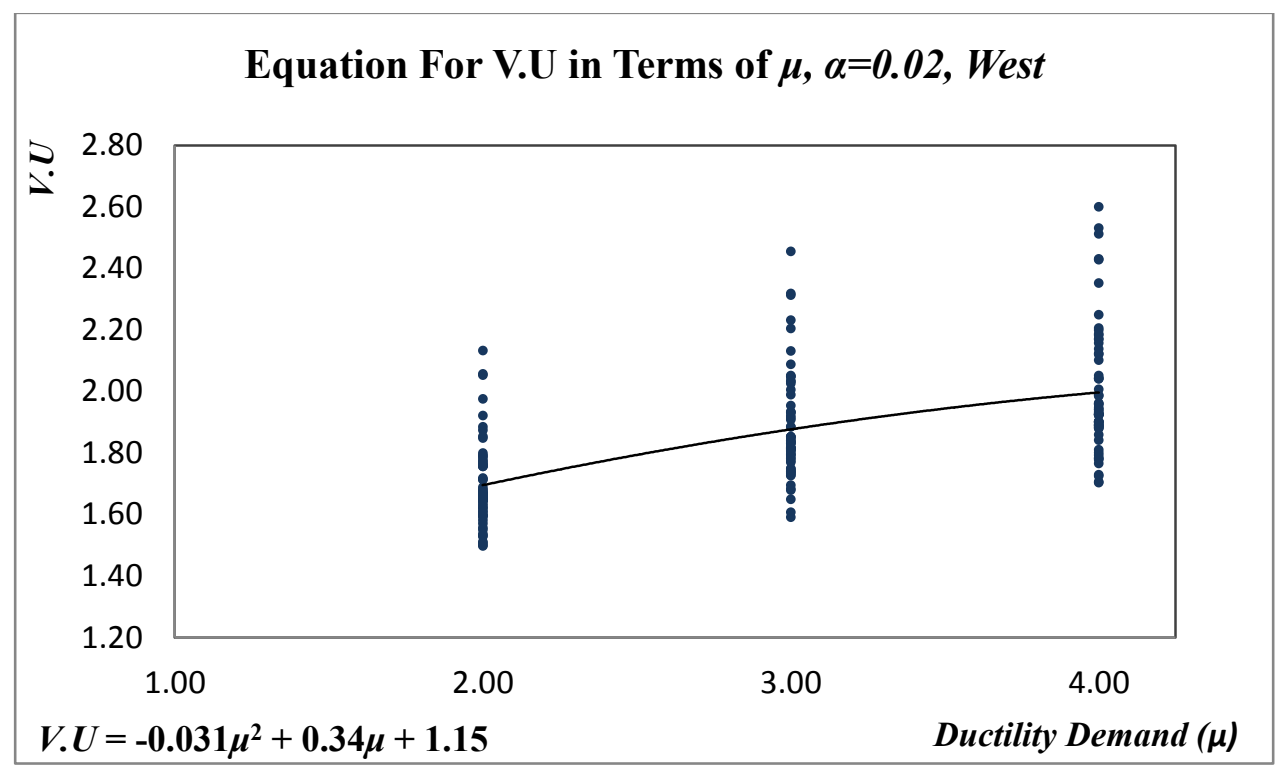

Figure 5.29 - V.U values mapped with respect to target ductility demands and the suggested equation for V.U for data corresponding to the western compatible spectrum and post-yield stiffness of 0.02 


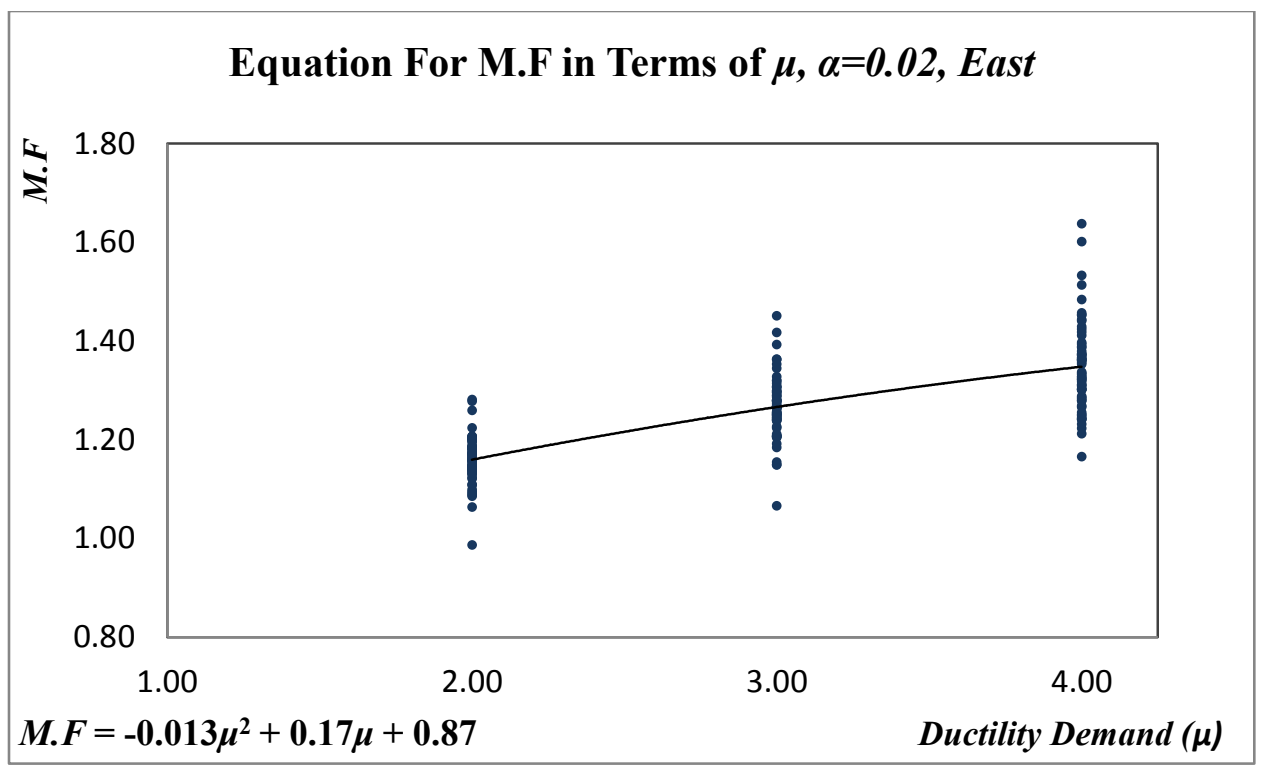

Figure 5.30 - M.F values mapped with respect to target ductility demands and the suggested equation for M.F for data corresponding to the eastern compatible spectrum and post-yield stiffness of 0.02

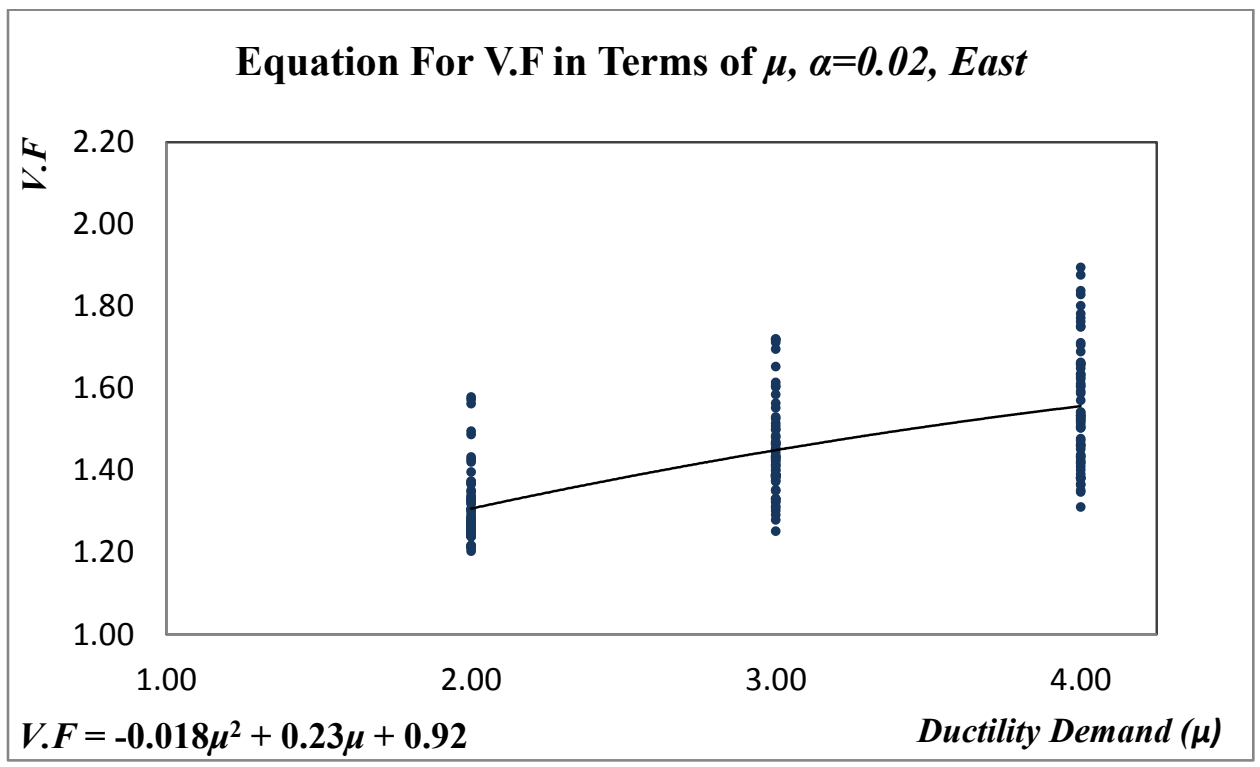

Figure 5.31 - V.F values mapped with respect to target ductility demands and the suggested equation for V.F for data corresponding to the eastern compatible spectrum and post-yield stiffness of 0.02 


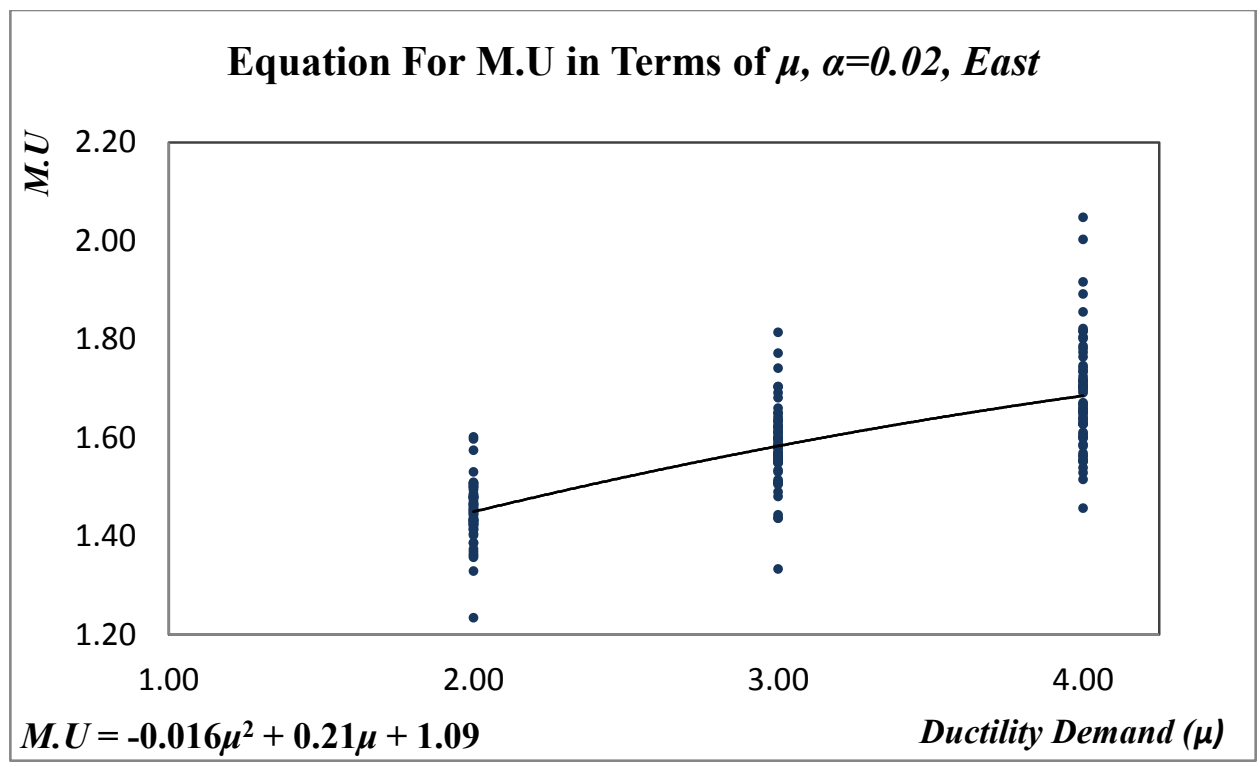

Figure 5.32 - M.U values mapped with respect to target ductility demands and the suggested equation for M.U for data corresponding to the eastern compatible spectrum and post-yield stiffness of 0.02

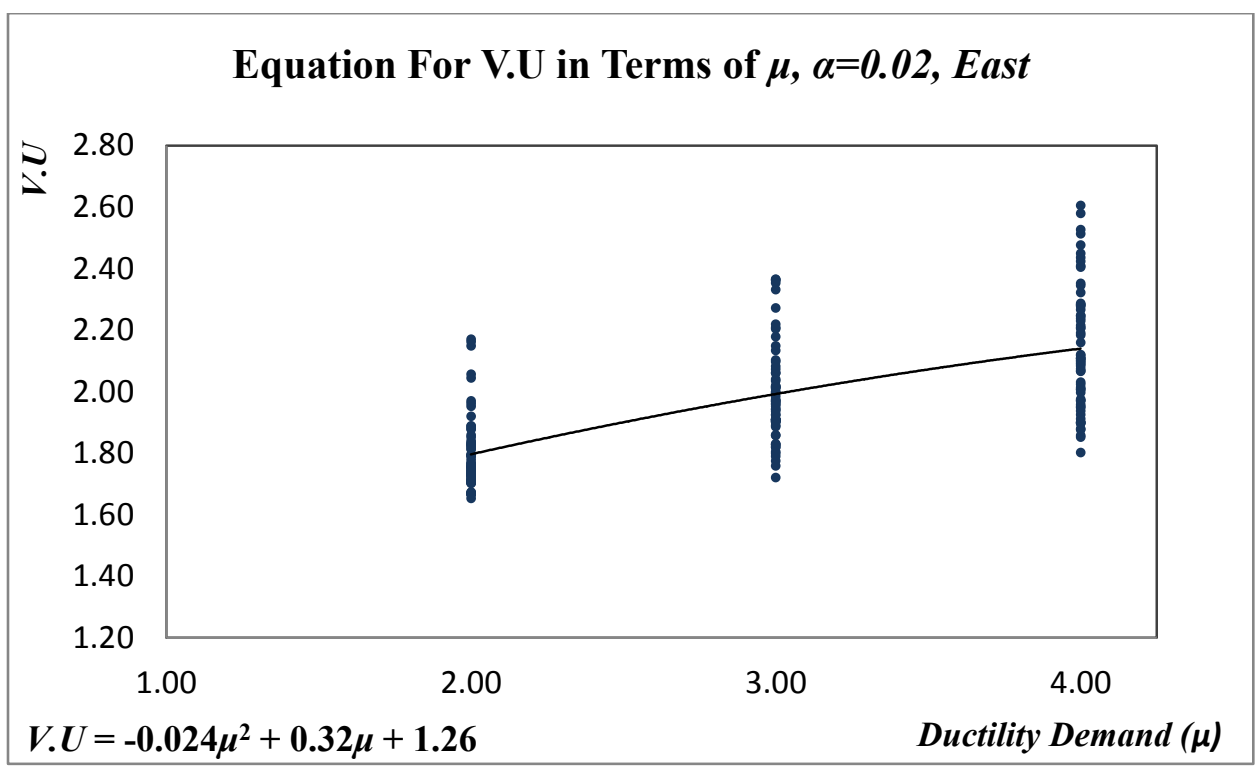

Figure 5.33 - V.U values mapped with respect to target ductility demands and the suggested equation for $V$.U for data corresponding to the eastern compatible spectrum and post-yield stiffness of 0.02 


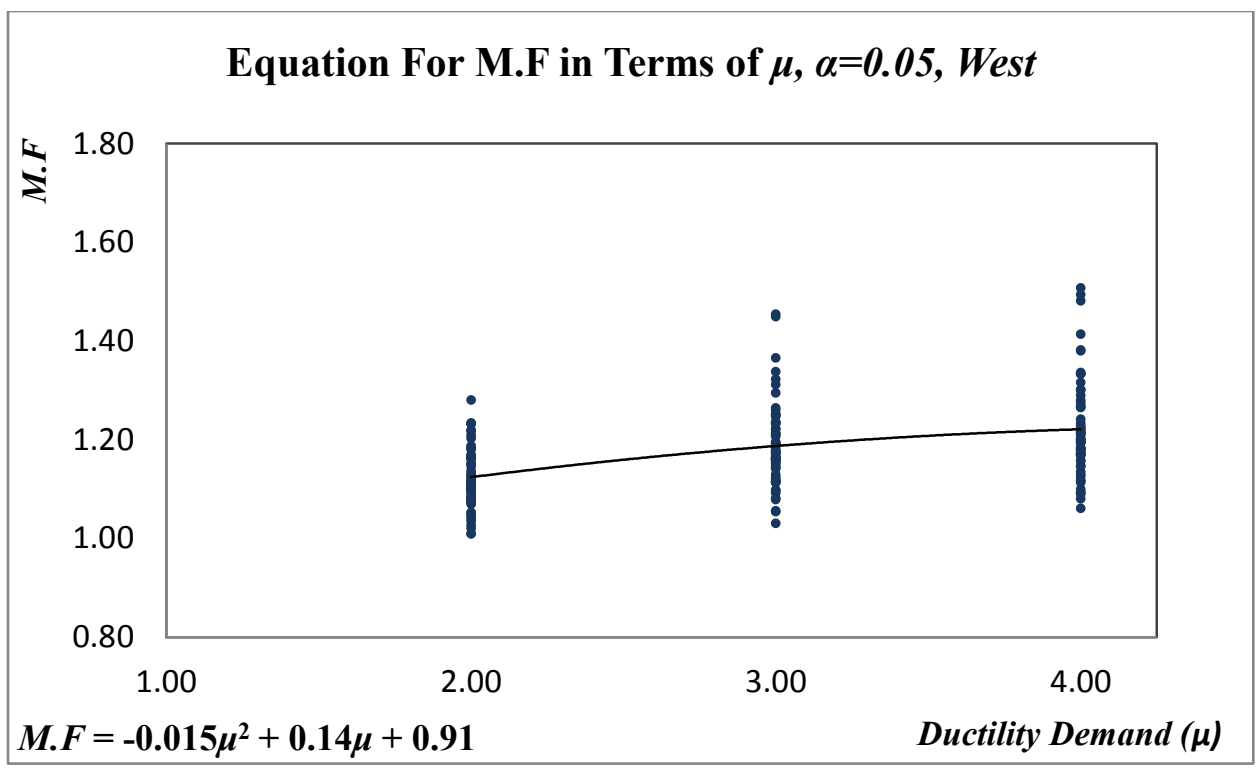

Figure 5.34 - M.F values mapped with respect to target ductility demands and the suggested equation for M.F for data corresponding to the western compatible spectrum and post-yield stiffness of 0.05

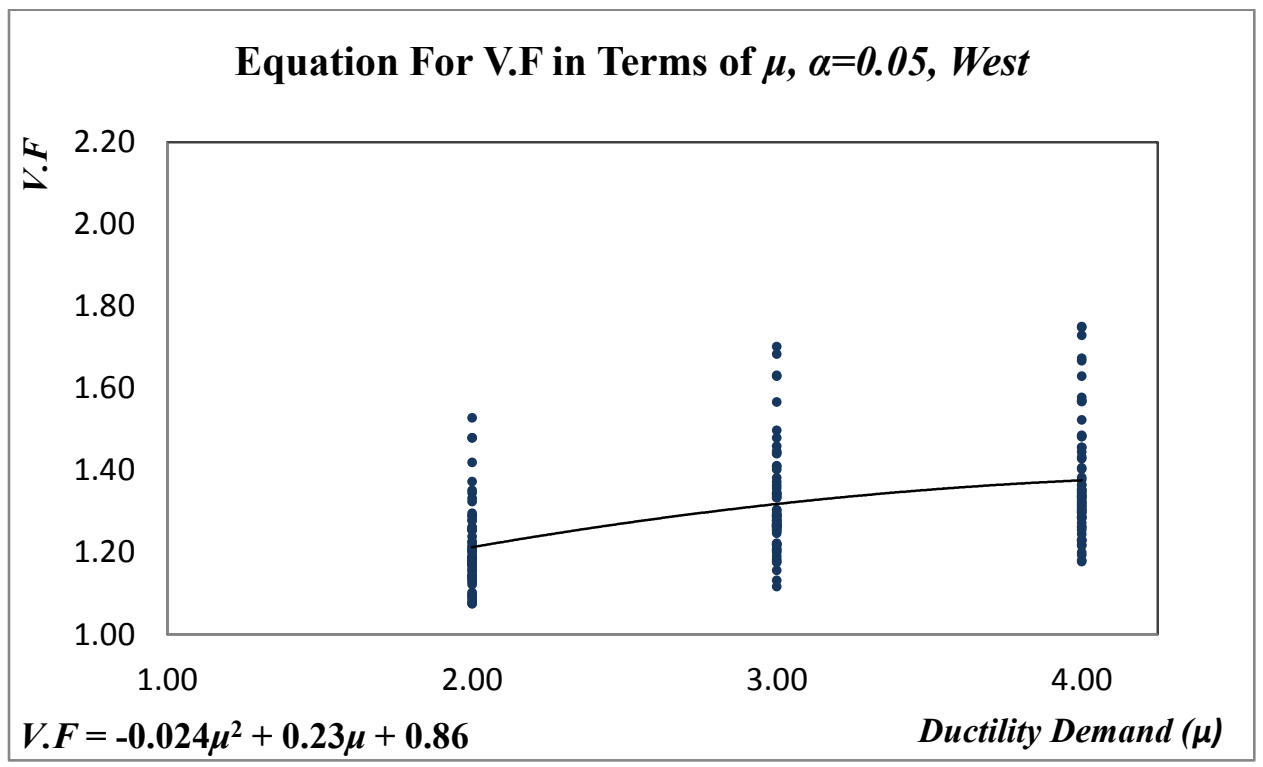

Figure 5.35 - V.F values mapped with respect to target ductility demands and the suggested equation for V.F for data corresponding to the western compatible spectrum and post-yield stiffness of 0.05 


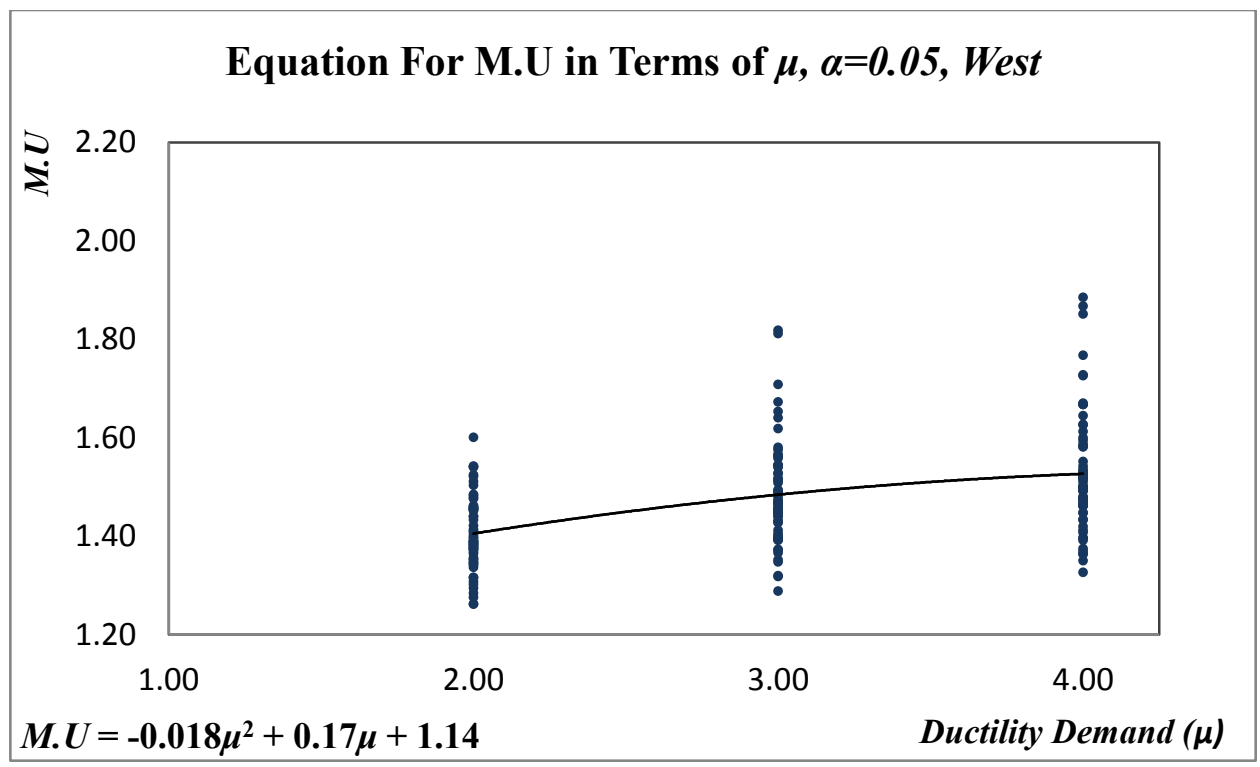

Figure 5.36 - M.U values mapped with respect to target ductility demands and the suggested equation for M.U for data corresponding to the western compatible spectrum and post-yield stiffness of 0.05

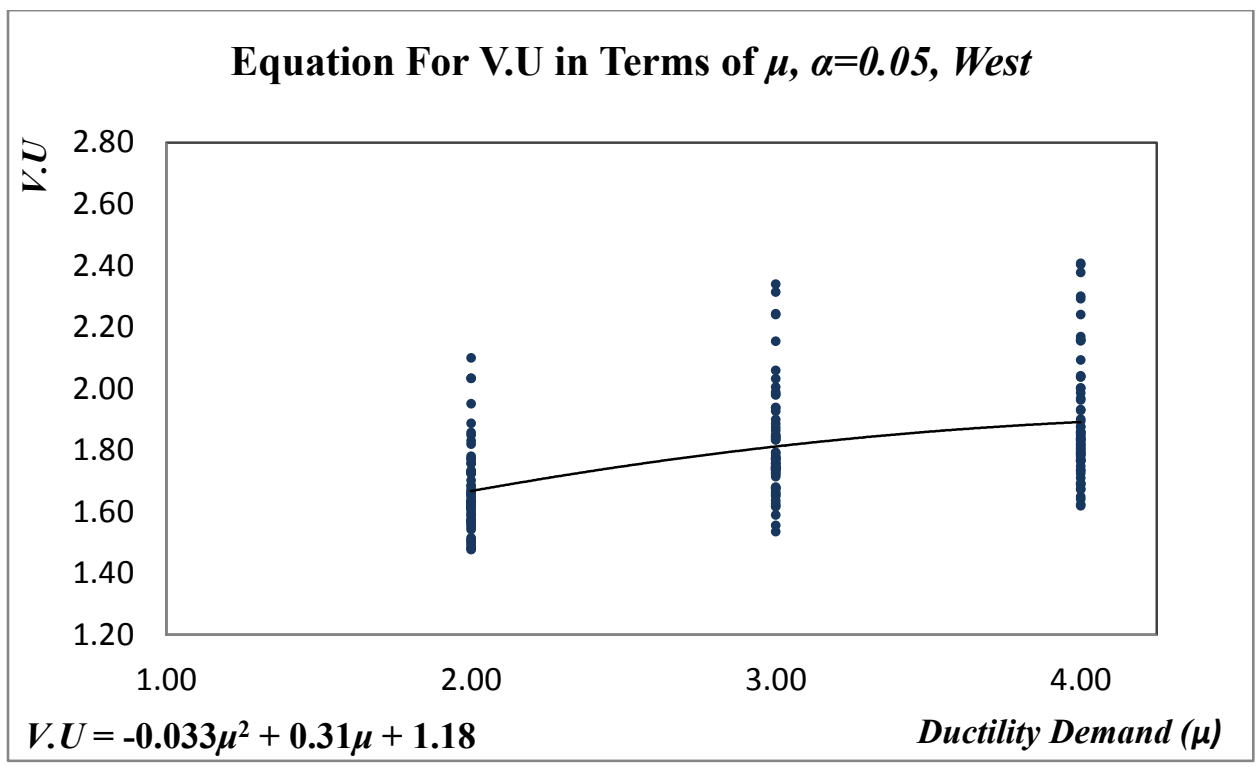

Figure 5.37 - V.U values mapped with respect to target ductility demands and the suggested equation for V.U for data corresponding to the western compatible spectrum and post-yield stiffness of 0.05 


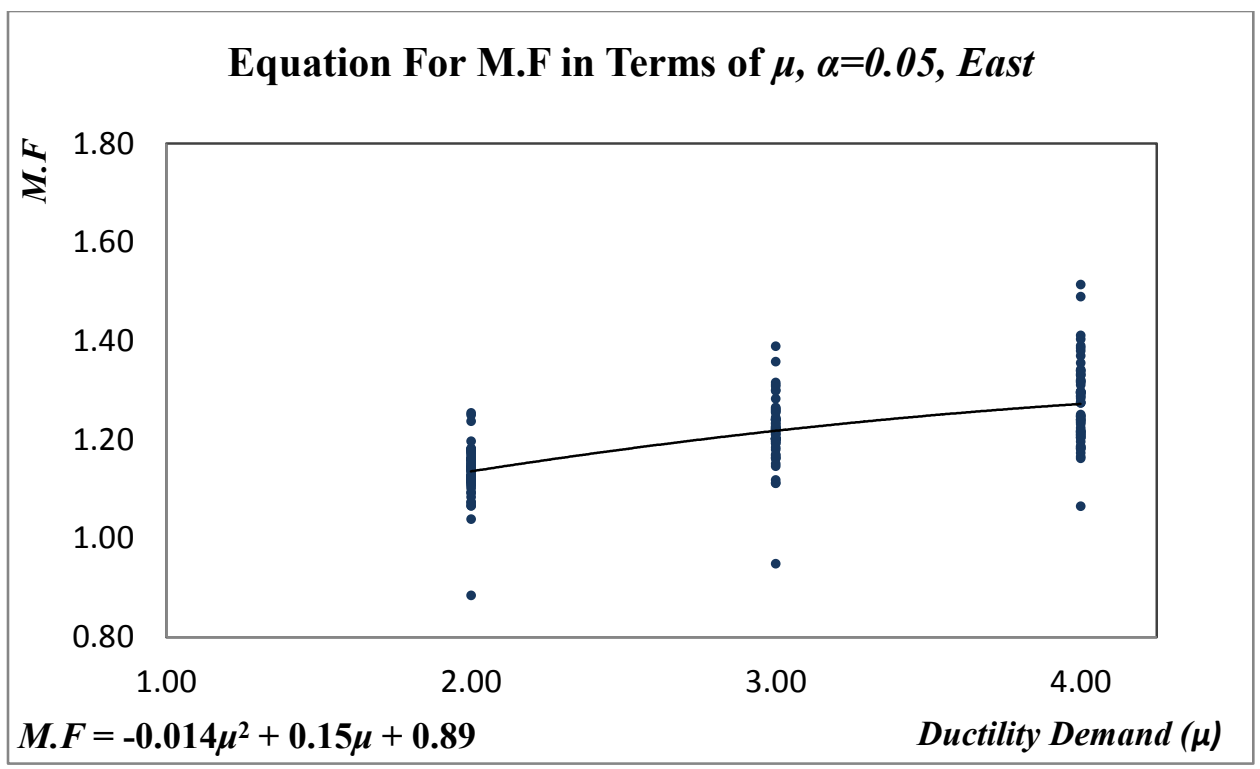

Figure 5.38 - M.F values mapped with respect to target ductility demands and the suggested equation for M.F for data corresponding to the eastern compatible spectrum and post-yield stiffness of 0.05

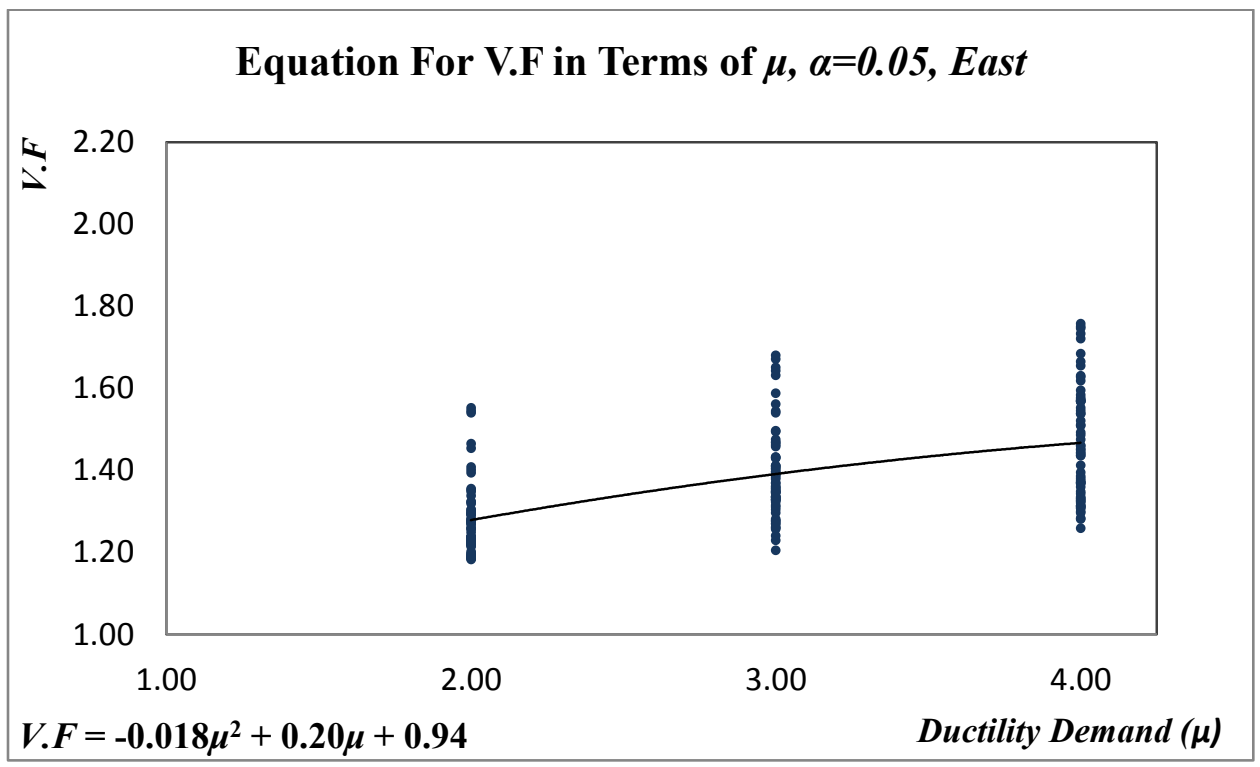

Figure 5.39 - V.F values mapped with respect to target ductility demands and the suggested equation for V.F for data corresponding to the eastern compatible spectrum and post-yield stiffness of 0.05 


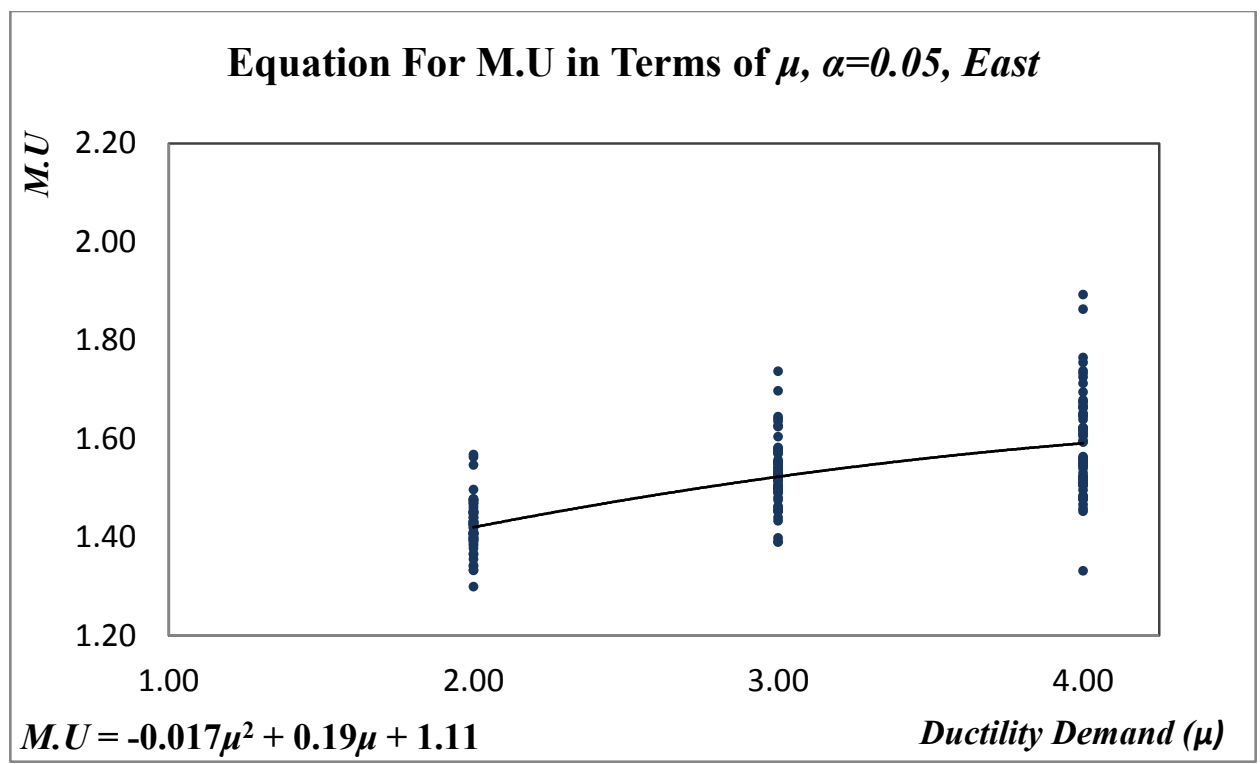

Figure 5.40 - M.U values mapped with respect to target ductility demands and the suggested equation for M.U for data corresponding to the eastern compatible spectrum and post-yield stiffness of 0.05

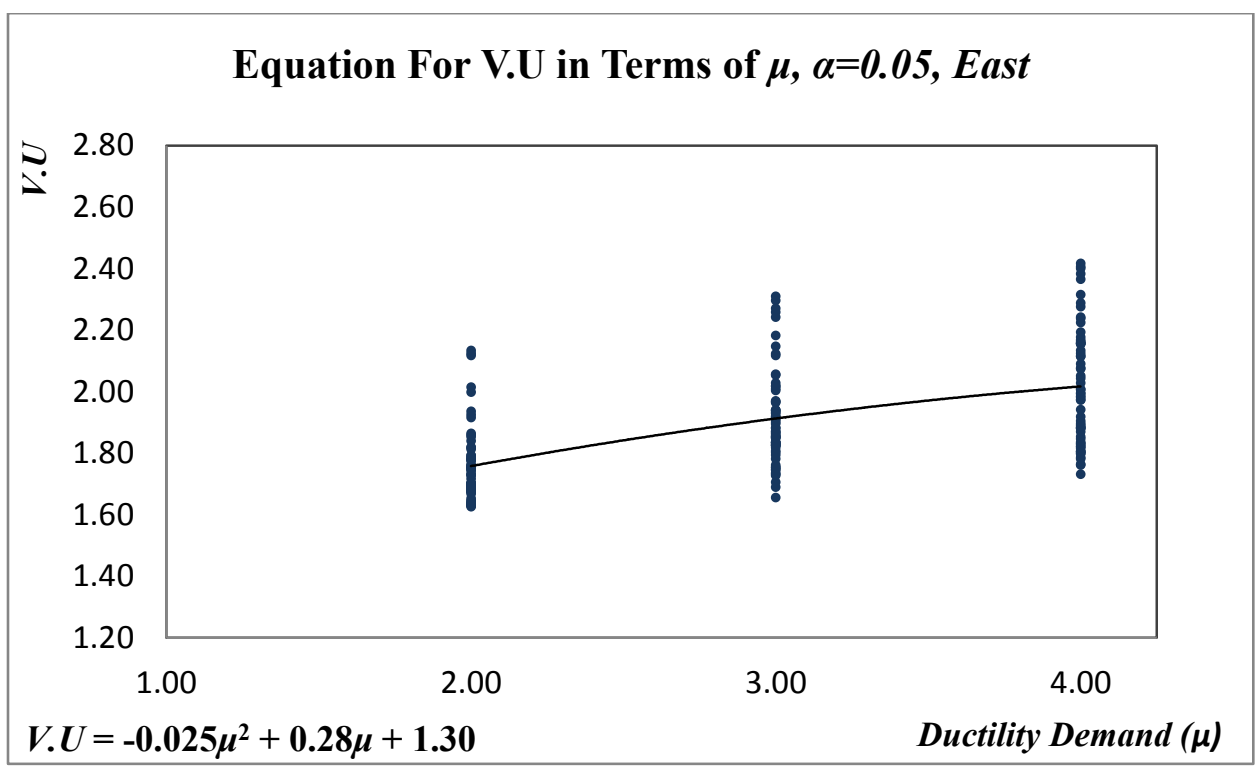

Figure 5.41 - V.U values mapped with respect to target ductility demands and the suggested equation for $V . U$ for data corresponding to the eastern compatible spectrum and post-yield stiffness of 0.05 


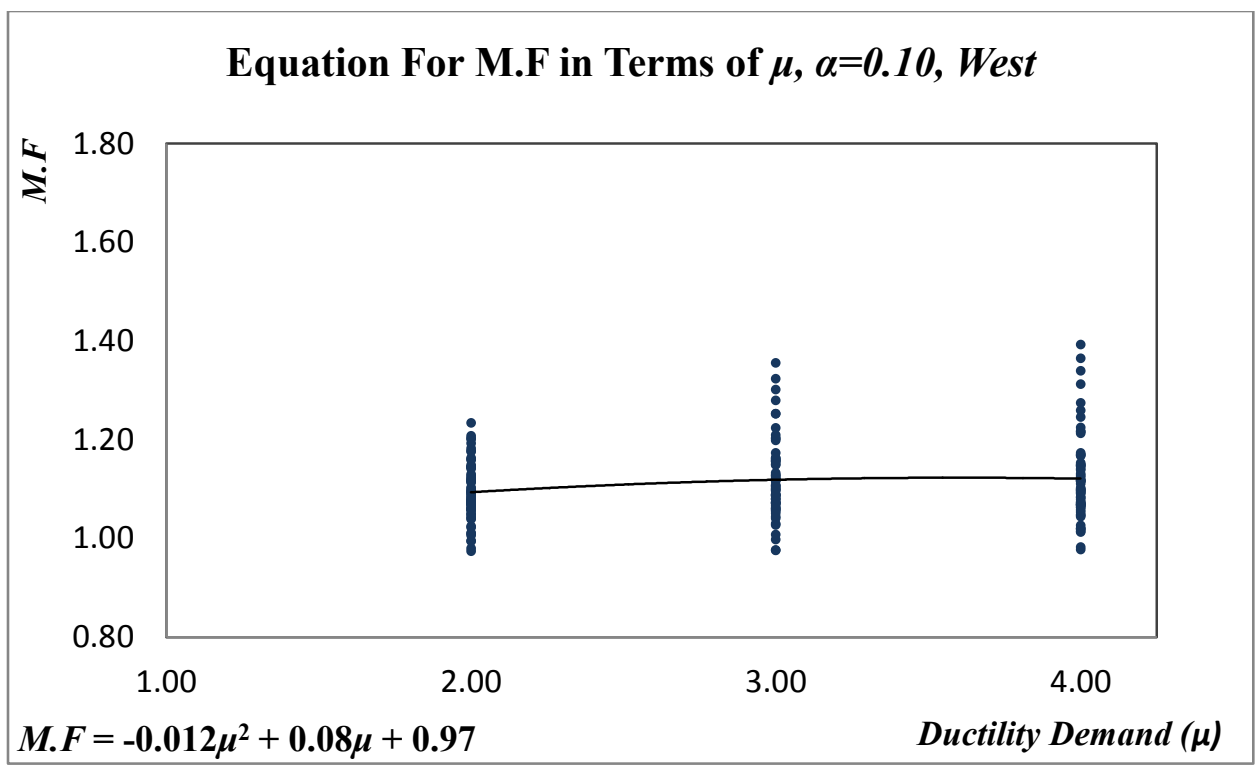

Figure 5.42 - M.F values mapped with respect to target ductility demands and the suggested equation for M.F for data corresponding to the western compatible spectrum and post-yield stiffness of 0.10

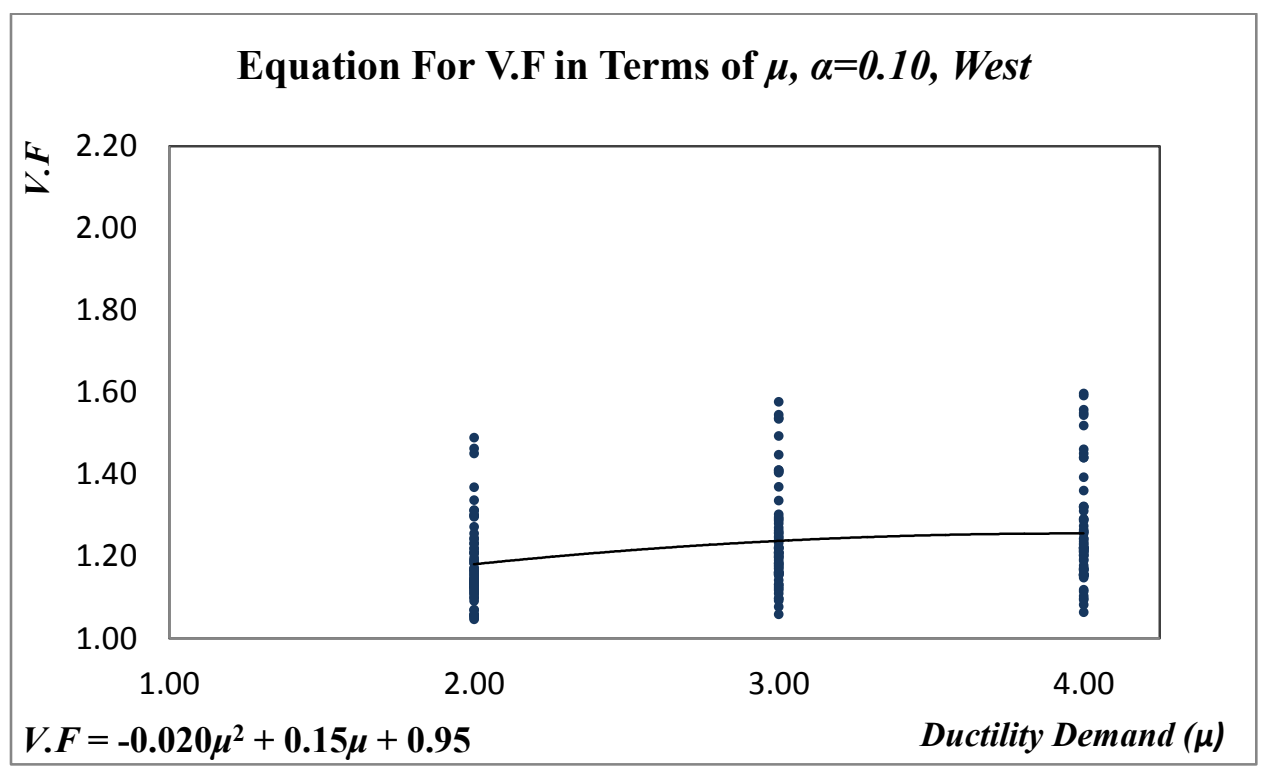

Figure 5.43 - V.F values mapped with respect to target ductility demands and the suggested equation for V.F for data corresponding to the western compatible spectrum and post-yield stiffness of 0.10 


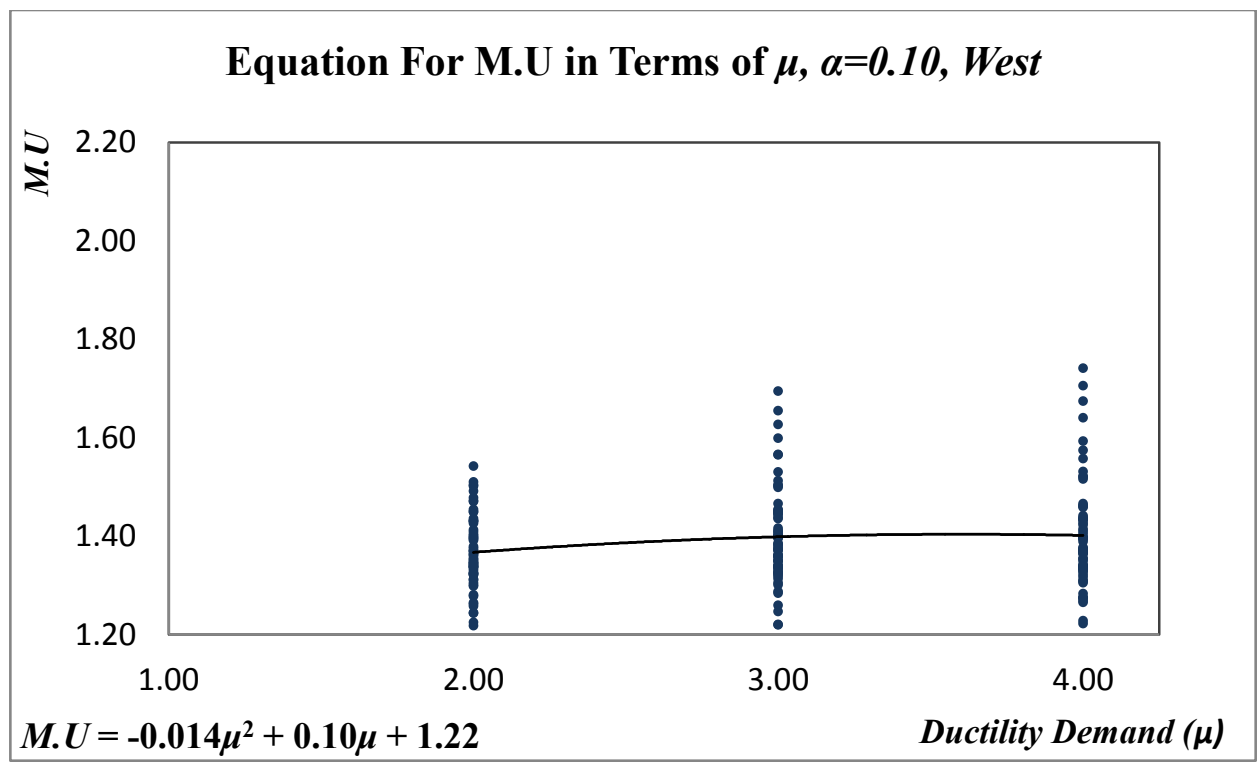

Figure 5.44 - M.U values mapped with respect to target ductility demands and the suggested equation for M.U for data corresponding to the western compatible spectrum and post-yield stiffness of 0.10

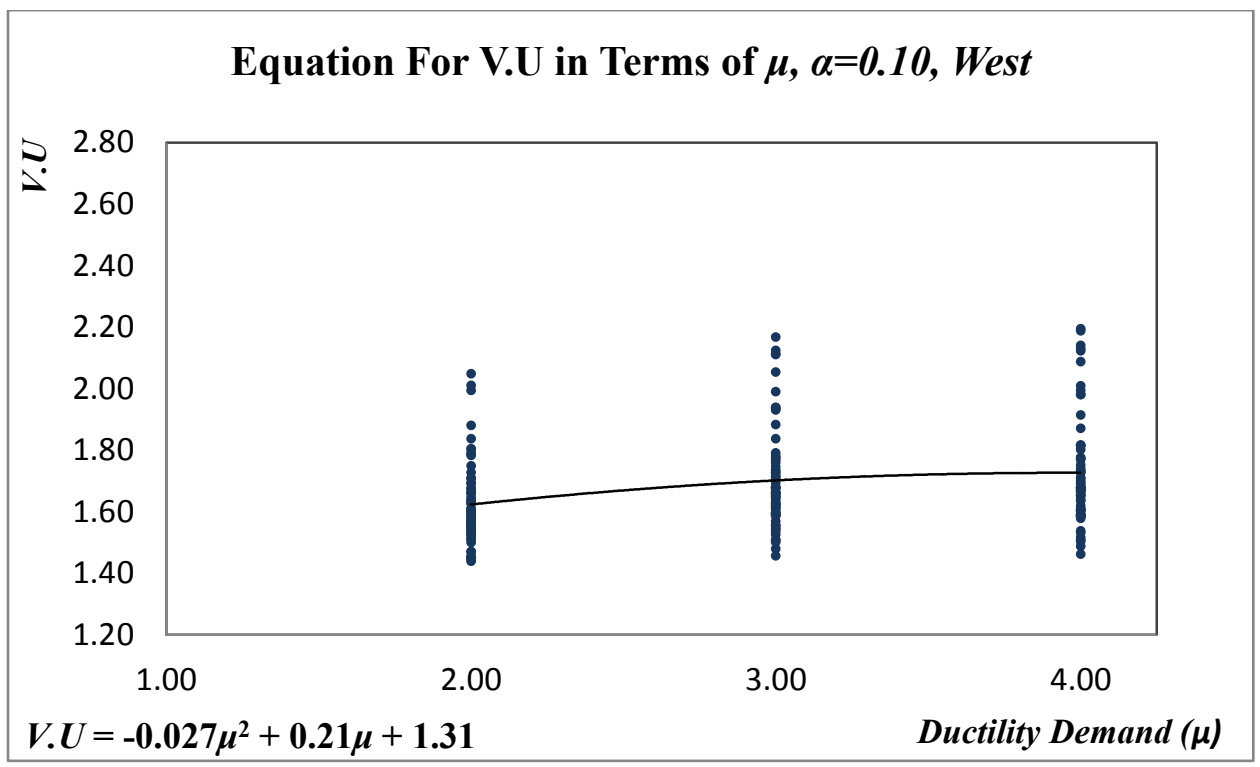

Figure 5.45 - V.U values mapped with respect to target ductility demands and the suggested equation for V.U for data corresponding to the western compatible spectrum and post-yield stiffness of 0.10 


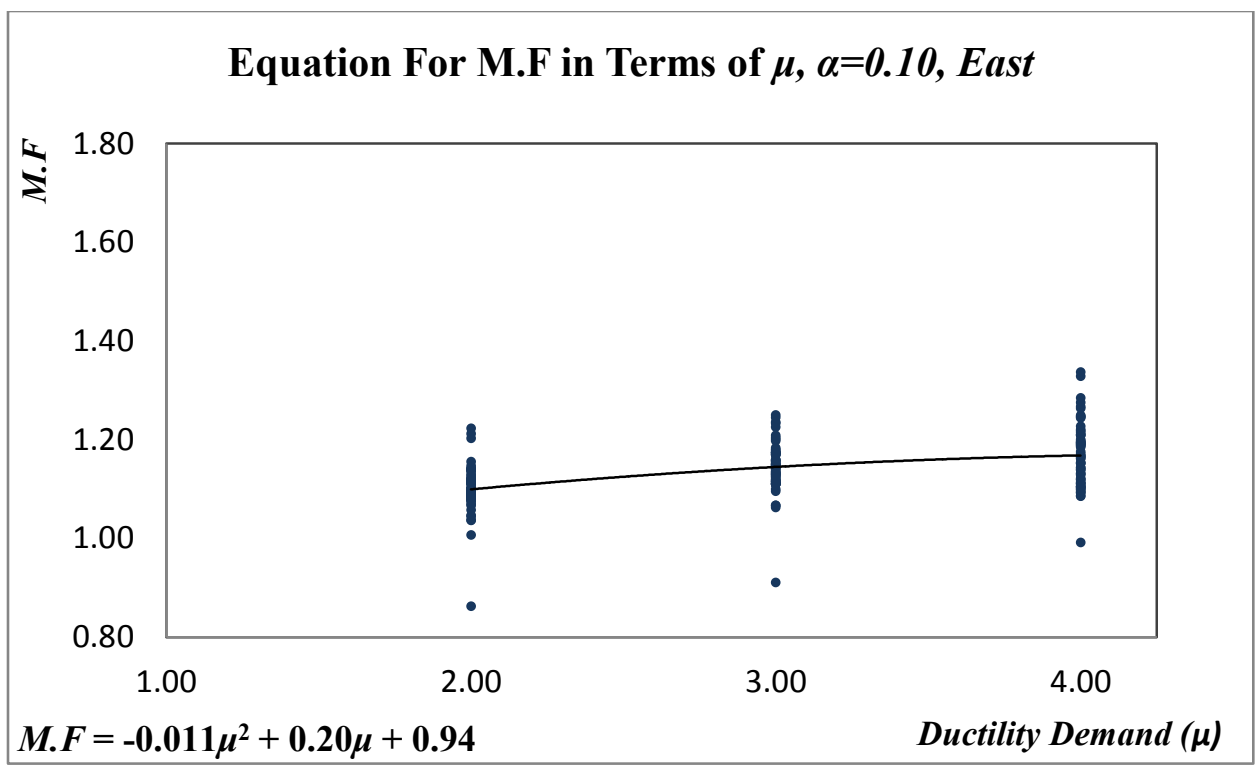

Figure 5.46 - M.F values mapped with respect to target ductility demands and the suggested equation for M.F for data corresponding to the eastern compatible spectrum and post-yield stiffness of 0.10

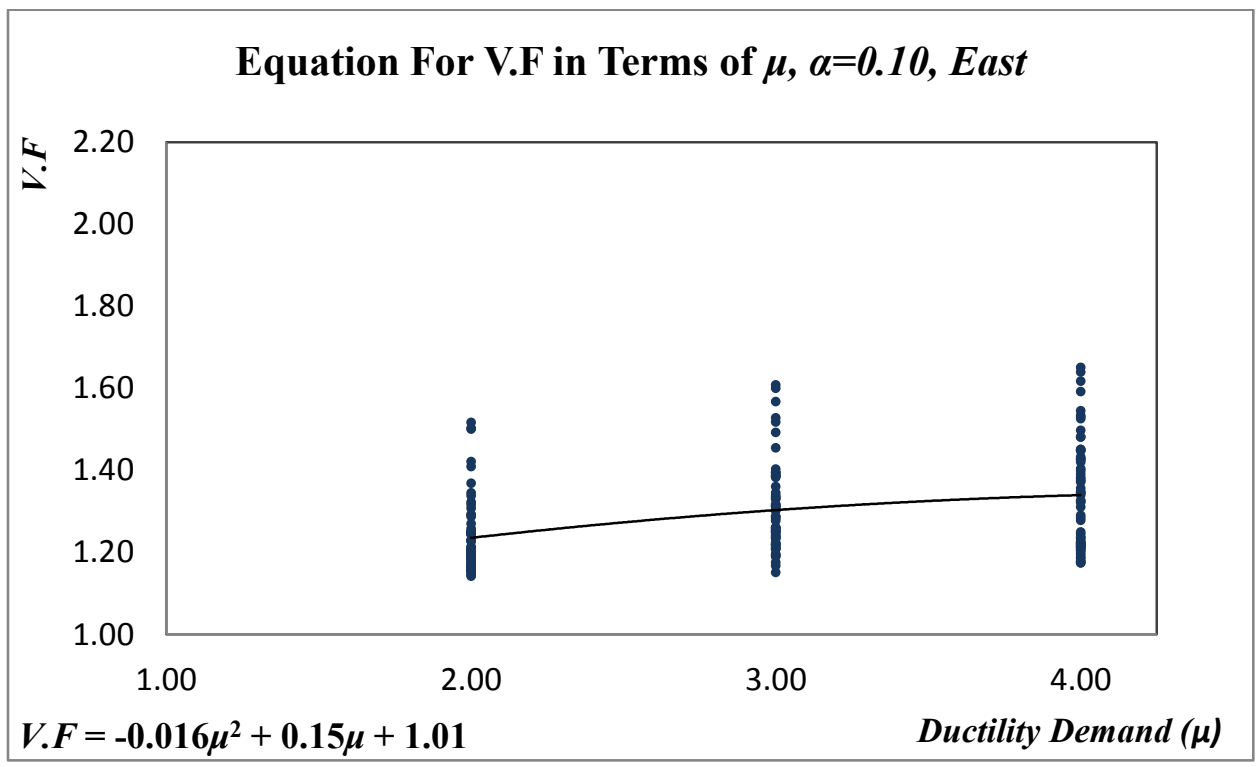

Figure 5.47 - V.F values mapped with respect to target ductility demands and the suggested equation for V.F for data corresponding to the eastern compatible spectrum and post-yield stiffness of 0.10 


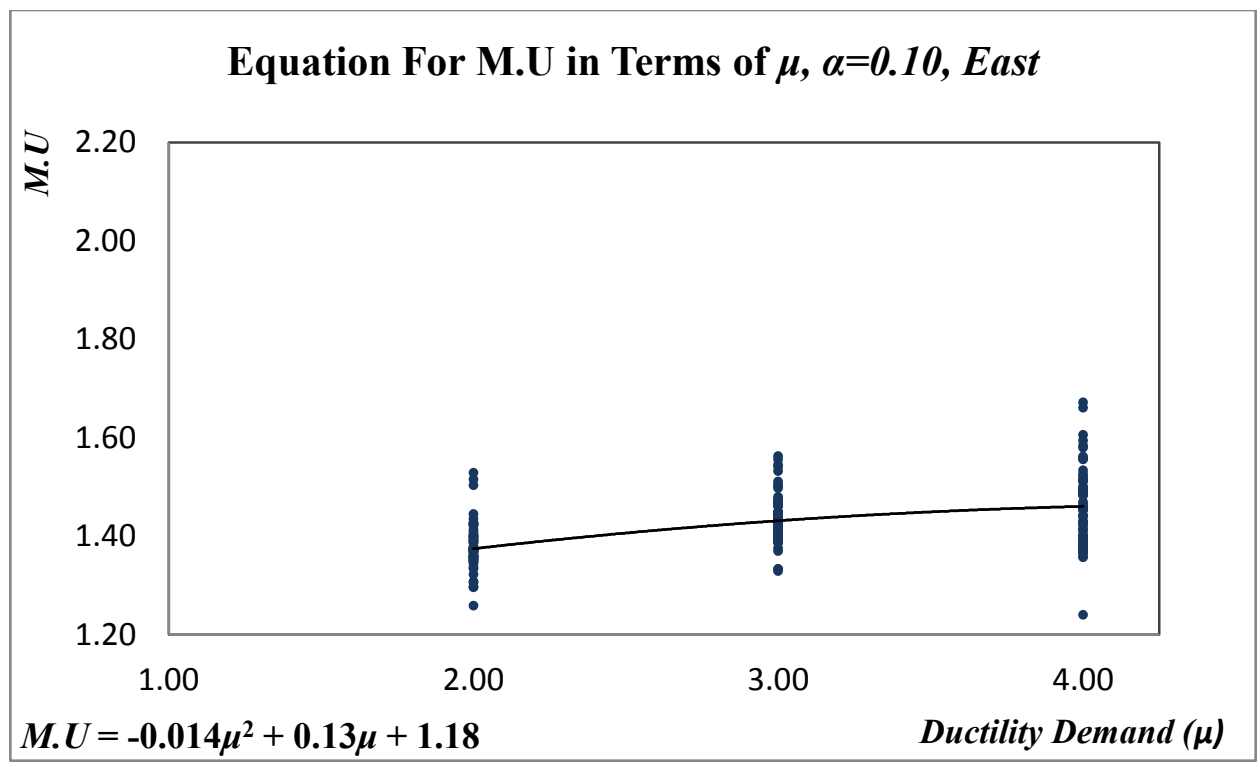

Figure 5.48 - M.U values mapped with respect to target ductility demands and the suggested equation for M.U for data corresponding to the eastern compatible spectrum and post-yield stiffness of 0.10

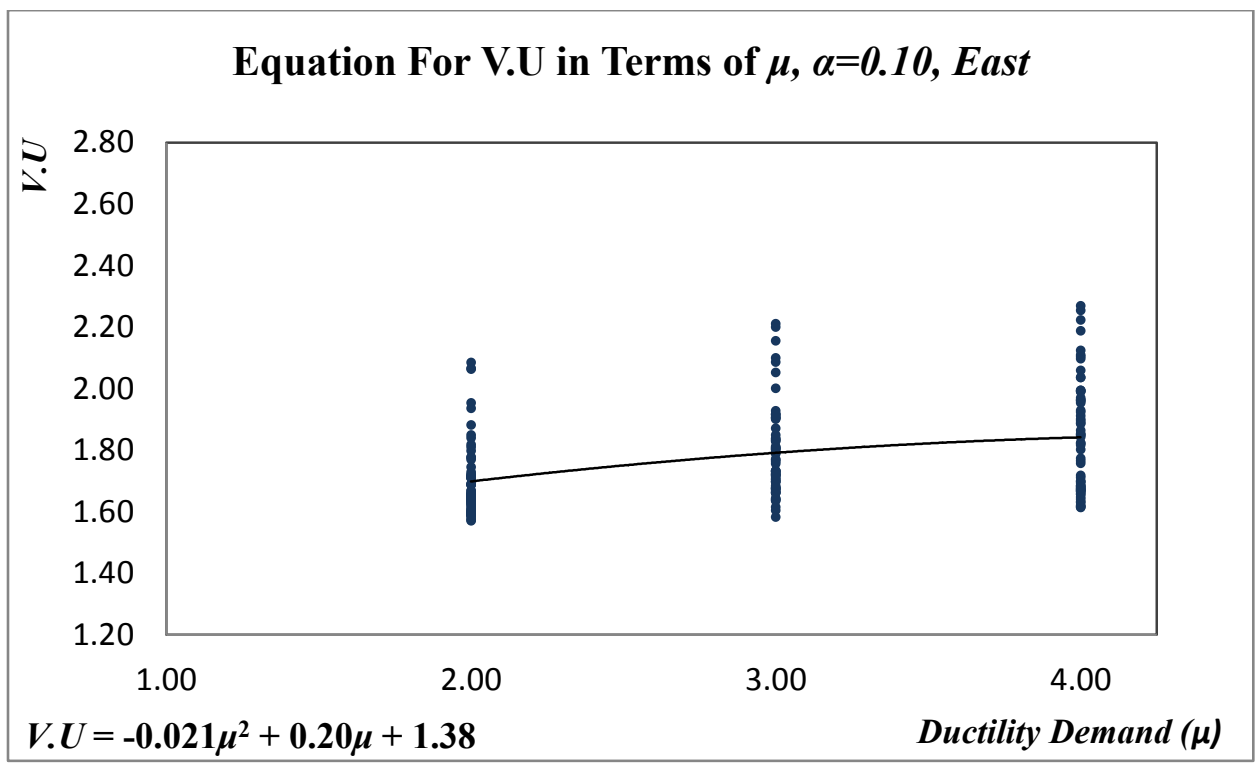

Figure 5.49 - V.U values mapped with respect to target ductility demands and the suggested equation for $V . U$ for data corresponding to the eastern compatible spectrum and post-yield stiffness of 0.10 
The suggested equations for the western compatible spectrum are as follow:

For $\alpha=0.00:$

$M . F=-0.019 \mu^{2}+0.21 \mu+0.82$

$V . F=-0.027 \mu^{2}+0.29 \mu+0.77$

$M . U=-0.024 \mu^{2}+0.26 \mu+1.03$

$V . U=-0.037 \mu^{2}+0.40 \mu+1.07$

For $\alpha=0.02$ :

$M . F=-0.014 \mu^{2}+0.16 \mu+0.89$

$V . F=-0.022 \mu^{2}+0.24 \mu+0.84$

$M . U=-0.018 \mu^{2}+0.20 \mu+1.11$

$V . U=-0.031 \mu^{2}+0.34 \mu+1.15$

For $\alpha=0.05$ :

$M . F=-0.015 \mu^{2}+0.14 \mu+0.91$

$V . F=-0.024 \mu^{2}+0.23 \mu+0.86$

$M . U=-0.018 \mu^{2}+0.17 \mu+1.14$ 
$V . U=-0.033 \mu^{2}+0.31 \mu+1.18$

For $\alpha=0.10$ :

$M . F=-0.012 \mu^{2}+0.08 \mu+0.97$

$V . F=-0.020 \mu^{2}+0.15 \mu+0.95$

$M . U=-0.014 \mu^{2}+0.10 \mu+1.22$

$V . U=-0.027 \mu^{2}+0.21 \mu+1.31$

The suggested equations for the eastern compatible spectrum are as follow:

For $\alpha=0.00$ :

$M . F=-0.013 \mu^{2}+0.19 \mu+0.84$

$V . F=-0.015 \mu^{2}+0.24 \mu+0.91$

$M . U=-0.014 \mu^{2}+0.22 \mu+1.08$

$V . U=-0.021 \mu^{2}+0.33 \mu+1.25$

For $\alpha=0.02$ :

$M . F=-0.013 \mu^{2}+0.17 \mu+0.87$

$V . F=-0.018 \mu^{2}+0.23 \mu+0.92$ 


$$
\begin{aligned}
& M . U=-0.016 \mu^{2}+0.21 \mu+1.09 \\
& V . U=-0.024 \mu^{2}+0.32 \mu+1.26
\end{aligned}
$$

For $\alpha=0.05$ :

$$
\begin{aligned}
& M . F=-0.014 \mu^{2}+0.15 \mu+0.89 \\
& V . F=-0.018 \mu^{2}+0.20 \mu+0.94 \\
& M . U=-0.017 \mu^{2}+0.19 \mu+1.11 \\
& V . U=-0.025 \mu^{2}+0.28 \mu+1.30
\end{aligned}
$$

For $\alpha=0.10$ :

$M . F=-0.011 \mu^{2}+0.20 \mu+0.94$

$V . F=-0.016 \mu^{2}+0.15 \mu+1.01$

$M . U=-0.014 \mu^{2}+0.13 \mu+1.18$

$V . U=-0.021 \mu^{2}+0.20 \mu+1.38$

The mappings of the ratio values as well as the fitted curves suggest that magnification in east is slightly greater than the magnification in the west. This is expected, because the spectral shape for the east is steeper causing greater contribution from the higher modes. As pointed out earlier, greater contribution from higher modes causes inertia forces to shift toward the mid-span, leading to greater bending moment towards mid-span and 
shear force at quarter span. However, the difference between the magnification values for east and west is not significant. We may combine the east and west data and come up with one set of expressions.

It can be seen that the magnification in the shear force at quarter span is much greater than the bending moment magnification at the mid-span. This confirms the validity of the choice of keeping them as separated quantities.

The mapped data points and the suggested equations confirm that post-yield hardening decrease the magnification factors. However since this decrease is not quite significant, conservatively data corresponding to $\alpha=0.00$ may be used for design purposes. This choice is justified by the observation that the magnification ratios are always the largest for no post-yield stiffness. The data also shows considerable scatter around the mean. However, given the uncertainty inherent in defining the seismic hazard, it would be reasonable to use the mean values in design. This would also suggest that a single set of equations may be considered appropriate both for the eastern and western regions.

In order to come up with consistent equations that can be used regardless of the type of spectrum, a second set of regressions are carried out. This time no distinction is made on the basis of the type of spectrum. The mappings as well as the fitted curves obtained from this set of analyses are presented in Figures 5.50 to 5.65. Of these Figures 5.50 to 5.53 and Equations 5.42 to 5.45 which represent the data corresponding to $\alpha=0.00$ can be used for design purposes. 


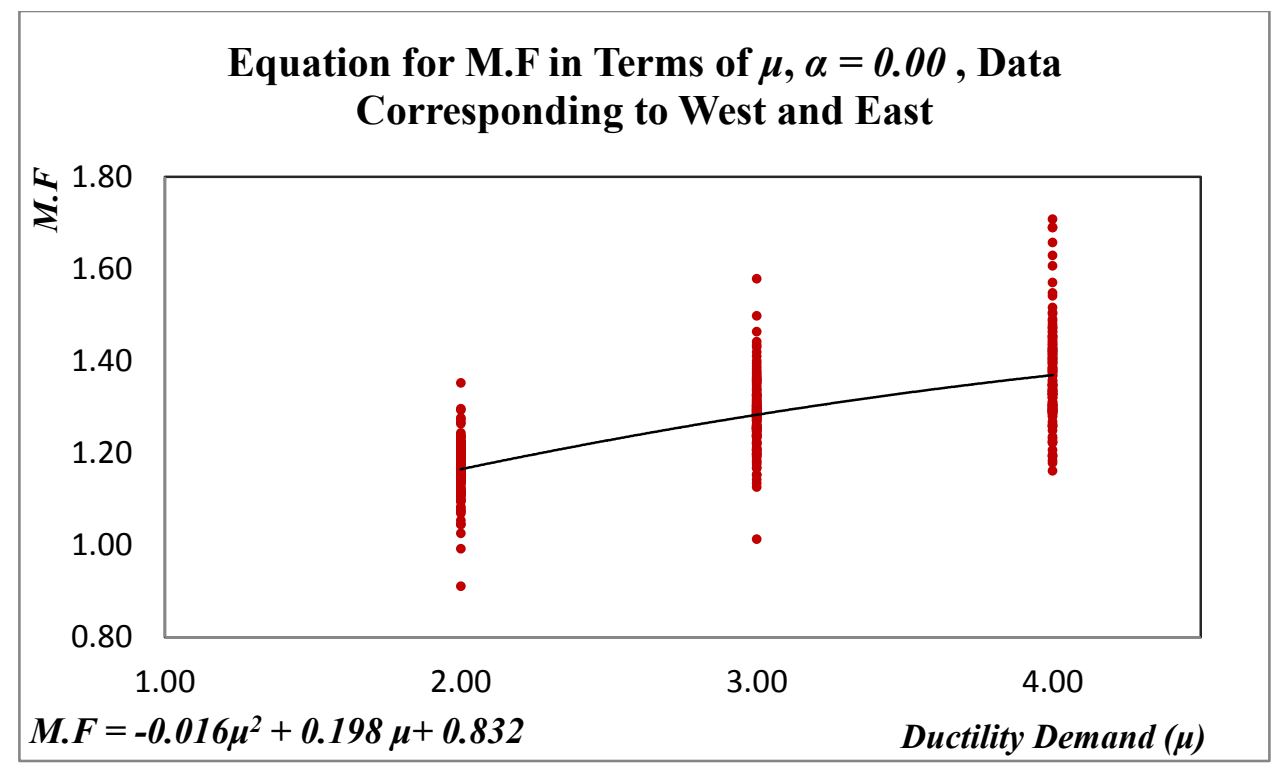

Figure 5.50 - M.F values mapped with respect to target ductility demands and the suggested equation for M.F for data corresponding to both spectra and post-yield stiffness of 0.00

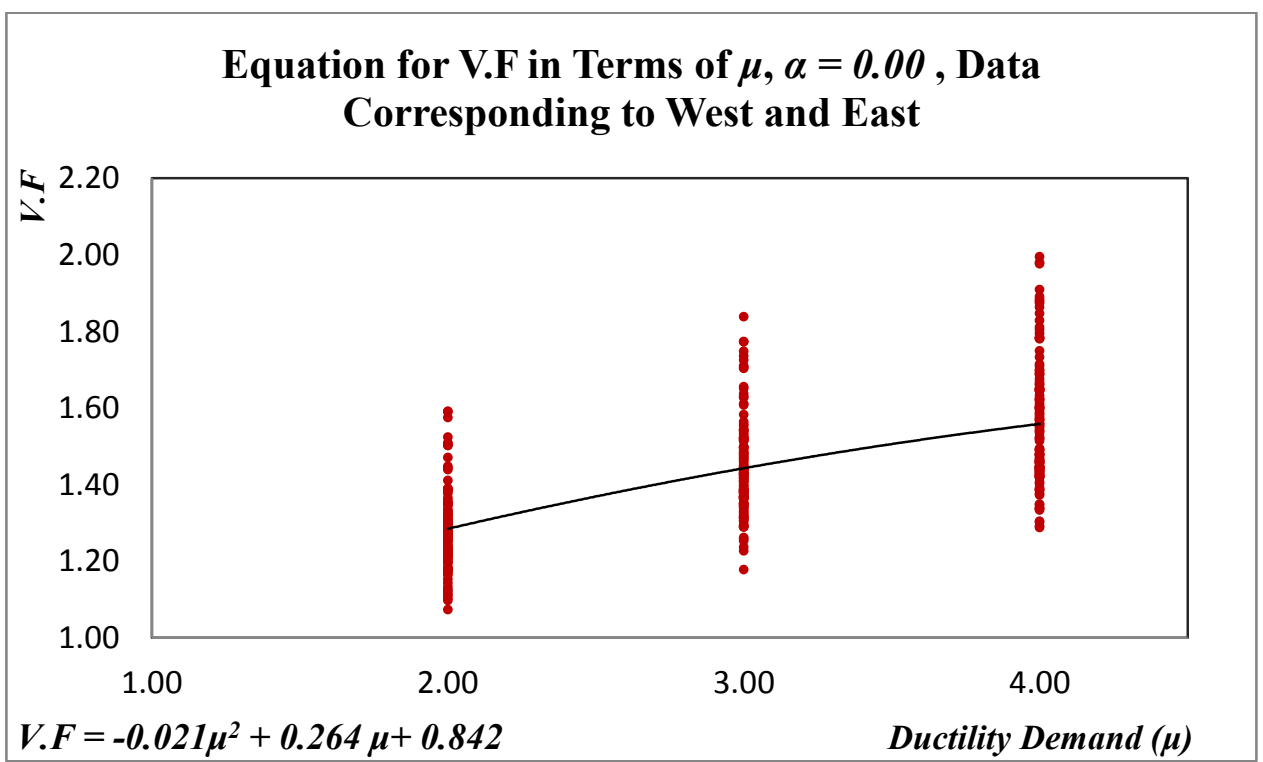

Figure 5.51 - V.F values mapped with respect to target ductility demands and the suggested equation for V.F for data corresponding to both spectra and post-yield stiffness of 0.00 


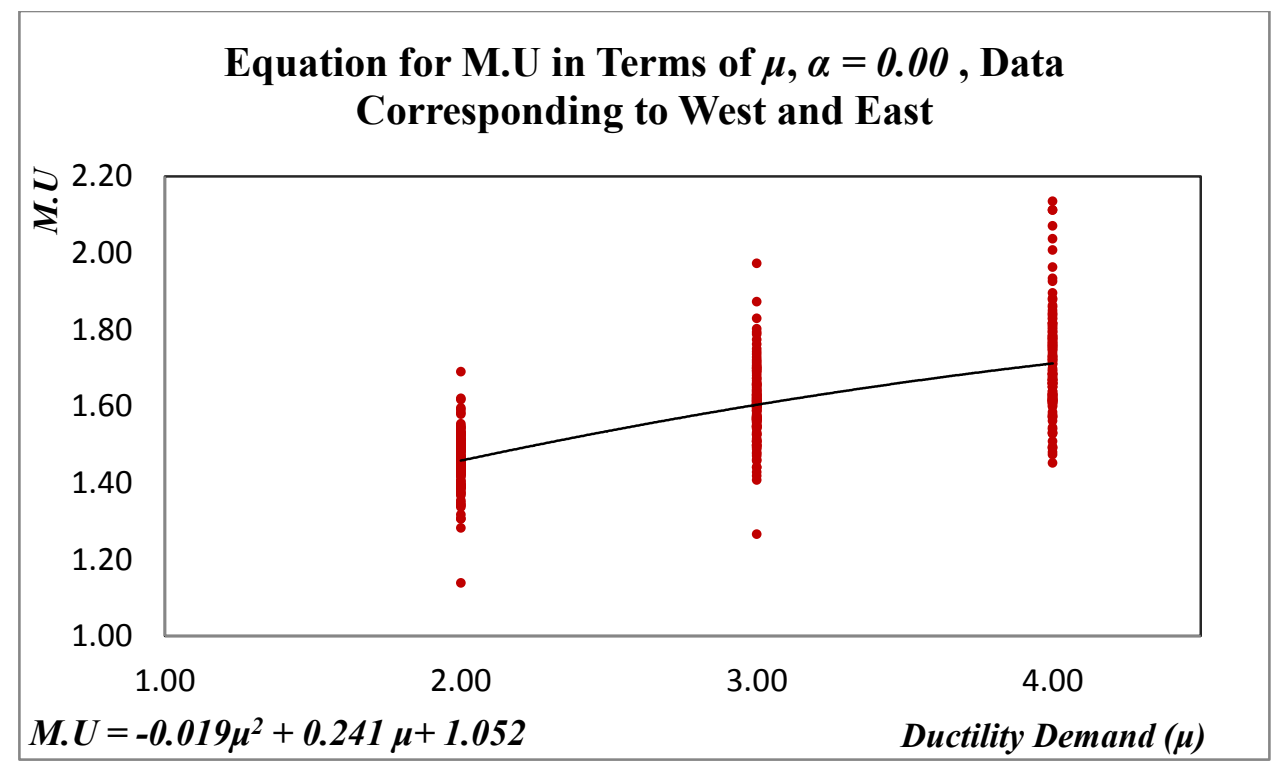

Figure 5.52 - M.U values mapped with respect to target ductility demands and the suggested equation for M.U for data corresponding to both spectra and post-yield stiffness of 0.00

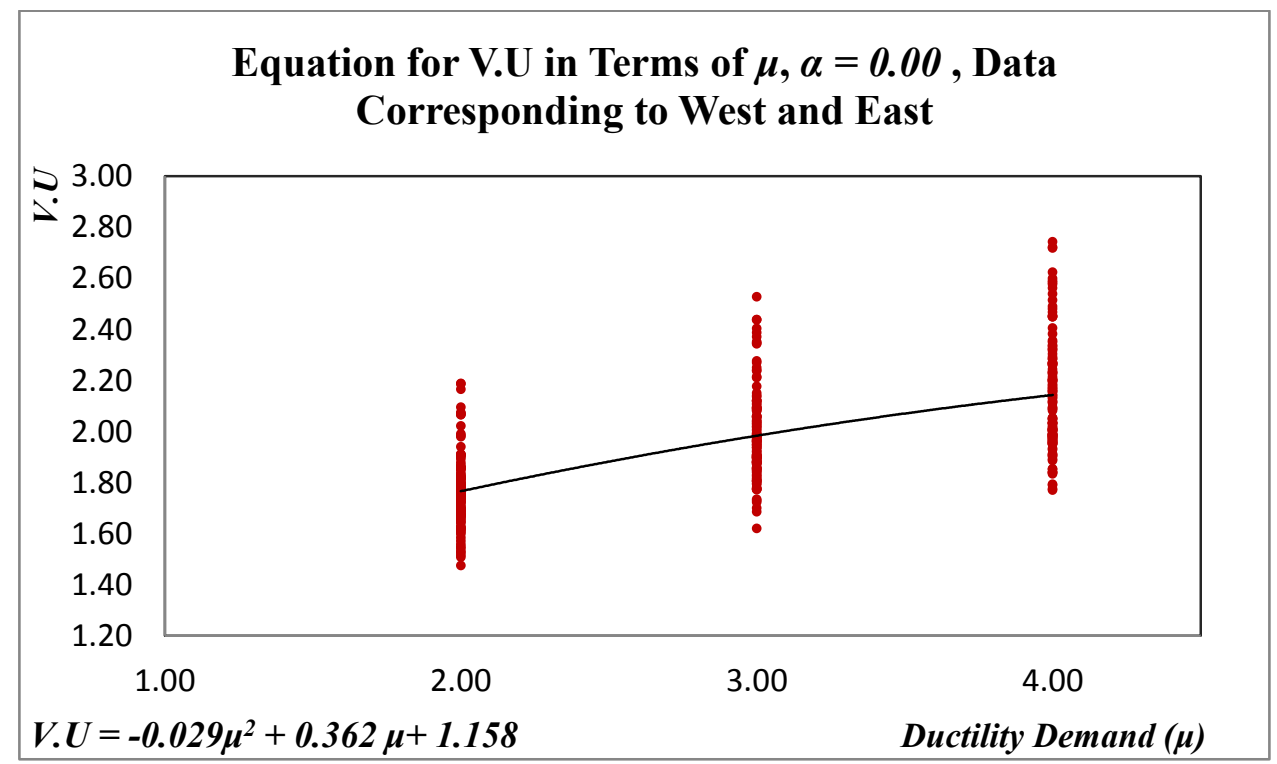

Figure 5.53 - V.U values mapped with respect to target ductility demands and the suggested equation for V.U for data corresponding to both spectra and post-yield stiffness of 0.00 


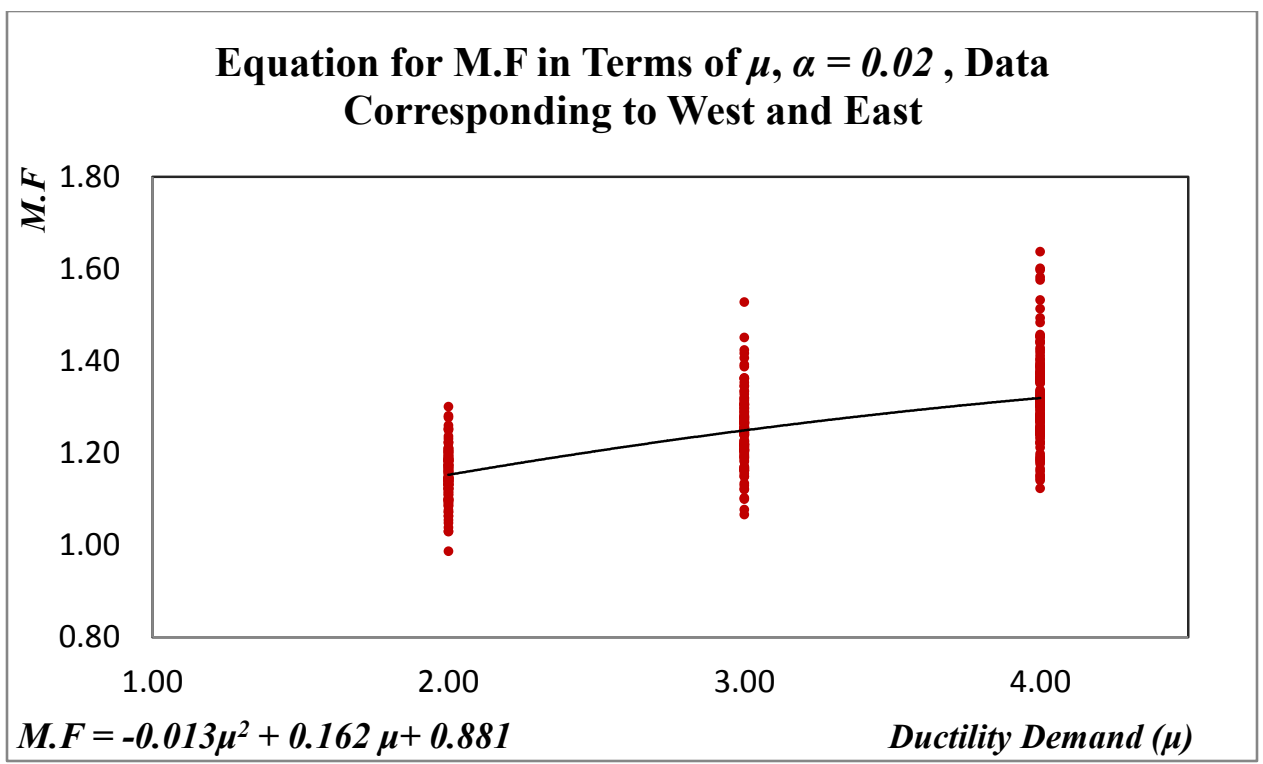

Figure 5.54 - M.F values mapped with respect to target ductility demands and the suggested equation for M.F for data corresponding to both spectra and post-yield stiffness of 0.02

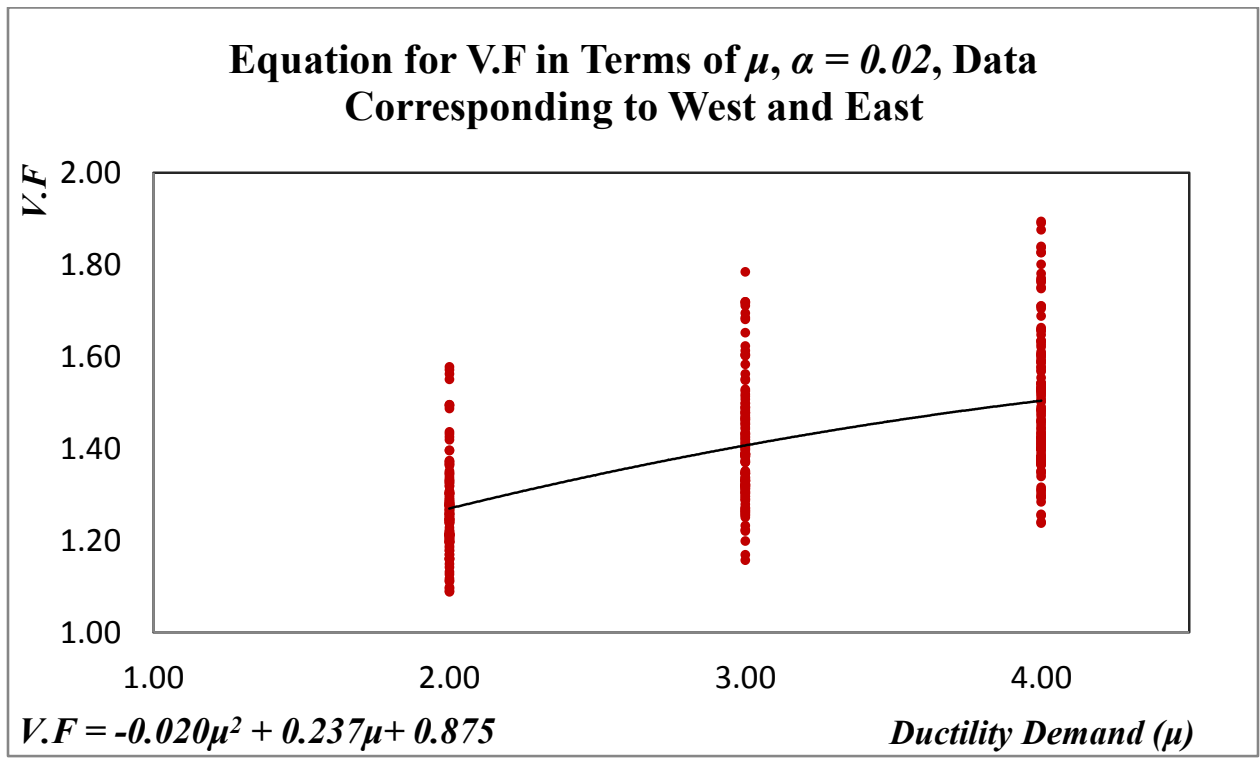

Figure 5.55 - V.F values mapped with respect to target ductility demands and the suggested equation for V.F for data corresponding to both spectra and post-yield stiffness of 0.02 


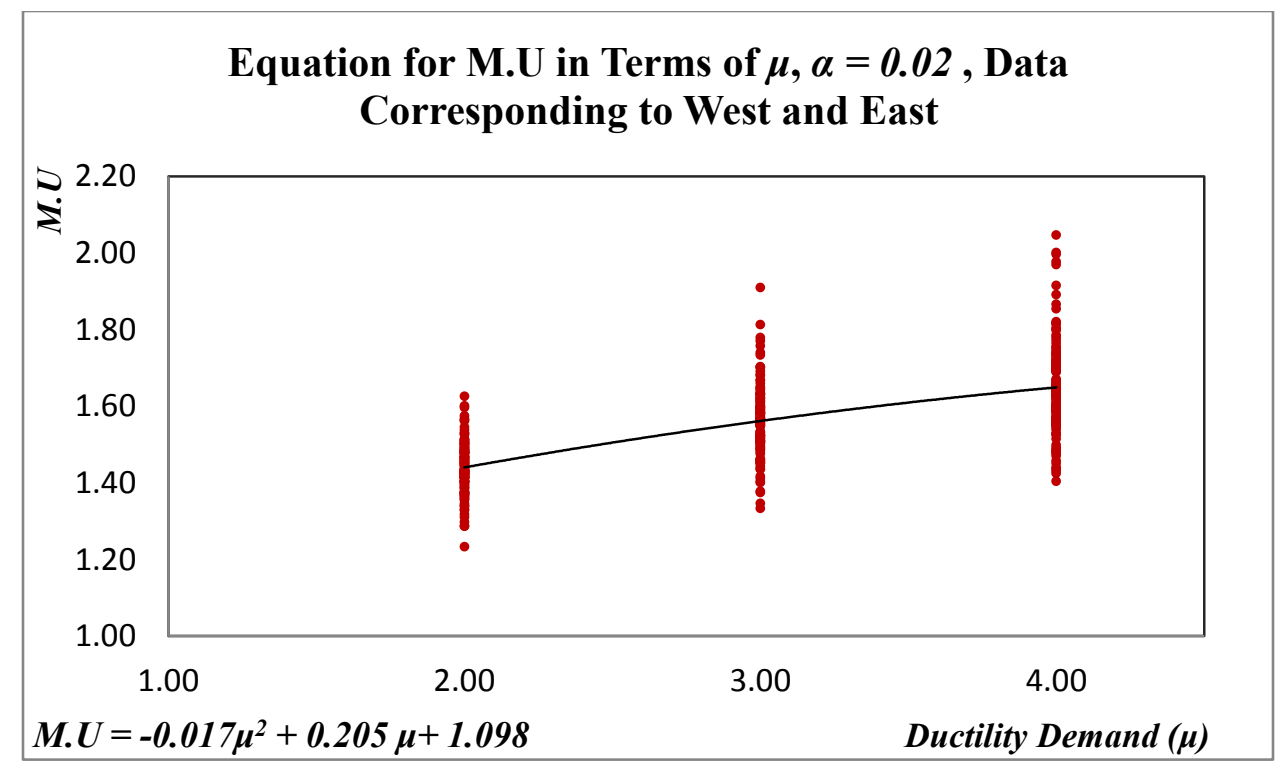

Figure 5.56 - M.U values mapped with respect to target ductility demands and the suggested equation for M.U for data corresponding to both spectra and post-yield stiffness of 0.02

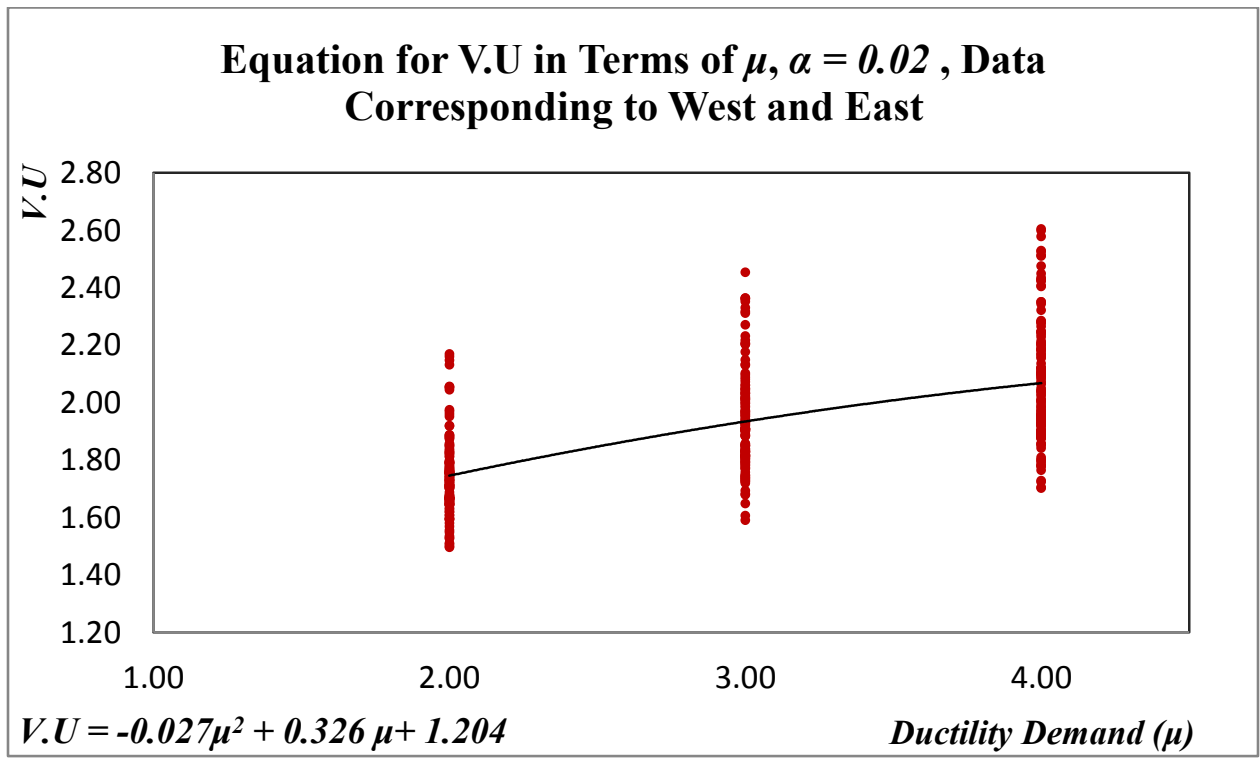

Figure 5.57 - V.U values mapped with respect to target ductility demands and the suggested equation for V.U for data corresponding to both spectra and post-yield stiffness of 0.02 


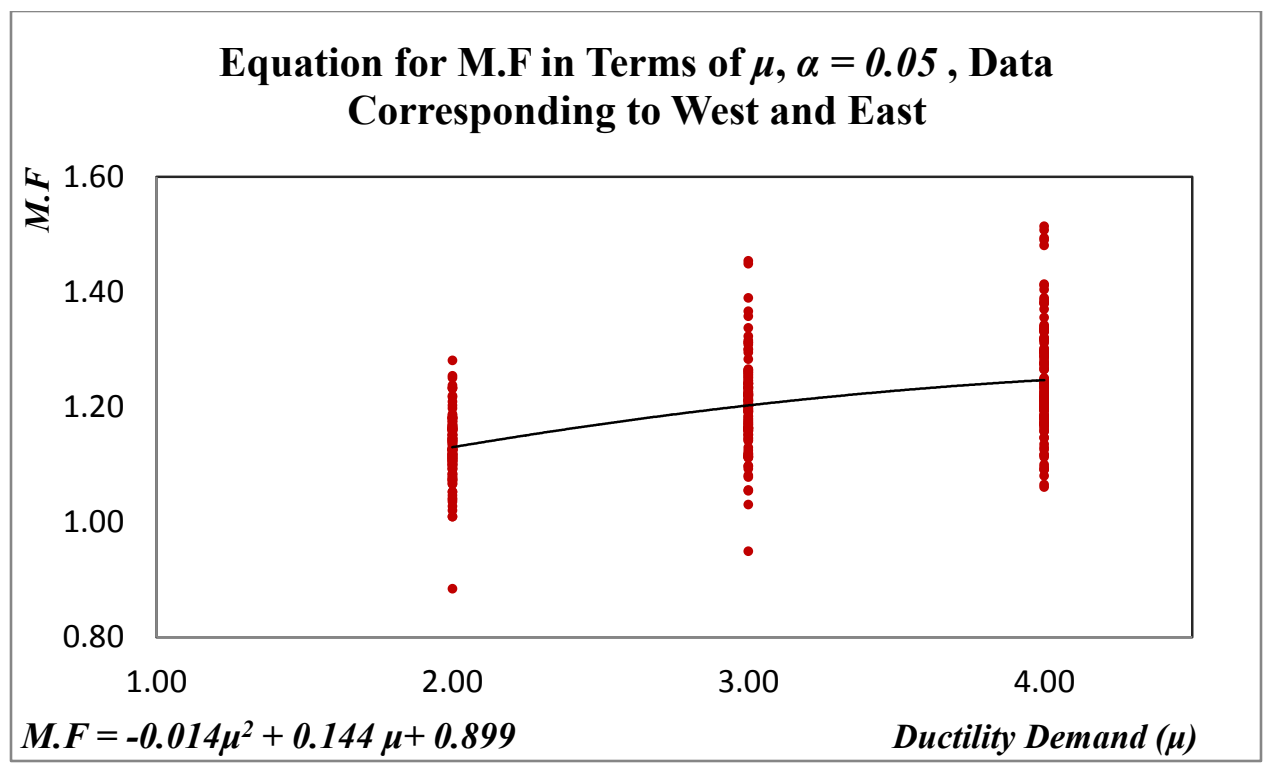

Figure 5.58 - M.F values mapped with respect to target ductility demands and the suggested equation for M.F for data corresponding to both spectra and post-yield stiffness of 0.05

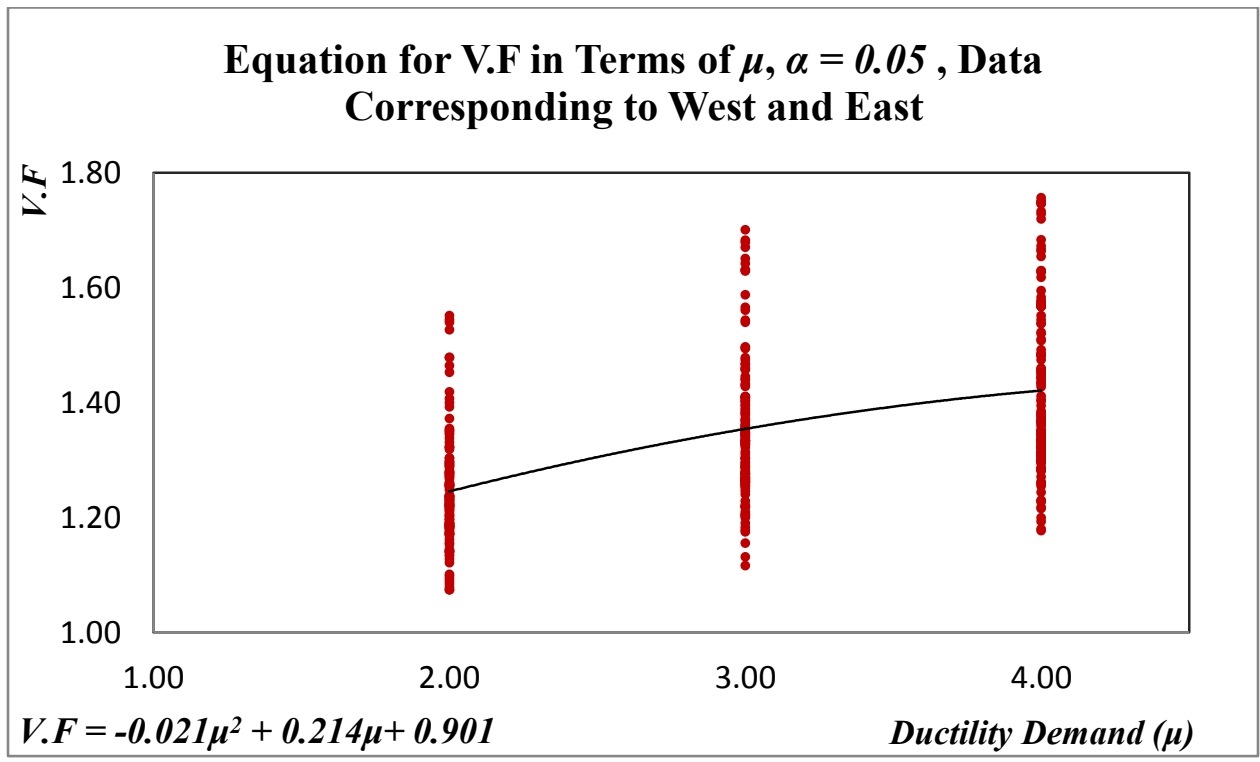

Figure 5.59 - V.F values mapped with respect to target ductility demands and the suggested equation for V.F for data corresponding to both spectra and post-yield stiffness of 0.05 


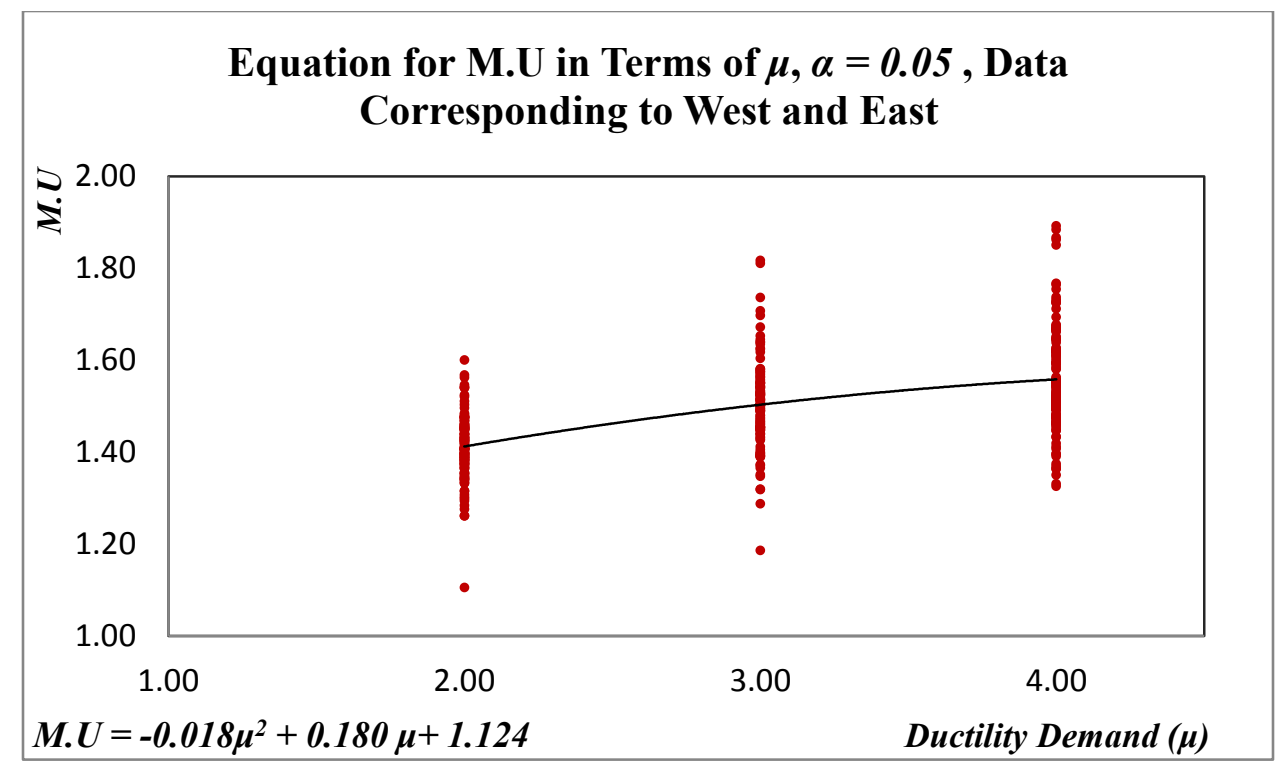

Figure 5.60 - M.U values mapped with respect to target ductility demands and the suggested equation for M.U for data corresponding to both spectra and post-yield stiffness of 0.05

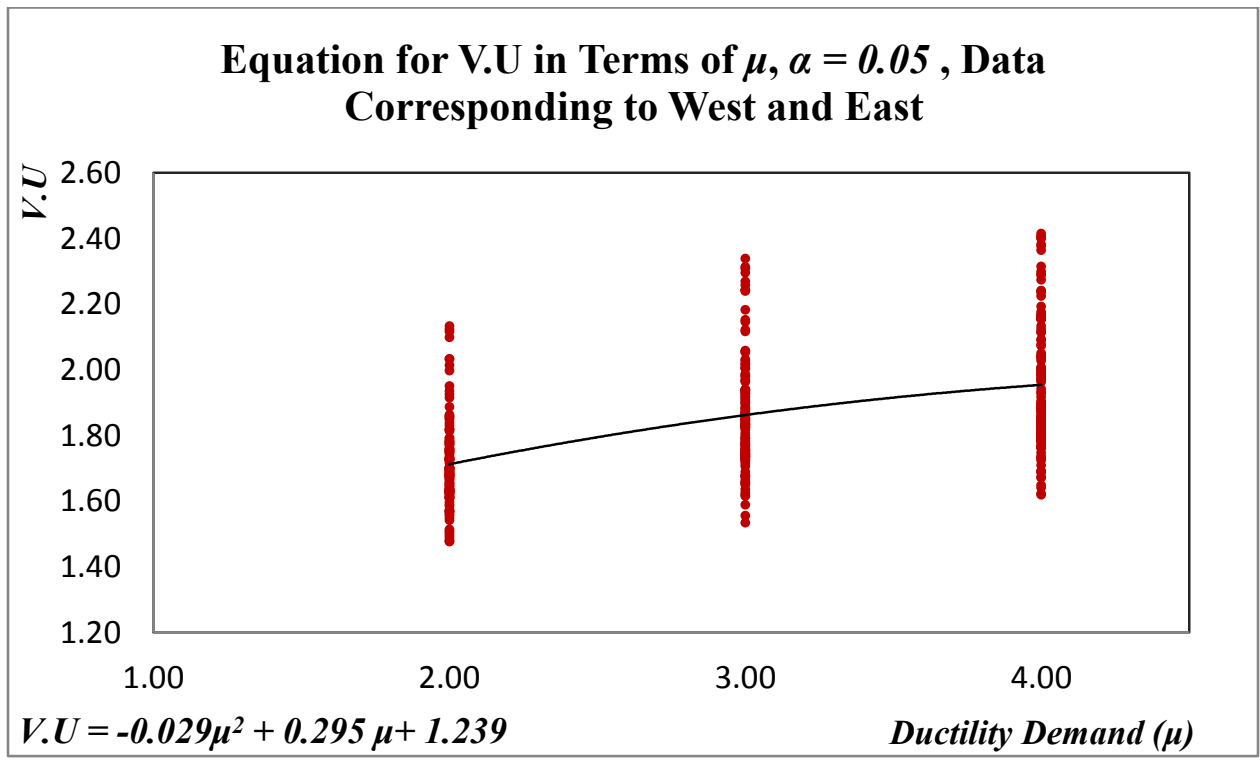

Figure 5.61 - V.U values mapped with respect to target ductility demands and the suggested equation for V.U for data corresponding to both spectra and post-yield stiffness of 0.05 


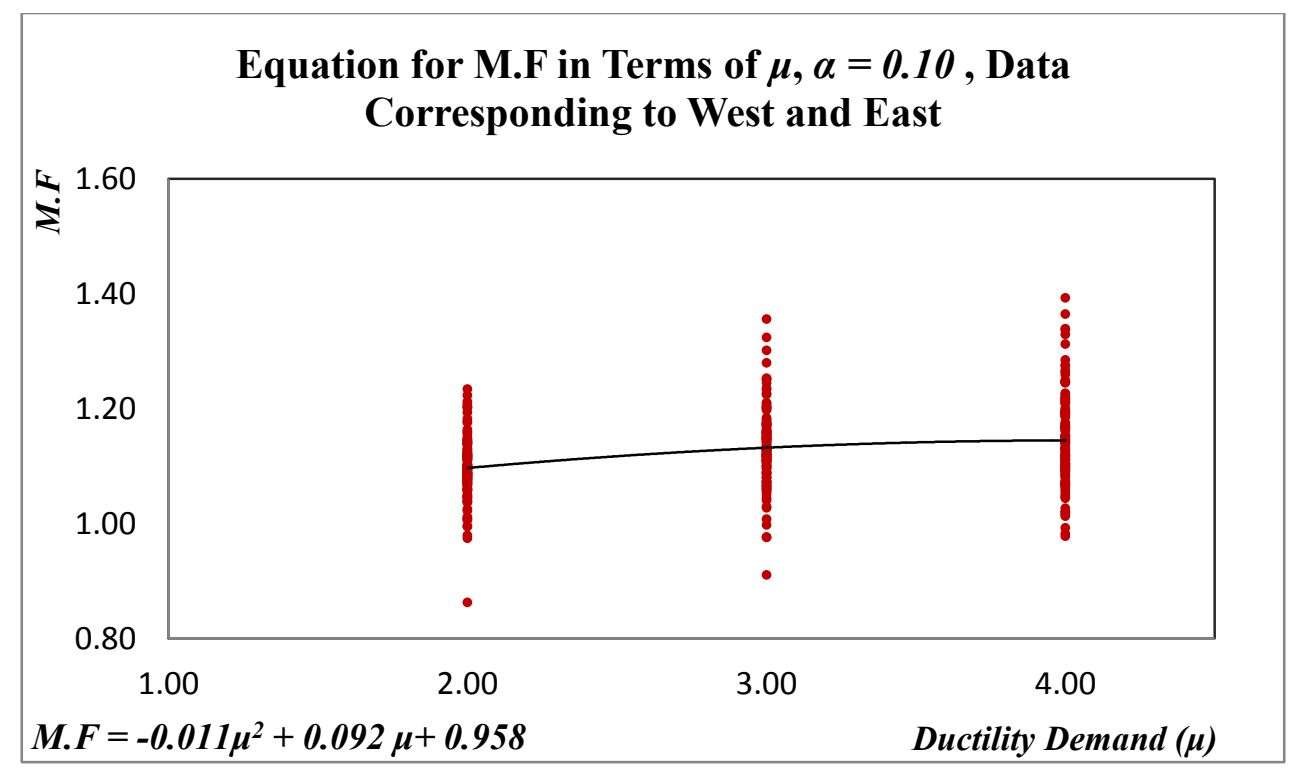

Figure 5.62 - M.F values mapped with respect to target ductility demands and the suggested equation for M.F for data corresponding to both spectra and post-yield stiffness of 0.10

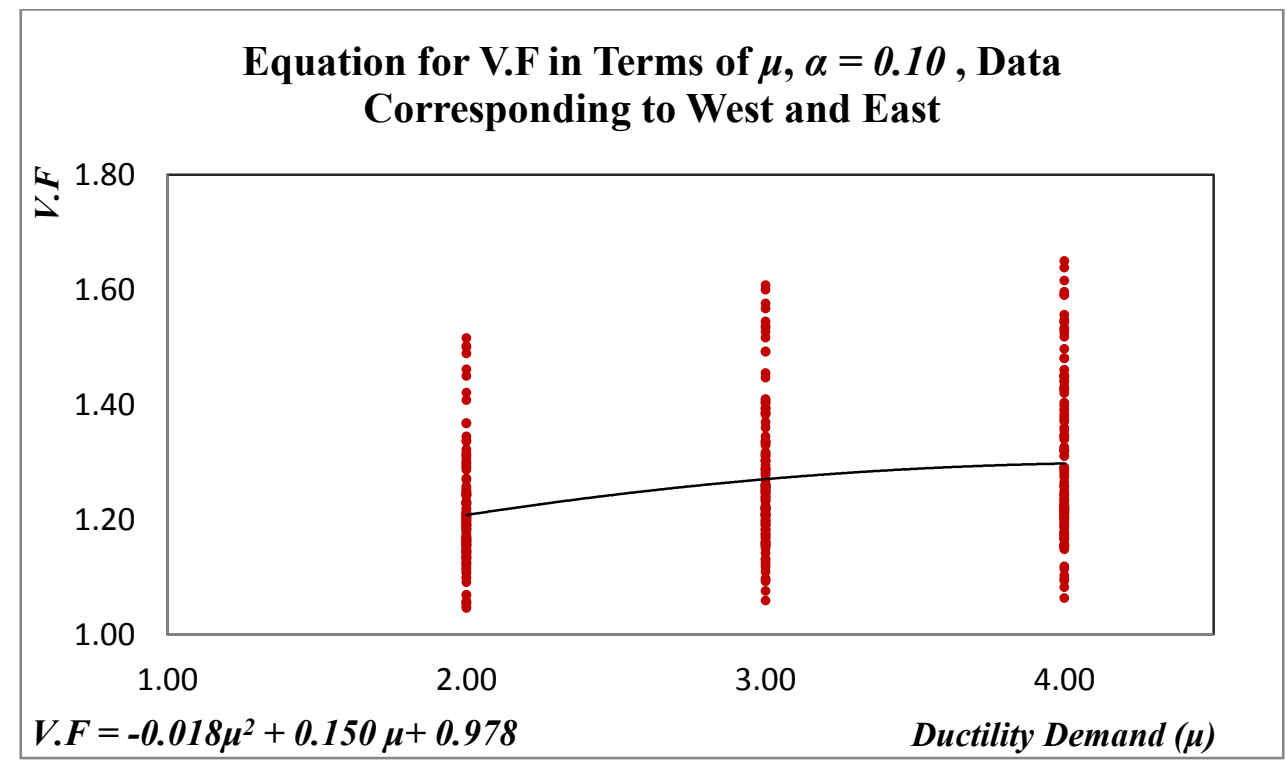

Figure 5.63 - V.F values mapped with respect to target ductility demands and the suggested equation for V.F for data corresponding to both spectra and post-yield stiffness of 0.10 


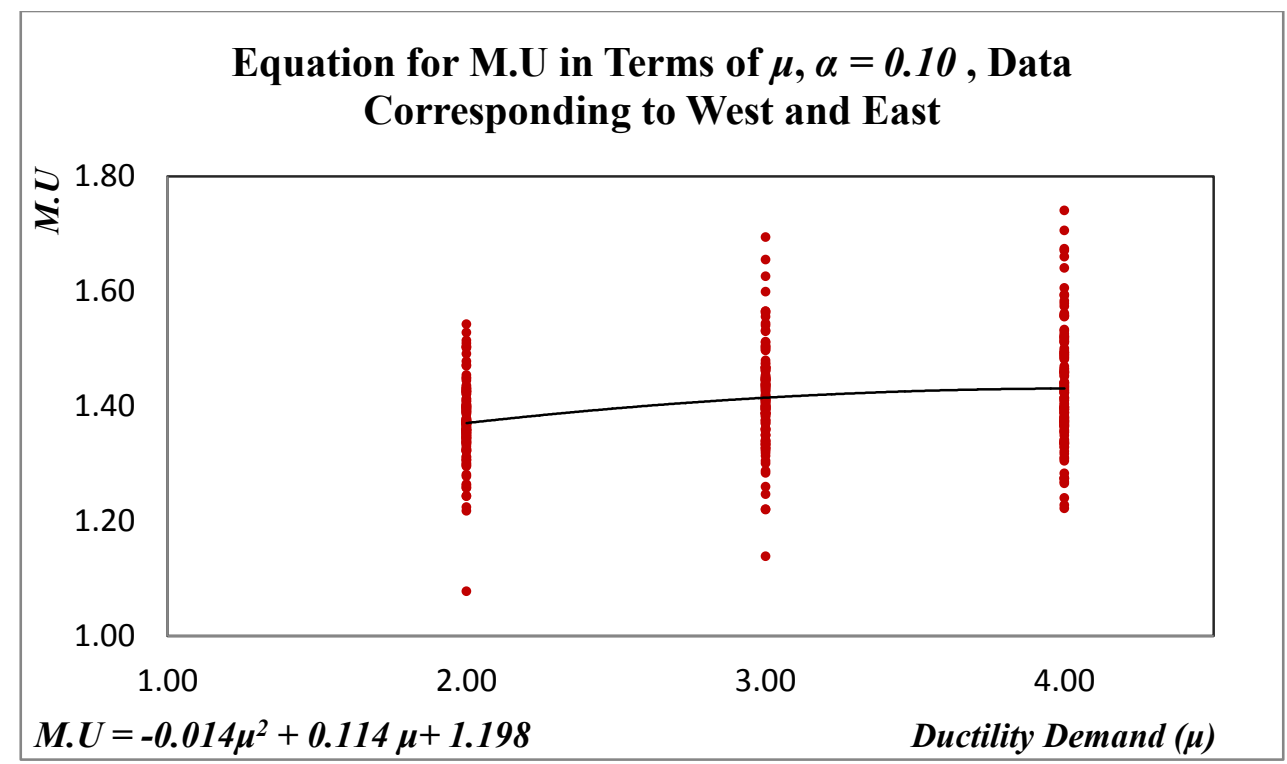

Figure 5.64 - M.U values mapped with respect to target ductility demands and the suggested equation for M.U for data corresponding to both spectra and post-yield stiffness of 0.10

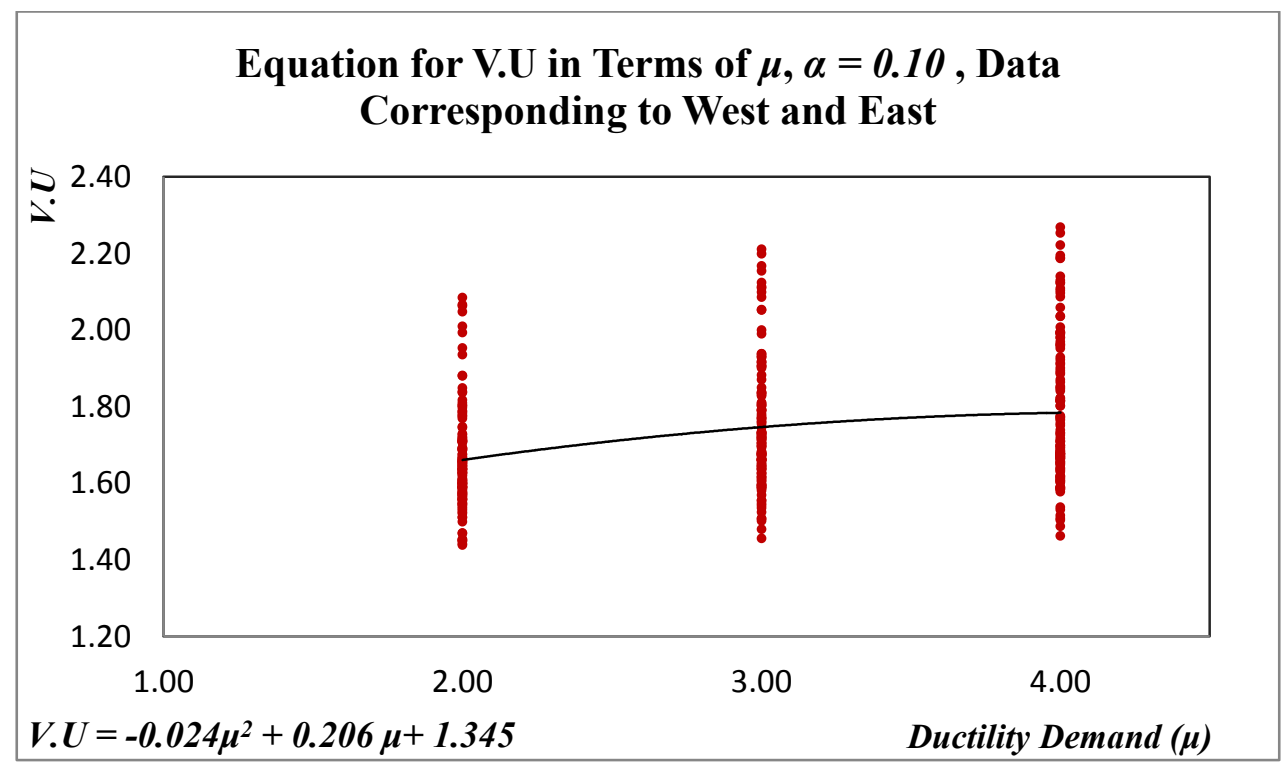

Figure 5.65 - V.U values mapped with respect to target ductility demands and the suggested equation for V.U for data corresponding to both spectra and post-yield stiffness of 0.10 
After combining the data corresponding to both eastern and western compatible spectra the suggested equations for predicting the magnification factors are as follows:

For $\alpha=0.00:$

$M . F=-0.016 \mu^{2}+0.198 \mu+0.832$

$V . F=-0.021 \mu^{2}+0.264 \mu+0.842$

$M . U=-0.019 \mu^{2}+0.241 \mu+1.052$

$V . U=-0.029 \mu^{2}+0.362 \mu+1.158$

For $\alpha=0.02$ :

$M . F=-0.013 \mu^{2}+0.162 \mu+0.881$

$V . F=-0.020 \mu^{2}+0.237 \mu+0.875$

$M . U=-0.017 \mu^{2}+0.205 \mu+1.098$

$V . U=-0.027 \mu^{2}+0.326 \mu+1.204$

For $\alpha=0.05$ :

$M . F=-0.014 \mu^{2}+0.144 \mu+0.899$

$V . F=-0.021 \mu^{2}+0.214 \mu+0.901$

$M . U=-0.018 \mu^{2}+0.180 \mu+1.124$ 
$V . U=-0.029 \mu^{2}+0.295 \mu+1.239$

For $\alpha=0.10$ :

$M . F=-0.011 \mu^{2}+0.092 \mu+0.958$

$V . F=-0.018 \mu^{2}+0.150 \mu+0.978$

$M . U=-0.014 \mu^{2}+0.114 \mu+1.198$

$V . U=-0.024 \mu^{2}+0.206 \mu+1.345$

The above equations can predict the magnification factor for the bending moment and shear force at the mid-span and quarter-span of the roofing system respectively in buildings with flexible diaphragm. For design purposes however, it would be appropriate to limit the variation of the equations as much as possible. Therefore, as stated earlier, conservatively the equations suggested for the elasto-plastic case may be used for design purposes. Among these equations, Equations 5.42 and 5.43 express the magnification factor for bending moment and shear force, respectively, with relation to those obtained when the total seismic load is applied according to the parabolic distribution suggested by FEMA. On the other hand, Equations 5.44 and 5.45 determine the aforementioned magnification factors in relation to the values obtained by applying the total seismic case in a uniform distribution. Either procedure can be used for determining the bending moment and shear force at mid-span and quarter-span, respectively. The parabolic distribution suggested by FEMA and the corresponding Equations 5.42 and 5.43 should be preferred since FEMA distribution is parabolic which at least partially accounts for the 
concentration of the seismic load towards the mid-span, the main reason for bending moment and shear force magnification in mid-span and quarter-span, respectively.

As pointed out earlier, in their research study, Humar and Popovski (2012) have shown that the FEMA parabolic distribution gives reasonable predictions for the elastic case $(\mu$ $=1.00)$. The results from the present study confirm such observation and shows that the nonlinearity of the system increases the contribution of higher modes and consequently the level of internal force magnifications. Equations 5.42 and 5.43 as which have been suggested here as appropriate for design are repeated in the following for ease of reference.

$$
\begin{aligned}
& M . F=-0.016 \mu^{2}+0.198 \mu+0.832 \\
& V . F=-0.021 \mu^{2}+0.264 \mu+0.842
\end{aligned}
$$

The magnification factors are calculated for different values of the ductility using Equations (5.42) and (5.43) and are presented in Table 5.1

Table $5.1-M . F$ and $V . F$ values for different ductility demands

\begin{tabular}{ccc}
\hline $\boldsymbol{\mu}$ & $\boldsymbol{M . F}$ & $\boldsymbol{V . F}$ \\
\hline 1.00 & 1.014 & 1.085 \\
\hline 2.00 & 1.164 & 1.286 \\
\hline 3.00 & 1.282 & 1.445 \\
\hline 4.00 & 1.368 & 1.562 \\
\hline
\end{tabular}

It can be observed that for $\mu=1.00$, the equations predict values that are fairly close to 1.00 which is consistent with the assumptions that were discussed earlier. For finalizing the design expressions it may prove beneficial to adjust the current equations to pass 
through the point $(\mu=1.00, M . F$ or $V . F=1.00)$. In other words, we want to refine the equations so that by setting $\mu=1.00$, we would have $M . F=1.00$ and $V . F=1.00$.

This can be done by using least squared errors. The value of $M . F$ for $\mu=1.00$ is 1.014 . Suppose we want to adjust the expression so that at $\mu=1.00, M . F=1.00$. The expression for any of the ratio values that would ensure that at $\mu=1.00$ the ratio parameter will take the value of 1 is $(\mu=1.00, M . F=1.00)$

$a \mu^{2}+b \mu+1-a-b$

The sum of squared errors between the values obtained from Equation 58 and those obtained from Equation 5.42 at $\mu=2,3$, and 4, is:

$$
\begin{aligned}
& E=(4 a+2 b+1-a-b-1.164)^{2}+(9 a+3 b+1-a-b-1.282)^{2} \\
& +(16 a+4 b+1-a-b-1.368)^{2}
\end{aligned}
$$

Now by setting $\frac{\partial E}{\partial a}=0$ and $\frac{\partial E}{\partial b}=0$ (least squared errors), we get the values a $=-0.0197$ and $b=0.2208$. The revised expression for magnification factor which will be used in the design, takes the form:

$$
M . F=-0.02 \mu^{2}+0.22 \mu+0.80
$$

Similarly, if we use the least squared errors method to force Equation 5.43 to give the value of one for $V . F(V . F=1.00)$ for $\mu=1.00$, the refined design equation would take the following form:

$V . F=-0.04 \mu^{2}+0.40 \mu+0.64$ 
In Figures 5.65 and 5.66 the original M.F equation and the original V.F equation are plotted together with the refined equations for M.F and $V . F$ that are recommended for design purposes.

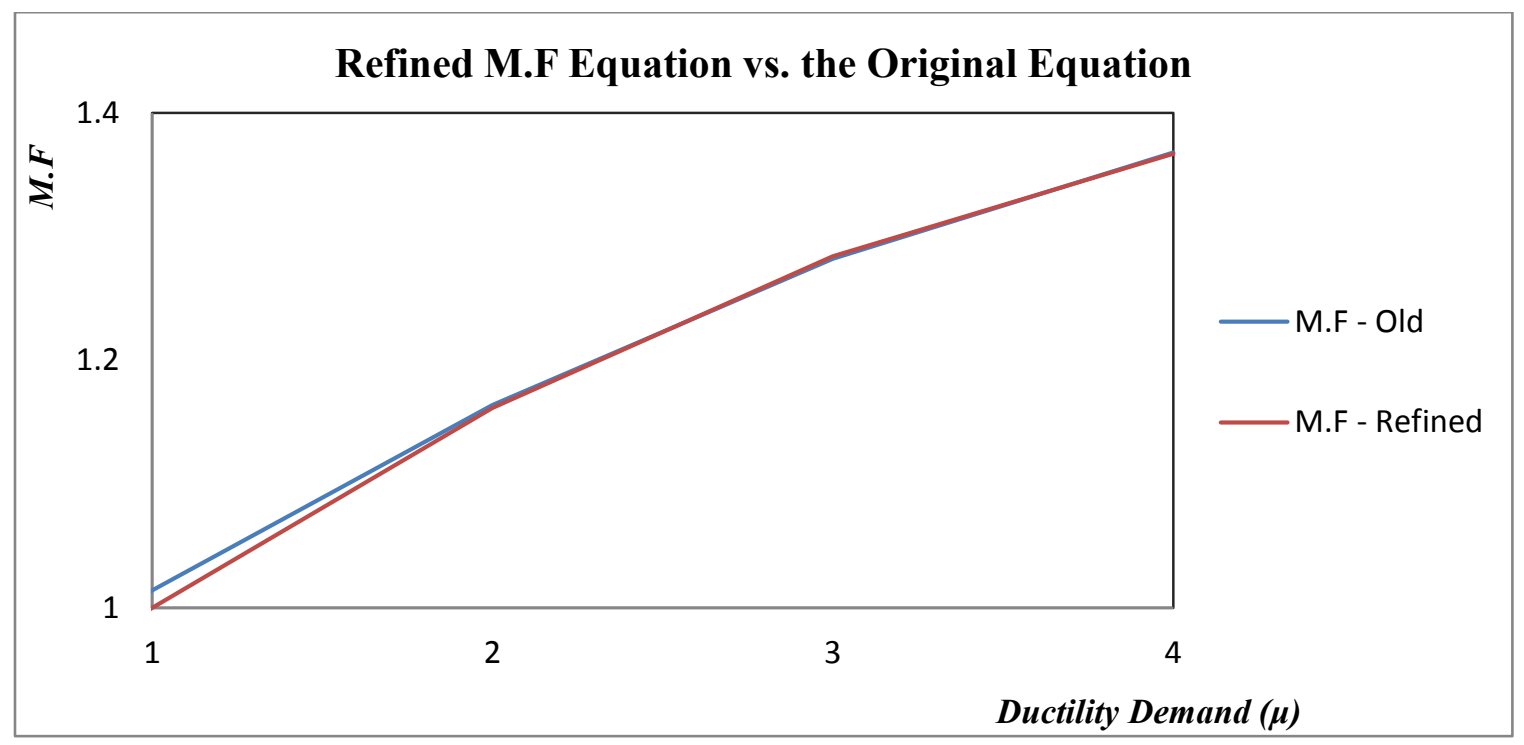

Figure 5.66 - The final design expression for M.F magnification factor vs. the original M.F equation

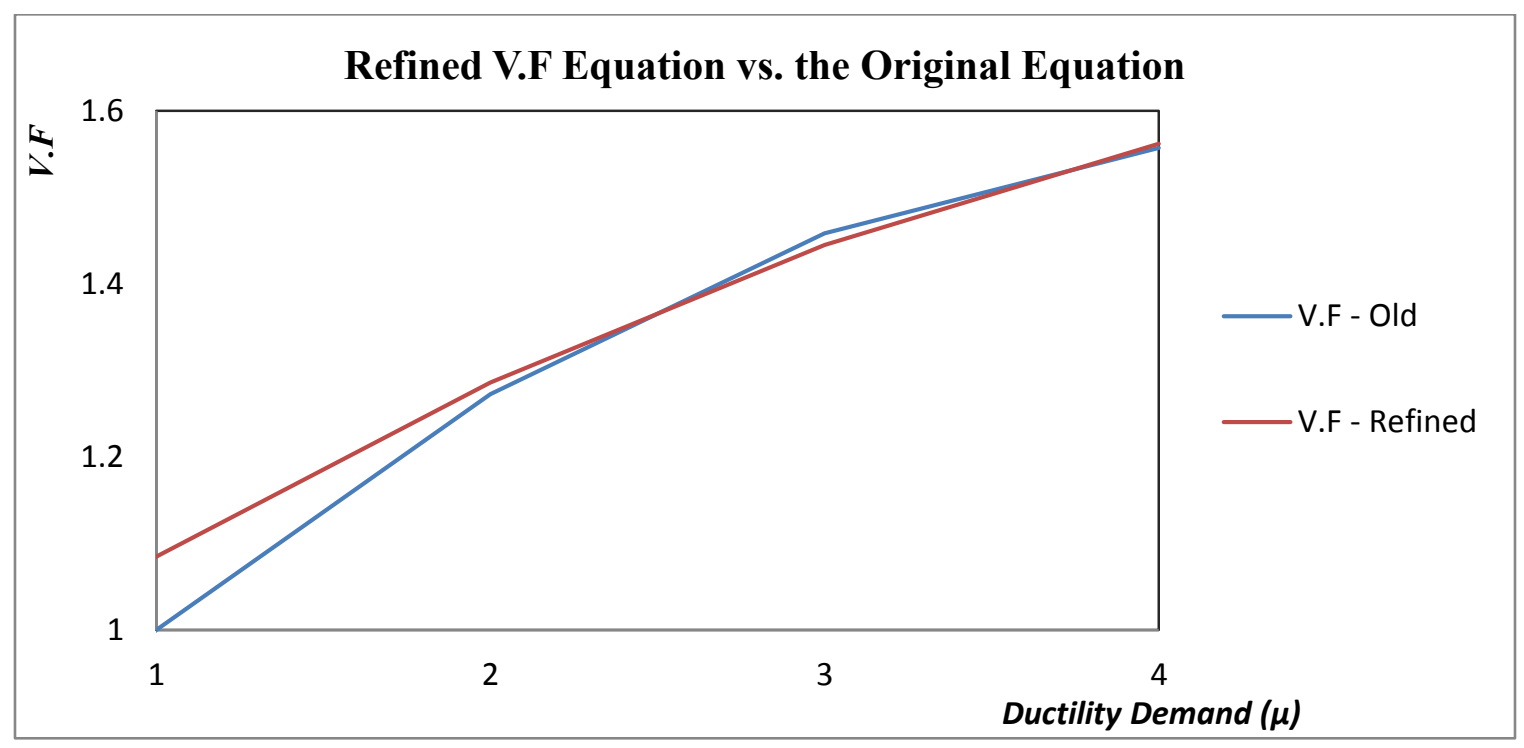

Figure 5.67 - The final design expression for V.F magnification factor vs. the original V.F equation 


\subsection{Conclusion and Recommendations}

The study presented in this chapter leads to the following conclusions

1. The flexibility of the diaphragm attracts greater contributions from the higher modes.

2. Greater contribution of higher modes is the main reason for the non-uniform distribution of seismic forces along the length of the diaphragm leading to a significant increase in bending moment at mid-span and shear force at the quarter span.

3. The contribution of higher modes and consequently the magnification of bending moments and shear forces is enlarged as the system is strained into the nonlinear range by using larger force reduction factors in design.

4. Magnification in the eastern region is slightly greater than that in the western region. This is expected, because the spectral shape for the east is steeper causing greater contribution from the higher modes. Greater contribution from higher modes causes inertia forces to shift toward the middle leading to greater bending moment near middle and the shear at quarter span. However, since the difference between the magnifications values for east and west is not large, the data for the two spectra are combined to give a single set of equations for both cases.

5. As the post yield stiffness or $\alpha$ increases the magnifications decrease. Again the differences are not large. Conservatively, and for simplicity the use of $\alpha=0$ is suggested for design purposes.

6. Quarter span shear magnification is much greater than mid-span moment magnification. Therefore, separate expressions are suggested, one set for 
predicting the magnification factor for bending moment and the other for the shear force.

7. The FEMA parabolic distribution of inertia forces provides good estimates of the bending moments when the LLRS is elastic. Nonlinearity in the LLRS further increases the mid-span bending moments and the in-span shears, but the parabolic distribution may be used as the base line pattern to which the magnifications proposed in this study may be applied.

8. While the FEMA parabolic load distribution provides bending moments and shear forces that are closer to the true values prior to the magnification of the results, if the simplicity of the uniform distribution makes it a useful alternative, the magnification factors proposed for that case may be applied.

9. Equations 5.6 to 5.9 provide magnification ratios that are most precise among the alternatives considered. However their lack of simplicity because of the number of parameters that must be considered makes other equations preferable.

10. Although Equations 5.6 to 5.9 may not be practical for design purposes, they provide a good understanding of the relation between the building characteristics and the level of magnification in the internal forces; shear forces and bending moments.

11. Based on Equations 5.6 to 5.9, it is observed that there is a direct relation between the force reduction factor (a measure of nonlinearity of the system) and the magnification factors. On the other hand, there is an inverse relation between the post-yield hardening effect and the magnification values. 
12. Since the effect of post-yield stiffness on the level of magnification is not significant, conservatively, the expressions obtained from considering elastoplastic behaviour for the LLRS, namely equations 5.42 to 5.45 can be used for design purposes as they give the highest magnification factors.

13. Since the parabolic distribution by FEMA is a better basis for design procedures, Equations 5.42 and 5.43 are further refined so that they give the magnification factor of 1.0 for the elastic case (when there is no nonlinearity in the system). Equations 5.60 and 5.61 are recommended as refined equations for a design which is based on the FEMA distribution of the seismic force.

\subsection{Design Procedure for Diaphragms in which the Diaphragm Remains Linearly Elastic while the LLRS May Yield}

In most of the design guidelines and provisions, the diaphragm is assumed to behave as a linear elastic component, while the LLRS may be strained into the inelastic range. It was not until recent years that some of the building codes allowed a design procedure for diaphragms in which the diaphragm could be permitted to yield when the building was subjected to earthquake loads. However, many engineers still assume a linear elastic behaviour for the diaphragm when designing buildings with flexible diaphragms. In addition, many design provisions still do not allow the diaphragms to behave nonlinearly. Therefore, it can be beneficial to develop design procedures for buildings with flexible diaphragms in which the diaphragm is expected to remain elastic during an earthquake. A simple design procedure is suggested here for such systems: 
1. Using the equations suggested by Humar and Popovski (2012), determine the fundamental period of the building when modelled as a beam/spring model. This involves the estimation of the frequencies of the components of the model. Thus

$$
\begin{aligned}
& \omega_{d}=\sqrt{\frac{E I}{m L^{4}}} \sqrt{\frac{1}{1+(E I / B G)(\pi / L)^{2}}} \\
& \omega_{b}=\sqrt{\frac{2 K_{b}}{m L}}
\end{aligned}
$$

in which $\omega_{d}$ is the frequency of the diaphragm (beam) on rigid supports and $\omega_{b}$ is the frequency of the LLRS (springs) supporting a perfectly rigid diaphragm. Since, in the beam-spring model, the diaphragm and the supporting LLRS act as two springs in series, the following equation based on the equivalent stiffness of two springs in series provides the frequency of the combined system (Humar and Popovski 2012).

$$
\omega=\frac{\omega_{b}+\omega_{d}}{\sqrt{\omega_{b}^{2}+\omega_{d}^{2}}}
$$

2. Determine the elastic base shear from the uniform hazard spectrum for the site $V_{e}=S(T) M$

where $S(\mathrm{~T})$ is the spectral acceleration for the period $T$ and $M$ is the total mass assigned to the level of the diaphragm. Equation 5.65 assumes that the entire mass of the diaphragm participates in the first mode. This is a conservative yet reasonable assumption. (Humar and Popovski 2012) 
3. Determine the drift ratio of the building using the following equations (Humar and Popovski 2012)

$u_{d}(L / 2)=\frac{5 w L^{4}}{384 E I}+\frac{w L^{2}}{8 B G^{\prime}}$

$u_{b}=\frac{w L}{2 K_{b}}$

$r=\frac{u_{d}(L / 2)}{u_{b}}$

in which $u_{d}$ is the diaphragm deformation at mid-span under a uniformly distributed lateral load $w$ per unit length, $u_{b}$ is the brace displacement and $r$ is the drift ratio of the system.

4. Using the following equations developed by Humar and Popovski (2013) and confirmed and extended in the current study, and the target ductility, calculate the force reduction factor for the design load.

$R_{y}=\kappa \mu$

$\kappa=-0.5 r+1.5$

The $\kappa$ value to be used in Equation 5.69 need not be less than the appropriate lower limit as recommended in Chapter 4 of the current study. Divide the elastic shear determined in Step 2 by the $R_{y}$ factor to obtain the design shear.

5. Distribute the seismic load determined in step 4, multiplied by the diaphragm design force factor $\Phi_{e}$ defined in Chapter 7, along the length of the diaphragm 
using parabolic distribution pattern suggested by FEMA 356. As an alternative, a uniform distribution of the seismic load can be used. Determine the mid-span bending moment and the quarter-span shear from the selected distribution of internal forces.

6. Based on the distribution pattern used in the previous step, select appropriate relationships from Equations 5.42 to 5.57 and Equations 5.60 and 5.61 to obtain the magnification factors to be applied to mid-span bending moment and quarterspan shear determined from either a uniform distribution of seismic loading or a parabolic distribution as suggested by FEMA 356. The use of Equations 5.60 and 5.61 and FEMA distribution pattern as the basis of the design is recommended.

7. By applying appropriate magnification factors to the moment and shear determined in Step 5, determine the critical bending moment at mid-span and the critical shear force at quarter-span and design the diaphragm for these actions. 


\section{Chapter 6 : Effect of Hysteretic Behaviour on the Seismic Response of Single-Storey Buildings}

\subsection{Introduction}

In the previous chapters, the influence of diaphragm flexibility on the seismic response of single-storey buildings with flexible diaphragms was investigated. The buildings studied had an un-topped steel deck diaphragm as the roofing system and concentric braced frames with HSS braces as the lateral load resisting system. The study expanded and improved on previously suggested methods for finding an appropriate force reduction factor for the seismic design of the lateral load resisting system in buildings with flexible diaphragms. In addition, the magnification of bending moment and shear force acting along the length of the diaphragm was studied and a set of equations were proposed for predicting these magnifications.

In the studies carried out so far, a bilinear force-displacement relationship was assumed for the lateral load resisting elements while a linear elastic behaviour was assumed for the diaphragm. Previous studies, such as the experimental research by Tremblay et al. (2003) and experimental data presented by Archambault et al. (1995), suggest that concentrically braced frames often exhibit pinched hysteretic behaviour when subjected to cyclic lateral loads. The validity and applicability of the results presented in the previous chapters must therefore be tested for buildings with concentric regular braces in which the lateral load resisting elements show pinching behaviour. 


\subsection{Chapter Outline}

In order to examine the validity of the results presented earlier, a selected set of buildings are analyzed for their response to several different ground motions. In the analyses, a Clough hysteretic model with and without pinching is considered for the lateral load resisting system. The results are then compared with those obtained from the previous analyses which were carried out on the same set of buildings but considering a bilinear behaviour for the lateral load resisting system. The comparisons are carried out for both the ductility demand values and the magnification in the shear force at quarter span and bending moment at mid span.

The selected buildings as well as the ground motions used in the study are first described. The assumptions made in the analyses are then outlined. Also, the analytical model to represent the hysteretic behaviour is explained. The applicability of the equations suggested in the previous chapters to buildings in which the lateral load resisting elements exhibit a hysteretic behaviour similar to Clough model with or without pinching behaviour is examined by comparing the two sets of response results. At the end, the conclusions and recommendations reached in this part of the study are presented.

\subsection{Selected Buildings}

A set of buildings is selected from the original building database which consists of 36 one-storey buildings designed by Tremblay and Steimer (1996). In order to include a wide range of drift ratios in the study, the buildings are analyzed in both orthogonal directions, that is, when the seismic load acts parallel to the short side of the building and when it acts parallel to the long side of the building. One building is selected from each 
building size (small, medium and large), so that the conclusions are valid for all building sizes. Buildings with heavy roofing systems are selected for the study to make the results more conservative. Such selection is based on the fact that as the seismic weight of the system, which in this case is proportional to the weight of the roofing system, increases the response to seismic ground motions becomes more critical.

Based on the assumptions outlined above, buildings SH1, MH1 and LH1 are selected for these sets of analyses. As mentioned earlier, the selected buildings are analyzed in both directions, making the total of selected buildings as six.

\subsection{Ground Motion Records}

Six of the ten ground motions generated by Atkinson (2009) that were introduced in previous chapters are used for this portion of the study. Three of the selected ground motions are compatible with the eastern (Montreal) uniform hazard spectrum while the rest are compatible with the western (Vancouver) spectrum. By doing so, the results

obtained become applicable to different spectral shapes and not just to one. Records M6C1, M6C2 and M6C26 with scaling factors of 0.78, 0.87 and 1.19, respectively, are selected to provide a match for the Vancouver UHS while records E6C1, E6C13 and E6C15 with scaling factors $0.55,0.74$ and 0.56 respectively, are used to match the UHS for Montreal. 


\subsection{Assumptions and Analytical Model}

\subsubsection{Selected Software}

In order to consider a Clough hysteretic behaviour, with or without pinching, for the lateral load resisting elements in the system, the structural system is modelled in OpenSees platform. The open system for earthquake engineering simulation (OpenSees) is an open source software developed at the Pacific Earthquake Engineering Research Center to assist researchers in studying performance-based design in earthquake engineering. OpenSees allows the modelling of different hysteretic behaviours including pinching behaviour. Also, using OpenSees makes it fairly simple and straightforward to apply earthquake records to the system and to post process the data.

\subsubsection{General Assumptions}

Most of the assumptions in modelling for this section are consistent with those outlined in Chapter 2. Some of the main assumptions are presented herein.

The buildings are modelled as deep beams, representing the diaphragm, supported by two springs, representing the lateral load resisting elements. It is assumed that the diaphragm's deformations are within the linear elastic range. Thus, an elastic forcedisplacement relationship is considered for the diaphragm. The deep beam (diaphragm) is divided into 20 elements interconnected at 21 nodes. Rayleigh damping of 5\% is assumed for the first and the third modes of vibration. For the dynamic analyses, a time step of $0.001 \mathrm{~s}$ is used. A more detailed discussion of the assumptions can be found in Chapter 2 . 
The assumption made in this part of the study that is different from the ones made in earlier parts is related to the behaviour of the lateral load resisting system under cyclic loading where a Clough model with and without pinching behaviour is considered. Details of the chosen pinching behaviour as well as the reasons for such choice are presented in the following section.

\subsection{The OpenSees Model}

\subsubsection{Nodes}

As pointed out earlier, the beam consists of 20 elements. Thus, 21 nodes are defined along the length of the diaphragm. Two additional nodes are located at the ends of the beam to allow modelling of zero-length elements representing the spring supports (braces) at each end. Figure 6.1 shows a schematic illustration of the model used in OpenSees. Note that for simplicity, only 7 nodes rather than 21 nodes are shown.

\subsubsection{Materials}

To account for shear deformations in the deep beam, an elastic uniaxialMaterial is defined in OpenSees to present the force-displacement relationship associated with shear

deformations. Similarly another elastic uniaxialMaterial is defined for presenting the moment-curvature relationship arising from flexural deformations. The stiffness corresponding to these material behaviours are based on the properties of the selected building. Next, using the section aggregator the two material behaviours are combined to be implemented in a section used for the interconnected beam elements representing the diaphragm. 
Hysteretic uniaxialMaterial is used to model the Clough hysteretic behaviour with and without pinching in the springs. For the selected material, the selection of the yield force is based on the appropriate force reduction factor which was determined previously. A value of 0.8 is used for the pinching factor for strain, defined as \$pinchX. The pinch factor for stress, defined as \$pinchY, is taken as 0.25 for high slenderness braces and 0.4 for intermediate slenderness. The reasons for such selection are explained in detail in the section titled "Modelling Pinching Behaviour in OpenSees"

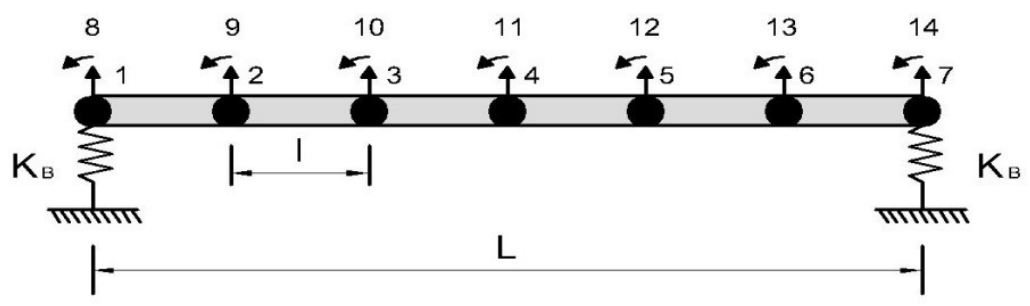

Figure 6.1 - Schematic illustration of the analytical model with 7 nodes

\subsubsection{Elements}

A nonlinearBeamColumn element with 5 integration points is used to represent the beam elements. Such selection will help to account for diaphragm nonlinearity, discussed in the next chapter, by just changing the material behaviour. A section aggregator is used to account for both shear and flexural deformations. 
A zeroLength element is used for the springs representing the LLRS. The previously defined hysteretic material which models the Clough hysteretic behaviour with or without pinching is assigned to the springs as their axial force-displacement relationship to account for the behaviour that the LLRS exhibits under cyclic lateral loads.

\subsubsection{Ground Motion}

Using a UniformExcitation pattern command, the ground motion time histories are applied to the model in the direction parallel to the axis of the defined springs.

\subsubsection{Dynamic Analysis}

A ConstraintHandler object is used in OpenSees to determine how the constraint equations are enforced; a plain constraint is used in the present study. RCM numberer is used as the DOF_Numberer object. UmfPack is selected as the solver. The convergence test comprises a tolerance of $1.0 \mathrm{e}-10$ and a maximum of 10 iterations. The Newton algorithm is used as the solution algorithm, which determines the required steps for solving nonlinear equations. To perform integration of the equations of motion, Newmark integrator is employed with \$gamma and \$beta factors equal to 0.5 and 0.25 , respectively. After setting the aforementioned parameters, a transient analysis command is used to perform dynamic analysis. 


\subsection{Modelling of Pinching Behaviour in OpenSees}

\subsubsection{Pinching Behaviour in HSS Concentric Braces}

In order to implement a reasonable and realistic pinching behaviour in the OpenSees model, one must first understand the hysteretic behaviour in concentric braces made of hollow structural sections. In their research study, Tremblay et al. (2003) examined the hysteretic behaviour of concentric hollow braces through a set of experimental tests. Figure 6.2 taken from the research paper by Tremblay et al. shows the hysteretic response of concentric hollow braces subjected to lateral loads.
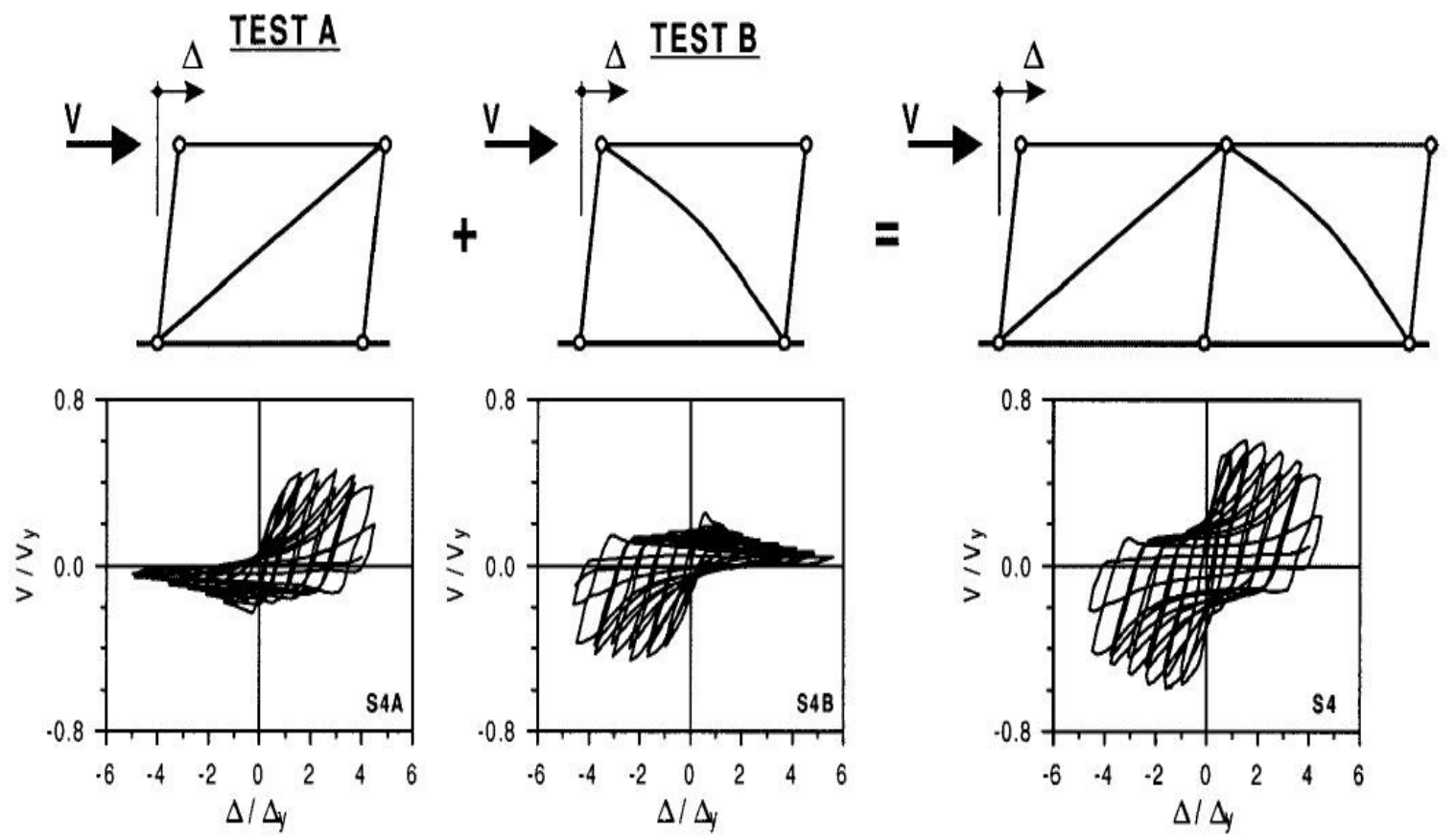

Figure 6.2 - The hysteretic response of concentric braces subjected to lateral loads, from Tremblay et al. (2003) with the authors' permission and, with permission from ASCE 
By observing the hysteretic response of a single brace it can be seen that the tensile capacity of the brace is significantly larger than its compressive capacity. This difference in capacity results in a pinched behaviour along the vertical (Force) axis in the response of the frame with two braces. Evidently, the severity of this effect is dependent on the slenderness of the member. The reason is that the tensile capacity is governed by the yield force while the compressive capacity is dictated by the buckling load which the member can carry, which is in turn based on the slenderness of the member.

Figure 6.2 also shows pinched behaviour along the horizontal axis (deformation). At each cycle in which the brace undergoes nonlinear deformation under tension the length of the brace increases, and therefore in the next loading cycle, the brace must deform to its new length before it provides tensile resistance. Thus, in each cycle of loading, the brace stiffens only after it has undergone significant deformation. The stiffening of the brace does not happen precisely when a deformation equal to the previous cycle is reached, but is rather a gradual process. Thus, at a certain fraction of the previous deformation the brace starts to stiffen showing tensile resistance.

\subsubsection{Implementing the Behaviour in OpenSees Platform}

In order to model the pinching behaviour in concentric hollow braces in one-storey buildings as explained above, the "Hysteretic uniaxialMaterial" material in OpenSees, developed by Michael Scott (Oregon State University) \& Filip Filippou (UC Berkeley), is

used. This material behaviour is based on the Clough hysteretic model with the option of inputting pinch factors for the vertical axis, known as force (PinchY), as well as the 
horizontal axis, known as displacement (PinchX). Obviously, such pinch factor values are less than one.

In Clough hysteretic behaviour without pinching, the unloading branch has the same slope as the initial loading branch until the deformation axis is reached. It is at this point, that the slope changes and the unloading direction aims for the point at which the previous maximum force was reached. Figure 6.3 shows the Clough hysteretic behaviour for a single element under displacement controlled analysis.

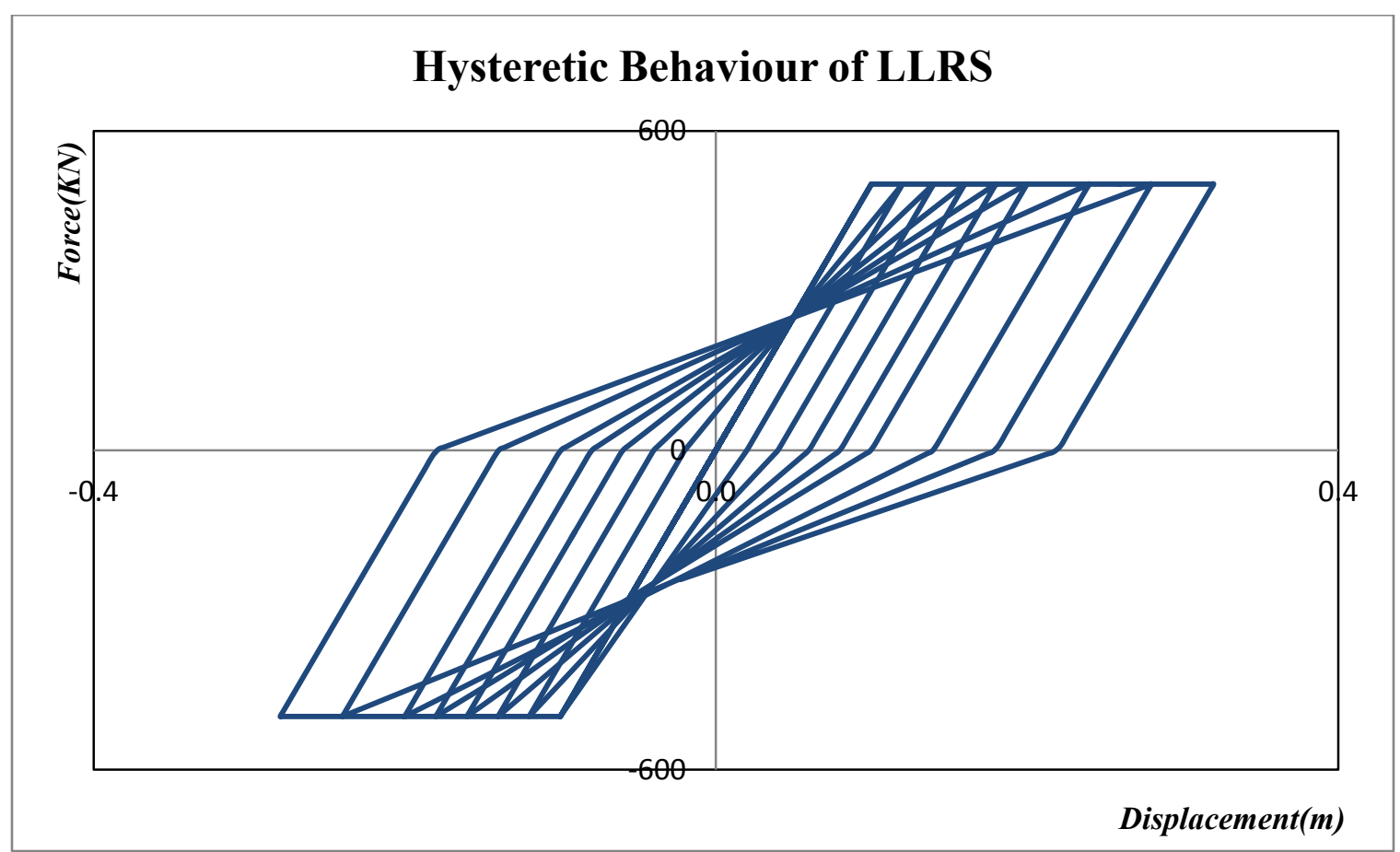

Figure 6.3 - The hysteretic response of a single element modelled in OpenSees with Clough model

After pinch factors for the vertical axis and the horizontal axis are introduced in OpenSees, unloading line does not follow the same path as in the Clough model. Instead of aiming for the previous maximum it aims for a point with the following coordinates: 
$\mathrm{X}($ deformation $)=$ Deformation associated with previous maximum force $\times$ Pinch $X$

$\mathrm{Y}($ Force $)=$ Previous maximum force $\times$ PinchY

Figure 6.4 illustrates the hysteretic behaviour of an element modelled in OpenSees with values of 0.8 and 0.2 for PinchX and PinchY, respectively.

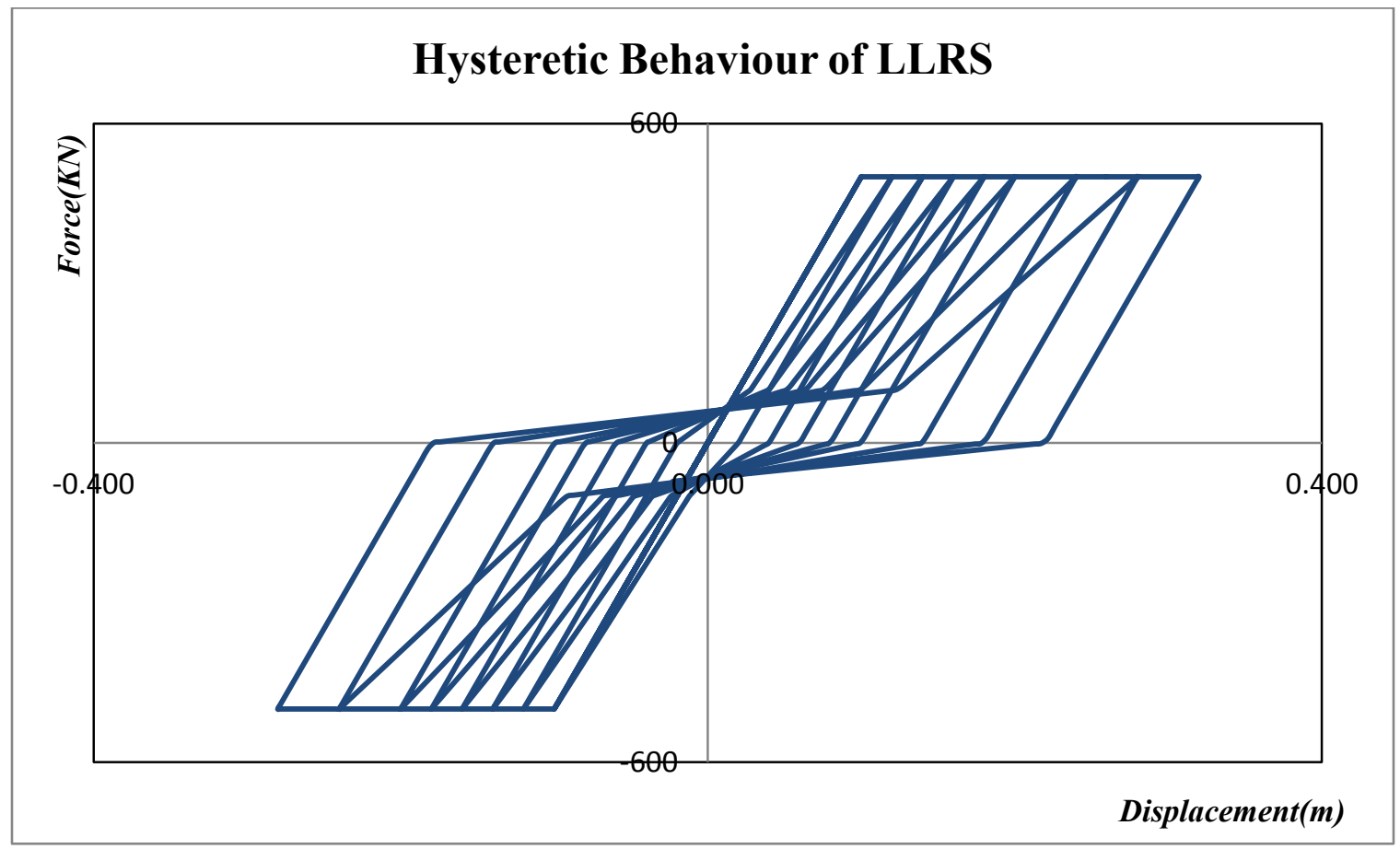

Figure 6.4 - Hysteretic response of an element with Clough hysteretic behaviour and pinching factors PinchX and PinchY equal to 0.8 and 0.2 , respectively

In Figure 6.5 the response based on Clough model with no pinching as well as the one based on Clough model with pinching are plotted on the same graph. The dimensions needed for defining the pinch factors in OpenSees are also shown on the figure:

$$
\operatorname{Pinch} X=\frac{x}{X}
$$


$\operatorname{Pinch} Y=\frac{y}{Y}$

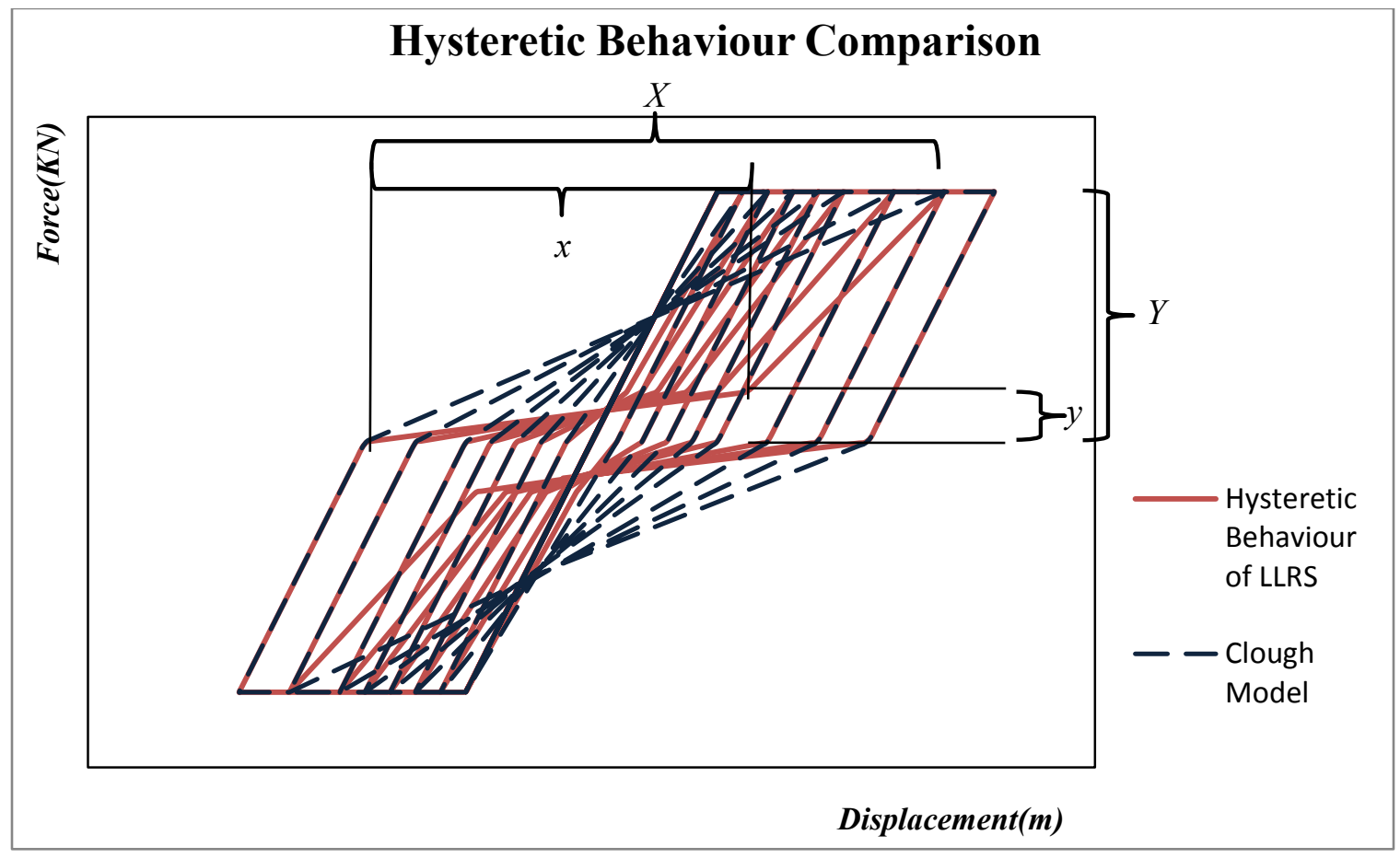

Figure 6.5 - Comparison between Clough model with pinching and Clough model without pinching

Based on the definition of PinchX and PinchY values in OpenSees, as indicated in Figure 6.5 , and by considering the hysteretic behaviour of concentric braces explained in Section 6.7.1, appropriate pinching factors are selected.

\section{PinchY Factor}

The PinchY factor which is imposed on the vertical axis (Force) is dependent on the slenderness of the brace element. Figure 6.2 shows that just before the brace stiffens in tension the force in the hysteretic behaviour for the frame with two braces is equal to the 
compressive capacity. When the frame reaches its ultimate capacity the force in the frame is equal to the sum of the tensile capacity and the compressive capacity. In the material model used in OpenSees, the unloading line changes slope when it meets the deformation axis. In Figure 6.2 however, it can be seen that the slope changes at a different point and below the deformation axis when unloading from tension and above the deformation axis when unloading from compression. This is on account of the fact that when the direction of the force is changing, the same brace that was in tension would now be strained in compression and the slope of the line would not change until the brace in compression has buckled. After buckling the frame would deform laterally showing no resistance until the other brace has attained enough tensile deformation to stiffen. This difference between the model in OpenSees and the actual behaviour is small and can be ignored. However, to account for this difference, the ultimate capacity is set equal to the sum of the tensile capacity and the compressive capacity and the force at the stiffening point is taken as twice the compressive capacity. By doing so, the area under the forcedeformation hysteretic curve would be roughly the same and in a sense we have shifted the hysteretic curve.

Figure 6.6 shows a schematic sketch of a part of the hysteretic behaviour before the stiffening of the tensile brace. The blue line shows the actual force deformation relationship while the dashed red line shows how this relationship is modelled. The area between the blue line and the $\mathrm{X}$ axis is seen to be approximately equal to the area between the dashed red line and the $\mathrm{X}$ axis. 


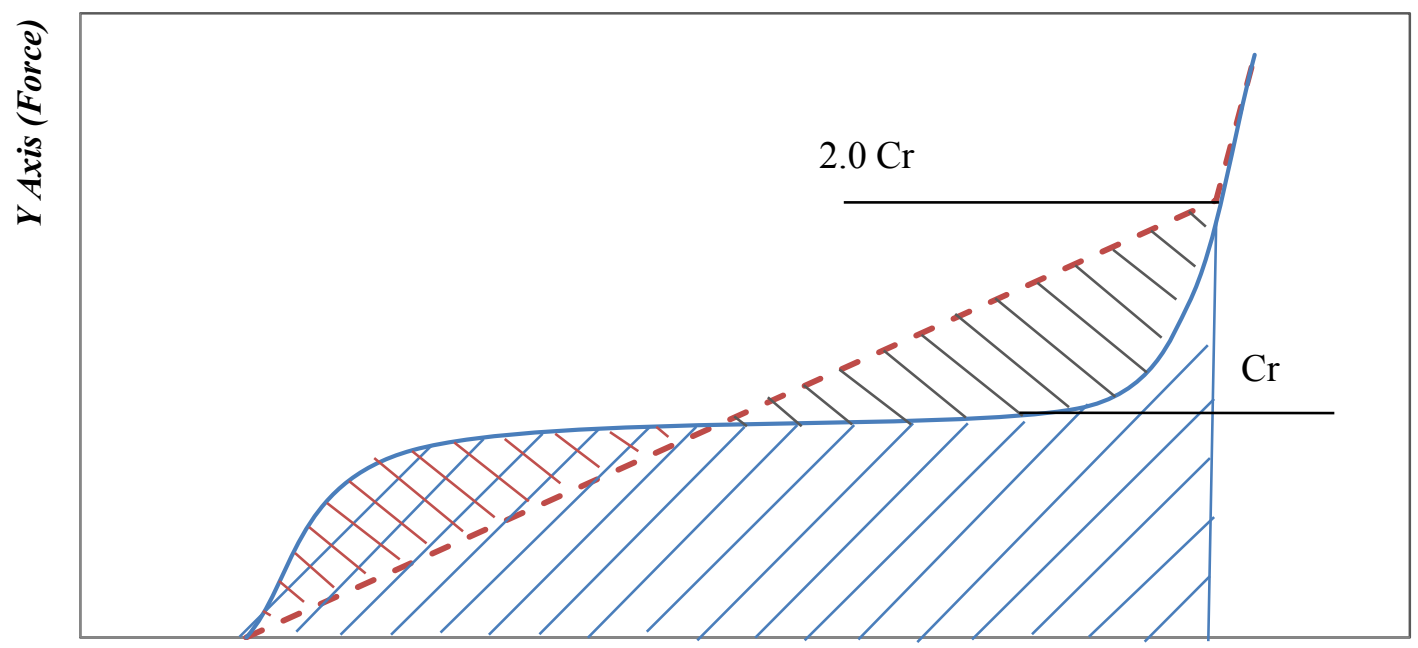

$X$ Axis (Deformation)

Figure 6.6 - Schematic illustration of the actual behaviour and the behaviour in the model before stiffening of the tensile brace

After establishing the basis for determining a proper PinchY factor as above, the PinchY factor in OpenSees is determined as follows:

Pinch $Y=\frac{2 C_{r}}{C_{r}+T_{r}}$

where $C_{r}$ is the bucking capacity of the brace and $T_{r}$ its tensile capacity.

According to the Canadian code for the design of steel structures (CSA S16-09) the buckling capacity of a brace is given by:

$C_{r}=\Phi A F_{y}\left(1+\lambda^{2 n}\right)^{-\frac{1}{n}}$

where

$\Phi=$ resistance factor 
$A=$ cross sectional area

$F_{y}=$ yield strength

$n=$ a parameter selected such that Eq. 6.4 matches the observed behaviour, 1.34 for hotrolled, fabricated structural sections and hollow structural sections

and $\lambda$ is given by

$\lambda=\sqrt{\frac{F_{y}}{F_{e}}}$

where

$$
F_{e}=\frac{\pi^{2} E}{\left(\frac{K L}{r}\right)^{2}}
$$

$E$ is the modulus of elasticity, $K$ is the effective length factor, and $r$ is the radius of gyration

Assuming $T_{r}=\Phi A F y$, Equation 6.4 takes the form:

$$
C_{r}=T_{r}\left(1+\lambda^{2 n}\right)^{-\frac{1}{n}}
$$

Substituting Equations 6.5 and 6.6 into Equation 6.7, and taking $E=200,000 \mathrm{MPa}, \mathrm{n}=$ 1.34 for HSS section and assuming that steel is of grade $350 \mathrm{~W}$, for which $F_{y}=350$, we get: 


$$
C_{r}=T_{r} \frac{5639.44}{\left[\left(\frac{K L}{r}\right)^{2.68}+106330.1\right]^{0.7462}}
$$

It can be seen that the compressive resistance is equal to the tensile resistance multiplied by a factor. This factor is clearly less than one and is dependent on the slenderness of the element. Knowing the relation between the compressive resistance and the tensile resistance, the PinchY factor can be determined in our model for different levels of slenderness.

In order to include the effect of slenderness of the braces in the current study, the PinchY factor is determined for two different ranges of slenderness; element with high slenderness $(K L / r=155$ to 200$)$ and intermediate slenderness $(K L / r=80$ to135) as follows:

Pinch $Y=0.25$ (For High Slenderness)

Pinch $Y=0.4$ (For Intermediate Slenderness)

\section{PinchX Factor}

The PinchX factor is imposed on the horizontal axis (Deformation) and is mostly dependent on the geometry. The reason for this behaviour can be traced to the fact that in any particular cycle in which the brace in tension is deforming in the nonlinear range it is getting longer. Therefore, in the cycle that follows, when the same brace undergoes tension, certain deformation must be reached before it starts to show tensile resistance. In other words, the brace in tension does not show any resistance until it has reached a certain deformation related to the maximum deformation reached in the earlier cycles. When that deformation is reached the tensile brace starts showing tensile resistance and 
this mechanism manifests itself as stiffening in the hysteretic curve. Evidently, the change from zero stiffness to full stiffness is not sudden but is rather a gradual process. Such behaviour imposes a pinching behaviour along the deformation axis (horizontal) and can be modelled using the PinchX factor in OpenSees.

In order to select a proper PinchX factor, the point at which the stiffening of the tensile brace occurs must be determined. PinchX factor is, in fact, the ratio of the displacement in the new cycle at which the tensile brace stiffens to the maximum displacement in the previous cycles. Previous experimental work reported in the literature has been studied to determine a reasonable value for PinchX. Archambault et al. (1995) carried out an experimental research to assess the hysteretic behaviour of concentric braces. The results of their study shows that the pinching factor for the deformation axis varies from 0.6 for random displacement time histories to 0.8 for displacement time histories with increasing amplitudes in cycles. Conservatively, a PinchX factor of 0.8 is chosen for the current study.

\subsubsection{Final Pinching Model in OpenSees}

Based on the foregoing discussion, in addition to the Clough hysteretic model, two pinching force-displacement hysteretic curves are chosen for the current study, one representing the behaviour of elements with high slenderness and the other modelling the hysteretic response of elements with intermediate slenderness. The PinchX factor and PinchY factor of 0.8 and 0.25 , respectively, are selected for elements with high slenderness while for elements with intermediate slenderness the PinchY factor is increased to 0.4 , PinchX factor remaining the same. 
To illustrate the final hysteretic models selected, the response of a single element modelled in OpenSees and subjected to a displacement controlled analysis is shown in Figures 6.7 and 6.8:

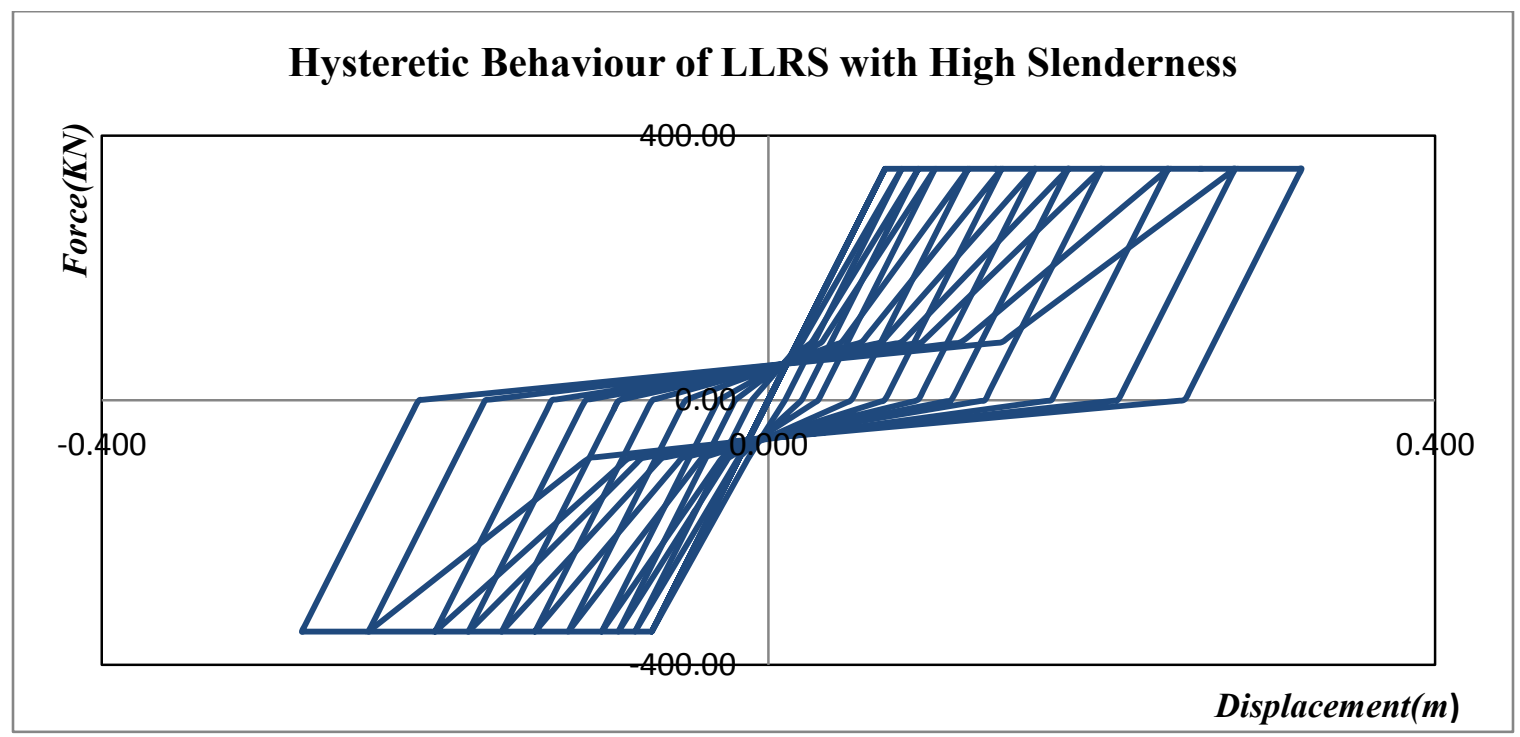

Figure 6.7 - Pinching hysteretic behaviour of LLRS with high slenderness

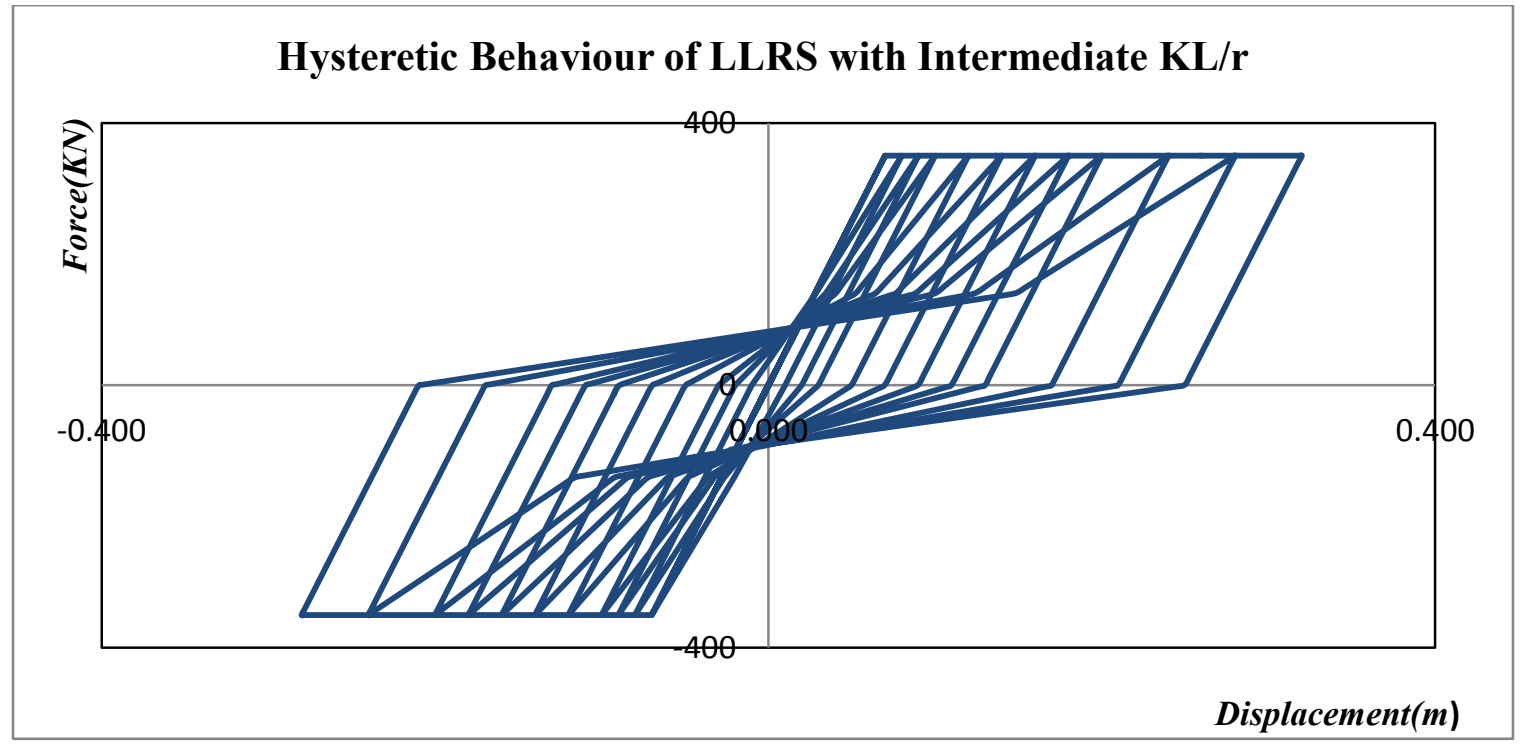

Figure 6.8 - Pinching hysteretic behaviour of LLRS with intermediate slenderness 


\subsection{Dynamic Analyses}

As stated earlier, the purpose of this part of the study is to compare the response of single storey buildings exhibiting Clough hysteretic behaviour with and without pinching in the model representing the LLRS, to those obtained in previous chapters, where a bilinear force displacement relationship was assumed for the LLRS. In the study presented in earlier chapters, three different values of the ductility demand, namely 2,3 , and 4 , were used as the target. A ductility target of 3 is used in this part of the study and, for compatibility, force reduction factors similar to those which led to the ductility demand of 3 in the previous sections are used again to determine the strength of LLRS with pinching behaviour.

After modelling the selected one-storey buildings using the assumptions outlined in the previous paragraphs, the buildings are subjected to the selected ground motions, once in a direction parallel to the short side of the system and a second time parallel to the long side. Rayleigh damping of 5\% is assumed for the first and the third modes and direct time step integration of the equations of motions is carried out. The analyses are first carried out considering elasto-plastic behaviour for the LLRS using a force reduction factor that leads to a ductility demand of 3 . Next, using the same force reduction factor, the analyses are repeated, once using a Clough model without pinching for the LLRS and next using a Clough model with pinching behaviour. When considering pinching, two separate sets of analyses are carried out; one in which the braces are assumed to have a high slenderness

and a second in which the braces have an intermediate slenderness. One would expect to observe an increase in the ductility demand compared to that in the elasto-plastic case when the Clough model and pinching behaviours are assigned to the LLRS. 


\subsection{Results of the Dynamic Analyses}

The response parameters studied include the maximum ductility demand on the LLRS, the magnification factors for the bending moment at the mid-span and the shear force at the quarter span of the diaphragm. The magnification factors are calculated with respect to both FEMA parabolic distribution and a uniform distribution of inertia forces. The notations used to represent the magnification factors are identical to those defined in previous chapters.

The complete set of the results of the analyses is presented in Appendix D. The results show that the magnifications in bending moment and shear force are slightly greater in the eastern region than in the western region. This is expected, because the spectral shape for the east is steeper causing greater contribution from the higher modes.

\subsection{Analysis of the Results}

The ductility demands as well as the force magnification factors for different hysteretic models are mapped with respect to the drift ratio to study the effect of using Clough model, pinching hysteretic model defined for intermediate slenderness, and pinching hysteretic behaviour in elements with high slenderness. 


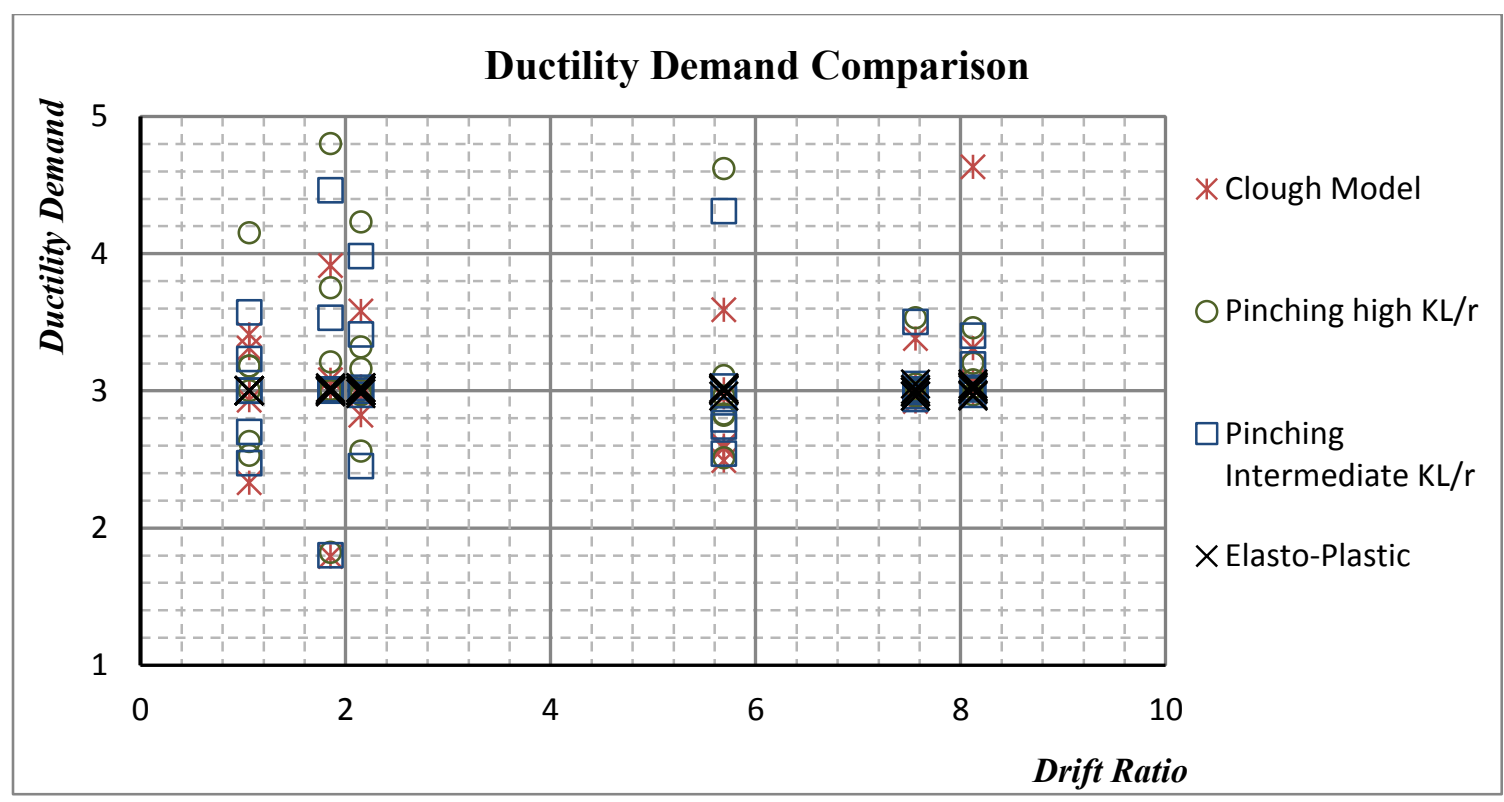

Figure 6.9 - Comparison between the maximum ductility demands on the LLRS considering different material behaviour

As would be expected, the ductility demand values for the elasto-plastic case are all equal to three. It can be seen that the ductility demand in other cases has increased in a majority of cases. Thus while the elasto-plastic behaviour provides a conservative estimate when it comes to force, for deformation and quantities associated with deformation, such as ductility, it may not be conservative. However, in some cases the Clough model as well as pinching cases cause a decrease in the ductility demand.

Whether the use of Clough model with or without pinching would cause an increase or a decrease in the ductility demand and force, depends mainly on the characteristics of the excitation. In order to understand this phenomenon, one must consider the hysteretic behaviours under random excitations and not a cyclic one. In the Clough model, with or without pinching, the force-displacement relationship prior to the yield point is the same 
as that in the elasto-plastic model. However, upon unloading, the Clough models with or without pinching follows a pattern quite different than that in the elasto-plastic model, showing greater deformation in reaching the same load. This alternative unloading pattern gives the element with the Clough model force-displacement relationship a better chance to deform towards the original length. Consequently, in the cycle following the first loading and first unloading the member with an elasto-plastic would have a much greater total deformation compared to that with the Clough model, since the former did not have the chance to deform back towards the original length as effectively. Such mechanism manifests itself under certain excitations. Under different excitations, the Clough model with or without pinching can cause an increase in the ductility demand. Thus, whether the Clough model with or without pinching would increase the ductility demand or decrease it, depends on the characteristics of the excitation and the structural system. In some cases, the ductility demand increases while in others it becomes smaller.

The results suggest that the ductility demand of 3 is roughly the mean value in the scatter distribution of the data points corresponding to the Clough model and pinching behaviour. The mean values of the ductility demands in the LLRS when its behaviour is represented by the Clough model without and with pinching behaviour are found to be $3.05,3.15$ and 3.2 respectively. The higher value corresponds to LLRS with braces of higher slenderness. Given the fact that most of the assumptions regarding the pinching model in this study are conservative, one can reason that the equations for predicting the force reduction factor in one-storey buildings with flexible diaphragms that were derived by Humar and Popovski (2013) and were improved in the current study can also be applied to buildings in which the LLRS exhibits pinching behaviour. 
It is important to point out that even when the Clough model is used without any pinching behavior, the value of the ductility demand fluctuates around 3, in some cases being greater than 3 and in others smaller, with the mean value of 3.05 .

Figures 6.10 and 6.11 show the scatter diagrams for moment magnifications. The first one is with respect to the parabolic distribution of the seismic forces and the second with respect to a uniform distribution. Figures 6.12 and 6.13 show the corresponding diagrams for the magnifications of the shear force. It can be observed that in all cases the increase in bending moment and shear force at mid span and quarter span, respectively, is more severe when the LLRS has an elasto-plastic force-displacement behaviour. Thus elastoplastic behaviour provides conservative estimates for internal forces and the quantities associated with such forces. Hence, the previously proposed equations for force magnification can be used for predicting the bending moment increase at mid span and the shear force magnification at quarter span in buildings even when the LLRS exhibits a pinched hysteretic behaviour or has a force-displacement relationship similar to the Clough model. 


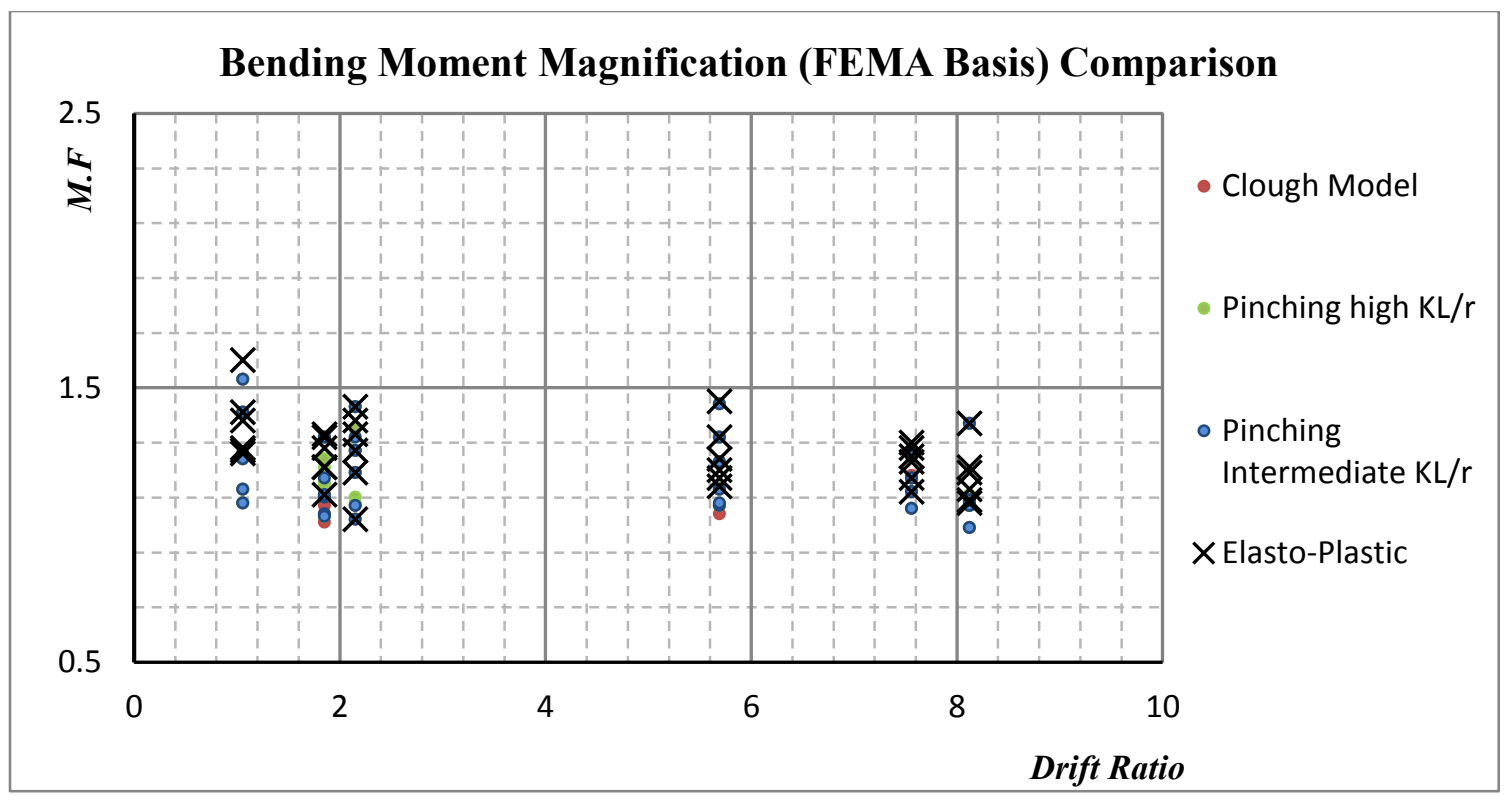

Figure 6.10 - Comparison between mid-span bending moment magnification ratios with respect to FEMA parabolic distribution of internal forces for different models to represent the hysteretic behaviour of the LLRS

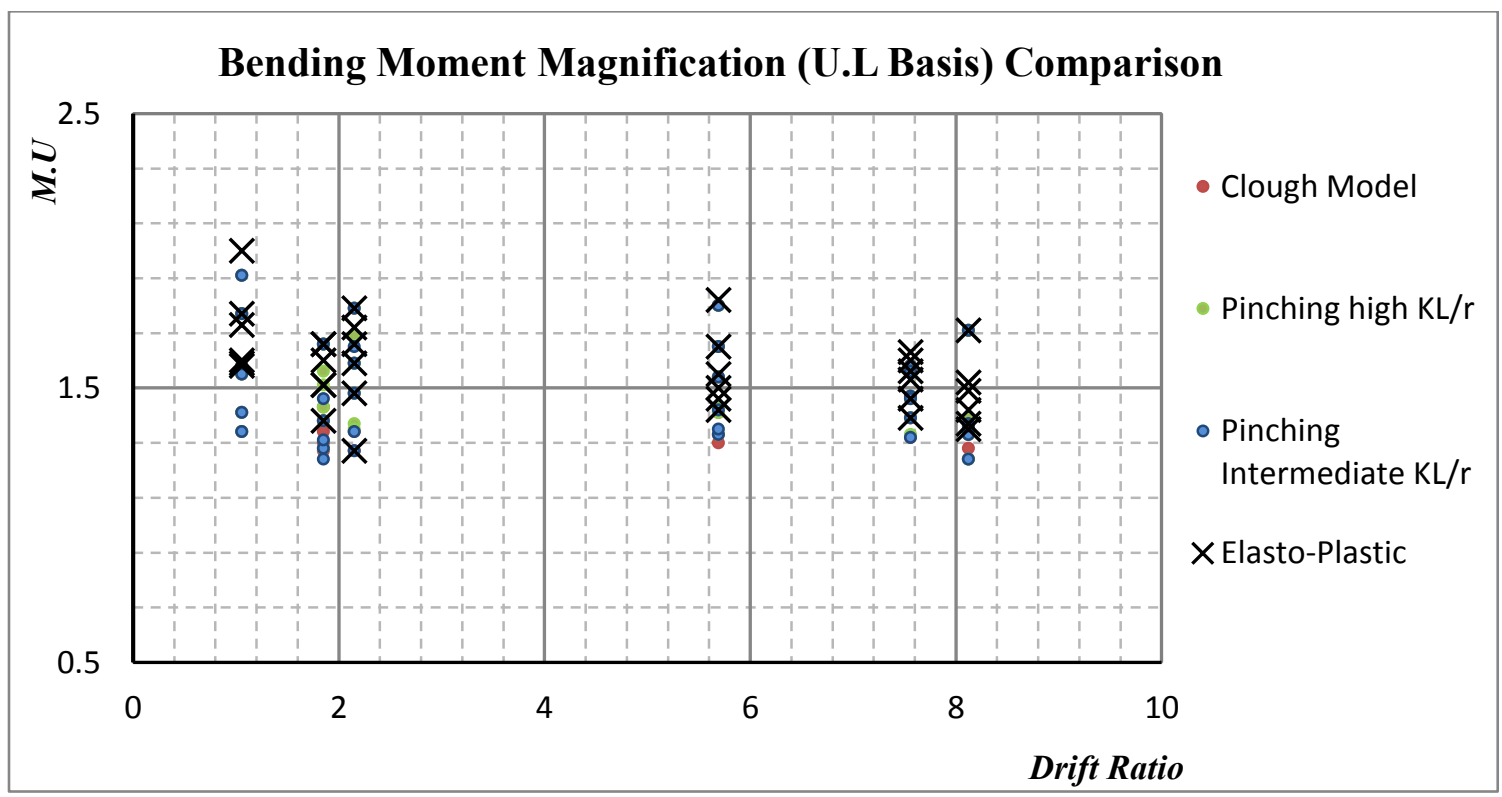

Figure 6.11 - Comparison between mid-span bending moment magnification ratios with respect to uniform distribution of internal forces for different models to represent the hysteretic behaviour of the LLRS 


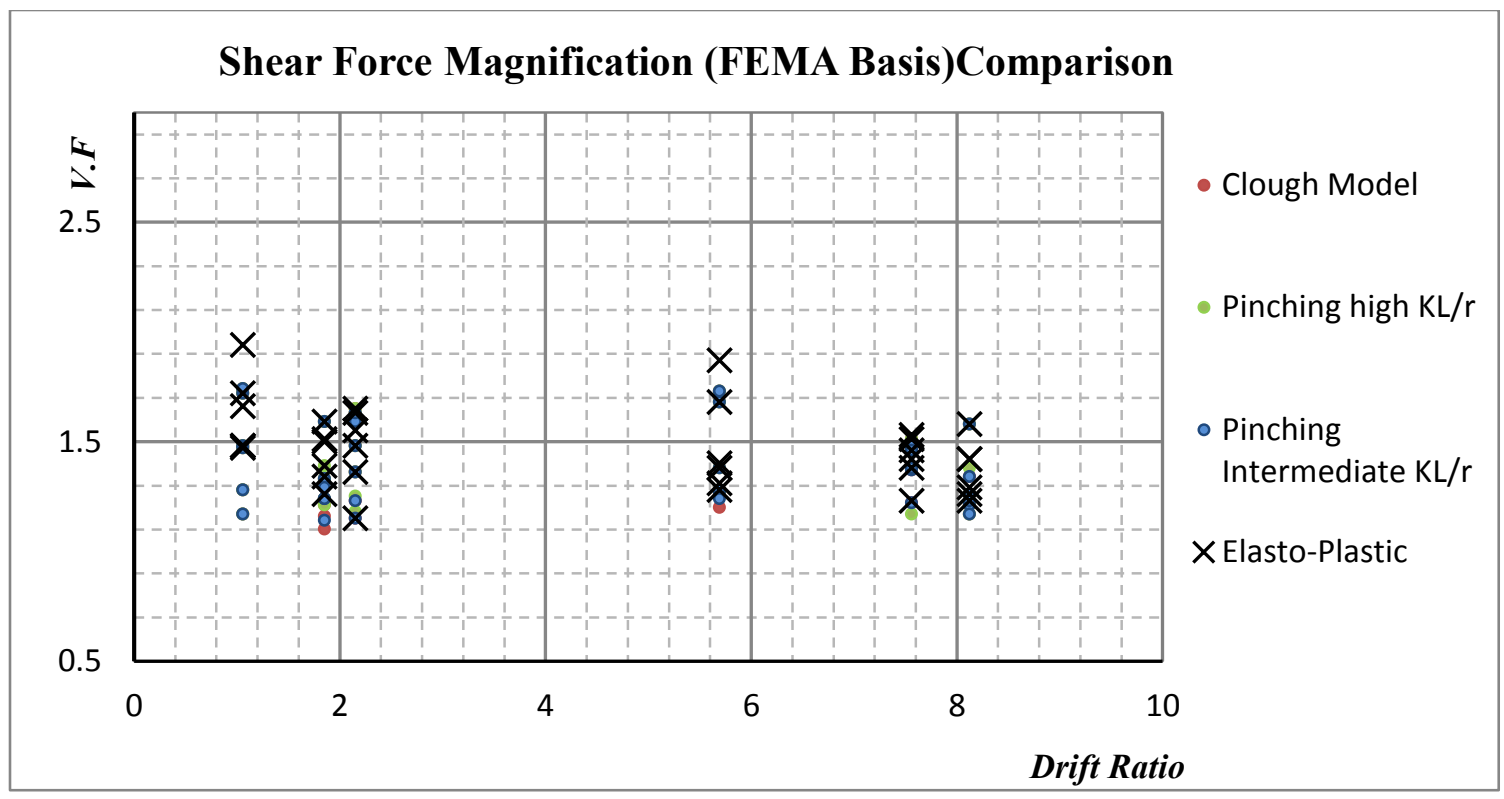

Figure 6.12 - Comparison between quarter span shear force magnification ratios with respect to FEMA parabolic distribution of internal forces for different models to represent the hysteretic behaviour of the LLRS

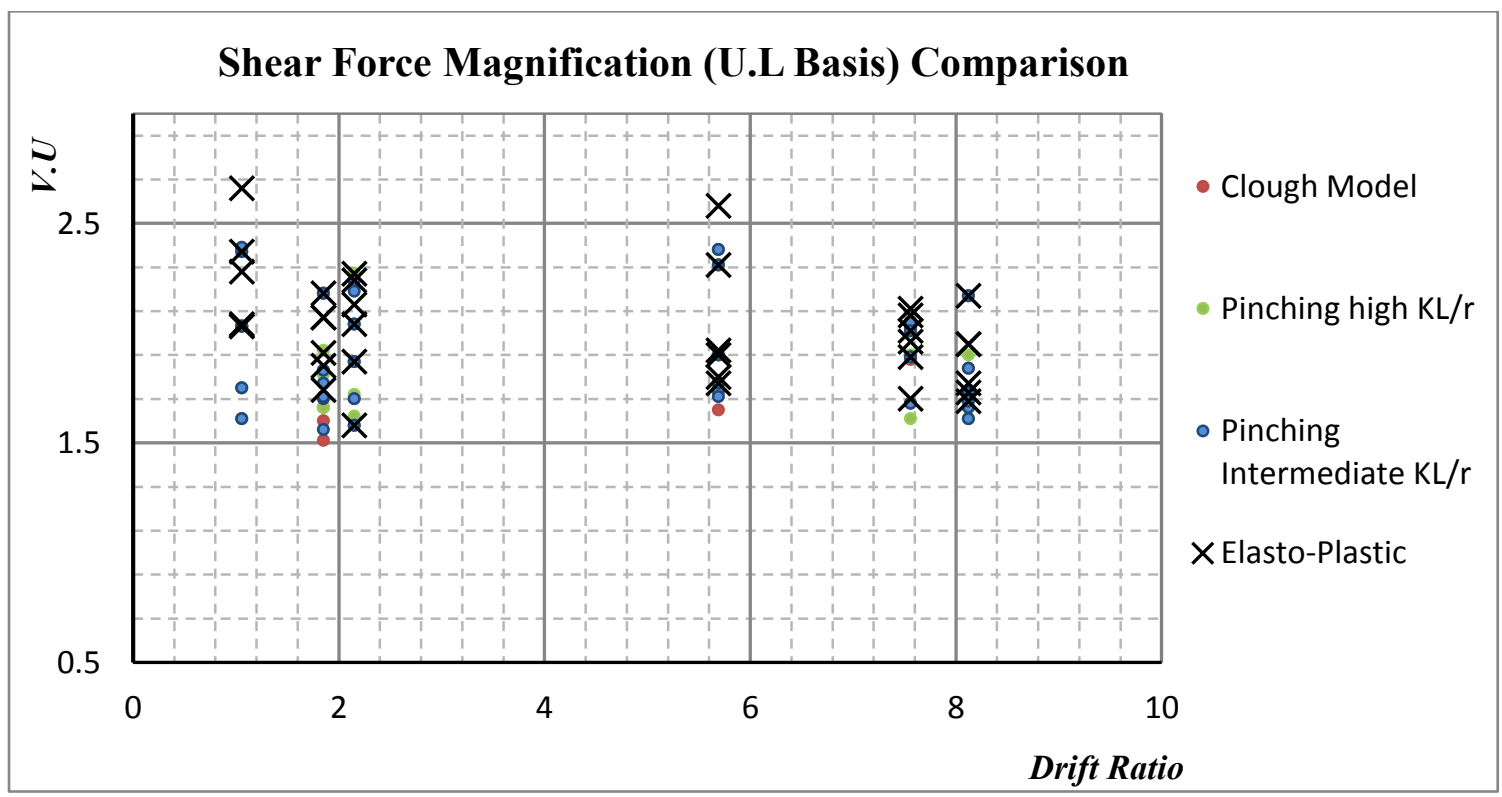

Figure 6.13 - Comparison between quarter span shear force magnification ratios with respect to uniform distribution of internal forces for different models to represent the hysteretic behaviour of the LLRS 
It is further observed that as the slenderness increases, the force magnification factors corresponding to pinching behaviour also increase. Nevertheless, the magnitudes of these magnifications are in all cases lower than those in the elasto-plastic case.

\section{Hysteretic Response of a Selected Building}

As stated earlier and as the results suggest, the pinching behaviour in LLRS can cause both a higher and a lower ductility demand depending on the characteristics of the excitation. In order to form a better understanding of how the pinching behaviour can have different effects on the ductility demand, the complete force displacement relationships of building LH1 responding to ground motions $\mathrm{M} 6 \mathrm{C} 1$ and $\mathrm{M} 6 \mathrm{C} 2$ are determined for 3 different hysteretic models assigned to the LLRS: elasto-plastic, pinching for low slenderness braces, and pinching for high slenderness braces. The response results are presented in Figures 6.14 to 6.16

As can be seen in Figure 6.14, a pinching behaviour in the LLRS causes an increase in the ductility demand on the LLRS. The ductility demand which was set to 3 for the elasto-plastic case increases to 4.62 and 4.31 for pinched behaviour with high slenderness and intermediate slenderness, respectively. 


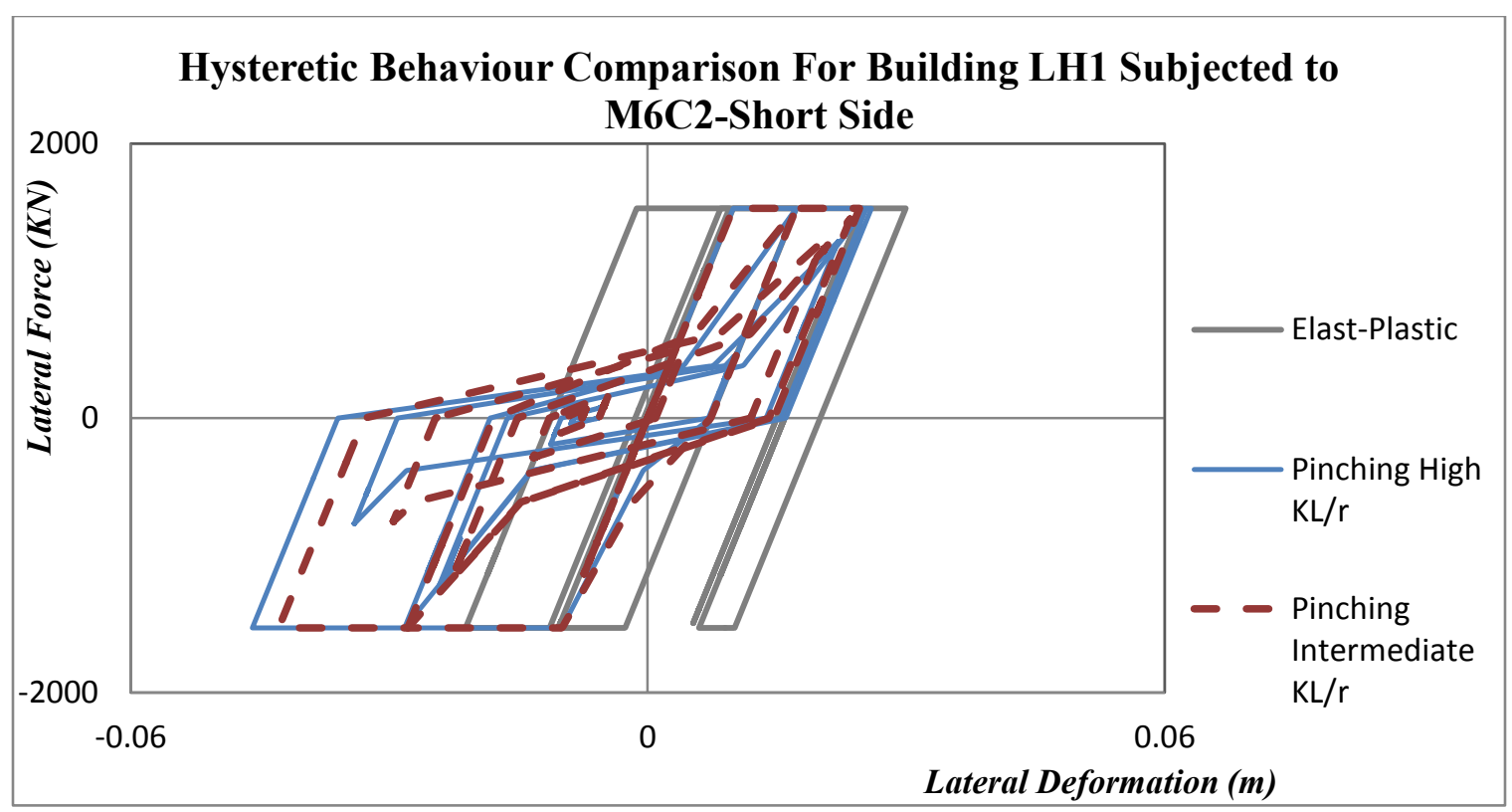

Figure 6.14 - Comparison of the hysteretic responses of the LLRS when represented by 3 different models - Building LH1 subjected to ground motion M6C2 parallel to the short side

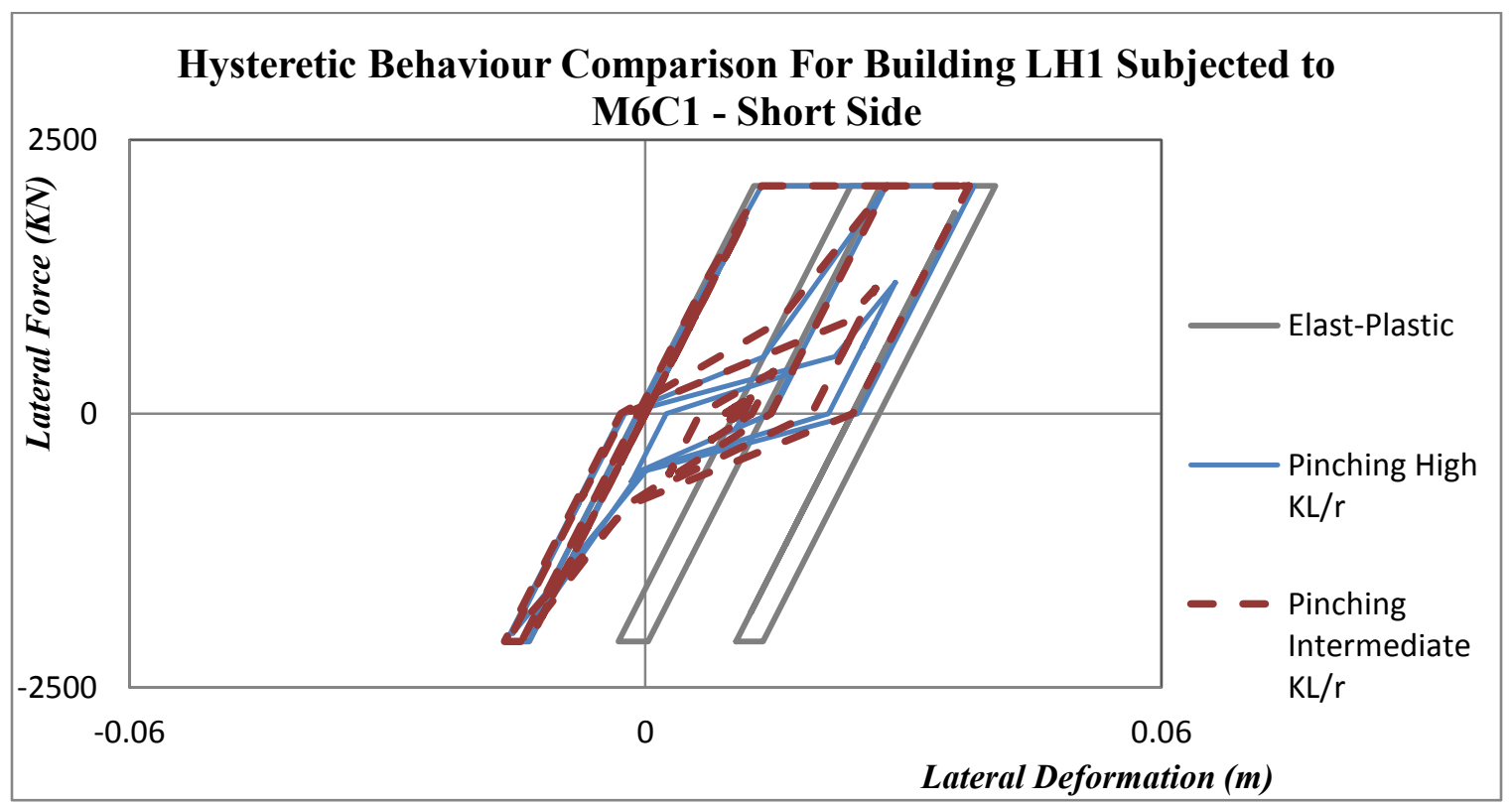

Figure 6.15 - Comparison of the hysteretic responses of the LLRS when represented by 3 different models - Building LH1 subjected to ground motion M6C1 parallel to the short side 


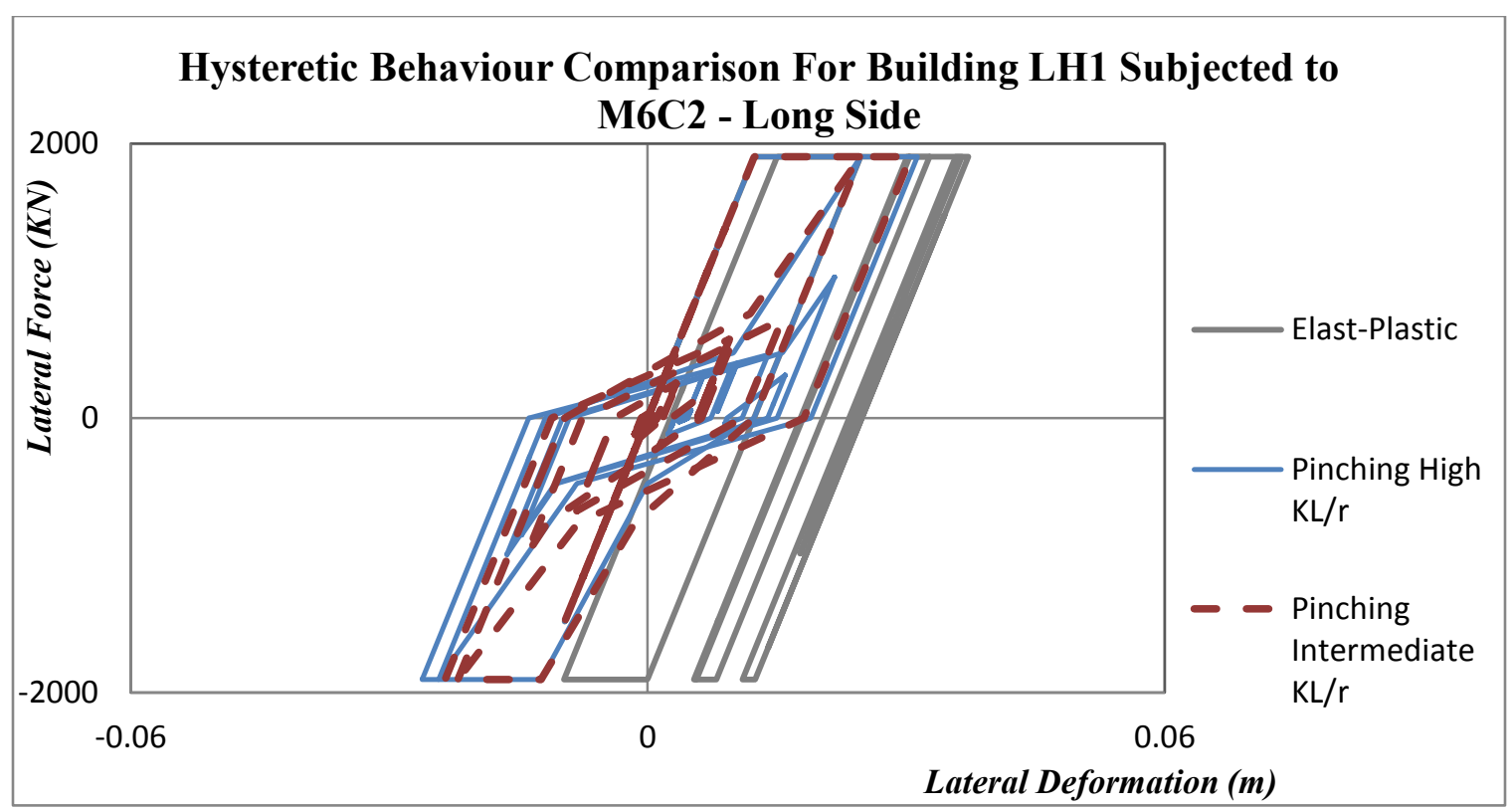

Figure 6.16 - Comparison of the hysteretic responses of the LLRS when represented by 3 different models - Building LH1 subjected to ground motion M6C2 parallel to the long side

It can be seen from Figure 6.15 that for building LH1 subjected to M6C1 ground motion parallel to the short side, pinching in the LLRS leads to approximately the same ductility demand as in the elasto-plastic case. The ductility demand in this case changes from 3 to 2.83 for the high slenderness pinching behaviour, which is, in fact, smaller than the ductility demand for the elasto-plastic case.

Figure 6.16 for the LLRS in building LH1 subjected to M6C2 ground motion shows even greater decrease in the ductility demand when pinching behaviour is considered. In this case the ductility demand reduces from the original value of 3 to 2.47 and 2.53 for the intermediate slenderness and high slenderness, respectively.

Figures 6.14 to 6.16 give a better understanding of how the Clough models with or without pinching either increase or decrease the ductility demand. It should be noted that 
higher slenderness in all cases would increase the ductility demand compared to the value corresponding to the intermediate case.

Based on the results of the analyses we can conclude that for predicting the internal forces, namely bending moments and shear forces, the elasto-plastic behaviour is always conservative and the results obtained from the previous analyses can be applied to buildings in which the lateral load resisting system exhibits pinching behaviour or a hysteretic behaviour similar to the Clough model.

The results for the ductility demand suggest that implementing the Clough model with or without pinching, while using the same force reduction factor that lead to the ductility demand of 3 for the elasto-plastic case, causes the values of ductility demand to fluctuate around 3 , being higher in some cases and lower in others. The mean value is fairly close to 3 . Thus, given the scatter of the results and the mean value being close to that for the elasto-plastic case, it may be reasoned that the results from the analyses based on elastoplastic case may be used to provide an estimate of the ductility demand even when the force-displacement relationship for the LLRS follows the Clough model without pinching, with pinching for intermediate slenderness, and with pinching for high slenderness. Additional studies using more ground motions and more buildings is recommended to further validate the findings regarding the ductility demand.

\subsection{Conclusions and Recommendations}

1. Concentric braces often exhibit pinching in their hysteretic behaviour. Therefore, the validity of the previously suggested equations that were derived based on the 
assumption of an elasto-plastic behaviour for the LLRS must be tested for buildings in which the LLRS shows a pinched hysteretic response.

2. The pinching behaviour in concentric braces is caused by buckling mechanism of the brace in compression and the increase in the length of the brace in tension in each cycle. The former can be related to the slenderness of the member and the latter depends mostly on geometry and the characteristics of the excitation.

3. When pinching is accounted for in the analytical model, the ductility demand values fluctuate around that determined from an elasto-plastic model, being greater in some cases and smaller in others.

4. Whether the ductility demand would increase or decrease, depends on the characteristics of the excitation and the structural system.

5. Use of the Clough hysteretic model with or without pinching, may decrease the ductility demand in comparison to the elasto-plastic case, because the member would have a better chance to deform back to the original length when the excitation allows it. As a consequence, in the subsequent cycles the overall deformation may at times be smaller than that in the elasto-plastic case.

6. Use of the Clough model with or without pinching may at times increase the ductility demand owing to a greater displacement in any one cycle.

7. The results of the current study show that the mean value of the ductility demands obtained from the response of different buildings subjected to ground motions compatible with the UHS for the western and eastern regions of Canada when a pinched behaviour for the LLRS is considered is more or less the same as that produced by an elasto-plastic behaviour. 
8. Given that most of the assumptions in the current study were conservative, equations previously proposed by Humar and Popovski can still be applied in buildings with concentric braces forming the LLRS.

9. The bending moment magnification and the shear force magnification at mid span and quarter span, respectively, are observed to be greater in the elasto-plastic case compared to that in the pinching cases. Consequently, the previously suggested equations for predicting the magnification factors in bending moment and shear force along the length of the diaphragm can conservatively be applied to singlestorey buildings with concentric braces forming the LLRS.

10. It is observed that the magnification in internal forces is higher for brace elements with high slenderness compared to the case when the braces have intermediate value of the slenderness. 


\section{Chapter 7 : Seismic Response of One-Storey Buildings with Nonlinear Flexible Diaphragm}

\subsection{Introduction}

In earlier chapters the effects of diaphragm flexibility on the seismic response of singlestorey buildings were investigated. Previous studies on the subject were reviewed and improvements were suggested in the equations proposed by Humar and Popovski (2013) to estimate the increase in ductility demand on the lateral load resisting system (LLRS) caused by the flexibility of the diaphragm, which itself remained elastic. In addition, the distribution of the bending moment and the shear force along the length of the flexible diaphragm was studied. It was observed that there was significant magnification in the bending moment and the shear force in the plane of the diaphragm at mid-span and quarter span, respectively. Appropriate methods were suggested to predict the amount of such magnifications.

Throughout the earlier study, it was assumed that the nonlinearity in the system was confined to the lateral load resisting system, which was assumed to consist of structural steel braces. In other words, it was assumed that the LLRS could be strained into the nonlinear range and act as the energy dissipating element while the diaphragm performed elastically. Such behaviour can be ensured by designing the diaphragm for the ultimate strength of the LLRS, an approach that is commonly referred to as capacity design. Increasing the thickness of the diaphragm as well as making the connections (side lap fasteners or deck to frame fasteners) stronger may be necessary for a capacity design approach. In fact, the roofing system must be designed to have a strength that is a certain 
factor times the yield force of the bracing system. This factor must be selected such that the roof diaphragm exhibits no nonlinearity. The selection of such a factor is an appropriate topic for study.

The common practice in the design of single-storey buildings consisting of corrugated steel decks supported by concentrically braced steel frames is to proportion the braces so as to act as the weak link that dissipates the earthquake energy. The elastic lateral loads are reduced by an appropriate force reduction factor that is equal to the product of the overstrength factor $R_{0}$ and the ductility factor $R_{d}$. This is satisfactory provided the lateral load resisting elements, in this case the braces, have enough ductile capacity to absorb the seismic energy even as they undergo large deformations. This widely accepted design approach has been the basis of the analytical results presented in the previous chapters. However, recent studies have pointed out that it is not necessary to design the bracings as the main energy dissipating system and that an alternative design practice may be possible, and at times advantageous, for one-storey buildings with flexible diaphragms. In this alternative approach the flexible roof diaphragm is relied upon to sustain the lateral load even as it is strained into the inelastic range.

As discussed earlier, if the braces are designed as the energy dissipating members, it must be ensured that the nonlinearity is confined to the braces and the steel deck diaphragms behave elastically during seismic events. In order to do so, a capacity design approach must be adopted for the design of steel deck panels to ensure that the roofing system remains elastic even as the bracing system reaches its yield capacity. Because of a comparative low seismic weight, the lateral load imposed on single-storey buildings is relatively small and a force-based design would lead to slender braces. However, in order 
to satisfy the slenderness limit specified by the steel design code, CSA S16-09, often heavier brace sections must be used leading to overdesigned brace members. Such selection of the braces requires an increase in the capacity of the diaphragm as well in order to match the ultimate capacity of the oversized braces. Consequently, the thickness of the diaphragm, the number of connections and their capacity must be increased far beyond what is needed for the design earthquake. As a result, the design ratio, that is the ratio of the force demand to the strength of the system, is often much less than 1.0. This makes the design approach uneconomical, as also pointed out in various studies.

In the alternative design approach, referred to earlier, the roofing system is allowed to be strained into the inelastic range and to dissipate all or part of the seismic energy imposed on the structure. It is then possible to design the braces to remain elastic and stay undamaged. Besides being a cost-efficient design procedure (Tremblay et al. 2002), it offers the advantage that the residual displacements, if any, are confined to the roofing system and the building stays intact for post-hazard occupancy. On the other hand, if the braces are used as the energy dissipating system, assuming that no self-centering mechanism has been incorporated, the structural system often shows residual displacements after the earthquake which may render the structure unfit for post-hazard occupancy.

The design approach in which the flexible diaphragm is the main energy dissipating element has been the subject of several previous studies such as that by Tremblay et al. (2002). These studies have been reviewed in the first chapter. Recently, codes and design guidelines have recognized this approach as well. The 2010 National Building Code of Canada (NRCC 2010) allows the steel deck roofing system to act as an energy dissipating 
member provided that the steel diaphragm is designed so that it can absorb the seismic energy by sustaining nonlinear deformations and showing ductile behaviour. The NBCC 2010 further specifies the seismic force for which the roofing system must be designed, by stating that the diaphragms, collectors, chords, struts and connections can be designed for a seismic design load equal to the elastic seismic load reduced by $R_{o} \times R_{d}=2.0$ (NBCC 2010 - Clause 4.1.8.15.).

A study of the performance of one-storey buildings in which the roof diaphragm acts as the main energy dissipating member can prove useful. It would be worthwhile investigating different aspects of the behavior of these buildings. Questions that must be addressed would include: whether or not the diaphragm flexibility leads to an increase in the ductility demand, as was the case when the braces were acting as the only energy dissipating members; what is an appropriate force reduction factor for obtaining the design seismic force; and whether there is a magnification in the internal forces.

It would be interesting also to evaluate the performance of one-storey buildings with flexible diaphragms in which both the braces and the steel decks are allowed to respond nonlinearly. In such structural systems, the question to be addressed would be to determine the design force for the roof deck diaphragm so that the ductility demand on the steel deck would be limited to an appropriate value.

The starting point for the study referred to in the preceding two paragraphs is to investigate the nonlinear behaviour of steel decks under lateral loads. Such investigation would include the hysteretic behaviour, effective stiffness, strength and stiffness degradation if any, and pinching in the hysteresis loop. 


\subsection{Chapter Outline}

In this chapter the hysteretic response of the steel deck diaphragms is investigated first. The investigation is based on previous experimental and analytical work on the subject, outlined and discussed in Chapter 1. Based on the review of the previous studies, an appropriate hysteretic behaviour is assigned to the steel diaphragm. Such selection is based on the mechanism in which the steel deck panels deform. The nonlinear forcedisplacement hysteretic behaviour so derived is then used to constitute the analytical model that represents the steel deck.

The performance of the system in which the steel deck diaphragm is designed to act as the sole energy dissipating member is examined and the response of the system to seismic forces is compared to the previous findings that were based upon systems in which the braces acted as the only energy dissipating system. An attempt is made to derive the force reduction factor for steel deck diaphragms that would lead to the achievement of specific target ductility in the diaphragm. At the end of this component of study, the potential advantages and disadvantages of having the diaphragm act as the energy dissipating element are reviewed.

In the second part of the study, an attempt is made to determine the seismic design force for the diaphragm expressed as a factor of the brace yielding force. The design force factor, denoted as $\Phi$, is found for two different performance levels in the roof diaphragm. First, the design force for the diaphragm that would ensure elastic behavior in the roofing system is found and expressed as a factor of the brace yield force $\left(\Phi_{e}\right)$. Second, the seismic design force factor $\left(\Phi_{d}\right)$ for steel deck panels that would lead to the roof 
diaphragm being strained into the nonlinear range but with a ductility demand limited to a a target dutility, selected here as 1.5 . In both cases, braces act as the main energy dissipating elements. In the former case, all of the earthquake energy is dissipated in the braces. In the latter case, a portion of the energy induced in the system during the earthquake is dissipated in the steel deck diaphragm. The performance of buildings in which both the brace system and the roof deck act nonlinearly and hence dissipate energy is reviewed.

In the sections that follow, first the shear behaviour of steel deck panels when subjected to seismic ground motions is discussed. Next, the buildings selected for this portion of the study are introduced. The earthquake records are briefly outlined. The analytical model and the selected hysteretic behaviour for the shear force-displacement are then explained. Finally, the results of the current study are presented and discussed and the conclusions reached are presented.

\section{3-Plane Shear Behaviour of the Diaphragm}

As stated earlier, when subjected to lateral loads the diaphragm behaves as a deep beam in which shear deformations play a major role. If the diaphragm is allowed to strain into the nonlinear range, it reaches its in-plane shear capacity first and then exhibits nonlinear shear deformations under the in-plane shear force imposed on the diaphragm. In other words, the diaphragm yields in shear and its nonlinear shear deformations contribute to the energy dissipation in the system. Thus, the in-plane shear capacity of the roofing system as well as its shear stiffness are of prime importance in modeling the hysteretic response of the steel deck diaphragms and the anticipated energy dissipation. 
In order to assess the in-plane shear behaviour of the diaphragm, one must understand the deformation mechanism of the steel deck diaphragm when subjected to in-plane shear caused by lateral loads. In an un-topped steel deck diaphragm the deformation mechanism is strongly affected by the connections. The different type of connections are therefore reviewed along with the type and nature of the fasteners that are commonly used

\subsubsection{Importance of Connections in Steel Deck Diaphragms}

As pointed out earlier, the in-plane shear behaviour of the diaphragm is of great importance in assessing the hysteretic response of the roofing system. Previous studies, such as the experimental studies by Rogers and Tremblay (2003a) on side lap fasteners and frame fasteners (2003b), the studies carried out by Essa et al. (2003) and Davies and Bryan (1982) and Massarelli et al. (2012) suggest that the in-plane shear behaviour of the diaphragm is dependent on the connections, and the shear behaviour of the corrugated deck, and its warping characteristics. Of these, the steel deck panels' stiffness and the warping stiffness of the panels have comparatively minor effect on the total shear behaviour of the diaphragm. Thus, the in-plane shear force-deformation behaviour of the steel deck diaphragms, their shear force capacity, shear stiffness and energy absorption capability are mainly dependent on the performance of the connections and the design of the roofing system is generally dictated by the type of connectors used (Rogers and Tremblay 2003a, b). Since the behaviour of the steel-deck diaphragm in the nonlinear

range is governed by the characteristics of the fasteners, the energy dissipation capability of the deck is mostly dependent on the energy absorption capability of the connections. 


\subsubsection{Types of Connectors}

Two different classifications exist for the connectors in corrugated steel deck systems; (1) depending on the connected members, and (2) depending on the method used to make the connection (weld, punch, etc.)

Connections in steel deck roofing systems are classified into two types based on the elements they connect: (1) Side Lap Fasteners which connect the steel deck panels together to make the roofing system and, (2) Deck to Frame Fasteners, also referred to as Frame Fasteners, which connect the roofing system to the underlying braced frame. The diaphragm panels rely on the former to act as one unit and to transfer the shear force between one another, while relying on the latter to transfer the lateral loads to the braced frame.

The connections in steel deck panels are further classified based on the method used for connecting the steel deck panels to one another and to the underlying frame. Different types of side lap connections are used for connecting the panels together: (1) the button punch side lap connector which is the most common type in Canada, (2) screwed side lap connectors which are more commonly used in the US construction practice and, (3) welded side lap connections that in some cases may be used to connect the longitudinal edges of deck sections (Rogers and Tremblay 2003a). Typically, the welded side lap connectors are able to carry the largest shear force and absorb the greatest amount of seismic energy (Rogers and Tremblay 2003a).

The steel deck structure can be attached to the underlying frame using alternative connection types such as: (1) arc-spot welds, (2) nails [powder-actuator fasteners (PAF)], 
and (3) screws. Among different types of deck to frame connectors, the weld connections are the most commonly used in Canada and in the United States practice. It must be noted that nailed as well as screwed connections are becoming more and more common (Rogers and Tremblay 2003a). Figure 7.1, taken from the paper by Rogers and Tremblay (2003a), shows the different types of connectors.

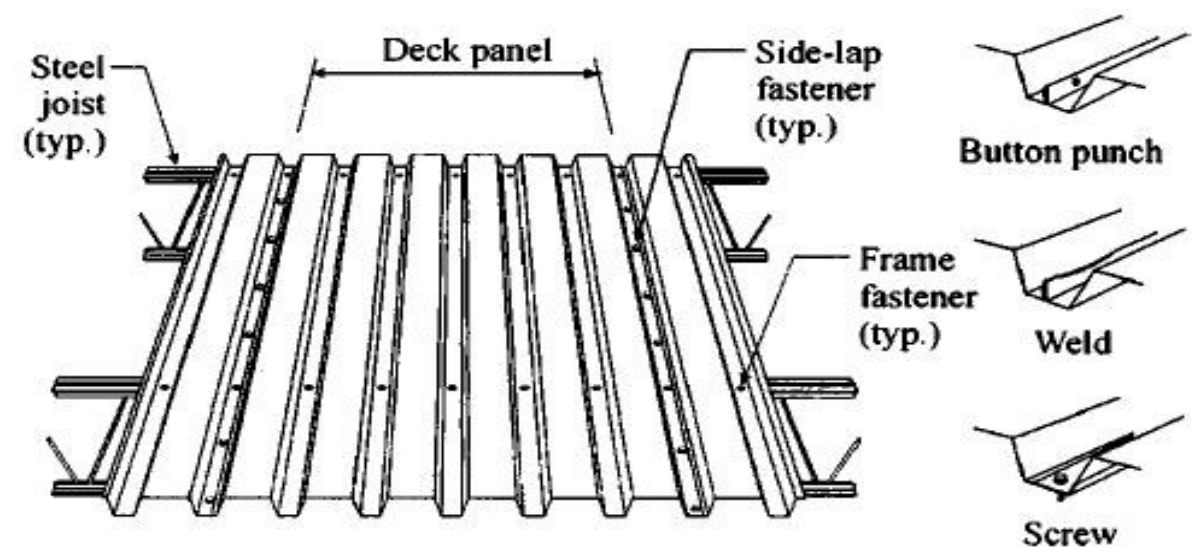

Figure 7.1 - Different connection types in steel deck diaphragms (Taken from Rogers and Tremblay 2003 with the authors' permission and, with permission from ASCE)

\subsubsection{Mechanism of Deformation in a Flexible Diaphragm Subjected to}

\section{Earthquake Loads}

When a single-storey building is subjected to earthquake ground motion, the relative movement at the base of the structure gives rise to inertia forces which are imposed on the structure at the roof level. These inertia forces are transmitted to the foundation through the structural action of the diaphragm and the vertical bracing system that supports the roof diaphragm. To limit the magnitude of the inertia forces that the structure must be designed to resist, one or both components of the lateral load resisting system are allowed to be strained into the nonlinear range. Alternative design strategies that may be used include the following: (1) braces acting as the sole energy dissipating 
member, (2) steel deck diaphragm acting as the main source of energy dissipation, and (3) a combination of the two aforementioned systems in which a portion of the seismic energy is dissipated in the braces and the rest is dissipated in the steel deck panels.

\section{Braces Acting as the Energy Dissipating Members}

If the braces are designed as the weak link in the system, the diaphragm is expected to remain elastic. The internal forces induced in the roof are transferred from one panel to another through side lap connectors. At locations where the diaphragm is supported by vertical bracing system the internal forces are transferred from the steel panel to the braces through deck to frame fasteners. The seismic force cannot, of course, exceed the brace capacity. When the brace capacity is reached, the earthquake energy is dissipated through ductile inelastic deformations of the braces. Since the seismic force is limited by the brace capacity, the seismic force in other components in the structural system (steel panels, side lap fasteners, and deck to frame fasteners) does not exceed the limit force. The inelastic action in the braces consists of the buckling of the compressive brace and the yielding of the tensile brace as discussed in greater detail in Chapter 6. The seismic load is further transferred from the braces to the foundations.

\section{Steel Deck Panels Acting as the Energy Dissipating Members}

In another design approach, the steel deck panels may be designed as the energy dissipating elements. Again, the inertia forces in the roof are transferred between panels and from panels to the vertical bracing system through the fasteners that make the connections. However, it is in the roofing where the nonlinearity occurs and the seismic energy dissipated through inelastic deformations in steel deck panels. The braces remain 
elastic as they deform under the lateral load transferred to them. The seismic force is limited by the capacity of the roofing system and the force in the rest of the components (braces, foundations, etc.) does not exceed that limit. Previous experimental and analytical studies have shown that the nonlinearity in the diaphragm occurs in shear. The ability of the diaphragm to dissipate the earthquake energy effectively is dependent on the in-plane shear stiffness, hysteretic behaviour and shear capacity of the diaphragm. Previous studies further illustrate that while the side lap fasteners, deck to frame fasteners, corrugated deck shear characteristics and warping nature of the panels all contribute to the effective shear behaviour of the roofing system, it is the type of side lap and deck to frame connections (weld, button punched, screw, etc.) that dominate the behaviour. During a seismic event, the connections will reach their ultimate capacity, after which the seismic force would remain practically constant in the panels and in the rest of the structural components (panels, braces and foundations) until load reversal takes place.

During an earthquake, nonlinearity can occur in the side lap fasteners or in the deck to frame fasteners, or in both. In addition, such nonlinearity can occur at different locations along the length of the diaphragm. As discussed in Chapter 5, in a flexible diaphragm the shear force at quarter span is considerably magnified and such magnification increases when the contribution of higher modes to the response becomes more pronounced. In fact, at times this magnification can be so significant that the shear force at quarter span would exceed that at the ends of the diaphragm span, in which case the nonlinearity is more likely to occur in the side lap fasteners near the quarter span. In other cases, the nonlinearity would be expected to occur in the deck to frame fasteners or side lap 
fasteners toward the ends of the diaphragm span. In general, the nonlinearity may occur at different locations along the length of the diaphragm.

\section{Braces as well as Steel Deck Acting Nonlinearly}

In systems in which both the braces and the steel deck panels act nonlinearly, the deformation mechanism in the structural system is a combination of the two mechanisms discussed in the preceding paragraphs and energy is dissipated in both the brace and the connections of the steel deck panels.

\subsubsection{Modelling of Shear Behaviour}

Information on the effective stiffness of diaphragm roofing system is available in design guidelines, such as those produced by CANAM (2007) and Steel Deck Institute (SDI) (2011). These guidelines provide the effective stiffness of diaphragm roofing systems based on the thickness of the deck as well as the type of both deck to deck and deck to frame fasteners. The catalogues produced by CANAM and SDI have become useful tools for the design of steel deck diaphragms and for assessing the effective shear stiffness as well as the strength of the system. However, for research in the area of performance based design where hysteretic behaviour of the system is often of importance, the information provided by the guidelines is not sufficient and previous experimental work such as that by Rogers and Tremblay (2003a, b) must be studied. Figure 7.2 taken from the study by Essa et al. (2003) illustrates different hysteretic behaviours for different diaphragm specimens having different thickness and various combinations of deck to deck and deck to frame fasteners. In all cases severe pinching and strength degradation 
can be observed. The hysteresis behaviour adopted in the present study is based on such observed behaviour and is described in detail in subsequent sections of this chapter.

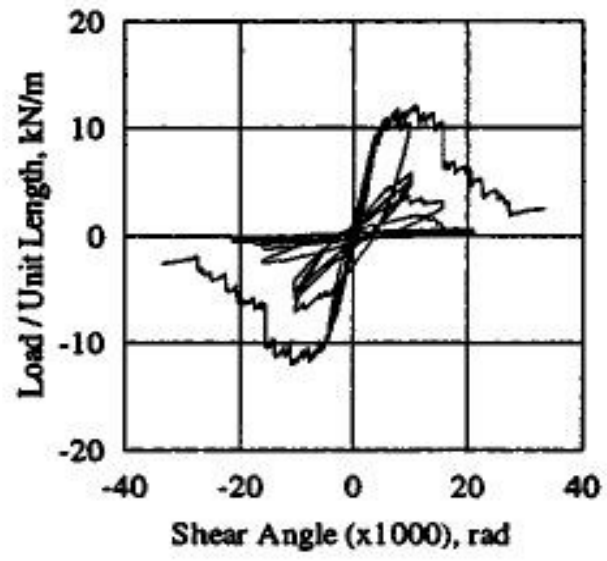

(a)

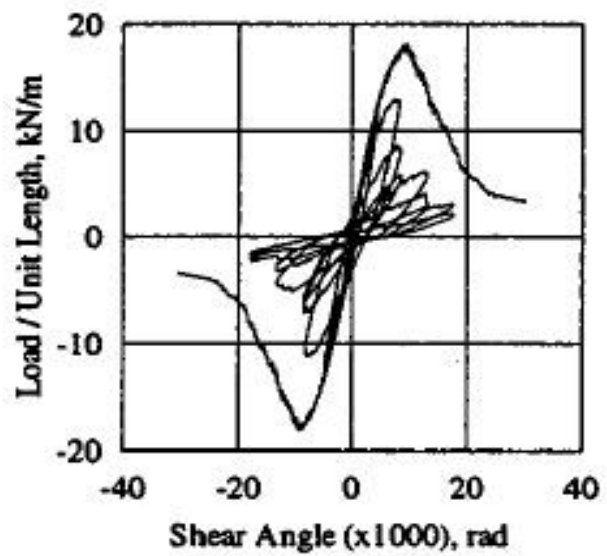

(c)

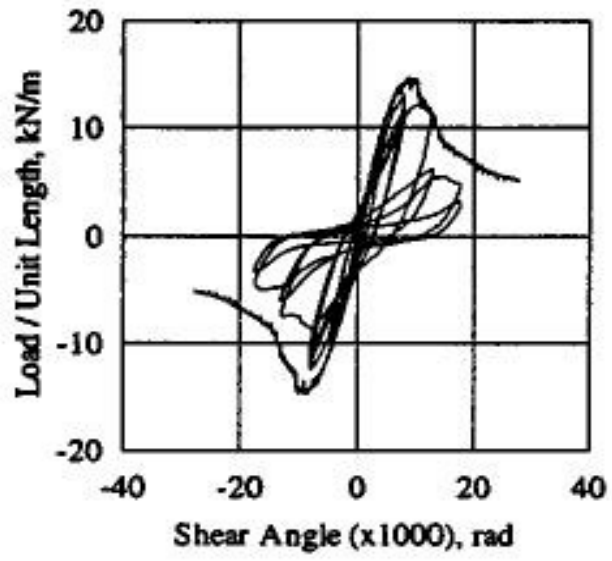

(b)

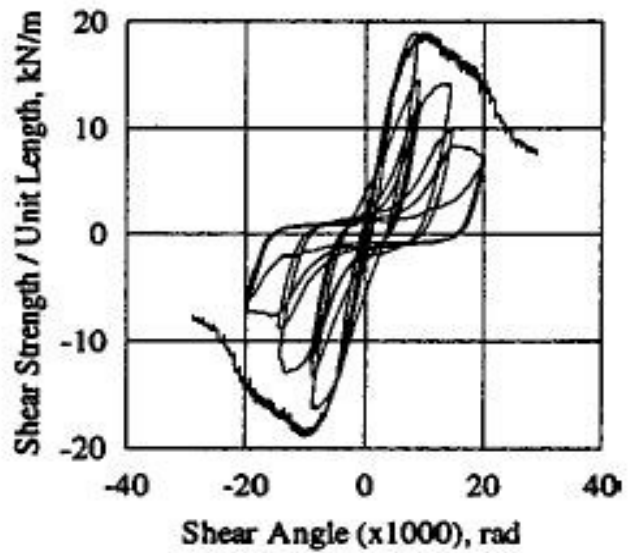

(d)

Figure 7.2 - Load deformation curves for different deck to deck and deck to frame fastener types, (a) welded deck to deck and deck to frame fastener - (b) welded deck to deck and deck to frame fasteners (c) screwed deck to deck and welded deck to frame fasteners - (d) screwed deck to deck and weld with washers deck to frame fasteners (taken from Essa et al. 2003 with the authors' permission and, with permission from ASCE) 


\subsection{Buildings Selected for Study}

A set of buildings is selected from the original building database referred to in Chapter 2 . The database consists of 36 one-storey buildings designed by Tremblay and Steimer (1996). In order to include a wide range of drift ratios in the study, the selected buildings are analyzed in both orthogonal directions, that is, when the seismic load acts parallel to the short side of the building and when it acts parallel to the long side of the building. One building is selected from each building size (small, medium and large), so that the conclusions are valid for all building sizes. Buildings with heavy as well as light roofing systems are selected so that the results would not be limited to just a particular seismic weight range. Figure 1.1 in Chapter 1 illustrates the typical lay out of the selected buildings.

Based on the considerations outlined, buildings SL1, SH1, ML1, MH1, LL1 and LH1 are selected for the sets of analyses, whose results are presented here. Initial sets of analyses showed a lack of data in the range of drift ratios from 2.5 to 5.5 and from 8 to 10 . In order to cover the entire drift range, buildings SH4, ML2, MH6 and LH5 with the earthquake acting parallel to the short side of the buildings were added to the selected buildings. The combined selection of buildings covered the range of drift ratios from 1.061 to 10.329.

In their design of the selected buildings Tremblay and Stiemer (1996) first determined the thickness of roof diaphragm based on the gravity loads. The effective shear stiffness $\left(G^{\prime}\right)$ was then assessed based on the provisions given by the Steel Deck Institute (SDI 1991). As stated earlier, the effective shear stiffness depends on the diaphragm thickness and the 
type of fasteners. In the selected buildings, weld connections were used for the deck to frame fasteners while screwed fasteners were used as the side lap fasteners.

\subsection{Ground Motion Records}

The ten ground motions generated by Atkinson (2009) that were introduced in previous chapters are used for this portion of the study. Five of the selected ground motions are compatible with the eastern (Montreal) uniform hazard spectrum while the rest are compatible with the western (Vancouver) spectrum. By doing so, the results obtained become applicable to different spectral shapes and not just to one. Records $\mathrm{M} 6 \mathrm{C} 1, \mathrm{M} 6 \mathrm{C} 2$, M6C26, M6C31 and M6C38 with scaling factors of 0.78, 0.87, 1.19, 0.99 and 1.43 respectively, are selected to provide a match for the Vancouver UHS while records E6C1, E6C13, E6C15, E6C18 and E6C42 with scaling factors 0.55, 0.74, 0.56, 0.61 and 1.01, respectively, are used to match the UHS for Montreal.

\subsection{Assumptions and Analytical Model}

In the following paragraphs some of the main assumptions made in the current study are outlined. In addition, the basis for the assumptions made in the finite element model developed in OpenSees platform is discussed.

\subsubsection{Selected Software}

As would be observed from Figure 7.2, the hysteretic behaviour of steel deck diaphragm exhibits severe pinching as well as some strength degradation regardless of the type of fasteners used. Therefore, for the finite element model, a software must be used which 
allows the modelling of a variety of materials with different hysteretic behaviours. The OpenSees platform allows such modelling as well as the representation of a pinched hysteretic behaviour, with or without strength degradation, for the steel deck panels.

\subsubsection{General Assumptions}

Most of the assumptions in modelling described in this section are consistent with those outlined in Chapter 2. Some of the main assumptions are outlined in the following paragraphs.

The buildings are modelled as deep beams, representing the diaphragm, supported by two springs, representing the braces. The deep beam (diaphragm) is divided into 20 elements interconnected at 21 nodes. Rayleigh damping of 5\% is assumed for the first and the third modes of vibration. For the dynamic analyses, a time step of $0.001 \mathrm{~s}$ is used. A more detailed discussion of the assumptions can be found in Chapter 2 .

The assumptions made in this part of the study that are different from the ones made in earlier parts are related to the behaviour of the braces as well as the diaphragm. In the first part of the study, which assesses the performance of buildings in which the steel deck diaphragm acts as the energy dissipating element, an elastic behaviour is assumed for the braces while a nonlinear behaviour with appropriate pinching and strength degradation characteristics is assumed for the steel deck diaphragm. The nonlinear hysteretic behaviour is discussed in detail in subsequent sections.

In the second part of the study, an attempt is made to find the diaphragm design force as a factor of the brace yield force for two performance levels in the roof diaphragm. The first factor $\Phi_{e}$, is determined such that the diaphragm would remain elastic while the brace acts 
as the main energy dissipating member. The second factor $\Phi_{d}$ is determined such that while the braces behave nonlinearly, the diaphragms also shows limited nonlinearity. In this part of the study, a bilinear behaviour with 0.05 post-yield stiffness ratio is assumed for the braces while a hysteretic nonlinear shear deformation behaviour with pinching and strength degradation is assumed for the diaphragm steel deck.

\subsubsection{Force - Deformation Behaviour in Shear for Steel Deck}

For assessing the shear behaviour of the steel deck panels, the strength, the initial stiffness and the hysteretic behaviour must be determined. For this purpose previous experimental studies are reviewed considering the design information related to the selected buildings as outlined in the paper by Tremblay and Steimer (1996). Appropriate effective shear stiffness is assigned to the diaphragm and a hysteretic behaviour is selected. The diaphragm yield force is determined for certain target ductility values.

\section{Effective Shear Stiffness}

The effective shear stiffness can be determined based on the thickness of the diaphragm and the type of fasteners, number of fasteners and the size of fasteners. CANAM provisions as well as Steel Deck Institute (SDI) guidelines are useful for such determination. In the design of the selected buildings arc spot welds were used for deck to frame fasteners while screwed connections were used for side lap fasteners. The effective shear stiffness for the selected buildings are determined based on diaphragm thickness and the mentioned fasteners and in accordance with the provisions given by SDI 1991. The G' values for the buildings are given in the 1996 paper by Tremblay and Steimer. In order to have a consistent value for initial shear stiffness, the same G' values 
are used throughout the study. This way the frequencies of the buildings remain unchanged even as the other characteristics are varied and do not affect the results in any way. The variations in the results of analyses thus depend only on the nonlinear behaviour assigned to the diaphragm. It may be noted that the same G' values are used in the analyses reported in the previous chapters for determining the shear stiffness for elastic behaviour of the roof diaphragm.

\section{Hysteretic Behaviour}

As discussed earlier, the hysteretic behaviour of the steel deck diaphragm is primarily dependent on the type of fasteners, more specifically on the type of both side lap fasteners and deck to frame fasteners. In their paper Essa et al. (2003) investigated the response of different specimens with different combinations of deck to deck and deck to frame fasteners under both monotonic and cycling loadings. Some of these results are shown in figure 7.2. Figure 7.2 (d) shows the hysteretic response of the diaphragm specimen in which arc spot welds were used as deck to frame fasteners and screws were used as the deck to deck fasteners which match the design assumptions for the selected buildings. Therefore, in the selection of an appropriate hysteretic behaviour which exhibits both pinching and strength degradation, an attempt is made to match the response to that shown in Figure 7.2 (d). The same force - deformation relationship is shown in Figure 7.3, but without the response to monotonic loading. The selected hysteretic behaviour is discussed in detail in the following sections. 


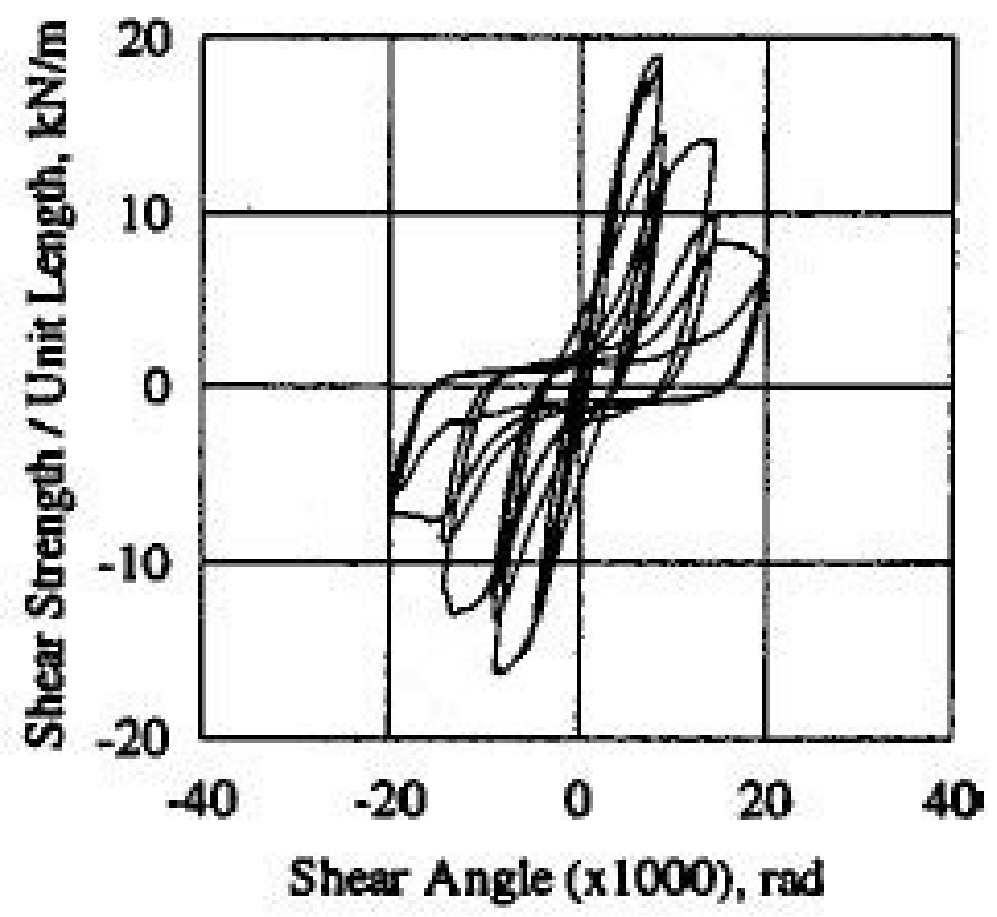

Figure 7.3 - Experimental force - deformation hysteretic curve in shear for the specimen with screwed deck to deck and welded deck to frame fasteners (taken from Essa et al. 2003 with the authors' permission and, with permission from

ASCE)

\subsubsection{Selected Model and Implementation of the Model in OpenSees Platform}

The advancement of research in the area of performance based design in earthquake engineering often requires the development of material response under cyclic loadings known as hysteretic behaviours. Hysteretic curves with different properties, such as pinching, strength deterioration, stiffness deterioration and etc. are often needed to match the response observed in experimental data. Experimental results have shown that the response of structural components to cyclic loadings is often subjected to deterioration of strength and stiffness. Such degradations in the hysteretic curves are dependent on numerous parameters affecting the response of the system. Therefore, over the past 
decades, the development of such hysteretic models has been the subject of numerous studies resulting in a rich database of material behaviours.

In their 2005 paper, Ibarra et al. developed a deterioration model referred to as IbarraMedina-Krawinkler (IMK) model. This model can simulate up to 4 modes of cyclic deterioration such as strength, postcapping strength, unloading stiffness and accelerated reloading stiffness deterioration (Ibarra et al. 2005). The model was modified to "Modified Ibarra-Medina-Krawinkler Deterioration Model with Peak-Oriented Hysteretic Response" by Lignos and Krawinkler (2009) to account for asymmetric hysteretic behaviour in structural components. Lignos and Krawinkler (2012) further advanced the model leading to the development of "Modified Ibarra-Medina-Krawinkler Deterioration Model with Pinched Hysteretic Response". The advantage of this model is that it can simulate 4 modes of deterioration, it accounts for asymmetric behaviour which can be useful in RC beams and can also account for pinching behaviour. While the model was originally developed for capturing moment-rotation relationship in beams, the authors point out that the model can be applied to any force-deformation relationship. Since the model is originally developed for moment-rotation relationship, in explaining the hysteretic behaviour, the strength is often regarded as bending moment strength and the deformation as rotation. To introduce the model the following description is presented in terms of moment-rotation relationship, although in the current study the model is used for capturing shear.

The modified IMK deterioration model with peak-oriented hysteretic response is defined by the backbone curve, specified by certain force and deformation values, and a set of rules and parameter values (Lignos and Krawinkler 2012). The backbone curve is defined 
by three strength parameters: (1) $M_{y}$ which is the effective yield moment, (2) $M_{c}$ which is the capping strength and is specified in terms of a post-yield stiffness ratio, and (3) $M_{r}$ which represents the residual moment. Four deformation parameters used in defining the backbone curve are: (1) $\theta_{y}$, known as the yield deformation, (2) $\theta_{p}$ the pre capping plastic deformation for monotonic loading, in other words the difference between the yield deformation and deformation at maximum moment, (3) $\theta_{p c}$ representing the post-capping plastic deformation (difference between deformation at maximum moment and deformation at complete loss of strength, and (4) $\theta_{u}$ representing the ultimate deformation capacity (Lignos and Krawinkler 2011). Figure 7.4 which is taken from Lignos and Krawinkler shows the backbone curve with the specified values.

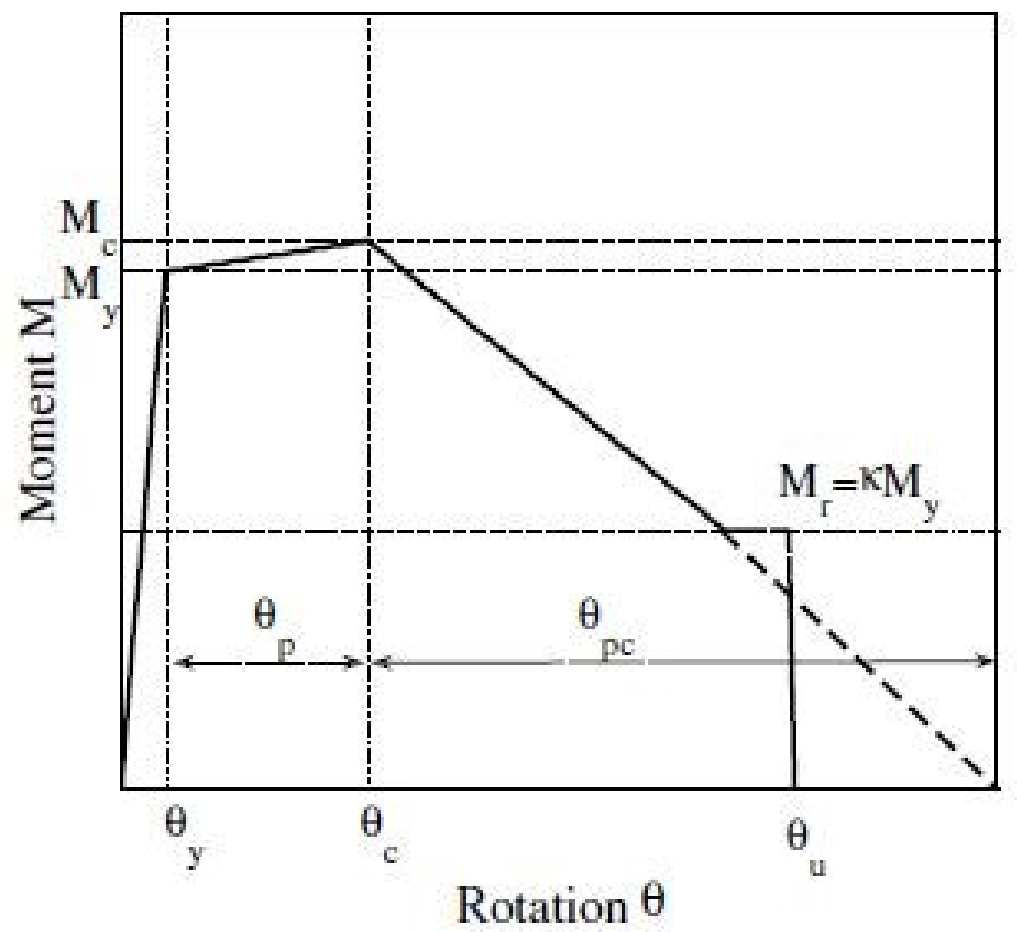

Figure 7.4 - Backbone curve for the modified IMK deterioration model with peak-oriented hysteretic response (taken from Lignos and Krawinkler 2011 with the authors' permission and, with permission from ASCE) 
Figure 7.5 taken from the OpenSees online command manual, which was originally presented in the research paper by Lignos and Krawinkler (2012), also illustrates the quantities described above.

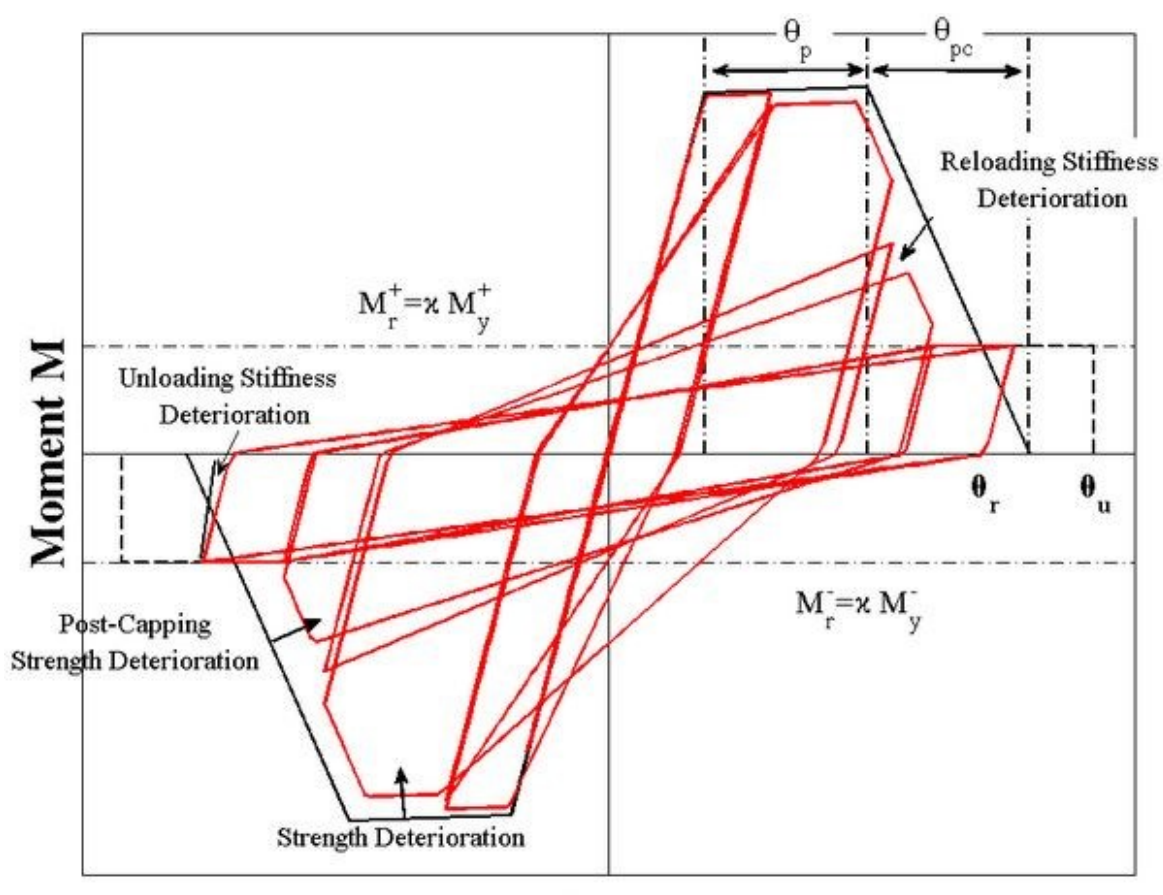

Chord Rotation $\theta$

Figure 7.5 - Illustration of the modified IMK deterioration model with peak-oriented response (taken from OpenSees command manual) (Lignos and Krawinkler 2012 with the authors' permission)

In defining the modified IMK deterioration model with pinched hysteretic response, which is used in the current study, the same parameters as defined above are used. In addition, three extra values are defined to specify the pinching of the hysteretic curve. Quantities $A_{\text {pinch }}, F_{p}{ }^{+}$and $F_{p}{ }^{-}$must be defined in order to establish the pinching behaviour. $A_{\text {pinch }}$ is defined as the ratio of the reloading stiffness to the previous loading or reloading stiffness. Parameters $F_{p}{ }^{+}$and $F_{p}{ }^{-}$are the ratios of the force at which reloading begins to the force corresponding to the maximum historic deformation demand at positive and 
negative values, respectively. Figure 7.6, taken from the OpenSees command manual and originally presented in the study paper by Lignos and Krawinkler (2012), illustrates the response of modified IMK deterioration model with pinched hysteretic response. Also, shown in the figure are certain measurements that would facilitate defining of quantities $A_{\text {pinch }}$ and $F_{p}^{+}$.

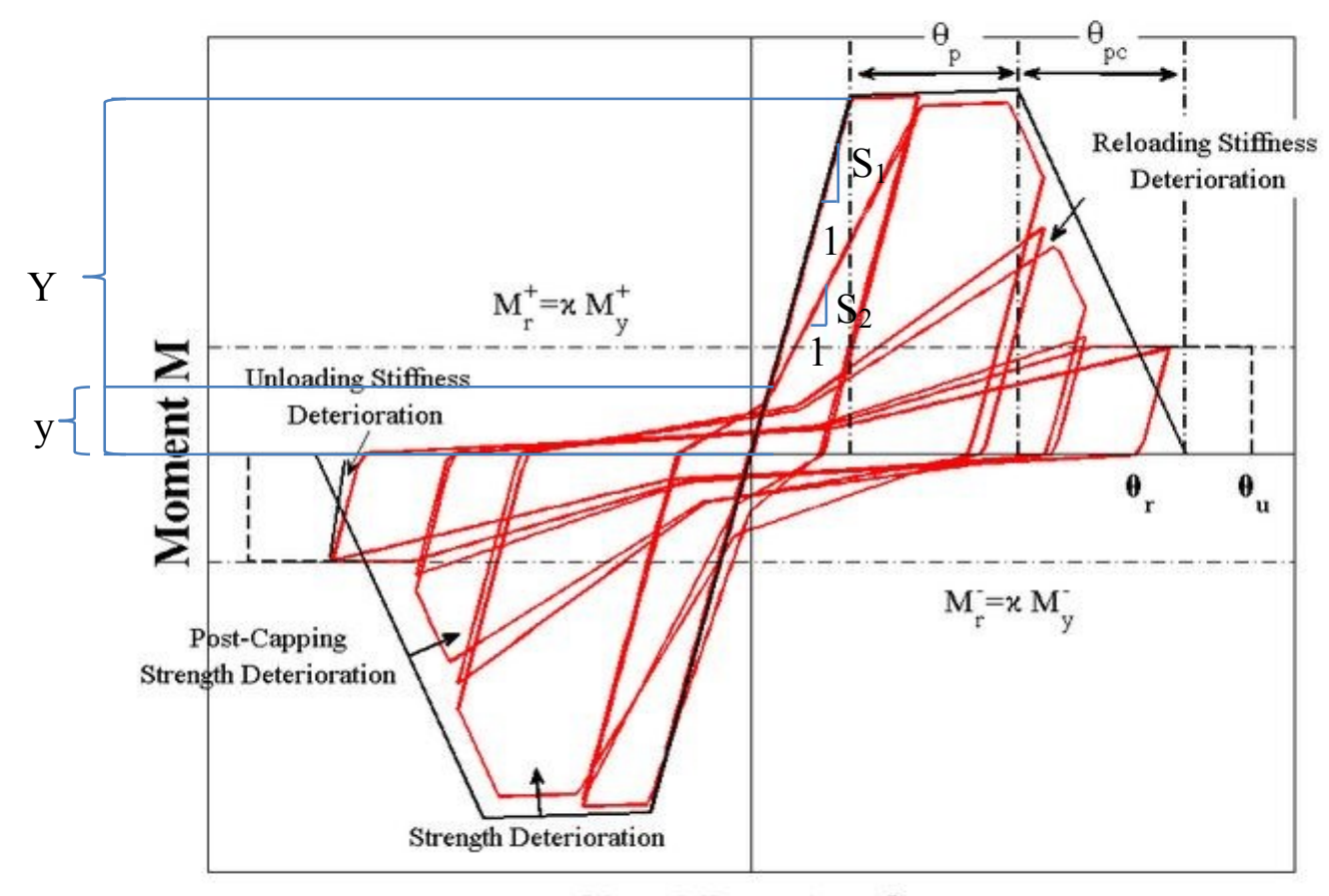

Chord Rotation $\theta$

Figure 7.6 - Illustration of the modified IMK deterioration model with pinched response (taken from OpenSees command manual) (Lignos and Krawinkler 2012 with the authors' permission)

In Figure 7.6, $S_{1}$ is the loading stiffness in one cycle and $S_{2}$ is the reloading stiffness in the following cycle, $\mathrm{Y}$ is the force corresponding to the maximum historic deformation demand and $\mathrm{y}$ is the force at which the reloading begins. Parameters $A_{\text {pinch }}$ and $F_{p}{ }^{+}$are defined as: 


$$
A_{\text {pinch }}=\frac{S_{2}}{S_{1}}
$$

$$
F_{p}^{+}=\frac{y}{Y}
$$

By specifying these pinching values as well as the previously defined force and deformation quantities, the definitions for the backbone curve in Modified IMK deterioration model with pinched hysteretic response is completed.

In order to obtain the final hysteretic behaviour, strength degradations as well as degradation of reloading stiffness and unloading stiffness, if any, must be specified. First the basis of the deteriorations for the model must be understood. The rates of the cyclic degradations are based on the rule that was originally developed by Rahnama and Krawinkler (1993). This rule, which is based on the hysteretic energy dissipation of structural components subjected to cyclic loadings, states that each structural component has a reference energy dissipation capacity which is an inherent property of the component and is not dependent on the loading history (Lignos and Krawinkler 2011). The following equations are all taken from Lignos and Krawinkler. Equation 7.3 gives the reference hysteretic energy dissipation capacity.

$$
E_{t}=\Lambda \cdot M_{y}
$$

where

$E_{t}=$ the reference hysteretic energy capacity,

$\Lambda=$ the reference cumulative rotation capacity and, 
$M_{y}=$ effective yield strength of the component.

Another parameter $\beta_{i}$ is defined as an energy-based deterioration parameter and is given by the following equation:

$$
\beta_{i}=\left(\frac{E_{i}}{E_{t}-\sum_{j=1}^{i-1} E_{j}}\right)^{c}
$$

where,

$E_{i}=$ hysteretic energy dissipation in excursion $i$,

$\Sigma E_{j}=$ cumulative energy dissipation in previous excursions,

$E_{t}=$ reference energy dissipation capacity given in Equation (7.3) and,

$c=$ empirical parameter, usually taken as 1.0

The cyclic strength deterioration, both basic deterioration and post-cap strength deterioration is defined by decreasing the two strength bounds at excursion $i$, by the rate given as:

$M_{i}=\left(1-\beta_{i}\right) \cdot M_{i-1}$

If necessary, the deterioration of stiffness can be specified in the same manner:

$$
K_{i}=\left(1-\beta_{i}\right) \cdot K_{i-1}
$$

Different rates of degradations for strength or stiffness can be modeled using different values of $\Lambda$ and $c$. However, the $c$ value is usually taken as 1.00 and $\Lambda$ is determined by 
calibration. The model is often calibrated based on the specimen under study and the empirical values are determined so that the analytical results match that of the experimental study.

Given the fact that the hysteretic response of the diaphragm specimen in shear in which screws are used as side lap connectors and arc spot welds are used as deck to frame fasteners exhibits sever pinching and strength degradation, the modified IMK deterioration model with pinched hysteretic response seems quite appropriate since it is capable of accounting for both pinching and degradations in strength and stiffness. Moreover, modified IMK deterioration model with pinched hysteretic response is implemented in both Drain D2X and OpenSees platform and can be used for finite element models conveniently.

\subsubsection{Hysteretic Model Calibration}

In order to calibrate the modified IMK pinched deterioration model with the experimental results of Essa et al. (2003) shown in Figure 7.7(b), the initial stiffness and the yield strength of the specimen are determined visually from Figure 7.7(b). Next, a single element is modelled in OpenSees. A displacement controlled analysis is carried out on the element in which the displacement time series is determined visually from the hysteretic response in Figure 7.7(b). The $c$ value is assumed to be 1.00. It is ensured that the deformations would not reach the pre-capping deformation (plastic deformation capacity) by giving $\theta_{p}$ in both positive and negative regions a large value (i.e. $200 \times \theta_{y}$ ). This way, the hysteretic behaviour would become elasto-plastic with strength deterioration and pinching. The degradation of reloading stiffness and unloading stiffness 
and the post-capping strength is assumed to be zero $(\Lambda$ corresponding to these values is set equal to zero). The reason for this assumption is that the stiffness deterioration is negligible and the post-capping region would not be reached since a large value is assigned to $\theta_{p}$.

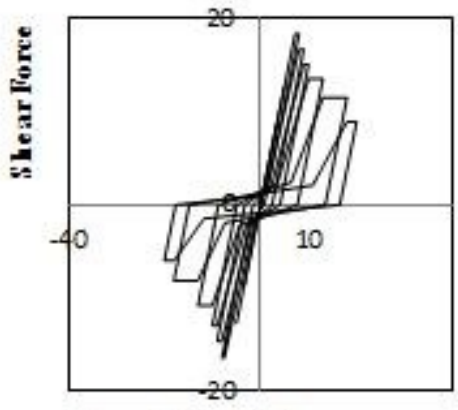

Shear Deformations

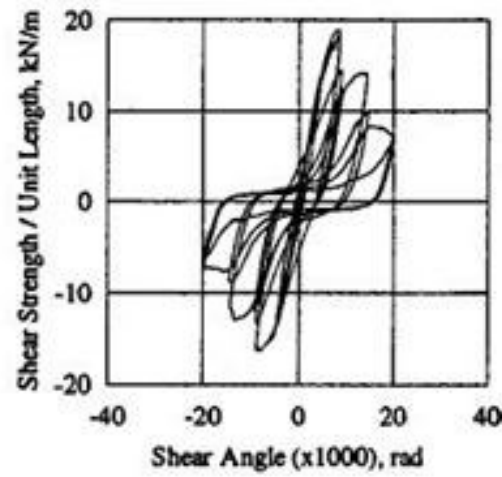

(b)

(a)

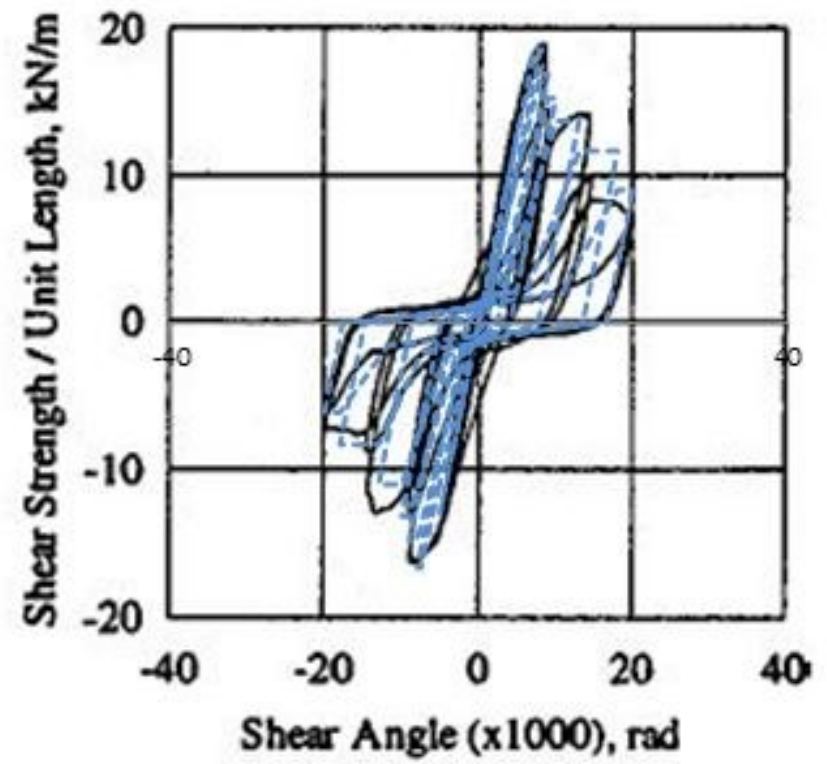

Figure 7.7 - The calibration of the analytical model based on the experimental results on the diaphragm specimen with screwed and weld sidelap and deck to frame fasteners (Essa et al 2003) -

(a) The response of the calibrated element under displacement controlled analysis (b) The experimental hysteretic curve (Essa et al 2003 with permission from ASCE) (c) The superimposed curves for assessing the calibration effectiveness 
Based on the hysteretic curve from experimental results, the stiffening in pinching approximately occurs at one third of the yield strength. Therefore, $F_{p}{ }^{+}$and $F_{p}{ }^{-}$are selected as 0.3 times the yield strength. In order to determine the $\Lambda$ value associated with cyclic strength deterioration and $A_{\text {pinch }}$ value several analysis runs are carried out on the element. After a few cases of trial and error and observations of the results, the optimized values for $A$ and $A_{\text {pinch }}$ are determined to be 0.04 and 0.75 respectively. With this selection, the analytical model matches the experimental results fairly well. Figure 7.7 shows the response of the calibrated model under displacement controlled analysis along with the hysteretic curve obtained from the experiment (Essa et al. 2003) as well as an illustration in which the two are superimposed.

Based on the calibration outlined above, the following parameter values are determined:

$\Lambda$ associated with cyclic strength deterioration $=0.04$

$\Lambda$ associated with post-capping cyclic strength deterioration $=0.00$

$\Lambda$ associated with accelerated reloading stiffness deterioration $=0.00$

$\Lambda$ associated with unloading stiffness deterioration $=0.00$

$F_{p}{ }^{+}=0.3 \times$ yield strength

$F_{p}^{-}=0.3 \times$ yield strength

$A_{\text {pinch }}=0.75$

A large value is assigned to each of $\theta_{p}, \theta_{p c}$, and $\theta_{u}$. This way the deformations will not reach the post-capping region. The $c$ values are set equal to the default value which is 1.0. 
Based on the outlined calibration, a finite element model is developed in the OpenSees platform.

\subsection{The OpenSees Model}

\subsubsection{Nodes}

As pointed out earlier, the deep beam, representing the diaphragm consists of 20 elements. Thus, 21 nodes are defined along the length of the diaphragm. Two additional nodes are located at the ends of the beam to allow modelling the zero-length elements that represent the spring supports (braces) at each end. Figure 7.8 shows a schematic illustration of the beam/spring model used in OpenSees. It may be noted that for simplicity, only 7 nodes rather than 21 nodes are shown.

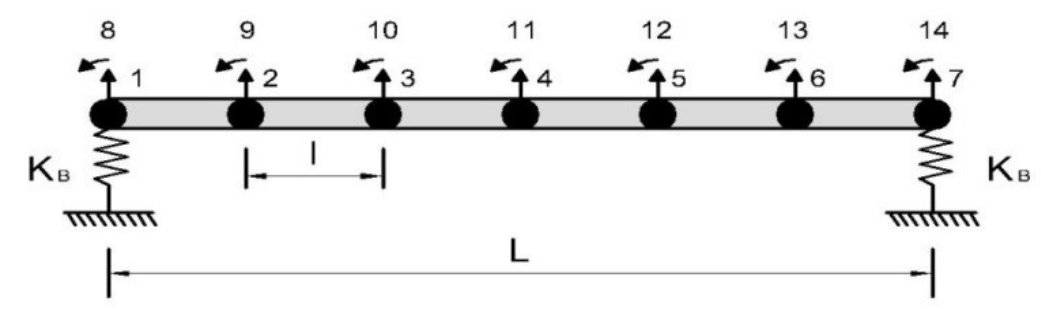

Figure 7.8 - Schematic illustration of the analytical model with 7 nodes

\subsubsection{Materials}

In this part of the study, the steel deck diaphragm may behave either in the elastic or the inelastic range. When the diaphragm is expected to behave in the inelastic range, nonlinear hysteretic relationship for shear must be used. Also, strength degradation as well as pinching must be modelled in the hysteretic behaviour of diaphragm in shear. The 
"modified IMK deterioration model with pinched hysteretic response" uniaxialMaterial is used in OpenSees to account for nonlinear shear deformations in the deep beam, as well as the hysteretic behaviour with pinching and strength degradation. The strength of the members corresponds to the target ductility demand and is obtained during an iterative set of analyses. Initial stiffness is determined from $\mathrm{G}^{\prime}$ and the dimensions of the building in a direction parallel to the seismic load. The values of the remaining variables are set as described under calibration. The elastic uniaxialMaterial model is used to derive the moment-curvature relationship under flexure. Next, using the section aggregator the two material behaviours are combined to model the interconnected beam elements representing the diaphragm.

When the braces are assumed to be elastic, elastic uniaxialMaterial is selected to model the behaviour of the braces. The stiffness of the braces is taken to be the same as the spring stiffness obtained in the design of the buildings (Tremblay and Steimer 1996).

When the braces are expected to be strained into the nonlinear range, the post-yield stiffness ratio for the braces is taken as 0.05 . The diaphragm seismic design force is determined for two different performance behaviours in the diaphragm: (1) elastic behaviour and (2) nonlinear behaviour with the $\mu \leq 1.5$. The Steel 02 uniaxialMaterial is assigned to the braces with the post-yield stiffness of 0.05 and an initial stiffness equal to that of the springs. 


\subsubsection{Elements}

A nonlinearBeamColumn element with 5 integration points is used to represent the beam elements. The section aggregator, referred to earlier, is used to account for both shear and flexural deformations in the elements.

A zeroLength element is used for the springs representing the LLRS. The elastic or the Steel 02 materials, referred to earlier, are assigned to the zero-length element to account for axial force-displacement relationship.

\subsubsection{Ground Motion}

Using a UniformExcitation pattern command, the ground motion time histories are applied to the model in the direction parallel to the axis of the defined springs.

\subsubsection{Dynamic Analysis}

The ConstraintHandler object is used in OpenSees to determine how the constraint equations are enforced; a plain constraint being used in the present study. RCM numberer is used as the DOF_Numberer object. UmfPack is selected as the solver. The convergence test comprises a tolerance of $1.0 \mathrm{e}-10$ and a maximum of 10 iterations. The Newton algorithm is used as the solution algorithm. To perform integration of the equations of motion, Newmark integrator is employed with \$gamma and \$beta factors equal to 0.5 and 0.25 , respectively. After setting the aforementioned parameters, a transient analysis command is used to perform dynamic analysis. 


\subsection{Hysteretic Response of the Example Building and the Behaviour in Shear}

In order to further illustrate the nature of shear deformations in the roof diaphragm, the hysteretic behaviour of the example building $\mathrm{SH} 1$ when subjected to $\mathrm{M} 6 \mathrm{C} 2$ ground motion is shown for three cases: (1) Target ductility of 1.5, (2) Target ductility of 2.0, and (3) when using a force reduction factor of 1.5.

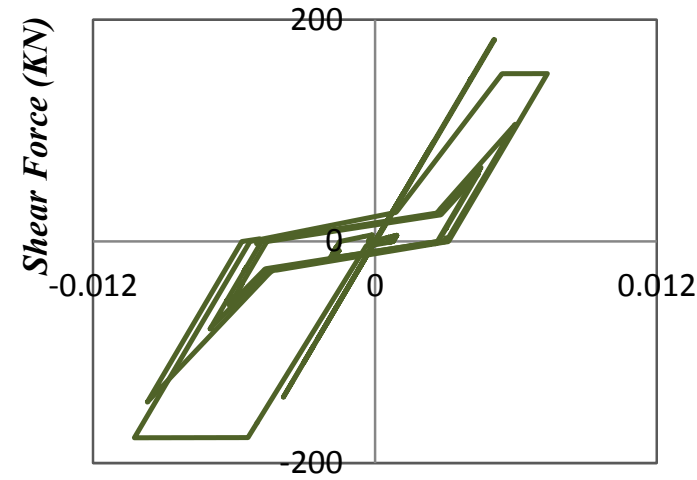

Shear Deformations (rad)

(a)

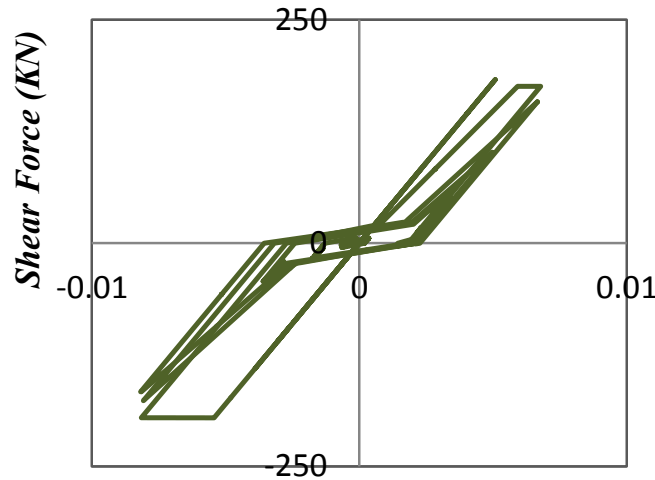

Shear Deformations (rad)

(b)

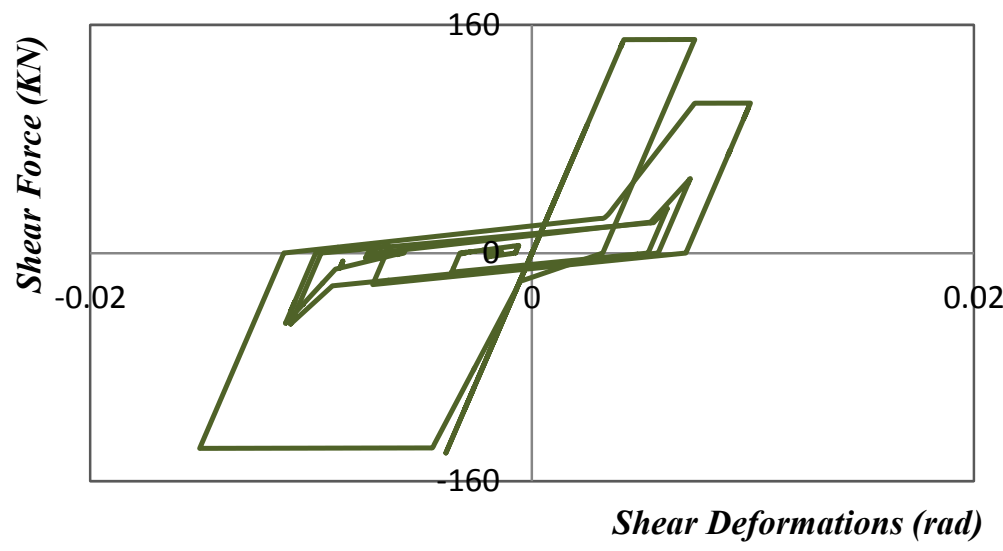

(c)

Figure 7.9 - Hysteretic shear behaviour in building SH1 subjected to M6C2 - (a) $\mu=2.0$, (b) $\mu=1.5$, and (c) $R_{d}=1.5$ 
As shown in Figure 7.9 (a) and (b), often strength degradation does not occur when the ductility demand values are limited to 2.0 or 1.5 . In Figure 7.9 (c) however, strength degradation is observable. Throughout the study when the target ductility was limited to 1.5 and 2.0, strength degradation was not observed and therefore did not contribute to any increase in the ductility demand. Therefore, in the final analytical model, the strength deterioration is neglected. The final hysteretic behaviour for the diaphragm steel decks is specified by setting the strength deterioration parameter equal to zero. Figure 7.10 shows the final hysteretic behaviour used in the analytical model. It can be seen that the hysteretic model exhibits sever pinching.

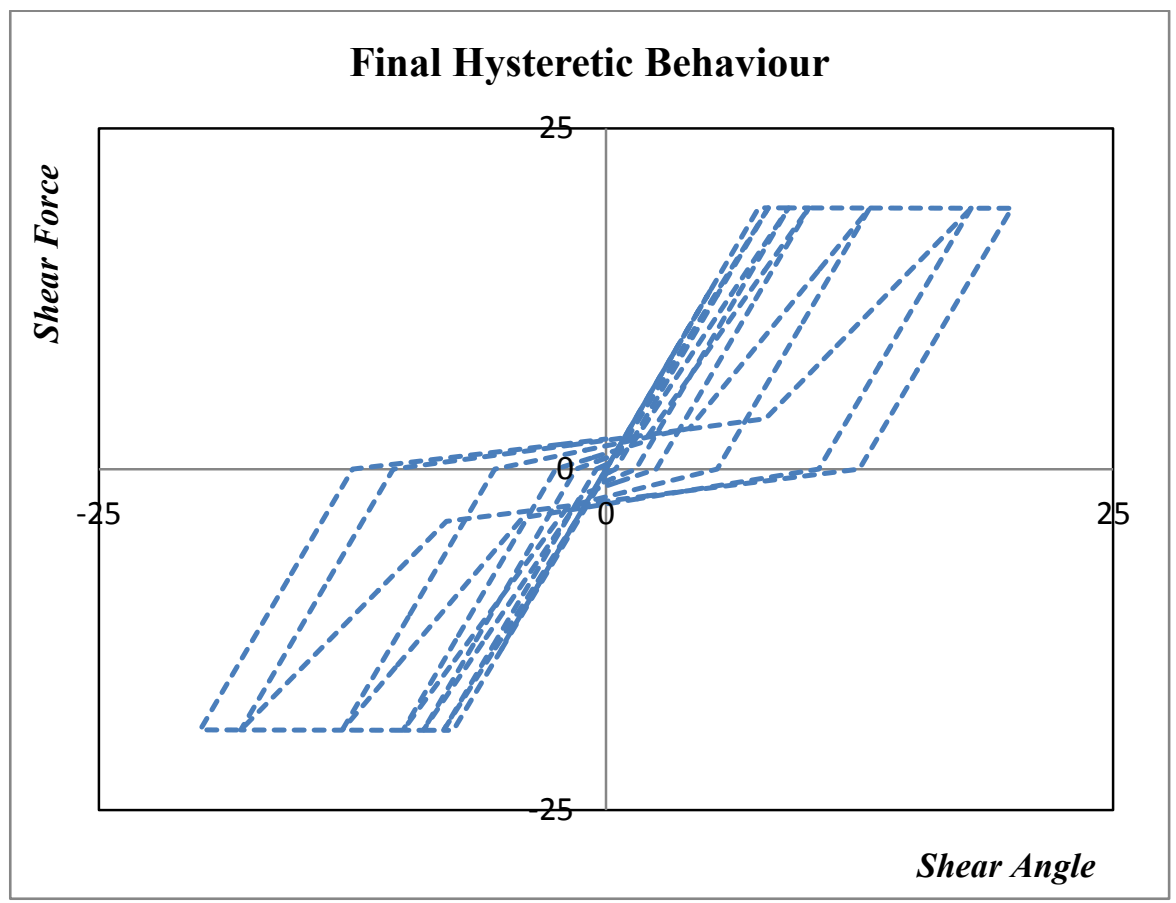

Figure 7.10 - Final shear hysteretic behaviour of the steel deck diaphragms 


\subsection{Diaphragm as a the Energy Dissipating Element}

As mentioned earlier, the common design approach for one-storey buildings with flexible diaphragms is to design the braces as the sole energy dissipating elements. In such a design approach, the diaphragm deformations are expected to remain in the elastic range while the braces dissipate the seismic energy through inelastic deformations. However, as discussed earlier there is interest in developing an alternative design approach in which the diaphragm, rather than the braces, is designed as the weak link in the system.

In this part of the study, an attempt is made to assess the performance of one-storey buildings with flexible diaphragms which are designed in accordance with the aforementioned alternative approach. For this purpose, the selected buildings are modelled in OpenSees platform. The nonlinear force-deformation relationship discussed in the previous sections is assigned to the diaphragm to account for the shear behaviour in the diaphragm, while an elastic behaviour is assumed for the braces. The buildings are subjected to the ground motions selected for the study and direct time step integration of the equations of motion is carried out.

\subsubsection{Dynamic Analyses Results}

In the first set of dynamic analyses, the entire structure is assumed to behave elastically and the elastic earthquake force imposed on the diaphragm is found. Next, the diaphragm is assigned a strength equal to the elastic seismic force divided by a ductility related force reduction factor $\left(R_{d}\right)$ of 1.5 , and the analyses are repeated to determine the ductility demand in the steel deck diaphragm to see whether the resulting ductility demand is acceptable. The reason for selecting a value of $R_{d}=1.5$ is as follows. The NBCC 2010 
(NBCC 2010 - Clause 4.1.8.15.) states that the diaphragms, collectors, chords, struts and connections must be designed for a seismic design load equal to the elastic seismic load reduced by a factor $R_{o} \times R_{d}=2.0$. Assuming a value of 1.3 for the over-strength related modification actor $\left(\mathrm{R}_{\mathrm{o}}\right)$, the $\mathrm{R}_{\mathrm{d}}$ consistent with this clause would be 1.5 .

When the analyses were carried out, it was observed that the ductility demand values associated with $\mathrm{R}_{\mathrm{d}}=1.5$ were much greater than 1.5 , at times exceeding 7 . Figure 7.11 shows a histogram, indicating the frequency of occurrence of different values of ductility demand for 160 cases when the ductility related force modification factor $R_{d}$ of 1.5 is selected to obtain the seismic design force for the diaphragm. It can be observed that in a substantial number of cases the ductility exceeds 2 and is significantly higher in many cases. It must be noted that in the analytical model the strength deterioration is neglected due to reasons outlined earlier, particularly since the ductility should be limited to a low value. If the ductility demand exceeds values of 1.5 and 2.0 strength degradation would be expected to occur leading to a further increase in the ductility demand on the diaphragm.

The levels of ductility observed in the analyses referred to in the preceding paragraph cannot be achieved in shear deformations. In fact, based on the equal displacement principle the force reduction factor of 1.5 is selected with the expectation that the ductility demand will also be 1.5. Obviously, this expectation is not fulfilled with a flexible diaphragm and an appropriate force reduction factor, which would be less than the target ductility of 1.5 , must be determined. 


\section{Frequency of Occurrence for Ductility Demand Values}

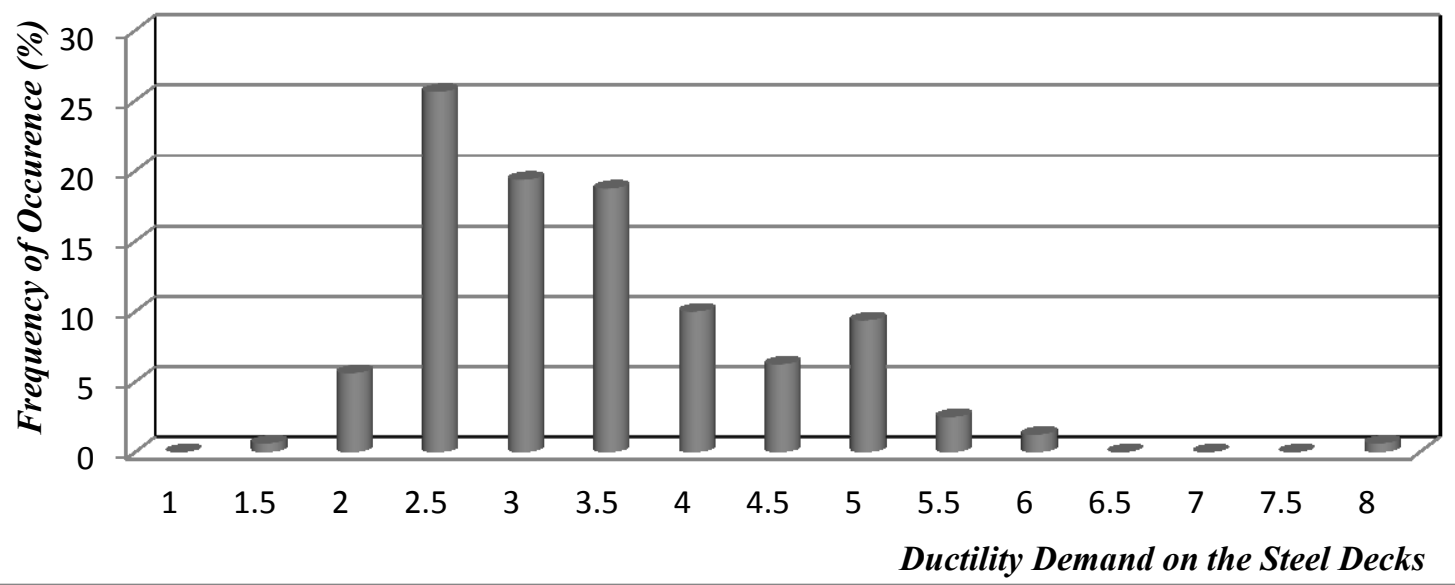

Figure 7.11 - The frequency of occurrence for the ductility demand on the diaphragm

To determine appropriate values for the force reduction factor, two target ductility demand values $(\mu=1.5$ and $\mu=2.0)$ are selected for the diaphragm. Next, using trial and error, the force reduction factor corresponding to these levels of ductility demand values are determined for each building. This involves a number of iterative analytical runs. In addition, the force magnification factors defined in Chapter 5 (M.F, V.F, M.U and V.U) are calculated for the buildings at each ductility level to investigate whether the nonlinearity in the diaphragm increases the contribution of higher modes and the internal force magnification values.

\subsubsection{Force Magnification Factors}

With 16 buildings, ten ground motion records and two targets of ductility demand, 320 cases were analyzed. The complete results obtained from this portion of the study are presented in Appendix E. In Figures 7.12 to 7.15 the magnification factor values have 
been plotted against the ductility demand in the diaphragm. The results presented in Appendix E and Figures 7.12 to 7.15 show that an increase in the nonlinearity (ductility demand) in the diaphragm, does not lead to any significant change in the force magnification factors.

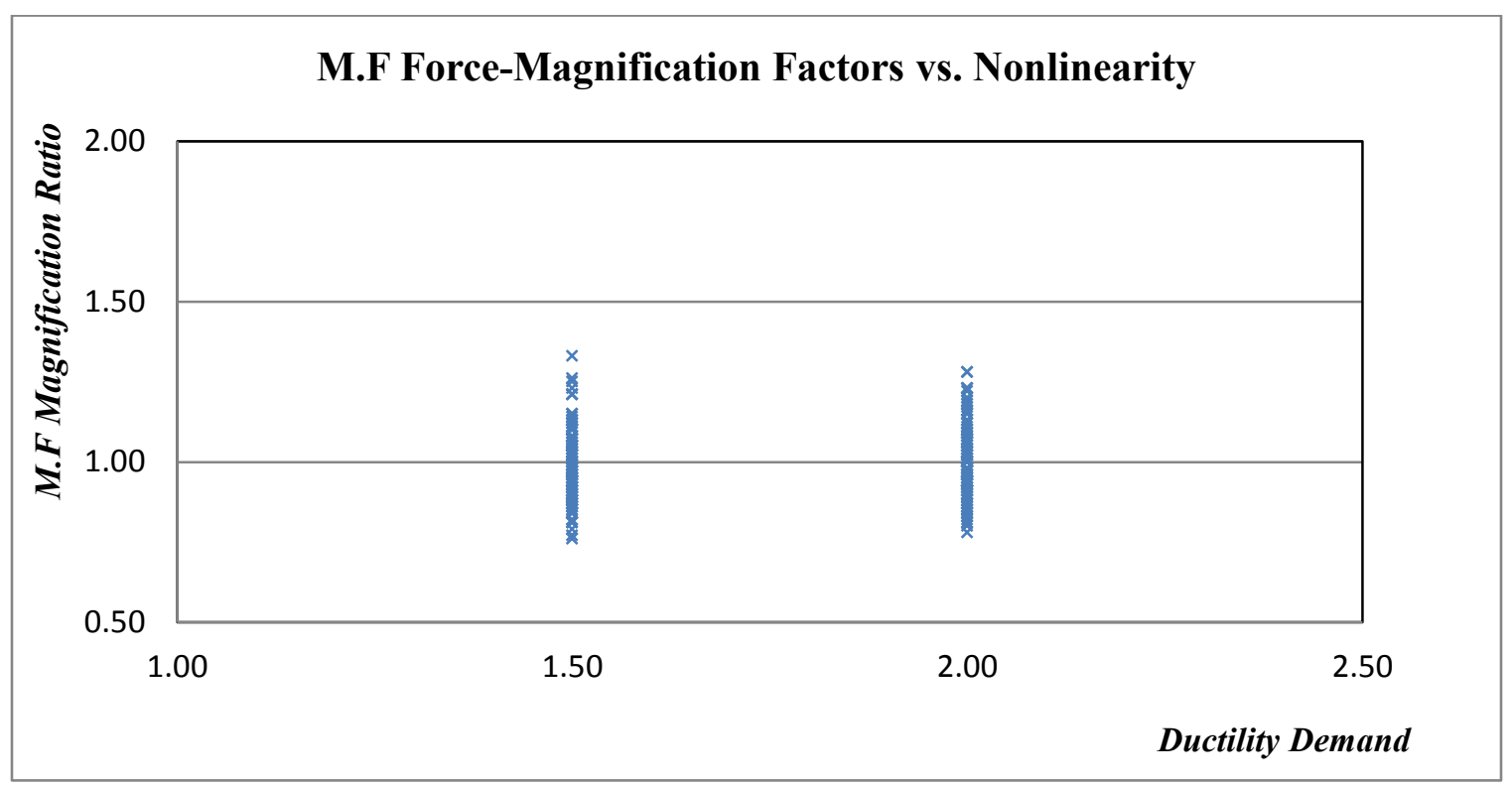

Figure 7.12 - M.F magnification factors vs. the ductility demand in the diaphragm 


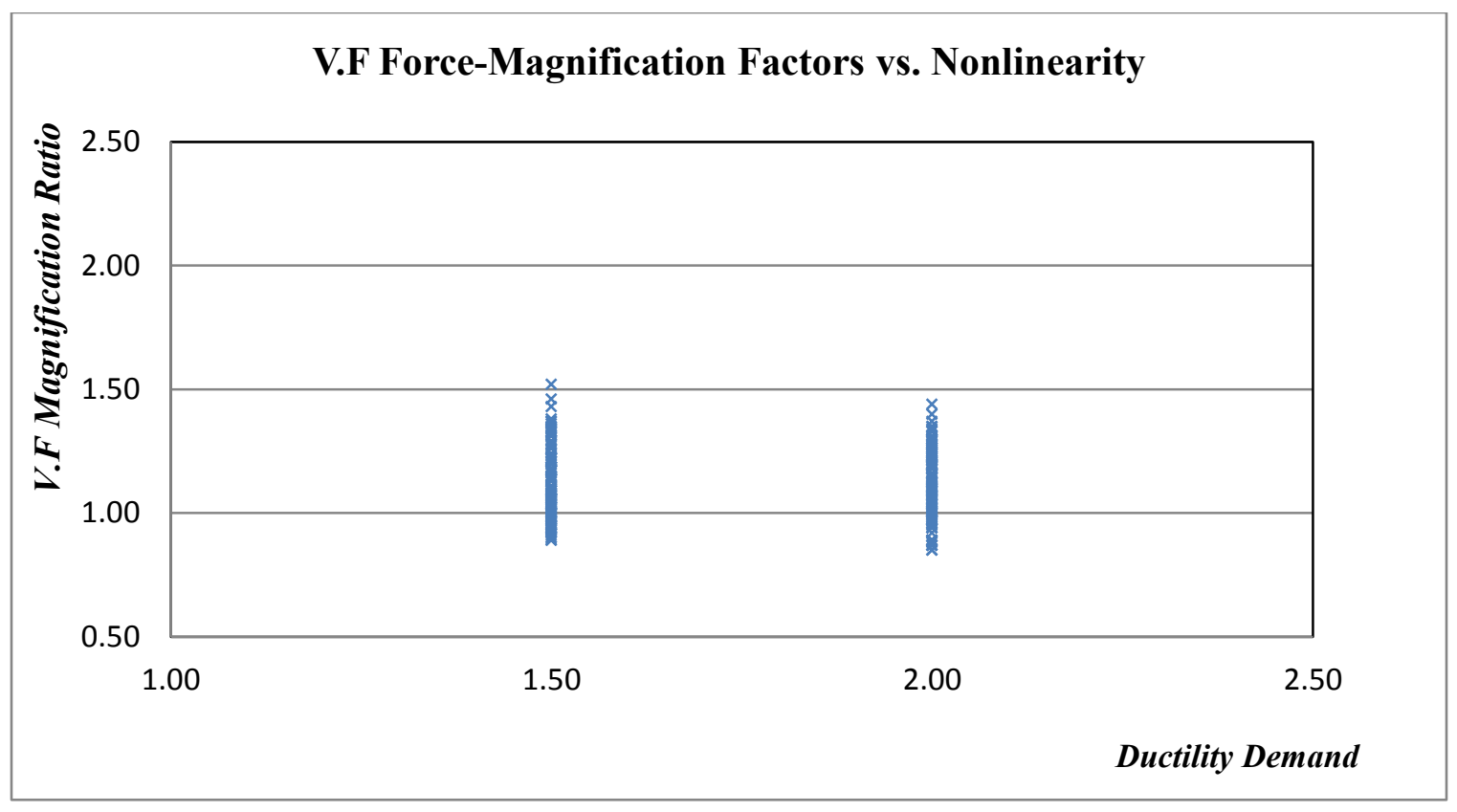

Figure 7.13 - V.F magnification factors vs. the ductility demand in the diaphragm

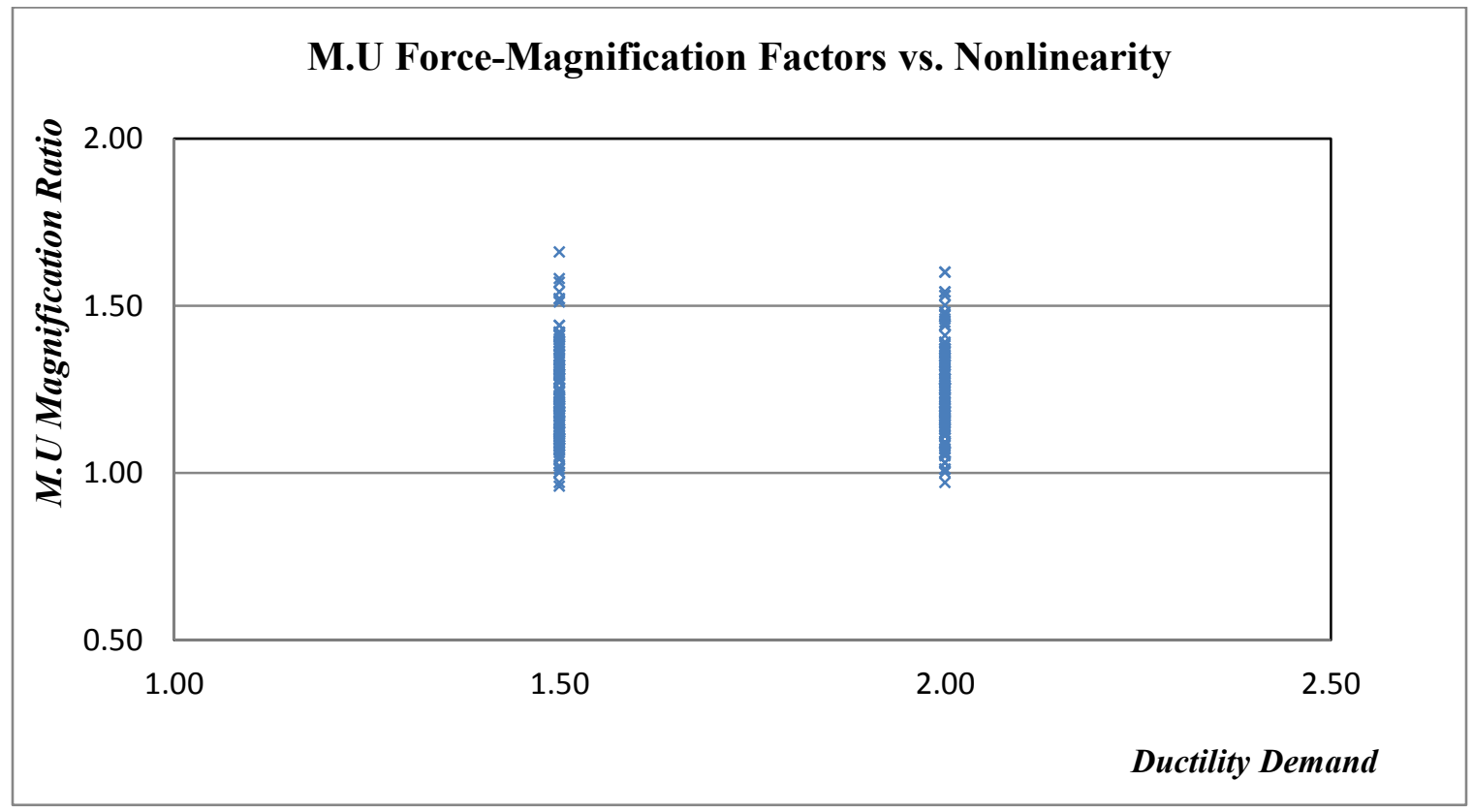

Figure 7.14 - M.U magnification factors vs. the ductility demand in the diaphragm 


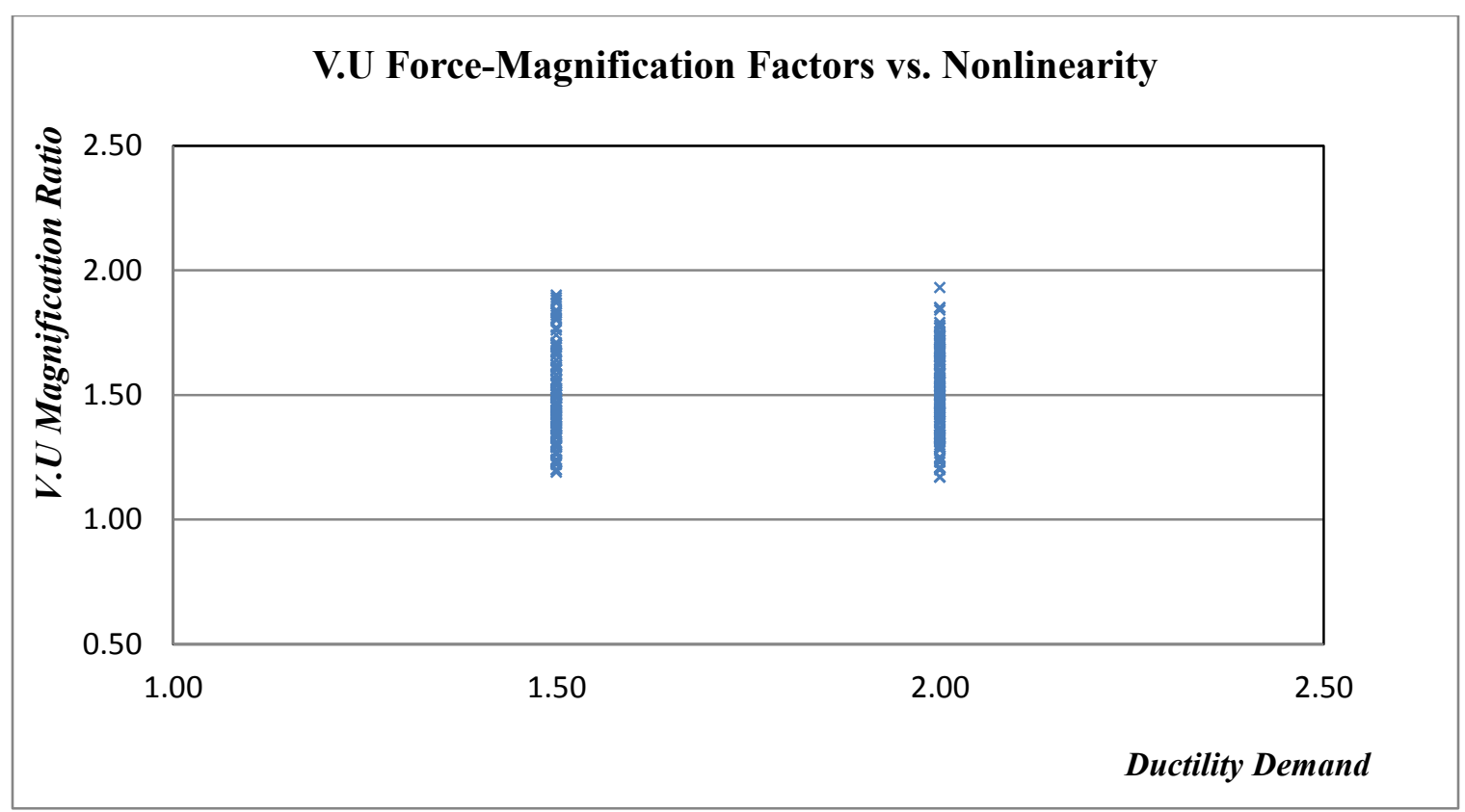

Figure 7.15 - V.U magnification factors vs. the ductility demand in the diaphragm

The results of the analyses show that the nonlinearity in the diaphragm does not lead to a greater contribution from the higher modes and the magnification values stay the same. Also, as found in studies reported in Chapter 5 where it was observed that for elastic braces, as is the case here, the FEMA parabolic distribution of the seismic force appears to give good prediction for the shear force at the quarter span and the bending moment at the mid-span.

Since the braces remain elastic, the contribution of higher modes is limited and the shear force at the quarter span is not as high as when the braces are strained in the nonlinear range. The nonlinearity in the diaphragm occurs mostly in the beam elements located at the two ends of the diaphragm where the shear force is at its maximum. Such behaviour was observed in most cases with only a few exceptions. In such exceptional cases the frequency content of the earthquake may have matched the frequency of higher modes in 
the buildings and hence the higher modes made a more significant contribution to the total response of the system. It may be noted that nonlinearity in the braces is not the main reason for an increase in the contribution of the higher modes, although it magnifies such behaviour. The main driving force for an increase in the contribution of higher modes is the frequency content of the earthquake and the frequency of higher modes in the building. This observation is based on the fact that nonlinear behaviour in the middle beam elements was observed mostly in those cases where the structural system was subjected to eastern ground motions. The reason is that the spectral shape for the east is steeper causing greater contribution from the higher modes

\subsubsection{Development of an Equation for Predicting the $\mathbf{R}_{\mathbf{d}}$ Factor}

The results of the analyses that provide values of $R_{d}$ factor that are appropriate for achieving a given target ductility demand suggest that $R_{d}$ is in direct proportion to the drift ratio of the structure. In order to better observe this correlation, the $R_{d}$ factors obtained from the analyses are plotted against the drift ratios for each target ductility demand. In addition, a regression fit is carried out on the results to develop an equation that would relate the ductility related force reduction factor to the drift ratio of the system. Figures 7.16 and 7.17 show plots of the data as well as the regression fits. 


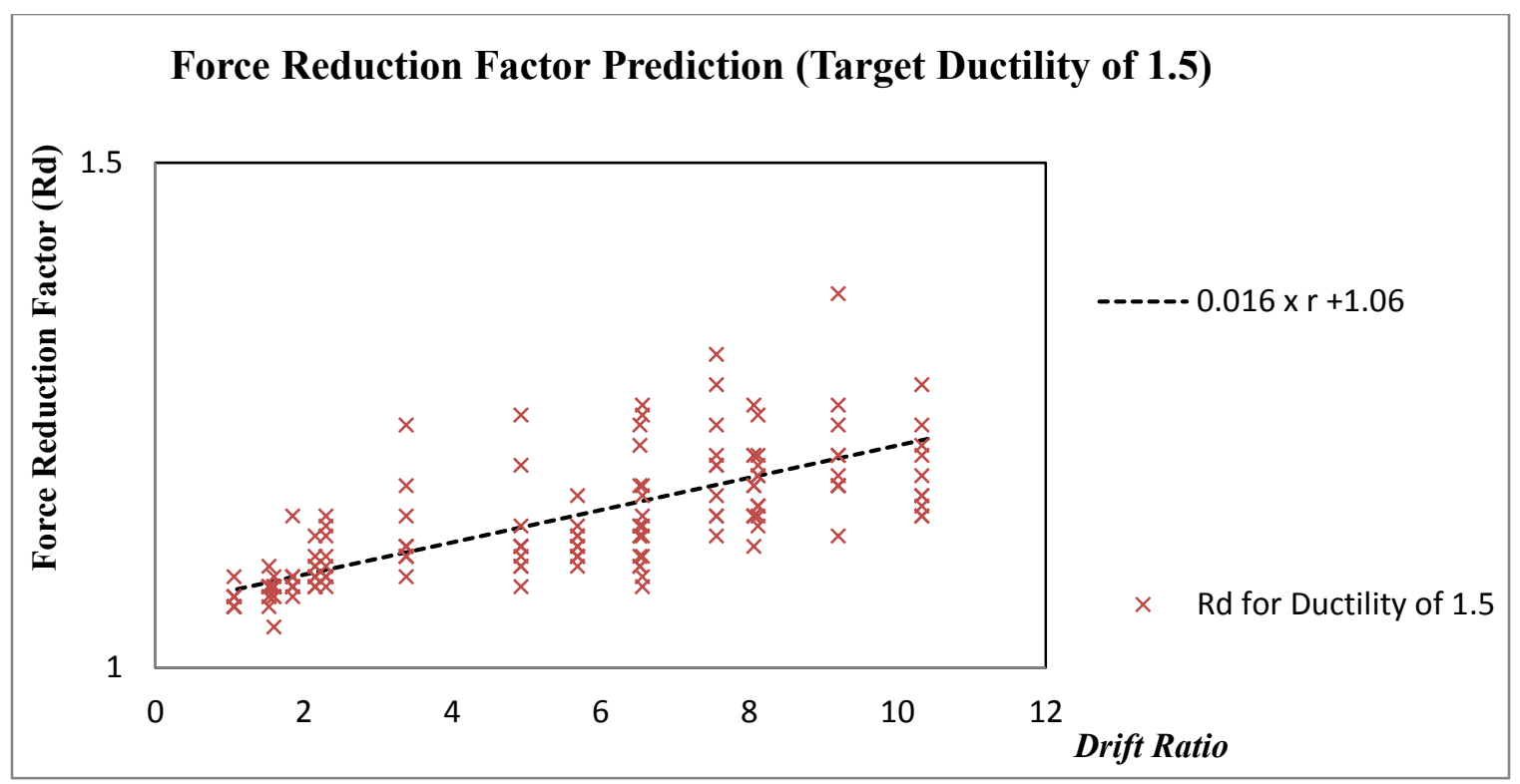

Figure 7.16 - Force reduction factors vs. drift ratio values for target ductility of 1.5

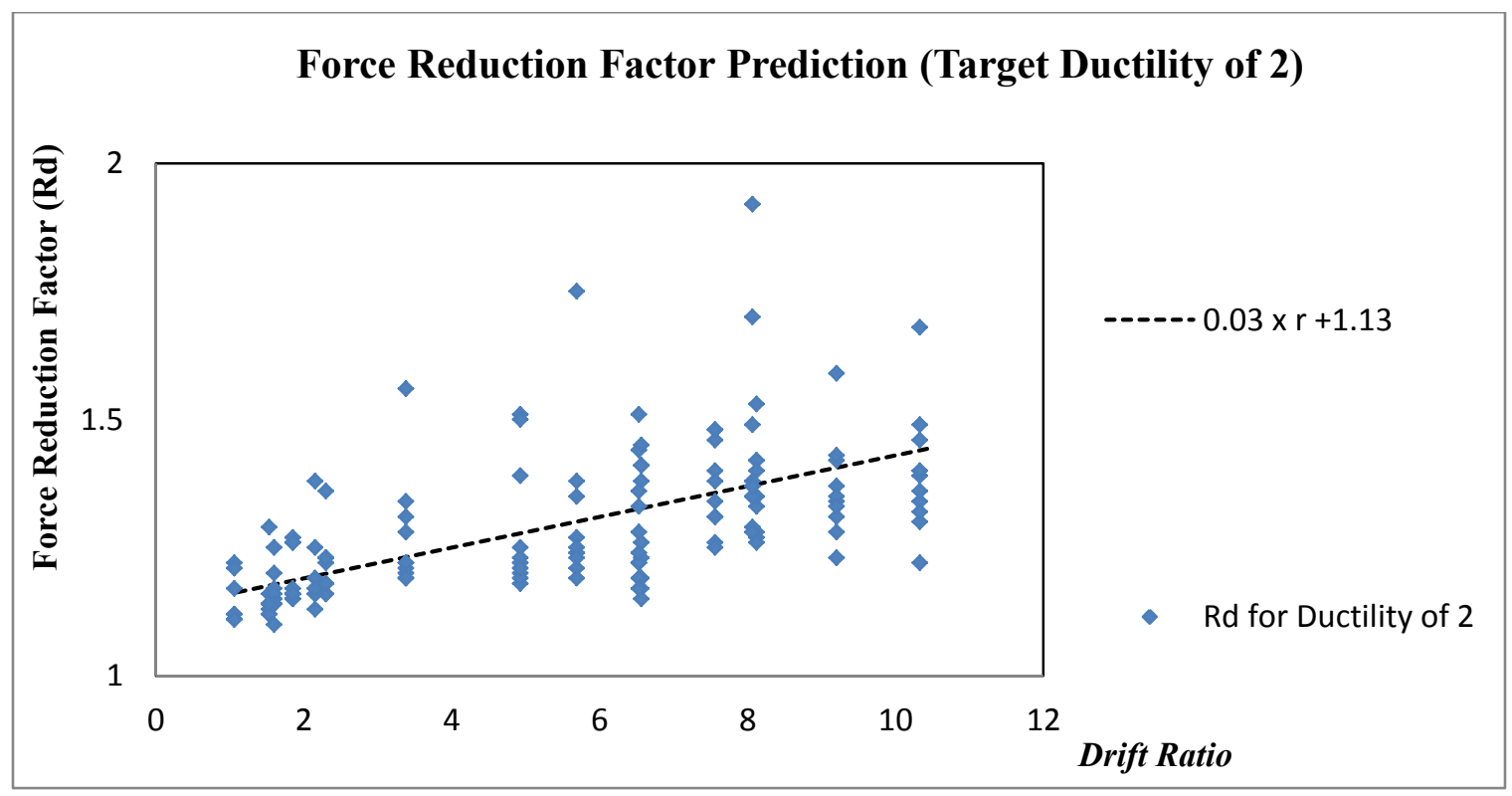

Figure 7.17 - Force reduction factors vs. drift ratio values for target ductility of 2.0 
Based on the regression fits carried out, two equations are suggested to predict the force modification factor appropriate for the design of the diaphragm. Each equation corresponds to a specific level of ductility demand on the roof deck diaphragm. The suggested equations are as follows:

$R_{d}=1.06+0.016 r$, for $\mu=1.5$

$R_{d}=1.13+0.03 r \quad$, for $\mu=2.0$

Figure 7.18 shows both sets of data and the suggested equations corresponding to both levels of ductility demand values. Although there is considerable scatter in the data, the suggested relationships may be considered as being appropriate for the purpose of design.

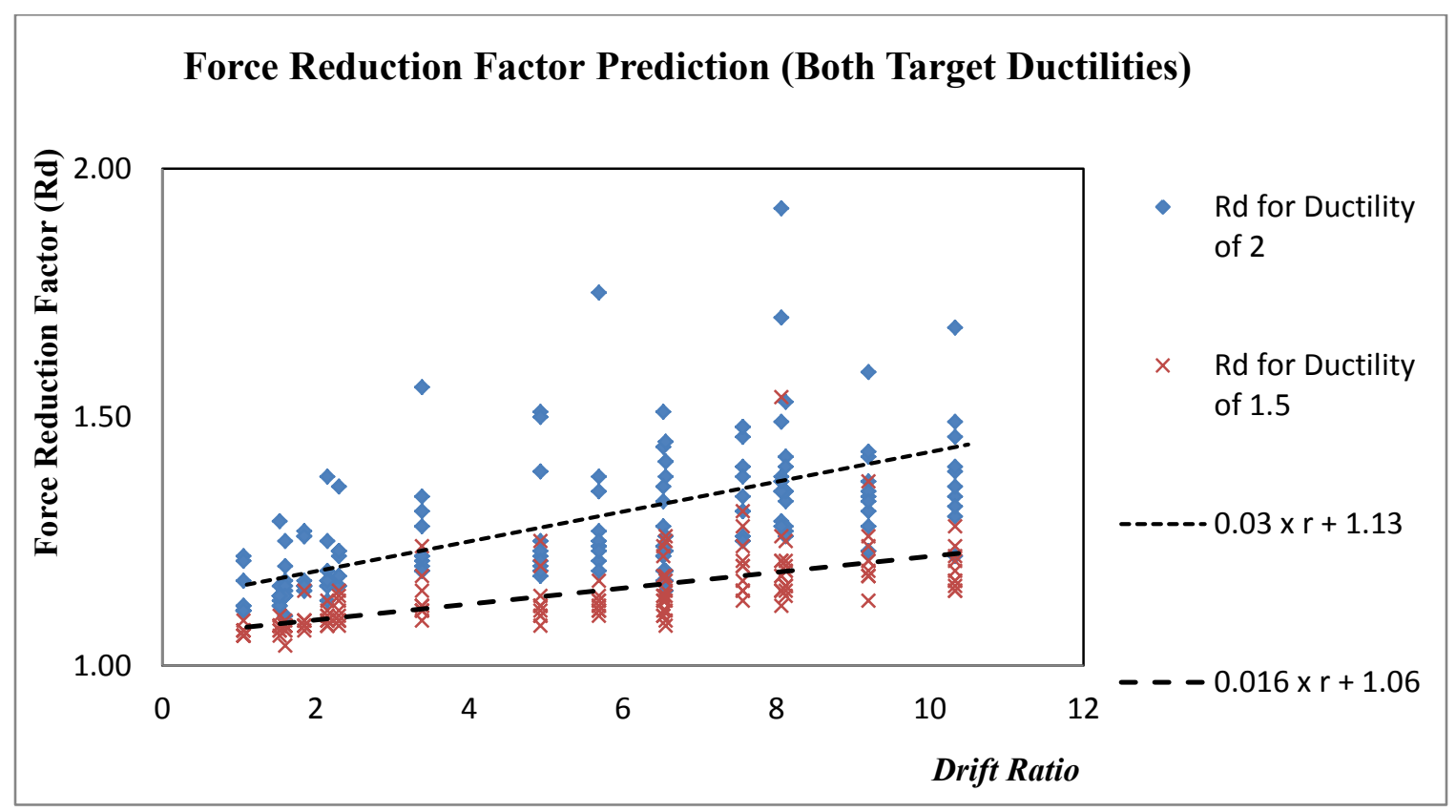

Figure 7.18 - Force reduction factor data vs. the drift ratio and suggested equations 


\subsubsection{Residual Displacements}

As mentioned earlier, one advantage of designing the roof diaphragm to be the energy dissipating member is that the residual displacements would be confined to the steel deck panels and the braced frame would stay intact making the building suitable for posthazard occupancy. To better illustrate such assessment, building $\mathrm{SH} 1$ is subjected to M6C2 record twice, first with the braces as the weak link and next with the roof diaphragm as the weaker element.

When the braces are designed to be the energy dissipating elements, a bilinear behaviour with a post-yield stiffness of 0.05 is assumed for the braces and the seismic elastic force is reduced to match a target ductility of 3.0. Figures 7.19 and 7.20 show, respectively, the displacement time history at the top of the bracing system and at various locations along the diaphragm. Figure 7.19 shows that there is a residual displacement in the braces.

Figures 7.21 and 7.22 show the displacement time-histories for the same building subjected to the same ground motion. However, this time, the diaphragm is acting as the energy dissipating member. The figures indicate that there is no residual displacement at the top of the bracing system. The building is therefore suitable for post-hazard occupancy. Such behaviour is expected since the braces remain elastic and was observed in all of the cases analysed. 


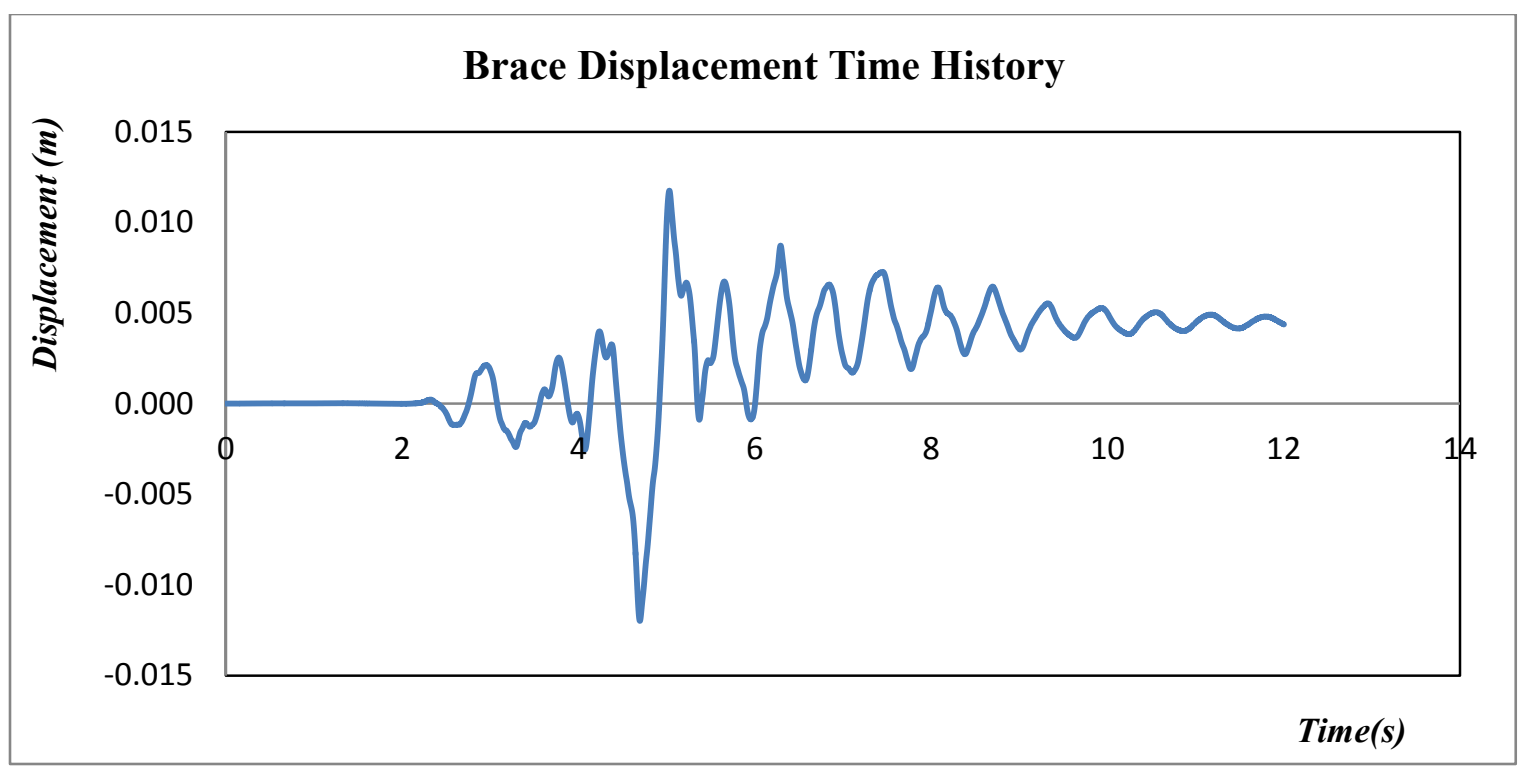

Figure 7.19 - The brace displacement time history in building SH1 subjected to M6C2 with the brace as the energy dissipating member and the target ductility of 3.0

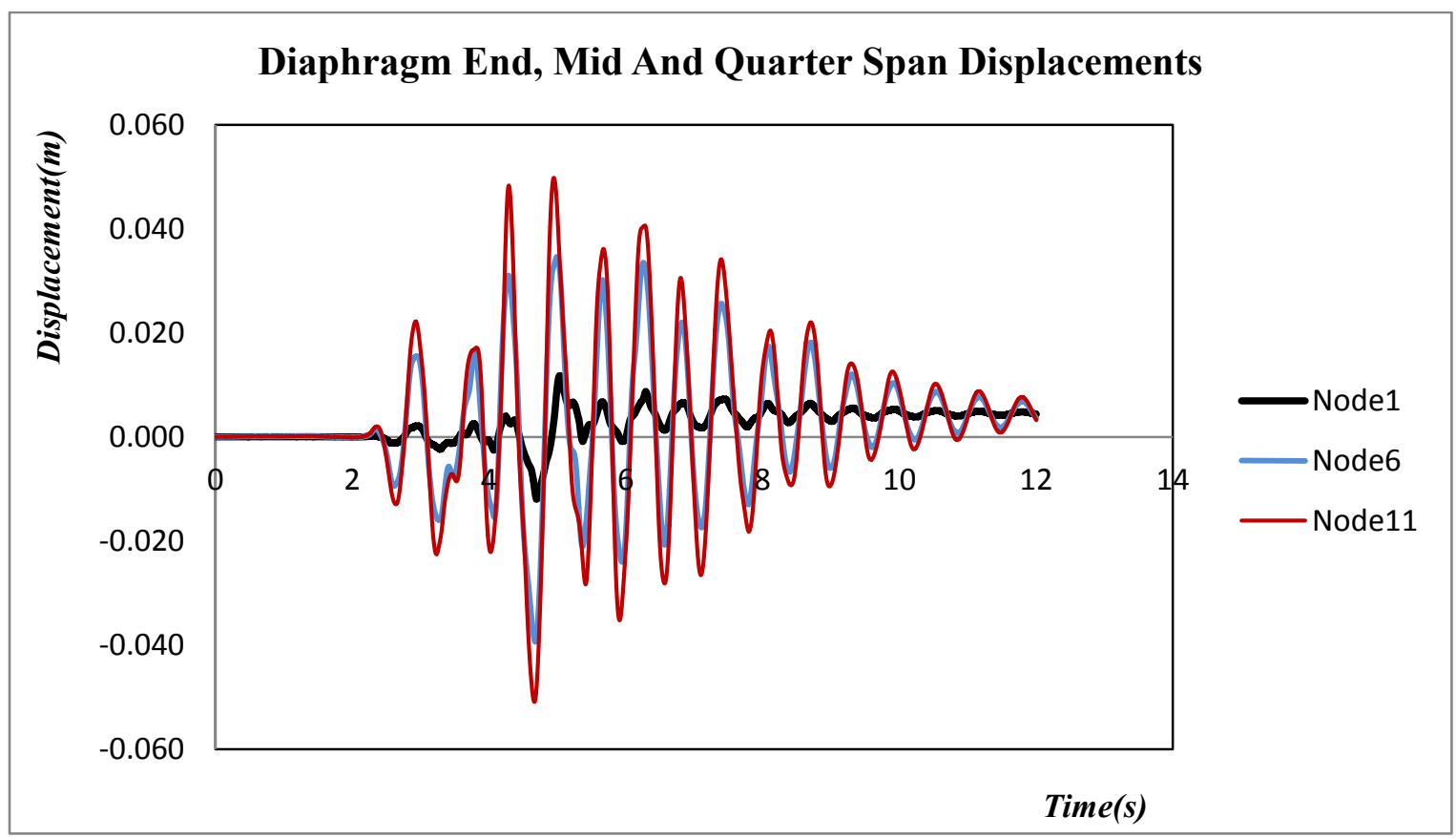

Figure 7.20 - Displacement time-histories at different locations along the length of the diaphragm in building SH1 with brace as the energy dissipating member and the target ductility of 3.0 


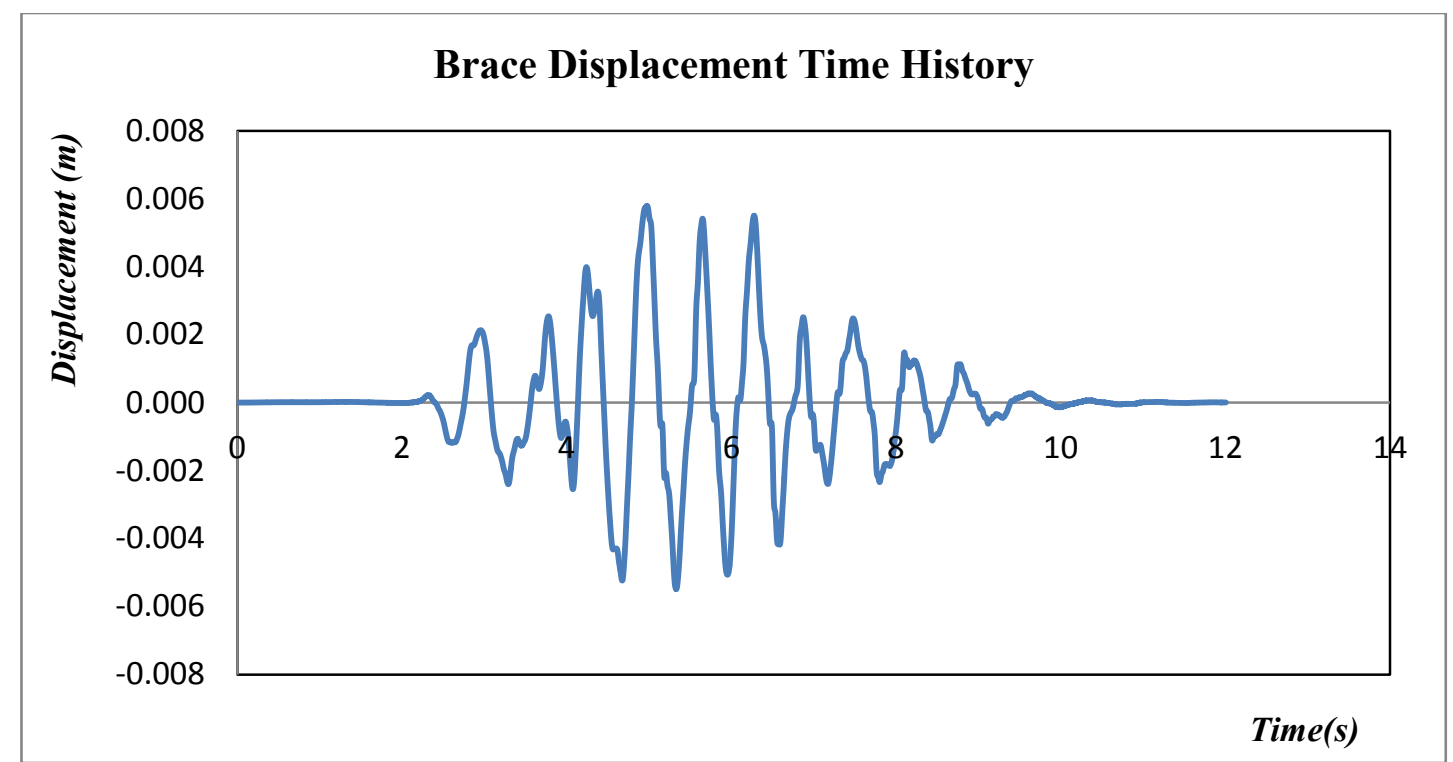

Figure 7.21 - The brace displacement time history in building SH1 subjected to M6C2 with the diaphragm as the energy dissipating member and the target ductility of 1.5

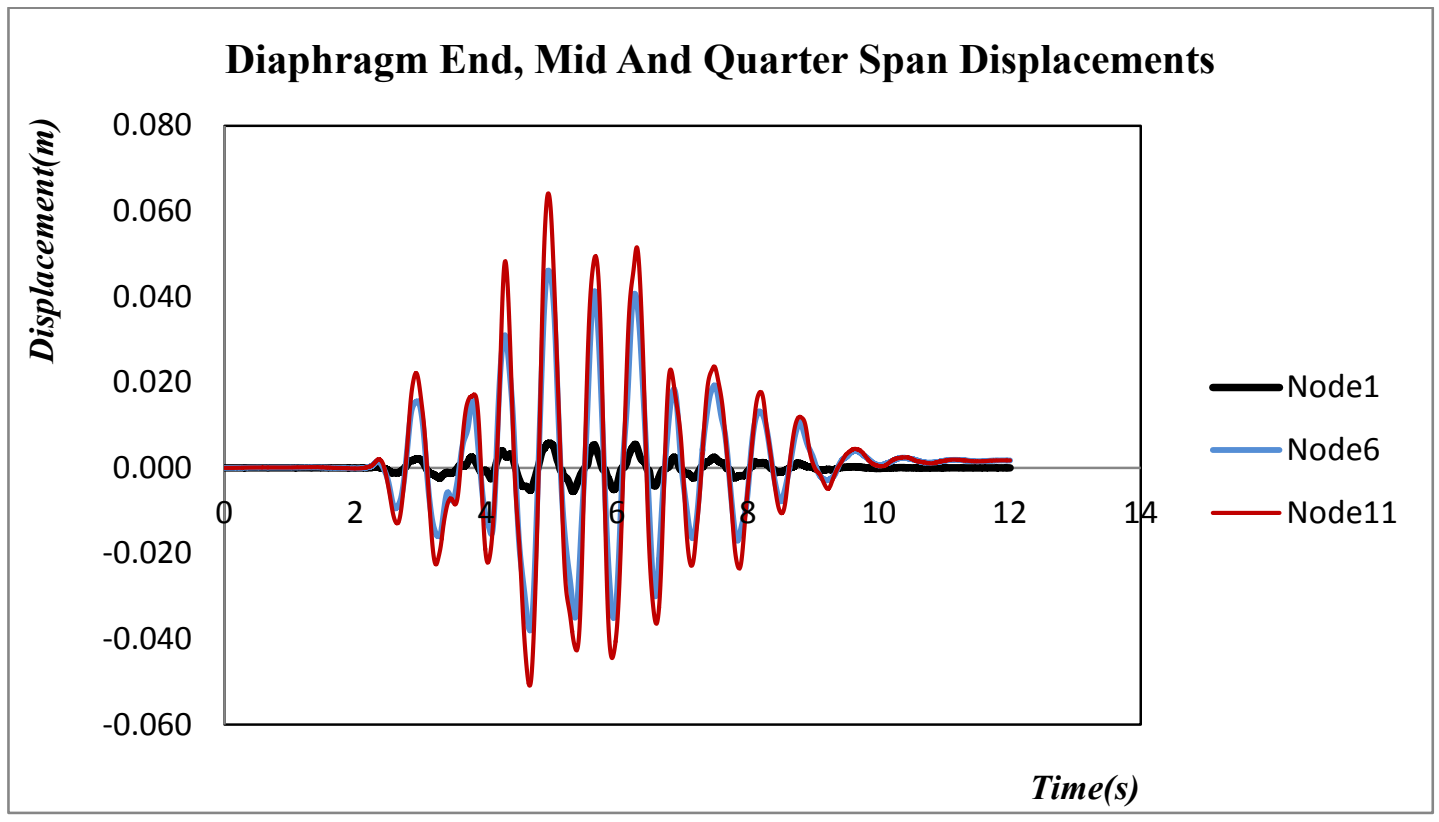

Figure 7.22 - Displacement time-histories at different locations along the length of the diaphragm in building SH1 with the diaphragm as the energy dissipating member and the target ductility of 1.5 


\subsection{Diaphragm Design Force Factors for Different Performance Levels}

As discussed earlier, in the design approach for one-storey buildings with flexible diaphragms in which the braces are treated as the main source of energy dissipation, the diaphragms, collectors, chords, struts and their connections must be designed not to yield under the ultimate load reached in the brace. Most materials including steel exhibit strain hardening. After the yield force is reached in the braces, the braces exhibit strain hardening and will show greater resistance as the deformations increase. Consequently, one cannot design the roofing system for the brace yield force and expect it to remain elastic. The diaphragm should, in fact, be designed for a force greater than the brace yield force. Such force can be considered as being equal to the yield force in the brace multiplied by a factor greater than one. Equation 7.9 illustrates this concept.

$F_{d}=\Phi_{e} \cdot F_{y}$

in which

$F_{d}=$ design force for the diaphragm

$\Phi_{e}=$ the design force factor that ensures the diaphragm would remain elastic

$F_{y}=$ the force at which the brace would yield

This concept is widely used in capacity design in earthquake engineering. For instance, in the seismic design of connections in steel structures, the CSA S16-09 introduces a factor designated as $R$ (clause 27.1.7) and requires that the beam-to-column connections be designed for $M_{d}$ given by (Clause 27.4.4.2): 
AISC 2005 has similar provisions. However, it specifies different $R$ values for different member types, ranging from 1.1 to 1.6.

Another example where this concept of capacity design is observed is in the seismic design of beams and columns. Most codes and guidelines suggest that the total moment capacity of the columns $\left(\boldsymbol{M}_{\boldsymbol{c}}\right)$ meeting at a joint should be 1.2 times the moment capacity of the beams framing into them $\left(\boldsymbol{M}_{\boldsymbol{b}}\right)$.

$M_{c}=1.2 M_{b}$

In this part of the study an attempt is made to find design force factor for the diaphragm which would ensure an elastic behaviour in the diaphragm when the braces are designed as the weak link in the system.

It may be acceptable to permit a limited extent of nonlinear action in the diaphragm even as the supporting braces are yielding. In such a case the design force factor need not be as large as that required to ensure elastic behaviour in the diaphragm. It would be of interest to find such revised value of the force factor. As an example, it would be useful to know what should be the value of the force factor when both the braces and the diaphragm are allowed to yield but the diaphragm ductility demand must be limited to 1.5 .

In this part of the study the question posed in the preceding paragraph is addressed by finding the design force factor that ensures that the ductility demand in the diaphragm does not exceed a specified limit and examining the performance of the resulting structural system. Equation 7.12 expresses the seismic design force for the diaphragm 
such that the diaphragm would exhibit nonlinearity but with ductility demand limited to 1.5 .

$F_{d}=\Phi_{d} \cdot F_{y}$

in which:

$\Phi_{d}=$ design force factor for the diaphragm leading to a ductility demand limited to 1.5

\subsubsection{Dynamic Analyses Results}

In order to account for strain hardening, a bilinear behaviour with post-yield stiffness of 0.05 is assumed for the braces. The diaphragm is modelled to perform elastically under bending moment but nonlinearly under shear force. The previously described nonlinear hysteretic behaviour is used to model nonlinear shear behaviour.

In the first set of dynamic analyses, that the diaphragm is assumed to behave elastically. Then the ductility related force reduction factor $\left(R_{y}\right)$, and hence the yield force $F_{y}$, corresponding to target ductility demands of 2.0, 3.0 and 4.0 in the braces is determined.

In the second set of nonlinear analyses, the diaphragm is modelled to respond, if necessary, in the nonlinear range. However it is assigned a yield force of $\Phi_{e} \times F_{y}$ just sufficient to ensure that it remains elastic under the applied seismic force. The $\Phi_{e}$ factor is determined by trial and error through repeated analyses. In the final analytical runs, the diaphragm design force is assumed to be $\Phi_{d} \times F_{y}$, where the $\Phi_{d}$ factor is such that the diaphragm would behave nonlinearly but with a ductility demand less than $1.5\left(\mu_{d} \leq 1.5\right)$. Again, $\Phi_{d}$ is determined by a process of trial and error over repeated analyses. 
In order to maintain a complete database, the previously selected 16 buildings, covering all drift ratio values, are used in the analysis. For the target ductility of 3.0, the buildings are subjected to all 10 ground motions, making a total of 160 cases. However, for target ductility values of 2.0 and 4.0 , the buildings are subjected to only five ground motions (M6C2, M6C26, M6C32, E6C18 and E6C42), making 80 cases for each target ductility demand. Three of the selected records are compatible with western spectrum while the rest are compatible with eastern spectrum. Theoretically when the braces and the diaphragm are both elastic $\Phi_{e}$ should be 1 . However, since the analytical model uses lumped mass representation, in which part of the mass is assigned as well to the end nodes of the beam representing the diaphragm, the inertia force on such mass leads to slightly different values for the brace force and the end shear in the diaphragm. The difference will become small when the model is refined and may be ignored. The design force factor for the case where the ductility demand in the diaphragm is less than $1.5\left(\Phi_{d}\right)$ is determined by dividing the diaphragm design force (defined in previous part of the study) by the brace elastic force. With 160 cases corresponding to $\mu=1.0,80$ cases corresponding to $\mu=2.0,160$ cases corresponding to $\mu=3.0$ and 80 cases corresponding to $\mu=4.0$, the complete data base consists of 480 cases. The entire set of results is presented in Appendix F.

\subsubsection{Relation between Design Force Factors, $\left(\Phi_{e}\right.$ and $\left.\Phi_{d}\right)$ and Building Properties}

Based on the results of the dynamic analyses, for each target of ductility demand the design force factors are plotted against the drift ratios. Figures 7.23 to 7.26 and 7.27 to 7.30 illustrate the $\Phi_{e}$ and $\Phi_{d}$ factors, respectively, as functions of the drift ratios for ductilities of 1.0 (elastic case), 2.0, 3.0 and 4.0 in the brace. 


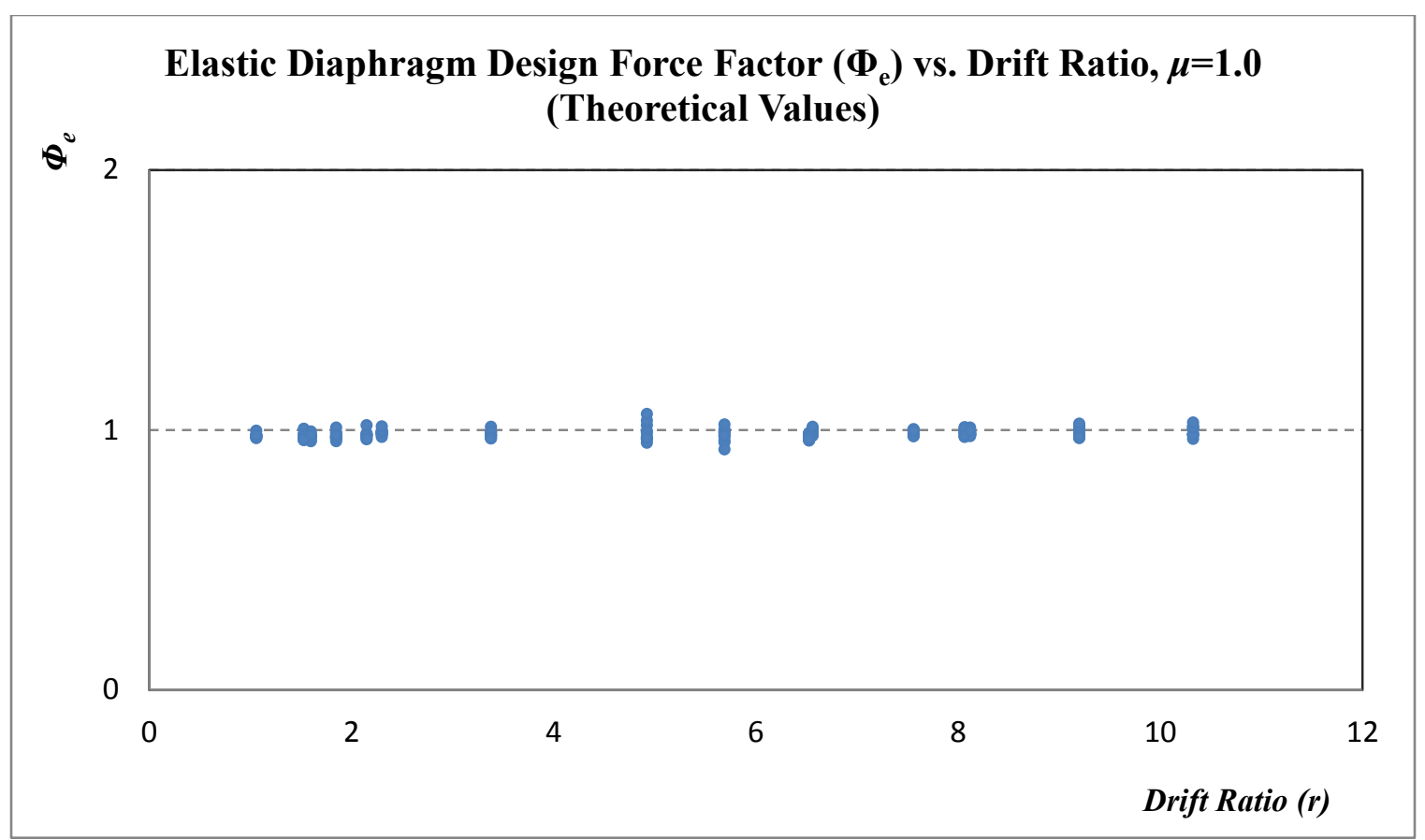

Figure 7.23 - Design force factor for elastic behaviour $\left(\Phi_{e}\right)$ vs. drift ratio for ductility of 1.0. The $\Phi_{e}$ factor should be 1 in this case. The slight deviation from that value is caused by lumped mass idealization. The mean value is still close to 1 .

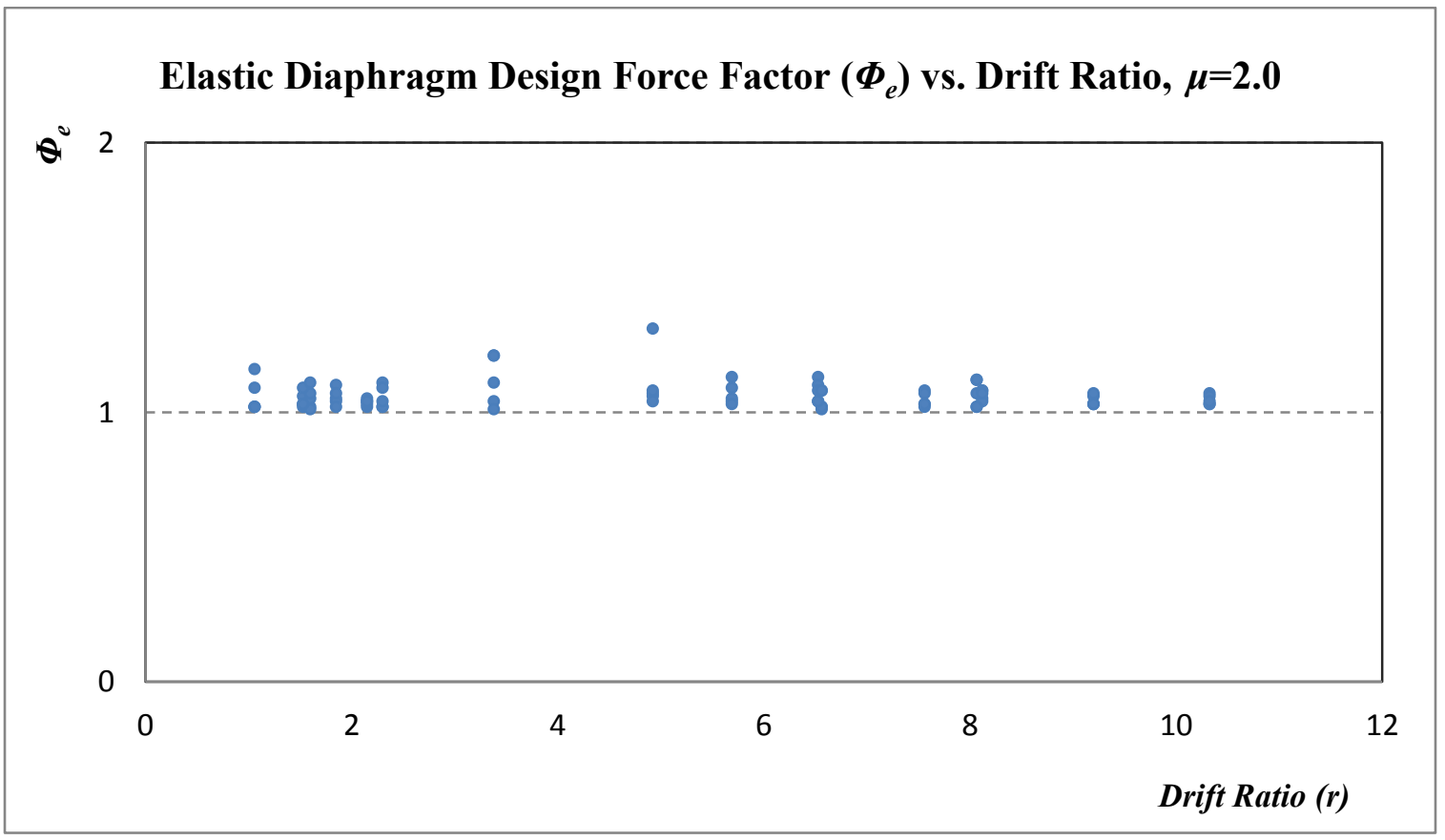

Figure 7.24 - Design force factor for elastic behaviour $\left(\Phi_{e}\right)$ vs. drift ratio for ductility of 2.0 


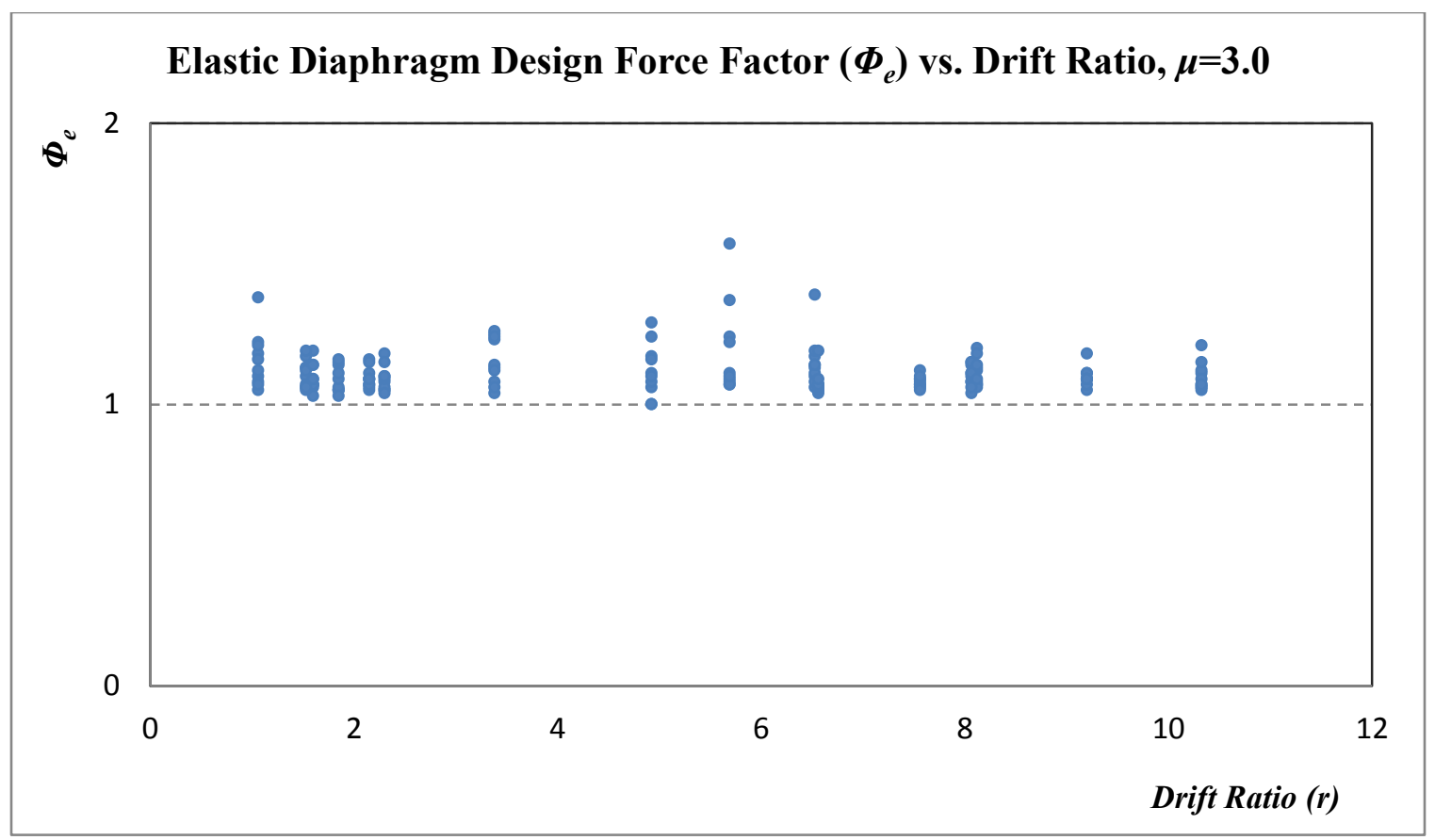

Figure 7.25 - Design force factor for elastic behaviour $\left(\Phi_{e}\right)$ vs. drift ratio for ductility of 3.0

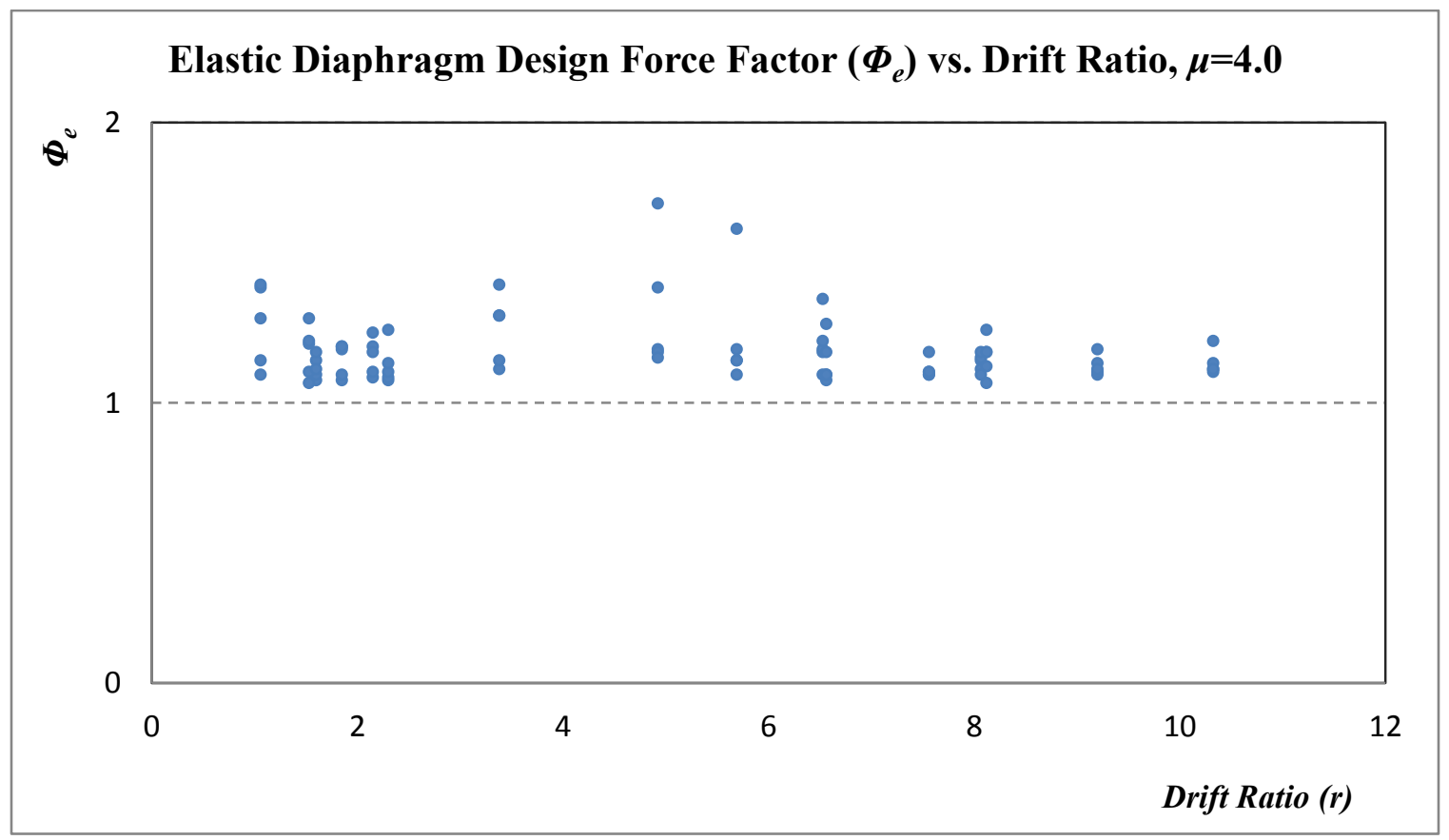

Figure 7.26 - Design force factor for elastic behaviour $\left(\Phi_{e}\right)$ vs. drift ratio for ductility of 4.0 


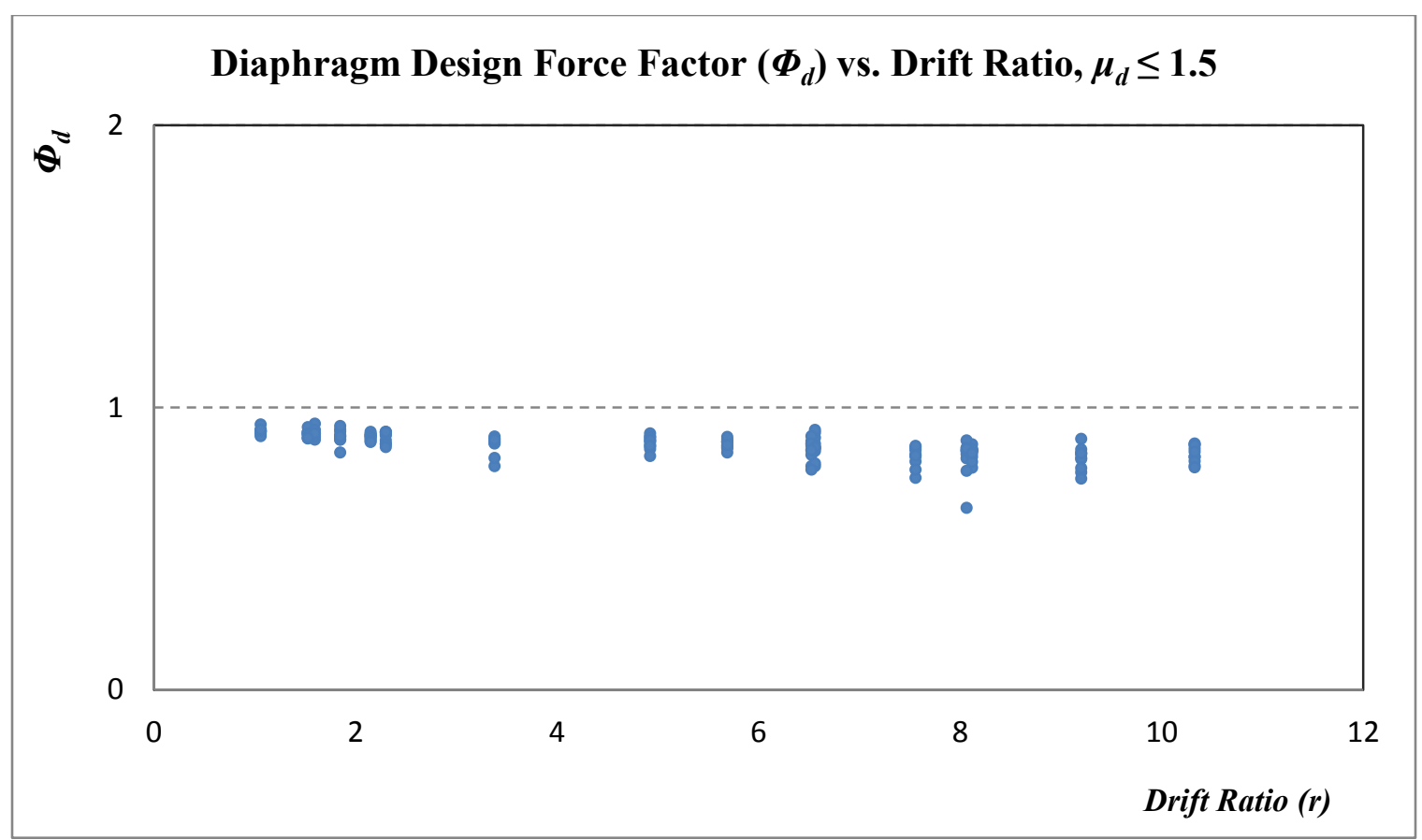

Figure 7.27 - Design force factor for nonlinear behaviour $\left(\Phi_{d}\right)$ vs. drift ratio for ductility of 1.0

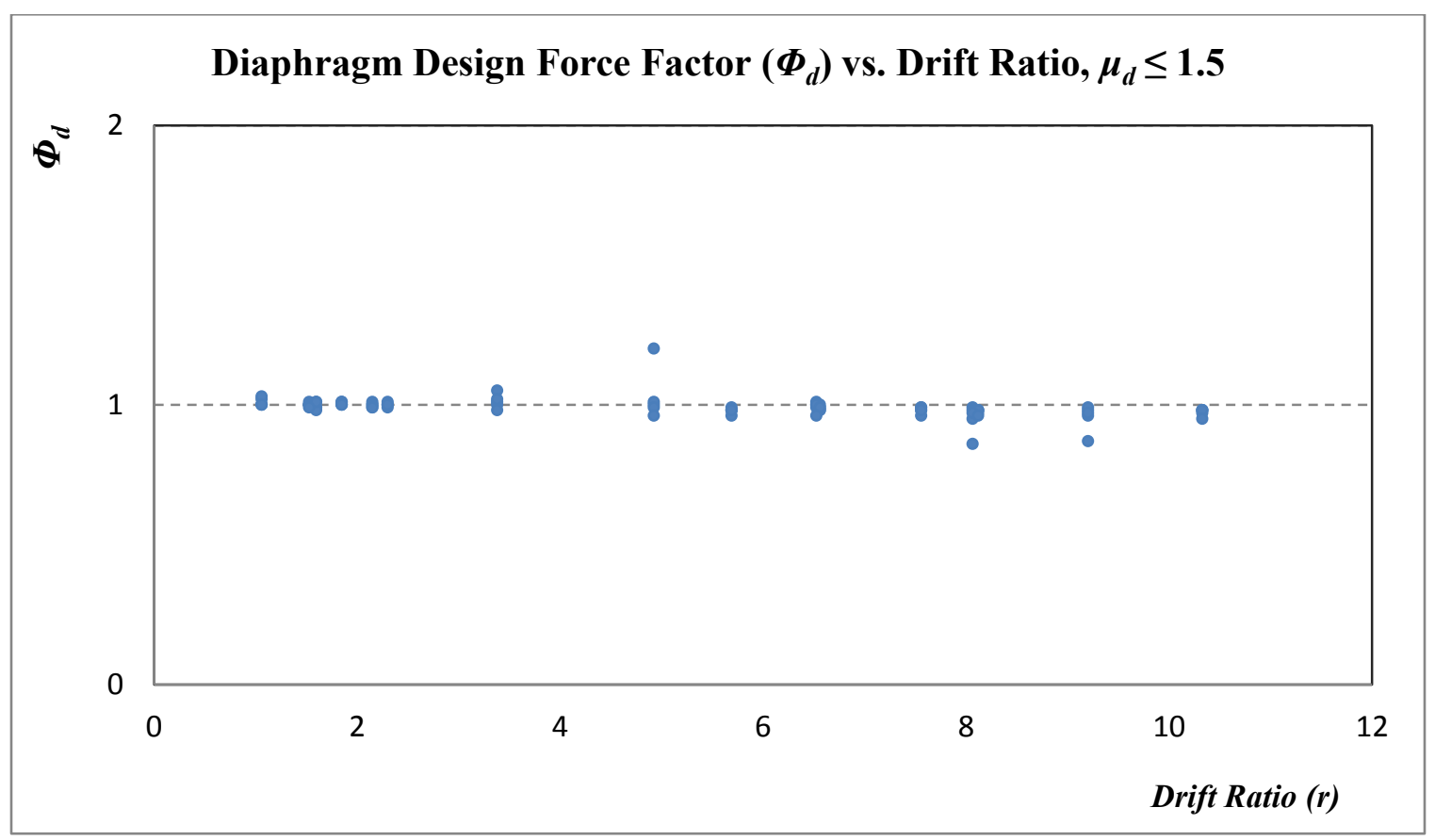

Figure 7.28 - Design force factor for nonlinear behaviour $\left(\Phi_{d}\right)$ vs. drift ratio for ductility of 2.0 


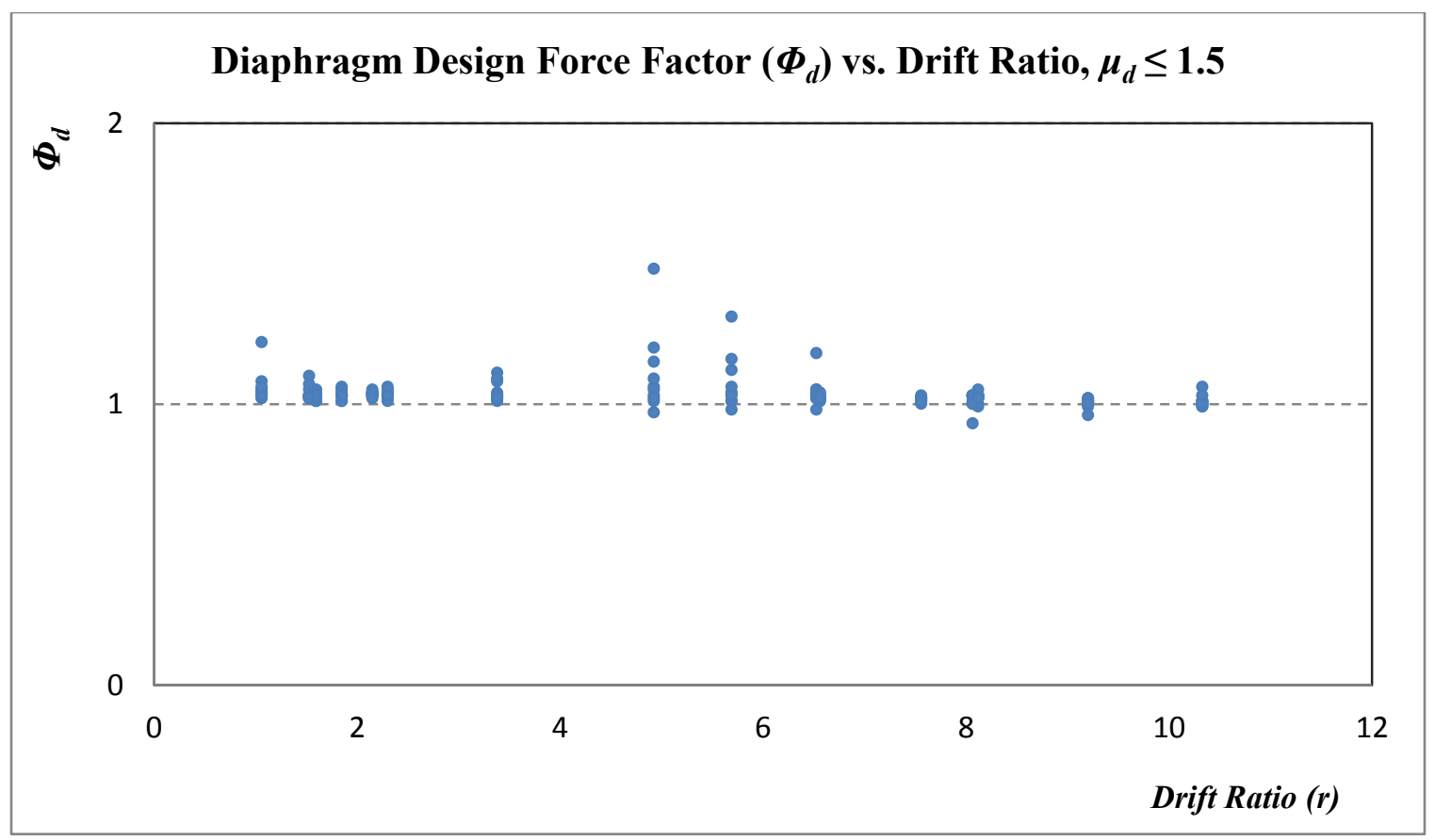

Figure 7.29 - Design force factor for nonlinear behaviour $\left(\Phi_{d}\right)$ vs. drift ratio for ductility of 3.0

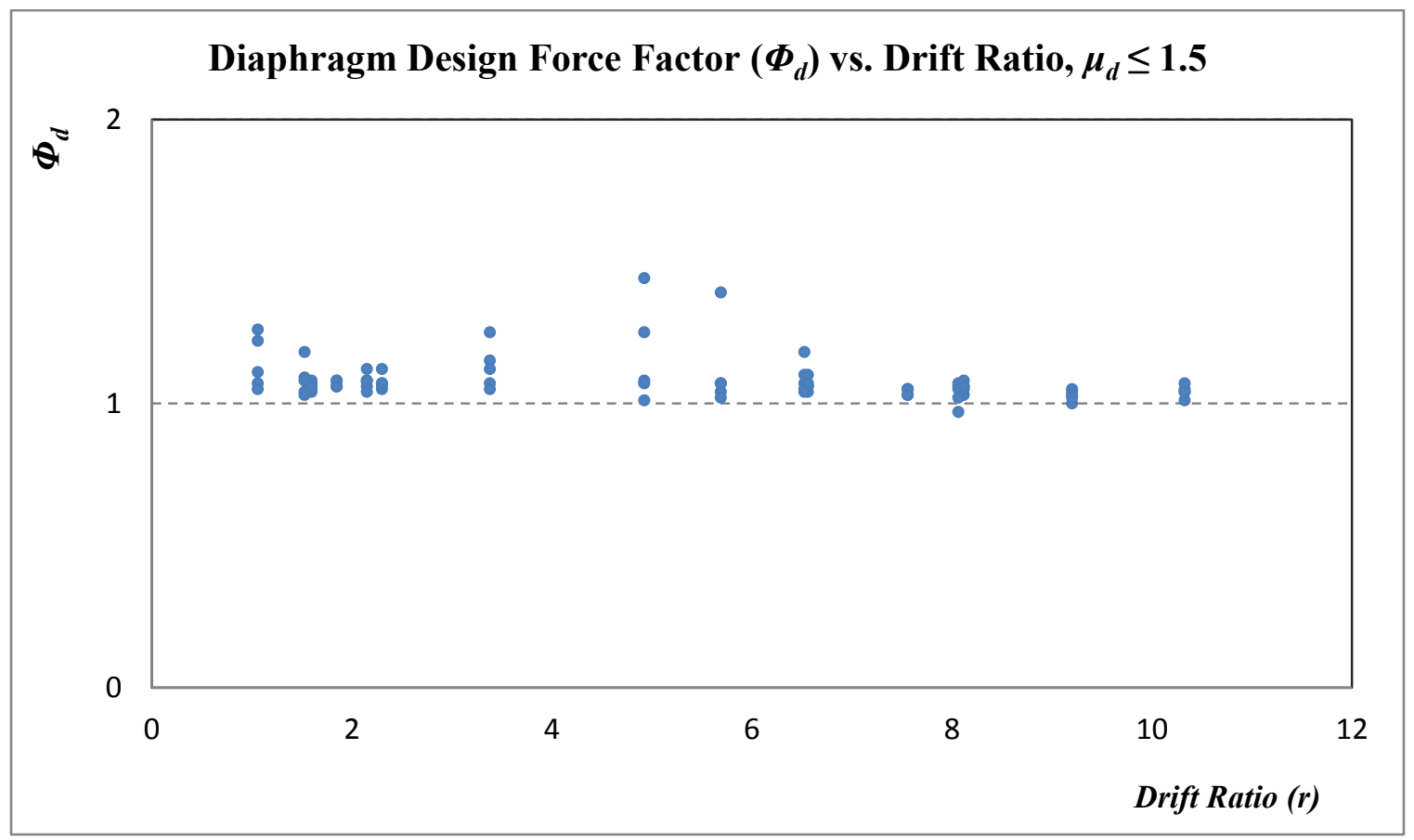

Figure 7.30 - Design force factor for nonlinear behaviour $\left(\Phi_{d}\right)$ vs. drift ratio for ductility of 4.0 
Figures 7.23 to 7.30 indicate that both design force factors $\left(\Phi_{e}\right.$ and $\left.\Phi_{d}\right)$ do not change with change in the drift ratio of the building. A mean value can be selected for each design factor, under each performance level (ductility in the brace). Table 7.1 shows the obtained mean values for the diaphragm design force based on the performance of the system.

Table 7.1 - Mean values for diaphragm design force factor

\begin{tabular}{ccc}
\hline Ductility & Elastic Design $\left(\boldsymbol{\Phi}_{e}\right)$ & Nonlinear Design $\left(\Phi_{d}\right)$ \\
\hline$\mu=1.0$ & 1.00 & 0.87 \\
$\mu=2.0$ & 1.06 & 0.99 \\
$\mu=3.0$ & 1.11 & 1.04 \\
$\mu=4.0$ & 1.18 & 1.08 \\
\hline
\end{tabular}

Figures 7.23 to 7.30 , as well as the mean values presented in Table 7.1 show that the design force factors are proportional to the ductility demand on the LLRS. In order to understand the reason for this one must first recognize the alternative locations at which the nonlinearity may occur in the diaphragm.

\subsubsection{Nonlinearity along the Length of the Diaphragm}

As pointed out earlier, the highest inelasticity may occur at either the diaphragm ends or somewhere along the length of the diaphragm. If the contribution of higher modes is limited and not significant, the nonlinearity is expected to take place at diaphragm ends since the shear force at the quarter span is less than that at the diaphragm ends. However, if the higher modes make a significant contribution to the total response of the system, the nonlinearity may occur somewhere along the length of the diaphragm and not at the diaphragm end. Thus, it is ultimately the contribution of higher modes that dictates the 
location along the length of the diaphragm at which the nonlinearity occurs. While the contributions of higher modes are mainly dependent on the frequency content of the earthquake and the frequency of the system, it was pointed out in Chapter 5 of the study that the higher ductility demands on the braces cause greater contribution from higher modes. The results from this part of the study are consistent with that assessment. For target ductility values of 2.0,3.0 and 4.0, it was observed that the nonlinearity occurred more frequently along the length of the diaphragm than at the diaphragm ends, in contrast to the case of elastic diaphragm when the largest shear was most often at the diaphragm ends.

Whether the nonlinearity occurs at diaphragm ends or somewhere along the length of the diaphragm, higher ductility demand values lead to larger values for diaphragm design force factors $\left(\Phi_{e}\right.$ and $\left.\Phi_{d}\right)$.

If the nonlinearity occurs at diaphragm ends, higher ductility demand shows that the brace force is greater than the yield value on account of strain hardening. Thus, greater force will be imposed on the roofing system by the brace, and a greater value for diaphragm design force factor must be used to ensure elasticity or limited ductility. On the other hand if inelasticity takes place somewhere along the length of the diaphragm, greater ductility demand values cause greater contribution from higher modes and hence greater shear force at quarter span. Thus, diaphragm design force factor must be increased for both performance levels of the diaphragm. The results of the study do indicate that when the nonlinearity occurs along the length of the diaphragm, greater strength is needed in the diaphragm to ensure an elastic behaviour or a nonlinear one with 
limited ductility. The reasons outlined above, show that the diaphragm design force factors must be proportional to the ductility demand in the brace.

\subsubsection{Development of the Equation Relating Diaphragm Design Force Factors to the Ductility Demand on the Brace}

Since greater ductility demand causes higher design force factors, an attempt has been made to relate the $\Phi_{e}$ and $\Phi_{d}$ values to the ductility demand on the LLRS. For this purpose, the design force factors obtained for each case (16 buildings, 10 ground motions and 4 target ductility demands) are plotted against the ductility demand values. In addition, a regression fit is carried out on the results and an equation is suggested to predict the design force factor for the diaphragm based on the ductility demand on the brace. Figures 7.31 and 7.32 show the procedure outlined above. In Figure 7.31, the regression line is constrained to pass through $(1,1)$.

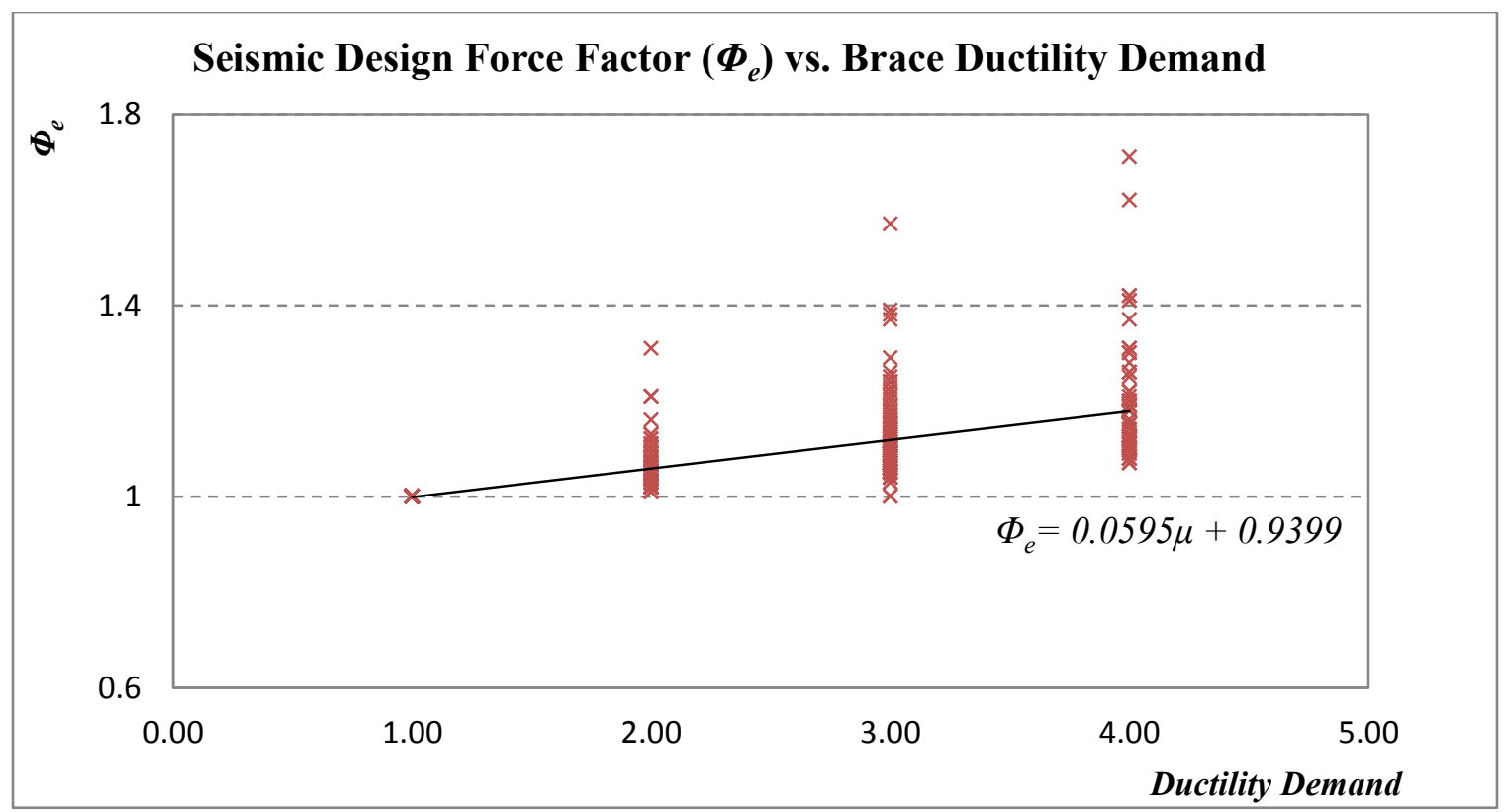

Figure 7.31 - $\Phi_{e}$ factor vs. the ductility demand on the brace when the diaphragm remains elastic and the suggested empirical equation 


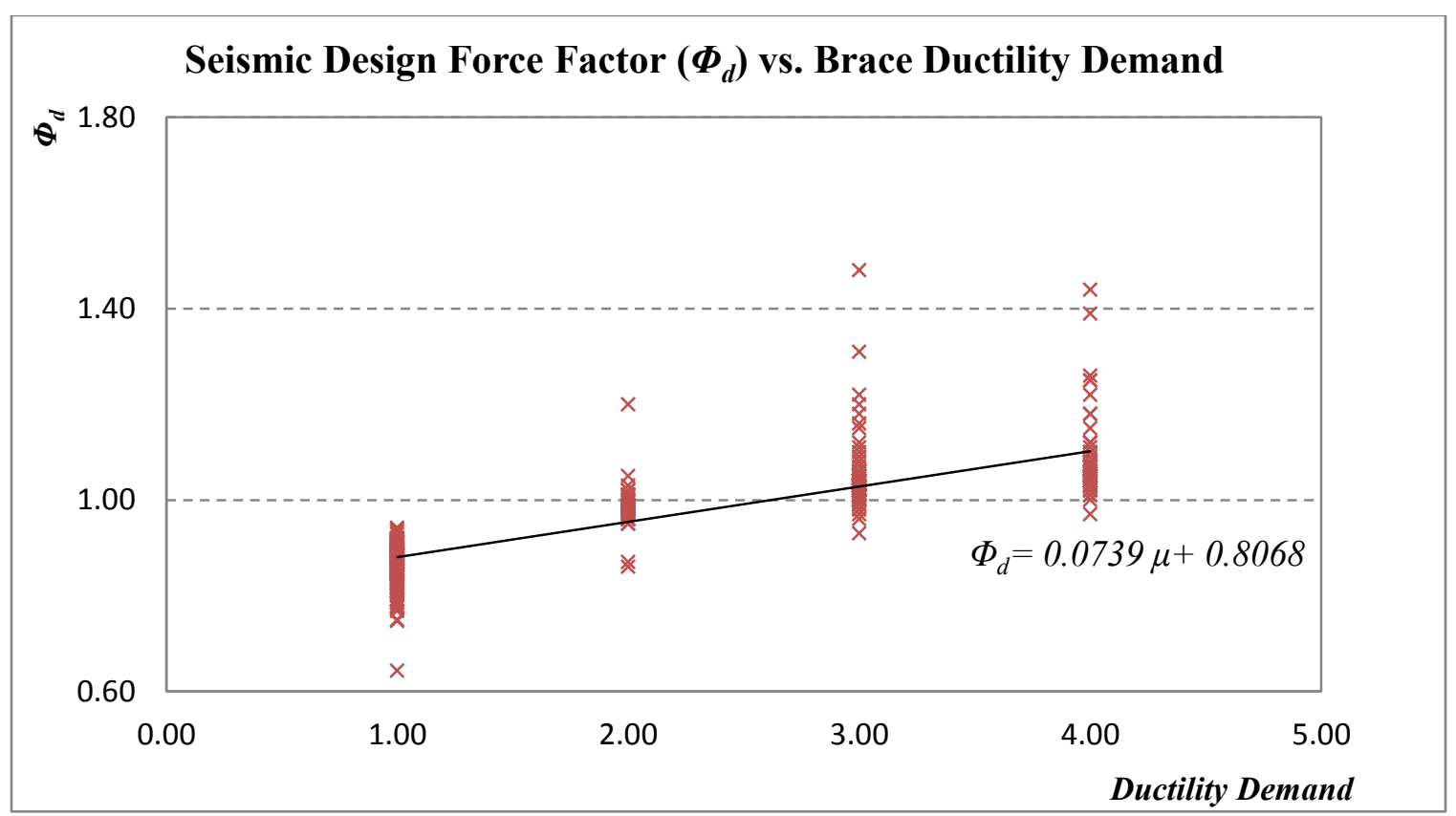

Figure 7.32 - $\Phi_{d}$ factor vs. the ductility demand on the brace when the ductility demand in the diaphragm is limited to 1.5 and the suggested empirical equation

The following equations are suggested for finding an appropriate design force in the diaphragm expressed as a factor of the brace yield force:

$\Phi_{e}=0.06 \mu+0.94$ (For Elastic Behaviour in the diaphragm)

$\Phi_{d}=0.074 \mu+0.81$ (For Nonlinear Behaviour in the diaphragm with $\mu_{d} \leq 1.5$ )

\subsubsection{Internal Force Magnification Factors}

As would be expected and as shown in Chapter 5, the greater contribution of higher modes caused by nonlinearity in the brace increases the magnification of internal forces as the target ductility for the braces is increased. However, the limited nonlinearity in the diaphragm does not make a very pronounced difference in the magnification factors, 
except for a slight decrease. The reason for this decrease is that by allowing the diaphragm to behave nonlinearly, the force associated with the yielding in diaphragm is decreased and therefore the maximum internal forces are decreased. However, this difference in the values is quite small and can be neglected in the design. It is of interest to note that this decrease due to nonlinearity in the diaphragm becomes more pronounced as the ductility demand in the brace becomes greater. For instance, the amount by which the internal forces are decreased by allowing nonlinearity in the diaphragm for ductility of brace equal to 3.0 is less than that corresponding to $\mu=4.0$.

The force magnification ratios (M.F, V.F, M.U and V.U) are plotted against the ductility demand in the diaphragm for each value of ductility in the brace separately. A regression fit is also carried out on the results to predict the slight decrease in the internal force magnifications. The plots are shown in Figures 7.33 to 7.44 .

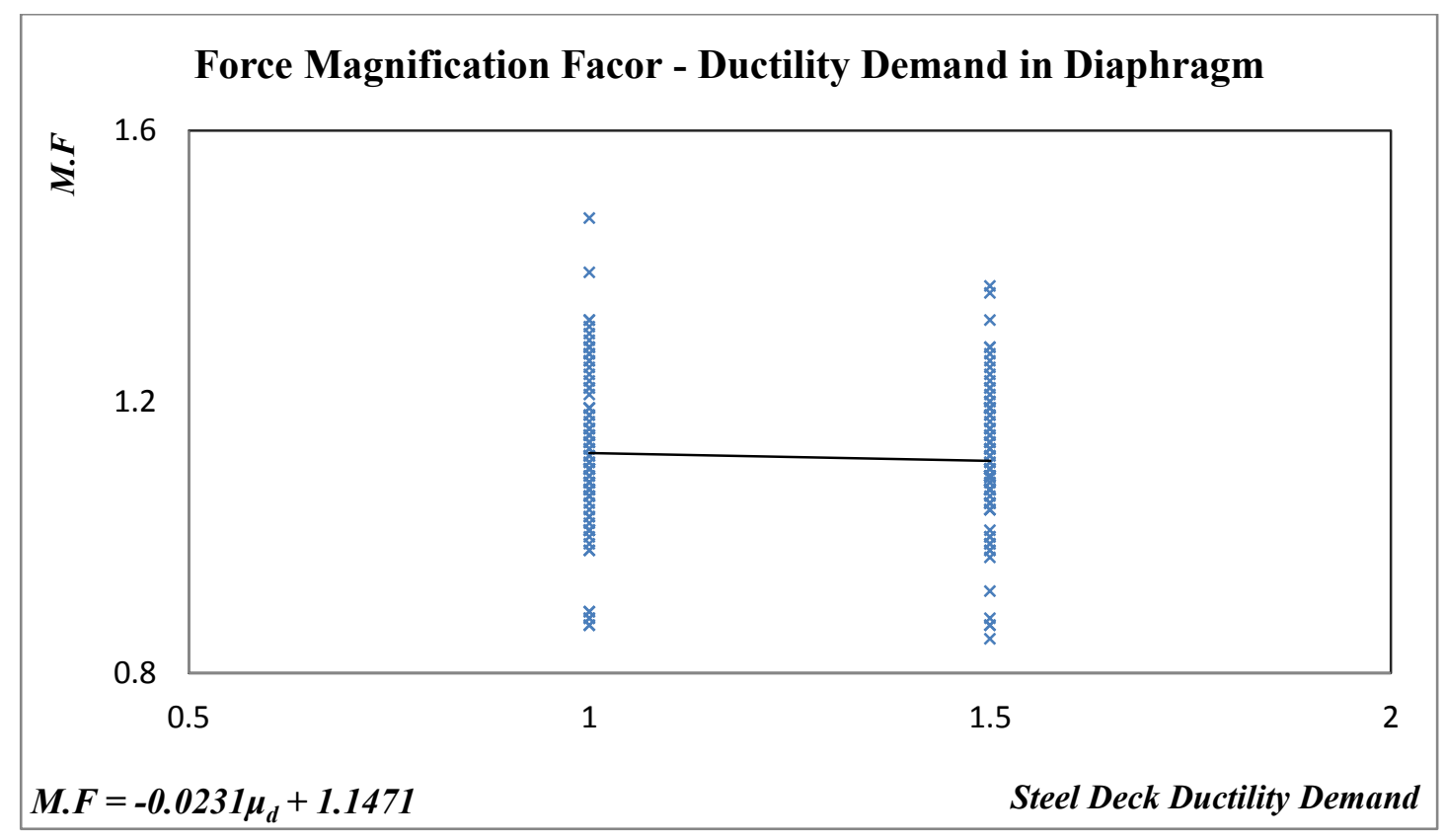

Figure 7.33 - Internal force magnification M.F vs. the ductility demand on the diaphragm with the suggested equation $(\mu=2.0)$ 


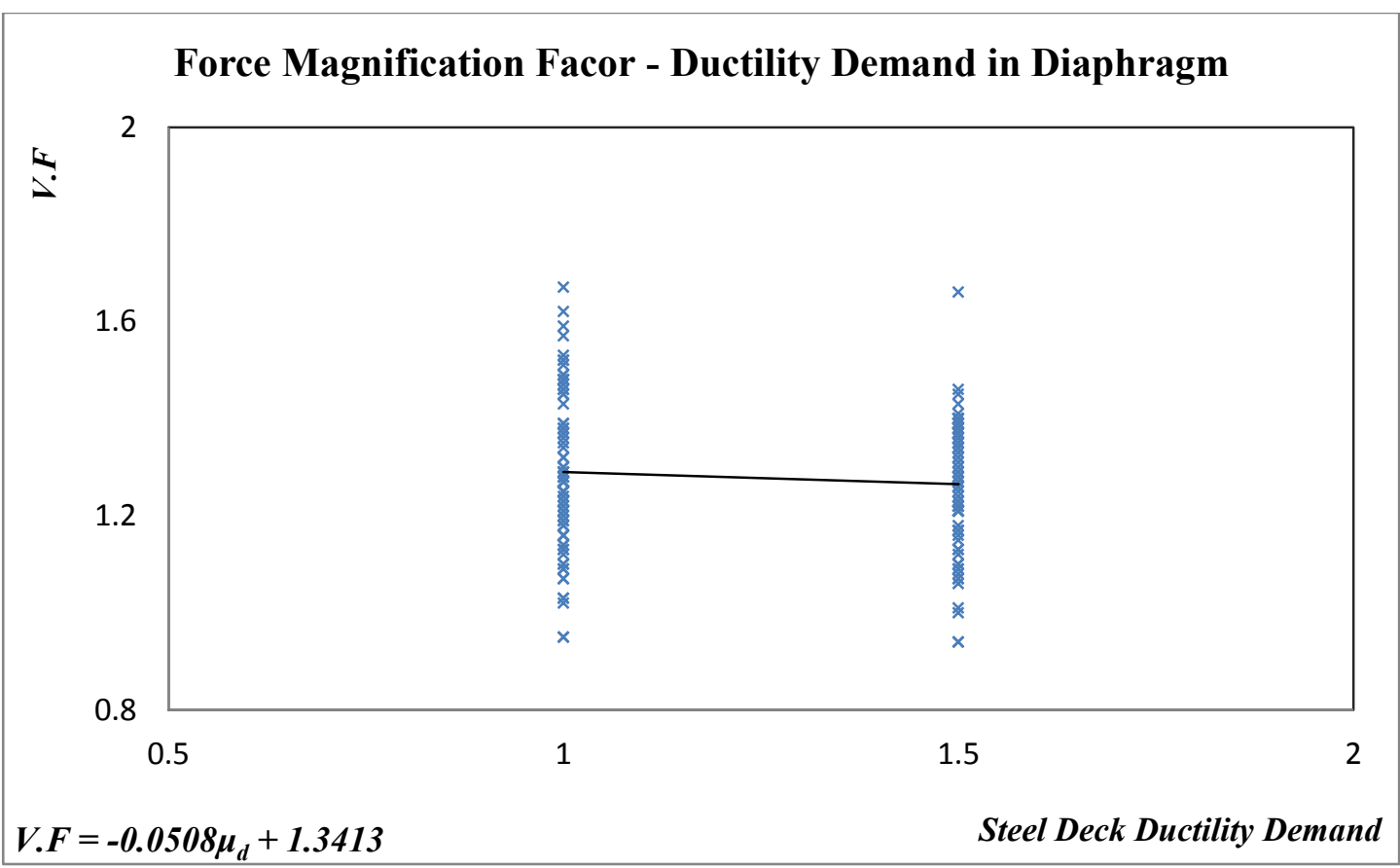

Figure 7.34 - Internal force magnification V.F vs. the ductility demand on the diaphragm with the suggested equation $(\mu=2.0)$

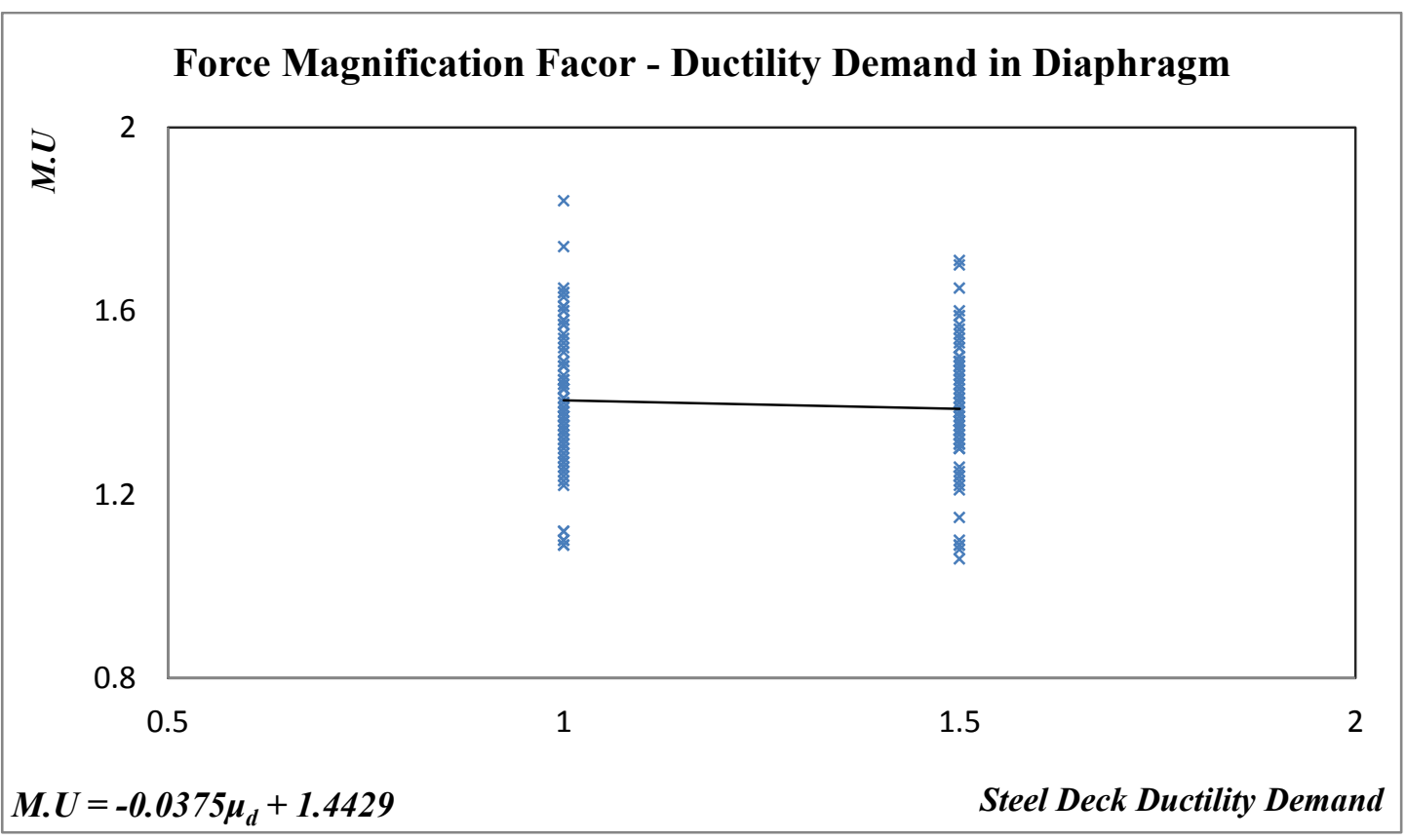

Figure 7.35 - Internal force magnification M.U vs. the ductility demand on the diaphragm with the suggested equation $(\mu=2.0)$ 


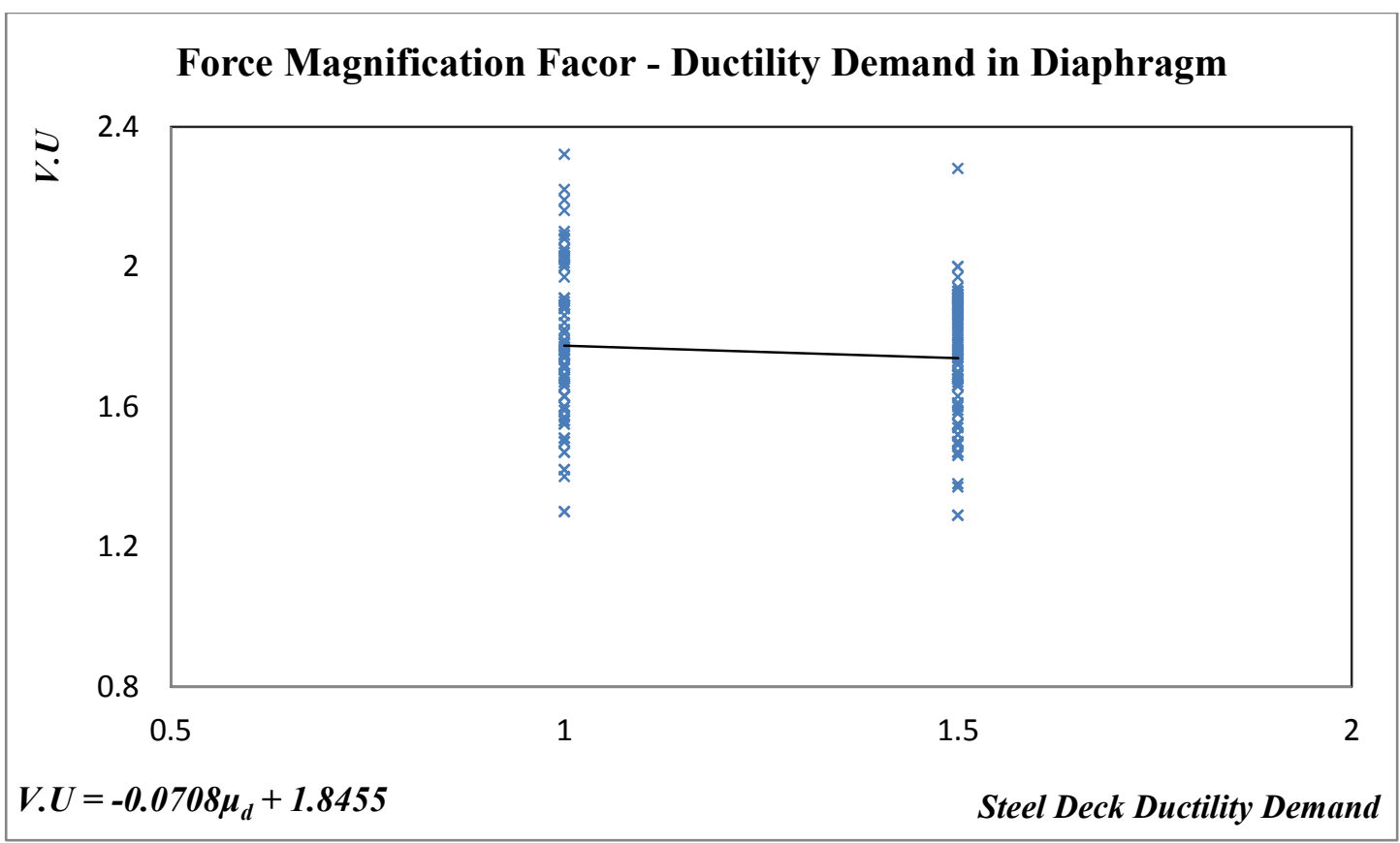

Figure 7.36 - Internal force magnification V.U vs. the ductility demand on the diaphragm with the suggested equation $(\mu=2.0)$

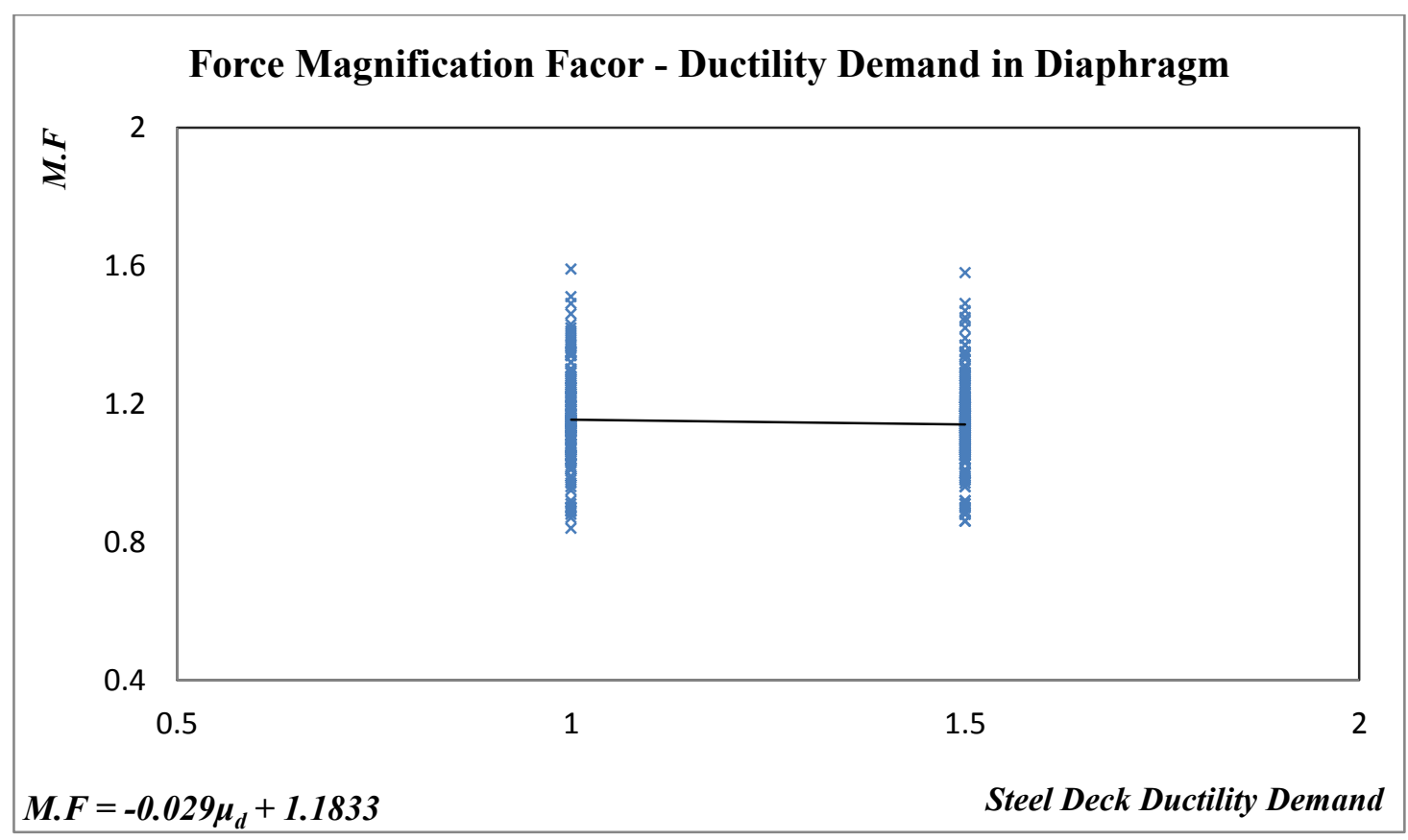

Figure 7.37 - Internal force magnification M.F vs. the ductility demand on the diaphragm with the suggested equation $(\mu=3.0)$ 


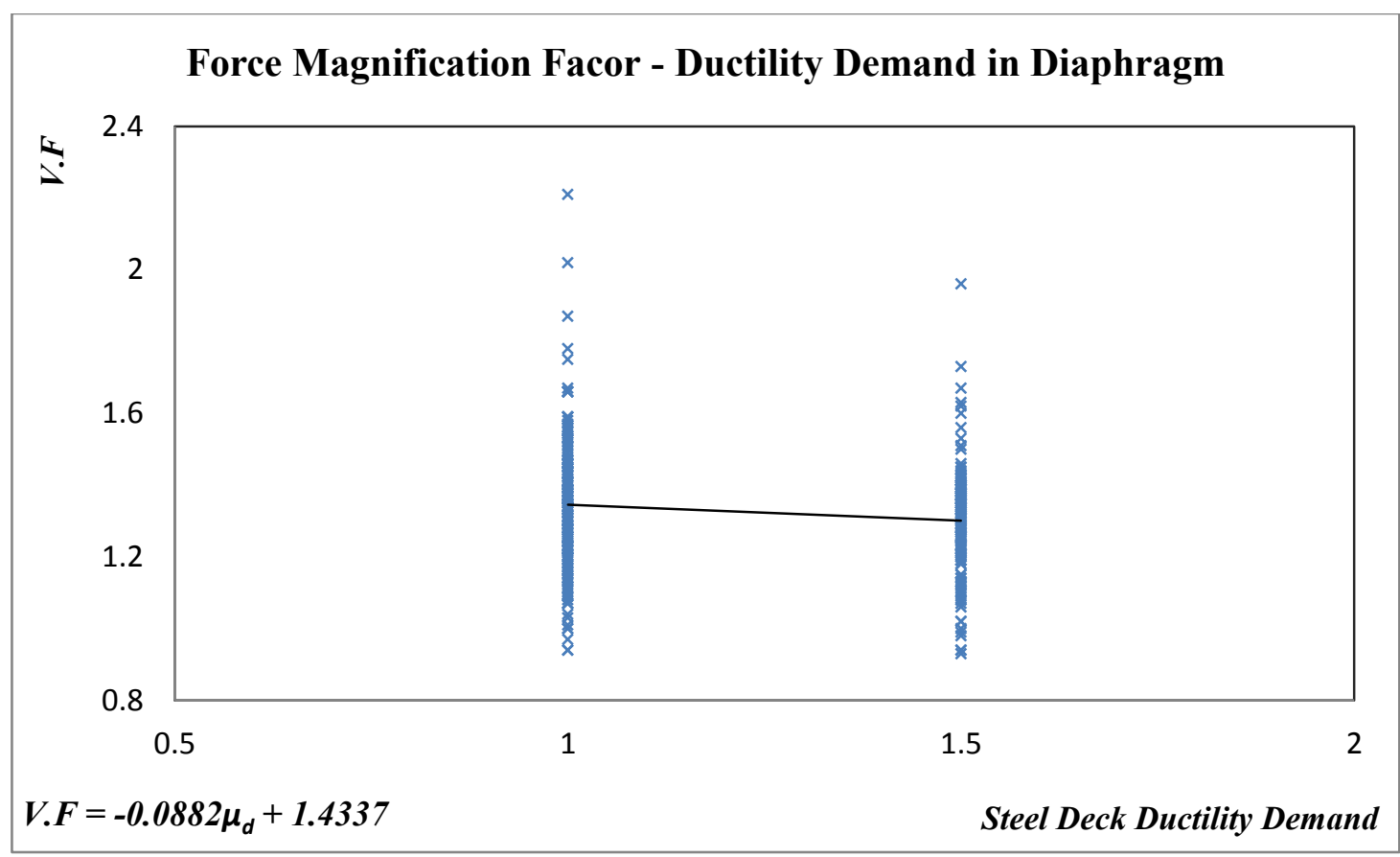

Figure 7.38 - Internal force magnification V.F vs. the ductility demand on the diaphragm with the suggested equation $(\mu=3.0)$

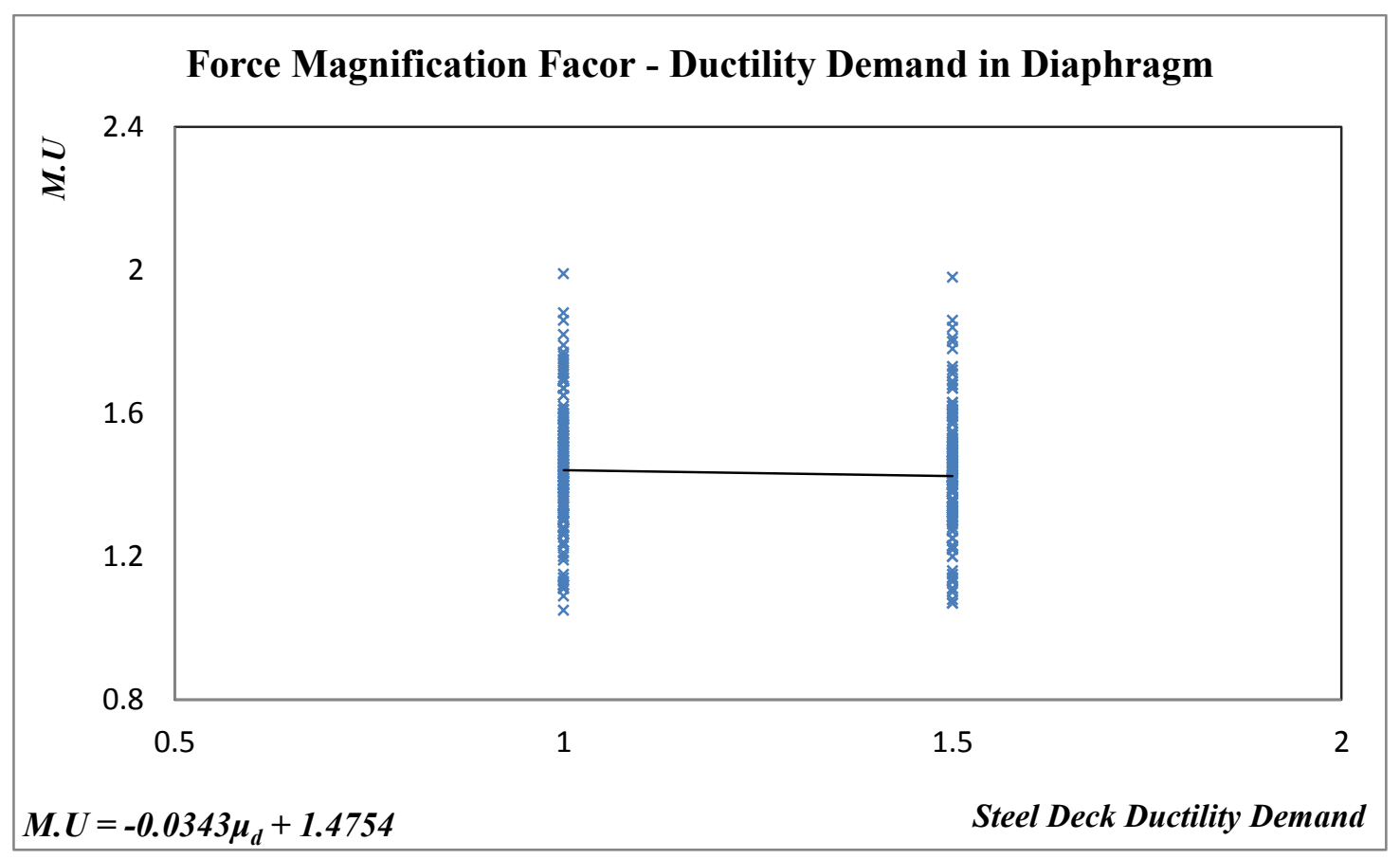

Figure 7.39 - Internal force magnification M.U vs. the ductility demand on the diaphragm with the suggested equation $(\mu=3.0)$ 


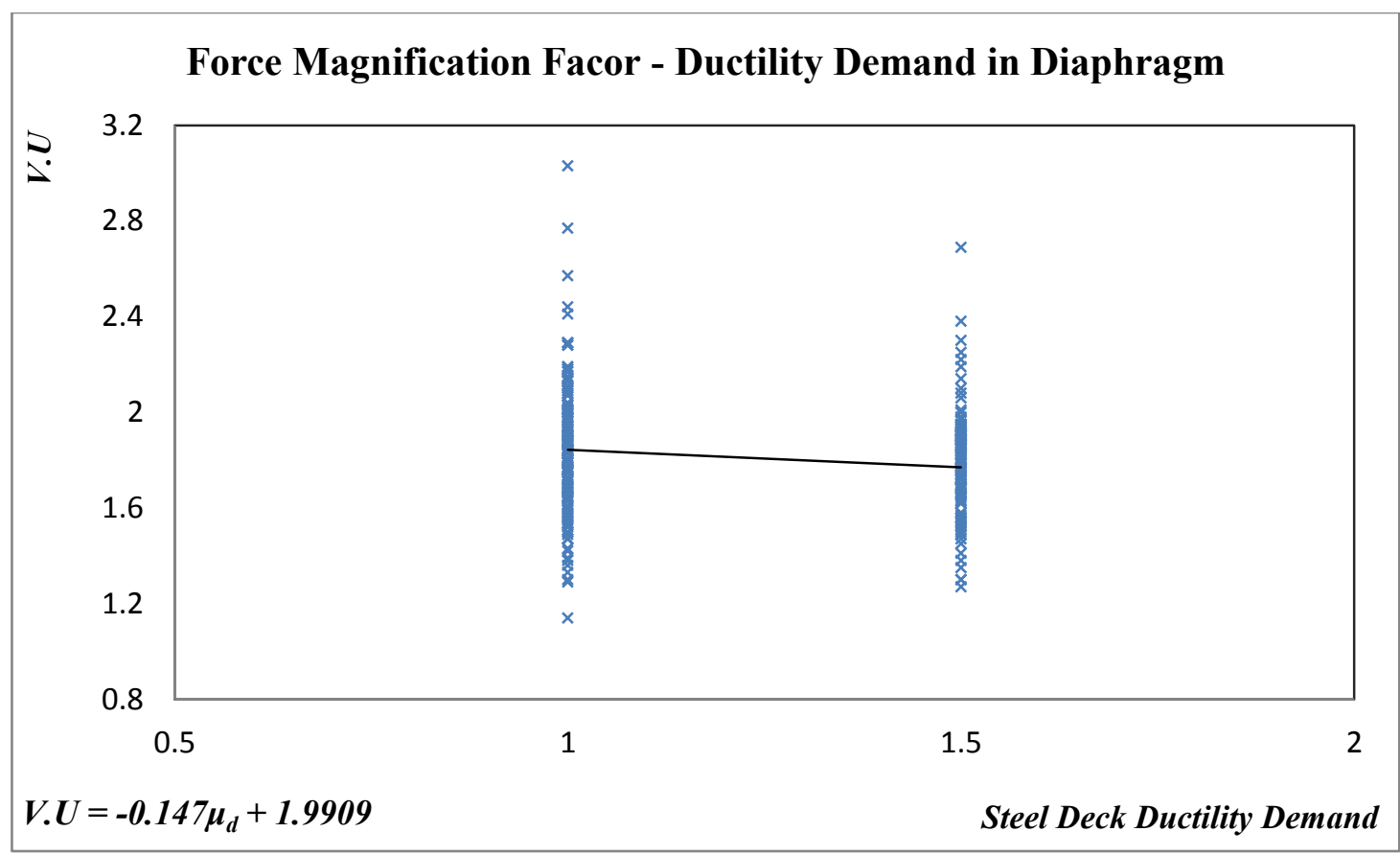

Figure 7.40 - Internal force magnification V.U vs. the ductility demand on the diaphragm with the suggested equation $(\mu=3.0)$

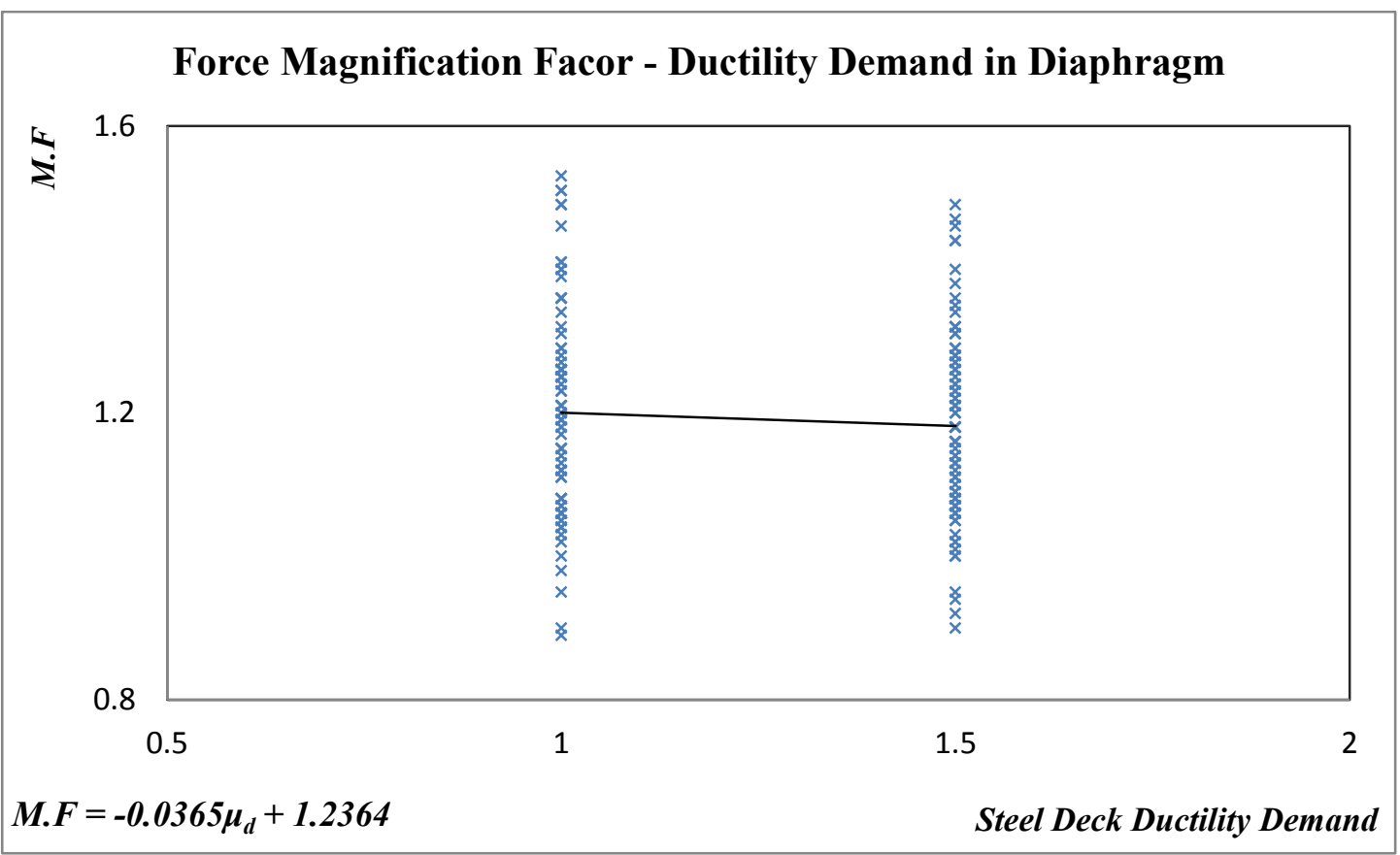

Figure 7.41 - Internal force magnification M.F vs. the ductility demand on the diaphragm with the suggested equation $(\mu=4.0)$ 


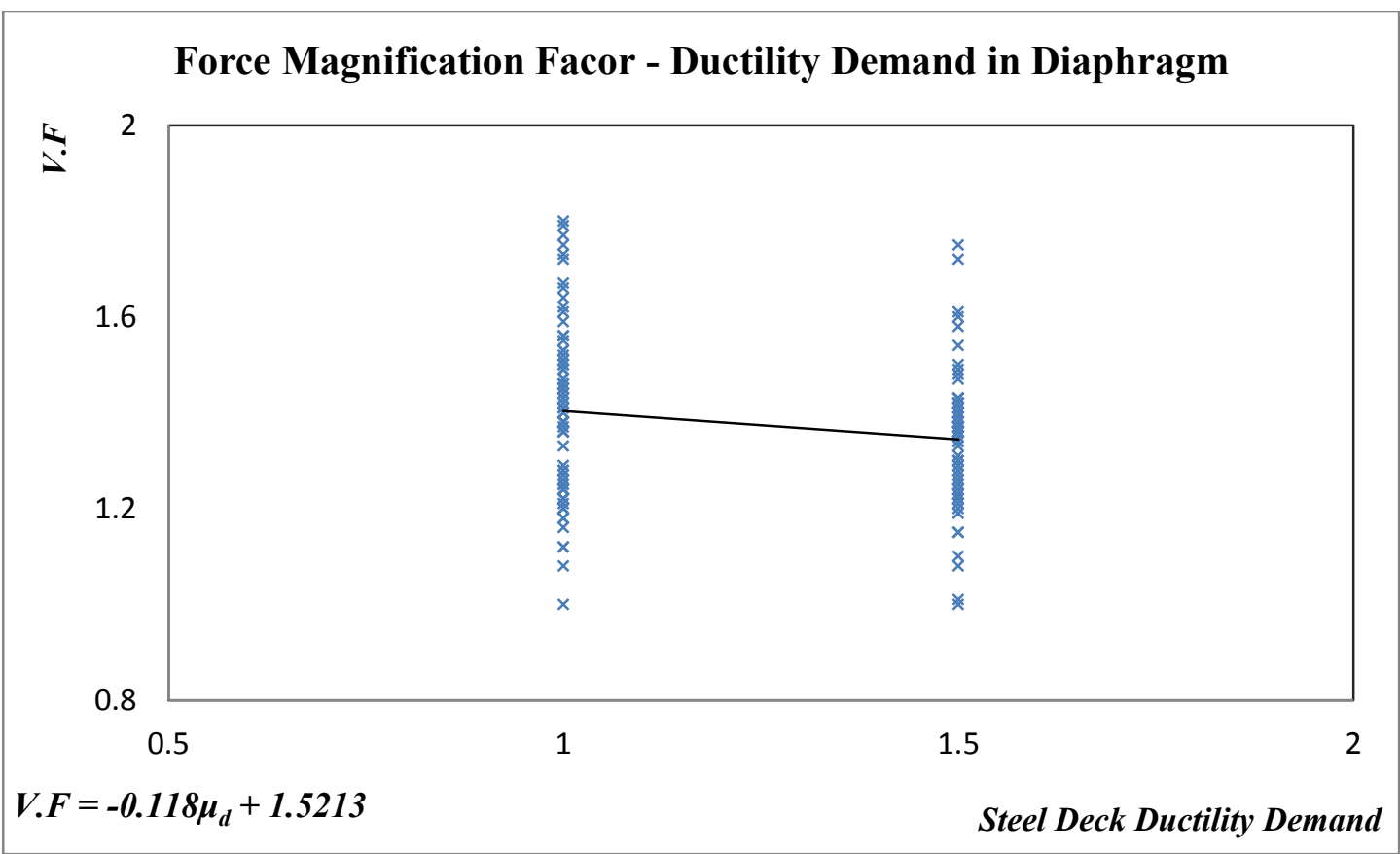

Figure 7.42 - Internal force magnification V.F vs. the ductility demand on the diaphragm with the suggested equation $(\mu=4.0)$

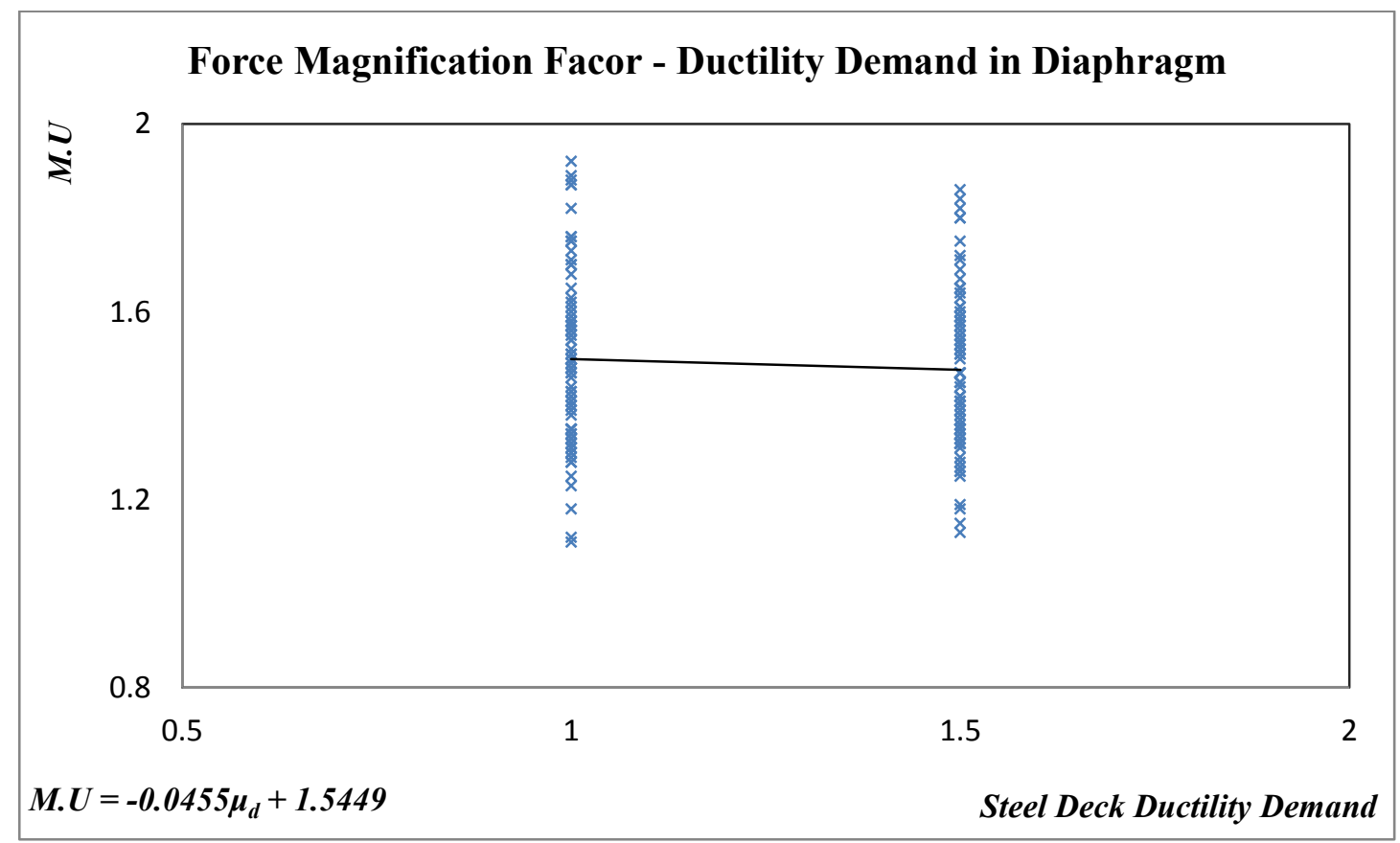

Figure 7.43 - Internal force magnification M.U vs. the ductility demand on the diaphragm with the suggested equation $(\mu=4.0)$ 


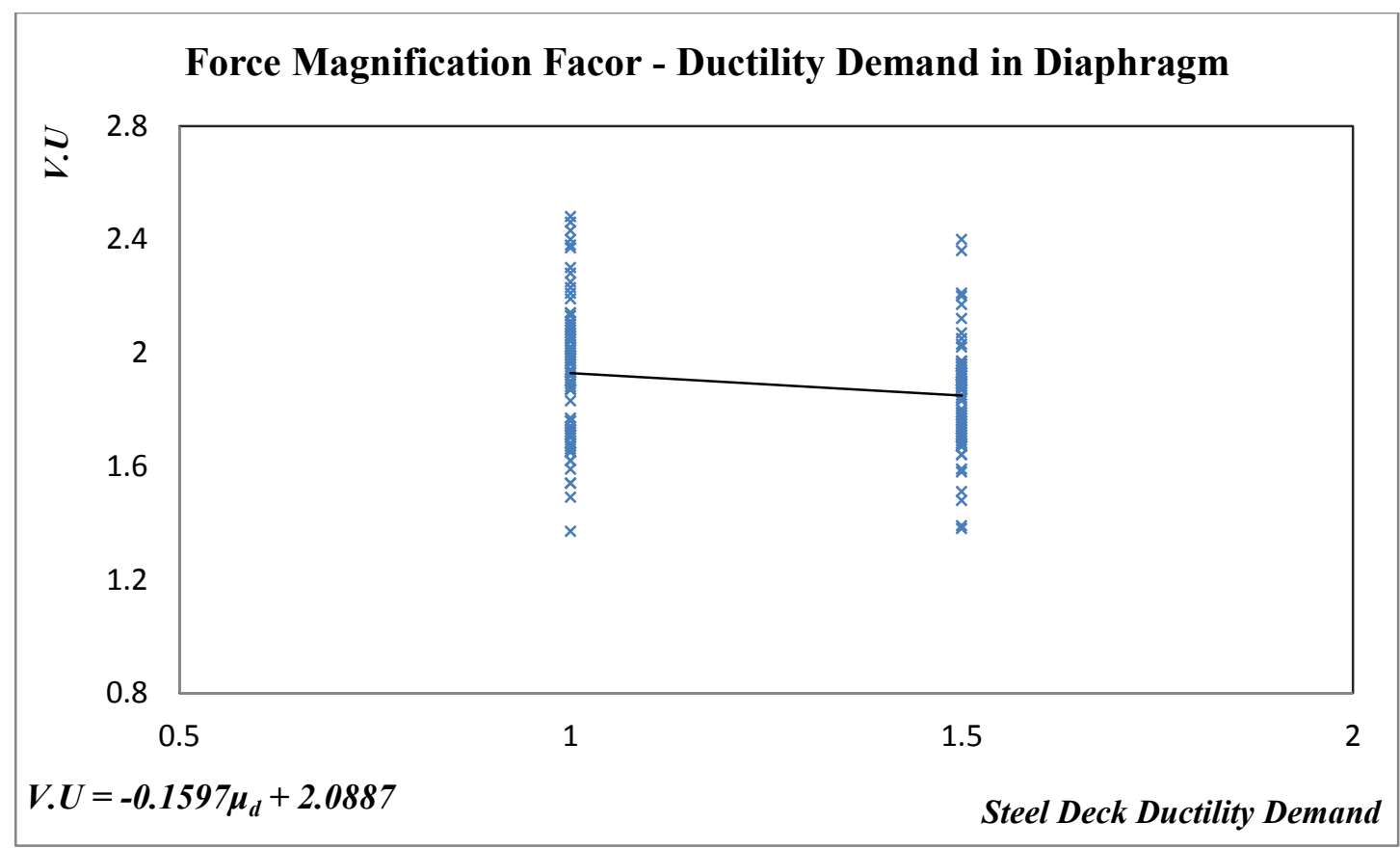

Figure 7.44 - Internal force magnification V.U vs. the ductility demand on the diaphragm with the suggested equation $(\mu=4.0)$

Although there is some reduction in the internal forces, this difference can be neglected in the design as it is insignificant. The equations suggested in Chapter 5 for the prediction of internal force magnification ratios can still be useful and will give acceptable and slightly conservative results.

\subsubsection{The Effect of Diaphragm Nonlinearity on the Ductility Demand in the Brace}

By allowing nonlinearity in the roof diaphragm, while keeping the braces as the main energy dissipating system, a portion of the seismic energy will be dissipated by the deformations in steel deck panels. Therefore, it is expected that the ductility demand on the braces would decrease. The results of the study illustrate that this reduction is more significant for buildings with greater drift ratio. In Figures 7.45 , to 7.47 the reduction in the ductility demand $\left(R_{\mu}\right)$ on the brace by allowing the diaphragm to behave nonlinearly 
is shown as a percentage of the target ductility demand for the braces. The results are shown for each target ductility demand separately.

A regression fit is also carried out on the results to propose an equation for the prediction of this reduction. It must be noted that a reduction of $50 \%, 66.66 \%$ and $75 \%$ means elastic behaviour for target ductility demand values of 2, 3 and 4 respectively. The elastic upper bounds are shown on the curves. The following equations are suggested to predict the percentage of the reduction in the ductility demand shown by $R_{\mu \text {. }}$

$R_{\mu}=3.59 \cdot r-0.6$ (For Target Ductility of 2.00)

$R_{\mu}=2.65 \cdot r+1.28$ (For Target Ductility of 3.00$)$

$R_{\mu}=2.73 \cdot r-0.56$ (For Target Ductility of 4.00 )

It can be noted from the results that as the flexibility of the diaphragm increases (higher drift ratio), the diaphragm dissipates the seismic energy more effectively and the ductility demand on the braces decreases more. 


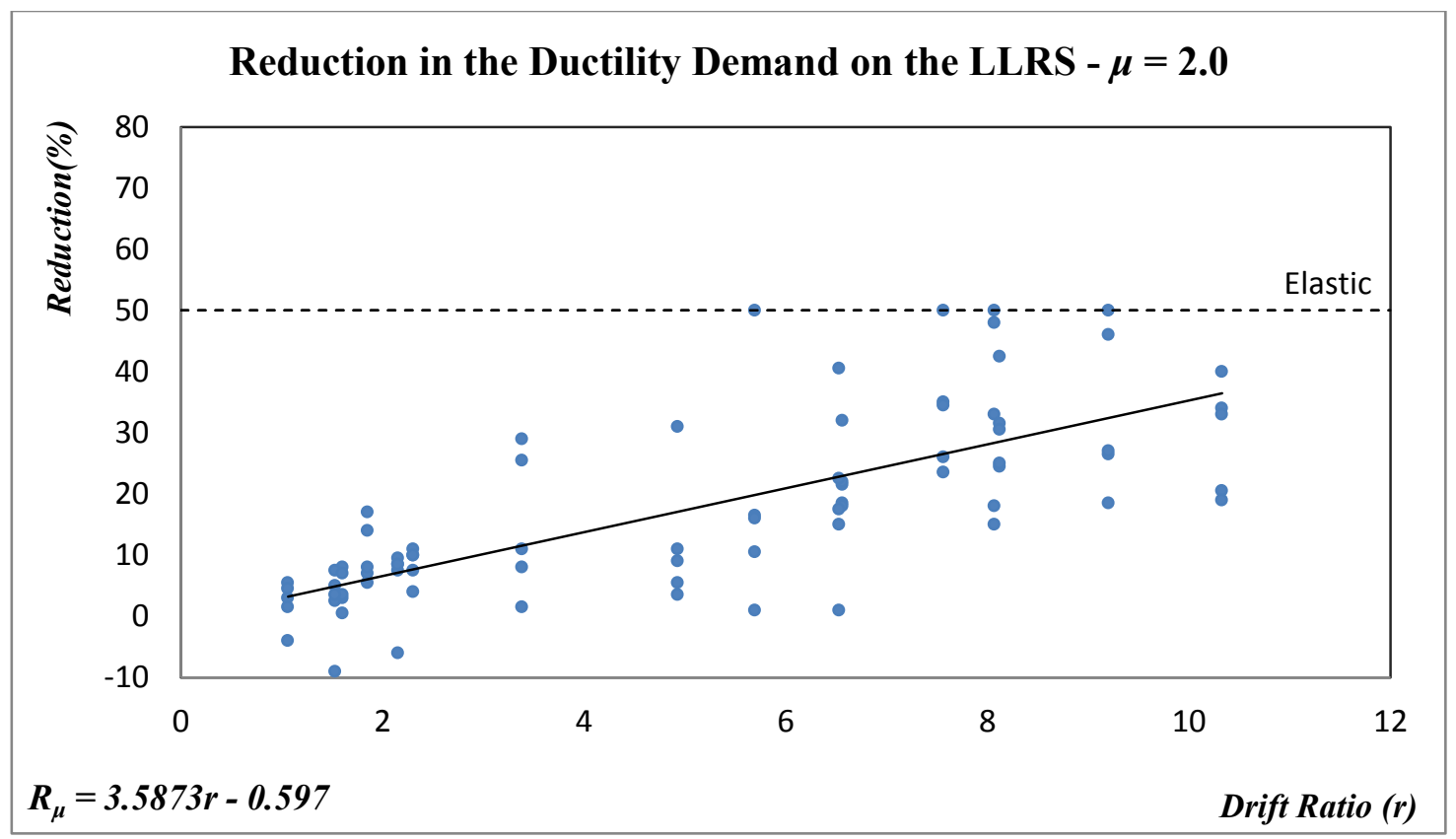

Figure 7.45 - The reduction in the ductility demand on the brace by allowing limited nonlinearity $\left(\mu_{d} \leq 1.5\right)$ in the roof diaphragm, target ductility of 2.00

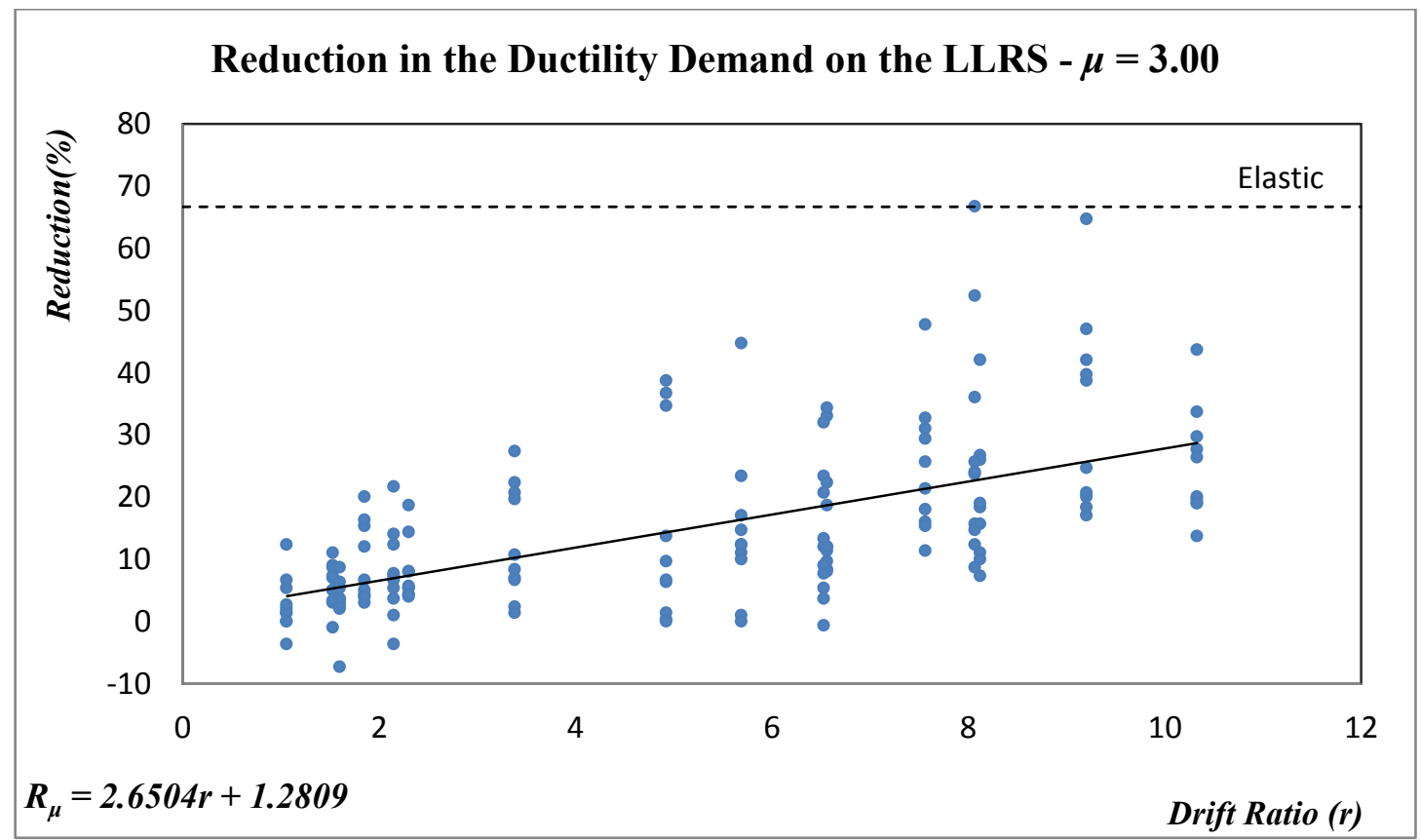

Figure 7.46 - The reduction in the ductility demand on the brace by allowing limited nonlinearity $\left(\mu_{d} \leq 1.5\right)$ in the roof diaphragm, target ductility of 3.00 


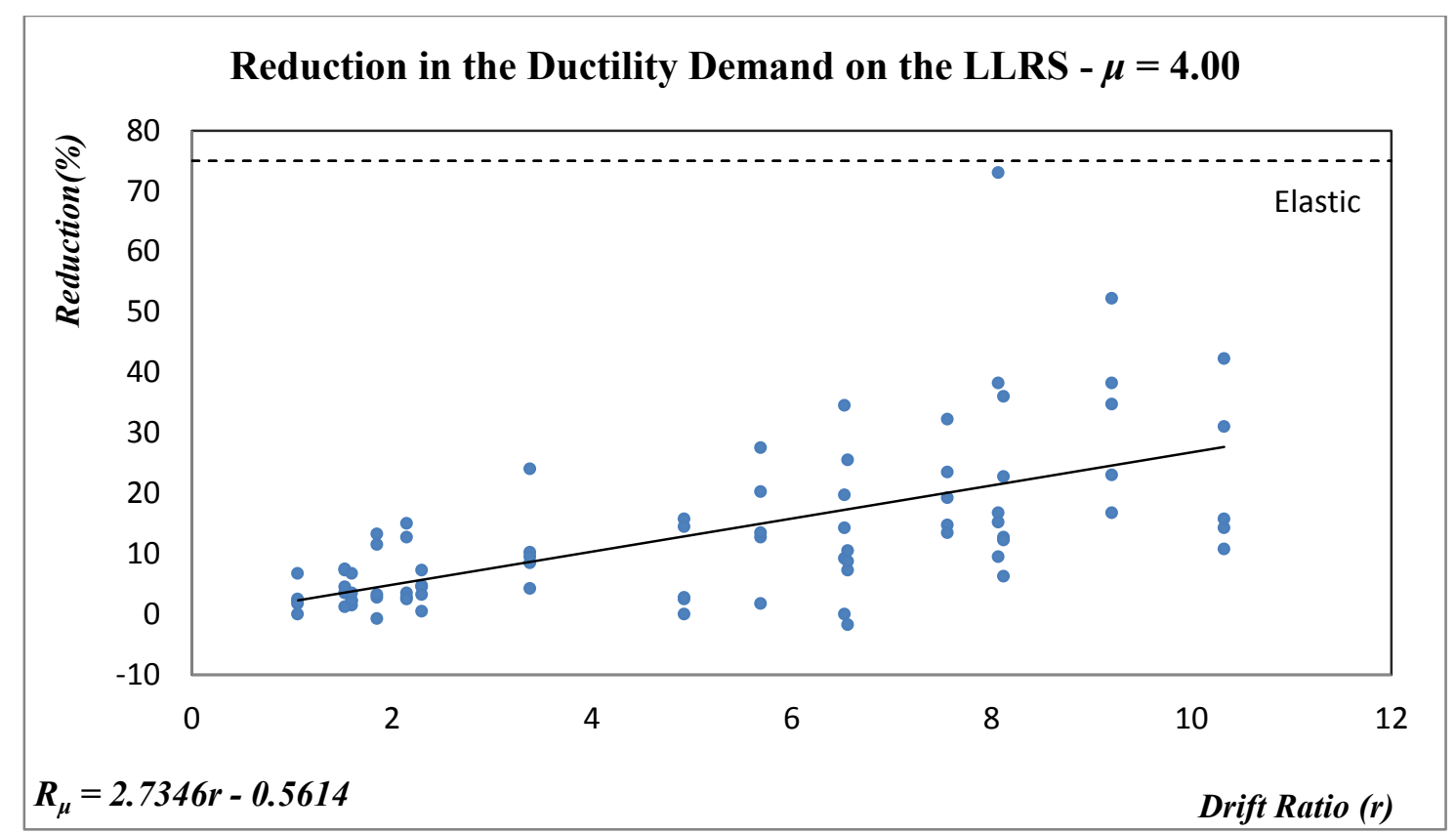

Figure 7.47 - The reduction in the ductility demand on the brace by allowing limited nonlinearity $\left(\mu_{d} \leq 1.5\right)$ in the roof diaphragm, target ductility of 4.00

\subsection{Selection of the Final Force Reduction Factor}

In the present study, methods have been suggested for finding a proper force reduction factor based on target ductility demand values. Equations have been proposed for finding the force reduction factor for different design approaches, such as designing the brace to be the energy dissipating element, the diaphragm to be the dissipating element, and both the brace and the diaphragm as the energy dissipating elements. For the final selection of the force reduction factor used in the design, NBCC 2010 states that the product of the ductility related force reduction factor and the over-strength related force reduction factor $\left(R_{d} \times R_{o}\right)$ must be used. In order to select appropriate values for these factors, it is useful to review the roles of $R_{d}$ and $R_{o}$. 


\subsubsection{Ductility-Related Force Reduction Factor $\left(\boldsymbol{R}_{d}\right)$}

The ductility-related force reduction factor is the classical meaning of the force reduction factor used in previous versions of NBCC and most of the design guidelines. In the design, a structural component capable of dissipating the seismic energy through inelastic deformations will be designed for a fraction of the elastic earthquake force. Usually, a capacity design approach must be adopted for other structural members to ensure that the nonlinearity is confined to the elements expected to deform in the inelastic range. Such element must be designed carefully and detailed according to the provisions given in codes and design standards to ensure a ductile behaviour and the ability to dissipate the seismic energy (Mitchell et al. 2003)

\subsubsection{Over-Strength Related Force Reduction Factor}

Experience of observed structural behaviour during past earthquakes and during laboratory experiments has shown that structures often have a strength that is larger than that computed from the nominal geometric and material properties. To account for this reserve of strength, NBCC 2010 specifies another reduction factor $R_{o}$ to be used to obtain the design force. Mitchell et al. (2003) have pointed out that there are several factors that contribute to the value of $R_{o}$. The over-strength force reduction factor is thus obtained from (Mitchell et al 2003):

$R_{o}=R_{\text {size }} R_{\phi} R_{\text {yield }} R_{\text {sh }} R_{\text {mech }}$

in which,

$R_{o}=$ Final over-strength related force reduction factor proposed for NBCC 2010 
$R_{\text {size }}=$ The over strength caused by restrictions in the selection of members and rounding up of the sizes and dimensions in the design

$R_{\phi}=$ A factor accounting for the difference between the nominal resistance and the factored resistance. $R_{\phi}$ can be defined as $1 / \phi$ when $\phi$ is the material resistance factor specified in CSA provisions

$R_{\text {yield }}=$ The ratio of the actual yield strength to the minimum specified yield strength

$R_{s h}=$ Over strength owing to strain hardening in the material

$R_{\text {mech }}=$ The over strength resulting from mobilization of the full capacity of the structure until a collapse mechanism is formed; it can be found from the following equation (Mitchell et al):

$R_{\text {mech }}=\frac{N+\beta_{c}}{N+1}$

where $N$ is the number of stories and $\beta_{c}$ is the ratio of the capacity of the column to the capacity of the beams (usually exceeding 1.2).

The factors outlined above are explained in detail in the 2003 paper by Mitchell et al. The authors also specify appropriate values of these factors for different structural systems based on both experimental and analytical studies.

\subsubsection{Selection of Design Force Reduction Factor}

In the present study, methods for calculating the ductility related force reduction factor $\left(R_{d}\right)$ to be applied in the design of single-storey buildings with flexible diaphragm have been suggested. Once an appropriate value has been selected for the overstrengh factor 
$R_{o}$, the two factors, $R_{d}$ and $R_{o}$, can be combined to obtain the final force reduction factor. In the design approach in which the brace is selected as the energy dissipating member, assuming a moderately-ductile concentrically braced frame, the values of the components as suggested by Mitchell et al are: $R_{\text {size }}=1.05, R_{\phi}=1.11, R_{\text {yield }}=1.10, R_{\text {sh }}=1.05, R_{\text {mech }}=$ 1.00 .

These aforementioned parameter values lead to $R_{o}=1.35$; the value specified in NBCC is 1.3 .

In the final part of the present study, the bracing system is assigned a post-yield stiffness ratio of 0.05 . It can thus be reasoned that the strain hardening component of the overstrength has already been accounted for. The other factors can still contribute to the over-strength force reduction factor. On setting $R_{s h}=1.0$, factor $R_{o}$ works out to 1.28 , which can be rounded off to 1.3 .

Mitchel et al. do not specify $R_{o}$ factors for the case when the diaphragm is the energy dissipating element. In the design, $R_{o}$ for the roof diaphragm can be conservatively assumed to be 1.3. However, studies on the magnitude of over-strength when the steel diaphragm is designed as an energy dissipating system will be useful.

It may be noted that the conclusions of this study are not affected by the selection of $R_{o}$. 


\subsection{Summary and Conclusions}

The common approach in the seismic design of one-storey buildings consisting of corrugated steel deck roof diaphragm supported by steel braced frames is to have the braces act as the main energy dissipating members. It has been noted that such design approach leads to over-designed members and often an uneconomical design (Tremblay et al. 2002). The study reported in this chapter investigates the seismic response of singlestorey buildings with flexible roof diaphragms designed to be strained into the nonlinear range. The objective of the investigation is to develop design methodologies for such buildings.

In developing the analytical model for the diaphragm previous analytical and experimental work on the force deformation relations for steel deck diaphragms are reviewed. These studies (Rogers and Tremblay 2003a, b and Essa et al 2003) show that the steel deck panels deform primarily in shear and the behaviour of the diaphragm in shear is determined mainly by the type of connections. Steel deck panels also exhibit severe pinching and strength degradation in their hysteretic response.

Two different cases are considered in the investigation; one in which the bracing system is elastic while the diaphragm is strained into the inelastic range, and the other in which both the diaphragm and the bracing system respond in the nonlinear range. A selected set of buildings are analysed for a suite of ground motions. The hysteresis behaviour for the diaphragm is selected based on the considerations cited in the preceding paragraph.

The following conclusions can be drawn from the study presented in this chapter:

1. The modified IMK deterioration model with pinched hysteretic response is found to give a fair prediction of the hysteretic behaviour of the steel deck panels. 
2. For low target ductility demands the strength deterioration does not get a chance to be triggered and can be disregarded in the analytical model.

3. When the braces remain elastic and the diaphragm is the main energy dissipating member, the nonlinearity of the diaphragm does not make a pronounced difference in the magnification of the internal forces. The FEMA force distribution provides fair predictions of the shear at quarter span and bending moment at mid span.

4. The force reduction factor for the diaphragm is observed to be proportional to the drift ratio of the building. Equations are suggested for predicting the force reduction factor for the case when the diaphragm is the only energy dissipating element.

5. When the diaphragm is the only energy dissipating element, residual displacements are not observed in the bracing system, but are confined to the roof. The building is therefore suitable for post-hazard occupancy

6. In the design approach in which the brace is designed to dissipate the seismic energy, the diaphragm must be designed to stay elastic. To ensure this the design force for the diaphragm must be larger than the brace yield force. The factor by which the diaphragm force should exceed the brace yield force, $\left(\Phi_{e}\right)$, is developed. Another factor $\left(\Phi_{d}\right)$ is determined for the case when the diaphragm is allowed limited nonlinearity while the bracing system remains the main energy dissipating element.

7. When the diaphragm responds in the inelastic range, the highest inelasticity can occur at either the diaphragm ends or somewhere along the length of the 
diaphragm. If the contribution of higher modes is limited and not significant, the nonlinearity is expected to take place at diaphragm ends since the shear force at the quarter span is less than that at the diaphragm ends. However, if the higher modes make a significant contribution to the total response of the system, the nonlinearity would occur somewhere along the length of the diaphragm and not at the diaphragm ends. The contribution of higher modes is mainly dependent on the frequency content of the earthquake and the frequency of the system, but as pointed out in Chapter 5 higher ductility demand on the braces also causes greater contribution from the higher modes. The results obtained in this part of the study are consistent with that assessment.

8. When the braces are designed for target ductility values of $2.0,3.0$ and 4.0 nonlinearity occurs more frequently along the length of the diaphragm and less at the diaphragm ends. This is in contrast to the case of elastic braces when the nonlinearity in the diaphragm is generally at the ends.

9. Nonlinearity along the length of the diaphragm was observed more frequently for eastern records. This is expected since the spectral shape for the east is steeper causing greater contribution from the higher modes

10. Whether the nonlinearity occurs at diaphragm ends, due to insignificant contribution from higher modes, or somewhere along the length of the diaphragm caused by greater contribution from higher modes, higher ductility demand values lead to larger values for diaphragm design force factors $\left(\Phi_{e}\right.$ and $\left.\Phi_{d}\right)$.

11. By allowing nonlinearity in the diaphragm, the ductility demand in the brace decreases as part of the seismic energy is dissipated in the diaphragm. This 
reduction is more severe in buildings with higher drift ratio. Such behaviour indicates that the higher the drift ratio the more effective is the energy dissipation in the diaphragm. 


\section{Chapter 8 : Summary, Conclusions and Recommendations}

\subsection{Summary}

Single-storey buildings with large footprints are used quite often for institutional, educational or commercial applications. The roof diaphragm for such buildings often consists of un-topped steel deck or wood panels. Such a diaphragm experiences significant in-plane deformation under lateral loads, in particular under seismic loads. Previous studies on the seismic response of single-storey buildings with flexible diaphragm have shown that the flexibility of the diaphragm has significant effect on the response of the building. The following effects of diaphragm flexibility have been identified:

1. The flexibility of the diaphragm lengthens the fundamental period of the system.

2. It leads to a significant increase in the ductility demand on the lateral load resisting system, particularly when the diaphragm is designed to remain elastic. Consequently, a smaller force reduction factor must be used to keep the ductility demand on the lateral load resisting system within a reasonable range that is achievable with current detailing practice.

3. It leads to an increase in shear forces and bending moments along the length of the diaphragm. In particular, the shear force at quarter span and the bending moment at mid span may increase significantly.

The objective of the present research is to carry out additional study on the effects outlined above 
For the purpose of this study a set of single-storey buildings whose design has been described in a previous study by Tremblay and Stiemer (1996) are analysed for their response to selected ground motion records. The buildings are located in various regions of Canada. Hence the ground motion records are selected so that they match the design uniform hazard spectra for two locations in Canada, Vancouver to represent the seismic environment of western Canada and Montreal to represent that of the eastern region. The building database as well as the ground motion records are described in Chapter 2. For the dynamic analyses the buildings are modelled by a deep beam representing the diaphragm, supported on two springs representing the lateral load resisting system (LLRS) consisting of concentric steel braces. Both flexural and shear deformations in the diaphragm are taken into account. The flexural resistance is contributed by the chord members at the edges of the diaphragm while the web consisting of the roof deck provides the shear resistance. It may be noted that while the results obtained and the conclusions reached in the present study are based mainly on the seismic time history analyses of building structures consisting of a steel deck diaphragm and a lateral load resisting system (LLRS) of concentric steel braces, most of the results are equally applicable to buildings in which a different type of LLRS is used.

In the first part of the study the diaphragm is assumed to remain elastic while the steel braces are strained into the inelastic range. Response analyses are carried out to determine the force reduction factors that would ensure that the ductility demand on the LLRS does not exceed the specified target ductilities. The procedure suggested by Humar and Popovski (2013) to determine such reduction factors, which was based on ground motions compatible with the western UHS is extended for eastern ground motions. The 
method by Humar and Popovski (2013) suggests values for a factor $\kappa$ to be used as a multiplier on the target ductility to determine the appropriate force reduction factor. It is found that with certain improvements the suggested expressions to find $\kappa$ are appropriate for all regions of Canada. A comparison is also made between the revised method and the method proposed by Tremblay. It is found that the latter is fairly conservative.

The response studies referred to in the preceding paragraph are based on the assumption that the LLRS possesses an elasto-plastic hysteretic relationship with no stiffness and no strength degradation. To examine the effect of strain hardening on the ductility demand, the dynamic analyses are repeated with a bilinear hysteretic relationship for the LLRS. Three different values are considered for the post-yield stiffness ratios, namely $0.02,0.05$ and 0.10 without any strength or stiffness degradation. The equation by Humar and Popovski (2013), as revised in this study is further refined and new lower limits on the $\kappa$ value are suggested for each post-yield stiffness ratio.

As stated earlier the flexibility of the diaphragm usually leads to magnification of internal forces, specifically shear force at quarter span and bending moment at mid span. However methods for predicting the extent of such magnification do not exist.

An investigation is carried out to determine the magnification of internal forces along the length of the diaphragm caused by the flexibility of the diaphragm. Based on a comprehensive set of analyses, methods are suggested for predicting the magnified values of the shear force at quarter span and the bending moment at mid span.

Concentric braces often exhibit strength and stiffness degradation and pinching behaviour in their hysteretic response to cyclic lateral loads. It is therefore useful to investigate the 
effect of such behaviour on the seismic response of buildings with flexible diaphragm. Based on the results of previous experimental studies on the response of concentric braces, an analytical model is proposed to capture the hysteretic behaviour of such elements to lateral cyclic loads. Nonlinear analyses are carried out on a limited number of buildings for their response to 6 different ground motions and the results are compared to those obtained for a bilinear hysteretic behaviour. The response results show that the mean value of the ductility demands is more or less the same as that produced by an elasto-plastic behaviour. The results further indicate that the bending moment magnification and the shear force magnification at mid span and quarter span, respectively, are greater in the elasto-plastic case compared to that in the pinching cases. Therefore, the previously suggested equations can conservatively be applied to singlestorey buildings with concentric braces forming the LLRS, even when the braces exhibit pinching behaviour.

Previous studies have shown that depending on the type of connections used for connecting the deck panels to each other and to the supporting frame, steel deck diaphragm do show some ductility capacity in shear. In the last stage of the present study an attempt is made to assess the performance of one-storey buildings in which the steel deck diaphragms are strained into the nonlinear range. With the assistance of previous experimental works, the hysteretic behaviour of steel deck diaphragms is defined and the key parameters that control its shape are identified. An analytical model is proposed to capture the severe pinching and the strength degradation that is often observed in the hysteretic response of diaphragms. 
The analytical model referred to in the preceding paragraph is used in the response analyses of selected buildings. Expressions for the prediction of appropriate force reduction factors, based on specific target ductility demand values, for the design of building structure including the LLRS and the diaphragm are proposed.

Design forces for the diaphragm are, in fact, determined for three different cases. (1) when the seismic energy is dissipated in the diaphragm and the braces remain elastic, (2) when the diaphragm remains just elastic while the braces dissipate the seismic energy, and (3) when both the diaphragm and the LLRS exhibit nonlinearity but the ductility demand on the diaphragm is limited to a specific value, chosen as 1.5 . In the first case, equations are suggested for finding an appropriate force reduction factor for the design of the diaphragm. In the two latter cases, however, equations are proposed for finding the diaphragm design force as a factor of the brace yield force.

\subsection{Conclusions}

The current study has led to several conclusions. Some of the more important conclusions are presented in the following paragraphs:

1. The flexibility of the diaphragm causes a significant increase in the ductility demand on the lateral load resisting system, particularly when the diaphragm is designed to remain elastic. In many instances such increase is quite large. It is therefore imperative that a smaller force reduction factor be used in the design in order to ensure that the ductility demands are achievable with the current detailing techniques. 
2. The equation and the method proposed by Humar and Popovski (2013) with the revisions proposed here provide a reasonable procedure for determining the force reduction factors for buildings with flexible diaphragms located within both the western and the eastern regions of Canada.

3. The relationship between force reduction factor, drift ratio, and ductility demand suggested by Tremblay generally leads to quite conservative seismic design forces. The equations proposed by Humar and Popovski and slightly modified as a result of this study provide more realistic and economic design.

4. Post-yield stiffness decreases the ductility demand on the LLRS, relative to the case for zero post-yield stiffness, and consequently increased force reduction factor may be used. However, the reduction in ductility demand is comparatively small and the expressions based on an elasto-plastic behaviour may be considered as being appropriate for design. If refinement for the effect of post-yield stiffness is desired, the values suggested in Table 4.1 or Equation 4.1 and 4.2 may be used. Also, systems with higher post-yield stiffness exhibit smaller residual displacements.

5. The flexibility of the diaphragm attracts greater contributions from the higher modes.

6. Greater contribution of higher modes is the main reason for the non-uniform distribution of seismic forces along the length of the diaphragm leading to a significant increase in bending moment at mid-span and shear force at the quarter span. 
7. The contribution of higher modes and consequently the magnification of bending moments and shear forces are enlarged as the excursion of the system into the nonlinear range is increased by using larger force reduction factors in design.

8. Magnification of internal forces is slightly greater in the eastern region than in the western region. This is expected, because the spectral shape for the east is steeper leading, to greater contribution from the higher modes. However, the difference between the magnifications values for east and west is not large; the single set of equations proposed here may be used for all regions.

9. Quarter span shear magnification is much greater than mid-span moment magnification. Therefore, separate expressions are suggested, one set for predicting the magnification factor for bending moment and the other for the shear force.

10. The FEMA parabolic distribution of inertia forces provides good estimates of the bending moments when the LLRS is elastic. Nonlinearity in the LLRS further increases the mid-span bending moments and the in-span shears, but the parabolic distribution may be used as the base line pattern to which the magnifications proposed in this study may be applied.

11. While the FEMA parabolic load distribution provides bending moments and shear forces that are closer to the true values prior to the magnification of the results, if the simplicity of the uniform distribution makes it a useful alternative, the magnification factors proposed for that case may be applied. 
12. Equations 5.6 to 5.9 provide magnification ratios that are most precise among the alternatives considered. However their lack of simplicity makes the alternatives more attractive.

13. Based on Equations 5.6 to 5.9, it is observed that there is a direct relation between the force reduction factor (a measure of nonlinearity of the system) and the magnification factors. On the other hand, there is an inverse relation between the post-yield hardening effect and the magnification values.

14. Since the effect of post-yield stiffness on the level of magnification is not significant, conservatively, the expressions obtained when elasto-plastic behaviour is considered for the LLRS, namely equations 5.60 and 5.61 can be used for design purposes as they are simpler and give the most conservative estimates for the magnification factors. It may be noted that Equations 5.60 and 5.61 are based on the FEMA distribution of the seismic force.

15. Concentric braces often exhibit pinching in their hysteretic behaviour. Therefore, the validity of the previously suggested equations that were derived based on the assumption of an elasto-plastic behaviour for the LLRS must be tested for buildings in which the LLRS shows a pinched hysteretic response.

16. The pinching behaviour in concentric braces is caused by buckling mechanism of the brace in compression and the increase in the length of the brace in tension in each cycle. The former can be related to the slenderness of the member and the latter depends mostly on geometry and the characteristics of the excitation.

17. When pinching is accounted for in the analytical model, the values of the ductility demand on the LLRS fluctuate around that determined from an elasto-plastic 
model, being greater in some cases and smaller in others. Whether the ductility demand would increase or decrease, depends on the characteristics of the excitation and the structural system. The results of the current study show that the mean value of the ductility demands obtained from the response of different buildings subjected to ground motions compatible with the UHS for the western and eastern regions of Canada when a pinched behaviour for the LLRS is considered is more or less the same as that produced by an elasto-plastic behaviour.

18. The bending moment magnification and the shear force magnification at mid span and quarter span, respectively, are observed to be greater in the elasto-plastic case compared to that in the pinching cases. Consequently, the previously suggested equations for predicting the magnification factors in bending moment and shear force along the length of the diaphragm can conservatively be applied to singlestorey buildings with concentric braces forming the LLRS, even when the braces exhibit pinching behaviour.

19. It is observed that the magnification in internal forces is higher for brace elements with high slenderness compared to the case when the braces have intermediate value of the slenderness.

20. The modified IMK deterioration model with pinched hysteretic response is found to give a fair prediction of the hysteretic behaviour of the steel deck panels.

21. For low target ductility demands the strength deterioration does not get a chance to be triggered and can be disregarded in the analytical model. 
22. When the braces remain elastic and the diaphragm is the main energy dissipating member, the nonlinearity of the diaphragm does not make a pronounced difference in the magnification of the internal forces, and the FEMA force distribution provides fair predictions of the shear at quarter span and bending moment at mid span.

23. When the diaphragm is the only energy dissipating element, the force reduction factor for the diaphragm is observed to be proportional to the drift ratio of the building. Equations are suggested for predicting the force reduction factor for this case.

24. By having the diaphragm as the only energy dissipating member, residual displacements are not observed in the bracing system, but are confined to the roof. The building is therefore suitable for post-hazard occupancy

25. In the design approach in which the brace is designed to dissipate the seismic energy, and the diaphragm is assumed to stay elastic, the design force for the diaphragm must be larger than the brace yield force. The factor by which the diaphragm force should exceed the brace yield force, $\left(\Phi_{e}\right)$, is developed. Another factor $\left(\Phi_{d}\right)$ is determined for the case when the diaphragm is allowed limited nonlinearity while the bracing system remains the main energy dissipating element.

26. When the diaphragm responds in the inelastic range, the highest inelasticity can occur at either the diaphragm ends or somewhere along the length of the diaphragm. If the contribution of higher modes is limited and not significant, the nonlinearity is expected to take place at diaphragm ends since the shear force at 
the quarter span is less than that at the diaphragm ends. However, if the higher modes make a significant contribution to the total response of the system, the nonlinearity would occur somewhere along the length of the diaphragm and not at the diaphragm ends. The contribution of higher modes is mainly dependent on the frequency content of the earthquake and the frequency of the system, but as observed earlier in the study higher ductility demand on the LLRS leads to greater contribution from the higher modes. The results obtained in this part of the study are consistent with that assessment.

27. When the braces are designed for target ductility values of $2.0,3.0$ and 4.0 nonlinearity occurs more frequently along the length of the diaphragm and less frequently at the diaphragm ends. This is in contrast to the case of elastic braces when the nonlinearity in the diaphragm is generally at the ends.

28. Nonlinearity along the length of the diaphragm was observed more frequently for eastern records. This is expected since the spectral shape for the east is steeper causing greater contribution from the higher modes

29. Whether the nonlinearity occurs at diaphragm ends, due to insignificant contribution from higher modes, or somewhere along the length of the diaphragm caused by greater contribution from higher modes, higher ductility demand values lead to larger values for diaphragm design force factors $\left(\Phi_{e}\right.$ and $\left.\Phi_{d}\right)$.

30. By allowing nonlinearity in the diaphragm, the ductility demand on the braces decreases since part of the seismic energy is dissipated in the diaphragm. This reduction is more severe in buildings with higher drift ratio values. Such 
behaviour indicates that the higher the drift ratio the more effective is the energy dissipation in the diaphragm.

\subsection{Recommendations}

Based on the findings and the conclusions reached in the current study, the author offers the following suggestions for future studies:

1. Additional studies on a more extensive database of buildings and for ground motions that are compatible with a series of uniform hazard spectra will be useful for confirming the validity of the conclusions arrived at in this study related to the increase in the ductility demand on bracing systems caused by the flexibility of the diaphragm. Additional studies on the effect of pinching and strength and stiffness degradation, when diaphragm and/or braces respond in the nonlinear range will be useful.

2. Further experimental studies are recommended for assessing the hysteretic behaviour of steel deck panels under cyclic lateral loads. The specimens must include a variety of deck to deck and deck to frame fasteners.

3. As shown in the present study, when the diaphragm is designed to respond in the nonlinear range, the nonlinearity may occur at different locations along the length of the diaphragm. In the current design practice, particularly in wide buildings, the deck panels are designed to be stronger towards the ends of the diaphragms. Given that nonlinearity is expected to take place at different locations, it is recommended that the steel deck panels be designed so that the strength and the 
stiffness stay consistent throughout the length of the diaphragm or at least for a significant length towards the ends of the diaphragm.

4. Further analytical studies using alternative software and finite element methods and experimental studies are suggested to validate the findings of the current study. 


\section{Appendix A}

Table A.1 presents the properties of the buildings included in the study when the load is applied parallel to the short side of the building. Table A.2 presents the properties of the selected buildings for the case in which the load is applied parallel to the long side of the building. Tables A.3 to A.22 present the results of the analyses explained in Chapter 3. 
Table A.1 - The properties of the buildings when the load acts parallel to the short side

\begin{tabular}{|c|c|c|c|c|c|c|c|c|}
\hline Buildings & $B$ & $L$ & $I$ & $G$ & $K b$ & $m$ & $T$ & $r$ \\
\hline SL1 & 15 & 30 & 0.556 & 2410 & 30700 & 1.529 & 0.426 & 6.563 \\
\hline SL3 & 15 & 30 & 0.663 & 2320 & 30700 & 1.529 & 0.432 & 6.779 \\
\hline SL4 & 15 & 30 & 0.853 & 2100 & 30700 & 1.529 & 0.45 & 7.436 \\
\hline SH1 & 15 & 30 & 0.663 & 2400 & 35400 & 3.379 & 0.628 & 7.563 \\
\hline $\mathrm{SH} 2$ & 15 & 30 & 0.766 & 2170 & 35400 & 3.379 & 0.655 & 8.319 \\
\hline SH3 & 15 & 30 & 0.853 & 2100 & 30700 & 3.379 & 0.669 & 7.436 \\
\hline SH4 & 15 & 30 & 0.977 & 1950 & 35400 & 3.379 & 0.685 & 9.204 \\
\hline SH5 & 15 & 30 & 0.977 & 1970 & 30700 & 3.379 & 0.687 & 7.902 \\
\hline SH6 & 15 & 30 & 0.766 & 2100 & 30700 & 3.379 & 0.667 & 7.45 \\
\hline ML1 & 30 & 60 & 2.223 & 3260 & 48600 & 3.058 & 0.741 & 8.069 \\
\hline ML2 & 30 & 60 & 2.246 & 2500 & 48600 & 3.058 & 0.841 & 10.329 \\
\hline ML3 & 30 & 60 & 2.65 & 2320 & 40200 & 3.058 & 0.86 & 9.09 \\
\hline ML4 & 30 & 60 & 3.411 & 2870 & 48600 & 3.058 & 0.773 & 8.868 \\
\hline ML5 & 30 & 60 & 3.411 & 2900 & 48600 & 3.058 & 0.768 & 8.78 \\
\hline ML6 & 30 & 60 & 2.246 & 2450 & 40200 & 3.058 & 0.843 & 8.708 \\
\hline MH1 & 30 & 60 & 2.651 & 5110 & 74900 & 6.758 & 0.89 & 8.123 \\
\hline MH2 & 30 & 60 & 3.065 & 4610 & 57200 & 6.758 & 0.938 & 6.729 \\
\hline MH3 & 30 & 60 & 3.411 & 2900 & 48600 & 6.758 & 1.144 & 8.78 \\
\hline MH4 & 30 & 60 & 3.906 & 4200 & 74900 & 6.758 & 0.953 & 9.456 \\
\hline MH5 & 30 & 60 & 3.906 & 4240 & 57200 & 6.758 & 0.964 & 7.157 \\
\hline MH6 & 30 & 60 & 3.065 & 8270 & 48600 & 6.758 & 1.014 & 3.384 \\
\hline LL1 & 60 & 120 & 8.982 & 13900 & 107000 & 6.116 & 0.907 & 6.529 \\
\hline LL2 & 60 & 120 & 8.982 & 13500 & 107000 & 6.116 & 0.914 & 6.643 \\
\hline LL3 & 60 & 120 & 10.6 & 8650 & 84700 & 6.116 & 1.032 & 6.694 \\
\hline LL4 & 60 & 120 & 13.64 & 12800 & 107000 & 6.116 & 0.873 & 5.945 \\
\hline LL5 & 60 & 120 & 13.64 & 13000 & 107000 & 6.116 & 0.869 & 5.88 \\
\hline LL6 & 60 & 120 & 8.982 & 4080 & 68600 & 6.116 & 1.379 & 10.125 \\
\hline LH1 & 60 & 120 & 17.9 & 20500 & 154000 & 13.517 & 1.061 & 5.692 \\
\hline LH2 & 60 & 120 & 13.9 & 22900 & 130000 & 13.517 & 1.088 & 4.943 \\
\hline LH3 & 60 & 120 & 13.69 & 13000 & 107000 & 13.517 & 1.291 & 5.88 \\
\hline LH4 & 60 & 120 & 15.62 & 20800 & 130000 & 13.517 & 1.094 & 4.998 \\
\hline LH5 & 60 & 120 & 15.62 & 20500 & 127000 & 13.517 & 1.1 & 4.927 \\
\hline LH6 & 60 & 120 & 12.26 & 12400 & 107000 & 13.517 & 1.327 & 6.278 \\
\hline
\end{tabular}


Table A.2 - The properties of the buildings when the load acts parallel to the long side

\begin{tabular}{|c|c|c|c|c|c|c|c|c|}
\hline Buildings & $B$ & $L$ & $I$ & $G$ & $K b$ & $m$ & $T$ & $r$ \\
\hline SL1 & 30 & 15 & 1.526 & 2410 & 30700 & 3.058 & 0.254 & 1.601 \\
\hline SL3 & 30 & 15 & 1.526 & 2320 & 30700 & 3.058 & 0.257 & 1.663 \\
\hline SL4 & 30 & 15 & 1.526 & 2100 & 30700 & 3.058 & 0.265 & 1.836 \\
\hline SH1 & 30 & 15 & 1.526 & 2400 & 35400 & 6.758 & 0.367 & 1.853 \\
\hline $\mathrm{SH} 2$ & 30 & 15 & 1.526 & 2170 & 35400 & 6.758 & 0.379 & 2.049 \\
\hline $\mathrm{SH} 3$ & 30 & 15 & 1.526 & 2100 & 30700 & 6.758 & 0.393 & 1.836 \\
\hline SH4 & 30 & 15 & 1.526 & 1950 & 35400 & 6.758 & 0.392 & 2.279 \\
\hline SH5 & 30 & 15 & 1.526 & 1970 & 30700 & 6.758 & 0.401 & 1.957 \\
\hline ML1 & 60 & 30 & 6.102 & 2670 & 48600 & 6.116 & 0.452 & 2.303 \\
\hline ML2 & 60 & 30 & 6.102 & 2430 & 48600 & 6.116 & 0.467 & 2.528 \\
\hline ML3 & 60 & 30 & 6.102 & 2320 & 40200 & 6.116 & 0.489 & 2.189 \\
\hline ML4 & 60 & 30 & 6.102 & 2490 & 48600 & 6.116 & 0.463 & 2.468 \\
\hline ML5 & 60 & 30 & 6.102 & 2370 & 48600 & 6.116 & 0.471 & 2.591 \\
\hline ML6 & 60 & 30 & 6.102 & 2410 & 40200 & 6.116 & 0.483 & 2.108 \\
\hline MH1 & 60 & 30 & 6.102 & 4440 & 74900 & 13.517 & 0.529 & 2.152 \\
\hline MH2 & 60 & 30 & 6.102 & 3880 & 57200 & 13.517 & 0.58 & 1.876 \\
\hline MH3 & 60 & 30 & 6.102 & 2550 & 48600 & 13.517 & 0.682 & 2.41 \\
\hline MH4 & 60 & 30 & 6.102 & 4200 & 74900 & 13.517 & 0.539 & 2.272 \\
\hline MH5 & 60 & 30 & 6.102 & 3550 & 57200 & 13.517 & 0.596 & 2.047 \\
\hline MH6 & 60 & 30 & 6.102 & 4590 & 48600 & 13.517 & 0.573 & 1.352 \\
\hline LL1 & 120 & 60 & 24.4 & 9500 & 107000 & 12.232 & 0.537 & 1.531 \\
\hline LL2 & 120 & 60 & 24.4 & 8740 & 107000 & 12.232 & 0.542 & 1.654 \\
\hline LL3 & 120 & 60 & 24.4 & 5220 & 84700 & 12.232 & 0.667 & 2.126 \\
\hline LL4 & 120 & 60 & 24.4 & 9430 & 107000 & 12.232 & 0.538 & 1.542 \\
\hline LL5 & 120 & 60 & 24.4 & 7310 & 107000 & 12.232 & 0.578 & 1.953 \\
\hline LL6 & 120 & 60 & 24.4 & 3940 & 68600 & 12.232 & 0.755 & 2.255 \\
\hline LH1 & 120 & 60 & 35.57 & 20500 & 154000 & 27.034 & 0.606 & 1.061 \\
\hline LH2 & 120 & 60 & 35.57 & 17300 & 130000 & 27.034 & 0.651 & 1.042 \\
\hline LH3 & 120 & 60 & 28.8 & 9760 & 107000 & 27.034 & 0.79 & 1.475 \\
\hline LH4 & 120 & 60 & 35.57 & 20800 & 130000 & 27.034 & 0.634 & 0.884 \\
\hline LH5 & 120 & 60 & 35.57 & 13100 & 127000 & 27.034 & 0.703 & 1.312 \\
\hline LH6 & 120 & 60 & 24.4 & 8240 & 107000 & 27.034 & 0.83 & 1.747 \\
\hline
\end{tabular}


Table A.3 - The response of the buildings subjected to $\mathrm{M} 6 \mathrm{C} 1$ ground motion parallel to the short side of the building with $\alpha=0.00$

\begin{tabular}{|c|c|c|c|c|c|}
\hline \multirow{2}{*}{ Building } & \multirow{2}{*}{$V e$} & \multirow{2}{*}{$\begin{array}{c}\text { Ductility }(\mu) \\
R_{y}=3\end{array}$} & \multicolumn{3}{|c|}{ Force Reduction Factor $\left(R_{y}\right)$} \\
\hline & & & $\mu=2$ & $\mu=3$ & $\mu=4$ \\
\hline SL1 & 131.5 & 13.78 & 1.25 & 1.37 & 1.50 \\
\hline SL3 & 133.1 & 14.10 & 1.25 & 1.38 & 1.47 \\
\hline SL4 & 142.1 & 14.09 & 1.30 & 1.41 & 1.50 \\
\hline SH1 & 316.8 & 9.39 & 1.21 & 1.38 & 1.51 \\
\hline $\mathrm{SH} 2$ & 283.9 & 10.62 & 1.32 & 1.41 & 1.49 \\
\hline $\mathrm{SH} 3$ & 264.2 & 10.72 & 1.30 & 1.39 & 1.48 \\
\hline SH4 & 260.9 & 11.29 & 1.29 & 1.40 & 1.49 \\
\hline SH5 & 262.9 & 10.09 & 1.31 & 1.42 & 1.54 \\
\hline SH6 & 263.0 & 10.78 & 1.30 & 1.38 & 1.48 \\
\hline ML1 & 508.8 & 6.41 & 1.43 & 1.59 & 1.71 \\
\hline ML2 & 435.8 & 8.21 & 1.15 & 1.48 & 1.58 \\
\hline ML3 & 417.7 & 8.15 & 1.19 & 1.48 & 1.60 \\
\hline ML4 & 490.8 & 6.85 & 1.43 & 1.58 & 1.71 \\
\hline ML5 & 493.7 & 6.76 & 1.43 & 1.58 & 1.71 \\
\hline ML6 & 428.7 & 7.61 & 1.18 & 1.50 & 1.61 \\
\hline MH1 & 878.8 & 8.26 & 1.35 & 1.49 & 1.61 \\
\hline MH2 & 832.1 & 7.75 & 1.14 & 1.49 & 1.62 \\
\hline MH3 & 500.5 & 10.78 & 1.43 & 1.82 & 2.01 \\
\hline MH4 & 832.5 & 8.46 & 1.13 & 1.45 & 1.61 \\
\hline MH5 & 851.3 & 6.65 & 1.37 & 1.58 & 1.73 \\
\hline MH6 & 1143.3 & 4.00 & 1.57 & 2.51 & 3.00 \\
\hline LL1 & 1523.3 & 7.45 & 1.27 & 1.43 & 1.73 \\
\hline LL2 & 1503.8 & 7.46 & 1.27 & 1.43 & 1.58 \\
\hline LL3 & 1496.1 & 9.01 & 1.46 & 1.66 & 1.77 \\
\hline LL4 & 1716.3 & 6.52 & 1.37 & 1.54 & 1.71 \\
\hline LL5 & 1735.1 & 6.38 & 1.37 & 1.55 & 1.73 \\
\hline LL6 & 469.1 & 20.08 & 1.27 & 1.39 & 1.48 \\
\hline LH1 & 2933.9 & 9.09 & 1.33 & 1.53 & 1.85 \\
\hline LH2 & 2472.9 & 11.27 & 1.29 & 1.60 & 1.85 \\
\hline LH3 & 1269.3 & 15.27 & 1.38 & 1.65 & 1.83 \\
\hline LH4 & 2409.6 & 11.00 & 1.26 & 1.53 & 1.87 \\
\hline LH5 & 2344.8 & 10.88 & 1.26 & 1.60 & 1.90 \\
\hline LH6 & 1212.9 & 14.92 & 1.31 & 1.54 & 1.68 \\
\hline
\end{tabular}


Table A.4 - The response of the buildings subjected to M6C2 ground motion parallel to the short side of the building with $\alpha=0.00$

\begin{tabular}{|c|c|c|c|c|c|}
\hline \multirow{2}{*}{ Building } & \multirow{2}{*}{$V e$} & \multirow{2}{*}{$\begin{array}{c}\text { Ductility }(\mu) \\
R_{y}=3\end{array}$} & \multicolumn{3}{|c|}{ Force Reduction Factor $\left(R_{y}\right)$} \\
\hline & & & $\mu=2$ & $\mu=3$ & $\mu=4$ \\
\hline SL1 & 135.5 & 16.86 & 1.21 & 1.33 & 1.44 \\
\hline SL3 & 137.2 & 18.38 & 1.20 & 1.31 & 1.42 \\
\hline SL4 & 138.2 & 21.56 & 1.18 & 1.26 & 1.33 \\
\hline SH1 & 226.8 & 8.43 & 1.19 & 1.61 & 1.73 \\
\hline $\mathrm{SH} 2$ & 223.9 & 7.92 & 1.20 & 1.62 & 1.72 \\
\hline $\mathrm{SH} 3$ & 223.3 & 6.55 & 1.23 & 1.69 & 1.80 \\
\hline SH4 & 216.4 & 9.59 & 1.24 & 1.35 & 1.83 \\
\hline SH5 & 215.9 & 8.83 & 1.25 & 1.38 & 1.87 \\
\hline SH6 & 223.2 & 6.40 & 1.23 & 1.35 & 1.80 \\
\hline ML1 & 276.6 & 21.94 & 1.22 & 1.31 & 1.37 \\
\hline ML2 & 211.1 & 36.12 & 1.25 & 1.38 & 1.45 \\
\hline ML3 & 234.5 & 29.69 & 1.18 & 1.50 & 1.58 \\
\hline ML4 & 231.1 & 28.63 & 1.17 & 1.27 & 1.36 \\
\hline ML5 & 235.5 & 27.77 & 1.18 & 1.27 & 1.36 \\
\hline ML6 & 221.4 & 31.37 & 1.22 & 1.45 & 1.52 \\
\hline MH1 & 583.3 & 21.50 & 1.21 & 1.63 & 1.73 \\
\hline MH2 & 687.7 & 9.57 & 1.51 & 1.73 & 1.91 \\
\hline MH3 & 580.2 & 17.05 & 1.46 & 1.80 & 1.89 \\
\hline MH4 & 697.4 & 9.40 & 1.25 & 1.63 & 1.79 \\
\hline MH5 & 706.7 & 7.17 & 1.48 & 1.69 & 1.91 \\
\hline MH6 & 568.5 & 12.62 & 1.33 & 1.46 & 1.67 \\
\hline LL1 & 1105.5 & 15.89 & 1.51 & 1.87 & 2.01 \\
\hline LL2 & 1108.6 & 16.18 & 1.49 & 1.85 & 1.99 \\
\hline LL3 & 1169.1 & 6.32 & 1.35 & 1.86 & 1.98 \\
\hline LL4 & 944.8 & 19.38 & 1.28 & 1.59 & 1.69 \\
\hline LL5 & 932.3 & 19.37 & 1.30 & 1.58 & 1.67 \\
\hline LL6 & 554.6 & 25.01 & 1.21 & 1.32 & 1.41 \\
\hline LH1 & 2431.3 & 6.74 & 1.34 & 1.66 & 1.77 \\
\hline LH2 & 2373.5 & 7.32 & 1.53 & 1.78 & 1.95 \\
\hline LH3 & 1828.0 & 15.74 & 1.43 & 1.60 & 1.71 \\
\hline LH4 & 2391.1 & 8.75 & 1.51 & 1.73 & 1.92 \\
\hline LH5 & 2440.7 & 9.28 & 1.52 & 1.75 & 1.95 \\
\hline LH6 & 1494.5 & 17.84 & 1.25 & 1.38 & 1.48 \\
\hline
\end{tabular}


Table A.5 - The response of the buildings subjected to M6C26 ground motion parallel to the short side of the building with $\alpha=0.00$

\begin{tabular}{|c|c|c|c|c|c|}
\hline \multirow{2}{*}{ Building } & \multirow{2}{*}{$V e$} & \multirow{2}{*}{$\begin{array}{c}\text { Ductility }(\mu) \\
R y=3\end{array}$} & \multicolumn{3}{|c|}{ Force Reduction Factor $(R y)$} \\
\hline & & & $\mu=2$ & $\mu=3$ & $\mu=4$ \\
\hline SL1 & 164.3 & 8.64 & 1.16 & 1.26 & 1.35 \\
\hline SL3 & 156.3 & 11.37 & 1.15 & 1.25 & 1.33 \\
\hline SL4 & 135.3 & 17.75 & 1.13 & 1.22 & 1.29 \\
\hline SH1 & 333.5 & 9.31 & 1.23 & 1.35 & 1.45 \\
\hline $\mathrm{SH} 2$ & 314.4 & 10.59 & 1.21 & 1.32 & 1.41 \\
\hline $\mathrm{SH} 3$ & 304.8 & 10.40 & 1.21 & 1.32 & 1.42 \\
\hline SH4 & 293.8 & 11.49 & 1.20 & 1.30 & 1.39 \\
\hline SH5 & 294.7 & 10.27 & 1.22 & 1.33 & 1.42 \\
\hline SH6 & 304.1 & 10.17 & 1.21 & 1.32 & 1.42 \\
\hline ML1 & 474.2 & 8.40 & 1.15 & 1.44 & 1.53 \\
\hline ML2 & 379.0 & 15.96 & 1.18 & 1.59 & 1.71 \\
\hline ML3 & 358.2 & 15.74 & 1.21 & 1.66 & 1.80 \\
\hline ML4 & 432.4 & 9.14 & 1.15 & 1.49 & 1.59 \\
\hline ML5 & 436.6 & 8.53 & 1.15 & 1.48 & 1.58 \\
\hline ML6 & 369.5 & 16.32 & 1.20 & 1.65 & 1.79 \\
\hline MH1 & 739.4 & 12.62 & 1.24 & 1.70 & 1.85 \\
\hline MH2 & 669.2 & 10.61 & 1.48 & 1.68 & 1.82 \\
\hline MH3 & 449.0 & 9.62 & 1.28 & 1.84 & 2.03 \\
\hline MH4 & 624.9 & 12.22 & 1.37 & 1.54 & 1.68 \\
\hline MH5 & 612.2 & 10.70 & 1.38 & 1.56 & 1.73 \\
\hline MH6 & 996.4 & 4.83 & 1.46 & 1.65 & 1.82 \\
\hline LL1 & 1154.3 & 12.88 & 1.28 & 1.41 & 1.87 \\
\hline LL2 & 1137.2 & 12.31 & 1.29 & 1.44 & 1.86 \\
\hline LL3 & 949.1 & 6.77 & 1.28 & 1.41 & 1.53 \\
\hline LL4 & 1226.8 & 13.01 & 1.22 & 1.68 & 1.83 \\
\hline LL5 & 1239.5 & 13.41 & 1.22 & 1.69 & 1.83 \\
\hline LL6 & 439.4 & 18.74 & 1.24 & 1.35 & 1.44 \\
\hline LH1 & 2064.4 & 4.91 & 1.24 & 1.42 & 1.90 \\
\hline LH2 & 2116.3 & 4.31 & 1.51 & 1.98 & 2.89 \\
\hline LH3 & 1321.5 & 5.97 & 1.16 & 1.75 & 1.95 \\
\hline LH4 & 1997.1 & 5.06 & 1.29 & 1.81 & 2.72 \\
\hline LH5 & 1961.0 & 5.12 & 1.29 & 1.87 & 2.67 \\
\hline LH6 & 1191.4 & 8.99 & 1.13 & 1.88 & 2.03 \\
\hline
\end{tabular}


Table A.6 - The response of the buildings subjected to M6C31 ground motion parallel to the short side of the building with $\alpha=0.00$

\begin{tabular}{|c|c|c|c|c|c|}
\hline \multirow{2}{*}{ Building } & \multirow{2}{*}{$V e$} & \multirow{2}{*}{$\frac{\text { Ductility }(\mu)}{R y=3}$} & \multicolumn{3}{|c|}{ Force Reduction Factor $(R y)$} \\
\hline & & & $\mu=2$ & $\mu=3$ & $\mu=4$ \\
\hline SL1 & 202.1 & 6.58 & 1.14 & 1.23 & 1.32 \\
\hline SL3 & 210.1 & 7.62 & 1.14 & 1.24 & 1.32 \\
\hline SL4 & 214.7 & 8.74 & 1.13 & 1.23 & 1.31 \\
\hline SH1 & 244.8 & 18.15 & 1.20 & 1.37 & 1.56 \\
\hline $\mathrm{SH} 2$ & 262.1 & 16.00 & 1.18 & 1.28 & 1.37 \\
\hline $\mathrm{SH} 3$ & 254.3 & 12.64 & 1.16 & 1.27 & 1.36 \\
\hline SH4 & 234.2 & 14.65 & 1.13 & 1.21 & 1.29 \\
\hline SH5 & 233.5 & 13.19 & 1.14 & 1.23 & 1.31 \\
\hline SH6 & 253.9 & 12.46 & 1.16 & 1.26 & 1.36 \\
\hline ML1 & 304.9 & 14.78 & 1.15 & 1.23 & 1.36 \\
\hline ML2 & 209.1 & 27.20 & 1.25 & 1.33 & 1.39 \\
\hline ML3 & 208.7 & 23.14 & 1.26 & 1.35 & 1.42 \\
\hline ML4 & 255.2 & 18.95 & 1.19 & 1.28 & 1.37 \\
\hline ML5 & 259.3 & 18.35 & 1.18 & 1.27 & 1.36 \\
\hline ML6 & 207.3 & 22.61 & 1.26 & 1.35 & 1.42 \\
\hline MH1 & 457.8 & 21.37 & 1.24 & 1.34 & 1.43 \\
\hline MH2 & 461.3 & 16.36 & 1.23 & 1.34 & 1.43 \\
\hline MH3 & 311.8 & 27.87 & 1.17 & 1.27 & 1.34 \\
\hline MH4 & 449.6 & 21.21 & 1.20 & 1.28 & 1.36 \\
\hline MH5 & 455.8 & 16.28 & 1.22 & 1.33 & 1.42 \\
\hline MH6 & 623.8 & 7.96 & 1.33 & 1.71 & 1.92 \\
\hline LL1 & 860.2 & 22.41 & 1.23 & 1.43 & 1.67 \\
\hline LL2 & 856.1 & 22.45 & 1.24 & 1.48 & 1.65 \\
\hline LL3 & 759.8 & 13.10 & 1.20 & 1.33 & 1.44 \\
\hline LL4 & 778.9 & 19.02 & 1.21 & 1.42 & 1.50 \\
\hline LL5 & 789.1 & 19.42 & 1.21 & 1.44 & 1.51 \\
\hline LL6 & 663.0 & 24.50 & 1.23 & 1.35 & 1.45 \\
\hline LH1 & 1584.7 & 10.56 & 1.25 & 1.39 & 1.53 \\
\hline LH2 & 1537.3 & 8.65 & 1.32 & 1.49 & 1.63 \\
\hline LH3 & 1278.1 & 20.10 & 1.22 & 1.37 & 1.47 \\
\hline LH4 & 1505.2 & 9.08 & 1.30 & 1.47 & 1.60 \\
\hline LH5 & 1489.8 & 9.01 & 1.30 & 1.48 & 1.61 \\
\hline LH6 & 1334.3 & 19.62 & 1.23 & 1.39 & 1.48 \\
\hline
\end{tabular}


Table A.7 - The response of the buildings subjected to M6C38 ground motion parallel to the short side of the building with $\alpha=0.00$

\begin{tabular}{|c|c|c|c|c|c|}
\hline \multirow{2}{*}{ Building } & \multirow{2}{*}{$V e$} & \multirow{2}{*}{$\begin{array}{c}\text { Ductility }(\mu) \\
R y=3\end{array}$} & \multicolumn{3}{|c|}{ Force Reduction Factor $(R y)$} \\
\hline & & & $\mu=2$ & $\mu=3$ & $\mu=4$ \\
\hline SL1 & 137.6 & 24.91 & 1.12 & 1.21 & 1.29 \\
\hline SL3 & 135.6 & 27.19 & 1.12 & 1.21 & 1.29 \\
\hline SL4 & 129.8 & 28.40 & 1.12 & 1.20 & 1.27 \\
\hline SH1 & 183.8 & 32.54 & 1.20 & 1.32 & 1.42 \\
\hline $\mathrm{SH} 2$ & 171.9 & 36.24 & 1.20 & 1.33 & 1.42 \\
\hline $\mathrm{SH} 3$ & 167.5 & 33.90 & 1.22 & 1.34 & 1.44 \\
\hline SH4 & 160.4 & 39.22 & 1.20 & 1.29 & 1.39 \\
\hline SH5 & 161.0 & 35.04 & 1.20 & 1.32 & 1.41 \\
\hline SH6 & 167.2 & 33.94 & 1.22 & 1.33 & 1.43 \\
\hline ML1 & 289.4 & 27.73 & 1.22 & 1.31 & 1.38 \\
\hline ML2 & 278.4 & 19.77 & 1.27 & 1.38 & 1.48 \\
\hline ML3 & 293.1 & 15.53 & 1.35 & 1.49 & 1.60 \\
\hline ML4 & 299.9 & 23.16 & 1.23 & 1.34 & 1.44 \\
\hline ML5 & 300.2 & 23.60 & 1.23 & 1.33 & 1.42 \\
\hline ML6 & 287.3 & 16.32 & 1.32 & 1.45 & 1.56 \\
\hline MH1 & 664.8 & 13.02 & 1.30 & 1.54 & 1.67 \\
\hline $\mathrm{MH} 2$ & 696.0 & 9.68 & 1.20 & 1.67 & 1.82 \\
\hline MH3 & 643.9 & 6.36 & 1.21 & 1.39 & 2.01 \\
\hline MH4 & 710.4 & 11.04 & 1.18 & 1.65 & 1.79 \\
\hline MH5 & 721.2 & 8.96 & 1.21 & 1.74 & 1.90 \\
\hline MH6 & 685.6 & 11.96 & 1.32 & 1.50 & 1.66 \\
\hline LL1 & 1158.1 & 12.03 & 1.39 & 1.55 & 1.68 \\
\hline LL2 & 1160.5 & 11.81 & 1.35 & 1.55 & 1.68 \\
\hline LL3 & 1296.9 & 7.55 & 1.23 & 1.78 & 1.96 \\
\hline LL4 & 1171.1 & 12.14 & 1.38 & 1.53 & 1.67 \\
\hline LL5 & 1164.4 & 12.24 & 1.37 & 1.52 & 1.65 \\
\hline LL6 & 920.3 & 7.91 & 1.45 & 1.79 & 2.32 \\
\hline LH1 & 2779.8 & 6.63 & 1.26 & 1.82 & 2.02 \\
\hline LH2 & 2604.5 & 5.65 & 1.56 & 1.80 & 2.01 \\
\hline LH3 & 2060.8 & 5.44 & 1.58 & 1.83 & 2.11 \\
\hline LH4 & 2742.0 & 5.62 & 1.63 & 1.88 & 2.11 \\
\hline LH5 & 2751.6 & 5.46 & 1.64 & 1.91 & 2.14 \\
\hline LH6 & 2027.0 & 5.62 & 1.46 & 1.78 & 2.04 \\
\hline
\end{tabular}


Table A. 8 - The response of the buildings subjected to $\mathrm{M} 6 \mathrm{C} 1$ ground motion parallel to the long side of the building with $\alpha=0.00$

\begin{tabular}{|c|c|c|c|c|c|}
\hline \multirow{2}{*}{ Building } & \multirow{2}{*}{$V e$} & \multirow{2}{*}{$\begin{array}{c}\text { Ductility }(\mu) \\
R y=3\end{array}$} & \multicolumn{3}{|c|}{ Force Reduction Factor $(R y)$} \\
\hline & & & $\mu=2$ & $\mu=3$ & $\mu=4$ \\
\hline SL1 & 206.3 & 5.23 & 1.32 & 1.51 & 1.75 \\
\hline SL3 & 199.9 & 5.17 & 1.31 & 1.49 & 1.69 \\
\hline SL4 & 178.7 & 5.55 & 1.25 & 1.40 & 1.53 \\
\hline SH1 & 420.5 & 4.99 & 1.35 & 2.37 & 2.70 \\
\hline $\mathrm{SH} 2$ & 382.9 & 4.71 & 1.35 & 2.50 & 2.81 \\
\hline $\mathrm{SH} 3$ & 335.6 & 5.16 & 1.57 & 2.09 & 2.63 \\
\hline SH4 & 335.0 & 5.74 & 1.51 & 2.02 & 2.44 \\
\hline SH5 & 314.5 & 5.86 & 1.46 & 1.95 & 2.41 \\
\hline ML1 & 620.3 & 12.17 & 1.46 & 2.26 & 2.52 \\
\hline ML2 & 599.4 & 7.20 & 1.39 & 2.07 & 2.30 \\
\hline ML3 & 582.6 & 7.67 & 1.30 & 1.96 & 2.20 \\
\hline ML4 & 609.2 & 6.76 & 1.42 & 2.14 & 2.38 \\
\hline ML5 & 586.2 & 7.73 & 1.34 & 2.01 & 2.23 \\
\hline ML6 & 579.7 & 7.46 & 1.32 & 1.97 & 2.22 \\
\hline MH1 & 1307.9 & 7.39 & 1.30 & 1.96 & 2.21 \\
\hline MH2 & 1306.9 & 7.09 & 1.34 & 2.17 & 2.46 \\
\hline MH3 & 1093.3 & 5.38 & 1.46 & 2.28 & 2.59 \\
\hline MH4 & 1298.1 & 7.80 & 1.29 & 1.96 & 2.21 \\
\hline MH5 & 1408.3 & 5.49 & 1.46 & 2.40 & 2.67 \\
\hline MH6 & 1288.8 & 6.80 & 1.31 & 2.19 & 2.48 \\
\hline LL1 & 2417.2 & 6.11 & 1.77 & 2.10 & 2.41 \\
\hline LL2 & 2376.2 & 6.87 & 1.56 & 2.08 & 2.38 \\
\hline LL3 & 2129.1 & 4.77 & 2.03 & 2.41 & 2.75 \\
\hline LL4 & 2414.6 & 6.28 & 1.77 & 2.10 & 2.40 \\
\hline LL5 & 2312.8 & 7.72 & 1.32 & 2.10 & 2.38 \\
\hline LL6 & 2063.5 & 3.64 & 1.62 & 2.75 & 3.14 \\
\hline LH1 & 6161.3 & 3.78 & 1.67 & 2.77 & 3.07 \\
\hline LH2 & 5375.9 & 3.34 & 2.38 & 2.84 & 3.26 \\
\hline LH3 & 4506.9 & 3.16 & 2.41 & 2.93 & 3.40 \\
\hline LH4 & 5840.1 & 3.62 & 2.37 & 2.76 & 3.14 \\
\hline LH5 & 4548.3 & 3.64 & 2.21 & 2.71 & 3.16 \\
\hline LH6 & 4230.9 & 3.52 & 2.33 & 2.71 & 3.20 \\
\hline
\end{tabular}


Table A.9 - The response of the buildings subjected to $\mathrm{M} 6 \mathrm{C} 2$ ground motion parallel to the long side of the building with $\alpha=0.00$

\begin{tabular}{|c|c|c|c|c|c|}
\hline \multirow{2}{*}{ Building } & \multirow{2}{*}{$V e$} & \multirow{2}{*}{$\begin{array}{c}\text { Ductility }(\mu) \\
R y=3\end{array}$} & \multicolumn{3}{|c|}{ Force Reduction Factor $(R y)$} \\
\hline & & & $\mu=2$ & $\mu=3$ & $\mu=4$ \\
\hline SL1 & 175.0 & 10.54 & 1.56 & 1.81 & 1.98 \\
\hline SL3 & 172.5 & 10.67 & 1.56 & 1.81 & 1.99 \\
\hline SL4 & 170.8 & 10.45 & 1.53 & 1.78 & 2.02 \\
\hline SH1 & 463.6 & 7.39 & 1.55 & 1.88 & 2.13 \\
\hline $\mathrm{SH} 2$ & 429.2 & 6.72 & 1.57 & 1.76 & 1.93 \\
\hline $\mathrm{SH} 3$ & 356.6 & 7.19 & 1.56 & 1.90 & 2.24 \\
\hline SH4 & 357.3 & 8.17 & 1.51 & 1.75 & 2.07 \\
\hline SH5 & 304.8 & 8.39 & 1.51 & 1.94 & 2.20 \\
\hline ML1 & 594.8 & 8.40 & 1.33 & 1.52 & 1.91 \\
\hline ML2 & 574.6 & 10.56 & 1.29 & 1.47 & 1.75 \\
\hline ML3 & 528.3 & 11.52 & 1.31 & 1.53 & 1.75 \\
\hline ML4 & 583.8 & 9.75 & 1.32 & 1.49 & 1.80 \\
\hline ML5 & 562.7 & 11.50 & 1.29 & 1.45 & 1.62 \\
\hline ML6 & 544.5 & 11.11 & 1.31 & 1.53 & 1.76 \\
\hline MH1 & 904.5 & 12.49 & 1.27 & 1.45 & 1.60 \\
\hline MH2 & 936.1 & 5.74 & 1.69 & 2.13 & 2.36 \\
\hline MH3 & 912.7 & 3.41 & 1.69 & 1.95 & 3.24 \\
\hline MH4 & 916.6 & 11.59 & 1.31 & 1.49 & 1.71 \\
\hline MH5 & 949.0 & 4.85 & 2.09 & 2.31 & 2.56 \\
\hline MH6 & 941.5 & 5.85 & 1.43 & 1.95 & 2.20 \\
\hline LL1 & 1684.8 & 8.99 & 1.35 & 1.58 & 1.77 \\
\hline LL2 & 1695.3 & 8.37 & 1.37 & 1.65 & 1.92 \\
\hline LL3 & 1695.5 & 3.59 & 1.63 & 1.92 & 3.16 \\
\hline LL4 & 1686.9 & 8.94 & 1.35 & 1.58 & 1.77 \\
\hline LL5 & 1684.6 & 6.34 & 1.60 & 2.07 & 2.29 \\
\hline LL6 & 1147.2 & 8.46 & 1.41 & 1.54 & 2.17 \\
\hline LH1 & 3848.9 & 3.88 & 1.69 & 2.17 & 3.04 \\
\hline LH2 & 3828.8 & 3.08 & 2.35 & 2.96 & 3.44 \\
\hline LH3 & 2290.5 & 7.33 & 1.56 & 1.95 & 2.10 \\
\hline LH4 & 3827.8 & 3.41 & 2.18 & 2.74 & 3.26 \\
\hline LH5 & 3575.9 & 3.73 & 1.71 & 2.24 & 3.20 \\
\hline LH6 & 1987.8 & 7.76 & 1.50 & 1.67 & 1.81 \\
\hline
\end{tabular}


Table A.10 - The response of the buildings subjected to M6C26 ground motion parallel to the long side of the building with $\alpha=0.00$

\begin{tabular}{|c|c|c|c|c|c|}
\hline \multirow{2}{*}{ Building } & \multirow{2}{*}{$V e$} & \multirow{2}{*}{$\begin{array}{c}\text { Ductility }(\mu) \\
R y=3\end{array}$} & \multicolumn{3}{|c|}{ Force Reduction Factor $(R y)$} \\
\hline & & & $\mu=2$ & $\mu=3$ & $\mu=4$ \\
\hline SL1 & 197.6 & 9.76 & 1.52 & 1.78 & 2.02 \\
\hline SL3 & 203.5 & 9.25 & 1.52 & 1.78 & 2.02 \\
\hline SL4 & 214.0 & 8.87 & 1.53 & 1.79 & 2.03 \\
\hline SH1 & 487.0 & 6.45 & 1.74 & 2.05 & 2.33 \\
\hline $\mathrm{SH} 2$ & 483.5 & 6.82 & 1.73 & 2.03 & 2.30 \\
\hline $\mathrm{SH} 3$ & 470.9 & 6.04 & 1.31 & 2.13 & 2.41 \\
\hline SH4 & 465.0 & 6.88 & 1.27 & 2.04 & 2.30 \\
\hline SH5 & 455.8 & 6.03 & 1.30 & 2.16 & 2.45 \\
\hline ML1 & 582.9 & 6.46 & 1.27 & 1.45 & 1.63 \\
\hline ML2 & 534.2 & 7.79 & 1.24 & 1.41 & 1.57 \\
\hline ML3 & 529.2 & 6.99 & 1.27 & 1.47 & 1.64 \\
\hline ML4 & 544.3 & 7.63 & 1.25 & 1.42 & 1.58 \\
\hline ML5 & 526.8 & 7.97 & 1.24 & 1.41 & 1.55 \\
\hline ML6 & 527.3 & 6.72 & 1.27 & 1.46 & 1.64 \\
\hline MH1 & 1263.7 & 5.26 & 1.31 & 1.51 & 1.70 \\
\hline MH2 & 1391.8 & 3.99 & 1.38 & 1.64 & 3.00 \\
\hline MH3 & 1271.5 & 5.25 & 1.35 & 1.57 & 2.65 \\
\hline MH4 & 1282.9 & 4.73 & 1.31 & 1.50 & 1.70 \\
\hline MH5 & 1417.4 & 4.21 & 1.38 & 1.69 & 2.92 \\
\hline MH6 & 1429.6 & 3.35 & 1.45 & 1.87 & 3.30 \\
\hline LL1 & 2377.5 & 3.69 & 1.37 & 1.62 & 2.20 \\
\hline LL2 & 2417.6 & 3.56 & 1.37 & 1.61 & 3.19 \\
\hline LL3 & 2410.1 & 4.78 & 1.38 & 1.82 & 2.74 \\
\hline LL4 & 2380.9 & 3.73 & 1.37 & 1.61 & 3.33 \\
\hline LL5 & 2503.4 & 4.09 & 1.37 & 1.59 & 2.97 \\
\hline LL6 & 1924.6 & 4.32 & 1.47 & 1.73 & 2.89 \\
\hline LH1 & 6150.0 & 3.06 & 1.55 & 2.98 & 3.49 \\
\hline LH2 & 5825.5 & 3.39 & 1.54 & 2.81 & 3.29 \\
\hline LH3 & 3856.6 & 3.40 & 1.59 & 2.81 & 3.26 \\
\hline LH4 & 6170.0 & 3.01 & 1.60 & 2.99 & 3.54 \\
\hline LH5 & 5117.4 & 3.94 & 1.49 & 2.60 & 3.05 \\
\hline LH6 & 3349.9 & 3.34 & 1.63 & 1.96 & 3.64 \\
\hline
\end{tabular}


Table A.11 - The response of the buildings subjected to M6C31 ground motion parallel to the long side of the building with $\alpha=0.00$

\begin{tabular}{|c|c|c|c|c|c|}
\hline \multirow{2}{*}{ Building } & \multirow{2}{*}{$V e$} & \multirow{2}{*}{$\begin{array}{c}\text { Ductility }(\mu) \\
R y=3\end{array}$} & \multicolumn{3}{|c|}{ Force Reduction Factor $(R y)$} \\
\hline & & & $\mu=2$ & $\mu=3$ & $\mu=4$ \\
\hline SL1 & 184.8 & 8.20 & 1.32 & 2.26 & 2.47 \\
\hline SL3 & 180.1 & 8.61 & 1.32 & 2.33 & 2.48 \\
\hline SL4 & 178.0 & 7.97 & 1.31 & 1.52 & 1.74 \\
\hline SH1 & 446.1 & 5.64 & 1.29 & 1.49 & 2.65 \\
\hline $\mathrm{SH} 2$ & 416.2 & 5.31 & 1.28 & 1.47 & 1.67 \\
\hline $\mathrm{SH} 3$ & 405.2 & 3.84 & 1.35 & 1.56 & 1.74 \\
\hline SH4 & 396.4 & 4.39 & 1.30 & 1.49 & 1.67 \\
\hline SH5 & 397.6 & 4.45 & 1.31 & 1.51 & 1.82 \\
\hline ML1 & 939.1 & 3.39 & 1.28 & 2.89 & 3.33 \\
\hline ML2 & 850.7 & 4.84 & 1.29 & 2.30 & 2.70 \\
\hline ML3 & 639.3 & 6.07 & 1.33 & 2.08 & 2.24 \\
\hline ML4 & 882.0 & 4.27 & 1.29 & 2.41 & 2.87 \\
\hline ML5 & 813.0 & 5.38 & 1.29 & 2.19 & 2.57 \\
\hline ML6 & 702.6 & 5.58 & 1.34 & 2.15 & 2.38 \\
\hline MH1 & 1026.3 & 6.64 & 1.34 & 1.90 & 2.03 \\
\hline MH2 & 829.2 & 7.43 & 1.35 & 1.96 & 2.12 \\
\hline MH3 & 1091.5 & 4.39 & 1.29 & 2.42 & 2.80 \\
\hline MH4 & 957.3 & 6.36 & 1.39 & 1.91 & 1.79 \\
\hline MH5 & 882.3 & 9.30 & 1.64 & 1.81 & 1.98 \\
\hline MH6 & 805.3 & 5.97 & 1.35 & 1.94 & 2.08 \\
\hline LL1 & 1747.3 & 5.23 & 1.48 & 1.84 & 2.22 \\
\hline LL2 & 1560.6 & 5.58 & 1.49 & 1.64 & 2.27 \\
\hline LL3 & 2143.2 & 4.60 & 1.34 & 1.64 & 2.70 \\
\hline LL4 & 1732.6 & 5.26 & 1.48 & 1.83 & 2.32 \\
\hline LL5 & 1474.9 & 7.15 & 1.34 & 1.95 & 2.09 \\
\hline LL6 & 1249.6 & 4.27 & 1.47 & 2.26 & 2.83 \\
\hline LH1 & 3731.5 & 4.83 & 1.57 & 1.81 & 2.09 \\
\hline LH2 & 4929.3 & 4.37 & 1.46 & 2.01 & 2.50 \\
\hline LH3 & 2466.2 & 3.73 & 2.03 & 2.64 & 3.13 \\
\hline LH4 & 4572.4 & 4.44 & 1.61 & 1.94 & 2.22 \\
\hline LH5 & 4364.4 & 2.55 & 1.99 & 3.24 & 3.80 \\
\hline LH6 & 1981.6 & 5.36 & 1.43 & 1.60 & 1.87 \\
\hline
\end{tabular}


Table A.12 - The response of the buildings subjected to M6C38 ground motion parallel to the long side of the building with $\alpha=0.00$

\begin{tabular}{|c|c|c|c|c|c|}
\hline \multirow{2}{*}{ Building } & \multirow{2}{*}{$V e$} & \multirow{2}{*}{$\begin{array}{c}\text { Ductility }(\mu) \\
R y=3\end{array}$} & \multicolumn{3}{|c|}{ Force Reduction Factor $(R y)$} \\
\hline & & & $\mu=2$ & $\mu=3$ & $\mu=4$ \\
\hline SL1 & 165.6 & 6.99 & 1.36 & 1.52 & 1.75 \\
\hline SL3 & 157.4 & 8.43 & 1.30 & 1.45 & 1.72 \\
\hline SL4 & 152.5 & 8.90 & 1.27 & 1.44 & 1.83 \\
\hline SH1 & 378.3 & 9.75 & 1.29 & 1.50 & 1.68 \\
\hline $\mathrm{SH} 2$ & 365.7 & 10.05 & 1.27 & 1.46 & 1.64 \\
\hline $\mathrm{SH} 3$ & 360.1 & 8.82 & 1.28 & 1.48 & 1.69 \\
\hline SH4 & 353.9 & 9.98 & 1.25 & 1.43 & 1.59 \\
\hline SH5 & 353.4 & 9.13 & 1.27 & 1.47 & 1.66 \\
\hline ML1 & 567.1 & 13.06 & 1.24 & 1.42 & 1.58 \\
\hline ML2 & 542.7 & 13.59 & 1.23 & 1.40 & 1.55 \\
\hline ML3 & 522.6 & 11.81 & 1.24 & 1.42 & 1.59 \\
\hline ML4 & 549.1 & 13.38 & 1.23 & 1.40 & 1.56 \\
\hline ML5 & 536.1 & 13.73 & 1.23 & 1.39 & 1.54 \\
\hline ML6 & 532.8 & 11.90 & 1.25 & 1.44 & 1.60 \\
\hline MH1 & 1041.2 & 9.00 & 1.29 & 1.49 & 1.69 \\
\hline MH2 & 911.2 & 8.89 & 1.34 & 1.58 & 1.79 \\
\hline MH3 & 697.7 & 13.38 & 1.33 & 1.55 & 1.69 \\
\hline MH4 & 1008.1 & 9.26 & 1.28 & 1.49 & 1.65 \\
\hline MH5 & 864.7 & 10.22 & 1.33 & 1.57 & 1.77 \\
\hline MH6 & 956.5 & 6.95 & 1.39 & 1.68 & 1.93 \\
\hline LL1 & 1918.8 & 7.17 & 1.36 & 1.62 & 1.84 \\
\hline LL2 & 1834.0 & 7.54 & 1.35 & 1.59 & 1.80 \\
\hline LL3 & 1310.3 & 13.05 & 1.35 & 1.58 & 1.75 \\
\hline LL4 & 1911.3 & 7.08 & 1.36 & 1.61 & 1.82 \\
\hline LL5 & 1656.1 & 9.03 & 1.33 & 1.56 & 1.76 \\
\hline LL6 & 1213.1 & 9.42 & 1.32 & 1.51 & 1.68 \\
\hline LH1 & 3545.2 & 6.65 & 1.45 & 1.78 & 2.05 \\
\hline LH2 & 3127.7 & 7.79 & 1.46 & 1.78 & 1.99 \\
\hline LH3 & 2731.4 & 6.98 & 1.45 & 1.75 & 2.00 \\
\hline LH4 & 3327.5 & 7.05 & 1.49 & 1.85 & 2.10 \\
\hline LH5 & 2828.2 & 8.22 & 1.43 & 1.65 & 1.82 \\
\hline LH6 & 2547.9 & 7.10 & 1.42 & 1.69 & 1.94 \\
\hline
\end{tabular}


Table A.13 - The response of the buildings subjected to E6C1 ground motion parallel to the short side of the building with $\alpha=0.00$

\begin{tabular}{|c|c|c|c|c|c|}
\hline \multirow{2}{*}{ Building } & \multirow{2}{*}{$V e$} & \multirow{2}{*}{$\frac{\text { Ductility }(\mu)}{R y=3}$} & \multicolumn{3}{|c|}{ Force Reduction Factor $(R y)$} \\
\hline & & & $\mu=2$ & $\mu=3$ & $\mu=4$ \\
\hline SL1 & 93.7 & 15.50 & 1.23 & 1.37 & 1.48 \\
\hline SL3 & 93.7 & 14.92 & 1.23 & 1.36 & 1.47 \\
\hline SL4 & 92.1 & 15.08 & 1.20 & 1.33 & 1.43 \\
\hline SH1 & 150.7 & 11.39 & 1.24 & 1.33 & 1.41 \\
\hline $\mathrm{SH} 2$ & 140.3 & 10.46 & 1.18 & 1.27 & 1.35 \\
\hline $\mathrm{SH} 3$ & 135.0 & 9.81 & 1.16 & 1.25 & 1.33 \\
\hline SH4 & 130.1 & 11.66 & 1.13 & 1.21 & 1.29 \\
\hline SH5 & 130.7 & 10.55 & 1.14 & 1.24 & 1.32 \\
\hline SH6 & 134.7 & 9.87 & 1.16 & 1.25 & 1.33 \\
\hline ML1 & 238.4 & 8.84 & 1.21 & 1.33 & 1.43 \\
\hline ML2 & 217.1 & 9.55 & 1.22 & 1.34 & 1.45 \\
\hline ML3 & 199.2 & 9.57 & 1.21 & 1.34 & 1.52 \\
\hline ML4 & 233.8 & 8.89 & 1.20 & 1.34 & 1.44 \\
\hline ML5 & 235.3 & 8.89 & 1.21 & 1.33 & 1.45 \\
\hline ML6 & 210.3 & 8.82 & 1.22 & 1.37 & 1.53 \\
\hline MH1 & 412.6 & 8.89 & 1.21 & 1.36 & 1.73 \\
\hline MH2 & 359.1 & 8.82 & 1.24 & 1.45 & 2.18 \\
\hline MH3 & 197.8 & 13.72 & 1.18 & 1.39 & 1.82 \\
\hline MH4 & 336.7 & 11.30 & 1.21 & 1.35 & 1.68 \\
\hline MH5 & 331.6 & 9.49 & 1.24 & 1.45 & 2.13 \\
\hline MH6 & 515.7 & 5.63 & 1.28 & 1.47 & 1.68 \\
\hline LL1 & 685.3 & 9.34 & 1.22 & 1.36 & 1.54 \\
\hline LL2 & 684.1 & 9.23 & 1.23 & 1.37 & 1.60 \\
\hline LL3 & 531.7 & 8.51 & 1.29 & 1.98 & 2.19 \\
\hline LL4 & 778.9 & 7.81 & 1.26 & 1.41 & 1.68 \\
\hline LL5 & 780.5 & 7.83 & 1.25 & 1.40 & 1.64 \\
\hline LL6 & 302.5 & 7.61 & 1.23 & 1.43 & 1.99 \\
\hline LH1 & 1111.2 & 7.90 & 1.42 & 2.03 & 2.26 \\
\hline LH2 & 1053.8 & 7.43 & 1.77 & 2.07 & 2.30 \\
\hline LH3 & 653.2 & 8.40 & 1.32 & 1.95 & 2.16 \\
\hline LH4 & 1020.8 & 7.47 & 1.75 & 2.04 & 2.28 \\
\hline LH5 & 998.5 & 7.64 & 1.74 & 2.03 & 2.27 \\
\hline LH6 & 641.5 & 7.73 & 1.34 & 2.05 & 2.26 \\
\hline
\end{tabular}


Table A.14 - The response of the buildings subjected to E6C13 ground motion parallel to the short side of the building with $\alpha=0.00$

\begin{tabular}{|c|c|c|c|c|c|}
\hline \multirow{2}{*}{ Building } & \multirow{2}{*}{$V e$} & \multirow{2}{*}{$\frac{\text { Ductility }(\mu)}{R y=3}$} & \multicolumn{3}{|c|}{ Force Reduction Factor $(R y)$} \\
\hline & & & $\mu=2$ & $\mu=3$ & $\mu=4$ \\
\hline SL1 & 96.0 & 9.18 & 1.34 & 1.49 & 1.63 \\
\hline SL3 & 90.5 & 9.27 & 1.32 & 1.47 & 1.60 \\
\hline SL4 & 74.2 & 10.55 & 1.26 & 1.39 & 1.49 \\
\hline SH1 & 89.1 & 12.26 & 1.42 & 1.62 & 1.82 \\
\hline $\mathrm{SH} 2$ & 90.2 & 13.06 & 1.30 & 1.51 & 1.67 \\
\hline $\mathrm{SH} 3$ & 89.9 & 12.66 & 1.30 & 1.49 & 1.64 \\
\hline SH4 & 91.4 & 13.81 & 1.28 & 1.43 & 1.56 \\
\hline SH5 & 91.7 & 12.81 & 1.29 & 1.46 & 1.60 \\
\hline SH6 & 89.8 & 12.42 & 1.30 & 1.49 & 1.64 \\
\hline ML1 & 163.0 & 9.57 & 1.25 & 1.40 & 1.55 \\
\hline ML2 & 142.5 & 9.33 & 1.22 & 1.35 & 1.56 \\
\hline ML3 & 137.5 & 9.79 & 1.24 & 1.88 & 1.97 \\
\hline ML4 & 153.1 & 8.96 & 1.22 & 1.35 & 1.47 \\
\hline ML5 & 153.6 & 8.35 & 1.22 & 1.35 & 1.47 \\
\hline ML6 & 140.8 & 8.65 & 1.24 & 1.43 & 2.18 \\
\hline MH1 & 307.7 & 8.18 & 1.34 & 1.57 & 1.68 \\
\hline MH2 & 334.2 & 5.71 & 1.22 & 1.54 & 2.53 \\
\hline MH3 & 239.4 & 8.45 & 1.17 & 1.59 & 2.21 \\
\hline MH4 & 342.0 & 6.35 & 1.18 & 1.79 & 2.44 \\
\hline MH5 & 351.3 & 5.02 & 1.20 & 1.82 & 2.49 \\
\hline MH6 & 361.9 & 5.95 & 1.36 & 1.62 & 1.85 \\
\hline LL1 & 619.8 & 5.57 & 1.23 & 1.98 & 2.58 \\
\hline LL2 & 633.1 & 5.48 & 1.24 & 2.08 & 2.61 \\
\hline LL3 & 627.5 & 5.14 & 1.42 & 1.64 & 1.99 \\
\hline LL4 & 559.7 & 6.56 & 1.29 & 1.66 & 2.38 \\
\hline LL5 & 565.5 & 6.43 & 1.29 & 1.60 & 2.41 \\
\hline LL6 & 300.7 & 10.12 & 1.26 & 1.43 & 1.62 \\
\hline LH1 & 1319.4 & 5.32 & 1.37 & 1.71 & 2.63 \\
\hline LH2 & 1206.6 & 5.84 & 1.31 & 2.10 & 2.43 \\
\hline LH3 & 823.6 & 6.85 & 1.36 & 2.06 & 2.33 \\
\hline LH4 & 1180.1 & 5.83 & 1.30 & 2.27 & 2.59 \\
\hline LH5 & 1153.7 & 5.96 & 1.29 & 2.25 & 2.56 \\
\hline LH6 & 772.2 & 7.36 & 1.32 & 2.05 & 2.29 \\
\hline
\end{tabular}


Table A.15 - The response of the buildings subjected to E6C15 ground motion parallel to the short side of the building with $\alpha=0.00$

\begin{tabular}{|c|c|c|c|c|c|}
\hline \multirow{2}{*}{ Building } & \multirow{2}{*}{$V e$} & \multirow{2}{*}{$\frac{\text { Ductility }(\mu)}{R y=3}$} & \multicolumn{3}{|c|}{ Force Reduction Factor $(R y)$} \\
\hline & & & $\mu=2$ & $\mu=3$ & $\mu=4$ \\
\hline SL1 & 71.8 & 21.12 & 1.36 & 1.68 & 1.77 \\
\hline SL3 & 71.9 & 19.55 & 1.37 & 1.68 & 1.79 \\
\hline SL4 & 71.4 & 17.90 & 1.39 & 1.71 & 1.81 \\
\hline SH1 & 168.7 & 8.66 & 1.24 & 1.36 & 1.82 \\
\hline $\mathrm{SH} 2$ & 136.4 & 9.43 & 1.20 & 1.44 & 1.60 \\
\hline $\mathrm{SH} 3$ & 120.4 & 9.69 & 1.18 & 1.39 & 1.52 \\
\hline SH4 & 104.7 & 15.60 & 1.17 & 1.26 & 1.36 \\
\hline SH5 & 103.7 & 14.44 & 1.18 & 1.28 & 1.39 \\
\hline SH6 & 120.1 & 9.86 & 1.18 & 1.39 & 1.53 \\
\hline ML1 & 147.6 & 22.94 & 1.32 & 1.40 & 1.48 \\
\hline ML2 & 120.6 & 23.72 & 1.15 & 1.35 & 1.41 \\
\hline ML3 & 123.2 & 17.63 & 1.27 & 1.35 & 1.41 \\
\hline ML4 & 131.5 & 26.71 & 1.28 & 1.38 & 1.47 \\
\hline ML5 & 132.0 & 26.77 & 1.27 & 1.37 & 1.46 \\
\hline ML6 & 120.5 & 19.77 & 1.26 & 1.34 & 1.41 \\
\hline MH1 & 283.6 & 13.39 & 1.30 & 1.39 & 1.48 \\
\hline MH2 & 286.3 & 10.31 & 1.29 & 1.43 & 1.53 \\
\hline MH3 & 163.6 & 13.81 & 1.30 & 1.80 & 1.94 \\
\hline MH4 & 276.6 & 13.14 & 1.27 & 1.40 & 1.50 \\
\hline MH5 & 270.9 & 11.55 & 1.28 & 1.42 & 1.52 \\
\hline MH6 & 319.9 & 13.92 & 1.45 & 1.65 & 1.83 \\
\hline LL1 & 644.0 & 7.45 & 1.40 & 1.60 & 1.87 \\
\hline LL2 & 640.2 & 7.51 & 1.38 & 1.57 & 1.81 \\
\hline LL3 & 410.6 & 11.00 & 1.27 & 1.46 & 1.97 \\
\hline LL4 & 636.8 & 7.64 & 1.45 & 1.63 & 1.86 \\
\hline LL5 & 638.3 & 7.67 & 1.45 & 1.64 & 1.89 \\
\hline LL6 & 271.3 & 13.10 & 1.32 & 1.53 & 1.70 \\
\hline LH1 & 812.3 & 9.20 & 1.28 & 1.73 & 1.88 \\
\hline LH2 & 701.6 & 9.03 & 1.37 & 1.57 & 1.78 \\
\hline LH3 & 660.9 & 7.92 & 1.58 & 1.86 & 2.08 \\
\hline LH4 & 695.3 & 9.11 & 1.41 & 1.59 & 1.80 \\
\hline LH5 & 667.2 & 9.35 & 1.37 & 1.56 & 1.79 \\
\hline LH6 & 621.0 & 9.06 & 1.45 & 1.70 & 1.92 \\
\hline
\end{tabular}


Table A.16 - The response of the buildings subjected to E6C18 ground motion parallel to the short side of the building with $\alpha=0.00$

\begin{tabular}{|c|c|c|c|c|c|}
\hline \multirow{2}{*}{ Building } & \multirow{2}{*}{$V e$} & \multirow{2}{*}{$\frac{\text { Ductility }(\mu)}{R y=3}$} & \multicolumn{3}{|c|}{ Force Reduction Factor $(R y)$} \\
\hline & & & $\mu=2$ & $\mu=3$ & $\mu=4$ \\
\hline SL1 & 90.4 & 12.13 & 1.28 & 1.41 & 1.56 \\
\hline SL3 & 82.3 & 15.42 & 1.27 & 1.40 & 1.52 \\
\hline SL4 & 64.4 & 27.47 & 1.20 & 1.44 & 1.55 \\
\hline SH1 & 124.1 & 9.71 & 1.19 & 1.32 & 1.43 \\
\hline $\mathrm{SH} 2$ & 125.3 & 7.01 & 1.21 & 1.36 & 1.48 \\
\hline $\mathrm{SH} 3$ & 125.2 & 6.52 & 1.24 & 1.40 & 1.53 \\
\hline SH4 & 122.3 & 7.82 & 1.23 & 1.38 & 1.51 \\
\hline SH5 & 122.3 & 7.14 & 1.26 & 1.41 & 1.55 \\
\hline SH6 & 125.1 & 6.54 & 1.24 & 1.40 & 1.54 \\
\hline ML1 & 197.8 & 8.42 & 1.25 & 1.40 & 1.58 \\
\hline ML2 & 152.6 & 12.56 & 1.22 & 1.66 & 1.82 \\
\hline ML3 & 137.6 & 11.96 & 1.45 & 1.64 & 1.80 \\
\hline ML4 & 179.1 & 10.35 & 1.22 & 1.38 & 2.04 \\
\hline ML5 & 180.1 & 10.11 & 1.21 & 1.37 & 2.07 \\
\hline ML6 & 146.0 & 11.37 & 1.48 & 1.67 & 1.84 \\
\hline MH1 & 280.1 & 10.95 & 1.45 & 1.65 & 1.83 \\
\hline MH2 & 249.6 & 9.19 & 1.56 & 1.79 & 1.99 \\
\hline MH3 & 202.5 & 5.18 & 1.63 & 1.97 & 2.40 \\
\hline MH4 & 242.8 & 10.68 & 1.26 & 1.75 & 1.94 \\
\hline MH5 & 237.4 & 9.02 & 1.59 & 1.82 & 2.03 \\
\hline MH6 & 419.6 & 5.10 & 1.37 & 2.34 & 2.67 \\
\hline LL1 & 507.2 & 8.91 & 1.58 & 1.83 & 2.04 \\
\hline LL2 & 499.4 & 9.05 & 1.56 & 1.81 & 2.03 \\
\hline LL3 & 365.8 & 7.72 & 1.64 & 1.90 & 2.14 \\
\hline LL4 & 573.8 & 8.18 & 1.64 & 1.90 & 2.13 \\
\hline LL5 & 579.6 & 8.16 & 1.65 & 1.90 & 2.14 \\
\hline LL6 & 200.3 & 8.59 & 1.31 & 1.47 & 1.67 \\
\hline LH1 & 751.5 & 7.16 & 1.66 & 1.95 & 2.22 \\
\hline LH2 & 701.8 & 6.86 & 1.65 & 1.97 & 2.25 \\
\hline LH3 & 680.8 & 3.69 & 2.16 & 2.68 & 3.13 \\
\hline LH4 & 699.5 & 6.60 & 1.70 & 2.04 & 2.34 \\
\hline LH5 & 693.0 & 6.37 & 1.71 & 2.05 & 2.34 \\
\hline LH6 & 642.6 & 3.95 & 2.06 & 2.57 & 3.03 \\
\hline
\end{tabular}


Table A.17 - The response of the buildings subjected to E6C42 ground motion parallel to the short side of the building with $\alpha=0.00$

\begin{tabular}{|c|c|c|c|c|c|}
\hline \multirow{2}{*}{ Building } & \multirow{2}{*}{$V e$} & \multirow{2}{*}{$\frac{\text { Ductility }(\mu)}{R y=3}$} & \multicolumn{3}{|c|}{ Force Reduction Factor $(R y)$} \\
\hline & & & $\mu=2$ & $\mu=3$ & $\mu=4$ \\
\hline SL1 & 72.6 & 8.54 & 1.23 & 1.38 & 1.51 \\
\hline SL3 & 70.0 & 7.55 & 1.22 & 1.37 & 1.53 \\
\hline SL4 & 62.2 & 8.47 & 1.24 & 1.71 & 2.11 \\
\hline SH1 & 115.8 & 10.07 & 1.24 & 1.34 & 1.42 \\
\hline $\mathrm{SH} 2$ & 106.4 & 10.96 & 1.18 & 1.27 & 1.35 \\
\hline $\mathrm{SH} 3$ & 108.6 & 9.81 & 1.24 & 1.34 & 1.43 \\
\hline SH4 & 109.5 & 11.75 & 1.27 & 1.37 & 1.46 \\
\hline SH5 & 109.7 & 10.41 & 1.28 & 1.39 & 1.49 \\
\hline SH6 & 108.8 & 9.97 & 1.24 & 1.34 & 1.44 \\
\hline ML1 & 192.8 & 10.32 & 1.35 & 1.50 & 1.63 \\
\hline ML2 & 156.3 & 12.26 & 1.28 & 1.43 & 1.54 \\
\hline ML3 & 140.7 & 13.18 & 1.26 & 1.38 & 1.49 \\
\hline ML4 & 182.9 & 9.93 & 1.32 & 1.50 & 1.64 \\
\hline ML5 & 184.1 & 10.40 & 1.33 & 1.50 & 1.64 \\
\hline ML6 & 149.9 & 11.57 & 1.29 & 1.43 & 1.54 \\
\hline MH1 & 323.9 & 12.81 & 1.40 & 1.52 & 1.63 \\
\hline MH2 & 328.4 & 11.83 & 1.28 & 1.64 & 1.76 \\
\hline MH3 & 298.8 & 12.62 & 1.52 & 1.72 & 1.90 \\
\hline MH4 & 314.1 & 15.76 & 1.41 & 1.55 & 1.65 \\
\hline MH5 & 313.1 & 13.40 & 1.44 & 1.60 & 1.72 \\
\hline MH6 & 403.3 & 5.40 & 1.43 & 1.66 & 1.90 \\
\hline LL1 & 731.6 & 9.04 & 1.33 & 1.72 & 2.19 \\
\hline LL2 & 716.9 & 9.36 & 1.32 & 1.68 & 2.15 \\
\hline LL3 & 586.4 & 11.77 & 1.56 & 1.75 & 1.90 \\
\hline LL4 & 664.7 & 9.54 & 1.55 & 1.74 & 1.91 \\
\hline LL5 & 669.3 & 9.58 & 1.56 & 1.74 & 1.91 \\
\hline LL6 & 426.9 & 11.02 & 1.46 & 1.67 & 1.86 \\
\hline LH1 & 1286.9 & 10.36 & 1.61 & 1.83 & 1.99 \\
\hline LH2 & 1265.6 & 9.34 & 1.66 & 1.89 & 2.08 \\
\hline LH3 & 1083.9 & 7.98 & 1.61 & 1.88 & 2.13 \\
\hline LH4 & 1246.3 & 9.49 & 1.60 & 1.84 & 2.04 \\
\hline LH5 & 1234.9 & 9.47 & 1.58 & 1.83 & 2.04 \\
\hline LH6 & 1045.4 & 7.98 & 1.61 & 1.88 & 2.13 \\
\hline
\end{tabular}


Table A.18 - The response of the buildings subjected to E6C1 ground motion parallel to the long side of the building with $\alpha=0.00$

\begin{tabular}{|c|c|c|c|c|c|}
\hline \multirow{2}{*}{ Building } & \multirow{2}{*}{$V e$} & \multirow{2}{*}{$\begin{array}{c}\text { Ductility }(\mu) \\
R y=3\end{array}$} & \multicolumn{3}{|c|}{ Force Reduction Factor $(R y)$} \\
\hline & & & $\mu=2$ & $\mu=3$ & $\mu=4$ \\
\hline SL1 & 90.2 & 11.58 & 1.64 & 2.08 & 2.22 \\
\hline SL3 & 92.6 & 12.60 & 1.75 & 2.08 & 2.21 \\
\hline SL4 & 97.0 & 12.35 & 1.36 & 1.73 & 2.16 \\
\hline SH1 & 197.4 & 9.37 & 1.36 & 1.58 & 1.78 \\
\hline $\mathrm{SH} 2$ & 198.5 & 9.16 & 1.33 & 1.54 & 1.72 \\
\hline $\mathrm{SH} 3$ & 201.3 & 8.28 & 1.34 & 1.56 & 1.76 \\
\hline SH4 & 198.9 & 9.42 & 1.31 & 1.50 & 1.68 \\
\hline SH5 & 201.7 & 8.34 & 1.33 & 1.55 & 1.73 \\
\hline ML1 & 376.8 & 7.71 & 1.36 & 1.60 & 1.79 \\
\hline ML2 & 372.8 & 7.40 & 1.34 & 1.56 & 1.74 \\
\hline ML3 & 362.9 & 6.09 & 1.33 & 1.55 & 1.77 \\
\hline ML4 & 374.4 & 7.31 & 1.34 & 1.57 & 1.75 \\
\hline ML5 & 370.6 & 7.16 & 1.33 & 1.55 & 1.73 \\
\hline ML6 & 365.7 & 6.07 & 1.35 & 1.57 & 1.77 \\
\hline MH1 & 774.8 & 5.86 & 1.38 & 1.65 & 1.88 \\
\hline MH2 & 723.6 & 5.02 & 1.41 & 1.67 & 1.89 \\
\hline MH3 & 558.6 & 5.13 & 1.24 & 1.42 & 1.59 \\
\hline MH4 & 758.2 & 6.08 & 1.38 & 1.63 & 1.86 \\
\hline MH5 & 695.5 & 5.14 & 1.39 & 1.62 & 1.83 \\
\hline MH6 & 752.7 & 4.32 & 1.47 & 1.78 & 2.22 \\
\hline LL1 & 1425.7 & 4.75 & 1.44 & 1.76 & 2.10 \\
\hline LL2 & 1401.2 & 4.89 & 1.43 & 1.74 & 2.06 \\
\hline LL3 & 1063.0 & 4.54 & 1.28 & 1.47 & 1.65 \\
\hline LL4 & 1424.4 & 4.74 & 1.44 & 1.76 & 2.10 \\
\hline LL5 & 1315.8 & 5.19 & 1.42 & 1.66 & 1.89 \\
\hline LL6 & 969.8 & 4.53 & 1.33 & 1.55 & 2.83 \\
\hline LH1 & 2972.3 & 3.28 & 1.52 & 1.87 & 3.91 \\
\hline LH2 & 2758.1 & 2.71 & 1.50 & 1.97 & 3.70 \\
\hline LH3 & 2042.7 & 3.94 & 1.38 & 2.61 & 3.01 \\
\hline LH4 & 2917.1 & 2.72 & 1.56 & 2.99 & 3.98 \\
\hline LH5 & 2367.9 & 3.57 & 1.38 & 1.65 & 3.19 \\
\hline LH6 & 1945.1 & 4.07 & 1.40 & 2.57 & 2.96 \\
\hline
\end{tabular}


Table A.19 - The response of the buildings subjected to E6C13 ground motion parallel to the long side of the building with $\alpha=0.00$

\begin{tabular}{|c|c|c|c|c|c|}
\hline \multirow{2}{*}{ Building } & \multirow{2}{*}{$V e$} & \multirow{2}{*}{$\begin{array}{c}\text { Ductility }(\mu) \\
R y=3\end{array}$} & \multicolumn{3}{|c|}{ Force Reduction Factor $(R y)$} \\
\hline & & & $\mu=2$ & $\mu=3$ & $\mu=4$ \\
\hline SL1 & 138.0 & 3.60 & 1.38 & 2.69 & 3.20 \\
\hline SL3 & 139.9 & 3.45 & 1.38 & 2.82 & 3.26 \\
\hline SL4 & 144.9 & 3.58 & 1.40 & 2.72 & 3.09 \\
\hline SH1 & 268.2 & 3.63 & 1.97 & 2.79 & 3.14 \\
\hline $\mathrm{SH} 2$ & 277.7 & 3.07 & 1.51 & 2.98 & 3.26 \\
\hline $\mathrm{SH} 3$ & 273.0 & 2.72 & 1.55 & 2.35 & 3.39 \\
\hline SH4 & 272.9 & 3.02 & 1.52 & 2.26 & 3.38 \\
\hline SH5 & 264.7 & 2.74 & 1.54 & 2.23 & 3.37 \\
\hline ML1 & 314.0 & 4.29 & 1.39 & 1.63 & 2.19 \\
\hline ML2 & 266.4 & 5.43 & 1.34 & 1.55 & 1.78 \\
\hline ML3 & 216.3 & 6.30 & 1.33 & 1.59 & 1.86 \\
\hline ML4 & 277.1 & 5.13 & 1.35 & 1.56 & 1.82 \\
\hline ML5 & 255.4 & 5.74 & 1.32 & 1.54 & 1.77 \\
\hline ML6 & 230.4 & 5.95 & 1.34 & 1.60 & 1.90 \\
\hline MH1 & 342.3 & 9.03 & 1.39 & 1.60 & 1.82 \\
\hline MH2 & 319.3 & 6.94 & 1.55 & 1.85 & 2.08 \\
\hline MH3 & 355.0 & 7.37 & 1.46 & 1.79 & 2.09 \\
\hline MH4 & 326.2 & 9.43 & 1.39 & 1.62 & 1.80 \\
\hline MH5 & 306.4 & 6.92 & 1.50 & 1.78 & 2.00 \\
\hline MH6 & 324.6 & 6.49 & 1.61 & 1.89 & 2.14 \\
\hline LL1 & 598.0 & 8.70 & 1.40 & 1.64 & 1.89 \\
\hline LL2 & 564.9 & 9.22 & 1.43 & 1.64 & 1.87 \\
\hline LL3 & 607.3 & 7.29 & 1.49 & 1.83 & 2.13 \\
\hline LL4 & 594.5 & 8.74 & 1.40 & 1.65 & 1.88 \\
\hline LL5 & 573.2 & 7.41 & 1.54 & 1.82 & 2.05 \\
\hline LL6 & 656.4 & 6.07 & 1.43 & 1.73 & 2.01 \\
\hline LH1 & 1215.7 & 5.94 & 1.59 & 1.88 & 2.15 \\
\hline LH2 & 1239.1 & 4.70 & 1.53 & 1.93 & 2.27 \\
\hline LH3 & 1392.0 & 5.61 & 1.49 & 1.86 & 2.19 \\
\hline LH4 & 1207.4 & 5.13 & 1.57 & 1.93 & 2.24 \\
\hline LH5 & 1325.5 & 6.23 & 1.52 & 1.90 & 2.23 \\
\hline LH6 & 1355.7 & 5.15 & 1.45 & 1.78 & 2.31 \\
\hline
\end{tabular}


Table A.20 - The response of the buildings subjected to E6C15 ground motion parallel to the long side of the building with $\alpha=0.00$

\begin{tabular}{|c|c|c|c|c|c|}
\hline \multirow{2}{*}{ Building } & \multirow{2}{*}{$V e$} & \multirow{2}{*}{$\begin{array}{c}\text { Ductility }(\mu) \\
R y=3\end{array}$} & \multicolumn{3}{|c|}{ Force Reduction Factor $(R y)$} \\
\hline & & & $\mu=2$ & $\mu=3$ & $\mu=4$ \\
\hline SL1 & 136.8 & 6.89 & 1.67 & 1.98 & 2.25 \\
\hline SL3 & 134.0 & 7.18 & 1.66 & 1.94 & 2.24 \\
\hline SL4 & 127.0 & 7.83 & 1.62 & 1.89 & 2.16 \\
\hline SH1 & 175.0 & 9.69 & 1.45 & 1.71 & 1.92 \\
\hline $\mathrm{SH} 2$ & 166.9 & 11.12 & 1.44 & 1.67 & 1.87 \\
\hline $\mathrm{SH} 3$ & 158.5 & 11.29 & 1.38 & 1.58 & 1.77 \\
\hline SH4 & 158.2 & 12.59 & 1.41 & 1.57 & 1.72 \\
\hline SH5 & 162.5 & 10.97 & 1.42 & 1.60 & 1.78 \\
\hline ML1 & 295.8 & 9.29 & 1.51 & 1.73 & 1.92 \\
\hline ML2 & 289.3 & 9.42 & 1.52 & 1.77 & 1.98 \\
\hline ML3 & 282.6 & 8.60 & 1.56 & 1.85 & 2.17 \\
\hline ML4 & 290.9 & 9.63 & 1.53 & 1.74 & 1.97 \\
\hline ML5 & 287.6 & 9.63 & 1.54 & 1.78 & 2.03 \\
\hline ML6 & 284.2 & 8.54 & 1.53 & 1.84 & 2.14 \\
\hline MH1 & 726.5 & 5.31 & 1.77 & 2.24 & 2.71 \\
\hline MH2 & 854.8 & 3.05 & 1.83 & 2.97 & 3.38 \\
\hline MH3 & 486.9 & 5.80 & 1.43 & 1.87 & 2.69 \\
\hline MH4 & 778.3 & 4.56 & 1.77 & 2.40 & 2.85 \\
\hline MH5 & 813.0 & 2.89 & 1.35 & 3.03 & 3.43 \\
\hline MH6 & 897.9 & 2.46 & 2.39 & 3.36 & 3.78 \\
\hline LL1 & 1414.7 & 3.84 & 2.02 & 2.79 & 3.07 \\
\hline LL2 & 1524.5 & 3.01 & 2.18 & 2.96 & 3.30 \\
\hline LL3 & 1034.0 & 3.96 & 1.79 & 2.31 & 3.01 \\
\hline LL4 & 1425.8 & 3.67 & 2.02 & 2.81 & 3.09 \\
\hline LL5 & 1565.1 & 3.18 & 1.82 & 2.96 & 3.41 \\
\hline LL6 & 597.0 & 11.09 & 1.45 & 1.67 & 2.13 \\
\hline LH1 & 3340.8 & 2.07 & 2.45 & 3.57 & 4.14 \\
\hline LH2 & 2668.6 & 2.61 & 2.16 & 3.20 & 3.70 \\
\hline LH3 & 1324.9 & 8.39 & 1.56 & 1.88 & 2.33 \\
\hline LH4 & 3067.4 & 2.07 & 2.26 & 3.60 & 4.25 \\
\hline LH5 & 1822.4 & 4.86 & 1.56 & 2.35 & 2.71 \\
\hline LH6 & 1288.9 & 7.88 & 1.50 & 1.81 & 2.41 \\
\hline
\end{tabular}


Table A.21 - The response of the buildings subjected to E6C18 ground motion parallel to the long side of the building with $\alpha=0.00$

\begin{tabular}{|c|c|c|c|c|c|}
\hline \multirow{2}{*}{ Building } & \multirow{2}{*}{$V e$} & \multirow{2}{*}{$\begin{array}{c}\text { Ductility }(\mu) \\
R y=3\end{array}$} & \multicolumn{3}{|c|}{ Force Reduction Factor $(R y)$} \\
\hline & & & $\mu=2$ & $\mu=3$ & $\mu=4$ \\
\hline SL1 & 183.6 & 5.19 & 1.37 & 2.31 & 2.62 \\
\hline SL3 & 185.1 & 5.16 & 1.36 & 2.28 & 2.62 \\
\hline SL4 & 187.9 & 5.31 & 1.35 & 2.29 & 2.61 \\
\hline SH1 & 353.9 & 4.23 & 1.34 & 2.27 & 2.59 \\
\hline $\mathrm{SH} 2$ & 336.3 & 3.83 & 1.34 & 2.21 & 4.02 \\
\hline $\mathrm{SH} 3$ & 310.4 & 3.05 & 1.39 & 2.76 & 4.14 \\
\hline SH4 & 309.1 & 3.40 & 1.35 & 2.41 & 3.84 \\
\hline SH5 & 290.3 & 2.71 & 1.41 & 3.25 & 3.85 \\
\hline ML1 & 277.4 & 9.30 & 1.48 & 1.73 & 1.94 \\
\hline ML2 & 243.2 & 11.95 & 1.54 & 1.70 & 1.81 \\
\hline ML3 & 234.9 & 12.53 & 1.60 & 1.81 & 1.95 \\
\hline ML4 & 248.6 & 11.28 & 1.52 & 1.69 & 1.80 \\
\hline ML5 & 239.4 & 12.96 & 1.55 & 1.73 & 1.82 \\
\hline ML6 & 234.5 & 11.78 & 1.60 & 1.78 & 1.91 \\
\hline MH1 & 542.7 & 10.65 & 1.54 & 1.82 & 2.06 \\
\hline MH2 & 541.7 & 8.38 & 1.41 & 1.68 & 1.93 \\
\hline MH3 & 505.9 & 3.35 & 1.38 & 1.65 & 3.28 \\
\hline MH4 & 535.1 & 10.75 & 1.48 & 1.74 & 1.95 \\
\hline MH5 & 535.3 & 7.59 & 1.37 & 1.63 & 1.87 \\
\hline MH6 & 550.8 & 7.53 & 1.49 & 1.80 & 2.09 \\
\hline LL1 & 989.4 & 9.00 & 1.54 & 1.86 & 2.13 \\
\hline LL2 & 985.6 & 8.79 & 1.50 & 1.79 & 2.06 \\
\hline LL3 & 923.2 & 3.99 & 1.37 & 1.64 & 2.11 \\
\hline LL4 & 989.3 & 9.00 & 1.54 & 1.85 & 2.13 \\
\hline LL5 & 977.5 & 8.79 & 1.41 & 1.68 & 1.91 \\
\hline LL6 & 786.1 & 4.09 & 1.41 & 2.56 & 2.98 \\
\hline LH1 & 2110.3 & 6.25 & 1.45 & 1.78 & 2.08 \\
\hline LH2 & 2008.9 & 3.83 & 1.44 & 1.79 & 3.18 \\
\hline LH3 & 1580.5 & 3.71 & 2.13 & 2.65 & 3.15 \\
\hline LH4 & 2048.8 & 4.63 & 1.46 & 1.82 & 2.16 \\
\hline LH5 & 1939.9 & 2.74 & 1.46 & 3.17 & 5.59 \\
\hline LH6 & 1411.4 & 4.31 & 1.94 & 2.42 & 2.87 \\
\hline
\end{tabular}


Table A.22 - The response of the buildings subjected to E6C42 ground motion parallel to the long side of the building with $\alpha=0.00$

\begin{tabular}{|c|c|c|c|c|c|}
\hline \multirow{2}{*}{ Building } & \multirow{2}{*}{$V e$} & \multirow{2}{*}{$\begin{array}{c}\text { Ductility }(\mu) \\
R y=3\end{array}$} & \multicolumn{3}{|c|}{ Force Reduction Factor $(R y)$} \\
\hline & & & $\mu=2$ & $\mu=3$ & $\mu=4$ \\
\hline SL1 & 172.3 & 5.20 & 1.85 & 2.18 & 2.48 \\
\hline SL3 & 165.3 & 5.00 & 1.86 & 2.18 & 2.51 \\
\hline SL4 & 142.9 & 4.55 & 1.90 & 2.20 & 2.67 \\
\hline SH1 & 224.2 & 4.90 & 1.37 & 1.65 & 2.56 \\
\hline $\mathrm{SH} 2$ & 199.8 & 4.93 & 1.35 & 1.59 & 1.99 \\
\hline $\mathrm{SH} 3$ & 183.4 & 3.89 & 1.35 & 1.60 & 1.87 \\
\hline SH4 & 178.1 & 4.32 & 1.31 & 1.52 & 1.72 \\
\hline SH5 & 176.8 & 3.57 & 1.34 & 1.58 & 1.77 \\
\hline ML1 & 270.0 & 4.64 & 1.84 & 2.39 & 2.87 \\
\hline ML2 & 232.6 & 6.04 & 1.36 & 2.08 & 2.38 \\
\hline ML3 & 246.2 & 4.68 & 1.36 & 1.63 & 2.00 \\
\hline ML4 & 242.7 & 5.89 & 1.47 & 2.20 & 2.54 \\
\hline ML5 & 231.9 & 5.69 & 1.34 & 1.88 & 2.41 \\
\hline ML6 & 241.9 & 4.63 & 1.36 & 1.73 & 2.24 \\
\hline MH1 & 584.0 & 4.54 & 1.37 & 1.62 & 2.40 \\
\hline MH2 & 555.6 & 3.92 & 1.37 & 1.85 & 3.02 \\
\hline MH3 & 423.0 & 4.32 & 1.27 & 1.46 & 1.72 \\
\hline MH4 & 582.5 & 4.57 & 1.37 & 1.61 & 2.84 \\
\hline MH5 & 536.4 & 4.00 & 1.36 & 1.76 & 3.00 \\
\hline MH6 & 562.1 & 3.53 & 1.41 & 2.02 & 3.19 \\
\hline LL1 & 1051.5 & 3.92 & 1.41 & 1.71 & 3.04 \\
\hline LL2 & 1047.9 & 3.92 & 1.40 & 1.69 & 3.02 \\
\hline LL3 & 780.7 & 4.29 & 1.26 & 1.45 & 1.81 \\
\hline LL4 & 1051.6 & 3.90 & 1.42 & 1.71 & 3.05 \\
\hline LL5 & 1005.8 & 4.04 & 1.36 & 1.76 & 2.98 \\
\hline LL6 & 748.6 & 4.18 & 1.46 & 1.72 & 2.16 \\
\hline LH1 & 2122.3 & 3.16 & 1.45 & 2.92 & 3.48 \\
\hline LH2 & 1879.5 & 3.07 & 1.42 & 2.96 & 3.50 \\
\hline LH3 & 1531.2 & 3.37 & 1.52 & 1.84 & 3.81 \\
\hline LH4 & 2003.8 & 2.93 & 1.47 & 3.04 & 3.75 \\
\hline LH5 & 1683.2 & 3.21 & 1.36 & 1.67 & 3.40 \\
\hline LH6 & 1390.8 & 3.65 & 1.49 & 1.81 & 3.70 \\
\hline
\end{tabular}




\section{Appendix B}

The regression fits on the results of analyses for different values of post-yield stiffness for the lateral load resisting system, explained in Chapter 4 are presented first. Next, the results of the analyses explained in Chapter 4 are presented in in a series of tables. Tables B.1 to B.20 present the results for buildings with post-yield stiffness ratio of 0.02 in their lateral load resisting system, Tables B.21 to B.40 present the results for post-yield stiffness ratio of 0.05 , Tables B.41 to B.60 present the results for post-yield stiffness ratio of 0.10 .

The results for each value of post-yield stiffness are presented for two different cases: (1) when the seismic load acts parallel to the short side of the building, and (2) when the seismic load acts parallel to the long side of the system. For each scenario, different targets of ductility demands, namely $\mu=2,3$ and 4, and two regions (Montreal spectrum and Vancouver spectrum) are considered. 


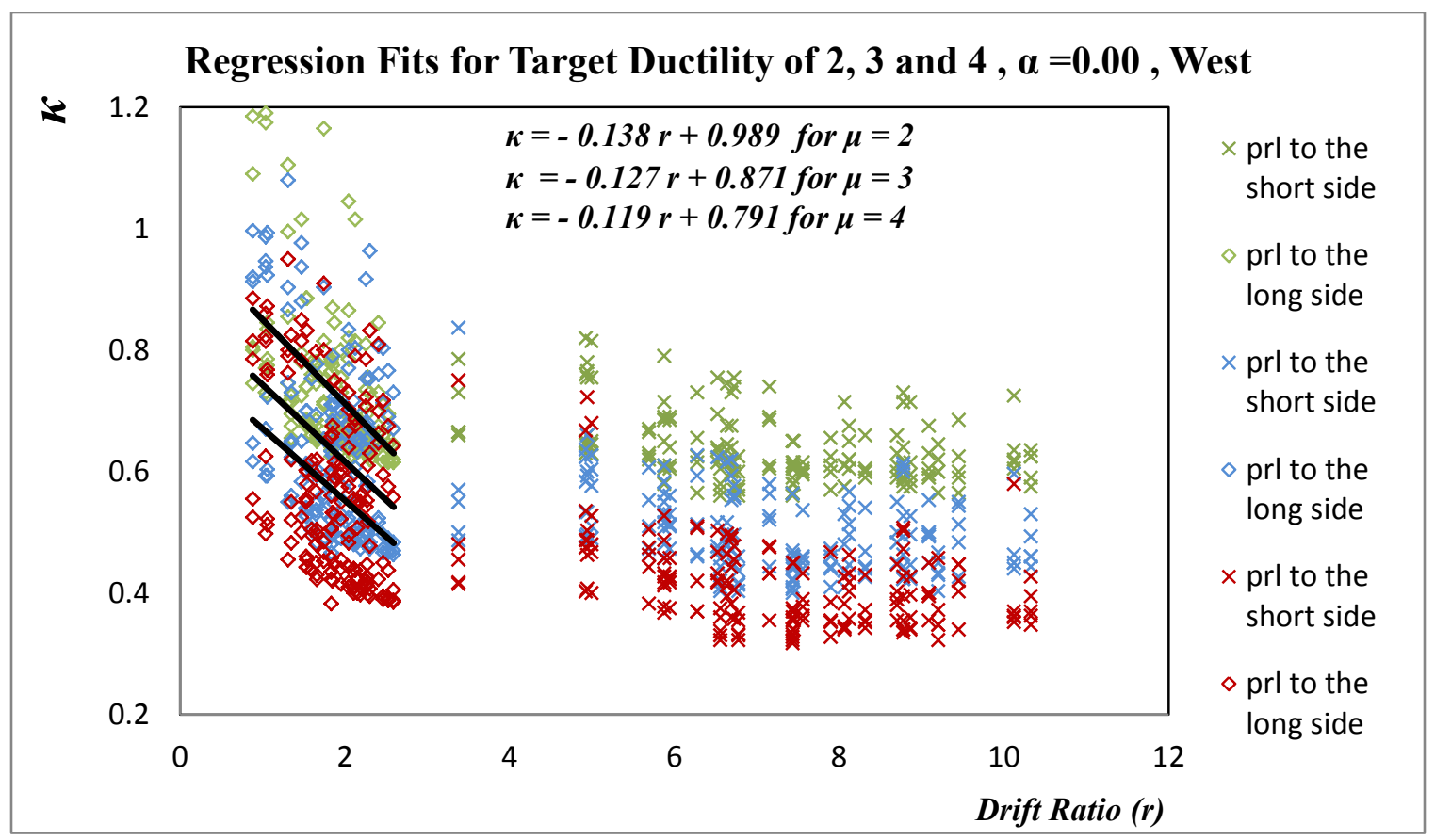

Figure B.1 - Regression fits and equations suggested for $\kappa$ for $\mu=2,3$ and $4-\alpha=0.00-$ Ground motions compatible with the UHS for Vancouver

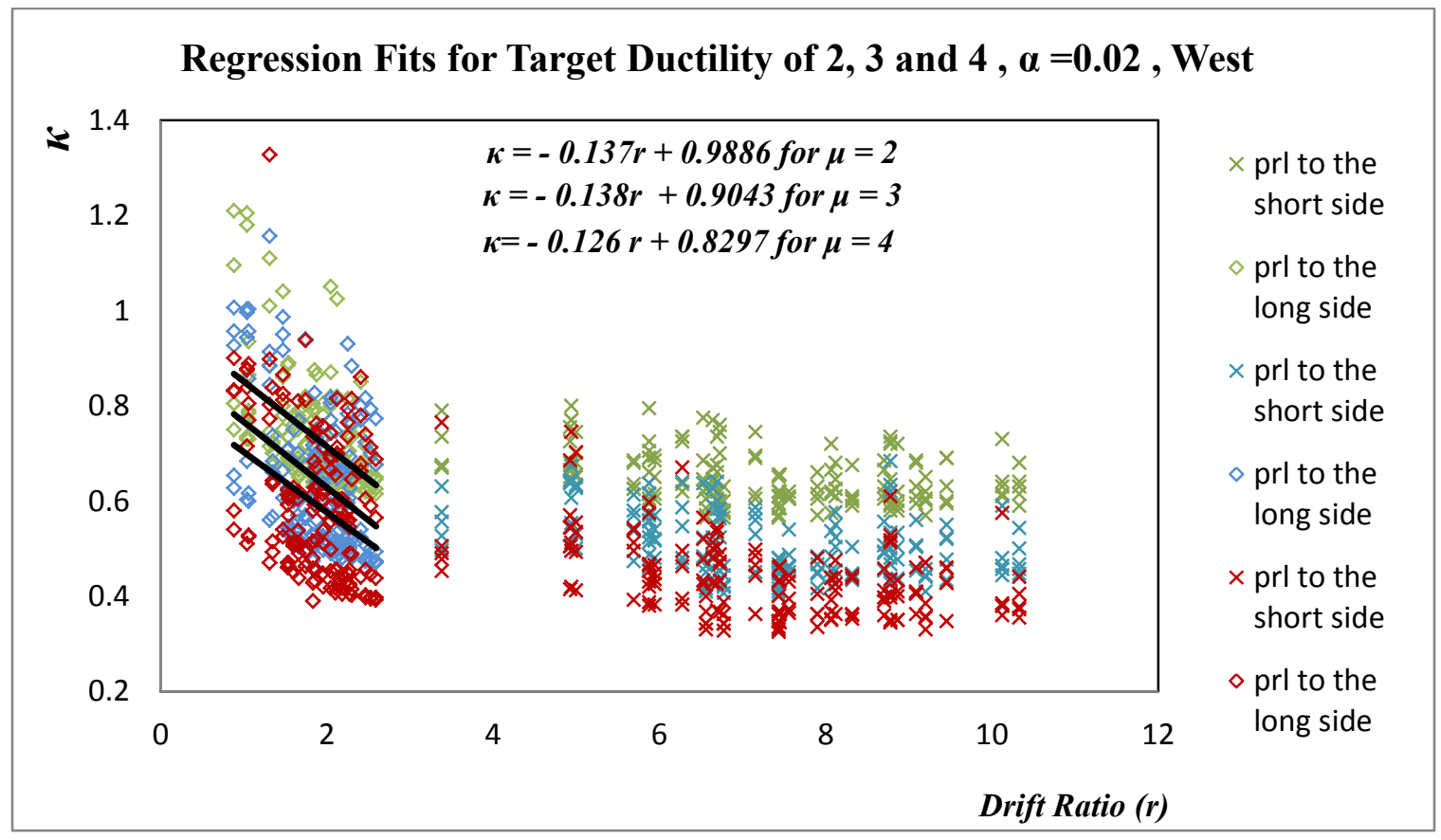

Figure B.2 - Regression fits and equations suggested for $\kappa$ for $\mu=2,3$ and $4-\alpha=0.02-$ Ground motions compatible with the UHS for Vancouver 


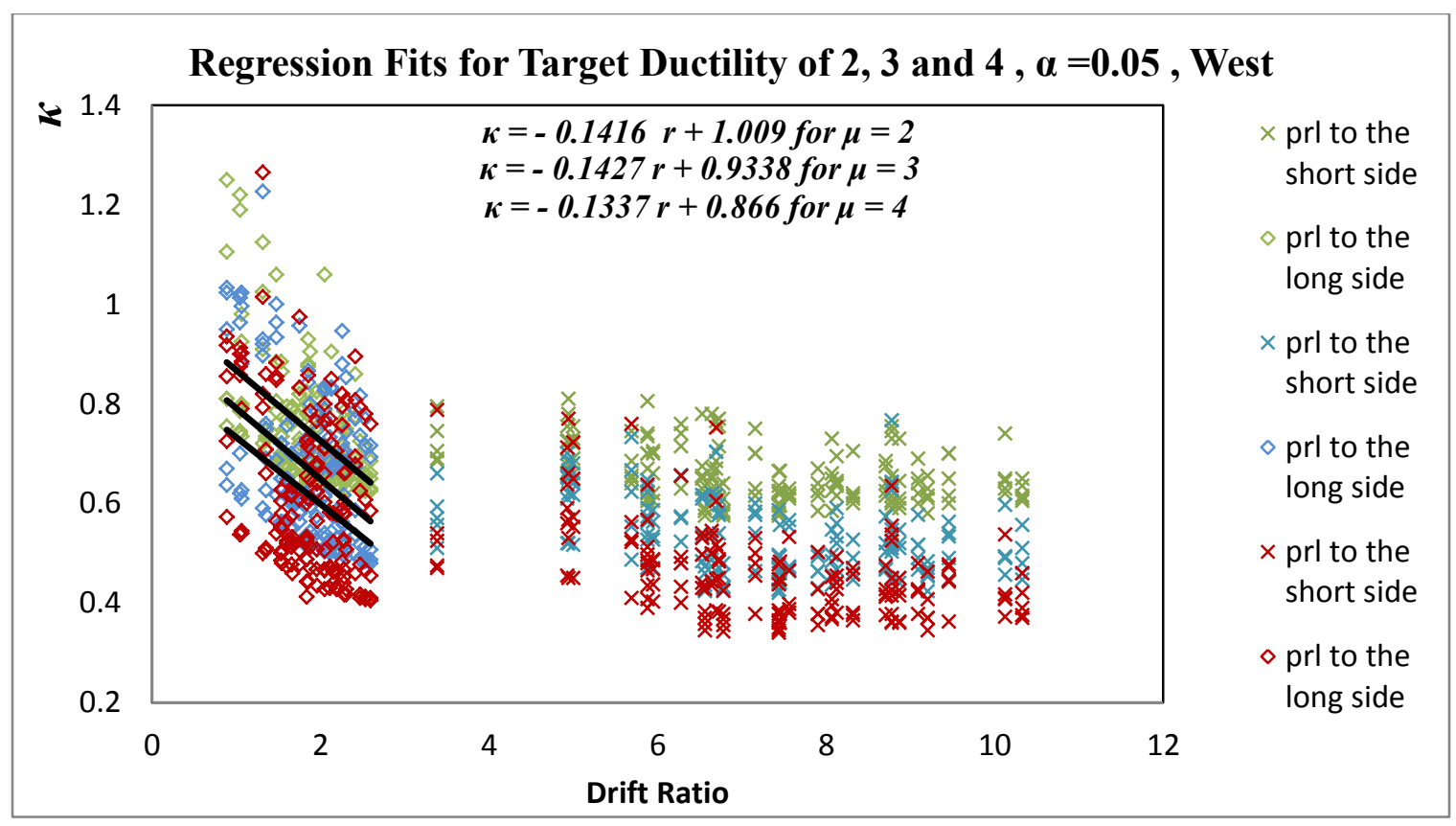

Figure B.3 - Regression fits and equations suggested for $\kappa$ for $\mu=2,3$ and $4-\alpha=0.05-$ Ground motions compatible with the UHS for Vancouver

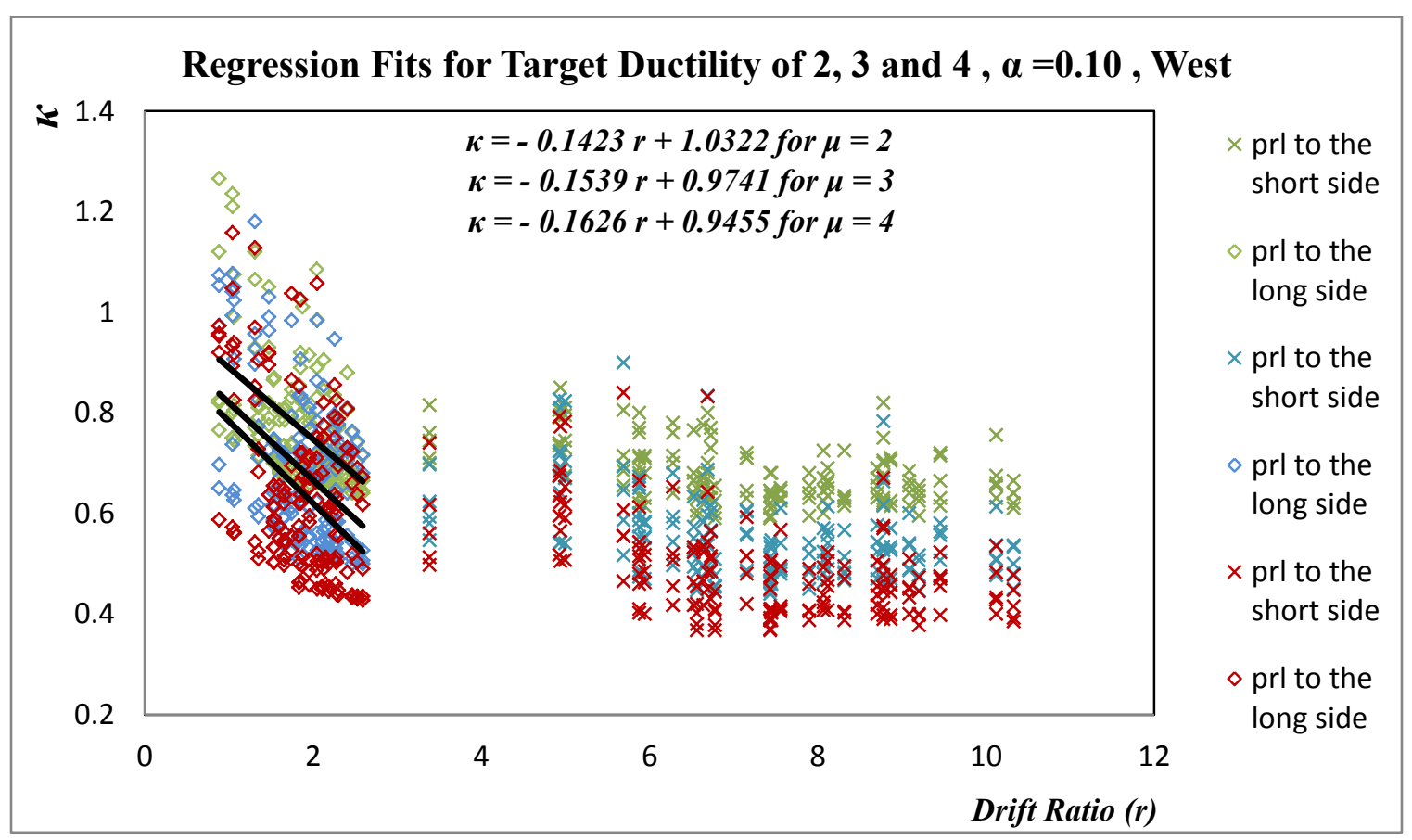

Figure B.4 - Regression fits and equations suggested for $\kappa$ for $\mu=2,3$ and $4-\alpha=0.10-$ Ground motions compatible with the UHS for Vancouver 


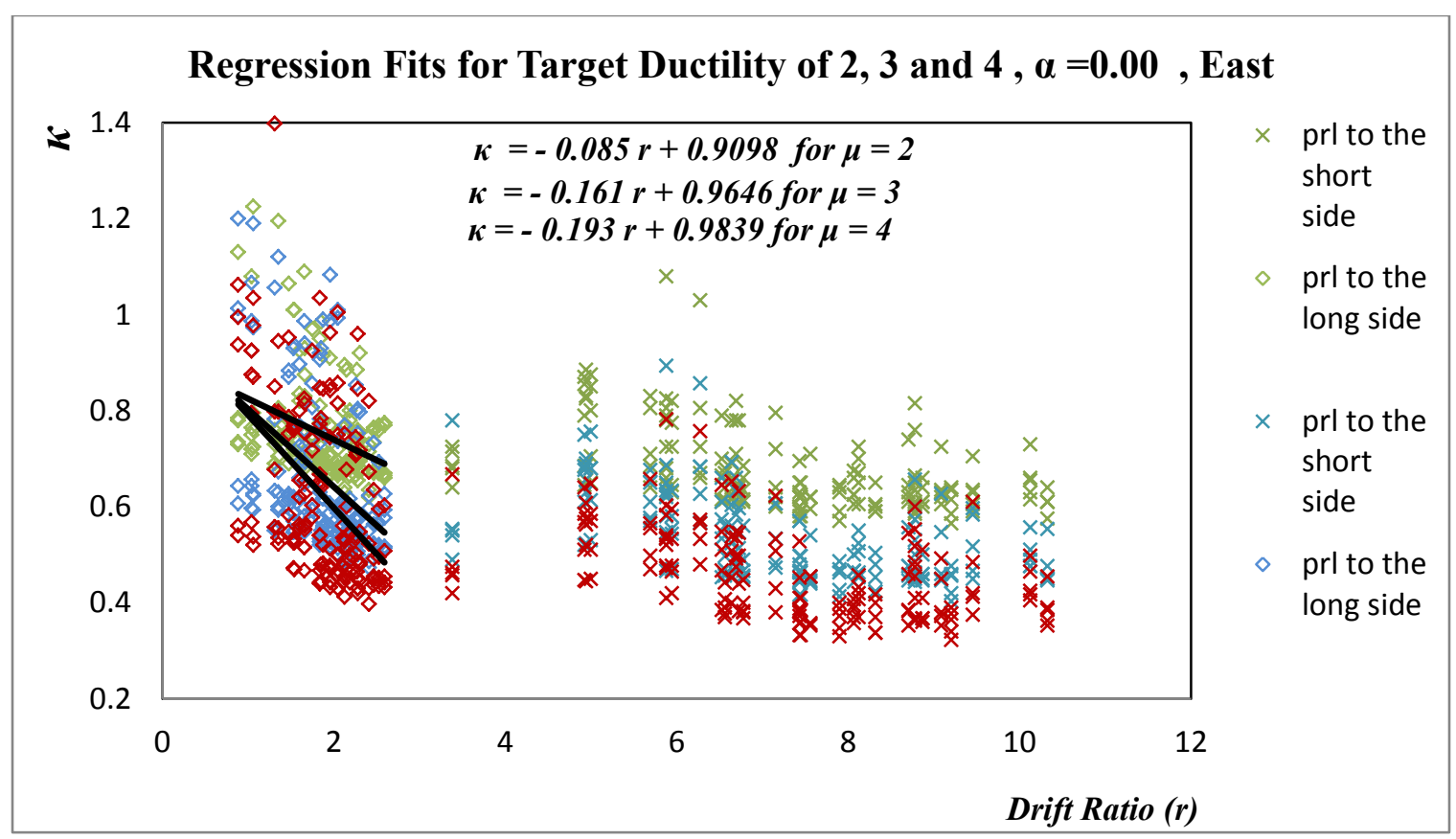

Figure B.5 - Regression fits and equations suggested for $\kappa$ for $\mu=2,3$ and $4-\alpha=0.00-$ Ground motions compatible with the UHS for Montreal

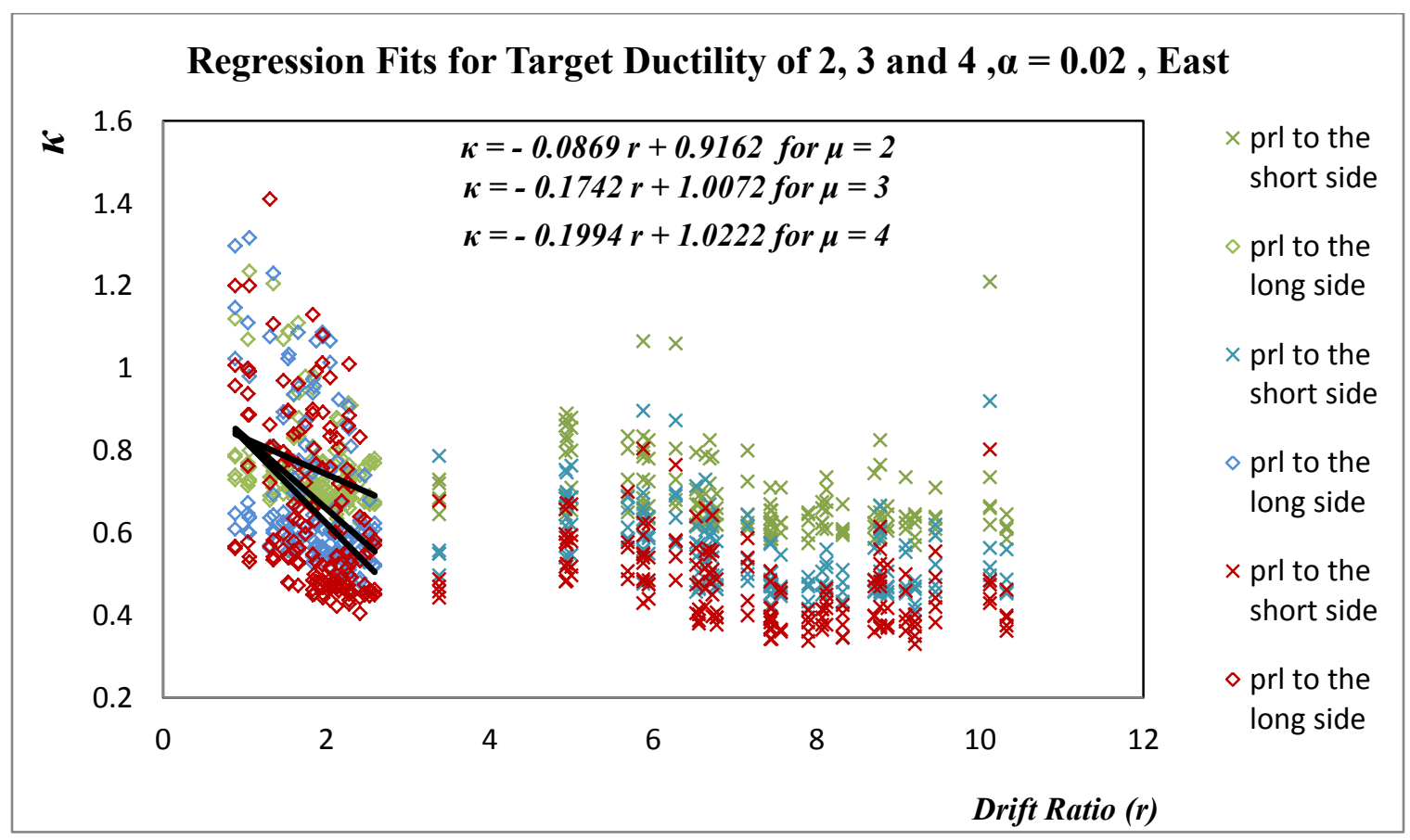

Figure B.6 - Regression fits and equations suggested for $\kappa$ for $\mu=2,3$ and $4-\alpha=0.02-$ Ground motions compatible with the UHS for Montreal 


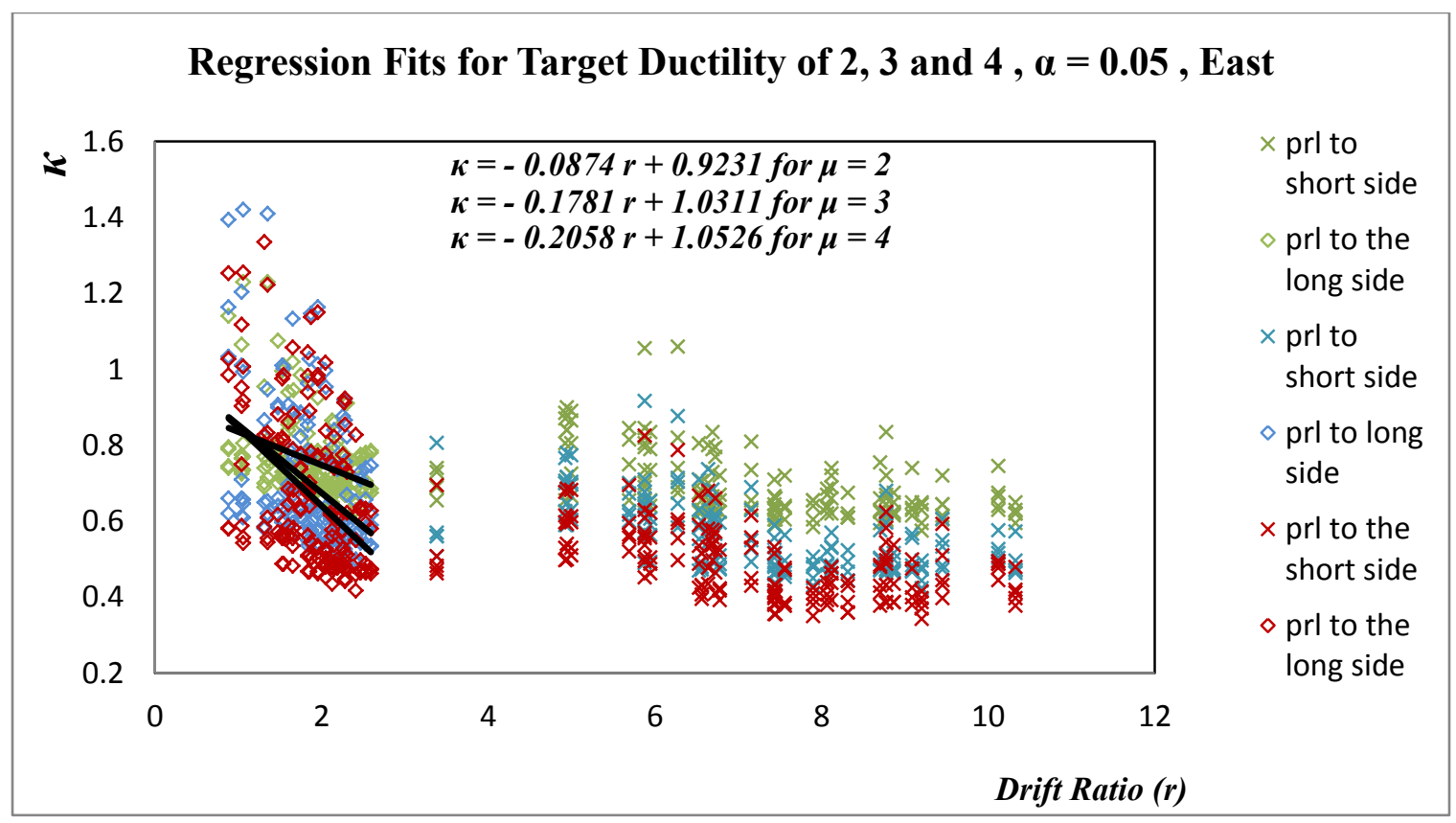

Figure B.7 - Regression fits and equations suggested for $\kappa$ for $\mu=2,3$ and $4-\alpha=0.05-$ Ground motions compatible with the UHS for Montreal

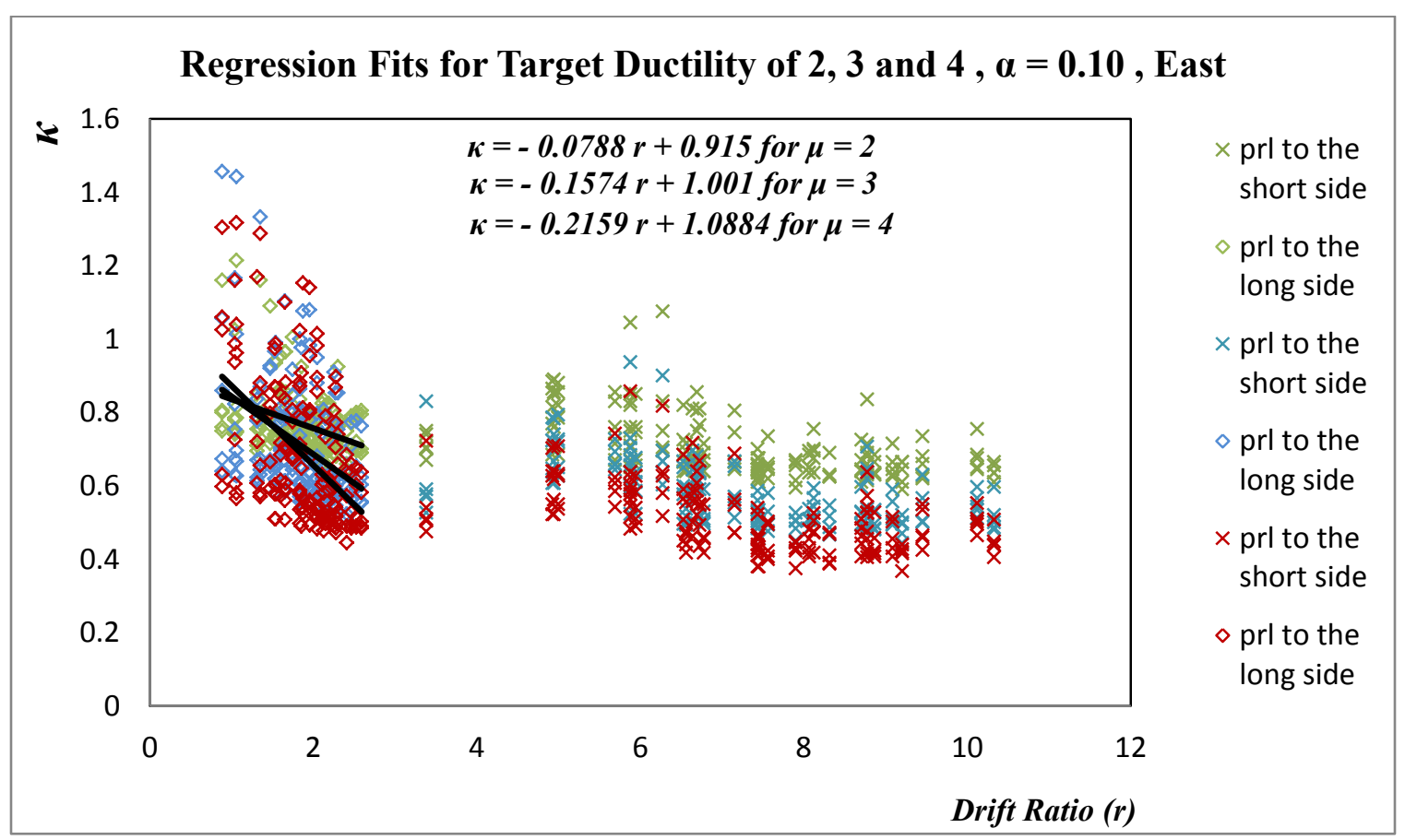

Figure B.8 - Regression fits and equations suggested for $\kappa$ for $\mu=2,3$ and $4-\alpha=0.10-$ Ground motions compatible with the UHS for Montreal 
Table B.1 - The response of the buildings subjected to M6C1 ground motion parallel to the short side of the building with $\alpha=0.02$

\begin{tabular}{|c|c|c|c|c|c|}
\hline \multirow{2}{*}{ Building } & \multirow{2}{*}{$V e$} & \multirow{2}{*}{$\frac{\text { Ductility }(\mu)}{R y=3}$} & \multicolumn{3}{|c|}{ Force Reduction Factor $(R y)$} \\
\hline & & & $\mu=2$ & $\mu=3$ & $\mu=4$ \\
\hline SL1 & 131.5 & 11.87 & 1.25 & 1.39 & 1.60 \\
\hline SL3 & 133.1 & 12.07 & 1.26 & 1.39 & 1.55 \\
\hline SL4 & 142.1 & 11.96 & 1.30 & 1.44 & 1.60 \\
\hline SH1 & 316.8 & 8.35 & 1.22 & 1.46 & 1.72 \\
\hline $\mathrm{SH} 2$ & 283.9 & 9.39 & 1.35 & 1.51 & 1.77 \\
\hline $\mathrm{SH} 3$ & 264.2 & 9.56 & 1.31 & 1.44 & 1.72 \\
\hline SH4 & 260.9 & 9.91 & 1.30 & 1.41 & 1.54 \\
\hline SH5 & 262.9 & 8.99 & 1.32 & 1.45 & 1.62 \\
\hline SH6 & 263.0 & 9.61 & 1.31 & 1.43 & 1.73 \\
\hline ML1 & 508.8 & 6.57 & 1.44 & 1.61 & 1.75 \\
\hline ML2 & 435.8 & 8.55 & 1.36 & 1.50 & 1.62 \\
\hline ML3 & 417.7 & 7.12 & 1.37 & 1.51 & 1.63 \\
\hline ML4 & 490.8 & 7.83 & 1.44 & 1.60 & 1.73 \\
\hline ML5 & 493.7 & 7.74 & 1.44 & 1.60 & 1.74 \\
\hline ML6 & 428.7 & 7.01 & 1.37 & 1.52 & 1.65 \\
\hline MH1 & 878.8 & 7.27 & 1.36 & 1.51 & 1.65 \\
\hline MH2 & 832.1 & 6.85 & 1.40 & 1.57 & 1.73 \\
\hline MH3 & 500.5 & 7.02 & 1.45 & 2.05 & 2.44 \\
\hline MH4 & 832.5 & 7.21 & 1.38 & 1.57 & 1.71 \\
\hline MH5 & 851.3 & 5.88 & 1.38 & 1.68 & 1.86 \\
\hline MH6 & 1143.3 & 3.83 & 1.58 & 1.89 & 3.06 \\
\hline LL1 & 1523.3 & 6.93 & 1.29 & 1.45 & 2.26 \\
\hline LL2 & 1503.8 & 6.97 & 1.28 & 1.45 & 2.00 \\
\hline LL3 & 1496.1 & 5.56 & 1.47 & 1.75 & 2.17 \\
\hline LL4 & 1716.3 & 6.06 & 1.37 & 1.56 & 1.79 \\
\hline LL5 & 1735.1 & 5.93 & 1.38 & 1.57 & 1.81 \\
\hline LL6 & 469.1 & 16.99 & 1.28 & 1.44 & 1.54 \\
\hline LH1 & 2933.9 & 5.80 & 1.37 & 1.74 & 2.16 \\
\hline LH2 & 2472.9 & 6.83 & 1.36 & 1.66 & 1.98 \\
\hline LH3 & 1269.3 & 11.77 & 1.41 & 1.68 & 1.86 \\
\hline LH4 & 2409.6 & 6.66 & 1.31 & 1.59 & 1.97 \\
\hline LH5 & 2344.8 & 6.47 & 1.33 & 1.63 & 2.02 \\
\hline LH6 & 1212.9 & 11.93 & 1.45 & 1.64 & 1.85 \\
\hline
\end{tabular}


Table B.2 - The response of the buildings subjected to M6C2 ground motion parallel to the short side of the building with $\alpha=0.02$

\begin{tabular}{|c|c|c|c|c|c|}
\hline \multirow{2}{*}{ Building } & \multirow{2}{*}{$V e$} & \multirow{2}{*}{$\begin{array}{c}\text { Ductility }(\mu) \\
R y=3\end{array}$} & \multicolumn{3}{|c|}{ Force Reduction Factor $(R y)$} \\
\hline & & & $\mu=2$ & $\mu=3$ & $\mu=4$ \\
\hline SL1 & 135.5 & 13.08 & 1.21 & 1.35 & 1.47 \\
\hline SL3 & 137.2 & 13.27 & 1.20 & 1.33 & 1.45 \\
\hline SL4 & 138.2 & 15.64 & 1.19 & 1.30 & 1.39 \\
\hline SH1 & 226.8 & 7.17 & 1.21 & 1.62 & 1.77 \\
\hline $\mathrm{SH} 2$ & 223.9 & 7.58 & 1.20 & 1.33 & 1.75 \\
\hline $\mathrm{SH} 3$ & 223.3 & 7.33 & 1.24 & 1.40 & 1.84 \\
\hline SH4 & 216.4 & 9.69 & 1.24 & 1.37 & 1.88 \\
\hline SH5 & 215.9 & 8.80 & 1.26 & 1.40 & 1.92 \\
\hline SH6 & 223.2 & 7.43 & 1.24 & 1.38 & 1.85 \\
\hline ML1 & 276.6 & 15.39 & 1.23 & 1.32 & 1.40 \\
\hline ML2 & 211.1 & 21.05 & 1.23 & 1.37 & 1.49 \\
\hline ML3 & 234.5 & 17.94 & 1.19 & 1.52 & 1.62 \\
\hline ML4 & 231.1 & 18.21 & 1.18 & 1.30 & 1.40 \\
\hline ML5 & 235.5 & 18.09 & 1.19 & 1.28 & 1.38 \\
\hline ML6 & 221.4 & 19.23 & 1.23 & 1.48 & 1.56 \\
\hline MH1 & 583.3 & 13.23 & 1.22 & 1.65 & 1.76 \\
\hline MH2 & 687.7 & 7.10 & 1.52 & 1.75 & 1.95 \\
\hline MH3 & 580.2 & 8.68 & 1.47 & 1.90 & 2.11 \\
\hline MH4 & 697.4 & 6.68 & 1.26 & 1.65 & 1.84 \\
\hline MH5 & 706.7 & 5.49 & 1.49 & 1.72 & 1.99 \\
\hline MH6 & 568.5 & 10.47 & 1.34 & 1.49 & 2.02 \\
\hline LL1 & 1105.5 & 10.20 & 1.55 & 1.91 & 2.08 \\
\hline LL2 & 1108.6 & 9.63 & 1.54 & 1.88 & 2.05 \\
\hline LL3 & 1169.1 & 4.93 & 1.35 & 1.92 & 2.14 \\
\hline LL4 & 944.8 & 13.20 & 1.30 & 1.62 & 1.74 \\
\hline LL5 & 932.3 & 13.43 & 1.32 & 1.61 & 1.72 \\
\hline LL6 & 554.6 & 15.62 & 1.22 & 1.33 & 1.44 \\
\hline LH1 & 2431.3 & 4.87 & 1.36 & 1.71 & 2.17 \\
\hline LH2 & 2373.5 & 5.33 & 1.60 & 1.93 & 2.18 \\
\hline LH3 & 1828.0 & 9.78 & 1.45 & 1.67 & 1.86 \\
\hline LH4 & 2391.1 & 5.75 & 1.49 & 1.89 & 2.22 \\
\hline LH5 & 2440.7 & 5.66 & 1.51 & 1.92 & 2.28 \\
\hline LH6 & 1494.5 & 10.75 & 1.27 & 1.44 & 1.58 \\
\hline
\end{tabular}


Table B.3 - The response of the buildings subjected to M6C26 ground motion parallel to the short side of the building with $\alpha=0.02$

\begin{tabular}{|c|c|c|c|c|c|}
\hline \multirow{2}{*}{ Building } & \multirow{2}{*}{$V e$} & \multirow{2}{*}{$\frac{\text { Ductility }(\mu)}{R y=3}$} & \multicolumn{3}{|c|}{ Force Reduction Factor $(R y)$} \\
\hline & & & $\mu=2$ & $\mu=3$ & $\mu=4$ \\
\hline SL1 & 164.3 & 10.41 & 1.17 & 1.27 & 1.37 \\
\hline SL3 & 156.3 & 11.47 & 1.16 & 1.27 & 1.37 \\
\hline SL4 & 135.3 & 16.49 & 1.14 & 1.23 & 1.32 \\
\hline SH1 & 333.5 & 8.21 & 1.24 & 1.38 & 1.49 \\
\hline $\mathrm{SH} 2$ & 314.4 & 9.16 & 1.22 & 1.34 & 1.44 \\
\hline $\mathrm{SH} 3$ & 304.8 & 9.05 & 1.22 & 1.34 & 1.45 \\
\hline SH4 & 293.8 & 9.78 & 1.21 & 1.32 & 1.42 \\
\hline SH5 & 294.7 & 8.92 & 1.23 & 1.35 & 1.45 \\
\hline SH6 & 304.1 & 8.87 & 1.22 & 1.34 & 1.45 \\
\hline ML1 & 474.2 & 7.54 & 1.33 & 1.45 & 1.57 \\
\hline ML2 & 379.0 & 13.12 & 1.18 & 1.63 & 1.76 \\
\hline ML3 & 358.2 & 11.85 & 1.22 & 1.68 & 1.84 \\
\hline ML4 & 432.4 & 9.86 & 1.16 & 1.51 & 1.64 \\
\hline ML5 & 436.6 & 9.38 & 1.16 & 1.50 & 1.62 \\
\hline ML6 & 369.5 & 11.85 & 1.21 & 1.67 & 1.83 \\
\hline MH1 & 739.4 & 10.68 & 1.25 & 1.73 & 1.90 \\
\hline MH2 & 669.2 & 9.14 & 1.49 & 1.71 & 1.89 \\
\hline MH3 & 449.0 & 8.59 & 1.29 & 1.86 & 2.07 \\
\hline MH4 & 624.9 & 10.88 & 1.38 & 1.56 & 1.71 \\
\hline MH5 & 612.2 & 8.88 & 1.39 & 1.59 & 1.77 \\
\hline MH6 & 996.4 & 4.56 & 1.47 & 1.67 & 1.91 \\
\hline LL1 & 1154.3 & 10.82 & 1.29 & 1.44 & 1.91 \\
\hline LL2 & 1137.2 & 10.71 & 1.29 & 1.77 & 1.92 \\
\hline LL3 & 949.1 & 6.85 & 1.32 & 1.51 & 1.70 \\
\hline LL4 & 1226.8 & 10.06 & 1.23 & 1.71 & 1.86 \\
\hline LL5 & 1239.5 & 10.01 & 1.23 & 1.71 & 1.87 \\
\hline LL6 & 439.4 & 12.04 & 1.26 & 1.39 & 1.52 \\
\hline LH1 & 2064.4 & 4.67 & 1.25 & 1.61 & 1.98 \\
\hline LH2 & 2116.3 & 4.03 & 1.54 & 2.02 & 2.98 \\
\hline LH3 & 1321.5 & 6.61 & 1.19 & 1.91 & 2.39 \\
\hline LH4 & 1997.1 & 4.70 & 1.36 & 1.88 & 2.81 \\
\hline LH5 & 1961.0 & 4.92 & 1.36 & 1.91 & 2.74 \\
\hline LH6 & 1191.4 & 8.02 & 1.47 & 1.76 & 1.98 \\
\hline
\end{tabular}


Table B.4 - The response of the buildings subjected to M6C31 ground motion parallel to the short side of the building with $\alpha=0.02$

\begin{tabular}{|c|c|c|c|c|c|}
\hline \multirow{2}{*}{ Building } & \multirow{2}{*}{$V e$} & \multirow{2}{*}{$\frac{\text { Ductility }(\mu)}{R y=3}$} & \multicolumn{3}{|c|}{ Force Reduction Factor $(R y)$} \\
\hline & & & $\mu=2$ & $\mu=3$ & $\mu=4$ \\
\hline SL1 & 202.1 & 7.71 & 1.14 & 1.25 & 1.37 \\
\hline SL3 & 210.1 & 7.70 & 1.15 & 1.26 & 1.37 \\
\hline SL4 & 214.7 & 8.29 & 1.14 & 1.25 & 1.34 \\
\hline SH1 & 244.8 & 14.23 & 1.21 & 1.39 & 1.58 \\
\hline $\mathrm{SH} 2$ & 262.1 & 11.32 & 1.18 & 1.30 & 1.41 \\
\hline $\mathrm{SH} 3$ & 254.3 & 9.61 & 1.17 & 1.28 & 1.39 \\
\hline SH4 & 234.2 & 10.59 & 1.14 & 1.23 & 1.32 \\
\hline SH5 & 233.5 & 9.34 & 1.14 & 1.25 & 1.34 \\
\hline SH6 & 253.9 & 9.46 & 1.17 & 1.28 & 1.38 \\
\hline ML1 & 304.9 & 11.49 & 1.15 & 1.26 & 1.41 \\
\hline ML2 & 209.1 & 17.66 & 1.26 & 1.34 & 1.42 \\
\hline ML3 & 208.7 & 14.95 & 1.26 & 1.36 & 1.45 \\
\hline ML4 & 255.2 & 13.60 & 1.20 & 1.31 & 1.40 \\
\hline ML5 & 259.3 & 12.96 & 1.19 & 1.30 & 1.40 \\
\hline ML6 & 207.3 & 15.35 & 1.26 & 1.36 & 1.44 \\
\hline MH1 & 457.8 & 13.83 & 1.25 & 1.36 & 1.45 \\
\hline MH2 & 461.3 & 12.15 & 1.24 & 1.36 & 1.47 \\
\hline MH3 & 311.8 & 16.82 & 1.18 & 1.29 & 1.38 \\
\hline MH4 & 449.6 & 14.78 & 1.20 & 1.30 & 1.39 \\
\hline MH5 & 455.8 & 12.37 & 1.23 & 1.35 & 1.45 \\
\hline MH6 & 623.8 & 6.62 & 1.35 & 1.73 & 1.96 \\
\hline LL1 & 860.2 & 14.70 & 1.24 & 1.43 & 1.73 \\
\hline LL2 & 856.1 & 14.71 & 1.25 & 1.45 & 1.71 \\
\hline LL3 & 759.8 & 10.60 & 1.21 & 1.34 & 1.48 \\
\hline LL4 & 778.9 & 14.25 & 1.21 & 1.44 & 1.53 \\
\hline LL5 & 789.1 & 13.91 & 1.21 & 1.46 & 1.55 \\
\hline LL6 & 663.0 & 15.80 & 1.23 & 1.37 & 1.52 \\
\hline LH1 & 1584.7 & 9.02 & 1.25 & 1.42 & 1.57 \\
\hline LH2 & 1537.3 & 7.41 & 1.33 & 1.52 & 1.68 \\
\hline LH3 & 1278.1 & 12.58 & 1.23 & 1.38 & 1.52 \\
\hline LH4 & 1505.2 & 7.93 & 1.30 & 1.50 & 1.65 \\
\hline LH5 & 1489.8 & 7.90 & 1.30 & 1.51 & 1.66 \\
\hline LH6 & 1334.3 & 12.83 & 1.24 & 1.41 & 1.53 \\
\hline
\end{tabular}


Table B.5 - The response of the buildings subjected to M6C38 ground motion parallel to the short side of the building with $\alpha=0.02$

\begin{tabular}{|c|c|c|c|c|c|}
\hline \multirow{2}{*}{ Building } & \multirow{2}{*}{$V e$} & \multirow{2}{*}{$\begin{array}{c}\text { Ductility }(\mu) \\
R y=3\end{array}$} & \multicolumn{3}{|c|}{ Force Reduction Factor $(R y)$} \\
\hline & & & $\mu=2$ & $\mu=3$ & $\mu=4$ \\
\hline SL1 & 137.6 & 19.99 & 1.13 & 1.23 & 1.32 \\
\hline SL3 & 135.6 & 20.16 & 1.13 & 1.22 & 1.31 \\
\hline SL4 & 129.8 & 21.39 & 1.13 & 1.21 & 1.30 \\
\hline SH1 & 183.8 & 20.72 & 1.21 & 1.35 & 1.46 \\
\hline $\mathrm{SH} 2$ & 171.9 & 21.88 & 1.21 & 1.34 & 1.45 \\
\hline $\mathrm{SH} 3$ & 167.5 & 20.42 & 1.22 & 1.35 & 1.47 \\
\hline SH4 & 160.4 & 23.13 & 1.20 & 1.33 & 1.43 \\
\hline SH5 & 161.0 & 21.15 & 1.21 & 1.34 & 1.45 \\
\hline SH6 & 167.2 & 20.46 & 1.22 & 1.36 & 1.47 \\
\hline ML1 & 289.4 & 18.74 & 1.24 & 1.36 & 1.47 \\
\hline ML2 & 278.4 & 17.51 & 1.28 & 1.40 & 1.51 \\
\hline ML3 & 293.1 & 14.12 & 1.36 & 1.51 & 1.64 \\
\hline ML4 & 299.9 & 16.84 & 1.24 & 1.43 & 1.60 \\
\hline ML5 & 300.2 & 16.92 & 1.24 & 1.40 & 1.59 \\
\hline ML6 & 287.3 & 14.76 & 1.33 & 1.47 & 1.59 \\
\hline MH1 & 664.8 & 12.07 & 1.29 & 1.57 & 1.70 \\
\hline MH2 & 696.0 & 9.20 & 1.20 & 1.69 & 1.87 \\
\hline MH3 & 643.9 & 6.30 & 1.22 & 1.44 & 2.05 \\
\hline MH4 & 710.4 & 10.42 & 1.19 & 1.43 & 1.83 \\
\hline MH5 & 721.2 & 8.60 & 1.21 & 1.76 & 1.94 \\
\hline MH6 & 685.6 & 10.30 & 1.34 & 1.58 & 1.81 \\
\hline LL1 & 1158.1 & 11.18 & 1.37 & 1.57 & 1.70 \\
\hline LL2 & 1160.5 & 11.01 & 1.34 & 1.57 & 1.71 \\
\hline LL3 & 1296.9 & 7.32 & 1.24 & 1.80 & 2.01 \\
\hline LL4 & 1171.1 & 11.25 & 1.39 & 1.55 & 1.70 \\
\hline LL5 & 1164.4 & 11.43 & 1.38 & 1.54 & 1.68 \\
\hline LL6 & 920.3 & 7.58 & 1.46 & 1.77 & 2.30 \\
\hline LH1 & 2779.8 & 6.52 & 1.27 & 1.84 & 2.05 \\
\hline LH2 & 2604.5 & 5.65 & 1.54 & 1.82 & 2.05 \\
\hline LH3 & 2060.8 & 4.96 & 1.59 & 1.85 & 2.30 \\
\hline LH4 & 2742.0 & 5.53 & 1.40 & 1.90 & 2.14 \\
\hline LH5 & 2751.6 & 5.38 & 1.45 & 1.93 & 2.18 \\
\hline LH6 & 2027.0 & 5.29 & 1.47 & 1.92 & 2.68 \\
\hline
\end{tabular}


Table B.6 - The response of the buildings subjected to M6C1 ground motion parallel to the long side of the building with $\alpha=0.02$

\begin{tabular}{|c|c|c|c|c|c|}
\hline \multirow{2}{*}{ Building } & \multirow{2}{*}{$V e$} & \multirow{2}{*}{$\begin{array}{c}\text { Ductility }(\mu) \\
R y=3\end{array}$} & \multicolumn{3}{|c|}{ Force Reduction Factor $(R y)$} \\
\hline & & & $\mu=2$ & $\mu=3$ & $\mu=4$ \\
\hline SL1 & 206.3 & 5.18 & 1.33 & 1.53 & 1.80 \\
\hline SL3 & 199.9 & 4.95 & 1.31 & 1.51 & 1.75 \\
\hline SL4 & 178.7 & 6.09 & 1.26 & 1.42 & 1.56 \\
\hline SH1 & 420.5 & 3.99 & 1.42 & 2.48 & 2.97 \\
\hline $\mathrm{SH} 2$ & 382.9 & 4.56 & 1.36 & 2.31 & 2.76 \\
\hline $\mathrm{SH} 3$ & 335.6 & 4.91 & 1.61 & 2.06 & 2.46 \\
\hline SH4 & 335.0 & 5.42 & 1.53 & 2.01 & 2.33 \\
\hline SH5 & 314.5 & 5.54 & 1.47 & 1.95 & 2.30 \\
\hline ML1 & 620.3 & 5.62 & 1.47 & 2.29 & 2.58 \\
\hline ML2 & 599.4 & 6.74 & 1.39 & 2.10 & 2.36 \\
\hline ML3 & 582.6 & 7.23 & 1.33 & 1.99 & 2.25 \\
\hline ML4 & 609.2 & 6.34 & 1.43 & 2.17 & 2.42 \\
\hline ML5 & 586.2 & 7.21 & 1.35 & 2.03 & 2.26 \\
\hline ML6 & 579.7 & 7.03 & 1.37 & 2.00 & 2.26 \\
\hline MH1 & 1307.9 & 6.98 & 1.32 & 1.99 & 2.25 \\
\hline MH2 & 1306.9 & 5.64 & 1.37 & 2.20 & 2.50 \\
\hline MH3 & 1093.3 & 5.15 & 1.50 & 2.31 & 2.65 \\
\hline MH4 & 1298.1 & 7.15 & 1.31 & 1.97 & 2.24 \\
\hline MH5 & 1408.3 & 4.65 & 1.49 & 2.42 & 2.78 \\
\hline MH6 & 1288.8 & 5.78 & 1.35 & 2.21 & 2.57 \\
\hline LL1 & 2417.2 & 5.74 & 1.77 & 2.12 & 2.45 \\
\hline LL2 & 2376.2 & 5.97 & 1.56 & 2.10 & 2.42 \\
\hline LL3 & 2129.1 & 4.58 & 2.05 & 2.45 & 2.80 \\
\hline LL4 & 2414.6 & 5.79 & 1.78 & 2.13 & 2.45 \\
\hline LL5 & 2312.8 & 6.05 & 1.34 & 2.13 & 2.41 \\
\hline LL6 & 2063.5 & 3.52 & 1.63 & 2.79 & 3.18 \\
\hline LH1 & 6161.3 & 3.36 & 1.73 & 2.87 & 3.22 \\
\hline LH2 & 5375.9 & 3.01 & 2.41 & 2.99 & 3.51 \\
\hline LH3 & 4506.9 & 3.09 & 1.72 & 2.96 & 3.46 \\
\hline LH4 & 5840.1 & 3.24 & 2.42 & 2.87 & 3.33 \\
\hline LH5 & 4548.3 & 3.56 & 2.22 & 2.74 & 3.21 \\
\hline LH6 & 4230.9 & 3.42 & 1.62 & 2.82 & 3.25 \\
\hline
\end{tabular}


Table B.7 - The response of the buildings subjected to M6C2 ground motion parallel to the long side of the building with $\alpha=0.02$

\begin{tabular}{|c|c|c|c|c|c|}
\hline \multirow{2}{*}{ Building } & \multirow{2}{*}{$V e$} & \multirow{2}{*}{$\begin{array}{c}\text { Ductility }(\mu) \\
R y=3\end{array}$} & \multicolumn{3}{|c|}{ Force Reduction Factor $(R y)$} \\
\hline & & & $\mu=2$ & $\mu=3$ & $\mu=4$ \\
\hline SL1 & 175.0 & 8.47 & 1.56 & 1.82 & 2.02 \\
\hline SL3 & 172.5 & 8.76 & 1.58 & 1.83 & 2.03 \\
\hline SL4 & 170.8 & 8.84 & 1.54 & 1.80 & 2.06 \\
\hline SH1 & 463.6 & 5.21 & 1.59 & 2.13 & 2.51 \\
\hline $\mathrm{SH} 2$ & 429.2 & 4.87 & 1.60 & 1.84 & 2.23 \\
\hline $\mathrm{SH} 3$ & 356.6 & 5.34 & 1.58 & 2.00 & 2.32 \\
\hline SH4 & 357.3 & 5.56 & 1.52 & 1.88 & 2.18 \\
\hline SH5 & 304.8 & 6.41 & 1.56 & 1.96 & 2.15 \\
\hline ML1 & 594.8 & 7.23 & 1.33 & 1.56 & 1.95 \\
\hline ML2 & 574.6 & 9.19 & 1.30 & 1.50 & 1.78 \\
\hline ML3 & 528.3 & 9.53 & 1.32 & 1.54 & 1.78 \\
\hline ML4 & 583.8 & 8.54 & 1.31 & 1.52 & 1.82 \\
\hline ML5 & 562.7 & 9.62 & 1.29 & 1.48 & 1.75 \\
\hline ML6 & 544.5 & 9.20 & 1.32 & 1.55 & 1.82 \\
\hline MH1 & 904.5 & 9.23 & 1.28 & 1.46 & 1.68 \\
\hline MH2 & 936.1 & 5.11 & 1.73 & 2.25 & 2.68 \\
\hline MH3 & 912.7 & 3.26 & 1.70 & 1.98 & 3.44 \\
\hline MH4 & 916.6 & 8.28 & 1.32 & 1.53 & 1.82 \\
\hline MH5 & 949.0 & 4.63 & 2.10 & 2.45 & 2.76 \\
\hline MH6 & 941.5 & 4.89 & 1.44 & 2.05 & 2.54 \\
\hline LL1 & 1684.8 & 7.21 & 1.36 & 1.60 & 1.84 \\
\hline LL2 & 1695.3 & 6.52 & 1.38 & 1.71 & 2.03 \\
\hline LL3 & 1695.5 & 3.40 & 1.64 & 1.94 & 3.26 \\
\hline LL4 & 1686.9 & 7.07 & 1.36 & 1.60 & 1.85 \\
\hline LL5 & 1684.6 & 5.31 & 1.61 & 2.19 & 2.46 \\
\hline LL6 & 1147.2 & 7.43 & 1.41 & 1.63 & 2.23 \\
\hline LH1 & 3848.9 & 3.79 & 1.87 & 2.57 & 3.08 \\
\hline LH2 & 3828.8 & 2.99 & 2.36 & 3.01 & 3.50 \\
\hline LH3 & 2290.5 & 6.69 & 1.56 & 1.99 & 2.16 \\
\hline LH4 & 3827.8 & 3.33 & 2.19 & 2.78 & 3.32 \\
\hline LH5 & 3575.9 & 3.45 & 1.77 & 2.53 & 3.59 \\
\hline LH6 & 1987.8 & 7.12 & 1.52 & 1.69 & 1.85 \\
\hline
\end{tabular}


Table B. 8 - The response of the buildings subjected to M6C26 ground motion parallel to the long side of the building with $\alpha=0.02$

\begin{tabular}{|c|c|c|c|c|c|}
\hline \multirow{2}{*}{ Building } & \multirow{2}{*}{$V e$} & \multirow{2}{*}{$\begin{array}{c}\text { Ductility }(\mu) \\
R y=3\end{array}$} & \multicolumn{3}{|c|}{ Force Reduction Factor $(R y)$} \\
\hline & & & $\mu=2$ & $\mu=3$ & $\mu=4$ \\
\hline SL1 & 197.6 & 8.98 & 1.52 & 1.80 & 2.05 \\
\hline SL3 & 203.5 & 8.57 & 1.53 & 1.80 & 2.05 \\
\hline SL4 & 214.0 & 8.33 & 1.54 & 1.82 & 2.06 \\
\hline SH1 & 487.0 & 6.17 & 1.75 & 2.09 & 2.37 \\
\hline $\mathrm{SH} 2$ & 483.5 & 6.48 & 1.74 & 2.05 & 2.35 \\
\hline $\mathrm{SH} 3$ & 470.9 & 5.77 & 1.32 & 2.15 & 2.46 \\
\hline SH4 & 465.0 & 6.51 & 1.28 & 2.08 & 2.34 \\
\hline SH5 & 455.8 & 5.75 & 1.30 & 2.19 & 2.49 \\
\hline ML1 & 582.9 & 6.56 & 1.28 & 1.47 & 1.66 \\
\hline ML2 & 534.2 & 7.75 & 1.25 & 1.43 & 1.59 \\
\hline ML3 & 529.2 & 7.00 & 1.28 & 1.49 & 1.67 \\
\hline ML4 & 544.3 & 7.54 & 1.25 & 1.44 & 1.61 \\
\hline ML5 & 526.8 & 7.96 & 1.25 & 1.42 & 1.59 \\
\hline ML6 & 527.3 & 6.76 & 1.28 & 1.48 & 1.67 \\
\hline MH1 & 1263.7 & 5.37 & 1.31 & 1.54 & 1.74 \\
\hline MH2 & 1391.8 & 3.85 & 1.39 & 1.68 & 3.05 \\
\hline MH3 & 1271.5 & 4.98 & 1.36 & 1.62 & 2.71 \\
\hline MH4 & 1282.9 & 5.17 & 1.31 & 1.53 & 1.78 \\
\hline MH5 & 1417.4 & 4.04 & 1.39 & 1.78 & 2.97 \\
\hline MH6 & 1429.6 & 3.27 & 1.45 & 1.98 & 3.35 \\
\hline LL1 & 2377.5 & 4.03 & 1.38 & 1.64 & 2.37 \\
\hline LL2 & 2417.6 & 3.87 & 1.38 & 1.62 & 3.24 \\
\hline LL3 & 2410.1 & 4.58 & 1.39 & 2.45 & 2.79 \\
\hline LL4 & 2380.9 & 4.06 & 1.38 & 1.64 & 2.28 \\
\hline LL5 & 2503.4 & 3.93 & 1.38 & 1.63 & 3.03 \\
\hline LL6 & 1924.6 & 4.15 & 1.48 & 1.79 & 2.94 \\
\hline LH1 & 6150.0 & 2.99 & 1.57 & 3.01 & 3.55 \\
\hline LH2 & 5825.5 & 3.31 & 1.55 & 2.83 & 3.35 \\
\hline LH3 & 3856.6 & 3.31 & 1.60 & 2.85 & 3.30 \\
\hline LH4 & 6170.0 & 2.95 & 1.61 & 3.02 & 3.60 \\
\hline LH5 & 5117.4 & 3.82 & 1.49 & 2.65 & 3.09 \\
\hline LH6 & 3349.9 & 3.42 & 1.64 & 2.02 & 3.75 \\
\hline
\end{tabular}


Table B.9 - The response of the buildings subjected to M6C31 ground motion parallel to the long side of the building with $\alpha=0.02$

\begin{tabular}{|c|c|c|c|c|c|}
\hline \multirow{2}{*}{ Building } & \multirow{2}{*}{$V e$} & \multirow{2}{*}{$\begin{array}{c}\text { Ductility }(\mu) \\
R y=3\end{array}$} & \multicolumn{3}{|c|}{ Force Reduction Factor $(R y)$} \\
\hline & & & $\mu=2$ & $\mu=3$ & $\mu=4$ \\
\hline SL1 & 184.8 & 6.34 & 1.33 & 2.25 & 2.51 \\
\hline SL3 & 180.1 & 6.92 & 1.33 & 2.31 & 2.48 \\
\hline SL4 & 178.0 & 6.81 & 1.32 & 1.53 & 1.83 \\
\hline SH1 & 446.1 & 4.94 & 1.30 & 1.51 & 2.73 \\
\hline $\mathrm{SH} 2$ & 416.2 & 4.93 & 1.29 & 1.49 & 2.66 \\
\hline $\mathrm{SH} 3$ & 405.2 & 3.92 & 1.36 & 1.58 & 2.94 \\
\hline SH4 & 396.4 & 4.48 & 1.31 & 1.51 & 1.69 \\
\hline SH5 & 397.6 & 4.13 & 1.33 & 1.53 & 2.85 \\
\hline ML1 & 939.1 & 3.50 & 1.28 & 2.65 & 3.26 \\
\hline ML2 & 850.7 & 4.30 & 1.30 & 2.38 & 2.85 \\
\hline ML3 & 639.3 & 5.09 & 1.34 & 1.66 & 2.42 \\
\hline ML4 & 882.0 & 4.06 & 1.29 & 2.45 & 2.96 \\
\hline ML5 & 813.0 & 4.56 & 1.30 & 2.32 & 2.75 \\
\hline ML6 & 702.6 & 4.62 & 1.35 & 2.24 & 2.62 \\
\hline MH1 & 1026.3 & 5.04 & 1.35 & 1.76 & 2.41 \\
\hline MH2 & 829.2 & 8.30 & 1.36 & 1.92 & 2.07 \\
\hline MH3 & 1091.5 & 3.72 & 1.30 & 2.40 & 3.12 \\
\hline MH4 & 957.3 & 6.07 & 1.41 & 1.82 & 1.96 \\
\hline MH5 & 882.3 & 9.05 & 1.64 & 1.82 & 1.99 \\
\hline MH6 & 805.3 & 6.28 & 1.35 & 1.91 & 2.06 \\
\hline LL1 & 1747.3 & 4.67 & 1.47 & 1.88 & 2.49 \\
\hline LL2 & 1560.6 & 6.08 & 1.50 & 1.67 & 2.15 \\
\hline LL3 & 2143.2 & 4.21 & 1.35 & 1.78 & 2.85 \\
\hline LL4 & 1732.6 & 4.73 & 1.47 & 1.86 & 2.42 \\
\hline LL5 & 1474.9 & 8.25 & 1.34 & 1.91 & 2.05 \\
\hline LL6 & 1249.6 & 3.87 & 1.48 & 2.35 & 3.06 \\
\hline LH1 & 3731.5 & 5.17 & 1.58 & 1.85 & 2.11 \\
\hline LH2 & 4929.3 & 4.11 & 1.46 & 2.05 & 2.86 \\
\hline LH3 & 2466.2 & 3.58 & 2.08 & 2.75 & 3.25 \\
\hline LH4 & 4572.4 & 4.21 & 1.61 & 1.96 & 2.32 \\
\hline LH5 & 4364.4 & 2.37 & 2.02 & 3.47 & 5.31 \\
\hline LH6 & 1981.6 & 4.76 & 1.44 & 1.63 & 1.98 \\
\hline
\end{tabular}


Table B.10 - The response of the buildings subjected to M6C38 ground motion parallel to the long side of the building with $\alpha=0.02$

\begin{tabular}{|c|c|c|c|c|c|}
\hline \multirow{2}{*}{ Building } & \multirow{2}{*}{$V e$} & \multirow{2}{*}{$\begin{array}{c}\text { Ductility }(\mu) \\
R y=3\end{array}$} & \multicolumn{3}{|c|}{ Force Reduction Factor $(R y)$} \\
\hline & & & $\mu=2$ & $\mu=3$ & $\mu=4$ \\
\hline SL1 & 165.6 & 7.52 & 1.37 & 1.58 & 2.03 \\
\hline SL3 & 157.4 & 8.30 & 1.30 & 1.56 & 1.96 \\
\hline SL4 & 152.5 & 8.75 & 1.28 & 1.53 & 1.92 \\
\hline SH1 & 378.3 & 8.42 & 1.30 & 1.51 & 1.73 \\
\hline $\mathrm{SH} 2$ & 365.7 & 8.58 & 1.27 & 1.47 & 1.67 \\
\hline $\mathrm{SH} 3$ & 360.1 & 8.09 & 1.28 & 1.51 & 1.71 \\
\hline SH4 & 353.9 & 9.12 & 1.26 & 1.45 & 1.62 \\
\hline SH5 & 353.4 & 8.36 & 1.28 & 1.49 & 1.68 \\
\hline ML1 & 567.1 & 9.71 & 1.25 & 1.44 & 1.61 \\
\hline ML2 & 542.7 & 9.63 & 1.23 & 1.42 & 1.58 \\
\hline ML3 & 522.6 & 8.77 & 1.25 & 1.44 & 1.62 \\
\hline ML4 & 549.1 & 9.63 & 1.24 & 1.42 & 1.59 \\
\hline ML5 & 536.1 & 9.95 & 1.23 & 1.41 & 1.57 \\
\hline ML6 & 532.8 & 8.51 & 1.26 & 1.45 & 1.63 \\
\hline MH1 & 1041.2 & 8.52 & 1.29 & 1.51 & 1.71 \\
\hline MH2 & 911.2 & 8.11 & 1.35 & 1.60 & 1.82 \\
\hline MH3 & 697.7 & 10.45 & 1.34 & 1.57 & 1.76 \\
\hline MH4 & 1008.1 & 8.96 & 1.29 & 1.50 & 1.70 \\
\hline MH5 & 864.7 & 8.67 & 1.35 & 1.59 & 1.81 \\
\hline MH6 & 956.5 & 6.56 & 1.40 & 1.70 & 1.97 \\
\hline LL1 & 1918.8 & 6.92 & 1.36 & 1.63 & 1.88 \\
\hline LL2 & 1834.0 & 7.33 & 1.36 & 1.62 & 1.85 \\
\hline LL3 & 1310.3 & 9.55 & 1.35 & 1.60 & 1.82 \\
\hline LL4 & 1911.3 & 6.99 & 1.36 & 1.63 & 1.88 \\
\hline LL5 & 1656.1 & 8.36 & 1.34 & 1.58 & 1.79 \\
\hline LL6 & 1213.1 & 8.88 & 1.35 & 1.57 & 1.79 \\
\hline LH1 & 3545.2 & 6.00 & 1.46 & 1.80 & 2.10 \\
\hline LH2 & 3127.7 & 6.27 & 1.47 & 1.81 & 2.04 \\
\hline LH3 & 2731.4 & 6.71 & 1.50 & 1.82 & 2.10 \\
\hline LH4 & 3327.5 & 5.92 & 1.50 & 1.88 & 2.16 \\
\hline LH5 & 2828.2 & 7.20 & 1.43 & 1.68 & 1.88 \\
\hline LH6 & 2547.9 & 6.89 & 1.43 & 1.71 & 1.97 \\
\hline
\end{tabular}


Table B.11 - The response of the buildings subjected to E6C1 ground motion parallel to the short side of the building with $\alpha=0.02$

\begin{tabular}{|c|c|c|c|c|c|}
\hline \multirow{2}{*}{ Building } & \multirow{2}{*}{$V e$} & \multirow{2}{*}{$\frac{\text { Ductility }(\mu)}{R y=3}$} & \multicolumn{3}{|c|}{ Force Reduction Factor $(R y)$} \\
\hline & & & $\mu=2$ & $\mu=3$ & $\mu=4$ \\
\hline SL1 & 93.7 & 12.46 & 1.24 & 1.39 & 1.52 \\
\hline SL3 & 93.7 & 12.53 & 1.24 & 1.39 & 1.51 \\
\hline SL4 & 92.1 & 12.76 & 1.21 & 1.35 & 1.48 \\
\hline SH1 & 150.7 & 12.54 & 1.25 & 1.36 & 1.44 \\
\hline $\mathrm{SH} 2$ & 140.3 & 13.38 & 1.20 & 1.29 & 1.38 \\
\hline $\mathrm{SH} 3$ & 135.0 & 11.39 & 1.17 & 1.27 & 1.37 \\
\hline SH4 & 130.1 & 13.42 & 1.14 & 1.24 & 1.32 \\
\hline SH5 & 130.7 & 11.46 & 1.15 & 1.25 & 1.35 \\
\hline SH6 & 134.7 & 11.44 & 1.17 & 1.27 & 1.37 \\
\hline ML1 & 238.4 & 9.02 & 1.22 & 1.35 & 1.46 \\
\hline ML2 & 217.1 & 8.57 & 1.22 & 1.36 & 1.50 \\
\hline ML3 & 199.2 & 8.63 & 1.22 & 1.36 & 1.60 \\
\hline ML4 & 233.8 & 8.05 & 1.21 & 1.35 & 1.48 \\
\hline ML5 & 235.3 & 8.04 & 1.22 & 1.35 & 1.48 \\
\hline ML6 & 210.3 & 7.98 & 1.23 & 1.38 & 1.60 \\
\hline MH1 & 412.6 & 8.08 & 1.22 & 1.38 & 1.80 \\
\hline MH2 & 359.1 & 8.08 & 1.24 & 1.49 & 2.22 \\
\hline MH3 & 197.8 & 12.16 & 1.18 & 1.44 & 1.88 \\
\hline MH4 & 336.7 & 10.08 & 1.22 & 1.37 & 1.77 \\
\hline MH5 & 331.6 & 8.65 & 1.25 & 1.50 & 2.16 \\
\hline MH6 & 515.7 & 5.26 & 1.29 & 1.49 & 1.77 \\
\hline LL1 & 685.3 & 8.28 & 1.23 & 1.37 & 1.62 \\
\hline LL2 & 684.1 & 8.17 & 1.24 & 1.39 & 1.69 \\
\hline LL3 & 531.7 & 7.80 & 1.30 & 2.01 & 2.24 \\
\hline LL4 & 778.9 & 7.00 & 1.27 & 1.43 & 1.76 \\
\hline LL5 & 780.5 & 7.02 & 1.26 & 1.43 & 1.72 \\
\hline LL6 & 302.5 & 6.99 & 1.24 & 1.45 & 1.94 \\
\hline LH1 & 1111.2 & 7.29 & 1.46 & 2.06 & 2.31 \\
\hline LH2 & 1053.8 & 6.90 & 1.78 & 2.10 & 2.35 \\
\hline LH3 & 653.2 & 7.77 & 1.34 & 1.98 & 2.20 \\
\hline LH4 & 1020.8 & 6.96 & 1.76 & 2.06 & 2.32 \\
\hline LH5 & 998.5 & 7.12 & 1.75 & 2.05 & 2.31 \\
\hline LH6 & 641.5 & 7.13 & 1.36 & 2.09 & 2.32 \\
\hline
\end{tabular}


Table B.12 - The response of the buildings subjected to E6C13 ground motion parallel to the short side of the building with $\alpha=0.02$

\begin{tabular}{|c|c|c|c|c|c|}
\hline \multirow{2}{*}{ Building } & \multirow{2}{*}{$V e$} & \multirow{2}{*}{$\begin{array}{c}\text { Ductility }(\mu) \\
R y=3\end{array}$} & \multicolumn{3}{|c|}{ Force Reduction Factor $(R y)$} \\
\hline & & & $\mu=2$ & $\mu=3$ & $\mu=4$ \\
\hline SL1 & 96.0 & 9.19 & 1.34 & 1.51 & 1.66 \\
\hline SL3 & 90.5 & 9.98 & 1.32 & 1.49 & 1.63 \\
\hline SL4 & 74.2 & 11.66 & 1.27 & 1.41 & 1.53 \\
\hline SH1 & 89.1 & 10.92 & 1.42 & 1.64 & 1.86 \\
\hline $\mathrm{SH} 2$ & 90.2 & 12.03 & 1.34 & 1.53 & 1.70 \\
\hline $\mathrm{SH} 3$ & 89.9 & 11.45 & 1.31 & 1.51 & 1.67 \\
\hline SH4 & 91.4 & 12.95 & 1.29 & 1.45 & 1.61 \\
\hline SH5 & 91.7 & 11.89 & 1.30 & 1.48 & 1.65 \\
\hline SH6 & 89.8 & 11.47 & 1.31 & 1.51 & 1.67 \\
\hline ML1 & 163.0 & 9.42 & 1.25 & 1.43 & 1.58 \\
\hline ML2 & 142.5 & 8.61 & 1.23 & 1.38 & 1.60 \\
\hline ML3 & 137.5 & 8.55 & 1.25 & 1.71 & 2.00 \\
\hline ML4 & 153.1 & 8.31 & 1.23 & 1.37 & 1.50 \\
\hline ML5 & 153.6 & 8.49 & 1.23 & 1.37 & 1.50 \\
\hline ML6 & 140.8 & 7.96 & 1.25 & 1.46 & 1.94 \\
\hline MH1 & 307.7 & 7.68 & 1.35 & 1.58 & 1.73 \\
\hline MH2 & 334.2 & 5.46 & 1.23 & 1.62 & 2.57 \\
\hline MH3 & 239.4 & 7.70 & 1.18 & 1.80 & 2.24 \\
\hline MH4 & 342.0 & 6.45 & 1.19 & 1.86 & 2.22 \\
\hline MH5 & 351.3 & 5.44 & 1.22 & 1.93 & 2.35 \\
\hline MH6 & 361.9 & 6.13 & 1.37 & 1.65 & 1.88 \\
\hline LL1 & 619.8 & 5.32 & 1.24 & 2.14 & 2.56 \\
\hline LL2 & 633.1 & 5.21 & 1.25 & 2.19 & 2.64 \\
\hline LL3 & 627.5 & 4.76 & 1.44 & 1.67 & 2.00 \\
\hline LL4 & 559.7 & 5.91 & 1.33 & 1.78 & 2.49 \\
\hline LL5 & 565.5 & 5.86 & 1.31 & 1.68 & 2.50 \\
\hline LL6 & 300.7 & 3.60 & 2.42 & 2.76 & 3.21 \\
\hline LH1 & 1319.4 & 4.53 & 1.38 & 1.75 & 2.80 \\
\hline LH2 & 1206.6 & 4.88 & 1.33 & 2.24 & 2.69 \\
\hline LH3 & 823.6 & 6.43 & 1.37 & 2.09 & 2.38 \\
\hline LH4 & 1180.1 & 5.02 & 1.31 & 2.29 & 2.68 \\
\hline LH5 & 1153.7 & 5.16 & 1.29 & 2.26 & 2.63 \\
\hline LH6 & 772.2 & 6.88 & 1.33 & 2.07 & 2.33 \\
\hline
\end{tabular}


Table B.13 - The response of the buildings subjected to E6C15 ground motion parallel to the short side of the building with $\alpha=0.02$

\begin{tabular}{|c|c|c|c|c|c|}
\hline \multirow{2}{*}{ Building } & \multirow{2}{*}{$V e$} & \multirow{2}{*}{$\frac{\text { Ductility }(\mu)}{R y=3}$} & \multicolumn{3}{|c|}{ Force Reduction Factor $(R y)$} \\
\hline & & & $\mu=2$ & $\mu=3$ & $\mu=4$ \\
\hline SL1 & 71.8 & 11.91 & 1.38 & 1.72 & 1.92 \\
\hline SL3 & 71.9 & 11.37 & 1.39 & 1.74 & 1.95 \\
\hline SL4 & 71.4 & 10.44 & 1.42 & 1.73 & 1.93 \\
\hline SH1 & 168.7 & 7.76 & 1.25 & 1.41 & 1.82 \\
\hline $\mathrm{SH} 2$ & 136.4 & 9.97 & 1.21 & 1.44 & 1.62 \\
\hline SH3 & 120.4 & 10.41 & 1.19 & 1.42 & 1.56 \\
\hline SH4 & 104.7 & 12.95 & 1.18 & 1.28 & 1.40 \\
\hline SH5 & 103.7 & 11.38 & 1.19 & 1.30 & 1.44 \\
\hline SH6 & 120.1 & 10.25 & 1.19 & 1.42 & 1.57 \\
\hline ML1 & 147.6 & 14.83 & 1.32 & 1.42 & 1.51 \\
\hline ML2 & 120.6 & 16.11 & 1.17 & 1.37 & 1.45 \\
\hline ML3 & 123.2 & 13.35 & 1.28 & 1.37 & 1.45 \\
\hline ML4 & 131.5 & 16.70 & 1.29 & 1.40 & 1.50 \\
\hline ML5 & 132.0 & 16.90 & 1.28 & 1.39 & 1.49 \\
\hline ML6 & 120.5 & 13.51 & 1.17 & 1.37 & 1.44 \\
\hline MH1 & 283.6 & 10.26 & 1.31 & 1.41 & 1.51 \\
\hline MH2 & 286.3 & 8.78 & 1.30 & 1.45 & 1.59 \\
\hline MH3 & 163.6 & 10.85 & 1.30 & 1.83 & 1.97 \\
\hline MH4 & 276.6 & 10.22 & 1.28 & 1.42 & 1.53 \\
\hline MH5 & 270.9 & 9.15 & 1.29 & 1.45 & 1.60 \\
\hline MH6 & 319.9 & 11.14 & 1.46 & 1.65 & 1.83 \\
\hline LL1 & 644.0 & 6.55 & 1.41 & 1.63 & 1.90 \\
\hline LL2 & 640.2 & 6.67 & 1.39 & 1.59 & 1.86 \\
\hline LL3 & 410.6 & 7.44 & 1.28 & 1.86 & 2.01 \\
\hline LL4 & 636.8 & 6.60 & 1.45 & 1.65 & 1.90 \\
\hline LL5 & 638.3 & 6.64 & 1.46 & 1.66 & 1.93 \\
\hline LL6 & 271.3 & 12.01 & 1.33 & 1.55 & 1.72 \\
\hline LH1 & 812.3 & 7.77 & 1.30 & 1.76 & 1.95 \\
\hline LH2 & 701.6 & 8.48 & 1.39 & 1.64 & 1.94 \\
\hline LH3 & 660.9 & 7.52 & 1.59 & 1.87 & 2.11 \\
\hline LH4 & 695.3 & 8.38 & 1.42 & 1.64 & 1.99 \\
\hline LH5 & 667.2 & 8.76 & 1.38 & 1.62 & 1.93 \\
\hline LH6 & 621.0 & 8.54 & 1.46 & 1.72 & 1.94 \\
\hline
\end{tabular}


Table B.14 - The response of the buildings subjected to E6C18 ground motion parallel to the short side of the building with $\alpha=0.02$

\begin{tabular}{|c|c|c|c|c|c|}
\hline \multirow{2}{*}{ Building } & \multirow{2}{*}{$V e$} & \multirow{2}{*}{$\frac{\text { Ductility }(\mu)}{R y=3}$} & \multicolumn{3}{|c|}{ Force Reduction Factor $(R y)$} \\
\hline & & & $\mu=2$ & $\mu=3$ & $\mu=4$ \\
\hline SL1 & 90.4 & 9.07 & 1.29 & 1.43 & 1.61 \\
\hline SL3 & 82.3 & 11.80 & 1.29 & 1.42 & 1.58 \\
\hline SL4 & 64.4 & 19.42 & 1.34 & 1.46 & 1.59 \\
\hline SH1 & 124.1 & 9.90 & 1.20 & 1.34 & 1.46 \\
\hline $\mathrm{SH} 2$ & 125.3 & 7.69 & 1.22 & 1.37 & 1.51 \\
\hline $\mathrm{SH} 3$ & 125.2 & 6.34 & 1.25 & 1.42 & 1.57 \\
\hline SH4 & 122.3 & 7.08 & 1.25 & 1.41 & 1.54 \\
\hline SH5 & 122.3 & 6.54 & 1.26 & 1.43 & 1.58 \\
\hline SH6 & 125.1 & 6.36 & 1.25 & 1.41 & 1.56 \\
\hline ML1 & 197.8 & 7.68 & 1.25 & 1.42 & 1.66 \\
\hline ML2 & 152.6 & 11.32 & 1.23 & 1.68 & 1.85 \\
\hline ML3 & 137.6 & 10.88 & 1.47 & 1.66 & 1.84 \\
\hline ML4 & 179.1 & 9.38 & 1.23 & 1.41 & 2.09 \\
\hline ML5 & 180.1 & 9.17 & 1.23 & 1.41 & 2.10 \\
\hline ML6 & 146.0 & 10.39 & 1.49 & 1.69 & 1.88 \\
\hline MH1 & 280.1 & 10.08 & 1.47 & 1.68 & 1.86 \\
\hline MH2 & 249.6 & 8.55 & 1.57 & 1.81 & 2.04 \\
\hline MH3 & 202.5 & 5.27 & 1.65 & 2.00 & 2.46 \\
\hline MH4 & 242.8 & 9.98 & 1.27 & 1.78 & 1.97 \\
\hline MH5 & 237.4 & 8.38 & 1.60 & 1.85 & 2.07 \\
\hline MH6 & 419.6 & 4.89 & 1.36 & 2.36 & 2.71 \\
\hline LL1 & 507.2 & 8.33 & 1.59 & 1.85 & 2.09 \\
\hline LL2 & 499.4 & 8.47 & 1.58 & 1.82 & 2.06 \\
\hline LL3 & 365.8 & 7.28 & 1.65 & 1.93 & 2.19 \\
\hline LL4 & 573.8 & 7.68 & 1.65 & 1.92 & 2.16 \\
\hline LL5 & 579.6 & 7.66 & 1.67 & 1.94 & 2.19 \\
\hline LL6 & 200.3 & 7.39 & 1.32 & 1.49 & 1.76 \\
\hline LH1 & 751.5 & 6.73 & 1.67 & 1.98 & 2.26 \\
\hline LH2 & 701.8 & 6.44 & 1.65 & 2.01 & 2.29 \\
\hline LH3 & 680.8 & 3.61 & 2.13 & 2.69 & 3.22 \\
\hline LH4 & 699.5 & 6.22 & 1.71 & 2.06 & 2.38 \\
\hline LH5 & 693.0 & 6.04 & 1.72 & 2.07 & 2.39 \\
\hline LH6 & 642.6 & 3.87 & 2.12 & 2.62 & 3.06 \\
\hline
\end{tabular}


Table B.15 - The response of the buildings subjected to E6C42 ground motion parallel to the short side of the building with $\alpha=0.02$

\begin{tabular}{|c|c|c|c|c|c|}
\hline \multirow{2}{*}{ Building } & \multirow{2}{*}{$V e$} & \multirow{2}{*}{$\frac{\text { Ductility }(\mu)}{R y=3}$} & \multicolumn{3}{|c|}{ Force Reduction Factor $(R y)$} \\
\hline & & & $\mu=2$ & $\mu=3$ & $\mu=4$ \\
\hline SL1 & 72.6 & 7.29 & 1.25 & 1.40 & 1.55 \\
\hline SL3 & 70.0 & 6.86 & 1.24 & 1.39 & 1.58 \\
\hline SL4 & 62.2 & 8.37 & 1.24 & 1.72 & 2.03 \\
\hline SH1 & 115.8 & 11.74 & 1.25 & 1.37 & 1.46 \\
\hline $\mathrm{SH} 2$ & 106.4 & 13.07 & 1.19 & 1.30 & 1.39 \\
\hline $\mathrm{SH} 3$ & 108.6 & 11.29 & 1.25 & 1.36 & 1.46 \\
\hline SH4 & 109.5 & 13.13 & 1.28 & 1.39 & 1.49 \\
\hline SH5 & 109.7 & 11.43 & 1.29 & 1.41 & 1.53 \\
\hline SH6 & 108.8 & 11.70 & 1.25 & 1.36 & 1.47 \\
\hline ML1 & 192.8 & 10.40 & 1.35 & 1.52 & 1.66 \\
\hline ML2 & 156.3 & 12.01 & 1.29 & 1.46 & 1.58 \\
\hline ML3 & 140.7 & 12.83 & 1.28 & 1.41 & 1.54 \\
\hline ML4 & 182.9 & 10.27 & 1.33 & 1.52 & 1.67 \\
\hline ML5 & 184.1 & 10.31 & 1.33 & 1.52 & 1.68 \\
\hline ML6 & 149.9 & 11.42 & 1.30 & 1.46 & 1.59 \\
\hline MH1 & 323.9 & 12.01 & 1.41 & 1.55 & 1.67 \\
\hline MH2 & 328.4 & 10.89 & 1.29 & 1.67 & 1.80 \\
\hline MH3 & 298.8 & 11.43 & 1.53 & 1.75 & 1.93 \\
\hline MH4 & 314.1 & 14.37 & 1.42 & 1.57 & 1.68 \\
\hline MH5 & 313.1 & 12.23 & 1.45 & 1.61 & 1.74 \\
\hline MH6 & 403.3 & 5.67 & 1.44 & 1.67 & 1.95 \\
\hline LL1 & 731.6 & 7.92 & 1.34 & 1.73 & 2.25 \\
\hline LL2 & 716.9 & 8.20 & 1.33 & 1.69 & 2.22 \\
\hline LL3 & 586.4 & 10.95 & 1.56 & 1.77 & 1.93 \\
\hline LL4 & 664.7 & 8.70 & 1.56 & 1.76 & 1.94 \\
\hline LL5 & 669.3 & 8.50 & 1.57 & 1.77 & 1.95 \\
\hline LL6 & 426.9 & 10.23 & 1.47 & 1.69 & 1.90 \\
\hline LH1 & 1286.9 & 9.49 & 1.61 & 1.84 & 2.03 \\
\hline LH2 & 1265.6 & 8.62 & 1.67 & 1.91 & 2.10 \\
\hline LH3 & 1083.9 & 7.58 & 1.61 & 1.90 & 2.16 \\
\hline LH4 & 1246.3 & 8.80 & 1.60 & 1.86 & 2.07 \\
\hline LH5 & 1234.9 & 8.80 & 1.59 & 1.85 & 2.07 \\
\hline LH6 & 1045.4 & 7.58 & 1.61 & 1.91 & 2.17 \\
\hline
\end{tabular}


Table B.16 - The response of the buildings subjected to E6C1 ground motion parallel to the long side of the building with $\alpha=0.02$

\begin{tabular}{|c|c|c|c|c|c|}
\hline \multirow{2}{*}{ Building } & \multirow{2}{*}{$V e$} & \multirow{2}{*}{$\begin{array}{c}\text { Ductility }(\mu) \\
R y=3\end{array}$} & \multicolumn{3}{|c|}{ Force Reduction Factor $(R y)$} \\
\hline & & & $\mu=2$ & $\mu=3$ & $\mu=4$ \\
\hline SL1 & 90.2 & 10.45 & 1.66 & 2.09 & 2.23 \\
\hline SL3 & 92.6 & 10.48 & 1.76 & 2.08 & 2.25 \\
\hline SL4 & 97.0 & 10.09 & 1.41 & 1.82 & 2.19 \\
\hline SH1 & 197.4 & 8.13 & 1.36 & 1.60 & 1.81 \\
\hline $\mathrm{SH} 2$ & 198.5 & 8.20 & 1.34 & 1.56 & 1.75 \\
\hline $\mathrm{SH} 3$ & 201.3 & 7.63 & 1.35 & 1.58 & 1.80 \\
\hline SH4 & 198.9 & 8.38 & 1.32 & 1.53 & 1.71 \\
\hline SH5 & 201.7 & 7.54 & 1.35 & 1.57 & 1.77 \\
\hline ML1 & 376.8 & 6.77 & 1.37 & 1.62 & 1.83 \\
\hline ML2 & 372.8 & 6.80 & 1.35 & 1.58 & 1.79 \\
\hline ML3 & 362.9 & 6.08 & 1.34 & 1.61 & 1.87 \\
\hline ML4 & 374.4 & 6.63 & 1.35 & 1.59 & 1.80 \\
\hline ML5 & 370.6 & 6.74 & 1.34 & 1.57 & 1.80 \\
\hline ML6 & 365.7 & 6.04 & 1.36 & 1.60 & 1.88 \\
\hline MH1 & 774.8 & 5.88 & 1.40 & 1.67 & 1.92 \\
\hline MH2 & 723.6 & 5.08 & 1.43 & 1.69 & 1.93 \\
\hline MH3 & 558.6 & 4.86 & 1.25 & 1.44 & 1.62 \\
\hline MH4 & 758.2 & 6.07 & 1.39 & 1.65 & 1.89 \\
\hline MH5 & 695.5 & 5.27 & 1.39 & 1.64 & 1.86 \\
\hline MH6 & 752.7 & 4.32 & 1.47 & 1.80 & 2.33 \\
\hline LL1 & 1425.7 & 4.71 & 1.44 & 1.77 & 2.16 \\
\hline LL2 & 1401.2 & 4.91 & 1.44 & 1.76 & 2.12 \\
\hline LL3 & 1063.0 & 4.34 & 1.29 & 1.49 & 1.69 \\
\hline LL4 & 1424.4 & 4.76 & 1.45 & 1.78 & 2.17 \\
\hline LL5 & 1315.8 & 5.12 & 1.42 & 1.68 & 1.94 \\
\hline LL6 & 969.8 & 4.32 & 1.34 & 1.57 & 2.89 \\
\hline LH1 & 2972.3 & 3.39 & 1.52 & 1.92 & 3.97 \\
\hline LH2 & 2758.1 & 2.83 & 1.51 & 2.02 & 3.75 \\
\hline LH3 & 2042.7 & 3.82 & 1.39 & 2.64 & 3.08 \\
\hline LH4 & 2917.1 & 2.84 & 1.57 & 3.44 & 4.03 \\
\hline LH5 & 2367.9 & 3.48 & 1.38 & 1.69 & 3.23 \\
\hline LH6 & 1945.1 & 3.93 & 1.40 & 2.61 & 3.01 \\
\hline
\end{tabular}


Table B.17 - The response of the buildings subjected to E6C13 ground motion parallel to the long side of the building with $\alpha=0.02$

\begin{tabular}{|c|c|c|c|c|c|}
\hline \multirow{2}{*}{ Building } & \multirow{2}{*}{$V e$} & \multirow{2}{*}{$\begin{array}{c}\text { Ductility }(\mu) \\
R y=3\end{array}$} & \multicolumn{3}{|c|}{ Force Reduction Factor $(R y)$} \\
\hline & & & $\mu=2$ & $\mu=3$ & $\mu=4$ \\
\hline SL1 & 138.0 & 3.27 & 1.39 & 2.81 & 3.36 \\
\hline SL3 & 139.9 & 3.09 & 1.39 & 2.86 & 3.38 \\
\hline SL4 & 144.9 & 3.04 & 1.40 & 2.92 & 3.56 \\
\hline SH1 & 268.2 & 3.24 & 1.96 & 2.87 & 3.22 \\
\hline $\mathrm{SH} 2$ & 277.7 & 2.84 & 1.52 & 3.04 & 3.42 \\
\hline $\mathrm{SH} 3$ & 273.0 & 2.78 & 1.56 & 2.42 & 3.60 \\
\hline SH4 & 272.9 & 3.22 & 1.53 & 2.25 & 3.54 \\
\hline SH5 & 264.7 & 3.23 & 1.55 & 2.27 & 3.57 \\
\hline ML1 & 314.0 & 4.76 & 1.40 & 1.65 & 2.15 \\
\hline ML2 & 266.4 & 5.63 & 1.34 & 1.58 & 1.84 \\
\hline ML3 & 216.3 & 6.22 & 1.35 & 1.61 & 1.89 \\
\hline ML4 & 277.1 & 5.38 & 1.35 & 1.59 & 1.90 \\
\hline ML5 & 255.4 & 5.98 & 1.34 & 1.56 & 1.81 \\
\hline ML6 & 230.4 & 5.46 & 1.35 & 1.63 & 1.94 \\
\hline MH1 & 342.3 & 8.56 & 1.40 & 1.63 & 1.84 \\
\hline MH2 & 319.3 & 6.88 & 1.55 & 1.86 & 2.12 \\
\hline MH3 & 355.0 & 7.00 & 1.48 & 1.81 & 2.13 \\
\hline MH4 & 326.2 & 8.81 & 1.41 & 1.63 & 1.84 \\
\hline MH5 & 306.4 & 7.83 & 1.50 & 1.80 & 2.05 \\
\hline MH6 & 324.6 & 5.89 & 1.62 & 1.93 & 2.17 \\
\hline LL1 & 598.0 & 7.55 & 1.41 & 1.67 & 1.92 \\
\hline LL2 & 564.9 & 7.97 & 1.43 & 1.67 & 1.89 \\
\hline LL3 & 607.3 & 6.98 & 1.49 & 1.85 & 2.18 \\
\hline LL4 & 594.5 & 7.65 & 1.41 & 1.67 & 1.91 \\
\hline LL5 & 573.2 & 7.29 & 1.54 & 1.84 & 2.09 \\
\hline LL6 & 656.4 & 5.99 & 1.43 & 1.76 & 2.06 \\
\hline LH1 & 1215.7 & 5.47 & 1.60 & 1.91 & 2.17 \\
\hline LH2 & 1239.1 & 5.05 & 1.54 & 1.94 & 2.31 \\
\hline LH3 & 1392.0 & 5.12 & 1.50 & 1.87 & 2.24 \\
\hline LH4 & 1207.4 & 4.79 & 1.58 & 1.94 & 2.27 \\
\hline LH5 & 1325.5 & 6.00 & 1.53 & 1.91 & 2.27 \\
\hline LH6 & 1355.7 & 4.66 & 1.46 & 1.80 & 2.39 \\
\hline
\end{tabular}


Table B.18 - The response of the buildings subjected to E6C15 ground motion parallel to the long side of the building with $\alpha=0.02$

\begin{tabular}{|c|c|c|c|c|c|}
\hline \multirow{2}{*}{ Building } & \multirow{2}{*}{$V e$} & \multirow{2}{*}{$\begin{array}{c}\text { Ductility }(\mu) \\
R y=3\end{array}$} & \multicolumn{3}{|c|}{ Force Reduction Factor $(R y)$} \\
\hline & & & $\mu=2$ & $\mu=3$ & $\mu=4$ \\
\hline SL1 & 136.8 & 6.46 & 1.67 & 2.00 & 2.32 \\
\hline SL3 & 134.0 & 6.63 & 1.67 & 1.99 & 2.26 \\
\hline SL4 & 127.0 & 7.13 & 1.62 & 1.93 & 2.19 \\
\hline SH1 & 175.0 & 8.45 & 1.45 & 1.74 & 2.00 \\
\hline $\mathrm{SH} 2$ & 166.9 & 9.44 & 1.45 & 1.72 & 1.95 \\
\hline $\mathrm{SH} 3$ & 158.5 & 9.39 & 1.42 & 1.63 & 1.85 \\
\hline SH4 & 158.2 & 10.12 & 1.45 & 1.64 & 1.81 \\
\hline SH5 & 162.5 & 9.10 & 1.45 & 1.66 & 1.87 \\
\hline ML1 & 295.8 & 6.84 & 1.50 & 1.80 & 2.11 \\
\hline ML2 & 289.3 & 6.22 & 1.53 & 1.82 & 2.21 \\
\hline ML3 & 282.6 & 5.62 & 1.56 & 2.02 & 2.43 \\
\hline ML4 & 290.9 & 6.71 & 1.52 & 1.81 & 2.19 \\
\hline ML5 & 287.6 & 6.22 & 1.54 & 1.83 & 2.28 \\
\hline ML6 & 284.2 & 6.03 & 1.53 & 1.99 & 2.41 \\
\hline MH1 & 726.5 & 3.46 & 1.75 & 2.77 & 3.23 \\
\hline MH2 & 854.8 & 2.76 & 1.37 & 3.20 & 3.97 \\
\hline MH3 & 486.9 & 4.38 & 1.45 & 1.89 & 2.56 \\
\hline MH4 & 778.3 & 3.36 & 1.83 & 2.72 & 3.44 \\
\hline MH5 & 813.0 & 2.81 & 1.36 & 3.20 & 3.91 \\
\hline MH6 & 897.9 & 2.32 & 2.41 & 3.69 & 4.43 \\
\hline LL1 & 1414.7 & 2.84 & 2.18 & 3.07 & 3.59 \\
\hline LL2 & 1524.5 & 2.58 & 2.22 & 3.26 & 3.85 \\
\hline LL3 & 1034.0 & 3.53 & 1.76 & 2.32 & 3.32 \\
\hline LL4 & 1425.8 & 2.68 & 2.18 & 3.10 & 3.58 \\
\hline LL5 & 1565.1 & 2.79 & 1.37 & 3.26 & 4.05 \\
\hline LL6 & 597.0 & 9.22 & 1.45 & 1.68 & 2.20 \\
\hline LH1 & 3340.8 & 2.20 & 2.47 & 3.95 & 4.80 \\
\hline LH2 & 2668.6 & 2.45 & 2.14 & 3.33 & 4.00 \\
\hline LH3 & 1324.9 & 7.50 & 1.59 & 1.95 & 2.39 \\
\hline LH4 & 3067.4 & 2.22 & 2.24 & 3.89 & 4.80 \\
\hline LH5 & 1822.4 & 4.30 & 1.61 & 2.43 & 2.89 \\
\hline LH6 & 1288.9 & 7.11 & 1.50 & 1.84 & 2.46 \\
\hline
\end{tabular}


Table B.19 - The response of the buildings subjected to E6C18 ground motion parallel to the long side of the building with $\alpha=0.02$

\begin{tabular}{|c|c|c|c|c|c|}
\hline \multirow{2}{*}{ Building } & \multirow{2}{*}{$V e$} & \multirow{2}{*}{$\begin{array}{c}\text { Ductility }(\mu) \\
R y=3\end{array}$} & \multicolumn{3}{|c|}{ Force Reduction Factor $(R y)$} \\
\hline & & & $\mu=2$ & $\mu=3$ & $\mu=4$ \\
\hline SL1 & 183.6 & 5.00 & 1.37 & 2.32 & 2.68 \\
\hline SL3 & 185.1 & 4.97 & 1.37 & 2.33 & 2.65 \\
\hline SL4 & 187.9 & 5.09 & 1.36 & 2.31 & 2.65 \\
\hline SH1 & 353.9 & 4.25 & 1.35 & 2.28 & 2.65 \\
\hline $\mathrm{SH} 2$ & 336.3 & 3.72 & 1.34 & 2.25 & 3.34 \\
\hline $\mathrm{SH} 3$ & 310.4 & 3.01 & 1.45 & 2.82 & 4.52 \\
\hline SH4 & 309.1 & 3.53 & 1.36 & 2.56 & 4.04 \\
\hline SH5 & 290.3 & 2.89 & 1.43 & 3.23 & 4.32 \\
\hline ML1 & 277.4 & 7.95 & 1.49 & 1.75 & 1.97 \\
\hline ML2 & 243.2 & 10.53 & 1.55 & 1.72 & 1.84 \\
\hline ML3 & 234.9 & 10.89 & 1.61 & 1.83 & 1.97 \\
\hline ML4 & 248.6 & 9.93 & 1.52 & 1.70 & 1.82 \\
\hline ML5 & 239.4 & 11.20 & 1.56 & 1.73 & 1.85 \\
\hline ML6 & 234.5 & 10.22 & 1.60 & 1.80 & 1.93 \\
\hline MH1 & 542.7 & 9.69 & 1.55 & 1.84 & 2.10 \\
\hline MH2 & 541.7 & 7.92 & 1.42 & 1.71 & 1.97 \\
\hline MH3 & 505.9 & 3.49 & 1.39 & 1.67 & 3.33 \\
\hline MH4 & 535.1 & 9.95 & 1.49 & 1.76 & 2.00 \\
\hline MH5 & 535.3 & 7.39 & 1.39 & 1.65 & 1.90 \\
\hline MH6 & 550.8 & 7.21 & 1.50 & 1.82 & 2.14 \\
\hline LL1 & 989.4 & 8.12 & 1.55 & 1.88 & 2.18 \\
\hline LL2 & 985.6 & 8.19 & 1.51 & 1.82 & 2.10 \\
\hline LL3 & 923.2 & 4.16 & 1.38 & 1.66 & 2.17 \\
\hline LL4 & 989.3 & 8.12 & 1.55 & 1.87 & 2.16 \\
\hline LL5 & 977.5 & 8.34 & 1.42 & 1.70 & 1.94 \\
\hline LL6 & 786.1 & 3.97 & 1.42 & 2.59 & 3.02 \\
\hline LH1 & 2110.3 & 6.05 & 1.46 & 1.80 & 2.12 \\
\hline LH2 & 2008.9 & 3.89 & 1.45 & 1.81 & 3.05 \\
\hline LH3 & 1580.5 & 3.63 & 2.14 & 2.68 & 3.19 \\
\hline LH4 & 2048.8 & 4.53 & 1.46 & 1.83 & 2.25 \\
\hline LH5 & 1939.9 & 2.73 & 1.47 & 3.23 & 5.64 \\
\hline LH6 & 1411.4 & 4.21 & 1.96 & 2.44 & 2.89 \\
\hline
\end{tabular}


Table B.20 - The response of the buildings subjected to E6C42 ground motion parallel to the long side of the building with $\alpha=0.02$

\begin{tabular}{|c|c|c|c|c|c|}
\hline \multirow{2}{*}{ Building } & \multirow{2}{*}{$V e$} & \multirow{2}{*}{$\begin{array}{c}\text { Ductility }(\mu) \\
R y=3\end{array}$} & \multicolumn{3}{|c|}{ Force Reduction Factor $(R y)$} \\
\hline & & & $\mu=2$ & $\mu=3$ & $\mu=4$ \\
\hline SL1 & 172.3 & 5.02 & 1.87 & 2.20 & 2.54 \\
\hline SL3 & 165.3 & 5.02 & 1.88 & 2.22 & 2.52 \\
\hline SL4 & 142.9 & 4.72 & 1.90 & 2.24 & 2.66 \\
\hline SH1 & 224.2 & 4.71 & 1.38 & 1.69 & 2.52 \\
\hline $\mathrm{SH} 2$ & 199.8 & 4.99 & 1.36 & 1.61 & 2.07 \\
\hline $\mathrm{SH} 3$ & 183.4 & 3.95 & 1.36 & 1.63 & 1.94 \\
\hline SH4 & 178.1 & 4.28 & 1.32 & 1.55 & 1.75 \\
\hline SH5 & 176.8 & 3.64 & 1.35 & 1.60 & 1.81 \\
\hline ML1 & 270.0 & 4.65 & 1.82 & 2.43 & 2.85 \\
\hline ML2 & 232.6 & 6.39 & 1.38 & 2.04 & 2.39 \\
\hline ML3 & 246.2 & 4.51 & 1.36 & 1.72 & 2.71 \\
\hline ML4 & 242.7 & 5.96 & 1.48 & 2.22 & 2.53 \\
\hline ML5 & 231.9 & 6.06 & 1.35 & 1.87 & 2.33 \\
\hline ML6 & 241.9 & 4.81 & 1.38 & 1.80 & 2.62 \\
\hline MH1 & 584.0 & 4.34 & 1.39 & 1.65 & 2.88 \\
\hline MH2 & 555.6 & 3.81 & 1.38 & 1.88 & 3.07 \\
\hline MH3 & 423.0 & 4.83 & 1.28 & 1.47 & 1.77 \\
\hline MH4 & 582.5 & 4.38 & 1.37 & 1.63 & 2.88 \\
\hline MH5 & 536.4 & 3.88 & 1.37 & 1.82 & 3.04 \\
\hline MH6 & 562.1 & 3.45 & 1.42 & 2.08 & 3.24 \\
\hline LL1 & 1051.5 & 3.80 & 1.42 & 1.73 & 3.14 \\
\hline LL2 & 1047.9 & 3.80 & 1.41 & 1.74 & 3.07 \\
\hline LL3 & 780.7 & 4.37 & 1.27 & 1.47 & 1.87 \\
\hline LL4 & 1051.6 & 3.77 & 1.42 & 1.74 & 3.11 \\
\hline LL5 & 1005.8 & 3.92 & 1.38 & 1.83 & 3.03 \\
\hline LL6 & 748.6 & 4.42 & 1.47 & 1.74 & 2.23 \\
\hline LH1 & 2122.3 & 3.11 & 1.45 & 2.94 & 3.55 \\
\hline LH2 & 1879.5 & 3.02 & 1.43 & 2.99 & 3.55 \\
\hline LH3 & 1531.2 & 3.52 & 1.53 & 1.88 & 3.88 \\
\hline LH4 & 2003.8 & 2.89 & 1.48 & 3.07 & 3.83 \\
\hline LH5 & 1683.2 & 3.43 & 1.36 & 1.71 & 3.45 \\
\hline LH6 & 1390.8 & 3.77 & 1.50 & 1.84 & 3.44 \\
\hline
\end{tabular}


Table B.21 - The response of the buildings subjected to M6C1 ground motion parallel to the short side of the building with $\alpha=0.05$

\begin{tabular}{|c|c|c|c|c|c|}
\hline \multirow{2}{*}{ Building } & \multirow{2}{*}{$V e$} & \multirow{2}{*}{$\frac{\text { Ductility }(\mu)}{R y=3}$} & \multicolumn{3}{|c|}{ Force Reduction Factor $(R y)$} \\
\hline & & & $\mu=2$ & $\mu=3$ & $\mu=4$ \\
\hline SL1 & 131.5 & 10.56 & 1.27 & 1.42 & 1.71 \\
\hline SL3 & 133.1 & 10.29 & 1.28 & 1.44 & 1.70 \\
\hline SL4 & 142.1 & 9.75 & 1.27 & 1.48 & 1.75 \\
\hline SH1 & 316.8 & 7.19 & 1.24 & 1.70 & 2.13 \\
\hline $\mathrm{SH} 2$ & 283.9 & 8.04 & 1.41 & 1.58 & 1.88 \\
\hline $\mathrm{SH} 3$ & 264.2 & 8.25 & 1.33 & 1.49 & 1.79 \\
\hline SH4 & 260.9 & 8.96 & 1.31 & 1.45 & 1.63 \\
\hline SH5 & 262.9 & 7.75 & 1.34 & 1.48 & 1.71 \\
\hline SH6 & 263.0 & 8.30 & 1.33 & 1.48 & 1.79 \\
\hline ML1 & 508.8 & 7.43 & 1.46 & 1.64 & 1.81 \\
\hline ML2 & 435.8 & 9.29 & 1.22 & 1.53 & 1.68 \\
\hline ML3 & 417.7 & 8.13 & 1.30 & 1.55 & 1.70 \\
\hline ML4 & 490.8 & 8.27 & 1.46 & 1.64 & 1.80 \\
\hline ML5 & 493.7 & 8.20 & 1.46 & 1.64 & 1.80 \\
\hline ML6 & 428.7 & 8.08 & 1.37 & 1.56 & 1.71 \\
\hline MH1 & 878.8 & 6.90 & 1.39 & 1.56 & 1.71 \\
\hline MH2 & 832.1 & 5.85 & 1.43 & 1.62 & 1.80 \\
\hline MH3 & 500.5 & 6.19 & 1.51 & 2.30 & 2.54 \\
\hline MH4 & 832.5 & 6.52 & 1.40 & 1.62 & 1.78 \\
\hline MH5 & 851.3 & 5.01 & 1.40 & 1.73 & 1.92 \\
\hline MH6 & 1143.3 & 3.60 & 1.59 & 1.98 & 3.15 \\
\hline LL1 & 1523.3 & 6.36 & 1.30 & 1.49 & 2.16 \\
\hline LL2 & 1503.8 & 6.37 & 1.30 & 1.49 & 2.18 \\
\hline LL3 & 1496.1 & 3.99 & 1.53 & 2.11 & 3.01 \\
\hline LL4 & 1716.3 & 5.48 & 1.40 & 1.60 & 1.95 \\
\hline LL5 & 1735.1 & 5.38 & 1.40 & 1.61 & 1.99 \\
\hline LL6 & 469.1 & 14.25 & 1.29 & 1.48 & 1.64 \\
\hline LH1 & 2933.9 & 3.93 & 1.49 & 2.20 & 3.04 \\
\hline LH2 & 2472.9 & 4.52 & 1.45 & 1.95 & 2.64 \\
\hline LH3 & 1269.3 & 10.33 & 1.47 & 1.72 & 1.92 \\
\hline LH4 & 2409.6 & 4.65 & 1.38 & 1.85 & 2.60 \\
\hline LH5 & 2344.8 & 4.67 & 1.37 & 1.83 & 2.55 \\
\hline LH6 & 1212.9 & 10.44 & 1.48 & 1.72 & 1.92 \\
\hline
\end{tabular}


Table B.22 - The response of the buildings subjected to M6C2 ground motion parallel to the short side of the building with $\alpha=0.05$

\begin{tabular}{|c|c|c|c|c|c|}
\hline \multirow{2}{*}{ Building } & \multirow{2}{*}{$V e$} & \multirow{2}{*}{$\frac{\text { Ductility }(\mu)}{R y=3}$} & \multicolumn{3}{|c|}{ Force Reduction Factor $(R y)$} \\
\hline & & & $\mu=2$ & $\mu=3$ & $\mu=4$ \\
\hline SL1 & 135.5 & 10.52 & 1.23 & 1.38 & 1.53 \\
\hline SL3 & 137.2 & 11.03 & 1.22 & 1.37 & 1.51 \\
\hline SL4 & 138.2 & 12.51 & 1.20 & 1.34 & 1.46 \\
\hline SH1 & 226.8 & 7.97 & 1.23 & 1.66 & 1.86 \\
\hline $\mathrm{SH} 2$ & 223.9 & 8.62 & 1.22 & 1.47 & 1.83 \\
\hline $\mathrm{SH} 3$ & 223.3 & 7.95 & 1.25 & 1.76 & 1.91 \\
\hline SH4 & 216.4 & 8.88 & 1.26 & 1.41 & 1.85 \\
\hline SH5 & 215.9 & 8.26 & 1.28 & 1.50 & 2.01 \\
\hline SH6 & 223.2 & 7.83 & 1.25 & 1.67 & 1.93 \\
\hline ML1 & 276.6 & 10.97 & 1.25 & 1.37 & 1.47 \\
\hline ML2 & 211.1 & 13.61 & 1.23 & 1.34 & 1.50 \\
\hline ML3 & 234.5 & 11.50 & 1.20 & 1.47 & 1.71 \\
\hline ML4 & 231.1 & 11.85 & 1.20 & 1.32 & 1.44 \\
\hline ML5 & 235.5 & 11.71 & 1.21 & 1.34 & 1.44 \\
\hline ML6 & 221.4 & 11.89 & 1.25 & 1.41 & 1.67 \\
\hline MH1 & 583.3 & 9.03 & 1.23 & 1.68 & 1.82 \\
\hline MH2 & 687.7 & 5.50 & 1.54 & 1.79 & 2.03 \\
\hline MH3 & 580.2 & 7.11 & 1.49 & 1.88 & 2.22 \\
\hline MH4 & 697.4 & 5.73 & 1.30 & 1.69 & 1.91 \\
\hline MH5 & 706.7 & 4.68 & 1.50 & 1.75 & 2.12 \\
\hline MH6 & 568.5 & 7.86 & 1.37 & 1.53 & 1.90 \\
\hline LL1 & 1105.5 & 7.23 & 1.56 & 1.85 & 2.12 \\
\hline LL2 & 1108.6 & 7.35 & 1.56 & 1.86 & 2.14 \\
\hline LL3 & 1169.1 & 4.93 & 1.37 & 2.11 & 2.42 \\
\hline LL4 & 944.8 & 9.11 & 1.32 & 1.69 & 1.87 \\
\hline LL5 & 932.3 & 9.24 & 1.34 & 1.69 & 1.86 \\
\hline LL6 & 554.6 & 13.18 & 1.24 & 1.37 & 1.49 \\
\hline LH1 & 2431.3 & 4.83 & 1.37 & 2.00 & 2.25 \\
\hline LH2 & 2373.5 & 4.54 & 1.62 & 2.00 & 2.27 \\
\hline LH3 & 1828.0 & 8.60 & 1.48 & 1.87 & 2.05 \\
\hline LH4 & 2391.1 & 4.75 & 1.51 & 2.04 & 2.29 \\
\hline LH5 & 2440.7 & 4.82 & 1.51 & 2.10 & 2.36 \\
\hline LH6 & 1494.5 & 9.87 & 1.31 & 1.57 & 1.73 \\
\hline
\end{tabular}


Table B.23 - The response of the buildings subjected to M6C26 ground motion parallel to the short side of the building with $\alpha=0.05$

\begin{tabular}{|c|c|c|c|c|c|}
\hline \multirow{2}{*}{ Building } & \multirow{2}{*}{$V e$} & \multirow{2}{*}{$\frac{\text { Ductility }(\mu)}{R y=3}$} & \multicolumn{3}{|c|}{ Force Reduction Factor $(R y)$} \\
\hline & & & $\mu=2$ & $\mu=3$ & $\mu=4$ \\
\hline SL1 & 164.3 & 10.63 & 1.18 & 1.31 & 1.43 \\
\hline SL3 & 156.3 & 11.72 & 1.17 & 1.30 & 1.42 \\
\hline SL4 & 135.3 & 15.01 & 1.16 & 1.28 & 1.38 \\
\hline SH1 & 333.5 & 9.14 & 1.26 & 1.41 & 1.55 \\
\hline $\mathrm{SH} 2$ & 314.4 & 9.79 & 1.24 & 1.38 & 1.50 \\
\hline $\mathrm{SH} 3$ & 304.8 & 8.65 & 1.24 & 1.38 & 1.51 \\
\hline SH4 & 293.8 & 10.33 & 1.23 & 1.36 & 1.48 \\
\hline SH5 & 294.7 & 8.96 & 1.25 & 1.39 & 1.51 \\
\hline SH6 & 304.1 & 9.00 & 1.24 & 1.38 & 1.51 \\
\hline ML1 & 474.2 & 8.53 & 1.32 & 1.49 & 1.63 \\
\hline ML2 & 379.0 & 11.12 & 1.21 & 1.67 & 1.84 \\
\hline ML3 & 358.2 & 9.99 & 1.24 & 1.73 & 1.92 \\
\hline ML4 & 432.4 & 9.42 & 1.18 & 1.56 & 1.70 \\
\hline ML5 & 436.6 & 9.34 & 1.18 & 1.55 & 1.70 \\
\hline ML6 & 369.5 & 10.24 & 1.23 & 1.72 & 1.90 \\
\hline MH1 & 739.4 & 9.17 & 1.28 & 1.77 & 1.97 \\
\hline MH2 & 669.2 & 8.21 & 1.51 & 1.75 & 1.95 \\
\hline MH3 & 449.0 & 7.49 & 1.31 & 1.93 & 2.15 \\
\hline MH4 & 624.9 & 9.66 & 1.40 & 1.60 & 1.77 \\
\hline MH5 & 612.2 & 8.32 & 1.40 & 1.62 & 1.82 \\
\hline MH6 & 996.4 & 4.21 & 1.49 & 1.71 & 2.16 \\
\hline LL1 & 1154.3 & 9.29 & 1.31 & 1.83 & 1.99 \\
\hline LL2 & 1137.2 & 9.20 & 1.31 & 1.83 & 2.00 \\
\hline LL3 & 949.1 & 7.05 & 1.34 & 1.55 & 1.79 \\
\hline LL4 & 1226.8 & 9.10 & 1.24 & 1.76 & 1.94 \\
\hline LL5 & 1239.5 & 8.82 & 1.24 & 1.77 & 1.94 \\
\hline LL6 & 439.4 & 9.42 & 1.30 & 1.48 & 1.67 \\
\hline LH1 & 2064.4 & 5.25 & 1.30 & 1.66 & 2.09 \\
\hline LH2 & 2116.3 & 3.94 & 1.56 & 2.06 & 3.08 \\
\hline LH3 & 1321.5 & 6.41 & 1.21 & 1.94 & 2.27 \\
\hline LH4 & 1997.1 & 4.26 & 1.46 & 1.92 & 2.89 \\
\hline LH5 & 1961.0 & 4.44 & 1.45 & 1.96 & 2.85 \\
\hline LH6 & 1191.4 & 7.44 & 1.43 & 1.71 & 1.97 \\
\hline
\end{tabular}


Table B.24 - The response of the buildings subjected to M6C31 ground motion parallel to the short side of the building with $\alpha=0.05$

\begin{tabular}{|c|c|c|c|c|c|}
\hline \multirow{2}{*}{ Building } & \multirow{2}{*}{$V e$} & \multirow{2}{*}{$\frac{\text { Ductility }(\mu)}{R y=3}$} & \multicolumn{3}{|c|}{ Force Reduction Factor $(R y)$} \\
\hline & & & $\mu=2$ & $\mu=3$ & $\mu=4$ \\
\hline SL1 & 202.1 & 8.34 & 1.17 & 1.29 & 1.48 \\
\hline SL3 & 210.1 & 8.12 & 1.16 & 1.29 & 1.47 \\
\hline SL4 & 214.7 & 8.97 & 1.16 & 1.28 & 1.40 \\
\hline SH1 & 244.8 & 12.72 & 1.22 & 1.42 & 1.59 \\
\hline $\mathrm{SH} 2$ & 262.1 & 10.59 & 1.20 & 1.34 & 1.46 \\
\hline $\mathrm{SH} 3$ & 254.3 & 9.15 & 1.19 & 1.32 & 1.44 \\
\hline SH4 & 234.2 & 10.60 & 1.16 & 1.27 & 1.38 \\
\hline SH5 & 233.5 & 9.47 & 1.17 & 1.29 & 1.42 \\
\hline SH6 & 253.9 & 9.08 & 1.19 & 1.32 & 1.44 \\
\hline ML1 & 304.9 & 10.91 & 1.17 & 1.31 & 1.49 \\
\hline ML2 & 209.1 & 14.54 & 1.27 & 1.38 & 1.48 \\
\hline ML3 & 208.7 & 11.53 & 1.28 & 1.40 & 1.51 \\
\hline ML4 & 255.2 & 12.76 & 1.22 & 1.35 & 1.45 \\
\hline ML5 & 259.3 & 12.71 & 1.21 & 1.34 & 1.45 \\
\hline ML6 & 207.3 & 12.56 & 1.28 & 1.40 & 1.50 \\
\hline MH1 & 457.8 & 10.55 & 1.27 & 1.40 & 1.52 \\
\hline MH2 & 461.3 & 8.63 & 1.27 & 1.41 & 1.53 \\
\hline MH3 & 311.8 & 14.44 & 1.20 & 1.35 & 1.51 \\
\hline MH4 & 449.6 & 10.23 & 1.23 & 1.34 & 1.45 \\
\hline MH5 & 455.8 & 8.87 & 1.26 & 1.39 & 1.51 \\
\hline MH6 & 623.8 & 5.93 & 1.38 & 1.78 & 2.10 \\
\hline LL1 & 860.2 & 10.50 & 1.26 & 1.45 & 1.72 \\
\hline LL2 & 856.1 & 10.50 & 1.26 & 1.46 & 1.74 \\
\hline LL3 & 759.8 & 8.00 & 1.23 & 1.39 & 1.54 \\
\hline LL4 & 778.9 & 11.34 & 1.21 & 1.41 & 1.61 \\
\hline LL5 & 789.1 & 11.35 & 1.22 & 1.41 & 1.63 \\
\hline LL6 & 663.0 & 13.22 & 1.26 & 1.47 & 1.63 \\
\hline LH1 & 1584.7 & 7.06 & 1.28 & 1.46 & 1.64 \\
\hline LH2 & 1537.3 & 6.02 & 1.35 & 1.56 & 1.80 \\
\hline LH3 & 1278.1 & 11.69 & 1.24 & 1.41 & 1.56 \\
\hline LH4 & 1505.2 & 6.41 & 1.32 & 1.55 & 1.80 \\
\hline LH5 & 1489.8 & 6.45 & 1.32 & 1.56 & 1.82 \\
\hline LH6 & 1334.3 & 11.50 & 1.26 & 1.44 & 1.60 \\
\hline
\end{tabular}


Table B.25 - The response of the buildings subjected to M6C38 ground motion parallel to the short side of the building with $\alpha=0.05$

\begin{tabular}{|c|c|c|c|c|c|}
\hline \multirow{2}{*}{ Building } & \multirow{2}{*}{$V e$} & \multirow{2}{*}{$\begin{array}{c}\text { Ductility }(\mu) \\
R y=3\end{array}$} & \multicolumn{3}{|c|}{ Force Reduction Factor $(R y)$} \\
\hline & & & $\mu=2$ & $\mu=3$ & $\mu=4$ \\
\hline SL1 & 137.6 & 17.04 & 1.15 & 1.27 & 1.38 \\
\hline SL3 & 135.6 & 17.15 & 1.15 & 1.27 & 1.37 \\
\hline SL4 & 129.8 & 17.98 & 1.15 & 1.26 & 1.36 \\
\hline SH1 & 183.8 & 16.69 & 1.23 & 1.39 & 1.52 \\
\hline $\mathrm{SH} 2$ & 171.9 & 17.37 & 1.23 & 1.38 & 1.52 \\
\hline $\mathrm{SH} 3$ & 167.5 & 16.61 & 1.24 & 1.40 & 1.54 \\
\hline SH4 & 160.4 & 18.24 & 1.22 & 1.37 & 1.48 \\
\hline SH5 & 161.0 & 17.07 & 1.23 & 1.39 & 1.51 \\
\hline SH6 & 167.2 & 16.65 & 1.24 & 1.40 & 1.53 \\
\hline ML1 & 289.4 & 15.41 & 1.30 & 1.45 & 1.58 \\
\hline ML2 & 278.4 & 14.73 & 1.30 & 1.44 & 1.56 \\
\hline ML3 & 293.1 & 12.25 & 1.38 & 1.55 & 1.69 \\
\hline ML4 & 299.9 & 13.90 & 1.29 & 1.53 & 1.66 \\
\hline ML5 & 300.2 & 13.98 & 1.28 & 1.53 & 1.66 \\
\hline ML6 & 287.3 & 12.77 & 1.35 & 1.51 & 1.65 \\
\hline MH1 & 664.8 & 10.77 & 1.29 & 1.60 & 1.76 \\
\hline MH2 & 696.0 & 8.52 & 1.22 & 1.73 & 1.93 \\
\hline MH3 & 643.9 & 6.31 & 1.23 & 1.51 & 2.10 \\
\hline MH4 & 710.4 & 9.44 & 1.20 & 1.47 & 1.89 \\
\hline MH5 & 721.2 & 7.96 & 1.23 & 1.80 & 2.00 \\
\hline MH6 & 685.6 & 9.26 & 1.41 & 1.67 & 1.88 \\
\hline LL1 & 1158.1 & 10.22 & 1.35 & 1.60 & 1.76 \\
\hline LL2 & 1160.5 & 10.09 & 1.34 & 1.61 & 1.77 \\
\hline LL3 & 1296.9 & 7.02 & 1.26 & 1.85 & 2.07 \\
\hline LL4 & 1171.1 & 19.48 & 1.41 & 1.58 & 1.75 \\
\hline LL5 & 1164.4 & 19.59 & 1.40 & 1.58 & 1.74 \\
\hline LL6 & 920.3 & 15.89 & 1.48 & 1.79 & 2.15 \\
\hline LH1 & 2779.8 & 11.81 & 1.31 & 1.87 & 2.11 \\
\hline LH2 & 2604.5 & 10.64 & 1.45 & 1.86 & 2.12 \\
\hline LH3 & 2060.8 & 11.07 & 1.61 & 1.90 & 2.55 \\
\hline LH4 & 2742.0 & 10.11 & 1.44 & 1.94 & 2.21 \\
\hline LH5 & 2751.6 & 9.84 & 1.45 & 1.97 & 2.24 \\
\hline LH6 & 2027.0 & 11.25 & 1.52 & 1.97 & 2.62 \\
\hline
\end{tabular}


Table B.26 - The response of the buildings subjected to M6C1 ground motion parallel to the long side of the building with $\alpha=0.05$

\begin{tabular}{|c|c|c|c|c|c|}
\hline \multirow{2}{*}{ Building } & \multirow{2}{*}{$V e$} & \multirow{2}{*}{$\begin{array}{c}\text { Ductility }(\mu) \\
R y=3\end{array}$} & \multicolumn{3}{|c|}{ Force Reduction Factor $(R y)$} \\
\hline & & & $\mu=2$ & $\mu=3$ & $\mu=4$ \\
\hline SL1 & 206.3 & 4.66 & 1.35 & 1.57 & 1.89 \\
\hline SL3 & 199.9 & 4.52 & 1.33 & 1.55 & 1.84 \\
\hline SL4 & 178.7 & 6.09 & 1.28 & 1.46 & 1.65 \\
\hline SH1 & 420.5 & 4.14 & 1.86 & 2.53 & 2.92 \\
\hline $\mathrm{SH} 2$ & 382.9 & 5.12 & 1.40 & 2.31 & 2.64 \\
\hline $\mathrm{SH} 3$ & 335.6 & 5.56 & 1.65 & 2.10 & 2.40 \\
\hline SH4 & 335.0 & 6.22 & 1.57 & 2.05 & 2.31 \\
\hline SH5 & 314.5 & 6.01 & 1.51 & 1.98 & 2.26 \\
\hline ML1 & 620.3 & 5.18 & 1.50 & 2.23 & 2.65 \\
\hline ML2 & 599.4 & 6.15 & 1.42 & 2.15 & 2.43 \\
\hline ML3 & 582.6 & 6.65 & 1.40 & 2.02 & 2.31 \\
\hline ML4 & 609.2 & 5.80 & 1.45 & 2.21 & 2.50 \\
\hline ML5 & 586.2 & 6.56 & 1.37 & 2.08 & 2.34 \\
\hline ML6 & 579.7 & 6.48 & 1.41 & 2.05 & 2.33 \\
\hline MH1 & 1307.9 & 6.46 & 1.37 & 2.03 & 2.32 \\
\hline MH2 & 1306.9 & 5.27 & 1.51 & 2.19 & 2.59 \\
\hline MH3 & 1093.3 & 4.85 & 1.55 & 2.36 & 2.71 \\
\hline MH4 & 1298.1 & 6.59 & 1.35 & 1.99 & 2.32 \\
\hline MH5 & 1408.3 & 4.37 & 1.57 & 2.48 & 2.85 \\
\hline MH6 & 1288.8 & 4.99 & 1.46 & 2.25 & 2.64 \\
\hline LL1 & 2417.2 & 5.42 & 1.77 & 2.16 & 2.51 \\
\hline LL2 & 2376.2 & 5.61 & 1.58 & 2.10 & 2.48 \\
\hline LL3 & 2129.1 & 4.34 & 1.81 & 2.49 & 2.88 \\
\hline LL4 & 2414.6 & 5.46 & 1.73 & 2.16 & 2.51 \\
\hline LL5 & 2312.8 & 5.64 & 1.45 & 2.04 & 2.49 \\
\hline LL6 & 2063.5 & 3.36 & 1.64 & 2.84 & 3.28 \\
\hline LH1 & 6161.3 & 3.01 & 1.85 & 2.99 & 3.54 \\
\hline LH2 & 5375.9 & 2.92 & 2.44 & 3.04 & 3.60 \\
\hline LH3 & 4506.9 & 2.99 & 1.77 & 3.00 & 3.53 \\
\hline LH4 & 5840.1 & 2.82 & 2.50 & 3.10 & 3.74 \\
\hline LH5 & 4548.3 & 3.43 & 2.25 & 2.79 & 3.28 \\
\hline LH6 & 4230.9 & 3.29 & 1.64 & 2.87 & 3.33 \\
\hline
\end{tabular}


Table B.27 - The response of the buildings subjected to M6C2 ground motion parallel to the long side of the building with $\alpha=0.05$

\begin{tabular}{|c|c|c|c|c|c|}
\hline \multirow{2}{*}{ Building } & \multirow{2}{*}{$V e$} & \multirow{2}{*}{$\begin{array}{c}\text { Ductility }(\mu) \\
R y=3\end{array}$} & \multicolumn{3}{|c|}{ Force Reduction Factor $(R y)$} \\
\hline & & & $\mu=2$ & $\mu=3$ & $\mu=4$ \\
\hline SL1 & 175.0 & 7.03 & 1.59 & 1.86 & 2.08 \\
\hline SL3 & 172.5 & 7.21 & 1.59 & 1.86 & 2.08 \\
\hline SL4 & 170.8 & 7.64 & 1.56 & 1.84 & 2.12 \\
\hline SH1 & 463.6 & 3.72 & 1.75 & 2.60 & 3.43 \\
\hline $\mathrm{SH} 2$ & 429.2 & 3.72 & 1.67 & 2.37 & 3.20 \\
\hline $\mathrm{SH} 3$ & 356.6 & 4.15 & 1.60 & 2.15 & 2.90 \\
\hline SH4 & 357.3 & 4.57 & 1.54 & 2.09 & 2.64 \\
\hline SH5 & 304.8 & 4.86 & 1.62 & 2.02 & 2.50 \\
\hline ML1 & 594.8 & 6.31 & 1.35 & 1.63 & 2.03 \\
\hline ML2 & 574.6 & 7.41 & 1.32 & 1.54 & 1.86 \\
\hline ML3 & 528.3 & 7.43 & 1.34 & 1.59 & 1.85 \\
\hline ML4 & 583.8 & 7.15 & 1.33 & 1.55 & 1.91 \\
\hline ML5 & 562.7 & 7.80 & 1.30 & 1.52 & 1.82 \\
\hline ML6 & 544.5 & 7.16 & 1.34 & 1.58 & 1.89 \\
\hline MH1 & 904.5 & 8.00 & 1.29 & 1.55 & 1.86 \\
\hline MH2 & 936.1 & 4.80 & 1.81 & 2.40 & 2.75 \\
\hline MH3 & 912.7 & 3.78 & 1.72 & 2.02 & 3.23 \\
\hline MH4 & 916.6 & 7.24 & 1.33 & 1.67 & 2.11 \\
\hline MH5 & 949.0 & 4.42 & 2.12 & 2.50 & 2.84 \\
\hline MH6 & 941.5 & 4.61 & 1.49 & 2.28 & 2.83 \\
\hline LL1 & 1684.8 & 6.53 & 1.37 & 1.69 & 2.00 \\
\hline LL2 & 1695.3 & 5.91 & 1.39 & 1.84 & 2.30 \\
\hline LL3 & 1695.5 & 3.16 & 1.66 & 2.00 & 3.40 \\
\hline LL4 & 1686.9 & 6.46 & 1.37 & 1.69 & 2.02 \\
\hline LL5 & 1684.6 & 4.99 & 1.65 & 2.35 & 2.70 \\
\hline LL6 & 1147.2 & 6.16 & 1.43 & 2.16 & 2.35 \\
\hline LH1 & 3848.9 & 3.66 & 1.96 & 2.64 & 3.16 \\
\hline LH2 & 3828.8 & 2.90 & 2.38 & 3.06 & 3.65 \\
\hline LH3 & 2290.5 & 5.65 & 1.57 & 2.06 & 2.26 \\
\hline LH4 & 3827.8 & 3.24 & 2.21 & 2.85 & 3.42 \\
\hline LH5 & 3575.9 & 3.22 & 1.82 & 2.76 & 4.06 \\
\hline LH6 & 1987.8 & 6.22 & 1.55 & 1.75 & 1.91 \\
\hline
\end{tabular}


Table B.28 - The response of the buildings subjected to M6C26 ground motion parallel to the long side of the building with $\alpha=0.05$

\begin{tabular}{|c|c|c|c|c|c|}
\hline \multirow{2}{*}{ Building } & \multirow{2}{*}{$V e$} & \multirow{2}{*}{$\begin{array}{c}\text { Ductility }(\mu) \\
R y=3\end{array}$} & \multicolumn{3}{|c|}{ Force Reduction Factor $(R y)$} \\
\hline & & & $\mu=2$ & $\mu=3$ & $\mu=4$ \\
\hline SL1 & 197.6 & 8.06 & 1.54 & 1.84 & 2.10 \\
\hline SL3 & 203.5 & 7.79 & 1.54 & 1.83 & 2.10 \\
\hline SL4 & 214.0 & 7.67 & 1.56 & 1.86 & 2.13 \\
\hline SH1 & 487.0 & 5.79 & 1.76 & 2.12 & 2.44 \\
\hline $\mathrm{SH} 2$ & 483.5 & 6.04 & 1.36 & 2.10 & 2.41 \\
\hline $\mathrm{SH} 3$ & 470.9 & 5.43 & 1.33 & 2.19 & 2.62 \\
\hline SH4 & 465.0 & 6.03 & 1.30 & 2.12 & 2.41 \\
\hline SH5 & 455.8 & 5.39 & 1.32 & 2.24 & 2.56 \\
\hline ML1 & 582.9 & 6.57 & 1.29 & 1.51 & 1.71 \\
\hline ML2 & 534.2 & 7.80 & 1.27 & 1.47 & 1.65 \\
\hline ML3 & 529.2 & 6.97 & 1.30 & 1.52 & 1.73 \\
\hline ML4 & 544.3 & 7.56 & 1.27 & 1.47 & 1.67 \\
\hline ML5 & 526.8 & 8.04 & 1.26 & 1.46 & 1.64 \\
\hline ML6 & 527.3 & 7.04 & 1.30 & 1.52 & 1.72 \\
\hline MH1 & 1263.7 & 5.66 & 1.33 & 1.57 & 1.85 \\
\hline MH2 & 1391.8 & 3.77 & 1.41 & 1.78 & 3.14 \\
\hline MH3 & 1271.5 & 4.64 & 1.38 & 1.76 & 2.78 \\
\hline MH4 & 1282.9 & 5.46 & 1.33 & 1.56 & 1.87 \\
\hline MH5 & 1417.4 & 3.83 & 1.41 & 1.85 & 3.06 \\
\hline MH6 & 1429.6 & 3.15 & 1.47 & 2.07 & 3.44 \\
\hline LL1 & 2377.5 & 4.31 & 1.40 & 1.67 & 2.42 \\
\hline LL2 & 2417.6 & 4.24 & 1.39 & 1.66 & 2.46 \\
\hline LL3 & 2410.1 & 4.30 & 1.40 & 2.50 & 2.88 \\
\hline LL4 & 2380.9 & 4.34 & 1.39 & 1.67 & 2.32 \\
\hline LL5 & 2503.4 & 3.93 & 1.40 & 1.72 & 3.06 \\
\hline LL6 & 1924.6 & 3.92 & 1.50 & 2.64 & 3.03 \\
\hline LH1 & 6150.0 & 2.90 & 1.59 & 3.07 & 3.61 \\
\hline LH2 & 5825.5 & 3.21 & 1.57 & 2.89 & 3.43 \\
\hline LH3 & 3856.6 & 3.20 & 1.61 & 2.89 & 3.42 \\
\hline LH4 & 6170.0 & 2.88 & 1.62 & 3.07 & 3.67 \\
\hline LH5 & 5117.4 & 3.67 & 1.51 & 2.69 & 3.17 \\
\hline LH6 & 3349.9 & 3.24 & 1.66 & 2.13 & 3.90 \\
\hline
\end{tabular}


Table B.29 - The response of the buildings subjected to M6C31 ground motion parallel to the long side of the building with $\alpha=0.05$

\begin{tabular}{|c|c|c|c|c|c|}
\hline \multirow{2}{*}{ Building } & \multirow{2}{*}{$V e$} & \multirow{2}{*}{$\begin{array}{c}\text { Ductility }(\mu) \\
R y=3\end{array}$} & \multicolumn{3}{|c|}{ Force Reduction Factor $(R y)$} \\
\hline & & & $\mu=2$ & $\mu=3$ & $\mu=4$ \\
\hline SL1 & 184.8 & 5.53 & 1.34 & 2.27 & 2.57 \\
\hline SL3 & 180.1 & 5.84 & 1.34 & 1.81 & 2.53 \\
\hline SL4 & 178.0 & 6.58 & 1.33 & 1.56 & 2.09 \\
\hline SH1 & 446.1 & 4.30 & 1.31 & 1.54 & 2.86 \\
\hline $\mathrm{SH} 2$ & 416.2 & 4.55 & 1.30 & 1.52 & 1.87 \\
\hline $\mathrm{SH} 3$ & 405.2 & 4.37 & 1.38 & 1.61 & 2.10 \\
\hline SH4 & 396.4 & 4.86 & 1.33 & 1.55 & 1.81 \\
\hline SH5 & 397.6 & 4.24 & 1.34 & 1.57 & 2.72 \\
\hline ML1 & 939.1 & 3.60 & 1.30 & 2.56 & 3.23 \\
\hline ML2 & 850.7 & 3.76 & 1.31 & 2.31 & 3.12 \\
\hline ML3 & 639.3 & 4.23 & 1.37 & 1.76 & 2.82 \\
\hline ML4 & 882.0 & 3.74 & 1.31 & 2.45 & 3.17 \\
\hline ML5 & 813.0 & 3.99 & 1.32 & 2.15 & 3.04 \\
\hline ML6 & 702.6 & 3.91 & 1.36 & 2.20 & 3.08 \\
\hline MH1 & 1026.3 & 6.08 & 1.37 & 1.72 & 2.09 \\
\hline MH2 & 829.2 & 7.72 & 1.38 & 1.89 & 2.05 \\
\hline MH3 & 1091.5 & 3.63 & 1.32 & 2.31 & 3.58 \\
\hline MH4 & 957.3 & 7.03 & 1.41 & 1.85 & 2.10 \\
\hline MH5 & 882.3 & 8.11 & 1.65 & 1.84 & 2.02 \\
\hline MH6 & 805.3 & 6.29 & 1.38 & 1.88 & 2.04 \\
\hline LL1 & 1747.3 & 5.61 & 1.47 & 1.95 & 2.21 \\
\hline LL2 & 1560.6 & 6.69 & 1.52 & 1.87 & 2.05 \\
\hline LL3 & 2143.2 & 3.95 & 1.37 & 2.02 & 3.00 \\
\hline LL4 & 1732.6 & 5.83 & 1.47 & 1.95 & 2.19 \\
\hline LL5 & 1474.9 & 7.89 & 1.36 & 1.87 & 2.03 \\
\hline LL6 & 1249.6 & 3.76 & 1.52 & 2.38 & 3.18 \\
\hline LH1 & 3731.5 & 5.04 & 1.60 & 1.88 & 2.16 \\
\hline LH2 & 4929.3 & 3.78 & 1.48 & 2.10 & 3.50 \\
\hline LH3 & 2466.2 & 3.44 & 2.12 & 2.80 & 3.39 \\
\hline LH4 & 4572.4 & 4.02 & 1.62 & 2.01 & 2.90 \\
\hline LH5 & 4364.4 & 2.35 & 2.05 & 3.68 & 5.06 \\
\hline LH6 & 1981.6 & 4.59 & 1.46 & 1.69 & 2.43 \\
\hline
\end{tabular}


Table B.30 - The response of the buildings subjected to M6C38 ground motion parallel to the long side of the building with $\alpha=0.05$

\begin{tabular}{|c|c|c|c|c|c|}
\hline \multirow{2}{*}{ Building } & \multirow{2}{*}{$V e$} & \multirow{2}{*}{$\begin{array}{c}\text { Ductility }(\mu) \\
R y=3\end{array}$} & \multicolumn{3}{|c|}{ Force Reduction Factor $(R y)$} \\
\hline & & & $\mu=2$ & $\mu=3$ & $\mu=4$ \\
\hline SL1 & 165.6 & 7.27 & 1.39 & 1.95 & 2.26 \\
\hline SL3 & 157.4 & 8.31 & 1.32 & 1.79 & 2.13 \\
\hline SL4 & 152.5 & 9.16 & 1.29 & 1.58 & 1.95 \\
\hline SH1 & 378.3 & 7.49 & 1.32 & 1.55 & 1.77 \\
\hline $\mathrm{SH} 2$ & 365.7 & 8.27 & 1.29 & 1.51 & 1.71 \\
\hline $\mathrm{SH} 3$ & 360.1 & 7.76 & 1.30 & 1.54 & 1.77 \\
\hline SH4 & 353.9 & 8.65 & 1.28 & 1.49 & 1.67 \\
\hline SH5 & 353.4 & 8.00 & 1.30 & 1.52 & 1.74 \\
\hline ML1 & 567.1 & 8.87 & 1.26 & 1.47 & 1.67 \\
\hline ML2 & 542.7 & 9.18 & 1.25 & 1.45 & 1.63 \\
\hline ML3 & 522.6 & 8.41 & 1.27 & 1.50 & 1.71 \\
\hline ML4 & 549.1 & 9.09 & 1.26 & 1.46 & 1.64 \\
\hline ML5 & 536.1 & 9.44 & 1.25 & 1.44 & 1.62 \\
\hline ML6 & 532.8 & 8.19 & 1.28 & 1.50 & 1.72 \\
\hline MH1 & 1041.2 & 8.13 & 1.31 & 1.54 & 1.76 \\
\hline MH2 & 911.2 & 7.72 & 1.36 & 1.63 & 1.88 \\
\hline MH3 & 697.7 & 9.52 & 1.36 & 1.61 & 1.84 \\
\hline MH4 & 1008.1 & 8.50 & 1.30 & 1.54 & 1.76 \\
\hline MH5 & 864.7 & 8.19 & 1.36 & 1.62 & 1.86 \\
\hline MH6 & 956.5 & 6.37 & 1.42 & 1.73 & 2.02 \\
\hline LL1 & 1918.8 & 6.73 & 1.38 & 1.66 & 1.94 \\
\hline LL2 & 1834.0 & 6.94 & 1.37 & 1.64 & 1.91 \\
\hline LL3 & 1310.3 & 8.79 & 1.37 & 1.63 & 1.88 \\
\hline LL4 & 1911.3 & 6.65 & 1.38 & 1.66 & 1.92 \\
\hline LL5 & 1656.1 & 7.94 & 1.35 & 1.62 & 1.87 \\
\hline LL6 & 1213.1 & 8.25 & 1.41 & 1.69 & 1.93 \\
\hline LH1 & 3545.2 & 5.78 & 1.47 & 1.83 & 2.18 \\
\hline LH2 & 3127.7 & 6.02 & 1.49 & 1.86 & 2.15 \\
\hline LH3 & 2731.4 & 6.35 & 1.52 & 1.85 & 2.16 \\
\hline LH4 & 3327.5 & 5.45 & 1.51 & 1.91 & 2.29 \\
\hline LH5 & 2828.2 & 6.82 & 1.44 & 1.77 & 2.00 \\
\hline LH6 & 2547.9 & 6.56 & 1.44 & 1.74 & 2.02 \\
\hline
\end{tabular}


Table B.31 - The response of the buildings subjected to E6C1 ground motion parallel to the short side of the building with $\alpha=0.05$

\begin{tabular}{|c|c|c|c|c|c|}
\hline \multirow{2}{*}{ Building } & \multirow{2}{*}{$V e$} & \multirow{2}{*}{$\frac{\text { Ductility }(\mu)}{R y=3}$} & \multicolumn{3}{|c|}{ Force Reduction Factor $(R y)$} \\
\hline & & & $\mu=2$ & $\mu=3$ & $\mu=4$ \\
\hline SL1 & 93.7 & 11.50 & 1.25 & 1.42 & 1.58 \\
\hline SL3 & 93.7 & 11.62 & 1.25 & 1.42 & 1.57 \\
\hline SL4 & 92.1 & 11.83 & 1.23 & 1.38 & 1.53 \\
\hline SH1 & 150.7 & 12.62 & 1.28 & 1.39 & 1.51 \\
\hline $\mathrm{SH} 2$ & 140.3 & 13.61 & 1.22 & 1.33 & 1.44 \\
\hline $\mathrm{SH} 3$ & 135.0 & 12.67 & 1.18 & 1.31 & 1.42 \\
\hline SH4 & 130.1 & 13.85 & 1.15 & 1.27 & 1.37 \\
\hline SH5 & 130.7 & 12.32 & 1.17 & 1.29 & 1.40 \\
\hline SH6 & 134.7 & 12.73 & 1.18 & 1.31 & 1.42 \\
\hline ML1 & 238.4 & 10.20 & 1.24 & 1.38 & 1.52 \\
\hline ML2 & 217.1 & 8.53 & 1.24 & 1.39 & 1.59 \\
\hline ML3 & 199.2 & 7.57 & 1.23 & 1.40 & 1.70 \\
\hline ML4 & 233.8 & 9.17 & 1.23 & 1.39 & 1.55 \\
\hline ML5 & 235.3 & 9.05 & 1.23 & 1.39 & 1.55 \\
\hline ML6 & 210.3 & 7.09 & 1.25 & 1.42 & 1.72 \\
\hline MH1 & 412.6 & 7.16 & 1.24 & 1.43 & 1.85 \\
\hline MH2 & 359.1 & 7.20 & 1.26 & 1.57 & 2.29 \\
\hline MH3 & 197.8 & 10.33 & 1.20 & 1.48 & 1.95 \\
\hline MH4 & 336.7 & 8.68 & 1.23 & 1.42 & 1.78 \\
\hline MH5 & 331.6 & 7.65 & 1.27 & 1.58 & 2.23 \\
\hline MH6 & 515.7 & 4.82 & 1.31 & 1.52 & 1.89 \\
\hline LL1 & 685.3 & 7.03 & 1.25 & 1.41 & 1.70 \\
\hline LL2 & 684.1 & 6.94 & 1.26 & 1.44 & 1.74 \\
\hline LL3 & 531.7 & 6.98 & 1.33 & 2.05 & 2.31 \\
\hline LL4 & 778.9 & 6.05 & 1.28 & 1.48 & 1.85 \\
\hline LL5 & 780.5 & 6.06 & 1.28 & 1.47 & 1.81 \\
\hline LL6 & 302.5 & 6.28 & 1.26 & 1.51 & 1.98 \\
\hline LH1 & 1111.2 & 6.56 & 1.50 & 2.10 & 2.38 \\
\hline LH2 & 1053.8 & 6.23 & 1.80 & 2.15 & 2.44 \\
\hline LH3 & 653.2 & 6.99 & 1.38 & 2.03 & 2.28 \\
\hline LH4 & 1020.8 & 6.31 & 1.78 & 2.11 & 2.39 \\
\hline LH5 & 998.5 & 6.46 & 1.77 & 2.10 & 2.39 \\
\hline LH6 & 641.5 & 6.40 & 1.38 & 2.14 & 2.38 \\
\hline
\end{tabular}


Table B.32 - The response of the buildings subjected to E6C13 ground motion parallel to the short side of the building with $\alpha=0.05$

\begin{tabular}{|c|c|c|c|c|c|}
\hline \multirow{2}{*}{ Building } & \multirow{2}{*}{$V e$} & \multirow{2}{*}{$\begin{array}{c}\text { Ductility }(\mu) \\
R y=3\end{array}$} & \multicolumn{3}{|c|}{ Force Reduction Factor $(R y)$} \\
\hline & & & $\mu=2$ & $\mu=3$ & $\mu=4$ \\
\hline SL1 & 96.0 & 8.71 & 1.36 & 1.55 & 1.72 \\
\hline SL3 & 90.5 & 9.33 & 1.34 & 1.53 & 1.69 \\
\hline SL4 & 74.2 & 11.22 & 1.29 & 1.45 & 1.59 \\
\hline SH1 & 89.1 & 9.63 & 1.44 & 1.69 & 1.91 \\
\hline $\mathrm{SH} 2$ & 90.2 & 10.80 & 1.35 & 1.57 & 1.77 \\
\hline $\mathrm{SH} 3$ & 89.9 & 10.56 & 1.33 & 1.55 & 1.73 \\
\hline SH4 & 91.4 & 11.57 & 1.30 & 1.49 & 1.66 \\
\hline SH5 & 91.7 & 10.80 & 1.31 & 1.52 & 1.70 \\
\hline SH6 & 89.8 & 10.58 & 1.32 & 1.55 & 1.73 \\
\hline ML1 & 163.0 & 9.26 & 1.28 & 1.46 & 1.63 \\
\hline ML2 & 142.5 & 7.55 & 1.24 & 1.42 & 1.68 \\
\hline ML3 & 137.5 & 7.76 & 1.27 & 1.67 & 2.00 \\
\hline ML4 & 153.1 & 8.82 & 1.25 & 1.41 & 1.55 \\
\hline ML5 & 153.6 & 8.84 & 1.25 & 1.41 & 1.55 \\
\hline ML6 & 140.8 & 7.24 & 1.26 & 1.51 & 1.92 \\
\hline MH1 & 307.7 & 7.04 & 1.38 & 1.59 & 1.85 \\
\hline MH2 & 334.2 & 5.12 & 1.24 & 1.82 & 2.64 \\
\hline MH3 & 239.4 & 6.91 & 1.21 & 1.86 & 2.33 \\
\hline MH4 & 342.0 & 5.91 & 1.21 & 1.65 & 2.37 \\
\hline MH5 & 351.3 & 5.40 & 1.26 & 2.07 & 2.46 \\
\hline MH6 & 361.9 & 5.90 & 1.39 & 1.68 & 1.93 \\
\hline LL1 & 619.8 & 5.02 & 1.26 & 2.13 & 2.67 \\
\hline LL2 & 633.1 & 5.89 & 1.28 & 2.21 & 2.72 \\
\hline LL3 & 627.5 & 5.07 & 1.46 & 1.72 & 2.15 \\
\hline LL4 & 559.7 & 5.79 & 1.31 & 1.77 & 2.49 \\
\hline LL5 & 565.5 & 5.68 & 1.31 & 1.72 & 2.52 \\
\hline LL6 & 300.7 & 7.93 & 1.28 & 1.48 & 1.95 \\
\hline LH1 & 1319.4 & 4.33 & 1.40 & 1.80 & 2.78 \\
\hline LH2 & 1206.6 & 4.68 & 1.35 & 2.34 & 2.74 \\
\hline LH3 & 823.6 & 5.91 & 1.40 & 2.13 & 2.44 \\
\hline LH4 & 1180.1 & 4.72 & 1.33 & 2.32 & 2.73 \\
\hline LH5 & 1153.7 & 4.84 & 1.31 & 2.30 & 2.71 \\
\hline LH6 & 772.2 & 6.27 & 1.35 & 2.11 & 2.42 \\
\hline
\end{tabular}


Table B.33 - The response of the buildings subjected to E6C15 ground motion parallel to the short side of the building with $\alpha=0.05$

\begin{tabular}{|c|c|c|c|c|c|}
\hline \multirow{2}{*}{ Building } & \multirow{2}{*}{$V e$} & \multirow{2}{*}{$\frac{\text { Ductility }(\mu)}{R y=3}$} & \multicolumn{3}{|c|}{ Force Reduction Factor $(R y)$} \\
\hline & & & $\mu=2$ & $\mu=3$ & $\mu=4$ \\
\hline SL1 & 71.8 & 9.78 & 1.41 & 1.80 & 2.06 \\
\hline SL3 & 71.9 & 9.30 & 1.44 & 1.79 & 2.10 \\
\hline SL4 & 71.4 & 9.34 & 1.42 & 1.77 & 2.13 \\
\hline SH1 & 168.7 & 6.99 & 1.27 & 1.49 & 1.89 \\
\hline $\mathrm{SH} 2$ & 136.4 & 8.62 & 1.22 & 1.48 & 1.72 \\
\hline $\mathrm{SH} 3$ & 120.4 & 9.54 & 1.21 & 1.44 & 1.67 \\
\hline SH4 & 104.7 & 12.24 & 1.19 & 1.33 & 1.48 \\
\hline SH5 & 103.7 & 11.53 & 1.21 & 1.35 & 1.52 \\
\hline SH6 & 120.1 & 9.59 & 1.21 & 1.45 & 1.66 \\
\hline ML1 & 147.6 & 14.49 & 1.34 & 1.46 & 1.57 \\
\hline ML2 & 120.6 & 17.89 & 1.19 & 1.41 & 1.51 \\
\hline ML3 & 123.2 & 15.30 & 1.26 & 1.41 & 1.52 \\
\hline ML4 & 131.5 & 16.12 & 1.30 & 1.43 & 1.55 \\
\hline ML5 & 132.0 & 16.52 & 1.29 & 1.42 & 1.54 \\
\hline ML6 & 120.5 & 15.61 & 1.19 & 1.41 & 1.51 \\
\hline MH1 & 283.6 & 12.36 & 1.32 & 1.45 & 1.57 \\
\hline MH2 & 286.3 & 7.96 & 1.31 & 1.48 & 1.64 \\
\hline MH3 & 163.6 & 9.10 & 1.32 & 1.86 & 2.04 \\
\hline MH4 & 276.6 & 8.86 & 1.29 & 1.45 & 1.59 \\
\hline MH5 & 270.9 & 6.78 & 1.31 & 1.48 & 1.72 \\
\hline MH6 & 319.9 & 8.47 & 1.48 & 1.68 & 1.85 \\
\hline LL1 & 644.0 & 6.81 & 1.42 & 1.66 & 1.95 \\
\hline LL2 & 640.2 & 6.53 & 1.40 & 1.63 & 1.91 \\
\hline LL3 & 410.6 & 6.47 & 1.29 & 1.83 & 2.17 \\
\hline LL4 & 636.8 & 7.45 & 1.47 & 1.69 & 1.95 \\
\hline LL5 & 638.3 & 7.47 & 1.47 & 1.70 & 1.98 \\
\hline LL6 & 271.3 & 10.79 & 1.35 & 1.58 & 1.78 \\
\hline LH1 & 812.3 & 6.91 & 1.31 & 1.85 & 2.26 \\
\hline LH2 & 701.6 & 7.67 & 1.43 & 1.77 & 2.00 \\
\hline LH3 & 660.9 & 6.97 & 1.61 & 1.91 & 2.15 \\
\hline LH4 & 695.3 & 7.62 & 1.45 & 1.81 & 2.04 \\
\hline LH5 & 667.2 & 7.82 & 1.40 & 1.77 & 1.99 \\
\hline LH6 & 621.0 & 7.95 & 1.48 & 1.75 & 1.99 \\
\hline
\end{tabular}


Table B.34 - The response of the buildings subjected to E6C18 ground motion parallel to the short side of the building with $\alpha=0.05$

\begin{tabular}{|c|c|c|c|c|c|}
\hline \multirow{2}{*}{ Building } & \multirow{2}{*}{$V e$} & \multirow{2}{*}{$\begin{array}{c}\text { Ductility }(\mu) \\
R y=3\end{array}$} & \multicolumn{3}{|c|}{ Force Reduction Factor $(R y)$} \\
\hline & & & $\mu=2$ & $\mu=3$ & $\mu=4$ \\
\hline SL1 & 90.4 & 8.56 & 1.31 & 1.47 & 1.73 \\
\hline SL3 & 82.3 & 10.24 & 1.31 & 1.46 & 1.69 \\
\hline SL4 & 64.4 & 16.33 & 1.33 & 1.51 & 1.65 \\
\hline SH1 & 124.1 & 10.19 & 1.21 & 1.36 & 1.51 \\
\hline $\mathrm{SH} 2$ & 125.3 & 8.61 & 1.24 & 1.40 & 1.55 \\
\hline $\mathrm{SH} 3$ & 125.2 & 7.30 & 1.27 & 1.45 & 1.61 \\
\hline SH4 & 122.3 & 7.72 & 1.26 & 1.44 & 1.59 \\
\hline SH5 & 122.3 & 9.76 & 1.28 & 1.46 & 1.64 \\
\hline SH6 & 125.1 & 7.32 & 1.26 & 1.45 & 1.62 \\
\hline ML1 & 197.8 & 6.79 & 1.27 & 1.45 & 1.76 \\
\hline ML2 & 152.6 & 9.88 & 1.26 & 1.72 & 1.92 \\
\hline ML3 & 137.6 & 9.81 & 1.48 & 1.70 & 1.90 \\
\hline ML4 & 179.1 & 8.24 & 1.24 & 1.50 & 2.15 \\
\hline ML5 & 180.1 & 8.06 & 1.24 & 1.47 & 2.18 \\
\hline ML6 & 146.0 & 9.41 & 1.51 & 1.73 & 1.94 \\
\hline MH1 & 280.1 & 9.23 & 1.48 & 1.71 & 1.92 \\
\hline MH2 & 249.6 & 7.77 & 1.59 & 1.85 & 2.09 \\
\hline MH3 & 202.5 & 5.06 & 1.67 & 2.04 & 2.50 \\
\hline MH4 & 242.8 & 8.83 & 1.29 & 1.82 & 2.04 \\
\hline MH5 & 237.4 & 7.60 & 1.62 & 1.89 & 2.12 \\
\hline MH6 & 419.6 & 4.62 & 1.38 & 2.42 & 2.78 \\
\hline LL1 & 507.2 & 7.60 & 1.61 & 1.89 & 2.14 \\
\hline LL2 & 499.4 & 7.73 & 1.60 & 1.86 & 2.13 \\
\hline LL3 & 365.8 & 6.90 & 1.67 & 1.97 & 2.23 \\
\hline LL4 & 573.8 & 7.04 & 1.67 & 1.96 & 2.23 \\
\hline LL5 & 579.6 & 7.01 & 1.69 & 1.98 & 2.24 \\
\hline LL6 & 200.3 & 6.38 & 1.33 & 1.55 & 1.91 \\
\hline LH1 & 751.5 & 6.29 & 1.69 & 2.02 & 2.23 \\
\hline LH2 & 701.8 & 5.94 & 1.68 & 2.05 & 2.37 \\
\hline LH3 & 680.8 & 3.51 & 2.11 & 2.75 & 3.30 \\
\hline LH4 & 699.5 & 5.81 & 1.73 & 2.09 & 2.45 \\
\hline LH5 & 693.0 & 5.65 & 1.74 & 2.12 & 2.46 \\
\hline LH6 & 642.6 & 3.76 & 2.12 & 2.63 & 3.15 \\
\hline
\end{tabular}


Table B.35 - The response of the buildings subjected to E6C42 ground motion parallel to the short side of the building with $\alpha=0.05$

\begin{tabular}{|c|c|c|c|c|c|}
\hline \multirow{2}{*}{ Building } & \multirow{2}{*}{$V e$} & \multirow{2}{*}{$\begin{array}{c}\text { Ductility }(\mu) \\
R y=3\end{array}$} & \multicolumn{3}{|c|}{ Force Reduction Factor $(R y)$} \\
\hline & & & $\mu=2$ & $\mu=3$ & $\mu=4$ \\
\hline SL1 & 72.6 & 6.81 & 1.26 & 1.44 & 1.63 \\
\hline SL3 & 70.0 & 6.71 & 1.25 & 1.43 & 1.67 \\
\hline SL4 & 62.2 & 8.43 & 1.27 & 1.67 & 2.06 \\
\hline SH1 & 115.8 & 11.83 & 1.27 & 1.41 & 1.54 \\
\hline $\mathrm{SH} 2$ & 106.4 & 13.44 & 1.22 & 1.33 & 1.44 \\
\hline $\mathrm{SH} 3$ & 108.6 & 11.79 & 1.26 & 1.39 & 1.52 \\
\hline SH4 & 109.5 & 12.55 & 1.29 & 1.42 & 1.55 \\
\hline SH5 & 109.7 & 11.25 & 1.31 & 1.44 & 1.58 \\
\hline SH6 & 108.8 & 11.73 & 1.27 & 1.40 & 1.53 \\
\hline ML1 & 192.8 & 9.93 & 1.37 & 1.56 & 1.71 \\
\hline ML2 & 156.3 & 11.05 & 1.30 & 1.49 & 1.65 \\
\hline ML3 & 140.7 & 11.80 & 1.30 & 1.45 & 1.60 \\
\hline ML4 & 182.9 & 9.67 & 1.35 & 1.55 & 1.74 \\
\hline ML5 & 184.1 & 9.68 & 1.34 & 1.55 & 1.73 \\
\hline ML6 & 149.9 & 10.43 & 1.32 & 1.50 & 1.65 \\
\hline MH1 & 323.9 & 10.67 & 1.44 & 1.60 & 1.75 \\
\hline MH2 & 328.4 & 9.78 & 1.54 & 1.70 & 1.86 \\
\hline MH3 & 298.8 & 10.00 & 1.44 & 1.79 & 1.99 \\
\hline MH4 & 314.1 & 12.42 & 1.44 & 1.60 & 1.73 \\
\hline MH5 & 313.1 & 10.87 & 1.47 & 1.65 & 1.80 \\
\hline MH6 & 403.3 & 5.88 & 1.46 & 1.71 & 2.03 \\
\hline LL1 & 731.6 & 6.68 & 1.35 & 1.75 & 2.36 \\
\hline LL2 & 716.9 & 6.91 & 1.35 & 1.72 & 2.32 \\
\hline LL3 & 586.4 & 9.65 & 1.58 & 1.80 & 1.99 \\
\hline LL4 & 664.7 & 7.51 & 1.58 & 1.82 & 2.02 \\
\hline LL5 & 669.3 & 7.50 & 1.59 & 1.82 & 2.02 \\
\hline LL6 & 426.9 & 9.26 & 1.49 & 1.73 & 1.95 \\
\hline LH1 & 1286.9 & 8.52 & 1.63 & 1.88 & 2.08 \\
\hline LH2 & 1265.6 & 7.79 & 1.68 & 1.94 & 2.16 \\
\hline LH3 & 1083.9 & 7.06 & 1.64 & 1.94 & 2.22 \\
\hline LH4 & 1246.3 & 8.12 & 1.61 & 1.89 & 2.12 \\
\hline LH5 & 1234.9 & 7.96 & 1.61 & 1.88 & 2.12 \\
\hline LH6 & 1045.4 & 7.06 & 1.64 & 1.94 & 2.22 \\
\hline
\end{tabular}


Table B.36 - The response of the buildings subjected to E6C1 ground motion parallel to the long side of the building with $\alpha=0.05$

\begin{tabular}{|c|c|c|c|c|c|}
\hline \multirow{2}{*}{ Building } & \multirow{2}{*}{$V e$} & \multirow{2}{*}{$\begin{array}{c}\text { Ductility }(\mu) \\
R y=3\end{array}$} & \multicolumn{3}{|c|}{ Force Reduction Factor $(R y)$} \\
\hline & & & $\mu=2$ & $\mu=3$ & $\mu=4$ \\
\hline SL1 & 90.2 & 9.80 & 1.68 & 2.09 & 2.25 \\
\hline SL3 & 92.6 & 9.36 & 1.73 & 2.07 & 2.26 \\
\hline SL4 & 97.0 & 8.59 & 1.43 & 1.88 & 2.25 \\
\hline SH1 & 197.4 & 7.66 & 1.38 & 1.63 & 1.87 \\
\hline $\mathrm{SH} 2$ & 198.5 & 7.86 & 1.35 & 1.59 & 1.81 \\
\hline $\mathrm{SH} 3$ & 201.3 & 7.29 & 1.37 & 1.61 & 1.87 \\
\hline SH4 & 198.9 & 8.02 & 1.34 & 1.56 & 1.77 \\
\hline SH5 & 201.7 & 7.35 & 1.36 & 1.60 & 1.85 \\
\hline ML1 & 376.8 & 6.58 & 1.38 & 1.65 & 1.90 \\
\hline ML2 & 372.8 & 6.64 & 1.36 & 1.62 & 1.86 \\
\hline ML3 & 362.9 & 5.99 & 1.36 & 1.64 & 1.91 \\
\hline ML4 & 374.4 & 6.48 & 1.37 & 1.63 & 1.88 \\
\hline ML5 & 370.6 & 6.59 & 1.35 & 1.61 & 1.85 \\
\hline ML6 & 365.7 & 5.95 & 1.37 & 1.66 & 1.92 \\
\hline MH1 & 774.8 & 5.72 & 1.41 & 1.70 & 1.97 \\
\hline MH2 & 723.6 & 5.08 & 1.44 & 1.72 & 2.01 \\
\hline MH3 & 558.6 & 4.92 & 1.26 & 1.47 & 1.67 \\
\hline MH4 & 758.2 & 5.96 & 1.40 & 1.68 & 1.94 \\
\hline MH5 & 695.5 & 5.39 & 1.42 & 1.68 & 1.92 \\
\hline MH6 & 752.7 & 4.34 & 1.49 & 1.83 & 2.44 \\
\hline LL1 & 1425.7 & 4.69 & 1.46 & 1.80 & 2.27 \\
\hline LL2 & 1401.2 & 4.86 & 1.46 & 1.79 & 2.22 \\
\hline LL3 & 1063.0 & 4.68 & 1.30 & 1.53 & 1.74 \\
\hline LL4 & 1424.4 & 4.73 & 1.46 & 1.81 & 2.25 \\
\hline LL5 & 1315.8 & 5.21 & 1.43 & 1.72 & 2.02 \\
\hline LL6 & 969.8 & 4.05 & 1.35 & 1.60 & 2.98 \\
\hline LH1 & 2972.3 & 3.48 & 1.54 & 1.97 & 4.03 \\
\hline LH2 & 2758.1 & 3.06 & 1.53 & 2.13 & 3.81 \\
\hline LH3 & 2042.7 & 3.66 & 1.40 & 2.70 & 3.15 \\
\hline LH4 & 2917.1 & 2.95 & 1.58 & 3.49 & 4.11 \\
\hline LH5 & 2367.9 & 3.36 & 1.40 & 1.74 & 3.31 \\
\hline LH6 & 1945.1 & 3.75 & 1.42 & 2.66 & 3.11 \\
\hline
\end{tabular}


Table B.37 - The response of the buildings subjected to E6C13 ground motion parallel to the long side of the building with $\alpha=0.05$

\begin{tabular}{|c|c|c|c|c|c|}
\hline \multirow{2}{*}{ Building } & \multirow{2}{*}{$V e$} & \multirow{2}{*}{$\begin{array}{c}\text { Ductility }(\mu) \\
R y=3\end{array}$} & \multicolumn{3}{|c|}{ Force Reduction Factor $(R y)$} \\
\hline & & & $\mu=2$ & $\mu=3$ & $\mu=4$ \\
\hline SL1 & 138.0 & 3.30 & 1.40 & 2.72 & 3.45 \\
\hline SL3 & 139.9 & 3.41 & 1.40 & 2.68 & 3.53 \\
\hline SL4 & 144.9 & 3.33 & 1.41 & 2.62 & 3.76 \\
\hline SH1 & 268.2 & 2.86 & 1.94 & 3.08 & 3.56 \\
\hline $\mathrm{SH} 2$ & 277.7 & 3.06 & 1.54 & 2.86 & 3.76 \\
\hline $\mathrm{SH} 3$ & 273.0 & 3.25 & 1.58 & 2.56 & 3.93 \\
\hline SH4 & 272.9 & 3.60 & 1.55 & 2.27 & 3.42 \\
\hline SH5 & 264.7 & 3.47 & 1.57 & 2.30 & 3.90 \\
\hline ML1 & 314.0 & 5.37 & 1.42 & 1.69 & 2.22 \\
\hline ML2 & 266.4 & 6.18 & 1.36 & 1.62 & 1.95 \\
\hline ML3 & 216.3 & 6.79 & 1.36 & 1.65 & 1.95 \\
\hline ML4 & 277.1 & 6.06 & 1.37 & 1.63 & 1.99 \\
\hline ML5 & 255.4 & 6.58 & 1.35 & 1.60 & 1.90 \\
\hline ML6 & 230.4 & 6.14 & 1.37 & 1.67 & 1.99 \\
\hline MH1 & 342.3 & 9.21 & 1.41 & 1.66 & 1.90 \\
\hline MH2 & 319.3 & 7.38 & 1.57 & 1.89 & 2.18 \\
\hline MH3 & 355.0 & 6.59 & 1.48 & 1.84 & 2.20 \\
\hline MH4 & 326.2 & 9.59 & 1.42 & 1.68 & 1.90 \\
\hline MH5 & 306.4 & 8.01 & 1.52 & 1.82 & 2.08 \\
\hline MH6 & 324.6 & 6.54 & 1.63 & 1.96 & 2.22 \\
\hline LL1 & 598.0 & 7.95 & 1.42 & 1.70 & 1.95 \\
\hline LL2 & 564.9 & 8.37 & 1.45 & 1.70 & 1.93 \\
\hline LL3 & 607.3 & 6.62 & 1.51 & 1.88 & 2.24 \\
\hline LL4 & 594.5 & 8.10 & 1.43 & 1.70 & 1.95 \\
\hline LL5 & 573.2 & 7.81 & 1.55 & 1.88 & 2.14 \\
\hline LL6 & 656.4 & 5.79 & 1.45 & 1.79 & 2.11 \\
\hline LH1 & 1215.7 & 5.75 & 1.61 & 1.94 & 2.23 \\
\hline LH2 & 1239.1 & 5.39 & 1.55 & 1.98 & 2.35 \\
\hline LH3 & 1392.0 & 4.98 & 1.52 & 1.92 & 2.29 \\
\hline LH4 & 1207.4 & 5.00 & 1.59 & 1.98 & 2.32 \\
\hline LH5 & 1325.5 & 5.80 & 1.54 & 1.95 & 2.34 \\
\hline LH6 & 1355.7 & 4.31 & 1.47 & 1.84 & 2.52 \\
\hline
\end{tabular}


Table B.38 - The response of the buildings subjected to E6C15 ground motion parallel to the long side of the building with $\alpha=0.05$

\begin{tabular}{|c|c|c|c|c|c|}
\hline \multirow{2}{*}{ Building } & \multirow{2}{*}{$V e$} & \multirow{2}{*}{$\begin{array}{c}\text { Ductility }(\mu) \\
R y=3\end{array}$} & \multicolumn{3}{|c|}{ Force Reduction Factor $(R y)$} \\
\hline & & & $\mu=2$ & $\mu=3$ & $\mu=4$ \\
\hline SL1 & 136.8 & 6.06 & 1.71 & 2.04 & 2.35 \\
\hline SL3 & 134.0 & 6.22 & 1.68 & 2.01 & 2.33 \\
\hline SL4 & 127.0 & 6.64 & 1.66 & 1.95 & 2.25 \\
\hline SH1 & 175.0 & 7.57 & 1.48 & 1.78 & 2.05 \\
\hline $\mathrm{SH} 2$ & 166.9 & 7.99 & 1.47 & 1.76 & 2.01 \\
\hline $\mathrm{SH} 3$ & 158.5 & 7.65 & 1.47 & 1.76 & 2.04 \\
\hline SH4 & 158.2 & 8.28 & 1.47 & 1.73 & 1.99 \\
\hline SH5 & 162.5 & 7.31 & 1.51 & 1.86 & 2.12 \\
\hline ML1 & 295.8 & 5.70 & 1.52 & 2.01 & 2.49 \\
\hline ML2 & 289.3 & 5.72 & 1.54 & 2.22 & 2.53 \\
\hline ML3 & 282.6 & 5.44 & 1.59 & 2.21 & 2.43 \\
\hline ML4 & 290.9 & 5.71 & 1.54 & 2.13 & 2.52 \\
\hline ML5 & 287.6 & 5.92 & 1.56 & 2.24 & 2.51 \\
\hline ML6 & 284.2 & 5.21 & 1.55 & 2.26 & 2.49 \\
\hline MH1 & 726.5 & 3.59 & 1.72 & 2.52 & 3.29 \\
\hline MH2 & 854.8 & 2.59 & 1.39 & 3.44 & 4.55 \\
\hline MH3 & 486.9 & 4.96 & 1.47 & 1.96 & 2.46 \\
\hline MH4 & 778.3 & 3.27 & 1.54 & 2.76 & 3.65 \\
\hline MH5 & 813.0 & 3.00 & 1.37 & 2.99 & 4.07 \\
\hline MH6 & 897.9 & 2.22 & 2.46 & 4.23 & 4.89 \\
\hline LL1 & 1414.7 & 3.03 & 1.99 & 3.03 & 3.90 \\
\hline LL2 & 1524.5 & 2.67 & 2.04 & 3.40 & 4.23 \\
\hline LL3 & 1034.0 & 3.94 & 1.73 & 2.38 & 3.01 \\
\hline LL4 & 1425.8 & 2.85 & 2.02 & 3.01 & 3.94 \\
\hline LL5 & 1565.1 & 2.60 & 1.85 & 3.49 & 4.60 \\
\hline LL6 & 597.0 & 7.35 & 1.47 & 1.71 & 1.94 \\
\hline LH1 & 3340.8 & 2.21 & 2.46 & 4.26 & 5.02 \\
\hline LH2 & 2668.6 & 2.46 & 2.13 & 3.61 & 4.47 \\
\hline LH3 & 1324.9 & 6.36 & 1.60 & 1.98 & 2.47 \\
\hline LH4 & 3067.4 & 2.31 & 2.28 & 4.18 & 5.01 \\
\hline LH5 & 1822.4 & 3.65 & 1.91 & 2.60 & 3.22 \\
\hline LH6 & 1288.9 & 6.20 & 1.52 & 1.88 & 2.54 \\
\hline
\end{tabular}


Table B.39 - The response of the buildings subjected to E6C18 ground motion parallel to the long side of the building with $\alpha=0.05$

\begin{tabular}{|c|c|c|c|c|c|}
\hline \multirow{2}{*}{ Building } & \multirow{2}{*}{$V e$} & \multirow{2}{*}{$\begin{array}{c}\text { Ductility }(\mu) \\
R y=3\end{array}$} & \multicolumn{3}{|c|}{ Force Reduction Factor $(R y)$} \\
\hline & & & $\mu=2$ & $\mu=3$ & $\mu=4$ \\
\hline SL1 & 183.6 & 4.73 & 1.39 & 2.37 & 2.74 \\
\hline SL3 & 185.1 & 4.70 & 1.38 & 2.37 & 2.73 \\
\hline SL4 & 187.9 & 4.81 & 1.37 & 2.36 & 2.71 \\
\hline SH1 & 353.9 & 4.06 & 1.36 & 2.31 & 2.81 \\
\hline $\mathrm{SH} 2$ & 336.3 & 3.70 & 1.36 & 2.28 & 3.35 \\
\hline $\mathrm{SH} 3$ & 310.4 & 3.05 & 1.49 & 2.89 & 4.18 \\
\hline SH4 & 309.1 & 3.41 & 1.37 & 2.60 & 3.69 \\
\hline SH5 & 290.3 & 2.92 & 1.49 & 3.04 & 3.94 \\
\hline ML1 & 277.4 & 6.92 & 1.51 & 1.77 & 2.05 \\
\hline ML2 & 243.2 & 8.96 & 1.56 & 1.75 & 1.87 \\
\hline ML3 & 234.9 & 9.49 & 1.62 & 1.85 & 2.00 \\
\hline ML4 & 248.6 & 8.46 & 1.55 & 1.73 & 1.86 \\
\hline ML5 & 239.4 & 9.57 & 1.57 & 1.77 & 1.89 \\
\hline ML6 & 234.5 & 8.95 & 1.62 & 1.82 & 1.97 \\
\hline MH1 & 542.7 & 8.77 & 1.57 & 1.89 & 2.15 \\
\hline MH2 & 541.7 & 7.39 & 1.44 & 1.73 & 2.01 \\
\hline MH3 & 505.9 & 3.87 & 1.40 & 1.70 & 3.31 \\
\hline MH4 & 535.1 & 9.00 & 1.51 & 1.80 & 2.06 \\
\hline MH5 & 535.3 & 6.94 & 1.40 & 1.69 & 1.95 \\
\hline MH6 & 550.8 & 6.70 & 1.52 & 1.86 & 2.18 \\
\hline LL1 & 989.4 & 7.48 & 1.57 & 1.91 & 2.22 \\
\hline LL2 & 985.6 & 7.61 & 1.53 & 1.85 & 2.14 \\
\hline LL3 & 923.2 & 4.37 & 1.39 & 1.69 & 2.31 \\
\hline LL4 & 989.3 & 7.48 & 1.56 & 1.91 & 2.22 \\
\hline LL5 & 977.5 & 7.69 & 1.43 & 1.74 & 2.01 \\
\hline LL6 & 786.1 & 3.81 & 1.44 & 2.63 & 3.10 \\
\hline LH1 & 2110.3 & 5.79 & 1.47 & 1.83 & 2.17 \\
\hline LH2 & 2008.9 & 4.00 & 1.46 & 1.84 & 3.00 \\
\hline LH3 & 1580.5 & 3.53 & 2.15 & 2.72 & 3.25 \\
\hline LH4 & 2048.8 & 4.52 & 1.48 & 1.86 & 2.33 \\
\hline LH5 & 1939.9 & 2.92 & 1.48 & 2.47 & 5.34 \\
\hline LH6 & 1411.4 & 4.07 & 1.97 & 2.47 & 2.95 \\
\hline
\end{tabular}


Table B.40 - The response of the buildings subjected to E6C42 ground motion parallel to the long side of the building with $\alpha=0.05$

\begin{tabular}{|c|c|c|c|c|c|}
\hline \multirow{2}{*}{ Building } & \multirow{2}{*}{$V e$} & \multirow{2}{*}{$\begin{array}{c}\text { Ductility }(\mu) \\
R y=3\end{array}$} & \multicolumn{3}{|c|}{ Force Reduction Factor $(R y)$} \\
\hline & & & $\mu=2$ & $\mu=3$ & $\mu=4$ \\
\hline SL1 & 172.3 & 4.76 & 1.88 & 2.25 & 2.58 \\
\hline SL3 & 165.3 & 4.75 & 1.89 & 2.26 & 2.63 \\
\hline SL4 & 142.9 & 4.80 & 1.44 & 2.31 & 2.69 \\
\hline SH1 & 224.2 & 4.84 & 1.40 & 1.74 & 2.56 \\
\hline $\mathrm{SH} 2$ & 199.8 & 4.99 & 1.38 & 1.64 & 2.11 \\
\hline $\mathrm{SH} 3$ & 183.4 & 4.03 & 1.37 & 1.65 & 2.03 \\
\hline SH4 & 178.1 & 4.54 & 1.34 & 1.58 & 1.79 \\
\hline SH5 & 176.8 & 3.83 & 1.37 & 1.63 & 1.87 \\
\hline ML1 & 270.0 & 4.42 & 1.82 & 2.47 & 2.92 \\
\hline ML2 & 232.6 & 6.56 & 1.41 & 1.97 & 2.38 \\
\hline ML3 & 246.2 & 4.87 & 1.38 & 1.74 & 2.48 \\
\hline ML4 & 242.7 & 6.13 & 1.51 & 2.19 & 2.55 \\
\hline ML5 & 231.9 & 6.28 & 1.37 & 1.85 & 2.32 \\
\hline ML6 & 241.9 & 5.14 & 1.39 & 1.84 & 2.47 \\
\hline MH1 & 584.0 & 4.08 & 1.40 & 1.69 & 2.96 \\
\hline MH2 & 555.6 & 3.66 & 1.40 & 1.95 & 3.13 \\
\hline MH3 & 423.0 & 5.44 & 1.29 & 1.50 & 1.84 \\
\hline MH4 & 582.5 & 4.12 & 1.39 & 1.66 & 2.97 \\
\hline MH5 & 536.4 & 3.72 & 1.39 & 1.91 & 3.11 \\
\hline MH6 & 562.1 & 3.34 & 1.43 & 2.84 & 3.33 \\
\hline LL1 & 1051.5 & 3.62 & 1.44 & 1.79 & 3.28 \\
\hline LL2 & 1047.9 & 3.63 & 1.43 & 1.83 & 3.16 \\
\hline LL3 & 780.7 & 5.00 & 1.29 & 1.51 & 1.92 \\
\hline LL4 & 1051.6 & 3.60 & 1.44 & 1.81 & 3.25 \\
\hline LL5 & 1005.8 & 3.76 & 1.39 & 1.91 & 3.09 \\
\hline LL6 & 748.6 & 4.71 & 1.48 & 1.78 & 2.30 \\
\hline LH1 & 2122.3 & 3.04 & 1.47 & 2.98 & 3.67 \\
\hline LH2 & 1879.5 & 2.99 & 1.45 & 3.03 & 3.61 \\
\hline LH3 & 1531.2 & 3.71 & 1.53 & 1.92 & 3.53 \\
\hline LH4 & 2003.8 & 2.83 & 1.49 & 3.10 & 3.94 \\
\hline LH5 & 1683.2 & 3.68 & 1.38 & 1.76 & 3.20 \\
\hline LH6 & 1390.8 & 3.95 & 1.51 & 1.89 & 2.97 \\
\hline
\end{tabular}


Table B.41 - The response of the buildings subjected to M6C1 ground motion parallel to the short side of the building with $\alpha=0.10$

\begin{tabular}{|c|c|c|c|c|c|}
\hline \multirow{2}{*}{ Building } & \multirow{2}{*}{$V e$} & \multirow{2}{*}{$\frac{\text { Ductility }(\mu)}{R y=3}$} & \multicolumn{3}{|c|}{ Force Reduction Factor $(R y)$} \\
\hline & & & $\mu=2$ & $\mu=3$ & $\mu=4$ \\
\hline SL1 & 131.5 & 9.39 & 1.30 & 1.53 & 1.82 \\
\hline SL3 & 133.1 & 9.09 & 1.31 & 1.53 & 1.78 \\
\hline SL4 & 142.1 & 8.32 & 1.29 & 1.54 & 1.80 \\
\hline SH1 & 316.8 & 6.37 & 1.29 & 1.83 & 2.27 \\
\hline $\mathrm{SH} 2$ & 283.9 & 7.89 & 1.45 & 1.70 & 1.98 \\
\hline $\mathrm{SH} 3$ & 264.2 & 8.12 & 1.36 & 1.60 & 1.90 \\
\hline SH4 & 260.9 & 9.15 & 1.31 & 1.52 & 1.78 \\
\hline SH5 & 262.9 & 8.35 & 1.36 & 1.56 & 1.84 \\
\hline SH6 & 263.0 & 8.18 & 1.36 & 1.59 & 1.89 \\
\hline ML1 & 508.8 & 7.26 & 1.45 & 1.71 & 1.97 \\
\hline ML2 & 435.8 & 8.76 & 1.28 & 1.61 & 1.79 \\
\hline ML3 & 417.7 & 8.08 & 1.33 & 1.62 & 1.81 \\
\hline ML4 & 490.8 & 7.63 & 1.42 & 1.71 & 1.91 \\
\hline ML5 & 493.7 & 7.71 & 1.40 & 1.71 & 1.91 \\
\hline ML6 & 428.7 & 7.96 & 1.34 & 1.63 & 1.82 \\
\hline MH1 & 878.8 & 7.36 & 1.38 & 1.62 & 1.82 \\
\hline MH2 & 832.1 & 5.72 & 1.46 & 1.69 & 1.94 \\
\hline MH3 & 500.5 & 5.19 & 1.64 & 2.35 & 2.68 \\
\hline MH4 & 832.5 & 6.86 & 1.43 & 1.69 & 1.90 \\
\hline MH5 & 851.3 & 5.35 & 1.42 & 1.81 & 2.06 \\
\hline MH6 & 1143.3 & 4.06 & 1.63 & 2.09 & 2.96 \\
\hline LL1 & 1523.3 & 5.86 & 1.33 & 1.58 & 2.09 \\
\hline LL2 & 1503.8 & 5.93 & 1.32 & 1.61 & 2.09 \\
\hline LL3 & 1496.1 & 3.46 & 1.60 & 2.50 & 3.33 \\
\hline LL4 & 1716.3 & 5.65 & 1.42 & 1.66 & 2.12 \\
\hline LL5 & 1735.1 & 5.60 & 1.43 & 1.66 & 2.12 \\
\hline LL6 & 469.1 & 11.25 & 1.32 & 1.53 & 1.73 \\
\hline LH1 & 2933.9 & 3.44 & 1.61 & 2.70 & 3.36 \\
\hline LH2 & 2472.9 & 3.86 & 1.62 & 2.43 & 3.09 \\
\hline LH3 & 1269.3 & 8.53 & 1.52 & 1.80 & 2.03 \\
\hline LH4 & 2409.6 & 3.77 & 1.62 & 2.47 & 3.13 \\
\hline LH5 & 2344.8 & 3.77 & 1.58 & 2.48 & 3.17 \\
\hline LH6 & 1212.9 & 8.61 & 1.52 & 1.78 & 2.03 \\
\hline
\end{tabular}


Table B.42 - The response of the buildings subjected to M6C2 ground motion parallel to the short side of the building with $\alpha=0.10$

\begin{tabular}{|c|c|c|c|c|c|}
\hline \multirow{2}{*}{ Building } & \multirow{2}{*}{$V e$} & \multirow{2}{*}{$\begin{array}{c}\text { Ductility }(\mu) \\
R y=3\end{array}$} & \multicolumn{3}{|c|}{ Force Reduction Factor $(R y)$} \\
\hline & & & $\mu=2$ & $\mu=3$ & $\mu=4$ \\
\hline SL1 & 135.5 & 7.84 & 1.26 & 1.45 & 1.68 \\
\hline SL3 & 137.2 & 8.21 & 1.25 & 1.43 & 1.64 \\
\hline SL4 & 138.2 & 8.87 & 1.24 & 1.40 & 1.55 \\
\hline SH1 & 226.8 & 8.05 & 1.25 & 1.62 & 1.98 \\
\hline $\mathrm{SH} 2$ & 223.9 & 7.96 & 1.25 & 1.54 & 1.88 \\
\hline $\mathrm{SH} 3$ & 223.3 & 7.27 & 1.29 & 1.62 & 2.01 \\
\hline SH4 & 216.4 & 7.71 & 1.29 & 1.55 & 1.89 \\
\hline SH5 & 215.9 & 7.10 & 1.31 & 1.62 & 1.96 \\
\hline SH6 & 223.2 & 7.28 & 1.28 & 1.64 & 2.03 \\
\hline ML1 & 276.6 & 9.31 & 1.25 & 1.44 & 1.66 \\
\hline ML2 & 211.1 & 12.37 & 1.22 & 1.35 & 1.54 \\
\hline ML3 & 234.5 & 10.19 & 1.23 & 1.46 & 1.73 \\
\hline ML4 & 231.1 & 10.86 & 1.23 & 1.38 & 1.59 \\
\hline ML5 & 235.5 & 10.67 & 1.24 & 1.39 & 1.59 \\
\hline ML6 & 221.4 & 10.64 & 1.26 & 1.41 & 1.67 \\
\hline MH1 & 583.3 & 7.95 & 1.27 & 1.70 & 2.02 \\
\hline MH2 & 687.7 & 5.19 & 1.51 & 1.85 & 2.26 \\
\hline MH3 & 580.2 & 6.14 & 1.50 & 1.85 & 2.30 \\
\hline MH4 & 697.4 & 5.89 & 1.33 & 1.74 & 2.09 \\
\hline MH5 & 706.7 & 4.84 & 1.42 & 1.82 & 2.37 \\
\hline MH6 & 568.5 & 5.85 & 1.40 & 1.64 & 2.05 \\
\hline LL1 & 1105.5 & 7.29 & 1.53 & 1.90 & 2.13 \\
\hline LL2 & 1108.6 & 7.22 & 1.55 & 1.88 & 2.13 \\
\hline LL3 & 1169.1 & 5.04 & 1.40 & 2.06 & 2.57 \\
\hline LL4 & 944.8 & 7.91 & 1.36 & 1.74 & 1.94 \\
\hline LL5 & 932.3 & 8.10 & 1.38 & 1.72 & 1.92 \\
\hline LL6 & 554.6 & 10.46 & 1.26 & 1.43 & 1.60 \\
\hline LH1 & 2431.3 & 5.07 & 1.39 & 2.08 & 2.43 \\
\hline LH2 & 2373.5 & 4.74 & 1.70 & 2.08 & 2.49 \\
\hline LH3 & 1828.0 & 7.06 & 1.54 & 1.95 & 2.17 \\
\hline LH4 & 2391.1 & 4.83 & 1.59 & 2.11 & 2.47 \\
\hline LH5 & 2440.7 & 4.70 & 1.61 & 2.17 & 2.54 \\
\hline LH6 & 1494.5 & 8.45 & 1.40 & 1.63 & 1.82 \\
\hline
\end{tabular}


Table B.43 - The response of the buildings subjected to M6C26 ground motion parallel to the short side of the building with $\alpha=0.10$

\begin{tabular}{|c|c|c|c|c|c|}
\hline \multirow{2}{*}{ Building } & \multirow{2}{*}{$V e$} & \multirow{2}{*}{$\frac{\text { Ductility }(\mu)}{R y=3}$} & \multicolumn{3}{|c|}{ Force Reduction Factor $(R y)$} \\
\hline & & & $\mu=2$ & $\mu=3$ & $\mu=4$ \\
\hline SL1 & 164.3 & 10.30 & 1.21 & 1.37 & 1.52 \\
\hline SL3 & 156.3 & 10.79 & 1.20 & 1.37 & 1.51 \\
\hline SL4 & 135.3 & 12.31 & 1.18 & 1.34 & 1.48 \\
\hline SH1 & 333.5 & 8.92 & 1.30 & 1.47 & 1.66 \\
\hline $\mathrm{SH} 2$ & 314.4 & 9.71 & 1.27 & 1.44 & 1.61 \\
\hline $\mathrm{SH} 3$ & 304.8 & 9.08 & 1.27 & 1.45 & 1.62 \\
\hline $\mathrm{SH} 4$ & 293.8 & 9.95 & 1.27 & 1.43 & 1.59 \\
\hline SH5 & 294.7 & 9.23 & 1.28 & 1.45 & 1.62 \\
\hline SH6 & 304.1 & 9.11 & 1.27 & 1.45 & 1.61 \\
\hline ML1 & 474.2 & 8.27 & 1.29 & 1.57 & 1.75 \\
\hline ML2 & 379.0 & 8.87 & 1.24 & 1.60 & 1.91 \\
\hline ML3 & 358.2 & 8.12 & 1.28 & 1.80 & 2.04 \\
\hline ML4 & 432.4 & 8.37 & 1.23 & 1.58 & 1.83 \\
\hline ML5 & 436.6 & 8.34 & 1.23 & 1.58 & 1.81 \\
\hline ML6 & 369.5 & 8.28 & 1.28 & 1.73 & 2.02 \\
\hline MH1 & 739.4 & 7.62 & 1.33 & 1.84 & 2.09 \\
\hline MH2 & 669.2 & 7.16 & 1.54 & 1.81 & 2.06 \\
\hline MH3 & 449.0 & 6.42 & 1.35 & 1.99 & 2.28 \\
\hline MH4 & 624.9 & 8.26 & 1.44 & 1.67 & 1.88 \\
\hline MH5 & 612.2 & 7.36 & 1.44 & 1.68 & 1.92 \\
\hline MH6 & 996.4 & 4.45 & 1.52 & 1.78 & 2.47 \\
\hline LL1 & 1154.3 & 7.50 & 1.33 & 1.72 & 2.13 \\
\hline LL2 & 1137.2 & 7.59 & 1.34 & 1.75 & 2.14 \\
\hline LL3 & 949.1 & 6.80 & 1.36 & 1.61 & 1.93 \\
\hline LL4 & 1226.8 & 7.59 & 1.26 & 1.75 & 2.07 \\
\hline LL5 & 1239.5 & 7.52 & 1.27 & 1.76 & 2.06 \\
\hline LL6 & 439.4 & 7.53 & 1.35 & 1.61 & 1.93 \\
\hline LH1 & 2064.4 & 5.64 & 1.43 & 1.76 & 2.22 \\
\hline LH2 & 2116.3 & 4.32 & 1.59 & 2.15 & 2.74 \\
\hline LH3 & 1321.5 & 5.31 & 1.36 & 2.02 & 2.45 \\
\hline LH4 & 1997.1 & 4.57 & 1.49 & 2.01 & 2.61 \\
\hline LH5 & 1961.0 & 4.32 & 1.48 & 2.05 & 2.70 \\
\hline LH6 & 1191.4 & 6.07 & 1.43 & 1.74 & 2.08 \\
\hline
\end{tabular}


Table B.44 - The response of the buildings subjected to M6C31 ground motion parallel to the short side of the building with $\alpha=0.10$

\begin{tabular}{|c|c|c|c|c|c|}
\hline \multirow{2}{*}{ Building } & \multirow{2}{*}{$V e$} & \multirow{2}{*}{$\begin{array}{c}\text { Ductility }(\mu) \\
R y=3\end{array}$} & \multicolumn{3}{|c|}{ Force Reduction Factor $(R y)$} \\
\hline & & & $\mu=2$ & $\mu=3$ & $\mu=4$ \\
\hline SL1 & 202.1 & 9.33 & 1.19 & 1.36 & 1.61 \\
\hline SL3 & 210.1 & 9.09 & 1.20 & 1.36 & 1.62 \\
\hline SL4 & 214.7 & 9.00 & 1.19 & 1.34 & 1.57 \\
\hline SH1 & 244.8 & 10.82 & 1.26 & 1.46 & 1.64 \\
\hline $\mathrm{SH} 2$ & 262.1 & 9.63 & 1.23 & 1.40 & 1.55 \\
\hline $\mathrm{SH} 3$ & 254.3 & 8.86 & 1.22 & 1.39 & 1.55 \\
\hline SH4 & 234.2 & 9.74 & 1.19 & 1.34 & 1.51 \\
\hline SH5 & 233.5 & 8.92 & 1.19 & 1.35 & 1.55 \\
\hline SH6 & 253.9 & 8.78 & 1.22 & 1.38 & 1.55 \\
\hline ML1 & 304.9 & 9.89 & 1.20 & 1.39 & 1.63 \\
\hline ML2 & 209.1 & 11.95 & 1.28 & 1.44 & 1.57 \\
\hline ML3 & 208.7 & 9.75 & 1.31 & 1.47 & 1.60 \\
\hline ML4 & 255.2 & 11.30 & 1.25 & 1.41 & 1.56 \\
\hline ML5 & 259.3 & 11.29 & 1.25 & 1.40 & 1.56 \\
\hline ML6 & 207.3 & 10.59 & 1.31 & 1.46 & 1.60 \\
\hline MH1 & 457.8 & 8.70 & 1.30 & 1.47 & 1.63 \\
\hline MH2 & 461.3 & 7.73 & 1.30 & 1.47 & 1.72 \\
\hline MH3 & 311.8 & 10.77 & 1.24 & 1.48 & 1.65 \\
\hline MH4 & 449.6 & 9.16 & 1.26 & 1.42 & 1.59 \\
\hline MH5 & 455.8 & 7.72 & 1.29 & 1.46 & 1.68 \\
\hline MH6 & 623.8 & 5.70 & 1.42 & 1.87 & 2.24 \\
\hline LL1 & 860.2 & 7.58 & 1.28 & 1.48 & 1.67 \\
\hline LL2 & 856.1 & 7.48 & 1.28 & 1.48 & 1.67 \\
\hline LL3 & 759.8 & 6.79 & 1.26 & 1.46 & 1.80 \\
\hline LL4 & 778.9 & 9.13 & 1.23 & 1.41 & 1.60 \\
\hline LL5 & 789.1 & 9.04 & 1.24 & 1.42 & 1.61 \\
\hline LL6 & 663.0 & 10.70 & 1.31 & 1.52 & 1.71 \\
\hline LH1 & 1584.7 & 6.18 & 1.31 & 1.55 & 1.86 \\
\hline LH2 & 1537.3 & 5.79 & 1.38 & 1.63 & 2.02 \\
\hline LH3 & 1278.1 & 10.16 & 1.27 & 1.46 & 1.64 \\
\hline LH4 & 1505.2 & 5.80 & 1.35 & 1.62 & 2.03 \\
\hline LH5 & 1489.8 & 5.83 & 1.35 & 1.62 & 2.07 \\
\hline LH6 & 1334.3 & 9.92 & 1.28 & 1.49 & 1.67 \\
\hline
\end{tabular}


Table B.45 - The response of the buildings subjected to M6C38 ground motion parallel to the short side of the building with $\alpha=0.10$

\begin{tabular}{|c|c|c|c|c|c|}
\hline \multirow{2}{*}{ Building } & \multirow{2}{*}{$V e$} & \multirow{2}{*}{$\begin{array}{c}\text { Ductility }(\mu) \\
R y=3\end{array}$} & \multicolumn{3}{|c|}{ Force Reduction Factor $(R y)$} \\
\hline & & & $\mu=2$ & $\mu=3$ & $\mu=4$ \\
\hline SL1 & 137.6 & 13.40 & 1.19 & 1.34 & 1.47 \\
\hline SL3 & 135.6 & 13.81 & 1.18 & 1.33 & 1.47 \\
\hline SL4 & 129.8 & 14.28 & 1.18 & 1.32 & 1.47 \\
\hline SH1 & 183.8 & 12.68 & 1.26 & 1.44 & 1.62 \\
\hline $\mathrm{SH} 2$ & 171.9 & 12.88 & 1.26 & 1.45 & 1.62 \\
\hline $\mathrm{SH} 3$ & 167.5 & 12.51 & 1.27 & 1.47 & 1.64 \\
\hline SH4 & 160.4 & 13.36 & 1.25 & 1.43 & 1.60 \\
\hline SH5 & 161.0 & 12.73 & 1.26 & 1.45 & 1.63 \\
\hline SH6 & 167.2 & 12.53 & 1.27 & 1.47 & 1.64 \\
\hline ML1 & 289.4 & 11.74 & 1.34 & 1.52 & 1.69 \\
\hline ML2 & 278.4 & 11.52 & 1.33 & 1.50 & 1.66 \\
\hline ML3 & 293.1 & 9.93 & 1.37 & 1.61 & 1.80 \\
\hline ML4 & 299.9 & 10.73 & 1.41 & 1.60 & 1.77 \\
\hline ML5 & 300.2 & 10.75 & 1.41 & 1.60 & 1.78 \\
\hline ML6 & 287.3 & 10.33 & 1.38 & 1.57 & 1.75 \\
\hline MH1 & 664.8 & 9.02 & 1.32 & 1.67 & 1.87 \\
\hline MH2 & 696.0 & 7.45 & 1.26 & 1.70 & 2.04 \\
\hline MH3 & 643.9 & 6.81 & 1.26 & 1.60 & 1.99 \\
\hline MH4 & 710.4 & 8.47 & 1.23 & 1.52 & 1.82 \\
\hline MH5 & 721.2 & 6.99 & 1.26 & 1.67 & 2.06 \\
\hline MH6 & 685.6 & 8.02 & 1.49 & 1.74 & 1.99 \\
\hline LL1 & 1158.1 & 8.81 & 1.36 & 1.65 & 1.85 \\
\hline LL2 & 1160.5 & 8.71 & 1.35 & 1.66 & 1.86 \\
\hline LL3 & 1296.9 & 6.38 & 1.29 & 1.79 & 2.17 \\
\hline LL4 & 1171.1 & 8.81 & 1.43 & 1.65 & 1.85 \\
\hline LL5 & 1164.4 & 8.92 & 1.42 & 1.63 & 1.84 \\
\hline LL6 & 920.3 & 6.45 & 1.51 & 1.84 & 2.14 \\
\hline LH1 & 2779.8 & 5.90 & 1.37 & 1.94 & 2.22 \\
\hline LH2 & 2604.5 & 5.48 & 1.45 & 1.92 & 2.26 \\
\hline LH3 & 2060.8 & 4.46 & 1.60 & 1.97 & 2.66 \\
\hline LH4 & 2742.0 & 5.25 & 1.46 & 2.01 & 2.36 \\
\hline LH5 & 2751.6 & 5.08 & 1.47 & 2.02 & 2.38 \\
\hline LH6 & 2027.0 & 4.76 & 1.56 & 2.04 & 2.61 \\
\hline
\end{tabular}


Table B.46 - The response of the buildings subjected to M6C1 ground motion parallel to the long side of the building with $\alpha=0.10$

\begin{tabular}{|c|c|c|c|c|c|}
\hline \multirow{2}{*}{ Building } & \multirow{2}{*}{$V e$} & \multirow{2}{*}{$\begin{array}{c}\text { Ductility }(\mu) \\
R y=3\end{array}$} & \multicolumn{3}{|c|}{ Force Reduction Factor $(R y)$} \\
\hline & & & $\mu=2$ & $\mu=3$ & $\mu=4$ \\
\hline SL1 & 206.3 & 4.46 & 1.37 & 1.65 & 2.20 \\
\hline SL3 & 199.9 & 4.43 & 1.35 & 1.61 & 2.06 \\
\hline SL4 & 178.7 & 5.57 & 1.31 & 1.53 & 1.81 \\
\hline SH1 & 420.5 & 4.31 & 1.84 & 2.50 & 2.88 \\
\hline $\mathrm{SH} 2$ & 382.9 & 5.04 & 1.97 & 2.35 & 2.69 \\
\hline $\mathrm{SH} 3$ & 335.6 & 5.61 & 1.71 & 2.16 & 2.48 \\
\hline SH4 & 335.0 & 6.08 & 1.57 & 2.12 & 2.39 \\
\hline SH5 & 314.5 & 6.06 & 1.56 & 2.04 & 2.33 \\
\hline ML1 & 620.3 & 4.61 & 1.54 & 2.22 & 2.78 \\
\hline ML2 & 599.4 & 5.39 & 1.48 & 2.23 & 2.55 \\
\hline ML3 & 582.6 & 5.88 & 1.48 & 2.09 & 2.43 \\
\hline ML4 & 609.2 & 5.11 & 1.52 & 2.29 & 2.64 \\
\hline ML5 & 586.2 & 5.73 & 1.43 & 2.15 & 2.47 \\
\hline ML6 & 579.7 & 5.75 & 1.49 & 2.13 & 2.44 \\
\hline MH1 & 1307.9 & 5.75 & 1.46 & 2.11 & 2.43 \\
\hline MH2 & 1306.9 & 4.79 & 1.64 & 2.11 & 2.69 \\
\hline MH3 & 1093.3 & 4.43 & 1.61 & 2.43 & 2.84 \\
\hline MH4 & 1298.1 & 5.84 & 1.44 & 2.06 & 2.44 \\
\hline MH5 & 1408.3 & 4.00 & 1.78 & 2.42 & 3.00 \\
\hline MH6 & 1288.8 & 4.62 & 1.58 & 2.32 & 2.73 \\
\hline LL1 & 2417.2 & 4.96 & 1.73 & 2.24 & 2.62 \\
\hline LL2 & 2376.2 & 5.11 & 1.61 & 2.14 & 2.59 \\
\hline LL3 & 2129.1 & 3.99 & 1.81 & 2.56 & 3.00 \\
\hline LL4 & 2414.6 & 5.00 & 1.74 & 2.24 & 2.62 \\
\hline LL5 & 2312.8 & 5.09 & 1.60 & 2.03 & 2.61 \\
\hline LL6 & 2063.5 & 3.14 & 1.67 & 2.84 & 3.42 \\
\hline LH1 & 6161.3 & 2.87 & 1.98 & 3.07 & 3.67 \\
\hline LH2 & 5375.9 & 2.79 & 2.47 & 3.12 & 3.73 \\
\hline LH3 & 4506.9 & 2.85 & 1.86 & 3.09 & 3.67 \\
\hline LH4 & 5840.1 & 2.66 & 2.53 & 3.22 & 3.89 \\
\hline LH5 & 4548.3 & 3.25 & 2.24 & 2.87 & 3.41 \\
\hline LH6 & 4230.9 & 3.09 & 1.66 & 2.95 & 3.46 \\
\hline
\end{tabular}


Table B.47 - The response of the buildings subjected to M6C2 ground motion parallel to the long side of the building with $\alpha=0.10$

\begin{tabular}{|c|c|c|c|c|c|}
\hline \multirow{2}{*}{ Building } & \multirow{2}{*}{$V e$} & \multirow{2}{*}{$\begin{array}{c}\text { Ductility }(\mu) \\
R y=3\end{array}$} & \multicolumn{3}{|c|}{ Force Reduction Factor $(R y)$} \\
\hline & & & $\mu=2$ & $\mu=3$ & $\mu=4$ \\
\hline SL1 & 175.0 & 5.71 & 1.61 & 1.92 & 2.29 \\
\hline SL3 & 172.5 & 5.93 & 1.62 & 1.92 & 2.25 \\
\hline SL4 & 170.8 & 6.28 & 1.59 & 1.91 & 2.22 \\
\hline SH1 & 463.6 & 3.42 & 2.05 & 2.72 & 4.10 \\
\hline $\mathrm{SH} 2$ & 429.2 & 3.02 & 1.78 & 2.95 & 4.23 \\
\hline $\mathrm{SH} 3$ & 356.6 & 3.46 & 1.66 & 2.50 & 3.41 \\
\hline SH4 & 357.3 & 3.78 & 1.58 & 2.25 & 3.30 \\
\hline SH5 & 304.8 & 4.42 & 1.58 & 2.08 & 2.86 \\
\hline ML1 & 594.8 & 5.69 & 1.38 & 1.75 & 2.22 \\
\hline ML2 & 574.6 & 6.38 & 1.35 & 1.60 & 2.01 \\
\hline ML3 & 528.3 & 6.44 & 1.36 & 1.63 & 2.00 \\
\hline ML4 & 583.8 & 6.24 & 1.36 & 1.63 & 2.08 \\
\hline ML5 & 562.7 & 6.68 & 1.33 & 1.58 & 1.96 \\
\hline ML6 & 544.5 & 6.36 & 1.36 & 1.63 & 2.01 \\
\hline MH1 & 904.5 & 6.49 & 1.33 & 1.76 & 2.05 \\
\hline MH2 & 936.1 & 4.37 & 2.02 & 2.48 & 2.88 \\
\hline MH3 & 912.7 & 4.09 & 1.76 & 2.12 & 2.92 \\
\hline MH4 & 916.6 & 5.99 & 1.36 & 1.94 & 2.23 \\
\hline MH5 & 949.0 & 3.99 & 2.17 & 2.59 & 3.01 \\
\hline MH6 & 941.5 & 4.28 & 1.62 & 2.48 & 2.91 \\
\hline LL1 & 1684.8 & 5.62 & 1.41 & 1.92 & 2.33 \\
\hline LL2 & 1695.3 & 5.22 & 1.44 & 2.16 & 2.54 \\
\hline LL3 & 1695.5 & 3.71 & 1.69 & 2.20 & 3.28 \\
\hline LL4 & 1686.9 & 5.57 & 1.41 & 1.94 & 2.36 \\
\hline LL5 & 1684.6 & 4.53 & 1.83 & 2.43 & 2.83 \\
\hline LL6 & 1147.2 & 5.04 & 1.46 & 2.30 & 2.55 \\
\hline LH1 & 3848.9 & 3.47 & 2.15 & 2.72 & 3.30 \\
\hline LH2 & 3828.8 & 2.71 & 2.42 & 3.23 & 4.19 \\
\hline LH3 & 2290.5 & 4.48 & 1.58 & 2.18 & 2.55 \\
\hline LH4 & 3827.8 & 3.10 & 2.24 & 2.92 & 3.68 \\
\hline LH5 & 3575.9 & 3.32 & 1.86 & 2.69 & 3.88 \\
\hline LH6 & 1987.8 & 4.76 & 1.59 & 1.84 & 2.22 \\
\hline
\end{tabular}


Table B.48 - The response of the buildings subjected to M6C26 ground motion parallel to the long side of the building with $\alpha=0.10$

\begin{tabular}{|c|c|c|c|c|c|}
\hline \multirow{2}{*}{ Building } & \multirow{2}{*}{$V e$} & \multirow{2}{*}{$\begin{array}{c}\text { Ductility }(\mu) \\
R y=3\end{array}$} & \multicolumn{3}{|c|}{ Force Reduction Factor $(R y)$} \\
\hline & & & $\mu=2$ & $\mu=3$ & $\mu=4$ \\
\hline SL1 & 197.6 & 7.00 & 1.57 & 1.89 & 2.20 \\
\hline SL3 & 203.5 & 6.86 & 1.57 & 1.89 & 2.19 \\
\hline SL4 & 214.0 & 6.79 & 1.59 & 1.92 & 2.22 \\
\hline SH1 & 487.0 & 5.26 & 1.78 & 2.19 & 2.55 \\
\hline $\mathrm{SH} 2$ & 483.5 & 5.44 & 1.43 & 2.17 & 2.51 \\
\hline $\mathrm{SH} 3$ & 470.9 & 4.94 & 1.38 & 2.25 & 2.64 \\
\hline SH4 & 465.0 & 5.39 & 1.32 & 2.20 & 2.52 \\
\hline SH5 & 455.8 & 4.88 & 1.34 & 2.30 & 2.68 \\
\hline ML1 & 582.9 & 6.47 & 1.31 & 1.56 & 1.79 \\
\hline ML2 & 534.2 & 7.50 & 1.29 & 1.52 & 1.74 \\
\hline ML3 & 529.2 & 6.85 & 1.33 & 1.58 & 1.82 \\
\hline ML4 & 544.3 & 7.25 & 1.30 & 1.53 & 1.75 \\
\hline ML5 & 526.8 & 7.62 & 1.29 & 1.52 & 1.74 \\
\hline ML6 & 527.3 & 6.77 & 1.32 & 1.58 & 1.82 \\
\hline MH1 & 1263.7 & 5.77 & 1.36 & 1.64 & 2.02 \\
\hline MH2 & 1391.8 & 4.28 & 1.43 & 1.91 & 2.77 \\
\hline MH3 & 1271.5 & 4.18 & 1.41 & 1.92 & 2.92 \\
\hline MH4 & 1282.9 & 5.67 & 1.36 & 1.63 & 2.05 \\
\hline MH5 & 1417.4 & 4.19 & 1.44 & 1.99 & 2.84 \\
\hline MH6 & 1429.6 & 3.60 & 1.51 & 2.13 & 3.62 \\
\hline LL1 & 2377.5 & 4.61 & 1.42 & 1.76 & 2.45 \\
\hline LL2 & 2417.6 & 4.54 & 1.42 & 1.77 & 2.48 \\
\hline LL3 & 2410.1 & 3.93 & 1.44 & 2.14 & 3.01 \\
\hline LL4 & 2380.9 & 4.63 & 1.42 & 1.74 & 2.45 \\
\hline LL5 & 2503.4 & 4.44 & 1.42 & 1.83 & 2.60 \\
\hline LL6 & 1924.6 & 3.60 & 1.53 & 2.39 & 3.17 \\
\hline LH1 & 6150.0 & 2.76 & 1.61 & 3.15 & 3.76 \\
\hline LH2 & 5825.5 & 3.05 & 1.60 & 2.98 & 3.57 \\
\hline LH3 & 3856.6 & 3.03 & 1.64 & 2.97 & 3.68 \\
\hline LH4 & 6170.0 & 2.76 & 1.65 & 3.16 & 3.81 \\
\hline LH5 & 5117.4 & 3.45 & 1.54 & 2.78 & 3.30 \\
\hline LH6 & 3349.9 & 3.14 & 1.69 & 2.38 & 4.15 \\
\hline
\end{tabular}


Table B.49 - The response of the buildings subjected to M6C31 ground motion parallel to the long side of the building with $\alpha=0.10$

\begin{tabular}{|c|c|c|c|c|c|}
\hline \multirow{2}{*}{ Building } & \multirow{2}{*}{$V e$} & \multirow{2}{*}{$\begin{array}{c}\text { Ductility }(\mu) \\
R y=3\end{array}$} & \multicolumn{3}{|c|}{ Force Reduction Factor $(R y)$} \\
\hline & & & $\mu=2$ & $\mu=3$ & $\mu=4$ \\
\hline SL1 & 184.8 & 5.66 & 1.36 & 2.18 & 2.62 \\
\hline SL3 & 180.1 & 5.98 & 1.37 & 1.89 & 2.55 \\
\hline SL4 & 178.0 & 6.41 & 1.36 & 1.63 & 2.22 \\
\hline SH1 & 446.1 & 4.69 & 1.34 & 1.68 & 2.48 \\
\hline $\mathrm{SH} 2$ & 416.2 & 5.32 & 1.33 & 1.58 & 2.02 \\
\hline $\mathrm{SH} 3$ & 405.2 & 4.67 & 1.41 & 1.66 & 2.30 \\
\hline SH4 & 396.4 & 5.46 & 1.37 & 1.62 & 2.01 \\
\hline SH5 & 397.6 & 4.85 & 1.37 & 1.66 & 2.38 \\
\hline ML1 & 939.1 & 3.80 & 1.33 & 2.33 & 3.15 \\
\hline ML2 & 850.7 & 4.20 & 1.34 & 2.11 & 2.76 \\
\hline ML3 & 639.3 & 4.79 & 1.38 & 1.83 & 2.45 \\
\hline ML4 & 882.0 & 4.15 & 1.34 & 2.18 & 2.89 \\
\hline ML5 & 813.0 & 4.46 & 1.34 & 2.04 & 2.69 \\
\hline ML6 & 702.6 & 4.38 & 1.39 & 2.02 & 2.72 \\
\hline MH1 & 1026.3 & 6.45 & 1.40 & 1.72 & 2.04 \\
\hline MH2 & 829.2 & 7.11 & 1.44 & 1.87 & 2.05 \\
\hline MH3 & 1091.5 & 3.78 & 1.34 & 2.17 & 3.23 \\
\hline MH4 & 957.3 & 7.23 & 1.43 & 1.75 & 2.02 \\
\hline MH5 & 882.3 & 7.05 & 1.66 & 1.87 & 2.08 \\
\hline MH6 & 805.3 & 6.23 & 1.63 & 1.85 & 2.04 \\
\hline LL1 & 1747.3 & 5.93 & 1.48 & 1.85 & 2.13 \\
\hline LL2 & 1560.6 & 6.66 & 1.47 & 1.78 & 2.03 \\
\hline LL3 & 2143.2 & 3.96 & 1.39 & 2.02 & 3.10 \\
\hline LL4 & 1732.6 & 6.01 & 1.48 & 1.85 & 2.13 \\
\hline LL5 & 1474.9 & 7.36 & 1.39 & 1.84 & 2.03 \\
\hline LL6 & 1249.6 & 3.89 & 1.55 & 2.37 & 3.00 \\
\hline LH1 & 3731.5 & 4.80 & 1.63 & 1.94 & 2.24 \\
\hline LH2 & 4929.3 & 3.54 & 1.50 & 2.21 & 4.63 \\
\hline LH3 & 2466.2 & 3.23 & 2.10 & 2.89 & 3.58 \\
\hline LH4 & 4572.4 & 3.72 & 1.64 & 2.09 & 3.83 \\
\hline LH5 & 4364.4 & 2.48 & 2.13 & 3.54 & 4.51 \\
\hline LH6 & 1981.6 & 4.32 & 1.48 & 1.83 & 2.78 \\
\hline
\end{tabular}


Table B.50 - The response of the buildings subjected to M6C38 ground motion parallel to the long side of the building with $\alpha=0.10$

\begin{tabular}{|c|c|c|c|c|c|}
\hline \multirow{2}{*}{ Building } & \multirow{2}{*}{$V e$} & \multirow{2}{*}{$\begin{array}{c}\text { Ductility }(\mu) \\
R y=3\end{array}$} & \multicolumn{3}{|c|}{ Force Reduction Factor $(R y)$} \\
\hline & & & $\mu=2$ & $\mu=3$ & $\mu=4$ \\
\hline SL1 & 165.6 & 7.23 & 1.41 & 2.01 & 2.30 \\
\hline SL3 & 157.4 & 8.07 & 1.36 & 1.84 & 2.17 \\
\hline SL4 & 152.5 & 8.73 & 1.32 & 1.65 & 2.00 \\
\hline SH1 & 378.3 & 7.03 & 1.34 & 1.61 & 1.86 \\
\hline $\mathrm{SH} 2$ & 365.7 & 7.53 & 1.32 & 1.56 & 1.80 \\
\hline $\mathrm{SH} 3$ & 360.1 & 7.15 & 1.33 & 1.60 & 1.85 \\
\hline SH4 & 353.9 & 8.02 & 1.30 & 1.55 & 1.76 \\
\hline SH5 & 353.4 & 7.34 & 1.32 & 1.59 & 1.83 \\
\hline ML1 & 567.1 & 8.10 & 1.29 & 1.53 & 1.75 \\
\hline ML2 & 542.7 & 8.35 & 1.28 & 1.51 & 1.72 \\
\hline ML3 & 522.6 & 7.75 & 1.30 & 1.55 & 1.78 \\
\hline ML4 & 549.1 & 8.28 & 1.28 & 1.50 & 1.73 \\
\hline ML5 & 536.1 & 8.55 & 1.28 & 1.50 & 1.71 \\
\hline ML6 & 532.8 & 7.57 & 1.31 & 1.56 & 1.80 \\
\hline MH1 & 1041.2 & 7.46 & 1.33 & 1.59 & 1.84 \\
\hline MH2 & 911.2 & 6.99 & 1.39 & 1.69 & 1.98 \\
\hline MH3 & 697.7 & 8.29 & 1.39 & 1.67 & 1.93 \\
\hline MH4 & 1008.1 & 7.73 & 1.33 & 1.59 & 1.84 \\
\hline MH5 & 864.7 & 7.34 & 1.38 & 1.68 & 1.95 \\
\hline MH6 & 956.5 & 5.92 & 1.43 & 1.78 & 2.10 \\
\hline LL1 & 1918.8 & 6.23 & 1.40 & 1.72 & 2.01 \\
\hline LL2 & 1834.0 & 6.51 & 1.39 & 1.69 & 1.99 \\
\hline LL3 & 1310.3 & 7.85 & 1.40 & 1.69 & 1.96 \\
\hline LL4 & 1911.3 & 6.28 & 1.40 & 1.71 & 2.01 \\
\hline LL5 & 1656.1 & 7.17 & 1.38 & 1.67 & 1.94 \\
\hline LL6 & 1213.1 & 7.36 & 1.46 & 1.75 & 2.03 \\
\hline LH1 & 3545.2 & 5.44 & 1.49 & 1.88 & 2.25 \\
\hline LH2 & 3127.7 & 5.64 & 1.50 & 1.91 & 2.29 \\
\hline LH3 & 2731.4 & 5.89 & 1.55 & 1.91 & 2.26 \\
\hline LH4 & 3327.5 & 5.17 & 1.53 & 1.95 & 2.35 \\
\hline LH5 & 2828.2 & 6.28 & 1.47 & 1.83 & 2.17 \\
\hline LH6 & 2547.9 & 6.10 & 1.47 & 1.80 & 2.11 \\
\hline
\end{tabular}


Table B.51 - The response of the buildings subjected to E6C1 ground motion parallel to the short side of the building with $\alpha=0.10$

\begin{tabular}{|c|c|c|c|c|c|}
\hline \multirow{2}{*}{ Building } & \multirow{2}{*}{$V e$} & \multirow{2}{*}{$\begin{array}{c}\text { Ductility }(\mu) \\
R y=3\end{array}$} & \multicolumn{3}{|c|}{ Force Reduction Factor $(R y)$} \\
\hline & & & $\mu=2$ & $\mu=3$ & $\mu=4$ \\
\hline SL1 & 93.7 & 9.94 & 1.28 & 1.48 & 1.67 \\
\hline SL3 & 93.7 & 10.08 & 1.28 & 1.49 & 1.67 \\
\hline SL4 & 92.1 & 10.29 & 1.27 & 1.45 & 1.64 \\
\hline SH1 & 150.7 & 11.05 & 1.32 & 1.48 & 1.63 \\
\hline $\mathrm{SH} 2$ & 140.3 & 11.97 & 1.25 & 1.40 & 1.55 \\
\hline $\mathrm{SH} 3$ & 135.0 & 11.51 & 1.22 & 1.37 & 1.52 \\
\hline SH4 & 130.1 & 12.33 & 1.18 & 1.33 & 1.47 \\
\hline SH5 & 130.7 & 11.46 & 1.19 & 1.35 & 1.50 \\
\hline SH6 & 134.7 & 11.56 & 1.22 & 1.37 & 1.52 \\
\hline ML1 & 238.4 & 9.65 & 1.27 & 1.45 & 1.63 \\
\hline ML2 & 217.1 & 8.64 & 1.27 & 1.46 & 1.75 \\
\hline ML3 & 199.2 & 7.79 & 1.26 & 1.48 & 1.82 \\
\hline ML4 & 233.8 & 8.97 & 1.26 & 1.44 & 1.69 \\
\hline ML5 & 235.3 & 8.95 & 1.26 & 1.45 & 1.70 \\
\hline ML6 & 210.3 & 7.64 & 1.28 & 1.49 & 1.85 \\
\hline MH1 & 412.6 & 6.94 & 1.27 & 1.54 & 1.90 \\
\hline MH2 & 359.1 & 6.16 & 1.28 & 1.64 & 2.19 \\
\hline MH3 & 197.8 & 8.25 & 1.23 & 1.54 & 1.90 \\
\hline MH4 & 336.7 & 7.11 & 1.25 & 1.51 & 1.86 \\
\hline MH5 & 331.6 & 6.46 & 1.29 & 1.66 & 2.19 \\
\hline MH6 & 515.7 & 5.64 & 1.34 & 1.58 & 2.02 \\
\hline LL1 & 685.3 & 7.49 & 1.28 & 1.49 & 1.80 \\
\hline LL2 & 684.1 & 7.21 & 1.29 & 1.52 & 1.85 \\
\hline LL3 & 531.7 & 6.00 & 1.37 & 1.97 & 2.43 \\
\hline LL4 & 778.9 & 6.49 & 1.31 & 1.57 & 1.96 \\
\hline LL5 & 780.5 & 6.65 & 1.30 & 1.57 & 1.93 \\
\hline LL6 & 302.5 & 6.35 & 1.29 & 1.60 & 2.01 \\
\hline LH1 & 1111.2 & 5.69 & 1.52 & 2.18 & 2.49 \\
\hline LH2 & 1053.8 & 5.39 & 1.78 & 2.22 & 2.56 \\
\hline LH3 & 653.2 & 6.09 & 1.42 & 2.11 & 2.41 \\
\hline LH4 & 1020.8 & 5.52 & 1.71 & 2.18 & 2.51 \\
\hline LH5 & 998.5 & 5.65 & 1.73 & 2.17 & 2.51 \\
\hline LH6 & 641.5 & 5.47 & 1.41 & 2.09 & 2.55 \\
\hline
\end{tabular}


Table B.52 - The response of the buildings subjected to E6C13 ground motion parallel to the short side of the building with $\alpha=0.10$

\begin{tabular}{|c|c|c|c|c|c|}
\hline \multirow{2}{*}{ Building } & \multirow{2}{*}{$V e$} & \multirow{2}{*}{$\begin{array}{c}\text { Ductility }(\mu) \\
R y=3\end{array}$} & \multicolumn{3}{|c|}{ Force Reduction Factor $(R y)$} \\
\hline & & & $\mu=2$ & $\mu=3$ & $\mu=4$ \\
\hline SL1 & 96.0 & 7.63 & 1.39 & 1.61 & 1.87 \\
\hline SL3 & 90.5 & 7.87 & 1.38 & 1.59 & 1.82 \\
\hline SL4 & 74.2 & 9.21 & 1.32 & 1.51 & 1.69 \\
\hline SH1 & 89.1 & 8.22 & 1.47 & 1.74 & 2.01 \\
\hline $\mathrm{SH} 2$ & 90.2 & 9.14 & 1.38 & 1.64 & 1.87 \\
\hline $\mathrm{SH} 3$ & 89.9 & 9.08 & 1.35 & 1.60 & 1.83 \\
\hline SH4 & 91.4 & 9.83 & 1.33 & 1.56 & 1.76 \\
\hline SH5 & 91.7 & 9.37 & 1.34 & 1.58 & 1.79 \\
\hline SH6 & 89.8 & 9.10 & 1.35 & 1.60 & 1.82 \\
\hline ML1 & 163.0 & 8.51 & 1.30 & 1.52 & 1.72 \\
\hline ML2 & 142.5 & 8.17 & 1.27 & 1.50 & 1.79 \\
\hline ML3 & 137.5 & 6.64 & 1.29 & 1.67 & 2.06 \\
\hline ML4 & 153.1 & 8.59 & 1.27 & 1.45 & 1.66 \\
\hline ML5 & 153.6 & 8.60 & 1.27 & 1.47 & 1.66 \\
\hline ML6 & 140.8 & 6.94 & 1.29 & 1.59 & 1.94 \\
\hline MH1 & 307.7 & 6.20 & 1.40 & 1.63 & 2.10 \\
\hline MH2 & 334.2 & 4.66 & 1.27 & 1.90 & 2.67 \\
\hline MH3 & 239.4 & 5.92 & 1.27 & 1.91 & 2.29 \\
\hline MH4 & 342.0 & 5.32 & 1.24 & 1.70 & 2.20 \\
\hline MH5 & 351.3 & 4.53 & 1.33 & 2.00 & 2.75 \\
\hline MH6 & 361.9 & 5.61 & 1.41 & 1.73 & 2.04 \\
\hline LL1 & 619.8 & 4.48 & 1.33 & 2.00 & 2.74 \\
\hline LL2 & 633.1 & 4.40 & 1.33 & 2.09 & 2.87 \\
\hline LL3 & 627.5 & 4.41 & 1.50 & 1.83 & 2.56 \\
\hline LL4 & 559.7 & 5.20 & 1.34 & 1.83 & 2.51 \\
\hline LL5 & 565.5 & 5.10 & 1.34 & 1.80 & 2.49 \\
\hline LL6 & 300.7 & 6.49 & 1.30 & 1.54 & 1.98 \\
\hline LH1 & 1319.4 & 4.04 & 1.43 & 1.98 & 2.97 \\
\hline LH2 & 1206.6 & 4.39 & 1.41 & 2.35 & 2.84 \\
\hline LH3 & 823.6 & 5.32 & 1.46 & 2.19 & 2.55 \\
\hline LH4 & 1180.1 & 4.40 & 1.37 & 2.38 & 2.83 \\
\hline LH5 & 1153.7 & 4.47 & 1.37 & 2.36 & 2.81 \\
\hline LH6 & 772.2 & 5.46 & 1.39 & 2.00 & 2.51 \\
\hline
\end{tabular}


Table B.53 - The response of the buildings subjected to E6C15 ground motion parallel to the short side of the building with $\alpha=0.10$

\begin{tabular}{|c|c|c|c|c|c|}
\hline \multirow{2}{*}{ Building } & \multirow{2}{*}{$V e$} & \multirow{2}{*}{$\begin{array}{c}\text { Ductility }(\mu) \\
R y=3\end{array}$} & \multicolumn{3}{|c|}{ Force Reduction Factor $(R y)$} \\
\hline & & & $\mu=2$ & $\mu=3$ & $\mu=4$ \\
\hline SL1 & 71.8 & 7.58 & 1.43 & 1.79 & 2.22 \\
\hline SL3 & 71.9 & 7.23 & 1.43 & 1.77 & 2.20 \\
\hline SL4 & 71.4 & 8.06 & 1.40 & 1.76 & 2.16 \\
\hline SH1 & 168.7 & 6.66 & 1.29 & 1.59 & 1.98 \\
\hline $\mathrm{SH} 2$ & 136.4 & 7.55 & 1.25 & 1.55 & 1.89 \\
\hline $\mathrm{SH} 3$ & 120.4 & 7.79 & 1.24 & 1.53 & 1.86 \\
\hline SH4 & 104.7 & 9.45 & 1.22 & 1.41 & 1.68 \\
\hline SH5 & 103.7 & 9.09 & 1.23 & 1.45 & 1.72 \\
\hline SH6 & 120.1 & 7.75 & 1.25 & 1.53 & 1.86 \\
\hline ML1 & 147.6 & 11.73 & 1.37 & 1.53 & 1.67 \\
\hline ML2 & 120.6 & 14.20 & 1.22 & 1.44 & 1.62 \\
\hline ML3 & 123.2 & 12.76 & 1.25 & 1.49 & 1.63 \\
\hline ML4 & 131.5 & 13.23 & 1.33 & 1.49 & 1.63 \\
\hline ML5 & 132.0 & 13.19 & 1.32 & 1.48 & 1.62 \\
\hline ML6 & 120.5 & 13.34 & 1.22 & 1.48 & 1.63 \\
\hline MH1 & 283.6 & 10.95 & 1.35 & 1.52 & 1.67 \\
\hline $\mathrm{MH} 2$ & 286.3 & 7.78 & 1.34 & 1.54 & 1.74 \\
\hline MH3 & 163.6 & 7.16 & 1.36 & 1.94 & 2.17 \\
\hline MH4 & 276.6 & 8.28 & 1.32 & 1.50 & 1.70 \\
\hline MH5 & 270.9 & 7.01 & 1.33 & 1.54 & 1.89 \\
\hline MH6 & 319.9 & 8.10 & 1.50 & 1.71 & 1.90 \\
\hline LL1 & 644.0 & 7.26 & 1.45 & 1.73 & 2.03 \\
\hline LL2 & 640.2 & 7.15 & 1.43 & 1.69 & 2.00 \\
\hline LL3 & 410.6 & 5.69 & 1.33 & 1.76 & 2.23 \\
\hline LL4 & 636.8 & 7.72 & 1.49 & 1.76 & 2.04 \\
\hline LL5 & 638.3 & 7.77 & 1.50 & 1.78 & 2.06 \\
\hline LL6 & 271.3 & 9.17 & 1.37 & 1.63 & 1.86 \\
\hline LH1 & 812.3 & 5.86 & 1.34 & 1.97 & 2.34 \\
\hline LH2 & 701.6 & 6.73 & 1.52 & 1.82 & 2.09 \\
\hline LH3 & 660.9 & 6.22 & 1.64 & 1.97 & 2.25 \\
\hline LH4 & 695.3 & 6.56 & 1.57 & 1.88 & 2.15 \\
\hline LH5 & 667.2 & 6.76 & 1.54 & 1.82 & 2.09 \\
\hline LH6 & 621.0 & 7.05 & 1.50 & 1.81 & 2.07 \\
\hline
\end{tabular}


Table B.54 - The response of the buildings subjected to E6C18 ground motion parallel to the short side of the building with $\alpha=0.10$

\begin{tabular}{|c|c|c|c|c|c|}
\hline \multirow{2}{*}{ Building } & \multirow{2}{*}{$V e$} & \multirow{2}{*}{$\begin{array}{c}\text { Ductility }(\mu) \\
R y=3\end{array}$} & \multicolumn{3}{|c|}{ Force Reduction Factor $(R y)$} \\
\hline & & & $\mu=2$ & $\mu=3$ & $\mu=4$ \\
\hline SL1 & 90.4 & 8.15 & 1.34 & 1.55 & 1.90 \\
\hline SL3 & 82.3 & 9.16 & 1.34 & 1.53 & 1.83 \\
\hline SL4 & 64.4 & 12.95 & 1.29 & 1.58 & 1.76 \\
\hline SH1 & 124.1 & 9.58 & 1.24 & 1.43 & 1.60 \\
\hline $\mathrm{SH} 2$ & 125.3 & 8.62 & 1.26 & 1.46 & 1.64 \\
\hline $\mathrm{SH} 3$ & 125.2 & 7.63 & 1.29 & 1.51 & 1.72 \\
\hline SH4 & 122.3 & 8.08 & 1.29 & 1.50 & 1.71 \\
\hline SH5 & 122.3 & 7.37 & 1.31 & 1.52 & 1.78 \\
\hline SH6 & 125.1 & 7.64 & 1.29 & 1.50 & 1.73 \\
\hline ML1 & 197.8 & 6.38 & 1.30 & 1.52 & 1.92 \\
\hline ML2 & 152.6 & 8.31 & 1.31 & 1.79 & 2.03 \\
\hline ML3 & 137.6 & 8.22 & 1.43 & 1.77 & 2.01 \\
\hline ML4 & 179.1 & 6.86 & 1.26 & 1.60 & 2.11 \\
\hline ML5 & 180.1 & 6.73 & 1.27 & 1.58 & 2.10 \\
\hline ML6 & 146.0 & 7.92 & 1.45 & 1.79 & 2.04 \\
\hline MH1 & 280.1 & 7.87 & 1.51 & 1.78 & 2.02 \\
\hline MH2 & 249.6 & 6.90 & 1.62 & 1.92 & 2.20 \\
\hline MH3 & 202.5 & 4.96 & 1.67 & 2.12 & 2.55 \\
\hline MH4 & 242.8 & 7.44 & 1.36 & 1.89 & 2.14 \\
\hline MH5 & 237.4 & 6.74 & 1.61 & 1.96 & 2.25 \\
\hline MH6 & 419.6 & 4.23 & 1.40 & 2.49 & 2.89 \\
\hline LL1 & 507.2 & 6.65 & 1.64 & 1.95 & 2.26 \\
\hline LL2 & 499.4 & 6.76 & 1.62 & 1.93 & 2.23 \\
\hline LL3 & 365.8 & 6.10 & 1.71 & 2.04 & 2.35 \\
\hline LL4 & 573.8 & 6.20 & 1.70 & 2.03 & 2.33 \\
\hline LL5 & 579.6 & 6.17 & 1.70 & 2.05 & 2.35 \\
\hline LL6 & 200.3 & 5.46 & 1.35 & 1.70 & 2.21 \\
\hline LH1 & 751.5 & 5.80 & 1.71 & 2.08 & 2.43 \\
\hline LH2 & 701.8 & 5.52 & 1.70 & 2.09 & 2.47 \\
\hline LH3 & 680.8 & 3.35 & 2.09 & 2.81 & 3.43 \\
\hline LH4 & 699.5 & 5.28 & 1.76 & 2.16 & 2.53 \\
\hline LH5 & 693.0 & 5.28 & 1.77 & 2.17 & 2.56 \\
\hline LH6 & 642.6 & 3.58 & 2.15 & 2.70 & 3.27 \\
\hline
\end{tabular}


Table B.55 - The response of the buildings subjected to E6C42 ground motion parallel to the short side of the building with $\alpha=0.10$

\begin{tabular}{|c|c|c|c|c|c|}
\hline \multirow{2}{*}{ Building } & \multirow{2}{*}{$V e$} & \multirow{2}{*}{$\begin{array}{c}\text { Ductility }(\mu) \\
R y=3\end{array}$} & \multicolumn{3}{|c|}{ Force Reduction Factor $(R y)$} \\
\hline & & & $\mu=2$ & $\mu=3$ & $\mu=4$ \\
\hline SL1 & 72.6 & 6.79 & 1.29 & 1.49 & 1.76 \\
\hline SL3 & 70.0 & 6.65 & 1.28 & 1.51 & 1.84 \\
\hline SL4 & 62.2 & 8.22 & 1.31 & 1.68 & 2.09 \\
\hline SH1 & 115.8 & 10.33 & 1.30 & 1.49 & 1.69 \\
\hline $\mathrm{SH} 2$ & 106.4 & 11.77 & 1.25 & 1.40 & 1.56 \\
\hline $\mathrm{SH} 3$ & 108.6 & 10.43 & 1.29 & 1.46 & 1.64 \\
\hline SH4 & 109.5 & 10.65 & 1.33 & 1.49 & 1.66 \\
\hline SH5 & 109.7 & 10.05 & 1.33 & 1.51 & 1.69 \\
\hline SH6 & 108.8 & 10.57 & 1.30 & 1.46 & 1.64 \\
\hline ML1 & 192.8 & 8.73 & 1.39 & 1.61 & 1.82 \\
\hline ML2 & 156.3 & 9.49 & 1.33 & 1.56 & 1.76 \\
\hline ML3 & 140.7 & 9.76 & 1.33 & 1.53 & 1.72 \\
\hline ML4 & 182.9 & 8.37 & 1.37 & 1.60 & 1.83 \\
\hline ML5 & 184.1 & 8.45 & 1.38 & 1.61 & 1.82 \\
\hline ML6 & 149.9 & 9.02 & 1.34 & 1.58 & 1.77 \\
\hline MH1 & 323.9 & 8.75 & 1.39 & 1.70 & 1.89 \\
\hline MH2 & 328.4 & 8.26 & 1.53 & 1.77 & 1.97 \\
\hline MH3 & 298.8 & 8.24 & 1.43 & 1.86 & 2.11 \\
\hline MH4 & 314.1 & 10.08 & 1.47 & 1.65 & 1.84 \\
\hline MH5 & 313.1 & 9.09 & 1.49 & 1.71 & 1.89 \\
\hline MH6 & 403.3 & 5.88 & 1.48 & 1.77 & 2.16 \\
\hline LL1 & 731.6 & 5.36 & 1.37 & 1.80 & 2.36 \\
\hline LL2 & 716.9 & 5.47 & 1.36 & 1.78 & 2.34 \\
\hline LL3 & 586.4 & 8.06 & 1.61 & 1.86 & 2.08 \\
\hline LL4 & 664.7 & 6.56 & 1.52 & 1.91 & 2.16 \\
\hline LL5 & 669.3 & 6.56 & 1.52 & 1.91 & 2.18 \\
\hline LL6 & 426.9 & 8.01 & 1.51 & 1.79 & 2.04 \\
\hline LH1 & 1286.9 & 7.43 & 1.66 & 1.93 & 2.17 \\
\hline LH2 & 1265.6 & 6.77 & 1.71 & 2.00 & 2.25 \\
\hline LH3 & 1083.9 & 6.34 & 1.66 & 2.00 & 2.31 \\
\hline LH4 & 1246.3 & 7.07 & 1.64 & 1.95 & 2.20 \\
\hline LH5 & 1234.9 & 7.08 & 1.63 & 1.94 & 2.20 \\
\hline LH6 & 1045.4 & 6.33 & 1.66 & 2.00 & 2.32 \\
\hline
\end{tabular}


Table B.56 - The response of the buildings subjected to E6C1 ground motion parallel to the long side of the building with $\alpha=0.10$

\begin{tabular}{|c|c|c|c|c|c|}
\hline \multirow{2}{*}{ Building } & \multirow{2}{*}{$V e$} & \multirow{2}{*}{$\begin{array}{c}\text { Ductility }(\mu) \\
R y=3\end{array}$} & \multicolumn{3}{|c|}{ Force Reduction Factor $(R y)$} \\
\hline & & & $\mu=2$ & $\mu=3$ & $\mu=4$ \\
\hline SL1 & 90.2 & 8.10 & 1.71 & 2.09 & 2.29 \\
\hline SL3 & 92.6 & 7.45 & 1.65 & 2.09 & 2.35 \\
\hline SL4 & 97.0 & 6.94 & 1.46 & 1.96 & 2.31 \\
\hline SH1 & 197.4 & 7.07 & 1.40 & 1.68 & 1.96 \\
\hline $\mathrm{SH} 2$ & 198.5 & 7.20 & 1.38 & 1.64 & 1.93 \\
\hline $\mathrm{SH} 3$ & 201.3 & 6.81 & 1.39 & 1.67 & 1.99 \\
\hline SH4 & 198.9 & 7.32 & 1.36 & 1.62 & 1.89 \\
\hline SH5 & 201.7 & 6.86 & 1.39 & 1.67 & 1.97 \\
\hline ML1 & 376.8 & 6.26 & 1.41 & 1.71 & 1.99 \\
\hline ML2 & 372.8 & 6.34 & 1.39 & 1.68 & 1.96 \\
\hline ML3 & 362.9 & 5.80 & 1.39 & 1.70 & 1.99 \\
\hline ML4 & 374.4 & 6.20 & 1.40 & 1.69 & 1.97 \\
\hline ML5 & 370.6 & 6.31 & 1.38 & 1.66 & 1.94 \\
\hline ML6 & 365.7 & 5.76 & 1.40 & 1.71 & 2.01 \\
\hline MH1 & 774.8 & 5.52 & 1.43 & 1.75 & 2.08 \\
\hline MH2 & 723.6 & 5.06 & 1.47 & 1.78 & 2.16 \\
\hline MH3 & 558.6 & 5.63 & 1.29 & 1.53 & 1.78 \\
\hline MH4 & 758.2 & 5.67 & 1.42 & 1.73 & 2.06 \\
\hline MH5 & 695.5 & 5.35 & 1.44 & 1.73 & 2.07 \\
\hline MH6 & 752.7 & 4.32 & 1.51 & 1.89 & 2.62 \\
\hline LL1 & 1425.7 & 4.62 & 1.48 & 1.86 & 2.41 \\
\hline LL2 & 1401.2 & 4.75 & 1.47 & 1.84 & 2.36 \\
\hline LL3 & 1063.0 & 5.13 & 1.33 & 1.59 & 1.91 \\
\hline LL4 & 1424.4 & 4.65 & 1.47 & 1.85 & 2.41 \\
\hline LL5 & 1315.8 & 5.10 & 1.46 & 1.77 & 2.16 \\
\hline LL6 & 969.8 & 4.37 & 1.38 & 1.70 & 2.54 \\
\hline LH1 & 2972.3 & 3.57 & 1.57 & 2.09 & 4.16 \\
\hline LH2 & 2758.1 & 3.27 & 1.56 & 2.26 & 3.95 \\
\hline LH3 & 2042.7 & 3.42 & 1.43 & 2.76 & 3.28 \\
\hline LH4 & 2917.1 & 3.12 & 1.60 & 2.58 & 4.24 \\
\hline LH5 & 2367.9 & 3.47 & 1.42 & 1.87 & 3.42 \\
\hline LH6 & 1945.1 & 3.50 & 1.44 & 2.75 & 3.23 \\
\hline
\end{tabular}


Table B.57 - The response of the buildings subjected to E6C13 ground motion parallel to the long side of the building with $\alpha=0.10$

\begin{tabular}{|c|c|c|c|c|c|}
\hline \multirow{2}{*}{ Building } & \multirow{2}{*}{$V e$} & \multirow{2}{*}{$\begin{array}{c}\text { Ductility }(\mu) \\
R y=3\end{array}$} & \multicolumn{3}{|c|}{ Force Reduction Factor $(R y)$} \\
\hline & & & $\mu=2$ & $\mu=3$ & $\mu=4$ \\
\hline SL1 & 138.0 & 3.64 & 1.42 & 2.36 & 3.39 \\
\hline SL3 & 139.9 & 3.50 & 1.42 & 2.36 & 3.53 \\
\hline SL4 & 144.9 & 3.61 & 1.44 & 2.21 & 3.53 \\
\hline SH1 & 268.2 & 3.21 & 1.85 & 2.93 & 3.63 \\
\hline $\mathrm{SH} 2$ & 277.7 & 3.38 & 1.57 & 2.64 & 3.43 \\
\hline $\mathrm{SH} 3$ & 273.0 & 3.41 & 1.60 & 2.59 & 3.49 \\
\hline SH4 & 272.9 & 3.82 & 1.58 & 2.30 & 3.09 \\
\hline SH5 & 264.7 & 3.72 & 1.59 & 2.34 & 3.23 \\
\hline ML1 & 314.0 & 5.47 & 1.45 & 1.75 & 2.28 \\
\hline ML2 & 266.4 & 6.36 & 1.40 & 1.68 & 2.06 \\
\hline ML3 & 216.3 & 6.76 & 1.40 & 1.72 & 2.05 \\
\hline ML4 & 277.1 & 6.12 & 1.40 & 1.69 & 2.11 \\
\hline ML5 & 255.4 & 6.66 & 1.38 & 1.67 & 2.01 \\
\hline ML6 & 230.4 & 6.30 & 1.40 & 1.74 & 2.08 \\
\hline MH1 & 342.3 & 8.03 & 1.43 & 1.72 & 1.99 \\
\hline MH2 & 319.3 & 6.70 & 1.59 & 1.94 & 2.26 \\
\hline MH3 & 355.0 & 5.96 & 1.50 & 1.90 & 2.29 \\
\hline MH4 & 326.2 & 8.17 & 1.44 & 1.74 & 2.01 \\
\hline MH5 & 306.4 & 7.21 & 1.55 & 1.86 & 2.16 \\
\hline MH6 & 324.6 & 6.29 & 1.66 & 2.02 & 2.32 \\
\hline LL1 & 598.0 & 7.74 & 1.44 & 1.74 & 2.04 \\
\hline LL2 & 564.9 & 7.97 & 1.48 & 1.75 & 2.03 \\
\hline LL3 & 607.3 & 6.09 & 1.53 & 1.95 & 2.32 \\
\hline LL4 & 594.5 & 7.67 & 1.44 & 1.74 & 2.04 \\
\hline LL5 & 573.2 & 6.88 & 1.58 & 1.92 & 2.21 \\
\hline LL6 & 656.4 & 5.52 & 1.47 & 1.85 & 2.19 \\
\hline LH1 & 1215.7 & 6.20 & 1.63 & 2.00 & 2.32 \\
\hline LH2 & 1239.1 & 5.54 & 1.57 & 2.02 & 2.42 \\
\hline LH3 & 1392.0 & 4.76 & 1.53 & 1.96 & 2.37 \\
\hline LH4 & 1207.4 & 5.59 & 1.61 & 2.02 & 2.39 \\
\hline LH5 & 1325.5 & 5.39 & 1.56 & 2.01 & 2.43 \\
\hline LH6 & 1355.7 & 4.27 & 1.50 & 1.88 & 2.72 \\
\hline
\end{tabular}


Table B.58 - The response of the buildings subjected to E6C15 ground motion parallel to the long side of the building with $\alpha=0.10$

\begin{tabular}{|c|c|c|c|c|c|}
\hline \multirow{2}{*}{ Building } & \multirow{2}{*}{$V e$} & \multirow{2}{*}{$\begin{array}{c}\text { Ductility }(\mu) \\
R y=3\end{array}$} & \multicolumn{3}{|c|}{ Force Reduction Factor $(R y)$} \\
\hline & & & $\mu=2$ & $\mu=3$ & $\mu=4$ \\
\hline SL1 & 136.8 & 5.51 & 1.73 & 2.11 & 2.46 \\
\hline SL3 & 134.0 & 5.63 & 1.71 & 2.08 & 2.43 \\
\hline SL4 & 127.0 & 5.98 & 1.67 & 2.03 & 2.35 \\
\hline SH1 & 175.0 & 6.84 & 1.50 & 1.82 & 2.16 \\
\hline $\mathrm{SH} 2$ & 166.9 & 7.14 & 1.50 & 1.80 & 2.13 \\
\hline $\mathrm{SH} 3$ & 158.5 & 6.90 & 1.49 & 1.81 & 2.14 \\
\hline SH4 & 158.2 & 7.33 & 1.50 & 1.80 & 2.07 \\
\hline SH5 & 162.5 & 6.56 & 1.57 & 1.90 & 2.24 \\
\hline ML1 & 295.8 & 5.07 & 1.56 & 2.26 & 2.64 \\
\hline ML2 & 289.3 & 5.58 & 1.58 & 2.33 & 2.59 \\
\hline ML3 & 282.6 & 5.44 & 1.65 & 2.18 & 2.48 \\
\hline ML4 & 290.9 & 5.34 & 1.57 & 2.32 & 2.64 \\
\hline ML5 & 287.6 & 5.74 & 1.59 & 2.29 & 2.55 \\
\hline ML6 & 284.2 & 5.39 & 1.61 & 2.23 & 2.52 \\
\hline MH1 & 726.5 & 3.77 & 1.64 & 2.35 & 3.16 \\
\hline MH2 & 854.8 & 2.84 & 1.45 & 3.23 & 4.61 \\
\hline MH3 & 486.9 & 4.90 & 1.50 & 2.06 & 2.55 \\
\hline MH4 & 778.3 & 3.49 & 1.50 & 2.56 & 3.47 \\
\hline MH5 & 813.0 & 3.06 & 1.43 & 2.85 & 4.06 \\
\hline MH6 & 897.9 & 2.39 & 2.32 & 4.00 & 5.15 \\
\hline LL1 & 1414.7 & 3.09 & 1.88 & 2.90 & 3.90 \\
\hline LL2 & 1524.5 & 2.75 & 1.93 & 3.31 & 4.40 \\
\hline LL3 & 1034.0 & 3.97 & 1.67 & 2.42 & 3.05 \\
\hline LL4 & 1425.8 & 3.02 & 1.87 & 2.97 & 3.95 \\
\hline LL5 & 1565.1 & 2.84 & 1.45 & 3.24 & 4.56 \\
\hline LL6 & 597.0 & 6.12 & 1.50 & 1.75 & 1.96 \\
\hline LH1 & 3340.8 & 2.25 & 2.43 & 4.33 & 5.27 \\
\hline LH2 & 2668.6 & 2.67 & 2.05 & 3.50 & 4.64 \\
\hline LH3 & 1324.9 & 5.06 & 1.64 & 2.01 & 2.66 \\
\hline LH4 & 3067.4 & 2.35 & 2.32 & 4.37 & 5.22 \\
\hline LH5 & 1822.4 & 3.79 & 1.70 & 2.51 & 3.15 \\
\hline LH6 & 1288.9 & 5.05 & 1.54 & 1.93 & 2.69 \\
\hline
\end{tabular}


Table B.59 - The response of the buildings subjected to E6C18 ground motion parallel to the long side of the building with $\alpha=0.10$

\begin{tabular}{|c|c|c|c|c|c|}
\hline \multirow{2}{*}{ Building } & \multirow{2}{*}{$V e$} & \multirow{2}{*}{$\begin{array}{c}\text { Ductility }(\mu) \\
R y=3\end{array}$} & \multicolumn{3}{|c|}{ Force Reduction Factor $(R y)$} \\
\hline & & & $\mu=2$ & $\mu=3$ & $\mu=4$ \\
\hline SL1 & 183.6 & 4.36 & 1.42 & 2.44 & 2.87 \\
\hline SL3 & 185.1 & 4.33 & 1.41 & 2.44 & 2.84 \\
\hline SL4 & 187.9 & 4.40 & 1.40 & 2.43 & 2.85 \\
\hline SH1 & 353.9 & 3.90 & 1.39 & 2.40 & 3.13 \\
\hline $\mathrm{SH} 2$ & 336.3 & 3.58 & 1.39 & 2.39 & 3.58 \\
\hline $\mathrm{SH} 3$ & 310.4 & 3.01 & 1.60 & 3.00 & 4.09 \\
\hline SH4 & 309.1 & 3.33 & 1.42 & 2.69 & 3.59 \\
\hline SH5 & 290.3 & 2.97 & 1.55 & 2.95 & 3.82 \\
\hline ML1 & 277.4 & 6.66 & 1.54 & 1.83 & 2.12 \\
\hline ML2 & 243.2 & 8.32 & 1.60 & 1.78 & 1.95 \\
\hline ML3 & 234.9 & 8.19 & 1.64 & 1.89 & 2.07 \\
\hline ML4 & 248.6 & 8.02 & 1.58 & 1.77 & 1.94 \\
\hline ML5 & 239.4 & 8.76 & 1.61 & 1.81 & 1.96 \\
\hline ML6 & 234.5 & 7.93 & 1.65 & 1.86 & 2.04 \\
\hline MH1 & 542.7 & 7.62 & 1.60 & 1.95 & 2.24 \\
\hline MH2 & 541.7 & 6.69 & 1.46 & 1.79 & 2.10 \\
\hline MH3 & 505.9 & 4.20 & 1.42 & 1.79 & 2.74 \\
\hline MH4 & 535.1 & 7.84 & 1.52 & 1.86 & 2.17 \\
\hline MH5 & 535.3 & 6.43 & 1.43 & 1.75 & 2.05 \\
\hline MH6 & 550.8 & 6.19 & 1.54 & 1.93 & 2.28 \\
\hline LL1 & 989.4 & 6.58 & 1.59 & 1.97 & 2.31 \\
\hline LL2 & 985.6 & 6.80 & 1.55 & 1.92 & 2.24 \\
\hline LL3 & 923.2 & 4.54 & 1.41 & 1.74 & 2.44 \\
\hline LL4 & 989.3 & 6.59 & 1.60 & 1.96 & 2.31 \\
\hline LL5 & 977.5 & 6.94 & 1.46 & 1.80 & 2.11 \\
\hline LL6 & 786.1 & 3.58 & 1.46 & 2.73 & 3.22 \\
\hline LH1 & 2110.3 & 5.43 & 1.50 & 1.88 & 2.26 \\
\hline LH2 & 2008.9 & 4.07 & 1.49 & 1.89 & 2.90 \\
\hline LH3 & 1580.5 & 3.38 & 2.18 & 2.78 & 3.41 \\
\hline LH4 & 2048.8 & 4.41 & 1.50 & 1.92 & 2.52 \\
\hline LH5 & 1939.9 & 3.20 & 1.51 & 2.33 & 4.68 \\
\hline LH6 & 1411.4 & 3.87 & 2.01 & 2.54 & 3.10 \\
\hline
\end{tabular}


Table B.60 - The response of the buildings subjected to E6C42 ground motion parallel to the long side of the building with $\alpha=0.10$

\begin{tabular}{|c|c|c|c|c|c|}
\hline \multirow{2}{*}{ Building } & \multirow{2}{*}{$V e$} & \multirow{2}{*}{$\begin{array}{c}\text { Ductility }(\mu) \\
R y=3\end{array}$} & \multicolumn{3}{|c|}{ Force Reduction Factor $(R y)$} \\
\hline & & & $\mu=2$ & $\mu=3$ & $\mu=4$ \\
\hline SL1 & 172.3 & 4.40 & 1.91 & 2.32 & 2.74 \\
\hline SL3 & 165.3 & 4.36 & 1.93 & 2.35 & 2.80 \\
\hline SL4 & 142.9 & 4.57 & 1.48 & 2.39 & 2.76 \\
\hline SH1 & 224.2 & 4.60 & 1.42 & 1.83 & 2.59 \\
\hline $\mathrm{SH} 2$ & 199.8 & 4.91 & 1.40 & 1.71 & 2.27 \\
\hline $\mathrm{SH} 3$ & 183.4 & 4.19 & 1.40 & 1.71 & 2.33 \\
\hline SH4 & 178.1 & 4.82 & 1.36 & 1.64 & 1.94 \\
\hline SH5 & 176.8 & 3.95 & 1.39 & 1.68 & 2.71 \\
\hline ML1 & 270.0 & 4.18 & 1.85 & 2.56 & 2.96 \\
\hline ML2 & 232.6 & 6.19 & 1.44 & 1.96 & 2.41 \\
\hline ML3 & 246.2 & 5.25 & 1.41 & 1.82 & 2.35 \\
\hline ML4 & 242.7 & 5.61 & 1.54 & 2.13 & 2.55 \\
\hline ML5 & 231.9 & 6.30 & 1.40 & 1.87 & 2.33 \\
\hline ML6 & 241.9 & 5.33 & 1.41 & 1.90 & 2.41 \\
\hline MH1 & 584.0 & 4.13 & 1.43 & 1.75 & 2.93 \\
\hline MH2 & 555.6 & 3.60 & 1.42 & 2.06 & 3.25 \\
\hline MH3 & 423.0 & 5.94 & 1.32 & 1.57 & 1.96 \\
\hline MH4 & 582.5 & 4.16 & 1.41 & 1.75 & 2.82 \\
\hline MH5 & 536.4 & 3.91 & 1.41 & 2.04 & 3.93 \\
\hline MH6 & 562.1 & 3.16 & 1.46 & 2.61 & 3.52 \\
\hline LL1 & 1051.5 & 3.53 & 1.47 & 2.00 & 3.48 \\
\hline LL2 & 1047.9 & 3.48 & 1.45 & 2.01 & 3.35 \\
\hline LL3 & 780.7 & 5.53 & 1.31 & 1.60 & 2.05 \\
\hline LL4 & 1051.6 & 3.49 & 1.46 & 1.95 & 3.47 \\
\hline LL5 & 1005.8 & 3.67 & 1.41 & 1.99 & 3.20 \\
\hline LL6 & 748.6 & 4.85 & 1.50 & 1.85 & 2.39 \\
\hline LH1 & 2122.3 & 2.92 & 1.49 & 3.04 & 3.85 \\
\hline LH2 & 1879.5 & 3.29 & 1.48 & 2.47 & 3.75 \\
\hline LH3 & 1531.2 & 3.93 & 1.56 & 2.01 & 3.19 \\
\hline LH4 & 2003.8 & 2.86 & 1.52 & 3.17 & 4.10 \\
\hline LH5 & 1683.2 & 4.04 & 1.39 & 1.84 & 2.88 \\
\hline LH6 & 1390.8 & 4.09 & 1.53 & 1.98 & 2.84 \\
\hline
\end{tabular}




\section{Appendix C}

The results of the analyses which led to the conclusions reached in Chapter 5 are presented. The ratio of the actual bending moments at the mid-span, and the actual shear forces at the quarter-span for buildings subjected to generated ground motions, determined from nonlinear time history analyses, to their corresponding values obtained from the application of the total seismic load with specific distribution patterns are presented in the following tables. The considered distribution patterns, as pointed out earlier, are a uniform distribution pattern and a parabolic distribution pattern as recommended by FEMA 356. For presentation purposes, symbols are used for these ratio values.

The presented results correspond to different post-yield stiffness values, ten ground motions, and three targets of ductility demand and two directions of the application of seismic load, all of which were specified earlier. 
Table C. 1 - The ratio of actual mid-span bending moments and quarter-span shear forces for buildings to the corresponding values obtained from FEMA parabolic distribution and a uniform distribution of seismic load when subjected to M6C1 ground motion and the Load is acting parallel to the short side, $\alpha=0.00$

\begin{tabular}{|c|c|c|c|c|c|c|c|c|c|c|c|c|}
\hline \multirow{2}{*}{ Building } & \multicolumn{4}{|c|}{$\mu=2$} & \multicolumn{4}{|c|}{$\mu=3$} & \multicolumn{4}{|c|}{$\mu=4$} \\
\hline & $M . F$ & V.F & $M . U$ & $V . U$ & $M . F$ & $V . F$ & $M . U$ & $V . U$ & M.F & V.F & $M . U$ & $V . U$ \\
\hline SL1 & 1.06 & 1.09 & 1.33 & 1.49 & 1.09 & 1.13 & 1.36 & 1.56 & 1.13 & 1.20 & 1.42 & 1.65 \\
\hline SL3 & 08 & 1.12 & 1.35 & 1.54 & 1.13 & 1.19 & 1.41 & 1.63 & 1.16 & 1.23 & 1.45 & 1.70 \\
\hline SL4 & 1.18 & 1.27 & 1.48 & 1.74 & 1.22 & 1.32 & 1.52 & 1.81 & 1.25 & 1.36 & 1.56 & 1.86 \\
\hline SH1 & 1.19 & 1.28 & 1.49 & 1.76 & 1.27 & 1.39 & 1.58 & 1.91 & 1.31 & 1.45 & 1.63 & 1.99 \\
\hline $\mathrm{SH} 2$ & 1.26 & 1.39 & 1.58 & 1.91 & 1.30 & 1.45 & 1.63 & 1.99 & 1.33 & 1.48 & 1.66 & 2.04 \\
\hline SH3 & 1.30 & 1.44 & 1.62 & 1.99 & 1.33 & 1.49 & 1.66 & 2.05 & 1.36 & 1.54 & 1.70 & 2.12 \\
\hline SH4 & 1.29 & 1.43 & 1.61 & 1.96 & 1.33 & 1.51 & 1.66 & 2.07 & 1.36 & 1.56 & 1.70 & 2.14 \\
\hline SH5 & 1.29 & 1.43 & 1.61 & 1.97 & 1.33 & 1.51 & 1.67 & 2.08 & 1.36 & 1.57 & 1.70 & 2.15 \\
\hline SH6 & 1.30 & 1.44 & 1.62 & 1.99 & 1.33 & 1.49 & 1.66 & 2.05 & 1.36 & 1.54 & 1.70 & 2.12 \\
\hline ML1 & 1.19 & 1.31 & 1.48 & 1.81 & 1.25 & 1.41 & 1.57 & 1.93 & 1.29 & 1.47 & 1.61 & 2.02 \\
\hline ML2 & 1.01 & 1.01 & 1.26 & 1.39 & 1.15 & 1.14 & 1.44 & 1.57 & 1.18 & 1.18 & 1.48 & 1.62 \\
\hline ML3 & 0.99 & 0.99 & 1.24 & 1.37 & 1.10 & 1.13 & 1.37 & 1.55 & 1.15 & 1.20 & 1.43 & 1.65 \\
\hline ML4 & 1.17 & 1.27 & 1.47 & 1.75 & 1.23 & 1.34 & 1.54 & 1.84 & 1.27 & 1.39 & 1.59 & 1.91 \\
\hline ML5 & 1.18 & 1.28 & 1.47 & 1.76 & 1.24 & 1.35 & 1.54 & 1.86 & 1.28 & 1.40 & 1.59 & 1.93 \\
\hline ML6 & 1.01 & 0.98 & 1.26 & 1.34 & 1.12 & 1.12 & 1.40 & 1.53 & 1.15 & 1.17 & 1.44 & 1.61 \\
\hline MH1 & 1.08 & 1.15 & 1.35 & 1.58 & 1.13 & 1.20 & 1.41 & 1.65 & 1.17 & 1.26 & 1.46 & 1.73 \\
\hline MH2 & 1.08 & 1.12 & 1.36 & 1.54 & 1.21 & 1.31 & 1.51 & 1.80 & 1.24 & 1.36 & 1.55 & 1.87 \\
\hline MH3 & 1.50 & 1.74 & 1.87 & 2.39 & 1.62 & 1.88 & 2.03 & 2.58 & 1.68 & 1.97 & 2.10 & 2.71 \\
\hline MH4 & 1.12 & 1.15 & 1.40 & 1.59 & 1.22 & 1.31 & 1.53 & 1.81 & 1.26 & 1.37 & 1.57 & 1.88 \\
\hline MH5 & 1.19 & 1.27 & 1.49 & 1.74 & 1.24 & 1.35 & 1.55 & 1.85 & 1.27 & 1.39 & 1.59 & 1.91 \\
\hline MH6 & 1.16 & 1.29 & 1.46 & 1.77 & 1.38 & 1.62 & 1.73 & 2.23 & 1.48 & 1.72 & 1.85 & 2.37 \\
\hline LL1 & 1.04 & 1.06 & 1.30 & 1.45 & 1.06 & 1.11 & 1.32 & 1.53 & 1.16 & 1.24 & 1.45 & 1.70 \\
\hline LL2 & 1.05 & 1.07 & 1.31 & 1.47 & 1.07 & 1.14 & 1.34 & 1.56 & 1.11 & 1.20 & 1.39 & 1.65 \\
\hline LL3 & 1.19 & 1.29 & 1.49 & 1.77 & 1.30 & 1.45 & 1.62 & 1.99 & 1.33 & 1.49 & 1.67 & 2.05 \\
\hline LL4 & 0.96 & 0.99 & 1.20 & 1.36 & 1.04 & 1.06 & 1.30 & 1.45 & 1.12 & 1.13 & 1.40 & 1.55 \\
\hline LL5 & 0.95 & 0.98 & 1.19 & 1.34 & 1.03 & 1.04 & 1.29 & 1.43 & 1.12 & 1.12 & 1.40 & 1.54 \\
\hline LL6 & 1.18 & 1.42 & 1.48 & 1.95 & 1.25 & 1.51 & 1.56 & 2.08 & 1.29 & 1.64 & 1.61 & 2.25 \\
\hline LH1 & 1.17 & 1.25 & 1.46 & 1.72 & 1.32 & 1.44 & 1.65 & 1.98 & 1.46 & 1.62 & 1.82 & 2.23 \\
\hline LH2 & 1.23 & 1.35 & 1.54 & 1.86 & 1.44 & 1.65 & 1.80 & 2.28 & 1.53 & 1.71 & 1.91 & 2.35 \\
\hline LH3 & 1.34 & 1.70 & 1.67 & 2.34 & 1.56 & 1.97 & 1.95 & 2.70 & 1.71 & 2.13 & 2.14 & 2.93 \\
\hline LH4 & 1.26 & 1.36 & 1.57 & 1.87 & 1.47 & 1.65 & 1.84 & 2.27 & 1.60 & 1.82 & 2.00 & 2.50 \\
\hline LH5 & 1.28 & 1.38 & 1.60 & 1.90 & 1.53 & 1.72 & 1.91 & 2.37 & 1.64 & 1.88 & 2.05 & 2.58 \\
\hline LH6 & 1.21 & 1.50 & 1.51 & 2.06 & 1.35 & 1.78 & 1.68 & 2.45 & 1.47 & 1.93 & 1.84 & 2.65 \\
\hline
\end{tabular}


Table C. 2 - The ratio of actual mid-span bending moments and quarter-span shear forces for buildings to the corresponding values obtained from FEMA parabolic distribution and a uniform distribution of seismic load when subjected to M6C2 ground motion and the Load is acting parallel to the short side, $\alpha=0.00$

\begin{tabular}{|c|c|c|c|c|c|c|c|c|c|c|c|c|}
\hline \multirow{2}{*}{ Building } & \multicolumn{4}{|c|}{$\mu=2$} & \multicolumn{4}{|c|}{$\mu=3$} & \multicolumn{4}{|c|}{$\mu=4$} \\
\hline & $M . F$ & $V . F$ & M.U & V.U & M.F & $V . F$ & $M . U$ & $V . U$ & M.F & $V . F$ & $M . U$ & V.U \\
\hline SL1 & 1.08 & 1.13 & 1.34 & 1.56 & 1.10 & 1.18 & 1.38 & 1.62 & 1.11 & 1.22 & 1.39 & 1.67 \\
\hline SL3 & 1.09 & 1.15 & 1.36 & 1.59 & 1.11 & 1.21 & 1.39 & 1.66 & 1.12 & 1.24 & 1.40 & 1.71 \\
\hline SL4 & 1.10 & 1.16 & 1.37 & 1.60 & 1.11 & 1.20 & 1.39 & 1.65 & 1.12 & 1.23 & 1.40 & 1.69 \\
\hline SH1 & 1.13 & 1.20 & 1.41 & 1.65 & 1.31 & 1.48 & 1.64 & 2.04 & 1.32 & 1.51 & 1.65 & 2.08 \\
\hline $\mathrm{SH} 2$ & 1.10 & 1.10 & 1.37 & 1.51 & 1.29 & 1.38 & 1.61 & 1.90 & 1.30 & 1.42 & 1.63 & 1.95 \\
\hline SH3 & 1.07 & 1.09 & 1.34 & 1.50 & 1.26 & 1.34 & 1.58 & 1.84 & 1.28 & 1.39 & 1.61 & 1.92 \\
\hline SH4 & 16 & 1.25 & 1.45 & 1.72 & 1.20 & 1.30 & 1.50 & 1.78 & 1.35 & 1.50 & 1.69 & 2.06 \\
\hline & 17 & 1.26 & 1.46 & 1.73 & 1.22 & 1.32 & 1.53 & 1.82 & 1.37 & 1.54 & 1.72 & 2.11 \\
\hline & 1.07 & 1.10 & 1.34 & 1.51 & 1.15 & 1.20 & 1.43 & 1.64 & 1.29 & 1.39 & 1.61 & 1.91 \\
\hline & 1.49 & 1.68 & 1.86 & 2.31 & 1.53 & 1.74 & 1.91 & 2.39 & 1.55 & 1.76 & 1.94 & 2.43 \\
\hline ML2 & 1.36 & 1.50 & 1.70 & 2.07 & 1.44 & 1.63 & 1.80 & 2.24 & 1.48 & 1.70 & 1.85 & 2.33 \\
\hline ML3 & 1.16 & 1.20 & 1.45 & 1.65 & 1.34 & 1.50 & 1.67 & 2.06 & 1.37 & 1.55 & 1.71 & 2.14 \\
\hline ML4 & 1.45 & 1.64 & 1.82 & 2.26 & 1.53 & 1.74 & 1.91 & 2.39 & 1.58 & 1.81 & 1.97 & 2.49 \\
\hline ML5 & 1.46 & 1.66 & 1.83 & 2.28 & 1.52 & 1.73 & 1.90 & 2.38 & 1.57 & 1.79 & 1.96 & 2.47 \\
\hline ML6 & 1.26 & 1.36 & 1.58 & 1.87 & 1.39 & 1.58 & 1.74 & 2.17 & 1.43 & 1.64 & 1.79 & 2.26 \\
\hline MH1 & 1.08 & 1.12 & 1.35 & 1.54 & 1.27 & 1.43 & 1.59 & 1.96 & 1.30 & 1.47 & 1.63 & 2.03 \\
\hline MH2 & 1.03 & 1.09 & 1.29 & 1.50 & 1.09 & 1.16 & 1.36 & 1.59 & 1.13 & 1.20 & 1.41 & 1.66 \\
\hline MH3 & 1.36 & 1.53 & 1.70 & 2.10 & 1.43 & 1.60 & 1.78 & 2.20 & 1.45 & 1.62 & 1.81 & 2.23 \\
\hline MH4 & 0.91 & 0.94 & 1.14 & 1.30 & 1.02 & 1.05 & 1.27 & 1.45 & 1.05 & 1.11 & 1.31 & 1.53 \\
\hline MH5 & 0.93 & 0.97 & 1.16 & 1.33 & 0.98 & 1.03 & 1.22 & 1.42 & 1.04 & 1.17 & 1.30 & 1.61 \\
\hline MH6 & 1.55 & 1.78 & 1.94 & 2.45 & 1.59 & 1.83 & 1.99 & 2.51 & 1.59 & 1.85 & 1.99 & 2.55 \\
\hline LL1 & 1.26 & 1.46 & 1.58 & 2.01 & 1.28 & 1.51 & 1.60 & 2.08 & 1.29 & 1.52 & 1.61 & 2.09 \\
\hline LL2 & 1.24 & 1.42 & 1.55 & 1.96 & 1.26 & 1.49 & 1.58 & 2.04 & 1.26 & 1.49 & 1.58 & 2.05 \\
\hline LL3 & 1.10 & 1.18 & 1.38 & 1.62 & 1.19 & 1.29 & 1.49 & 1.77 & 1.21 & 1.30 & 1.51 & 1.79 \\
\hline LL4 & 1.31 & 1.48 & 1.64 & 2.03 & 1.42 & 1.67 & 1.77 & 2.29 & 1.43 & 1.69 & 1.79 & 2.32 \\
\hline LL5 & 1.34 & 1.51 & 1.67 & 2.08 & 1.43 & 1.68 & 1.79 & 2.31 & 1.44 & 1.69 & 1.80 & 2.33 \\
\hline LL6 & 1.35 & 1.70 & 1.69 & 2.34 & 1.43 & 1.76 & 1.79 & 2.42 & 1.49 & 1.81 & 1.86 & 2.49 \\
\hline LH1 & 1.20 & 1.31 & 1.50 & 1.80 & 1.25 & 1.40 & 1.57 & 1.92 & 1.27 & 1.42 & 1.59 & 1.95 \\
\hline LH2 & 1.32 & 1.45 & 1.64 & 2.00 & 1.36 & 1.51 & 1.70 & 2.08 & 1.40 & 1.54 & 1.75 & 2.12 \\
\hline LH3 & 1.25 & 1.51 & 1.56 & 2.07 & 1.33 & 1.58 & 1.67 & 2.17 & 1.37 & 1.61 & 1.71 & 2.21 \\
\hline LH4 & 1.29 & 1.44 & 1.62 & 1.97 & 1.34 & 1.50 & 1.68 & 2.07 & 1.39 & 1.55 & 1.73 & 2.13 \\
\hline LH5 & 1.30 & 1.44 & 1.62 & 1.99 & 1.35 & 1.51 & 1.69 & 2.07 & 1.40 & 1.55 & 1.75 & 2.13 \\
\hline LH6 & 1.21 & 1.47 & 1.51 & 2.02 & 1.32 & 1.60 & 1.65 & 2.20 & 1.39 & 1.67 & 1.74 & 2.29 \\
\hline
\end{tabular}


Table C.3 - The ratio of actual mid-span bending moments and quarter-span shear forces for buildings to the corresponding values obtained from FEMA parabolic distribution and a uniform distribution of seismic load when subjected to M6C26 ground motion and the Load is acting parallel to the short side, $\alpha=0.00$

\begin{tabular}{|c|c|c|c|c|c|c|c|c|c|c|c|c|}
\hline \multirow{2}{*}{ Building } & \multicolumn{4}{|c|}{$\mu=2$} & \multicolumn{4}{|c|}{$\mu=3$} & \multicolumn{4}{|c|}{$\mu=4$} \\
\hline & $M . F$ & $V . F$ & M.U & V.U & $M . F$ & $V . F$ & $M . U$ & V.U & $M . F$ & V.F & $M . U$ & $V . U$ \\
\hline SL1 & 1.11 & 1.17 & 1.39 & 1.60 & 1.16 & 1.24 & 1.45 & 1.71 & 1.19 & 1.30 & 1.49 & 1.79 \\
\hline SL3 & 12 & 1.17 & 1.39 & 1.61 & 1.16 & 1.24 & 1.45 & 1.71 & 1.20 & 1.30 & 1.49 & 1.78 \\
\hline SL4 & 13 & 1.19 & 1.41 & 1.63 & 1.17 & 1.25 & 1.46 & 1.72 & 1.20 & 1.30 & 1.50 & 1.78 \\
\hline SH1 & 15 & 1.21 & 1.44 & 1.66 & 1.19 & 1.27 & 1.49 & 1.75 & 1.22 & 1.31 & 1.53 & 1.81 \\
\hline $\mathrm{SH} 2$ & 1.17 & 1.25 & 1.47 & 1.72 & 1.21 & 1.31 & 1.51 & 1.80 & 1.24 & 1.35 & 1.55 & 1.86 \\
\hline SH3 & 1.17 & 1.26 & 1.47 & 1.73 & 1.21 & 1.32 & 1.52 & 1.82 & 1.23 & 1.36 & 1.54 & 1.87 \\
\hline SH4 & 1.16 & 1.25 & 1.45 & 1.72 & 1.20 & 1.31 & 1.50 & 1.80 & 1.23 & 1.36 & 1.54 & 1.87 \\
\hline SH5 & 1.16 & 1.25 & 1.45 & 1.72 & 1.20 & 1.32 & 1.50 & 1.81 & 1.22 & 1.36 & 1.53 & 1.86 \\
\hline SH6 & 1.18 & 1.26 & 1.47 & 1.73 & 1.21 & 1.32 & 1.51 & 1.81 & 1.23 & 1.36 & 1.54 & 1.87 \\
\hline ML1 & 1.06 & 1.09 & 1.33 & 1.51 & 1.20 & 1.30 & 1.50 & 1.78 & 1.23 & 1.35 & 1.54 & 1.85 \\
\hline ML2 & 0.94 & 1.02 & 1.18 & 1.41 & 1.13 & 1.25 & 1.41 & 1.71 & 1.16 & 1.34 & 1.45 & 1.84 \\
\hline & 0.91 & 0.97 & 1.13 & 1.34 & 1.08 & 1.22 & 1.35 & 1.68 & 1.16 & 1.33 & 1.44 & 1.83 \\
\hline ML4 & 1.03 & 1.08 & 1.28 & 1.49 & 1.19 & 1.30 & 1.49 & 1.78 & 1.22 & 1.34 & 1.53 & 1.84 \\
\hline ML5 & 1.03 & 1.08 & 1.29 & 1.49 & 1.18 & 1.29 & 1.48 & 1.78 & 1.22 & 1.34 & 1.53 & 1.84 \\
\hline ML6 & 0.93 & 1.01 & 1.16 & 1.39 & 1.10 & 1.26 & 1.37 & 1.73 & 1.15 & 1.36 & 1.43 & 1.87 \\
\hline MH1 & 0.94 & 1.03 & 1.18 & 1.41 & 1.17 & 1.28 & 1.47 & 1.76 & 1.25 & 1.36 & 1.56 & 1.87 \\
\hline MH2 & 1.02 & 1.08 & 1.27 & 1.48 & 1.11 & 1.22 & 1.39 & 1.68 & 1.18 & 1.33 & 1.47 & 1.83 \\
\hline MH3 & 1.13 & 1.42 & 1.41 & 1.96 & 1.44 & 1.67 & 1.79 & 2.30 & 1.50 & 1.72 & 1.87 & 2.37 \\
\hline MH4 & 1.01 & 1.05 & 1.27 & 1.44 & 1.07 & 1.18 & 1.33 & 1.62 & 1.10 & 1.28 & 1.37 & 1.77 \\
\hline MH5 & 1.02 & 1.08 & 1.27 & 1.49 & 1.07 & 1.23 & 1.34 & 1.69 & 1.11 & 1.35 & 1.39 & 1.86 \\
\hline MH6 & 1.18 & 1.27 & 1.47 & 1.75 & 1.24 & 1.36 & 1.55 & 1.86 & 1.31 & 1.46 & 1.64 & 2.00 \\
\hline LL1 & 1.17 & 1.24 & 1.46 & 1.70 & 1.25 & 1.36 & 1.56 & 1.87 & 1.41 & 1.57 & 1.77 & 2.16 \\
\hline & 1.18 & 1.26 & 1.48 & 1.73 & 1.27 & 1.38 & 1.58 & 1.90 & 1.40 & 1.58 & 1.75 & 2.18 \\
\hline LL3 & 1.11 & 1.19 & 1.38 & 1.64 & 1.15 & 1.30 & 1.44 & 1.79 & 1.19 & 1.41 & 1.49 & 1.94 \\
\hline LL4 & 1.10 & 1.20 & 1.38 & 1.65 & 1.30 & 1.45 & 1.63 & 2.00 & 1.34 & 1.57 & 1.68 & 2.16 \\
\hline LL5 & 1.10 & 1.19 & 1.38 & 1.64 & 1.32 & 1.45 & 1.65 & 1.99 & 1.36 & 1.57 & 1.70 & 2.16 \\
\hline LL6 & 1.53 & 1.83 & 1.91 & 2.52 & 1.66 & 2.00 & 2.08 & 2.74 & 1.74 & 2.08 & 2.17 & 2.86 \\
\hline LH1 & 1.12 & 1.23 & 1.39 & 1.69 & 1.18 & 1.36 & 1.48 & 1.87 & 1.42 & 1.67 & 1.78 & 2.29 \\
\hline LH2 & 1.20 & 1.46 & 1.50 & 2.00 & 1.47 & 1.73 & 1.84 & 2.38 & 1.71 & 1.94 & 2.13 & 2.67 \\
\hline LH3 & 1.36 & 1.71 & 1.70 & 2.35 & 1.79 & 2.14 & 2.23 & 2.94 & 1.86 & 2.20 & 2.33 & 3.02 \\
\hline LH4 & 1.10 & 1.30 & 1.37 & 1.79 & 1.43 & 1.67 & 1.79 & 2.30 & 1.71 & 1.92 & 2.14 & 2.64 \\
\hline LH5 & 1.11 & 1.33 & 1.38 & 1.83 & 1.48 & 1.72 & 1.85 & 2.36 & 1.70 & 1.91 & 2.13 & 2.62 \\
\hline LH6 & 1.39 & 1.76 & 1.74 & 2.42 & 1.89 & 2.23 & 2.36 & 3.07 & 1.93 & 2.26 & 2.42 & 3.10 \\
\hline
\end{tabular}


Table C.4 - The ratio of actual mid-span bending moments and quarter-span shear forces for buildings to the corresponding values obtained from FEMA parabolic distribution and a uniform distribution of seismic load when subjected to M6C31 ground motion and the Load is acting parallel to the short side, $\alpha=0.00$

\begin{tabular}{|c|c|c|c|c|c|c|c|c|c|c|c|c|}
\hline \multirow{2}{*}{ Building } & \multicolumn{4}{|c|}{$\mu=2$} & \multicolumn{4}{|c|}{$\mu=3$} & \multicolumn{4}{|c|}{$\mu=4$} \\
\hline & $M . F$ & $V . F$ & $M . U$ & $V . U$ & M.F & $V . F$ & $M . U$ & $V . U$ & M.F & $V . F$ & $M . U$ & V.U \\
\hline SL1 & 1.13 & 1.20 & 1.41 & 1.65 & 1.17 & 1.26 & 1.46 & 1.73 & 1.20 & 1.31 & 1.50 & 1.80 \\
\hline SL3 & 14 & 1.21 & 1.43 & 1.67 & 1.19 & 1.29 & 1.49 & 1.77 & 1.22 & 1.34 & 1.53 & 1.84 \\
\hline SL4 & 1.16 & 1.22 & 1.45 & 1.68 & 1.22 & 1.31 & 1.52 & 1.80 & 1.26 & 1.37 & 1.57 & 1.89 \\
\hline SH1 & 1.14 & 1.18 & 1.42 & 1.63 & 1.25 & 1.29 & 1.56 & 1.77 & 1.35 & 1.41 & 1.69 & 1.94 \\
\hline $\mathrm{SH} 2$ & 1.20 & 1.24 & 1.50 & 1.70 & 1.28 & 1.34 & 1.60 & 1.84 & 1.34 & 1.42 & 1.67 & 1.95 \\
\hline SH3 & 1.22 & 1.29 & 1.52 & 1.77 & 1.30 & 1.41 & 1.63 & 1.94 & 1.36 & 1.49 & 1.70 & 2.05 \\
\hline SH4 & 1.24 & 1.35 & 1.55 & 1.86 & 1.29 & 1.43 & 1.61 & 1.97 & 1.35 & 1.51 & 1.69 & 2.08 \\
\hline & 24 & 1.36 & 1.55 & 1.86 & 1.30 & 1.45 & 1.63 & 1.99 & 1.36 & 1.53 & 1.70 & 2.10 \\
\hline & 1.22 & 1.29 & 1.52 & 1.78 & 1.29 & 1.40 & 1.62 & 1.93 & 1.36 & 1.50 & 1.70 & 2.06 \\
\hline & 28 & 1.45 & 1.61 & 2.00 & 1.33 & 1.53 & 1.66 & 2.10 & 1.38 & 1.59 & 1.72 & 2.19 \\
\hline ML2 & 1.25 & 1.33 & 1.56 & 1.83 & 1.30 & 1.43 & 1.62 & 1.96 & 1.33 & 1.50 & 1.66 & 2.07 \\
\hline ML3 & 1.16 & 1.25 & 1.44 & 1.72 & 1.20 & 1.35 & 1.51 & 1.86 & 1.25 & 1.44 & 1.56 & 1.98 \\
\hline ML4 & 1.25 & 1.40 & 1.56 & 1.92 & 1.27 & 1.43 & 1.59 & 1.97 & 1.30 & 1.50 & 1.63 & 2.06 \\
\hline ML5 & 1.25 & 1.39 & 1.56 & 1.91 & 1.27 & 1.44 & 1.59 & 1.98 & 1.31 & 1.51 & 1.63 & 2.08 \\
\hline ML6 & 1.22 & 1.31 & 1.52 & 1.80 & 1.27 & 1.42 & 1.59 & 1.95 & 1.31 & 1.50 & 1.63 & 2.07 \\
\hline MH1 & 1.13 & 1.18 & 1.42 & 1.63 & 1.19 & 1.28 & 1.49 & 1.75 & 1.24 & 1.38 & 1.56 & 1.90 \\
\hline MH2 & 1.17 & 1.21 & 1.46 & 1.66 & 1.21 & 1.29 & 1.52 & 1.77 & 1.25 & 1.35 & 1.56 & 1.85 \\
\hline MH3 & 1.23 & 1.44 & 1.53 & 1.98 & 1.28 & 1.48 & 1.60 & 2.04 & 1.32 & 1.52 & 1.64 & 2.09 \\
\hline MH4 & 1.20 & 1.26 & 1.50 & 1.74 & 1.23 & 1.32 & 1.53 & 1.81 & 1.25 & 1.37 & 1.56 & 1.88 \\
\hline MH5 & 19 & 1.27 & 1.49 & 1.75 & 1.23 & 1.34 & 1.54 & 1.84 & 1.26 & 1.39 & 1.57 & 1.91 \\
\hline MH6 & 1.30 & 1.45 & 1.63 & 2.00 & 1.50 & 1.68 & 1.87 & 2.31 & 1.63 & 1.84 & 2.04 & 2.54 \\
\hline LL1 & 1.20 & 1.26 & 1.50 & 1.73 & 1.36 & 1.46 & 1.70 & 2.01 & 1.43 & 1.57 & 1.79 & 2.16 \\
\hline LL2 & 1.22 & 1.29 & 1.53 & 1.78 & 1.39 & 1.51 & 1.73 & 2.08 & 1.43 & 1.58 & 1.79 & 2.17 \\
\hline LL3 & 1.05 & 1.25 & 1.32 & 1.71 & 1.10 & 1.37 & 1.37 & 1.88 & 1.17 & 1.47 & 1.46 & 2.02 \\
\hline LL4 & 1.32 & 1.41 & 1.65 & 1.94 & 1.41 & 1.52 & 1.77 & 2.10 & 1.44 & 1.55 & 1.80 & 2.13 \\
\hline LL5 & 1.31 & 1.39 & 1.64 & 1.91 & 1.42 & 1.53 & 1.78 & 2.10 & 1.45 & 1.55 & 1.81 & 2.14 \\
\hline LL6 & 1.02 & 1.40 & 1.28 & 1.93 & 1.06 & 1.46 & 1.33 & 2.00 & 1.08 & 1.50 & 1.36 & 2.06 \\
\hline LH1 & 1.13 & 1.37 & 1.41 & 1.88 & 1.19 & 1.50 & 1.48 & 2.06 & 1.26 & 1.61 & 1.58 & 2.22 \\
\hline LH2 & 1.20 & 1.46 & 1.50 & 2.01 & 1.24 & 1.57 & 1.55 & 2.15 & 1.26 & 1.64 & 1.57 & 2.25 \\
\hline LH3 & 1.13 & 1.40 & 1.42 & 1.93 & 1.27 & 1.55 & 1.59 & 2.13 & 1.36 & 1.66 & 1.70 & 2.29 \\
\hline LH4 & 1.18 & 1.38 & 1.47 & 1.90 & 1.21 & 1.52 & 1.52 & 2.09 & 1.23 & 1.59 & 1.54 & 2.18 \\
\hline LH5 & 1.18 & 1.36 & 1.48 & 1.88 & 1.22 & 1.50 & 1.53 & 2.06 & 1.24 & 1.58 & 1.55 & 2.17 \\
\hline LH6 & 1.07 & 1.48 & 1.34 & 2.03 & 1.17 & 1.61 & 1.46 & 2.21 & 1.24 & 1.63 & 1.56 & 2.23 \\
\hline
\end{tabular}


Table C.5 - The ratio of actual mid-span bending moments and quarter-span shear forces for buildings to the corresponding values obtained from FEMA parabolic distribution and a uniform distribution of seismic load when subjected to M6C 38 ground motion and the Load is acting parallel to the short side, $\alpha=0.00$

\begin{tabular}{|c|c|c|c|c|c|c|c|c|c|c|c|c|}
\hline \multirow{2}{*}{ Building } & \multicolumn{4}{|c|}{$\mu=2$} & \multicolumn{4}{|c|}{$\mu=3$} & \multicolumn{4}{|c|}{$\mu=4$} \\
\hline & $M . F$ & V.F & $M . U$ & $V . U$ & $M . F$ & $V . F$ & $M . U$ & $V . U$ & $M . F$ & V.F & $M . U$ & $V . U$ \\
\hline SL1 & 1.21 & 1.31 & 1.51 & 1.80 & 1.25 & 1.37 & 1.56 & 1.88 & 1.28 & 1.41 & 1.60 & 1.94 \\
\hline SL3 & .21 & 1.32 & 1.51 & 1.82 & 1.25 & 1.37 & 1.56 & 1.88 & 1.28 & 1.41 & 1.60 & 1.94 \\
\hline SL4 & .21 & 1.32 & 1.52 & 1.82 & 1.25 & 1.38 & 1.57 & 1.90 & 1.29 & 1.42 & 1.61 & 1.96 \\
\hline SH1 & 1.18 & 1.22 & 1.47 & 1.67 & 1.26 & 1.33 & 1.58 & 1.82 & 1.33 & 1.41 & 1.66 & 1.94 \\
\hline $\mathrm{SH} 2$ & 1.19 & 1.22 & 1.49 & 1.67 & 1.28 & 1.33 & 1.61 & 1.83 & 1.35 & 1.42 & 1.69 & 1.95 \\
\hline SH3 & 1.21 & 1.23 & 1.51 & 1.70 & 1.30 & 1.34 & 1.62 & 1.85 & 1.36 & 1.43 & 1.71 & 1.96 \\
\hline SH4 & 1.21 & 1.22 & 1.51 & 1.68 & 1.28 & 1.31 & 1.60 & 1.80 & 1.35 & 1.40 & 1.69 & 1.92 \\
\hline SH5 & 1.21 & 1.22 & 1.51 & 1.67 & 1.30 & 1.33 & 1.62 & 1.83 & 1.37 & 1.41 & 1.71 & 1.94 \\
\hline SH6 & 1.21 & 1.23 & 1.51 & 1.70 & 1.29 & 1.33 & 1.61 & 1.83 & 1.36 & 1.42 & 1.70 & 1.95 \\
\hline ML1 & 1.16 & 1.15 & 1.45 & 1.58 & 1.23 & 1.23 & 1.54 & 1.70 & 1.28 & 1.30 & 1.61 & 1.78 \\
\hline ML2 & 1.17 & 1.23 & 1.47 & 1.69 & 1.27 & 1.30 & 1.59 & 1.78 & 1.36 & 1.39 & 1.70 & 1.92 \\
\hline ML3 & 1.16 & 1.21 & 1.45 & 1.67 & 1.28 & 1.34 & 1.60 & 1.84 & 1.37 & 1.43 & 1.71 & 1.97 \\
\hline ML4 & 1.11 & 1.09 & 1.39 & 1.50 & 1.20 & 1.18 & 1.50 & 1.62 & 1.28 & 1.27 & 1.60 & 1.74 \\
\hline ML5 & 1.11 & 1.08 & 1.39 & 1.49 & 1.20 & 1.18 & 1.50 & 1.62 & 1.27 & 1.26 & 1.59 & 1.73 \\
\hline ML6 & 1.17 & 1.21 & 1.46 & 1.66 & 1.28 & 1.32 & 1.60 & 1.82 & 1.38 & 1.43 & 1.72 & 1.96 \\
\hline MH1 & 1.04 & 1.10 & 1.30 & 1.51 & 1.24 & 1.30 & 1.54 & 1.79 & 1.34 & 1.41 & 1.67 & 1.93 \\
\hline MH2 & 0.92 & 0.99 & 1.15 & 1.36 & 1.22 & 1.33 & 1.53 & 1.83 & 1.33 & 1.45 & 1.66 & 1.99 \\
\hline MH3 & 0.96 & 0.93 & 1.20 & 1.27 & 1.06 & 1.04 & 1.33 & 1.43 & 1.26 & 1.39 & 1.58 & 1.91 \\
\hline MH4 & 0.89 & 0.96 & 1.11 & 1.32 & 1.14 & 1.27 & 1.43 & 1.74 & 1.24 & 1.38 & 1.55 & 1.89 \\
\hline MH5 & 0.90 & 0.97 & 1.12 & 1.33 & 1.19 & 1.32 & 1.49 & 1.82 & 1.29 & 1.44 & 1.62 & 1.98 \\
\hline MH6 & 1.14 & 1.15 & 1.43 & 1.58 & 1.27 & 1.31 & 1.58 & 1.79 & 1.36 & 1.44 & 1.71 & 1.98 \\
\hline LL1 & 1.03 & 1.06 & 1.29 & 1.46 & 1.15 & 1.18 & 1.44 & 1.63 & 1.23 & 1.29 & 1.54 & 1.78 \\
\hline LL2 & 1.02 & 1.02 & 1.27 & 1.41 & 1.14 & 1.17 & 1.43 & 1.61 & 1.23 & 1.28 & 1.53 & 1.76 \\
\hline LL3 & 0.89 & 0.86 & 1.12 & 1.18 & 1.03 & 1.16 & 1.29 & 1.59 & 1.13 & 1.27 & 1.41 & 1.75 \\
\hline LL4 & 1.10 & 1.13 & 1.38 & 1.55 & 1.22 & 1.23 & 1.52 & 1.70 & 1.31 & 1.35 & 1.64 & 1.86 \\
\hline LL5 & 1.11 & 1.16 & 1.39 & 1.59 & 1.22 & 1.24 & 1.52 & 1.70 & 1.31 & 1.35 & 1.64 & 1.86 \\
\hline LL6 & 1.09 & 1.19 & 1.37 & 1.64 & 1.29 & 1.43 & 1.62 & 1.96 & 1.47 & 1.73 & 1.84 & 2.37 \\
\hline LH1 & 0.96 & 0.93 & 1.19 & 1.28 & 1.01 & 1.15 & 1.26 & 1.59 & 1.12 & 1.28 & 1.40 & 1.75 \\
\hline LH2 & 1.04 & 1.04 & 1.29 & 1.42 & 1.05 & 1.13 & 1.31 & 1.56 & 1.10 & 1.27 & 1.37 & 1.75 \\
\hline LH3 & 1.12 & 1.21 & 1.40 & 1.67 & 1.20 & 1.32 & 1.50 & 1.82 & 1.28 & 1.43 & 1.60 & 1.96 \\
\hline LH4 & 1.01 & 1.01 & 1.26 & 1.39 & 1.04 & 1.16 & 1.30 & 1.59 & 1.11 & 1.30 & 1.38 & 1.78 \\
\hline LH5 & 1.01 & 1.01 & 1.27 & 1.39 & 1.04 & 1.17 & 1.31 & 1.61 & 1.12 & 1.31 & 1.39 & 1.81 \\
\hline LH6 & 1.07 & 1.15 & 1.34 & 1.58 & 1.19 & 1.32 & 1.49 & 1.81 & 1.27 & 1.43 & 1.59 & 1.97 \\
\hline
\end{tabular}


Table C.6 - The ratio of actual mid-span bending moments and quarter-span shear forces for buildings to the corresponding values obtained from FEMA parabolic distribution and a uniform distribution of seismic load when subjected to M6C1 ground motion and the Load is acting parallel to the long side, $\alpha=0.00$

\begin{tabular}{|c|c|c|c|c|c|c|c|c|c|c|c|c|}
\hline \multirow{2}{*}{ Building } & \multicolumn{4}{|c|}{$\mu=2$} & \multicolumn{4}{|c|}{$\mu=3$} & \multicolumn{4}{|c|}{$\mu=4$} \\
\hline & $M . F$ & $V . F$ & $M . U$ & V.U & M.F & $V . F$ & $M . U$ & $V . U$ & M.F & V.F & M.U & $V . U$ \\
\hline SL1 & 1.04 & 1.06 & 1.30 & 1.45 & 1.07 & 1.11 & 1.33 & 1.52 & 1.10 & 1.16 & 1.38 & 1.60 \\
\hline SL3 & 04 & 1.06 & 1.30 & 1.45 & 1.07 & 1.12 & 1.34 & 1.53 & 1.10 & 1.16 & 1.37 & 1.59 \\
\hline SL4 & 03 & 1.04 & 1.29 & 1.43 & 1.05 & 1.08 & 1.32 & 1.49 & 1.10 & 1.16 & 1.38 & 1.59 \\
\hline SH1 & 14 & 1.19 & 1.42 & 1.64 & 1.30 & 1.46 & 1.63 & 2.01 & 1.30 & 1.46 & 1.63 & 2.01 \\
\hline & 1.13 & 1.18 & 1.42 & 1.62 & 1.24 & 1.38 & 1.56 & 1.90 & 1.27 & 1.41 & 1.58 & 1.94 \\
\hline $\mathrm{SH} 3$ & 1.10 & 1.14 & 1.37 & 1.57 & 1.17 & 1.26 & 1.46 & 1.73 & 1.29 & 1.43 & 1.61 & 1.97 \\
\hline SH4 & 1.12 & 1.16 & 1.40 & 1.59 & 1.21 & 1.30 & 1.51 & 1.79 & 1.30 & 1.45 & 1.63 & 1.99 \\
\hline SH5 & 1.10 & 1.13 & 1.37 & 1.56 & 1.17 & 1.24 & 1.46 & 1.70 & 1.29 & 1.43 & 1.61 & 1.97 \\
\hline ML1 & 1.15 & 1.22 & 1.43 & 1.68 & 1.33 & 1.48 & 1.66 & 2.03 & 1.37 & 1.53 & 1.72 & 2.11 \\
\hline ML2 & 1.18 & 1.27 & 1.47 & 1.75 & 1.34 & 1.49 & 1.67 & 2.04 & 1.37 & 1.54 & 1.72 & 2.11 \\
\hline ML3 & 1.10 & 1.17 & 1.38 & 1.61 & 1.30 & 1.44 & 1.63 & 1.98 & 1.36 & 1.51 & 1.70 & 2.08 \\
\hline ML4 & 1.18 & 1.27 & 1.47 & 1.74 & 1.34 & 1.49 & 1.67 & 2.05 & 1.37 & 1.54 & 1.72 & 2.12 \\
\hline ML5 & 1.17 & 1.26 & 1.46 & 1.74 & 1.32 & 1.47 & 1.65 & 2.03 & 1.37 & 1.53 & 1.71 & 2.10 \\
\hline ML6 & 1.13 & 1.21 & 1.42 & 1.67 & 1.31 & 1.45 & 1.63 & 1.99 & 1.35 & 1.51 & 1.69 & 2.07 \\
\hline & 08 & 1.15 & 1.35 & 1.58 & 1.34 & 1.49 & 1.67 & 2.05 & 1.40 & 1.57 & 1.75 & 2.15 \\
\hline & 22 & 1.32 & 1.53 & 1.81 & 1.34 & 1.51 & 1.67 & 2.07 & 1.38 & 1.53 & 1.72 & 2.10 \\
\hline & 1.30 & 1.41 & 1.62 & 1.93 & 1.53 & 1.76 & 1.91 & 2.43 & 1.60 & 1.87 & 2.00 & 2.57 \\
\hline MH4 & 1.08 & 1.15 & 1.36 & 1.58 & 1.38 & 1.54 & 1.72 & 2.12 & 1.43 & 1.61 & 1.79 & 2.21 \\
\hline MH5 & 1.21 & 1.30 & 1.52 & 1.79 & 1.35 & 1.50 & 1.68 & 2.07 & 1.40 & 1.58 & 1.75 & 2.18 \\
\hline MH6 & 1.17 & 1.23 & 1.46 & 1.69 & 1.31 & 1.41 & 1.64 & 1.95 & 1.32 & 1.47 & 1.65 & 2.02 \\
\hline LL1 & 1.25 & 1.36 & 1.56 & 1.87 & 1.31 & 1.43 & 1.64 & 1.97 & 1.34 & 1.47 & 1.68 & 2.02 \\
\hline LL2 & 1.20 & 1.29 & 1.50 & 1.78 & 1.34 & 1.46 & 1.67 & 2.00 & 1.33 & 1.48 & 1.66 & 2.04 \\
\hline LL3 & 1.39 & 1.53 & 1.74 & 2.10 & 1.47 & 1.65 & 1.84 & 2.27 & 1.55 & 1.77 & 1.94 & 2.44 \\
\hline LL4 & 1.25 & 1.37 & 1.57 & 1.88 & 1.32 & 1.43 & 1.65 & 1.97 & 1.34 & 1.47 & 1.68 & 2.02 \\
\hline LL5 & 1.23 & 1.33 & 1.54 & 1.82 & 1.35 & 1.51 & 1.68 & 2.08 & 1.37 & 1.54 & 1.72 & 2.12 \\
\hline LL6 & 1.23 & 1.37 & 1.53 & 1.88 & 1.49 & 1.74 & 1.86 & 2.40 & 1.53 & 1.77 & 1.91 & 2.43 \\
\hline LH1 & 1.08 & 1.13 & 1.35 & 1.55 & 1.28 & 1.38 & 1.60 & 1.90 & 1.28 & 1.42 & 1.61 & 1.95 \\
\hline LH2 & 1.26 & 1.37 & 1.58 & 1.88 & 1.31 & 1.42 & 1.64 & 1.95 & 1.32 & 1.43 & 1.65 & 1.97 \\
\hline LH3 & 1.33 & 1.53 & 1.66 & 2.10 & 1.45 & 1.69 & 1.81 & 2.33 & 1.54 & 1.82 & 1.93 & 2.50 \\
\hline LH4 & 1.23 & 1.30 & 1.54 & 1.79 & 1.25 & 1.32 & 1.57 & 1.82 & 1.25 & 1.32 & 1.56 & 1.81 \\
\hline LH5 & 1.35 & 1.47 & 1.69 & 2.02 & 1.44 & 1.60 & 1.80 & 2.19 & 1.54 & 1.73 & 1.92 & 2.38 \\
\hline LH6 & 1.30 & 1.41 & 1.62 & 1.94 & 1.43 & 1.61 & 1.78 & 2.22 & 1.56 & 1.82 & 1.95 & 2.50 \\
\hline
\end{tabular}


Table C.7 - The ratio of actual mid-span bending moments and quarter-span shear forces for buildings to the corresponding values obtained from FEMA parabolic distribution and a uniform distribution of seismic load when subjected to M6C2 ground motion and the Load is acting parallel to the long side, $\alpha=0.00$

\begin{tabular}{|c|c|c|c|c|c|c|c|c|c|c|c|c|}
\hline \multirow{2}{*}{ Building } & \multicolumn{4}{|c|}{$\mu=2$} & \multicolumn{4}{|c|}{$\mu=3$} & \multicolumn{4}{|c|}{$\mu=4$} \\
\hline & $M . F$ & $V . F$ & $M . U$ & V.U & M.F & $V . F$ & $M . U$ & $V . U$ & M.F & V.F & M.U & $V . U$ \\
\hline SL1 & 1.12 & 1.17 & 1.40 & 1.61 & 1.17 & 1.25 & 1.47 & 1.72 & 1.21 & 1.30 & 1.51 & 1.78 \\
\hline SL3 & 09 & 1.13 & 1.36 & 1.55 & 1.15 & 1.23 & 1.44 & 1.69 & 1.18 & 1.27 & 1.47 & 1.75 \\
\hline & 11 & 1.12 & 1.38 & 1.54 & 1.09 & 1.10 & 1.37 & 1.52 & 1.15 & 1.23 & 1.44 & 1.69 \\
\hline SH1 & 11 & 1.15 & 1.39 & 1.57 & 1.22 & 1.30 & 1.52 & 1.78 & 1.28 & 1.39 & 1.60 & 1.91 \\
\hline & 1.09 & 1.14 & 1.37 & 1.57 & 1.13 & 1.19 & 1.41 & 1.64 & 1.16 & 1.24 & 1.45 & 1.71 \\
\hline $\mathrm{SH} 3$ & 1.14 & 1.23 & 1.42 & 1.69 & 1.20 & 1.31 & 1.50 & 1.81 & 1.23 & 1.34 & 1.53 & 1.85 \\
\hline SH4 & 1.12 & 1.21 & 1.40 & 1.66 & 1.19 & 1.30 & 1.49 & 1.79 & 1.23 & 1.35 & 1.53 & 1.86 \\
\hline SH5 & 1.15 & 1.25 & 1.44 & 1.71 & 1.21 & 1.33 & 1.51 & 1.83 & 1.27 & 1.40 & 1.58 & 1.93 \\
\hline IL1 & 1.06 & 1.13 & 1.33 & 1.55 & 1.07 & 1.14 & 1.34 & 1.57 & 1.19 & 1.28 & 1.48 & 1.76 \\
\hline ML2 & 1.11 & 1.18 & 1.38 & 1.62 & 1.16 & 1.24 & 1.44 & 1.71 & 1.23 & 1.34 & 1.54 & 1.84 \\
\hline ML3 & 1.18 & 1.27 & 1.47 & 1.75 & 1.26 & 1.39 & 1.57 & 1.91 & 1.32 & 1.47 & 1.64 & 2.02 \\
\hline ML4 & 1.10 & 1.16 & 1.37 & 1.60 & 1.14 & 1.22 & 1.42 & 1.67 & 1.22 & 1.33 & 1.53 & 1.82 \\
\hline ML5 & 1.13 & 1.21 & 1.41 & 1.66 & 1.17 & 1.27 & 1.47 & 1.75 & 1.22 & 1.33 & 1.52 & 1.83 \\
\hline ML6 & 1.16 & 1.25 & 1.45 & 1.72 & 1.23 & 1.35 & 1.53 & 1.85 & 1.29 & 1.44 & 1.62 & 1.98 \\
\hline & 20 & 1.26 & 1.50 & 1.74 & 1.30 & 1.42 & 1.62 & 1.96 & 1.36 & 1.52 & 1.70 & 2.09 \\
\hline & 26 & 1.35 & 1.58 & 1.86 & 1.45 & 1.65 & 1.81 & 2.27 & 1.52 & 1.75 & 1.90 & 2.41 \\
\hline & 1.26 & 1.36 & 1.57 & 1.88 & 1.32 & 1.44 & 1.65 & 1.99 & 1.49 & 1.71 & 1.86 & 2.35 \\
\hline MH4 & 1.17 & 1.22 & 1.46 & 1.68 & 1.27 & 1.37 & 1.59 & 1.89 & 1.37 & 1.53 & 1.71 & 2.10 \\
\hline MH5 & 1.46 & 1.66 & 1.82 & 2.29 & 1.53 & 1.76 & 1.91 & 2.42 & 1.60 & 1.84 & 2.00 & 2.53 \\
\hline MH6 & 1.07 & 1.08 & 1.34 & 1.49 & 1.29 & 1.42 & 1.62 & 1.96 & 1.38 & 1.56 & 1.73 & 2.15 \\
\hline LL1 & 1.15 & 1.20 & 1.44 & 1.64 & 1.28 & 1.39 & 1.60 & 1.91 & 1.35 & 1.51 & 1.69 & 2.08 \\
\hline LL2 & 1.12 & 1.14 & 1.40 & 1.57 & 1.27 & 1.37 & 1.59 & 1.88 & 1.38 & 1.55 & 1.73 & 2.13 \\
\hline LL3 & 1.22 & 1.30 & 1.53 & 1.79 & 1.28 & 1.47 & 1.60 & 2.02 & 1.48 & 1.72 & 1.85 & 2.36 \\
\hline LL4 & 1.15 & 1.19 & 1.44 & 1.64 & 1.28 & 1.39 & 1.60 & 1.90 & 1.34 & 1.50 & 1.68 & 2.06 \\
\hline LL5 & 1.20 & 1.26 & 1.50 & 1.74 & 1.42 & 1.60 & 1.77 & 2.19 & 1.50 & 1.73 & 1.88 & 2.37 \\
\hline LL6 & 1.50 & 1.71 & 1.87 & 2.35 & 1.54 & 1.76 & 1.93 & 2.42 & 1.71 & 2.01 & 2.14 & 2.76 \\
\hline LH1 & 1.14 & 1.17 & 1.42 & 1.61 & 1.32 & 1.45 & 1.65 & 2.00 & 1.58 & 1.81 & 1.97 & 2.49 \\
\hline LH2 & 1.42 & 1.58 & 1.78 & 2.17 & 1.54 & 1.79 & 1.93 & 2.46 & 1.56 & 1.81 & 1.95 & 2.49 \\
\hline LH3 & 1.53 & 1.75 & 1.91 & 2.41 & 1.63 & 1.89 & 2.04 & 2.60 & 1.69 & 1.98 & 2.11 & 2.72 \\
\hline LH4 & 1.31 & 1.45 & 1.64 & 2.00 & 1.47 & 1.66 & 1.84 & 2.28 & 1.58 & 1.79 & 1.97 & 2.47 \\
\hline LH5 & 1.17 & 1.26 & 1.46 & 1.73 & 1.27 & 1.40 & 1.59 & 1.92 & 1.38 & 1.58 & 1.73 & 2.17 \\
\hline LH6 & 1.47 & 1.64 & 1.84 & 2.26 & 1.51 & 1.70 & 1.89 & 2.34 & 1.54 & 1.76 & 1.93 & 2.41 \\
\hline
\end{tabular}


Table C.8 - The ratio of actual mid-span bending moments and quarter-span shear forces for buildings to the corresponding values obtained from FEMA parabolic distribution and a uniform distribution of seismic load when subjected to M6C26 ground motion and the Load is acting parallel to the long side, $\alpha=0.00$

\begin{tabular}{|c|c|c|c|c|c|c|c|c|c|c|c|c|}
\hline \multirow{2}{*}{ Building } & \multicolumn{4}{|c|}{$\mu=2$} & \multicolumn{4}{|c|}{$\mu=3$} & \multicolumn{4}{|c|}{$\mu=4$} \\
\hline & $M . F$ & $V . F$ & $M . U$ & V.U & M.F & $V . F$ & $M . U$ & $V . U$ & M.F & V.F & M.U & $V . U$ \\
\hline SL1 & 1.15 & 1.21 & 1.44 & 1.66 & 1.23 & 1.31 & 1.54 & 1.80 & 1.27 & 1.36 & 1.58 & 1.86 \\
\hline SL3 & 15 & 1.20 & 1.44 & 1.65 & 1.24 & 1.32 & 1.54 & 1.81 & 1.27 & 1.38 & 1.59 & 1.89 \\
\hline & 16 & 1.22 & 1.45 & 1.68 & 1.24 & 1.34 & 1.55 & 1.85 & 1.29 & 1.41 & 1.61 & 1.94 \\
\hline SH1 & 18 & 1.23 & 1.47 & 1.70 & 1.22 & 1.28 & 1.53 & 1.76 & 1.23 & 1.35 & 1.54 & 1.85 \\
\hline & 1.22 & 1.30 & 1.52 & 1.79 & 1.28 & 1.37 & 1.60 & 1.89 & 1.30 & 1.42 & 1.63 & 1.95 \\
\hline $\mathrm{SH} 3$ & 1.06 & 1.11 & 1.33 & 1.52 & 1.32 & 1.46 & 1.65 & 2.01 & 1.37 & 1.51 & 1.72 & 2.08 \\
\hline $\mathrm{SH} 4$ & 1.06 & 1.11 & 1.33 & 1.53 & 1.32 & 1.47 & 1.65 & 2.02 & 1.37 & 1.53 & 1.72 & 2.11 \\
\hline SH5 & 1.07 & 1.13 & 1.34 & 1.55 & 1.35 & 1.52 & 1.69 & 2.08 & 1.42 & 1.60 & 1.77 & 2.21 \\
\hline IL1 & 1.12 & 1.18 & 1.40 & 1.62 & 1.17 & 1.26 & 1.46 & 1.74 & 1.21 & 1.33 & 1.51 & 1.82 \\
\hline ML2 & 1.11 & 1.18 & 1.39 & 1.62 & 1.15 & 1.24 & 1.44 & 1.70 & 1.18 & 1.28 & 1.47 & 1.76 \\
\hline ML3 & 1.07 & 1.12 & 1.34 & 1.54 & 1.10 & 1.16 & 1.37 & 1.60 & 1.12 & 1.20 & 1.39 & 1.65 \\
\hline ML4 & 1.12 & 1.19 & 1.40 & 1.63 & 1.16 & 1.25 & 1.45 & 1.72 & 1.19 & 1.30 & 1.49 & 1.78 \\
\hline ML5 & 1.11 & 1.17 & 1.38 & 1.61 & 1.14 & 1.23 & 1.43 & 1.69 & 1.16 & 1.26 & 1.46 & 1.73 \\
\hline ML6 & 1.09 & 1.14 & 1.36 & 1.57 & 1.11 & 1.19 & 1.39 & 1.63 & 1.13 & 1.22 & 1.42 & 1.68 \\
\hline MH1 & 1.04 & 1.08 & 1.29 & 1.48 & 1.06 & 1.12 & 1.33 & 1.54 & 1.09 & 1.17 & 1.36 & 1.60 \\
\hline & & 1.08 & 1.32 & 1.49 & 1.08 & 1.15 & 1.35 & 1.58 & 1.44 & 1.63 & 1.80 & 2.24 \\
\hline & 1.16 & 1.23 & 1.45 & 1.69 & 1.16 & 1.28 & 1.45 & 1.76 & 1.37 & 1.56 & 1.71 & 2.14 \\
\hline MH4 & 1.03 & 1.08 & 1.29 & 1.48 & 1.06 & 1.12 & 1.33 & 1.54 & 1.10 & 1.18 & 1.37 & 1.62 \\
\hline MH5 & 1.08 & 1.10 & 1.35 & 1.51 & 1.10 & 1.17 & 1.37 & 1.61 & 1.44 & 1.64 & 1.80 & 2.25 \\
\hline MH6 & 1.06 & 1.11 & 1.32 & 1.52 & 1.15 & 1.24 & 1.44 & 1.71 & 1.44 & 1.64 & 1.81 & 2.26 \\
\hline LL1 & 1.06 & 1.10 & 1.33 & 1.52 & 1.09 & 1.15 & 1.36 & 1.58 & 1.24 & 1.37 & 1.55 & 1.89 \\
\hline LL2 & 1.06 & 1.10 & 1.32 & 1.52 & 1.09 & 1.15 & 1.36 & 1.59 & 1.45 & 1.65 & 1.81 & 2.27 \\
\hline LL3 & 1.16 & 1.21 & 1.44 & 1.66 & 1.23 & 1.36 & 1.54 & 1.87 & 1.42 & 1.59 & 1.77 & 2.18 \\
\hline LL4 & 1.06 & 1.10 & 1.32 & 1.52 & 1.09 & 1.15 & 1.36 & 1.58 & 1.47 & 1.68 & 1.84 & 2.31 \\
\hline LL5 & 1.05 & 1.10 & 1.32 & 1.52 & 1.08 & 1.15 & 1.35 & 1.58 & 1.45 & 1.64 & 1.81 & 2.26 \\
\hline LL6 & 1.13 & 1.22 & 1.42 & 1.67 & 1.24 & 1.35 & 1.55 & 1.85 & 1.65 & 1.96 & 2.06 & 2.69 \\
\hline LH1 & 1.11 & 1.18 & 1.38 & 1.62 & 1.40 & 1.59 & 1.76 & 2.18 & 1.45 & 1.65 & 1.81 & 2.27 \\
\hline LH2 & 1.12 & 1.19 & 1.40 & 1.64 & 1.41 & 1.60 & 1.77 & 2.21 & 1.47 & 1.68 & 1.84 & 2.31 \\
\hline LH3 & 1.16 & 1.25 & 1.45 & 1.72 & 1.62 & 1.91 & 2.02 & 2.63 & 1.75 & 2.10 & 2.18 & 2.88 \\
\hline LH4 & 1.12 & 1.20 & 1.40 & 1.65 & 1.40 & 1.57 & 1.75 & 2.16 & 1.43 & 1.61 & 1.78 & 2.21 \\
\hline LH5 & 1.12 & 1.19 & 1.40 & 1.63 & 1.43 & 1.61 & 1.78 & 2.21 & 1.49 & 1.69 & 1.86 & 2.32 \\
\hline LH6 & 1.15 & 1.29 & 1.44 & 1.78 & 1.38 & 1.56 & 1.73 & 2.14 & 1.96 & 2.35 & 2.45 & 3.22 \\
\hline
\end{tabular}


Table C.9 - The ratio of actual mid-span bending moments and quarter-span shear forces for buildings to the corresponding values obtained from FEMA parabolic distribution and a uniform distribution of seismic load when subjected to M6C31 ground motion and the Load is acting parallel to the long side, $\alpha=0.00$

\begin{tabular}{|c|c|c|c|c|c|c|c|c|c|c|c|c|}
\hline \multirow{2}{*}{ Building } & \multicolumn{4}{|c|}{$\mu=2$} & \multicolumn{4}{|c|}{$\mu=3$} & \multicolumn{4}{|c|}{$\mu=4$} \\
\hline & $M . F$ & $V . F$ & $M . U$ & V.U & M.F & $V . F$ & $M . U$ & $V . U$ & M.F & V.F & M.U & $V . U$ \\
\hline SL1 & 1.05 & 1.06 & 1.31 & 1.46 & 1.36 & 1.54 & 1.70 & 2.12 & 1.38 & 1.57 & 1.73 & 2.16 \\
\hline SL3 & 04 & 1.05 & 1.30 & 1.45 & 1.36 & 1.55 & 1.71 & 2.14 & 1.37 & 1.56 & 1.71 & 2.15 \\
\hline & 04 & 1.05 & 1.30 & 1.45 & 1.12 & 1.18 & 1.40 & 1.62 & 1.21 & 1.31 & 1.51 & 1.80 \\
\hline SH1 & 14 & 1.22 & 1.42 & 1.67 & 1.19 & 1.29 & 1.48 & 1.78 & 1.43 & 1.63 & 1.78 & 2.24 \\
\hline & 1.15 & 1.24 & 1.44 & 1.70 & 1.19 & 1.29 & 1.49 & 1.77 & 1.23 & 1.34 & 1.54 & 1.84 \\
\hline $\mathrm{SH} 3$ & 1.12 & 1.19 & 1.40 & 1.64 & 1.15 & 1.23 & 1.44 & 1.69 & 1.19 & 1.29 & 1.49 & 1.77 \\
\hline $\mathrm{SH} 4$ & 1.11 & 1.18 & 1.39 & 1.62 & 1.13 & 1.21 & 1.42 & 1.66 & 1.16 & 1.25 & 1.45 & 1.72 \\
\hline SH5 & 1.08 & 1.13 & 1.34 & 1.55 & 1.10 & 1.16 & 1.37 & 1.60 & 1.23 & 1.36 & 1.54 & 1.87 \\
\hline IL1 & 1.17 & 1.24 & 1.46 & 1.71 & 1.46 & 1.63 & 1.83 & 2.23 & 1.49 & 1.65 & 1.86 & 2.26 \\
\hline ML2 & 1.16 & 1.22 & 1.45 & 1.68 & 1.38 & 1.55 & 1.72 & 2.13 & 1.41 & 1.59 & 1.76 & 2.19 \\
\hline ML3 & 1.16 & 1.22 & 1.45 & 1.68 & 1.33 & 1.45 & 1.66 & 1.99 & 1.36 & 1.51 & 1.70 & 2.07 \\
\hline ML4 & 1.17 & 1.23 & 1.46 & 1.69 & 1.38 & 1.56 & 1.73 & 2.14 & 1.42 & 1.60 & 1.78 & 2.20 \\
\hline ML5 & 1.16 & 1.21 & 1.45 & 1.67 & 1.37 & 1.54 & 1.72 & 2.11 & 1.41 & 1.60 & 1.77 & 2.20 \\
\hline ML6 & 1.16 & 1.21 & 1.45 & 1.67 & 1.32 & 1.45 & 1.66 & 2.00 & 1.37 & 1.54 & 1.71 & 2.12 \\
\hline MH1 & .06 & 1.10 & 1.32 & 1.51 & 1.29 & 1.44 & 1.62 & 1.98 & 1.34 & 1.51 & 1.68 & 2.08 \\
\hline & 01 & 1.03 & 1.26 & 1.41 & 1.37 & 1.50 & 1.72 & 2.06 & 1.46 & 1.63 & 1.83 & 2.24 \\
\hline & 1.21 & 1.28 & 1.51 & 1.76 & 1.37 & 1.55 & 1.72 & 2.13 & 1.39 & 1.56 & 1.74 & 2.14 \\
\hline MH4 & 1.06 & 1.10 & 1.33 & 1.51 & 1.31 & 1.46 & 1.64 & 2.01 & 1.26 & 1.39 & 1.57 & 1.91 \\
\hline MH5 & 1.21 & 1.28 & 1.52 & 1.76 & 1.30 & 1.41 & 1.62 & 1.94 & 1.38 & 1.54 & 1.73 & 2.11 \\
\hline MH6 & 1.02 & 1.04 & 1.28 & 1.43 & 1.28 & 1.40 & 1.59 & 1.93 & 1.35 & 1.50 & 1.68 & 2.06 \\
\hline LL1 & 1.13 & 1.21 & 1.42 & 1.66 & 1.28 & 1.42 & 1.60 & 1.96 & 1.34 & 1.51 & 1.67 & 2.08 \\
\hline LL2 & 1.13 & 1.21 & 1.41 & 1.66 & 1.20 & 1.31 & 1.50 & 1.80 & 1.38 & 1.58 & 1.73 & 2.17 \\
\hline LL3 & 1.15 & 1.19 & 1.44 & 1.63 & 1.30 & 1.41 & 1.62 & 1.94 & 1.47 & 1.69 & 1.84 & 2.32 \\
\hline LL4 & 1.13 & 1.20 & 1.41 & 1.66 & 1.27 & 1.41 & 1.59 & 1.94 & 1.33 & 1.51 & 1.67 & 2.07 \\
\hline LL5 & 1.01 & 1.04 & 1.26 & 1.42 & 1.36 & 1.48 & 1.70 & 2.04 & 1.44 & 1.60 & 1.80 & 2.19 \\
\hline LL6 & 1.28 & 1.40 & 1.61 & 1.92 & 1.75 & 2.06 & 2.18 & 2.84 & 1.86 & 2.22 & 2.33 & 3.05 \\
\hline LH1 & 1.07 & 1.11 & 1.34 & 1.53 & 1.15 & 1.22 & 1.43 & 1.68 & 1.25 & 1.37 & 1.57 & 1.88 \\
\hline LH2 & 1.09 & 1.14 & 1.36 & 1.56 & 1.27 & 1.40 & 1.58 & 1.92 & 1.38 & 1.56 & 1.73 & 2.15 \\
\hline LH3 & 1.57 & 1.76 & 1.97 & 2.42 & 1.85 & 2.24 & 2.31 & 3.07 & 2.00 & 2.44 & 2.50 & 3.36 \\
\hline LH4 & 1.11 & 1.16 & 1.39 & 1.60 & 1.19 & 1.28 & 1.49 & 1.75 & 1.26 & 1.37 & 1.57 & 1.88 \\
\hline LH5 & 1.27 & 1.42 & 1.59 & 1.95 & 1.47 & 1.70 & 1.84 & 2.34 & 1.50 & 1.72 & 1.87 & 2.37 \\
\hline LH6 & 1.23 & 1.32 & 1.54 & 1.82 & 1.37 & 1.54 & 1.72 & 2.11 & 1.67 & 1.90 & 2.09 & 2.62 \\
\hline
\end{tabular}


Table C.10 - The ratio of actual mid-span bending moments and quarter-span shear forces for buildings to the corresponding values obtained from FEMA parabolic distribution and a uniform distribution of seismic load when subjected to M6C38 ground motion and the Load is acting parallel to the long side, $\alpha=0.00$

\begin{tabular}{|c|c|c|c|c|c|c|c|c|c|c|c|c|}
\hline \multirow{2}{*}{ Building } & \multicolumn{4}{|c|}{$\mu=2$} & \multicolumn{4}{|c|}{$\mu=3$} & \multicolumn{4}{|c|}{$\mu=4$} \\
\hline & $M . F$ & $V . F$ & $M . U$ & $V . U$ & M.F & $V . F$ & $M . U$ & $V . U$ & M.F & V.F & $M . U$ & $V . U$ \\
\hline SL1 & 1.12 & 1.17 & 1.40 & 1.61 & 1.16 & 1.24 & 1.45 & 1.70 & 1.21 & 1.31 & 1.51 & 1.80 \\
\hline SL3 & 11 & 1.17 & 1.38 & 1.61 & 1.15 & 1.24 & 1.44 & 1.70 & 1.21 & 1.33 & 1.52 & 1.83 \\
\hline SL4 & 05 & 1.08 & 1.31 & 1.49 & 1.13 & 1.19 & 1.41 & 1.63 & 1.22 & 1.35 & 1.53 & 1.85 \\
\hline SH1 & 13 & 1.21 & 1.42 & 1.67 & 1.17 & 1.27 & 1.47 & 1.74 & 1.20 & 1.30 & 1.50 & 1.79 \\
\hline $\mathrm{SH} 2$ & 1.15 & 1.24 & 1.44 & 1.70 & 1.19 & 1.29 & 1.49 & 1.77 & 1.22 & 1.33 & 1.53 & 1.83 \\
\hline $\mathrm{SH} 3$ & 1.16 & 1.25 & 1.45 & 1.72 & 1.20 & 1.30 & 1.50 & 1.79 & 1.24 & 1.35 & 1.55 & 1.86 \\
\hline SH4 & 1.16 & 1.25 & 1.45 & 1.72 & 1.20 & 1.30 & 1.50 & 1.79 & 1.22 & 1.34 & 1.53 & 1.84 \\
\hline H5 & 1.16 & 1.25 & 1.45 & 1.72 & 1.21 & 1.32 & 1.51 & 1.81 & 1.24 & 1.36 & 1.55 & 1.86 \\
\hline ML1 & 1.20 & 1.31 & 1.50 & 1.80 & 1.26 & 1.39 & 1.57 & 1.91 & 1.30 & 1.46 & 1.63 & 2.00 \\
\hline ML2 & 1.20 & 1.31 & 1.51 & 1.81 & 1.26 & 1.40 & 1.58 & 1.92 & 1.31 & 1.47 & 1.64 & 2.01 \\
\hline ML3 & 1.19 & 1.29 & 1.49 & 1.78 & 1.26 & 1.39 & 1.57 & 1.92 & 1.31 & 1.47 & 1.64 & 2.02 \\
\hline ML4 & 1.20 & 1.31 & 1.51 & 1.81 & 1.26 & 1.40 & 1.58 & 1.92 & 1.30 & 1.46 & 1.63 & 2.00 \\
\hline ML5 & 1.20 & 1.31 & 1.51 & 1.80 & 1.26 & 1.40 & 1.58 & 1.92 & 1.31 & 1.46 & 1.64 & 2.01 \\
\hline ML6 & 1.19 & 1.30 & 1.49 & 1.78 & 1.26 & 1.40 & 1.58 & 1.93 & 1.31 & 1.47 & 1.64 & 2.02 \\
\hline MH1 & 1.19 & 1.29 & 1.49 & 1.77 & 1.27 & 1.41 & 1.59 & 1.94 & 1.34 & 1.50 & 1.67 & 2.07 \\
\hline & 1.17 & 1.26 & 1.47 & 1.73 & 1.27 & 1.40 & 1.59 & 1.93 & 1.35 & 1.52 & 1.69 & 2.09 \\
\hline MH3 & 1.21 & 1.27 & 1.52 & 1.74 & 1.34 & 1.45 & 1.67 & 1.99 & 1.42 & 1.56 & 1.77 & 2.14 \\
\hline MH4 & 1.18 & 1.28 & 1.48 & 1.75 & 1.27 & 1.41 & 1.59 & 1.94 & 1.33 & 1.49 & 1.66 & 2.05 \\
\hline MH5 & 1.17 & 1.24 & 1.46 & 1.71 & 1.28 & 1.41 & 1.60 & 1.94 & 1.36 & 1.53 & 1.70 & 2.10 \\
\hline MH6 & 1.17 & 1.26 & 1.46 & 1.73 & 1.27 & 1.42 & 1.59 & 1.95 & 1.35 & 1.53 & 1.69 & 2.10 \\
\hline LL1 & 1.20 & 1.30 & 1.50 & 1.79 & 1.30 & 1.44 & 1.62 & 1.99 & 1.36 & 1.53 & 1.70 & 2.11 \\
\hline LL2 & 1.19 & 1.29 & 1.49 & 1.77 & 1.29 & 1.43 & 1.61 & 1.97 & 1.35 & 1.53 & 1.69 & 2.10 \\
\hline LL3 & 1.20 & 1.27 & 1.50 & 1.74 & 1.33 & 1.45 & 1.66 & 1.99 & 1.41 & 1.57 & 1.76 & 2.16 \\
\hline L4 & 1.20 & 1.30 & 1.50 & 1.78 & 1.29 & 1.44 & 1.61 & 1.98 & 1.35 & 1.53 & 1.69 & 2.10 \\
\hline LL5 & 1.18 & 1.26 & 1.47 & 1.74 & 1.27 & 1.41 & 1.59 & 1.93 & 1.35 & 1.52 & 1.69 & 2.09 \\
\hline LL6 & 1.18 & 1.20 & 1.47 & 1.65 & 1.30 & 1.36 & 1.62 & 1.87 & 1.40 & 1.51 & 1.75 & 2.07 \\
\hline LH1 & 1.17 & 1.25 & 1.46 & 1.73 & 1.29 & 1.43 & 1.61 & 1.97 & 1.37 & 1.55 & 1.71 & 2.13 \\
\hline LH2 & 1.15 & 1.23 & 1.44 & 1.69 & 1.27 & 1.41 & 1.59 & 1.94 & 1.35 & 1.51 & 1.68 & 2.08 \\
\hline LH3 & 1.18 & 1.22 & 1.47 & 1.68 & 1.35 & 1.46 & 1.68 & 2.01 & 1.47 & 1.64 & 1.84 & 2.26 \\
\hline LH4 & 1.16 & 1.25 & 1.46 & 1.71 & 1.28 & 1.42 & 1.60 & 1.95 & 1.35 & 1.52 & 1.68 & 2.09 \\
\hline LH5 & 1.19 & 1.26 & 1.49 & 1.73 & 1.29 & 1.41 & 1.61 & 1.94 & 1.37 & 1.53 & 1.71 & 2.10 \\
\hline LH6 & 1.23 & 1.27 & 1.54 & 1.74 & 1.40 & 1.51 & 1.76 & 2.07 & 1.55 & 1.72 & 1.94 & 2.37 \\
\hline
\end{tabular}


Table C.11 - The ratio of actual mid-span bending moments and quarter-span shear forces for buildings to the corresponding values obtained from FEMA parabolic distribution and a uniform distribution of seismic load when subjected to E6C1 ground motion and the Load is acting parallel to the short side, $\alpha=0.00$

\begin{tabular}{|c|c|c|c|c|c|c|c|c|c|c|c|c|}
\hline \multirow{2}{*}{ Building } & \multicolumn{4}{|c|}{$\mu=2$} & \multicolumn{4}{|c|}{$\mu=3$} & \multicolumn{4}{|c|}{$\mu=4$} \\
\hline & M.F & $V . F$ & $M . U$ & $V . U$ & $M . F$ & $V . F$ & $M . U$ & $V . U$ & $M . F$ & V.F & $M . U$ & $V . U$ \\
\hline SL1 & 0.95 & 0.96 & 1.19 & 1.32 & 0.97 & 0.98 & 1.21 & 1.34 & 0.99 & 1.00 & 1.23 & 1.37 \\
\hline SL3 & .94 & 0.95 & 1.18 & 1.31 & 0.96 & 0.96 & 1.20 & 1.33 & 0.98 & 0.98 & 1.22 & 1.35 \\
\hline SL4 & 0.92 & 0.93 & 1.15 & 1.28 & 0.94 & 0.95 & 1.17 & 1.30 & 0.97 & 0.97 & 1.21 & 1.34 \\
\hline SH1 & 1.28 & 1.44 & 1.60 & 1.98 & 1.31 & 1.47 & 1.63 & 2.03 & 1.34 & 1.52 & 1.67 & 2.09 \\
\hline $\mathrm{SH} 2$ & 1.29 & 1.45 & 1.61 & 2.00 & 1.31 & 1.49 & 1.64 & 2.05 & 1.33 & 1.51 & 1.66 & 2.08 \\
\hline SH3 & 1.28 & 1.44 & 1.60 & 1.98 & 1.30 & 1.48 & 1.63 & 2.04 & 1.32 & 1.51 & 1.65 & 2.08 \\
\hline SH4 & 1.25 & 1.41 & 1.57 & 1.93 & 1.27 & 1.45 & 1.59 & 1.99 & 1.31 & 1.48 & 1.63 & 2.03 \\
\hline SH5 & 1.25 & 1.40 & 1.56 & 1.92 & 1.27 & 1.45 & 1.59 & 2.00 & 1.31 & 1.48 & 1.64 & 2.03 \\
\hline SH6 & 1.28 & 1.44 & 1.60 & 1.98 & 1.31 & 1.49 & 1.63 & 2.04 & 1.32 & 1.51 & 1.66 & 2.08 \\
\hline ML1 & 1.15 & 1.22 & 1.43 & 1.67 & 1.24 & 1.29 & 1.55 & 1.78 & 1.31 & 1.38 & 1.64 & 1.89 \\
\hline ML2 & 1.03 & 1.10 & 1.29 & 1.52 & 1.12 & 1.21 & 1.40 & 1.66 & 1.21 & 1.32 & 1.52 & 1.81 \\
\hline ML3 & 1.05 & 1.12 & 1.31 & 1.55 & 1.15 & 1.24 & 1.44 & 1.71 & 1.27 & 1.37 & 1.58 & 1.88 \\
\hline ML4 & 1.08 & 1.11 & 1.34 & 1.53 & 1.18 & 1.24 & 1.48 & 1.70 & 1.26 & 1.34 & 1.57 & 1.84 \\
\hline ML5 & 1.08 & 1.12 & 1.35 & 1.54 & 1.18 & 1.24 & 1.48 & 1.70 & 1.27 & 1.34 & 1.58 & 1.85 \\
\hline ML6 & 1.04 & 1.11 & 1.30 & 1.53 & 1.15 & 1.24 & 1.44 & 1.71 & 1.26 & 1.37 & 1.57 & 1.88 \\
\hline MH1 & 1.03 & 1.09 & 1.29 & 1.50 & 1.14 & 1.22 & 1.43 & 1.68 & 1.34 & 1.42 & 1.67 & 1.95 \\
\hline MH2 & 1.07 & 1.11 & 1.34 & 1.52 & 1.23 & 1.27 & 1.54 & 1.74 & 1.48 & 1.56 & 1.85 & 2.14 \\
\hline MH3 & 1.23 & 1.47 & 1.54 & 2.02 & 1.39 & 1.62 & 1.74 & 2.23 & 1.63 & 1.81 & 2.04 & 2.50 \\
\hline MH4 & 1.08 & 1.09 & 1.35 & 1.49 & 1.20 & 1.21 & 1.49 & 1.67 & 1.37 & 1.41 & 1.72 & 1.94 \\
\hline MH5 & 1.09 & 1.11 & 1.36 & 1.53 & 1.25 & 1.29 & 1.56 & 1.78 & 1.46 & 1.60 & 1.83 & 2.20 \\
\hline MH6 & 1.16 & 1.19 & 1.45 & 1.64 & 1.28 & 1.36 & 1.60 & 1.87 & 1.38 & 1.52 & 1.73 & 2.09 \\
\hline LL1 & 1.00 & 1.11 & 1.25 & 1.53 & 1.08 & 1.24 & 1.36 & 1.70 & 1.20 & 1.31 & 1.50 & 1.81 \\
\hline LL2 & 0.98 & 1.12 & 1.23 & 1.53 & 1.07 & 1.24 & 1.33 & 1.70 & 1.21 & 1.31 & 1.51 & 1.80 \\
\hline LL3 & 1.04 & 1.25 & 1.29 & 1.72 & 1.35 & 1.67 & 1.69 & 2.30 & 1.42 & 1.77 & 1.78 & 2.43 \\
\hline LL4 & 1.01 & 1.03 & 1.26 & 1.42 & 1.12 & 1.16 & 1.39 & 1.60 & 1.28 & 1.29 & 1.60 & 1.77 \\
\hline LL5 & 1.02 & 1.04 & 1.27 & 1.43 & 1.12 & 1.16 & 1.40 & 1.59 & 1.27 & 1.26 & 1.58 & 1.73 \\
\hline LL6 & 1.08 & 1.41 & 1.34 & 1.93 & 1.24 & 1.61 & 1.55 & 2.21 & 1.61 & 1.95 & 2.02 & 2.68 \\
\hline LH1 & 1.14 & 1.37 & 1.43 & 1.88 & 1.40 & 1.72 & 1.75 & 2.37 & 1.48 & 1.85 & 1.85 & 2.55 \\
\hline LH2 & 1.34 & 1.56 & 1.67 & 2.14 & 1.43 & 1.73 & 1.79 & 2.38 & 1.50 & 1.85 & 1.88 & 2.55 \\
\hline LH3 & 1.41 & 1.82 & 1.76 & 2.50 & 1.78 & 2.16 & 2.23 & 2.97 & 1.88 & 2.27 & 2.35 & 3.12 \\
\hline LH4 & 1.35 & 1.62 & 1.69 & 2.23 & 1.46 & 1.80 & 1.83 & 2.48 & 1.54 & 1.93 & 1.93 & 2.65 \\
\hline LH5 & 1.36 & 1.64 & 1.70 & 2.25 & 1.48 & 1.83 & 1.85 & 2.52 & 1.56 & 1.95 & 1.95 & 2.68 \\
\hline LH6 & 1.38 & 1.81 & 1.73 & 2.49 & 1.82 & 2.21 & 2.27 & 3.04 & 1.90 & 2.31 & 2.38 & 3.17 \\
\hline
\end{tabular}


Table C.12 - The ratio of actual mid-span bending moments and quarter-span shear forces for buildings to the corresponding values obtained from FEMA parabolic distribution and a uniform distribution of seismic load when subjected to E6C13 ground motion and the Load is acting parallel to the short side, $\alpha=0.00$

\begin{tabular}{|c|c|c|c|c|c|c|c|c|c|c|c|c|}
\hline \multirow{2}{*}{ Building } & \multicolumn{4}{|c|}{$\mu=2$} & \multicolumn{4}{|c|}{$\mu=3$} & \multicolumn{4}{|c|}{$\mu=4$} \\
\hline & M.F & $V . F$ & $M . U$ & $V . U$ & $M . F$ & $V . F$ & $M . U$ & $V . U$ & $M . F$ & V.F & $M . U$ & $V . U$ \\
\hline SL1 & 1.11 & 1.20 & 1.38 & 1.65 & 1.15 & 1.28 & 1.44 & 1.76 & 1.20 & 1.34 & 1.50 & 1.84 \\
\hline SL3 & 12 & 1.22 & 1.40 & 1.68 & 1.18 & 1.31 & 1.47 & 1.80 & 1.22 & 1.37 & 1.52 & 1.88 \\
\hline SL4 & .19 & 1.32 & 1.49 & 1.82 & 1.25 & 1.38 & 1.57 & 1.90 & 1.29 & 1.42 & 1.62 & 1.95 \\
\hline SH1 & 1.06 & 1.23 & 1.32 & 1.69 & 1.17 & 1.39 & 1.46 & 1.91 & 1.29 & 1.54 & 1.62 & 2.11 \\
\hline $\mathrm{SH} 2$ & 0.97 & 1.16 & 1.22 & 1.59 & 1.04 & 1.23 & 1.30 & 1.69 & 1.10 & 1.35 & 1.38 & 1.86 \\
\hline SH3 & 0.97 & 1.12 & 1.21 & 1.53 & 1.01 & 1.20 & 1.27 & 1.64 & 1.05 & 1.31 & 1.32 & 1.80 \\
\hline SH4 & 0.94 & 1.09 & 1.18 & 1.49 & 0.98 & 1.09 & 1.22 & 1.50 & 1.00 & 1.18 & 1.25 & 1.62 \\
\hline SH5 & 0.94 & 1.07 & 1.18 & 1.48 & 0.98 & 1.12 & 1.22 & 1.53 & 0.99 & 1.20 & 1.24 & 1.65 \\
\hline SH6 & 0.97 & 1.12 & 1.21 & 1.53 & 1.01 & 1.19 & 1.26 & 1.64 & 1.05 & 1.31 & 1.32 & 1.81 \\
\hline ML1 & 0.95 & 1.03 & 1.19 & 1.42 & 0.98 & 1.04 & 1.23 & 1.43 & 1.00 & 1.11 & 1.25 & 1.52 \\
\hline ML2 & 0.96 & 1.00 & 1.20 & 1.37 & 1.02 & 1.10 & 1.27 & 1.52 & 1.10 & 1.24 & 1.37 & 1.70 \\
\hline ML3 & 1.01 & 1.19 & 1.26 & 1.64 & 1.31 & 1.56 & 1.64 & 2.15 & 1.34 & 1.60 & 1.67 & 2.20 \\
\hline ML4 & 0.94 & 1.00 & 1.18 & 1.37 & 0.96 & 1.03 & 1.20 & 1.42 & 0.97 & 1.06 & 1.22 & 1.46 \\
\hline ML5 & 0.94 & 0.99 & 1.17 & 1.37 & 0.96 & 1.03 & 1.20 & 1.42 & 0.97 & 1.06 & 1.22 & 1.45 \\
\hline ML6 & 0.97 & 1.11 & 1.21 & 1.52 & 1.08 & 1.23 & 1.35 & 1.69 & 1.34 & 1.70 & 1.67 & 2.34 \\
\hline MH1 & 1.11 & 1.31 & 1.39 & 1.81 & 1.22 & 1.44 & 1.53 & 1.99 & 1.27 & 1.49 & 1.58 & 2.05 \\
\hline MH2 & 1.05 & 1.09 & 1.32 & 1.50 & 1.16 & 1.28 & 1.46 & 1.77 & 1.53 & 1.91 & 1.91 & 2.62 \\
\hline MH3 & 1.27 & 1.42 & 1.59 & 1.95 & 1.48 & 1.82 & 1.85 & 2.51 & 1.52 & 2.10 & 1.89 & 2.88 \\
\hline MH4 & 0.98 & 1.03 & 1.23 & 1.42 & 1.20 & 1.49 & 1.50 & 2.04 & 1.49 & 1.86 & 1.86 & 2.56 \\
\hline MH5 & 0.96 & 1.03 & 1.21 & 1.41 & 1.20 & 1.50 & 1.50 & 2.06 & 1.48 & 1.86 & 1.85 & 2.56 \\
\hline MH6 & 0.97 & 1.00 & 1.22 & 1.37 & 0.97 & 1.13 & 1.21 & 1.56 & 0.97 & 1.28 & 1.21 & 1.76 \\
\hline LL1 & 1.07 & 1.26 & 1.34 & 1.73 & 1.30 & 1.35 & 1.62 & 1.85 & 1.43 & 1.56 & 1.79 & 2.14 \\
\hline LL2 & 1.04 & 1.23 & 1.30 & 1.69 & 1.31 & 1.35 & 1.64 & 1.86 & 1.43 & 1.52 & 1.79 & 2.10 \\
\hline LL3 & 1.07 & 1.09 & 1.34 & 1.50 & 1.14 & 1.14 & 1.43 & 1.56 & 1.21 & 1.30 & 1.51 & 1.78 \\
\hline LL4 & 1.09 & 1.31 & 1.36 & 1.79 & 1.16 & 1.41 & 1.45 & 1.94 & 1.33 & 1.64 & 1.66 & 2.25 \\
\hline LL5 & 1.09 & 1.28 & 1.36 & 1.76 & 1.14 & 1.40 & 1.43 & 1.92 & 1.33 & 1.65 & 1.66 & 2.27 \\
\hline LL6 & 0.95 & 1.32 & 1.18 & 1.81 & 1.04 & 1.49 & 1.30 & 2.05 & 1.15 & 1.68 & 1.44 & 2.31 \\
\hline LH1 & 1.14 & 1.23 & 1.42 & 1.69 & 1.18 & 1.28 & 1.47 & 1.75 & 1.33 & 1.60 & 1.66 & 2.20 \\
\hline LH2 & 1.19 & 1.29 & 1.48 & 1.78 & 1.29 & 1.42 & 1.61 & 1.96 & 1.34 & 1.49 & 1.68 & 2.05 \\
\hline LH3 & 1.05 & 1.43 & 1.32 & 1.96 & 1.48 & 1.79 & 1.85 & 2.46 & 1.61 & 1.87 & 2.02 & 2.57 \\
\hline LH4 & 1.21 & 1.33 & 1.52 & 1.82 & 1.30 & 1.50 & 1.62 & 2.06 & 1.37 & 1.69 & 1.71 & 2.32 \\
\hline LH5 & 1.22 & 1.34 & 1.53 & 1.84 & 1.31 & 1.52 & 1.64 & 2.10 & 1.35 & 1.69 & 1.69 & 2.32 \\
\hline LH6 & 0.99 & 1.39 & 1.23 & 1.91 & 1.44 & 1.80 & 1.81 & 2.48 & 1.56 & 1.85 & 1.94 & 2.55 \\
\hline
\end{tabular}


Table C.13 - The ratio of actual mid-span bending moments and quarter-span shear forces for buildings to the corresponding values obtained from FEMA parabolic distribution and a uniform distribution of seismic load when subjected to E6C15 ground motion and the Load is acting parallel to the short side, $\alpha=0.00$

\begin{tabular}{|c|c|c|c|c|c|c|c|c|c|c|c|c|}
\hline \multirow{2}{*}{ Building } & \multicolumn{4}{|c|}{$\mu=2$} & \multicolumn{4}{|c|}{$\mu=3$} & \multicolumn{4}{|c|}{$\mu=4$} \\
\hline & $M . F$ & $V . F$ & $M . U$ & V.U & $M . F$ & $V . F$ & $M . U$ & V.U & M.F & V.F & $M . U$ & $V . U$ \\
\hline SL1 & 1.33 & 1.53 & 1.66 & 2.10 & 1.48 & 1.67 & 1.85 & 2.29 & 1.51 & 1.70 & 1.88 & 2.33 \\
\hline SL3 & .32 & 1.53 & 1.65 & 2.10 & 1.47 & 1.66 & 1.84 & 2.28 & 1.51 & 1.70 & 1.88 & 2.33 \\
\hline SL4 & .28 & 1.51 & 1.60 & 2.07 & 1.47 & 1.66 & 1.83 & 2.29 & 1.50 & 1.69 & 1.87 & 2.33 \\
\hline SH1 & 1.22 & 1.31 & 1.53 & 1.80 & 1.29 & 1.42 & 1.62 & 1.95 & 1.42 & 1.62 & 1.77 & 2.23 \\
\hline $\mathrm{SH} 2$ & 1.30 & 1.46 & 1.62 & 2.00 & 1.44 & 1.64 & 1.80 & 2.26 & 1.48 & 1.69 & 1.84 & 2.33 \\
\hline SH3 & 1.32 & 1.52 & 1.66 & 2.09 & 1.47 & 1.69 & 1.83 & 2.32 & 1.49 & 1.72 & 1.87 & 2.36 \\
\hline SH4 & 1.34 & 1.54 & 1.67 & 2.11 & 1.43 & 1.65 & 1.79 & 2.28 & 1.49 & 1.73 & 1.86 & 2.38 \\
\hline SH5 & 1.34 & 1.54 & 1.68 & 2.12 & 1.45 & 1.68 & 1.81 & 2.30 & 1.50 & 1.74 & 1.87 & 2.40 \\
\hline SH6 & 1.32 & 1.51 & 1.65 & 2.08 & 1.46 & 1.68 & 1.82 & 2.31 & 1.49 & 1.71 & 1.86 & 2.35 \\
\hline ML1 & 1.35 & 1.57 & 1.69 & 2.16 & 1.37 & 1.58 & 1.72 & 2.18 & 1.44 & 1.67 & 1.80 & 2.29 \\
\hline $\mathrm{L} 2$ & 1.32 & 1.54 & 1.65 & 2.11 & 1.48 & 1.67 & 1.85 & 2.30 & 1.50 & 1.69 & 1.88 & 2.33 \\
\hline ML3 & 1.36 & 1.51 & 1.70 & 2.08 & 1.39 & 1.56 & 1.74 & 2.14 & 1.42 & 1.59 & 1.77 & 2.19 \\
\hline ML4 & 1.33 & 1.56 & 1.67 & 2.15 & 1.41 & 1.66 & 1.77 & 2.28 & 1.50 & 1.77 & 1.88 & 2.43 \\
\hline ML5 & 1.34 & 1.57 & 1.67 & 2.15 & 1.40 & 1.63 & 1.75 & 2.25 & 1.49 & 1.75 & 1.86 & 2.40 \\
\hline ML6 & 1.41 & 1.57 & 1.76 & 2.16 & 1.44 & 1.62 & 1.81 & 2.22 & 1.47 & 1.64 & 1.84 & 2.26 \\
\hline MH1 & 1.35 & 1.48 & 1.69 & 2.04 & 1.41 & 1.57 & 1.76 & 2.17 & 1.45 & 1.63 & 1.81 & 2.24 \\
\hline MH2 & 1.25 & 1.30 & 1.56 & 1.79 & 1.35 & 1.44 & 1.68 & 1.98 & 1.42 & 1.55 & 1.77 & 2.13 \\
\hline MH3 & 1.33 & 1.52 & 1.66 & 2.09 & 1.59 & 1.93 & 1.99 & 2.66 & 1.66 & 2.01 & 2.07 & 2.77 \\
\hline MH4 & 1.24 & 1.29 & 1.56 & 1.77 & 1.35 & 1.42 & 1.69 & 1.95 & 1.43 & 1.53 & 1.78 & 2.10 \\
\hline MH5 & 1.26 & 1.30 & 1.58 & 1.79 & 1.37 & 1.44 & 1.72 & 1.98 & 1.45 & 1.55 & 1.81 & 2.13 \\
\hline H6 & 35 & 1.69 & 1.69 & 2.32 & 1.48 & 1.81 & 1.85 & 2.48 & 1.60 & 1.91 & 2.01 & 2.62 \\
\hline L1 & 1.15 & 1.31 & 1.43 & 1.80 & 1.30 & 1.48 & 1.63 & 2.04 & 1.48 & 1.70 & 1.85 & 2.33 \\
\hline LL2 & 1.14 & 1.31 & 1.42 & 1.80 & 1.29 & 1.49 & 1.62 & 2.05 & 1.46 & 1.69 & 1.82 & 2.32 \\
\hline LL3 & 1.16 & 1.26 & 1.45 & 1.74 & 1.31 & 1.46 & 1.64 & 2.00 & 1.52 & 1.84 & 1.90 & 2.53 \\
\hline LL4 & 1.28 & 1.49 & 1.60 & 2.06 & 1.43 & 1.68 & 1.79 & 2.31 & 1.60 & 1.88 & 2.00 & 2.59 \\
\hline LL5 & 1.27 & 1.48 & 1.58 & 2.03 & 1.43 & 1.67 & 1.78 & 2.29 & 1.61 & 1.89 & 2.01 & 2.60 \\
\hline LL6 & 0.92 & 1.13 & 1.15 & 1.55 & 1.02 & 1.30 & 1.28 & 1.79 & 1.14 & 1.45 & 1.42 & 1.99 \\
\hline LH1 & 1.22 & 1.40 & 1.53 & 1.92 & 1.49 & 1.81 & 1.86 & 2.48 & 1.56 & 1.88 & 1.95 & 2.59 \\
\hline LH2 & 1.36 & 1.67 & 1.69 & 2.29 & 1.49 & 1.86 & 1.86 & 2.56 & 1.62 & 1.99 & 2.03 & 2.74 \\
\hline LH3 & 1.15 & 1.42 & 1.44 & 1.95 & 1.36 & 1.68 & 1.70 & 2.31 & 1.48 & 1.80 & 1.86 & 2.48 \\
\hline LH4 & 1.39 & 1.69 & 1.74 & 2.32 & 1.49 & 1.83 & 1.87 & 2.52 & 1.63 & 1.96 & 2.04 & 2.69 \\
\hline LH5 & 1.38 & 1.68 & 1.72 & 2.31 & 1.51 & 1.85 & 1.89 & 2.54 & 1.67 & 1.98 & 2.08 & 2.72 \\
\hline LH6 & 1.06 & 1.31 & 1.32 & 1.81 & 1.24 & 1.54 & 1.55 & 2.12 & 1.39 & 1.72 & 1.74 & 2.36 \\
\hline
\end{tabular}


Table C.14 - The ratio of actual mid-span bending moments and quarter-span shear forces for buildings to the corresponding values obtained from FEMA parabolic distribution and a uniform distribution of seismic load when subjected to E6C18 ground motion and the Load is acting parallel to the short side, $\alpha=0.00$

\begin{tabular}{|c|c|c|c|c|c|c|c|c|c|c|c|c|}
\hline \multirow{2}{*}{ Building } & \multicolumn{4}{|c|}{$\mu=2$} & \multicolumn{4}{|c|}{$\mu=3$} & \multicolumn{4}{|c|}{$\mu=4$} \\
\hline & $M . F$ & V.F & $M . U$ & $V . U$ & $M . F$ & V.F & $M . U$ & $V . U$ & M.F & V.F & M.U & $V . U$ \\
\hline SL1 & 1.17 & 1.27 & 1.47 & 1.74 & 1.23 & 1.36 & 1.54 & 1.86 & 1.32 & 1.42 & 1.65 & 1.96 \\
\hline SL3 & 21 & .32 & 1.51 & 1.82 & 1.25 & 1.39 & 1.56 & 1.92 & 1.32 & 1.44 & 1.66 & 1.98 \\
\hline SL4 & 1.24 & 1.38 & 1.56 & 1.89 & 1.31 & 1.49 & 1.64 & 2.04 & 1.39 & 1.54 & 1.74 & 2.12 \\
\hline SH1 & 1.06 & 1.12 & 1.32 & 1.54 & 1.16 & 1.18 & 1.45 & 1.62 & 1.24 & 1.25 & 1.55 & 1.72 \\
\hline $\mathrm{SH} 2$ & 1.04 & 1.07 & 1.30 & 1.48 & 1.16 & 1.20 & 1.46 & 1.65 & 1.26 & 1.30 & 1.57 & 1.79 \\
\hline SH3 & 1.05 & 1.10 & 1.31 & 1.52 & 1.18 & 1.24 & 1.48 & 1.71 & 1.29 & 1.36 & 1.61 & 1.87 \\
\hline SH4 & 1.04 & 1.11 & 1.30 & 1.52 & 1.16 & 1.24 & 1.45 & 1.70 & 1.27 & 1.35 & 1.59 & 1.86 \\
\hline SH5 & 1.05 & 1.13 & 1.32 & 1.55 & 1.19 & 1.27 & 1.49 & 1.75 & 1.30 & 1.39 & 1.62 & 1.91 \\
\hline SH6 & 1.05 & 1.10 & 1.31 & 1.52 & 1.18 & 1.24 & 1.48 & 1.71 & 1.29 & 1.36 & 1.61 & 1.87 \\
\hline ML1 & 1.01 & 1.12 & 1.26 & 1.53 & 1.13 & 1.25 & 1.41 & 1.72 & 1.25 & 1.38 & 1.57 & 1.89 \\
\hline ML2 & 1.00 & 1.26 & 1.26 & 1.74 & 1.24 & 1.47 & 1.55 & 2.02 & 1.30 & 1.53 & 1.63 & 2.11 \\
\hline ML3 & 15 & 1.44 & 1.44 & 1.98 & 1.24 & 1.53 & 1.55 & 2.10 & 1.30 & 1.59 & 1.62 & 2.19 \\
\hline ML4 & 1.00 & 1.17 & 1.25 & 1.60 & 1.13 & 1.32 & 1.41 & 1.81 & 1.45 & 1.60 & 1.82 & 2.20 \\
\hline ML5 & 1.00 & 1.17 & 1.26 & 1.61 & 1.13 & 1.32 & 1.42 & 1.81 & 1.47 & 1.62 & 1.84 & 2.22 \\
\hline ML6 & 1.16 & 1.42 & 1.45 & 1.96 & 1.24 & 1.49 & 1.55 & 2.05 & 1.30 & 1.55 & 1.63 & 2.14 \\
\hline MH1 & 1.18 & 1.45 & 1.48 & 1.99 & 1.26 & 1.50 & 1.58 & 2.07 & 1.31 & 1.57 & 1.64 & 2.15 \\
\hline MH2 & 1.29 & 1.54 & 1.61 & 2.11 & 1.38 & 1.59 & 1.72 & 2.19 & 1.45 & 1.67 & 1.81 & 2.30 \\
\hline MH3 & 0.97 & 1.47 & 1.21 & 2.02 & 1.06 & 1.64 & 1.33 & 2.26 & 1.13 & 1.86 & 1.41 & 2.56 \\
\hline MH4 & 1.11 & 1.43 & 1.39 & 1.96 & 1.37 & 1.62 & 1.71 & 2.23 & 1.45 & 1.69 & 1.81 & 2.32 \\
\hline MH5 & 1.32 & 1.60 & 1.64 & 2.20 & 1.41 & 1.66 & 1.76 & 2.28 & 1.48 & 1.72 & 1.85 & 2.36 \\
\hline MH6 & 1.06 & 1.24 & 1.33 & 1.71 & 1.47 & 1.58 & 1.84 & 2.17 & 1.55 & 1.68 & 1.93 & 2.31 \\
\hline LL1 & 1.38 & 1.84 & 1.73 & 2.53 & 1.46 & 1.93 & 1.82 & 2.65 & 1.51 & 1.98 & 1.89 & 2.73 \\
\hline LL2 & 1.36 & 1.83 & 1.70 & 2.52 & 1.45 & 1.93 & 1.81 & 2.66 & 1.51 & 2.00 & 1.89 & 2.75 \\
\hline LL3 & 1.45 & 1.92 & 1.82 & 2.63 & 1.57 & 2.04 & 1.97 & 2.80 & 1.66 & 2.13 & 2.07 & 2.93 \\
\hline LL4 & 1.30 & 1.66 & 1.63 & 2.28 & 1.38 & 1.74 & 1.72 & 2.39 & 1.42 & 1.77 & 1.77 & 2.44 \\
\hline LL5 & 1.31 & 1.67 & 1.64 & 2.30 & 1.37 & 1.73 & 1.72 & 2.38 & 1.43 & 1.78 & 1.78 & 2.45 \\
\hline LL6 & 0.67 & 1.45 & 0.84 & 1.99 & 0.77 & 1.62 & 0.96 & 2.23 & 0.88 & 1.81 & 1.10 & 2.48 \\
\hline LH1 & 1.51 & 2.06 & 1.89 & 2.83 & 1.63 & 2.19 & 2.04 & 3.02 & 1.75 & 2.33 & 2.19 & 3.21 \\
\hline LH2 & 1.54 & 2.18 & 1.92 & 3.00 & 1.68 & 2.36 & 2.10 & 3.24 & 1.79 & 2.50 & 2.24 & 3.43 \\
\hline LH3 & 1.03 & 1.88 & 1.28 & 2.59 & 1.22 & 2.12 & 1.53 & 2.92 & 1.37 & 2.27 & 1.71 & 3.12 \\
\hline LH4 & 1.56 & 2.19 & 1.95 & 3.01 & 1.72 & 2.38 & 2.15 & 3.27 & 1.83 & 2.53 & 2.29 & 3.47 \\
\hline LH5 & 1.57 & 2.21 & 1.96 & 3.03 & 1.72 & 2.40 & 2.15 & 3.29 & 1.84 & 2.56 & 2.30 & 3.52 \\
\hline LH6 & 0.99 & 1.87 & 1.24 & 2.57 & 1.19 & 2.12 & 1.49 & 2.91 & 1.33 & 2.28 & 1.66 & 3.13 \\
\hline
\end{tabular}


Table C.15 - The ratio of actual mid-span bending moments and quarter-span shear forces for buildings to the corresponding values obtained from FEMA parabolic distribution and a uniform distribution of seismic load when subjected to E6C42 ground motion and the Load is acting parallel to the short side, $\alpha=0.00$

\begin{tabular}{|c|c|c|c|c|c|c|c|c|c|c|c|c|}
\hline \multirow{2}{*}{ Building } & \multicolumn{4}{|c|}{$\mu=2$} & \multicolumn{4}{|c|}{$\mu=3$} & \multicolumn{4}{|c|}{$\mu=4$} \\
\hline & M.F & $V . F$ & $M . U$ & $V . U$ & $M . F$ & $V . F$ & $M . U$ & $V . U$ & $M . F$ & V.F & $M . U$ & $V . U$ \\
\hline SL1 & 1.26 & 1.32 & 1.57 & 1.81 & 1.36 & 1.44 & 1.70 & 1.98 & 1.45 & 1.56 & 1.82 & 2.15 \\
\hline SL3 & .29 & 1.36 & 1.61 & 1.87 & 1.40 & 1.50 & 1.75 & 2.07 & 1.50 & 1.63 & 1.88 & 2.24 \\
\hline SL4 & .35 & 1.50 & 1.69 & 2.06 & 1.52 & 1.69 & 1.90 & 2.32 & 1.60 & 1.77 & 2.00 & 2.44 \\
\hline SH1 & 1.20 & 1.22 & 1.50 & 1.67 & 1.26 & 1.30 & 1.58 & 1.79 & 1.32 & 1.37 & 1.65 & 1.88 \\
\hline $\mathrm{SH} 2$ & 1.18 & 1.19 & 1.48 & 1.64 & 1.24 & 1.27 & 1.55 & 1.74 & 1.30 & 1.34 & 1.63 & 1.84 \\
\hline SH3 & 1.17 & 1.17 & 1.46 & 1.61 & 1.24 & 1.27 & 1.56 & 1.74 & 1.30 & 1.34 & 1.63 & 1.84 \\
\hline SH4 & 1.15 & 1.14 & 1.43 & 1.56 & 1.22 & 1.22 & 1.52 & 1.68 & 1.28 & 1.30 & 1.60 & 1.78 \\
\hline SH5 & 1.14 & 1.14 & 1.43 & 1.56 & 1.23 & 1.24 & 1.53 & 1.70 & 1.29 & 1.32 & 1.62 & 1.81 \\
\hline SH6 & 1.18 & 1.18 & 1.47 & 1.63 & 1.24 & 1.26 & 1.55 & 1.73 & 1.30 & 1.34 & 1.63 & 1.84 \\
\hline ML1 & 1.10 & 1.23 & 1.38 & 1.70 & 1.16 & 1.28 & 1.45 & 1.77 & 1.25 & 1.33 & 1.56 & 1.84 \\
\hline ML2 & 1.25 & 1.49 & 1.56 & 2.04 & 1.33 & 1.59 & 1.66 & 2.18 & 1.37 & 1.66 & 1.72 & 2.29 \\
\hline ML3 & 1.30 & 1.56 & 1.62 & 2.14 & 1.36 & 1.68 & 1.71 & 2.31 & 1.41 & 1.78 & 1.77 & 2.45 \\
\hline ML4 & 1.15 & 1.32 & 1.43 & 1.81 & 1.23 & 1.40 & 1.54 & 1.93 & 1.29 & 1.46 & 1.61 & 2.01 \\
\hline ML5 & 1.15 & 1.32 & 1.44 & 1.81 & 1.22 & 1.39 & 1.53 & 1.91 & 1.28 & 1.46 & 1.60 & 2.01 \\
\hline ML6 & 1.29 & 1.54 & 1.61 & 2.12 & 1.36 & 1.65 & 1.69 & 2.27 & 1.40 & 1.74 & 1.75 & 2.39 \\
\hline MH1 & 1.31 & 1.61 & 1.64 & 2.22 & 1.34 & 1.69 & 1.68 & 2.33 & 1.37 & 1.76 & 1.72 & 2.41 \\
\hline MH2 & 1.14 & 1.38 & 1.42 & 1.90 & 1.28 & 1.57 & 1.60 & 2.16 & 1.30 & 1.62 & 1.63 & 2.23 \\
\hline MH3 & 0.88 & 0.92 & 1.09 & 1.27 & 0.93 & 1.02 & 1.16 & 1.41 & 0.98 & 1.14 & 1.23 & 1.56 \\
\hline MH4 & 1.19 & 1.43 & 1.49 & 1.97 & 1.25 & 1.49 & 1.56 & 2.04 & 1.27 & 1.52 & 1.58 & 2.09 \\
\hline MH5 & 1.19 & 1.43 & 1.49 & 1.96 & 1.25 & 1.49 & 1.56 & 2.05 & 1.26 & 1.53 & 1.58 & 2.10 \\
\hline MH6 & 1.25 & 1.41 & 1.56 & 1.95 & 1.31 & 1.51 & 1.64 & 2.08 & 1.37 & 1.59 & 1.72 & 2.19 \\
\hline LL1 & 1.10 & 1.44 & 1.38 & 1.97 & 1.34 & 1.72 & 1.67 & 2.37 & 1.44 & 1.91 & 1.80 & 2.62 \\
\hline LL2 & 1.09 & 1.42 & 1.36 & 1.96 & 1.30 & 1.69 & 1.63 & 2.32 & 1.44 & 1.91 & 1.79 & 2.63 \\
\hline LL3 & 0.99 & 1.24 & 1.24 & 1.71 & 1.04 & 1.30 & 1.30 & 1.78 & 1.07 & 1.35 & 1.34 & 1.85 \\
\hline LL4 & 1.38 & 1.72 & 1.72 & 2.37 & 1.44 & 1.83 & 1.80 & 2.51 & 1.48 & 1.92 & 1.85 & 2.64 \\
\hline LL5 & 1.39 & 1.73 & 1.73 & 2.38 & 1.43 & 1.82 & 1.79 & 2.50 & 1.49 & 1.91 & 1.86 & 2.63 \\
\hline LL6 & 0.94 & 1.12 & 1.18 & 1.54 & 0.99 & 1.18 & 1.24 & 1.62 & 1.03 & 1.23 & 1.29 & 1.69 \\
\hline LH1 & 0.93 & 1.19 & 1.17 & 1.64 & 0.97 & 1.26 & 1.21 & 1.73 & 1.02 & 1.31 & 1.27 & 1.80 \\
\hline LH2 & 0.95 & 1.18 & 1.19 & 1.62 & 0.98 & 1.25 & 1.22 & 1.72 & 0.99 & 1.31 & 1.24 & 1.81 \\
\hline LH3 & 0.91 & 1.07 & 1.14 & 1.47 & 0.95 & 1.12 & 1.19 & 1.55 & 1.01 & 1.18 & 1.26 & 1.63 \\
\hline LH4 & 0.95 & 1.12 & 1.19 & 1.55 & 0.98 & 1.17 & 1.22 & 1.61 & 1.03 & 1.22 & 1.28 & 1.68 \\
\hline LH5 & 0.95 & 1.10 & 1.19 & 1.51 & 0.98 & 1.14 & 1.22 & 1.57 & 1.03 & 1.19 & 1.29 & 1.64 \\
\hline LH6 & 0.96 & 1.13 & 1.20 & 1.55 & 1.00 & 1.20 & 1.25 & 1.65 & 1.04 & 1.26 & 1.30 & 1.73 \\
\hline
\end{tabular}


Table C.16 - The ratio of actual mid-span bending moments and quarter-span shear forces for buildings to the corresponding values obtained from FEMA parabolic distribution and a uniform distribution of seismic load when subjected to E6C1 ground motion and the Load is acting parallel to the long side, $\alpha=0.00$

\begin{tabular}{|c|c|c|c|c|c|c|c|c|c|c|c|c|}
\hline \multirow{2}{*}{ Building } & \multicolumn{4}{|c|}{$\mu=2$} & \multicolumn{4}{|c|}{$\mu=3$} & \multicolumn{4}{|c|}{$\mu=4$} \\
\hline & $M . F$ & $V . F$ & $M . U$ & V.U & $M . F$ & $V . F$ & $M . U$ & $V . U$ & $M . F$ & $V . F$ & $M . U$ & $V . U$ \\
\hline SL1 & 1.24 & 1.33 & 1.55 & 1.84 & 1.40 & 1.56 & 1.75 & 2.15 & 1.45 & 1.62 & 1.81 & 2.22 \\
\hline SL3 & .28 & 1.38 & 1.60 & 1.90 & 1.38 & 1.55 & 1.73 & 2.13 & 1.41 & 1.57 & 1.76 & 2.16 \\
\hline SL4 & 12 & 1.16 & 1.40 & 1.60 & 1.22 & 1.33 & 1.52 & 1.83 & 1.38 & 1.55 & 1.73 & 2.14 \\
\hline SH1 & 1.04 & 1.06 & 1.30 & 1.46 & 1.11 & 1.18 & 1.39 & 1.62 & 1.15 & 1.22 & 1.43 & 1.67 \\
\hline $\mathrm{SH} 2$ & 1.04 & 1.07 & 1.30 & 1.46 & 1.08 & 1.14 & 1.36 & 1.56 & 1.11 & 1.16 & 1.38 & 1.60 \\
\hline $\mathrm{SH} 3$ & 1.03 & 1.06 & 1.29 & 1.45 & 1.06 & 1.10 & 1.33 & 1.52 & 1.08 & 1.13 & 1.35 & 1.55 \\
\hline $\mathrm{SH} 4$ & 1.02 & 1.04 & 1.27 & 1.42 & 1.04 & 1.07 & 1.30 & 1.47 & 1.05 & 1.09 & 1.31 & 1.50 \\
\hline SH5 & 1.01 & 1.03 & 1.26 & 1.41 & 1.03 & 1.06 & 1.29 & 1.46 & 1.04 & 1.09 & 1.30 & 1.49 \\
\hline ML1 & 0.92 & 0.90 & 1.15 & 1.24 & 0.96 & 0.93 & 1.20 & 1.28 & 0.99 & 0.98 & 1.25 & 1.35 \\
\hline ML2 & 0.92 & 0.90 & 1.15 & 1.23 & 0.96 & 0.93 & 1.20 & 1.28 & 1.04 & 1.00 & 1.30 & 1.37 \\
\hline ML3 & 0.92 & 0.89 & 1.15 & 1.23 & 1.01 & 0.97 & 1.27 & 1.33 & 1.11 & 1.08 & 1.39 & 1.48 \\
\hline ML4 & 0.92 & 0.90 & 1.15 & 1.24 & 0.95 & 0.93 & 1.19 & 1.28 & 1.03 & 0.99 & 1.28 & 1.36 \\
\hline ML5 & 0.92 & 0.89 & 1.15 & 1.23 & 0.98 & 0.94 & 1.22 & 1.29 & 1.05 & 1.01 & 1.31 & 1.38 \\
\hline ML6 & 0.92 & 0.88 & 1.15 & 1.22 & 0.99 & 0.95 & 1.24 & 1.30 & 1.09 & 1.05 & 1.36 & 1.44 \\
\hline MH1 & 1.07 & 1.08 & 1.34 & 1.48 & 1.20 & 1.25 & 1.51 & 1.72 & 1.30 & 1.40 & 1.63 & 1.92 \\
\hline & 1.20 & 1.30 & 1.50 & 1.78 & 1.30 & 1.45 & 1.62 & 1.99 & 1.37 & 1.55 & 1.72 & 2.14 \\
\hline MH3 & 1.25 & 1.39 & 1.56 & 1.91 & 1.27 & 1.43 & 1.59 & 1.97 & 1.33 & 1.52 & 1.66 & 2.09 \\
\hline MH4 & 1.12 & 1.15 & 1.40 & 1.59 & 1.24 & 1.32 & 1.55 & 1.81 & 1.34 & 1.46 & 1.67 & 2.01 \\
\hline MH5 & 1.23 & 1.35 & 1.53 & 1.86 & 1.31 & 1.48 & 1.64 & 2.03 & 1.38 & 1.57 & 1.72 & 2.16 \\
\hline MH6 & 1.13 & 1.19 & 1.42 & 1.64 & 1.26 & 1.37 & 1.58 & 1.89 & 1.37 & 1.55 & 1.71 & 2.13 \\
\hline LL1 & 0.01 & 1.00 & 1.26 & 1.38 & 1.15 & 1.17 & 1.44 & 1.61 & 1.28 & 1.36 & 1.61 & 1.87 \\
\hline LL2 & 1.08 & 1.09 & 1.35 & 1.50 & 1.21 & 1.28 & 1.52 & 1.75 & 1.33 & 1.45 & 1.66 & 1.99 \\
\hline LL3 & 1.26 & 1.40 & 1.58 & 1.93 & 1.30 & 1.46 & 1.62 & 2.01 & 1.36 & 1.55 & 1.70 & 2.13 \\
\hline LL4 & 1.02 & 1.01 & 1.27 & 1.39 & 1.16 & 1.18 & 1.45 & 1.63 & 1.29 & 1.37 & 1.61 & 1.88 \\
\hline LL5 & 1.19 & 1.29 & 1.49 & 1.77 & 1.30 & 1.44 & 1.62 & 1.98 & 1.39 & 1.57 & 1.73 & 2.16 \\
\hline LL6 & 1.17 & 1.23 & 1.46 & 1.68 & 1.27 & 1.37 & 1.59 & 1.89 & 1.59 & 1.82 & 1.99 & 2.50 \\
\hline LH1 & 1.12 & 1.17 & 1.40 & 1.61 & 1.26 & 1.37 & 1.57 & 1.89 & 1.68 & 1.98 & 2.11 & 2.73 \\
\hline LH2 & 1.20 & 1.29 & 1.50 & 1.78 & 1.34 & 1.52 & 1.67 & 2.08 & 1.67 & 1.95 & 2.09 & 2.68 \\
\hline LH3 & 1.15 & 1.21 & 1.43 & 1.66 & 1.47 & 1.68 & 1.84 & 2.31 & 1.53 & 1.75 & 1.92 & 2.40 \\
\hline LH4 & 1.12 & 1.18 & 1.40 & 1.63 & 1.56 & 1.81 & 1.95 & 2.49 & 1.68 & 1.99 & 2.10 & 2.73 \\
\hline LH5 & 1.23 & 1.34 & 1.53 & 1.84 & 1.32 & 1.48 & 1.65 & 2.04 & 1.57 & 1.78 & 1.96 & 2.44 \\
\hline LH6 & 1.12 & 1.13 & 1.41 & 1.55 & 1.49 & 1.70 & 1.86 & 2.33 & 1.54 & 1.75 & 1.93 & 2.41 \\
\hline
\end{tabular}


Table C.17 - The ratio of actual mid-span bending moments and quarter-span shear forces for buildings to the corresponding values obtained from FEMA parabolic distribution and a uniform distribution of seismic load when subjected to E6C13 ground motion and the Load is acting parallel to the long side, $\alpha=0.00$

\begin{tabular}{|c|c|c|c|c|c|c|c|c|c|c|c|c|}
\hline \multirow{2}{*}{ Building } & \multicolumn{4}{|c|}{$\mu=2$} & \multicolumn{4}{|c|}{$\mu=3$} & \multicolumn{4}{|c|}{$\mu=4$} \\
\hline & $M . F$ & $V . F$ & $M . U$ & V.U & M.F & $V . F$ & $M . U$ & $V . U$ & M.F & V.F & M.U & $V . U$ \\
\hline SL1 & 1.18 & 1.26 & 1.47 & 1.73 & 1.43 & 1.61 & 1.78 & 2.21 & 1.50 & 1.72 & 1.87 & 2.36 \\
\hline SL3 & 17 & 1.24 & 1.46 & 1.71 & 1.46 & 1.66 & 1.82 & 2.29 & 1.53 & 1.77 & 1.91 & 2.43 \\
\hline & 14 & 1.19 & 1.42 & 1.64 & 1.46 & 1.65 & 1.82 & 2.27 & 1.52 & 1.74 & 1.90 & 2.40 \\
\hline SH1 & 1.28 & 1.41 & 1.59 & 1.94 & 1.34 & 1.50 & 1.68 & 2.06 & 1.34 & 1.49 & 1.68 & 2.05 \\
\hline & 1.04 & 1.05 & 1.30 & 1.44 & 1.36 & 1.52 & 1.70 & 2.09 & 1.36 & 1.53 & 1.70 & 2.10 \\
\hline $\mathrm{SH} 3$ & 0.98 & 0.99 & 1.22 & 1.37 & 1.27 & 1.40 & 1.59 & 1.93 & 1.34 & 1.50 & 1.67 & 2.06 \\
\hline $\mathrm{SH} 4$ & 1.00 & 0.98 & 1.25 & 1.35 & 1.29 & 1.41 & 1.62 & 1.93 & 1.39 & 1.56 & 1.73 & 2.15 \\
\hline SH5 & 1.01 & 0.99 & 1.26 & 1.37 & 1.25 & 1.35 & 1.56 & 1.86 & 1.36 & 1.51 & 1.70 & 2.08 \\
\hline IL1 & 1.17 & 1.31 & 1.47 & 1.80 & 1.22 & 1.40 & 1.53 & 1.92 & 1.33 & 1.51 & 1.66 & 2.07 \\
\hline ML2 & 1.22 & 1.38 & 1.53 & 1.90 & 1.29 & 1.46 & 1.61 & 2.01 & 1.35 & 1.52 & 1.69 & 2.09 \\
\hline ML3 & 1.31 & 1.50 & 1.63 & 2.06 & 1.38 & 1.58 & 1.73 & 2.18 & 1.45 & 1.63 & 1.81 & 2.24 \\
\hline ML4 & 1.21 & 1.35 & 1.51 & 1.86 & 1.27 & 1.44 & 1.59 & 1.98 & 1.34 & 1.50 & 1.67 & 2.06 \\
\hline ML5 & 1.24 & 1.41 & 1.55 & 1.94 & 1.31 & 1.50 & 1.64 & 2.06 & 1.37 & 1.55 & 1.72 & 2.13 \\
\hline ML6 & 1.28 & 1.46 & 1.60 & 2.01 & 1.35 & 1.55 & 1.69 & 2.13 & 1.42 & 1.60 & 1.78 & 2.20 \\
\hline & 40 & 1.57 & 1.75 & 2.16 & 1.42 & 1.56 & 1.77 & 2.14 & 1.45 & 1.68 & 1.81 & 2.31 \\
\hline & 1.35 & 1.52 & 1.68 & 2.09 & 1.47 & 1.71 & 1.83 & 2.36 & 1.55 & 1.84 & 1.94 & 2.52 \\
\hline & 1.03 & 1.17 & 1.29 & 1.60 & 1.21 & 1.43 & 1.52 & 1.97 & 1.39 & 1.65 & 1.74 & 2.27 \\
\hline MH4 & 1.38 & 1.52 & 1.72 & 2.09 & 1.40 & 1.61 & 1.74 & 2.22 & 1.49 & 1.74 & 1.86 & 2.39 \\
\hline MH5 & 1.36 & 1.53 & 1.70 & 2.10 & 1.48 & 1.73 & 1.85 & 2.38 & 1.55 & 1.83 & 1.93 & 2.52 \\
\hline MH6 & 1.32 & 1.49 & 1.65 & 2.04 & 1.45 & 1.68 & 1.82 & 2.31 & 1.53 & 1.79 & 1.91 & 2.46 \\
\hline LL1 & 1.41 & 1.60 & 1.76 & 2.20 & 1.44 & 1.60 & 1.80 & 2.20 & 1.46 & 1.69 & 1.82 & 2.32 \\
\hline LL2 & 1.39 & 1.54 & 1.73 & 2.11 & 1.42 & 1.64 & 1.78 & 2.25 & 1.49 & 1.74 & 1.87 & 2.40 \\
\hline LL3 & 1.10 & 1.27 & 1.38 & 1.74 & 1.35 & 1.56 & 1.69 & 2.15 & 1.50 & 1.76 & 1.87 & 2.42 \\
\hline LL4 & 1.41 & 1.59 & 1.76 & 2.19 & 1.44 & 1.60 & 1.80 & 2.20 & 1.46 & 1.70 & 1.82 & 2.33 \\
\hline LL5 & 1.35 & 1.52 & 1.69 & 2.09 & 1.49 & 1.73 & 1.86 & 2.39 & 1.54 & 1.82 & 1.93 & 2.50 \\
\hline LL6 & 0.94 & 0.99 & 1.18 & 1.36 & 0.97 & 1.21 & 1.21 & 1.67 & 1.12 & 1.41 & 1.40 & 1.93 \\
\hline LH1 & 1.32 & 1.45 & 1.64 & 1.99 & 1.43 & 1.64 & 1.79 & 2.26 & 1.53 & 1.78 & 1.91 & 2.45 \\
\hline LH2 & 1.22 & 1.30 & 1.52 & 1.79 & 1.41 & 1.59 & 1.76 & 2.18 & 1.51 & 1.76 & 1.89 & 2.41 \\
\hline LH3 & 0.98 & 0.99 & 1.23 & 1.37 & 1.00 & 1.23 & 1.25 & 1.69 & 1.22 & 1.46 & 1.53 & 2.01 \\
\hline LH4 & 1.25 & 1.35 & 1.57 & 1.86 & 1.40 & 1.58 & 1.75 & 2.18 & 1.49 & 1.73 & 1.86 & 2.38 \\
\hline LH5 & 1.09 & 1.19 & 1.37 & 1.64 & 1.30 & 1.47 & 1.63 & 2.03 & 1.45 & 1.67 & 1.81 & 2.30 \\
\hline LH6 & 0.97 & 1.14 & 1.21 & 1.56 & 1.18 & 1.38 & 1.47 & 1.89 & 1.33 & 1.55 & 1.66 & 2.13 \\
\hline
\end{tabular}


Table C.18 - The ratio of actual mid-span bending moments and quarter-span shear forces for buildings to the corresponding values obtained from FEMA parabolic distribution and a uniform distribution of seismic load when subjected to E6C15 ground motion and the Load is acting parallel to the long side, $\alpha=0.00$

\begin{tabular}{|c|c|c|c|c|c|c|c|c|c|c|c|c|}
\hline \multirow{2}{*}{ Building } & \multicolumn{4}{|c|}{$\mu=2$} & \multicolumn{4}{|c|}{$\mu=3$} & \multicolumn{4}{|c|}{$\mu=4$} \\
\hline & $M . F$ & $V . F$ & $M . U$ & V.U & M.F & $V . F$ & $M . U$ & $V . U$ & M.F & V.F & M.U & $V . U$ \\
\hline SL1 & 1.29 & 1.45 & 1.61 & 1.99 & 1.37 & 1.55 & 1.71 & 2.13 & 1.40 & 1.58 & 1.75 & 2.17 \\
\hline SL3 & 29 & 1.44 & 1.61 & 1.98 & 1.36 & 1.53 & 1.70 & 2.11 & 1.38 & 1.58 & 1.73 & 2.17 \\
\hline & 26 & 1.41 & 1.57 & 1.93 & 1.35 & 1.52 & 1.69 & 2.09 & 1.37 & 1.55 & 1.71 & 2.14 \\
\hline & 1.28 & 1.43 & 1.60 & 1.97 & 1.37 & 1.55 & 1.71 & 2.14 & 1.42 & 1.62 & 1.77 & 2.23 \\
\hline & 1.33 & 1.49 & 1.66 & 2.06 & 1.40 & 1.60 & 1.75 & 2.20 & 1.46 & 1.67 & 1.83 & 2.30 \\
\hline $\mathrm{SH} 3$ & 1.33 & 1.49 & 1.66 & 2.05 & 1.39 & 1.58 & 1.74 & 2.17 & 1.45 & 1.66 & 1.81 & 2.28 \\
\hline $\mathrm{SH} 4$ & 1.36 & 1.54 & 1.70 & 2.11 & 1.42 & 1.61 & 1.77 & 2.22 & 1.46 & 1.66 & 1.82 & 2.29 \\
\hline SH5 & 1.34 & 1.51 & 1.67 & 2.07 & 1.40 & 1.59 & 1.75 & 2.18 & 1.45 & 1.65 & 1.81 & 2.27 \\
\hline IL1 & 1.37 & 1.54 & 1.72 & 2.12 & 1.44 & 1.62 & 1.80 & 2.23 & 1.48 & 1.67 & 1.84 & 2.29 \\
\hline ML2 & 1.38 & 1.55 & 1.72 & 2.13 & 1.45 & 1.64 & 1.82 & 2.25 & 1.49 & 1.68 & 1.87 & 2.31 \\
\hline ML3 & 1.38 & 1.56 & 1.72 & 2.14 & 1.45 & 1.64 & 1.82 & 2.25 & 1.52 & 1.71 & 1.90 & 2.35 \\
\hline ML4 & 1.38 & 1.56 & 1.73 & 2.14 & 1.44 & 1.63 & 1.81 & 2.24 & 1.49 & 1.68 & 1.87 & 2.31 \\
\hline ML5 & 1.39 & 1.57 & 1.74 & 2.16 & 1.45 & 1.64 & 1.82 & 2.25 & 1.51 & 1.70 & 1.89 & 2.33 \\
\hline ML6 & 1.38 & 1.55 & 1.72 & 2.13 & 1.45 & 1.64 & 1.82 & 2.25 & 1.52 & 1.71 & 1.90 & 2.35 \\
\hline & 28 & 1.47 & 1.60 & 2.02 & 1.45 & 1.64 & 1.81 & 2.25 & 1.52 & 1.70 & 1.90 & 2.34 \\
\hline & 1.29 & 1.40 & 1.62 & 1.93 & 1.50 & 1.72 & 1.88 & 2.36 & 1.55 & 1.77 & 1.94 & 2.44 \\
\hline & 1.40 & 1.61 & 1.75 & 2.21 & 1.45 & 1.69 & 1.81 & 2.32 & 1.61 & 1.84 & 2.02 & 2.53 \\
\hline MH4 & 1.22 & 1.35 & 1.53 & 1.85 & 1.43 & 1.63 & 1.79 & 2.24 & 1.51 & 1.69 & 1.88 & 2.33 \\
\hline MH5 & 1.14 & 1.21 & 1.43 & 1.66 & 1.53 & 1.74 & 1.91 & 2.39 & 1.55 & 1.76 & 1.94 & 2.42 \\
\hline MH6 & 1.38 & 1.52 & 1.73 & 2.09 & 1.52 & 1.73 & 1.90 & 2.38 & 1.56 & 1.79 & 1.95 & 2.46 \\
\hline LL1 & 1.35 & 1.51 & 1.68 & 2.08 & 1.52 & 1.71 & 1.90 & 2.35 & 1.55 & 1.73 & 1.93 & 2.39 \\
\hline LL2 & 1.32 & 1.49 & 1.65 & 2.05 & 1.50 & 1.69 & 1.88 & 2.32 & 1.55 & 1.74 & 1.94 & 2.39 \\
\hline LL3 & 1.39 & 1.61 & 1.74 & 2.21 & 1.46 & 1.69 & 1.82 & 2.33 & 1.61 & 1.83 & 2.01 & 2.52 \\
\hline LL4 & 1.34 & 1.50 & 1.67 & 2.06 & 1.52 & 1.71 & 1.90 & 2.35 & 1.56 & 1.75 & 1.94 & 2.40 \\
\hline LL5 & 1.28 & 1.38 & 1.60 & 1.90 & 1.51 & 1.73 & 1.89 & 2.37 & 1.56 & 1.79 & 1.95 & 2.46 \\
\hline LL6 & 1.36 & 1.65 & 1.70 & 2.27 & 1.49 & 1.76 & 1.86 & 2.42 & 1.68 & 1.91 & 2.09 & 2.63 \\
\hline LH1 & 1.33 & 1.47 & 1.67 & 2.03 & 1.56 & 1.79 & 1.95 & 2.46 & 1.61 & 1.86 & 2.02 & 2.56 \\
\hline LH2 & 1.40 & 1.60 & 1.75 & 2.21 & 1.63 & 1.90 & 2.03 & 2.61 & 1.72 & 2.02 & 2.15 & 2.78 \\
\hline LH3 & 1.39 & 1.65 & 1.73 & 2.27 & 1.56 & 1.82 & 1.95 & 2.50 & 1.71 & 1.96 & 2.14 & 2.69 \\
\hline LH4 & 1.28 & 1.39 & 1.61 & 1.91 & 1.56 & 1.77 & 1.95 & 2.44 & 1.64 & 1.87 & 2.05 & 2.57 \\
\hline LH5 & 1.30 & 1.44 & 1.63 & 1.98 & 1.57 & 1.79 & 1.96 & 2.47 & 1.64 & 1.87 & 2.06 & 2.57 \\
\hline LH6 & 1.44 & 1.65 & 1.80 & 2.27 & 1.57 & 1.84 & 1.96 & 2.53 & 1.73 & 1.97 & 2.17 & 2.71 \\
\hline
\end{tabular}


Table C.19 - The ratio of actual mid-span bending moments and quarter-span shear forces for buildings to the corresponding values obtained from FEMA parabolic distribution and a uniform distribution of seismic load when subjected to E6C18 ground motion and the Load is acting parallel to the long side, $\alpha=0.00$

\begin{tabular}{|c|c|c|c|c|c|c|c|c|c|c|c|c|}
\hline \multirow{2}{*}{ Building } & \multicolumn{4}{|c|}{$\mu=2$} & \multicolumn{4}{|c|}{$\mu=3$} & \multicolumn{4}{|c|}{$\mu=4$} \\
\hline & $M . F$ & $V . F$ & $M . U$ & $V . U$ & M.F & $V . F$ & $M . U$ & $V . U$ & M.F & V.F & $M . U$ & $V . U$ \\
\hline SL1 & 1.02 & 1.03 & 1.27 & 1.41 & 1.24 & 1.29 & 1.54 & 1.78 & 1.26 & 1.39 & 1.57 & 1.91 \\
\hline SL3 & 02 & 1.04 & 1.28 & 1.43 & 1.26 & 1.32 & 1.57 & 1.81 & 1.26 & 1.37 & 1.57 & 1.89 \\
\hline SL4 & 1.03 & 1.05 & 1.29 & 1.44 & 1.31 & 1.42 & 1.64 & 1.95 & 1.34 & 1.44 & 1.68 & 1.98 \\
\hline SH1 & 1.13 & 1.18 & 1.41 & 1.62 & 1.40 & 1.58 & 1.75 & 2.18 & 1.47 & 1.67 & 1.83 & 2.29 \\
\hline $\mathrm{SH} 2$ & 1.13 & 1.16 & 1.41 & 1.60 & 1.36 & 1.52 & 1.70 & 2.10 & 1.49 & 1.64 & 1.86 & 2.26 \\
\hline $\mathrm{SH} 3$ & 1.12 & 1.16 & 1.40 & 1.59 & 1.35 & 1.51 & 1.69 & 2.08 & 1.44 & 1.60 & 1.80 & 2.20 \\
\hline SH4 & 1.13 & 1.16 & 1.41 & 1.60 & 1.32 & 1.48 & 1.66 & 2.03 & 1.43 & 1.64 & 1.79 & 2.25 \\
\hline H5 & 1.12 & 1.17 & 1.41 & 1.60 & 1.36 & 1.51 & 1.70 & 2.08 & 1.39 & 1.59 & 1.74 & 2.18 \\
\hline ML1 & 1.31 & 1.47 & 1.64 & 2.02 & 1.39 & 1.58 & 1.74 & 2.18 & 1.39 & 1.58 & 1.74 & 2.17 \\
\hline ML2 & 1.35 & 1.53 & 1.68 & 2.10 & 1.40 & 1.60 & 1.75 & 2.20 & 1.41 & 1.62 & 1.76 & 2.22 \\
\hline ML3 & 1.30 & 1.46 & 1.62 & 2.00 & 1.39 & 1.58 & 1.74 & 2.17 & 1.41 & 1.61 & 1.77 & 2.21 \\
\hline ML4 & 1.34 & 1.52 & 1.68 & 2.10 & 1.40 & 1.60 & 1.75 & 2.20 & 1.42 & 1.62 & 1.77 & 2.23 \\
\hline ML5 & 1.33 & 1.51 & 1.67 & 2.08 & 1.40 & 1.61 & 1.75 & 2.21 & 1.42 & 1.63 & 1.78 & 2.24 \\
\hline ML6 & 1.33 & 1.50 & 1.66 & 2.06 & 1.39 & 1.57 & 1.74 & 2.16 & 1.40 & 1.59 & 1.76 & 2.18 \\
\hline MH1 & 1.28 & 1.43 & 1.60 & 1.96 & 1.37 & 1.55 & 1.71 & 2.13 & 1.39 & 1.60 & 1.74 & 2.20 \\
\hline & 1.14 & 1.23 & 1.42 & 1.69 & 1.18 & 1.33 & 1.48 & 1.83 & 1.29 & 1.37 & 1.62 & 1.89 \\
\hline MH3 & 1.13 & 1.17 & 1.41 & 1.61 & 1.32 & 1.39 & 1.65 & 1.91 & 1.82 & 2.12 & 2.27 & 2.91 \\
\hline MH4 & 1.26 & 1.40 & 1.57 & 1.92 & 1.34 & 1.51 & 1.67 & 2.08 & 1.35 & 1.56 & 1.69 & 2.14 \\
\hline MH5 & 1.09 & 1.18 & 1.37 & 1.62 & 1.22 & 1.29 & 1.53 & 1.77 & 1.34 & 1.40 & 1.67 & 1.93 \\
\hline MH6 & 1.16 & 1.24 & 1.45 & 1.71 & 1.18 & 1.30 & 1.47 & 1.79 & 1.26 & 1.38 & 1.57 & 1.89 \\
\hline LL1 & 1.26 & 1.37 & 1.58 & 1.88 & 1.30 & 1.45 & 1.63 & 1.99 & 1.32 & 1.48 & 1.74 & 2.17 \\
\hline LL2 & 1.25 & 1.36 & 1.56 & 1.87 & 1.28 & 1.44 & 1.60 & 1.98 & 1.30 & 1.47 & 1.62 & 2.01 \\
\hline LL3 & 1.10 & 1.10 & 1.37 & 1.51 & 1.27 & 1.30 & 1.58 & 1.79 & 1.50 & 1.61 & 1.87 & 2.22 \\
\hline L4 & 1.26 & 1.37 & 1.58 & 1.88 & 1.30 & 1.45 & 1.62 & 1.99 & 1.33 & 1.49 & 1.66 & 2.04 \\
\hline LL5 & 1.15 & 1.25 & 1.44 & 1.71 & 1.18 & 1.34 & 1.48 & 1.84 & 1.28 & 1.38 & 1.60 & 1.90 \\
\hline LL6 & 1.13 & 1.23 & 1.41 & 1.69 & 1.53 & 1.67 & 1.92 & 2.30 & 1.61 & 1.80 & 2.01 & 2.47 \\
\hline LH1 & 1.08 & 1.14 & 1.35 & 1.57 & 1.17 & 1.28 & 1.47 & 1.76 & 1.27 & 1.41 & 1.59 & 1.94 \\
\hline LH2 & 1.02 & 1.03 & 1.28 & 1.42 & 1.18 & 1.26 & 1.47 & 1.73 & 1.47 & 1.70 & 1.84 & 2.33 \\
\hline LH3 & 1.28 & 1.38 & 1.60 & 1.89 & 1.37 & 1.48 & 1.71 & 2.03 & 1.45 & 1.62 & 1.81 & 2.23 \\
\hline LH4 & 1.06 & 1.12 & 1.33 & 1.54 & 1.16 & 1.26 & 1.45 & 1.73 & 1.25 & 1.38 & 1.56 & 1.90 \\
\hline LH5 & 1.06 & 1.15 & 1.32 & 1.58 & 1.55 & 1.79 & 1.94 & 2.46 & 1.86 & 2.20 & 2.33 & 3.03 \\
\hline LH6 & 1.30 & 1.44 & 1.63 & 1.98 & 1.35 & 1.48 & 1.68 & 2.04 & 1.40 & 1.51 & 1.75 & 2.08 \\
\hline
\end{tabular}


Table C.20 - The ratio of actual mid-span bending moments and quarter-span shear forces for buildings to the corresponding values obtained from FEMA parabolic distribution and a uniform distribution of seismic load when subjected to E6C42 ground motion and the Load is acting parallel to the long side, $\alpha=0.00$

\begin{tabular}{|c|c|c|c|c|c|c|c|c|c|c|c|c|}
\hline \multirow{2}{*}{ Building } & \multicolumn{4}{|c|}{$\mu=2$} & \multicolumn{4}{|c|}{$\mu=3$} & \multicolumn{4}{|c|}{$\mu=4$} \\
\hline & $M . F$ & $V . F$ & $M . U$ & $V . U$ & M.F & $V . F$ & $M . U$ & $V . U$ & M.F & V.F & $M . U$ & $V . U$ \\
\hline SL1 & 1.32 & 1.44 & 1.65 & 1.98 & 1.35 & 1.48 & 1.69 & 2.03 & 1.36 & 1.54 & 1.70 & 2.11 \\
\hline SL3 & 35 & 1.50 & 1.69 & 2.06 & 1.40 & 1.55 & 1.75 & 2.13 & 1.40 & 1.58 & 1.75 & 2.17 \\
\hline SL4 & 40 & 1.59 & 1.75 & 2.19 & 1.46 & 1.68 & 1.83 & 2.30 & 1.48 & 1.72 & 1.85 & 2.36 \\
\hline SH1 & 1.20 & 1.25 & 1.51 & 1.72 & 1.36 & 1.48 & 1.69 & 2.04 & 1.54 & 1.77 & 1.92 & 2.43 \\
\hline $\mathrm{SH} 2$ & 1.29 & 1.38 & 1.61 & 1.90 & 1.42 & 1.59 & 1.78 & 2.18 & 1.51 & 1.74 & 1.89 & 2.39 \\
\hline $\mathrm{SH} 3$ & 1.30 & 1.43 & 1.62 & 1.96 & 1.41 & 1.59 & 1.76 & 2.19 & 1.48 & 1.69 & 1.86 & 2.33 \\
\hline SH4 & 1.30 & 1.43 & 1.63 & 1.97 & 1.39 & 1.56 & 1.74 & 2.14 & 1.47 & 1.66 & 1.84 & 2.29 \\
\hline H5 & 1.27 & 1.40 & 1.59 & 1.93 & 1.37 & 1.53 & 1.71 & 2.11 & 1.44 & 1.63 & 1.80 & 2.24 \\
\hline ML1 & 1.35 & 1.51 & 1.68 & 2.07 & 1.37 & 1.55 & 1.71 & 2.13 & 1.51 & 1.71 & 1.88 & 2.36 \\
\hline ML2 & 1.24 & 1.40 & 1.55 & 1.93 & 1.31 & 1.51 & 1.64 & 2.08 & 1.39 & 1.54 & 1.74 & 2.11 \\
\hline ML3 & 1.06 & 1.21 & 1.33 & 1.66 & 1.11 & 1.31 & 1.38 & 1.80 & 1.14 & 1.31 & 1.43 & 1.80 \\
\hline ML4 & 1.27 & 1.43 & 1.59 & 1.97 & 1.35 & 1.53 & 1.68 & 2.11 & 1.44 & 1.61 & 1.80 & 2.21 \\
\hline ML5 & 1.20 & 1.36 & 1.51 & 1.86 & 1.25 & 1.47 & 1.56 & 2.02 & 1.38 & 1.50 & 1.73 & 2.07 \\
\hline ML6 & 1.10 & 1.24 & 1.37 & 1.71 & 1.13 & 1.35 & 1.42 & 1.85 & 1.24 & 1.35 & 1.55 & 1.86 \\
\hline MH1 & 0.93 & 0.93 & 1.16 & 1.27 & 1.02 & 1.02 & 1.27 & 1.40 & 1.24 & 1.32 & 1.55 & 1.81 \\
\hline & 1.04 & 1.07 & 1.30 & 1.47 & 1.19 & 1.29 & 1.49 & 1.77 & 1.50 & 1.71 & 1.88 & 2.35 \\
\hline MH3 & 1.17 & 1.22 & 1.46 & 1.68 & 1.27 & 1.37 & 1.58 & 1.88 & 1.38 & 1.54 & 1.73 & 2.12 \\
\hline MH4 & 0.96 & 0.96 & 1.20 & 1.32 & 1.04 & 1.06 & 1.31 & 1.46 & 1.34 & 1.49 & 1.67 & 2.05 \\
\hline MH5 & 1.09 & 1.13 & 1.36 & 1.55 & 1.23 & 1.34 & 1.54 & 1.85 & 1.70 & 2.01 & 2.13 & 2.76 \\
\hline MH6 & 0.99 & 1.02 & 1.24 & 1.40 & 1.16 & 1.25 & 1.45 & 1.73 & 1.45 & 1.61 & 1.82 & 2.22 \\
\hline LL1 & 0.94 & 0.94 & 1.17 & 1.29 & 0.99 & 1.01 & 1.24 & 1.39 & 1.37 & 1.53 & 1.71 & 2.10 \\
\hline LL2 & 0.96 & 0.97 & 1.20 & 1.34 & 1.02 & 1.06 & 1.27 & 1.45 & 1.36 & 1.50 & 1.70 & 2.07 \\
\hline LL3 & 1.17 & 1.24 & 1.46 & 1.71 & 1.27 & 1.40 & 1.58 & 1.92 & 1.41 & 1.69 & 1.76 & 2.32 \\
\hline L4 & 0.94 & 0.94 & 1.17 & 1.30 & 0.99 & 1.01 & 1.24 & 1.39 & 1.38 & 1.55 & 1.72 & 2.12 \\
\hline LL5 & 1.03 & 1.06 & 1.28 & 1.46 & 1.15 & 1.23 & 1.44 & 1.70 & 1.46 & 1.67 & 1.83 & 2.30 \\
\hline LL6 & 1.18 & 1.32 & 1.48 & 1.82 & 1.26 & 1.40 & 1.58 & 1.92 & 1.44 & 1.61 & 1.80 & 2.22 \\
\hline LH1 & 1.04 & 1.09 & 1.30 & 1.50 & 1.39 & 1.57 & 1.74 & 2.16 & 1.64 & 1.89 & 2.05 & 2.59 \\
\hline LH2 & 1.10 & 1.19 & 1.37 & 1.64 & 1.94 & 2.26 & 2.43 & 3.10 & 2.08 & 2.48 & 2.61 & 3.41 \\
\hline LH3 & 1.29 & 1.43 & 1.61 & 1.97 & 1.35 & 1.52 & 1.68 & 2.09 & 1.79 & 2.13 & 2.24 & 2.93 \\
\hline LH4 & 1.07 & 1.13 & 1.34 & 1.56 & 1.53 & 1.71 & 1.92 & 2.35 & 1.80 & 2.09 & 2.25 & 2.88 \\
\hline LH5 & 1.21 & 1.37 & 1.52 & 1.89 & 1.43 & 1.65 & 1.79 & 2.27 & 2.02 & 2.36 & 2.53 & 3.25 \\
\hline LH6 & 1.38 & 1.57 & 1.73 & 2.16 & 1.47 & 1.66 & 1.83 & 2.29 & 2.03 & 2.45 & 2.54 & 3.37 \\
\hline
\end{tabular}


Table C.21 - The ratio of actual mid-span bending moments and quarter-span shear forces for buildings to the corresponding values obtained from FEMA parabolic distribution and a uniform distribution of seismic load when subjected to M6C1 ground motion and the Load is acting parallel to the short side, $\alpha=0.02$

\begin{tabular}{|c|c|c|c|c|c|c|c|c|c|c|c|c|}
\hline \multirow{2}{*}{ Building } & \multicolumn{4}{|c|}{$\mu=2$} & \multicolumn{4}{|c|}{$\mu=3$} & \multicolumn{4}{|c|}{$\mu=4$} \\
\hline & $M . F$ & V.F & $M . U$ & V.U & $M . F$ & $V . F$ & $M . U$ & $V . U$ & M.F & V.F & M.U & $V . U$ \\
\hline SL1 & 1.04 & 1.06 & 1.30 & 1.46 & 1.05 & 1.09 & 1.31 & 1.50 & 1.11 & 1.17 & 1.38 & 1.61 \\
\hline SL3 & 06 & 1.10 & 1.32 & 1.51 & 1.08 & 1.14 & 1.35 & 1.57 & 1.11 & 1.19 & 1.38 & 1.64 \\
\hline SL4 & 1.16 & 1.24 & 1.45 & 1.70 & 1.17 & 1.27 & 1.46 & 1.74 & 1.19 & 1.30 & 1.49 & 1.78 \\
\hline SH1 & 1.18 & 1.27 & 1.47 & 1.74 & 1.25 & 1.37 & 1.56 & 1.89 & 1.29 & 1.44 & 1.62 & 1.98 \\
\hline $\mathrm{SH} 2$ & 1.25 & 1.38 & 1.56 & 1.90 & 1.28 & 1.44 & 1.60 & 1.98 & 1.30 & 1.50 & 1.63 & 2.06 \\
\hline SH3 & 1.27 & 1.43 & 1.59 & 1.96 & 1.29 & 1.47 & 1.61 & 2.02 & 1.33 & 1.54 & 1.66 & 2.12 \\
\hline SH4 & 1.26 & 1.41 & 1.58 & 1.94 & 1.28 & 1.46 & 1.60 & 2.01 & 1.29 & 1.49 & 1.61 & 2.05 \\
\hline SH5 & 1.27 & 1.42 & 1.58 & 1.95 & 1.29 & 1.47 & 1.61 & 2.03 & 1.31 & 1.51 & 1.63 & 2.08 \\
\hline SH6 & 1.27 & 1.43 & 1.59 & 1.96 & 1.29 & 1.47 & 1.61 & 2.02 & 1.33 & 1.54 & 1.66 & 2.12 \\
\hline ML1 & 1.18 & 1.30 & 1.47 & 1.79 & 1.23 & 1.38 & 1.53 & 1.90 & 1.25 & 1.44 & 1.57 & 1.97 \\
\hline ML2 & 1.09 & 1.09 & 1.37 & 1.50 & 1.12 & 1.13 & 1.40 & 1.55 & 1.13 & 1.15 & 1.42 & 1.58 \\
\hline ML3 & 1.04 & 1.06 & 1.31 & 1.46 & 1.07 & 1.10 & 1.34 & 1.51 & 1.10 & 1.16 & 1.38 & 1.59 \\
\hline ML4 & 1.16 & 1.26 & 1.45 & 1.74 & 1.20 & 1.32 & 1.50 & 1.82 & 1.23 & 1.36 & 1.53 & 1.87 \\
\hline ML5 & 1.16 & 1.27 & 1.45 & 1.75 & 1.21 & 1.33 & 1.51 & 1.83 & 1.23 & 1.37 & 1.54 & 1.88 \\
\hline ML6 & 1.07 & 1.04 & 1.34 & 1.42 & 1.09 & 1.09 & 1.37 & 1.50 & 1.10 & 1.13 & 1.38 & 1.55 \\
\hline MH1 & 1.07 & 1.13 & 1.33 & 1.55 & 1.10 & 1.17 & 1.38 & 1.60 & 1.13 & 1.21 & 1.41 & 1.67 \\
\hline MH2 & 1.17 & 1.25 & 1.46 & 1.72 & 1.18 & 1.29 & 1.47 & 1.78 & 1.19 & 1.34 & 1.48 & 1.84 \\
\hline MH3 & 1.49 & 1.73 & 1.87 & 2.38 & 1.62 & 1.91 & 2.03 & 2.63 & 1.69 & 1.97 & 2.11 & 2.71 \\
\hline MH4 & 1.18 & 1.27 & 1.48 & 1.75 & 1.20 & 1.31 & 1.50 & 1.80 & 1.20 & 1.33 & 1.50 & 1.82 \\
\hline MH5 & 1.17 & 1.25 & 1.47 & 1.72 & 1.20 & 1.32 & 1.50 & 1.81 & 1.22 & 1.38 & 1.52 & 1.90 \\
\hline MH6 & 1.15 & 1.27 & 1.44 & 1.75 & 1.22 & 1.41 & 1.52 & 1.93 & 1.38 & 1.60 & 1.72 & 2.20 \\
\hline LL1 & 1.01 & 1.04 & 1.26 & 1.43 & 1.02 & 1.08 & 1.28 & 1.48 & 1.38 & 1.49 & 1.73 & 2.05 \\
\hline LL2 & 1.02 & 1.05 & 1.28 & 1.45 & 1.04 & 1.10 & 1.30 & 1.51 & 1.27 & 1.34 & 1.59 & 1.84 \\
\hline LL3 & 1.18 & 1.27 & 1.47 & 1.75 & 1.28 & 1.43 & 1.60 & 1.96 & 1.36 & 1.53 & 1.70 & 2.10 \\
\hline LL4 & 0.95 & 0.97 & 1.19 & 1.34 & 1.01 & 1.03 & 1.27 & 1.41 & 1.09 & 1.11 & 1.36 & 1.53 \\
\hline LL5 & 0.94 & 0.96 & 1.18 & 1.32 & 1.01 & 1.01 & 1.26 & 1.39 & 1.09 & 1.10 & 1.36 & 1.52 \\
\hline LL6 & 1.17 & 1.40 & 1.47 & 1.93 & 1.23 & 1.53 & 1.54 & 2.10 & 1.26 & 1.64 & 1.58 & 2.26 \\
\hline LH1 & 1.18 & 1.26 & 1.47 & 1.73 & 1.37 & 1.53 & 1.71 & 2.10 & 1.46 & 1.64 & 1.82 & 2.25 \\
\hline LH2 & 1.26 & 1.40 & 1.57 & 1.92 & 1.41 & 1.59 & 1.76 & 2.19 & 1.49 & 1.67 & 1.86 & 2.30 \\
\hline LH3 & 1.33 & 1.70 & 1.67 & 2.34 & 1.54 & 1.94 & 1.92 & 2.66 & 1.63 & 2.02 & 2.04 & 2.78 \\
\hline LH4 & 1.27 & 1.39 & 1.59 & 1.91 & 1.45 & 1.62 & 1.81 & 2.23 & 1.53 & 1.75 & 1.92 & 2.41 \\
\hline LH5 & 1.31 & 1.43 & 1.64 & 1.97 & 1.48 & 1.68 & 1.86 & 2.31 & 1.58 & 1.81 & 1.98 & 2.49 \\
\hline LH6 & 1.27 & 1.62 & 1.59 & 2.23 & 1.37 & 1.81 & 1.72 & 2.49 & 1.53 & 1.97 & 1.91 & 2.71 \\
\hline
\end{tabular}


Table C.22 - The ratio of actual mid-span bending moments and quarter-span shear forces for buildings to the corresponding values obtained from FEMA parabolic distribution and a uniform distribution of seismic load when subjected to M6C2 ground motion and the Load is acting parallel to the short side, $\alpha=0.02$

\begin{tabular}{|c|c|c|c|c|c|c|c|c|c|c|c|c|}
\hline \multirow{2}{*}{ Building } & \multicolumn{4}{|c|}{$\mu=2$} & \multicolumn{4}{|c|}{$\mu=3$} & \multicolumn{4}{|c|}{$\mu=4$} \\
\hline & $M . F$ & V.F & $M . U$ & $V . U$ & $M . F$ & $V . F$ & $M . U$ & $V . U$ & M.F & V.F & $M . U$ & $V . U$ \\
\hline SL1 & 1.06 & 1.12 & 1.33 & 1.53 & 1.08 & 1.15 & 1.35 & 1.58 & 1.09 & 1.17 & 1.36 & 1.61 \\
\hline SL3 & .07 & 1.14 & 1.34 & 1.56 & 1.09 & 1.18 & 1.37 & 1.62 & 1.09 & 1.20 & 1.37 & 1.65 \\
\hline SL4 & 1.09 & 1.15 & 1.36 & 1.58 & 1.10 & 1.19 & 1.37 & 1.63 & 1.10 & 1.20 & 1.37 & 1.65 \\
\hline SH1 & 1.12 & 1.20 & 1.40 & 1.65 & 1.26 & 1.44 & 1.57 & 1.98 & 1.26 & 1.45 & 1.57 & 2.00 \\
\hline $\mathrm{SH} 2$ & 1.08 & 1.08 & 1.35 & 1.48 & 1.14 & 1.17 & 1.43 & 1.61 & 1.24 & 1.37 & 1.55 & 1.88 \\
\hline SH3 & 1.06 & 1.08 & 1.33 & 1.48 & 1.13 & 1.19 & 1.41 & 1.63 & 1.24 & 1.34 & 1.55 & 1.85 \\
\hline SH4 & 1.13 & 1.21 & 1.41 & 1.66 & 1.14 & 1.23 & 1.42 & 1.70 & 1.31 & 1.40 & 1.64 & 1.93 \\
\hline SH5 & 1.14 & 1.23 & 1.43 & 1.69 & 1.16 & 1.26 & 1.45 & 1.73 & 1.32 & 1.44 & 1.65 & 1.98 \\
\hline SH6 & 1.06 & 1.07 & 1.32 & 1.48 & 1.12 & 1.17 & 1.40 & 1.61 & 1.25 & 1.35 & 1.56 & 1.85 \\
\hline ML1 & 1.45 & 1.63 & 1.81 & 2.24 & 1.46 & 1.66 & 1.83 & 2.28 & 1.46 & 1.66 & 1.83 & 2.29 \\
\hline ML2 & 1.32 & 1.45 & 1.65 & 2.00 & 1.38 & 1.55 & 1.73 & 2.14 & 1.42 & 1.63 & 1.78 & 2.25 \\
\hline ML3 & 15 & 1.19 & 1.43 & 1.63 & 1.30 & 1.45 & 1.62 & 1.99 & 1.30 & 1.49 & 1.63 & 2.05 \\
\hline ML4 & 1.43 & 1.62 & 1.78 & 2.22 & 1.46 & 1.66 & 1.83 & 2.29 & 1.49 & 1.70 & 1.86 & 2.34 \\
\hline ML5 & 1.43 & 1.62 & 1.79 & 2.23 & 1.46 & 1.66 & 1.83 & 2.29 & 1.48 & 1.69 & 1.85 & 2.33 \\
\hline ML6 & 1.24 & 1.34 & 1.55 & 1.84 & 1.35 & 1.53 & 1.69 & 2.11 & 1.37 & 1.57 & 1.71 & 2.16 \\
\hline MH1 & 1.07 & 1.11 & 1.34 & 1.52 & 1.22 & 1.38 & 1.52 & 1.90 & 1.22 & 1.40 & 1.52 & 1.92 \\
\hline MH2 & 1.01 & 1.08 & 1.26 & 1.48 & 1.04 & 1.12 & 1.30 & 1.54 & 1.03 & 1.13 & 1.29 & 1.56 \\
\hline MH3 & 1.34 & 1.51 & 1.68 & 2.08 & 1.40 & 1.57 & 1.75 & 2.15 & 1.39 & 1.55 & 1.74 & 2.13 \\
\hline MH4 & 0.90 & 0.94 & 1.13 & 1.29 & 0.97 & 1.02 & 1.22 & 1.41 & 0.98 & 1.08 & 1.23 & 1.48 \\
\hline MH5 & 0.92 & 0.96 & 1.15 & 1.32 & 0.93 & 1.00 & 1.16 & 1.38 & 1.01 & 1.13 & 1.26 & 1.56 \\
\hline MH6 & 1.52 & 1.75 & 1.90 & 2.40 & 1.53 & 1.77 & 1.92 & 2.44 & 1.58 & 1.88 & 1.97 & 2.58 \\
\hline LL1 & 1.25 & 1.45 & 1.57 & 2.00 & 1.23 & 1.46 & 1.54 & 2.01 & 1.20 & 1.43 & 1.49 & 1.96 \\
\hline LL2 & 1.23 & 1.42 & 1.53 & 1.95 & 1.21 & 1.43 & 1.51 & 1.97 & 1.18 & 1.40 & 1.48 & 1.93 \\
\hline LL3 & 1.08 & 1.14 & 1.35 & 1.57 & 1.14 & 1.23 & 1.43 & 1.69 & 1.16 & 1.27 & 1.45 & 1.75 \\
\hline LL4 & 1.30 & 1.46 & 1.63 & 2.01 & 1.36 & 1.60 & 1.70 & 2.20 & 1.33 & 1.58 & 1.67 & 2.17 \\
\hline LL5 & 1.33 & 1.50 & 1.66 & 2.06 & 1.37 & 1.60 & 1.71 & 2.21 & 1.35 & 1.60 & 1.69 & 2.20 \\
\hline LL6 & 1.33 & 1.68 & 1.67 & 2.31 & 1.39 & 1.71 & 1.74 & 2.36 & 1.43 & 1.74 & 1.79 & 2.39 \\
\hline LH1 & 1.17 & 1.27 & 1.46 & 1.75 & 1.21 & 1.34 & 1.51 & 1.84 & 1.27 & 1.42 & 1.59 & 1.95 \\
\hline $\mathrm{LH} 2$ & 1.31 & 1.45 & 1.64 & 1.99 & 1.33 & 1.48 & 1.66 & 2.03 & 1.33 & 1.48 & 1.67 & 2.04 \\
\hline LH3 & 1.24 & 1.49 & 1.55 & 2.05 & 1.31 & 1.55 & 1.64 & 2.13 & 1.34 & 1.57 & 1.68 & 2.16 \\
\hline LH4 & 1.27 & 1.40 & 1.58 & 1.92 & 1.33 & 1.48 & 1.66 & 2.04 & 1.34 & 1.50 & 1.68 & 2.06 \\
\hline LH5 & 1.26 & 1.40 & 1.58 & 1.93 & 1.33 & 1.49 & 1.67 & 2.05 & 1.35 & 1.50 & 1.69 & 2.06 \\
\hline LH6 & 1.20 & 1.46 & 1.50 & 2.01 & 1.31 & 1.58 & 1.64 & 2.17 & 1.37 & 1.63 & 1.72 & 2.24 \\
\hline
\end{tabular}


Table C.23 - The ratio of actual mid-span bending moments and quarter-span shear forces for buildings to the corresponding values obtained from FEMA parabolic distribution and a uniform distribution of seismic load when subjected to M6C26 ground motion and the Load is acting parallel to the short side, $\alpha=0.02$

\begin{tabular}{|c|c|c|c|c|c|c|c|c|c|c|c|c|}
\hline \multirow{2}{*}{ Building } & \multicolumn{4}{|c|}{$\mu=2$} & \multicolumn{4}{|c|}{$\mu=3$} & \multicolumn{4}{|c|}{$\mu=4$} \\
\hline & $M . F$ & $V . F$ & M.U & V.U & $M . F$ & $V . F$ & M.U & $V . U$ & $M . F$ & $V . F$ & $M . U$ & $V . U$ \\
\hline SL1 & 1.10 & 1.15 & 1.37 & 1.58 & 1.12 & 1.20 & 1.40 & 1.65 & 1.14 & 1.24 & 1.43 & 1.70 \\
\hline SL3 & 10 & 1.15 & 1.38 & 1.58 & 1.13 & 1.21 & 1.41 & 1.66 & 1.15 & 1.24 & 1.44 & 1.71 \\
\hline SL4 & .12 & 1.18 & 1.40 & 1.62 & 1.14 & 1.22 & 1.42 & 1.67 & 1.15 & 1.24 & 1.44 & 1.71 \\
\hline SH1 & .13 & 1.19 & 1.41 & 1.64 & 1.15 & 1.24 & 1.43 & 1.71 & 1.15 & 1.27 & 1.44 & 1.74 \\
\hline $\mathrm{SH} 2$ & 1.15 & 1.23 & 1.44 & 1.69 & 1.16 & 1.27 & 1.46 & 1.75 & 1.17 & 1.29 & 1.46 & 1.78 \\
\hline $\mathrm{SH} 3$ & 1.15 & 1.24 & 1.44 & 1.70 & 1.16 & 1.28 & 1.45 & 1.76 & 1.16 & 1.30 & 1.46 & 1.79 \\
\hline SH4 & 1.14 & 1.23 & 1.43 & 1.69 & 1.16 & 1.28 & 1.45 & 1.75 & 1.16 & 1.30 & 1.45 & 1.78 \\
\hline SH5 & 1.14 & 1.23 & 1.42 & 1.69 & 1.16 & 1.28 & 1.45 & 1.76 & 1.16 & 1.30 & 1.45 & 1.79 \\
\hline SH6 & 1.15 & 1.24 & 1.44 & 1.71 & 1.17 & 1.28 & 1.46 & 1.76 & 1.17 & 1.30 & 1.46 & 1.79 \\
\hline ML1 & 1.13 & 1.21 & 1.42 & 1.66 & 1.17 & 1.26 & 1.46 & 1.73 & 1.19 & 1.29 & 1.49 & 1.78 \\
\hline ML2 & 0.93 & 1.00 & 1.16 & 1.38 & 1.11 & 1.23 & 1.39 & 1.69 & 1.13 & 1.30 & 1.41 & 1.78 \\
\hline L3 & 0.90 & 0.96 & 1.12 & 1.32 & 1.05 & 1.19 & 1.31 & 1.64 & 1.10 & 1.28 & 1.38 & 1.76 \\
\hline ML4 & 1.01 & 1.07 & 1.27 & 1.47 & 1.16 & 1.26 & 1.45 & 1.73 & 1.18 & 1.29 & 1.48 & 1.77 \\
\hline ML5 & 1.02 & 1.07 & 1.28 & 1.48 & 1.16 & 1.26 & 1.45 & 1.73 & 1.18 & 1.29 & 1.48 & 1.77 \\
\hline ML6 & 0.92 & 1.00 & 1.15 & 1.37 & 1.08 & 1.23 & 1.35 & 1.69 & 1.11 & 1.32 & 1.39 & 1.81 \\
\hline MH1 & 0.93 & 1.02 & 1.17 & 1.40 & 1.13 & 1.23 & 1.42 & 1.69 & 1.18 & 1.30 & 1.47 & 1.78 \\
\hline $\mathrm{MH} 2$ & 1.01 & 1.07 & 1.26 & 1.47 & 1.07 & 1.19 & 1.33 & 1.64 & 1.11 & 1.29 & 1.39 & 1.78 \\
\hline MH3 & 1.11 & 1.41 & 1.39 & 1.94 & 1.40 & 1.64 & 1.75 & 2.25 & 1.46 & 1.67 & 1.82 & 2.30 \\
\hline MH4 & 1.00 & 1.04 & 1.25 & 1.43 & 1.02 & 1.15 & 1.28 & 1.58 & 1.04 & 1.24 & 1.30 & 1.70 \\
\hline MH5 & 1.00 & 1.07 & 1.25 & 1.47 & 1.03 & 1.20 & 1.29 & 1.65 & 1.07 & 1.31 & 1.34 & 1.80 \\
\hline MH6 & 1.16 & 1.25 & 1.45 & 1.72 & 1.20 & 1.31 & 1.50 & 1.81 & 1.29 & 1.44 & 1.61 & 1.98 \\
\hline LL1 & 1.15 & 1.22 & 1.44 & 1.68 & 1.22 & 1.33 & 1.53 & 1.83 & 1.37 & 1.52 & 1.71 & 2.08 \\
\hline LL2 & 1.17 & 1.24 & 1.46 & 1.70 & 1.34 & 1.45 & 1.67 & 2.00 & 1.35 & 1.54 & 1.69 & 2.11 \\
\hline LL3 & 1.09 & 1.20 & 1.37 & 1.65 & 1.12 & 1.34 & 1.40 & 1.84 & 1.20 & 1.46 & 1.50 & 2.01 \\
\hline LL4 & 1.09 & 1.18 & 1.36 & 1.63 & 1.28 & 1.42 & 1.60 & 1.95 & 1.30 & 1.51 & 1.62 & 2.07 \\
\hline LL5 & 1.09 & 1.18 & 1.36 & 1.62 & 1.29 & 1.41 & 1.61 & 1.94 & 1.31 & 1.51 & 1.63 & 2.07 \\
\hline LL6 & 1.53 & 1.84 & 1.91 & 2.52 & 1.65 & 1.98 & 2.06 & 2.72 & 1.71 & 2.04 & 2.13 & 2.80 \\
\hline LH1 & 1.10 & 1.21 & 1.38 & 1.66 & 1.22 & 1.47 & 1.52 & 2.03 & 1.38 & 1.60 & 1.72 & 2.21 \\
\hline LH2 & 1.20 & 1.46 & 1.50 & 2.01 & 1.42 & 1.68 & 1.78 & 2.31 & 1.67 & 1.90 & 2.09 & 2.61 \\
\hline LH3 & 1.37 & 1.71 & 1.71 & 2.36 & 1.80 & 2.15 & 2.25 & 2.95 & 1.93 & 2.23 & 2.41 & 3.07 \\
\hline LH4 & 1.12 & 1.35 & 1.40 & 1.86 & 1.41 & 1.64 & 1.76 & 2.26 & 1.67 & 1.88 & 2.09 & 2.58 \\
\hline LH5 & 1.14 & 1.38 & 1.43 & 1.89 & 1.44 & 1.68 & 1.81 & 2.30 & 1.68 & 1.88 & 2.10 & 2.59 \\
\hline LH6 & 1.67 & 2.08 & 2.09 & 2.85 & 1.80 & 2.18 & 2.26 & 2.99 & 1.84 & 2.18 & 2.30 & 2.99 \\
\hline
\end{tabular}


Table C.24 - The ratio of actual mid-span bending moments and quarter-span shear forces for buildings to the corresponding values obtained from FEMA parabolic distribution and a uniform distribution of seismic load when subjected to M6C31 ground motion and the Load is acting parallel to the short side, $\alpha=0.02$

\begin{tabular}{|c|c|c|c|c|c|c|c|c|c|c|c|c|}
\hline \multirow{2}{*}{ Building } & \multicolumn{4}{|c|}{$\mu=2$} & \multicolumn{4}{|c|}{$\mu=3$} & \multicolumn{4}{|c|}{$\mu=4$} \\
\hline & $M . F$ & V.F & $M . U$ & $V . U$ & $M . F$ & $V . F$ & $M . U$ & $V . U$ & M.F & V.F & $M . U$ & $V . U$ \\
\hline SL1 & 1.11 & 1.18 & 1.39 & 1.63 & 1.14 & 1.22 & 1.42 & 1.68 & 1.16 & 1.26 & 1.45 & 1.74 \\
\hline SL3 & 13 & 1.20 & 1.42 & 1.66 & 1.16 & 1.25 & 1.45 & 1.72 & 1.18 & 1.29 & 1.48 & 1.77 \\
\hline SL4 & 15 & 1.21 & 1.43 & 1.66 & 1.18 & 1.28 & 1.48 & 1.75 & 1.21 & 1.32 & 1.51 & 1.81 \\
\hline SH1 & 1.12 & 1.17 & 1.40 & 1.60 & 1.21 & 1.25 & 1.51 & 1.72 & 1.28 & 1.35 & 1.60 & 1.85 \\
\hline $\mathrm{SH} 2$ & 1.18 & 1.21 & 1.47 & 1.67 & 1.24 & 1.30 & 1.55 & 1.79 & 1.29 & 1.37 & 1.61 & 1.89 \\
\hline SH3 & 1.20 & 1.28 & 1.51 & 1.76 & 1.26 & 1.37 & 1.58 & 1.88 & 1.31 & 1.43 & 1.63 & 1.97 \\
\hline SH4 & 1.22 & 1.34 & 1.53 & 1.84 & 1.26 & 1.40 & 1.58 & 1.93 & 1.30 & 1.45 & 1.62 & 2.00 \\
\hline SH5 & 1.22 & 1.33 & 1.52 & 1.83 & 1.27 & 1.41 & 1.59 & 1.94 & 1.30 & 1.46 & 1.63 & 2.01 \\
\hline SH6 & 1.20 & 1.28 & 1.50 & 1.76 & 1.26 & 1.36 & 1.58 & 1.88 & 1.31 & 1.43 & 1.63 & 1.97 \\
\hline ML1 & 1.26 & 1.42 & 1.58 & 1.96 & 1.30 & 1.49 & 1.63 & 2.05 & 1.32 & 1.52 & 1.65 & 2.08 \\
\hline ML2 & 22 & 1.31 & 1.53 & 1.81 & 1.24 & 1.39 & 1.55 & 1.91 & 1.24 & 1.46 & 1.55 & 2.01 \\
\hline ML3 & 13 & 1.23 & 1.41 & 1.69 & 1.15 & 1.31 & 1.44 & 1.81 & 1.16 & 1.39 & 1.45 & 1.91 \\
\hline ML4 & 1.22 & 1.36 & 1.52 & 1.88 & 1.23 & 1.40 & 1.54 & 1.92 & 1.26 & 1.45 & 1.57 & 1.99 \\
\hline ML5 & 1.21 & 1.36 & 1.52 & 1.87 & 1.24 & 1.41 & 1.54 & 1.94 & 1.26 & 1.45 & 1.58 & 2.00 \\
\hline ML6 & 1.19 & 1.29 & 1.48 & 1.77 & 1.20 & 1.38 & 1.50 & 1.89 & 1.21 & 1.44 & 1.51 & 1.98 \\
\hline MH1 & 1.11 & 1.16 & 1.39 & 1.59 & 1.14 & 1.25 & 1.43 & 1.72 & 1.16 & 1.32 & 1.45 & 1.82 \\
\hline MH2 & 1.15 & 1.20 & 1.44 & 1.65 & 1.18 & 1.26 & 1.47 & 1.73 & 1.19 & 1.30 & 1.49 & 1.79 \\
\hline MH3 & 1.21 & 1.42 & 1.52 & 1.95 & 1.26 & 1.45 & 1.58 & 2.00 & 1.28 & 1.48 & 1.60 & 2.04 \\
\hline MH4 & 1.18 & 1.25 & 1.48 & 1.72 & 1.19 & 1.29 & 1.49 & 1.77 & 1.20 & 1.32 & 1.50 & 1.81 \\
\hline MH5 & 1.18 & 1.26 & 1.48 & 1.73 & 1.20 & 1.32 & 1.50 & 1.81 & 1.20 & 1.35 & 1.50 & 1.85 \\
\hline MH6 & 1.28 & 1.43 & 1.60 & 1.97 & 1.45 & 1.63 & 1.81 & 2.24 & 1.57 & 1.79 & 1.96 & 2.46 \\
\hline LL1 & 1.19 & 1.24 & 1.48 & 1.71 & 1.32 & 1.41 & 1.64 & 1.94 & 1.39 & 1.55 & 1.74 & 2.13 \\
\hline LL2 & 1.21 & 1.28 & 1.52 & 1.76 & 1.33 & 1.45 & 1.66 & 1.99 & 1.39 & 1.55 & 1.73 & 2.13 \\
\hline LL3 & 1.04 & 1.23 & 1.30 & 1.70 & 1.07 & 1.33 & 1.34 & 1.83 & 1.14 & 1.42 & 1.42 & 1.96 \\
\hline LL4 & 1.30 & 1.39 & 1.62 & 1.91 & 1.37 & 1.49 & 1.71 & 2.04 & 1.37 & 1.50 & 1.72 & 2.06 \\
\hline LL5 & 1.29 & 1.37 & 1.61 & 1.88 & 1.38 & 1.50 & 1.72 & 2.06 & 1.39 & 1.51 & 1.74 & 2.08 \\
\hline LL6 & 1.00 & 1.37 & 1.25 & 1.89 & 1.02 & 1.43 & 1.28 & 1.97 & 1.03 & 1.50 & 1.29 & 2.06 \\
\hline LH1 & 1.12 & 1.35 & 1.40 & 1.85 & 1.17 & 1.47 & 1.46 & 2.03 & 1.23 & 1.57 & 1.54 & 2.16 \\
\hline LH2 & 1.19 & 1.44 & 1.49 & 1.98 & 1.22 & 1.54 & 1.52 & 2.12 & 1.23 & 1.62 & 1.54 & 2.22 \\
\hline LH3 & 1.12 & 1.39 & 1.40 & 1.91 & 1.24 & 1.51 & 1.54 & 2.08 & 1.33 & 1.62 & 1.66 & 2.22 \\
\hline LH4 & 1.17 & 1.36 & 1.46 & 1.87 & 1.20 & 1.49 & 1.50 & 2.06 & 1.21 & 1.57 & 1.51 & 2.15 \\
\hline LH5 & 1.17 & 1.34 & 1.46 & 1.85 & 1.20 & 1.47 & 1.50 & 2.03 & 1.21 & 1.55 & 1.51 & 2.13 \\
\hline LH6 & 1.06 & 1.46 & 1.33 & 2.00 & 1.14 & 1.55 & 1.43 & 2.13 & 1.21 & 1.54 & 1.52 & 2.11 \\
\hline
\end{tabular}


Table C.25 - The ratio of actual mid-span bending moments and quarter-span shear forces for buildings to the corresponding values obtained from FEMA parabolic distribution and a uniform distribution of seismic load when subjected to M6C 38 ground motion and the Load is acting parallel to the short side, $\alpha=0.02$

\begin{tabular}{|c|c|c|c|c|c|c|c|c|c|c|c|c|}
\hline \multirow{2}{*}{ Building } & \multicolumn{4}{|c|}{$\mu=2$} & \multicolumn{4}{|c|}{$\mu=3$} & \multicolumn{4}{|c|}{$\mu=4$} \\
\hline & $M . F$ & $V . F$ & M.U & V.U & $M . F$ & $V . F$ & $M . U$ & $V . U$ & $M . F$ & V.F & $M . U$ & $V . U$ \\
\hline SL1 & 1.19 & 1.30 & 1.49 & 1.78 & 1.21 & 1.33 & 1.52 & 1.83 & 1.23 & 1.35 & 1.54 & 1.85 \\
\hline SL3 & 20 & 1.30 & 1.49 & 1.79 & 1.22 & 1.33 & 1.52 & 1.83 & 1.23 & 1.35 & 1.54 & 1.86 \\
\hline SL4 & .20 & 1.30 & 1.50 & 1.79 & 1.22 & 1.34 & 1.53 & 1.84 & 1.24 & 1.36 & 1.55 & 1.88 \\
\hline SH1 & .16 & 1.20 & 1.45 & 1.65 & 1.24 & 1.30 & 1.54 & 1.79 & 1.28 & 1.36 & 1.60 & 1.87 \\
\hline $\mathrm{SH} 2$ & 1.18 & 1.20 & 1.47 & 1.65 & 1.25 & 1.29 & 1.56 & 1.78 & 1.30 & 1.36 & 1.62 & 1.88 \\
\hline SH3 & 1.19 & 1.21 & 1.49 & 1.66 & 1.26 & 1.30 & 1.58 & 1.79 & 1.31 & 1.37 & 1.64 & 1.89 \\
\hline SH4 & 1.19 & 1.20 & 1.49 & 1.65 & 1.26 & 1.29 & 1.58 & 1.77 & 1.31 & 1.35 & 1.64 & 1.86 \\
\hline SH5 & 1.19 & 1.20 & 1.49 & 1.66 & 1.27 & 1.30 & 1.58 & 1.79 & 1.32 & 1.36 & 1.65 & 1.88 \\
\hline SH6 & 1.19 & 1.21 & 1.49 & 1.66 & 1.26 & 1.31 & 1.58 & 1.80 & 1.31 & 1.38 & 1.64 & 1.89 \\
\hline ML1 & 1.16 & 1.15 & 1.45 & 1.58 & 1.23 & 1.24 & 1.53 & 1.70 & 1.28 & 1.31 & 1.60 & 1.80 \\
\hline ML2 & 1.16 & 1.21 & 1.45 & 1.66 & 1.24 & 1.27 & 1.55 & 1.74 & 1.31 & 1.34 & 1.64 & 1.84 \\
\hline L3 & 1.14 & 1.19 & 1.43 & 1.64 & 1.25 & 1.30 & 1.56 & 1.79 & 1.32 & 1.39 & 1.65 & 1.91 \\
\hline ML4 & 1.10 & 1.07 & 1.37 & 1.48 & 1.22 & 1.21 & 1.53 & 1.67 & 1.32 & 1.33 & 1.65 & 1.83 \\
\hline ML5 & 1.10 & 1.08 & 1.38 & 1.48 & 1.21 & 1.20 & 1.51 & 1.64 & 1.32 & 1.33 & 1.65 & 1.82 \\
\hline ML6 & 1.16 & 1.19 & 1.45 & 1.64 & 1.25 & 1.29 & 1.57 & 1.78 & 1.33 & 1.37 & 1.66 & 1.89 \\
\hline MH1 & 1.01 & 1.07 & 1.27 & 1.47 & 1.21 & 1.27 & 1.51 & 1.75 & 1.29 & 1.35 & 1.61 & 1.86 \\
\hline MH2 & 0.91 & 0.97 & 1.13 & 1.34 & 1.19 & 1.29 & 1.48 & 1.78 & 1.29 & 1.40 & 1.61 & 1.93 \\
\hline MH3 & 0.95 & 0.92 & 1.18 & 1.26 & 1.05 & 1.03 & 1.31 & 1.41 & 1.21 & 1.34 & 1.51 & 1.84 \\
\hline MH4 & 0.88 & 0.95 & 1.10 & 1.30 & 1.00 & 1.07 & 1.25 & 1.47 & 1.20 & 1.33 & 1.50 & 1.82 \\
\hline MH5 & 0.88 & 0.95 & 1.10 & 1.31 & 1.15 & 1.28 & 1.44 & 1.76 & 1.24 & 1.38 & 1.56 & 1.90 \\
\hline MH6 & 1.14 & 1.15 & 1.42 & 1.58 & 1.27 & 1.32 & 1.59 & 1.82 & 1.38 & 1.48 & 1.73 & 2.03 \\
\hline LL1 & 1.01 & 1.03 & 1.26 & 1.41 & 1.12 & 1.15 & 1.40 & 1.58 & 1.18 & 1.24 & 1.48 & 1.71 \\
\hline & 0.99 & 0.99 & 1.24 & 1.37 & 1.11 & 1.14 & 1.39 & 1.57 & 1.18 & 1.23 & 1.47 & 1.69 \\
\hline LL3 & 0.89 & 0.86 & 1.11 & 1.18 & 1.00 & 1.13 & 1.25 & 1.55 & 1.09 & 1.23 & 1.37 & 1.69 \\
\hline LL4 & 1.09 & 1.11 & 1.36 & 1.52 & 1.18 & 1.21 & 1.48 & 1.66 & 1.26 & 1.30 & 1.57 & 1.79 \\
\hline LL5 & 1.10 & 1.13 & 1.37 & 1.56 & 1.19 & 1.21 & 1.48 & 1.67 & 1.26 & 1.30 & 1.57 & 1.79 \\
\hline LL6 & 1.08 & 1.17 & 1.35 & 1.62 & 1.24 & 1.36 & 1.54 & 1.87 & 1.39 & 1.63 & 1.73 & 2.25 \\
\hline LH1 & 0.95 & 0.93 & 1.18 & 1.28 & 0.98 & 1.12 & 1.23 & 1.54 & 1.07 & 1.22 & 1.34 & 1.68 \\
\hline LH2 & 1.01 & 1.00 & 1.26 & 1.37 & 0.99 & 1.10 & 1.24 & 1.51 & 1.05 & 1.22 & 1.31 & 1.67 \\
\hline LH3 & 1.10 & 1.19 & 1.37 & 1.63 & 1.15 & 1.26 & 1.43 & 1.73 & 1.24 & 1.39 & 1.55 & 1.91 \\
\hline LH4 & 0.98 & 0.96 & 1.23 & 1.32 & 0.99 & 1.13 & 1.23 & 1.55 & 1.06 & 1.24 & 1.33 & 1.71 \\
\hline LH5 & 0.98 & 0.95 & 1.23 & 1.31 & 0.99 & 1.14 & 1.24 & 1.57 & 1.07 & 1.26 & 1.33 & 1.73 \\
\hline LH6 & 1.06 & 1.14 & 1.33 & 1.56 & 1.17 & 1.31 & 1.47 & 1.80 & 1.32 & 1.52 & 1.64 & 2.09 \\
\hline
\end{tabular}


Table C.26 - The ratio of actual mid-span bending moments and quarter-span shear forces for buildings to the corresponding values obtained from FEMA parabolic distribution and a uniform distribution of seismic load when subjected to $\mathrm{M} 6 \mathrm{C} 1$ ground motion and the Load is acting parallel to the long side, $\alpha=0.02$

\begin{tabular}{|c|c|c|c|c|c|c|c|c|c|c|c|c|}
\hline \multirow{2}{*}{ Building } & \multicolumn{4}{|c|}{$\mu=2$} & \multicolumn{4}{|c|}{$\mu=3$} & \multicolumn{4}{|c|}{$\mu=4$} \\
\hline & $M . F$ & $V . F$ & $M . U$ & V.U & M.F & $V . F$ & $M . U$ & $V . U$ & M.F & V.F & M.U & $V . U$ \\
\hline SL1 & 1.02 & 1.04 & 1.28 & 1.43 & 1.05 & 1.09 & 1.32 & 1.50 & 1.08 & 1.14 & 1.35 & 1.56 \\
\hline SL3 & 03 & 1.04 & 1.28 & 1.44 & 1.06 & 1.10 & 1.32 & 1.51 & 1.07 & 1.13 & 1.34 & 1.55 \\
\hline & 02 & 1.03 & 1.28 & 1.42 & 1.03 & 1.06 & 1.29 & 1.46 & 1.04 & 1.10 & 1.30 & 1.51 \\
\hline SH1 & 15 & 1.22 & 1.44 & 1.68 & 1.26 & 1.41 & 1.58 & 1.94 & 1.24 & 1.38 & 1.55 & 1.90 \\
\hline & 1.12 & 1.16 & 1.40 & 1.60 & 1.19 & 1.31 & 1.49 & 1.81 & 1.21 & 1.34 & 1.51 & 1.84 \\
\hline $\mathrm{SH} 3$ & 1.09 & 1.14 & 1.36 & 1.57 & 1.12 & 1.21 & 1.40 & 1.66 & 1.17 & 1.29 & 1.47 & 1.77 \\
\hline SH4 & 1.09 & 1.14 & 1.37 & 1.57 & 1.15 & 1.23 & 1.43 & 1.69 & 1.18 & 1.30 & 1.48 & 1.79 \\
\hline SH5 & 1.08 & 1.11 & 1.36 & 1.53 & 1.12 & 1.18 & 1.40 & 1.63 & 1.18 & 1.29 & 1.47 & 1.77 \\
\hline IL1 & 1.12 & 1.20 & 1.40 & 1.65 & 1.26 & 1.41 & 1.58 & 1.94 & 1.28 & 1.43 & 1.60 & 1.97 \\
\hline ML2 & 1.14 & 1.24 & 1.43 & 1.70 & 1.28 & 1.42 & 1.59 & 1.95 & 1.29 & 1.45 & 1.61 & 1.99 \\
\hline ML3 & 1.08 & 1.16 & 1.36 & 1.59 & 1.23 & 1.37 & 1.54 & 1.88 & 1.28 & 1.43 & 1.60 & 1.97 \\
\hline ML4 & 1.15 & 1.24 & 1.43 & 1.71 & 1.27 & 1.42 & 1.59 & 1.95 & 1.29 & 1.45 & 1.61 & 1.99 \\
\hline ML5 & 1.15 & 1.24 & 1.44 & 1.71 & 1.26 & 1.40 & 1.58 & 1.93 & 1.28 & 1.44 & 1.60 & 1.98 \\
\hline ML6 & 1.11 & 1.20 & 1.39 & 1.65 & 1.25 & 1.38 & 1.56 & 1.90 & 1.29 & 1.44 & 1.62 & 1.98 \\
\hline & 07 & 1.13 & 1.34 & 1.56 & 1.30 & 1.45 & 1.62 & 1.99 & 1.33 & 1.49 & 1.66 & 2.05 \\
\hline & 20 & 1.31 & 1.50 & 1.80 & 1.28 & 1.45 & 1.60 & 1.99 & 1.29 & 1.47 & 1.61 & 2.02 \\
\hline & 1.29 & 1.41 & 1.61 & 1.93 & 1.46 & 1.67 & 1.82 & 2.30 & 1.48 & 1.71 & 1.84 & 2.36 \\
\hline MH4 & 1.07 & 1.14 & 1.34 & 1.56 & 1.31 & 1.48 & 1.64 & 2.03 & 1.34 & 1.52 & 1.68 & 2.09 \\
\hline MH5 & 1.20 & 1.29 & 1.50 & 1.78 & 1.30 & 1.46 & 1.62 & 2.00 & 1.32 & 1.51 & 1.65 & 2.08 \\
\hline MH6 & 1.16 & 1.23 & 1.44 & 1.69 & 1.25 & 1.38 & 1.57 & 1.89 & 1.25 & 1.41 & 1.56 & 1.94 \\
\hline LL1 & 1.22 & 1.34 & 1.52 & 1.84 & 1.27 & 1.39 & 1.58 & 1.91 & 1.25 & 1.37 & 1.57 & 1.89 \\
\hline LL2 & 1.17 & 1.27 & 1.47 & 1.75 & 1.29 & 1.42 & 1.61 & 1.95 & 1.25 & 1.39 & 1.56 & 1.91 \\
\hline LL3 & 1.36 & 1.49 & 1.69 & 2.05 & 1.39 & 1.56 & 1.74 & 2.14 & 1.43 & 1.62 & 1.79 & 2.23 \\
\hline LL4 & 1.22 & 1.34 & 1.53 & 1.84 & 1.26 & 1.38 & 1.58 & 1.90 & 1.25 & 1.37 & 1.57 & 1.89 \\
\hline LL5 & 1.21 & 1.31 & 1.51 & 1.81 & 1.28 & 1.47 & 1.60 & 2.02 & 1.30 & 1.47 & 1.62 & 2.02 \\
\hline LL6 & 1.22 & 1.36 & 1.52 & 1.86 & 1.42 & 1.67 & 1.77 & 2.30 & 1.44 & 1.71 & 1.80 & 2.35 \\
\hline LH1 & 1.09 & 1.14 & 1.36 & 1.57 & 1.24 & 1.35 & 1.55 & 1.86 & 1.22 & 1.35 & 1.53 & 1.85 \\
\hline LH2 & 1.24 & 1.35 & 1.54 & 1.85 & 1.26 & 1.39 & 1.58 & 1.91 & 1.26 & 1.39 & 1.57 & 1.91 \\
\hline LH3 & 1.12 & 1.23 & 1.40 & 1.68 & 1.37 & 1.62 & 1.71 & 2.22 & 1.41 & 1.70 & 1.77 & 2.33 \\
\hline LH4 & 1.21 & 1.29 & 1.51 & 1.77 & 1.21 & 1.29 & 1.52 & 1.78 & 1.19 & 1.27 & 1.49 & 1.74 \\
\hline LH5 & 1.30 & 1.42 & 1.63 & 1.95 & 1.36 & 1.50 & 1.70 & 2.06 & 1.38 & 1.55 & 1.73 & 2.13 \\
\hline LH6 & 1.05 & 1.09 & 1.31 & 1.51 & 1.41 & 1.60 & 1.76 & 2.20 & 1.49 & 1.74 & 1.87 & 2.39 \\
\hline
\end{tabular}


Table C.27 - The ratio of actual mid-span bending moments and quarter-span shear forces for buildings to the corresponding values obtained from FEMA parabolic distribution and a uniform distribution of seismic load when subjected to M6C2 ground motion and the Load is acting parallel to the long side, $\alpha=0.02$

\begin{tabular}{|c|c|c|c|c|c|c|c|c|c|c|c|c|}
\hline \multirow{2}{*}{ Building } & \multicolumn{4}{|c|}{$\mu=2$} & \multicolumn{4}{|c|}{$\mu=3$} & \multicolumn{4}{|c|}{$\mu=4$} \\
\hline & $M . F$ & $V . F$ & $M . U$ & V.U & M.F & $V . F$ & $M . U$ & $V . U$ & M.F & V.F & M.U & $V . U$ \\
\hline SL1 & 1.10 & 1.15 & 1.38 & 1.58 & 1.13 & 1.21 & 1.41 & 1.67 & 1.13 & 1.23 & 1.41 & 1.69 \\
\hline SL3 & 08 & 1.12 & 1.35 & 1.55 & 1.10 & 1.18 & 1.38 & 1.63 & 1.10 & 1.20 & 1.38 & 1.65 \\
\hline & 09 & 1.11 & 1.36 & 1.52 & 1.06 & 1.08 & 1.33 & 1.48 & 1.07 & 1.16 & 1.34 & 1.59 \\
\hline & 11 & 1.15 & 1.39 & 1.59 & 1.23 & 1.35 & 1.54 & 1.85 & 1.28 & 1.43 & 1.61 & 1.97 \\
\hline & 1.08 & 1.13 & 1.35 & 1.55 & 1.12 & 1.19 & 1.40 & 1.63 & 1.16 & 1.28 & 1.45 & 1.76 \\
\hline $\mathrm{SH} 3$ & 1.12 & 1.21 & 1.40 & 1.67 & 1.17 & 1.29 & 1.47 & 1.77 & 1.19 & 1.30 & 1.49 & 1.79 \\
\hline $\mathrm{SH} 4$ & 1.10 & 1.19 & 1.38 & 1.63 & 1.17 & 1.28 & 1.46 & 1.76 & 1.16 & 1.27 & 1.45 & 1.75 \\
\hline SH5 & 1.14 & 1.23 & 1.42 & 1.69 & 1.18 & 1.29 & 1.47 & 1.78 & 1.18 & 1.30 & 1.48 & 1.79 \\
\hline IL1 & 1.05 & 1.12 & 1.32 & 1.54 & 1.05 & 1.13 & 1.31 & 1.55 & 1.14 & 1.22 & 1.42 & 1.68 \\
\hline ML2 & 1.09 & 1.16 & 1.37 & 1.60 & 1.13 & 1.21 & 1.41 & 1.66 & 1.18 & 1.28 & 1.48 & 1.76 \\
\hline ML3 & 1.16 & 1.26 & 1.45 & 1.73 & 1.22 & 1.34 & 1.52 & 1.85 & 1.26 & 1.40 & 1.58 & 1.93 \\
\hline ML4 & 1.08 & 1.14 & 1.35 & 1.57 & 1.11 & 1.18 & 1.38 & 1.62 & 1.17 & 1.26 & 1.47 & 1.74 \\
\hline ML5 & 1.11 & 1.19 & 1.39 & 1.63 & 1.14 & 1.24 & 1.43 & 1.70 & 1.20 & 1.30 & 1.50 & 1.79 \\
\hline ML6 & 1.15 & 1.23 & 1.43 & 1.70 & 1.19 & 1.31 & 1.49 & 1.80 & 1.24 & 1.37 & 1.55 & 1.89 \\
\hline MH1 & 18 & 1.25 & 1.48 & 1.72 & 1.26 & 1.38 & 1.58 & 1.90 & 1.33 & 1.50 & 1.66 & 2.06 \\
\hline & 26 & 1.36 & 1.57 & 1.87 & 1.44 & 1.65 & 1.80 & 2.27 & 1.54 & 1.79 & 1.93 & 2.46 \\
\hline & 1.24 & 1.34 & 1.55 & 1.85 & 1.29 & 1.40 & 1.61 & 1.92 & 1.47 & 1.68 & 1.83 & 2.31 \\
\hline MH4 & 1.15 & 1.20 & 1.44 & 1.65 & 1.24 & 1.35 & 1.55 & 1.86 & 1.35 & 1.52 & 1.68 & 2.09 \\
\hline MH5 & 1.45 & 1.65 & 1.81 & 2.26 & 1.52 & 1.75 & 1.90 & 2.40 & 1.57 & 1.80 & 1.97 & 2.48 \\
\hline MH6 & 1.06 & 1.07 & 1.33 & 1.47 & 1.29 & 1.43 & 1.61 & 1.96 & 1.41 & 1.62 & 1.76 & 2.23 \\
\hline LL1 & 1.14 & 1.18 & 1.43 & 1.63 & 1.23 & 1.34 & 1.54 & 1.85 & 1.32 & 1.48 & 1.65 & 2.04 \\
\hline LL2 & 1.10 & 1.13 & 1.38 & 1.55 & 1.24 & 1.35 & 1.55 & 1.86 & 1.36 & 1.54 & 1.70 & 2.11 \\
\hline LL3 & 1.20 & 1.28 & 1.50 & 1.77 & 1.25 & 1.44 & 1.56 & 1.97 & 1.43 & 1.66 & 1.79 & 2.28 \\
\hline LL4 & 1.14 & 1.18 & 1.42 & 1.62 & 1.24 & 1.34 & 1.54 & 1.84 & 1.31 & 1.47 & 1.64 & 2.03 \\
\hline LL5 & 1.19 & 1.26 & 1.49 & 1.73 & 1.42 & 1.61 & 1.77 & 2.21 & 1.48 & 1.70 & 1.85 & 2.34 \\
\hline LL6 & 1.46 & 1.68 & 1.83 & 2.30 & 1.50 & 1.73 & 1.87 & 2.38 & 1.63 & 1.91 & 2.03 & 2.63 \\
\hline LH1 & 1.19 & 1.26 & 1.48 & 1.73 & 1.39 & 1.57 & 1.73 & 2.16 & 1.50 & 1.74 & 1.88 & 2.39 \\
\hline LH2 & 1.40 & 1.56 & 1.75 & 2.15 & 1.50 & 1.73 & 1.87 & 2.38 & 1.51 & 1.75 & 1.89 & 2.40 \\
\hline LH3 & 1.50 & 1.73 & 1.88 & 2.38 & 1.55 & 1.79 & 1.93 & 2.46 & 1.54 & 1.80 & 1.93 & 2.47 \\
\hline LH4 & 1.28 & 1.41 & 1.60 & 1.94 & 1.42 & 1.61 & 1.77 & 2.21 & 1.48 & 1.70 & 1.85 & 2.34 \\
\hline LH5 & 1.15 & 1.23 & 1.44 & 1.70 & 1.26 & 1.39 & 1.58 & 1.91 & 1.38 & 1.59 & 1.73 & 2.18 \\
\hline LH6 & 1.44 & 1.61 & 1.80 & 2.21 & 1.44 & 1.62 & 1.80 & 2.23 & 1.41 & 1.60 & 1.76 & 2.21 \\
\hline
\end{tabular}


Table C.28 - The ratio of actual mid-span bending moments and quarter-span shear forces for buildings to the corresponding values obtained from FEMA parabolic distribution and a uniform distribution of seismic load when subjected to M6C26 ground motion and the Load is acting parallel to the long side, $\alpha=0.02$

\begin{tabular}{|c|c|c|c|c|c|c|c|c|c|c|c|c|}
\hline \multirow{2}{*}{ Building } & \multicolumn{4}{|c|}{$\mu=2$} & \multicolumn{4}{|c|}{$\mu=3$} & \multicolumn{4}{|c|}{$\mu=4$} \\
\hline & $M . F$ & $V . F$ & $M . U$ & $V . U$ & $M . F$ & $V . F$ & $M . U$ & $V . U$ & $M . F$ & $V . F$ & $M . U$ & $V . U$ \\
\hline SL1 & 1.13 & 1.19 & 1.42 & 1.64 & 1.19 & 1.28 & 1.49 & 1.76 & 1.21 & 1.32 & 1.52 & 1.81 \\
\hline SL3 & 14 & 1.20 & 1.42 & 1.65 & 1.19 & 1.29 & 1.49 & 1.77 & 1.22 & 1.33 & 1.52 & 1.83 \\
\hline SL4 & 15 & 1.21 & 1.44 & 1.67 & 1.20 & 1.31 & 1.50 & 1.80 & 1.21 & 1.35 & 1.52 & 1.85 \\
\hline SH1 & 16 & 1.22 & 1.45 & 1.67 & 1.17 & 1.25 & 1.47 & 1.72 & 1.18 & 1.28 & 1.47 & 1.76 \\
\hline $\mathrm{SH} 2$ & 18 & 1.27 & 1.48 & 1.75 & 1.22 & 1.33 & 1.52 & 1.83 & 1.23 & 1.35 & 1.53 & 1.86 \\
\hline $\mathrm{SH} 3$ & 1.04 & 1.09 & 1.30 & 1.50 & 1.27 & 1.41 & 1.59 & 1.94 & 1.29 & 1.45 & 1.61 & 1.99 \\
\hline SH4 & 1.05 & 1.09 & 1.31 & 1.50 & 1.25 & 1.39 & 1.56 & 1.92 & 1.27 & 1.43 & 1.59 & 1.96 \\
\hline SH5 & 1.05 & 1.11 & 1.32 & 1.52 & 1.29 & 1.45 & 1.61 & 1.99 & 1.30 & 1.48 & 1.62 & 2.03 \\
\hline ML1 & 1.10 & 1.16 & 1.38 & 1.60 & 1.14 & 1.23 & 1.42 & 1.68 & 1.16 & 1.26 & 1.44 & 1.74 \\
\hline ML2 & 1.10 & 1.16 & 1.38 & 1.60 & 1.12 & 1.20 & 1.40 & 1.65 & 1.13 & 1.22 & 1.41 & 1.68 \\
\hline ML3 & 1.06 & 1.11 & 1.33 & 1.52 & 1.07 & 1.13 & 1.34 & 1.55 & 1.07 & 1.14 & 1.34 & 1.57 \\
\hline ML4 & 1.10 & 1.17 & 1.38 & 1.60 & 1.13 & 1.21 & 1.41 & 1.67 & 1.14 & 1.24 & 1.42 & 1.70 \\
\hline ML5 & 1.10 & 1.16 & 1.37 & 1.59 & 1.11 & 1.19 & 1.39 & 1.63 & 1.12 & 1.21 & 1.40 & 1.66 \\
\hline ML6 & 1.08 & 1.13 & 1.34 & 1.55 & 1.09 & 1.15 & 1.36 & 1.58 & 1.09 & 1.16 & 1.36 & 1.60 \\
\hline MH1 & 1.02 & 1.06 & 1.28 & 1.46 & 1.04 & 1.09 & 1.29 & 1.50 & 1.05 & 1.12 & 1.31 & 1.54 \\
\hline & 1.04 & 1.07 & 1.30 & 1.47 & 1.06 & 1.12 & 1.33 & 1.54 & 1.34 & 1.52 & 1.67 & 2.09 \\
\hline MH3 & 1.14 & 1.22 & 1.43 & 1.67 & 1.15 & 1.26 & 1.44 & 1.73 & 1.32 & 1.50 & 1.65 & 2.06 \\
\hline MH4 & 1.02 & 1.06 & 1.28 & 1.46 & 1.04 & 1.09 & 1.30 & 1.50 & 1.07 & 1.14 & 1.33 & 1.57 \\
\hline MH5 & 1.07 & 1.09 & 1.33 & 1.50 & 1.09 & 1.17 & 1.36 & 1.60 & 1.35 & 1.54 & 1.69 & 2.11 \\
\hline MH6 & 1.04 & 1.09 & 1.31 & 1.50 & 1.14 & 1.23 & 1.43 & 1.70 & 1.34 & 1.53 & 1.67 & 2.10 \\
\hline LL1 & 1.05 & 1.09 & 1.31 & 1.50 & 1.06 & 1.12 & 1.33 & 1.53 & 1.22 & 1.35 & 1.52 & 1.85 \\
\hline LL2 & 1.05 & 1.09 & 1.31 & 1.50 & 1.06 & 1.12 & 1.33 & 1.54 & 1.36 & 1.55 & 1.70 & 2.13 \\
\hline LL3 & 1.14 & 1.20 & 1.43 & 1.64 & 1.31 & 1.46 & 1.63 & 2.01 & 1.31 & 1.47 & 1.64 & 2.02 \\
\hline LL4 & 1.05 & 1.09 & 1.31 & 1.50 & 1.06 & 1.12 & 1.33 & 1.54 & 1.20 & 1.32 & 1.50 & 1.82 \\
\hline LL5 & 1.04 & 1.09 & 1.30 & 1.49 & 1.06 & 1.12 & 1.33 & 1.55 & 1.36 & 1.55 & 1.70 & 2.13 \\
\hline LL6 & 1.12 & 1.20 & 1.40 & 1.65 & 1.23 & 1.34 & 1.53 & 1.84 & 1.58 & 1.87 & 1.98 & 2.57 \\
\hline LH1 & 1.09 & 1.16 & 1.37 & 1.59 & 1.34 & 1.52 & 1.68 & 2.09 & 1.35 & 1.54 & 1.68 & 2.12 \\
\hline LH2 & 1.10 & 1.18 & 1.38 & 1.62 & 1.35 & 1.54 & 1.69 & 2.12 & 1.36 & 1.57 & 1.70 & 2.16 \\
\hline LH3 & 1.14 & 1.23 & 1.43 & 1.69 & 1.57 & 1.86 & 1.97 & 2.55 & 1.66 & 1.99 & 2.08 & 2.73 \\
\hline LH4 & 1.11 & 1.19 & 1.39 & 1.63 & 1.33 & 1.51 & 1.66 & 2.08 & 1.34 & 1.53 & 1.67 & 2.11 \\
\hline LH5 & 1.10 & 1.17 & 1.37 & 1.60 & 1.35 & 1.52 & 1.69 & 2.09 & 1.38 & 1.57 & 1.73 & 2.16 \\
\hline LH6 & 1.13 & 1.27 & 1.42 & 1.75 & 1.36 & 1.53 & 1.70 & 2.11 & 1.87 & 2.23 & 2.34 & 3.06 \\
\hline
\end{tabular}


Table C.29 - The ratio of actual mid-span bending moments and quarter-span shear forces for buildings to the corresponding values obtained from FEMA parabolic distribution and a uniform distribution of seismic load when subjected to M6C31 ground motion and the Load is acting parallel to the long side, $\alpha=0.02$

\begin{tabular}{|c|c|c|c|c|c|c|c|c|c|c|c|c|}
\hline \multirow{2}{*}{ Building } & \multicolumn{4}{|c|}{$\mu=2$} & \multicolumn{4}{|c|}{$\mu=3$} & \multicolumn{4}{|c|}{$\mu=4$} \\
\hline & $M . F$ & $V . F$ & $M . U$ & $V . U$ & M.F & $V . F$ & $M . U$ & $V . U$ & M.F & V.F & $M . U$ & $V . U$ \\
\hline SL1 & 1.03 & 1.04 & 1.28 & 1.44 & 1.31 & 1.48 & 1.64 & 2.03 & 1.33 & 1.51 & 1.66 & 2.07 \\
\hline SL3 & 03 & 1.04 & 1.28 & 1.43 & 1.33 & 1.51 & 1.66 & 2.07 & 1.32 & 1.50 & 1.65 & 2.07 \\
\hline SL4 & .02 & 1.04 & 1.28 & 1.42 & 1.08 & 1.15 & 1.36 & 1.58 & 1.18 & 1.29 & 1.48 & 1.78 \\
\hline SH1 & 12 & 1.20 & 1.40 & 1.65 & 1.16 & 1.25 & 1.44 & 1.72 & 1.32 & 1.51 & 1.65 & 2.08 \\
\hline $\mathrm{SH} 2$ & 1.14 & 1.22 & 1.42 & 1.68 & 1.16 & 1.25 & 1.45 & 1.72 & 1.34 & 1.53 & 1.67 & 2.11 \\
\hline $\mathrm{SH} 3$ & 1.11 & 1.18 & 1.39 & 1.62 & 1.12 & 1.19 & 1.40 & 1.63 & 1.38 & 1.60 & 1.72 & 2.19 \\
\hline SH4 & 1.10 & 1.16 & 1.37 & 1.60 & 1.11 & 1.17 & 1.38 & 1.61 & 1.12 & 1.20 & 1.41 & 1.65 \\
\hline H5 & 1.06 & 1.11 & 1.33 & 1.53 & 1.07 & 1.13 & 1.34 & 1.55 & 1.43 & 1.65 & 1.79 & 2.27 \\
\hline ML1 & 1.48 & 1.22 & 1.44 & 1.67 & 1.38 & 1.54 & 1.73 & 2.12 & 1.39 & 1.54 & 1.74 & 2.12 \\
\hline ML2 & 1.15 & 1.21 & 1.43 & 1.66 & 1.34 & 1.51 & 1.68 & 2.07 & 1.36 & 1.53 & 1.70 & 2.10 \\
\hline ML3 & 1.14 & 1.20 & 1.43 & 1.65 & 1.21 & 1.33 & 1.51 & 1.83 & 1.37 & 1.53 & 1.71 & 2.11 \\
\hline ML4 & 1.15 & 1.20 & 1.43 & 1.66 & 1.34 & 1.51 & 1.68 & 2.07 & 1.36 & 1.52 & 1.69 & 2.09 \\
\hline ML5 & 1.14 & 1.20 & 1.43 & 1.65 & 1.35 & 1.51 & 1.68 & 2.08 & 1.36 & 1.53 & 1.71 & 2.11 \\
\hline ML6 & 14 & 1.20 & 1.43 & 1.65 & 1.32 & 1.46 & 1.65 & 2.01 & 1.37 & 1.54 & 1.71 & 2.11 \\
\hline MH1 & 1.04 & 1.08 & 1.31 & 1.49 & 1.18 & 1.30 & 1.48 & 1.78 & 1.30 & 1.45 & 1.63 & 1.99 \\
\hline & 1.00 & 1.02 & 1.25 & 1.40 & 1.29 & 1.40 & 1.61 & 1.93 & 1.35 & 1.50 & 1.69 & 2.06 \\
\hline MH3 & 1.19 & 1.26 & 1.49 & 1.74 & 1.33 & 1.49 & 1.66 & 2.05 & 1.38 & 1.54 & 1.72 & 2.12 \\
\hline MH4 & 1.06 & 1.09 & 1.32 & 1.50 & 1.20 & 1.33 & 1.50 & 1.83 & 1.23 & 1.38 & 1.54 & 1.90 \\
\hline MH5 & 1.19 & 1.26 & 1.49 & 1.73 & 1.26 & 1.37 & 1.57 & 1.88 & 1.32 & 1.46 & 1.64 & 2.01 \\
\hline MH6 & 1.01 & 1.02 & 1.26 & 1.41 & 1.21 & 1.33 & 1.51 & 1.83 & 1.26 & 1.40 & 1.57 & 1.92 \\
\hline LL1 & 1.11 & 1.18 & 1.39 & 1.62 & 1.22 & 1.36 & 1.53 & 1.88 & 1.30 & 1.44 & 1.63 & 1.99 \\
\hline LL2 & 1.11 & 1.18 & 1.39 & 1.63 & 1.15 & 1.26 & 1.44 & 1.73 & 1.31 & 1.49 & 1.64 & 2.05 \\
\hline LL3 & 1.14 & 1.17 & 1.42 & 1.61 & 1.29 & 1.43 & 1.61 & 1.96 & 1.41 & 1.62 & 1.76 & 2.22 \\
\hline L4 & 1.10 & 1.17 & 1.38 & 1.61 & 1.22 & 1.36 & 1.53 & 1.87 & 1.28 & 1.42 & 1.60 & 1.95 \\
\hline LL5 & 0.99 & 1.02 & 1.24 & 1.40 & 1.27 & 1.38 & 1.59 & 1.90 & 1.33 & 1.46 & 1.66 & 2.01 \\
\hline LL6 & 1.27 & 1.38 & 1.59 & 1.90 & 1.69 & 2.01 & 2.12 & 2.76 & 1.85 & 2.21 & 2.31 & 3.04 \\
\hline LH1 & 1.06 & 1.09 & 1.32 & 1.50 & 1.13 & 1.20 & 1.41 & 1.65 & 1.20 & 1.31 & 1.50 & 1.80 \\
\hline LH2 & 1.07 & 1.11 & 1.33 & 1.53 & 1.23 & 1.36 & 1.54 & 1.87 & 1.36 & 1.55 & 1.70 & 2.13 \\
\hline LH3 & 1.56 & 1.76 & 1.95 & 2.42 & 1.83 & 2.22 & 2.29 & 3.05 & 1.93 & 2.36 & 2.41 & 3.25 \\
\hline LH4 & 1.09 & 1.14 & 1.37 & 1.57 & 1.16 & 1.24 & 1.44 & 1.70 & 1.22 & 1.33 & 1.52 & 1.83 \\
\hline LH5 & 1.25 & 1.39 & 1.57 & 1.92 & 1.44 & 1.66 & 1.80 & 2.28 & 1.45 & 1.65 & 1.81 & 2.27 \\
\hline LH6 & 1.21 & 1.31 & 1.52 & 1.80 & 1.35 & 1.52 & 1.69 & 2.08 & 1.66 & 1.92 & 2.07 & 2.64 \\
\hline
\end{tabular}


Table C.30 - The ratio of actual mid-span bending moments and quarter-span shear forces for buildings to the corresponding values obtained from FEMA parabolic distribution and a uniform distribution of seismic load when subjected to M6C38 ground motion and the Load is acting parallel to the long side, $\alpha=0.02$

\begin{tabular}{|c|c|c|c|c|c|c|c|c|c|c|c|c|}
\hline \multirow{2}{*}{ Building } & \multicolumn{4}{|c|}{$\mu=2$} & \multicolumn{4}{|c|}{$\mu=3$} & \multicolumn{4}{|c|}{$\mu=4$} \\
\hline & $M . F$ & $V . F$ & $M . U$ & V.U & $M . F$ & V.F & $M . U$ & $V . U$ & M.F & V.F & M.U & $V . U$ \\
\hline SL1 & 1.10 & 1.16 & 1.37 & 1.59 & 1.13 & 1.22 & 1.41 & 1.68 & 1.19 & 1.33 & 1.49 & 1.82 \\
\hline SL3 & .09 & 1.15 & 1.37 & 1.58 & 1.15 & 1.24 & 1.44 & 1.71 & 1.21 & 1.33 & 1.51 & 1.83 \\
\hline SL4 & 1.04 & 1.07 & 1.30 & 1.47 & 1.13 & 1.19 & 1.41 & 1.64 & 1.19 & 1.31 & 1.49 & 1.80 \\
\hline SH1 & 1.12 & 1.19 & 1.40 & 1.64 & 1.14 & 1.22 & 1.42 & 1.68 & 1.15 & 1.24 & 1.44 & 1.71 \\
\hline $\mathrm{SH} 2$ & 1.13 & 1.22 & 1.42 & 1.67 & 1.16 & 1.25 & 1.45 & 1.72 & 1.17 & 1.26 & 1.46 & 1.74 \\
\hline SH3 & 1.14 & 1.23 & 1.43 & 1.69 & 1.17 & 1.27 & 1.46 & 1.74 & 1.18 & 1.28 & 1.48 & 1.77 \\
\hline SH4 & 1.14 & 1.23 & 1.43 & 1.69 & 1.17 & 1.26 & 1.46 & 1.74 & 1.18 & 1.28 & 1.47 & 1.76 \\
\hline SH5 & 1.15 & 1.24 & 1.43 & 1.70 & 1.17 & 1.27 & 1.47 & 1.75 & 1.19 & 1.29 & 1.49 & 1.78 \\
\hline ML1 & 1.18 & 1.29 & 1.48 & 1.77 & 1.22 & 1.35 & 1.53 & 1.85 & 1.25 & 1.39 & 1.56 & 1.91 \\
\hline ML2 & 1.19 & 1.29 & 1.48 & 1.77 & 1.23 & 1.36 & 1.53 & 1.86 & 1.25 & 1.40 & 1.57 & 1.92 \\
\hline ML3 & 1.18 & 1.28 & 1.47 & 1.75 & 1.22 & 1.35 & 1.52 & 1.85 & 1.26 & 1.40 & 1.57 & 1.93 \\
\hline ML4 & 1.19 & 1.29 & 1.48 & 1.77 & 1.23 & 1.36 & 1.53 & 1.86 & 1.25 & 1.39 & 1.56 & 1.91 \\
\hline ML5 & 1.19 & 1.29 & 1.48 & 1.77 & 1.23 & 1.36 & 1.53 & 1.86 & 1.25 & 1.40 & 1.57 & 1.92 \\
\hline ML6 & 1.18 & 1.28 & 1.48 & 1.76 & 1.23 & 1.35 & 1.53 & 1.86 & 1.25 & 1.40 & 1.57 & 1.93 \\
\hline MH1 & 1.17 & 1.27 & 1.47 & 1.74 & 1.24 & 1.37 & 1.55 & 1.89 & 1.28 & 1.44 & 1.60 & 1.97 \\
\hline MH2 & 1.16 & 1.24 & 1.45 & 1.70 & 1.24 & 1.36 & 1.54 & 1.87 & 1.29 & 1.45 & 1.62 & 1.99 \\
\hline MH3 & 1.20 & 1.25 & 1.50 & 1.72 & 1.31 & 1.41 & 1.63 & 1.95 & 1.38 & 1.52 & 1.72 & 2.09 \\
\hline MH4 & 1.17 & 1.26 & 1.46 & 1.73 & 1.23 & 1.36 & 1.54 & 1.87 & 1.28 & 1.43 & 1.60 & 1.97 \\
\hline MH5 & 1.16 & 1.23 & 1.45 & 1.69 & 1.25 & 1.37 & 1.56 & 1.88 & 1.31 & 1.46 & 1.63 & 2.01 \\
\hline MH6 & 1.16 & 1.24 & 1.45 & 1.71 & 1.23 & 1.37 & 1.54 & 1.88 & 1.30 & 1.46 & 1.62 & 2.01 \\
\hline LL1 & 1.18 & 1.28 & 1.48 & 1.75 & 1.25 & 1.39 & 1.57 & 1.91 & 1.31 & 1.47 & 1.64 & 2.03 \\
\hline LL2 & 1.18 & 1.27 & 1.47 & 1.75 & 1.25 & 1.39 & 1.56 & 1.91 & 1.30 & 1.46 & 1.63 & 2.01 \\
\hline LL3 & 1.18 & 1.24 & 1.48 & 1.71 & 1.29 & 1.41 & 1.61 & 1.93 & 1.37 & 1.53 & 1.71 & 2.10 \\
\hline LL4 & 1.18 & 1.27 & 1.47 & 1.75 & 1.26 & 1.39 & 1.57 & 1.92 & 1.30 & 1.47 & 1.63 & 2.02 \\
\hline LL5 & 1.16 & 1.25 & 1.45 & 1.72 & 1.24 & 1.37 & 1.55 & 1.89 & 1.29 & 1.45 & 1.62 & 2.00 \\
\hline LL6 & 1.17 & 1.20 & 1.47 & 1.65 & 1.29 & 1.36 & 1.61 & 1.87 & 1.38 & 1.50 & 1.72 & 2.07 \\
\hline LH1 & 1.16 & 1.24 & 1.45 & 1.70 & 1.25 & 1.38 & 1.56 & 1.90 & 1.31 & 1.48 & 1.64 & 2.04 \\
\hline LH2 & 1.14 & 1.21 & 1.43 & 1.67 & 1.24 & 1.37 & 1.55 & 1.88 & 1.30 & 1.46 & 1.62 & 2.00 \\
\hline LH3 & 1.18 & 1.23 & 1.48 & 1.70 & 1.33 & 1.45 & 1.66 & 1.99 & 1.44 & 1.61 & 1.79 & 2.21 \\
\hline LH4 & 1.14 & 1.22 & 1.43 & 1.68 & 1.24 & 1.38 & 1.55 & 1.89 & 1.30 & 1.47 & 1.63 & 2.02 \\
\hline LH5 & 1.17 & 1.24 & 1.46 & 1.70 & 1.26 & 1.38 & 1.58 & 1.90 & 1.32 & 1.48 & 1.65 & 2.03 \\
\hline LH6 & 1.22 & 1.25 & 1.52 & 1.72 & 1.37 & 1.48 & 1.71 & 2.03 & 1.48 & 1.64 & 1.85 & 2.26 \\
\hline
\end{tabular}


Table C.31 - The ratio of actual mid-span bending moments and quarter-span shear forces for buildings to the corresponding values obtained from FEMA parabolic distribution and a uniform distribution of seismic load when subjected to E6C1 ground motion and the Load is acting parallel to the short side, $\alpha=0.02$

\begin{tabular}{|c|c|c|c|c|c|c|c|c|c|c|c|c|}
\hline \multirow{2}{*}{ Building } & \multicolumn{4}{|c|}{$\mu=2$} & \multicolumn{4}{|c|}{$\mu=3$} & \multicolumn{4}{|c|}{$\mu=4$} \\
\hline & M.F & $V . F$ & $M . U$ & $V . U$ & $M . F$ & $V . F$ & $M . U$ & $V . U$ & $M . F$ & V.F & $M . U$ & $V . U$ \\
\hline SL1 & 0.95 & 0.95 & 1.18 & 1.30 & 0.95 & 0.94 & 1.19 & 1.30 & 0.95 & 0.95 & 1.19 & 1.30 \\
\hline SL3 & .93 & 0.94 & 1.17 & 1.29 & 0.94 & 0.94 & 1.18 & 1.29 & 0.94 & 0.94 & 1.18 & 1.29 \\
\hline SL4 & 0.91 & 0.92 & 1.14 & 1.26 & 0.92 & 0.92 & 1.15 & 1.27 & 0.93 & 0.93 & 1.16 & 1.28 \\
\hline SH1 & 1.26 & 1.41 & 1.57 & 1.94 & 1.27 & 1.43 & 1.59 & 1.97 & 1.29 & 1.46 & 1.61 & 2.01 \\
\hline $\mathrm{SH} 2$ & 1.27 & 1.43 & 1.59 & 1.97 & 1.27 & 1.44 & 1.59 & 1.98 & 1.28 & 1.44 & 1.60 & 1.97 \\
\hline SH3 & 1.25 & 1.41 & 1.57 & 1.94 & 1.26 & 1.43 & 1.57 & 1.96 & 1.27 & 1.42 & 1.59 & 1.96 \\
\hline SH4 & 1.23 & 1.38 & 1.54 & 1.90 & 1.23 & 1.40 & 1.54 & 1.93 & 1.26 & 1.40 & 1.57 & 1.92 \\
\hline SH5 & 1.23 & 1.38 & 1.53 & 1.89 & 1.23 & 1.40 & 1.54 & 1.93 & 1.26 & 1.39 & 1.58 & 1.92 \\
\hline SH6 & 1.26 & 1.41 & 1.57 & 1.94 & 1.26 & 1.43 & 1.57 & 1.96 & 1.28 & 1.43 & 1.59 & 1.96 \\
\hline ML1 & 1.14 & 1.21 & 1.42 & 1.66 & 1.21 & 1.27 & 1.51 & 1.75 & 1.26 & 1.33 & 1.57 & 1.83 \\
\hline ML2 & 1.01 & 1.08 & 1.27 & 1.49 & 1.09 & 1.18 & 1.37 & 1.62 & 1.17 & 1.27 & 1.46 & 1.75 \\
\hline ML3 & 1.04 & 1.11 & 1.30 & 1.53 & 1.12 & 1.21 & 1.40 & 1.66 & 1.24 & 1.34 & 1.55 & 1.84 \\
\hline ML4 & 1.06 & 1.10 & 1.33 & 1.51 & 1.15 & 1.20 & 1.43 & 1.65 & 1.21 & 1.29 & 1.52 & 1.78 \\
\hline ML5 & 1.07 & 1.11 & 1.34 & 1.53 & 1.15 & 1.21 & 1.44 & 1.66 & 1.21 & 1.29 & 1.52 & 1.78 \\
\hline ML6 & 1.02 & 1.10 & 1.28 & 1.51 & 1.12 & 1.21 & 1.40 & 1.67 & 1.22 & 1.33 & 1.53 & 1.83 \\
\hline MH1 & 1.02 & 1.08 & 1.27 & 1.48 & 1.12 & 1.20 & 1.40 & 1.65 & 1.30 & 1.38 & 1.63 & 1.90 \\
\hline MH2 & 1.05 & 1.09 & 1.32 & 1.49 & 1.21 & 1.24 & 1.51 & 1.70 & 1.43 & 1.50 & 1.78 & 2.06 \\
\hline MH3 & 1.21 & 1.44 & 1.51 & 1.98 & 1.39 & 1.60 & 1.74 & 2.20 & 1.58 & 1.75 & 1.98 & 2.41 \\
\hline MH4 & 1.07 & 1.07 & 1.33 & 1.48 & 1.17 & 1.18 & 1.46 & 1.63 & 1.34 & 1.38 & 1.67 & 1.90 \\
\hline MH5 & 1.08 & 1.11 & 1.36 & 1.52 & 1.23 & 1.28 & 1.54 & 1.75 & 1.42 & 1.55 & 1.77 & 2.13 \\
\hline MH6 & 1.14 & 1.18 & 1.43 & 1.62 & 1.24 & 1.32 & 1.55 & 1.82 & 1.35 & 1.49 & 1.68 & 2.04 \\
\hline LL1 & 0.99 & 1.10 & 1.23 & 1.51 & 1.05 & 1.21 & 1.32 & 1.66 & 1.17 & 1.25 & 1.47 & 1.72 \\
\hline LL2 & 0.97 & 1.11 & 1.21 & 1.52 & 1.04 & 1.21 & 1.30 & 1.66 & 1.19 & 1.25 & 1.48 & 1.72 \\
\hline LL3 & 1.02 & 1.24 & 1.28 & 1.70 & 1.33 & 1.63 & 1.66 & 2.24 & 1.39 & 1.71 & 1.74 & 2.35 \\
\hline LL4 & 1.00 & 1.03 & 1.25 & 1.41 & 1.09 & 1.13 & 1.36 & 1.56 & 1.25 & 1.27 & 1.57 & 1.75 \\
\hline LL5 & 1.00 & 1.02 & 1.25 & 1.40 & 1.10 & 1.14 & 1.37 & 1.56 & 1.24 & 1.25 & 1.55 & 1.72 \\
\hline LL6 & 1.07 & 1.39 & 1.33 & 1.92 & 1.22 & 1.58 & 1.52 & 2.17 & 1.51 & 1.83 & 1.89 & 2.52 \\
\hline LH1 & 1.14 & 1.36 & 1.43 & 1.88 & 1.37 & 1.67 & 1.71 & 2.30 & 1.43 & 1.77 & 1.78 & 2.43 \\
\hline LH2 & 1.32 & 1.54 & 1.66 & 2.12 & 1.41 & 1.69 & 1.76 & 2.32 & 1.46 & 1.78 & 1.83 & 2.45 \\
\hline LH3 & 1.39 & 1.80 & 1.74 & 2.47 & 1.75 & 2.12 & 2.19 & 2.92 & 1.80 & 2.18 & 2.26 & 3.00 \\
\hline LH4 & 1.34 & 1.60 & 1.67 & 2.20 & 1.43 & 1.75 & 1.79 & 2.41 & 1.49 & 1.85 & 1.87 & 2.54 \\
\hline LH5 & 1.35 & 1.62 & 1.69 & 2.23 & 1.45 & 1.77 & 1.81 & 2.44 & 1.51 & 1.87 & 1.89 & 2.57 \\
\hline LH6 & 1.37 & 1.79 & 1.71 & 2.46 & 1.79 & 2.17 & 2.23 & 2.99 & 1.85 & 2.24 & 2.31 & 3.09 \\
\hline
\end{tabular}


Table C.32 - The ratio of actual mid-span bending moments and quarter-span shear forces for buildings to the corresponding values obtained from FEMA parabolic distribution and a uniform distribution of seismic load when subjected to E6C13 ground motion and the Load is acting parallel to the short side, $\alpha=0.02$

\begin{tabular}{|c|c|c|c|c|c|c|c|c|c|c|c|c|}
\hline \multirow{2}{*}{ Building } & \multicolumn{4}{|c|}{$\mu=2$} & \multicolumn{4}{|c|}{$\mu=3$} & \multicolumn{4}{|c|}{$\mu=4$} \\
\hline & $M . F$ & V.F & $M . U$ & V.U & $M . F$ & $V . F$ & $M . U$ & $V . U$ & M.F & V.F & M.U & $V . U$ \\
\hline SL1 & 1.08 & 1.17 & 1.35 & 1.61 & 1.11 & 1.23 & 1.39 & 1.70 & 1.15 & 1.25 & 1.43 & 1.73 \\
\hline SL3 & 10 & 1.19 & 1.37 & 1.64 & 1.15 & 1.26 & 1.43 & 1.73 & 1.17 & 1.28 & 1.47 & 1.75 \\
\hline SL4 & 1.19 & 1.31 & 1.48 & 1.80 & 1.23 & 1.35 & 1.53 & 1.85 & 1.25 & 1.36 & 1.57 & 1.87 \\
\hline SH1 & 1.04 & 1.21 & 1.30 & 1.66 & 1.13 & 1.35 & 1.41 & 1.85 & 1.25 & 1.49 & 1.56 & 2.04 \\
\hline $\mathrm{SH} 2$ & 0.98 & 1.15 & 1.22 & 1.58 & 1.02 & 1.19 & 1.27 & 1.63 & 1.06 & 1.31 & 1.33 & 1.80 \\
\hline SH3 & 0.96 & 1.11 & 1.20 & 1.52 & 0.99 & 1.15 & 1.24 & 1.58 & 1.01 & 1.25 & 1.26 & 1.72 \\
\hline SH4 & 0.93 & 1.08 & 1.16 & 1.48 & 0.96 & 1.07 & 1.20 & 1.47 & 0.98 & 1.14 & 1.22 & 1.57 \\
\hline SH5 & 0.94 & 1.06 & 1.17 & 1.46 & 0.96 & 1.07 & 1.20 & 1.48 & 0.97 & 1.17 & 1.22 & 1.60 \\
\hline SH6 & 0.96 & 1.11 & 1.20 & 1.52 & 0.99 & 1.15 & 1.24 & 1.58 & 1.01 & 1.25 & 1.26 & 1.72 \\
\hline ML1 & 0.94 & 1.02 & 1.18 & 1.40 & 0.97 & 1.01 & 1.21 & 1.39 & 0.97 & 1.05 & 1.22 & 1.44 \\
\hline ML2 & 0.95 & 0.99 & 1.19 & 1.36 & 0.99 & 1.08 & 1.24 & 1.49 & 1.06 & 1.20 & 1.33 & 1.64 \\
\hline ML3 & 1.00 & 1.17 & 1.24 & 1.61 & 1.21 & 1.41 & 1.51 & 1.93 & 1.27 & 1.53 & 1.59 & 2.10 \\
\hline ML4 & 0.93 & 0.98 & 1.17 & 1.35 & 0.95 & 1.00 & 1.18 & 1.38 & 0.95 & 1.01 & 1.19 & 1.40 \\
\hline ML5 & 0.93 & 0.98 & 1.17 & 1.35 & 0.95 & 1.00 & 1.18 & 1.38 & 0.95 & 1.01 & 1.19 & 1.39 \\
\hline ML6 & 0.96 & 1.09 & 1.19 & 1.50 & 1.06 & 1.20 & 1.32 & 1.65 & 1.20 & 1.47 & 1.49 & 2.02 \\
\hline MH1 & 1.09 & 1.29 & 1.37 & 1.78 & 1.18 & 1.39 & 1.47 & 1.91 & 1.21 & 1.42 & 1.51 & 1.95 \\
\hline MH2 & 1.04 & 1.08 & 1.30 & 1.48 & 1.14 & 1.30 & 1.43 & 1.78 & 1.46 & 1.83 & 1.82 & 2.52 \\
\hline MH3 & 1.27 & 1.42 & 1.59 & 1.95 & 1.47 & 1.92 & 1.83 & 2.64 & 1.45 & 1.98 & 1.82 & 2.72 \\
\hline MH4 & 0.97 & 1.02 & 1.21 & 1.40 & 1.20 & 1.49 & 1.50 & 2.05 & 1.34 & 1.68 & 1.68 & 2.31 \\
\hline MH5 & 0.96 & 1.02 & 1.20 & 1.41 & 1.21 & 1.51 & 1.52 & 2.08 & 1.37 & 1.72 & 1.72 & 2.36 \\
\hline MH6 & 0.97 & 1.00 & 1.21 & 1.38 & 0.96 & 1.09 & 1.20 & 1.50 & 0.94 & 1.24 & 1.18 & 1.70 \\
\hline LL1 & 1.05 & 1.23 & 1.32 & 1.70 & 1.30 & 1.35 & 1.63 & 1.85 & 1.33 & 1.46 & 1.66 & 2.01 \\
\hline LL2 & 1.02 & 1.21 & 1.28 & 1.66 & 1.30 & 1.33 & 1.62 & 1.83 & 1.33 & 1.46 & 1.67 & 2.01 \\
\hline LL3 & 1.06 & 1.07 & 1.33 & 1.47 & 1.11 & 1.10 & 1.39 & 1.51 & 1.15 & 1.24 & 1.44 & 1.71 \\
\hline LL4 & 1.08 & 1.28 & 1.35 & 1.76 & 1.16 & 1.38 & 1.45 & 1.90 & 1.24 & 1.61 & 1.55 & 2.21 \\
\hline LL5 & 1.08 & 1.25 & 1.35 & 1.72 & 1.14 & 1.36 & 1.42 & 1.87 & 1.24 & 1.62 & 1.55 & 2.23 \\
\hline LL6 & 1.37 & 1.95 & 1.71 & 2.68 & 1.42 & 1.96 & 1.77 & 2.69 & 1.40 & 1.91 & 1.75 & 2.63 \\
\hline LH1 & 1.11 & 1.19 & 1.38 & 1.64 & 1.13 & 1.20 & 1.42 & 1.65 & 1.28 & 1.61 & 1.61 & 2.21 \\
\hline LH2 & 1.16 & 1.27 & 1.45 & 1.74 & 1.25 & 1.37 & 1.56 & 1.88 & 1.31 & 1.51 & 1.64 & 2.07 \\
\hline LH3 & 1.03 & 1.41 & 1.29 & 1.94 & 1.45 & 1.75 & 1.81 & 2.41 & 1.52 & 1.76 & 1.90 & 2.42 \\
\hline LH4 & 1.19 & 1.30 & 1.48 & 1.79 & 1.24 & 1.48 & 1.55 & 2.03 & 1.29 & 1.65 & 1.61 & 2.26 \\
\hline LH5 & 1.19 & 1.31 & 1.49 & 1.79 & 1.27 & 1.50 & 1.58 & 2.07 & 1.30 & 1.67 & 1.62 & 2.30 \\
\hline LH6 & 0.97 & 1.38 & 1.21 & 1.90 & 1.40 & 1.75 & 1.75 & 2.40 & 1.49 & 1.75 & 1.86 & 2.41 \\
\hline
\end{tabular}


Table C.33 - The ratio of actual mid-span bending moments and quarter-span shear forces for buildings to the corresponding values obtained from FEMA parabolic distribution and a uniform distribution of seismic load when subjected to E6C15 ground motion and the Load is acting parallel to the short side, $\alpha=0.02$

\begin{tabular}{|c|c|c|c|c|c|c|c|c|c|c|c|c|}
\hline \multirow{2}{*}{ Building } & \multicolumn{4}{|c|}{$\mu=2$} & \multicolumn{4}{|c|}{$\mu=3$} & \multicolumn{4}{|c|}{$\mu=4$} \\
\hline & $M . F$ & V.F & $M . U$ & $V . U$ & $M . F$ & $V . F$ & $M . U$ & V.U & M.F & V.F & $M . U$ & $V . U$ \\
\hline SL1 & 1.31 & 1.50 & 1.64 & 2.07 & 1.47 & 1.65 & 1.84 & 2.27 & 1.49 & 1.66 & 1.87 & 2.29 \\
\hline SL3 & 30 & 1.50 & 1.63 & 2.07 & 1.47 & 1.65 & 1.83 & 2.26 & 1.50 & 1.67 & 1.87 & 2.29 \\
\hline SL4 & 1.28 & 1.50 & 1.60 & 2.07 & 1.43 & 1.61 & 1.79 & 2.22 & 1.49 & 1.66 & 1.86 & 2.29 \\
\hline SH1 & 1.21 & 1.30 & 1.51 & 1.78 & 1.27 & 1.40 & 1.58 & 1.92 & 1.35 & 1.53 & 1.68 & 2.10 \\
\hline $\mathrm{SH} 2$ & 1.28 & 1.44 & 1.60 & 1.98 & 1.38 & 1.58 & 1.73 & 2.18 & 1.40 & 1.61 & 1.75 & 2.21 \\
\hline SH3 & 1.31 & 1.50 & 1.64 & 2.06 & 1.42 & 1.63 & 1.77 & 2.24 & 1.42 & 1.64 & 1.78 & 2.25 \\
\hline SH4 & 1.33 & 1.53 & 1.66 & 2.10 & 1.40 & 1.63 & 1.75 & 2.24 & 1.43 & 1.66 & 1.79 & 2.28 \\
\hline SH5 & 1.34 & 1.53 & 1.67 & 2.11 & 1.41 & 1.64 & 1.77 & 2.26 & 1.43 & 1.66 & 1.79 & 2.28 \\
\hline SH6 & 1.31 & 1.50 & 1.63 & 2.06 & 1.42 & 1.63 & 1.77 & 2.25 & 1.43 & 1.64 & 1.78 & 2.25 \\
\hline ML1 & 1.34 & 1.55 & 1.67 & 2.13 & 1.35 & 1.55 & 1.68 & 2.14 & 1.40 & 1.61 & 1.75 & 2.22 \\
\hline ML2 & 32 & 1.53 & 1.65 & 2.11 & 1.45 & 1.63 & 1.81 & 2.25 & 1.46 & 1.64 & 1.83 & 2.26 \\
\hline ML3 & 34 & 1.49 & 1.68 & 2.05 & 1.36 & 1.51 & 1.70 & 2.08 & 1.37 & 1.53 & 1.71 & 2.10 \\
\hline ML4 & 1.32 & 1.54 & 1.65 & 2.11 & 1.39 & 1.62 & 1.73 & 2.23 & 1.46 & 1.72 & 1.83 & 2.37 \\
\hline ML5 & 1.32 & 1.54 & 1.65 & 2.12 & 1.37 & 1.60 & 1.72 & 2.20 & 1.45 & 1.70 & 1.81 & 2.33 \\
\hline ML6 & 1.33 & 1.50 & 1.66 & 2.06 & 1.41 & 1.57 & 1.76 & 2.16 & 1.42 & 1.58 & 1.78 & 2.17 \\
\hline MH1 & 1.33 & 1.46 & 1.67 & 2.01 & 1.37 & 1.53 & 1.71 & 2.10 & 1.39 & 1.56 & 1.73 & 2.14 \\
\hline MH2 & 1.23 & 1.29 & 1.54 & 1.77 & 1.31 & 1.41 & 1.64 & 1.94 & 1.38 & 1.52 & 1.73 & 2.09 \\
\hline MH3 & 1.31 & 1.50 & 1.64 & 2.06 & 1.53 & 1.89 & 1.92 & 2.60 & 1.60 & 1.94 & 2.00 & 2.67 \\
\hline MH4 & 1.23 & 1.27 & 1.54 & 1.75 & 1.31 & 1.38 & 1.64 & 1.90 & 1.37 & 1.47 & 1.71 & 2.02 \\
\hline MH5 & 1.25 & 1.29 & 1.56 & 1.77 & 1.35 & 1.42 & 1.68 & 1.95 & 1.42 & 1.52 & 1.77 & 2.09 \\
\hline MH6 & 1.34 & 1.67 & 1.67 & 2.29 & 1.44 & 1.76 & 1.80 & 2.42 & 1.51 & 1.79 & 1.88 & 2.46 \\
\hline LL1 & 1.13 & 1.29 & 1.42 & 1.77 & 1.27 & 1.45 & 1.59 & 1.99 & 1.41 & 1.63 & 1.77 & 2.24 \\
\hline LL2 & 1.13 & 1.30 & 1.41 & 1.78 & 1.26 & 1.45 & 1.57 & 1.99 & 1.41 & 1.63 & 1.76 & 2.24 \\
\hline LL3 & 1.16 & 1.26 & 1.45 & 1.73 & 1.45 & 1.74 & 1.81 & 2.39 & 1.47 & 1.78 & 1.84 & 2.45 \\
\hline LL4 & 1.26 & 1.47 & 1.57 & 2.02 & 1.39 & 1.63 & 1.74 & 2.24 & 1.54 & 1.81 & 1.92 & 2.49 \\
\hline LL5 & 1.25 & 1.46 & 1.57 & 2.01 & 1.39 & 1.62 & 1.73 & 2.22 & 1.54 & 1.81 & 1.92 & 2.49 \\
\hline LL6 & 0.91 & 1.11 & 1.14 & 1.53 & 1.00 & 1.27 & 1.25 & 1.74 & 1.09 & 1.39 & 1.36 & 1.91 \\
\hline LH1 & 1.21 & 1.38 & 1.51 & 1.90 & 1.46 & 1.76 & 1.82 & 2.43 & 1.52 & 1.83 & 1.90 & 2.52 \\
\hline $\mathrm{LH} 2$ & 1.35 & 1.67 & 1.69 & 2.30 & 1.49 & 1.85 & 1.86 & 2.54 & 1.63 & 1.99 & 2.04 & 2.73 \\
\hline LH3 & 1.14 & 1.41 & 1.43 & 1.94 & 1.31 & 1.62 & 1.64 & 2.22 & 1.42 & 1.72 & 1.77 & 2.36 \\
\hline LH4 & 1.38 & 1.67 & 1.72 & 2.29 & 1.49 & 1.81 & 1.86 & 2.49 & 1.67 & 1.97 & 2.09 & 2.71 \\
\hline LH5 & 1.37 & 1.67 & 1.71 & 2.30 & 1.50 & 1.82 & 1.88 & 2.50 & 1.67 & 1.97 & 2.09 & 2.71 \\
\hline LH6 & 1.05 & 1.30 & 1.31 & 1.79 & 1.21 & 1.51 & 1.51 & 2.07 & 1.32 & 1.63 & 1.65 & 2.24 \\
\hline
\end{tabular}


Table C.34 - The ratio of actual mid-span bending moments and quarter-span shear forces for buildings to the corresponding values obtained from FEMA parabolic distribution and a uniform distribution of seismic load when subjected to E6C18 ground motion and the Load is acting parallel to the short side, $\alpha=0.02$

\begin{tabular}{|c|c|c|c|c|c|c|c|c|c|c|c|c|}
\hline \multirow{2}{*}{ Building } & \multicolumn{4}{|c|}{$\mu=2$} & \multicolumn{4}{|c|}{$\mu=3$} & \multicolumn{4}{|c|}{$\mu=4$} \\
\hline & $M . F$ & V.F & $M . U$ & $V . U$ & $M . F$ & V.F & $M . U$ & $V . U$ & M.F & V.F & M.U & $V . U$ \\
\hline SL1 & 1.15 & 1.25 & 1.44 & 1.72 & 1.20 & 1.31 & 1.50 & 1.80 & 1.27 & 1.37 & 1.58 & 1.89 \\
\hline SL3 & 19 & 1.30 & 1.48 & 1.79 & 1.22 & 1.35 & 1.52 & 1.85 & 1.28 & 1.39 & 1.60 & 1.91 \\
\hline SL4 & 1.27 & 1.43 & 1.59 & 1.96 & 1.28 & 1.43 & 1.60 & 1.97 & 1.34 & 1.49 & 1.67 & 2.05 \\
\hline SH1 & 1.04 & 1.11 & 1.30 & 1.53 & 1.13 & 1.16 & 1.41 & 1.59 & 1.19 & 1.20 & 1.48 & 1.65 \\
\hline $\mathrm{SH} 2$ & 1.03 & 1.06 & 1.29 & 1.46 & 1.13 & 1.17 & 1.41 & 1.61 & 1.21 & 1.26 & 1.51 & 1.73 \\
\hline SH3 & 1.04 & 1.09 & 1.30 & 1.50 & 1.16 & 1.21 & 1.44 & 1.67 & 1.24 & 1.31 & 1.55 & 1.80 \\
\hline SH4 & 1.03 & 1.10 & 1.28 & 1.51 & 1.14 & 1.22 & 1.42 & 1.67 & 1.22 & 1.30 & 1.53 & 1.79 \\
\hline SH5 & 1.03 & 1.11 & 1.29 & 1.52 & 1.16 & 1.24 & 1.44 & 1.70 & 1.25 & 1.34 & 1.56 & 1.84 \\
\hline SH6 & 1.04 & 1.09 & 1.30 & 1.50 & 1.15 & 1.21 & 1.44 & 1.66 & 1.24 & 1.31 & 1.55 & 1.80 \\
\hline ML1 & 0.99 & 1.09 & 1.24 & 1.50 & 1.10 & 1.22 & 1.38 & 1.68 & 1.23 & 1.34 & 1.54 & 1.85 \\
\hline ML2 & 0.99 & 1.25 & 1.24 & 1.72 & 1.22 & 1.45 & 1.52 & 1.99 & 1.26 & 1.48 & 1.58 & 2.04 \\
\hline ML3 & 15 & 1.43 & 1.43 & 1.97 & 1.22 & 1.50 & 1.52 & 2.06 & 1.26 & 1.54 & 1.58 & 2.12 \\
\hline ML4 & 0.99 & 1.16 & 1.24 & 1.59 & 1.11 & 1.30 & 1.39 & 1.78 & 1.42 & 1.57 & 1.77 & 2.16 \\
\hline ML5 & 1.00 & 1.16 & 1.25 & 1.59 & 1.11 & 1.28 & 1.38 & 1.76 & 1.42 & 1.58 & 1.78 & 2.17 \\
\hline ML6 & 1.14 & 1.40 & 1.43 & 1.93 & 1.21 & 1.46 & 1.52 & 2.01 & 1.26 & 1.50 & 1.58 & 2.07 \\
\hline MH1 & 1.17 & 1.44 & 1.47 & 1.98 & 1.24 & 1.48 & 1.55 & 2.03 & 1.27 & 1.51 & 1.59 & 2.08 \\
\hline MH2 & 1.28 & 1.53 & 1.59 & 2.10 & 1.35 & 1.57 & 1.69 & 2.16 & 1.40 & 1.61 & 1.75 & 2.22 \\
\hline MH3 & 0.96 & 1.46 & 1.20 & 2.01 & 1.04 & 1.61 & 1.30 & 2.21 & 1.09 & 1.79 & 1.37 & 2.46 \\
\hline MH4 & 1.10 & 1.41 & 1.37 & 1.94 & 1.34 & 1.60 & 1.68 & 2.20 & 1.40 & 1.65 & 1.75 & 2.26 \\
\hline MH5 & 1.30 & 1.59 & 1.63 & 2.18 & 1.39 & 1.64 & 1.73 & 2.26 & 1.44 & 1.68 & 1.80 & 2.31 \\
\hline MH6 & 1.03 & 1.20 & 1.29 & 1.65 & 1.44 & 1.56 & 1.80 & 2.15 & 1.49 & 1.65 & 1.87 & 2.27 \\
\hline LL1 & 1.36 & 1.83 & 1.71 & 2.51 & 1.43 & 1.91 & 1.79 & 2.62 & 1.47 & 1.95 & 1.84 & 2.68 \\
\hline LL2 & 1.36 & 1.83 & 1.70 & 2.52 & 1.43 & 1.91 & 1.78 & 2.63 & 1.47 & 1.96 & 1.84 & 2.70 \\
\hline LL3 & 1.43 & 1.90 & 1.79 & 2.61 & 1.54 & 2.01 & 1.93 & 2.77 & 1.61 & 2.09 & 2.01 & 2.87 \\
\hline LL4 & 1.29 & 1.66 & 1.61 & 2.28 & 1.35 & 1.72 & 1.69 & 2.37 & 1.39 & 1.75 & 1.73 & 2.41 \\
\hline LL5 & 1.30 & 1.66 & 1.62 & 2.28 & 1.36 & 1.72 & 1.70 & 2.36 & 1.39 & 1.75 & 1.74 & 2.41 \\
\hline LL6 & 0.67 & 1.43 & 0.83 & 1.97 & 0.75 & 1.57 & 0.93 & 2.16 & 0.87 & 1.78 & 1.09 & 2.44 \\
\hline LH1 & 1.49 & 2.04 & 1.86 & 2.80 & 1.61 & 2.18 & 2.02 & 3.00 & 1.69 & 2.27 & 2.11 & 3.12 \\
\hline LH2 & 1.52 & 2.16 & 1.90 & 2.97 & 1.65 & 2.33 & 2.06 & 3.20 & 1.74 & 2.45 & 2.18 & 3.36 \\
\hline LH3 & 1.01 & 1.83 & 1.26 & 2.52 & 1.19 & 2.06 & 1.49 & 2.83 & 1.28 & 2.13 & 1.60 & 2.92 \\
\hline LH4 & 1.55 & 2.18 & 1.94 & 3.00 & 1.69 & 2.35 & 2.11 & 3.24 & 1.78 & 2.47 & 2.22 & 3.40 \\
\hline LH5 & 1.56 & 2.20 & 1.95 & 3.02 & 1.68 & 2.36 & 2.10 & 3.25 & 1.79 & 2.50 & 2.24 & 3.44 \\
\hline LH6 & 1.00 & 1.88 & 1.25 & 2.58 & 1.15 & 2.04 & 1.43 & 2.81 & 1.24 & 2.14 & 1.55 & 2.95 \\
\hline
\end{tabular}


Table C.35 - The ratio of actual mid-span bending moments and quarter-span shear forces for buildings to the corresponding values obtained from FEMA parabolic distribution and a uniform distribution of seismic load when subjected to E6C42 ground motion and the Load is acting parallel to the short side, $\alpha=0.02$

\begin{tabular}{|c|c|c|c|c|c|c|c|c|c|c|c|c|}
\hline \multirow{2}{*}{ Building } & \multicolumn{4}{|c|}{$\mu=2$} & \multicolumn{4}{|c|}{$\mu=3$} & \multicolumn{4}{|c|}{$\mu=4$} \\
\hline & $M . F$ & $V . F$ & $M . U$ & V.U & $M . F$ & $V . F$ & M.U & V.U & $M . F$ & $V . F$ & $M . U$ & $V . U$ \\
\hline SL1 & 1.25 & 1.31 & 1.56 & 1.80 & 1.32 & 1.41 & 1.66 & 1.93 & 1.39 & 1.50 & 1.74 & 2.06 \\
\hline SL3 & 28 & 1.35 & 1.60 & 1.86 & 1.36 & 1.46 & 1.71 & 2.01 & 1.44 & 1.56 & 1.80 & 2.15 \\
\hline SL4 & .33 & 1.47 & 1.66 & 2.02 & 1.47 & 1.63 & 1.84 & 2.25 & 1.51 & 1.67 & 1.89 & 2.30 \\
\hline SH1 & 1.18 & 1.20 & 1.48 & 1.66 & 1.24 & 1.28 & 1.55 & 1.76 & 1.27 & 1.32 & 1.59 & 1.82 \\
\hline $\mathrm{SH} 2$ & 1.17 & 1.18 & 1.46 & 1.62 & 1.22 & 1.24 & 1.52 & 1.71 & 1.26 & 1.30 & 1.57 & 1.78 \\
\hline $\mathrm{SH} 3$ & 1.16 & 1.17 & 1.45 & 1.61 & 1.21 & 1.23 & 1.51 & 1.69 & 1.26 & 1.29 & 1.57 & 1.78 \\
\hline SH4 & 1.13 & 1.13 & 1.42 & 1.55 & 1.19 & 1.19 & 1.49 & 1.64 & 1.23 & 1.25 & 1.54 & 1.72 \\
\hline SH5 & 1.13 & 1.13 & 1.42 & 1.55 & 1.20 & 1.21 & 1.50 & 1.66 & 1.24 & 1.27 & 1.55 & 1.74 \\
\hline SH6 & 1.16 & 1.17 & 1.45 & 1.60 & 1.21 & 1.23 & 1.51 & 1.70 & 1.26 & 1.29 & 1.57 & 1.78 \\
\hline ML1 & 1.07 & 1.20 & 1.34 & 1.65 & 1.12 & 1.20 & 1.41 & 1.65 & 1.20 & 1.22 & 1.50 & 1.68 \\
\hline ML2 & 1.22 & 1.45 & 1.53 & 1.99 & 1.29 & 1.52 & 1.61 & 2.09 & 1.32 & 1.57 & 1.65 & 2.16 \\
\hline L3 & 1.29 & 1.55 & 1.62 & 2.13 & 1.34 & 1.64 & 1.68 & 2.26 & 1.36 & 1.69 & 1.71 & 2.33 \\
\hline ML4 & 1.13 & 1.28 & 1.41 & 1.77 & 1.18 & 1.33 & 1.48 & 1.83 & 1.21 & 1.35 & 1.51 & 1.85 \\
\hline ML5 & 1.12 & 1.27 & 1.40 & 1.75 & 1.17 & 1.31 & 1.46 & 1.80 & 1.19 & 1.32 & 1.48 & 1.82 \\
\hline ML6 & 1.26 & 1.50 & 1.58 & 2.07 & 1.32 & 1.60 & 1.66 & 2.20 & 1.34 & 1.63 & 1.68 & 2.25 \\
\hline MH1 & 1.30 & 1.59 & 1.62 & 2.19 & 1.33 & 1.67 & 1.66 & 2.30 & 1.34 & 1.71 & 1.68 & 2.35 \\
\hline $\mathrm{MH} 2$ & 1.13 & 1.37 & 1.41 & 1.88 & 1.26 & 1.55 & 1.58 & 2.14 & 1.27 & 1.59 & 1.59 & 2.18 \\
\hline MH3 & 0.86 & 0.91 & 1.08 & 1.26 & 0.90 & 1.00 & 1.12 & 1.38 & 0.94 & 1.09 & 1.18 & 1.50 \\
\hline MH4 & 1.19 & 1.42 & 1.48 & 1.96 & 1.22 & 1.47 & 1.53 & 2.01 & 1.23 & 1.49 & 1.54 & 2.05 \\
\hline MH5 & 1.18 & 1.42 & 1.48 & 1.95 & 1.22 & 1.46 & 1.52 & 2.01 & 1.23 & 1.49 & 1.53 & 2.05 \\
\hline MH6 & 1.22 & 1.36 & 1.52 & 1.88 & 1.25 & 1.42 & 1.56 & 1.95 & 1.30 & 1.48 & 1.63 & 2.03 \\
\hline LL1 & 1.08 & 1.41 & 1.35 & 1.94 & 1.29 & 1.66 & 1.61 & 2.29 & 1.42 & 1.88 & 1.78 & 2.58 \\
\hline LL2 & 1.07 & 1.40 & 1.34 & 1.92 & 1.26 & 1.63 & 1.58 & 2.25 & 1.40 & 1.87 & 1.76 & 2.57 \\
\hline LL3 & 0.98 & 1.23 & 1.22 & 1.69 & 1.03 & 1.28 & 1.28 & 1.77 & 1.04 & 1.32 & 1.30 & 1.81 \\
\hline LL4 & 1.36 & 1.70 & 1.70 & 2.34 & 1.41 & 1.79 & 1.76 & 2.46 & 1.44 & 1.85 & 1.80 & 2.54 \\
\hline LL5 & 1.37 & 1.71 & 1.71 & 2.35 & 1.42 & 1.79 & 1.78 & 2.47 & 1.45 & 1.86 & 1.82 & 2.55 \\
\hline LL6 & 0.93 & 1.09 & 1.16 & 1.50 & 0.95 & 1.12 & 1.19 & 1.54 & 0.96 & 1.12 & 1.20 & 1.54 \\
\hline LH1 & 0.91 & 1.19 & 1.14 & 1.63 & 0.96 & 1.25 & 1.20 & 1.71 & 0.98 & 1.28 & 1.22 & 1.76 \\
\hline LH2 & 0.94 & 1.17 & 1.18 & 1.61 & 0.93 & 1.24 & 1.16 & 1.71 & 0.95 & 1.28 & 1.18 & 1.77 \\
\hline LH3 & 0.89 & 1.03 & 1.11 & 1.42 & 0.90 & 1.05 & 1.13 & 1.45 & 0.96 & 1.07 & 1.21 & 1.47 \\
\hline LH4 & 0.93 & 1.12 & 1.17 & 1.53 & 0.92 & 1.16 & 1.15 & 1.60 & 0.99 & 1.20 & 1.23 & 1.65 \\
\hline LH5 & 0.93 & 1.10 & 1.17 & 1.51 & 0.92 & 1.14 & 1.14 & 1.56 & 0.99 & 1.17 & 1.24 & 1.61 \\
\hline LH6 & 0.93 & 1.09 & 1.17 & 1.49 & 0.95 & 1.12 & 1.19 & 1.54 & 0.97 & 1.14 & 1.22 & 1.57 \\
\hline
\end{tabular}


Table C.36 - The ratio of actual mid-span bending moments and quarter-span shear forces for buildings to the corresponding values obtained from FEMA parabolic distribution and a uniform distribution of seismic load when subjected to E6C1 ground motion and the Load is acting parallel to the long side, $\alpha=0.02$

\begin{tabular}{|c|c|c|c|c|c|c|c|c|c|c|c|c|}
\hline \multirow{2}{*}{ Building } & \multicolumn{4}{|c|}{$\mu=2$} & \multicolumn{4}{|c|}{$\mu=3$} & \multicolumn{4}{|c|}{$\mu=4$} \\
\hline & M.F & $V . F$ & $M . U$ & $V . U$ & $M . F$ & $V . F$ & $M . U$ & $V . U$ & $M . F$ & $V . F$ & $M . U$ & $V . U$ \\
\hline SL1 & 1.23 & 1.34 & 1.54 & 1.84 & 1.30 & 1.47 & 1.63 & 2.02 & 1.35 & 1.54 & 1.68 & 2.11 \\
\hline SL3 & 1.26 & 1.37 & 1.58 & 1.89 & 1.30 & 1.46 & 1.62 & 2.00 & 1.33 & 1.51 & 1.66 & 2.08 \\
\hline SL4 & 1.13 & 1.19 & 1.41 & 1.64 & 1.21 & 1.33 & 1.51 & 1.83 & 1.29 & 1.45 & 1.62 & 2.00 \\
\hline SH1 & 1.02 & 1.04 & 1.28 & 1.43 & 1.08 & 1.14 & 1.35 & 1.56 & 1.09 & 1.15 & 1.36 & 1.59 \\
\hline $\mathrm{SH} 2$ & 1.03 & 1.05 & 1.28 & 1.44 & 1.05 & 1.10 & 1.31 & 1.51 & 1.06 & 1.11 & 1.32 & 1.52 \\
\hline SH3 & 1.02 & 1.04 & 1.27 & 1.43 & 1.03 & 1.07 & 1.29 & 1.47 & 1.03 & 1.08 & 1.29 & 1.48 \\
\hline SH4 & 1.00 & 1.02 & 1.25 & 1.40 & 1.01 & 1.04 & 1.26 & 1.42 & 1.01 & 1.04 & 1.26 & 1.43 \\
\hline SH5 & 1.00 & 1.01 & 1.25 & 1.39 & 1.00 & 1.03 & 1.25 & 1.41 & 1.00 & 1.03 & 1.25 & 1.42 \\
\hline ML1 & 0.91 & 0.90 & 1.14 & 1.23 & 0.94 & 0.93 & 1.17 & 1.27 & 0.96 & 0.95 & 1.20 & 1.31 \\
\hline ML2 & 0.91 & 0.89 & 1.14 & 1.22 & 0.94 & 0.90 & 1.17 & 1.24 & 1.00 & 0.96 & 1.25 & 1.32 \\
\hline ML3 & 0.91 & 0.88 & 1.14 & 1.21 & 1.00 & 0.96 & 1.25 & 1.32 & 1.10 & 1.08 & 1.37 & 1.48 \\
\hline L4 & 0.92 & 0.89 & 1.14 & 1.23 & 0.93 & 0.91 & 1.16 & 1.25 & 0.99 & 0.95 & 1.23 & 1.31 \\
\hline ML5 & 0.91 & 0.89 & 1.14 & 1.22 & 0.95 & 0.91 & 1.19 & 1.25 & 1.02 & 0.97 & 1.27 & 1.34 \\
\hline ML6 & 0.91 & 0.88 & 1.14 & 1.21 & 0.97 & 0.92 & 1.21 & 1.27 & 1.07 & 1.04 & 1.34 & 1.44 \\
\hline MH1 & 1.06 & 1.07 & 1.33 & 1.47 & 1.17 & 1.22 & 1.46 & 1.68 & 1.26 & 1.35 & 1.57 & 1.86 \\
\hline MH2 & 1.18 & 1.28 & 1.48 & 1.76 & 1.26 & 1.41 & 1.58 & 1.93 & 1.32 & 1.50 & 1.65 & 2.06 \\
\hline MH3 & 1.24 & 1.37 & 1.55 & 1.89 & 1.24 & 1.40 & 1.55 & 1.93 & 1.28 & 1.45 & 1.60 & 2.00 \\
\hline MH4 & 1.11 & 1.14 & 1.38 & 1.57 & 1.20 & 1.28 & 1.51 & 1.76 & 1.28 & 1.40 & 1.60 & 1.92 \\
\hline MH5 & 1.21 & 1.33 & 1.51 & 1.82 & 1.27 & 1.43 & 1.59 & 1.97 & 1.32 & 1.50 & 1.65 & 2.07 \\
\hline MH6 & 1.11 & 1.17 & 1.39 & 1.61 & 1.23 & 1.34 & 1.54 & 1.84 & 1.32 & 1.49 & 1.65 & 2.05 \\
\hline LL1 & 0.99 & 0.98 & 1.24 & 1.35 & 1.11 & 1.13 & 1.39 & 1.55 & 1.23 & 1.30 & 1.53 & 1.79 \\
\hline LL2 & 1.06 & 1.07 & 1.32 & 1.47 & 1.18 & 1.24 & 1.48 & 1.71 & 1.28 & 1.40 & 1.60 & 1.92 \\
\hline LL3 & 1.25 & 1.39 & 1.57 & 1.91 & 1.26 & 1.42 & 1.58 & 1.95 & 1.30 & 1.48 & 1.63 & 2.04 \\
\hline LL4 & 1.00 & 0.99 & 1.25 & 1.36 & 1.12 & 1.14 & 1.40 & 1.57 & 1.23 & 1.32 & 1.54 & 1.81 \\
\hline LL5 & 1.17 & 1.26 & 1.47 & 1.74 & 1.26 & 1.40 & 1.58 & 1.92 & 1.32 & 1.49 & 1.65 & 2.05 \\
\hline LL6 & 1.15 & 1.21 & 1.44 & 1.66 & 1.23 & 1.33 & 1.54 & 1.83 & 1.51 & 1.73 & 1.88 & 2.38 \\
\hline LH1 & 1.10 & 1.15 & 1.37 & 1.58 & 1.22 & 1.33 & 1.52 & 1.83 & 1.56 & 1.84 & 1.95 & 2.53 \\
\hline LH2 & 1.18 & 1.27 & 1.48 & 1.75 & 1.31 & 1.48 & 1.64 & 2.03 & 1.57 & 1.82 & 1.96 & 2.51 \\
\hline LH3 & 1.13 & 1.19 & 1.41 & 1.64 & 1.42 & 1.62 & 1.77 & 2.23 & 1.43 & 1.63 & 1.79 & 2.24 \\
\hline LH4 & 1.10 & 1.16 & 1.38 & 1.59 & 1.56 & 1.83 & 1.95 & 2.52 & 1.58 & 1.87 & 1.97 & 2.57 \\
\hline LH5 & 1.21 & 1.31 & 1.51 & 1.81 & 1.28 & 1.43 & 1.60 & 1.97 & 1.46 & 1.65 & 1.82 & 2.27 \\
\hline LH6 & 1.10 & 1.11 & 1.38 & 1.52 & 1.44 & 1.64 & 1.80 & 2.26 & 1.46 & 1.67 & 1.83 & 2.30 \\
\hline
\end{tabular}


Table C.37 - The ratio of actual mid-span bending moments and quarter-span shear forces for buildings to the corresponding values obtained from FEMA parabolic distribution and a uniform distribution of seismic load when subjected to E6C13 ground motion and the Load is acting parallel to the long side, $\alpha=0.02$

\begin{tabular}{|c|c|c|c|c|c|c|c|c|c|c|c|c|}
\hline \multirow{2}{*}{ Building } & \multicolumn{4}{|c|}{$\mu=2$} & \multicolumn{4}{|c|}{$\mu=3$} & \multicolumn{4}{|c|}{$\mu=4$} \\
\hline & $M . F$ & $V . F$ & $M . U$ & V.U & M.F & $V . F$ & $M . U$ & $V . U$ & M.F & V.F & M.U & $V . U$ \\
\hline SL1 & 1.16 & 1.24 & 1.45 & 1.71 & 1.38 & 1.57 & 1.73 & 2.15 & 1.44 & 1.66 & 1.80 & 2.29 \\
\hline SL3 & 16 & 1.23 & 1.44 & 1.69 & 1.42 & 1.62 & 1.77 & 2.22 & 1.45 & 1.68 & 1.82 & 2.32 \\
\hline & 12 & 1.17 & 1.40 & 1.61 & 1.44 & 1.65 & 1.80 & 2.26 & 1.49 & 1.72 & 1.86 & 2.37 \\
\hline SH1 & 1.25 & 1.38 & 1.57 & 1.90 & 1.32 & 1.46 & 1.65 & 2.01 & 1.31 & 1.43 & 1.64 & 1.97 \\
\hline & 1.04 & 1.04 & 1.29 & 1.43 & 1.34 & 1.49 & 1.67 & 2.04 & 1.32 & 1.46 & 1.66 & 2.01 \\
\hline $\mathrm{SH} 3$ & 0.97 & 0.98 & 1.21 & 1.35 & 1.25 & 1.37 & 1.56 & 1.89 & 1.31 & 1.45 & 1.64 & 2.00 \\
\hline $\mathrm{SH} 4$ & 0.99 & 0.98 & 1.24 & 1.34 & 1.25 & 1.35 & 1.56 & 1.86 & 1.34 & 1.49 & 1.68 & 2.05 \\
\hline SH5 & 0.99 & 0.97 & 1.24 & 1.34 & 1.21 & 1.32 & 1.52 & 1.81 & 1.31 & 1.46 & 1.64 & 2.00 \\
\hline IL1 & 1.16 & 1.29 & 1.44 & 1.77 & 1.19 & 1.35 & 1.48 & 1.86 & 1.26 & 1.44 & 1.57 & 1.98 \\
\hline ML2 & 1.21 & 1.36 & 1.51 & 1.87 & 1.26 & 1.42 & 1.58 & 1.95 & 1.30 & 1.45 & 1.63 & 2.00 \\
\hline ML3 & 1.30 & 1.48 & 1.62 & 2.04 & 1.35 & 1.54 & 1.69 & 2.11 & 1.38 & 1.53 & 1.73 & 2.11 \\
\hline ML4 & 1.19 & 1.33 & 1.49 & 1.83 & 1.24 & 1.40 & 1.55 & 1.92 & 1.29 & 1.44 & 1.62 & 1.98 \\
\hline ML5 & 1.23 & 1.39 & 1.54 & 1.91 & 1.28 & 1.45 & 1.60 & 1.99 & 1.32 & 1.47 & 1.65 & 2.03 \\
\hline ML6 & 1.26 & 1.44 & 1.58 & 1.97 & 1.32 & 1.50 & 1.65 & 2.06 & 1.35 & 1.51 & 1.69 & 2.08 \\
\hline & 38 & 1.54 & 1.73 & 2.12 & 1.38 & 1.55 & 1.73 & 2.13 & 1.39 & 1.62 & 1.74 & 2.22 \\
\hline & 35 & 1.52 & 1.69 & 2.09 & 1.42 & 1.65 & 1.77 & 2.27 & 1.50 & 1.76 & 1.87 & 2.42 \\
\hline & 1.03 & 1.15 & 1.28 & 1.57 & 1.17 & 1.38 & 1.46 & 1.90 & 1.33 & 1.58 & 1.67 & 2.18 \\
\hline MH4 & 1.36 & 1.49 & 1.70 & 2.05 & 1.38 & 1.59 & 1.72 & 2.18 & 1.40 & 1.64 & 1.76 & 2.25 \\
\hline MH5 & 1.33 & 1.49 & 1.66 & 2.05 & 1.42 & 1.66 & 1.78 & 2.29 & 1.50 & 1.77 & 1.87 & 2.43 \\
\hline MH6 & 1.32 & 1.48 & 1.65 & 2.04 & 1.40 & 1.62 & 1.75 & 2.23 & 1.46 & 1.71 & 1.83 & 2.35 \\
\hline LL1 & 1.39 & 1.57 & 1.74 & 2.16 & 1.39 & 1.53 & 1.74 & 2.11 & 1.39 & 1.61 & 1.74 & 2.21 \\
\hline LL2 & 1.37 & 1.51 & 1.71 & 2.07 & 1.38 & 1.58 & 1.72 & 2.18 & 1.43 & 1.67 & 1.79 & 2.29 \\
\hline LL3 & 1.09 & 1.24 & 1.36 & 1.71 & 1.30 & 1.50 & 1.62 & 2.07 & 1.42 & 1.68 & 1.78 & 2.31 \\
\hline LL4 & 1.39 & 1.56 & 1.73 & 2.15 & 1.39 & 1.53 & 1.73 & 2.10 & 1.39 & 1.61 & 1.74 & 2.22 \\
\hline LL5 & 1.35 & 1.52 & 1.69 & 2.09 & 1.44 & 1.67 & 1.80 & 2.30 & 1.48 & 1.74 & 1.86 & 2.40 \\
\hline LL6 & 0.94 & 0.97 & 1.17 & 1.33 & 0.93 & 1.17 & 1.16 & 1.60 & 1.06 & 1.33 & 1.33 & 1.83 \\
\hline LH1 & 1.28 & 1.42 & 1.61 & 1.95 & 1.39 & 1.59 & 1.74 & 2.19 & 1.45 & 1.68 & 1.81 & 2.31 \\
\hline LH2 & 1.21 & 1.30 & 1.51 & 1.78 & 1.35 & 1.52 & 1.69 & 2.09 & 1.45 & 1.68 & 1.81 & 2.31 \\
\hline LH3 & 0.98 & 0.98 & 1.23 & 1.35 & 0.96 & 1.18 & 1.21 & 1.63 & 1.13 & 1.38 & 1.42 & 1.90 \\
\hline LH4 & 1.24 & 1.33 & 1.55 & 1.83 & 1.35 & 1.52 & 1.69 & 2.10 & 1.42 & 1.65 & 1.78 & 2.27 \\
\hline LH5 & 1.09 & 1.17 & 1.36 & 1.61 & 1.27 & 1.44 & 1.59 & 1.98 & 1.41 & 1.63 & 1.76 & 2.24 \\
\hline LH6 & 0.95 & 1.11 & 1.18 & 1.52 & 1.11 & 1.29 & 1.39 & 1.78 & 1.30 & 1.50 & 1.62 & 2.07 \\
\hline
\end{tabular}


Table C.38 - The ratio of actual mid-span bending moments and quarter-span shear forces for buildings to the corresponding values obtained from FEMA parabolic distribution and a uniform distribution of seismic load when subjected to E6C15 ground motion and the Load is acting parallel to the long side, $\alpha=0.02$

\begin{tabular}{|c|c|c|c|c|c|c|c|c|c|c|c|c|}
\hline \multirow{2}{*}{ Building } & \multicolumn{4}{|c|}{$\mu=2$} & \multicolumn{4}{|c|}{$\mu=3$} & \multicolumn{4}{|c|}{$\mu=4$} \\
\hline & $M . F$ & $V . F$ & $M . U$ & $V . U$ & M.F & $V . F$ & $M . U$ & $V . U$ & M.F & V.F & $M . U$ & $V . U$ \\
\hline SL1 & 1.26 & 1.42 & 1.58 & 1.95 & 1.29 & 1.47 & 1.61 & 2.02 & 1.31 & 1.51 & 1.63 & 2.07 \\
\hline SL3 & 26 & 1.41 & 1.57 & 1.94 & 1.29 & 1.47 & 1.61 & 2.02 & 1.32 & 1.51 & 1.64 & 2.08 \\
\hline SL4 & 23 & 1.38 & 1.54 & 1.89 & 1.27 & 1.45 & 1.59 & 1.99 & 1.30 & 1.49 & 1.62 & 2.05 \\
\hline SH1 & 1.26 & 1.41 & 1.58 & 1.93 & 1.33 & 1.50 & 1.66 & 2.06 & 1.38 & 1.57 & 1.72 & 2.16 \\
\hline $\mathrm{SH} 2$ & 1.31 & 1.47 & 1.64 & 2.02 & 1.37 & 1.56 & 1.71 & 2.14 & 1.41 & 1.61 & 1.76 & 2.21 \\
\hline $\mathrm{SH} 3$ & 1.32 & 1.49 & 1.65 & 2.04 & 1.37 & 1.56 & 1.72 & 2.14 & 1.41 & 1.61 & 1.77 & 2.21 \\
\hline SH4 & 1.36 & 1.53 & 1.69 & 2.10 & 1.40 & 1.59 & 1.75 & 2.18 & 1.42 & 1.61 & 1.77 & 2.21 \\
\hline H5 & 1.33 & 1.50 & 1.66 & 2.06 & 1.39 & 1.57 & 1.73 & 2.16 & 1.41 & 1.61 & 1.77 & 2.21 \\
\hline ML1 & 1.36 & 1.52 & 1.70 & 2.09 & 1.42 & 1.60 & 1.78 & 2.20 & 1.48 & 1.66 & 1.85 & 2.28 \\
\hline ML2 & 1.36 & 1.53 & 1.70 & 2.10 & 1.43 & 1.60 & 1.78 & 2.20 & 1.49 & 1.66 & 1.86 & 2.28 \\
\hline ML3 & 1.36 & 1.53 & 1.70 & 2.10 & 1.46 & 1.64 & 1.83 & 2.25 & 1.53 & 1.70 & 1.91 & 2.34 \\
\hline ML4 & 1.36 & 1.53 & 1.70 & 2.11 & 1.41 & 1.58 & 1.76 & 2.18 & 1.50 & 1.68 & 1.88 & 2.31 \\
\hline ML5 & 1.37 & 1.54 & 1.71 & 2.12 & 1.44 & 1.61 & 1.79 & 2.21 & 1.49 & 1.66 & 1.87 & 2.29 \\
\hline ML6 & 1.36 & 1.53 & 1.70 & 2.11 & 1.47 & 1.64 & 1.83 & 2.26 & 1.52 & 1.70 & 1.91 & 2.34 \\
\hline MH1 & 1.25 & 1.42 & 1.56 & 1.96 & 1.52 & 1.69 & 1.90 & 2.33 & 1.53 & 1.71 & 1.92 & 2.35 \\
\hline & 1.11 & 1.16 & 1.38 & 1.59 & 1.48 & 1.70 & 1.85 & 2.34 & 1.54 & 1.78 & 1.93 & 2.44 \\
\hline MH3 & 1.38 & 1.58 & 1.72 & 2.18 & 1.41 & 1.64 & 1.76 & 2.25 & 1.53 & 1.75 & 1.91 & 2.40 \\
\hline MH4 & 1.22 & 1.37 & 1.52 & 1.89 & 1.46 & 1.63 & 1.82 & 2.25 & 1.56 & 1.74 & 1.95 & 2.39 \\
\hline MH5 & 1.13 & 1.19 & 1.41 & 1.64 & 1.48 & 1.67 & 1.85 & 2.30 & 1.53 & 1.72 & 1.91 & 2.37 \\
\hline MH6 & 1.36 & 1.50 & 1.70 & 2.06 & 1.49 & 1.70 & 1.86 & 2.33 & 1.56 & 1.75 & 1.95 & 2.40 \\
\hline LL1 & 1.37 & 1.55 & 1.71 & 2.12 & 1.51 & 1.69 & 1.89 & 2.32 & 1.57 & 1.75 & 1.96 & 2.41 \\
\hline LL2 & 1.32 & 1.50 & 1.65 & 2.06 & 1.50 & 1.68 & 1.88 & 2.31 & 1.55 & 1.73 & 1.94 & 2.37 \\
\hline LL3 & 1.36 & 1.57 & 1.70 & 2.16 & 1.42 & 1.65 & 1.78 & 2.27 & 1.61 & 1.82 & 2.01 & 2.50 \\
\hline LL4 & 1.38 & 1.55 & 1.72 & 2.14 & 1.51 & 1.69 & 1.89 & 2.32 & 1.55 & 1.72 & 1.94 & 2.37 \\
\hline LL5 & 1.10 & 1.14 & 1.37 & 1.56 & 1.50 & 1.72 & 1.87 & 2.36 & 1.55 & 1.80 & 1.94 & 2.47 \\
\hline LL6 & 1.34 & 1.62 & 1.67 & 2.23 & 1.45 & 1.72 & 1.81 & 2.36 & 1.63 & 1.86 & 2.04 & 2.55 \\
\hline LH1 & 1.32 & 1.46 & 1.65 & 2.01 & 1.55 & 1.74 & 1.93 & 2.39 & 1.63 & 1.82 & 2.03 & 2.51 \\
\hline LH2 & 1.37 & 1.57 & 1.72 & 2.16 & 1.58 & 1.84 & 1.97 & 2.53 & 1.62 & 1.89 & 2.03 & 2.60 \\
\hline LH3 & 1.39 & 1.65 & 1.74 & 2.27 & 1.54 & 1.78 & 1.92 & 2.45 & 1.64 & 1.87 & 2.05 & 2.57 \\
\hline LH4 & 1.25 & 1.36 & 1.57 & 1.87 & 1.56 & 1.77 & 1.95 & 2.43 & 1.63 & 1.85 & 2.04 & 2.54 \\
\hline LH5 & 1.30 & 1.46 & 1.63 & 2.00 & 1.54 & 1.76 & 1.93 & 2.41 & 1.62 & 1.84 & 2.03 & 2.53 \\
\hline LH6 & 1.41 & 1.62 & 1.77 & 2.23 & 1.53 & 1.79 & 1.91 & 2.46 & 1.67 & 1.90 & 2.09 & 2.61 \\
\hline
\end{tabular}


Table C.39 - The ratio of actual mid-span bending moments and quarter-span shear forces for buildings to the corresponding values obtained from FEMA parabolic distribution and a uniform distribution of seismic load when subjected to E6C18 ground motion and the Load is acting parallel to the long side, $\alpha=0.02$

\begin{tabular}{|c|c|c|c|c|c|c|c|c|c|c|c|c|}
\hline \multirow{2}{*}{ Building } & \multicolumn{4}{|c|}{$\mu=2$} & \multicolumn{4}{|c|}{$\mu=3$} & \multicolumn{4}{|c|}{$\mu=4$} \\
\hline & $M . F$ & $V . F$ & $M . U$ & $V . U$ & M.F & $V . F$ & $M . U$ & $V . U$ & M.F & V.F & $M . U$ & $V . U$ \\
\hline SL1 & 1.00 & 1.01 & 1.25 & 1.39 & 1.19 & 1.26 & 1.49 & 1.73 & 1.21 & 1.33 & 1.51 & 1.83 \\
\hline SL3 & 01 & 1.03 & 1.27 & 1.42 & 1.20 & 1.29 & 1.51 & 1.77 & 1.20 & 1.32 & 1.50 & 1.81 \\
\hline SL4 & 02 & 1.04 & 1.28 & 1.43 & 1.27 & 1.38 & 1.59 & 1.90 & 1.28 & 1.39 & 1.59 & 1.91 \\
\hline SH1 & 11 & 1.16 & 1.39 & 1.59 & 1.35 & 1.53 & 1.68 & 2.10 & 1.36 & 1.55 & 1.70 & 2.13 \\
\hline $\mathrm{SH} 2$ & 1.11 & 1.14 & 1.38 & 1.57 & 1.29 & 1.45 & 1.61 & 1.99 & 1.38 & 1.54 & 1.73 & 2.12 \\
\hline $\mathrm{SH} 3$ & 1.11 & 1.16 & 1.39 & 1.60 & 1.31 & 1.46 & 1.63 & 2.00 & 1.40 & 1.57 & 1.74 & 2.16 \\
\hline SH4 & 1.11 & 1.15 & 1.39 & 1.58 & 1.27 & 1.42 & 1.59 & 1.96 & 1.39 & 1.59 & 1.74 & 2.19 \\
\hline H5 & 1.11 & 1.16 & 1.39 & 1.59 & 1.33 & 1.47 & 1.67 & 2.02 & 1.41 & 1.61 & 1.76 & 2.22 \\
\hline ML1 & 1.28 & 1.44 & 1.60 & 1.99 & 1.34 & 1.54 & 1.67 & 2.11 & 1.33 & 1.52 & 1.66 & 2.09 \\
\hline ML2 & 1.31 & 1.49 & 1.64 & 2.05 & 1.33 & 1.53 & 1.67 & 2.11 & 1.33 & 1.54 & 1.66 & 2.12 \\
\hline ML3 & 1.27 & 1.43 & 1.59 & 1.97 & 1.33 & 1.52 & 1.66 & 2.09 & 1.33 & 1.54 & 1.66 & 2.11 \\
\hline ML4 & 1.32 & 1.49 & 1.64 & 2.05 & 1.33 & 1.53 & 1.67 & 2.11 & 1.32 & 1.53 & 1.65 & 2.11 \\
\hline ML5 & 1.31 & 1.49 & 1.64 & 2.04 & 1.33 & 1.53 & 1.66 & 2.10 & 1.33 & 1.55 & 1.67 & 2.13 \\
\hline ML6 & 1.29 & 1.45 & 1.61 & 1.99 & 1.33 & 1.52 & 1.66 & 2.09 & 1.32 & 1.52 & 1.65 & 2.09 \\
\hline MH1 & 1.25 & 1.40 & 1.56 & 1.93 & 1.31 & 1.50 & 1.64 & 2.06 & 1.33 & 1.54 & 1.66 & 2.12 \\
\hline & 1.13 & 1.21 & 1.41 & 1.67 & 1.15 & 1.30 & 1.44 & 1.79 & 1.24 & 1.34 & 1.55 & 1.84 \\
\hline MH3 & 1.11 & 1.16 & 1.39 & 1.59 & 1.28 & 1.35 & 1.60 & 1.86 & 1.74 & 2.05 & 2.17 & 2.82 \\
\hline MH4 & 1.22 & 1.37 & 1.53 & 1.88 & 1.29 & 1.47 & 1.61 & 2.02 & 1.31 & 1.51 & 1.64 & 2.08 \\
\hline MH5 & 1.09 & 1.17 & 1.36 & 1.61 & 1.19 & 1.26 & 1.49 & 1.74 & 1.28 & 1.35 & 1.60 & 1.85 \\
\hline MH6 & 1.14 & 1.22 & 1.43 & 1.68 & 1.15 & 1.27 & 1.43 & 1.75 & 1.21 & 1.32 & 1.51 & 1.82 \\
\hline LL1 & 1.24 & 1.35 & 1.55 & 1.86 & 1.28 & 1.42 & 1.59 & 1.95 & 1.28 & 1.44 & 1.59 & 1.98 \\
\hline LL2 & 1.23 & 1.35 & 1.54 & 1.85 & 1.26 & 1.41 & 1.57 & 1.94 & 1.24 & 1.42 & 1.55 & 1.96 \\
\hline LL3 & 1.07 & 1.08 & 1.34 & 1.48 & 1.24 & 1.27 & 1.55 & 1.75 & 1.44 & 1.56 & 1.80 & 2.14 \\
\hline L4 & 1.24 & 1.36 & 1.55 & 1.86 & 1.27 & 1.42 & 1.59 & 1.95 & 1.27 & 1.44 & 1.59 & 1.97 \\
\hline LL5 & 1.14 & 1.23 & 1.42 & 1.69 & 1.16 & 1.31 & 1.45 & 1.81 & 1.22 & 1.34 & 1.52 & 1.84 \\
\hline LL6 & 1.12 & 1.22 & 1.40 & 1.68 & 1.50 & 1.65 & 1.87 & 2.27 & 1.55 & 1.76 & 1.94 & 2.42 \\
\hline LH1 & 1.07 & 1.13 & 1.33 & 1.55 & 1.14 & 1.24 & 1.43 & 1.71 & 1.21 & 1.34 & 1.51 & 1.84 \\
\hline LH2 & 1.01 & 1.02 & 1.26 & 1.41 & 1.13 & 1.21 & 1.42 & 1.67 & 1.39 & 1.60 & 1.74 & 2.20 \\
\hline LH3 & 1.27 & 1.37 & 1.59 & 1.88 & 1.33 & 1.46 & 1.67 & 2.01 & 1.40 & 1.58 & 1.75 & 2.18 \\
\hline LH4 & 1.05 & 1.10 & 1.31 & 1.51 & 1.13 & 1.22 & 1.41 & 1.68 & 1.19 & 1.32 & 1.49 & 1.82 \\
\hline LH5 & 1.04 & 1.13 & 1.30 & 1.55 & 1.50 & 1.74 & 1.88 & 2.39 & 1.78 & 2.12 & 2.23 & 2.92 \\
\hline LH6 & 1.30 & 1.45 & 1.63 & 1.99 & 1.33 & 1.47 & 1.66 & 2.02 & 1.36 & 1.49 & 1.70 & 2.05 \\
\hline
\end{tabular}


Table C.40 - The ratio of actual mid-span bending moments and quarter-span shear forces for buildings to the corresponding values obtained from FEMA parabolic distribution and a uniform distribution of seismic load when subjected to E6C42 ground motion and the Load is acting parallel to the long side, $\alpha=0.02$

\begin{tabular}{|c|c|c|c|c|c|c|c|c|c|c|c|c|}
\hline \multirow{2}{*}{ Building } & \multicolumn{4}{|c|}{$\mu=2$} & \multicolumn{4}{|c|}{$\mu=3$} & \multicolumn{4}{|c|}{$\mu=4$} \\
\hline & $M . F$ & $V . F$ & $M . U$ & V.U & M.F & $V . F$ & $M . U$ & $V . U$ & M.F & V.F & M.U & $V . U$ \\
\hline SL1 & 1.29 & 1.42 & 1.61 & 1.95 & 1.31 & 1.45 & 1.64 & 1.99 & 1.31 & 1.48 & 1.64 & 2.03 \\
\hline SL3 & 32 & 1.47 & 1.65 & 2.02 & 1.34 & 1.51 & 1.68 & 2.08 & 1.34 & 1.51 & 1.68 & 2.08 \\
\hline & 37 & 1.55 & 1.71 & 2.14 & 1.41 & 1.62 & 1.76 & 2.23 & 1.41 & 1.64 & 1.76 & 2.26 \\
\hline SH1 & 18 & 1.24 & 1.48 & 1.70 & 1.31 & 1.44 & 1.64 & 1.98 & 1.47 & 1.68 & 1.83 & 2.31 \\
\hline & 1.27 & 1.37 & 1.59 & 1.88 & 1.38 & 1.54 & 1.73 & 2.12 & 1.44 & 1.65 & 1.80 & 2.26 \\
\hline $\mathrm{SH} 3$ & 1.28 & 1.41 & 1.61 & 1.94 & 1.37 & 1.55 & 1.72 & 2.13 & 1.40 & 1.59 & 1.75 & 2.18 \\
\hline $\mathrm{SH} 4$ & 1.28 & 1.41 & 1.60 & 1.93 & 1.36 & 1.51 & 1.69 & 2.08 & 1.41 & 1.59 & 1.77 & 2.19 \\
\hline SH5 & 1.25 & 1.38 & 1.57 & 1.90 & 1.33 & 1.49 & 1.67 & 2.05 & 1.38 & 1.55 & 1.72 & 2.13 \\
\hline ML1 & 1.32 & 1.48 & 1.65 & 2.04 & 1.33 & 1.51 & 1.67 & 2.08 & 1.39 & 1.59 & 1.74 & 2.19 \\
\hline ML2 & 1.23 & 1.39 & 1.54 & 1.91 & 1.25 & 1.48 & 1.56 & 2.03 & 1.27 & 1.43 & 1.59 & 1.97 \\
\hline ML3 & 1.05 & 1.19 & 1.31 & 1.64 & 1.08 & 1.29 & 1.35 & 1.78 & 1.30 & 1.45 & 1.62 & 1.99 \\
\hline ML4 & 1.26 & 1.42 & 1.57 & 1.95 & 1.28 & 1.50 & 1.60 & 2.06 & 1.32 & 1.49 & 1.66 & 2.05 \\
\hline ML5 & 1.19 & 1.34 & 1.49 & 1.85 & 1.20 & 1.44 & 1.50 & 1.98 & 1.24 & 1.43 & 1.54 & 1.96 \\
\hline ML6 & 1.09 & 1.23 & 1.36 & 1.70 & 1.11 & 1.33 & 1.39 & 1.83 & 1.27 & 1.41 & 1.58 & 1.94 \\
\hline & 92 & 0.91 & 1.15 & 1.26 & 0.99 & 0.99 & 1.24 & 1.36 & 1.29 & 1.45 & 1.62 & 1.99 \\
\hline & 1.03 & 1.05 & 1.28 & 1.45 & 1.16 & 1.26 & 1.45 & 1.73 & 1.45 & 1.65 & 1.82 & 2.27 \\
\hline & 1.15 & 1.21 & 1.44 & 1.66 & 1.23 & 1.33 & 1.54 & 1.82 & 1.33 & 1.48 & 1.66 & 2.04 \\
\hline MH4 & 0.94 & 0.94 & 1.18 & 1.30 & 1.01 & 1.02 & 1.26 & 1.40 & 1.28 & 1.43 & 1.60 & 1.96 \\
\hline MH5 & 1.08 & 1.11 & 1.34 & 1.53 & 1.20 & 1.31 & 1.50 & 1.81 & 1.66 & 1.95 & 2.07 & 2.68 \\
\hline MH6 & 0.98 & 1.00 & 1.22 & 1.38 & 1.14 & 1.24 & 1.42 & 1.70 & 1.40 & 1.59 & 1.75 & 2.18 \\
\hline LL1 & 0.92 & 0.93 & 1.16 & 1.27 & 0.96 & 0.98 & 1.20 & 1.35 & 1.35 & 1.52 & 1.68 & 2.08 \\
\hline LL2 & 0.95 & 0.96 & 1.18 & 1.31 & 0.99 & 1.03 & 1.24 & 1.42 & 1.32 & 1.46 & 1.65 & 2.00 \\
\hline LL3 & 1.15 & 1.23 & 1.44 & 1.69 & 1.23 & 1.34 & 1.54 & 1.84 & 1.35 & 1.58 & 1.69 & 2.17 \\
\hline LL4 & 0.93 & 0.93 & 1.16 & 1.27 & 0.96 & 0.99 & 1.20 & 1.36 & 1.34 & 1.51 & 1.67 & 2.07 \\
\hline LL5 & 1.01 & 1.04 & 1.27 & 1.44 & 1.14 & 1.22 & 1.42 & 1.68 & 1.39 & 1.57 & 1.74 & 2.16 \\
\hline LL6 & 1.15 & 1.28 & 1.44 & 1.76 & 1.22 & 1.31 & 1.53 & 1.80 & 1.39 & 1.57 & 1.74 & 2.16 \\
\hline LH1 & 1.03 & 1.07 & 1.29 & 1.47 & 1.34 & 1.49 & 1.68 & 2.05 & 1.58 & 1.84 & 1.97 & 2.53 \\
\hline LH2 & 1.08 & 1.17 & 1.35 & 1.61 & 1.88 & 2.21 & 2.36 & 3.04 & 1.98 & 2.38 & 2.48 & 3.28 \\
\hline LH3 & 1.26 & 1.40 & 1.57 & 1.93 & 1.29 & 1.45 & 1.61 & 2.00 & 1.66 & 1.98 & 2.08 & 2.72 \\
\hline LH4 & 1.06 & 1.11 & 1.32 & 1.53 & 1.49 & 1.69 & 1.86 & 2.32 & 1.74 & 2.06 & 2.18 & 2.83 \\
\hline LH5 & 1.18 & 1.32 & 1.47 & 1.82 & 1.37 & 1.55 & 1.71 & 2.13 & 1.92 & 2.24 & 2.40 & 3.08 \\
\hline LH6 & 1.35 & 1.54 & 1.69 & 2.12 & 1.41 & 1.62 & 1.76 & 2.23 & 1.87 & 2.25 & 2.34 & 3.09 \\
\hline
\end{tabular}


Table C.41 - The ratio of actual mid-span bending moments and quarter-span shear forces for buildings to the corresponding values obtained from FEMA parabolic distribution and a uniform distribution of seismic load when subjected to M6C1 ground motion and the Load is acting parallel to the short side, $\alpha=0.05$

\begin{tabular}{|c|c|c|c|c|c|c|c|c|c|c|c|c|}
\hline \multirow{2}{*}{ Building } & \multicolumn{4}{|c|}{$\mu=2$} & \multicolumn{4}{|c|}{$\mu=3$} & \multicolumn{4}{|c|}{$\mu=4$} \\
\hline & $M . F$ & V.F & $M . U$ & $V . U$ & $M . F$ & $V . F$ & $M . U$ & $V . U$ & M.F & V.F & M.U & $V . U$ \\
\hline SL1 & 1.01 & 1.04 & 1.27 & 1.43 & 1.00 & 1.04 & 1.25 & 1.43 & 1.05 & 1.11 & 1.32 & 1.52 \\
\hline SL3 & 03 & 1.07 & 1.29 & 1.48 & 1.02 & 1.08 & 1.27 & 1.49 & 1.05 & 1.12 & 1.32 & 1.55 \\
\hline SL4 & 1.12 & 1.19 & 1.40 & 1.63 & 1.11 & 1.20 & 1.39 & 1.65 & 1.12 & 1.20 & 1.40 & 1.65 \\
\hline SH1 & 1.16 & 1.25 & 1.45 & 1.72 & 1.24 & 1.37 & 1.55 & 1.89 & 1.26 & 1.41 & 1.58 & 1.93 \\
\hline $\mathrm{SH} 2$ & 1.23 & 1.38 & 1.54 & 1.90 & 1.22 & 1.39 & 1.52 & 1.91 & 1.21 & 1.40 & 1.51 & 1.93 \\
\hline $\mathrm{SH} 3$ & 1.24 & 1.40 & 1.55 & 1.92 & 1.22 & 1.41 & 1.53 & 1.93 & 1.22 & 1.42 & 1.52 & 1.96 \\
\hline SH4 & 1.23 & 1.39 & 1.54 & 1.91 & 1.22 & 1.40 & 1.52 & 1.93 & 1.20 & 1.39 & 1.50 & 1.92 \\
\hline SH5 & 1.24 & 1.40 & 1.55 & 1.92 & 1.22 & 1.41 & 1.53 & 1.94 & 1.21 & 1.41 & 1.51 & 1.94 \\
\hline SH6 & 1.24 & 1.40 & 1.55 & 1.92 & 1.22 & 1.41 & 1.53 & 1.93 & 1.22 & 1.43 & 1.53 & 1.96 \\
\hline ML1 & 1.16 & 1.29 & 1.45 & 1.77 & 1.19 & 1.35 & 1.49 & 1.86 & 1.18 & 1.38 & 1.48 & 1.90 \\
\hline ML2 & 1.00 & 1.00 & 1.25 & 1.38 & 1.08 & 1.11 & 1.35 & 1.53 & 1.07 & 1.13 & 1.34 & 1.55 \\
\hline ML3 & 1.00 & 1.00 & 1.24 & 1.38 & 1.04 & 1.06 & 1.30 & 1.46 & 1.05 & 1.11 & 1.32 & 1.52 \\
\hline ML4 & 1.14 & 1.25 & 1.43 & 1.72 & 1.17 & 1.30 & 1.46 & 1.78 & 1.16 & 1.33 & 1.45 & 1.82 \\
\hline ML5 & 1.15 & 1.26 & 1.43 & 1.73 & 1.17 & 1.31 & 1.46 & 1.80 & 1.17 & 1.34 & 1.46 & 1.84 \\
\hline ML6 & 1.05 & 1.02 & 1.31 & 1.40 & 1.05 & 1.07 & 1.32 & 1.47 & 1.05 & 1.09 & 1.31 & 1.49 \\
\hline MH1 & 1.05 & 1.11 & 1.31 & 1.53 & 1.07 & 1.12 & 1.34 & 1.54 & 1.08 & 1.16 & 1.35 & 1.60 \\
\hline MH2 & 1.13 & 1.23 & 1.42 & 1.69 & 1.12 & 1.24 & 1.40 & 1.71 & 1.14 & 1.28 & 1.42 & 1.76 \\
\hline MH3 & 1.49 & 1.71 & 1.86 & 2.35 & 1.62 & 1.89 & 2.02 & 2.59 & 1.59 & 1.84 & 1.99 & 2.53 \\
\hline MH4 & 1.15 & 1.25 & 1.44 & 1.72 & 1.13 & 1.25 & 1.41 & 1.72 & 1.12 & 1.26 & 1.40 & 1.73 \\
\hline MH5 & 1.14 & 1.23 & 1.43 & 1.69 & 1.14 & 1.26 & 1.43 & 1.74 & 1.16 & 1.31 & 1.45 & 1.81 \\
\hline MH6 & 1.14 & 1.26 & 1.42 & 1.73 & 1.20 & 1.39 & 1.50 & 1.91 & 1.30 & 1.48 & 1.62 & 2.03 \\
\hline LL1 & 0.99 & 1.01 & 1.24 & 1.39 & 0.99 & 1.03 & 1.24 & 1.41 & 1.23 & 1.31 & 1.54 & 1.80 \\
\hline LL2 & 1.00 & 1.03 & 1.25 & 1.42 & 1.01 & 1.05 & 1.26 & 1.45 & 1.26 & 1.35 & 1.57 & 1.86 \\
\hline LL3 & 1.18 & 1.29 & 1.48 & 1.77 & 1.32 & 1.44 & 1.65 & 1.99 & 1.60 & 1.84 & 2.00 & 2.53 \\
\hline LL4 & 0.93 & 0.95 & 1.17 & 1.31 & 0.98 & 0.98 & 1.23 & 1.35 & 1.06 & 1.12 & 1.32 & 1.54 \\
\hline LL5 & 0.92 & 0.94 & 1.16 & 1.29 & 0.97 & 0.97 & 1.22 & 1.33 & 1.06 & 1.12 & 1.33 & 1.54 \\
\hline LL6 & 1.15 & 1.38 & 1.44 & 1.89 & 1.20 & 1.51 & 1.50 & 2.07 & 1.23 & 1.62 & 1.53 & 2.22 \\
\hline LH1 & 1.23 & 1.34 & 1.54 & 1.84 & 1.43 & 1.61 & 1.79 & 2.21 & 1.64 & 1.88 & 2.04 & 2.58 \\
\hline LH2 & 1.28 & 1.44 & 1.61 & 1.99 & 1.43 & 1.61 & 1.78 & 2.21 & 1.62 & 1.89 & 2.03 & 2.60 \\
\hline LH3 & 1.35 & 1.72 & 1.68 & 2.37 & 1.50 & 1.90 & 1.88 & 2.61 & 1.54 & 1.88 & 1.92 & 2.59 \\
\hline LH4 & 1.29 & 1.42 & 1.62 & 1.96 & 1.45 & 1.65 & 1.81 & 2.27 & 1.62 & 1.87 & 2.03 & 2.58 \\
\hline LH5 & 1.30 & 1.43 & 1.63 & 1.97 & 1.47 & 1.68 & 1.83 & 2.31 & 1.62 & 1.87 & 2.02 & 2.57 \\
\hline LH6 & 1.27 & 1.63 & 1.58 & 2.24 & 1.38 & 1.80 & 1.73 & 2.48 & 1.47 & 1.90 & 1.84 & 2.62 \\
\hline
\end{tabular}


Table C.42 - The ratio of actual mid-span bending moments and quarter-span shear forces for buildings to the corresponding values obtained from FEMA parabolic distribution and a uniform distribution of seismic load when subjected to M6C2 ground motion and the Load is acting parallel to the short side, $\alpha=0.05$

\begin{tabular}{|c|c|c|c|c|c|c|c|c|c|c|c|c|}
\hline \multirow{2}{*}{ Building } & \multicolumn{4}{|c|}{$\mu=2$} & \multicolumn{4}{|c|}{$\mu=3$} & \multicolumn{4}{|c|}{$\mu=4$} \\
\hline & $M . F$ & V.F & $M . U$ & $V . U$ & $M . F$ & $V . F$ & $M . U$ & $V . U$ & M.F & V.F & $M . U$ & $V . U$ \\
\hline SL1 & 1.05 & 1.10 & 1.31 & 1.51 & 1.04 & 1.12 & 1.30 & 1.53 & 1.02 & 1.12 & 1.28 & 1.54 \\
\hline SL3 & 06 & 1.12 & 1.32 & 1.54 & 1.05 & 1.14 & 1.32 & 1.57 & 1.04 & 1.15 & 1.29 & 1.58 \\
\hline SL4 & 1.07 & 1.13 & 1.33 & 1.56 & 1.06 & 1.16 & 1.32 & 1.59 & 1.03 & 1.16 & 1.29 & 1.60 \\
\hline SH1 & 1.10 & 1.18 & 1.38 & 1.63 & 1.20 & 1.38 & 1.50 & 1.90 & 1.16 & 1.37 & 1.45 & 1.89 \\
\hline $\mathrm{SH} 2$ & 1.06 & 1.07 & 1.33 & 1.47 & 1.13 & 1.20 & 1.42 & 1.65 & 1.15 & 1.31 & 1.44 & 1.81 \\
\hline SH3 & 1.04 & 1.05 & 1.30 & 1.45 & 1.20 & 1.25 & 1.50 & 1.72 & 1.19 & 1.29 & 1.49 & 1.77 \\
\hline SH4 & 1.09 & 1.17 & 1.37 & 1.61 & 1.10 & 1.20 & 1.37 & 1.64 & 1.23 & 1.29 & 1.53 & 1.78 \\
\hline SH5 & 1.11 & 1.19 & 1.39 & 1.64 & 1.14 & 1.25 & 1.43 & 1.71 & 1.27 & 1.33 & 1.59 & 1.83 \\
\hline SH6 & 1.04 & 1.06 & 1.30 & 1.45 & 1.17 & 1.23 & 1.47 & 1.69 & 1.20 & 1.29 & 1.49 & 1.78 \\
\hline ML1 & 1.42 & 1.59 & 1.78 & 2.19 & 1.38 & 1.55 & 1.72 & 2.13 & 1.34 & 1.51 & 1.68 & 2.07 \\
\hline ML2 & 1.28 & 1.41 & 1.59 & 1.94 & 1.28 & 1.44 & 1.61 & 1.98 & 1.31 & 1.50 & 1.63 & 2.06 \\
\hline ML3 & 12 & 1.17 & 1.40 & 1.61 & 1.21 & 1.33 & 1.51 & 1.83 & 1.25 & 1.44 & 1.56 & 1.98 \\
\hline ML4 & 1.39 & 1.57 & 1.74 & 2.15 & 1.39 & 1.57 & 1.74 & 2.16 & 1.36 & 1.54 & 1.70 & 2.11 \\
\hline ML5 & 1.40 & 1.57 & 1.75 & 2.16 & 1.38 & 1.55 & 1.72 & 2.14 & 1.37 & 1.54 & 1.71 & 2.12 \\
\hline ML6 & 1.22 & 1.32 & 1.53 & 1.82 & 1.25 & 1.40 & 1.57 & 1.92 & 1.30 & 1.51 & 1.62 & 2.08 \\
\hline MH1 & 1.05 & 1.09 & 1.32 & 1.50 & 1.17 & 1.33 & 1.47 & 1.83 & 1.14 & 1.33 & 1.43 & 1.83 \\
\hline MH2 & 0.99 & 1.07 & 1.24 & 1.47 & 0.98 & 1.08 & 1.23 & 1.49 & 0.98 & 1.07 & 1.23 & 1.48 \\
\hline MH3 & 1.31 & 1.47 & 1.63 & 2.02 & 1.32 & 1.47 & 1.65 & 2.03 & 1.29 & 1.43 & 1.61 & 1.96 \\
\hline MH4 & 0.90 & 0.94 & 1.12 & 1.29 & 0.93 & 1.00 & 1.17 & 1.38 & 0.92 & 1.04 & 1.15 & 1.43 \\
\hline MH5 & 0.90 & 0.96 & 1.12 & 1.31 & 0.89 & 0.97 & 1.11 & 1.34 & 0.97 & 1.08 & 1.21 & 1.48 \\
\hline MH6 & 1.48 & 1.70 & 1.85 & 2.34 & 1.44 & 1.67 & 1.80 & 2.30 & 1.46 & 1.72 & 1.82 & 2.37 \\
\hline LL1 & 1.23 & 1.42 & 1.54 & 1.96 & 1.19 & 1.41 & 1.48 & 1.93 & 1.11 & 1.34 & 1.39 & 1.84 \\
\hline LL2 & 1.20 & 1.39 & 1.50 & 1.91 & 1.15 & 1.37 & 1.44 & 1.89 & 1.11 & 1.30 & 1.39 & 1.79 \\
\hline LL3 & 1.05 & 1.14 & 1.31 & 1.57 & 1.08 & 1.20 & 1.35 & 1.65 & 1.14 & 1.23 & 1.42 & 1.69 \\
\hline LL4 & 1.29 & 1.45 & 1.61 & 1.99 & 1.32 & 1.55 & 1.65 & 2.13 & 1.23 & 1.47 & 1.54 & 2.02 \\
\hline LL5 & 1.31 & 1.48 & 1.64 & 2.03 & 1.32 & 1.55 & 1.65 & 2.13 & 1.25 & 1.48 & 1.56 & 2.04 \\
\hline LL6 & 1.31 & 1.65 & 1.64 & 2.27 & 1.35 & 1.66 & 1.68 & 2.28 & 1.37 & 1.67 & 1.72 & 2.30 \\
\hline LH1 & 1.14 & 1.25 & 1.42 & 1.72 & 1.18 & 1.31 & 1.47 & 1.80 & 1.16 & 1.30 & 1.45 & 1.78 \\
\hline $\mathrm{LH} 2$ & 1.27 & 1.40 & 1.59 & 1.93 & 1.25 & 1.39 & 1.57 & 1.91 & 1.22 & 1.36 & 1.53 & 1.87 \\
\hline LH3 & 1.23 & 1.47 & 1.53 & 2.02 & 1.30 & 1.52 & 1.63 & 2.08 & 1.31 & 1.50 & 1.64 & 2.06 \\
\hline LH4 & 1.24 & 1.35 & 1.55 & 1.85 & 1.27 & 1.41 & 1.58 & 1.94 & 1.23 & 1.38 & 1.54 & 1.89 \\
\hline LH5 & 1.24 & 1.35 & 1.55 & 1.86 & 1.27 & 1.42 & 1.59 & 1.96 & 1.24 & 1.39 & 1.55 & 1.91 \\
\hline LH6 & 1.21 & 1.47 & 1.51 & 2.02 & 1.32 & 1.57 & 1.65 & 2.15 & 1.34 & 1.56 & 1.68 & 2.15 \\
\hline
\end{tabular}


Table C.43 - The ratio of actual mid-span bending moments and quarter-span shear forces for buildings to the corresponding values obtained from FEMA parabolic distribution and a uniform distribution of seismic load when subjected to M6C26 ground motion and the Load is acting parallel to the short side, $\alpha=0.05$

\begin{tabular}{|c|c|c|c|c|c|c|c|c|c|c|c|c|}
\hline \multirow{2}{*}{ Building } & \multicolumn{4}{|c|}{$\mu=2$} & \multicolumn{4}{|c|}{$\mu=3$} & \multicolumn{4}{|c|}{$\mu=4$} \\
\hline & $M . F$ & V.F & $M . U$ & $V . U$ & $M . F$ & $V . F$ & M.U & V.U & $M . F$ & $V . F$ & $M . U$ & $V . U$ \\
\hline SL1 & 1.08 & 1.13 & 1.35 & 1.55 & 1.09 & 1.16 & 1.36 & 1.60 & 1.09 & 1.17 & 1.36 & 1.61 \\
\hline SL3 & .08 & 1.13 & 1.35 & 1.56 & 1.09 & 1.17 & 1.37 & 1.60 & 1.09 & 1.18 & 1.36 & 1.62 \\
\hline SL4 & .10 & 1.15 & 1.37 & 1.59 & 1.10 & 1.17 & 1.38 & 1.61 & 1.10 & 1.18 & 1.38 & 1.62 \\
\hline SH1 & 1.10 & 1.17 & 1.38 & 1.61 & 1.09 & 1.19 & 1.36 & 1.63 & 1.06 & 1.18 & 1.33 & 1.63 \\
\hline $\mathrm{SH} 2$ & 1.12 & 1.20 & 1.40 & 1.65 & 1.10 & 1.22 & 1.38 & 1.67 & 1.08 & 1.21 & 1.35 & 1.66 \\
\hline $\mathrm{SH} 3$ & 1.12 & 1.22 & 1.40 & 1.67 & 1.11 & 1.23 & 1.38 & 1.69 & 1.08 & 1.21 & 1.35 & 1.66 \\
\hline SH4 & 1.12 & 1.21 & 1.39 & 1.66 & 1.10 & 1.22 & 1.37 & 1.68 & 1.09 & 1.21 & 1.36 & 1.66 \\
\hline SH5 & 1.12 & 1.21 & 1.39 & 1.67 & 1.10 & 1.22 & 1.37 & 1.68 & 1.09 & 1.21 & 1.36 & 1.66 \\
\hline SH6 & 1.12 & 1.21 & 1.40 & 1.67 & 1.10 & 1.22 & 1.38 & 1.68 & 1.08 & 1.21 & 1.35 & 1.66 \\
\hline ML1 & 1.10 & 1.17 & 1.38 & 1.61 & 1.13 & 1.21 & 1.41 & 1.67 & 1.14 & 1.23 & 1.42 & 1.69 \\
\hline ML2 & 0.92 & 1.00 & 1.15 & 1.37 & 1.08 & 1.19 & 1.35 & 1.64 & 1.09 & 1.25 & 1.36 & 1.72 \\
\hline L3 & 0.89 & 0.94 & 1.11 & 1.30 & 1.03 & 1.16 & 1.28 & 1.60 & 1.05 & 1.23 & 1.32 & 1.69 \\
\hline ML4 & 1.00 & 1.06 & 1.25 & 1.45 & 1.13 & 1.21 & 1.41 & 1.67 & 1.13 & 1.22 & 1.42 & 1.68 \\
\hline ML5 & 1.01 & 1.06 & 1.26 & 1.46 & 1.13 & 1.21 & 1.41 & 1.67 & 1.13 & 1.22 & 1.42 & 1.68 \\
\hline ML6 & 0.91 & 0.98 & 1.13 & 1.35 & 1.05 & 1.19 & 1.32 & 1.64 & 1.07 & 1.27 & 1.34 & 1.74 \\
\hline MH1 & 0.93 & 1.01 & 1.16 & 1.39 & 1.09 & 1.19 & 1.37 & 1.64 & 1.11 & 1.25 & 1.39 & 1.71 \\
\hline $\mathrm{MH} 2$ & 0.98 & 1.05 & 1.23 & 1.44 & 1.02 & 1.16 & 1.27 & 1.59 & 1.03 & 1.24 & 1.29 & 1.71 \\
\hline MH3 & 1.10 & 1.39 & 1.37 & 1.91 & 1.37 & 1.61 & 1.72 & 2.21 & 1.41 & 1.63 & 1.76 & 2.24 \\
\hline MH4 & 0.97 & 1.02 & 1.22 & 1.41 & 0.97 & 1.11 & 1.22 & 1.53 & 0.99 & 1.18 & 1.24 & 1.63 \\
\hline MH5 & 0.97 & 1.05 & 1.22 & 1.45 & 0.98 & 1.16 & 1.22 & 1.60 & 1.02 & 1.24 & 1.27 & 1.71 \\
\hline MH6 & 1.14 & 1.23 & 1.43 & 1.69 & 1.16 & 1.26 & 1.45 & 1.74 & 1.32 & 1.51 & 1.65 & 2.07 \\
\hline LL1 & 1.14 & 1.20 & 1.42 & 1.65 & 1.31 & 1.40 & 1.64 & 1.93 & 1.31 & 1.46 & 1.63 & 2.00 \\
\hline LL2 & 1.15 & 1.22 & 1.44 & 1.68 & 1.30 & 1.42 & 1.62 & 1.95 & 1.29 & 1.48 & 1.62 & 2.03 \\
\hline LL3 & 1.05 & 1.18 & 1.31 & 1.62 & 1.06 & 1.30 & 1.33 & 1.79 & 1.16 & 1.40 & 1.45 & 1.92 \\
\hline LL4 & 1.07 & 1.16 & 1.34 & 1.60 & 1.24 & 1.38 & 1.55 & 1.90 & 1.24 & 1.45 & 1.55 & 2.00 \\
\hline LL5 & 1.07 & 1.16 & 1.34 & 1.59 & 1.25 & 1.38 & 1.56 & 1.90 & 1.25 & 1.45 & 1.56 & 1.99 \\
\hline LL6 & 1.53 & 1.84 & 1.92 & 2.53 & 1.62 & 1.93 & 2.02 & 2.65 & 1.65 & 1.95 & 2.06 & 2.68 \\
\hline LH1 & 1.08 & 1.19 & 1.36 & 1.64 & 1.18 & 1.42 & 1.48 & 1.96 & 1.33 & 1.55 & 1.67 & 2.13 \\
\hline LH2 & 1.18 & 1.44 & 1.48 & 1.97 & 1.38 & 1.63 & 1.73 & 2.24 & 1.62 & 1.85 & 2.02 & 2.55 \\
\hline LH3 & 1.35 & 1.69 & 1.69 & 2.32 & 1.75 & 2.10 & 2.19 & 2.89 & 1.80 & 2.13 & 2.25 & 2.92 \\
\hline LH4 & 1.16 & 1.40 & 1.45 & 1.93 & 1.36 & 1.58 & 1.70 & 2.18 & 1.62 & 1.83 & 2.03 & 2.52 \\
\hline LH5 & 1.18 & 1.43 & 1.48 & 1.96 & 1.40 & 1.63 & 1.76 & 2.24 & 1.63 & 1.83 & 2.03 & 2.52 \\
\hline LH6 & 1.60 & 2.00 & 2.01 & 2.75 & 1.70 & 2.08 & 2.12 & 2.86 & 1.72 & 2.06 & 2.15 & 2.83 \\
\hline
\end{tabular}


Table C.44 - The ratio of actual mid-span bending moments and quarter-span shear forces for buildings to the corresponding values obtained from FEMA parabolic distribution and a uniform distribution of seismic load when subjected to M6C31 ground motion and the Load is acting parallel to the short side, $\alpha=0.05$

\begin{tabular}{|c|c|c|c|c|c|c|c|c|c|c|c|c|}
\hline \multirow{2}{*}{ Building } & \multicolumn{4}{|c|}{$\mu=2$} & \multicolumn{4}{|c|}{$\mu=3$} & \multicolumn{4}{|c|}{$\mu=4$} \\
\hline & $M . F$ & $V . F$ & $M . U$ & V.U & $M . F$ & $V . F$ & M.U & $V . U$ & $M . F$ & V.F & $M . U$ & $V . U$ \\
\hline SL1 & 1.10 & 1.16 & 1.37 & 1.60 & 1.11 & 1.18 & 1.38 & 1.63 & 1.12 & 1.22 & 1.41 & 1.67 \\
\hline SL3 & 11 & 1.18 & 1.39 & 1.62 & 1.12 & 1.20 & 1.40 & 1.65 & 1.14 & 1.24 & 1.43 & 1.70 \\
\hline SL4 & 13 & 1.19 & 1.41 & 1.64 & 1.14 & 1.23 & 1.43 & 1.69 & 1.15 & 1.25 & 1.44 & 1.72 \\
\hline SH1 & .10 & 1.14 & 1.38 & 1.57 & 1.16 & 1.19 & 1.45 & 1.64 & 1.18 & 1.24 & 1.48 & 1.70 \\
\hline $\mathrm{SH} 2$ & 1.16 & 1.20 & 1.45 & 1.64 & 1.20 & 1.27 & 1.50 & 1.74 & 1.23 & 1.31 & 1.53 & 1.80 \\
\hline $\mathrm{SH} 3$ & 1.19 & 1.26 & 1.48 & 1.74 & 1.22 & 1.32 & 1.53 & 1.82 & 1.24 & 1.36 & 1.56 & 1.87 \\
\hline SH4 & 1.20 & 1.32 & 1.50 & 1.81 & 1.23 & 1.36 & 1.53 & 1.87 & 1.24 & 1.39 & 1.55 & 1.91 \\
\hline SH5 & 1.20 & 1.32 & 1.50 & 1.82 & 1.23 & 1.37 & 1.54 & 1.88 & 1.24 & 1.39 & 1.55 & 1.91 \\
\hline SH6 & 1.19 & 1.26 & 1.48 & 1.74 & 1.22 & 1.33 & 1.53 & 1.83 & 1.24 & 1.36 & 1.55 & 1.87 \\
\hline ML1 & 1.24 & 1.40 & 1.55 & 1.93 & 1.26 & 1.43 & 1.57 & 1.97 & 1.25 & 1.42 & 1.56 & 1.95 \\
\hline ML2 & 1.19 & 1.29 & 1.49 & 1.78 & 1.17 & 1.36 & 1.46 & 1.87 & 1.13 & 1.40 & 1.41 & 1.92 \\
\hline L3 & 1.10 & 1.21 & 1.37 & 1.67 & 1.09 & 1.28 & 1.36 & 1.76 & 1.06 & 1.34 & 1.33 & 1.84 \\
\hline ML4 & 1.18 & 1.32 & 1.48 & 1.82 & 1.20 & 1.36 & 1.50 & 1.87 & 1.21 & 1.39 & 1.51 & 1.91 \\
\hline ML5 & 1.19 & 1.33 & 1.48 & 1.82 & 1.20 & 1.36 & 1.50 & 1.88 & 1.21 & 1.39 & 1.51 & 1.90 \\
\hline ML6 & 1.16 & 1.27 & 1.45 & 1.74 & 1.14 & 1.34 & 1.42 & 1.85 & 1.10 & 1.39 & 1.38 & 1.92 \\
\hline MH1 & 1.08 & 1.14 & 1.35 & 1.57 & 1.08 & 1.22 & 1.34 & 1.68 & 1.06 & 1.28 & 1.33 & 1.76 \\
\hline $\mathrm{MH} 2$ & 1.13 & 1.19 & 1.42 & 1.63 & 1.12 & 1.21 & 1.40 & 1.67 & 1.10 & 1.22 & 1.38 & 1.67 \\
\hline MH3 & 1.20 & 1.40 & 1.50 & 1.92 & 1.23 & 1.42 & 1.54 & 1.95 & 1.26 & 1.45 & 1.57 & 1.99 \\
\hline MH4 & 1.16 & 1.23 & 1.45 & 1.70 & 1.15 & 1.26 & 1.44 & 1.73 & 1.12 & 1.25 & 1.41 & 1.72 \\
\hline MH5 & 1.16 & 1.25 & 1.45 & 1.72 & 1.15 & 1.27 & 1.44 & 1.75 & 1.12 & 1.27 & 1.41 & 1.75 \\
\hline MH6 & 1.26 & 1.40 & 1.57 & 1.93 & 1.41 & 1.58 & 1.76 & 2.18 & 1.52 & 1.76 & 1.90 & 2.42 \\
\hline LL1 & 1.17 & 1.22 & 1.46 & 1.68 & 1.26 & 1.36 & 1.57 & 1.86 & 1.30 & 1.44 & 1.63 & 1.98 \\
\hline LL2 & 1.19 & 1.26 & 1.49 & 1.73 & 1.27 & 1.39 & 1.58 & 1.91 & 1.31 & 1.45 & 1.63 & 1.99 \\
\hline LL3 & 1.02 & 1.22 & 1.28 & 1.67 & 1.05 & 1.30 & 1.31 & 1.79 & 1.10 & 1.37 & 1.37 & 1.89 \\
\hline LL4 & 1.26 & 1.35 & 1.58 & 1.86 & 1.28 & 1.37 & 1.60 & 1.89 & 1.25 & 1.39 & 1.56 & 1.91 \\
\hline LL5 & 1.26 & 1.34 & 1.57 & 1.84 & 1.28 & 1.38 & 1.60 & 1.89 & 1.26 & 1.39 & 1.57 & 1.91 \\
\hline LL6 & 0.98 & 1.37 & 1.23 & 1.88 & 0.98 & 1.43 & 1.22 & 1.96 & 1.02 & 1.45 & 1.27 & 2.00 \\
\hline LH1 & 1.11 & 1.34 & 1.38 & 1.84 & 1.14 & 1.44 & 1.43 & 1.97 & 1.20 & 1.52 & 1.49 & 2.09 \\
\hline LH2 & 1.18 & 1.42 & 1.47 & 1.96 & 1.19 & 1.52 & 1.49 & 2.09 & 1.21 & 1.60 & 1.52 & 2.19 \\
\hline LH3 & 1.10 & 1.37 & 1.37 & 1.88 & 1.19 & 1.46 & 1.49 & 2.01 & 1.25 & 1.52 & 1.57 & 2.09 \\
\hline LH4 & 1.15 & 1.34 & 1.44 & 1.84 & 1.17 & 1.46 & 1.46 & 2.01 & 1.18 & 1.57 & 1.48 & 2.16 \\
\hline LH5 & 1.16 & 1.32 & 1.45 & 1.82 & 1.17 & 1.46 & 1.47 & 2.00 & 1.18 & 1.56 & 1.48 & 2.15 \\
\hline LH6 & 1.04 & 1.42 & 1.29 & 1.95 & 1.10 & 1.47 & 1.38 & 2.02 & 1.17 & 1.47 & 1.46 & 2.02 \\
\hline
\end{tabular}


Table C.45 - The ratio of actual mid-span bending moments and quarter-span shear forces for buildings to the corresponding values obtained from FEMA parabolic distribution and a uniform distribution of seismic load when subjected to M6C 38 ground motion and the Load is acting parallel to the short side, $\alpha=0.05$

\begin{tabular}{|c|c|c|c|c|c|c|c|c|c|c|c|c|}
\hline \multirow{2}{*}{ Building } & \multicolumn{4}{|c|}{$\mu=2$} & \multicolumn{4}{|c|}{$\mu=3$} & \multicolumn{4}{|c|}{$\mu=4$} \\
\hline & $M . F$ & V.F & $M . U$ & V.U & $M . F$ & V.F & $M . U$ & $V . U$ & M.F & V.F & M.U & $V . U$ \\
\hline SL1 & 1.17 & 1.27 & 1.47 & 1.75 & 1.18 & 1.28 & 1.47 & 1.76 & 1.17 & 1.27 & 1.47 & 1.75 \\
\hline SL3 & 18 & 1.28 & 1.47 & 1.76 & 1.18 & 1.28 & 1.48 & 1.77 & 1.18 & 1.28 & 1.47 & 1.76 \\
\hline SL4 & 18 & 1.28 & 1.48 & 1.76 & 1.18 & 1.29 & 1.48 & 1.78 & 1.18 & 1.29 & 1.48 & 1.77 \\
\hline SH1 & 1.15 & 1.19 & 1.43 & 1.63 & 1.20 & 1.26 & 1.50 & 1.73 & 1.23 & 1.30 & 1.53 & 1.79 \\
\hline $\mathrm{SH} 2$ & 1.16 & 1.18 & 1.45 & 1.63 & 1.21 & 1.26 & 1.51 & 1.73 & 1.24 & 1.31 & 1.55 & 1.80 \\
\hline SH3 & 1.17 & 1.19 & 1.46 & 1.64 & 1.23 & 1.27 & 1.53 & 1.75 & 1.26 & 1.32 & 1.57 & 1.82 \\
\hline SH4 & 1.17 & 1.18 & 1.46 & 1.62 & 1.23 & 1.26 & 1.53 & 1.73 & 1.25 & 1.30 & 1.56 & 1.78 \\
\hline SH5 & 1.18 & 1.19 & 1.47 & 1.63 & 1.23 & 1.27 & 1.54 & 1.74 & 1.26 & 1.31 & 1.58 & 1.81 \\
\hline SH6 & 1.17 & 1.19 & 1.46 & 1.64 & 1.23 & 1.27 & 1.53 & 1.75 & 1.26 & 1.32 & 1.57 & 1.81 \\
\hline ML1 & 17 & 1.17 & 1.46 & 1.61 & 1.22 & 1.24 & 1.53 & 1.70 & 1.26 & 1.29 & 1.57 & 1.78 \\
\hline ML2 & 14 & 1.17 & 1.43 & 1.62 & 1.21 & 1.23 & 1.51 & 1.69 & 1.25 & 1.28 & 1.56 & 1.76 \\
\hline ML3 & 13 & 1.18 & 1.41 & 1.62 & 1.21 & 1.26 & 1.51 & 1.74 & 1.26 & 1.32 & 1.57 & 1.81 \\
\hline ML4 & 11 & 1.09 & 1.38 & 1.49 & 1.23 & 1.23 & 1.54 & 1.69 & 1.26 & 1.28 & 1.58 & 1.76 \\
\hline ML5 & 1.10 & 1.07 & 1.37 & 1.48 & 1.23 & 1.23 & 1.54 & 1.69 & 1.26 & 1.28 & 1.58 & 1.76 \\
\hline ML6 & 1.14 & 1.18 & 1.43 & 1.62 & 1.22 & 1.26 & 1.52 & 1.73 & 1.27 & 1.31 & 1.58 & 1.81 \\
\hline MH1 & 0.99 & 1.04 & 1.23 & 1.43 & 1.17 & 1.23 & 1.46 & 1.69 & 1.23 & 1.30 & 1.54 & 1.78 \\
\hline MH2 & 0.89 & 0.96 & 1.12 & 1.32 & 1.15 & 1.25 & 1.44 & 1.73 & 1.22 & 1.33 & 1.53 & 1.83 \\
\hline MH3 & 0.93 & 0.90 & 1.16 & 1.23 & 1.02 & 1.01 & 1.27 & 1.38 & 1.13 & 1.27 & 1.41 & 1.74 \\
\hline MH4 & 0.86 & 0.93 & 1.08 & 1.28 & 0.97 & 1.04 & 1.21 & 1.43 & 1.15 & 1.27 & 1.43 & 1.75 \\
\hline MH5 & 0.87 & 0.94 & 1.09 & 1.29 & 1.12 & 1.25 & 1.40 & 1.71 & 1.18 & 1.32 & 1.48 & 1.81 \\
\hline MH6 & 1.15 & 1.17 & 1.44 & 1.61 & 1.26 & 1.33 & 1.58 & 1.82 & 1.32 & 1.42 & 1.65 & 1.95 \\
\hline LL1 & 0.98 & 0.99 & 1.23 & 1.36 & 1.08 & 1.12 & 1.35 & 1.54 & 1.12 & 1.19 & 1.40 & 1.63 \\
\hline LL2 & 0.97 & 0.96 & 1.21 & 1.33 & 1.07 & 1.10 & 1.34 & 1.52 & 1.12 & 1.17 & 1.40 & 1.61 \\
\hline LL3 & 0.87 & 0.86 & 1.09 & 1.18 & 0.97 & 1.10 & 1.22 & 1.51 & 1.04 & 1.17 & 1.30 & 1.61 \\
\hline LL4 & 1.07 & 1.08 & 1.34 & 1.48 & 1.14 & 1.17 & 1.43 & 1.60 & 1.19 & 1.24 & 1.49 & 1.71 \\
\hline LL5 & 1.08 & 1.10 & 1.35 & 1.51 & 1.15 & 1.17 & 1.43 & 1.61 & 1.19 & 1.24 & 1.49 & 1.71 \\
\hline LL6 & 1.06 & 1.16 & 1.33 & 1.59 & 1.18 & 1.31 & 1.48 & 1.80 & 1.25 & 1.45 & 1.56 & 2.00 \\
\hline LH1 & 0.93 & 0.92 & 1.17 & 1.26 & 0.95 & 1.08 & 1.19 & 1.49 & 1.02 & 1.17 & 1.28 & 1.60 \\
\hline LH2 & 0.99 & 0.96 & 1.23 & 1.31 & 0.92 & 1.06 & 1.16 & 1.46 & 1.00 & 1.16 & 1.25 & 1.59 \\
\hline LH3 & 1.06 & 1.15 & 1.33 & 1.57 & 1.09 & 1.18 & 1.36 & 1.63 & 1.17 & 1.31 & 1.46 & 1.80 \\
\hline LH4 & 0.96 & 0.94 & 1.20 & 1.29 & 0.93 & 1.09 & 1.16 & 1.50 & 1.02 & 1.19 & 1.27 & 1.63 \\
\hline LH5 & 0.96 & 0.94 & 1.20 & 1.29 & 0.93 & 1.10 & 1.17 & 1.51 & 1.01 & 1.19 & 1.27 & 1.64 \\
\hline LH6 & 1.05 & 1.12 & 1.31 & 1.54 & 1.11 & 1.24 & 1.39 & 1.70 & 1.20 & 1.37 & 1.50 & 1.88 \\
\hline
\end{tabular}


Table C.46 - The ratio of actual mid-span bending moments and quarter-span shear forces for buildings to the corresponding values obtained from FEMA parabolic distribution and a uniform distribution of seismic load when subjected to $\mathrm{M} 6 \mathrm{C} 1$ ground motion and the Load is acting parallel to the long side, $\alpha=0.05$

\begin{tabular}{|c|c|c|c|c|c|c|c|c|c|c|c|c|}
\hline \multirow{2}{*}{ Building } & \multicolumn{4}{|c|}{$\mu=2$} & \multicolumn{4}{|c|}{$\mu=3$} & \multicolumn{4}{|c|}{$\mu=4$} \\
\hline & $M . F$ & $V . F$ & $M . U$ & V.U & M.F & $V . F$ & $M . U$ & $V . U$ & M.F & V.F & M.U & $V . U$ \\
\hline SL1 & 1.00 & 1.02 & 1.26 & 1.40 & 1.02 & 1.05 & 1.27 & 1.44 & 1.04 & 1.09 & 1.29 & 1.50 \\
\hline SL3 & 01 & 1.02 & 1.26 & 1.41 & 1.02 & 1.06 & 1.28 & 1.45 & 1.03 & 1.08 & 1.29 & 1.49 \\
\hline & 00 & 1.03 & 1.25 & 1.41 & 1.00 & 1.03 & 1.25 & 1.41 & 1.00 & 1.06 & 1.25 & 1.45 \\
\hline SH1 & 1.21 & 1.34 & 1.52 & 1.84 & 1.19 & 1.32 & 1.49 & 1.82 & 1.14 & 1.26 & 1.43 & 1.74 \\
\hline & 1.10 & 1.15 & 1.37 & 1.58 & 1.12 & 1.23 & 1.40 & 1.69 & 1.06 & 1.17 & 1.33 & 1.60 \\
\hline $\mathrm{SH} 3$ & 1.06 & 1.12 & 1.32 & 1.54 & 1.05 & 1.13 & 1.31 & 1.56 & 1.04 & 1.12 & 1.30 & 1.54 \\
\hline SH4 & 1.07 & 1.13 & 1.34 & 1.55 & 1.08 & 1.16 & 1.35 & 1.60 & 1.06 & 1.15 & 1.32 & 1.59 \\
\hline SH5 & 1.05 & 1.08 & 1.31 & 1.49 & 1.05 & 1.10 & 1.32 & 1.52 & 1.06 & 1.14 & 1.33 & 1.57 \\
\hline IL1 & 1.09 & 1.16 & 1.36 & 1.60 & 1.16 & 1.30 & 1.46 & 1.79 & 1.17 & 1.32 & 1.46 & 1.82 \\
\hline ML2 & 1.11 & 1.20 & 1.39 & 1.65 & 1.19 & 1.32 & 1.49 & 1.81 & 1.17 & 1.32 & 1.47 & 1.82 \\
\hline ML3 & 1.06 & 1.14 & 1.32 & 1.56 & 1.15 & 1.27 & 1.44 & 1.75 & 1.18 & 1.32 & 1.47 & 1.82 \\
\hline ML4 & 1.11 & 1.20 & 1.39 & 1.66 & 1.19 & 1.32 & 1.49 & 1.82 & 1.19 & 1.34 & 1.48 & 1.84 \\
\hline ML5 & 1.11 & 1.20 & 1.38 & 1.65 & 1.17 & 1.29 & 1.46 & 1.78 & 1.18 & 1.33 & 1.48 & 1.83 \\
\hline ML6 & 1.09 & 1.18 & 1.36 & 1.62 & 1.17 & 1.29 & 1.46 & 1.77 & 1.18 & 1.32 & 1.47 & 1.82 \\
\hline & .07 & 1.13 & 1.33 & 1.55 & 1.20 & 1.35 & 1.50 & 1.86 & 1.22 & 1.39 & 1.52 & 1.92 \\
\hline & 20 & 1.33 & 1.50 & 1.83 & 1.23 & 1.41 & 1.54 & 1.93 & 1.20 & 1.37 & 1.50 & 1.88 \\
\hline & 1.26 & 1.40 & 1.58 & 1.92 & 1.34 & 1.54 & 1.68 & 2.12 & 1.34 & 1.55 & 1.67 & 2.14 \\
\hline MH4 & 1.06 & 1.13 & 1.33 & 1.55 & 1.22 & 1.38 & 1.53 & 1.90 & 1.23 & 1.42 & 1.53 & 1.96 \\
\hline MH5 & 1.17 & 1.29 & 1.47 & 1.77 & 1.24 & 1.40 & 1.55 & 1.92 & 1.23 & 1.41 & 1.53 & 1.93 \\
\hline MH6 & 1.15 & 1.25 & 1.44 & 1.72 & 1.18 & 1.32 & 1.48 & 1.81 & 1.14 & 1.30 & 1.43 & 1.79 \\
\hline LL1 & 1.17 & 1.29 & 1.46 & 1.77 & 1.21 & 1.35 & 1.51 & 1.85 & 1.16 & 1.30 & 1.45 & 1.78 \\
\hline LL2 & 1.14 & 1.24 & 1.43 & 1.70 & 1.22 & 1.36 & 1.52 & 1.88 & 1.18 & 1.32 & 1.47 & 1.81 \\
\hline LL3 & 1.26 & 1.38 & 1.58 & 1.90 & 1.28 & 1.43 & 1.60 & 1.97 & 1.26 & 1.42 & 1.57 & 1.95 \\
\hline LL4 & 1.16 & 1.27 & 1.45 & 1.75 & 1.20 & 1.34 & 1.50 & 1.84 & 1.16 & 1.30 & 1.45 & 1.79 \\
\hline LL5 & 1.19 & 1.32 & 1.49 & 1.82 & 1.24 & 1.42 & 1.55 & 1.95 & 1.21 & 1.38 & 1.52 & 1.89 \\
\hline LL6 & 1.20 & 1.34 & 1.50 & 1.84 & 1.32 & 1.57 & 1.65 & 2.16 & 1.31 & 1.54 & 1.64 & 2.12 \\
\hline LH1 & 1.10 & 1.16 & 1.37 & 1.59 & 1.17 & 1.29 & 1.47 & 1.77 & 1.13 & 1.25 & 1.41 & 1.72 \\
\hline LH2 & 1.19 & 1.31 & 1.49 & 1.80 & 1.19 & 1.33 & 1.49 & 1.83 & 1.16 & 1.32 & 1.45 & 1.81 \\
\hline LH3 & 1.11 & 1.23 & 1.39 & 1.68 & 1.26 & 1.51 & 1.58 & 2.08 & 1.32 & 1.51 & 1.65 & 2.07 \\
\hline LH4 & 1.18 & 1.27 & 1.47 & 1.74 & 1.14 & 1.24 & 1.43 & 1.71 & 1.10 & 1.21 & 1.38 & 1.66 \\
\hline LH5 & 1.25 & 1.36 & 1.56 & 1.87 & 1.23 & 1.36 & 1.54 & 1.87 & 1.21 & 1.40 & 1.51 & 1.92 \\
\hline LH6 & 1.04 & 1.08 & 1.30 & 1.49 & 1.35 & 1.53 & 1.69 & 2.11 & 1.41 & 1.63 & 1.76 & 2.24 \\
\hline
\end{tabular}


Table C.47 - The ratio of actual mid-span bending moments and quarter-span shear forces for buildings to the corresponding values obtained from FEMA parabolic distribution and a uniform distribution of seismic load when subjected to M6C2 ground motion and the Load is acting parallel to the long side, $\alpha=0.05$

\begin{tabular}{|c|c|c|c|c|c|c|c|c|c|c|c|c|}
\hline \multirow{2}{*}{ Building } & \multicolumn{4}{|c|}{$\mu=2$} & \multicolumn{4}{|c|}{$\mu=3$} & \multicolumn{4}{|c|}{$\mu=4$} \\
\hline & $M . F$ & $V . F$ & $M . U$ & V.U & M.F & $V . F$ & $M . U$ & $V . U$ & M.F & V.F & M.U & $V . U$ \\
\hline SL1 & 1.07 & 1.12 & 1.34 & 1.55 & 1.08 & 1.16 & 1.35 & 1.60 & 1.05 & 1.15 & 1.32 & 1.58 \\
\hline SL3 & 06 & 1.10 & 1.33 & 1.52 & 1.05 & 1.13 & 1.32 & 1.55 & 1.03 & 1.12 & 1.28 & 1.54 \\
\hline & .06 & 1.09 & 1.33 & 1.50 & 1.01 & 1.05 & 1.26 & 1.44 & 1.01 & 1.09 & 1.26 & 1.49 \\
\hline SH1 & 1.13 & 1.19 & 1.41 & 1.64 & 1.25 & 1.39 & 1.57 & 1.91 & 1.32 & 1.51 & 1.65 & 2.07 \\
\hline & 1.07 & 1.12 & 1.34 & 1.54 & 1.14 & 1.23 & 1.43 & 1.68 & 1.25 & 1.40 & 1.56 & 1.93 \\
\hline $\mathrm{SH} 3$ & 1.09 & 1.18 & 1.37 & 1.62 & 1.13 & 1.23 & 1.41 & 1.69 & 1.22 & 1.36 & 1.52 & 1.87 \\
\hline SH4 & 1.08 & 1.16 & 1.35 & 1.59 & 1.12 & 1.22 & 1.40 & 1.67 & 1.18 & 1.28 & 1.47 & 1.76 \\
\hline SH5 & 1.11 & 1.20 & 1.39 & 1.65 & 1.13 & 1.23 & 1.41 & 1.70 & 1.19 & 1.31 & 1.48 & 1.80 \\
\hline IL1 & 1.04 & 1.10 & 1.30 & 1.51 & 1.03 & 1.11 & 1.28 & 1.52 & 1.08 & 1.15 & 1.35 & 1.58 \\
\hline ML2 & 1.08 & 1.14 & 1.35 & 1.57 & 1.09 & 1.16 & 1.36 & 1.59 & 1.13 & 1.20 & 1.41 & 1.66 \\
\hline ML3 & 1.14 & 1.23 & 1.43 & 1.70 & 1.17 & 1.29 & 1.47 & 1.77 & 1.20 & 1.32 & 1.50 & 1.82 \\
\hline ML4 & 1.06 & 1.12 & 1.33 & 1.53 & 1.07 & 1.13 & 1.33 & 1.55 & 1.12 & 1.19 & 1.40 & 1.64 \\
\hline ML5 & 1.09 & 1.16 & 1.36 & 1.60 & 1.11 & 1.18 & 1.38 & 1.63 & 1.14 & 1.22 & 1.42 & 1.68 \\
\hline ML6 & 1.12 & 1.21 & 1.40 & 1.66 & 1.15 & 1.25 & 1.44 & 1.73 & 1.18 & 1.30 & 1.48 & 1.78 \\
\hline MH1 & 16 & 1.23 & 1.45 & 1.69 & 1.23 & 1.36 & 1.54 & 1.88 & 1.30 & 1.48 & 1.63 & 2.03 \\
\hline & 26 & 1.37 & 1.57 & 1.88 & 1.42 & 1.62 & 1.78 & 2.23 & 1.46 & 1.68 & 1.83 & 2.31 \\
\hline & 1.22 & 1.32 & 1.52 & 1.81 & 1.24 & 1.34 & 1.55 & 1.84 & 1.34 & 1.53 & 1.67 & 2.11 \\
\hline MH4 & 1.13 & 1.18 & 1.41 & 1.62 & 1.24 & 1.37 & 1.55 & 1.88 & 1.34 & 1.53 & 1.67 & 2.10 \\
\hline MH5 & 1.42 & 1.61 & 1.77 & 2.21 & 1.47 & 1.67 & 1.83 & 2.30 & 1.49 & 1.69 & 1.86 & 2.33 \\
\hline MH6 & 1.05 & 1.07 & 1.32 & 1.48 & 1.30 & 1.46 & 1.63 & 2.01 & 1.38 & 1.59 & 1.73 & 2.19 \\
\hline LL1 & 1.12 & 1.16 & 1.40 & 1.60 & 1.21 & 1.33 & 1.51 & 1.83 & 1.28 & 1.45 & 1.60 & 1.99 \\
\hline LL2 & 1.08 & 1.11 & 1.35 & 1.52 & 1.25 & 1.38 & 1.56 & 1.89 & 1.34 & 1.53 & 1.68 & 2.11 \\
\hline LL3 & 1.16 & 1.26 & 1.46 & 1.73 & 1.21 & 1.40 & 1.52 & 1.92 & 1.38 & 1.58 & 1.73 & 2.18 \\
\hline LL4 & 1.12 & 1.16 & 1.40 & 1.59 & 1.22 & 1.33 & 1.52 & 1.83 & 1.28 & 1.45 & 1.60 & 1.99 \\
\hline LL5 & 1.18 & 1.25 & 1.48 & 1.72 & 1.41 & 1.60 & 1.76 & 2.20 & 1.45 & 1.66 & 1.81 & 2.29 \\
\hline LL6 & 1.41 & 1.62 & 1.77 & 2.22 & 1.53 & 1.81 & 1.92 & 2.49 & 1.49 & 1.76 & 1.87 & 2.42 \\
\hline LH1 & 1.19 & 1.28 & 1.49 & 1.76 & 1.34 & 1.52 & 1.68 & 2.08 & 1.40 & 1.61 & 1.75 & 2.22 \\
\hline LH2 & 1.38 & 1.53 & 1.72 & 2.11 & 1.44 & 1.66 & 1.80 & 2.28 & 1.44 & 1.66 & 1.81 & 2.28 \\
\hline LH3 & 1.45 & 1.69 & 1.82 & 2.32 & 1.45 & 1.68 & 1.81 & 2.31 & 1.39 & 1.62 & 1.74 & 2.22 \\
\hline LH4 & 1.23 & 1.35 & 1.54 & 1.86 & 1.37 & 1.55 & 1.71 & 2.13 & 1.40 & 1.60 & 1.74 & 2.19 \\
\hline LH5 & 1.13 & 1.20 & 1.42 & 1.66 & 1.21 & 1.35 & 1.51 & 1.86 & 1.38 & 1.59 & 1.73 & 2.19 \\
\hline LH6 & 1.40 & 1.57 & 1.75 & 2.17 & 1.34 & 1.51 & 1.67 & 2.07 & 1.25 & 1.42 & 1.56 & 1.96 \\
\hline
\end{tabular}


Table C.48 - The ratio of actual mid-span bending moments and quarter-span shear forces for buildings to the corresponding values obtained from FEMA parabolic distribution and a uniform distribution of seismic load when subjected to M6C26 ground motion and the Load is acting parallel to the long side, $\alpha=0.05$

\begin{tabular}{|c|c|c|c|c|c|c|c|c|c|c|c|c|}
\hline \multirow{2}{*}{ Building } & \multicolumn{4}{|c|}{$\mu=2$} & \multicolumn{4}{|c|}{$\mu=3$} & \multicolumn{4}{|c|}{$\mu=4$} \\
\hline & $M . F$ & $V . F$ & $M . U$ & V.U & M.F & $V . F$ & $M . U$ & $V . U$ & M.F & V.F & M.U & $V . U$ \\
\hline SL1 & 1.10 & 1.17 & 1.38 & 1.60 & 1.13 & 1.22 & 1.41 & 1.68 & 1.13 & 1.24 & 1.41 & 1.70 \\
\hline SL3 & 10 & 1.16 & 1.38 & 1.60 & 1.13 & 1.22 & 1.41 & 1.68 & 1.13 & 1.25 & 1.42 & 1.72 \\
\hline SL4 & 12 & 1.19 & 1.40 & 1.64 & 1.13 & 1.24 & 1.41 & 1.71 & 1.11 & 1.24 & 1.39 & 1.71 \\
\hline SH1 & 12 & 1.19 & 1.39 & 1.63 & 1.11 & 1.20 & 1.38 & 1.66 & 1.11 & 1.20 & 1.39 & 1.65 \\
\hline & 1.02 & 1.05 & 1.28 & 1.45 & 1.15 & 1.27 & 1.44 & 1.74 & 1.16 & 1.27 & 1.45 & 1.75 \\
\hline $\mathrm{SH} 3$ & 1.02 & 1.07 & 1.28 & 1.47 & 1.19 & 1.32 & 1.48 & 1.82 & 1.21 & 1.35 & 1.51 & 1.86 \\
\hline SH4 & 1.03 & 1.07 & 1.28 & 1.47 & 1.19 & 1.31 & 1.48 & 1.80 & 1.20 & 1.34 & 1.51 & 1.84 \\
\hline SH5 & 1.03 & 1.09 & 1.29 & 1.49 & 1.21 & 1.35 & 1.51 & 1.86 & 1.23 & 1.38 & 1.54 & 1.90 \\
\hline ML1 & 1.08 & 1.14 & 1.35 & 1.57 & 1.09 & 1.18 & 1.37 & 1.62 & 1.09 & 1.19 & 1.37 & 1.63 \\
\hline ML2 & 1.08 & 1.14 & 1.35 & 1.57 & 1.08 & 1.15 & 1.35 & 1.58 & 1.07 & 1.15 & 1.34 & 1.58 \\
\hline ML3 & 1.05 & 1.08 & 1.31 & 1.49 & 1.03 & 1.08 & 1.29 & 1.48 & 1.02 & 1.07 & 1.27 & 1.47 \\
\hline ML4 & 1.08 & 1.14 & 1.36 & 1.57 & 1.09 & 1.16 & 1.36 & 1.60 & 1.08 & 1.16 & 1.35 & 1.59 \\
\hline ML5 & 1.08 & 1.13 & 1.35 & 1.55 & 1.07 & 1.14 & 1.34 & 1.56 & 1.06 & 1.13 & 1.33 & 1.55 \\
\hline ML6 & 1.06 & 1.10 & 1.32 & 1.52 & 1.05 & 1.10 & 1.31 & 1.51 & 1.03 & 1.09 & 1.29 & 1.50 \\
\hline MH1 & 1.01 & 1.04 & 1.26 & 1.43 & 1.00 & 1.04 & 1.25 & 1.43 & 1.01 & 1.07 & 1.26 & 1.47 \\
\hline & & 1.04 & 1.26 & 1.43 & 1.04 & 1.10 & 1.30 & 1.51 & 1.21 & 1.38 & 1.51 & 1.89 \\
\hline & 1.12 & 1.20 & 1.40 & 1.65 & 1.13 & 1.25 & 1.41 & 1.72 & 1.26 & 1.41 & 1.57 & 1.93 \\
\hline MH4 & 1.01 & 1.04 & 1.26 & 1.43 & 1.00 & 1.04 & 1.25 & 1.43 & 1.02 & 1.09 & 1.28 & 1.49 \\
\hline MH5 & 1.04 & 1.07 & 1.30 & 1.48 & 1.06 & 1.13 & 1.32 & 1.55 & 1.22 & 1.38 & 1.52 & 1.89 \\
\hline MH6 & 1.03 & 1.07 & 1.28 & 1.47 & 1.10 & 1.19 & 1.38 & 1.64 & 1.21 & 1.38 & 1.51 & 1.90 \\
\hline LL1 & 1.03 & 1.06 & 1.29 & 1.46 & 1.02 & 1.07 & 1.28 & 1.47 & 1.12 & 1.23 & 1.40 & 1.70 \\
\hline LL2 & 1.03 & 1.06 & 1.29 & 1.46 & 1.02 & 1.07 & 1.28 & 1.47 & 1.14 & 1.26 & 1.43 & 1.73 \\
\hline LL3 & 1.11 & 1.18 & 1.39 & 1.62 & 1.24 & 1.38 & 1.55 & 1.90 & 1.20 & 1.33 & 1.50 & 1.83 \\
\hline LL4 & 1.03 & 1.06 & 1.29 & 1.46 & 1.02 & 1.07 & 1.28 & 1.47 & 1.11 & 1.21 & 1.39 & 1.67 \\
\hline LL5 & 1.02 & 1.06 & 1.28 & 1.46 & 1.04 & 1.10 & 1.30 & 1.51 & 1.23 & 1.39 & 1.53 & 1.91 \\
\hline LL6 & 1.10 & 1.17 & 1.38 & 1.61 & 1.46 & 1.70 & 1.83 & 2.34 & 1.50 & 1.76 & 1.88 & 2.42 \\
\hline LH1 & 1.07 & 1.13 & 1.34 & 1.56 & 1.24 & 1.41 & 1.55 & 1.94 & 1.21 & 1.40 & 1.51 & 1.92 \\
\hline LH2 & 1.08 & 1.15 & 1.35 & 1.58 & 1.25 & 1.44 & 1.57 & 1.97 & 1.24 & 1.44 & 1.55 & 1.98 \\
\hline LH3 & 1.12 & 1.20 & 1.40 & 1.66 & 1.52 & 1.78 & 1.90 & 2.45 & 1.58 & 1.88 & 1.97 & 2.58 \\
\hline LH4 & 1.09 & 1.16 & 1.36 & 1.59 & 1.25 & 1.43 & 1.56 & 1.96 & 1.21 & 1.41 & 1.52 & 1.94 \\
\hline LH5 & 1.08 & 1.14 & 1.35 & 1.57 & 1.26 & 1.42 & 1.57 & 1.95 & 1.25 & 1.41 & 1.57 & 1.94 \\
\hline LH6 & 1.12 & 1.26 & 1.40 & 1.73 & 1.36 & 1.54 & 1.70 & 2.11 & 1.77 & 2.08 & 2.21 & 2.86 \\
\hline
\end{tabular}


Table C.49 - The ratio of actual mid-span bending moments and quarter-span shear forces for buildings to the corresponding values obtained from FEMA parabolic distribution and a uniform distribution of seismic load when subjected to M6C31 ground motion and the Load is acting parallel to the long side, $\alpha=0.05$

\begin{tabular}{|c|c|c|c|c|c|c|c|c|c|c|c|c|}
\hline \multirow{2}{*}{ Building } & \multicolumn{4}{|c|}{$\mu=2$} & \multicolumn{4}{|c|}{$\mu=3$} & \multicolumn{4}{|c|}{$\mu=4$} \\
\hline & $M . F$ & $V . F$ & $M . U$ & $V . U$ & M.F & $V . F$ & $M . U$ & $V . U$ & M.F & V.F & $M . U$ & $V . U$ \\
\hline SL1 & 1.01 & 1.03 & 1.26 & 1.41 & 1.26 & 1.42 & 1.58 & 1.95 & 1.25 & 1.41 & 1.57 & 1.94 \\
\hline SL3 & 00 & 1.02 & 1.26 & 1.41 & 1.13 & 1.24 & 1.42 & 1.70 & 1.24 & 1.40 & 1.55 & 1.93 \\
\hline SL4 & 1.00 & 1.02 & 1.25 & 1.40 & 1.04 & 1.10 & 1.30 & 1.51 & 1.16 & 1.29 & 1.45 & 1.77 \\
\hline SH1 & 1.10 & 1.17 & 1.38 & 1.61 & 1.11 & 1.20 & 1.39 & 1.65 & 1.18 & 1.34 & 1.48 & 1.85 \\
\hline $\mathrm{SH} 2$ & 1.12 & 1.19 & 1.40 & 1.64 & 1.12 & 1.19 & 1.40 & 1.64 & 1.15 & 1.25 & 1.44 & 1.72 \\
\hline $\mathrm{SH} 3$ & 1.09 & 1.15 & 1.36 & 1.58 & 1.08 & 1.14 & 1.35 & 1.56 & 1.15 & 1.27 & 1.44 & 1.74 \\
\hline SH4 & 1.08 & 1.14 & 1.35 & 1.56 & 1.07 & 1.12 & 1.34 & 1.54 & 1.09 & 1.17 & 1.36 & 1.61 \\
\hline H5 & 1.05 & 1.09 & 1.31 & 1.50 & 1.05 & 1.10 & 1.31 & 1.51 & 1.25 & 1.45 & 1.57 & 1.99 \\
\hline ML1 & 1.13 & 1.19 & 1.41 & 1.64 & 1.30 & 1.44 & 1.62 & 1.99 & 1.27 & 1.40 & 1.58 & 1.92 \\
\hline ML2 & 1.12 & 1.18 & 1.40 & 1.63 & 1.27 & 1.42 & 1.59 & 1.95 & 1.29 & 1.43 & 1.62 & 1.97 \\
\hline ML3 & 1.13 & 1.19 & 1.41 & 1.64 & 1.16 & 1.28 & 1.45 & 1.76 & 1.32 & 1.48 & 1.65 & 2.03 \\
\hline ML4 & 1.13 & 1.19 & 1.41 & 1.63 & 1.29 & 1.44 & 1.61 & 1.98 & 1.28 & 1.42 & 1.61 & 1.95 \\
\hline ML5 & 1.12 & 1.18 & 1.40 & 1.63 & 1.26 & 1.39 & 1.57 & 1.92 & 1.30 & 1.44 & 1.62 & 1.98 \\
\hline ML6 & 12 & 1.17 & 1.40 & 1.61 & 1.25 & 1.37 & 1.56 & 1.88 & 1.31 & 1.46 & 1.64 & 2.01 \\
\hline MH1 & 1.03 & 1.06 & 1.28 & 1.46 & 1.11 & 1.20 & 1.38 & 1.65 & 1.18 & 1.34 & 1.48 & 1.84 \\
\hline $\mathrm{H} 2$ & 0.99 & 1.00 & 1.23 & 1.37 & 1.19 & 1.29 & 1.49 & 1.78 & 1.23 & 1.37 & 1.54 & 1.88 \\
\hline MH3 & 1.17 & 1.25 & 1.46 & 1.71 & 1.26 & 1.42 & 1.58 & 1.95 & 1.34 & 1.48 & 1.67 & 2.04 \\
\hline MH4 & 1.03 & 1.06 & 1.29 & 1.46 & 1.15 & 1.28 & 1.44 & 1.75 & 1.18 & 1.33 & 1.47 & 1.83 \\
\hline MH5 & 1.15 & 1.22 & 1.44 & 1.67 & 1.20 & 1.31 & 1.50 & 1.80 & 1.23 & 1.37 & 1.54 & 1.88 \\
\hline MH6 & 0.99 & 1.01 & 1.23 & 1.38 & 1.12 & 1.23 & 1.41 & 1.69 & 1.15 & 1.27 & 1.44 & 1.75 \\
\hline LL1 & 1.08 & 1.15 & 1.35 & 1.57 & 1.18 & 1.31 & 1.47 & 1.81 & 1.18 & 1.33 & 1.48 & 1.83 \\
\hline LL2 & 1.09 & 1.16 & 1.36 & 1.60 & 1.16 & 1.29 & 1.45 & 1.78 & 1.18 & 1.33 & 1.47 & 1.83 \\
\hline LL3 & 1.11 & 1.15 & 1.39 & 1.58 & 1.28 & 1.44 & 1.59 & 1.98 & 1.32 & 1.50 & 1.65 & 2.06 \\
\hline L4 & 1.08 & 1.14 & 1.35 & 1.57 & 1.18 & 1.31 & 1.47 & 1.80 & 1.18 & 1.34 & 1.48 & 1.84 \\
\hline LL5 & 0.98 & 1.00 & 1.23 & 1.37 & 1.17 & 1.27 & 1.47 & 1.75 & 1.21 & 1.34 & 1.52 & 1.84 \\
\hline LL6 & 1.26 & 1.38 & 1.58 & 1.90 & 1.64 & 1.95 & 2.05 & 2.68 & 1.75 & 2.10 & 2.19 & 2.88 \\
\hline LH1 & 1.04 & 1.08 & 1.30 & 1.48 & 1.08 & 1.15 & 1.35 & 1.58 & 1.13 & 1.23 & 1.41 & 1.70 \\
\hline LH2 & 1.05 & 1.10 & 1.31 & 1.51 & 1.18 & 1.30 & 1.48 & 1.79 & 1.31 & 1.48 & 1.63 & 2.03 \\
\hline LH3 & 1.55 & 1.76 & 1.94 & 2.43 & 1.76 & 2.14 & 2.21 & 2.94 & 1.82 & 2.23 & 2.28 & 3.06 \\
\hline LH4 & 1.07 & 1.11 & 1.33 & 1.53 & 1.11 & 1.18 & 1.38 & 1.63 & 1.26 & 1.42 & 1.57 & 1.95 \\
\hline LH5 & 1.22 & 1.36 & 1.53 & 1.88 & 1.37 & 1.56 & 1.71 & 2.14 & 1.39 & 1.59 & 1.74 & 2.18 \\
\hline LH6 & 1.18 & 1.30 & 1.47 & 1.78 & 1.33 & 1.50 & 1.67 & 2.06 & 1.71 & 2.04 & 2.14 & 2.81 \\
\hline
\end{tabular}


Table C.50 - The ratio of actual mid-span bending moments and quarter-span shear forces for buildings to the corresponding values obtained from FEMA parabolic distribution and a uniform distribution of seismic load when subjected to M6C38 ground motion and the Load is acting parallel to the long side, $\alpha=0.05$

\begin{tabular}{|c|c|c|c|c|c|c|c|c|c|c|c|c|}
\hline \multirow{2}{*}{ Building } & \multicolumn{4}{|c|}{$\mu=2$} & \multicolumn{4}{|c|}{$\mu=3$} & \multicolumn{4}{|c|}{$\mu=4$} \\
\hline & $M . F$ & $V . F$ & $M . U$ & V.U & M.F & $V . F$ & $M . U$ & $V . U$ & M.F & V.F & M.U & $V . U$ \\
\hline SL1 & 1.08 & 1.14 & 1.34 & 1.56 & 1.14 & 1.25 & 1.42 & 1.71 & 1.12 & 1.27 & 1.40 & 1.74 \\
\hline SL3 & 08 & 1.13 & 1.35 & 1.56 & 1.16 & 1.25 & 1.45 & 1.72 & 1.13 & 1.24 & 1.41 & 1.70 \\
\hline & 02 & 1.05 & 1.28 & 1.45 & 1.10 & 1.17 & 1.37 & 1.60 & 1.13 & 1.23 & 1.41 & 1.69 \\
\hline SH1 & 10 & 1.17 & 1.37 & 1.61 & 1.10 & 1.17 & 1.37 & 1.61 & 1.09 & 1.16 & 1.37 & 1.60 \\
\hline & 1.12 & 1.19 & 1.40 & 1.64 & 1.12 & 1.20 & 1.40 & 1.64 & 1.11 & 1.18 & 1.39 & 1.63 \\
\hline $\mathrm{SH} 3$ & 1.12 & 1.20 & 1.40 & 1.66 & 1.13 & 1.21 & 1.41 & 1.67 & 1.12 & 1.20 & 1.40 & 1.65 \\
\hline SH4 & 1.13 & 1.21 & 1.41 & 1.66 & 1.13 & 1.21 & 1.41 & 1.66 & 1.12 & 1.20 & 1.40 & 1.65 \\
\hline SH5 & 1.13 & 1.21 & 1.41 & 1.67 & 1.13 & 1.22 & 1.41 & 1.68 & 1.13 & 1.21 & 1.41 & 1.67 \\
\hline ML1 & 1.16 & 1.26 & 1.45 & 1.74 & 1.18 & 1.29 & 1.47 & 1.78 & 1.18 & 1.30 & 1.48 & 1.79 \\
\hline ML2 & 1.17 & 1.27 & 1.46 & 1.74 & 1.18 & 1.30 & 1.48 & 1.79 & 1.19 & 1.31 & 1.48 & 1.80 \\
\hline ML3 & 1.16 & 1.25 & 1.45 & 1.73 & 1.19 & 1.31 & 1.48 & 1.80 & 1.20 & 1.33 & 1.50 & 1.83 \\
\hline ML4 & 1.17 & 1.27 & 1.46 & 1.74 & 1.18 & 1.30 & 1.48 & 1.78 & 1.19 & 1.31 & 1.48 & 1.80 \\
\hline ML5 & 1.17 & 1.27 & 1.46 & 1.74 & 1.18 & 1.30 & 1.48 & 1.79 & 1.19 & 1.31 & 1.49 & 1.81 \\
\hline ML6 & 1.16 & 1.26 & 1.45 & 1.73 & 1.18 & 1.30 & 1.48 & 1.79 & 1.20 & 1.33 & 1.49 & 1.82 \\
\hline & 15 & 1.24 & 1.44 & 1.71 & 1.19 & 1.31 & 1.49 & 1.80 & 1.21 & 1.35 & 1.51 & 1.86 \\
\hline & & 1.21 & 1.41 & 1.66 & 1.19 & 1.31 & 1.49 & 1.80 & 1.22 & 1.37 & 1.53 & 1.88 \\
\hline & 1.17 & 1.23 & 1.47 & 1.69 & 1.26 & 1.36 & 1.57 & 1.87 & 1.31 & 1.45 & 1.64 & 1.99 \\
\hline MH4 & 1.15 & 1.23 & 1.43 & 1.70 & 1.19 & 1.31 & 1.48 & 1.80 & 1.21 & 1.35 & 1.51 & 1.86 \\
\hline MH5 & 1.13 & 1.21 & 1.42 & 1.66 & 1.19 & 1.31 & 1.49 & 1.80 & 1.23 & 1.38 & 1.54 & 1.89 \\
\hline MH6 & 1.13 & 1.21 & 1.42 & 1.67 & 1.19 & 1.31 & 1.49 & 1.81 & 1.22 & 1.37 & 1.52 & 1.88 \\
\hline LL1 & 1.16 & 1.25 & 1.45 & 1.72 & 1.21 & 1.34 & 1.51 & 1.84 & 1.23 & 1.38 & 1.54 & 1.90 \\
\hline LL2 & 1.15 & 1.24 & 1.44 & 1.71 & 1.20 & 1.33 & 1.50 & 1.82 & 1.23 & 1.38 & 1.54 & 1.89 \\
\hline LL3 & 1.16 & 1.23 & 1.45 & 1.69 & 1.24 & 1.36 & 1.55 & 1.86 & 1.30 & 1.45 & 1.62 & 1.99 \\
\hline LL4 & 1.16 & 1.25 & 1.45 & 1.72 & 1.20 & 1.33 & 1.51 & 1.83 & 1.23 & 1.38 & 1.54 & 1.90 \\
\hline LL5 & 1.14 & 1.22 & 1.42 & 1.68 & 1.19 & 1.32 & 1.49 & 1.81 & 1.23 & 1.38 & 1.54 & 1.89 \\
\hline LL6 & 1.18 & 1.22 & 1.48 & 1.68 & 1.29 & 1.38 & 1.61 & 1.90 & 1.35 & 1.48 & 1.69 & 2.04 \\
\hline LH1 & 1.13 & 1.21 & 1.42 & 1.67 & 1.20 & 1.33 & 1.50 & 1.83 & 1.24 & 1.40 & 1.55 & 1.92 \\
\hline LH2 & 1.12 & 1.19 & 1.40 & 1.63 & 1.20 & 1.32 & 1.49 & 1.81 & 1.23 & 1.38 & 1.53 & 1.90 \\
\hline LH3 & 1.17 & 1.22 & 1.46 & 1.67 & 1.28 & 1.39 & 1.59 & 1.91 & 1.36 & 1.53 & 1.70 & 2.10 \\
\hline LH4 & 1.12 & 1.20 & 1.40 & 1.64 & 1.19 & 1.32 & 1.49 & 1.81 & 1.24 & 1.39 & 1.55 & 1.91 \\
\hline LH5 & 1.14 & 1.21 & 1.43 & 1.67 & 1.23 & 1.36 & 1.54 & 1.87 & 1.27 & 1.42 & 1.58 & 1.96 \\
\hline LH6 & 1.19 & 1.23 & 1.49 & 1.69 & 1.31 & 1.41 & 1.64 & 1.94 & 1.40 & 1.56 & 1.75 & 2.14 \\
\hline
\end{tabular}


Table C.51 - The ratio of actual mid-span bending moments and quarter-span shear forces for buildings to the corresponding values obtained from FEMA parabolic distribution and a uniform distribution of seismic load when subjected to E6C1 ground motion and the Load is acting parallel to the short side, $\alpha=0.05$

\begin{tabular}{|c|c|c|c|c|c|c|c|c|c|c|c|c|}
\hline \multirow{2}{*}{ Building } & \multicolumn{4}{|c|}{$\mu=2$} & \multicolumn{4}{|c|}{$\mu=3$} & \multicolumn{4}{|c|}{$\mu=4$} \\
\hline & $M . F$ & V.F & $M . U$ & V.U & $M . F$ & $V . F$ & $M . U$ & V.U & $M . F$ & V.F & $M . U$ & $V . U$ \\
\hline SL1 & 0.93 & 0.93 & 1.17 & 1.28 & 0.93 & 0.90 & 1.16 & 1.24 & 0.92 & 0.89 & 1.15 & 1.23 \\
\hline SL3 & .92 & 0.92 & 1.16 & 1.26 & 0.92 & 0.90 & 1.15 & 1.23 & 0.91 & 0.88 & 1.13 & 1.21 \\
\hline SL4 & 0.90 & 0.90 & 1.13 & 1.24 & 0.89 & 0.88 & 1.12 & 1.22 & 0.89 & 0.87 & 1.11 & 1.20 \\
\hline SH1 & 1.23 & 1.37 & 1.54 & 1.89 & 1.24 & 1.38 & 1.54 & 1.90 & 1.24 & 1.39 & 1.54 & 1.91 \\
\hline $\mathrm{SH} 2$ & 1.24 & 1.39 & 1.55 & 1.91 & 1.22 & 1.35 & 1.53 & 1.86 & 1.22 & 1.37 & 1.53 & 1.88 \\
\hline $\mathrm{SH} 3$ & 1.22 & 1.38 & 1.53 & 1.89 & 1.21 & 1.35 & 1.51 & 1.85 & 1.22 & 1.35 & 1.52 & 1.86 \\
\hline SH4 & 1.20 & 1.35 & 1.50 & 1.86 & 1.19 & 1.33 & 1.49 & 1.83 & 1.20 & 1.32 & 1.50 & 1.81 \\
\hline SH5 & 1.20 & 1.34 & 1.50 & 1.85 & 1.19 & 1.33 & 1.49 & 1.83 & 1.20 & 1.32 & 1.50 & 1.82 \\
\hline SH6 & 1.22 & 1.38 & 1.53 & 1.89 & 1.21 & 1.34 & 1.51 & 1.85 & 1.22 & 1.35 & 1.52 & 1.86 \\
\hline ML1 & 1.12 & 1.20 & 1.40 & 1.64 & 1.17 & 1.24 & 1.46 & 1.71 & 1.19 & 1.27 & 1.49 & 1.74 \\
\hline ML2 & 1.00 & 1.07 & 1.25 & 1.47 & 1.06 & 1.15 & 1.33 & 1.57 & 1.13 & 1.23 & 1.41 & 1.69 \\
\hline L3 & 1.02 & 1.09 & 1.27 & 1.50 & 1.08 & 1.17 & 1.35 & 1.61 & 1.19 & 1.29 & 1.48 & 1.77 \\
\hline ML4 & 1.05 & 1.09 & 1.31 & 1.50 & 1.11 & 1.17 & 1.39 & 1.61 & 1.16 & 1.25 & 1.45 & 1.71 \\
\hline ML5 & 1.05 & 1.09 & 1.31 & 1.49 & 1.12 & 1.17 & 1.39 & 1.61 & 1.16 & 1.25 & 1.45 & 1.71 \\
\hline ML6 & 1.01 & 1.08 & 1.26 & 1.49 & 1.08 & 1.17 & 1.35 & 1.62 & 1.18 & 1.29 & 1.48 & 1.78 \\
\hline MH1 & 1.00 & 1.07 & 1.26 & 1.47 & 1.08 & 1.16 & 1.35 & 1.59 & 1.22 & 1.31 & 1.53 & 1.80 \\
\hline $\mathrm{MH} 2$ & 1.04 & 1.07 & 1.30 & 1.47 & 1.17 & 1.20 & 1.47 & 1.65 & 1.35 & 1.42 & 1.68 & 1.95 \\
\hline MH3 & 1.19 & 1.42 & 1.48 & 1.95 & 1.34 & 1.54 & 1.68 & 2.12 & 1.53 & 1.69 & 1.91 & 2.32 \\
\hline MH4 & 1.05 & 1.05 & 1.31 & 1.45 & 1.13 & 1.15 & 1.42 & 1.59 & 1.24 & 1.28 & 1.55 & 1.76 \\
\hline MH5 & 1.06 & 1.09 & 1.33 & 1.49 & 1.19 & 1.24 & 1.49 & 1.71 & 1.34 & 1.46 & 1.68 & 2.01 \\
\hline MH6 & 1.12 & 1.16 & 1.40 & 1.59 & 1.19 & 1.27 & 1.49 & 1.75 & 1.29 & 1.43 & 1.61 & 1.97 \\
\hline LL1 & 0.97 & 1.08 & 1.21 & 1.49 & 1.02 & 1.17 & 1.28 & 1.60 & 1.12 & 1.18 & 1.40 & 1.62 \\
\hline LL2 & 0.95 & 1.09 & 1.19 & 1.50 & 1.02 & 1.16 & 1.27 & 1.60 & 1.12 & 1.19 & 1.41 & 1.64 \\
\hline LL3 & 1.01 & 1.22 & 1.26 & 1.68 & 1.29 & 1.56 & 1.61 & 2.15 & 1.33 & 1.61 & 1.66 & 2.21 \\
\hline LL4 & 0.98 & 1.01 & 1.23 & 1.39 & 1.06 & 1.10 & 1.32 & 1.51 & 1.20 & 1.24 & 1.50 & 1.70 \\
\hline LL5 & 0.99 & 1.01 & 1.24 & 1.39 & 1.06 & 1.10 & 1.32 & 1.51 & 1.19 & 1.22 & 1.49 & 1.68 \\
\hline LL6 & 1.05 & 1.37 & 1.31 & 1.88 & 1.19 & 1.53 & 1.49 & 2.10 & 1.41 & 1.72 & 1.77 & 2.36 \\
\hline LH1 & 1.13 & 1.35 & 1.42 & 1.85 & 1.33 & 1.61 & 1.67 & 2.21 & 1.37 & 1.66 & 1.71 & 2.28 \\
\hline LH2 & 1.30 & 1.50 & 1.62 & 2.06 & 1.37 & 1.62 & 1.71 & 2.22 & 1.40 & 1.67 & 1.75 & 2.30 \\
\hline LH3 & 1.39 & 1.78 & 1.73 & 2.44 & 1.70 & 2.06 & 2.13 & 2.84 & 1.74 & 2.11 & 2.18 & 2.90 \\
\hline LH4 & 1.32 & 1.57 & 1.65 & 2.15 & 1.39 & 1.68 & 1.74 & 2.31 & 1.43 & 1.74 & 1.78 & 2.39 \\
\hline LH5 & 1.34 & 1.59 & 1.67 & 2.19 & 1.41 & 1.70 & 1.76 & 2.34 & 1.44 & 1.76 & 1.80 & 2.42 \\
\hline LH6 & 1.35 & 1.76 & 1.69 & 2.42 & 1.75 & 2.13 & 2.18 & 2.93 & 1.78 & 2.18 & 2.23 & 2.99 \\
\hline
\end{tabular}


Table C.52 - The ratio of actual mid-span bending moments and quarter-span shear forces for buildings to the corresponding values obtained from FEMA parabolic distribution and a uniform distribution of seismic load when subjected to E6C13 ground motion and the Load is acting parallel to the short side, $\alpha=0.05$

\begin{tabular}{|c|c|c|c|c|c|c|c|c|c|c|c|c|}
\hline \multirow{2}{*}{ Building } & \multicolumn{4}{|c|}{$\mu=2$} & \multicolumn{4}{|c|}{$\mu=3$} & \multicolumn{4}{|c|}{$\mu=4$} \\
\hline & $M . F$ & V.F & $M . U$ & V.U & $M . F$ & V.F & $M . U$ & $V . U$ & M.F & V.F & M.U & $V . U$ \\
\hline SL1 & 1.05 & 1.15 & 1.31 & 1.58 & 1.08 & 1.18 & 1.35 & 1.63 & 1.10 & 1.16 & 1.37 & 1.59 \\
\hline SL3 & 1.08 & 1.16 & 1.35 & 1.59 & 1.11 & 1.17 & 1.38 & 1.61 & 1.12 & 1.17 & 1.40 & 1.61 \\
\hline SL4 & 1.17 & 1.29 & 1.46 & 1.77 & 1.19 & 1.29 & 1.49 & 1.78 & 1.20 & 1.29 & 1.50 & 1.77 \\
\hline SH1 & 1.02 & 1.19 & 1.28 & 1.64 & 1.10 & 1.31 & 1.37 & 1.80 & 1.20 & 1.43 & 1.50 & 1.96 \\
\hline $\mathrm{SH} 2$ & 0.97 & 1.13 & 1.21 & 1.56 & 0.99 & 1.15 & 1.24 & 1.58 & 1.01 & 1.24 & 1.27 & 1.71 \\
\hline SH3 & 0.95 & 1.09 & 1.18 & 1.50 & 0.97 & 1.12 & 1.22 & 1.54 & 0.98 & 1.21 & 1.23 & 1.66 \\
\hline SH4 & 0.92 & 1.06 & 1.15 & 1.46 & 0.94 & 1.05 & 1.18 & 1.44 & 0.95 & 1.11 & 1.19 & 1.52 \\
\hline SH5 & 0.92 & 1.05 & 1.15 & 1.45 & 0.94 & 1.04 & 1.18 & 1.43 & 0.95 & 1.13 & 1.18 & 1.55 \\
\hline SH6 & 0.95 & 1.09 & 1.18 & 1.50 & 0.97 & 1.12 & 1.22 & 1.53 & 0.98 & 1.21 & 1.23 & 1.66 \\
\hline ML1 & 0.93 & 1.00 & 1.17 & 1.38 & 0.94 & 0.98 & 1.18 & 1.35 & 0.94 & 1.01 & 1.18 & 1.39 \\
\hline ML2 & 0.93 & 0.97 & 1.17 & 1.33 & 0.96 & 1.04 & 1.20 & 1.44 & 1.00 & 1.14 & 1.25 & 1.57 \\
\hline ML3 & 0.98 & 1.14 & 1.22 & 1.57 & 1.13 & 1.29 & 1.41 & 1.77 & 1.18 & 1.39 & 1.47 & 1.91 \\
\hline ML4 & 0.92 & 0.96 & 1.15 & 1.32 & 0.93 & 0.96 & 1.16 & 1.33 & 0.92 & 0.96 & 1.15 & 1.32 \\
\hline ML5 & 0.92 & 0.96 & 1.15 & 1.32 & 0.93 & 0.96 & 1.16 & 1.32 & 0.92 & 0.96 & 1.15 & 1.32 \\
\hline ML6 & 0.94 & 1.06 & 1.17 & 1.46 & 1.02 & 1.15 & 1.28 & 1.58 & 1.10 & 1.32 & 1.37 & 1.81 \\
\hline MH1 & 1.07 & 1.27 & 1.34 & 1.75 & 1.11 & 1.31 & 1.39 & 1.80 & 1.12 & 1.31 & 1.40 & 1.80 \\
\hline MH2 & 1.02 & 1.05 & 1.28 & 1.45 & 1.13 & 1.34 & 1.41 & 1.85 & 1.37 & 1.75 & 1.71 & 2.41 \\
\hline MH3 & 1.26 & 1.41 & 1.58 & 1.94 & 1.39 & 1.83 & 1.74 & 2.51 & 1.39 & 1.84 & 1.74 & 2.53 \\
\hline MH4 & 0.96 & 1.01 & 1.20 & 1.38 & 1.07 & 1.29 & 1.34 & 1.77 & 1.31 & 1.65 & 1.64 & 2.27 \\
\hline MH5 & 0.96 & 1.03 & 1.20 & 1.41 & 1.21 & 1.51 & 1.51 & 2.08 & 1.32 & 1.66 & 1.65 & 2.29 \\
\hline MH6 & 0.96 & 1.00 & 1.20 & 1.38 & 0.95 & 1.05 & 1.18 & 1.45 & 0.92 & 1.17 & 1.14 & 1.61 \\
\hline LL1 & 1.04 & 1.22 & 1.30 & 1.67 & 1.22 & 1.27 & 1.52 & 1.74 & 1.21 & 1.42 & 1.52 & 1.95 \\
\hline LL2 & 1.02 & 1.19 & 1.27 & 1.64 & 1.22 & 1.25 & 1.52 & 1.72 & 1.20 & 1.38 & 1.50 & 1.90 \\
\hline LL3 & 1.04 & 1.04 & 1.30 & 1.43 & 1.06 & 1.06 & 1.33 & 1.46 & 1.07 & 1.23 & 1.34 & 1.69 \\
\hline LL4 & 1.06 & 1.23 & 1.33 & 1.69 & 1.11 & 1.30 & 1.39 & 1.79 & 1.15 & 1.49 & 1.43 & 2.05 \\
\hline LL5 & 1.07 & 1.21 & 1.33 & 1.66 & 1.11 & 1.29 & 1.38 & 1.77 & 1.15 & 1.51 & 1.43 & 2.07 \\
\hline LL6 & 0.92 & 1.28 & 1.15 & 1.76 & 0.97 & 1.40 & 1.21 & 1.93 & 1.14 & 1.69 & 1.43 & 2.32 \\
\hline LH1 & 1.07 & 1.14 & 1.33 & 1.57 & 1.08 & 1.12 & 1.35 & 1.54 & 1.19 & 1.49 & 1.48 & 2.05 \\
\hline LH2 & 1.13 & 1.23 & 1.41 & 1.69 & 1.18 & 1.31 & 1.47 & 1.80 & 1.19 & 1.42 & 1.49 & 1.96 \\
\hline LH3 & 1.00 & 1.39 & 1.25 & 1.91 & 1.36 & 1.67 & 1.70 & 2.29 & 1.40 & 1.62 & 1.75 & 2.23 \\
\hline LH4 & 1.14 & 1.25 & 1.43 & 1.72 & 1.15 & 1.41 & 1.44 & 1.94 & 1.22 & 1.57 & 1.52 & 2.16 \\
\hline LH5 & 1.15 & 1.26 & 1.44 & 1.73 & 1.15 & 1.44 & 1.43 & 1.98 & 1.23 & 1.60 & 1.54 & 2.20 \\
\hline LH6 & 0.94 & 1.36 & 1.18 & 1.87 & 1.31 & 1.66 & 1.64 & 2.28 & 1.36 & 1.61 & 1.70 & 2.21 \\
\hline
\end{tabular}


Table C.53 - The ratio of actual mid-span bending moments and quarter-span shear forces for buildings to the corresponding values obtained from FEMA parabolic distribution and a uniform distribution of seismic load when subjected to E6C15 ground motion and the Load is acting parallel to the short side, $\alpha=0.05$

\begin{tabular}{|c|c|c|c|c|c|c|c|c|c|c|c|c|}
\hline \multirow{2}{*}{ Building } & \multicolumn{4}{|c|}{$\mu=2$} & \multicolumn{4}{|c|}{$\mu=3$} & \multicolumn{4}{|c|}{$\mu=4$} \\
\hline & $M . F$ & V.F & $M . U$ & $V . U$ & $M . F$ & $V . F$ & $M . U$ & V.U & M.F & V.F & $M . U$ & $V . U$ \\
\hline SL1 & 1.30 & 1.49 & 1.62 & 2.05 & 1.44 & 1.61 & 1.81 & 2.21 & 1.47 & 1.61 & 1.84 & 2.22 \\
\hline SL3 & 31 & 1.51 & 1.64 & 2.07 & 1.44 & 1.60 & 1.80 & 2.20 & 1.47 & 1.62 & 1.84 & 2.23 \\
\hline SL4 & 25 & 1.47 & 1.56 & 2.02 & 1.41 & 1.59 & 1.76 & 2.18 & 1.48 & 1.63 & 1.85 & 2.24 \\
\hline SH1 & 1.18 & 1.27 & 1.48 & 1.75 & 1.23 & 1.37 & 1.54 & 1.88 & 1.27 & 1.42 & 1.59 & 1.96 \\
\hline $\mathrm{SH} 2$ & 1.25 & 1.41 & 1.57 & 1.94 & 1.32 & 1.51 & 1.65 & 2.08 & 1.32 & 1.50 & 1.65 & 2.06 \\
\hline SH3 & 1.29 & 1.48 & 1.62 & 2.04 & 1.35 & 1.56 & 1.69 & 2.14 & 1.34 & 1.52 & 1.67 & 2.09 \\
\hline SH4 & 1.30 & 1.50 & 1.63 & 2.06 & 1.35 & 1.57 & 1.69 & 2.16 & 1.35 & 1.55 & 1.68 & 2.13 \\
\hline SH5 & 1.32 & 1.51 & 1.65 & 2.08 & 1.36 & 1.58 & 1.69 & 2.17 & 1.35 & 1.55 & 1.68 & 2.13 \\
\hline SH6 & 1.29 & 1.48 & 1.61 & 2.04 & 1.35 & 1.55 & 1.69 & 2.14 & 1.34 & 1.52 & 1.67 & 2.09 \\
\hline ML1 & 1.31 & 1.50 & 1.63 & 2.07 & 1.31 & 1.51 & 1.64 & 2.07 & 1.34 & 1.55 & 1.67 & 2.14 \\
\hline ML2 & 30 & 1.51 & 1.63 & 2.07 & 1.41 & 1.58 & 1.76 & 2.18 & 1.40 & 1.56 & 1.75 & 2.15 \\
\hline ML3 & 31 & 1.45 & 1.63 & 1.99 & 1.32 & 1.46 & 1.65 & 2.01 & 1.32 & 1.45 & 1.65 & 2.00 \\
\hline ML4 & 1.29 & 1.50 & 1.61 & 2.06 & 1.34 & 1.58 & 1.68 & 2.17 & 1.40 & 1.65 & 1.75 & 2.26 \\
\hline ML5 & 1.29 & 1.50 & 1.61 & 2.06 & 1.33 & 1.55 & 1.66 & 2.13 & 1.38 & 1.63 & 1.73 & 2.23 \\
\hline ML6 & 1.31 & 1.47 & 1.64 & 2.02 & 1.37 & 1.52 & 1.72 & 2.09 & 1.37 & 1.50 & 1.71 & 2.07 \\
\hline MH1 & 1.31 & 1.44 & 1.64 & 1.97 & 1.32 & 1.47 & 1.65 & 2.02 & 1.32 & 1.47 & 1.65 & 2.02 \\
\hline MH2 & 1.20 & 1.26 & 1.51 & 1.73 & 1.27 & 1.37 & 1.58 & 1.88 & 1.31 & 1.45 & 1.63 & 1.99 \\
\hline MH3 & 1.29 & 1.48 & 1.61 & 2.03 & 1.48 & 1.81 & 1.85 & 2.49 & 1.53 & 1.84 & 1.91 & 2.54 \\
\hline MH4 & 1.21 & 1.25 & 1.51 & 1.72 & 1.26 & 1.34 & 1.58 & 1.84 & 1.30 & 1.40 & 1.62 & 1.92 \\
\hline MH5 & 1.23 & 1.27 & 1.54 & 1.75 & 1.29 & 1.37 & 1.62 & 1.88 & 1.36 & 1.47 & 1.70 & 2.03 \\
\hline MH6 & 1.32 & 1.64 & 1.64 & 2.25 & 1.37 & 1.66 & 1.71 & 2.29 & 1.40 & 1.66 & 1.75 & 2.28 \\
\hline LL1 & 1.11 & 1.26 & 1.39 & 1.74 & 1.23 & 1.40 & 1.53 & 1.92 & 1.33 & 1.54 & 1.67 & 2.12 \\
\hline LL2 & 1.10 & 1.27 & 1.38 & 1.75 & 1.22 & 1.41 & 1.53 & 1.93 & 1.33 & 1.55 & 1.67 & 2.13 \\
\hline LL3 & 1.13 & 1.23 & 1.42 & 1.70 & 1.37 & 1.62 & 1.71 & 2.23 & 1.44 & 1.71 & 1.80 & 2.35 \\
\hline LL4 & 1.24 & 1.45 & 1.55 & 1.99 & 1.35 & 1.58 & 1.69 & 2.17 & 1.44 & 1.70 & 1.80 & 2.33 \\
\hline LL5 & 1.23 & 1.43 & 1.54 & 1.97 & 1.34 & 1.57 & 1.68 & 2.16 & 1.45 & 1.70 & 1.81 & 2.34 \\
\hline LL6 & 0.90 & 1.09 & 1.12 & 1.50 & 0.96 & 1.22 & 1.21 & 1.68 & 1.04 & 1.32 & 1.30 & 1.81 \\
\hline LH1 & 1.19 & 1.36 & 1.48 & 1.87 & 1.41 & 1.72 & 1.77 & 2.36 & 1.56 & 1.83 & 1.95 & 2.52 \\
\hline LH2 & 1.34 & 1.67 & 1.67 & 2.30 & 1.49 & 1.83 & 1.86 & 2.52 & 1.55 & 1.89 & 1.94 & 2.59 \\
\hline LH3 & 1.12 & 1.38 & 1.40 & 1.90 & 1.26 & 1.56 & 1.58 & 2.14 & 1.33 & 1.61 & 1.67 & 2.21 \\
\hline LH4 & 1.36 & 1.65 & 1.70 & 2.27 & 1.52 & 1.82 & 1.90 & 2.51 & 1.58 & 1.86 & 1.97 & 2.55 \\
\hline LH5 & 1.35 & 1.65 & 1.68 & 2.27 & 1.52 & 1.82 & 1.90 & 2.50 & 1.58 & 1.85 & 1.97 & 2.55 \\
\hline LH6 & 1.03 & 1.28 & 1.29 & 1.76 & 1.16 & 1.44 & 1.45 & 1.98 & 1.25 & 1.53 & 1.56 & 2.10 \\
\hline
\end{tabular}


Table C.54 - The ratio of actual mid-span bending moments and quarter-span shear forces for buildings to the corresponding values obtained from FEMA parabolic distribution and a uniform distribution of seismic load when subjected to E6C18 ground motion and the Load is acting parallel to the short side, $\alpha=0.05$

\begin{tabular}{|c|c|c|c|c|c|c|c|c|c|c|c|c|}
\hline \multirow{2}{*}{ Building } & \multicolumn{4}{|c|}{$\mu=2$} & \multicolumn{4}{|c|}{$\mu=3$} & \multicolumn{4}{|c|}{$\mu=4$} \\
\hline & $M . F$ & V.F & $M . U$ & $V . U$ & $M . F$ & V.F & $M . U$ & $V . U$ & M.F & V.F & M.U & $V . U$ \\
\hline SL1 & 1.13 & 1.22 & 1.41 & 1.68 & 1.16 & 1.24 & 1.45 & 1.70 & 1.20 & 1.32 & 1.51 & 1.81 \\
\hline SL3 & 16 & 1.27 & 1.45 & 1.75 & 1.18 & 1.27 & 1.47 & 1.75 & 1.22 & 1.33 & 1.52 & 1.83 \\
\hline SL4 & 1.24 & 1.38 & 1.55 & 1.90 & 1.24 & 1.36 & 1.56 & 1.88 & 1.27 & 1.42 & 1.58 & 1.95 \\
\hline SH1 & 1.03 & 1.10 & 1.28 & 1.51 & 1.08 & 1.10 & 1.36 & 1.52 & 1.13 & 1.15 & 1.41 & 1.58 \\
\hline $\mathrm{SH} 2$ & 1.02 & 1.05 & 1.27 & 1.44 & 1.09 & 1.13 & 1.36 & 1.55 & 1.15 & 1.20 & 1.44 & 1.64 \\
\hline SH3 & 1.02 & 1.08 & 1.28 & 1.48 & 1.11 & 1.17 & 1.39 & 1.61 & 1.18 & 1.25 & 1.48 & 1.72 \\
\hline SH4 & 1.01 & 1.08 & 1.26 & 1.48 & 1.10 & 1.17 & 1.37 & 1.61 & 1.16 & 1.24 & 1.45 & 1.71 \\
\hline SH5 & 1.02 & 1.10 & 1.28 & 1.51 & 1.12 & 1.19 & 1.40 & 1.64 & 1.19 & 1.27 & 1.49 & 1.75 \\
\hline SH6 & 1.02 & 1.07 & 1.27 & 1.47 & 1.11 & 1.17 & 1.39 & 1.61 & 1.18 & 1.25 & 1.48 & 1.72 \\
\hline ML1 & 0.97 & 1.08 & 1.22 & 1.48 & 1.06 & 1.17 & 1.33 & 1.62 & 1.20 & 1.31 & 1.50 & 1.80 \\
\hline ML2 & 0.99 & 1.23 & 1.23 & 1.70 & 1.18 & 1.41 & 1.48 & 1.94 & 1.21 & 1.43 & 1.52 & 1.97 \\
\hline ML3 & 13 & 1.41 & 1.41 & 1.94 & 1.18 & 1.46 & 1.48 & 2.00 & 1.21 & 1.48 & 1.52 & 2.04 \\
\hline ML4 & 0.98 & 1.14 & 1.22 & 1.57 & 1.10 & 1.27 & 1.38 & 1.75 & 1.35 & 1.52 & 1.69 & 2.09 \\
\hline ML5 & 0.98 & 1.14 & 1.22 & 1.57 & 1.09 & 1.25 & 1.36 & 1.72 & 1.37 & 1.54 & 1.71 & 2.11 \\
\hline ML6 & 1.13 & 1.39 & 1.41 & 1.91 & 1.18 & 1.42 & 1.48 & 1.96 & 1.21 & 1.45 & 1.52 & 1.99 \\
\hline MH1 & 1.16 & 1.43 & 1.45 & 1.96 & 1.20 & 1.45 & 1.51 & 2.00 & 1.23 & 1.47 & 1.53 & 2.02 \\
\hline MH2 & 1.26 & 1.52 & 1.58 & 2.08 & 1.32 & 1.55 & 1.65 & 2.13 & 1.35 & 1.57 & 1.68 & 2.16 \\
\hline MH3 & 0.95 & 1.44 & 1.19 & 1.98 & 1.02 & 1.57 & 1.27 & 2.16 & 1.04 & 1.69 & 1.30 & 2.32 \\
\hline MH4 & 1.08 & 1.39 & 1.35 & 1.91 & 1.31 & 1.57 & 1.64 & 2.16 & 1.34 & 1.60 & 1.68 & 2.20 \\
\hline MH5 & 1.29 & 1.57 & 1.61 & 2.16 & 1.35 & 1.61 & 1.68 & 2.21 & 1.39 & 1.64 & 1.73 & 2.26 \\
\hline MH6 & 1.01 & 1.18 & 1.26 & 1.63 & 1.39 & 1.54 & 1.74 & 2.11 & 1.42 & 1.61 & 1.78 & 2.21 \\
\hline LL1 & 1.35 & 1.81 & 1.69 & 2.49 & 1.40 & 1.88 & 1.75 & 2.58 & 1.42 & 1.90 & 1.77 & 2.62 \\
\hline LL2 & 1.34 & 1.81 & 1.67 & 2.49 & 1.39 & 1.88 & 1.74 & 2.59 & 1.41 & 1.92 & 1.77 & 2.63 \\
\hline LL3 & 1.41 & 1.88 & 1.77 & 2.58 & 1.50 & 1.97 & 1.87 & 2.71 & 1.54 & 2.02 & 1.93 & 2.78 \\
\hline LL4 & 1.28 & 1.65 & 1.59 & 2.26 & 1.32 & 1.70 & 1.65 & 2.33 & 1.34 & 1.72 & 1.68 & 2.37 \\
\hline LL5 & 1.28 & 1.65 & 1.60 & 2.26 & 1.32 & 1.70 & 1.65 & 2.33 & 1.34 & 1.72 & 1.68 & 2.36 \\
\hline LL6 & 0.65 & 1.40 & 0.82 & 1.93 & 0.73 & 1.54 & 0.92 & 2.12 & 0.84 & 1.71 & 1.06 & 2.35 \\
\hline LH1 & 1.47 & 2.02 & 1.84 & 2.78 & 1.57 & 2.14 & 1.96 & 2.94 & 1.61 & 2.19 & 2.01 & 3.01 \\
\hline LH2 & 1.50 & 2.14 & 1.87 & 2.94 & 1.60 & 2.28 & 2.00 & 3.13 & 1.67 & 2.37 & 2.09 & 3.26 \\
\hline LH3 & 0.99 & 1.79 & 1.24 & 2.45 & 1.10 & 1.92 & 1.37 & 2.63 & 1.17 & 1.97 & 1.46 & 2.71 \\
\hline LH4 & 1.53 & 2.15 & 1.91 & 2.96 & 1.63 & 2.29 & 2.04 & 3.15 & 1.71 & 2.40 & 2.13 & 3.29 \\
\hline LH5 & 1.54 & 2.18 & 1.92 & 2.99 & 1.64 & 2.31 & 2.05 & 3.18 & 1.72 & 2.43 & 2.15 & 3.34 \\
\hline LH6 & 0.97 & 1.82 & 1.21 & 2.50 & 1.09 & 1.95 & 1.37 & 2.69 & 1.13 & 1.97 & 1.42 & 2.71 \\
\hline
\end{tabular}


Table C.55 - The ratio of actual mid-span bending moments and quarter-span shear forces for buildings to the corresponding values obtained from FEMA parabolic distribution and a uniform distribution of seismic load when subjected to E6C42 ground motion and the Load is acting parallel to the short side, $\alpha=0.05$

\begin{tabular}{|c|c|c|c|c|c|c|c|c|c|c|c|c|}
\hline \multirow{2}{*}{ Building } & \multicolumn{4}{|c|}{$\mu=2$} & \multicolumn{4}{|c|}{$\mu=3$} & \multicolumn{4}{|c|}{$\mu=4$} \\
\hline & $M . F$ & V.F & $M . U$ & $V . U$ & $M . F$ & $V . F$ & $M . U$ & $V . U$ & M.F & V.F & $M . U$ & $V . U$ \\
\hline SL1 & 1.22 & 1.28 & 1.53 & 1.76 & 1.29 & 1.37 & 1.61 & 1.88 & 1.34 & 1.44 & 1.67 & 1.97 \\
\hline SL3 & 26 & 1.33 & 1.57 & 1.83 & 1.32 & 1.42 & 1.65 & 1.95 & 1.37 & 1.49 & 1.71 & 2.05 \\
\hline SL4 & 1.31 & 1.45 & 1.64 & 1.99 & 1.40 & 1.54 & 1.74 & 2.12 & 1.40 & 1.53 & 1.75 & 2.10 \\
\hline SH1 & 1.17 & 1.19 & 1.46 & 1.63 & 1.20 & 1.24 & 1.50 & 1.71 & 1.22 & 1.27 & 1.52 & 1.75 \\
\hline $\mathrm{SH} 2$ & 1.16 & 1.17 & 1.45 & 1.61 & 1.18 & 1.20 & 1.48 & 1.66 & 1.20 & 1.24 & 1.50 & 1.71 \\
\hline SH3 & 1.14 & 1.15 & 1.43 & 1.58 & 1.17 & 1.19 & 1.46 & 1.64 & 1.20 & 1.23 & 1.49 & 1.70 \\
\hline SH4 & 1.11 & 1.10 & 1.39 & 1.52 & 1.15 & 1.16 & 1.44 & 1.59 & 1.17 & 1.19 & 1.47 & 1.64 \\
\hline SH5 & 1.12 & 1.11 & 1.39 & 1.53 & 1.15 & 1.17 & 1.44 & 1.60 & 1.18 & 1.20 & 1.47 & 1.66 \\
\hline SH6 & 1.14 & 1.15 & 1.43 & 1.58 & 1.17 & 1.19 & 1.46 & 1.64 & 1.20 & 1.23 & 1.49 & 1.70 \\
\hline ML1 & 1.04 & 1.16 & 1.30 & 1.59 & 1.09 & 1.12 & 1.36 & 1.53 & 1.13 & 1.16 & 1.42 & 1.59 \\
\hline ML2 & 1.20 & 1.41 & 1.49 & 1.94 & 1.23 & 1.43 & 1.53 & 1.97 & 1.23 & 1.43 & 1.54 & 1.97 \\
\hline ML3 & 27 & 1.52 & 1.59 & 2.09 & 1.29 & 1.56 & 1.61 & 2.14 & 1.29 & 1.56 & 1.61 & 2.15 \\
\hline ML4 & 1.09 & 1.23 & 1.37 & 1.69 & 1.11 & 1.22 & 1.39 & 1.68 & 1.10 & 1.19 & 1.38 & 1.64 \\
\hline ML5 & 1.09 & 1.23 & 1.36 & 1.69 & 1.10 & 1.21 & 1.38 & 1.66 & 1.10 & 1.17 & 1.38 & 1.61 \\
\hline ML6 & 1.24 & 1.47 & 1.55 & 2.01 & 1.27 & 1.50 & 1.59 & 2.07 & 1.26 & 1.50 & 1.58 & 2.06 \\
\hline MH1 & 1.29 & 1.58 & 1.61 & 2.17 & 1.30 & 1.63 & 1.63 & 2.24 & 1.29 & 1.62 & 1.61 & 2.23 \\
\hline MH2 & 1.23 & 1.50 & 1.54 & 2.06 & 1.23 & 1.53 & 1.54 & 2.10 & 1.23 & 1.54 & 1.54 & 2.12 \\
\hline MH3 & 0.82 & 0.87 & 1.03 & 1.19 & 0.88 & 0.97 & 1.10 & 1.33 & 0.89 & 1.03 & 1.12 & 1.42 \\
\hline MH4 & 1.17 & 1.40 & 1.46 & 1.93 & 1.19 & 1.43 & 1.48 & 1.97 & 1.18 & 1.44 & 1.48 & 1.99 \\
\hline MH5 & 1.17 & 1.40 & 1.46 & 1.93 & 1.18 & 1.43 & 1.48 & 1.97 & 1.18 & 1.45 & 1.47 & 1.99 \\
\hline MH6 & 1.17 & 1.30 & 1.47 & 1.79 & 1.15 & 1.28 & 1.44 & 1.76 & 1.23 & 1.34 & 1.54 & 1.85 \\
\hline LL1 & 1.06 & 1.39 & 1.33 & 1.91 & 1.24 & 1.60 & 1.55 & 2.20 & 1.38 & 1.82 & 1.73 & 2.50 \\
\hline LL2 & 1.05 & 1.37 & 1.32 & 1.89 & 1.22 & 1.58 & 1.53 & 2.18 & 1.36 & 1.81 & 1.70 & 2.48 \\
\hline LL3 & 0.97 & 1.22 & 1.21 & 1.68 & 1.00 & 1.26 & 1.24 & 1.73 & 0.99 & 1.28 & 1.24 & 1.76 \\
\hline LL4 & 1.34 & 1.67 & 1.68 & 2.30 & 1.37 & 1.70 & 1.71 & 2.34 & 1.36 & 1.70 & 1.70 & 2.33 \\
\hline LL5 & 1.35 & 1.67 & 1.68 & 2.30 & 1.37 & 1.70 & 1.71 & 2.33 & 1.37 & 1.69 & 1.71 & 2.33 \\
\hline LL6 & 0.90 & 1.05 & 1.13 & 1.44 & 0.89 & 1.03 & 1.12 & 1.41 & 0.89 & 1.00 & 1.11 & 1.37 \\
\hline LH1 & 0.90 & 1.18 & 1.13 & 1.62 & 0.93 & 1.22 & 1.16 & 1.68 & 0.93 & 1.25 & 1.16 & 1.72 \\
\hline $\mathrm{LH} 2$ & 0.92 & 1.16 & 1.15 & 1.60 & 0.87 & 1.22 & 1.09 & 1.68 & 0.90 & 1.25 & 1.12 & 1.72 \\
\hline LH3 & 0.86 & 0.99 & 1.08 & 1.36 & 0.87 & 0.95 & 1.09 & 1.31 & 0.91 & 1.02 & 1.14 & 1.40 \\
\hline LH4 & 0.91 & 1.11 & 1.13 & 1.52 & 0.87 & 1.14 & 1.09 & 1.57 & 0.93 & 1.17 & 1.16 & 1.61 \\
\hline LH5 & 0.90 & 1.09 & 1.12 & 1.49 & 0.87 & 1.12 & 1.08 & 1.54 & 0.93 & 1.15 & 1.17 & 1.57 \\
\hline LH6 & 0.91 & 1.05 & 1.14 & 1.44 & 0.89 & 1.02 & 1.11 & 1.41 & 0.92 & 0.99 & 1.15 & 1.36 \\
\hline
\end{tabular}


Table C.56 - The ratio of actual mid-span bending moments and quarter-span shear forces for buildings to the corresponding values obtained from FEMA parabolic distribution and a uniform distribution of seismic load when subjected to E6C1 ground motion and the Load is acting parallel to the long side, $\alpha=0.05$

\begin{tabular}{|c|c|c|c|c|c|c|c|c|c|c|c|c|}
\hline \multirow{2}{*}{ Building } & \multicolumn{4}{|c|}{$\mu=2$} & \multicolumn{4}{|c|}{$\mu=3$} & \multicolumn{4}{|c|}{$\mu=4$} \\
\hline & $M . F$ & $V . F$ & $M . U$ & V.U & $M . F$ & $V . F$ & $M . U$ & $V . U$ & $M . F$ & $V . F$ & $M . U$ & $V . U$ \\
\hline SL1 & 1.20 & 1.32 & 1.50 & 1.81 & 1.21 & 1.34 & 1.51 & 1.84 & 1.19 & 1.34 & 1.48 & 1.84 \\
\hline SL3 & .22 & 1.32 & 1.52 & 1.82 & 1.21 & 1.34 & 1.52 & 1.84 & 1.18 & 1.35 & 1.48 & 1.85 \\
\hline SL4 & 11 & 1.17 & 1.39 & 1.61 & 1.15 & 1.27 & 1.44 & 1.74 & 1.13 & 1.26 & 1.42 & 1.73 \\
\hline SH1 & 1.00 & 1.02 & 1.25 & 1.40 & 1.03 & 1.08 & 1.29 & 1.49 & 1.02 & 1.08 & 1.28 & 1.48 \\
\hline $\mathrm{SH} 2$ & 1.00 & 1.03 & 1.25 & 1.41 & 1.01 & 1.05 & 1.26 & 1.44 & 0.99 & 1.04 & 1.24 & 1.43 \\
\hline $\mathrm{SH} 3$ & 1.00 & 1.01 & 1.24 & 1.39 & 0.99 & 1.02 & 1.24 & 1.40 & 0.97 & 1.01 & 1.21 & 1.39 \\
\hline $\mathrm{SH} 4$ & 0.98 & 1.00 & 1.23 & 1.37 & 0.97 & 0.99 & 1.21 & 1.36 & 0.95 & 0.97 & 1.19 & 1.34 \\
\hline SH5 & 0.98 & 0.99 & 1.23 & 1.36 & 0.96 & 0.98 & 1.20 & 1.34 & 0.94 & 0.97 & 1.18 & 1.33 \\
\hline ML1 & 0.89 & 0.88 & 1.12 & 1.21 & 0.88 & 0.87 & 1.10 & 1.20 & 0.93 & 0.89 & 1.14 & 1.23 \\
\hline ML2 & 0.89 & 0.88 & 1.12 & 1.20 & 0.90 & 0.87 & 1.13 & 1.19 & 0.95 & 0.91 & 1.19 & 1.26 \\
\hline ML3 & 0.90 & 0.87 & 1.12 & 1.19 & 0.96 & 0.92 & 1.21 & 1.27 & 1.03 & 1.02 & 1.29 & 1.40 \\
\hline ML4 & 0.89 & 0.88 & 1.12 & 1.21 & 0.89 & 0.87 & 1.12 & 1.19 & 0.94 & 0.90 & 1.17 & 1.24 \\
\hline L5 & 0.89 & 0.87 & 1.12 & 1.20 & 0.91 & 0.87 & 1.14 & 1.20 & 0.97 & 0.93 & 1.21 & 1.27 \\
\hline ML6 & 0.90 & 0.87 & 1.12 & 1.20 & 0.94 & 0.90 & 1.18 & 1.23 & 1.01 & 0.98 & 1.26 & 1.35 \\
\hline MH1 & 1.04 & 1.05 & 1.30 & 1.44 & 1.12 & 1.17 & 1.41 & 1.61 & 1.18 & 1.27 & 1.48 & 1.74 \\
\hline & 1.16 & 1.26 & 1.45 & 1.73 & 1.21 & 1.35 & 1.52 & 1.85 & 1.24 & 1.40 & 1.55 & 1.92 \\
\hline MH3 & 1.22 & 1.35 & 1.53 & 1.86 & 1.21 & 1.37 & 1.51 & 1.88 & 1.21 & 1.37 & 1.51 & 1.88 \\
\hline MH4 & 1.08 & 1.11 & 1.35 & 1.53 & 1.16 & 1.24 & 1.45 & 1.70 & 1.21 & 1.32 & 1.51 & 1.82 \\
\hline MH5 & 1.19 & 1.30 & 1.48 & 1.79 & 1.23 & 1.37 & 1.53 & 1.88 & 1.25 & 1.41 & 1.56 & 1.94 \\
\hline MH6 & 1.09 & 1.14 & 1.37 & 1.57 & 1.18 & 1.28 & 1.47 & 1.76 & 1.25 & 1.41 & 1.56 & 1.94 \\
\hline LL1 & 0.97 & 0.96 & 1.22 & 1.33 & 1.07 & 1.09 & 1.34 & 1.49 & 1.16 & 1.25 & 1.45 & 1.72 \\
\hline LL2 & 1.04 & 1.05 & 1.30 & 1.45 & 1.13 & 1.19 & 1.42 & 1.64 & 1.20 & 1.32 & 1.50 & 1.82 \\
\hline LL3 & 1.23 & 1.37 & 1.54 & 1.88 & 1.22 & 1.37 & 1.52 & 1.88 & 1.23 & 1.39 & 1.54 & 1.91 \\
\hline LL4 & 0.98 & 0.97 & 1.23 & 1.34 & 1.08 & 1.10 & 1.35 & 1.51 & 1.17 & 1.26 & 1.46 & 1.73 \\
\hline LL5 & 1.15 & 1.24 & 1.44 & 1.70 & 1.21 & 1.34 & 1.51 & 1.84 & 1.24 & 1.40 & 1.55 & 1.93 \\
\hline LL6 & 1.13 & 1.18 & 1.41 & 1.62 & 1.19 & 1.28 & 1.48 & 1.76 & 1.41 & 1.62 & 1.76 & 2.22 \\
\hline LH1 & 1.08 & 1.13 & 1.35 & 1.55 & 1.17 & 1.29 & 1.47 & 1.77 & 1.42 & 1.67 & 1.78 & 2.29 \\
\hline LH2 & 1.16 & 1.25 & 1.45 & 1.72 & 1.27 & 1.42 & 1.59 & 1.96 & 1.42 & 1.64 & 1.78 & 2.26 \\
\hline LH3 & 1.10 & 1.16 & 1.38 & 1.60 & 1.33 & 1.52 & 1.67 & 2.10 & 1.32 & 1.51 & 1.65 & 2.08 \\
\hline LH4 & 1.08 & 1.14 & 1.35 & 1.56 & 1.45 & 1.70 & 1.81 & 2.34 & 1.43 & 1.69 & 1.78 & 2.33 \\
\hline LH5 & 1.19 & 1.29 & 1.48 & 1.78 & 1.23 & 1.37 & 1.53 & 1.88 & 1.33 & 1.49 & 1.66 & 2.05 \\
\hline LH6 & 1.09 & 1.09 & 1.36 & 1.50 & 1.36 & 1.57 & 1.70 & 2.15 & 1.34 & 1.54 & 1.68 & 2.12 \\
\hline
\end{tabular}


Table C.57 - The ratio of actual mid-span bending moments and quarter-span shear forces for buildings to the corresponding values obtained from FEMA parabolic distribution and a uniform distribution of seismic load when subjected to E6C13 ground motion and the Load is acting parallel to the long side, $\alpha=0.05$

\begin{tabular}{|c|c|c|c|c|c|c|c|c|c|c|c|c|}
\hline \multirow{2}{*}{ Building } & \multicolumn{4}{|c|}{$\mu=2$} & \multicolumn{4}{|c|}{$\mu=3$} & \multicolumn{4}{|c|}{$\mu=4$} \\
\hline & $M . F$ & $V . F$ & $M . U$ & V.U & M.F & $V . F$ & $M . U$ & $V . U$ & M.F & V.F & M.U & $V . U$ \\
\hline SL1 & 1.13 & 1.22 & 1.42 & 1.67 & 1.31 & 1.49 & 1.64 & 2.05 & 1.31 & 1.50 & 1.63 & 2.07 \\
\hline SL3 & 13 & 1.20 & 1.41 & 1.65 & 1.33 & 1.52 & 1.66 & 2.09 & 1.33 & 1.55 & 1.66 & 2.13 \\
\hline & 10 & 1.15 & 1.37 & 1.58 & 1.34 & 1.54 & 1.68 & 2.12 & 1.36 & 1.58 & 1.69 & 2.17 \\
\hline SH1 & 1.22 & 1.34 & 1.53 & 1.84 & 1.30 & 1.41 & 1.62 & 1.93 & 1.28 & 1.36 & 1.60 & 1.87 \\
\hline & 1.02 & 1.02 & 1.27 & 1.40 & 1.28 & 1.41 & 1.59 & 1.94 & 1.30 & 1.39 & 1.62 & 1.91 \\
\hline $\mathrm{SH} 3$ & 0.95 & 0.97 & 1.18 & 1.33 & 1.21 & 1.33 & 1.51 & 1.83 & 1.28 & 1.38 & 1.60 & 1.90 \\
\hline SH4 & 0.97 & 0.96 & 1.21 & 1.32 & 1.18 & 1.28 & 1.48 & 1.77 & 1.27 & 1.40 & 1.59 & 1.92 \\
\hline SH5 & 0.97 & 0.95 & 1.21 & 1.31 & 1.17 & 1.27 & 1.46 & 1.74 & 1.28 & 1.40 & 1.61 & 1.93 \\
\hline ML1 & 1.12 & 1.24 & 1.40 & 1.70 & 1.15 & 1.28 & 1.44 & 1.76 & 1.17 & 1.31 & 1.46 & 1.80 \\
\hline ML2 & 1.19 & 1.33 & 1.49 & 1.83 & 1.22 & 1.36 & 1.53 & 1.87 & 1.23 & 1.34 & 1.54 & 1.85 \\
\hline ML3 & 1.27 & 1.45 & 1.59 & 1.99 & 1.30 & 1.46 & 1.62 & 2.01 & 1.29 & 1.40 & 1.61 & 1.93 \\
\hline ML4 & 1.17 & 1.31 & 1.47 & 1.80 & 1.20 & 1.34 & 1.50 & 1.84 & 1.22 & 1.32 & 1.52 & 1.82 \\
\hline ML5 & 1.21 & 1.36 & 1.52 & 1.88 & 1.24 & 1.39 & 1.55 & 1.91 & 1.25 & 1.37 & 1.56 & 1.88 \\
\hline ML6 & 1.24 & 1.41 & 1.56 & 1.94 & 1.27 & 1.43 & 1.59 & 1.96 & 1.27 & 1.39 & 1.58 & 1.90 \\
\hline & 36 & 1.51 & 1.70 & 2.07 & 1.32 & 1.48 & 1.65 & 2.03 & 1.31 & 1.51 & 1.63 & 2.07 \\
\hline & 31 & 1.48 & 1.64 & 2.04 & 1.38 & 1.60 & 1.72 & 2.20 & 1.40 & 1.64 & 1.75 & 2.25 \\
\hline & 1.02 & 1.12 & 1.27 & 1.53 & 1.13 & 1.33 & 1.41 & 1.83 & 1.24 & 1.48 & 1.55 & 2.04 \\
\hline MH4 & 1.34 & 1.46 & 1.67 & 2.01 & 1.33 & 1.53 & 1.66 & 2.10 & 1.36 & 1.57 & 1.69 & 2.16 \\
\hline MH5 & 1.31 & 1.47 & 1.63 & 2.02 & 1.39 & 1.61 & 1.73 & 2.22 & 1.43 & 1.68 & 1.79 & 2.31 \\
\hline MH6 & 1.30 & 1.45 & 1.62 & 2.00 & 1.35 & 1.55 & 1.69 & 2.14 & 1.39 & 1.62 & 1.74 & 2.22 \\
\hline LL1 & 1.36 & 1.52 & 1.70 & 2.09 & 1.32 & 1.46 & 1.65 & 2.01 & 1.32 & 1.52 & 1.65 & 2.09 \\
\hline LL2 & 1.34 & 1.47 & 1.67 & 2.02 & 1.33 & 1.53 & 1.66 & 2.10 & 1.34 & 1.55 & 1.68 & 2.14 \\
\hline LL3 & 1.08 & 1.23 & 1.35 & 1.69 & 1.25 & 1.45 & 1.56 & 1.99 & 1.34 & 1.58 & 1.68 & 2.18 \\
\hline LL4 & 1.36 & 1.52 & 1.70 & 2.08 & 1.32 & 1.46 & 1.65 & 2.01 & 1.33 & 1.53 & 1.66 & 2.10 \\
\hline LL5 & 1.33 & 1.49 & 1.66 & 2.05 & 1.38 & 1.60 & 1.72 & 2.20 & 1.42 & 1.66 & 1.78 & 2.28 \\
\hline LL6 & 0.93 & 0.95 & 1.16 & 1.31 & 0.90 & 1.13 & 1.13 & 1.55 & 1.01 & 1.26 & 1.26 & 1.74 \\
\hline LH1 & 1.27 & 1.39 & 1.58 & 1.92 & 1.34 & 1.53 & 1.68 & 2.11 & 1.37 & 1.59 & 1.72 & 2.18 \\
\hline LH2 & 1.17 & 1.26 & 1.46 & 1.73 & 1.30 & 1.46 & 1.62 & 2.01 & 1.36 & 1.58 & 1.70 & 2.17 \\
\hline LH3 & 0.97 & 0.99 & 1.22 & 1.36 & 0.93 & 1.14 & 1.17 & 1.57 & 1.07 & 1.31 & 1.33 & 1.80 \\
\hline LH4 & 1.22 & 1.31 & 1.52 & 1.80 & 1.32 & 1.48 & 1.65 & 2.04 & 1.34 & 1.55 & 1.68 & 2.13 \\
\hline LH5 & 1.08 & 1.15 & 1.35 & 1.59 & 1.22 & 1.39 & 1.53 & 1.91 & 1.30 & 1.51 & 1.63 & 2.08 \\
\hline LH6 & 0.92 & 1.08 & 1.15 & 1.48 & 1.04 & 1.21 & 1.30 & 1.66 & 1.21 & 1.41 & 1.51 & 1.93 \\
\hline
\end{tabular}


Table C.58 - The ratio of actual mid-span bending moments and quarter-span shear forces for buildings to the corresponding values obtained from FEMA parabolic distribution and a uniform distribution of seismic load when subjected to E6C15 ground motion and the Load is acting parallel to the long side, $\alpha=0.05$

\begin{tabular}{|c|c|c|c|c|c|c|c|c|c|c|c|c|}
\hline \multirow{2}{*}{ Building } & \multicolumn{4}{|c|}{$\mu=2$} & \multicolumn{4}{|c|}{$\mu=3$} & \multicolumn{4}{|c|}{$\mu=4$} \\
\hline & $M . F$ & $V . F$ & $M . U$ & $V . U$ & M.F & $V . F$ & $M . U$ & $V . U$ & M.F & V.F & $M . U$ & $V . U$ \\
\hline SL1 & 1.22 & 1.38 & 1.53 & 1.89 & 1.23 & 1.41 & 1.54 & 1.94 & 1.20 & 1.39 & 1.50 & 1.92 \\
\hline SL3 & 23 & 1.39 & 1.54 & 1.90 & 1.23 & 1.40 & 1.53 & 1.93 & 1.18 & 1.37 & 1.48 & 1.89 \\
\hline SL4 & 19 & 1.34 & 1.49 & 1.84 & 1.20 & 1.37 & 1.50 & 1.88 & 1.16 & 1.35 & 1.46 & 1.85 \\
\hline SH1 & 1.24 & 1.38 & 1.55 & 1.90 & 1.28 & 1.44 & 1.60 & 1.98 & 1.31 & 1.47 & 1.63 & 2.03 \\
\hline $\mathrm{SH} 2$ & 1.29 & 1.44 & 1.61 & 1.98 & 1.33 & 1.49 & 1.66 & 2.05 & 1.35 & 1.52 & 1.68 & 2.09 \\
\hline $\mathrm{SH} 3$ & 1.31 & 1.47 & 1.63 & 2.02 & 1.35 & 1.52 & 1.68 & 2.09 & 1.37 & 1.54 & 1.71 & 2.12 \\
\hline SH4 & 1.33 & 1.50 & 1.67 & 2.06 & 1.37 & 1.54 & 1.71 & 2.12 & 1.38 & 1.55 & 1.73 & 2.13 \\
\hline H5 & 1.32 & 1.49 & 1.65 & 2.04 & 1.37 & 1.54 & 1.71 & 2.12 & 1.39 & 1.56 & 1.73 & 2.14 \\
\hline ML1 & 1.32 & 1.48 & 1.65 & 2.04 & 1.42 & 1.59 & 1.78 & 2.18 & 1.46 & 1.61 & 1.83 & 2.22 \\
\hline ML2 & 1.34 & 1.51 & 1.67 & 2.07 & 1.47 & 1.62 & 1.83 & 2.23 & 1.48 & 1.62 & 1.85 & 2.23 \\
\hline ML3 & 1.34 & 1.51 & 1.67 & 2.07 & 1.46 & 1.62 & 1.83 & 2.23 & 1.46 & 1.61 & 1.83 & 2.22 \\
\hline ML4 & 1.33 & 1.50 & 1.67 & 2.06 & 1.46 & 1.62 & 1.82 & 2.22 & 1.48 & 1.62 & 1.85 & 2.23 \\
\hline ML5 & 1.34 & 1.51 & 1.67 & 2.07 & 1.47 & 1.63 & 1.84 & 2.24 & 1.48 & 1.63 & 1.85 & 2.24 \\
\hline ML6 & 1.33 & 1.49 & 1.66 & 2.05 & 1.47 & 1.63 & 1.84 & 2.24 & 1.47 & 1.63 & 1.84 & 2.23 \\
\hline MH1 & 1.19 & 1.36 & 1.49 & 1.87 & 1.39 & 1.55 & 1.74 & 2.13 & 1.49 & 1.64 & 1.86 & 2.25 \\
\hline & 1.09 & 1.14 & 1.36 & 1.57 & 1.44 & 1.63 & 1.80 & 2.24 & 1.52 & 1.68 & 1.90 & 2.31 \\
\hline MH3 & 1.35 & 1.55 & 1.68 & 2.13 & 1.35 & 1.55 & 1.68 & 2.14 & 1.38 & 1.58 & 1.72 & 2.17 \\
\hline MH4 & 1.11 & 1.19 & 1.39 & 1.64 & 1.42 & 1.59 & 1.78 & 2.18 & 1.51 & 1.66 & 1.89 & 2.29 \\
\hline MH5 & 1.11 & 1.17 & 1.38 & 1.61 & 1.38 & 1.56 & 1.73 & 2.15 & 1.48 & 1.64 & 1.85 & 2.26 \\
\hline MH6 & 1.32 & 1.46 & 1.65 & 2.01 & 1.50 & 1.67 & 1.87 & 2.29 & 1.51 & 1.67 & 1.89 & 2.30 \\
\hline LL1 & 1.27 & 1.43 & 1.59 & 1.96 & 1.45 & 1.61 & 1.81 & 2.21 & 1.52 & 1.67 & 1.90 & 2.30 \\
\hline LL2 & 1.20 & 1.34 & 1.50 & 1.84 & 1.48 & 1.65 & 1.86 & 2.26 & 1.52 & 1.68 & 1.90 & 2.30 \\
\hline LL3 & 1.32 & 1.52 & 1.64 & 2.09 & 1.36 & 1.56 & 1.70 & 2.15 & 1.46 & 1.65 & 1.82 & 2.27 \\
\hline LL4 & 1.28 & 1.44 & 1.60 & 1.97 & 1.46 & 1.63 & 1.83 & 2.24 & 1.52 & 1.67 & 1.90 & 2.30 \\
\hline LL5 & 1.22 & 1.33 & 1.52 & 1.83 & 1.45 & 1.65 & 1.82 & 2.26 & 1.53 & 1.69 & 1.91 & 2.32 \\
\hline LL6 & 1.32 & 1.59 & 1.65 & 2.19 & 1.38 & 1.63 & 1.73 & 2.24 & 1.42 & 1.64 & 1.78 & 2.26 \\
\hline LH1 & 1.28 & 1.42 & 1.60 & 1.95 & 1.53 & 1.71 & 1.91 & 2.35 & 1.55 & 1.73 & 1.94 & 2.38 \\
\hline LH2 & 1.33 & 1.51 & 1.66 & 2.07 & 1.51 & 1.75 & 1.89 & 2.41 & 1.57 & 1.75 & 1.96 & 2.41 \\
\hline LH3 & 1.36 & 1.61 & 1.70 & 2.22 & 1.46 & 1.68 & 1.82 & 2.32 & 1.55 & 1.76 & 1.94 & 2.43 \\
\hline LH4 & 1.22 & 1.32 & 1.53 & 1.82 & 1.53 & 1.72 & 1.91 & 2.36 & 1.57 & 1.75 & 1.96 & 2.41 \\
\hline LH5 & 1.37 & 1.59 & 1.72 & 2.18 & 1.53 & 1.73 & 1.92 & 2.38 & 1.58 & 1.77 & 1.97 & 2.44 \\
\hline LH6 & 1.39 & 1.59 & 1.73 & 2.19 & 1.46 & 1.70 & 1.83 & 2.34 & 1.58 & 1.79 & 1.97 & 2.46 \\
\hline
\end{tabular}


Table C.59 - The ratio of actual mid-span bending moments and quarter-span shear forces for buildings to the corresponding values obtained from FEMA parabolic distribution and a uniform distribution of seismic load when subjected to E6C18 ground motion and the Load is acting parallel to the long side, $\alpha=0.05$

\begin{tabular}{|c|c|c|c|c|c|c|c|c|c|c|c|c|}
\hline \multirow{2}{*}{ Building } & \multicolumn{4}{|c|}{$\mu=2$} & \multicolumn{4}{|c|}{$\mu=3$} & \multicolumn{4}{|c|}{$\mu=4$} \\
\hline & $M . F$ & $V . F$ & $M . U$ & V.U & M.F & $V . F$ & $M . U$ & $V . U$ & M.F & V.F & M.U & $V . U$ \\
\hline SL1 & 0.98 & 1.00 & 1.23 & 1.37 & 1.12 & 1.21 & 1.40 & 1.66 & 1.14 & 1.25 & 1.42 & 1.72 \\
\hline SL3 & 99 & 1.00 & 1.24 & 1.38 & 1.14 & 1.24 & 1.42 & 1.70 & 1.13 & 1.24 & 1.41 & 1.70 \\
\hline & 00 & 1.02 & 1.25 & 1.40 & 1.18 & 1.31 & 1.48 & 1.80 & 1.17 & 1.31 & 1.46 & 1.80 \\
\hline SH1 & 1.09 & 1.14 & 1.37 & 1.56 & 1.26 & 1.43 & 1.58 & 1.96 & 1.23 & 1.40 & 1.54 & 1.92 \\
\hline & 1.09 & 1.12 & 1.36 & 1.54 & 1.22 & 1.37 & 1.53 & 1.88 & 1.26 & 1.43 & 1.57 & 1.97 \\
\hline $\mathrm{SH} 3$ & 1.10 & 1.15 & 1.37 & 1.59 & 1.26 & 1.39 & 1.57 & 1.91 & 1.30 & 1.44 & 1.62 & 1.98 \\
\hline $\mathrm{SH} 4$ & 1.09 & 1.12 & 1.36 & 1.54 & 1.23 & 1.37 & 1.54 & 1.89 & 1.27 & 1.44 & 1.58 & 1.98 \\
\hline SH5 & 1.10 & 1.16 & 1.37 & 1.59 & 1.22 & 1.36 & 1.53 & 1.87 & 1.29 & 1.46 & 1.61 & 2.01 \\
\hline ML1 & 1.24 & 1.40 & 1.55 & 1.93 & 1.27 & 1.44 & 1.59 & 1.99 & 1.22 & 1.40 & 1.53 & 1.93 \\
\hline ML2 & 1.28 & 1.45 & 1.60 & 1.99 & 1.24 & 1.43 & 1.55 & 1.96 & 1.21 & 1.40 & 1.51 & 1.93 \\
\hline ML3 & 1.22 & 1.37 & 1.53 & 1.89 & 1.25 & 1.43 & 1.56 & 1.97 & 1.22 & 1.42 & 1.52 & 1.95 \\
\hline ML4 & 1.27 & 1.45 & 1.59 & 1.99 & 1.24 & 1.43 & 1.55 & 1.96 & 1.21 & 1.40 & 1.52 & 1.93 \\
\hline ML5 & 1.27 & 1.43 & 1.59 & 1.97 & 1.24 & 1.42 & 1.55 & 1.96 & 1.21 & 1.40 & 1.51 & 1.93 \\
\hline ML6 & 1.24 & 1.40 & 1.56 & 1.93 & 1.23 & 1.42 & 1.54 & 1.95 & 1.20 & 1.40 & 1.50 & 1.93 \\
\hline & 21 & 1.35 & 1.51 & 1.86 & 1.21 & 1.40 & 1.52 & 1.92 & 1.21 & 1.42 & 1.51 & 1.95 \\
\hline & & 1.20 & 1.38 & 1.64 & 1.13 & 1.27 & 1.41 & 1.75 & 1.17 & 1.29 & 1.46 & 1.78 \\
\hline & 1.09 & 1.14 & 1.36 & 1.56 & 1.24 & 1.31 & 1.55 & 1.80 & 1.62 & 1.93 & 2.03 & 2.66 \\
\hline MH4 & 1.18 & 1.32 & 1.47 & 1.82 & 1.20 & 1.38 & 1.50 & 1.90 & 1.19 & 1.39 & 1.49 & 1.92 \\
\hline MH5 & 1.07 & 1.16 & 1.34 & 1.59 & 1.14 & 1.23 & 1.43 & 1.70 & 1.22 & 1.28 & 1.52 & 1.76 \\
\hline MH6 & 1.12 & 1.20 & 1.40 & 1.66 & 1.12 & 1.24 & 1.40 & 1.70 & 1.14 & 1.24 & 1.42 & 1.70 \\
\hline LL1 & 1.20 & 1.33 & 1.50 & 1.82 & 1.21 & 1.37 & 1.52 & 1.89 & 1.21 & 1.38 & 1.51 & 1.90 \\
\hline LL2 & 1.19 & 1.32 & 1.49 & 1.82 & 1.20 & 1.37 & 1.50 & 1.88 & 1.18 & 1.37 & 1.48 & 1.88 \\
\hline LL3 & 1.05 & 1.06 & 1.32 & 1.46 & 1.19 & 1.22 & 1.48 & 1.68 & 1.36 & 1.50 & 1.71 & 2.06 \\
\hline LL4 & 1.20 & 1.32 & 1.50 & 1.82 & 1.21 & 1.37 & 1.52 & 1.89 & 1.20 & 1.38 & 1.50 & 1.90 \\
\hline LL5 & 1.12 & 1.21 & 1.40 & 1.66 & 1.13 & 1.28 & 1.42 & 1.76 & 1.15 & 1.29 & 1.44 & 1.78 \\
\hline LL6 & 1.10 & 1.20 & 1.37 & 1.65 & 1.45 & 1.62 & 1.81 & 2.23 & 1.47 & 1.70 & 1.83 & 2.34 \\
\hline LH1 & 1.04 & 1.10 & 1.31 & 1.51 & 1.10 & 1.19 & 1.37 & 1.63 & 1.14 & 1.25 & 1.42 & 1.72 \\
\hline LH2 & 0.99 & 1.00 & 1.23 & 1.37 & 1.09 & 1.17 & 1.36 & 1.60 & 1.28 & 1.47 & 1.60 & 2.03 \\
\hline LH3 & 1.26 & 1.36 & 1.58 & 1.87 & 1.29 & 1.43 & 1.61 & 1.97 & 1.32 & 1.52 & 1.65 & 2.10 \\
\hline LH4 & 1.03 & 1.07 & 1.29 & 1.48 & 1.08 & 1.17 & 1.35 & 1.61 & 1.12 & 1.24 & 1.40 & 1.70 \\
\hline LH5 & 1.02 & 1.11 & 1.27 & 1.53 & 1.30 & 1.43 & 1.62 & 1.96 & 1.65 & 1.98 & 2.06 & 2.72 \\
\hline LH6 & 1.29 & 1.44 & 1.61 & 1.98 & 1.30 & 1.45 & 1.63 & 1.99 & 1.30 & 1.46 & 1.63 & 2.01 \\
\hline
\end{tabular}


Table C.60 - The ratio of actual mid-span bending moments and quarter-span shear forces for buildings to the corresponding values obtained from FEMA parabolic distribution and a uniform distribution of seismic load when subjected to E6C42 ground motion and the Load is acting parallel to the long side, $\alpha=0.05$

\begin{tabular}{|c|c|c|c|c|c|c|c|c|c|c|c|c|}
\hline \multirow{2}{*}{ Building } & \multicolumn{4}{|c|}{$\mu=2$} & \multicolumn{4}{|c|}{$\mu=3$} & \multicolumn{4}{|c|}{$\mu=4$} \\
\hline & $M . F$ & $V . F$ & $M . U$ & V.U & M.F & $V . F$ & $M . U$ & $V . U$ & M.F & V.F & M.U & $V . U$ \\
\hline & 1.26 & 1.39 & 1.57 & 1.92 & 1.23 & 1.39 & 1.54 & 1.92 & 1.24 & 1.39 & 1.55 & 1.91 \\
\hline SL3 & 28 & 1.44 & 1.60 & 1.98 & 1.27 & 1.45 & 1.58 & 1.99 & 1.25 & 1.42 & 1.57 & 1.96 \\
\hline & 20 & 1.31 & 1.49 & 1.80 & 1.30 & 1.49 & 1.62 & 2.05 & 1.26 & 1.48 & 1.58 & 2.04 \\
\hline & 16 & 1.22 & 1.45 & 1.68 & 1.26 & 1.39 & 1.58 & 1.92 & 1.36 & 1.55 & 1.70 & 2.13 \\
\hline & 1.25 & 1.34 & 1.56 & 1.85 & 1.33 & 1.48 & 1.66 & 2.03 & 1.35 & 1.53 & 1.69 & 2.10 \\
\hline $\mathrm{SH} 3$ & 1.26 & 1.38 & 1.57 & 1.89 & 1.32 & 1.48 & 1.65 & 2.04 & 1.32 & 1.47 & 1.65 & 2.02 \\
\hline $\mathrm{SH} 4$ & 1.26 & 1.38 & 1.57 & 1.90 & 1.31 & 1.45 & 1.63 & 2.00 & 1.33 & 1.49 & 1.67 & 2.05 \\
\hline SH5 & 1.23 & 1.35 & 1.54 & 1.86 & 1.28 & 1.43 & 1.60 & 1.96 & 1.30 & 1.45 & 1.62 & 2.00 \\
\hline IL1 & 1.30 & 1.46 & 1.63 & 2.01 & 1.28 & 1.45 & 1.60 & 2.00 & 1.28 & 1.46 & 1.60 & 2.01 \\
\hline ML2 & 1.20 & 1.36 & 1.50 & 1.86 & 1.18 & 1.40 & 1.48 & 1.92 & 1.16 & 1.34 & 1.45 & 1.84 \\
\hline ML3 & 1.04 & 1.18 & 1.30 & 1.62 & 1.04 & 1.24 & 1.30 & 1.70 & 1.16 & 1.26 & 1.46 & 1.74 \\
\hline ML4 & 1.23 & 1.39 & 1.53 & 1.91 & 1.22 & 1.40 & 1.52 & 1.92 & 1.20 & 1.35 & 1.50 & 1.86 \\
\hline ML5 & 1.16 & 1.32 & 1.45 & 1.81 & 1.15 & 1.37 & 1.44 & 1.88 & 1.12 & 1.33 & 1.41 & 1.83 \\
\hline ML6 & 1.08 & 1.22 & 1.35 & 1.67 & 1.07 & 1.28 & 1.34 & 1.75 & 1.13 & 1.23 & 1.42 & 1.69 \\
\hline & 0.90 & 0.90 & 1.13 & 1.23 & 0.96 & 0.95 & 1.19 & 1.31 & 1.23 & 1.38 & 1.54 & 1.90 \\
\hline & 1.01 & 1.03 & 1.26 & 1.42 & 1.12 & 1.22 & 1.40 & 1.67 & 1.37 & 1.60 & 1.71 & 2.20 \\
\hline & 1.13 & 1.19 & 1.41 & 1.63 & 1.18 & 1.28 & 1.48 & 1.75 & 1.26 & 1.40 & 1.57 & 1.92 \\
\hline MH4 & 0.93 & 0.92 & 1.16 & 1.27 & 0.97 & 0.99 & 1.22 & 1.36 & 1.21 & 1.35 & 1.51 & 1.85 \\
\hline MH5 & 1.06 & 1.09 & 1.32 & 1.50 & 1.17 & 1.28 & 1.46 & 1.76 & 1.56 & 1.87 & 1.95 & 2.58 \\
\hline MH6 & 0.96 & 0.98 & 1.20 & 1.35 & 1.18 & 1.29 & 1.48 & 1.77 & 1.30 & 1.52 & 1.63 & 2.09 \\
\hline LL1 & 0.91 & 0.90 & 1.14 & 1.24 & 0.93 & 0.95 & 1.16 & 1.31 & 1.27 & 1.43 & 1.58 & 1.96 \\
\hline LL2 & 0.93 & 0.94 & 1.16 & 1.29 & 0.97 & 1.01 & 1.21 & 1.39 & 1.23 & 1.38 & 1.54 & 1.90 \\
\hline LL3 & 1.13 & 1.20 & 1.41 & 1.65 & 1.19 & 1.29 & 1.48 & 1.78 & 1.27 & 1.43 & 1.59 & 1.97 \\
\hline LL4 & 0.91 & 0.91 & 1.14 & 1.25 & 0.93 & 0.96 & 1.17 & 1.32 & 1.26 & 1.42 & 1.57 & 1.95 \\
\hline LL5 & 1.00 & 1.02 & 1.25 & 1.41 & 1.10 & 1.19 & 1.38 & 1.63 & 1.31 & 1.50 & 1.63 & 2.07 \\
\hline LL6 & 1.11 & 1.21 & 1.38 & 1.67 & 1.18 & 1.25 & 1.48 & 1.72 & 1.30 & 1.47 & 1.63 & 2.02 \\
\hline LH1 & 1.01 & 1.05 & 1.26 & 1.44 & 1.27 & 1.44 & 1.59 & 1.98 & 1.47 & 1.74 & 1.83 & 2.40 \\
\hline LH2 & 1.06 & 1.16 & 1.33 & 1.60 & 1.78 & 2.14 & 2.23 & 2.94 & 1.82 & 2.22 & 2.27 & 3.05 \\
\hline LH3 & 1.21 & 1.34 & 1.51 & 1.85 & 1.20 & 1.36 & 1.50 & 1.87 & 1.52 & 1.81 & 1.89 & 2.49 \\
\hline LH4 & 1.04 & 1.09 & 1.30 & 1.50 & 1.41 & 1.63 & 1.76 & 2.24 & 1.60 & 1.92 & 2.00 & 2.64 \\
\hline LH5 & 1.14 & 1.26 & 1.43 & 1.74 & 1.27 & 1.42 & 1.58 & 1.95 & 1.71 & 2.00 & 2.14 & 2.74 \\
\hline LH6 & 1.30 & 1.49 & 1.63 & 2.05 & 1.33 & 1.55 & 1.66 & 2.14 & 1.59 & 1.94 & 1.99 & 2.67 \\
\hline
\end{tabular}


Table C.61 - The ratio of actual mid-span bending moments and quarter-span shear forces for buildings to the corresponding values obtained from FEMA parabolic distribution and a uniform distribution of seismic load when subjected to M6C1 ground motion and the Load is acting parallel to the short side, $\alpha=0.10$

\begin{tabular}{|c|c|c|c|c|c|c|c|c|c|c|c|c|}
\hline \multirow{2}{*}{ Building } & \multicolumn{4}{|c|}{$\mu=2$} & \multicolumn{4}{|c|}{$\mu=3$} & \multicolumn{4}{|c|}{$\mu=4$} \\
\hline & M.F & $V . F$ & $M . U$ & $V . U$ & $M . F$ & $V . F$ & $M . U$ & $V . U$ & $M . F$ & V.F & $M . U$ & $V . U$ \\
\hline SL1 & 0.97 & 1.01 & 1.22 & 1.39 & 0.96 & 0.98 & 1.20 & 1.34 & 0.94 & 1.00 & 1.18 & 1.37 \\
\hline SL3 & .99 & 1.03 & 1.24 & 1.42 & 0.97 & 1.00 & 1.21 & 1.37 & 0.97 & 1.00 & 1.21 & 1.38 \\
\hline SL4 & 1.08 & 1.13 & 1.35 & 1.56 & 1.04 & 1.09 & 1.30 & 1.50 & 1.02 & 1.05 & 1.28 & 1.44 \\
\hline SH1 & 1.13 & 1.22 & 1.42 & 1.68 & 1.16 & 1.27 & 1.45 & 1.75 & 1.11 & 1.22 & 1.39 & 1.68 \\
\hline $\mathrm{SH} 2$ & 1.19 & 1.33 & 1.49 & 1.82 & 1.14 & 1.29 & 1.43 & 1.77 & 1.08 & 1.23 & 1.35 & 1.70 \\
\hline SH3 & 1.20 & 1.35 & 1.49 & 1.85 & 1.14 & 1.31 & 1.43 & 1.80 & 1.09 & 1.26 & 1.36 & 1.73 \\
\hline SH4 & 1.18 & 1.33 & 1.47 & 1.83 & 1.14 & 1.31 & 1.42 & 1.80 & 1.09 & 1.25 & 1.36 & 1.72 \\
\hline SH5 & 1.19 & 1.35 & 1.49 & 1.85 & 1.14 & 1.31 & 1.43 & 1.80 & 1.09 & 1.26 & 1.37 & 1.73 \\
\hline SH6 & 1.19 & 1.35 & 1.49 & 1.85 & 1.15 & 1.31 & 1.43 & 1.80 & 1.09 & 1.26 & 1.36 & 1.73 \\
\hline ML1 & 1.11 & 1.24 & 1.39 & 1.70 & 1.12 & 1.30 & 1.39 & 1.79 & 1.07 & 1.25 & 1.34 & 1.72 \\
\hline ML2 & 0.99 & 0.99 & 1.23 & 1.36 & 1.03 & 1.09 & 1.28 & 1.50 & 0.99 & 1.10 & 1.24 & 1.51 \\
\hline ML3 & 0.96 & 0.97 & 1.20 & 1.33 & 1.00 & 1.01 & 1.25 & 1.40 & 0.99 & 1.04 & 1.24 & 1.44 \\
\hline ML4 & 1.08 & 1.18 & 1.35 & 1.63 & 1.10 & 1.27 & 1.38 & 1.74 & 1.06 & 1.22 & 1.32 & 1.68 \\
\hline ML5 & 1.07 & 1.18 & 1.34 & 1.62 & 1.11 & 1.27 & 1.38 & 1.75 & 1.06 & 1.23 & 1.32 & 1.69 \\
\hline ML6 & 0.99 & 0.97 & 1.23 & 1.33 & 1.00 & 1.04 & 1.25 & 1.43 & 0.98 & 1.04 & 1.23 & 1.44 \\
\hline MH1 & 1.01 & 1.06 & 1.27 & 1.46 & 1.03 & 1.06 & 1.29 & 1.46 & 1.02 & 1.10 & 1.27 & 1.51 \\
\hline MH2 & 1.09 & 1.19 & 1.37 & 1.63 & 1.07 & 1.16 & 1.34 & 1.59 & 1.09 & 1.22 & 1.36 & 1.68 \\
\hline MH3 & 1.47 & 1.67 & 1.84 & 2.30 & 1.53 & 1.76 & 1.91 & 2.42 & 1.49 & 1.68 & 1.86 & 2.31 \\
\hline MH4 & 1.10 & 1.20 & 1.38 & 1.65 & 1.08 & 1.17 & 1.35 & 1.61 & 1.08 & 1.18 & 1.34 & 1.63 \\
\hline MH5 & 1.10 & 1.19 & 1.37 & 1.63 & 1.09 & 1.19 & 1.36 & 1.63 & 1.11 & 1.25 & 1.38 & 1.72 \\
\hline MH6 & 1.11 & 1.24 & 1.39 & 1.70 & 1.15 & 1.35 & 1.43 & 1.86 & 1.10 & 1.33 & 1.38 & 1.83 \\
\hline LL1 & 0.97 & 0.97 & 1.21 & 1.34 & 0.96 & 0.97 & 1.20 & 1.33 & 1.06 & 1.12 & 1.32 & 1.54 \\
\hline LL2 & 0.98 & 0.99 & 1.23 & 1.37 & 0.97 & 0.99 & 1.22 & 1.37 & 1.08 & 1.14 & 1.34 & 1.57 \\
\hline LL3 & 1.16 & 1.29 & 1.45 & 1.77 & 1.37 & 1.55 & 1.72 & 2.14 & 1.48 & 1.67 & 1.85 & 2.29 \\
\hline LL4 & 0.91 & 0.92 & 1.14 & 1.27 & 0.93 & 0.92 & 1.16 & 1.27 & 1.01 & 1.08 & 1.26 & 1.49 \\
\hline LL5 & 0.90 & 0.91 & 1.13 & 1.25 & 0.92 & 0.91 & 1.15 & 1.25 & 1.00 & 1.06 & 1.25 & 1.46 \\
\hline LL6 & 1.13 & 1.35 & 1.41 & 1.86 & 1.15 & 1.46 & 1.44 & 2.01 & 1.16 & 1.54 & 1.45 & 2.11 \\
\hline LH1 & 1.25 & 1.38 & 1.56 & 1.90 & 1.52 & 1.76 & 1.90 & 2.42 & 1.49 & 1.69 & 1.86 & 2.33 \\
\hline LH2 & 1.32 & 1.50 & 1.64 & 2.06 & 1.52 & 1.78 & 1.90 & 2.44 & 1.48 & 1.71 & 1.85 & 2.35 \\
\hline LH3 & 1.33 & 1.71 & 1.66 & 2.35 & 1.45 & 1.81 & 1.81 & 2.49 & 1.43 & 1.73 & 1.79 & 2.38 \\
\hline LH4 & 1.38 & 1.55 & 1.72 & 2.14 & 1.54 & 1.79 & 1.93 & 2.46 & 1.48 & 1.70 & 1.85 & 2.34 \\
\hline LH5 & 1.38 & 1.56 & 1.73 & 2.15 & 1.54 & 1.78 & 1.93 & 2.45 & 1.49 & 1.72 & 1.86 & 2.36 \\
\hline LH6 & 1.24 & 1.59 & 1.55 & 2.19 & 1.34 & 1.74 & 1.67 & 2.39 & 1.39 & 1.76 & 1.74 & 2.42 \\
\hline
\end{tabular}


Table C.62 - The ratio of actual mid-span bending moments and quarter-span shear forces for buildings to the corresponding values obtained from FEMA parabolic distribution and a uniform distribution of seismic load when subjected to M6C2 ground motion and the Load is acting parallel to the short side, $\alpha=0.10$

\begin{tabular}{|c|c|c|c|c|c|c|c|c|c|c|c|c|}
\hline \multirow{2}{*}{ Building } & \multicolumn{4}{|c|}{$\mu=2$} & \multicolumn{4}{|c|}{$\mu=3$} & \multicolumn{4}{|c|}{$\mu=4$} \\
\hline & M.F & $V . F$ & $M . U$ & $V . U$ & $M . F$ & $V . F$ & $M . U$ & $V . U$ & $M . F$ & V.F & $M . U$ & $V . U$ \\
\hline SL1 & 1.00 & 1.07 & 1.25 & 1.47 & 0.96 & 1.05 & 1.20 & 1.44 & 0.94 & 1.03 & 1.18 & 1.42 \\
\hline SL3 & 1.02 & 1.09 & 1.27 & 1.50 & 0.98 & 1.08 & 1.22 & 1.49 & 0.93 & 1.06 & 1.16 & 1.45 \\
\hline SL4 & 1.03 & 1.11 & 1.29 & 1.53 & 1.00 & 1.11 & 1.25 & 1.52 & 0.98 & 1.08 & 1.23 & 1.48 \\
\hline SH1 & 1.07 & 1.15 & 1.34 & 1.58 & 1.10 & 1.26 & 1.37 & 1.73 & 1.08 & 1.27 & 1.35 & 1.74 \\
\hline $\mathrm{SH} 2$ & 1.04 & 1.04 & 1.30 & 1.43 & 1.06 & 1.15 & 1.33 & 1.58 & 1.05 & 1.19 & 1.31 & 1.64 \\
\hline SH3 & 1.02 & 1.04 & 1.27 & 1.42 & 1.07 & 1.12 & 1.34 & 1.54 & 1.12 & 1.20 & 1.41 & 1.65 \\
\hline SH4 & 1.06 & 1.11 & 1.32 & 1.53 & 1.08 & 1.16 & 1.34 & 1.60 & 1.13 & 1.18 & 1.41 & 1.63 \\
\hline SH5 & 1.06 & 1.12 & 1.33 & 1.54 & 1.11 & 1.19 & 1.38 & 1.64 & 1.15 & 1.21 & 1.44 & 1.66 \\
\hline SH6 & 1.01 & 1.03 & 1.27 & 1.42 & 1.08 & 1.12 & 1.35 & 1.54 & 1.13 & 1.20 & 1.41 & 1.65 \\
\hline ML1 & 1.36 & 1.50 & 1.70 & 2.06 & 1.29 & 1.41 & 1.62 & 1.94 & 1.24 & 1.35 & 1.55 & 1.86 \\
\hline ML2 & 1.22 & 1.35 & 1.53 & 1.85 & 1.17 & 1.30 & 1.46 & 1.78 & 1.15 & 1.30 & 1.44 & 1.78 \\
\hline ML3 & 1.09 & 1.14 & 1.36 & 1.57 & 1.11 & 1.22 & 1.38 & 1.68 & 1.12 & 1.30 & 1.40 & 1.79 \\
\hline ML4 & 1.34 & 1.50 & 1.68 & 2.06 & 1.29 & 1.42 & 1.62 & 1.95 & 1.26 & 1.38 & 1.58 & 1.90 \\
\hline ML5 & 1.34 & 1.49 & 1.68 & 2.05 & 1.30 & 1.42 & 1.62 & 1.95 & 1.27 & 1.39 & 1.58 & 1.90 \\
\hline ML6 & 1.17 & 1.28 & 1.46 & 1.76 & 1.14 & 1.27 & 1.43 & 1.75 & 1.14 & 1.32 & 1.43 & 1.82 \\
\hline MH1 & 1.03 & 1.07 & 1.29 & 1.47 & 1.10 & 1.24 & 1.37 & 1.70 & 1.08 & 1.28 & 1.36 & 1.75 \\
\hline MH2 & 0.96 & 1.04 & 1.20 & 1.43 & 0.93 & 1.05 & 1.16 & 1.45 & 0.93 & 1.03 & 1.17 & 1.41 \\
\hline MH3 & 1.25 & 1.41 & 1.56 & 1.93 & 1.22 & 1.35 & 1.52 & 1.86 & 1.17 & 1.27 & 1.46 & 1.74 \\
\hline MH4 & 0.88 & 0.93 & 1.10 & 1.28 & 0.89 & 0.98 & 1.12 & 1.35 & 0.87 & 0.98 & 1.09 & 1.35 \\
\hline MH5 & 0.86 & 0.92 & 1.08 & 1.27 & 0.84 & 0.94 & 1.05 & 1.29 & 0.93 & 1.01 & 1.16 & 1.39 \\
\hline MH6 & 1.41 & 1.60 & 1.76 & 2.20 & 1.33 & 1.52 & 1.67 & 2.10 & 1.28 & 1.52 & 1.61 & 2.09 \\
\hline LL1 & 1.19 & 1.35 & 1.49 & 1.86 & 1.12 & 1.32 & 1.40 & 1.82 & 1.04 & 1.25 & 1.30 & 1.72 \\
\hline LL2 & 1.17 & 1.33 & 1.46 & 1.83 & 1.09 & 1.29 & 1.37 & 1.77 & 1.01 & 1.22 & 1.27 & 1.68 \\
\hline LL3 & 1.02 & 1.13 & 1.28 & 1.56 & 1.03 & 1.08 & 1.28 & 1.48 & 1.06 & 1.13 & 1.32 & 1.55 \\
\hline LL4 & 1.26 & 1.42 & 1.57 & 1.95 & 1.26 & 1.46 & 1.57 & 2.01 & 1.17 & 1.39 & 1.46 & 1.92 \\
\hline LL5 & 1.28 & 1.45 & 1.60 & 1.99 & 1.27 & 1.48 & 1.59 & 2.04 & 1.18 & 1.40 & 1.48 & 1.93 \\
\hline LL6 & 1.28 & 1.60 & 1.60 & 2.20 & 1.29 & 1.59 & 1.61 & 2.19 & 1.29 & 1.58 & 1.61 & 2.17 \\
\hline LH1 & 1.11 & 1.25 & 1.39 & 1.71 & 1.08 & 1.14 & 1.35 & 1.57 & 1.05 & 1.15 & 1.31 & 1.58 \\
\hline LH2 & 1.23 & 1.35 & 1.54 & 1.85 & 1.14 & 1.25 & 1.43 & 1.72 & 1.08 & 1.21 & 1.34 & 1.66 \\
\hline LH3 & 1.21 & 1.44 & 1.51 & 1.98 & 1.24 & 1.41 & 1.54 & 1.95 & 1.22 & 1.38 & 1.53 & 1.90 \\
\hline LH4 & 1.22 & 1.32 & 1.52 & 1.82 & 1.15 & 1.27 & 1.44 & 1.75 & 1.08 & 1.21 & 1.36 & 1.66 \\
\hline LH5 & 1.22 & 1.32 & 1.52 & 1.82 & 1.16 & 1.29 & 1.45 & 1.77 & 1.09 & 1.21 & 1.36 & 1.66 \\
\hline LH6 & 1.22 & 1.48 & 1.53 & 2.03 & 1.25 & 1.47 & 1.56 & 2.02 & 1.24 & 1.44 & 1.56 & 1.98 \\
\hline
\end{tabular}


Table C.63 - The ratio of actual mid-span bending moments and quarter-span shear forces for buildings to the corresponding values obtained from FEMA parabolic distribution and a uniform distribution of seismic load when subjected to M6C26 ground motion and the Load is acting parallel to the short side, $\alpha=0.10$

\begin{tabular}{|c|c|c|c|c|c|c|c|c|c|c|c|c|}
\hline \multirow{2}{*}{ Building } & \multicolumn{4}{|c|}{$\mu=2$} & \multicolumn{4}{|c|}{$\mu=3$} & \multicolumn{4}{|c|}{$\mu=4$} \\
\hline & $M . F$ & $V . F$ & $M . U$ & V.U & $M . F$ & $V . F$ & $M . U$ & $V . U$ & $M . F$ & V.F & $M . U$ & $V . U$ \\
\hline SL1 & 1.05 & 1.10 & 1.31 & 1.51 & 1.04 & 1.10 & 1.30 & 1.51 & 1.02 & 1.08 & 1.28 & 1.49 \\
\hline SL3 & 1.06 & 1.10 & 1.32 & 1.52 & 1.04 & 1.10 & 1.30 & 1.52 & 1.03 & 1.09 & 1.28 & 1.50 \\
\hline SL4 & 1.08 & 1.12 & 1.34 & 1.55 & 1.06 & 1.11 & 1.32 & 1.53 & 1.04 & 1.09 & 1.30 & 1.49 \\
\hline SH1 & 1.06 & 1.13 & 1.32 & 1.55 & 1.01 & 1.10 & 1.26 & 1.52 & 1.00 & 1.06 & 1.25 & 1.45 \\
\hline $\mathrm{SH} 2$ & 1.08 & 1.16 & 1.35 & 1.59 & 1.03 & 1.13 & 1.29 & 1.56 & 1.02 & 1.08 & 1.27 & 1.48 \\
\hline SH3 & 1.08 & 1.17 & 1.35 & 1.61 & 1.03 & 1.14 & 1.29 & 1.56 & 1.02 & 1.08 & 1.28 & 1.49 \\
\hline SH4 & 1.08 & 1.17 & 1.35 & 1.61 & 1.04 & 1.14 & 1.30 & 1.57 & 1.03 & 1.09 & 1.29 & 1.49 \\
\hline SH5 & 1.08 & 1.17 & 1.34 & 1.61 & 1.04 & 1.14 & 1.30 & 1.56 & 1.03 & 1.09 & 1.29 & 1.50 \\
\hline SH6 & 1.08 & 1.17 & 1.35 & 1.61 & 1.03 & 1.13 & 1.29 & 1.56 & 1.02 & 1.09 & 1.28 & 1.49 \\
\hline ML1 & 1.04 & 1.10 & 1.31 & 1.51 & 1.09 & 1.15 & 1.36 & 1.58 & 1.08 & 1.14 & 1.34 & 1.57 \\
\hline ML2 & 0.91 & 0.98 & 1.13 & 1.34 & 0.99 & 1.05 & 1.24 & 1.44 & 1.03 & 1.16 & 1.29 & 1.59 \\
\hline $\mathrm{M}$ & 0.88 & 0.93 & 1.09 & 1.27 & 0.98 & 1.11 & 1.23 & 1.53 & 0.98 & 1.16 & 1.23 & 1.59 \\
\hline ML4 & 0.99 & 1.05 & 1.24 & 1.44 & 1.07 & 1.13 & 1.33 & 1.55 & 1.08 & 1.16 & 1.35 & 1.59 \\
\hline ML5 & 0.99 & 1.05 & 1.24 & 1.44 & 1.07 & 1.13 & 1.34 & 1.56 & 1.08 & 1.15 & 1.35 & 1.58 \\
\hline ML6 & 0.89 & 0.96 & 1.12 & 1.33 & 1.00 & 1.11 & 1.25 & 1.52 & 1.02 & 1.19 & 1.27 & 1.63 \\
\hline MH1 & 0.92 & 1.00 & 1.15 & 1.38 & 1.03 & 1.14 & 1.29 & 1.57 & 1.01 & 1.17 & 1.26 & 1.60 \\
\hline MH2 & 0.95 & 1.02 & 1.19 & 1.41 & 0.95 & 1.10 & 1.18 & 1.52 & 0.95 & 1.16 & 1.19 & 1.59 \\
\hline MH3 & 1.08 & 1.36 & 1.35 & 1.87 & 1.32 & 1.57 & 1.66 & 2.16 & 1.35 & 1.58 & 1.69 & 2.18 \\
\hline MH4 & 0.94 & 1.00 & 1.18 & 1.37 & 0.92 & 1.06 & 1.15 & 1.46 & 0.93 & 1.11 & 1.16 & 1.52 \\
\hline MH5 & 0.94 & 1.03 & 1.18 & 1.41 & 0.92 & 1.10 & 1.15 & 1.51 & 0.95 & 1.16 & 1.18 & 1.60 \\
\hline MH6 & 1.11 & 1.19 & 1.39 & 1.63 & 1.11 & 1.19 & 1.38 & 1.63 & 1.27 & 1.47 & 1.58 & 2.02 \\
\hline LL1 & 1.11 & 1.17 & 1.39 & 1.61 & 1.18 & 1.26 & 1.48 & 1.74 & 1.23 & 1.38 & 1.54 & 1.90 \\
\hline & 1.12 & 1.19 & 1.40 & 1.64 & 1.18 & 1.26 & 1.48 & 1.73 & 1.22 & 1.40 & 1.53 & 1.92 \\
\hline LL3 & 1.00 & 1.15 & 1.25 & 1.58 & 1.02 & 1.24 & 1.27 & 1.71 & 1.09 & 1.29 & 1.36 & 1.78 \\
\hline LL4 & 1.05 & 1.13 & 1.31 & 1.55 & 1.17 & 1.27 & 1.46 & 1.74 & 1.18 & 1.37 & 1.47 & 1.89 \\
\hline LL5 & 1.05 & 1.13 & 1.31 & 1.55 & 1.18 & 1.27 & 1.47 & 1.74 & 1.18 & 1.37 & 1.48 & 1.88 \\
\hline LL6 & 1.52 & 1.83 & 1.90 & 2.51 & 1.56 & 1.84 & 1.95 & 2.53 & 1.58 & 1.87 & 1.98 & 2.57 \\
\hline LH1 & 1.05 & 1.26 & 1.31 & 1.73 & 1.14 & 1.36 & 1.42 & 1.87 & 1.23 & 1.44 & 1.54 & 1.98 \\
\hline LH2 & 1.15 & 1.39 & 1.43 & 1.91 & 1.31 & 1.55 & 1.63 & 2.13 & 1.41 & 1.66 & 1.77 & 2.28 \\
\hline LH3 & 1.41 & 1.75 & 1.76 & 2.40 & 1.69 & 2.05 & 2.11 & 2.81 & 1.70 & 2.04 & 2.12 & 2.80 \\
\hline LH4 & 1.13 & 1.37 & 1.42 & 1.88 & 1.30 & 1.52 & 1.63 & 2.09 & 1.42 & 1.64 & 1.78 & 2.26 \\
\hline LH5 & 1.15 & 1.38 & 1.43 & 1.90 & 1.33 & 1.55 & 1.66 & 2.13 & 1.46 & 1.68 & 1.83 & 2.31 \\
\hline LH6 & 1.54 & 1.93 & 1.93 & 2.65 & 1.60 & 1.98 & 2.00 & 2.72 & 1.59 & 1.93 & 1.99 & 2.66 \\
\hline
\end{tabular}


Table C.64 - The ratio of actual mid-span bending moments and quarter-span shear forces for buildings to the corresponding values obtained from FEMA parabolic distribution and a uniform distribution of seismic load when subjected to M6C31 ground motion and the Load is acting parallel to the short side, $\alpha=0.10$

\begin{tabular}{|c|c|c|c|c|c|c|c|c|c|c|c|c|}
\hline \multirow{2}{*}{ Building } & \multicolumn{4}{|c|}{$\mu=2$} & \multicolumn{4}{|c|}{$\mu=3$} & \multicolumn{4}{|c|}{$\mu=4$} \\
\hline & $M . F$ & $V . F$ & $M . U$ & V.U & $M . F$ & $V . F$ & $M . U$ & $V . U$ & $M . F$ & V.F & $M . U$ & $V . U$ \\
\hline SL1 & 1.07 & 1.13 & 1.34 & 1.56 & 1.06 & 1.12 & 1.33 & 1.55 & 1.05 & 1.11 & 1.31 & 1.53 \\
\hline SL3 & .09 & 1.15 & 1.36 & 1.58 & 1.08 & 1.14 & 1.35 & 1.57 & 1.06 & 1.14 & 1.33 & 1.56 \\
\hline SL4 & .10 & 1.16 & 1.37 & 1.60 & 1.09 & 1.17 & 1.37 & 1.61 & 1.08 & 1.17 & 1.35 & 1.61 \\
\hline SH1 & 1.08 & 1.12 & 1.35 & 1.54 & 1.09 & 1.12 & 1.36 & 1.54 & 1.08 & 1.12 & 1.35 & 1.54 \\
\hline $\mathrm{SH} 2$ & 1.13 & 1.17 & 1.41 & 1.61 & 1.15 & 1.21 & 1.44 & 1.66 & 1.15 & 1.22 & 1.44 & 1.68 \\
\hline SH3 & 1.16 & 1.23 & 1.45 & 1.70 & 1.17 & 1.27 & 1.46 & 1.74 & 1.17 & 1.27 & 1.46 & 1.74 \\
\hline SH4 & 1.18 & 1.29 & 1.47 & 1.77 & 1.18 & 1.30 & 1.47 & 1.79 & 1.16 & 1.28 & 1.45 & 1.75 \\
\hline SH5 & 1.18 & 1.29 & 1.47 & 1.77 & 1.18 & 1.31 & 1.47 & 1.80 & 1.16 & 1.27 & 1.45 & 1.75 \\
\hline SH6 & 1.16 & 1.24 & 1.45 & 1.70 & 1.17 & 1.27 & 1.46 & 1.74 & 1.16 & 1.27 & 1.46 & 1.74 \\
\hline ML1 & 1.22 & 1.37 & 1.52 & 1.88 & 1.20 & 1.35 & 1.50 & 1.85 & 1.16 & 1.31 & 1.46 & 1.81 \\
\hline ML2 & 1.15 & 1.24 & 1.44 & 1.71 & 1.10 & 1.30 & 1.38 & 1.79 & 1.05 & 1.33 & 1.31 & 1.82 \\
\hline L3 & 1.07 & 1.19 & 1.33 & 1.64 & 1.01 & 1.24 & 1.27 & 1.70 & 0.97 & 1.27 & 1.21 & 1.74 \\
\hline ML4 & 1.16 & 1.29 & 1.45 & 1.77 & 1.16 & 1.30 & 1.45 & 1.78 & 1.15 & 1.32 & 1.43 & 1.82 \\
\hline ML5 & 1.17 & 1.30 & 1.46 & 1.78 & 1.16 & 1.30 & 1.45 & 1.79 & 1.15 & 1.32 & 1.44 & 1.82 \\
\hline ML6 & 1.12 & 1.24 & 1.40 & 1.71 & 1.05 & 1.29 & 1.32 & 1.77 & 1.01 & 1.32 & 1.27 & 1.81 \\
\hline MH1 & 1.05 & 1.12 & 1.31 & 1.55 & 1.00 & 1.18 & 1.25 & 1.62 & 0.95 & 1.21 & 1.19 & 1.66 \\
\hline MH2 & 1.09 & 1.15 & 1.37 & 1.58 & 1.05 & 1.14 & 1.31 & 1.57 & 1.01 & 1.11 & 1.27 & 1.53 \\
\hline MH3 & 1.18 & 1.37 & 1.48 & 1.88 & 1.23 & 1.38 & 1.54 & 1.90 & 1.23 & 1.36 & 1.54 & 1.87 \\
\hline MH4 & 1.13 & 1.21 & 1.41 & 1.66 & 1.08 & 1.18 & 1.35 & 1.62 & 1.04 & 1.13 & 1.30 & 1.55 \\
\hline MH5 & 1.12 & 1.22 & 1.40 & 1.68 & 1.08 & 1.20 & 1.35 & 1.64 & 1.03 & 1.15 & 1.29 & 1.58 \\
\hline MH6 & 1.22 & 1.35 & 1.53 & 1.86 & 1.34 & 1.52 & 1.68 & 2.09 & 1.42 & 1.66 & 1.77 & 2.29 \\
\hline LL1 & 1.13 & 1.19 & 1.42 & 1.64 & 1.17 & 1.27 & 1.46 & 1.75 & 1.14 & 1.24 & 1.43 & 1.71 \\
\hline & 1.15 & 1.22 & 1.44 & 1.68 & 1.17 & 1.29 & 1.47 & 1.77 & 1.14 & 1.24 & 1.42 & 1.70 \\
\hline LL3 & 1.01 & 1.19 & 1.26 & 1.64 & 1.02 & 1.26 & 1.27 & 1.73 & 1.05 & 1.28 & 1.31 & 1.76 \\
\hline LL4 & 1.21 & 1.30 & 1.52 & 1.79 & 1.16 & 1.23 & 1.46 & 1.69 & 1.11 & 1.21 & 1.39 & 1.66 \\
\hline LL5 & 1.21 & 1.29 & 1.51 & 1.78 & 1.17 & 1.24 & 1.46 & 1.70 & 1.12 & 1.22 & 1.40 & 1.67 \\
\hline LL6 & 0.95 & 1.34 & 1.19 & 1.85 & 0.92 & 1.34 & 1.14 & 1.84 & 0.95 & 1.32 & 1.18 & 1.81 \\
\hline LH1 & 1.09 & 1.31 & 1.36 & 1.80 & 1.11 & 1.40 & 1.39 & 1.92 & 1.13 & 1.44 & 1.41 & 1.98 \\
\hline LH2 & 1.16 & 1.39 & 1.45 & 1.92 & 1.16 & 1.46 & 1.45 & 2.01 & 1.16 & 1.54 & 1.45 & 2.11 \\
\hline LH3 & 1.07 & 1.34 & 1.34 & 1.84 & 1.13 & 1.38 & 1.42 & 1.90 & 1.15 & 1.39 & 1.44 & 1.91 \\
\hline LH4 & 1.13 & 1.31 & 1.42 & 1.80 & 1.13 & 1.41 & 1.42 & 1.93 & 1.15 & 1.55 & 1.43 & 2.12 \\
\hline LH5 & 1.14 & 1.30 & 1.42 & 1.78 & 1.14 & 1.40 & 1.42 & 1.92 & 1.14 & 1.53 & 1.42 & 2.11 \\
\hline LH6 & 1.00 & 1.38 & 1.25 & 1.89 & 1.05 & 1.36 & 1.31 & 1.87 & 1.08 & 1.40 & 1.36 & 1.93 \\
\hline
\end{tabular}


Table C.65 - The ratio of actual mid-span bending moments and quarter-span shear forces for buildings to the corresponding values obtained from FEMA parabolic distribution and a uniform distribution of seismic load when subjected to M6C 38 ground motion and the Load is acting parallel to the short side, $\alpha=0.10$

\begin{tabular}{|c|c|c|c|c|c|c|c|c|c|c|c|c|}
\hline \multirow{2}{*}{ Building } & \multicolumn{4}{|c|}{$\mu=2$} & \multicolumn{4}{|c|}{$\mu=3$} & \multicolumn{4}{|c|}{$\mu=4$} \\
\hline & M.F & $V . F$ & $M . U$ & $V . U$ & $M . F$ & $V . F$ & $M . U$ & $V . U$ & $M . F$ & V.F & $M . U$ & $V . U$ \\
\hline SL1 & 1.15 & 1.24 & 1.44 & 1.70 & 1.13 & 1.21 & 1.41 & 1.67 & 1.11 & 1.18 & 1.39 & 1.63 \\
\hline SL3 & 15 & 1.24 & 1.44 & 1.71 & 1.14 & 1.22 & 1.42 & 1.68 & 1.12 & 1.19 & 1.39 & 1.63 \\
\hline SL4 & .16 & 1.25 & 1.44 & 1.72 & 1.14 & 1.23 & 1.42 & 1.69 & 1.12 & 1.20 & 1.40 & 1.65 \\
\hline SH1 & 1.12 & 1.16 & 1.40 & 1.59 & 1.14 & 1.20 & 1.43 & 1.65 & 1.15 & 1.22 & 1.44 & 1.68 \\
\hline $\mathrm{SH} 2$ & 1.13 & 1.16 & 1.41 & 1.59 & 1.16 & 1.20 & 1.45 & 1.66 & 1.17 & 1.23 & 1.46 & 1.69 \\
\hline SH3 & 1.14 & 1.16 & 1.43 & 1.60 & 1.17 & 1.22 & 1.46 & 1.67 & 1.18 & 1.24 & 1.48 & 1.71 \\
\hline SH4 & 1.14 & 1.16 & 1.43 & 1.59 & 1.17 & 1.20 & 1.46 & 1.65 & 1.18 & 1.23 & 1.48 & 1.68 \\
\hline SH5 & 1.15 & 1.16 & 1.44 & 1.60 & 1.18 & 1.21 & 1.47 & 1.67 & 1.19 & 1.24 & 1.49 & 1.70 \\
\hline SH6 & 1.14 & 1.17 & 1.43 & 1.60 & 1.17 & 1.22 & 1.46 & 1.67 & 1.18 & 1.24 & 1.48 & 1.71 \\
\hline ML1 & 1.15 & 1.15 & 1.44 & 1.59 & 1.17 & 1.19 & 1.47 & 1.64 & 1.18 & 1.22 & 1.48 & 1.68 \\
\hline ML2 & 1.12 & 1.14 & 1.40 & 1.57 & 1.15 & 1.18 & 1.44 & 1.62 & 1.17 & 1.21 & 1.46 & 1.66 \\
\hline ML3 & 1.07 & 1.12 & 1.34 & 1.54 & 1.15 & 1.21 & 1.44 & 1.66 & 1.18 & 1.24 & 1.47 & 1.71 \\
\hline ML4 & 1.15 & 1.14 & 1.44 & 1.56 & 1.17 & 1.18 & 1.47 & 1.62 & 1.19 & 1.21 & 1.48 & 1.67 \\
\hline ML5 & 1.15 & 1.14 & 1.44 & 1.56 & 1.18 & 1.18 & 1.47 & 1.62 & 1.19 & 1.21 & 1.48 & 1.67 \\
\hline ML6 & 1.11 & 1.15 & 1.39 & 1.58 & 1.16 & 1.20 & 1.45 & 1.65 & 1.18 & 1.23 & 1.48 & 1.70 \\
\hline MH1 & 0.96 & 1.02 & 1.21 & 1.40 & 1.12 & 1.18 & 1.40 & 1.62 & 1.15 & 1.22 & 1.44 & 1.67 \\
\hline MH2 & 0.88 & 0.94 & 1.10 & 1.30 & 1.04 & 1.13 & 1.30 & 1.56 & 1.15 & 1.25 & 1.43 & 1.72 \\
\hline MH3 & 0.91 & 0.88 & 1.13 & 1.21 & 0.97 & 0.97 & 1.21 & 1.33 & 0.99 & 1.07 & 1.24 & 1.47 \\
\hline MH4 & 0.84 & 0.91 & 1.05 & 1.25 & 0.91 & 0.99 & 1.14 & 1.36 & 0.98 & 1.09 & 1.22 & 1.49 \\
\hline MH5 & 0.85 & 0.92 & 1.06 & 1.26 & 0.95 & 1.06 & 1.19 & 1.45 & 1.09 & 1.21 & 1.36 & 1.66 \\
\hline MH6 & 1.15 & 1.18 & 1.44 & 1.62 & 1.20 & 1.26 & 1.50 & 1.74 & 1.22 & 1.32 & 1.53 & 1.82 \\
\hline LL1 & 0.94 & 0.95 & 1.17 & 1.30 & 1.02 & 1.06 & 1.27 & 1.46 & 1.04 & 1.11 & 1.30 & 1.52 \\
\hline LL2 & 0.93 & 0.93 & 1.16 & 1.28 & 1.01 & 1.05 & 1.27 & 1.44 & 1.04 & 1.10 & 1.30 & 1.51 \\
\hline LL3 & 0.86 & 0.86 & 1.07 & 1.18 & 0.87 & 0.97 & 1.08 & 1.34 & 0.97 & 1.09 & 1.21 & 1.50 \\
\hline LL4 & 1.04 & 1.05 & 1.30 & 1.44 & 1.09 & 1.12 & 1.36 & 1.53 & 1.11 & 1.16 & 1.39 & 1.60 \\
\hline LL5 & 1.04 & 1.05 & 1.31 & 1.45 & 1.09 & 1.12 & 1.36 & 1.54 & 1.11 & 1.17 & 1.39 & 1.60 \\
\hline LL6 & 1.04 & 1.13 & 1.30 & 1.55 & 1.10 & 1.24 & 1.38 & 1.70 & 1.11 & 1.29 & 1.38 & 1.78 \\
\hline LH1 & 0.91 & 0.90 & 1.14 & 1.24 & 0.90 & 1.03 & 1.13 & 1.41 & 0.95 & 1.08 & 1.18 & 1.49 \\
\hline LH2 & 0.95 & 0.93 & 1.19 & 1.28 & 0.87 & 1.01 & 1.09 & 1.39 & 0.94 & 1.09 & 1.17 & 1.50 \\
\hline LH3 & 1.02 & 1.08 & 1.27 & 1.48 & 1.00 & 1.08 & 1.25 & 1.48 & 1.06 & 1.20 & 1.32 & 1.66 \\
\hline LH4 & 0.94 & 0.93 & 1.17 & 1.27 & 0.88 & 1.03 & 1.10 & 1.42 & 0.95 & 1.11 & 1.19 & 1.53 \\
\hline LH5 & 0.94 & 0.93 & 1.17 & 1.28 & 0.88 & 1.04 & 1.10 & 1.42 & 0.95 & 1.12 & 1.18 & 1.54 \\
\hline LH6 & 1.01 & 1.07 & 1.26 & 1.47 & 1.03 & 1.13 & 1.29 & 1.55 & 1.06 & 1.19 & 1.33 & 1.63 \\
\hline
\end{tabular}


Table C.66 - The ratio of actual mid-span bending moments and quarter-span shear forces for buildings to the corresponding values obtained from FEMA parabolic distribution and a uniform distribution of seismic load when subjected to $\mathrm{M} 6 \mathrm{C} 1$ ground motion and the Load is acting parallel to the long side, $\alpha=0.10$

\begin{tabular}{|c|c|c|c|c|c|c|c|c|c|c|c|c|}
\hline \multirow{2}{*}{ Building } & \multicolumn{4}{|c|}{$\mu=2$} & \multicolumn{4}{|c|}{$\mu=3$} & \multicolumn{4}{|c|}{$\mu=4$} \\
\hline & $M . F$ & $V . F$ & $M . U$ & V.U & M.F & $V . F$ & $M . U$ & $V . U$ & M.F & V.F & M.U & $V . U$ \\
\hline SL1 & 0.98 & 0.99 & 1.22 & 1.36 & 0.98 & 1.01 & 1.23 & 1.39 & 1.00 & 1.05 & 1.26 & 1.45 \\
\hline SL3 & 98 & 0.99 & 1.23 & 1.36 & 0.99 & 1.01 & 1.24 & 1.39 & 1.00 & 1.04 & 1.25 & 1.43 \\
\hline & 0.98 & 1.01 & 1.22 & 1.39 & 0.98 & 0.99 & 1.23 & 1.37 & 0.97 & 1.01 & 1.22 & 1.39 \\
\hline SH1 & 16 & 1.28 & 1.45 & 1.75 & 1.11 & 1.22 & 1.38 & 1.68 & 1.03 & 1.13 & 1.28 & 1.55 \\
\hline & 1.11 & 1.22 & 1.39 & 1.68 & 1.02 & 1.12 & 1.28 & 1.53 & 0.94 & 1.01 & 1.17 & 1.39 \\
\hline $\mathrm{SH} 3$ & 1.01 & 1.09 & 1.26 & 1.50 & 0.98 & 1.02 & 1.22 & 1.41 & 0.95 & 1.01 & 1.19 & 1.38 \\
\hline $\mathrm{SH} 4$ & 1.01 & 1.08 & 1.26 & 1.49 & 0.99 & 1.03 & 1.23 & 1.42 & 0.97 & 1.03 & 1.21 & 1.41 \\
\hline SH5 & 1.00 & 1.04 & 1.25 & 1.44 & 0.99 & 1.03 & 1.23 & 1.41 & 0.97 & 1.03 & 1.22 & 1.42 \\
\hline IL1 & 1.03 & 1.11 & 1.29 & 1.52 & 1.07 & 1.14 & 1.33 & 1.56 & 1.05 & 1.15 & 1.31 & 1.59 \\
\hline ML2 & 1.07 & 1.15 & 1.33 & 1.58 & 1.07 & 1.16 & 1.34 & 1.60 & 1.04 & 1.16 & 1.29 & 1.59 \\
\hline ML3 & 1.02 & 1.09 & 1.28 & 1.49 & 1.05 & 1.14 & 1.31 & 1.57 & 1.04 & 1.18 & 1.30 & 1.62 \\
\hline ML4 & 1.07 & 1.15 & 1.33 & 1.58 & 1.09 & 1.19 & 1.36 & 1.64 & 1.03 & 1.16 & 1.29 & 1.59 \\
\hline ML5 & 1.06 & 1.14 & 1.33 & 1.57 & 1.07 & 1.16 & 1.34 & 1.59 & 1.04 & 1.16 & 1.30 & 1.59 \\
\hline ML6 & 1.04 & 1.12 & 1.30 & 1.54 & 1.06 & 1.16 & 1.32 & 1.60 & 1.04 & 1.17 & 1.29 & 1.61 \\
\hline & .05 & 1.12 & 1.31 & 1.54 & 1.09 & 1.22 & 1.36 & 1.68 & 1.07 & 1.23 & 1.33 & 1.69 \\
\hline & & 1.31 & 1.46 & 1.80 & 1.12 & 1.31 & 1.40 & 1.80 & 1.10 & 1.25 & 1.38 & 1.72 \\
\hline & 1.22 & 1.37 & 1.52 & 1.88 & 1.22 & 1.42 & 1.52 & 1.96 & 1.15 & 1.36 & 1.43 & 1.87 \\
\hline MH4 & 1.05 & 1.12 & 1.31 & 1.54 & 1.10 & 1.24 & 1.38 & 1.71 & 1.07 & 1.25 & 1.34 & 1.72 \\
\hline MH5 & 1.15 & 1.28 & 1.43 & 1.76 & 1.14 & 1.27 & 1.43 & 1.75 & 1.09 & 1.25 & 1.37 & 1.71 \\
\hline MH6 & 1.13 & 1.24 & 1.41 & 1.71 & 1.10 & 1.25 & 1.37 & 1.72 & 1.04 & 1.15 & 1.30 & 1.58 \\
\hline LL1 & 1.10 & 1.19 & 1.37 & 1.64 & 1.11 & 1.26 & 1.38 & 1.74 & 1.05 & 1.20 & 1.31 & 1.65 \\
\hline LL2 & 1.09 & 1.18 & 1.36 & 1.63 & 1.11 & 1.28 & 1.39 & 1.75 & 1.05 & 1.22 & 1.31 & 1.67 \\
\hline LL3 & 1.20 & 1.33 & 1.50 & 1.82 & 1.16 & 1.31 & 1.45 & 1.80 & 1.10 & 1.24 & 1.37 & 1.71 \\
\hline LL4 & 1.09 & 1.19 & 1.37 & 1.63 & 1.11 & 1.27 & 1.39 & 1.75 & 1.06 & 1.20 & 1.32 & 1.65 \\
\hline LL5 & 1.18 & 1.32 & 1.47 & 1.82 & 1.13 & 1.32 & 1.41 & 1.81 & 1.10 & 1.26 & 1.37 & 1.73 \\
\hline LL6 & 1.17 & 1.31 & 1.46 & 1.80 & 1.22 & 1.46 & 1.53 & 2.01 & 1.21 & 1.39 & 1.51 & 1.91 \\
\hline LH1 & 1.07 & 1.14 & 1.34 & 1.57 & 1.07 & 1.21 & 1.34 & 1.66 & 1.08 & 1.16 & 1.35 & 1.60 \\
\hline LH2 & 1.13 & 1.25 & 1.41 & 1.72 & 1.08 & 1.23 & 1.35 & 1.69 & 1.01 & 1.18 & 1.26 & 1.62 \\
\hline LH3 & 1.11 & 1.22 & 1.38 & 1.68 & 1.18 & 1.36 & 1.47 & 1.86 & 1.21 & 1.37 & 1.52 & 1.89 \\
\hline LH4 & 1.12 & 1.22 & 1.40 & 1.68 & 1.05 & 1.18 & 1.31 & 1.62 & 1.04 & 1.13 & 1.30 & 1.56 \\
\hline LH5 & 1.18 & 1.28 & 1.47 & 1.76 & 1.08 & 1.24 & 1.35 & 1.71 & 1.04 & 1.22 & 1.30 & 1.68 \\
\hline LH6 & 1.01 & 1.07 & 1.26 & 1.47 & 1.27 & 1.44 & 1.59 & 1.98 & 1.29 & 1.49 & 1.62 & 2.05 \\
\hline
\end{tabular}


Table C.67 - The ratio of actual mid-span bending moments and quarter-span shear forces for buildings to the corresponding values obtained from FEMA parabolic distribution and a uniform distribution of seismic load when subjected to M6C2 ground motion and the Load is acting parallel to the long side, $\alpha=0.10$

\begin{tabular}{|c|c|c|c|c|c|c|c|c|c|c|c|c|}
\hline \multirow{2}{*}{ Building } & \multicolumn{4}{|c|}{$\mu=2$} & \multicolumn{4}{|c|}{$\mu=3$} & \multicolumn{4}{|c|}{$\mu=4$} \\
\hline & $M . F$ & $V . F$ & $M . U$ & V.U & M.F & $V . F$ & $M . U$ & $V . U$ & M.F & V.F & M.U & $V . U$ \\
\hline SL1 & 1.04 & 1.08 & 1.30 & 1.48 & 1.00 & 1.07 & 1.25 & 1.47 & 0.95 & 1.03 & 1.18 & 1.42 \\
\hline SL3 & 03 & 1.06 & 1.29 & 1.46 & 0.99 & 1.05 & 1.24 & 1.45 & 0.93 & 1.01 & 1.16 & 1.38 \\
\hline & 02 & 1.06 & 1.27 & 1.46 & 0.94 & 1.00 & 1.18 & 1.37 & 0.90 & 0.95 & 1.13 & 1.30 \\
\hline SH1 & 15 & 1.24 & 1.43 & 1.71 & 1.14 & 1.27 & 1.42 & 1.75 & 1.22 & 1.42 & 1.52 & 1.96 \\
\hline & 1.05 & 1.10 & 1.32 & 1.51 & 1.12 & 1.25 & 1.40 & 1.71 & 1.26 & 1.46 & 1.58 & 2.01 \\
\hline $\mathrm{SH} 3$ & 1.05 & 1.13 & 1.31 & 1.55 & 1.11 & 1.21 & 1.39 & 1.66 & 1.14 & 1.32 & 1.42 & 1.81 \\
\hline $\mathrm{SH} 4$ & 1.04 & 1.11 & 1.30 & 1.53 & 1.08 & 1.16 & 1.35 & 1.60 & 1.14 & 1.32 & 1.43 & 1.81 \\
\hline SH5 & 1.06 & 1.14 & 1.33 & 1.56 & 1.08 & 1.17 & 1.35 & 1.61 & 1.11 & 1.26 & 1.39 & 1.73 \\
\hline ML1 & 1.01 & 1.08 & 1.26 & 1.48 & 0.98 & 1.07 & 1.23 & 1.47 & 1.01 & 1.09 & 1.27 & 1.50 \\
\hline ML2 & 1.05 & 1.10 & 1.31 & 1.52 & 1.03 & 1.09 & 1.29 & 1.49 & 1.07 & 1.12 & 1.33 & 1.54 \\
\hline ML3 & 1.11 & 1.19 & 1.39 & 1.64 & 1.11 & 1.21 & 1.39 & 1.66 & 1.13 & 1.22 & 1.41 & 1.68 \\
\hline ML4 & 1.03 & 1.08 & 1.29 & 1.48 & 1.02 & 1.07 & 1.28 & 1.47 & 1.06 & 1.11 & 1.32 & 1.53 \\
\hline ML5 & 1.06 & 1.12 & 1.33 & 1.55 & 1.05 & 1.11 & 1.31 & 1.53 & 1.08 & 1.14 & 1.35 & 1.56 \\
\hline ML6 & 1.09 & 1.17 & 1.36 & 1.61 & 1.09 & 1.17 & 1.36 & 1.61 & 1.12 & 1.20 & 1.40 & 1.66 \\
\hline & 14 & 1.21 & 1.42 & 1.66 & 1.23 & 1.38 & 1.54 & 1.89 & 1.23 & 1.39 & 1.54 & 1.91 \\
\hline & 29 & 1.44 & 1.62 & 1.98 & 1.34 & 1.52 & 1.68 & 2.10 & 1.35 & 1.53 & 1.69 & 2.10 \\
\hline & 1.19 & 1.27 & 1.48 & 1.75 & 1.17 & 1.25 & 1.46 & 1.72 & 1.16 & 1.34 & 1.45 & 1.84 \\
\hline MH4 & 1.10 & 1.15 & 1.37 & 1.58 & 1.25 & 1.40 & 1.57 & 1.93 & 1.26 & 1.42 & 1.57 & 1.95 \\
\hline MH5 & 1.38 & 1.56 & 1.72 & 2.15 & 1.39 & 1.57 & 1.74 & 2.16 & 1.38 & 1.54 & 1.73 & 2.12 \\
\hline MH6 & 1.06 & 1.11 & 1.33 & 1.52 & 1.26 & 1.42 & 1.57 & 1.95 & 1.27 & 1.44 & 1.59 & 1.98 \\
\hline LL1 & 1.09 & 1.14 & 1.36 & 1.57 & 1.21 & 1.35 & 1.51 & 1.85 & 1.24 & 1.39 & 1.54 & 1.91 \\
\hline LL2 & 1.06 & 1.10 & 1.33 & 1.51 & 1.26 & 1.41 & 1.57 & 1.94 & 1.27 & 1.44 & 1.58 & 1.98 \\
\hline LL3 & 1.11 & 1.21 & 1.38 & 1.66 & 1.19 & 1.38 & 1.48 & 1.89 & 1.28 & 1.45 & 1.60 & 2.00 \\
\hline LL4 & 1.09 & 1.13 & 1.36 & 1.56 & 1.22 & 1.36 & 1.52 & 1.87 & 1.24 & 1.40 & 1.55 & 1.92 \\
\hline LL5 & 1.21 & 1.31 & 1.51 & 1.80 & 1.33 & 1.50 & 1.66 & 2.07 & 1.33 & 1.51 & 1.67 & 2.08 \\
\hline LL6 & 1.34 & 1.52 & 1.67 & 2.09 & 1.39 & 1.67 & 1.74 & 2.29 & 1.31 & 1.59 & 1.64 & 2.18 \\
\hline LH1 & 1.21 & 1.31 & 1.51 & 1.80 & 1.26 & 1.42 & 1.58 & 1.95 & 1.27 & 1.44 & 1.59 & 1.98 \\
\hline LH2 & 1.33 & 1.48 & 1.66 & 2.03 & 1.36 & 1.55 & 1.70 & 2.13 & 1.36 & 1.53 & 1.70 & 2.11 \\
\hline LH3 & 1.39 & 1.62 & 1.74 & 2.22 & 1.34 & 1.58 & 1.68 & 2.18 & 1.25 & 1.46 & 1.56 & 2.01 \\
\hline LH4 & 1.20 & 1.30 & 1.50 & 1.79 & 1.28 & 1.44 & 1.60 & 1.99 & 1.28 & 1.44 & 1.60 & 1.98 \\
\hline LH5 & 1.10 & 1.17 & 1.38 & 1.61 & 1.12 & 1.24 & 1.40 & 1.71 & 1.22 & 1.39 & 1.53 & 1.92 \\
\hline LH6 & 1.35 & 1.54 & 1.69 & 2.12 & 1.22 & 1.39 & 1.53 & 1.91 & 1.18 & 1.33 & 1.48 & 1.83 \\
\hline
\end{tabular}


Table C.68 - The ratio of actual mid-span bending moments and quarter-span shear forces for buildings to the corresponding values obtained from FEMA parabolic distribution and a uniform distribution of seismic load when subjected to M6C26 ground motion and the Load is acting parallel to the long side, $\alpha=0.10$

\begin{tabular}{|c|c|c|c|c|c|c|c|c|c|c|c|c|}
\hline \multirow{2}{*}{ Building } & \multicolumn{4}{|c|}{$\mu=2$} & \multicolumn{4}{|c|}{$\mu=3$} & \multicolumn{4}{|c|}{$\mu=4$} \\
\hline & $M . F$ & $V . F$ & $M . U$ & $V . U$ & M.F & $V . F$ & $M . U$ & $V . U$ & M.F & V.F & $M . U$ & $V . U$ \\
\hline SL1 & 1.07 & 1.13 & 1.34 & 1.55 & 1.03 & 1.11 & 1.28 & 1.52 & 0.99 & 1.09 & 1.23 & 1.50 \\
\hline SL3 & 07 & 1.13 & 1.34 & 1.55 & 1.03 & 1.13 & 1.29 & 1.55 & 1.00 & 1.11 & 1.25 & 1.53 \\
\hline SL4 & .08 & 1.15 & 1.35 & 1.58 & 1.05 & 1.16 & 1.32 & 1.60 & 1.00 & 1.12 & 1.25 & 1.54 \\
\hline SH1 & 1.06 & 1.13 & 1.32 & 1.56 & 1.04 & 1.12 & 1.30 & 1.54 & 1.02 & 1.09 & 1.28 & 1.50 \\
\hline $\mathrm{SH} 2$ & 1.01 & 1.04 & 1.26 & 1.43 & 1.09 & 1.17 & 1.36 & 1.61 & 1.07 & 1.16 & 1.34 & 1.59 \\
\hline $\mathrm{SH} 3$ & 1.00 & 1.04 & 1.25 & 1.44 & 1.12 & 1.23 & 1.40 & 1.68 & 1.12 & 1.23 & 1.40 & 1.68 \\
\hline SH4 & 1.00 & 1.04 & 1.25 & 1.43 & 1.13 & 1.23 & 1.41 & 1.69 & 1.12 & 1.22 & 1.40 & 1.68 \\
\hline H5 & 1.00 & 1.05 & 1.26 & 1.44 & 1.15 & 1.26 & 1.43 & 1.73 & 1.14 & 1.26 & 1.43 & 1.73 \\
\hline ML1 & 1.05 & 1.10 & 1.31 & 1.52 & 1.04 & 1.10 & 1.26 & 1.51 & 1.01 & 1.08 & 1.27 & 1.49 \\
\hline ML2 & 1.05 & 1.10 & 1.32 & 1.51 & 1.03 & 1.08 & 1.28 & 1.48 & 1.00 & 1.04 & 1.25 & 1.43 \\
\hline ML3 & 1.02 & 1.05 & 1.28 & 1.44 & 0.99 & 1.01 & 1.23 & 1.39 & 0.95 & 0.97 & 1.19 & 1.33 \\
\hline ML4 & 1.06 & 1.11 & 1.32 & 1.52 & 1.03 & 1.09 & 1.29 & 1.50 & 1.00 & 1.05 & 1.25 & 1.45 \\
\hline ML5 & 1.05 & 1.09 & 1.31 & 1.50 & 1.02 & 1.06 & 1.28 & 1.46 & 0.99 & 1.03 & 1.24 & 1.41 \\
\hline ML6 & 1.03 & 1.06 & 1.29 & 1.46 & 1.00 & 1.03 & 1.25 & 1.42 & 0.96 & 0.99 & 1.21 & 1.36 \\
\hline MH1 & 0.99 & 1.00 & 1.23 & 1.38 & 0.96 & 0.98 & 1.19 & 1.34 & 0.95 & 0.99 & 1.18 & 1.36 \\
\hline $\mathrm{H} 2$ & 0.99 & 1.01 & 1.23 & 1.39 & 0.99 & 1.04 & 1.24 & 1.43 & 1.02 & 1.13 & 1.28 & 1.55 \\
\hline MH3 & 1.06 & 1.16 & 1.33 & 1.60 & 1.07 & 1.18 & 1.33 & 1.63 & 1.18 & 1.29 & 1.48 & 1.77 \\
\hline MH4 & 0.99 & 1.00 & 1.23 & 1.38 & 0.96 & 0.98 & 1.20 & 1.35 & 0.96 & 1.01 & 1.20 & 1.39 \\
\hline MH5 & 0.99 & 1.04 & 1.24 & 1.44 & 1.01 & 1.06 & 1.26 & 1.46 & 1.04 & 1.15 & 1.29 & 1.58 \\
\hline MH6 & 1.00 & 1.03 & 1.25 & 1.42 & 1.03 & 1.09 & 1.28 & 1.51 & 1.04 & 1.18 & 1.30 & 1.62 \\
\hline LL1 & 1.00 & 1.03 & 1.26 & 1.41 & 0.98 & 1.00 & 1.22 & 1.38 & 0.99 & 1.07 & 1.24 & 1.47 \\
\hline LL2 & 1.00 & 1.03 & 1.26 & 1.41 & 0.98 & 1.02 & 1.23 & 1.40 & 1.00 & 1.09 & 1.26 & 1.50 \\
\hline LL3 & 1.06 & 1.14 & 1.33 & 1.57 & 1.09 & 1.19 & 1.36 & 1.64 & 1.12 & 1.21 & 1.40 & 1.66 \\
\hline L4 & 1.01 & 1.03 & 1.26 & 1.41 & 0.98 & 1.00 & 1.22 & 1.38 & 0.99 & 1.07 & 1.24 & 1.48 \\
\hline LL5 & 1.00 & 1.03 & 1.25 & 1.41 & 1.00 & 1.04 & 1.24 & 1.43 & 1.02 & 1.12 & 1.28 & 1.53 \\
\hline LL6 & 1.07 & 1.14 & 1.34 & 1.56 & 1.28 & 1.47 & 1.60 & 2.02 & 1.39 & 1.61 & 1.74 & 2.21 \\
\hline LH1 & 1.04 & 1.09 & 1.30 & 1.50 & 1.11 & 1.26 & 1.39 & 1.73 & 1.04 & 1.19 & 1.30 & 1.64 \\
\hline LH2 & 1.05 & 1.11 & 1.32 & 1.53 & 1.12 & 1.27 & 1.39 & 1.75 & 1.04 & 1.21 & 1.30 & 1.66 \\
\hline LH3 & 1.09 & 1.16 & 1.36 & 1.60 & 1.43 & 1.67 & 1.79 & 2.30 & 1.46 & 1.70 & 1.82 & 2.34 \\
\hline LH4 & 1.05 & 1.12 & 1.32 & 1.54 & 1.11 & 1.27 & 1.39 & 1.75 & 1.03 & 1.21 & 1.29 & 1.66 \\
\hline LH5 & 1.05 & 1.10 & 1.31 & 1.52 & 1.17 & 1.29 & 1.46 & 1.77 & 1.17 & 1.29 & 1.46 & 1.77 \\
\hline LH6 & 1.09 & 1.22 & 1.36 & 1.68 & 1.35 & 1.56 & 1.68 & 2.15 & 1.63 & 1.88 & 2.03 & 2.58 \\
\hline
\end{tabular}


Table C.69 - The ratio of actual mid-span bending moments and quarter-span shear forces for buildings to the corresponding values obtained from FEMA parabolic distribution and a uniform distribution of seismic load when subjected to M6C31 ground motion and the Load is acting parallel to the long side, $\alpha=0.10$

\begin{tabular}{|c|c|c|c|c|c|c|c|c|c|c|c|c|}
\hline \multirow{2}{*}{ Building } & \multicolumn{4}{|c|}{$\mu=2$} & \multicolumn{4}{|c|}{$\mu=3$} & \multicolumn{4}{|c|}{$\mu=4$} \\
\hline & $M . F$ & $V . F$ & $M . U$ & $V . U$ & M.F & $V . F$ & $M . U$ & $V . U$ & M.F & V.F & $M . U$ & $V . U$ \\
\hline SL1 & 0.97 & 0.99 & 1.22 & 1.37 & 1.17 & 1.29 & 1.46 & 1.78 & 1.17 & 1.30 & 1.47 & 1.79 \\
\hline SL3 & 97 & 0.99 & 1.21 & 1.36 & 1.06 & 1.16 & 1.33 & 1.59 & 1.14 & 1.27 & 1.42 & 1.74 \\
\hline SL4 & 97 & 0.99 & 1.21 & 1.36 & 0.98 & 1.04 & 1.23 & 1.43 & 1.05 & 1.17 & 1.32 & 1.61 \\
\hline SH1 & 1.07 & 1.13 & 1.34 & 1.56 & 1.07 & 1.15 & 1.34 & 1.58 & 1.05 & 1.16 & 1.31 & 1.59 \\
\hline $\mathrm{SH} 2$ & 1.09 & 1.15 & 1.36 & 1.59 & 1.06 & 1.12 & 1.33 & 1.54 & 1.05 & 1.13 & 1.32 & 1.56 \\
\hline $\mathrm{SH} 3$ & 1.07 & 1.11 & 1.33 & 1.53 & 1.03 & 1.07 & 1.29 & 1.47 & 1.02 & 1.12 & 1.28 & 1.54 \\
\hline SH4 & 1.06 & 1.10 & 1.32 & 1.51 & 1.03 & 1.05 & 1.28 & 1.45 & 1.02 & 1.09 & 1.27 & 1.49 \\
\hline H5 & 1.03 & 1.06 & 1.28 & 1.45 & 1.01 & 1.06 & 1.27 & 1.45 & 1.04 & 1.16 & 1.30 & 1.60 \\
\hline ML1 & 1.09 & 1.16 & 1.37 & 1.60 & 1.17 & 1.29 & 1.46 & 1.77 & 1.14 & 1.25 & 1.42 & 1.72 \\
\hline ML2 & 1.09 & 1.15 & 1.36 & 1.58 & 1.14 & 1.26 & 1.43 & 1.74 & 1.14 & 1.26 & 1.43 & 1.73 \\
\hline ML3 & 1.09 & 1.15 & 1.36 & 1.58 & 1.08 & 1.19 & 1.36 & 1.63 & 1.13 & 1.25 & 1.41 & 1.72 \\
\hline ML4 & 1.09 & 1.15 & 1.36 & 1.59 & 1.16 & 1.28 & 1.45 & 1.76 & 1.14 & 1.26 & 1.43 & 1.73 \\
\hline ML5 & 1.09 & 1.15 & 1.36 & 1.58 & 1.13 & 1.25 & 1.42 & 1.71 & 1.14 & 1.26 & 1.42 & 1.73 \\
\hline ML6 & 1.08 & 1.14 & 1.36 & 1.57 & 1.10 & 1.19 & 1.37 & 1.64 & 1.14 & 1.27 & 1.43 & 1.74 \\
\hline MH1 & 1.00 & 1.03 & 1.25 & 1.42 & 1.04 & 1.11 & 1.30 & 1.53 & 1.06 & 1.17 & 1.32 & 1.61 \\
\hline & 0.97 & 0.98 & 1.21 & 1.35 & 1.08 & 1.17 & 1.35 & 1.61 & 1.09 & 1.21 & 1.37 & 1.66 \\
\hline MH3 & 1.13 & 1.21 & 1.42 & 1.66 & 1.17 & 1.31 & 1.47 & 1.80 & 1.15 & 1.27 & 1.44 & 1.75 \\
\hline MH4 & 1.00 & 1.03 & 1.25 & 1.41 & 1.05 & 1.13 & 1.31 & 1.56 & 1.05 & 1.16 & 1.31 & 1.60 \\
\hline MH5 & 1.09 & 1.16 & 1.37 & 1.60 & 1.11 & 1.21 & 1.39 & 1.66 & 1.11 & 1.23 & 1.39 & 1.70 \\
\hline MH6 & 1.01 & 1.08 & 1.27 & 1.48 & 1.02 & 1.11 & 1.28 & 1.52 & 1.03 & 1.12 & 1.28 & 1.54 \\
\hline LL1 & 1.05 & 1.10 & 1.31 & 1.52 & 1.07 & 1.17 & 1.34 & 1.60 & 1.06 & 1.18 & 1.33 & 1.62 \\
\hline LL2 & 1.02 & 1.07 & 1.28 & 1.47 & 1.05 & 1.15 & 1.32 & 1.59 & 1.05 & 1.16 & 1.31 & 1.60 \\
\hline LL3 & 1.08 & 1.12 & 1.35 & 1.54 & 1.18 & 1.32 & 1.47 & 1.81 & 1.18 & 1.32 & 1.47 & 1.82 \\
\hline L4 & 1.04 & 1.10 & 1.31 & 1.51 & 1.07 & 1.16 & 1.33 & 1.60 & 1.06 & 1.18 & 1.33 & 1.62 \\
\hline LL5 & 0.96 & 0.97 & 1.20 & 1.34 & 1.06 & 1.14 & 1.32 & 1.57 & 1.07 & 1.18 & 1.34 & 1.62 \\
\hline LL6 & 1.23 & 1.34 & 1.53 & 1.84 & 1.51 & 1.78 & 1.88 & 2.45 & 1.54 & 1.83 & 1.92 & 2.51 \\
\hline LH1 & 1.00 & 1.04 & 1.26 & 1.43 & 1.01 & 1.08 & 1.26 & 1.48 & 1.03 & 1.12 & 1.28 & 1.54 \\
\hline LH2 & 1.02 & 1.06 & 1.27 & 1.45 & 1.12 & 1.23 & 1.40 & 1.70 & 1.19 & 1.41 & 1.49 & 1.93 \\
\hline LH3 & 1.45 & 1.65 & 1.81 & 2.26 & 1.62 & 1.96 & 2.03 & 2.70 & 1.67 & 2.03 & 2.08 & 2.79 \\
\hline LH4 & 1.02 & 1.07 & 1.28 & 1.47 & 1.04 & 1.10 & 1.30 & 1.52 & 1.22 & 1.41 & 1.52 & 1.93 \\
\hline LH5 & 1.18 & 1.32 & 1.47 & 1.81 & 1.22 & 1.38 & 1.52 & 1.90 & 1.22 & 1.36 & 1.52 & 1.86 \\
\hline LH6 & 1.12 & 1.26 & 1.40 & 1.73 & 1.32 & 1.51 & 1.65 & 2.07 & 1.58 & 1.86 & 1.97 & 2.55 \\
\hline
\end{tabular}


Table C.70 - The ratio of actual mid-span bending moments and quarter-span shear forces for buildings to the corresponding values obtained from FEMA parabolic distribution and a uniform distribution of seismic load when subjected to M6C38 ground motion and the Load is acting parallel to the long side, $\alpha=0.10$

\begin{tabular}{|c|c|c|c|c|c|c|c|c|c|c|c|c|}
\hline \multirow{2}{*}{ Building } & \multicolumn{4}{|c|}{$\mu=2$} & \multicolumn{4}{|c|}{$\mu=3$} & \multicolumn{4}{|c|}{$\mu=4$} \\
\hline & $M . F$ & $V . F$ & $M . U$ & $V . U$ & M.F & $V . F$ & $M . U$ & $V . U$ & M.F & V.F & $M . U$ & $V . U$ \\
\hline SL1 & 1.05 & 1.10 & 1.32 & 1.51 & 1.04 & 1.15 & 1.31 & 1.58 & 0.99 & 1.11 & 1.24 & 1.52 \\
\hline SL3 & 07 & 1.12 & 1.34 & 1.54 & 1.06 & 1.14 & 1.33 & 1.57 & 1.01 & 1.09 & 1.26 & 1.50 \\
\hline SL4 & 99 & 1.02 & 1.24 & 1.41 & 1.03 & 1.10 & 1.29 & 1.52 & 0.99 & 1.07 & 1.23 & 1.47 \\
\hline SH1 & 1.07 & 1.13 & 1.34 & 1.55 & 1.05 & 1.10 & 1.31 & 1.51 & 1.02 & 1.06 & 1.27 & 1.46 \\
\hline $\mathrm{SH} 2$ & 1.09 & 1.15 & 1.36 & 1.59 & 1.07 & 1.12 & 1.33 & 1.54 & 1.04 & 1.08 & 1.30 & 1.49 \\
\hline $\mathrm{SH} 3$ & 1.09 & 1.16 & 1.37 & 1.60 & 1.07 & 1.13 & 1.34 & 1.56 & 1.04 & 1.09 & 1.30 & 1.50 \\
\hline SH4 & 1.10 & 1.17 & 1.37 & 1.61 & 1.08 & 1.13 & 1.34 & 1.56 & 1.05 & 1.09 & 1.31 & 1.50 \\
\hline H5 & 1.10 & 1.17 & 1.37 & 1.61 & 1.08 & 1.14 & 1.35 & 1.57 & 1.05 & 1.10 & 1.31 & 1.52 \\
\hline ML1 & 1.13 & 1.22 & 1.41 & 1.68 & 1.12 & 1.21 & 1.40 & 1.67 & 1.10 & 1.19 & 1.38 & 1.64 \\
\hline ML2 & 1.13 & 1.23 & 1.42 & 1.69 & 1.12 & 1.22 & 1.40 & 1.68 & 1.10 & 1.20 & 1.38 & 1.65 \\
\hline ML3 & 1.13 & 1.22 & 1.41 & 1.68 & 1.12 & 1.23 & 1.40 & 1.69 & 1.11 & 1.21 & 1.39 & 1.67 \\
\hline ML4 & 1.13 & 1.23 & 1.42 & 1.69 & 1.12 & 1.22 & 1.40 & 1.68 & 1.10 & 1.20 & 1.38 & 1.65 \\
\hline ML5 & 1.13 & 1.23 & 1.42 & 1.69 & 1.12 & 1.22 & 1.41 & 1.68 & 1.11 & 1.20 & 1.38 & 1.65 \\
\hline ML6 & 13 & 1.22 & 1.41 & 1.68 & 1.12 & 1.23 & 1.41 & 1.69 & 1.11 & 1.21 & 1.38 & 1.66 \\
\hline MH1 & 1.12 & 1.20 & 1.40 & 1.65 & 1.12 & 1.23 & 1.41 & 1.69 & 1.12 & 1.23 & 1.40 & 1.70 \\
\hline & 1.10 & 1.17 & 1.38 & 1.62 & 1.12 & 1.23 & 1.40 & 1.69 & 1.13 & 1.25 & 1.41 & 1.72 \\
\hline MH3 & 1.14 & 1.20 & 1.43 & 1.65 & 1.19 & 1.29 & 1.49 & 1.78 & 1.21 & 1.34 & 1.51 & 1.84 \\
\hline MH4 & 1.11 & 1.20 & 1.39 & 1.64 & 1.12 & 1.23 & 1.40 & 1.69 & 1.12 & 1.24 & 1.40 & 1.70 \\
\hline MH5 & 1.10 & 1.17 & 1.38 & 1.61 & 1.13 & 1.23 & 1.41 & 1.70 & 1.14 & 1.26 & 1.42 & 1.74 \\
\hline MH6 & 1.10 & 1.17 & 1.37 & 1.61 & 1.12 & 1.23 & 1.40 & 1.69 & 1.12 & 1.25 & 1.40 & 1.71 \\
\hline LL1 & 1.12 & 1.21 & 1.40 & 1.66 & 1.14 & 1.25 & 1.42 & 1.72 & 1.13 & 1.25 & 1.42 & 1.72 \\
\hline LL2 & 1.12 & 1.20 & 1.40 & 1.65 & 1.13 & 1.24 & 1.42 & 1.71 & 1.13 & 1.25 & 1.41 & 1.72 \\
\hline LL3 & 1.13 & 1.19 & 1.41 & 1.64 & 1.17 & 1.28 & 1.47 & 1.76 & 1.19 & 1.33 & 1.49 & 1.83 \\
\hline L4 & 1.12 & 1.21 & 1.40 & 1.66 & 1.14 & 1.25 & 1.42 & 1.72 & 1.13 & 1.25 & 1.42 & 1.72 \\
\hline LL5 & 1.11 & 1.18 & 1.38 & 1.63 & 1.13 & 1.24 & 1.41 & 1.70 & 1.13 & 1.26 & 1.42 & 1.73 \\
\hline LL6 & 1.16 & 1.20 & 1.45 & 1.65 & 1.22 & 1.31 & 1.52 & 1.80 & 1.25 & 1.37 & 1.56 & 1.89 \\
\hline LH1 & 1.10 & 1.17 & 1.37 & 1.61 & 1.13 & 1.24 & 1.41 & 1.70 & 1.14 & 1.27 & 1.42 & 1.74 \\
\hline LH2 & 1.08 & 1.14 & 1.35 & 1.57 & 1.12 & 1.23 & 1.40 & 1.70 & 1.14 & 1.28 & 1.43 & 1.76 \\
\hline LH3 & 1.13 & 1.19 & 1.42 & 1.63 & 1.20 & 1.31 & 1.50 & 1.81 & 1.24 & 1.39 & 1.55 & 1.92 \\
\hline LH4 & 1.08 & 1.15 & 1.35 & 1.58 & 1.12 & 1.23 & 1.40 & 1.69 & 1.13 & 1.26 & 1.42 & 1.73 \\
\hline LH5 & 1.11 & 1.17 & 1.39 & 1.61 & 1.16 & 1.28 & 1.45 & 1.76 & 1.19 & 1.34 & 1.49 & 1.84 \\
\hline LH6 & 1.15 & 1.19 & 1.44 & 1.64 & 1.24 & 1.34 & 1.55 & 1.84 & 1.29 & 1.43 & 1.61 & 1.97 \\
\hline
\end{tabular}


Table C.71 - The ratio of actual mid-span bending moments and quarter-span shear forces for buildings to the corresponding values obtained from FEMA parabolic distribution and a uniform distribution of seismic load when subjected to E6C1 ground motion and the Load is acting parallel to the short side, $\alpha=0.10$

\begin{tabular}{|c|c|c|c|c|c|c|c|c|c|c|c|c|}
\hline \multirow{2}{*}{ Building } & \multicolumn{4}{|c|}{$\mu=2$} & \multicolumn{4}{|c|}{$\mu=3$} & \multicolumn{4}{|c|}{$\mu=4$} \\
\hline & $M . F$ & V.F & $M . U$ & V.U & $M . F$ & $V . F$ & M.U & $V . U$ & $M . F$ & $V . F$ & $M . U$ & $V . U$ \\
\hline SL1 & 0.92 & 0.90 & 1.15 & 1.24 & 0.90 & 0.85 & 1.13 & 1.17 & 0.88 & 0.83 & 1.10 & 1.14 \\
\hline SL3 & .91 & 0.89 & 1.14 & 1.23 & 0.89 & 0.84 & 1.12 & 1.16 & 0.87 & 0.81 & 1.09 & 1.12 \\
\hline SL4 & 0.89 & 0.88 & 1.11 & 1.20 & 0.87 & 0.84 & 1.09 & 1.15 & 0.85 & 0.80 & 1.06 & 1.11 \\
\hline SH1 & 1.21 & 1.34 & 1.51 & 1.84 & 1.19 & 1.32 & 1.49 & 1.81 & 1.17 & 1.29 & 1.47 & 1.78 \\
\hline $\mathrm{SH} 2$ & 1.20 & 1.33 & 1.50 & 1.84 & 1.18 & 1.29 & 1.47 & 1.77 & 1.16 & 1.27 & 1.45 & 1.75 \\
\hline $\mathrm{SH} 3$ & 1.18 & 1.32 & 1.48 & 1.82 & 1.16 & 1.27 & 1.45 & 1.74 & 1.15 & 1.26 & 1.43 & 1.73 \\
\hline SH4 & 1.16 & 1.30 & 1.45 & 1.79 & 1.14 & 1.23 & 1.43 & 1.70 & 1.13 & 1.24 & 1.42 & 1.70 \\
\hline SH5 & 1.16 & 1.29 & 1.45 & 1.78 & 1.14 & 1.23 & 1.43 & 1.69 & 1.13 & 1.24 & 1.42 & 1.71 \\
\hline SH6 & 1.18 & 1.32 & 1.48 & 1.82 & 1.16 & 1.27 & 1.45 & 1.74 & 1.15 & 1.26 & 1.44 & 1.74 \\
\hline ML1 & 1.09 & 1.18 & 1.36 & 1.62 & 1.11 & 1.18 & 1.39 & 1.63 & 1.11 & 1.19 & 1.39 & 1.64 \\
\hline ML2 & 0.98 & 1.05 & 1.22 & 1.44 & 1.01 & 1.10 & 1.27 & 1.51 & 1.07 & 1.18 & 1.33 & 1.62 \\
\hline L3 & 0.99 & 1.07 & 1.24 & 1.46 & 1.04 & 1.13 & 1.30 & 1.55 & 1.10 & 1.22 & 1.38 & 1.67 \\
\hline ML4 & 1.02 & 1.06 & 1.28 & 1.46 & 1.06 & 1.12 & 1.32 & 1.54 & 1.09 & 1.19 & 1.36 & 1.64 \\
\hline ML5 & 1.02 & 1.06 & 1.28 & 1.46 & 1.06 & 1.12 & 1.33 & 1.54 & 1.10 & 1.20 & 1.37 & 1.65 \\
\hline ML6 & 0.98 & 1.06 & 1.23 & 1.45 & 1.03 & 1.13 & 1.29 & 1.55 & 1.10 & 1.22 & 1.37 & 1.68 \\
\hline MH1 & 0.98 & 1.04 & 1.22 & 1.43 & 1.04 & 1.12 & 1.30 & 1.53 & 1.11 & 1.21 & 1.39 & 1.67 \\
\hline $\mathrm{MH} 2$ & 1.01 & 1.04 & 1.26 & 1.43 & 1.11 & 1.14 & 1.39 & 1.57 & 1.19 & 1.27 & 1.49 & 1.75 \\
\hline MH3 & 1.16 & 1.39 & 1.45 & 1.90 & 1.28 & 1.46 & 1.59 & 2.01 & 1.38 & 1.54 & 1.72 & 2.11 \\
\hline MH4 & 1.02 & 1.03 & 1.27 & 1.41 & 1.08 & 1.11 & 1.36 & 1.52 & 1.13 & 1.17 & 1.42 & 1.61 \\
\hline MH5 & 1.03 & 1.06 & 1.29 & 1.45 & 1.13 & 1.18 & 1.41 & 1.62 & 1.20 & 1.29 & 1.50 & 1.78 \\
\hline MH6 & 1.09 & 1.13 & 1.36 & 1.55 & 1.13 & 1.20 & 1.41 & 1.66 & 1.18 & 1.33 & 1.48 & 1.82 \\
\hline LL1 & 0.95 & 1.06 & 1.18 & 1.45 & 0.98 & 1.09 & 1.22 & 1.50 & 1.04 & 1.10 & 1.30 & 1.51 \\
\hline LL2 & 0.93 & 1.06 & 1.16 & 1.46 & 0.97 & 1.10 & 1.22 & 1.51 & 1.04 & 1.10 & 1.30 & 1.51 \\
\hline LL3 & 0.99 & 1.20 & 1.24 & 1.64 & 1.18 & 1.40 & 1.47 & 1.93 & 1.25 & 1.47 & 1.56 & 2.02 \\
\hline LL4 & 0.96 & 0.99 & 1.20 & 1.35 & 1.02 & 1.04 & 1.27 & 1.43 & 1.10 & 1.16 & 1.38 & 1.60 \\
\hline LL5 & 0.96 & 0.98 & 1.20 & 1.35 & 1.03 & 1.04 & 1.28 & 1.43 & 1.10 & 1.16 & 1.38 & 1.59 \\
\hline LL6 & 1.02 & 1.34 & 1.28 & 1.84 & 1.15 & 1.46 & 1.43 & 2.01 & 1.28 & 1.57 & 1.60 & 2.16 \\
\hline LH1 & 1.10 & 1.30 & 1.37 & 1.79 & 1.28 & 1.51 & 1.60 & 2.08 & 1.29 & 1.52 & 1.61 & 2.09 \\
\hline LH2 & 1.25 & 1.43 & 1.56 & 1.96 & 1.32 & 1.52 & 1.65 & 2.08 & 1.32 & 1.53 & 1.65 & 2.10 \\
\hline LH3 & 1.36 & 1.73 & 1.70 & 2.38 & 1.65 & 2.00 & 2.06 & 2.75 & 1.66 & 2.01 & 2.07 & 2.77 \\
\hline LH4 & 1.25 & 1.47 & 1.56 & 2.02 & 1.34 & 1.58 & 1.67 & 2.17 & 1.34 & 1.59 & 1.68 & 2.18 \\
\hline LH5 & 1.27 & 1.50 & 1.59 & 2.06 & 1.35 & 1.60 & 1.69 & 2.20 & 1.36 & 1.61 & 1.70 & 2.21 \\
\hline LH6 & 1.32 & 1.72 & 1.65 & 2.36 & 1.62 & 2.00 & 2.03 & 2.75 & 1.70 & 2.09 & 2.13 & 2.88 \\
\hline
\end{tabular}


Table C.72 - The ratio of actual mid-span bending moments and quarter-span shear forces for buildings to the corresponding values obtained from FEMA parabolic distribution and a uniform distribution of seismic load when subjected to E6C13 ground motion and the Load is acting parallel to the short side, $\alpha=0.10$

\begin{tabular}{|c|c|c|c|c|c|c|c|c|c|c|c|c|}
\hline \multirow{2}{*}{ Building } & \multicolumn{4}{|c|}{$\mu=2$} & \multicolumn{4}{|c|}{$\mu=3$} & \multicolumn{4}{|c|}{$\mu=4$} \\
\hline & M.F & $V . F$ & $M . U$ & $V . U$ & $M . F$ & $V . F$ & $M . U$ & $V . U$ & $M . F$ & V.F & $M . U$ & $V . U$ \\
\hline SL1 & 1.03 & 1.12 & 1.29 & 1.54 & 1.03 & 1.12 & 1.29 & 1.55 & 1.03 & 1.10 & 1.29 & 1.52 \\
\hline SL3 & 1.06 & 1.12 & 1.32 & 1.54 & 1.06 & 1.10 & 1.33 & 1.51 & 1.06 & 1.09 & 1.32 & 1.50 \\
\hline SL4 & 1.15 & 1.25 & 1.43 & 1.72 & 1.15 & 1.23 & 1.43 & 1.69 & 1.14 & 1.19 & 1.42 & 1.64 \\
\hline SH1 & 1.00 & 1.17 & 1.25 & 1.61 & 1.05 & 1.25 & 1.32 & 1.72 & 1.10 & 1.32 & 1.38 & 1.81 \\
\hline $\mathrm{SH} 2$ & 0.95 & 1.11 & 1.18 & 1.53 & 0.96 & 1.11 & 1.21 & 1.52 & 0.97 & 1.16 & 1.21 & 1.60 \\
\hline SH3 & 0.93 & 1.08 & 1.16 & 1.48 & 0.94 & 1.07 & 1.18 & 1.47 & 0.95 & 1.13 & 1.18 & 1.55 \\
\hline SH4 & 0.90 & 1.05 & 1.13 & 1.44 & 0.91 & 1.02 & 1.14 & 1.41 & 0.91 & 1.04 & 1.14 & 1.43 \\
\hline SH5 & 0.91 & 1.03 & 1.14 & 1.42 & 0.92 & 1.01 & 1.15 & 1.39 & 0.91 & 1.05 & 1.14 & 1.44 \\
\hline SH6 & 0.93 & 1.08 & 1.16 & 1.48 & 0.95 & 1.07 & 1.18 & 1.47 & 0.95 & 1.13 & 1.18 & 1.55 \\
\hline ML1 & 0.92 & 0.98 & 1.15 & 1.35 & 0.92 & 0.96 & 1.14 & 1.33 & 0.91 & 0.96 & 1.13 & 1.31 \\
\hline ML2 & 0.92 & 0.94 & 1.15 & 1.30 & 0.92 & 1.00 & 1.16 & 1.38 & 0.92 & 1.12 & 1.16 & 1.53 \\
\hline ML3 & 0.95 & 1.10 & 1.19 & 1.51 & 1.04 & 1.19 & 1.30 & 1.63 & 1.07 & 1.24 & 1.33 & 1.71 \\
\hline ML4 & 0.91 & 0.94 & 1.13 & 1.29 & 0.90 & 0.91 & 1.13 & 1.26 & 0.89 & 0.91 & 1.11 & 1.24 \\
\hline ML5 & 0.91 & 0.94 & 1.13 & 1.29 & 0.90 & 0.91 & 1.13 & 1.25 & 0.89 & 0.91 & 1.11 & 1.25 \\
\hline ML6 & 0.92 & 1.03 & 1.15 & 1.41 & 0.96 & 1.10 & 1.20 & 1.51 & 0.99 & 1.21 & 1.23 & 1.67 \\
\hline MH1 & 1.03 & 1.22 & 1.29 & 1.67 & 1.03 & 1.21 & 1.29 & 1.66 & 1.05 & 1.27 & 1.31 & 1.75 \\
\hline MH2 & 0.99 & 1.03 & 1.24 & 1.41 & 1.08 & 1.29 & 1.35 & 1.77 & 1.22 & 1.56 & 1.52 & 2.14 \\
\hline MH3 & 1.24 & 1.40 & 1.55 & 1.92 & 1.29 & 1.68 & 1.62 & 2.31 & 1.25 & 1.65 & 1.57 & 2.27 \\
\hline MH4 & 0.94 & 0.99 & 1.17 & 1.36 & 0.99 & 1.21 & 1.24 & 1.67 & 1.11 & 1.39 & 1.38 & 1.91 \\
\hline MH5 & 0.95 & 1.04 & 1.18 & 1.42 & 1.09 & 1.36 & 1.36 & 1.87 & 1.26 & 1.62 & 1.58 & 2.22 \\
\hline MH6 & 0.95 & 1.00 & 1.18 & 1.38 & 0.93 & 0.99 & 1.16 & 1.37 & 0.90 & 1.08 & 1.12 & 1.48 \\
\hline LL1 & 1.03 & 1.19 & 1.29 & 1.64 & 1.13 & 1.21 & 1.41 & 1.67 & 1.10 & 1.29 & 1.37 & 1.77 \\
\hline LL2 & 1.00 & 1.16 & 1.25 & 1.60 & 1.12 & 1.18 & 1.40 & 1.63 & 1.09 & 1.29 & 1.36 & 1.77 \\
\hline LL3 & 1.01 & 1.00 & 1.26 & 1.38 & 0.99 & 1.03 & 1.24 & 1.41 & 1.01 & 1.28 & 1.27 & 1.77 \\
\hline LL4 & 1.04 & 1.17 & 1.31 & 1.61 & 1.06 & 1.20 & 1.32 & 1.65 & 1.03 & 1.34 & 1.28 & 1.84 \\
\hline LL5 & 1.05 & 1.16 & 1.31 & 1.59 & 1.06 & 1.19 & 1.33 & 1.64 & 1.03 & 1.32 & 1.28 & 1.82 \\
\hline LL6 & 0.90 & 1.24 & 1.13 & 1.71 & 0.93 & 1.34 & 1.16 & 1.84 & 1.03 & 1.52 & 1.29 & 2.09 \\
\hline LH1 & 1.02 & 1.06 & 1.28 & 1.46 & 1.00 & 1.05 & 1.24 & 1.45 & 1.11 & 1.43 & 1.38 & 1.97 \\
\hline LH2 & 1.07 & 1.16 & 1.34 & 1.59 & 1.05 & 1.21 & 1.31 & 1.66 & 1.07 & 1.33 & 1.34 & 1.82 \\
\hline LH3 & 0.98 & 1.37 & 1.23 & 1.89 & 1.22 & 1.54 & 1.52 & 2.12 & 1.22 & 1.46 & 1.53 & 2.01 \\
\hline LH4 & 1.08 & 1.17 & 1.35 & 1.60 & 1.06 & 1.36 & 1.33 & 1.87 & 1.12 & 1.46 & 1.40 & 2.01 \\
\hline LH5 & 1.09 & 1.18 & 1.37 & 1.63 & 1.07 & 1.39 & 1.34 & 1.91 & 1.14 & 1.50 & 1.42 & 2.06 \\
\hline LH6 & 0.91 & 1.33 & 1.13 & 1.83 & 1.10 & 1.50 & 1.37 & 2.07 & 1.18 & 1.44 & 1.48 & 1.98 \\
\hline
\end{tabular}


Table C.73 - The ratio of actual mid-span bending moments and quarter-span shear forces for buildings to the corresponding values obtained from FEMA parabolic distribution and a uniform distribution of seismic load when subjected to E6C15 ground motion and the Load is acting parallel to the short side, $\alpha=0.10$

\begin{tabular}{|c|c|c|c|c|c|c|c|c|c|c|c|c|}
\hline \multirow{2}{*}{ Building } & \multicolumn{4}{|c|}{$\mu=2$} & \multicolumn{4}{|c|}{$\mu=3$} & \multicolumn{4}{|c|}{$\mu=4$} \\
\hline & $M . F$ & $V . F$ & M.U & $V . U$ & $M . F$ & $V . F$ & M.U & V.U & $M . F$ & $V . F$ & $M . U$ & $V . U$ \\
\hline SL1 & 1.27 & 1.45 & 1.58 & 1.99 & 1.35 & 1.50 & 1.69 & 2.06 & 1.41 & 1.54 & 1.77 & 2.11 \\
\hline SL3 & 25 & 1.43 & 1.56 & 1.97 & 1.33 & 1.48 & 1.66 & 2.03 & 1.42 & 1.54 & 1.77 & 2.12 \\
\hline SL4 & 19 & 1.39 & 1.48 & 1.92 & 1.30 & 1.46 & 1.62 & 2.01 & 1.38 & 1.52 & 1.72 & 2.08 \\
\hline SH1 & .15 & 1.24 & 1.44 & 1.71 & 1.17 & 1.30 & 1.46 & 1.79 & 1.17 & 1.28 & 1.46 & 1.76 \\
\hline $\mathrm{SH} 2$ & 1.23 & 1.38 & 1.53 & 1.90 & 1.24 & 1.41 & 1.55 & 1.94 & 1.22 & 1.35 & 1.53 & 1.86 \\
\hline $\mathrm{SH} 3$ & 1.26 & 1.45 & 1.58 & 1.99 & 1.27 & 1.45 & 1.59 & 1.99 & 1.24 & 1.37 & 1.55 & 1.89 \\
\hline SH4 & 1.27 & 1.47 & 1.59 & 2.02 & 1.28 & 1.48 & 1.60 & 2.03 & 1.25 & 1.40 & 1.57 & 1.93 \\
\hline SH5 & 1.28 & 1.47 & 1.60 & 2.03 & 1.28 & 1.47 & 1.60 & 2.02 & 1.25 & 1.40 & 1.57 & 1.92 \\
\hline SH6 & 1.27 & 1.45 & 1.58 & 1.99 & 1.27 & 1.45 & 1.59 & 1.99 & 1.24 & 1.37 & 1.55 & 1.89 \\
\hline ML1 & 1.27 & 1.45 & 1.59 & 2.00 & 1.26 & 1.44 & 1.58 & 1.98 & 1.26 & 1.46 & 1.57 & 2.01 \\
\hline ML2 & 1.27 & 1.47 & 1.59 & 2.02 & 1.33 & 1.49 & 1.66 & 2.05 & 1.33 & 1.47 & 1.66 & 2.02 \\
\hline L3 & 1.25 & 1.38 & 1.56 & 1.89 & 1.28 & 1.39 & 1.60 & 1.92 & 1.26 & 1.37 & 1.58 & 1.88 \\
\hline ML4 & 1.25 & 1.46 & 1.57 & 2.01 & 1.29 & 1.51 & 1.61 & 2.08 & 1.30 & 1.53 & 1.63 & 2.10 \\
\hline ML5 & 1.25 & 1.44 & 1.57 & 1.99 & 1.28 & 1.49 & 1.59 & 2.05 & 1.30 & 1.52 & 1.62 & 2.09 \\
\hline ML6 & 1.27 & 1.43 & 1.59 & 1.96 & 1.33 & 1.45 & 1.66 & 2.00 & 1.30 & 1.41 & 1.63 & 1.94 \\
\hline MH1 & 1.28 & 1.40 & 1.60 & 1.93 & 1.26 & 1.39 & 1.58 & 1.91 & 1.24 & 1.36 & 1.56 & 1.88 \\
\hline $\mathrm{MH} 2$ & 1.17 & 1.23 & 1.46 & 1.69 & 1.20 & 1.30 & 1.50 & 1.79 & 1.21 & 1.35 & 1.52 & 1.86 \\
\hline MH3 & 1.27 & 1.45 & 1.58 & 1.99 & 1.41 & 1.72 & 1.76 & 2.36 & 1.42 & 1.70 & 1.78 & 2.34 \\
\hline MH4 & 1.17 & 1.22 & 1.47 & 1.68 & 1.20 & 1.27 & 1.50 & 1.75 & 1.21 & 1.32 & 1.52 & 1.81 \\
\hline MH5 & 1.19 & 1.23 & 1.49 & 1.70 & 1.23 & 1.30 & 1.53 & 1.79 & 1.26 & 1.40 & 1.58 & 1.92 \\
\hline MH6 & 1.28 & 1.58 & 1.59 & 2.18 & 1.28 & 1.55 & 1.60 & 2.14 & 1.27 & 1.51 & 1.59 & 2.07 \\
\hline LL1 & 1.08 & 1.23 & 1.35 & 1.69 & 1.16 & 1.33 & 1.45 & 1.83 & 1.22 & 1.42 & 1.53 & 1.96 \\
\hline LL2 & 1.08 & 1.24 & 1.35 & 1.70 & 1.16 & 1.33 & 1.45 & 1.83 & 1.23 & 1.43 & 1.53 & 1.97 \\
\hline LL3 & 1.11 & 1.21 & 1.38 & 1.66 & 1.24 & 1.44 & 1.55 & 1.99 & 1.30 & 1.55 & 1.62 & 2.13 \\
\hline LL4 & 1.20 & 1.40 & 1.50 & 1.93 & 1.28 & 1.50 & 1.60 & 2.07 & 1.31 & 1.53 & 1.64 & 2.10 \\
\hline LL5 & 1.19 & 1.39 & 1.49 & 1.91 & 1.28 & 1.50 & 1.60 & 2.06 & 1.31 & 1.53 & 1.63 & 2.10 \\
\hline LL6 & 0.87 & 1.06 & 1.09 & 1.46 & 0.92 & 1.16 & 1.15 & 1.59 & 0.96 & 1.22 & 1.20 & 1.67 \\
\hline LH1 & 1.16 & 1.33 & 1.45 & 1.83 & 1.36 & 1.64 & 1.70 & 2.25 & 1.43 & 1.69 & 1.79 & 2.32 \\
\hline LH2 & 1.33 & 1.67 & 1.66 & 2.30 & 1.39 & 1.72 & 1.74 & 2.36 & 1.44 & 1.75 & 1.79 & 2.41 \\
\hline LH3 & 1.09 & 1.35 & 1.36 & 1.85 & 1.19 & 1.46 & 1.49 & 2.01 & 1.23 & 1.48 & 1.54 & 2.03 \\
\hline LH4 & 1.36 & 1.67 & 1.70 & 2.29 & 1.43 & 1.72 & 1.79 & 2.36 & 1.46 & 1.72 & 1.82 & 2.36 \\
\hline LH5 & 1.38 & 1.69 & 1.72 & 2.32 & 1.43 & 1.71 & 1.79 & 2.35 & 1.47 & 1.73 & 1.84 & 2.38 \\
\hline LH6 & 1.00 & 1.24 & 1.25 & 1.71 & 1.10 & 1.37 & 1.38 & 1.88 & 1.15 & 1.40 & 1.43 & 1.92 \\
\hline
\end{tabular}


Table C.74 - The ratio of actual mid-span bending moments and quarter-span shear forces for buildings to the corresponding values obtained from FEMA parabolic distribution and a uniform distribution of seismic load when subjected to E6C18 ground motion and the Load is acting parallel to the short side, $\alpha=0.10$

\begin{tabular}{|c|c|c|c|c|c|c|c|c|c|c|c|c|}
\hline \multirow{2}{*}{ Building } & \multicolumn{4}{|c|}{$\mu=2$} & \multicolumn{4}{|c|}{$\mu=3$} & \multicolumn{4}{|c|}{$\mu=4$} \\
\hline & $M . F$ & $V . F$ & M.U & V.U & M.F & $V . F$ & $M . U$ & V.U & M.F & $V . F$ & M.U & $V . U$ \\
\hline SL1 & 1.08 & 1.17 & 1.36 & 1.61 & 1.11 & 1.18 & 1.38 & 1.63 & 1.11 & 1.22 & 1.39 & 1.68 \\
\hline SL3 & 1.12 & 1.22 & 1.40 & 1.68 & 1.13 & 1.21 & 1.41 & 1.66 & 1.13 & 1.25 & 1.42 & 1.71 \\
\hline SL4 & 1.19 & 1.31 & 1.48 & 1.80 & 1.19 & 1.31 & 1.49 & 1.80 & 1.18 & 1.31 & 1.47 & 1.80 \\
\hline SH1 & 1.00 & 1.05 & 1.25 & 1.45 & 1.04 & 1.04 & 1.29 & 1.44 & 1.06 & 1.08 & 1.32 & 1.49 \\
\hline $\mathrm{SH} 2$ & 0.99 & 1.02 & 1.23 & 1.40 & 1.04 & 1.08 & 1.30 & 1.48 & 1.07 & 1.11 & 1.33 & 1.53 \\
\hline SH3 & 0.99 & 1.04 & 1.24 & 1.44 & 1.06 & 1.12 & 1.33 & 1.54 & 1.10 & 1.17 & 1.38 & 1.60 \\
\hline SH4 & 0.98 & 1.05 & 1.23 & 1.45 & 1.05 & 1.12 & 1.31 & 1.54 & 1.10 & 1.17 & 1.37 & 1.61 \\
\hline SH5 & 1.00 & 1.07 & 1.25 & 1.47 & 1.07 & 1.14 & 1.33 & 1.57 & 1.13 & 1.20 & 1.41 & 1.66 \\
\hline & 0.99 & 1.04 & 1.24 & 1.44 & 1.06 & 1.11 & 1.32 & 1.53 & 1.10 & 1.17 & 1.38 & 1.60 \\
\hline & 95 & 1.05 & 1.19 & 1.45 & 1.02 & 1.12 & 1.27 & 1.55 & 1.13 & 1.24 & 1.42 & 1.70 \\
\hline & 0.97 & 1.21 & 1.22 & 1.66 & 1.14 & 1.36 & 1.42 & 1.88 & 1.15 & 1.37 & 1.43 & 1.89 \\
\hline ML3 & 1.06 & 1.33 & 1.33 & 1.83 & 1.14 & 1.40 & 1.42 & 1.93 & 1.15 & 1.41 & 1.44 & 1.94 \\
\hline ML4 & 0.95 & 1.11 & 1.19 & 1.52 & 1.07 & 1.22 & 1.33 & 1.68 & 1.21 & 1.39 & 1.52 & 1.91 \\
\hline ML5 & 0.95 & 1.11 & 1.19 & 1.52 & 1.06 & 1.21 & 1.33 & 1.67 & 1.21 & 1.38 & 1.51 & 1.89 \\
\hline ML6 & 1.06 & 1.31 & 1.32 & 1.80 & 1.14 & 1.38 & 1.42 & 1.89 & 1.15 & 1.38 & 1.43 & 1.90 \\
\hline MH1 & 1.14 & 1.41 & 1.42 & 1.94 & 1.16 & 1.42 & 1.45 & 1.96 & 1.17 & 1.43 & 1.46 & 1.97 \\
\hline MH2 & 1.24 & 1.50 & 1.55 & 2.06 & 1.27 & 1.51 & 1.58 & 2.08 & 1.28 & 1.53 & 1.60 & 2.10 \\
\hline MH3 & 0.92 & 1.39 & 1.15 & 1.91 & 0.97 & 1.49 & 1.21 & 2.05 & 0.97 & 1.55 & 1.22 & 2.14 \\
\hline MH4 & 1.07 & 1.37 & 1.34 & 1.88 & 1.26 & 1.54 & 1.57 & 2.11 & 1.28 & 1.55 & 1.59 & 2.13 \\
\hline $\mathrm{H} 5$ & 1.24 & 1.52 & 1.55 & 2.10 & 1.30 & 1.57 & 1.62 & 2.16 & 1.31 & 1.59 & 1.64 & 2.19 \\
\hline & 0.98 & 1.15 & 1.23 & 1.58 & 1.33 & 1.50 & 1.66 & 2.06 & 1.32 & 1.55 & 1.65 & 2.13 \\
\hline & 1.32 & 1.79 & 1.65 & 2.46 & 1.35 & 1.83 & 1.68 & 2.52 & 1.35 & 1.85 & 1.69 & 2.54 \\
\hline LL2 & 1.31 & 1.79 & 1.64 & 2.46 & 1.34 & 1.84 & 1.67 & 2.52 & 1.35 & 1.86 & 1.68 & 2.55 \\
\hline LL3 & 1.39 & 1.85 & 1.73 & 2.55 & 1.44 & 1.92 & 1.80 & 2.64 & 1.46 & 1.95 & 1.83 & 2.68 \\
\hline LL4 & 1.25 & 1.62 & 1.56 & 2.23 & 1.27 & 1.66 & 1.59 & 2.28 & 1.28 & 1.67 & 1.60 & 2.30 \\
\hline LL5 & 1.24 & 1.61 & 1.55 & 2.21 & 1.28 & 1.66 & 1.60 & 2.28 & 1.28 & 1.67 & 1.60 & 2.30 \\
\hline LL6 & 0.63 & 1.36 & 0.79 & 1.87 & 0.72 & 1.50 & 0.90 & 2.06 & 0.85 & 1.69 & 1.06 & 2.32 \\
\hline LH1 & 1.44 & 1.99 & 1.80 & 2.73 & 1.50 & 2.07 & 1.87 & 2.84 & 1.53 & 2.12 & 1.91 & 2.91 \\
\hline LH2 & 1.47 & 2.10 & 1.83 & 2.89 & 1.54 & 2.21 & 1.92 & 3.04 & 1.58 & 2.27 & 1.97 & 3.12 \\
\hline LH3 & 0.96 & 1.71 & 1.20 & 2.36 & 0.99 & 1.76 & 1.24 & 2.42 & 1.02 & 1.78 & 1.28 & 2.45 \\
\hline LH4 & 1.50 & 2.12 & 1.87 & 2.92 & 1.57 & 2.23 & 1.96 & 3.06 & 1.60 & 2.29 & 2.01 & 3.15 \\
\hline LH5 & 1.50 & 2.14 & 1.88 & 2.94 & 1.58 & 2.25 & 1.97 & 3.09 & 1.60 & 2.30 & 2.00 & 3.17 \\
\hline LH6 & 0.93 & 1.76 & 1.16 & 2.42 & 0.99 & 1.79 & 1.23 & 2.46 & 1.00 & 1.79 & 1.25 & 2.46 \\
\hline
\end{tabular}


Table C.75 - The ratio of actual mid-span bending moments and quarter-span shear forces for buildings to the corresponding values obtained from FEMA parabolic distribution and a uniform distribution of seismic load when subjected to E6C42 ground motion and the Load is acting parallel to the short side, $\alpha=0.10$

\begin{tabular}{|c|c|c|c|c|c|c|c|c|c|c|c|c|}
\hline \multirow{2}{*}{ Building } & \multicolumn{4}{|c|}{$\mu=2$} & \multicolumn{4}{|c|}{$\mu=3$} & \multicolumn{4}{|c|}{$\mu=4$} \\
\hline & M.F & $V . F$ & $M . U$ & $V . U$ & $M . F$ & $V . F$ & $M . U$ & $V . U$ & $M . F$ & V.F & $M . U$ & $V . U$ \\
\hline SL1 & 1.19 & 1.25 & 1.49 & 1.72 & 1.23 & 1.30 & 1.53 & 1.78 & 1.25 & 1.34 & 1.57 & 1.85 \\
\hline SL3 & .22 & 1.29 & 1.53 & 1.78 & 1.26 & 1.35 & 1.58 & 1.86 & 1.28 & 1.38 & 1.60 & 1.90 \\
\hline SL4 & 1.28 & 1.41 & 1.60 & 1.94 & 1.29 & 1.41 & 1.62 & 1.94 & 1.27 & 1.36 & 1.59 & 1.87 \\
\hline SH1 & 1.14 & 1.16 & 1.42 & 1.59 & 1.16 & 1.19 & 1.45 & 1.64 & 1.16 & 1.21 & 1.45 & 1.67 \\
\hline $\mathrm{SH} 2$ & 1.13 & 1.14 & 1.42 & 1.57 & 1.14 & 1.16 & 1.42 & 1.60 & 1.13 & 1.18 & 1.42 & 1.62 \\
\hline SH3 & 1.11 & 1.12 & 1.39 & 1.54 & 1.12 & 1.14 & 1.40 & 1.57 & 1.12 & 1.17 & 1.41 & 1.61 \\
\hline SH4 & 1.08 & 1.08 & 1.36 & 1.49 & 1.10 & 1.10 & 1.37 & 1.52 & 1.10 & 1.12 & 1.37 & 1.54 \\
\hline SH5 & 1.08 & 1.08 & 1.36 & 1.49 & 1.10 & 1.11 & 1.37 & 1.53 & 1.10 & 1.14 & 1.38 & 1.56 \\
\hline SH6 & 1.11 & 1.12 & 1.39 & 1.54 & 1.12 & 1.14 & 1.40 & 1.57 & 1.12 & 1.17 & 1.40 & 1.61 \\
\hline ML1 & 1.00 & 1.12 & 1.25 & 1.54 & 1.03 & 1.04 & 1.29 & 1.43 & 1.05 & 1.08 & 1.32 & 1.49 \\
\hline ML2 & 1.15 & 1.34 & 1.44 & 1.84 & 1.15 & 1.30 & 1.44 & 1.79 & 1.13 & 1.25 & 1.41 & 1.72 \\
\hline ML3 & 1.24 & 1.47 & 1.55 & 2.03 & 1.23 & 1.45 & 1.53 & 1.99 & 1.18 & 1.38 & 1.48 & 1.89 \\
\hline ML4 & 1.06 & 1.18 & 1.32 & 1.62 & 1.03 & 1.11 & 1.28 & 1.53 & 1.02 & 1.05 & 1.28 & 1.45 \\
\hline ML5 & 1.05 & 1.16 & 1.31 & 1.60 & 1.02 & 1.10 & 1.27 & 1.51 & 1.03 & 1.05 & 1.29 & 1.44 \\
\hline ML6 & 1.19 & 1.40 & 1.49 & 1.93 & 1.19 & 1.38 & 1.49 & 1.90 & 1.16 & 1.33 & 1.45 & 1.82 \\
\hline MH1 & 1.21 & 1.47 & 1.51 & 2.02 & 1.24 & 1.52 & 1.55 & 2.09 & 1.20 & 1.45 & 1.50 & 2.00 \\
\hline MH2 & 1.18 & 1.44 & 1.48 & 1.98 & 1.19 & 1.49 & 1.49 & 2.04 & 1.15 & 1.42 & 1.44 & 1.95 \\
\hline MH3 & 0.79 & 0.84 & 0.99 & 1.16 & 0.85 & 0.93 & 1.06 & 1.27 & 0.84 & 0.97 & 1.04 & 1.33 \\
\hline MH4 & 1.14 & 1.38 & 1.43 & 1.90 & 1.14 & 1.39 & 1.43 & 1.91 & 1.11 & 1.34 & 1.39 & 1.85 \\
\hline MH5 & 1.14 & 1.38 & 1.42 & 1.89 & 1.14 & 1.39 & 1.42 & 1.91 & 1.10 & 1.34 & 1.38 & 1.84 \\
\hline MH6 & 1.11 & 1.21 & 1.38 & 1.66 & 1.08 & 1.13 & 1.35 & 1.56 & 1.14 & 1.26 & 1.43 & 1.73 \\
\hline LL1 & 1.03 & 1.34 & 1.29 & 1.85 & 1.16 & 1.50 & 1.45 & 2.07 & 1.24 & 1.59 & 1.55 & 2.18 \\
\hline LL2 & 1.02 & 1.33 & 1.28 & 1.83 & 1.15 & 1.49 & 1.44 & 2.04 & 1.23 & 1.58 & 1.53 & 2.17 \\
\hline LL3 & 0.94 & 1.20 & 1.18 & 1.65 & 0.95 & 1.22 & 1.19 & 1.68 & 0.94 & 1.23 & 1.18 & 1.70 \\
\hline LL4 & 1.25 & 1.55 & 1.56 & 2.13 & 1.27 & 1.52 & 1.59 & 2.10 & 1.22 & 1.46 & 1.53 & 2.01 \\
\hline LL5 & 1.26 & 1.56 & 1.58 & 2.14 & 1.28 & 1.53 & 1.60 & 2.10 & 1.23 & 1.46 & 1.53 & 2.01 \\
\hline LL6 & 0.88 & 1.02 & 1.10 & 1.40 & 0.84 & 0.99 & 1.05 & 1.36 & 0.84 & 0.96 & 1.05 & 1.32 \\
\hline LH1 & 0.88 & 1.16 & 1.10 & 1.60 & 0.89 & 1.19 & 1.11 & 1.64 & 0.88 & 1.21 & 1.10 & 1.66 \\
\hline LH2 & 0.90 & 1.15 & 1.13 & 1.57 & 0.83 & 1.19 & 1.04 & 1.63 & 0.83 & 1.21 & 1.03 & 1.66 \\
\hline LH3 & 0.83 & 0.94 & 1.04 & 1.30 & 0.83 & 0.88 & 1.03 & 1.20 & 0.84 & 0.94 & 1.05 & 1.29 \\
\hline LH4 & 0.88 & 1.09 & 1.10 & 1.50 & 0.83 & 1.12 & 1.03 & 1.54 & 0.85 & 1.14 & 1.06 & 1.56 \\
\hline LH5 & 0.87 & 1.07 & 1.09 & 1.47 & 0.82 & 1.10 & 1.03 & 1.51 & 0.86 & 1.11 & 1.07 & 1.53 \\
\hline LH6 & 0.88 & 1.00 & 1.10 & 1.37 & 0.84 & 0.92 & 1.05 & 1.26 & 0.85 & 0.91 & 1.07 & 1.25 \\
\hline
\end{tabular}


Table C.76 - The ratio of actual mid-span bending moments and quarter-span shear forces for buildings to the corresponding values obtained from FEMA parabolic distribution and a linear distribution of seismic load when subjected to E6C1 ground motion and the Load is acting parallel to the long side, $\alpha=0.10$

\begin{tabular}{|c|c|c|c|c|c|c|c|c|c|c|c|c|}
\hline \multirow{2}{*}{ Building } & \multicolumn{4}{|c|}{$\mu=2$} & \multicolumn{4}{|c|}{$\mu=3$} & \multicolumn{4}{|c|}{$\mu=4$} \\
\hline & $M . F$ & $V . F$ & $M . U$ & V.U & M.F & $V . F$ & $M . U$ & $V . U$ & M.F & V.F & M.U & $V . U$ \\
\hline SL1 & 1.15 & 1.27 & 1.44 & 1.74 & 1.11 & 1.23 & 1.39 & 1.69 & 1.07 & 1.19 & 1.34 & 1.64 \\
\hline SL3 & 13 & 1.22 & 1.41 & 1.67 & 1.10 & 1.21 & 1.38 & 1.67 & 1.05 & 1.17 & 1.32 & 1.61 \\
\hline & 1.07 & 1.14 & 1.34 & 1.56 & 1.06 & 1.16 & 1.33 & 1.60 & 1.04 & 1.13 & 1.30 & 1.55 \\
\hline SH1 & 0.97 & 0.99 & 1.21 & 1.36 & 0.96 & 1.01 & 1.20 & 1.38 & 0.93 & 0.97 & 1.16 & 1.33 \\
\hline & 0.97 & 0.99 & 1.21 & 1.36 & 0.95 & 0.97 & 1.18 & 1.34 & 0.91 & 0.93 & 1.13 & 1.29 \\
\hline $\mathrm{SH} 3$ & 0.96 & 0.98 & 1.21 & 1.34 & 0.93 & 0.95 & 1.16 & 1.30 & 0.89 & 0.91 & 1.11 & 1.25 \\
\hline SH4 & 0.96 & 0.96 & 1.20 & 1.32 & 0.92 & 0.92 & 1.15 & 1.26 & 0.87 & 0.88 & 1.09 & 1.21 \\
\hline SH5 & 0.95 & 0.95 & 1.19 & 1.31 & 0.91 & 0.91 & 1.14 & 1.25 & 0.87 & 0.88 & 1.08 & 1.21 \\
\hline IL1 & 0.88 & 0.84 & 1.10 & 1.16 & 0.83 & 0.81 & 1.04 & 1.12 & 0.84 & 0.82 & 1.04 & 1.12 \\
\hline ML2 & 0.85 & 0.83 & 1.07 & 1.14 & 0.85 & 0.82 & 1.07 & 1.12 & 0.87 & 0.84 & 1.09 & 1.16 \\
\hline ML3 & 0.87 & 0.84 & 1.08 & 1.15 & 0.91 & 0.87 & 1.14 & 1.20 & 0.95 & 0.94 & 1.18 & 1.29 \\
\hline ML4 & 0.86 & 0.83 & 1.08 & 1.15 & 0.84 & 0.81 & 1.05 & 1.12 & 0.86 & 0.83 & 1.08 & 1.14 \\
\hline ML5 & 0.85 & 0.83 & 1.06 & 1.14 & 0.86 & 0.82 & 1.08 & 1.13 & 0.89 & 0.85 & 1.11 & 1.17 \\
\hline ML6 & 0.85 & 0.83 & 1.06 & 1.14 & 0.89 & 0.85 & 1.11 & 1.17 & 0.92 & 0.91 & 1.15 & 1.25 \\
\hline & 01 & 1.01 & 1.26 & 1.39 & 1.06 & 1.10 & 1.32 & 1.52 & 1.09 & 1.17 & 1.36 & 1.61 \\
\hline & & 1.22 & 1.41 & 1.67 & 1.15 & 1.27 & 1.43 & 1.74 & 1.14 & 1.27 & 1.42 & 1.75 \\
\hline & 1.16 & 1.31 & 1.46 & 1.80 & 1.14 & 1.26 & 1.42 & 1.74 & 1.14 & 1.27 & 1.42 & 1.74 \\
\hline MH4 & 1.05 & 1.08 & 1.31 & 1.48 & 1.09 & 1.17 & 1.37 & 1.60 & 1.11 & 1.21 & 1.39 & 1.67 \\
\hline MH5 & 1.15 & 1.26 & 1.44 & 1.73 & 1.16 & 1.29 & 1.45 & 1.77 & 1.15 & 1.27 & 1.43 & 1.75 \\
\hline MH6 & 1.06 & 1.11 & 1.32 & 1.52 & 1.11 & 1.20 & 1.39 & 1.65 & 1.15 & 1.29 & 1.44 & 1.77 \\
\hline LL1 & 0.94 & 0.93 & 1.18 & 1.28 & 1.01 & 1.03 & 1.26 & 1.41 & 1.07 & 1.16 & 1.34 & 1.59 \\
\hline LL2 & 1.00 & 1.02 & 1.25 & 1.40 & 1.07 & 1.12 & 1.33 & 1.54 & 1.10 & 1.22 & 1.38 & 1.68 \\
\hline LL3 & 1.17 & 1.32 & 1.47 & 1.82 & 1.16 & 1.28 & 1.45 & 1.76 & 1.14 & 1.25 & 1.42 & 1.72 \\
\hline LL4 & 0.94 & 0.93 & 1.18 & 1.28 & 1.01 & 1.04 & 1.27 & 1.42 & 1.07 & 1.16 & 1.34 & 1.60 \\
\hline LL5 & 1.12 & 1.20 & 1.40 & 1.65 & 1.14 & 1.26 & 1.43 & 1.73 & 1.14 & 1.27 & 1.43 & 1.75 \\
\hline LL6 & 1.09 & 1.15 & 1.37 & 1.57 & 1.13 & 1.23 & 1.41 & 1.69 & 1.21 & 1.39 & 1.51 & 1.91 \\
\hline LH1 & 1.05 & 1.10 & 1.31 & 1.51 & 1.11 & 1.22 & 1.39 & 1.68 & 1.24 & 1.44 & 1.56 & 1.98 \\
\hline LH2 & 1.13 & 1.21 & 1.41 & 1.67 & 1.20 & 1.33 & 1.50 & 1.83 & 1.22 & 1.39 & 1.53 & 1.92 \\
\hline LH3 & 1.07 & 1.13 & 1.34 & 1.56 & 1.24 & 1.41 & 1.55 & 1.94 & 1.17 & 1.34 & 1.47 & 1.84 \\
\hline LH4 & 1.05 & 1.10 & 1.31 & 1.51 & 1.21 & 1.37 & 1.51 & 1.88 & 1.23 & 1.45 & 1.54 & 1.99 \\
\hline LH5 & 1.15 & 1.25 & 1.44 & 1.71 & 1.16 & 1.27 & 1.45 & 1.75 & 1.18 & 1.31 & 1.48 & 1.80 \\
\hline LH6 & 1.05 & 1.06 & 1.31 & 1.45 & 1.27 & 1.46 & 1.58 & 2.01 & 1.21 & 1.40 & 1.52 & 1.92 \\
\hline
\end{tabular}


Table C.77 - The ratio of actual mid-span bending moments and quarter-span shear forces for buildings to the corresponding values obtained from FEMA parabolic distribution and a uniform distribution of seismic load when subjected to E6C13 ground motion and the Load is acting parallel to the long side, $\alpha=0.10$

\begin{tabular}{|c|c|c|c|c|c|c|c|c|c|c|c|c|}
\hline \multirow{2}{*}{ Building } & \multicolumn{4}{|c|}{$\mu=2$} & \multicolumn{4}{|c|}{$\mu=3$} & \multicolumn{4}{|c|}{$\mu=4$} \\
\hline & $M . F$ & $V . F$ & $M . U$ & V.U & M.F & $V . F$ & $M . U$ & $V . U$ & M.F & V.F & M.U & $V . U$ \\
\hline SL1 & 1.09 & 1.18 & 1.37 & 1.62 & 1.15 & 1.32 & 1.44 & 1.82 & 1.14 & 1.34 & 1.42 & 1.84 \\
\hline SL3 & .09 & 1.16 & 1.36 & 1.60 & 1.16 & 1.33 & 1.45 & 1.84 & 1.15 & 1.35 & 1.43 & 1.86 \\
\hline SL4 & 06 & 1.12 & 1.33 & 1.53 & 1.15 & 1.32 & 1.44 & 1.81 & 1.19 & 1.41 & 1.49 & 1.94 \\
\hline SH1 & 15 & 1.25 & 1.44 & 1.72 & 1.22 & 1.32 & 1.52 & 1.81 & 1.22 & 1.29 & 1.52 & 1.77 \\
\hline & 0.99 & 1.00 & 1.24 & 1.38 & 1.16 & 1.28 & 1.45 & 1.76 & 1.17 & 1.25 & 1.46 & 1.72 \\
\hline $\mathrm{SH} 3$ & 0.91 & 0.93 & 1.14 & 1.28 & 1.11 & 1.22 & 1.39 & 1.68 & 1.14 & 1.24 & 1.43 & 1.70 \\
\hline SH4 & 0.93 & 0.94 & 1.16 & 1.29 & 1.10 & 1.19 & 1.37 & 1.64 & 1.12 & 1.22 & 1.39 & 1.68 \\
\hline SH5 & 0.92 & 0.92 & 1.16 & 1.27 & 1.07 & 1.17 & 1.34 & 1.61 & 1.11 & 1.21 & 1.39 & 1.67 \\
\hline ML1 & 1.10 & 1.19 & 1.38 & 1.64 & 1.10 & 1.19 & 1.38 & 1.63 & 1.06 & 1.11 & 1.32 & 1.52 \\
\hline ML2 & 1.17 & 1.30 & 1.46 & 1.78 & 1.16 & 1.28 & 1.46 & 1.75 & 1.12 & 1.18 & 1.40 & 1.62 \\
\hline ML3 & 1.25 & 1.40 & 1.56 & 1.93 & 1.22 & 1.35 & 1.53 & 1.85 & 1.17 & 1.23 & 1.46 & 1.69 \\
\hline ML4 & 1.15 & 1.27 & 1.44 & 1.74 & 1.15 & 1.25 & 1.43 & 1.72 & 1.11 & 1.16 & 1.38 & 1.59 \\
\hline ML5 & 1.19 & 1.32 & 1.48 & 1.82 & 1.18 & 1.30 & 1.48 & 1.79 & 1.14 & 1.21 & 1.43 & 1.66 \\
\hline ML6 & 1.22 & 1.37 & 1.52 & 1.88 & 1.20 & 1.32 & 1.50 & 1.82 & 1.14 & 1.21 & 1.43 & 1.66 \\
\hline & 1.33 & 1.45 & 1.66 & 2.00 & 1.26 & 1.38 & 1.57 & 1.90 & 1.21 & 1.38 & 1.51 & 1.90 \\
\hline & 26 & 1.42 & 1.58 & 1.95 & 1.30 & 1.49 & 1.63 & 2.05 & 1.31 & 1.51 & 1.63 & 2.07 \\
\hline & 1.01 & 1.09 & 1.26 & 1.49 & 1.06 & 1.25 & 1.33 & 1.72 & 1.14 & 1.36 & 1.43 & 1.88 \\
\hline MH4 & 1.31 & 1.42 & 1.64 & 1.95 & 1.26 & 1.44 & 1.58 & 1.98 & 1.25 & 1.43 & 1.56 & 1.96 \\
\hline MH5 & 1.27 & 1.43 & 1.59 & 1.97 & 1.30 & 1.49 & 1.62 & 2.05 & 1.31 & 1.52 & 1.64 & 2.08 \\
\hline MH6 & 1.26 & 1.40 & 1.57 & 1.93 & 1.28 & 1.46 & 1.60 & 2.01 & 1.27 & 1.46 & 1.59 & 2.00 \\
\hline LL1 & 1.31 & 1.45 & 1.64 & 1.99 & 1.22 & 1.37 & 1.53 & 1.89 & 1.21 & 1.37 & 1.51 & 1.89 \\
\hline LL2 & 1.30 & 1.42 & 1.63 & 1.95 & 1.25 & 1.42 & 1.56 & 1.95 & 1.25 & 1.43 & 1.57 & 1.97 \\
\hline LL3 & 1.06 & 1.20 & 1.33 & 1.64 & 1.19 & 1.38 & 1.48 & 1.89 & 1.23 & 1.45 & 1.54 & 2.00 \\
\hline LL4 & 1.31 & 1.45 & 1.64 & 1.99 & 1.23 & 1.38 & 1.53 & 1.89 & 1.21 & 1.38 & 1.51 & 1.90 \\
\hline LL5 & 1.28 & 1.43 & 1.60 & 1.97 & 1.30 & 1.49 & 1.63 & 2.05 & 1.33 & 1.53 & 1.66 & 2.10 \\
\hline LL6 & 0.92 & 0.96 & 1.15 & 1.32 & 0.89 & 1.06 & 1.11 & 1.46 & 0.94 & 1.18 & 1.17 & 1.62 \\
\hline LH1 & 1.22 & 1.34 & 1.53 & 1.85 & 1.27 & 1.44 & 1.58 & 1.97 & 1.26 & 1.45 & 1.58 & 1.99 \\
\hline LH2 & 1.14 & 1.23 & 1.43 & 1.69 & 1.21 & 1.37 & 1.52 & 1.88 & 1.25 & 1.43 & 1.56 & 1.97 \\
\hline LH3 & 0.97 & 1.00 & 1.21 & 1.37 & 0.91 & 1.08 & 1.14 & 1.48 & 0.98 & 1.20 & 1.22 & 1.65 \\
\hline LH4 & 1.17 & 1.25 & 1.46 & 1.72 & 1.23 & 1.37 & 1.53 & 1.89 & 1.23 & 1.40 & 1.54 & 1.93 \\
\hline LH5 & 1.07 & 1.12 & 1.34 & 1.54 & 1.15 & 1.31 & 1.44 & 1.80 & 1.19 & 1.38 & 1.49 & 1.90 \\
\hline LH6 & 0.89 & 1.01 & 1.12 & 1.39 & 0.96 & 1.10 & 1.19 & 1.52 & 1.10 & 1.34 & 1.37 & 1.84 \\
\hline
\end{tabular}


Table C.78 - The ratio of actual mid-span bending moments and quarter-span shear forces for buildings to the corresponding values obtained from FEMA parabolic distribution and a uniform distribution of seismic load when subjected to E6C15 ground motion and the Load is acting parallel to the long side, $\alpha=0.10$

\begin{tabular}{|c|c|c|c|c|c|c|c|c|c|c|c|c|}
\hline \multirow{2}{*}{ Building } & \multicolumn{4}{|c|}{$\mu=2$} & \multicolumn{4}{|c|}{$\mu=3$} & \multicolumn{4}{|c|}{$\mu=4$} \\
\hline & $M . F$ & $V . F$ & $M . U$ & V.U & M.F & $V . F$ & $M . U$ & $V . U$ & M.F & V.F & M.U & $V . U$ \\
\hline SL1 & 1.17 & 1.32 & 1.47 & 1.81 & 1.10 & 1.26 & 1.37 & 1.73 & 1.04 & 1.20 & 1.30 & 1.65 \\
\hline SL3 & 17 & 1.31 & 1.46 & 1.79 & 1.10 & 1.26 & 1.37 & 1.73 & 1.04 & 1.17 & 1.31 & 1.61 \\
\hline & 17 & 1.30 & 1.46 & 1.79 & 1.09 & 1.25 & 1.36 & 1.72 & 1.04 & 1.18 & 1.30 & 1.62 \\
\hline SH1 & 21 & 1.33 & 1.51 & 1.83 & 1.22 & 1.35 & 1.52 & 1.86 & 1.21 & 1.34 & 1.52 & 1.85 \\
\hline & 1.25 & 1.39 & 1.57 & 1.92 & 1.26 & 1.40 & 1.58 & 1.93 & 1.25 & 1.39 & 1.57 & 1.91 \\
\hline $\mathrm{SH} 3$ & 1.27 & 1.42 & 1.59 & 1.95 & 1.28 & 1.43 & 1.60 & 1.96 & 1.28 & 1.41 & 1.59 & 1.94 \\
\hline $\mathrm{SH} 4$ & 1.30 & 1.45 & 1.62 & 2.00 & 1.30 & 1.45 & 1.63 & 1.99 & 1.29 & 1.43 & 1.62 & 1.96 \\
\hline SH5 & 1.30 & 1.45 & 1.62 & 2.00 & 1.31 & 1.45 & 1.63 & 2.00 & 1.30 & 1.43 & 1.62 & 1.96 \\
\hline IL1 & 1.28 & 1.43 & 1.60 & 1.97 & 1.40 & 1.53 & 1.75 & 2.10 & 1.39 & 1.51 & 1.74 & 2.07 \\
\hline ML2 & 1.29 & 1.45 & 1.61 & 1.99 & 1.42 & 1.55 & 1.78 & 2.13 & 1.39 & 1.50 & 1.73 & 2.06 \\
\hline ML3 & 1.31 & 1.47 & 1.64 & 2.02 & 1.36 & 1.49 & 1.70 & 2.05 & 1.34 & 1.46 & 1.68 & 2.01 \\
\hline ML4 & 1.30 & 1.45 & 1.62 & 2.00 & 1.42 & 1.54 & 1.77 & 2.12 & 1.41 & 1.52 & 1.76 & 2.09 \\
\hline ML5 & 1.30 & 1.46 & 1.62 & 2.00 & 1.42 & 1.55 & 1.78 & 2.13 & 1.39 & 1.51 & 1.74 & 2.07 \\
\hline ML6 & 1.29 & 1.45 & 1.61 & 1.99 & 1.39 & 1.52 & 1.74 & 2.09 & 1.35 & 1.47 & 1.69 & 2.01 \\
\hline & 1.09 & 1.24 & 1.36 & 1.71 & 1.28 & 1.43 & 1.60 & 1.96 & 1.36 & 1.48 & 1.70 & 2.04 \\
\hline & 1.07 & 1.12 & 1.33 & 1.55 & 1.30 & 1.46 & 1.63 & 2.01 & 1.42 & 1.55 & 1.77 & 2.13 \\
\hline & 1.30 & 1.50 & 1.62 & 2.06 & 1.27 & 1.44 & 1.59 & 1.98 & 1.27 & 1.45 & 1.59 & 2.00 \\
\hline MH4 & 1.06 & 1.13 & 1.33 & 1.55 & 1.27 & 1.42 & 1.58 & 1.95 & 1.36 & 1.48 & 1.70 & 2.04 \\
\hline MH5 & 1.09 & 1.15 & 1.36 & 1.58 & 1.25 & 1.41 & 1.56 & 1.94 & 1.35 & 1.49 & 1.69 & 2.05 \\
\hline MH6 & 1.23 & 1.36 & 1.54 & 1.87 & 1.39 & 1.53 & 1.74 & 2.11 & 1.43 & 1.56 & 1.79 & 2.15 \\
\hline LL1 & 1.15 & 1.29 & 1.44 & 1.77 & 1.35 & 1.49 & 1.69 & 2.05 & 1.42 & 1.55 & 1.77 & 2.13 \\
\hline LL2 & 1.13 & 1.22 & 1.41 & 1.67 & 1.37 & 1.51 & 1.72 & 2.08 & 1.43 & 1.55 & 1.78 & 2.13 \\
\hline LL3 & 1.25 & 1.43 & 1.56 & 1.97 & 1.28 & 1.46 & 1.60 & 2.00 & 1.31 & 1.48 & 1.64 & 2.03 \\
\hline LL4 & 1.13 & 1.27 & 1.42 & 1.74 & 1.35 & 1.49 & 1.68 & 2.04 & 1.41 & 1.54 & 1.77 & 2.12 \\
\hline LL5 & 1.06 & 1.11 & 1.32 & 1.52 & 1.32 & 1.47 & 1.65 & 2.02 & 1.44 & 1.57 & 1.79 & 2.16 \\
\hline LL6 & 1.28 & 1.54 & 1.59 & 2.12 & 1.29 & 1.51 & 1.61 & 2.08 & 1.28 & 1.48 & 1.60 & 2.03 \\
\hline LH1 & 1.20 & 1.33 & 1.50 & 1.83 & 1.46 & 1.61 & 1.82 & 2.22 & 1.46 & 1.60 & 1.82 & 2.20 \\
\hline LH2 & 1.24 & 1.40 & 1.55 & 1.93 & 1.40 & 1.57 & 1.75 & 2.15 & 1.47 & 1.62 & 1.83 & 2.23 \\
\hline LH3 & 1.32 & 1.56 & 1.65 & 2.15 & 1.36 & 1.58 & 1.70 & 2.17 & 1.47 & 1.66 & 1.84 & 2.28 \\
\hline LH4 & 1.16 & 1.28 & 1.45 & 1.76 & 1.46 & 1.63 & 1.83 & 2.24 & 1.46 & 1.62 & 1.83 & 2.22 \\
\hline LH5 & 1.24 & 1.43 & 1.55 & 1.96 & 1.38 & 1.56 & 1.73 & 2.14 & 1.43 & 1.59 & 1.79 & 2.19 \\
\hline LH6 & 1.34 & 1.54 & 1.67 & 2.11 & 1.36 & 1.58 & 1.70 & 2.17 & 1.47 & 1.66 & 1.83 & 2.28 \\
\hline
\end{tabular}


Table C.79 - The ratio of actual mid-span bending moments and quarter-span shear forces for buildings to the corresponding values obtained from FEMA parabolic distribution and a uniform distribution of seismic load when subjected to E6C18 ground motion and the Load is acting parallel to the long side, $\alpha=0.10$

\begin{tabular}{|c|c|c|c|c|c|c|c|c|c|c|c|c|}
\hline \multirow{2}{*}{ Building } & \multicolumn{4}{|c|}{$\mu=2$} & \multicolumn{4}{|c|}{$\mu=3$} & \multicolumn{4}{|c|}{$\mu=4$} \\
\hline & $M . F$ & $V . F$ & $M . U$ & V.U & M.F & $V . F$ & $M . U$ & $V . U$ & M.F & V.F & M.U & $V . U$ \\
\hline SL1 & 0.95 & 0.97 & 1.19 & 1.33 & 1.05 & 1.13 & 1.31 & 1.56 & 1.05 & 1.14 & 1.31 & 1.56 \\
\hline SL3 & 96 & 0.97 & 1.20 & 1.34 & 1.04 & 1.15 & 1.30 & 1.58 & 1.04 & 1.13 & 1.30 & 1.56 \\
\hline & 0.97 & 0.99 & 1.21 & 1.35 & 1.08 & 1.22 & 1.35 & 1.67 & 1.04 & 1.17 & 1.31 & 1.60 \\
\hline SH1 & 1.06 & 1.10 & 1.32 & 1.51 & 1.15 & 1.30 & 1.44 & 1.78 & 1.13 & 1.27 & 1.41 & 1.75 \\
\hline & 1.06 & 1.10 & 1.32 & 1.51 & 1.13 & 1.25 & 1.41 & 1.72 & 1.15 & 1.29 & 1.43 & 1.77 \\
\hline $\mathrm{SH} 3$ & 1.08 & 1.15 & 1.35 & 1.59 & 1.14 & 1.27 & 1.42 & 1.74 & 1.14 & 1.28 & 1.43 & 1.75 \\
\hline $\mathrm{SH} 4$ & 1.06 & 1.10 & 1.32 & 1.51 & 1.15 & 1.28 & 1.43 & 1.76 & 1.14 & 1.28 & 1.43 & 1.76 \\
\hline SH5 & 1.07 & 1.13 & 1.33 & 1.56 & 1.14 & 1.25 & 1.43 & 1.72 & 1.15 & 1.28 & 1.43 & 1.76 \\
\hline ML1 & 1.19 & 1.34 & 1.48 & 1.84 & 1.19 & 1.33 & 1.49 & 1.83 & 1.12 & 1.21 & 1.40 & 1.67 \\
\hline ML2 & 1.21 & 1.36 & 1.52 & 1.88 & 1.13 & 1.29 & 1.42 & 1.77 & 1.09 & 1.20 & 1.37 & 1.65 \\
\hline ML3 & 1.17 & 1.30 & 1.46 & 1.79 & 1.11 & 1.27 & 1.39 & 1.75 & 1.06 & 1.20 & 1.32 & 1.64 \\
\hline ML4 & 1.21 & 1.37 & 1.51 & 1.88 & 1.14 & 1.29 & 1.43 & 1.77 & 1.12 & 1.23 & 1.40 & 1.70 \\
\hline ML5 & 1.20 & 1.35 & 1.50 & 1.85 & 1.12 & 1.28 & 1.40 & 1.76 & 1.08 & 1.20 & 1.34 & 1.65 \\
\hline ML6 & 1.18 & 1.32 & 1.47 & 1.82 & 1.11 & 1.27 & 1.39 & 1.75 & 1.04 & 1.21 & 1.30 & 1.66 \\
\hline & 14 & 1.27 & 1.42 & 1.75 & 1.10 & 1.26 & 1.37 & 1.73 & 1.10 & 1.22 & 1.37 & 1.68 \\
\hline & & 1.16 & 1.33 & 1.60 & 1.05 & 1.22 & 1.31 & 1.67 & 1.08 & 1.21 & 1.35 & 1.67 \\
\hline & 1.05 & 1.10 & 1.32 & 1.51 & 1.17 & 1.24 & 1.46 & 1.70 & 1.35 & 1.55 & 1.68 & 2.14 \\
\hline MH4 & 1.12 & 1.25 & 1.40 & 1.72 & 1.08 & 1.25 & 1.35 & 1.71 & 1.07 & 1.20 & 1.34 & 1.65 \\
\hline MH5 & 1.05 & 1.14 & 1.31 & 1.56 & 1.08 & 1.19 & 1.35 & 1.63 & 1.12 & 1.18 & 1.39 & 1.62 \\
\hline MH6 & 1.07 & 1.17 & 1.34 & 1.61 & 1.04 & 1.18 & 1.30 & 1.63 & 1.05 & 1.16 & 1.31 & 1.60 \\
\hline LL1 & 1.14 & 1.27 & 1.42 & 1.75 & 1.10 & 1.27 & 1.37 & 1.75 & 1.12 & 1.24 & 1.40 & 1.71 \\
\hline LL2 & 1.13 & 1.27 & 1.41 & 1.74 & 1.09 & 1.26 & 1.36 & 1.74 & 1.08 & 1.23 & 1.35 & 1.69 \\
\hline LL3 & 1.02 & 1.02 & 1.28 & 1.41 & 1.12 & 1.16 & 1.40 & 1.59 & 1.25 & 1.39 & 1.56 & 1.91 \\
\hline LL4 & 1.13 & 1.27 & 1.42 & 1.74 & 1.10 & 1.27 & 1.37 & 1.75 & 1.11 & 1.24 & 1.39 & 1.71 \\
\hline LL5 & 1.08 & 1.18 & 1.34 & 1.62 & 1.05 & 1.22 & 1.31 & 1.68 & 1.06 & 1.20 & 1.33 & 1.65 \\
\hline LL6 & 1.06 & 1.16 & 1.33 & 1.60 & 1.37 & 1.58 & 1.71 & 2.17 & 1.35 & 1.61 & 1.69 & 2.22 \\
\hline LH1 & 1.02 & 1.06 & 1.27 & 1.46 & 1.04 & 1.11 & 1.29 & 1.53 & 1.05 & 1.14 & 1.31 & 1.57 \\
\hline LH2 & 0.96 & 0.97 & 1.20 & 1.33 & 1.03 & 1.10 & 1.28 & 1.52 & 1.14 & 1.29 & 1.42 & 1.78 \\
\hline LH3 & 1.24 & 1.35 & 1.55 & 1.85 & 1.22 & 1.40 & 1.53 & 1.92 & 1.21 & 1.44 & 1.51 & 1.98 \\
\hline LH4 & 1.00 & 1.04 & 1.25 & 1.43 & 1.02 & 1.09 & 1.27 & 1.50 & 1.03 & 1.13 & 1.29 & 1.56 \\
\hline LH5 & 0.98 & 1.07 & 1.23 & 1.48 & 1.17 & 1.26 & 1.46 & 1.73 & 1.44 & 1.74 & 1.80 & 2.39 \\
\hline LH6 & 1.27 & 1.43 & 1.59 & 1.97 & 1.27 & 1.43 & 1.59 & 1.97 & 1.26 & 1.43 & 1.58 & 1.96 \\
\hline
\end{tabular}


Table C.80 - The ratio of actual mid-span bending moments and quarter-span shear forces for buildings to the corresponding values obtained from FEMA parabolic distribution and a uniform distribution of seismic load when subjected to E6C42 ground motion and the Load is acting parallel to the long side, $\alpha=0.10$

\begin{tabular}{|c|c|c|c|c|c|c|c|c|c|c|c|c|}
\hline \multirow{2}{*}{ Building } & \multicolumn{4}{|c|}{$\mu=2$} & \multicolumn{4}{|c|}{$\mu=3$} & \multicolumn{4}{|c|}{$\mu=4$} \\
\hline & $M . F$ & $V . F$ & $M . U$ & V.U & M.F & $V . F$ & $M . U$ & $V . U$ & M.F & V.F & M.U & $V . U$ \\
\hline SL1 & 1.19 & 1.33 & 1.49 & 1.84 & 1.16 & 1.29 & 1.45 & 1.77 & 1.14 & 1.26 & 1.43 & 1.73 \\
\hline SL3 & 22 & 1.37 & 1.53 & 1.89 & 1.18 & 1.32 & 1.47 & 1.81 & 1.15 & 1.26 & 1.43 & 1.73 \\
\hline & 16 & 1.27 & 1.45 & 1.75 & 1.21 & 1.35 & 1.51 & 1.85 & 1.15 & 1.27 & 1.44 & 1.75 \\
\hline SH1 & 13 & 1.19 & 1.41 & 1.64 & 1.19 & 1.33 & 1.49 & 1.82 & 1.23 & 1.38 & 1.54 & 1.90 \\
\hline & 1.21 & 1.30 & 1.51 & 1.79 & 1.25 & 1.39 & 1.56 & 1.92 & 1.24 & 1.36 & 1.55 & 1.87 \\
\hline $\mathrm{SH} 3$ & 1.22 & 1.34 & 1.53 & 1.84 & 1.25 & 1.39 & 1.56 & 1.91 & 1.21 & 1.29 & 1.52 & 1.77 \\
\hline $\mathrm{SH} 4$ & 1.22 & 1.34 & 1.53 & 1.84 & 1.24 & 1.37 & 1.55 & 1.88 & 1.22 & 1.32 & 1.52 & 1.82 \\
\hline SH5 & 1.20 & 1.31 & 1.50 & 1.80 & 1.22 & 1.34 & 1.52 & 1.84 & 1.19 & 1.26 & 1.49 & 1.73 \\
\hline IL1 & 1.26 & 1.41 & 1.58 & 1.94 & 1.23 & 1.40 & 1.54 & 1.92 & 1.19 & 1.35 & 1.49 & 1.85 \\
\hline ML2 & 1.16 & 1.31 & 1.45 & 1.81 & 1.09 & 1.24 & 1.36 & 1.71 & 1.05 & 1.16 & 1.31 & 1.59 \\
\hline ML3 & 1.01 & 1.14 & 1.26 & 1.57 & 0.94 & 1.10 & 1.17 & 1.52 & 0.99 & 1.05 & 1.24 & 1.45 \\
\hline ML4 & 1.18 & 1.34 & 1.48 & 1.84 & 1.13 & 1.29 & 1.42 & 1.77 & 1.08 & 1.20 & 1.35 & 1.65 \\
\hline ML5 & 1.12 & 1.27 & 1.40 & 1.74 & 1.05 & 1.23 & 1.31 & 1.69 & 1.01 & 1.12 & 1.26 & 1.55 \\
\hline ML6 & 1.04 & 1.18 & 1.31 & 1.62 & 0.97 & 1.13 & 1.21 & 1.56 & 1.02 & 1.10 & 1.27 & 1.51 \\
\hline & 0.88 & 0.87 & 1.10 & 1.19 & 0.90 & 0.90 & 1.12 & 1.23 & 1.08 & 1.17 & 1.35 & 1.60 \\
\hline & & 1.00 & 1.23 & 1.38 & 1.06 & 1.14 & 1.32 & 1.57 & 1.13 & 1.36 & 1.41 & 1.87 \\
\hline & 1.09 & 1.15 & 1.37 & 1.58 & 1.12 & 1.21 & 1.40 & 1.67 & 1.15 & 1.28 & 1.44 & 1.76 \\
\hline MH4 & 0.90 & 0.89 & 1.13 & 1.23 & 0.92 & 0.94 & 1.15 & 1.29 & 1.04 & 1.13 & 1.30 & 1.55 \\
\hline MH5 & 1.03 & 1.06 & 1.28 & 1.45 & 1.11 & 1.21 & 1.39 & 1.66 & 1.30 & 1.56 & 1.62 & 2.15 \\
\hline MH6 & 0.94 & 0.95 & 1.17 & 1.30 & 1.05 & 1.14 & 1.32 & 1.56 & 1.09 & 1.28 & 1.37 & 1.76 \\
\hline LL1 & 0.89 & 0.87 & 1.12 & 1.20 & 0.90 & 0.92 & 1.12 & 1.26 & 1.17 & 1.31 & 1.46 & 1.80 \\
\hline LL2 & 0.91 & 0.90 & 1.14 & 1.24 & 0.94 & 0.98 & 1.17 & 1.35 & 1.14 & 1.27 & 1.42 & 1.74 \\
\hline LL3 & 1.10 & 1.17 & 1.37 & 1.60 & 1.13 & 1.23 & 1.41 & 1.69 & 1.17 & 1.30 & 1.46 & 1.78 \\
\hline LL4 & 0.90 & 0.88 & 1.12 & 1.21 & 0.90 & 0.93 & 1.12 & 1.27 & 1.16 & 1.30 & 1.45 & 1.79 \\
\hline LL5 & 0.97 & 0.99 & 1.21 & 1.36 & 1.04 & 1.11 & 1.30 & 1.53 & 1.08 & 1.29 & 1.35 & 1.77 \\
\hline LL6 & 1.05 & 1.12 & 1.31 & 1.54 & 1.11 & 1.19 & 1.39 & 1.63 & 1.18 & 1.34 & 1.48 & 1.84 \\
\hline LH1 & 0.99 & 1.01 & 1.23 & 1.39 & 1.13 & 1.31 & 1.42 & 1.80 & 1.24 & 1.47 & 1.55 & 2.02 \\
\hline LH2 & 1.03 & 1.12 & 1.29 & 1.55 & 1.34 & 1.59 & 1.67 & 2.19 & 1.61 & 1.98 & 2.02 & 2.72 \\
\hline LH3 & 1.14 & 1.25 & 1.42 & 1.72 & 1.11 & 1.22 & 1.39 & 1.67 & 1.27 & 1.50 & 1.59 & 2.06 \\
\hline LH4 & 1.01 & 1.05 & 1.27 & 1.45 & 1.25 & 1.47 & 1.56 & 2.02 & 1.36 & 1.65 & 1.70 & 2.27 \\
\hline LH5 & 1.06 & 1.16 & 1.33 & 1.60 & 1.14 & 1.26 & 1.42 & 1.73 & 1.40 & 1.63 & 1.75 & 2.24 \\
\hline LH6 & 1.23 & 1.40 & 1.54 & 1.92 & 1.19 & 1.41 & 1.49 & 1.93 & 1.30 & 1.59 & 1.62 & 2.18 \\
\hline
\end{tabular}




\section{Appendix D}

Table D.1 - Ductility demand on the lateral load resisting system and internal force magnification factors for selected buildings subjected to $\mathrm{M} 6 \mathrm{C} 1$ ground motion -

load parallel to the short side $-\mu=3.00$

\begin{tabular}{|c|c|c|c|c|c|}
\hline LLRS Behaviour & \multicolumn{5}{|c|}{ Elastoplastic } \\
\hline Buildings & Ductility Demand $(\mu)$ & $M . F$ & V.F & $M . U$ & $V . F$ \\
\hline SH1 $(\mathrm{R}=1.29)$ & 2.99 & 1.23 & 1.42 & 1.53 & 1.96 \\
\hline MH1 $(\mathrm{R}=1.43)$ & 3.01 & 1.08 & 1.26 & 1.35 & 1.73 \\
\hline LH1 $(\mathrm{R}=1.41)$ & 3.01 & 1.24 & 1.38 & 1.55 & 1.9 \\
\hline LLRS Behaviour & \multicolumn{5}{|c|}{ Clough Model } \\
\hline Buildings & Ductility Demand $(\mu)$ & $M . F$ & $V . F$ & M.U & $V . F$ \\
\hline SH1 $(\mathrm{R}=1.29)$ & 2.92 & 1.18 & 1.37 & 1.47 & 1.88 \\
\hline MH1 (R=1.43) & 3.01 & 1.07 & 1.26 & 1.28 & 1.73 \\
\hline LH1 $(\mathrm{R}=1.41)$ & 2.6 & 1.23 & 1.38 & 1.54 & 1.9 \\
\hline LLRS Behaviour & \multicolumn{5}{|c|}{ Pinching Behaviour ( $\mathrm{kL} / \mathrm{r}=80$ to 135$)$} \\
\hline Buildings & Ductility Demand $(\mu)$ & $M . F$ & $V . F$ & $M . U$ & V.F \\
\hline SH1 $(\mathrm{R}=1.29)$ & 2.94 & 1.17 & 1.37 & 1.47 & 1.89 \\
\hline MH1 $(\mathrm{R}=1.43)$ & 3.01 & 1.08 & 1.26 & 1.35 & 1.73 \\
\hline LH1 $(\mathrm{R}=1.41)$ & 2.79 & 1.23 & 1.38 & 1.54 & 1.9 \\
\hline LLRS Behaviour & \multicolumn{5}{|c|}{ Pinching Behaviour $(\mathrm{kL} / \mathrm{r}=155$ to 200$)$} \\
\hline Buildings & Ductility Demand $(\mu)$ & $M . F$ & $V . F$ & $M . U$ & $V . F$ \\
\hline SH1 $(\mathrm{R}=1.29)$ & 2.99 & 1.17 & 1.38 & 1.47 & 1.9 \\
\hline MH1 (R=1.43) & 3.08 & 1.08 & 1.26 & 1.35 & 1.73 \\
\hline LH1 $(\mathrm{R}=1.41)$ & 2.83 & 1.23 & 1.38 & 1.54 & 1.9 \\
\hline
\end{tabular}


Table D.2 - Ductility demand on the lateral load resisting system and internal force magnification factors for selected buildings subjected to M6C2 ground motion load parallel to the short side $-\mu=3.00$

\begin{tabular}{|c|c|c|c|c|c|}
\hline LLRS Behaviour & \multicolumn{5}{|c|}{ Elastoplastic } \\
\hline Buildings & Ductility Demand $(\mu)$ & $M . F$ & $V . F$ & M.U & V.F \\
\hline SH1 $(\mathrm{R}=1.55)$ & 2.96 & 1.28 & 1.53 & 1.6 & 2.11 \\
\hline MH1 $(\mathrm{R}=1.57)$ & 3.03 & 1.21 & 1.42 & 1.52 & 1.95 \\
\hline LH1 (R=1.59) & 3.01 & 1.2 & 1.4 & 1.5 & 1.92 \\
\hline LLRS Behaviour & \multicolumn{5}{|c|}{ Clough Model } \\
\hline Buildings & Ductility Demand $(\mu)$ & $M . F$ & $V . F$ & M.U & V.F \\
\hline SH1 $(\mathrm{R}=1.55)$ & 3.38 & 1.17 & 1.47 & 1.46 & 2.02 \\
\hline MH1 $(\mathrm{R}=1.57)$ & 3.31 & 1.07 & 1.21 & 1.33 & 1.66 \\
\hline LH1 $(\mathrm{R}=1.59)$ & 3.59 & 1.13 & 1.26 & 1.41 & 1.73 \\
\hline LLRS Behaviour & \multicolumn{5}{|c|}{ Pinching Behaviour $(\mathrm{kL} / \mathrm{r}=80$ to 135$)$} \\
\hline Buildings & Ductility Demand $(\mu)$ & $M . F$ & V.F & M.U & $V . F$ \\
\hline SH1 $(\mathrm{R}=1.55)$ & 3.5 & 1.17 & 1.47 & 1.46 & 2.02 \\
\hline MH1 $(\mathrm{R}=1.57)$ & 3.4 & 1.07 & 1.21 & 1.33 & 1.66 \\
\hline LH1 $(\mathrm{R}=1.59)$ & 4.31 & 1.13 & 1.27 & 1.42 & 1.74 \\
\hline LLRS Behaviour & \multicolumn{5}{|c|}{ Pinching Behaviour $(\mathrm{kL} / \mathrm{r}=155$ to 200$)$} \\
\hline Buildings & Ductility Demand $(\mu)$ & M.F & V.F & M.U & $V . F$ \\
\hline SH1 $(\mathrm{R}=1.55)$ & 3.53 & 1.17 & 1.47 & 1.46 & 2.02 \\
\hline MH1 $(\mathrm{R}=1.57)$ & 3.46 & 1.07 & 1.21 & 1.33 & 1.66 \\
\hline LH1 $(\mathrm{R}=1.59)$ & 4.62 & 1.14 & 1.28 & 1.43 & 1.76 \\
\hline
\end{tabular}


Table D.3 - Ductility demand on the lateral load resisting system and internal force magnification factors for selected buildings subjected to M6C26 ground motion load parallel to the short side $-\mu=3.00$

\begin{tabular}{cccccc}
\hline LLRS Behaviour & \multicolumn{5}{c}{ Elastoplastic } \\
\hline Buildings & Ductility Demand $(\mu)$ & $M . F$ & $V . F$ & $M . U$ & $V . F$ \\
\hline SH1 (R=1.29) & 2.96 & 1.17 & 1.23 & 1.46 & 1.7 \\
MH1 (R=1.61) & 2.97 & 1.13 & 1.29 & 1.42 & 1.77 \\
LH1 (R=1.33) & 3.02 & 1.14 & 1.28 & 1.42 & 1.77 \\
\hline LLRS Behaviour & \multicolumn{5}{c}{ Clough Model } \\
\hline Buildings & Ductility Demand $(\mu)$ & $M . F$ & $V . F$ & $M . U$ & $V . F$ \\
\hline SH1 (R=1.29) & 2.96 & 1.06 & 1.22 & 1.32 & 1.68 \\
MH1 (R=1.61) & 2.97 & 0.99 & 1.17 & 1.24 & 1.61 \\
LH1 (R=1.33) & 2.49 & 1.07 & 1.28 & 1.33 & 1.77 \\
\hline LLRS Behaviour & Pinching Behaviour $(\mathrm{kL} / \mathrm{r}=80$ to 135$)$ \\
\hline Buildings & Ductility Demand $(\mu)$ & $M . F$ & $V . F$ & $M . U$ & $V . F$ \\
\hline SH1 (R=1.29) & 2.96 & 1.06 & 1.22 & 1.32 & 1.68 \\
MH1 (R=1.61) & 2.97 & 0.99 & 1.17 & 1.24 & 1.61 \\
LH1 (R=1.33) & 2.54 & 1.07 & 1.28 & 1.33 & 1.77 \\
\hline LLRS Behaviour & Pinching Behaviour $(\mathrm{kL} / \mathrm{r}=155$ to 200$)$ \\
\hline Buildings & Ductility Demand $(\mu)$ & $M . F$ & $V . F$ & $M . U$ & $V . F$ \\
\hline SH1 (R=1.29) & 2.96 & 1.06 & 1.17 & 1.33 & 1.61 \\
MH1 (R=1.61) & 2.97 & 0.99 & 1.17 & 1.24 & 1.61 \\
LH1 (R=1.33) & 2.51 & 1.07 & 1.28 & 1.33 & 1.77 \\
\hline
\end{tabular}


Table D.4 - Ductility demand on the lateral load resisting system and internal force magnification factors for selected buildings subjected to $\mathrm{M} 6 \mathrm{C} 1$ ground motion load parallel to the long side $-\mu=3.00$

\begin{tabular}{cccccc}
\hline LLRS Behaviour & \multicolumn{5}{c}{ Elastoplastic } \\
\hline Buildings & Ductility Demand $(\mu)$ & $M . F$ & $V . F$ & $M . U$ & $V . F$ \\
\hline SH1 $(\mathrm{R}=2.34)$ & 3 & 1.28 & 1.51 & 1.6 & 2.07 \\
MH1 $(\mathrm{R}=1.90)$ & 3 & 1.33 & 1.55 & 1.66 & 2.13 \\
LH1 (R=2.66) & 3 & 1.27 & 1.48 & 1.59 & 2.04 \\
\hline LLRS Behaviour & \multicolumn{5}{c}{ Clough Model } \\
\hline Buildings & Ductility Demand $(\mu)$ & $M . F$ & $V . F$ & $M . U$ & $V . F$ \\
\hline SH1 $(\mathrm{R}=2.34)$ & 3.08 & 1.01 & 1.1 & 1.27 & 1.51 \\
MH1 $(\mathrm{R}=1.90)$ & 3 & 1.07 & 1.23 & 1.34 & 1.7 \\
LH1 (R=2.66) & 3.31 & 1.13 & 1.28 & 1.41 & 1.75 \\
\hline LLRS Behaviour & Pinching Behaviour $(\mathrm{kL} / \mathrm{r}=80$ to 135$)$ \\
\hline Buildings & Ductility Demand $(\mu)$ & $M . F$ & $V . F$ & $M . U$ & $V . F$ \\
\hline SH1 $(\mathrm{R}=2.34)$ & 3.53 & 1.04 & 1.24 & 1.24 & 1.7 \\
MH1 $(\mathrm{R}=1.90)$ & 3.02 & 1.07 & 1.23 & 1.34 & 1.7 \\
LH1 (R=2.66) & 3.23 & 1.13 & 1.28 & 1.41 & 1.75 \\
\hline LLRS Behaviour & \multicolumn{5}{c}{ Pinching Behaviour $(\mathrm{kL} / \mathrm{r}=155$ to 200$)$} \\
\hline Buildings & Ductility Demand $(\mu)$ & $M . F$ & $V . F$ & $M . U$ & $V . F$ \\
\hline SH1 $(\mathrm{R}=2.34)$ & 3.75 & 1.21 & 1.32 & 1.51 & 1.81 \\
MH1 (R=1.90) & 3.16 & 1.1 & 1.25 & 1.37 & 1.72 \\
LH1 (R=2.66) & 3.18 & 1.13 & 1.28 & 1.41 & 1.75 \\
\hline
\end{tabular}


Table D.5 - Ductility demand on the lateral load resisting system and internal force magnification factors for selected buildings subjected to M6C2 ground motion load parallel to the long side $-\mu=3.00$

\begin{tabular}{|c|c|c|c|c|c|}
\hline LLRS Behaviour & \multicolumn{5}{|c|}{ Elastoplastic } \\
\hline Buildings & Ductility Demand $(\mu)$ & M.F & V.F & $M . U$ & V.F \\
\hline SH1 $(\mathrm{R}=1.66)$ & 3.02 & 1.21 & 1.39 & 1.51 & 1.91 \\
\hline MH1 $(\mathrm{R}=1.39)$ & 3 & 1.27 & 1.48 & 1.59 & 2.04 \\
\hline LH1 $(\mathrm{R}=2.02)$ & 3 & 1.26 & 1.48 & 1.58 & 2.03 \\
\hline LLRS Behaviour & \multicolumn{5}{|c|}{ Clough Model } \\
\hline Buildings & Ductility Demand $(\mu)$ & M.F & V.F & $M . U$ & V.F \\
\hline SH1 $(\mathrm{R}=1.66)$ & 1.79 & 1.04 & 1.16 & 1.3 & 1.6 \\
\hline MH1 $(\mathrm{R}=1.39)$ & 2.82 & 1.27 & 1.48 & 1.59 & 2.04 \\
\hline LH1 $(\mathrm{R}=2.02)$ & 2.33 & 1.26 & 1.48 & 1.58 & 2.03 \\
\hline LLRS Behaviour & \multicolumn{5}{|c|}{ Pinching Behaviour $(\mathrm{kL} / \mathrm{r}=80$ to 135$)$} \\
\hline Buildings & Ductility Demand $(\mu)$ & $M . F$ & V.F & $M . U$ & V.F \\
\hline SH1 $(\mathrm{R}=1.66)$ & 1.8 & 1.03 & 1.14 & 1.28 & 1.56 \\
\hline MH1 $(\mathrm{R}=1.39)$ & 3.41 & 1.27 & 1.48 & 1.59 & 2.04 \\
\hline LH1 $(\mathrm{R}=2.02)$ & 2.47 & 1.26 & 1.48 & 1.58 & 2.03 \\
\hline LLRS Behaviour & \multicolumn{5}{|c|}{ Pinching Behaviour $(\mathrm{kL} / \mathrm{r}=155$ to 200$)$} \\
\hline Buildings & Ductility Demand $(\mu)$ & M.F & $V . F$ & $M . U$ & V.F \\
\hline SH1 $(\mathrm{R}=1.66)$ & 1.82 & 1.03 & 1.21 & 1.28 & 1.66 \\
\hline MH1 (R=1.39) & 3.32 & 1.27 & 1.48 & 1.59 & 2.04 \\
\hline LH1 $(\mathrm{R}=2.02)$ & 2.53 & 1.26 & 1.48 & 1.58 & 2.03 \\
\hline
\end{tabular}


Table D.6 - Ductility demand on the lateral load resisting system and internal force magnification factors for selected buildings subjected to M6C26 ground motion load parallel to the long side $-\mu=3.00$

\begin{tabular}{|c|c|c|c|c|c|}
\hline LLRS Behaviour & \multicolumn{5}{|c|}{ Elastoplastic } \\
\hline Buildings & Ductility Demand $(\mu)$ & M.F & V.F & $M . U$ & V.F \\
\hline SH1 $(\mathrm{R}=1.97)$ & 3 & 1.21 & 1.34 & 1.51 & 1.85 \\
\hline MH1 $(\mathrm{R}=1.46)$ & 3.02 & 1.02 & 1.15 & 1.27 & 1.58 \\
\hline LH1 $(\mathrm{R}=2.88)$ & 3 & 1.38 & 1.66 & 1.73 & 2.28 \\
\hline LLRS Behaviour & \multicolumn{5}{|c|}{ Clough Model } \\
\hline Buildings & Ductility Demand $(\mu)$ & M.F & V.F & $M . U$ & V.F \\
\hline SH1 $(\mathrm{R}=1.97)$ & 3 & 1.15 & 1.33 & 1.43 & 1.83 \\
\hline MH1 $(\mathrm{R}=1.46)$ & 2.97 & 1.02 & 1.15 & 1.27 & 1.58 \\
\hline LH1 $(\mathrm{R}=2.88)$ & 3 & 1.08 & 1.17 & 1.34 & 1.61 \\
\hline LLRS Behaviour & \multicolumn{5}{|c|}{ Pinching Behaviour $(\mathrm{kL} / \mathrm{r}=80$ to 135$)$} \\
\hline Buildings & Ductility Demand $(\mu)$ & M.F & V.F & $M . U$ & V.F \\
\hline SH1 $(\mathrm{R}=1.97)$ & 3 & 1.1 & 1.33 & 1.31 & 1.83 \\
\hline MH1 $(\mathrm{R}=1.46)$ & 2.97 & 1.02 & 1.15 & 1.27 & 1.58 \\
\hline LH1 $(\mathrm{R}=2.88)$ & 3 & 1.08 & 1.17 & 1.34 & 1.61 \\
\hline LLRS Behaviour & \multicolumn{5}{|c|}{ Pinching Behaviour $(\mathrm{kL} / \mathrm{r}=155$ to 200$)$} \\
\hline Buildings & Ductility Demand $(\mu)$ & M.F & $V . F$ & $M . U$ & V.F \\
\hline SH1 $(\mathrm{R}=1.97)$ & 3 & 1.15 & 1.33 & 1.43 & 1.83 \\
\hline MH1 $(\mathrm{R}=1.46)$ & 2.97 & 1.08 & 1.18 & 1.35 & 1.62 \\
\hline LH1 $(\mathrm{R}=2.88)$ & 3.18 & 1.08 & 1.17 & 1.34 & 1.61 \\
\hline
\end{tabular}


Table D.7 - Ductility demand on the lateral load resisting system and internal force magnification factors for selected buildings subjected to E6C1 ground motion - load parallel to the short side $-\mu=3.00$

\begin{tabular}{|c|c|c|c|c|c|}
\hline LLRS Behaviour & \multicolumn{5}{|c|}{ Elastoplastic } \\
\hline Buildings & Ductility Demand $(\mu)$ & M.F & V.F & $M . U$ & V.F \\
\hline SH1 $(\mathrm{R}=1.29)$ & 3.01 & 1.3 & 1.51 & 1.63 & 2.08 \\
\hline MH1 $(\mathrm{R}=1.29)$ & 3.02 & 1.09 & 1.23 & 1.37 & 1.69 \\
\hline LH1 $(\mathrm{R}=1.93)$ & 3.01 & 1.32 & 1.68 & 1.65 & 2.31 \\
\hline LLRS Behaviour & \multicolumn{5}{|c|}{ Clough Model } \\
\hline Buildings & Ductility Demand $(\mu)$ & M.F & V.F & $M . U$ & V.F \\
\hline SH1 $(\mathrm{R}=1.29)$ & 3.01 & 1.26 & 1.51 & 1.58 & 2.08 \\
\hline MH1 $(\mathrm{R}=1.29)$ & 3.02 & 1.09 & 1.23 & 1.37 & 1.69 \\
\hline LH1 $(\mathrm{R}=1.93)$ & 3.01 & 1.32 & 1.68 & 1.65 & 2.31 \\
\hline LLRS Behaviour & \multicolumn{5}{|c|}{ Pinching Behaviour $(\mathrm{kL} / \mathrm{r}=80$ to 135$)$} \\
\hline Buildings & Ductility Demand $(\mu)$ & $M . F$ & V.F & $M . U$ & V.F \\
\hline SH1 $(\mathrm{R}=1.29)$ & 3.01 & 1.26 & 1.49 & 1.58 & 2.05 \\
\hline MH1 $(\mathrm{R}=1.29)$ & 3.02 & 1.09 & 1.23 & 1.37 & 1.69 \\
\hline LH1 $(\mathrm{R}=1.93)$ & 3.03 & 1.32 & 1.68 & 1.65 & 2.31 \\
\hline LLRS Behaviour & \multicolumn{5}{|c|}{ Pinching Behaviour $(\mathrm{kL} / \mathrm{r}=155$ to 200$)$} \\
\hline Buildings & Ductility Demand $(\mu)$ & M.F & $V . F$ & $M . U$ & V.F \\
\hline SH1 $(\mathrm{R}=1.29)$ & 3.01 & 1.26 & 1.51 & 1.58 & 2.08 \\
\hline MH1 $(\mathrm{R}=1.29)$ & 3.02 & 1.09 & 1.23 & 1.37 & 1.69 \\
\hline LH1 $(\mathrm{R}=1.93)$ & 3.11 & 1.32 & 1.68 & 1.65 & 2.31 \\
\hline
\end{tabular}


Table D.8 - Ductility demand on the lateral load resisting system and internal force magnification factors for selected buildings subjected to E6C13 ground motion load parallel to the short side $-\mu=3.00$

\begin{tabular}{cccccc}
\hline LLRS Behaviour & \multicolumn{5}{c}{ Elastoplastic } \\
\hline Buildings & Ductility Demand $(\mu)$ & $M . F$ & $V . F$ & $M . U$ & $V . F$ \\
\hline SH1 (R=1.52) & 2.99 & 1.12 & 1.38 & 1.39 & 1.89 \\
MH1 (R=1.50) & 2.96 & 1.19 & 1.42 & 1.49 & 1.95 \\
LH1 (R=1.6) & 3 & 1.17 & 1.31 & 1.46 & 1.8 \\
\hline LLRS Behaviour & \multicolumn{5}{c}{ Clough Model } \\
\hline Buildings & Ductility Demand $(\mu)$ & $M . F$ & $V . F$ & $M . U$ & $V . F$ \\
\hline SH1 (R=1.52) & 2.99 & 1.12 & 1.38 & 1.39 & 1.89 \\
MH1 (R=1.50) & 4.63 & 1.07 & 1.34 & 1.34 & 1.84 \\
LH1 (R=1.6) & 2.95 & 1.04 & 1.2 & 1.3 & 1.65 \\
\hline LLRS Behaviour & Pinching Behaviour $(\mathrm{kL} / \mathrm{r}=80$ to 135$)$ \\
\hline Buildings & Ductility Demand $(\mu)$ & $M . F$ & $V . F$ & $M . U$ & $V . F$ \\
\hline SH1 (R=1.52) & 2.99 & 1.12 & 1.38 & 1.39 & 1.89 \\
MH1 (R=1.50) & 5.35 & 1.09 & 1.34 & 1.36 & 1.84 \\
LH1 (R=1.6) & 2.94 & 1.08 & 1.24 & 1.35 & 1.71 \\
\hline LLRS Behaviour & Pinching Behaviour $(\mathrm{kL} / \mathrm{r}=155$ to 200$)$ \\
\hline Buildings & Ductility Demand $(\mu)$ & $M . F$ & $V . F$ & $M . U$ & $V . F$ \\
\hline SH1 (R=1.52) & 2.99 & 1.12 & 1.38 & 1.39 & 1.89 \\
MH1 (R=1.50) & 5.45 & 1.11 & 1.38 & 1.39 & 1.9 \\
LH1 (R=1.6) & 2.96 & 1.13 & 1.27 & 1.41 & 1.75 \\
\hline
\end{tabular}


Table D.9 - Ductility demand on the lateral load resisting system and internal force magnification factors for selected buildings subjected to E6C15 ground motion load parallel to the short side $-\mu=3.00$

\begin{tabular}{cccccc}
\hline LLRS Behaviour & \multicolumn{5}{c}{ Elastoplastic } \\
\hline Buildings & Ductility Demand $(\mu)$ & $M . F$ & $V . F$ & $M . U$ & $V . F$ \\
\hline SH1 (R=1.29) & 3.05 & 1.25 & 1.46 & 1.56 & 2.01 \\
MH1 (R=1.35) & 3.05 & 1.37 & 1.58 & 1.71 & 2.17 \\
LH1 (R=1.68) & 2.96 & 1.45 & 1.87 & 1.82 & 2.58 \\
\hline LLRS Behaviour & \multicolumn{5}{c}{ Clough Model } \\
\hline Buildings & Ductility Demand $(\mu)$ & $M . F$ & $V . F$ & $M . U$ & $V . F$ \\
\hline SH1 (R=1.29) & 3.05 & 1.25 & 1.46 & 1.56 & 2.01 \\
MH1 (R=1.35) & 3.07 & 1.37 & 1.58 & 1.71 & 2.17 \\
LH1 (R=1.68) & 2.58 & 1.44 & 1.73 & 1.8 & 2.38 \\
\hline LLRS Behaviour & Pinching Behaviour $(\mathrm{kL} / \mathrm{r}=80$ to 135$)$ \\
\hline Buildings & Ductility Demand $(\mu)$ & $M . F$ & $V . F$ & $M . U$ & $V . F$ \\
\hline SH1 (R=1.29) & 3.05 & 1.25 & 1.46 & 1.56 & 2.01 \\
MH1 (R=1.35) & 3.19 & 1.37 & 1.58 & 1.71 & 2.17 \\
LH1 (R=1.68) & 2.74 & 1.44 & 1.73 & 1.8 & 2.38 \\
\hline LLRS Behaviour & Pinching Behaviour $(\mathrm{kL} / \mathrm{r}=155$ to 200$)$ \\
\hline Buildings & Ductility Demand $(\mu)$ & $M . F$ & $V . F$ & $M . U$ & $V . F$ \\
\hline SH1 (R=1.29) & 3.05 & 1.25 & 1.46 & 1.56 & 2.01 \\
MH1 (R=1.35) & 3.2 & 1.37 & 1.58 & 1.71 & 2.17 \\
LH1 (R=1.68) & 2.82 & 1.44 & 1.73 & 1.8 & 2.38 \\
\hline
\end{tabular}


Table D.10 - Ductility demand on the lateral load resisting system and internal force magnification factors for selected buildings subjected to E6C1 ground motion - load parallel to the long side $-\mu=3.00$

\begin{tabular}{|c|c|c|c|c|c|}
\hline LLRS Behaviour & \multicolumn{5}{|c|}{ Elastoplastic } \\
\hline Buildings & Ductility Demand $(\mu)$ & M.F & V.F & $M . U$ & V.F \\
\hline SH1 $(\mathrm{R}=1.54)$ & 3.01 & 1.11 & 1.26 & 1.38 & 1.74 \\
\hline MH1 $(\mathrm{R}=1.59)$ & 3 & 1.19 & 1.36 & 1.48 & 1.87 \\
\hline LH1 $(\mathrm{R}=1.81)$ & 3 & 1.28 & 1.47 & 1.6 & 2.03 \\
\hline LLRS Behaviour & \multicolumn{5}{|c|}{ Clough Model } \\
\hline Buildings & Ductility Demand $(\mu)$ & M.F & V.F & $M . U$ & V.F \\
\hline SH1 $(\mathrm{R}=1.54)$ & 3.01 & 1.11 & 1.24 & 1.38 & 1.71 \\
\hline MH1 $(\mathrm{R}=1.59)$ & 3.01 & 1.19 & 1.36 & 1.48 & 1.87 \\
\hline LH1 $(\mathrm{R}=1.81)$ & 3 & 1.24 & 1.47 & 1.55 & 2.03 \\
\hline LLRS Behaviour & \multicolumn{5}{|c|}{ Pinching Behaviour $(\mathrm{kL} / \mathrm{r}=80$ to 135$)$} \\
\hline Buildings & Ductility Demand $(\mu)$ & $M . F$ & V.F & $M . U$ & V.F \\
\hline $\mathrm{SH} 1(\mathrm{R}=1.54)$ & 3.01 & 1.11 & 1.24 & 1.38 & 1.71 \\
\hline MH1 $(\mathrm{R}=1.59)$ & 3 & 1.19 & 1.36 & 1.48 & 1.87 \\
\hline LH1 $(\mathrm{R}=1.81)$ & 3 & 1.24 & 1.47 & 1.55 & 2.03 \\
\hline LLRS Behaviour & \multicolumn{5}{|c|}{ Pinching Behaviour $(\mathrm{kL} / \mathrm{r}=155$ to 200$)$} \\
\hline Buildings & Ductility Demand $(\mu)$ & M.F & $V . F$ & $M . U$ & V.F \\
\hline $\mathrm{SH} 1(\mathrm{R}=1.54)$ & 3.01 & 1.11 & 1.24 & 1.38 & 1.71 \\
\hline MH1 $(\mathrm{R}=1.59)$ & 3 & 1.19 & 1.36 & 1.48 & 1.87 \\
\hline LH1 $(\mathrm{R}=1.81)$ & 3 & 1.24 & 1.47 & 1.55 & 2.03 \\
\hline
\end{tabular}


Table D.11 - Ductility demand on the lateral load resisting system and internal force magnification factors for selected buildings subjected to E6C13 ground motion load parallel to the long side $-\mu=3.00$

\begin{tabular}{cccccc}
\hline LLRS Behaviour & \multicolumn{5}{c}{ Elastoplastic } \\
\hline Buildings & Ductility Demand $(\mu)$ & $M . F$ & $V . F$ & $M . U$ & $V . F$ \\
\hline SH1 (R=2.72) & 3 & 1.33 & 1.5 & 1.66 & 2.07 \\
MH1 ( $=1.46)$ & 2.98 & 1.38 & 1.65 & 1.72 & 2.27 \\
LH1 (R=1.84) & 3 & 1.41 & 1.72 & 1.77 & 2.37 \\
\hline LLRS Behaviour & \multicolumn{5}{c}{ Clough Model } \\
\hline Buildings & Ductility Demand $(\mu)$ & $M . F$ & $V . F$ & $M . U$ & $V . F$ \\
\hline SH1 (R=2.72) & 3.91 & 1.07 & 1.21 & 1.34 & 1.66 \\
MH1 (R=1.46) & 3.03 & 1.35 & 1.65 & 1.69 & 2.27 \\
LH1 (R=1.84) & 3.41 & 1.41 & 1.72 & 1.77 & 2.37 \\
\hline LLRS Behaviour & Pinching Behaviour $(\mathrm{kL} / \mathrm{r}=80$ to 135$)$ \\
\hline Buildings & Ductility Demand $(\mu)$ & $M . F$ & $V . F$ & $M . U$ & $V . F$ \\
\hline SH1 (R=2.72) & 4.46 & 1.17 & 1.29 & 1.46 & 1.77 \\
MH1 (R=1.46) & 2.45 & 1.32 & 1.59 & 1.65 & 2.19 \\
LH1 (R=1.84) & 3.57 & 1.41 & 1.72 & 1.77 & 2.37 \\
\hline LLRS Behaviour & Pinching Behaviour $(\mathrm{kL} / \mathrm{r}=155$ to 200$)$ \\
\hline Buildings & Ductility Demand $(\mu)$ & $M . F$ & $V . F$ & $M . U$ & $V . F$ \\
\hline SH1 (R=2.72) & 4.8 & 1.24 & 1.39 & 1.56 & 1.92 \\
MH1 (R=1.46) & 2.56 & 1.35 & 1.65 & 1.69 & 2.27 \\
LH1 (R=1.84) & 4.15 & 1.41 & 1.72 & 1.77 & 2.37 \\
\hline
\end{tabular}


Table D.12 - Ductility demand on the lateral load resisting system and internal force magnification factors for selected buildings subjected to E6C15 ground motion load parallel to the long side $-\mu=3.00$

\begin{tabular}{cccccc}
\hline LLRS Behaviour & \multicolumn{5}{c}{ Elastoplastic } \\
\hline Buildings & Ductility Demand $(\mu)$ & $M . F$ & $V . F$ & $M . U$ & $V . F$ \\
\hline SH1 (R=1.65) & 2.99 & 1.32 & 1.59 & 1.66 & 2.18 \\
MH1 (R=2.16) & 3.02 & 1.43 & 1.63 & 1.79 & 2.24 \\
LH1 (R=3.55) & 3 & 1.6 & 1.94 & 2 & 2.66 \\
\hline LLRS Behaviour & \multicolumn{5}{c}{ Clough Model } \\
\hline Buildings & Ductility Demand $(\mu)$ & $M . F$ & $V . F$ & $M . U$ & $V . F$ \\
\hline SH1 (R=1.65) & 2.99 & 1.32 & 1.59 & 1.66 & 2.18 \\
MH1 (R=2.16) & 3.58 & 1.43 & 1.63 & 1.79 & 2.24 \\
LH1 (R=3.55) & 2.93 & 1.53 & 1.74 & 1.91 & 2.39 \\
\hline LLRS Behaviour & Pinching Behaviour $(\mathrm{kL} / \mathrm{r}=80$ to 135$)$ \\
\hline Buildings & Ductility Demand $(\mu)$ & $M . F$ & $V . F$ & $M . U$ & $V . F$ \\
\hline SH1 (R=1.65) & 3.01 & 1.32 & 1.59 & 1.66 & 2.18 \\
MH1 (R=2.16) & 3.98 & 1.43 & 1.63 & 1.79 & 2.24 \\
LH1 (R=3.55) & 2.7 & 1.53 & 1.74 & 1.91 & 2.39 \\
\hline LLRS Behaviour & Pinching Behaviour $(\mathrm{kL} / \mathrm{r}=155$ to 200$)$ \\
\hline Buildings & Ductility Demand $(\mu)$ & $M . F$ & $V . F$ & $M . U$ & $V . F$ \\
\hline SH1 (R=1.65) & 3.21 & 1.32 & 1.59 & 1.66 & 2.18 \\
MH1 (R=2.16) & 4.23 & 1.43 & 1.63 & 1.79 & 2.24 \\
LH1 (R=3.55) & 2.63 & 1.53 & 1.74 & 1.91 & 2.39 \\
\hline
\end{tabular}




\section{Appendix E}

In the following tables the results of the analyses outlined in Chapter 7 are presented

Table E. 1 - Proper $R_{d}$ for steel deck panels acting as the main source of energy dissipation (weak diaphragm) - short side -ductility of 1.5 - M6C1

\begin{tabular}{|c|c|c|c|c|c|c|c|}
\hline \multirow{2}{*}{ Buildings } & \multirow{2}{*}{$\begin{array}{c}\text { Deck } \\
\text { Elastic } \\
\text { Force }\end{array}$} & \multirow{2}{*}{$\begin{array}{c}\mu_{d} \text { on Deck } \\
\text { Panels for } \\
R d=1.5\end{array}$} & \multirow{2}{*}{$\begin{array}{c}R_{d} \\
\left(\mu_{d}=1.5\right)\end{array}$} & \multicolumn{4}{|c|}{ Internal Force Magnification Ratios } \\
\hline & & & & $M . F$ & $V . F$ & $M . U$ & $V . U$ \\
\hline SL1 & 131.65 & 3.75 & 1.13 & 0.96 & 1.06 & 1.20 & 1.45 \\
\hline SH1 & 320.50 & 2.10 & 1.20 & 1.11 & 1.26 & 1.39 & 1.74 \\
\hline SH4 & 255.66 & 2.51 & 1.26 & 1.10 & 1.34 & 1.37 & 1.85 \\
\hline ML1 & 502.44 & 2.05 & 1.15 & 0.94 & 1.08 & 1.17 & 1.49 \\
\hline ML2 & 443.07 & 2.04 & 1.16 & 0.92 & 0.96 & 1.15 & 1.32 \\
\hline MH1 & 876.76 & 2.38 & 1.14 & 0.95 & 1.09 & 1.19 & 1.50 \\
\hline MH6 & 1135.99 & 2.31 & 1.11 & 0.84 & 0.96 & 1.06 & 1.32 \\
\hline LL1 & 1540.74 & 3.43 & 1.13 & 0.94 & 1.04 & 1.17 & 1.43 \\
\hline LH1 & 3009.10 & 2.31 & 1.12 & 0.92 & 1.02 & 1.15 & 1.41 \\
\hline LH5 & 2495.46 & 1.97 & 1.12 & 1.02 & 1.14 & 1.27 & 1.56 \\
\hline
\end{tabular}

Table E. 2 - Proper $R_{d}$ for steel deck panels acting as the main source of energy dissipation (weak diaphragm) - short side -ductility of 1.5 - M6C2

\begin{tabular}{|c|c|c|c|c|c|c|c|}
\hline \multirow{2}{*}{ Buildings } & \multirow{2}{*}{$\begin{array}{c}\text { Deck } \\
\text { Elastic } \\
\text { Force }\end{array}$} & \multirow{2}{*}{$\begin{array}{c}\mu_{d} \text { on Deck } \\
\text { Panels for } \\
\text { Rd }=1.5\end{array}$} & \multirow{2}{*}{$\begin{array}{c}R_{d} \\
\left(\mu_{d}=1.5\right)\end{array}$} & \multicolumn{4}{|c|}{ Internal Force Magnification Ratios } \\
\hline & & & & $M . F$ & V.F & $M . U$ & $V . U$ \\
\hline SL1 & 134.25 & 3.66 & 1.14 & 0.94 & 1.07 & 1.18 & 1.47 \\
\hline SH1 & 223.96 & 2.08 & 1.15 & 1.06 & 1.23 & 1.33 & 1.69 \\
\hline SH4 & 215.84 & 2.35 & 1.21 & 1.04 & 1.17 & 1.30 & 1.60 \\
\hline ML1 & 282.78 & 3.05 & 1.21 & 1.21 & 1.33 & 1.52 & 1.83 \\
\hline ML2 & 212.82 & 3.54 & 1.15 & 1.05 & 1.22 & 1.32 & 1.67 \\
\hline MH1 & 579.85 & 2.61 & 1.16 & 0.90 & 0.99 & 1.12 & 1.36 \\
\hline MH6 & 580.95 & 2.84 & $1.15(4)$ & 1.33 & 1.43 & 1.66 & 1.97 \\
\hline LL1 & 1086.37 & 2.85 & 1.13 & 1.05 & 1.22 & 1.31 & 1.68 \\
\hline LH1 & 2499.19 & 3.97 & 1.17 & 1.05 & 1.24 & 1.31 & 1.70 \\
\hline LH5 & 2422.78 & 4.40 & 1.08 & 1.10 & 1.35 & 1.38 & 1.86 \\
\hline
\end{tabular}


Table E. 3 - Proper $R_{d}$ for steel deck panels acting as the main source of energy dissipation (weak diaphragm) - short side -ductility of 1.5 - M6C26

\begin{tabular}{|c|c|c|c|c|c|c|c|}
\hline \multirow{2}{*}{ Buildings } & \multirow{2}{*}{$\begin{array}{c}\text { Deck } \\
\text { Elastic } \\
\text { Force }\end{array}$} & \multirow{2}{*}{$\begin{array}{c}\mu_{d} \text { on Deck } \\
\text { Panels for } \\
R d=1.5\end{array}$} & \multirow{2}{*}{$\begin{array}{c}R_{d} \\
\left(\mu_{d}=1.5\right)\end{array}$} & \multicolumn{4}{|c|}{ Internal Force Magnification Ratios } \\
\hline & & & & $M . F$ & $V . F$ & $M . U$ & $V . U$ \\
\hline SL1 & 163.47 & 4.10 & 1.11 & 1.04 & 1.15 & 1.29 & 1.58 \\
\hline SH1 & 336.81 & 2.94 & 1.21 & 1.02 & 1.17 & 1.28 & 1.60 \\
\hline SH4 & 291.21 & 3.09 & 1.18 & 1.03 & 1.22 & 1.29 & 1.67 \\
\hline ML1 & 471.18 & 2.50 & 1.12 & 0.99 & 1.09 & 1.23 & 1.50 \\
\hline ML2 & 374.61 & 2.20 & 1.15 & 0.87 & 1.00 & 1.08 & 1.37 \\
\hline MH1 & 739.26 & 2.11 & 1.16 & 0.81 & 0.90 & 1.00 & 1.23 \\
\hline MH6 & 986.90 & 2.59 & 1.09 & 0.94 & 1.03 & 1.17 & 1.41 \\
\hline LL1 & 1159.74 & 2.63 & 1.18 & 1.05 & 1.17 & 1.31 & 1.60 \\
\hline LH1 & 2038.29 & 3.35 & 1.11 & 0.96 & 1.10 & 1.20 & 1.51 \\
\hline LH5 & 1941.37 & 2.94 & 1.10 & 0.93 & 1.06 & 1.16 & 1.45 \\
\hline
\end{tabular}

Table E. 4 - Proper $R_{d}$ for steel deck panels acting as the main source of energy dissipation (weak diaphragm) - short side -ductility of 1.5 M6C31

\begin{tabular}{|c|c|c|c|c|c|c|c|}
\hline \multirow{2}{*}{ Buildings } & \multirow{2}{*}{$\begin{array}{c}\text { Deck } \\
\text { Elastic } \\
\text { Force }\end{array}$} & \multirow{2}{*}{$\begin{array}{c}\mu_{d} \text { on Deck } \\
\text { Panels for } \\
R d=1.5\end{array}$} & \multirow{2}{*}{$\begin{array}{c}R_{d} \\
\left(\mu_{d}=1.5\right)\end{array}$} & \multicolumn{4}{|c|}{ Internal Force Magnification Ratios } \\
\hline & & & & $M . F$ & $V . F$ & $M . U$ & $V . U$ \\
\hline SL1 & 202.00 & 3.64 & 1.09 & 1.06 & 1.18 & 1.32 & 1.62 \\
\hline SH1 & 245.50 & 3.01 & 1.15 & 1.03 & 1.13 & 1.28 & 1.55 \\
\hline SH4 & 237.87 & 2.55 & 1.19 & 1.13 & 1.33 & 1.42 & 1.83 \\
\hline ML1 & 305.15 & 1.64 & 1.21 & 1.12 & 1.34 & 1.40 & 1.84 \\
\hline ML2 & 205.80 & 2.95 & 1.19 & 1.13 & 1.22 & 1.42 & 1.68 \\
\hline MH1 & 462.06 & 3.05 & 1.20 & 0.96 & 1.05 & 1.20 & 1.44 \\
\hline MH6 & 629.63 & 2.50 & 1.11 & 1.11 & 1.29 & 1.39 & 1.78 \\
\hline LL1 & 870.58 & 2.48 & 1.14 & 1.05 & 1.17 & 1.32 & 1.61 \\
\hline LH1 & 1618.01 & 3.26 & 1.13 & 0.96 & 1.15 & 1.20 & 1.58 \\
\hline LH5 & 1516.05 & 3.65 & 1.11 & 1.01 & 1.22 & 1.27 & 1.67 \\
\hline
\end{tabular}


Table E.5 - Proper $R_{d}$ for steel deck panels acting as the main source of energy dissipation (weak diaphragm) - short side - ductility of 1.5 - M6C38

\begin{tabular}{|c|c|c|c|c|c|c|c|}
\hline \multirow{2}{*}{ Buildings } & \multirow{2}{*}{$\begin{array}{c}\text { Deck } \\
\text { Elastic } \\
\text { Force }\end{array}$} & \multirow{2}{*}{$\begin{array}{c}\mu_{d} \text { on Deck } \\
\text { Panels for } \\
R d=1.5\end{array}$} & \multirow{2}{*}{$\begin{array}{c}R_{d} \\
\left(\mu_{d}=1.5\right)\end{array}$} & \multicolumn{4}{|c|}{ Internal Force Magnification Ratios } \\
\hline & & & & $M . F$ & $V . F$ & $M . U$ & $V . U$ \\
\hline SL1 & 136.99 & 4.42 & 1.08 & 1.14 & 1.29 & 1.42 & 1.77 \\
\hline SH1 & 184.84 & 2.52 & 1.17 & 1.06 & 1.17 & 1.32 & 1.61 \\
\hline SH4 & 163.97 & 2.17 & 1.21 & 1.07 & 1.16 & 1.34 & 1.60 \\
\hline ML1 & 291.23 & 2.19 & 1.15 & 1.00 & 1.07 & 1.25 & 1.47 \\
\hline ML2 & 275.08 & 2.09 & 1.24 & 1.05 & 1.11 & 1.32 & 1.52 \\
\hline MH1 & 663.23 & 2.39 & 1.15 & 0.87 & 0.97 & 1.09 & 1.33 \\
\hline MH6 & 693.28 & 2.41 & 1.12 & 0.87 & 0.95 & 1.09 & 1.31 \\
\hline LL1 & 1136.37 & 3.77 & 1.11 & 0.87 & 0.98 & 1.09 & 1.35 \\
\hline LH1 & 2700.07 & 3.32 & 1.11 & 0.88 & 0.98 & 1.08 & 1.34 \\
\hline LH5 & 2687.17 & 3.30 & 1.10 & 0.88 & 1.03 & 1.10 & 1.41 \\
\hline
\end{tabular}

Table E.6 - Proper $R_{d}$ for steel deck panels acting as the main source of energy dissipation (weak diaphragm) - short side -ductility of 2.0 - M6C1

\begin{tabular}{cccccccc}
\hline & \multirow{2}{*}{$\begin{array}{c}\text { Deck } \\
\text { Buildings }\end{array}$} & $\begin{array}{c}\mu_{d} \text { on Deck } \\
\text { Elastic } \\
\text { Fonels for }\end{array}$ & $\begin{array}{c}R_{d} \\
\text { Rd }=1.5\end{array}$ & & \multicolumn{3}{c}{ Internal Force Magnification Ratios } \\
\cline { 5 - 8 } & $\left.\mu_{d}=2.0\right)$ & & M.F & V.F & M.U & V.U \\
\hline SL1 & 131.65 & 3.75 & 1.19 & 1.00 & 1.11 & 1.25 & 1.53 \\
SH1 & 320.50 & 2.10 & 1.40 & 1.09 & 1.27 & 1.36 & 1.74 \\
SH4 & 255.66 & 2.51 & 1.37 & 1.07 & 1.34 & 1.34 & 1.84 \\
ML1 & 502.44 & 2.05 & 1.49 & 1.00 & 1.21 & 1.25 & 1.67 \\
ML2 & 443.07 & 2.04 & 1.49 & 0.94 & 1.04 & 1.18 & 1.43 \\
MH1 & 876.76 & 2.38 & 1.35 & 0.96 & 1.10 & 1.20 & 1.51 \\
MH6 & 1135.99 & 2.31 & 1.20 & 0.89 & 1.00 & 1.11 & 1.38 \\
LL1 & 1540.74 & 3.43 & 1.22 & 0.91 & 1.00 & 1.13 & 1.37 \\
LH1 & 3009.10 & 2.31 & 1.24 & 0.97 & 1.07 & 1.21 & 1.46 \\
LH5 & 2495.46 & 1.97 & 1.51 & 1.11 & 1.22 & 1.38 & 1.68 \\
\hline
\end{tabular}


Table E. 7 - Proper $R_{d}$ for steel deck panels acting as the main source of energy dissipation (weak diaphragm) - short side -ductility of 2.0 - M6C2

\begin{tabular}{|c|c|c|c|c|c|c|c|}
\hline \multirow{2}{*}{ Buildings } & \multirow{2}{*}{$\begin{array}{c}\text { Deck } \\
\text { Elastic } \\
\text { Force }\end{array}$} & \multirow{2}{*}{$\begin{array}{c}\mu_{d} \text { on Deck } \\
\text { Panels for } \\
R d=1.5\end{array}$} & \multirow{2}{*}{$\begin{array}{c}R_{d} \\
\left(\mu_{d}=2.0\right)\end{array}$} & \multicolumn{4}{|c|}{ Internal Force Magnification Ratios } \\
\hline & & & & $M . F$ & $V . F$ & $M . U$ & $V . U$ \\
\hline SL1 & 134.25 & 3.66 & 1.23 & 0.91 & 1.05 & 1.13 & 1.44 \\
\hline SH1 & 223.96 & 2.08 & 1.46 & 1.07 & 1.29 & 1.33 & 1.77 \\
\hline SH4 & 215.84 & 2.35 & 1.31 & 1.01 & 1.16 & 1.26 & 1.59 \\
\hline ML1 & 282.78 & 3.05 & 1.29 & 1.15 & 1.29 & 1.44 & 1.77 \\
\hline ML2 & 212.82 & 3.54 & 1.22 & 0.94 & 1.15 & 1.18 & 1.58 \\
\hline MH1 & 579.85 & 2.61 & 1.27 & 0.81 & 0.94 & 1.01 & 1.29 \\
\hline MH6 & 580.95 & 2.84 & 1.34 & 1.19 & 1.30 & 1.48 & 1.79 \\
\hline LL1 & 1086.37 & 2.85 & 1.33 & 1.01 & 1.19 & 1.26 & 1.64 \\
\hline LH1 & 2499.19 & 3.97 & 1.27 & 1.00 & 1.18 & 1.25 & 1.62 \\
\hline LH5 & 2422.78 & 4.40 & 1.22 & 1.07 & 1.28 & 1.33 & 1.76 \\
\hline
\end{tabular}

Table E.8 - Proper $R_{d}$ for steel deck panels acting as the main source of energy dissipation (weak diaphragm) - short side -ductility of 2.0 - M6C26

\begin{tabular}{|c|c|c|c|c|c|c|c|}
\hline \multirow{2}{*}{ Buildings } & \multirow{2}{*}{$\begin{array}{c}\text { Deck } \\
\text { Elastic } \\
\text { Force }\end{array}$} & \multirow{2}{*}{$\begin{array}{c}\mu_{d} \text { on Deck } \\
\text { Panels for } \\
R d=1.5\end{array}$} & \multirow{2}{*}{$\begin{array}{c}R_{d} \\
\left(\mu_{d}=2.0\right)\end{array}$} & \multicolumn{4}{|c|}{ Internal Force Magnification Ratios } \\
\hline & & & & $M . F$ & $V . F$ & $M . U$ & $V . U$ \\
\hline SL1 & 163.47 & 4.10 & 1.19 & 1.06 & 1.19 & 1.32 & 1.64 \\
\hline SH1 & 336.81 & 2.94 & 1.31 & 0.98 & 1.14 & 1.22 & 1.57 \\
\hline SH4 & 291.21 & 3.09 & 1.28 & 1.02 & 1.20 & 1.28 & 1.65 \\
\hline ML1 & 471.18 & 2.50 & 1.38 & 1.60 & 1.21 & 1.32 & 1.66 \\
\hline ML2 & 374.61 & 2.20 & 1.30 & 0.94 & 1.08 & 1.18 & 1.49 \\
\hline MH1 & 739.26 & 2.11 & 1.42 & 0.85 & 0.97 & 1.06 & 1.33 \\
\hline MH6 & 986.90 & 2.59 & 1.19 & 1.00 & 1.10 & 1.24 & 1.59 \\
\hline LL1 & 1159.74 & 2.63 & 1.28 & 1.06 & 1.18 & 1.33 & 1.62 \\
\hline LH1 & 2038.29 & 3.35 & 1.21 & 0.93 & 1.06 & 1.16 & 1.46 \\
\hline LH5 & 1941.37 & 2.94 & 1.18 & 0.94 & 1.12 & 1.17 & 1.54 \\
\hline
\end{tabular}


Table E.9 - Proper $R_{d}$ for steel deck panels acting as the main source of energy dissipation (weak diaphragm) - short side - ductility of 2.0 - M6C31

\begin{tabular}{|c|c|c|c|c|c|c|c|}
\hline \multirow{2}{*}{ Buildings } & \multirow{2}{*}{$\begin{array}{c}\text { Deck } \\
\text { Elastic } \\
\text { Force }\end{array}$} & \multirow{2}{*}{$\begin{array}{c}\mu_{d} \text { on Deck } \\
\text { Panels for } \\
R d=1.5\end{array}$} & \multirow{2}{*}{$\begin{array}{c}R_{d} \\
\left(\mu_{d}=2.0\right)\end{array}$} & \multicolumn{4}{|c|}{ Internal Force Magnification Ratios } \\
\hline & & & & $M . F$ & V.F & $M . U$ & $V . U$ \\
\hline SL1 & 202.00 & 3.64 & 1.17 & 1.06 & 1.18 & 1.32 & 1.63 \\
\hline SH1 & 245.50 & 3.01 & 1.26 & 1.08 & 1.20 & 1.35 & 1.65 \\
\hline SH4 & 237.87 & 2.55 & 1.34 & 1.16 & 1.31 & 1.44 & 1.80 \\
\hline ML1 & 305.15 & 1.64 & 1.92 & 1.12 & 1.25 & 1.39 & 1.72 \\
\hline ML2 & 205.80 & 2.95 & 1.34 & 1.01 & 1.20 & 1.27 & 1.65 \\
\hline MH1 & 462.06 & 3.05 & 1.28 & 0.93 & 1.02 & 1.16 & 1.41 \\
\hline MH6 & 629.63 & 2.50 & 1.31 & 1.12 & 1.31 & 1.39 & 1.80 \\
\hline LL1 & 870.58 & 2.48 & 1.44 & 1.04 & 1.22 & 1.30 & 1.68 \\
\hline LH1 & 1618.01 & 3.26 & 1.24 & 0.93 & 1.09 & 1.16 & 1.50 \\
\hline LH5 & 1516.05 & 3.65 & 1.20 & 0.98 & 1.16 & 1.23 & 1.59 \\
\hline
\end{tabular}

Table E.10 - Proper $R_{d}$ for steel deck panels acting as the main source of energy dissipation (weak diaphragm) - short side -ductility of 2.0 - M6C38

\begin{tabular}{|c|c|c|c|c|c|c|c|}
\hline \multirow{2}{*}{ Buildings } & \multirow{2}{*}{$\begin{array}{c}\text { Deck } \\
\text { Elastic } \\
\text { Force }\end{array}$} & \multirow{2}{*}{$\begin{array}{c}\mu_{d} \text { on Deck } \\
\text { Panels for } \\
R d=1.5\end{array}$} & \multirow{2}{*}{$\begin{array}{c}R_{d} \\
\left(\mu_{d}=2.0\right)\end{array}$} & \multicolumn{4}{|c|}{ Internal Force Magnification Ratios } \\
\hline & & & & $M . F$ & $V . F$ & $M . U$ & $V . U$ \\
\hline SL1 & 136.99 & 4.42 & 1.15 & 1.13 & 1.28 & 1.41 & 1.75 \\
\hline SH1 & 184.84 & 2.52 & 1.31 & 1.10 & 1.22 & 1.37 & 1.68 \\
\hline SH4 & 163.97 & 2.17 & 1.42 & 1.16 & 1.25 & 1.45 & 1.72 \\
\hline ML1 & 291.23 & 2.19 & 1.28 & 1.03 & 1.11 & 1.29 & 1.52 \\
\hline ML2 & 275.08 & 2.09 & 1.46 & 1.16 & 1.27 & 1.44 & 1.75 \\
\hline MH1 & 663.23 & 2.39 & 1.33 & 0.96 & 1.07 & 1.20 & 1.47 \\
\hline MH6 & 693.28 & 2.41 & 1.21 & 0.92 & 1.00 & 1.15 & 1.37 \\
\hline LL1 & 1136.37 & 3.77 & 1.22 & 0.90 & 0.94 & 1.12 & 1.29 \\
\hline LH1 & 2700.07 & 3.32 & 1.19 & 0.87 & 0.96 & 1.08 & 1.32 \\
\hline LH5 & 2687.17 & 3.30 & 1.19 & 0.87 & 0.98 & 1.09 & 1.35 \\
\hline
\end{tabular}


Table E.11 - Proper $R_{d}$ for steel deck panels acting as the main source of energy dissipation (weak diaphragm) - long side -ductility of 1.5 - M6C1

\begin{tabular}{|c|c|c|c|c|c|c|c|}
\hline \multirow{2}{*}{ Buildings } & \multirow{2}{*}{$\begin{array}{c}\text { Deck } \\
\text { Elastic } \\
\text { Force }\end{array}$} & \multirow{2}{*}{$\begin{array}{c}\mu_{d} \text { on Deck } \\
\text { Panels for } \\
R d=1.5\end{array}$} & \multirow{2}{*}{$\begin{array}{c}R_{d} \\
\left(\mu_{d}=1.5\right)\end{array}$} & \multicolumn{4}{|c|}{ Internal Force Magnification Ratios } \\
\hline & & & & $M . F$ & $V . F$ & $M . U$ & V.U \\
\hline SL1 & 209.88 & 3.99 & 1.09 & 0.90 & 0.95 & 1.12 & 1.30 \\
\hline SH1 & 429.19 & 2.92 & 1.08 & 0.92 & 0.98 & 1.15 & 1.35 \\
\hline ML1 & 629.11 & 2.70 & 1.09 & 0.97 & 1.05 & 1.21 & 1.45 \\
\hline MH1 & 1300.08 & 3.79 & 1.08 & 0.97 & 1.06 & 1.22 & 1.46 \\
\hline LL1 & 2412.95 & 3.73 & 1.07 & 0.95 & 1.01 & 1.18 & 1.39 \\
\hline LH1 & 6344.10 & 3.03 & 1.07 & 0.88 & 0.93 & 1.10 & 1.27 \\
\hline
\end{tabular}

Table E.12 - Proper $R_{d}$ for steel deck panels acting as the main source of energy dissipation (weak diaphragm) - long side -ductility of 1.5 - M6C2

\begin{tabular}{|c|c|c|c|c|c|c|c|}
\hline \multirow{2}{*}{ Buildings } & \multirow{2}{*}{$\begin{array}{c}\text { Deck } \\
\text { Elastic } \\
\text { Force }\end{array}$} & \multirow{2}{*}{$\begin{array}{c}\mu_{d} \text { on Deck } \\
\text { Panels for } \\
\text { Rd }=1.5\end{array}$} & \multirow{2}{*}{$\begin{array}{c}R_{d} \\
\left(\mu_{d}=1.5\right)\end{array}$} & \multicolumn{4}{|c|}{ Internal Force Magnification Ratios } \\
\hline & & & & $M . F$ & $V . F$ & $M . U$ & $V . U$ \\
\hline SL1 & 174.31 & 3.26 & 1.08 & 0.93 & 1.00 & 1.17 & 1.37 \\
\hline SH1 & 475.44 & 3.13 & 1.09 & 0.91 & 0.97 & 1.14 & 1.33 \\
\hline ML1 & 605.11 & 4.22 & 1.13 & 0.95 & 1.03 & 1.18 & 1.42 \\
\hline MH1 & 918.03 & 4.84 & 1.09 & 0.99 & 1.08 & 1.24 & 1.49 \\
\hline LL1 & 1702.84 & 5.56 & 1.08 & 0.89 & 0.95 & 1.11 & 1.31 \\
\hline LH1 & 3869.11 & 4.51 & 1.06 & 0.90 & 0.94 & 1.12 & 1.29 \\
\hline
\end{tabular}

Table E.13 - Proper $R_{d}$ for steel deck panels acting as the main source of energy dissipation (weak diaphragm) - long side -ductility of 1.5 - M6C26

\begin{tabular}{cccccccc}
\hline \multirow{2}{*}{ Buildings } & $\begin{array}{c}\text { Deck } \\
\text { Elastic } \\
\text { Force }\end{array}$ & $\begin{array}{c}\mu_{d} \text { on Deck } \\
\text { Panels for } \\
R d=1.5\end{array}$ & $\begin{array}{c}R_{d} \\
\left(\mu_{d}=1.5\right)\end{array}$ & \multicolumn{2}{c}{ Internal Force Magnification Ratios } \\
\cline { 5 - 8 } & 196.45 & 3.58 & 1.07 & 0.89 & 0.94 & 1.12 & 1.29 \\
\hline SL1 & 19.5 & 1.08 & 0.88 & 0.92 & 1.10 & 1.27 \\
SH1 & 486.54 & 3.47 & 1.08 & 1.00 & 1.08 & 1.25 & 1.49 \\
ML1 & 575.91 & 4.71 & 1.09 & 0.95 & 1.03 & 1.18 & 1.42 \\
MH1 & 1250.38 & 4.75 & 1.10 & 0.98 & 1.06 & 1.22 & 1.46 \\
LL1 & 2372.76 & 4.92 & 1.06 & 0.89 & 0.94 & 1.12 & 1.29 \\
LH1 & 6194.09 & 4.04 & 1.06 & \\
\hline
\end{tabular}


Table E.14 - Proper $R_{d}$ for steel deck panels acting as the main source of energy dissipation (weak diaphragm) - long side -ductility of 1.5 - M6C31

\begin{tabular}{cccccccc}
\hline \multirow{2}{*}{ Buildings } & \multirow{2}{*}{$\begin{array}{c}\text { Deck } \\
\text { Elastic } \\
\text { Force }\end{array}$} & $\begin{array}{c}\mu_{d} \text { on Deck } \\
\text { Panels for } \\
R d=1.5\end{array}$ & $\begin{array}{c}R_{d} \\
\left(\mu_{d}=1.5\right)\end{array}$ & \multicolumn{2}{c}{ Internal Force Magnification Ratios } \\
\cline { 5 - 8 } & 181.35 & 5.30 & 1.07 & 0.86 & 0.90 & 1.08 & 1.24 \\
\hline SL1 & 187.6 & 4.86 & 1.07 & 1.03 & 1.14 & 1.29 & 1.56 \\
SH1 & 447.69 & 3.33 & 1.09 & 1.01 & 1.10 & 1.27 & 1.51 \\
ML1 & 959.85 & 3.05 & 1.09 & 0.91 & 0.96 & 1.13 & 1.33 \\
MH1 & 1026.04 & 3.91 & 1.08 & 0.96 & 1.03 & 1.20 & 1.42 \\
LL1 & 1734.43 & 7.98 & 1.07 & 0.91 & 0.97 & 1.13 & 1.34 \\
LH1 & 3838.30 & & & & & & \\
\hline
\end{tabular}

Table E.15 - Proper $R_{d}$ for steel deck panels acting as the main source of energy dissipation (weak diaphragm) - long side -ductility of 1.5 - M6C38

\begin{tabular}{|c|c|c|c|c|c|c|c|}
\hline \multirow{2}{*}{ Buildings } & \multirow{2}{*}{$\begin{array}{c}\text { Deck } \\
\text { Elastic } \\
\text { Force }\end{array}$} & \multirow{2}{*}{$\begin{array}{c}\mu_{d} \text { on Deck } \\
\text { Panels for } \\
\text { Rd }=1.5\end{array}$} & \multirow{2}{*}{$\begin{array}{c}R_{d} \\
\left(\mu_{d}=1.5\right)\end{array}$} & \multicolumn{4}{|c|}{ Internal Force Magnification Ratio } \\
\hline & & & & M.F & V.F & $M . U$ & $V . U$ \\
\hline SL1 & 169.93 & 3.37 & 1.04 & 0.94 & 1.01 & 1.18 & 1.39 \\
\hline SH1 & 378.09 & 4.93 & 1.08 & 1.03 & 1.14 & 1.28 & 1.56 \\
\hline ML1 & 566.19 & 4.62 & 1.09 & 1.10 & 1.23 & 1.37 & 1.69 \\
\hline MH1 & 1033.92 & 4.29 & 1.08 & 1.05 & 1.16 & 1.31 & 1.60 \\
\hline LL1 & 1894.80 & 4.50 & 1.07 & 1.00 & 1.10 & 1.26 & 1.52 \\
\hline LH1 & 3488.16 & 5.03 & 1.07 & 0.93 & 0.99 & 1.16 & 1.36 \\
\hline
\end{tabular}

Table E.16 - Proper $R_{d}$ for steel deck panels acting as the main source of energy dissipation (weak diaphragm) - long side -ductility of 2.0 - M6C1

\begin{tabular}{cccccccc}
\hline & \multirow{2}{*}{$\begin{array}{c}\text { Deck } \\
\text { Buildings }\end{array}$} & $\begin{array}{c}\mu_{d} \text { on Deck } \\
\text { Elastic } \\
\text { Fonels for }\end{array}$ & $\begin{array}{c}R_{d} \\
\left(\mu_{d}=2.0\right)\end{array}$ & \multicolumn{3}{c}{ Internal Force Magnification Ratios } \\
\cline { 5 - 8 } & Rd $=1.5$ & & & M.F & $V . F$ & $M . U$ & V.U \\
\hline SL1 & 209.88 & 3.99 & 1.15 & 0.92 & 0.97 & 1.14 & 1.34 \\
SH1 & 429.19 & 2.92 & 1.26 & 1.01 & 1.12 & 1.26 & 1.54 \\
ML1 & 629.11 & 2.70 & 1.18 & 0.98 & 1.08 & 1.22 & 1.49 \\
MH1 & 1300.08 & 3.79 & 1.16 & 0.97 & 1.07 & 1.22 & 1.48 \\
LL1 & 2412.95 & 3.73 & 1.13 & 0.96 & 1.04 & 1.20 & 1.42 \\
LH1 & 6344.10 & 3.03 & 1.22 & 0.91 & 0.97 & 1.14 & 1.34 \\
\hline
\end{tabular}


Table E.17 - Proper $R_{d}$ for steel deck panels acting as the main source of energy dissipation (weak diaphragm) - long side -ductility of 2.0 - M6C2

\begin{tabular}{cccccccc}
\hline \multirow{2}{*}{ Buildings } & $\begin{array}{c}\text { Deck } \\
\text { Elastic } \\
\text { Force }\end{array}$ & $\begin{array}{c}\mu_{d} \text { on Deck } \\
\text { Panels for } \\
R d=1.5\end{array}$ & $\begin{array}{c}R_{d} \\
\left(\mu_{d}=2.0\right)\end{array}$ & \multicolumn{2}{c}{ Internal Force Magnification Ratios } \\
\cline { 5 - 8 } & 174.31 & 3.26 & 1.14 & 0.95 & 1.03 & 1.19 & 1.41 \\
\hline SL1 & $17 . F$ & 1.17 & 0.92 & 0.99 & 1.15 & 1.36 \\
SH1 & 475.44 & 3.13 & 1.22 & 0.94 & 1.01 & 1.17 & 1.39 \\
ML1 & 605.11 & 4.22 & 1.13 & 1.00 & 1.10 & 1.25 & 1.51 \\
MH1 & 918.03 & 4.84 & 1.14 & 0.91 & 0.98 & 1.14 & 1.34 \\
LL1 & 1702.84 & 5.56 & 1.11 & 0.90 & 0.94 & 1.12 & 1.29 \\
LH1 & 3869.11 & 4.51 & & & & &
\end{tabular}

Table E.18 - Proper $R_{d}$ for steel deck panels acting as the main source of energy dissipation (weak diaphragm) - long side -ductility of 2.0 - M6C26

\begin{tabular}{cccccccc}
\hline \multirow{2}{*}{ Buildings } & $\begin{array}{c}\text { Deck } \\
\text { Elastic } \\
\text { Force }\end{array}$ & $\begin{array}{c}\mu_{d} \text { on Deck } \\
\text { Panels for } \\
R d=1.5\end{array}$ & $\begin{array}{c}R_{d} \\
\left(\mu_{d}=2.0\right)\end{array}$ & \multicolumn{2}{c}{ Internal Force Magnification Ratios } \\
\cline { 5 - 8 } & 196.45 & 3.58 & 1.10 & 0.90 & 0.95 & 1.12 & 1.31 \\
\hline SL1 & 19.5 & 1.15 & 0.89 & 0.93 & 1.11 & 1.29 \\
SH1 & 486.54 & 3.47 & 1.16 & 1.01 & 1.11 & 1.27 & 1.53 \\
ML1 & 575.91 & 4.71 & 1.16 & 1.01 & 0.94 & 1.39 & 1.17 \\
MH1 & 1250.38 & 4.75 & 1.16 & 0.97 & 1.05 & 1.21 & 1.44 \\
LL1 & 2372.76 & 4.92 & 1.11 & 0.91 & 0.96 & 1.13 & 1.32 \\
LH1 & 6194.09 & 4.04 & & & & & \\
\hline
\end{tabular}

Table E.19 - Proper $R_{d}$ for steel deck panels acting as the main source of energy dissipation (weak diaphragm) - long side -ductility of 2.0 - M6C31

\begin{tabular}{|c|c|c|c|c|c|c|c|}
\hline \multirow{2}{*}{ Buildings } & \multirow{2}{*}{$\begin{array}{c}\text { Deck } \\
\text { Elastic } \\
\text { Force }\end{array}$} & \multirow{2}{*}{$\begin{array}{c}\mu_{d} \text { on Deck } \\
\text { Panels for } \\
R d=1.5\end{array}$} & \multirow{2}{*}{$\begin{array}{c}R_{d} \\
\left(\mu_{d}=2.0\right)\end{array}$} & \multicolumn{4}{|c|}{ Internal Force Magnification Ratios } \\
\hline & & & & $M . F$ & $V . F$ & $M . U$ & $V . U$ \\
\hline SL1 & 181.35 & 5.30 & 1.14 & 0.90 & 0.95 & 1.12 & 1.31 \\
\hline SH1 & 447.69 & 4.86 & 1.16 & 1.04 & 1.16 & 1.30 & 1.59 \\
\hline ML1 & 959.85 & 3.33 & 1.18 & 1.04 & 1.16 & 1.30 & 1.59 \\
\hline MH1 & 1026.04 & 3.05 & 1.17 & 0.91 & 0.98 & 1.14 & 1.35 \\
\hline LL1 & 1734.43 & 3.91 & 1.14 & 0.96 & 1.04 & 1.20 & 1.43 \\
\hline LH1 & 3838.30 & 7.98 & 1.12 & 0.90 & 0.97 & 1.13 & 1.33 \\
\hline
\end{tabular}


Table E.20 - Proper $R_{d}$ for steel deck panels acting as the main source of energy dissipation (weak diaphragm) - long side -ductility of 2.0 - M6C38

\begin{tabular}{|c|c|c|c|c|c|c|c|}
\hline \multirow{2}{*}{ Buildings } & \multirow{2}{*}{$\begin{array}{c}\text { Deck } \\
\text { Elastic } \\
\text { Force }\end{array}$} & \multirow{2}{*}{$\begin{array}{c}\mu_{d} \text { on Deck } \\
\text { Panels for } \\
R d=1.5\end{array}$} & \multirow{2}{*}{$\begin{array}{c}R_{d} \\
\left(\mu_{d}=2.0\right)\end{array}$} & \multicolumn{4}{|c|}{ Internal Force Magnification Ratios } \\
\hline & & & & M.F & $V . F$ & M.U & $V . U$ \\
\hline SL1 & 169.93 & 3.37 & 1.20 & 0.98 & 1.08 & 1.22 & 1.48 \\
\hline SH1 & 378.09 & 4.93 & 1.15 & 1.02 & 1.14 & 1.28 & 1.56 \\
\hline ML1 & 566.19 & 4.62 & 1.16 & 1.10 & 1.24 & 1.37 & 1.71 \\
\hline MH1 & 1033.92 & 4.29 & 1.17 & 1.06 & 1.20 & 1.33 & 1.64 \\
\hline LL1 & 1894.80 & 4.50 & 1.14 & 1.03 & 1.15 & 1.29 & 1.58 \\
\hline LH1 & 3488.16 & 5.03 & 1.11 & 0.94 & 1.01 & 1.17 & 1.39 \\
\hline
\end{tabular}


Table E.21 - Proper $R_{d}$ for steel deck panels acting as the main source of energy dissipation (weak diaphragm) - short side -ductility of 1.5 - E6C1

\begin{tabular}{cccccccc}
\hline & \multirow{2}{*}{$\begin{array}{c}\text { Deck } \\
\text { Buildings }\end{array}$} & $\begin{array}{c}\mu_{d} \text { on Deck } \\
\text { Elastic } \\
\text { Fonels for }\end{array}$ & $\begin{array}{c}R_{d} \\
\text { Rd }=1.5\end{array}$ & $\left.\mu_{d}=1.5\right)$ & \multicolumn{3}{c}{ Internal Force Magnification Ratios } \\
\cline { 5 - 8 } & & & & M.F & V.F & M.U & V.U \\
\hline SL1 & 93.13 & 3.28 & 1.15 & 0.85 & 0.92 & 1.07 & 1.26 \\
SH1 & 152.09 & 2.92 & 1.24 & 1.15 & 1.37 & 1.44 & 1.88 \\
SH4 & 131.34 & 2.79 & 1.13 & 1.08 & 1.28 & 1.35 & 1.75 \\
ML1 & 234.67 & 2.51 & 1.15 & 1.05 & 1.15 & 1.31 & 1.58 \\
ML2 & 215.51 & 2.46 & 1.17 & 0.94 & 1.07 & 1.17 & 1.47 \\
MH1 & 424.92 & 2.34 & 1.19 & 0.89 & 1.00 & 1.11 & 1.37 \\
MH6 & 511.30 & 3.45 & 1.11 & 0.97 & 1.01 & 1.21 & 1.39 \\
LL1 & 681.11 & 3.65 & 1.10 & 0.88 & 1.02 & 1.10 & 1.41 \\
LH1 & 1117.58 & 2.36 & 1.12 & 0.86 & 1.13 & 1.08 & 1.56 \\
LH5 & 1011.30 & 2.24 & 1.12 & 0.90 & 1.17 & 1.13 & 1.62 \\
\hline
\end{tabular}

Table E.22 - Proper $R_{d}$ for steel deck panels acting as the main source of energy dissipation (weak diaphragm) - short side -ductility of 1.5 - E6C13

\begin{tabular}{|c|c|c|c|c|c|c|c|}
\hline \multirow{2}{*}{ Buildings } & \multirow{2}{*}{$\begin{array}{c}\text { Deck } \\
\text { Elastic } \\
\text { Force }\end{array}$} & \multirow{2}{*}{$\begin{array}{c}\mu_{d} \text { on Deck } \\
\text { Panels for } \\
R d=1.5\end{array}$} & \multirow{2}{*}{$\begin{array}{c}R_{d} \\
\left(\mu_{d}=1.5\right)\end{array}$} & \multicolumn{4}{|c|}{ Internal Force Magnification Ratios } \\
\hline & & & & M.F & $V . F$ & $M . U$ & $V . U$ \\
\hline SL1 & 97.14 & 2.34 & 1.26 & 0.96 & 1.09 & 1.20 & 1.50 \\
\hline SH1 & 89.34 & 2.07 & 1.31 & 0.88 & 1.11 & 1.10 & 1.52 \\
\hline SH4 & 90.78 & 2.45 & 1.18 & 0.82 & 0.98 & 1.02 & 1.35 \\
\hline ML1 & 167.36 & 2.52 & 1.18 & 0.81 & 0.89 & 1.01 & 1.23 \\
\hline ML2 & 142.48 & 2.27 & $1.21(2)$ & 0.86 & 1.00 & 1.07 & 1.37 \\
\hline MH1 & 310.37 & 3.23 & 1.16 & 0.88 & 1.05 & 1.10 & 1.45 \\
\hline MH6 & 371.37 & 3.27 & 1.12 & 0.84 & 0.93 & 1.04 & 1.28 \\
\hline LL1 & 610.81 & 3.44 & 1.10 & 0.91 & 1.09 & 1.13 & 1.50 \\
\hline LH1 & 1301.02 & 2.52 & 1.11 & 0.98 & 1.09 & 1.23 & 1.50 \\
\hline LH5 & 1134.76 & 3.06 & 1.14 & 1.10 & 1.27 & 1.38 & 1.75 \\
\hline
\end{tabular}


Table E.23 - Proper $R_{d}$ for steel deck panels acting as the main source of energy dissipation (weak diaphragm) - short side -ductility of 1.5 - E6C15

\begin{tabular}{cccccccc}
\hline & \multirow{2}{*}{$\begin{array}{c}\text { Deck } \\
\text { Buildings }\end{array}$} & $\begin{array}{c}\mu_{d} \text { on Deck } \\
\text { Elastic } \\
\text { Fonels for }\end{array}$ & $\begin{array}{c}R_{d} \\
\text { Porce }\end{array}$ & \begin{tabular}{c}
$\left.R_{d}=1.5\right)$ \\
\cline { 5 - 8 }
\end{tabular} & & & \multicolumn{3}{c}{ Internal Force Magnification Ratios } \\
\cline { 5 - 8 } SL1 & 71.70 & 2.59 & 1.17 & 1.07 & 1.28 & 1.34 & 1.76 \\
SH1 & 170.66 & 2.02 & 1.28 & 1.12 & 1.31 & 1.41 & 1.80 \\
SH4 & 107.63 & 1.83 & 1.37 & 1.23 & 1.35 & 1.54 & 1.85 \\
ML1 & 147.96 & 1.44 & 1.54 & 1.26 & 1.36 & 1.58 & 1.87 \\
ML2 & 122.92 & 2.41 & 1.17 & 1.21 & 1.37 & 1.52 & 1.88 \\
MH1 & 281.97 & 2.08 & 1.25 & 1.21 & 1.32 & 1.51 & 1.81 \\
MH6 & 323.47 & 1.75 & 1.24 & 1.15 & 1.34 & 1.44 & 1.84 \\
LL1 & 643.48 & 2.60 & 1.22 & 0.91 & 1.06 & 1.13 & 1.47 \\
LH1 & 850.54 & $1.63(7$ th $)$ & 1.14 & 0.97 & 1.21 & 1.21 & 1.66 \\
LH5 & 715.13 & $1.98(7$ th $)$ & $1.25(7)$ & 1.25 & 1.52 & 1.57 & 2.09 \\
\hline
\end{tabular}

Table E.24 - Proper $R_{d}$ for steel deck panels acting as the main source of energy dissipation (weak diaphragm) - short side -ductility of 1.5 - E6C18

\begin{tabular}{|c|c|c|c|c|c|c|c|}
\hline \multirow{2}{*}{ Buildings } & \multirow{2}{*}{$\begin{array}{c}\text { Deck } \\
\text { Elastic } \\
\text { Force }\end{array}$} & \multirow{2}{*}{$\begin{array}{c}\mu_{d} \text { on Deck } \\
\text { Panels for } \\
R d=1.5\end{array}$} & \multirow{2}{*}{$\begin{array}{c}R_{d} \\
\left(\mu_{d}=1.5\right)\end{array}$} & \multicolumn{4}{|c|}{ Internal Force Magnification Ratios } \\
\hline & & & & $M . F$ & V.F & $M . U$ & $V . U$ \\
\hline SL1 & 91.45 & 2.10 & 1.25 & 1.01 & 1.18 & 1.27 & 1.62 \\
\hline SH1 & 122.16 & 2.71 & 1.13 & 0.95 & 1.00 & 1.19 & 1.37 \\
\hline SH4 & 122.28 & 2.10 & 1.18 & 0.90 & 1.00 & 1.13 & 1.37 \\
\hline ML1 & 202.91 & 2.33 & 1.18 & 0.84 & 0.95 & 1.04 & 1.30 \\
\hline ML2 & 158.62 & 1.72 & 1.28 & 0.94 & 1.18 & 1.18 & 1.63 \\
\hline MH1 & 279.50 & 1.92 & 1.21 & 0.98 & 1.21 & 1.22 & 1.67 \\
\hline MH6 & 426.80 & 3.10 & 1.12 & 0.77 & 0.91 & 0.97 & 1.26 \\
\hline LL1 & 504.33 & 1.97 & $1.24(61)$ & 1.10 & 1.33 & 1.38 & 1.82 \\
\hline LH1 & 717.14 & 2.16 & $1.1(6)$ & 1.10 & 1.38 & 1.38 & 1.90 \\
\hline LH5 & $764.84(6)$ & 2.13 & $1.2(6)$ & 1.13 & 1.46 & 1.41 & 2.01 \\
\hline
\end{tabular}


Table E.25 - Proper $R_{d}$ for steel deck panels acting as the main source of energy dissipation (weak diaphragm) - short side - ductility of 1.5 - E6C42

\begin{tabular}{|c|c|c|c|c|c|c|c|}
\hline \multirow{2}{*}{ Buildings } & \multirow{2}{*}{$\begin{array}{c}\text { Deck } \\
\text { Elastic } \\
\text { Force }\end{array}$} & \multirow{2}{*}{$\begin{array}{c}\mu_{d} \text { on Deck } \\
\text { Panels for } \\
\text { Rd }=1.5\end{array}$} & \multirow{2}{*}{$\begin{array}{c}R_{d} \\
\left(\mu_{d}=1.5\right)\end{array}$} & \multicolumn{4}{|c|}{ Internal Force Magnification Ratios } \\
\hline & & & & $M . F$ & V.F & $M . U$ & V.U \\
\hline SL1 & 74.37 & 2.21 & 1.18 & 1.08 & 1.20 & 1.36 & 1.64 \\
\hline SH1 & 116.11 & 2.54 & 1.20 & 1.02 & 1.08 & 1.28 & 1.49 \\
\hline SH4 & 108.47 & 2.72 & 1.24 & 1.00 & 1.04 & 1.25 & 1.43 \\
\hline ML1 & 190.69 & 2.57 & 1.26 & 0.94 & 1.10 & 1.18 & 1.51 \\
\hline ML2 & 161.99 & 2.28 & 1.22 & 0.94 & 1.21 & 1.18 & 1.67 \\
\hline MH1 & 328.63 & 2.23 & 1.19 & 0.97 & 1.22 & 1.22 & 1.67 \\
\hline MH6 & 401.86 & 3.16 & 1.18 & 1.05 & 1.26 & 1.31 & 1.73 \\
\hline LL1 & 728.28 & 2.75 & 1.14 & 0.84 & 1.10 & 1.05 & 1.51 \\
\hline LH1 & 1300.99 & 2.68 & 1.13 & 0.76 & 0.89 & 0.96 & 1.23 \\
\hline LH5 & 1241.73 & 2.58 & 1.12 & 0.79 & 0.94 & 1.00 & 1.30 \\
\hline
\end{tabular}

Table E.26 - Proper $R_{d}$ for steel deck panels acting as the main source of energy dissipation (weak diaphragm) - short side - ductility of 2.0 - E6C1

\begin{tabular}{|c|c|c|c|c|c|c|c|}
\hline \multirow{2}{*}{ Buildings } & \multirow{2}{*}{$\begin{array}{c}\text { Deck } \\
\text { Elastic } \\
\text { Force }\end{array}$} & \multirow{2}{*}{$\begin{array}{c}\mu_{d} \text { on Deck } \\
\text { Panels for } \\
R d=1.5\end{array}$} & \multirow{2}{*}{$\begin{array}{c}R_{d} \\
\left(\mu_{d}=2.0\right)\end{array}$} & \multicolumn{4}{|c|}{ Internal Force Magnification Ratios } \\
\hline & & & & M.F & $V . F$ & $M . U$ & $V . U$ \\
\hline SL1 & 93.13 & 3.28 & 1.26 & 0.84 & 0.85 & 1.06 & 1.23 \\
\hline SH1 & 152.09 & 2.92 & 1.34 & 1.17 & 1.35 & 1.46 & 1.85 \\
\hline SH4 & 131.34 & 2.79 & 1.23 & 1.07 & 1.23 & 1.34 & 1.69 \\
\hline ML1 & 234.67 & 2.51 & 1.35 & 1.08 & 1.23 & 1.35 & 1.69 \\
\hline ML2 & 215.51 & 2.46 & 1.32 & 0.94 & 1.09 & 1.17 & 1.49 \\
\hline MH1 & 424.92 & 2.34 & 1.35 & 1.02 & 1.16 & 1.28 & 1.59 \\
\hline MH6 & 511.30 & 3.45 & 1.19 & 1.00 & 1.07 & 1.25 & 1.47 \\
\hline LL1 & 681.11 & 3.65 & 1.19 & 0.85 & 1.03 & 1.06 & 1.41 \\
\hline LH1 & 1117.58 & 2.36 & 1.25 & 0.91 & 1.20 & 1.14 & 1.65 \\
\hline LH5 & 1011.30 & 2.24 & 1.25 & 0.97 & 1.26 & 1.21 & 1.73 \\
\hline
\end{tabular}


Table E.27 - Proper $R_{d}$ for steel deck panels acting as the main source of energy dissipation (weak diaphragm) - short side -ductility of 2.0 - E6C13

\begin{tabular}{|c|c|c|c|c|c|c|c|}
\hline \multirow{2}{*}{ Buildings } & \multirow{2}{*}{$\begin{array}{c}\text { Deck } \\
\text { Elastic } \\
\text { Force }\end{array}$} & \multirow{2}{*}{$\begin{array}{c}\mu_{d} \text { on Deck } \\
\text { Panels for } \\
R d=1.5\end{array}$} & \multirow{2}{*}{$\begin{array}{c}R_{d} \\
\left(\mu_{d}=2.0\right)\end{array}$} & \multicolumn{4}{|c|}{ Internal Force Magnification Ratios } \\
\hline & & & & $M . F$ & $V . F$ & $M . U$ & $V . U$ \\
\hline SL1 & 97.14 & 2.34 & 1.41 & 0.97 & 1.09 & 1.21 & 1.50 \\
\hline SH1 & 89.34 & 2.07 & 1.48 & 0.87 & 1.07 & 1.09 & 1.47 \\
\hline SH4 & 90.78 & 2.45 & 1.35 & 0.78 & 0.89 & 0.97 & 1.22 \\
\hline ML1 & 167.36 & 2.52 & 1.35 & 0.80 & 0.85 & 1.00 & 1.17 \\
\hline ML2 & 142.48 & 2.27 & $1.39(2)$ & 0.87 & 1.08 & 1.09 & 1.49 \\
\hline MH1 & 310.37 & 3.23 & 1.26 & 0.90 & 1.04 & 1.12 & 1.43 \\
\hline MH6 & 371.37 & 3.27 & 1.22 & 0.81 & 0.88 & 1.01 & 1.21 \\
\hline LL1 & 610.81 & 3.44 & 1.17 & 0.94 & 1.13 & 1.17 & 1.55 \\
\hline LH1 & 1301.02 & 2.52 & 1.35 & 0.98 & 1.09 & 1.22 & 1.50 \\
\hline LH5 & 1134.76 & 3.06 & 1.23 & 1.06 & 1.23 & 1.33 & 1.70 \\
\hline
\end{tabular}

Table E.28 - Proper $R_{d}$ for steel deck panels acting as the main source of energy dissipation (weak diaphragm) - short side -ductility of 2.0 - E6C15

\begin{tabular}{|c|c|c|c|c|c|c|c|}
\hline \multirow{2}{*}{ Buildings } & \multirow{2}{*}{$\begin{array}{l}\text { Deck } \\
\text { Elastic } \\
\text { Force }\end{array}$} & \multirow{2}{*}{$\begin{array}{c}\mu_{d} \text { on Deck } \\
\text { Panels for } \\
R d=1.5\end{array}$} & \multirow{2}{*}{$\begin{array}{c}R_{d} \\
\left(\mu_{d}=2.0\right)\end{array}$} & \multicolumn{4}{|c|}{ Internal Force Magnification Ratios } \\
\hline & & & & $M . F$ & V.F & $M . U$ & $V . U$ \\
\hline SL1 & 71.70 & 2.59 & 1.38 & 1.15 & 1.30 & 1.44 & 1.79 \\
\hline SH1 & 170.66 & 2.02 & 1.48 & 1.09 & 1.30 & 1.37 & 1.78 \\
\hline SH4 & 107.63 & 1.83 & 1.59 & 1.22 & 1.33 & 1.53 & 1.83 \\
\hline ML1 & 147.96 & 1.44 & 1.70 & 1.23 & 1.29 & 1.54 & 1.77 \\
\hline ML2 & 122.92 & 2.41 & 1.36 & 1.18 & 1.24 & 1.47 & 1.70 \\
\hline MH1 & 281.97 & 2.08 & 1.42 & 1.23 & 1.35 & 1.53 & 1.85 \\
\hline MH6 & 323.47 & 1.75 & 1.56 & 1.23 & 1.30 & 1.54 & 1.79 \\
\hline LL1 & 643.48 & 2.60 & 1.36 & 1.02 & 1.19 & 1.28 & 1.63 \\
\hline LH1 & 850.54 & $1.63(7 \mathrm{th})$ & $1.75(7 \mathrm{th})$ & 1.28 & 1.40 & 1.60 & 1.93 \\
\hline LH5 & 715.13 & $1.98(7 \mathrm{th})$ & $1.5(7 \mathrm{th})$ & 1.28 & 1.44 & 1.60 & 1.98 \\
\hline
\end{tabular}


Table E.29 - Proper $R_{d}$ for steel deck panels acting as the main source of energy dissipation (weak diaphragm) - short side -ductility of 2.0 - E6C18

\begin{tabular}{|c|c|c|c|c|c|c|c|}
\hline \multirow{2}{*}{ Buildings } & \multirow{2}{*}{$\begin{array}{c}\text { Deck } \\
\text { Elastic } \\
\text { Force }\end{array}$} & \multirow{2}{*}{$\begin{array}{c}\mu_{d} \text { on Deck } \\
\text { Panels for } \\
R d=1.5\end{array}$} & \multirow{2}{*}{$\begin{array}{c}R_{d} \\
\left(\mu_{d}=2.0\right)\end{array}$} & \multicolumn{4}{|c|}{ Internal Force Magnification Ratios } \\
\hline & & & & $M . F$ & V.F & $M . U$ & $V . U$ \\
\hline SL1 & 91.45 & 2.10 & 1.45 & 1.08 & 1.25 & 1.34 & 1.72 \\
\hline SH1 & 122.16 & 2.71 & 1.25 & 0.99 & 1.05 & 1.23 & 1.45 \\
\hline SH4 & 122.28 & 2.10 & 1.43 & 1.08 & 1.19 & 1.35 & 1.64 \\
\hline ML1 & 202.91 & 2.33 & 1.37 & 0.92 & 1.04 & 1.15 & 1.43 \\
\hline ML2 & 158.62 & 1.72 & 1.68 & 0.96 & 1.15 & 1.20 & 1.59 \\
\hline MH1 & 279.50 & 1.92 & 1.53 & 0.97 & 1.16 & 1.21 & 1.60 \\
\hline MH6 & 426.80 & 3.10 & 1.22 & 0.80 & 0.95 & 1.00 & 1.30 \\
\hline LL1 & 504.33 & 1.97 & 1.51 & 1.09 & 1.19 & 1.36 & 1.64 \\
\hline LH1 & 717.14 & 2.16 & $1.38(6 \mathrm{th})$ & 1.11 & 1.25 & 1.38 & 1.72 \\
\hline LH5 & $764.84(6)$ & 2.13 & $1.39(6)$ & 1.20 & 1.42 & 1.51 & 1.95 \\
\hline
\end{tabular}

Table E.30 - Proper $R_{d}$ for steel deck panels acting as the main source of energy dissipation (weak diaphragm) - short side -ductility of 2.0 - E6C42

\begin{tabular}{|c|c|c|c|c|c|c|c|}
\hline \multirow{2}{*}{ Buildings } & \multirow{2}{*}{$\begin{array}{c}\text { Deck } \\
\text { Elastic } \\
\text { Force }\end{array}$} & \multirow{2}{*}{$\begin{array}{c}\mu_{d} \text { on Deck } \\
\text { Panels for } \\
R d=1.5\end{array}$} & \multirow{2}{*}{$\begin{array}{c}R_{d} \\
\left(\mu_{d}=2.0\right)\end{array}$} & \multicolumn{4}{|c|}{ Internal Force Magnification Ratios } \\
\hline & & & & $M . F$ & $V . F$ & $M . U$ & $V . U$ \\
\hline SL1 & 74.37 & 2.21 & 1.41 & 1.15 & 1.25 & 1.44 & 1.71 \\
\hline SH1 & 116.11 & 2.54 & 1.38 & 1.09 & 1.20 & 1.36 & 1.65 \\
\hline SH4 & 108.47 & 2.72 & 1.33 & 1.01 & 1.06 & 1.26 & 1.45 \\
\hline ML1 & 190.69 & 2.57 & 1.37 & 0.96 & 1.13 & 1.20 & 1.56 \\
\hline ML2 & 161.99 & 2.28 & 1.40 & 0.86 & 1.11 & 1.08 & 1.53 \\
\hline MH1 & 328.63 & 2.23 & 1.40 & 0.93 & 1.18 & 1.17 & 1.62 \\
\hline MH6 & 401.86 & 3.16 & 1.28 & 1.03 & 1.25 & 1.29 & 1.72 \\
\hline LL1 & 728.28 & 2.75 & 1.24 & 0.86 & 1.13 & 1.08 & 1.56 \\
\hline LH1 & 1300.99 & 2.68 & 1.23 & 0.73 & 0.88 & 0.91 & 1.20 \\
\hline LH5 & 1241.73 & 2.58 & 1.21 & 0.76 & 0.90 & 0.95 & 1.24 \\
\hline
\end{tabular}


Table E.31 - Proper $R_{d}$ for steel deck panels acting as the main source of energy dissipation (weak diaphragm) - long side -ductility of 1.5 - E6C1

\begin{tabular}{|c|c|c|c|c|c|c|c|}
\hline \multirow{2}{*}{ Buildings } & \multirow{2}{*}{$\begin{array}{l}\text { Deck } \\
\text { Elastic } \\
\text { Force }\end{array}$} & \multirow{2}{*}{$\begin{array}{c}\mu_{d} \text { on Deck } \\
\text { Panels for } \\
\text { Rd }=1.5\end{array}$} & \multirow{2}{*}{$\begin{array}{c}R_{d} \\
\left(\mu_{d}=1.5\right)\end{array}$} & \multicolumn{4}{|c|}{ Internal Force Magnification Ratios } \\
\hline & & & & $M . F$ & $V . F$ & $M . U$ & $V . U$ \\
\hline SL1 & 91.65 & 2.21 & 1.08 & 0.96 & 1.05 & 1.21 & 1.44 \\
\hline SH1 & 192.89 & 4.93 & 1.09 & 0.83 & 0.87 & 1.03 & 1.19 \\
\hline ML1 & 373.35 & 3.82 & 1.10 & 0.84 & 0.92 & 1.05 & 1.27 \\
\hline MH1 & 770.85 & 3.34 & 1.10 & 0.86 & 0.94 & 1.07 & 1.24 \\
\hline LL1 & 1401.03 & 4.39 & 1.08 & 0.89 & 0.97 & 1.11 & 1.34 \\
\hline LH1 & 3007.83 & 4.58 & 1.09 & 0.94 & 1.02 & 1.18 & 1.40 \\
\hline
\end{tabular}

Table E.32 - Proper $R_{d}$ for steel deck panels acting as the main source of energy dissipation (weak diaphragm) - long side -ductility of 1.5 - E6C13

\begin{tabular}{cccccccc}
\hline \multirow{2}{*}{ Buildings } & $\begin{array}{c}\text { Deck } \\
\text { Elastic } \\
\text { Force }\end{array}$ & $\begin{array}{c}\mu_{d} \text { on Deck } \\
\text { Panels for } \\
\text { Rd }=1.5\end{array}$ & $\begin{array}{c}R_{d} \\
\left(\mu_{d}=1.5\right)\end{array}$ & \multicolumn{3}{c}{ Internal Force Magnification Ratios } \\
\cline { 5 - 8 } & 134.95 & 4.46 & 1.08 & 0.91 & 1.01 & 1.14 & 1.40 \\
\hline SL1 & 13.5 & 1.08 & 0.82 & 0.87 & 1.03 & 1.20 \\
SH1 & 266.89 & 3.09 & 1.14 & 1.00 & 1.13 & 1.25 & 1.56 \\
ML1 & 319.53 & 3.22 & 1.13 & 1.18 & 1.33 & 1.47 & 1.83 \\
MH1 & 349.18 & 3.09 & 1.08 & 1.20 & 1.37 & 1.50 & 1.89 \\
LL1 & 598.43 & 4.09 & 1.06 & 1.09 & 1.24 & 1.36 & 1.70 \\
LH1 & 1259.12 & 5.28 & 1.06 & \\
\hline
\end{tabular}

Table E.33 - Proper $R_{d}$ for steel deck panels acting as the main source of energy dissipation (weak diaphragm) - long side -ductility of 1.5 - E6C15

\begin{tabular}{cccccccc}
\hline \multirow{2}{*}{ Buildings } & $\begin{array}{c}\text { Deck } \\
\text { Elastic } \\
\text { Force }\end{array}$ & $\begin{array}{c}\mu_{d} \text { on Deck } \\
\text { Panels for } \\
R d=1.5\end{array}$ & $\begin{array}{c}R_{d} \\
\left(\mu_{d}=1.5\right)\end{array}$ & \multicolumn{3}{c}{ Internal Force Magnification Ratios } \\
\cline { 5 - 8 } & 138.15 & 2.56 & 1.08 & 1.02 & 1.12 & 1.27 & 1.54 \\
\hline SL1 & 172.26 & 2.97 & 1.15 & 1.10 & 1.24 & 1.37 & 1.70 \\
SH1 & 17.5 & 1.15 & 1.02 & 1.18 & 1.28 & 1.62 \\
ML1 & 299.45 & 2.79 & 1.08 & 0.96 & 1.05 & 1.19 & 1.44 \\
MH1 & 725.54 & 4.75 & 1.06 & 0.85 & 0.89 & 1.06 & 1.22 \\
LL1 & 1414.74 & 4.76 & 1.06 & 0.92 & 0.97 & 1.15 & 1.34 \\
LH1 & 3412.38 & 4.52 & & & & & \\
\hline
\end{tabular}


Table E.34 - Proper $R_{d}$ for steel deck panels acting as the main source of energy dissipation (weak diaphragm) - long side -ductility of 1.5 - E6C18

\begin{tabular}{cccccccc}
\hline \multirow{2}{*}{ Buildings } & \multirow{2}{*}{$\begin{array}{c}\text { Deck } \\
\text { Elastic } \\
\text { Force }\end{array}$} & $\begin{array}{c}\mu_{d} \text { on Deck } \\
\text { Panels for } \\
\text { Rd }=1.5\end{array}$ & $\begin{array}{c}R_{d} \\
\left(\mu_{d}=1.5\right)\end{array}$ & \multicolumn{3}{c}{ Internal Force Magnification Ratios } \\
\cline { 5 - 8 } & 181.49 & 4.59 & 1.08 & 0.86 & 0.89 & 1.07 & 1.23 \\
\hline SL1 & 18.5 & 1.08 & 0.94 & 1.00 & 1.17 & 1.37 \\
SH1 & 356.76 & 3.58 & 1.09 & 1.06 & 1.19 & 1.32 & 1.64 \\
ML1 & 276.94 & 2.23 & 1.11 & 1.02 & 1.15 & 1.28 & 1.58 \\
MH1 & 550.09 & 2.49 & 1.08 & 1.00 & 1.10 & 1.25 & 1.52 \\
LL1 & 1010.10 & 3.17 & 1.07 & 0.92 & 0.98 & 1.15 & 1.35 \\
LH1 & 2108.12 & 5.23 & & & & & \\
\hline
\end{tabular}

Table E.35 - Proper $R_{d}$ for steel deck panels acting as the main source of energy dissipation (weak diaphragm) - long side -ductility of 1.5 - E6C42

\begin{tabular}{cccccccc}
\hline \multirow{2}{*}{ Buildings } & $\begin{array}{c}\text { Deck } \\
\text { Elastic } \\
\text { Force }\end{array}$ & $\begin{array}{c}\mu_{d} \text { on Deck } \\
\text { Panels for } \\
R d=1.5\end{array}$ & $\begin{array}{c}R_{d} \\
\left(\mu_{d}=1.5\right)\end{array}$ & \multicolumn{3}{c}{ Internal Force Magnification Ratios } \\
\cline { 5 - 8 } & 175.73 & 2.91 & 1.08 & 0.98 & 1.06 & 1.22 & 1.46 \\
\hline SL1 & 224.90 & 3.40 & 1.09 & 0.94 & 1.01 & 1.17 & 1.39 \\
SH1 & 278.96 & 2.32 & 1.11 & 1.08 & 1.25 & 1.36 & 1.71 \\
ML1 & 27.9 & 1.10 & 0.83 & 0.87 & 1.03 & 1.20 \\
MH1 & 577.93 & 3.79 & 1.08 & 0.88 & 0.95 & 1.09 & 1.30 \\
LL1 & 1041.11 & 4.68 & 1.06 & 0.95 & 1.02 & 1.19 & 1.41 \\
LH1 & 2130.46 & 5.57 & & & & & \\
\hline
\end{tabular}

Table E.36 - Proper $R_{d}$ for steel deck panels acting as the main source of energy dissipation (weak diaphragm) - long side -ductility of 2.0 - E6C1

\begin{tabular}{cccccccc}
\hline \multirow{2}{*}{ Buildings } & $\begin{array}{c}\text { Deck } \\
\text { Elastic } \\
\text { Force }\end{array}$ & $\begin{array}{c}\mu_{d} \text { on Deck } \\
\text { Panels for } \\
R d=1.5\end{array}$ & $\begin{array}{c}R_{d} \\
\left(\mu_{d}=2.0\right)\end{array}$ & \multicolumn{3}{c}{ Internal Force Magnification Ratios } \\
\cline { 5 - 8 } & & & & $M . F$ & $V . F$ & $M . U$ & $V . U$ \\
\hline SL1 & 91.65 & 2.21 & 1.17 & 1.04 & 1.16 & 1.30 & 1.59 \\
SH1 & 192.89 & 4.93 & 1.16 & 0.86 & 0.90 & 1.07 & 1.24 \\
ML1 & 373.35 & 3.82 & 1.18 & 0.81 & 0.88 & 1.02 & 1.21 \\
MH1 & 770.85 & 3.34 & 1.19 & 0.89 & 0.97 & 1.11 & 1.33 \\
LL1 & 1401.03 & 4.39 & 1.14 & 0.88 & 0.97 & 1.10 & 1.34 \\
LH1 & 3007.83 & 4.58 & 1.17 & 0.90 & 0.91 & 1.12 & 1.33 \\
\hline
\end{tabular}


Table E.37 - Proper $R_{d}$ for steel deck panels acting as the main source of energy dissipation (weak diaphragm) - long side - ductility of 2.0 - E6C13

\begin{tabular}{|c|c|c|c|c|c|c|c|}
\hline \multirow{2}{*}{ Buildings } & \multirow{2}{*}{$\begin{array}{c}\text { Deck } \\
\text { Elastic } \\
\text { Force }\end{array}$} & \multirow{2}{*}{$\begin{array}{c}\mu_{d} \text { on Deck } \\
\text { Panels for } \\
R d=1.5\end{array}$} & \multirow{2}{*}{$\begin{array}{c}R_{d} \\
\left(\mu_{d}=2.0\right)\end{array}$} & \multicolumn{4}{|c|}{ Internal Force Magnification Ratios } \\
\hline & & & & $M . F$ & $V . F$ & $M . U$ & $V . U$ \\
\hline SL1 & 134.95 & 4.46 & 1.15 & 0.95 & 1.06 & 1.19 & 1.46 \\
\hline SH1 & 266.89 & 3.09 & 1.27 & 0.92 & 0.96 & 1.14 & 1.32 \\
\hline ML1 & 319.53 & 3.22 & 1.23 & 0.99 & 1.13 & 1.24 & 1.55 \\
\hline MH1 & 349.18 & 3.09 & 1.25 & 1.12 & 1.26 & 1.40 & 1.73 \\
\hline LL1 & 598.43 & 4.09 & 1.12 & 1.18 & 1.34 & 1.48 & 1.84 \\
\hline LH1 & 1259.12 & 5.28 & 1.21 & 1.08 & 1.24 & 1.35 & 1.71 \\
\hline
\end{tabular}

Table E.38 - Proper $R_{d}$ for steel deck panels acting as the main source of energy dissipation (weak diaphragm) - long side -ductility of 2.0 - E6C15

\begin{tabular}{cccccccc}
\hline \multirow{2}{*}{ Buildings } & \multirow{2}{*}{$\begin{array}{c}\text { Deck } \\
\text { Elastic } \\
\text { Force }\end{array}$} & $\begin{array}{c}\mu_{d} \text { on Deck } \\
\text { Panels for } \\
R d=1.5\end{array}$ & $\begin{array}{c}R_{d} \\
\left(\mu_{d}=2.0\right)\end{array}$ & \multicolumn{2}{c}{ Internal Force Magnification Ratios } \\
\cline { 5 - 8 } & 138.15 & 2.56 & 1.16 & 1.01 & 1.13 & 1.26 & 1.55 \\
\hline SL1 & 172.26 & 2.97 & 1.26 & 1.11 & 1.26 & 1.39 & 1.74 \\
SH1 & 176 & 1.23 & 1.07 & 1.24 & 1.34 & 1.70 \\
ML1 & 299.45 & 2.79 & 1.16 & 1.00 & 1.11 & 1.25 & 1.52 \\
MH1 & 725.54 & 4.75 & 1.12 & 0.87 & 0.91 & 1.08 & 1.25 \\
LL1 & 1414.74 & 4.76 & 1.11 & 0.89 & 0.94 & 1.11 & 1.30 \\
LH1 & 3412.38 & 4.52 & & & &
\end{tabular}

Table E.39 - Proper $R_{d}$ for steel deck panels acting as the main source of energy dissipation (weak diaphragm) - long side -ductility of 2.0 - E6C18

\begin{tabular}{|c|c|c|c|c|c|c|c|}
\hline \multirow{2}{*}{ Buildings } & \multirow{2}{*}{$\begin{array}{c}\text { Deck } \\
\text { Elastic } \\
\text { Force }\end{array}$} & \multirow{2}{*}{$\begin{array}{c}\mu_{d} \text { on Deck } \\
\text { Panels for } \\
R d=1.5\end{array}$} & \multirow{2}{*}{$\begin{array}{c}R_{d} \\
\left(\mu_{d}=2.0\right)\end{array}$} & \multicolumn{4}{|c|}{ Internal Force Magnification Ratios } \\
\hline & & & & $M . F$ & V.F & $M . U$ & $V . U$ \\
\hline SL1 & 181.49 & 4.59 & 1.14 & 0.87 & 0.91 & 1.09 & 1.25 \\
\hline SH1 & 356.76 & 3.58 & 1.16 & 0.96 & 1.04 & 1.20 & 1.43 \\
\hline ML1 & 276.94 & 2.23 & 1.36 & 1.03 & 1.21 & 1.29 & 1.67 \\
\hline MH1 & 550.09 & 2.49 & 1.38 & 1.05 & 1.22 & 1.31 & 1.68 \\
\hline LL1 & 1010.10 & 3.17 & 1.29 & 1.00 & 1.14 & 1.26 & 1.57 \\
\hline LH1 & 2108.12 & 5.23 & 1.12 & 0.92 & 0.98 & 1.15 & 1.34 \\
\hline
\end{tabular}


Table E.40 - Proper $R_{d}$ for steel deck panels acting as the main source of energy dissipation (weak diaphragm) - long side -ductility of 2.0 - E6C42

\begin{tabular}{cccccccc}
\hline \multirow{2}{*}{ Buildings } & $\begin{array}{c}\text { Deck } \\
\text { Elastic } \\
\text { Force }\end{array}$ & $\begin{array}{c}\mu_{d} \text { on Deck } \\
\text { Panels for } \\
R d=1.5\end{array}$ & $\begin{array}{c}R_{d} \\
\left(\mu_{d}=2.0\right)\end{array}$ & \multicolumn{2}{c}{ Internal Force Magnification Ratios } \\
\cline { 5 - 8 } & 175.73 & 2.91 & 1.25 & 1.00 & 1.10 & 1.25 & 1.51 \\
\hline SL1 & 224.90 & 3.40 & 1.16 & 0.97 & 1.03 & 1.21 & 1.42 \\
SH1 & 278.96 & 2.32 & 1.23 & 1.08 & 1.25 & 1.36 & 1.72 \\
ML1 & 27.9 & 1.17 & 0.82 & 0.85 & 1.03 & 1.17 \\
MH1 & 577.93 & 3.79 & 1.14 & 0.87 & 0.93 & 1.08 & 1.28 \\
LL1 & 1041.11 & 4.68 & 1.11 & 0.95 & 1.02 & 1.18 & 1.40 \\
LH1 & 2130.46 & 5.57 & & & &
\end{tabular}




\section{Appendix F}

Table F.1 - Diaphragm design factors for linear and nonlinear performance with limited ductility to 1.5 - brace $\mu=2.0-\mathrm{M} 6 \mathrm{C} 2$ record - short side

\begin{tabular}{|c|c|c|c|c|c|c|c|c|c|}
\hline \multirow{3}{*}{ Buildings } & \multirow{3}{*}{$\begin{array}{c}\text { Brace } \\
\text { Elastic } \\
\text { Force }\end{array}$} & \multirow{3}{*}{$\begin{array}{c}\text { Brace } \\
R_{y} \\
(\mu=2)\end{array}$} & \multicolumn{7}{|c|}{ Roof Diaphragm Design Factor $(\phi)$ Determination } \\
\hline & & & \multirow{2}{*}{$\begin{array}{c}\phi \text { - Elastic } \\
\text { Diaphragm }\end{array}$} & \multicolumn{6}{|c|}{ Nonlinear Diaphragm with Ductility $\leq 1.5$} \\
\hline & & & & $\phi$ & $\mu($ Brace $)$ & M.F & V.F & M.U & V.U \\
\hline SL1 & 137.47 & 1.20 & 1.01 & 0.98 & 1.63 & 1.00 & 1.13 & 1.24 & 1.55 \\
\hline SH1 & 229.83 & 1.20 & 1.02 & 0.98 & 1.30 & 1.10 & 1.30 & 1.38 & 1.78 \\
\hline SH4 & 218.55 & 1.24 & 1.03 & 0.98 & 1.47 & 1.10 & 1.24 & 1.37 & 1.70 \\
\hline ML1 & 280.41 & 1.24 & 1.12 & 0.99 & 1.64 & 1.28 & 1.40 & 1.60 & 1.92 \\
\hline ML2 & 212.98 & 1.19 & 1.03 & 0.98 & 1.59 & 1.17 & 1.35 & 1.46 & 1.86 \\
\hline MH1 & 591.08 & 1.19 & 1.05 & 0.97 & 1.37 & 0.97 & 1.08 & 1.21 & 1.49 \\
\hline MH6 & 575.21 & 1.35 & 1.21 & 1.05 & 1.97 & 1.37 & 1.46 & 1.71 & 2.00 \\
\hline LL1 & 1134.30 & 1.55 & 1.13 & 1.01 & 1.98 & 1.19 & 1.37 & 1.49 & 1.88 \\
\hline LH1 & 2498.02 & 1.34 & 1.04 & 0.98 & 1.79 & 1.11 & 1.26 & 1.39 & 1.74 \\
\hline LH5 & 2507.27 & 1.52 & 1.07 & 1.00 & 1.93 & 1.19 & 1.39 & 1.49 & 1.91 \\
\hline
\end{tabular}

Table F.2 - Diaphragm design factors for linear and nonlinear performance with limited ductility to 1.5 - brace $\mu=2.0$ - M6C26 record - short side

\begin{tabular}{|c|c|c|c|c|c|c|c|c|c|}
\hline \multirow{3}{*}{ Buildings } & \multirow{3}{*}{$\begin{array}{c}\text { Brace } \\
\text { Elastic } \\
\text { Force }\end{array}$} & \multirow{3}{*}{$\begin{array}{c}\text { Brace } \\
R_{y} \\
(\mu=2)\end{array}$} & \multicolumn{7}{|c|}{ Roof Diaphragm Design Factor $(\phi)$ Determination } \\
\hline & & & \multirow{2}{*}{$\begin{array}{c}\phi-\text { Elastic } \\
\text { Diaphragm }\end{array}$} & \multicolumn{6}{|c|}{ Nonlinear Diaphragm with Ductility $\leq 1.5$} \\
\hline & & & & $\phi$ & $\mu($ Brace $)$ & M.F & V.F & M.U & V.U \\
\hline SL1 & 165.22 & 1.16 & 1.02 & 0.99 & 1.57 & 1.07 & 1.21 & 1.34 & 1.66 \\
\hline SH1 & 337.30 & 1.24 & 1.03 & 0.99 & 1.48 & 1.07 & 1.22 & 1.33 & 1.68 \\
\hline SH4 & 295.74 & 1.21 & 1.03 & 0.99 & 1.46 & 1.08 & 1.28 & 1.35 & 1.76 \\
\hline ML1 & 477.20 & 1.15 & 1.02 & 0.98 & 1.34 & 1.04 & 1.16 & 1.30 & 1.59 \\
\hline ML2 & 382.10 & 1.17 & 1.03 & 0.97 & 1.32 & 0.92 & 1.06 & 1.15 & 1.46 \\
\hline MH1 & 750.17 & 1.22 & 1.04 & 0.98 & 1.51 & 0.87 & 1.01 & 1.09 & 1.38 \\
\hline MH6 & 1009.86 & 1.47 & 1.04 & 1.01 & 1.78 & 1.13 & 1.29 & 1.41 & 1.77 \\
\hline LL1 & 1182.33 & 1.31 & 1.04 & 1.00 & 1.70 & 1.12 & 1.26 & 1.40 & 1.74 \\
\hline LH1 & 2127.93 & 1.27 & 1.05 & 0.99 & 1.67 & 1.05 & 1.17 & 1.31 & 1.61 \\
\hline LH5 & 2016.26 & 1.43 & 1.06 & 1.01 & 1.82 & 1.16 & 1.39 & 1.45 & 1.91 \\
\hline
\end{tabular}


Table F.3 - Diaphragm design factors for linear and nonlinear performance with limited ductility to 1.5 - brace $\mu=2.0$ - M6C 31 record - short side

\begin{tabular}{|c|c|c|c|c|c|c|c|c|c|}
\hline \multirow{3}{*}{ Buildings } & \multirow{3}{*}{$\begin{array}{c}\text { Brace } \\
\text { Elastic } \\
\text { Force }\end{array}$} & \multirow{3}{*}{$\begin{array}{c}\text { Brace } \\
\mathrm{R}_{\mathrm{y}} \\
(\mu=2)\end{array}$} & \multicolumn{7}{|c|}{ Roof Diaphragm Design Factor $(\phi)$ Determination } \\
\hline & & & \multirow{2}{*}{$\begin{array}{l}\phi-\text { Elastic } \\
\text { Diaphragm }\end{array}$} & \multicolumn{6}{|c|}{ Nonlinear Diaphragm with Ductility $\leq 1.5$} \\
\hline & & & & $\phi$ & $\mu($ Brace $)$ & M.F & V.F & M.U & V.U \\
\hline SL1 & 203.86 & 1.14 & 1.02 & 1.00 & 1.64 & 1.09 & 1.22 & 1.36 & 1.68 \\
\hline SH1 & 248.69 & 1.20 & 1.03 & 0.99 & 1.53 & 1.09 & 1.21 & 1.36 & 1.67 \\
\hline SH4 & 234.99 & 1.13 & 1.06 & 0.96 & 1.08 & 1.18 & 1.38 & 1.47 & 1.90 \\
\hline ML1 & 307.90 & 1.16 & 1.12 & 0.95 & 1.04 & 1.15 & 1.38 & 1.44 & 1.89 \\
\hline ML2 & 209.80 & 1.24 & 1.07 & 0.98 & 1.34 & 1.18 & 1.32 & 1.47 & 1.82 \\
\hline MH1 & 467.44 & 1.25 & 1.08 & 0.98 & 1.50 & 1.05 & 1.15 & 1.32 & 1.58 \\
\hline MH6 & 637.26 & 1.40 & 1.11 & 1.00 & 1.49 & 1.20 & 1.40 & 1.50 & 1.93 \\
\hline LL1 & 883.13 & 1.24 & 1.10 & 0.99 & 1.19 & 1.19 & 1.34 & 1.48 & 1.84 \\
\hline LH1 & 1634.19 & 1.27 & 1.03 & 0.98 & 1.68 & 1.07 & 1.28 & 1.33 & 1.76 \\
\hline LH5 & 1525.82 & 1.29 & 1.04 & 0.99 & 1.78 & 1.11 & 1.30 & 1.39 & 1.79 \\
\hline
\end{tabular}

Table F.4 - Diaphragm design factors for linear and nonlinear performance with limited ductility to 1.5 - brace $\mu=2.0$ - E6C15 record - short side

\begin{tabular}{|c|c|c|c|c|c|c|c|c|c|}
\hline \multirow{3}{*}{ Buildings } & \multirow{3}{*}{$\begin{array}{l}\text { Brace } \\
\text { Elastic } \\
\text { Force }\end{array}$} & \multirow{3}{*}{$\begin{array}{c}\text { Brace } \\
R_{y} \\
(\mu=2)\end{array}$} & \multicolumn{7}{|c|}{ Roof Diaphragm Design Factor $(\phi)$ Determination } \\
\hline & & & \multirow{2}{*}{$\begin{array}{l}\phi-\text { Elastic } \\
\text { Diaphragm }\end{array}$} & \multicolumn{6}{|c|}{ Nonlinear Diaphragm with Ductility $\leq 1.5$} \\
\hline & & & & $\phi$ & $\mu($ Brace $)$ & M.F & V.F & M.U & V.U \\
\hline SL1 & 72.53 & 1.36 & 1.08 & 0.98 & 1.56 & 1.22 & 1.38 & 1.53 & 1.91 \\
\hline SH1 & 171.13 & 1.24 & 1.08 & 0.96 & 1.00 & 1.14 & 1.33 & 1.43 & 1.83 \\
\hline $\mathrm{SH} 4$ & 105.18 & 1.16 & 1.07 & 0.87 & 1.00 & 1.23 & 1.35 & 1.54 & 1.85 \\
\hline ML1 & 149.41 & 1.33 & 1.07 & 0.86 & 1.00 & 1.26 & 1.36 & 1.57 & 1.87 \\
\hline ML2 & 121.33 & 1.16 & 1.06 & 0.98 & 1.20 & 1.27 & 1.41 & 1.59 & 1.94 \\
\hline MH1 & 287.16 & 1.31 & 1.08 & 0.98 & 1.15 & 1.28 & 1.39 & 1.59 & 1.90 \\
\hline MH6 & 329.74 & 1.50 & 1.21 & 1.02 & 1.42 & 1.32 & 1.45 & 1.65 & 2.00 \\
\hline LL1 & 667.35 & 1.41 & 1.08 & 1.00 & 1.55 & 1.09 & 1.27 & 1.36 & 1.74 \\
\hline LH1 & 833.79 & 1.30 & 1.13 & 0.96 & 1.00 & 1.16 & 1.40 & 1.45 & 1.92 \\
\hline LH5 & 691.51 & 1.43 & 1.31 & 1.20 & 1.38 & 1.36 & 1.66 & 1.70 & 2.28 \\
\hline
\end{tabular}


Table F.5 - Diaphragm design factors for linear and nonlinear performance with limited ductility to 1.5 - brace $\mu=2.0$ - E6C42 record - short side

\begin{tabular}{|c|c|c|c|c|c|c|c|c|c|}
\hline \multirow{3}{*}{ Buildings } & \multirow{3}{*}{$\begin{array}{c}\text { Brace } \\
\text { Elastic } \\
\text { Force }\end{array}$} & \multirow{3}{*}{$\begin{array}{c}\text { Brace } \\
R_{y} \\
(\mu=2)\end{array}$} & \multicolumn{7}{|c|}{ Roof Diaphragm Design Factor $(\phi)$ Determination } \\
\hline & & & \multirow{2}{*}{$\begin{array}{l}\phi-\text { Elastic } \\
\text { Diaphragm }\end{array}$} & \multicolumn{6}{|c|}{ Nonlinear Diaphragm with Ductility $\leq 1.5$} \\
\hline & & & & $\phi$ & $\mu($ Brace $)$ & M.F & V.F & M.U & V.U \\
\hline SL1 & 73.56 & 1.23 & 1.08 & 0.99 & 1.36 & 1.19 & 1.31 & 1.49 & 1.80 \\
\hline SH1 & 116.73 & 1.24 & 1.07 & 0.98 & 1.31 & 1.14 & 1.21 & 1.42 & 1.66 \\
\hline $\mathrm{SH} 4$ & 111.51 & 1.29 & 1.03 & 0.97 & 1.63 & 1.09 & 1.13 & 1.36 & 1.55 \\
\hline ML1 & 195.28 & 1.34 & 1.02 & 0.97 & 1.70 & 1.04 & 1.21 & 1.30 & 1.66 \\
\hline ML2 & 157.78 & 1.23 & 1.04 & 0.95 & 1.62 & 1.08 & 1.34 & 1.35 & 1.84 \\
\hline MH1 & 329.99 & 1.32 & 1.07 & 0.96 & 1.39 & 1.13 & 1.37 & 1.41 & 1.88 \\
\hline MH6 & 415.21 & 1.46 & 1.01 & 0.98 & 1.84 & 1.15 & 1.32 & 1.44 & 1.81 \\
\hline LL1 & 754.07 & 1.32 & 1.04 & 0.96 & 1.65 & 1.05 & 1.35 & 1.31 & 1.86 \\
\hline LH1 & 1330.71 & 1.63 & 1.09 & 0.98 & 1.98 & 1.09 & 1.09 & 1.09 & 1.50 \\
\hline LH5 & 1284.05 & 1.60 & 1.08 & 0.96 & 1.89 & 0.85 & 1.00 & 1.06 & 1.37 \\
\hline
\end{tabular}

Table F.6 - Diaphragm design factors for linear and nonlinear performance with limited ductility to 1.5 - brace $\mu=3.0$ - M6C1 record - short side

\begin{tabular}{|c|c|c|c|c|c|c|c|c|c|}
\hline \multirow{3}{*}{ Buildings } & \multirow{3}{*}{$\begin{array}{c}\text { Brace } \\
\text { Elastic } \\
\text { Force }\end{array}$} & \multirow{3}{*}{$\begin{array}{c}\text { Brace } \\
\mathrm{R}_{\mathrm{y}} \\
(\mu=3)\end{array}$} & \multicolumn{7}{|c|}{ Roof Diaphragm Design Factor $(\phi)$ Determination } \\
\hline & & & \multirow{2}{*}{$\begin{array}{l}\phi \text { - Elastic } \\
\text { Diaphragm }\end{array}$} & \multicolumn{6}{|c|}{ Nonlinear Diaphragm with Ductility $\leq 1.5$} \\
\hline & & & & $\phi$ & $\mu($ Brace $)$ & M.F & V.F & M.U & V.U \\
\hline SL1 & 133.91 & 1.40 & 1.05 & 1.02 & 2.76 & 0.97 & 1.13 & 1.22 & 1.56 \\
\hline SH1 & 320.86 & 1.72 & 1.07 & 1.03 & 2.52 & 1.14 & 1.34 & 1.42 & 1.85 \\
\hline SH4 & 264.05 & 1.41 & 1.09 & 0.99 & 2.26 & 1.13 & 1.35 & 1.42 & 1.86 \\
\hline ML1 & 516.49 & 1.60 & 1.04 & 1.00 & 2.63 & 1.11 & 1.34 & 1.39 & 1.85 \\
\hline ML2 & 438.79 & 1.48 & 1.07 & 1.01 & 2.41 & 1.00 & 1.12 & 1.25 & 1.54 \\
\hline MH1 & 886.09 & 1.51 & 1.08 & 1.03 & 2.67 & 1.03 & 1.19 & 1.29 & 1.63 \\
\hline MH6 & 1175.66 & 1.88 & 1.04 & 1.01 & 2.79 & 1.13 & 1.35 & 1.41 & 1.85 \\
\hline LL1 & 1565.21 & 1.44 & 1.06 & 1.03 & 2.64 & 0.98 & 1.08 & 1.23 & 1.49 \\
\hline LH1 & 3032.80 & 2.09 & 1.24 & 1.12 & 2.30 & 1.39 & 1.53 & 1.73 & 2.10 \\
\hline LH5 & 2455.20 & 1.71 & 1.29 & 1.15 & 1.96 & 1.42 & 1.60 & 1.78 & 2.19 \\
\hline
\end{tabular}


Table F.7 - Diaphragm design factors for linear and nonlinear performance with limited ductility to 1.5 - brace $\mu=3.0$ - M6C2 record - short side

\begin{tabular}{|c|c|c|c|c|c|c|c|c|c|}
\hline \multirow{3}{*}{ Buildings } & \multirow{3}{*}{$\begin{array}{c}\text { Brace } \\
\text { Elastic } \\
\text { Force }\end{array}$} & \multirow{3}{*}{$\begin{array}{c}\text { Brace } \\
R_{y} \\
(\mu=3)\end{array}$} & \multicolumn{7}{|c|}{ Roof Diaphragm Design Factor $(\phi)$ Determination } \\
\hline & & & \multirow{2}{*}{$\begin{array}{l}\phi-\text { Elastic } \\
\text { Diaphragm }\end{array}$} & \multicolumn{6}{|c|}{ Nonlinear Diaphragm with Ductility $\leq 1.5$} \\
\hline & & & & $\phi$ & $\mu($ Brace $)$ & M.F & V.F & M.U & V.U \\
\hline SL1 & 137.47 & 1.34 & 1.04 & 1.01 & 2.66 & 0.98 & 1.12 & 1.22 & 1.54 \\
\hline SH1 & 229.83 & 1.63 & 1.07 & 1.02 & 2.54 & 1.12 & 1.38 & 1.40 & 1.89 \\
\hline SH4 & 218.55 & 1.36 & 1.05 & 1.00 & 2.40 & 1.09 & 1.25 & 1.36 & 1.72 \\
\hline ML1 & 280.41 & 1.34 & 1.14 & 1.03 & 2.53 & 1.28 & 1.39 & 1.60 & 1.91 \\
\hline ML2 & 212.98 & 1.29 & 1.06 & 0.99 & 2.40 & 1.13 & 1.33 & 1.41 & 1.82 \\
\hline MH1 & 591.08 & 1.64 & 1.07 & 0.99 & 2.78 & 1.00 & 1.19 & 1.25 & 1.64 \\
\hline MH6 & 575.21 & 1.50 & 1.23 & 1.08 & 2.93 & 1.34 & 1.43 & 1.68 & 1.97 \\
\hline LL1 & 1134.30 & 1.85 & 1.13 & 1.02 & 2.89 & 1.10 & 1.31 & 1.38 & 1.80 \\
\hline LH1 & 2498.02 & 1.96 & 1.07 & 1.04 & 2.97 & 1.10 & 1.28 & 1.38 & 1.75 \\
\hline LH5 & 2507.27 & 2.05 & 1.17 & 1.06 & 2.96 & 1.21 & 1.40 & 1.52 & 1.93 \\
\hline
\end{tabular}

Table F.8 - Diaphragm design factors for linear and nonlinear performance with limited ductility to 1.5 - brace $\mu=3.0$ - M6C26 record - short side

\begin{tabular}{|c|c|c|c|c|c|c|c|c|c|}
\hline \multirow{3}{*}{ Buildings } & \multirow{3}{*}{$\begin{array}{c}\text { Brace } \\
\text { Elastic } \\
\text { Force }\end{array}$} & \multirow{3}{*}{$\begin{array}{c}\text { Brace } \\
R_{y} \\
(\mu=3)\end{array}$} & \multicolumn{7}{|c|}{ Roof Diaphragm Design Factor $(\phi)$ Determination } \\
\hline & & & \multirow{2}{*}{$\begin{array}{l}\phi \text { - Elastic } \\
\text { Diaphragm }\end{array}$} & \multicolumn{6}{|c|}{ Nonlinear Diaphragm with Ductility $\leq 1.5$} \\
\hline & & & & $\phi$ & $\mu($ Brace $)$ & M.F & V.F & M.U & V.U \\
\hline SL1 & 165.22 & 1.28 & 1.07 & 1.03 & 2.75 & 1.07 & 1.22 & 1.33 & 1.68 \\
\hline SH1 & 337.30 & 1.37 & 1.06 & 1.02 & 2.46 & 1.04 & 1.22 & 1.30 & 1.68 \\
\hline SH4 & 295.74 & 1.32 & 1.07 & 1.02 & 2.38 & 1.06 & 1.27 & 1.32 & 1.75 \\
\hline ML1 & 477.20 & 1.45 & 1.08 & 1.03 & 2.56 & 1.10 & 1.26 & 1.38 & 1.74 \\
\hline ML2 & 382.10 & 1.46 & 1.05 & 1.00 & 2.43 & 1.00 & 1.13 & 1.25 & 1.56 \\
\hline MH1 & 750.17 & 1.71 & 1.07 & 1.03 & 2.53 & 0.96 & 1.13 & 1.20 & 1.55 \\
\hline MH6 & 1009.86 & 1.68 & 1.08 & 1.04 & 2.75 & 1.14 & 1.31 & 1.42 & 1.79 \\
\hline LL1 & 1182.33 & 1.47 & 1.10 & 1.05 & 2.84 & 1.18 & 1.34 & 1.47 & 1.85 \\
\hline LH1 & 2127.93 & 1.60 & 1.09 & 1.04 & 2.63 & 1.15 & 1.39 & 1.44 & 1.91 \\
\hline LH5 & 2016.26 & 1.88 & 1.16 & 1.05 & 1.90 & 1.35 & 1.46 & 1.69 & 2.01 \\
\hline
\end{tabular}


Table F.9 - Diaphragm design factors for linear and nonlinear performance with limited ductility to 1.5 - brace $\mu=3.0$ - M6C 31 record - short side

\begin{tabular}{|c|c|c|c|c|c|c|c|c|c|}
\hline \multirow{3}{*}{ Buildings } & \multirow{3}{*}{$\begin{array}{c}\text { Brace } \\
\text { Elastic } \\
\text { Force }\end{array}$} & \multirow{3}{*}{$\begin{array}{c}\text { Brace } \\
R_{y} \\
(\mu=3)\end{array}$} & \multicolumn{7}{|c|}{ Roof Diaphragm Design Factor $(\phi)$ Determination } \\
\hline & & & \multirow{2}{*}{$\begin{array}{l}\phi \text { - Elastic } \\
\text { Diaphragm }\end{array}$} & \multicolumn{6}{|c|}{ Nonlinear Diaphragm with Ductility $\leq 1.5$} \\
\hline & & & & $\phi$ & $\mu($ Brace $)$ & M.F & V.F & M.U & V.U \\
\hline SL1 & 203.86 & 1.25 & 1.06 & 1.03 & 2.64 & 1.08 & 1.23 & 1.35 & 1.69 \\
\hline SH1 & 248.69 & 1.36 & 1.06 & 1.01 & 2.36 & 1.13 & 1.25 & 1.42 & 1.72 \\
\hline SH4 & 234.99 & 1.23 & 1.09 & 1.00 & 1.59 & 1.22 & 1.41 & 1.52 & 1.94 \\
\hline ML1 & 307.90 & 1.28 & 1.15 & 1.01 & 1.43 & 1.20 & 1.44 & 1.50 & 1.98 \\
\hline ML2 & 209.80 & 1.34 & 1.11 & 1.01 & 1.99 & 1.16 & 1.40 & 1.45 & 1.92 \\
\hline MH1 & 467.44 & 1.37 & 1.12 & 1.03 & 2.20 & 1.05 & 1.23 & 1.31 & 1.69 \\
\hline MH6 & 637.26 & 1.79 & 1.25 & 1.11 & 2.33 & 1.37 & 1.51 & 1.71 & 2.08 \\
\hline LL1 & 883.13 & 1.41 & 1.17 & 1.04 & 2.30 & 1.25 & 1.42 & 1.57 & 1.95 \\
\hline LH1 & 1634.19 & 1.45 & 1.08 & 1.01 & 2.56 & 1.06 & 1.32 & 1.33 & 1.81 \\
\hline LH5 & 1525.82 & 1.51 & 1.06 & 1.03 & 2.81 & 1.11 & 1.28 & 1.38 & 1.77 \\
\hline
\end{tabular}

Table F.10 - Diaphragm design factors for linear and nonlinear performance with limited ductility to 1.5 - brace $\mu=3.0$ - M6C38 record - short side

\begin{tabular}{|c|c|c|c|c|c|c|c|c|c|}
\hline \multirow{3}{*}{ Buildings } & \multirow{3}{*}{$\begin{array}{c}\text { Brace } \\
\text { Elastic } \\
\text { Force }\end{array}$} & \multirow{3}{*}{$\begin{array}{c}\text { Brace } \\
R_{y} \\
(\mu=3)\end{array}$} & \multicolumn{7}{|c|}{ Roof Diaphragm Design Factor $(\phi)$ Determination } \\
\hline & & & \multirow{2}{*}{$\begin{array}{l}\phi \text { - Elastic } \\
\text { Diaphragm }\end{array}$} & \multicolumn{6}{|c|}{ Nonlinear Diaphragm with Ductility $\leq 1.5$} \\
\hline & & & & $\phi$ & $\mu($ Brace $)$ & M.F & V.F & M.U & V.U \\
\hline SL1 & 137.93 & 1.23 & 1.07 & 1.04 & 2.64 & 1.16 & 1.31 & 1.45 & 1.80 \\
\hline SH1 & 185.24 & 1.34 & 1.08 & 1.02 & 2.23 & 1.17 & 1.30 & 1.46 & 1.79 \\
\hline SH4 & 161.75 & 1.32 & 1.11 & 1.01 & 1.84 & 1.20 & 1.30 & 1.50 & 1.79 \\
\hline ML1 & 295.69 & 1.43 & 1.08 & 1.03 & 2.29 & 1.19 & 1.29 & 1.49 & 1.77 \\
\hline ML2 & 280.13 & 1.39 & 1.12 & 0.99 & 2.11 & 1.19 & 1.28 & 1.49 & 1.76 \\
\hline MH1 & 676.11 & 1.55 & 1.06 & 1.00 & 2.70 & 1.13 & 1.23 & 1.42 & 1.69 \\
\hline MH6 & 708.60 & 1.68 & 1.13 & 1.03 & 2.38 & 1.22 & 1.35 & 1.53 & 1.86 \\
\hline LL1 & 1165.03 & 1.54 & 1.11 & 1.02 & 2.60 & 1.05 & 1.22 & 1.31 & 1.68 \\
\hline LH1 & 2838.64 & 1.82 & 1.07 & 1.01 & 2.67 & 0.92 & 1.08 & 1.16 & 1.49 \\
\hline LH5 & 2829.69 & 1.92 & 1.08 & 1.01 & 2.71 & 0.91 & 1.10 & 1.14 & 1.51 \\
\hline
\end{tabular}


Table F.11 - Diaphragm design factors for linear and nonlinear performance with limited ductility to 1.5 - brace $\mu=3.0$ - E6C1 record - short side

\begin{tabular}{|c|c|c|c|c|c|c|c|c|c|}
\hline \multirow{3}{*}{ Buildings } & \multirow{3}{*}{$\begin{array}{c}\text { Brace } \\
\text { Elastic } \\
\text { Force }\end{array}$} & \multirow{3}{*}{$\begin{array}{c}\text { Brace } \\
R_{y} \\
(\mu=3)\end{array}$} & \multicolumn{7}{|c|}{ Roof Diaphragm Design Factor $(\phi)$ Determination } \\
\hline & & & \multirow{2}{*}{$\begin{array}{l}\phi \text { - Elastic } \\
\text { Diaphragm }\end{array}$} & \multicolumn{6}{|c|}{ Nonlinear Diaphragm with Ductility $\leq 1.5$} \\
\hline & & & & $\phi$ & $\mu($ Brace $)$ & M.F & V.F & M.U & V.U \\
\hline SL1 & 94.82 & 1.38 & 1.05 & 1.01 & 2.71 & 0.88 & 0.94 & 1.11 & 1.30 \\
\hline SH1 & 151.75 & 1.37 & 1.09 & 1.02 & 2.07 & 1.21 & 1.40 & 1.51 & 1.93 \\
\hline SH4 & 130.88 & 1.23 & 1.11 & 1.00 & 1.74 & 1.16 & 1.33 & 1.45 & 1.83 \\
\hline ML1 & 241.62 & 1.35 & 1.10 & 1.00 & 1.92 & 1.14 & 1.29 & 1.42 & 1.77 \\
\hline ML2 & 223.61 & 1.36 & 1.06 & 1.01 & 2.17 & 1.04 & 1.21 & 1.30 & 1.66 \\
\hline MH1 & 421.93 & 1.37 & 1.13 & 1.03 & 2.22 & 1.06 & 1.20 & 1.32 & 1.65 \\
\hline MH6 & 524.05 & 1.49 & 1.14 & 1.02 & 2.41 & 1.14 & 1.27 & 1.42 & 1.74 \\
\hline LL1 & 689.98 & 1.36 & 1.08 & 1.04 & 2.73 & 1.00 & 1.23 & 1.25 & 1.69 \\
\hline LH1 & 1136.43 & 2.04 & 1.22 & 1.06 & 2.70 & 1.23 & 1.42 & 1.54 & 1.95 \\
\hline LH5 & 1022.93 & 2.06 & 1.24 & 1.09 & 2.80 & 1.29 & 1.45 & 1.62 & 2.00 \\
\hline
\end{tabular}

Table F.12 - Diaphragm design factors for linear and nonlinear performance with limited ductility to 1.5 - brace $\mu=3.0$ - E6C13 record - short side

\begin{tabular}{|c|c|c|c|c|c|c|c|c|c|}
\hline \multirow{3}{*}{ Buildings } & \multirow{3}{*}{$\begin{array}{c}\text { Brace } \\
\text { Elastic } \\
\text { Force }\end{array}$} & \multirow{3}{*}{$\begin{array}{c}\text { Brace } \\
\mathrm{R}_{\mathrm{y}} \\
(\mu=3)\end{array}$} & \multicolumn{7}{|c|}{ Roof Diaphragm Design Factor $(\phi)$ Determination } \\
\hline & & & \multirow{2}{*}{$\begin{array}{l}\phi \text { - Elastic } \\
\text { Diaphragm }\end{array}$} & \multicolumn{6}{|c|}{ Nonlinear Diaphragm with Ductility $\leq 1.5$} \\
\hline & & & & $\phi$ & $\mu($ Brace $)$ & M.F & V.F & M.U & V.U \\
\hline SL1 & 97.40 & 1.50 & 1.06 & 1.02 & 2.44 & 1.04 & 1.22 & 1.30 & 1.68 \\
\hline SH1 & 90.92 & 1.61 & 1.05 & 1.00 & 2.66 & 1.07 & 1.31 & 1.34 & 1.80 \\
\hline SH4 & 93.89 & 1.43 & 1.09 & 1.02 & 2.49 & 0.88 & 1.02 & 1.10 & 1.41 \\
\hline ML1 & 167.54 & 1.41 & 1.11 & 1.03 & 2.23 & 0.90 & 0.98 & 1.13 & 1.35 \\
\hline ML2 & 145.60 & 1.36 & 1.21 & 1.06 & 1.69 & 0.92 & 1.10 & 1.15 & 1.52 \\
\hline MH1 & 317.05 & 1.57 & 1.20 & 1.05 & 3.55 & 1.04 & 1.21 & 1.30 & 1.67 \\
\hline MH6 & 374.02 & 1.63 & 1.12 & 1.03 & 2.68 & 0.90 & 1.06 & 1.13 & 1.45 \\
\hline LL1 & 637.04 & 1.95 & 1.19 & 1.05 & 2.77 & 1.19 & 1.30 & 1.40 & 1.79 \\
\hline LH1 & 1337.81 & 1.68 & 1.11 & 1.03 & 2.49 & 1.04 & 1.19 & 1.30 & 1.63 \\
\hline LH5 & 1168.11 & 2.18 & 1.11 & 1.02 & 2.99 & 1.12 & 1.35 & 1.40 & 1.86 \\
\hline
\end{tabular}


Table F.13 - Diaphragm design factors for linear and nonlinear performance with limited ductility to 1.5 - brace $\mu=3.0$ - E6C15 record - short side

\begin{tabular}{|c|c|c|c|c|c|c|c|c|c|}
\hline \multirow{3}{*}{ Buildings } & \multirow{3}{*}{$\begin{array}{c}\text { Brace } \\
\text { Elastic } \\
\text { Force }\end{array}$} & \multirow{3}{*}{$\begin{array}{c}\text { Brace } \\
R_{y} \\
(\mu=3)\end{array}$} & \multicolumn{7}{|c|}{ Roof Diaphragm Design Factor $(\phi)$ Determination } \\
\hline & & & \multirow{2}{*}{$\begin{array}{l}\phi \text { - Elastic } \\
\text { Diaphragm }\end{array}$} & \multicolumn{6}{|c|}{ Nonlinear Diaphragm with Ductility $\leq 1.5$} \\
\hline & & & & $\phi$ & $\mu($ Brace $)$ & M.F & V.F & M.U & V.U \\
\hline SL1 & 72.53 & 1.72 & 1.19 & 1.04 & 2.33 & 1.26 & 1.37 & 1.58 & 1.89 \\
\hline SH1 & 171.13 & 1.40 & 1.12 & 1.01 & 1.57 & 1.20 & 1.40 & 1.50 & 1.92 \\
\hline SH4 & 105.18 & 1.27 & 1.18 & 0.96 & 1.06 & 1.27 & 1.34 & 1.59 & 1.91 \\
\hline ML1 & 149.41 & 1.43 & 1.15 & 0.93 & 1.00 & 1.26 & 1.36 & 1.57 & 1.87 \\
\hline ML2 & 121.33 & 1.38 & 1.09 & 1.03 & 2.21 & 1.35 & 1.42 & 1.68 & 1.94 \\
\hline MH1 & 287.16 & 1.42 & 1.14 & 1.02 & 1.74 & 1.29 & 1.41 & 1.61 & 1.93 \\
\hline MH6 & 329.74 & 1.67 & 1.26 & 1.09 & 2.18 & 1.37 & 1.50 & 1.72 & 2.06 \\
\hline LL1 & 667.35 & 1.61 & 1.14 & 1.03 & 2.38 & 1.19 & 1.39 & 1.49 & 1.91 \\
\hline LH1 & 833.79 & 1.88 & 1.37 & 1.16 & 1.66 & 1.45 & 1.63 & 1.81 & 2.25 \\
\hline LH5 & 691.51 & 1.75 & 1.60 & 1.35 & 1.84 & 1.44 & 1.67 & 1.80 & 2.30 \\
\hline
\end{tabular}

Table F.14 - diaphragm design factors for linear and nonlinear performance with limited ductility to 1.5 - brace $\mu=3.0$ - E6C18 record - short side

\begin{tabular}{|c|c|c|c|c|c|c|c|c|c|}
\hline \multirow{3}{*}{ Buildings } & \multirow{3}{*}{$\begin{array}{c}\text { Brace } \\
\text { Elastic } \\
\text { Force }\end{array}$} & \multirow{3}{*}{$\begin{array}{c}\text { Brace } \\
\mathrm{R}_{\mathrm{y}} \\
(\mu=3)\end{array}$} & \multicolumn{7}{|c|}{ Roof Diaphragm Design Factor $(\phi)$ Determination } \\
\hline & & & \multirow{2}{*}{$\begin{array}{c}\phi \text { - Elastic } \\
\text { Diaphragm }\end{array}$} & \multicolumn{6}{|c|}{ Nonlinear Diaphragm with Ductility $\leq 1.5$} \\
\hline & & & & $\phi$ & $\mu($ Brace $)$ & M.F & V.F & M.U & V.U \\
\hline SL1 & 91.40 & 1.43 & 1.07 & 1.02 & 2.01 & 1.14 & 1.33 & 1.42 & 1.82 \\
\hline SH1 & 125.30 & 1.31 & 1.09 & 1.02 & 2.12 & 1.07 & 1.15 & 1.34 & 1.58 \\
\hline SH4 & 123.84 & 1.37 & 1.09 & 1.01 & 1.81 & 1.09 & 1.20 & 1.36 & 1.65 \\
\hline ML1 & 201.69 & 1.40 & 1.11 & 1.03 & 2.28 & 1.05 & 1.19 & 1.31 & 1.63 \\
\hline ML2 & 157.53 & 1.67 & 1.15 & 1.00 & 2.43 & 1.06 & 1.28 & 1.32 & 1.75 \\
\hline MH1 & 286.59 & 1.65 & 1.09 & 1.02 & 2.45 & 1.13 & 1.36 & 1.41 & 1.87 \\
\hline MH6 & 430.75 & 2.38 & 1.24 & 1.03 & 2.96 & 1.28 & 1.36 & 1.60 & 1.88 \\
\hline LL1 & 522.76 & 1.85 & 1.39 & 1.18 & 3.02 & 1.33 & 1.56 & 1.67 & 2.14 \\
\hline LH1 & 777.01 & 2.00 & 1.57 & 1.31 & 3.00 & 1.49 & 1.73 & 1.86 & 2.38 \\
\hline LH5 & 721.88 & 2.12 & 1.00 & 1.48 & 3.00 & 1.58 & 1.96 & 1.98 & 2.69 \\
\hline
\end{tabular}


Table F.15 - diaphragm design factors for linear and nonlinear performance with limited ductility to 1.5 - brace $\mu=3.0$ - E6C42 record - short side

\begin{tabular}{|c|c|c|c|c|c|c|c|c|c|}
\hline \multirow{3}{*}{ Buildings } & \multirow{3}{*}{$\begin{array}{c}\text { Brace } \\
\text { Elastic } \\
\text { Force }\end{array}$} & \multirow{3}{*}{$\begin{array}{c}\text { Brace } \\
R_{y} \\
(\mu=3)\end{array}$} & \multicolumn{7}{|c|}{ Roof Diaphragm Design Factor $(\phi)$ Determination } \\
\hline & & & \multirow{2}{*}{$\begin{array}{l}\phi \text { - Elastic } \\
\text { Diaphragm }\end{array}$} & \multicolumn{6}{|c|}{ Nonlinear Diaphragm with Ductility $\leq 1.5$} \\
\hline & & & & $\phi$ & $\mu($ Brace $)$ & M.F & V.F & M.U & V.U \\
\hline SL1 & 73.56 & 1.39 & 1.09 & 1.02 & 1.97 & 1.24 & 1.36 & 1.55 & 1.87 \\
\hline SH1 & 116.73 & 1.38 & 1.10 & 1.01 & 2.02 & 1.16 & 1.28 & 1.45 & 1.76 \\
\hline $\mathrm{SH} 4$ & 111.51 & 1.40 & 1.09 & 1.00 & 2.45 & 1.11 & 1.18 & 1.39 & 1.62 \\
\hline ML1 & 195.28 & 1.50 & 1.06 & 1.00 & 2.74 & 1.05 & 1.14 & 1.31 & 1.57 \\
\hline ML2 & 157.78 & 1.41 & 1.07 & 1.00 & 2.59 & 1.12 & 1.35 & 1.40 & 1.85 \\
\hline MH1 & 329.99 & 1.56 & 1.18 & 1.02 & 2.43 & 1.17 & 1.38 & 1.46 & 1.90 \\
\hline MH6 & 415.21 & 1.70 & 1.06 & 1.02 & 2.80 & 1.12 & 1.26 & 1.40 & 1.74 \\
\hline LL1 & 754.07 & 1.64 & 1.14 & 0.98 & 2.04 & 1.14 & 1.36 & 1.42 & 1.86 \\
\hline LH1 & 1330.71 & 1.86 & 1.10 & 0.98 & 2.63 & 0.86 & 1.00 & 1.07 & 1.38 \\
\hline LH5 & 1284.05 & 1.87 & 1.10 & 0.97 & 2.59 & 0.86 & 0.94 & 1.07 & 1.30 \\
\hline
\end{tabular}

Table F.16 - Diaphragm design factors for linear and nonlinear performance with limited ductility to 1.5 - brace $\mu=4.0$ - M6C2 record - short side

\begin{tabular}{|c|c|c|c|c|c|c|c|c|c|}
\hline \multirow{3}{*}{ Buildings } & \multirow{3}{*}{$\begin{array}{c}\text { Brace } \\
\text { Elastic } \\
\text { Force }\end{array}$} & \multirow{3}{*}{$\begin{array}{c}\text { Brace } \\
R_{y} \\
(\mu=4)\end{array}$} & \multicolumn{7}{|c|}{ Roof Diaphragm Design Factor $(\phi)$ Determination } \\
\hline & & & \multirow{2}{*}{$\begin{array}{l}\phi-\text { Elastic } \\
\text { Diaphragm }\end{array}$} & \multicolumn{6}{|c|}{ Nonlinear Diaphragm with Ductility $\leq 1.5$} \\
\hline & & & & $\phi$ & $\mu($ Brace $)$ & M.F & V.F & M.U & V.U \\
\hline SL1 & 137.47 & 1.47 & 1.08 & 1.04 & 3.71 & 0.94 & 1.10 & 1.18 & 1.51 \\
\hline SH1 & 229.83 & 1.78 & 1.11 & 1.05 & 3.41 & 1.15 & 1.36 & 1.44 & 1.87 \\
\hline $\mathrm{SH} 4$ & 218.55 & 1.68 & 1.10 & 1.00 & 2.47 & 1.18 & 1.34 & 1.47 & 1.84 \\
\hline ML1 & 280.41 & 1.43 & 1.15 & 1.06 & 3.33 & 1.26 & 1.38 & 1.57 & 1.90 \\
\hline ML2 & 212.98 & 1.37 & 1.11 & 1.01 & 3.43 & 1.11 & 1.30 & 1.38 & 1.79 \\
\hline MH1 & 591.08 & 1.76 & 1.07 & 1.03 & 3.75 & 1.02 & 1.23 & 1.27 & 1.70 \\
\hline MH6 & 575.21 & 1.72 & 1.31 & 1.15 & 3.83 & 1.35 & 1.47 & 1.69 & 2.02 \\
\hline LL1 & 1134.30 & 2.08 & 1.10 & 1.04 & 3.63 & 1.08 & 1.27 & 1.35 & 1.75 \\
\hline LH1 & 2498.02 & 2.17 & 1.10 & 1.07 & 3.93 & 1.08 & 1.26 & 1.35 & 1.73 \\
\hline LH5 & 2507.27 & 2.29 & 1.19 & 1.08 & 3.90 & 1.18 & 1.37 & 1.47 & 1.89 \\
\hline
\end{tabular}


Table F.17 - Diaphragm design factors for linear and nonlinear performance with limited ductility to 1.5 - brace $\mu=4.0$ - M6C26 record - short side

\begin{tabular}{|c|c|c|c|c|c|c|c|c|c|}
\hline \multirow{3}{*}{ Buildings } & \multirow{3}{*}{$\begin{array}{c}\text { Brace } \\
\text { Elastic } \\
\text { Force }\end{array}$} & \multirow{3}{*}{$\begin{array}{c}\text { Brace } \\
\mathrm{R}_{\mathrm{y}} \\
(\mu=4)\end{array}$} & \multicolumn{7}{|c|}{ Roof Diaphragm Design Factor $(\phi)$ Determination } \\
\hline & & & \multirow{2}{*}{$\begin{array}{l}\phi \text { - Elastic } \\
\text { Diaphragm }\end{array}$} & \multicolumn{6}{|c|}{ Nonlinear Diaphragm with Ductility $\leq 1.5$} \\
\hline & & & & $\phi$ & $\mu($ Brace $)$ & M.F & V.F & M.U & V.U \\
\hline SL1 & 165.22 & 1.38 & 1.10 & 1.06 & 3.65 & 1.06 & 1.22 & 1.32 & 1.68 \\
\hline SH1 & 337.30 & 1.49 & 1.10 & 1.05 & 3.46 & 1.01 & 1.20 & 1.26 & 1.64 \\
\hline SH4 & 295.74 & 1.42 & 1.11 & 1.05 & 3.33 & 1.05 & 1.24 & 1.31 & 1.71 \\
\hline ML1 & 477.20 & 1.57 & 1.12 & 1.07 & 3.62 & 1.10 & 1.27 & 1.37 & 1.74 \\
\hline ML2 & 382.10 & 1.74 & 1.12 & 1.05 & 3.37 & 1.03 & 1.21 & 1.29 & 1.67 \\
\hline MH1 & 750.17 & 1.88 & 1.13 & 1.06 & 3.49 & 1.00 & 1.19 & 1.26 & 1.64 \\
\hline MH6 & 1009.86 & 1.94 & 1.12 & 1.07 & 3.66 & 1.21 & 1.37 & 1.51 & 1.89 \\
\hline LL1 & 1182.33 & 1.98 & 1.18 & 1.10 & 3.43 & 1.27 & 1.43 & 1.59 & 1.96 \\
\hline LH1 & 2127.93 & 1.98 & 1.19 & 1.07 & 2.90 & 1.29 & 1.42 & 1.61 & 1.95 \\
\hline LH5 & 2016.26 & 2.78 & 1.41 & 1.25 & 4.00 & 1.49 & 1.58 & 1.86 & 2.17 \\
\hline
\end{tabular}

Table F.18 - Diaphragm design factors for linear and nonlinear performance with limited ductility to 1.5 - brace $\mu=4.0$ - M6C31 record - short side

\begin{tabular}{|c|c|c|c|c|c|c|c|c|c|}
\hline \multirow{3}{*}{ Buildings } & \multirow{3}{*}{$\begin{array}{c}\text { Brace } \\
\text { Elastic } \\
\text { Force }\end{array}$} & \multirow{3}{*}{$\begin{array}{c}\text { Brace } \\
R_{y} \\
(\mu=4)\end{array}$} & \multicolumn{7}{|c|}{ Roof Diaphragm Design Factor $(\phi)$ Determination } \\
\hline & & & \multirow{2}{*}{$\begin{array}{l}\phi-\text { Elastic } \\
\text { Diaphragm }\end{array}$} & \multicolumn{6}{|c|}{ Nonlinear Diaphragm with Ductility $\leq 1.5$} \\
\hline & & & & $\phi$ & $\mu($ Brace $)$ & M.F & V.F & M.U & V.U \\
\hline SL1 & 203.86 & 1.37 & 1.10 & 1.06 & 3.58 & 1.08 & 1.23 & 1.35 & 1.70 \\
\hline SH1 & 248.69 & 1.51 & 1.10 & 1.03 & 3.23 & 1.14 & 1.28 & 1.42 & 1.76 \\
\hline SH4 & 234.99 & 1.23 & 1.14 & 1.04 & 2.61 & 1.22 & 1.40 & 1.53 & 1.92 \\
\hline ML1 & 307.90 & 1.44 & 1.16 & 1.05 & 2.47 & 1.23 & 1.42 & 1.54 & 1.96 \\
\hline ML2 & 209.80 & 1.42 & 1.22 & 1.07 & 2.76 & 1.13 & 1.43 & 1.42 & 1.97 \\
\hline MH1 & 467.44 & 1.48 & 1.18 & 1.08 & 3.09 & 1.02 & 1.29 & 1.28 & 1.77 \\
\hline MH6 & 637.26 & 2.06 & 1.42 & 1.25 & 3.59 & 1.44 & 1.61 & 1.80 & 2.21 \\
\hline LL1 & 883.13 & 1.59 & 1.19 & 1.07 & 3.21 & 1.27 & 1.40 & 1.58 & 1.93 \\
\hline LH1 & 1634.19 & 1.61 & 1.15 & 1.04 & 3.49 & 1.06 & 1.35 & 1.32 & 1.85 \\
\hline LH5 & 1525.82 & 1.74 & 1.18 & 1.07 & 3.89 & 1.09 & 1.36 & 1.37 & 1.87 \\
\hline
\end{tabular}


Table F.19 - Diaphragm design factors for linear and nonlinear performance with limited ductility to 1.5 - brace $\mu=4.0$ - E6C15 record - short side

\begin{tabular}{|c|c|c|c|c|c|c|c|c|c|}
\hline \multirow{3}{*}{ Buildings } & \multirow{3}{*}{$\begin{array}{l}\text { Brace } \\
\text { Elastic } \\
\text { Force }\end{array}$} & \multirow{3}{*}{$\begin{array}{c}\text { Brace } \\
R_{y} \\
(\mu=4)\end{array}$} & \multicolumn{7}{|c|}{ Roof Diaphragm Design Factor $(\phi)$ Determination } \\
\hline & & & \multirow{2}{*}{$\begin{array}{l}\phi \text { - Elastic } \\
\text { Diaphragm }\end{array}$} & \multicolumn{6}{|c|}{ Nonlinear Diaphragm with Ductility $\leq 1.5$} \\
\hline & & & & $\phi$ & $\mu($ Brace $)$ & M.F & V.F & M.U & V.U \\
\hline SL1 & 72.53 & 1.99 & 1.28 & 1.10 & 4.07 & 1.28 & 1.39 & 1.60 & 1.91 \\
\hline SH1 & 171.13 & 1.86 & 1.11 & 1.05 & 3.06 & 1.24 & 1.38 & 1.55 & 1.90 \\
\hline SH4 & 105.18 & 1.43 & 1.19 & 1.03 & 1.91 & 1.32 & 1.43 & 1.65 & 1.97 \\
\hline ML1 & 149.41 & 1.53 & 1.18 & 0.97 & 1.08 & 1.31 & 1.41 & 1.63 & 1.93 \\
\hline ML2 & 121.33 & 1.47 & 1.14 & 1.04 & 2.31 & 1.36 & 1.42 & 1.71 & 1.95 \\
\hline MH1 & 287.16 & 1.52 & 1.18 & 1.05 & 2.56 & 1.28 & 1.40 & 1.59 & 1.93 \\
\hline MH6 & 329.74 & 1.83 & 1.31 & 1.12 & 3.04 & 1.38 & 1.48 & 1.72 & 2.03 \\
\hline LL1 & 667.35 & 1.87 & 1.22 & 1.05 & 2.62 & 1.31 & 1.41 & 1.64 & 1.94 \\
\hline LH1 & 833.79 & 2.21 & 1.62 & 1.39 & 3.19 & 1.44 & 1.75 & 1.80 & 2.40 \\
\hline LH5 & 691.51 & 1.96 & 1.71 & 1.44 & 3.37 & 1.46 & 1.72 & 1.82 & 2.36 \\
\hline
\end{tabular}

Table F.20 - Diaphragm design factors for linear and nonlinear performance with limited ductility to 1.5 - brace $\mu=4.0$ - E6C42 record - short side

\begin{tabular}{|c|c|c|c|c|c|c|c|c|c|}
\hline \multirow{3}{*}{ Buildings } & \multirow{3}{*}{$\begin{array}{l}\text { Brace } \\
\text { Elastic } \\
\text { Force }\end{array}$} & \multirow{3}{*}{$\begin{array}{c}\text { Brace } \\
R_{y} \\
(\mu=4)\end{array}$} & \multicolumn{7}{|c|}{ Roof Diaphragm Design Factor $(\phi)$ Determination } \\
\hline & & & \multirow{2}{*}{$\begin{array}{l}\phi-\text { Elastic } \\
\text { Diaphragm }\end{array}$} & \multicolumn{6}{|c|}{ Nonlinear Diaphragm with Ductility $\leq 1.5$} \\
\hline & & & & $\phi$ & $\mu($ Brace $)$ & M.F & V.F & M.U & V.U \\
\hline SL1 & 73.56 & 1.54 & 1.18 & 1.07 & 2.98 & 1.26 & 1.38 & 1.58 & 1.90 \\
\hline SH1 & 116.73 & 1.48 & 1.18 & 1.03 & 2.71 & 1.16 & 1.30 & 1.45 & 1.78 \\
\hline SH4 & 111.51 & 1.50 & 1.12 & 1.02 & 3.08 & 1.13 & 1.22 & 1.41 & 1.68 \\
\hline ML1 & 195.28 & 1.64 & 1.10 & 1.02 & 3.39 & 1.09 & 1.15 & 1.36 & 1.58 \\
\hline ML2 & 157.78 & 1.56 & 1.12 & 1.04 & 3.57 & 1.13 & 1.34 & 1.41 & 1.84 \\
\hline MH1 & 329.99 & 1.70 & 1.26 & 1.06 & 3.51 & 1.16 & 1.37 & 1.45 & 1.88 \\
\hline MH6 & 415.21 & 1.98 & 1.15 & 1.05 & 3.62 & 1.18 & 1.29 & 1.47 & 1.78 \\
\hline LL1 & 754.07 & 2.32 & 1.37 & 1.18 & 4.00 & 1.26 & 1.49 & 1.57 & 2.05 \\
\hline LH1 & 1330.71 & 2.04 & 1.15 & 1.02 & 3.46 & 0.90 & 1.00 & 1.13 & 1.38 \\
\hline LH5 & 1284.05 & 2.09 & 1.16 & 1.01 & 3.42 & 0.92 & 1.01 & 1.15 & 1.39 \\
\hline
\end{tabular}


Table F.21 - Diaphragm design factors for linear and nonlinear performance with limited ductility to 1.5 - brace $\mu=2.0$ - M6C2 record - long side

\begin{tabular}{|c|c|c|c|c|c|c|c|c|c|}
\hline \multirow{3}{*}{ Buildings } & \multirow{3}{*}{$\begin{array}{c}\text { Brace } \\
\text { Elastic } \\
\text { Force }\end{array}$} & \multirow{3}{*}{$\begin{array}{c}\text { Brace } \\
\mathrm{R}_{\mathrm{y}} \\
(\mu=2)\end{array}$} & \multicolumn{7}{|c|}{ Roof Diaphragm Design Factor $(\phi)$ Determination } \\
\hline & & & \multirow{2}{*}{$\begin{array}{c}\phi \text { - Elastic } \\
\text { Diaphragm }\end{array}$} & \multicolumn{6}{|c|}{ Nonlinear Diaphragm with Ductility $\leq 1.5$} \\
\hline & & & & $\phi$ & $\mu($ Brace $)$ & M.F & V.F & M.U & V.U \\
\hline SL1 & 179.03 & 1.59 & 1.02 & 0.99 & 1.94 & 1.05 & 1.17 & 1.31 & 1.60 \\
\hline SH1 & 488.50 & 1.76 & 1.05 & 1.01 & 1.89 & 1.10 & 1.26 & 1.37 & 1.73 \\
\hline ML1 & 615.67 & 1.34 & 1.02 & 0.99 & 1.80 & 0.99 & 1.09 & 1.24 & 1.50 \\
\hline MH1 & 937.20 & 1.29 & 1.05 & 1.01 & 1.83 & 1.13 & 1.29 & 1.42 & 1.78 \\
\hline LL1 & 1745.36 & 1.37 & 1.06 & 1.01 & 1.90 & 1.09 & 1.23 & 1.36 & 1.69 \\
\hline LH1 & 3995.76 & 2.01 & 1.09 & 1.02 & 1.89 & 1.16 & 1.34 & 1.46 & 1.85 \\
\hline
\end{tabular}

Table F.22 - Diaphragm design factors for linear and nonlinear performance with limited ductility to 1.5 - brace $\mu=2.0$ - M6C26 record - long side

\begin{tabular}{|c|c|c|c|c|c|c|c|c|c|}
\hline \multirow{3}{*}{ Buildings } & \multirow{3}{*}{$\begin{array}{c}\text { Brace } \\
\text { Elastic } \\
\text { Force }\end{array}$} & \multirow{3}{*}{$\begin{array}{c}\text { Brace } \\
R_{y} \\
(\mu=2)\end{array}$} & \multicolumn{7}{|c|}{ Roof Diaphragm Design Factor $(\phi)$ Determination } \\
\hline & & & \multirow{2}{*}{$\begin{array}{l}\phi-\text { Elastic } \\
\text { Diaphragm }\end{array}$} & \multicolumn{6}{|c|}{ Nonlinear Diaphragm with Ductility $\leq 1.5$} \\
\hline & & & & $\phi$ & $\mu($ Brace $)$ & M.F & V.F & M.U & V.U \\
\hline SL1 & 202.44 & 1.53 & 1.05 & 0.99 & 1.99 & 1.07 & 1.23 & 1.34 & 1.69 \\
\hline SH1 & 500.73 & 1.76 & 1.04 & 1.00 & 1.86 & 1.07 & 1.23 & 1.34 & 1.69 \\
\hline ML1 & 585.71 & 1.27 & 1.02 & 1.00 & 1.85 & 1.07 & 1.21 & 1.34 & 1.66 \\
\hline MH1 & 1282.07 & 1.32 & 1.02 & 0.99 & 1.85 & 0.98 & 1.07 & 1.23 & 1.47 \\
\hline LL1 & 2423.53 & 1.39 & 1.02 & 1.00 & 1.95 & 1.01 & 1.10 & 1.26 & 1.52 \\
\hline LH1 & 6386.35 & 1.59 & 1.02 & 1.00 & 1.94 & 1.06 & 1.18 & 1.32 & 1.63 \\
\hline
\end{tabular}

Table F.23 - Diaphragm design factors for linear and nonlinear performance with limited ductility to 1.5 - brace $\mu=2.0$ - M6C31 record - long side

\begin{tabular}{|c|c|c|c|c|c|c|c|c|c|}
\hline \multirow{3}{*}{ Buildings } & \multirow{3}{*}{$\begin{array}{l}\text { Brace } \\
\text { Elastic } \\
\text { Force }\end{array}$} & \multirow{3}{*}{$\begin{array}{c}\text { Brace } \\
R_{y} \\
(\mu=2)\end{array}$} & \multicolumn{7}{|c|}{ Roof Diaphragm Design Factor $(\phi)$ Determination } \\
\hline & & & \multirow{2}{*}{$\begin{array}{l}\phi-\text { Elastic } \\
\text { Diaphragm }\end{array}$} & \multicolumn{6}{|c|}{ Nonlinear Diaphragm with Ductility $\leq 1.5$} \\
\hline & & & & $\phi$ & $\mu($ Brace $)$ & M.F & V.F & M.U & V.U \\
\hline SL1 & 189.42 & 1.33 & 1.01 & 0.98 & 1.84 & 1.00 & 1.10 & 1.25 & 1.52 \\
\hline SH1 & 454.44 & 1.29 & 1.02 & 1.00 & 1.84 & 1.10 & 1.25 & 1.38 & 1.72 \\
\hline ML1 & 978.00 & 1.29 & 1.04 & 1.00 & 1.78 & 1.12 & 1.27 & 1.40 & 1.75 \\
\hline MH1 & 1044.74 & 1.34 & 1.03 & 1.00 & 1.81 & 0.99 & 1.12 & 1.24 & 1.54 \\
\hline LL1 & 1764.29 & 1.46 & 1.03 & 1.00 & 1.85 & 1.07 & 1.22 & 1.34 & 1.68 \\
\hline LH1 & 3925.45 & 1.64 & 1.02 & 1.00 & 2.08 & 1.04 & 1.17 & 1.30 & 1.61 \\
\hline
\end{tabular}


Table F.24 - Diaphragm design factors for linear and nonlinear performance with limited ductility to 1.5 - brace $\mu=2.0$ - E6C15 record - long side

\begin{tabular}{|c|c|c|c|c|c|c|c|c|c|}
\hline \multirow{3}{*}{ Buildings } & \multirow{3}{*}{$\begin{array}{c}\text { Brace } \\
\text { Elastic } \\
\text { Force }\end{array}$} & \multirow{3}{*}{$\begin{array}{c}\text { Brace } \\
R_{y} \\
(\mu=2)\end{array}$} & \multicolumn{7}{|c|}{ Roof Diaphragm Design Factor $(\phi)$ Determination } \\
\hline & & & \multirow{2}{*}{$\begin{array}{c}\phi-\text { Elastic } \\
\text { Diaphragm }\end{array}$} & \multicolumn{6}{|c|}{ Nonlinear Diaphragm with Ductility $\leq 1.5$} \\
\hline & & & & $\phi$ & $\mu($ Brace $)$ & M.F & V.F & M.U & V.U \\
\hline SL1 & 139.11 & 1.69 & 1.07 & 1.01 & 1.93 & 1.19 & 1.39 & 1.48 & 1.92 \\
\hline SH1 & 178.32 & 1.46 & 1.10 & 1.01 & 1.66 & 1.19 & 1.37 & 1.49 & 1.89 \\
\hline ML1 & 303.03 & 1.52 & 1.11 & 1.01 & 1.92 & 1.25 & 1.40 & 1.56 & 1.93 \\
\hline MH1 & 753.83 & 1.70 & 1.04 & 1.00 & 2.12 & 1.09 & 1.36 & 1.36 & 1.87 \\
\hline LL1 & 1473.38 & 2.04 & 1.09 & 1.00 & 2.18 & 1.21 & 1.37 & 1.52 & 1.89 \\
\hline LH1 & 3507.12 & 2.50 & 1.16 & 1.03 & 1.91 & 1.24 & 1.43 & 1.55 & 1.97 \\
\hline
\end{tabular}

Table F.25 - Diaphragm design factors for linear and nonlinear performance with limited ductility to 1.5 - brace $\mu=2.0$ - E6C42 record - long side

\begin{tabular}{|c|c|c|c|c|c|c|c|c|c|}
\hline \multirow{3}{*}{ Buildings } & \multirow{3}{*}{$\begin{array}{c}\text { Brace } \\
\text { Elastic } \\
\text { Force }\end{array}$} & \multirow{3}{*}{$\begin{array}{c}\text { Brace } \\
\mathrm{R}_{\mathrm{y}} \\
(\mu=2)\end{array}$} & \multicolumn{7}{|c|}{ Roof Diaphragm Design Factor $(\phi)$ Determination } \\
\hline & & & \multirow{2}{*}{$\begin{array}{c}\phi-\text { Elastic } \\
\text { Diaphragm }\end{array}$} & \multicolumn{6}{|c|}{ Nonlinear Diaphragm with Ductility $\leq 1.5$} \\
\hline & & & & $\phi$ & $\mu($ Brace $)$ & M.F & V.F & M.U & V.U \\
\hline SL1 & 179.40 & 1.89 & 1.11 & 1.01 & 1.86 & 1.20 & 1.39 & 1.50 & 1.91 \\
\hline SH1 & 230.21 & 1.38 & 1.07 & 1.00 & 1.72 & 1.14 & 1.28 & 1.42 & 1.75 \\
\hline ML1 & 275.59 & 1.80 & 1.09 & 1.00 & 1.80 & 1.22 & 1.40 & 1.52 & 1.92 \\
\hline MH1 & 599.19 & 1.38 & 1.04 & 0.99 & 1.83 & 0.87 & 0.94 & 1.08 & 1.29 \\
\hline LL1 & 1081.02 & 1.43 & 1.03 & 0.99 & 1.93 & 0.88 & 0.94 & 1.10 & 1.29 \\
\hline LH1 & 2182.47 & 1.46 & 1.02 & 1.00 & 1.97 & 0.98 & 1.07 & 1.22 & 1.47 \\
\hline
\end{tabular}

Table F.26 - Diaphragm design factors for linear and nonlinear performance with limited ductility to 1.5 - brace $\mu=3.0$ - M6C1 record - long side

\begin{tabular}{|c|c|c|c|c|c|c|c|c|c|}
\hline \multirow{3}{*}{ Buildings } & \multirow{3}{*}{$\begin{array}{c}\text { Brace } \\
\text { Elastic } \\
\text { Force }\end{array}$} & \multirow{3}{*}{$\begin{array}{c}\text { Brace } \\
R_{y} \\
(\mu=3)\end{array}$} & \multicolumn{7}{|c|}{ Roof Diaphragm Design Factor $(\phi)$ Determination } \\
\hline & & & \multirow{2}{*}{$\begin{array}{l}\phi \text { - Elastic } \\
\text { Diaphragm }\end{array}$} & \multicolumn{6}{|c|}{ Nonlinear Diaphragm with Ductility $\leq 1.5$} \\
\hline & & & & $\phi$ & $\mu($ Brace $)$ & M.F & V.F & M.U & V.U \\
\hline SL1 & 214.98 & 1.57 & 1.06 & 1.04 & 2.92 & 1.01 & 1.13 & 1.27 & 1.55 \\
\hline SH1 & 441.96 & 2.51 & 1.05 & 1.01 & 2.40 & 1.19 & 1.37 & 1.48 & 1.88 \\
\hline ML1 & 638.08 & 2.26 & 1.08 & 1.05 & 2.76 & 1.13 & 1.35 & 1.42 & 1.86 \\
\hline MH1 & 1330.29 & 2.01 & 1.09 & 1.04 & 2.97 & 1.16 & 1.36 & 1.45 & 1.87 \\
\hline LL1 & 2475.26 & 2.14 & 1.10 & 1.02 & 2.85 & 1.11 & 1.27 & 1.39 & 1.74 \\
\hline LH1 & 6561.23 & 3.10 & 1.10 & 1.05 & 3.00 & 1.15 & 1.34 & 1.43 & 1.85 \\
\hline
\end{tabular}


Table F.27 - Diaphragm design factors for linear and nonlinear performance with limited ductility to 1.5 - brace $\mu=3.0$ - M6C2 record - long side

\begin{tabular}{|c|c|c|c|c|c|c|c|c|c|}
\hline \multirow{3}{*}{ Buildings } & \multirow{3}{*}{$\begin{array}{c}\text { Brace } \\
\text { Elastic } \\
\text { Force }\end{array}$} & \multirow{3}{*}{$\begin{array}{c}\text { Brace } \\
\mathrm{R}_{\mathrm{y}} \\
(\mu=3)\end{array}$} & \multicolumn{7}{|c|}{ Roof Diaphragm Design Factor $(\phi)$ Determination } \\
\hline & & & \multirow{2}{*}{$\begin{array}{c}\phi \text { - Elastic } \\
\text { Diaphragm }\end{array}$} & \multicolumn{6}{|c|}{ Nonlinear Diaphragm with Ductility $\leq 1.5$} \\
\hline & & & & $\phi$ & $\mu($ Brace $)$ & M.F & V.F & M.U & V.U \\
\hline SL1 & 179.03 & 1.85 & 1.03 & 1.01 & 2.89 & 1.00 & 1.14 & 1.25 & 1.56 \\
\hline SH1 & 488.50 & 2.60 & 1.14 & 1.06 & 2.91 & 1.19 & 1.39 & 1.49 & 1.91 \\
\hline ML1 & 615.67 & 1.59 & 1.05 & 1.02 & 2.84 & 0.99 & 1.11 & 1.23 & 1.53 \\
\hline MH1 & 937.20 & 1.56 & 1.11 & 1.04 & 2.63 & 1.20 & 1.39 & 1.49 & 1.92 \\
\hline LL1 & 1745.36 & 1.71 & 1.13 & 1.05 & 2.85 & 1.17 & 1.37 & 1.47 & 1.89 \\
\hline LH1 & 3995.76 & 2.65 & 1.18 & 1.04 & 2.63 & 1.31 & 1.40 & 1.63 & 1.92 \\
\hline
\end{tabular}

Table F.28 - Diaphragm design factors for linear and nonlinear performance with limited ductility to 1.5 - brace $\mu=3.0$ - M6C26 record - long side

\begin{tabular}{|c|c|c|c|c|c|c|c|c|c|}
\hline \multirow{3}{*}{ Buildings } & \multirow{3}{*}{$\begin{array}{l}\text { Brace } \\
\text { Elastic } \\
\text { Force }\end{array}$} & \multirow{3}{*}{$\begin{array}{c}\text { Brace } \\
\mathrm{R}_{\mathrm{y}} \\
(\mu=3)\end{array}$} & \multicolumn{7}{|c|}{ Roof Diaphragm Design Factor $(\phi)$ Determination } \\
\hline & & & \multirow{2}{*}{$\begin{array}{l}\phi-\text { Elastic } \\
\text { Diaphragm }\end{array}$} & \multicolumn{6}{|c|}{ Nonlinear Diaphragm with Ductility $\leq 1.5$} \\
\hline & & & & $\phi$ & $\mu($ Brace $)$ & M.F & V.F & M.U & V.U \\
\hline SL1 & 202.44 & 1.81 & 1.07 & 1.01 & 2.94 & 1.08 & 1.25 & 1.35 & 1.72 \\
\hline SH1 & 500.73 & 2.09 & 1.05 & 1.03 & 2.87 & 1.07 & 1.24 & 1.34 & 1.70 \\
\hline ML1 & 585.71 & 1.48 & 1.05 & 1.03 & 2.88 & 1.08 & 1.23 & 1.35 & 1.69 \\
\hline MH1 & 1282.07 & 1.54 & 1.05 & 1.03 & 2.89 & 0.96 & 1.07 & 1.20 & 1.47 \\
\hline LL1 & 2423.53 & 1.65 & 1.05 & 1.03 & 2.91 & 0.99 & 1.09 & 1.23 & 1.50 \\
\hline LH1 & 6386.35 & 3.08 & 1.12 & 1.04 & 2.94 & 1.17 & 1.38 & 1.46 & 1.90 \\
\hline
\end{tabular}

Table F.29 - Diaphragm design factors for linear and nonlinear performance with limited ductility to 1.5 - brace $\mu=3.0$ - M6C31 record - long side

\begin{tabular}{|c|c|c|c|c|c|c|c|c|c|}
\hline \multirow{3}{*}{ Buildings } & \multirow{3}{*}{$\begin{array}{c}\text { Brace } \\
\text { Elastic } \\
\text { Force }\end{array}$} & \multirow{3}{*}{$\begin{array}{c}\text { Brace } \\
R_{y} \\
(\mu=3)\end{array}$} & \multicolumn{7}{|c|}{ Roof Diaphragm Design Factor $(\phi)$ Determination } \\
\hline & & & \multirow{2}{*}{$\begin{array}{c}\phi \text { - Elastic } \\
\text { Diaphragm }\end{array}$} & \multicolumn{6}{|c|}{ Nonlinear Diaphragm with Ductility $\leq 1.5$} \\
\hline & & & & $\phi$ & $\mu($ Brace $)$ & M.F & V.F & M.U & V.U \\
\hline SL1 & 189.42 & 2.24 & 1.14 & 1.04 & 3.22 & 1.18 & 1.36 & 1.47 & 1.87 \\
\hline SH1 & 454.44 & 1.49 & 1.05 & 1.03 & 2.80 & 1.10 & 1.26 & 1.38 & 1.73 \\
\hline ML1 & 978.00 & 2.50 & 1.10 & 1.03 & 2.57 & 1.27 & 1.39 & 1.59 & 1.91 \\
\hline MH1 & 1044.74 & 1.67 & 1.07 & 1.03 & 2.80 & 1.05 & 1.23 & 1.31 & 1.69 \\
\hline LL1 & 1764.29 & 1.91 & 1.07 & 1.02 & 2.67 & 1.08 & 1.26 & 1.35 & 1.73 \\
\hline LH1 & 3925.45 & 1.91 & 1.05 & 1.02 & 2.96 & 1.07 & 1.23 & 1.33 & 1.68 \\
\hline
\end{tabular}


Table F.30 - Diaphragm design factors for linear and nonlinear performance with limited ductility to 1.5 - brace $\mu=3.0$ - M6C 38 record - long side

\begin{tabular}{|c|c|c|c|c|c|c|c|c|c|}
\hline \multirow{3}{*}{ Buildings } & \multirow{3}{*}{$\begin{array}{c}\text { Brace } \\
\text { Elastic } \\
\text { Force }\end{array}$} & \multirow{3}{*}{$\begin{array}{c}\text { Brace } \\
\mathrm{R}_{\mathrm{y}} \\
(\mu=3)\end{array}$} & \multicolumn{7}{|c|}{ Roof Diaphragm Design Factor $(\phi)$ Determination } \\
\hline & & & \multirow{2}{*}{$\begin{array}{c}\phi \text { - Elastic } \\
\text { Diaphragm }\end{array}$} & \multicolumn{6}{|c|}{ Nonlinear Diaphragm with Ductility $\leq 1.5$} \\
\hline & & & & $\phi$ & $\mu($ Brace $)$ & M.F & V.F & M.U & V.U \\
\hline SL1 & 173.45 & 1.96 & 1.07 & 1.04 & 2.93 & 1.11 & 1.29 & 1.39 & 1.77 \\
\hline SH1 & 382.82 & 1.52 & 1.06 & 1.03 & 2.85 & 1.07 & 1.20 & 1.34 & 1.66 \\
\hline ML1 & 571.24 & 1.43 & 1.06 & 1.04 & 2.84 & 1.15 & 1.32 & 1.44 & 1.81 \\
\hline MH1 & 1048.81 & 1.51 & 1.06 & 1.03 & 2.77 & 1.16 & 1.33 & 1.45 & 1.83 \\
\hline LL1 & 1939.68 & 1.63 & 1.06 & 1.03 & 2.79 & 1.17 & 1.35 & 1.46 & 1.86 \\
\hline LH1 & 3580.19 & 1.81 & 1.08 & 1.03 & 2.84 & 1.15 & 1.34 & 1.44 & 1.84 \\
\hline
\end{tabular}

Table F.31 - Diaphragm design factors for linear and nonlinear performance with limited ductility to 1.5 - brace $\mu=3.0$ - E6C1 record - long side

\begin{tabular}{|c|c|c|c|c|c|c|c|c|c|}
\hline \multirow{3}{*}{ Buildings } & \multirow{3}{*}{$\begin{array}{l}\text { Brace } \\
\text { Elastic } \\
\text { Force }\end{array}$} & \multirow{3}{*}{$\begin{array}{c}\text { Brace } \\
R_{y} \\
(\mu=3)\end{array}$} & \multicolumn{7}{|c|}{ Roof Diaphragm Design Factor $(\phi)$ Determination } \\
\hline & & & \multirow{2}{*}{$\begin{array}{c}\phi-\text { Elastic } \\
\text { Diaphragm }\end{array}$} & \multicolumn{6}{|c|}{ Nonlinear Diaphragm with Ductility $\leq 1.5$} \\
\hline & & & & $\phi$ & $\mu($ Brace $)$ & M.F & V.F & M.U & V.U \\
\hline SL1 & 93.57 & 2.06 & 1.07 & 1.05 & 2.91 & 1.19 & 1.37 & 1.49 & 1.89 \\
\hline SH1 & 199.84 & 1.60 & 1.03 & 1.01 & 2.88 & 1.03 & 1.15 & 1.28 & 1.58 \\
\hline ML1 & 384.51 & 1.63 & 1.04 & 1.01 & 2.83 & 0.86 & 0.93 & 1.08 & 1.27 \\
\hline MH1 & 790.48 & 1.67 & 1.07 & 1.03 & 2.58 & 1.09 & 1.26 & 1.36 & 1.74 \\
\hline LL1 & 1459.87 & 1.78 & 1.13 & 1.03 & 2.78 & 1.04 & 1.18 & 1.30 & 1.62 \\
\hline LH1 & 3073.35 & 1.93 & 1.16 & 1.04 & 2.80 & 1.11 & 1.31 & 1.39 & 1.80 \\
\hline
\end{tabular}

Table F.32 - Diaphragm design factors for linear and nonlinear performance with limited ductility to 1.5 - brace $\mu=3.0$ - E6C13 record - long side

\begin{tabular}{|c|c|c|c|c|c|c|c|c|c|}
\hline \multirow{3}{*}{ Buildings } & \multirow{3}{*}{$\begin{array}{c}\text { Brace } \\
\text { Elastic } \\
\text { Force }\end{array}$} & \multirow{3}{*}{$\begin{array}{c}\text { Brace } \\
R_{y} \\
(\mu=3)\end{array}$} & \multicolumn{7}{|c|}{ Roof Diaphragm Design Factor $(\phi)$ Determination } \\
\hline & & & \multirow{2}{*}{$\begin{array}{c}\phi \text { - Elastic } \\
\text { Diaphragm }\end{array}$} & \multicolumn{6}{|c|}{ Nonlinear Diaphragm with Ductility $\leq 1.5$} \\
\hline & & & & $\phi$ & $\mu($ Brace $)$ & M.F & V.F & M.U & V.U \\
\hline SL1 & 141.22 & 2.72 & 1.19 & 1.03 & 2.81 & 1.22 & 1.37 & 1.53 & 1.89 \\
\hline SH1 & 279.48 & 3.09 & 1.09 & 1.05 & 2.88 & 1.28 & 1.40 & 1.60 & 1.92 \\
\hline ML1 & 322.29 & 1.66 & 1.09 & 1.04 & 2.76 & 1.12 & 1.29 & 1.40 & 1.77 \\
\hline MH1 & 343.31 & 1.62 & 1.16 & 1.04 & 2.35 & 1.31 & 1.42 & 1.63 & 1.95 \\
\hline LL1 & 596.82 & 1.64 & 1.17 & 1.07 & 2.73 & 1.30 & 1.43 & 1.62 & 1.97 \\
\hline LH1 & 1265.51 & 1.97 & 1.22 & 1.08 & 3.11 & 1.23 & 1.42 & 1.54 & 1.95 \\
\hline
\end{tabular}


Table F.33 - Diaphragm design factors for linear and nonlinear performance with limited ductility to 1.5 - brace $\mu=3.0$ - E6C15 record - long side

\begin{tabular}{|c|c|c|c|c|c|c|c|c|c|}
\hline \multirow{3}{*}{ Buildings } & \multirow{3}{*}{$\begin{array}{c}\text { Brace } \\
\text { Elastic } \\
\text { Force }\end{array}$} & \multirow{3}{*}{$\begin{array}{c}\text { Brace } \\
\mathrm{R}_{\mathrm{y}} \\
(\mu=3)\end{array}$} & \multicolumn{7}{|c|}{ Roof Diaphragm Design Factor $(\phi)$ Determination } \\
\hline & & & \multirow{2}{*}{$\begin{array}{c}\phi \text { - Elastic } \\
\text { Diaphragm }\end{array}$} & \multicolumn{6}{|c|}{ Nonlinear Diaphragm with Ductility $\leq 1.5$} \\
\hline & & & & $\phi$ & $\mu($ Brace $)$ & M.F & V.F & M.U & V.U \\
\hline SL1 & 139.11 & 2.01 & 1.09 & 1.04 & 2.89 & 1.18 & 1.38 & 1.48 & 1.90 \\
\hline SH1 & 178.32 & 1.75 & 1.15 & 1.04 & 2.54 & 1.21 & 1.39 & 1.51 & 1.92 \\
\hline ML1 & 303.03 & 2.01 & 1.18 & 1.06 & 2.87 & 1.29 & 1.41 & 1.61 & 1.94 \\
\hline MH1 & 753.83 & 2.45 & 1.15 & 1.05 & 3.11 & 1.28 & 1.38 & 1.60 & 1.90 \\
\hline LL1 & 1473.38 & 2.97 & 1.19 & 1.10 & 3.03 & 1.34 & 1.45 & 1.67 & 2.00 \\
\hline LH1 & 3507.12 & 4.36 & 1.38 & 1.22 & 2.96 & 1.47 & 1.62 & 1.84 & 2.22 \\
\hline
\end{tabular}

Table F.34 - Diaphragm design factors for linear and nonlinear performance with limited ductility to 1.5 - brace $\mu=3.0$ - E6C18 record - long side

\begin{tabular}{|c|c|c|c|c|c|c|c|c|c|}
\hline \multirow{3}{*}{ Buildings } & \multirow{3}{*}{$\begin{array}{c}\text { Brace } \\
\text { Elastic } \\
\text { Force }\end{array}$} & \multirow{3}{*}{$\begin{array}{c}\text { Brace } \\
\mathrm{R}_{\mathrm{y}} \\
(\mu=3)\end{array}$} & \multicolumn{7}{|c|}{ Roof Diaphragm Design Factor $(\phi)$ Determination } \\
\hline & & & \multirow{2}{*}{$\begin{array}{c}\phi-\text { Elastic } \\
\text { Diaphragm }\end{array}$} & \multicolumn{6}{|c|}{ Nonlinear Diaphragm with Ductility $\leq 1.5$} \\
\hline & & & & $\phi$ & $\mu($ Brace $)$ & M.F & V.F & M.U & V.U \\
\hline SL1 & 187.54 & 2.34 & 1.09 & 1.02 & 2.84 & 1.09 & 1.25 & 1.36 & 1.72 \\
\hline SH1 & 353.92 & 2.28 & 1.11 & 1.03 & 2.64 & 1.23 & 1.38 & 1.53 & 1.94 \\
\hline ML1 & 278.26 & 1.74 & 1.15 & 1.03 & 2.44 & 1.22 & 1.40 & 1.52 & 1.92 \\
\hline MH1 & 562.83 & 1.90 & 1.09 & 1.04 & 2.84 & 1.19 & 1.39 & 1.48 & 1.90 \\
\hline LL1 & 1036.68 & 1.95 & 1.12 & 1.03 & 2.74 & 1.15 & 1.32 & 1.44 & 1.82 \\
\hline LH1 & 2151.43 & 1.81 & 1.07 & 1.03 & 2.92 & 1.05 & 1.21 & 1.31 & 1.66 \\
\hline
\end{tabular}

Table F.35 - Diaphragm design factors for linear and nonlinear performance with limited ductility to 1.5 - brace $\mu=3.0$ - E6C42 record - long side

\begin{tabular}{|c|c|c|c|c|c|c|c|c|c|}
\hline \multirow{3}{*}{ Buildings } & \multirow{3}{*}{$\begin{array}{c}\text { Brace } \\
\text { Elastic } \\
\text { Force }\end{array}$} & \multirow{3}{*}{$\begin{array}{c}\text { Brace } \\
R_{y} \\
(\mu=3)\end{array}$} & \multicolumn{7}{|c|}{ Roof Diaphragm Design Factor $(\phi)$ Determination } \\
\hline & & & \multirow{2}{*}{$\begin{array}{c}\phi \text { - Elastic } \\
\text { Diaphragm }\end{array}$} & \multicolumn{6}{|c|}{ Nonlinear Diaphragm with Ductility $\leq 1.5$} \\
\hline & & & & $\phi$ & $\mu($ Brace $)$ & M.F & V.F & M.U & V.U \\
\hline SL1 & 179.40 & 2.10 & 1.14 & 1.03 & 2.74 & 1.19 & 1.37 & 1.49 & 1.89 \\
\hline SH1 & 230.21 & 1.72 & 1.16 & 1.03 & 2.51 & 1.21 & 1.39 & 1.51 & 1.91 \\
\hline ML1 & 275.59 & 2.42 & 1.10 & 1.03 & 2.87 & 1.15 & 1.37 & 1.43 & 1.88 \\
\hline MH1 & 599.19 & 1.65 & 1.09 & 1.02 & 2.78 & 0.91 & 1.02 & 1.14 & 1.41 \\
\hline LL1 & 1081.02 & 1.74 & 1.06 & 1.02 & 2.90 & 0.89 & 0.99 & 1.11 & 1.36 \\
\hline LH1 & 2182.47 & 2.97 & 1.21 & 1.06 & 3.00 & 1.17 & 1.40 & 1.46 & 1.93 \\
\hline
\end{tabular}


Table F.36 - Diaphragm design factors for linear and nonlinear performance with limited ductility to 1.5 - brace $\mu=4.0$ - M6C2 record - long side

\begin{tabular}{|c|c|c|c|c|c|c|c|c|c|}
\hline \multirow{3}{*}{ Buildings } & \multirow{3}{*}{$\begin{array}{c}\text { Brace } \\
\text { Elastic } \\
\text { Force }\end{array}$} & \multirow{3}{*}{$\begin{array}{c}\text { Brace } \\
\mathrm{R}_{\mathrm{y}} \\
(\mu=4)\end{array}$} & \multicolumn{7}{|c|}{ Roof Diaphragm Design Factor $(\phi)$ Determination } \\
\hline & & & \multirow{2}{*}{$\begin{array}{c}\phi \text { - Elastic } \\
\text { Diaphragm }\end{array}$} & \multicolumn{6}{|c|}{ Nonlinear Diaphragm with Ductility $\leq 1.5$} \\
\hline & & & & $\phi$ & $\mu($ Brace $)$ & M.F & V.F & M.U & V.U \\
\hline SL1 & 179.03 & 2.04 & 1.08 & 1.05 & 3.91 & 1.00 & 1.15 & 1.25 & 1.59 \\
\hline SH1 & 488.50 & 3.25 & 1.20 & 1.08 & 3.89 & 1.18 & 1.37 & 1.47 & 1.89 \\
\hline ML1 & 615.67 & 2.01 & 1.08 & 1.05 & 3.81 & 1.06 & 1.22 & 1.32 & 1.67 \\
\hline MH1 & 937.20 & 1.88 & 1.20 & 1.08 & 3.40 & 1.24 & 1.40 & 1.55 & 1.93 \\
\hline LL1 & 1745.36 & 2.02 & 1.21 & 1.09 & 3.71 & 1.21 & 1.40 & 1.52 & 1.92 \\
\hline LH1 & 3995.76 & 3.16 & 1.30 & 1.11 & 3.73 & 1.32 & 1.42 & 1.65 & 1.95 \\
\hline
\end{tabular}

Table F.37 - Diaphragm design factors for linear and nonlinear performance with limited ductility to 1.5 - brace $\mu=4.0$ - M6C26 record - long side

\begin{tabular}{|c|c|c|c|c|c|c|c|c|c|}
\hline \multirow{3}{*}{ Buildings } & \multirow{3}{*}{$\begin{array}{l}\text { Brace } \\
\text { Elastic } \\
\text { Force }\end{array}$} & \multirow{3}{*}{$\begin{array}{c}\text { Brace } \\
R_{y} \\
(\mu=4)\end{array}$} & \multicolumn{7}{|c|}{ Roof Diaphragm Design Factor $(\phi)$ Determination } \\
\hline & & & \multirow{2}{*}{$\begin{array}{c}\phi-\text { Elastic } \\
\text { Diaphragm }\end{array}$} & \multicolumn{6}{|c|}{ Nonlinear Diaphragm with Ductility $\leq 1.5$} \\
\hline & & & & $\phi$ & $\mu($ Brace $)$ & M.F & V.F & M.U & V.U \\
\hline SL1 & 202.44 & 2.07 & 1.10 & 1.04 & 3.94 & 1.07 & 1.24 & 1.33 & 1.71 \\
\hline SH1 & 500.73 & 2.40 & 1.08 & 1.06 & 3.87 & 1.08 & 1.25 & 1.35 & 1.72 \\
\hline ML1 & 585.71 & 1.66 & 1.09 & 1.06 & 3.82 & 1.07 & 1.23 & 1.33 & 1.69 \\
\hline MH1 & 1282.07 & 1.78 & 1.09 & 1.06 & 3.86 & 0.95 & 1.08 & 1.19 & 1.48 \\
\hline LL1 & 2423.53 & 2.23 & 1.07 & 1.04 & 3.86 & 1.05 & 1.21 & 1.32 & 1.67 \\
\hline LH1 & 6386.35 & 3.63 & 1.15 & 1.07 & 3.91 & 1.14 & 1.36 & 1.42 & 1.87 \\
\hline
\end{tabular}

Table F.38 - Diaphragm design factors for linear and nonlinear performance with limited ductility to 1.5 - brace $\mu=4.0$ - M6C31 record - long side

\begin{tabular}{|c|c|c|c|c|c|c|c|c|c|}
\hline \multirow{3}{*}{ Buildings } & \multirow{3}{*}{$\begin{array}{c}\text { Brace } \\
\text { Elastic } \\
\text { Force }\end{array}$} & \multirow{3}{*}{$\begin{array}{c}\text { Brace } \\
\mathrm{R}_{\mathrm{y}} \\
(\mu=4)\end{array}$} & \multicolumn{7}{|c|}{ Roof Diaphragm Design Factor $(\phi)$ Determination } \\
\hline & & & \multirow{2}{*}{$\begin{array}{l}\phi \text { - Elastic } \\
\text { Diaphragm }\end{array}$} & \multicolumn{6}{|c|}{ Nonlinear Diaphragm with Ductility $\leq 1.5$} \\
\hline & & & & $\phi$ & $\mu($ Brace $)$ & M.F & V.F & M.U & V.U \\
\hline SL1 & 189.42 & 2.49 & 1.15 & 1.08 & 3.91 & 1.20 & 1.37 & 1.51 & 1.89 \\
\hline SH1 & 454.44 & 2.70 & 1.10 & 1.06 & 4.03 & 1.11 & 1.33 & 1.39 & 1.83 \\
\hline ML1 & 978.00 & 3.20 & 1.11 & 1.07 & 3.71 & 1.25 & 1.37 & 1.56 & 1.88 \\
\hline MH1 & 1044.74 & 2.06 & 1.11 & 1.04 & 3.49 & 1.07 & 1.26 & 1.34 & 1.73 \\
\hline LL1 & 1764.29 & 2.14 & 1.11 & 1.03 & 3.70 & 1.11 & 1.30 & 1.39 & 1.79 \\
\hline LH1 & 3925.45 & 2.17 & 1.10 & 1.05 & 3.90 & 1.07 & 1.25 & 1.34 & 1.73 \\
\hline
\end{tabular}


Table F.39 - Diaphragm design factors for linear and nonlinear performance with limited ductility to 1.5 - brace $\mu=4.0$ - E6C15 record - long side

\begin{tabular}{|c|c|c|c|c|c|c|c|c|c|}
\hline \multirow{3}{*}{ Buildings } & \multirow{3}{*}{$\begin{array}{c}\text { Brace } \\
\text { Elastic } \\
\text { Force }\end{array}$} & \multirow{3}{*}{$\begin{array}{c}\text { Brace } \\
\mathrm{R}_{\mathrm{y}} \\
(\mu=4)\end{array}$} & \multicolumn{7}{|c|}{ Roof Diaphragm Design Factor $(\phi)$ Determination } \\
\hline & & & \multirow{2}{*}{$\begin{array}{c}\phi \text { - Elastic } \\
\text { Diaphragm }\end{array}$} & \multicolumn{6}{|c|}{ Nonlinear Diaphragm with Ductility $\leq 1.5$} \\
\hline & & & & $\phi$ & $\mu($ Brace $)$ & M.F & V.F & M.U & V.U \\
\hline SL1 & 139.11 & 2.32 & 1.12 & 1.07 & 3.86 & 1.16 & 1.36 & 1.45 & 1.87 \\
\hline SH1 & 178.32 & 2.02 & 1.19 & 1.08 & 3.54 & 1.21 & 1.39 & 1.52 & 1.92 \\
\hline ML1 & 303.03 & 2.46 & 1.26 & 1.12 & 3.87 & 1.32 & 1.43 & 1.65 & 1.96 \\
\hline MH1 & 753.83 & 3.18 & 1.25 & 1.12 & 3.89 & 1.34 & 1.42 & 1.67 & 1.96 \\
\hline LL1 & 1473.38 & 3.89 & 1.30 & 1.18 & 3.82 & 1.40 & 1.50 & 1.75 & 2.07 \\
\hline LH1 & 3507.12 & 5.11 & 1.41 & 1.26 & 3.93 & 1.47 & 1.60 & 1.84 & 2.20 \\
\hline
\end{tabular}

Table F.40 - Diaphragm design factors for linear and nonlinear performance with limited ductility to 1.5 - Brace $\mu=4.0$ - E6C42 record - Long side

\begin{tabular}{|c|c|c|c|c|c|c|c|c|c|}
\hline \multirow{3}{*}{ Buildings } & \multirow{3}{*}{$\begin{array}{c}\text { Brace } \\
\text { Elastic } \\
\text { Force }\end{array}$} & \multirow{3}{*}{$\begin{array}{c}\text { Brace } \\
\mathrm{R}_{\mathrm{y}} \\
(\mu=4)\end{array}$} & \multicolumn{7}{|c|}{ Roof Diaphragm Design Factor $(\phi)$ Determination } \\
\hline & & & \multirow{2}{*}{$\begin{array}{c}\phi \text { - Elastic } \\
\text { Diaphragm }\end{array}$} & \multicolumn{6}{|c|}{ Nonlinear Diaphragm with Ductility $\leq 1.5$} \\
\hline & & & & $\phi$ & $\mu($ Brace $)$ & M.F & V.F & M.U & V.U \\
\hline SL1 & 179.40 & 2.57 & 1.18 & 1.06 & 3.73 & 1.20 & 1.36 & 1.50 & 1.87 \\
\hline SH1 & 230.21 & 2.65 & 1.20 & 1.06 & 3.47 & 1.29 & 1.37 & 1.61 & 1.89 \\
\hline ML1 & 275.59 & 2.82 & 1.14 & 1.07 & 3.98 & 1.12 & 1.31 & 1.40 & 1.80 \\
\hline MH1 & 599.19 & 2.91 & 1.18 & 1.08 & 3.90 & 1.22 & 1.37 & 1.53 & 1.89 \\
\hline LL1 & 1081.02 & 3.21 & 1.22 & 1.08 & 3.95 & 1.23 & 1.36 & 1.54 & 1.87 \\
\hline LH1 & 2182.47 & 3.62 & 1.42 & 1.22 & 4.00 & 1.28 & 1.54 & 1.59 & 2.12 \\
\hline
\end{tabular}




\section{References}

[1] Adebar, P. Guan, Z., and Elwood, K. 2004. Displacement-based design of concrete tilt-up frames accounting for flexible diaphragms. $13^{\text {th }}$ World Conference on Earthquake Engineering. Vancouver, B.C. Paper No. 1054.

[2] AISC. 2005a. "ANSI/AISC 341-05, Seismic provisions for structural steel buildings", American Institute of Steel Construction, Inc. Chicago, Illinois.

[3] AISC. 2005b. "ANSI/AISC 360-05, Specification for structural steel buildings", American Institute of Steel Construction, Inc. Chicago, Illinois.

[4] Archambault, M-H, Tremblay, R., and Filiatrault, A. 1995. E'tude du comportement se'ismique des contreventements ductiles en $\mathrm{X}$ avec profile's tubulaires en acier. Rep. No. EPM/GCS-1995-09, E'cole Polytechnique, Montreal (in French).

[5] ASCE. 2005. ASCE 7-05 Standard minimum design loads for buildings and other structures. American Society of Civil Engineers. Reston, VA.

[6] Atkinson, G.M. 2009. Earthquake time histories compatible with the 2005 National Building Code of Canada Uniform Hazard Spectrum. Canadian Journal of Civil Engineering, 36(6): 991-1000.

[7] Black, R.G., Wenger, W.A., and Popov, E.P. 1980. Inelastic buckling of steel struts under cyclic load and reversal. Rep. No. UCB/EERC- 80/40, Earthquake Engineering Research Center, University of California, Berkeley, California. 
[8] Button, M.R., Kelly, T.E, and Jones, L.R. 1984. Influence of diaphragm flexibility on the seismic response of buildings. $8^{\text {th }}$ World Conference on Earthquake Engineering, San Francisco, California, 4: 759-766.

[9] Canadian Institute of Steel Construction. 2012. Handbook of Steel Construction, tenth edition.

[10] CANAM. 2007, Steel deck diaphragm design manual.

[11] CSA-S16-09. 2009. Canadian Standard Association. Design of steel structures.

[12] Chen, J. 2010. An experimental study of strain rate effects on mild steel. MASc thesis. Department of Civil and Environmental Engineering, Carleton University, Ottawa, Ontario, Canada.

[13] Chen, L., and Trica, L. 2013. Simulating the seismic response of concentrically braced frames using physical theory brace models. Open Journal of Civil Engineering, 3: 69-81.

[14] Davies, J.M., and Bryan, E.R. 1982. Manual of stressed skin diaphragm design. Wiley, New York.

[15] Dolce, M., Lorusso, V.D., and Masi, A. 1994. Seismic response of building structures with flexible inelastic diaphragm. The Structural Design of Tall Buildings, $\mathbf{3}$ : 87-106.

[16] Essa, H.S., Tremblay, R., and Rogers, C.A. 2003. Behavior of roof deck diaphragms under quasistatic cyclic loading. Journal of Structural Engineering (C ASCE, 129: 16581666. 
[17] FEMA. 2000. FEMA 356 Prestandard and commentary for the seismic rehabilitation of buildings. Federal Emergency Management Agency, Washington D.C.

[18] FEMA. 2006. FEMA 356 Prestandard and commentary for the seismic rehabilitation of buildings. Federal Emergency Management Agency, Washington D.C.

[19] Humar, J. and Popovski, M. 2012. Seismic response of single-storey buildings with flexible diaphragms. Paper STR 1014, $3^{\text {rd }}$ International Structural Specialty Conference, Edmonton, Alberta.

[20] Humar, J. and Popovski, M. 2013. Seismic response of single-storey buildings with flexible diaphragms. Canadian Journal of Civil Engineering, 40(9): 875-886

[21] Ibarra, L. F., Medina, R., and Krawinkler, H. 2002. Collapse assessment of deteriorating SDOF systems. Proc. 12th European Conf. on Earthquake Engineering, Paper 665, Elsevier Science, London.

[22] Ibarra, L. F., and Krawinkler, H. 2005. Global collapse of frame structures under seismic excitations. Rep. No. TB 152, The John A. Blume Earthquake Engineering Center, Stanford Univ., Stanford, CA.

[23] Ibarra, L. F., Medina, R. A., and Krawinkler, H. 2005. Hysteretic models that incorporate strength and stiffness deterioration. Earthquake Engineering and Structural Dynamics, 34(12), 1489-1511.

[24] ICC. 2006. International Building Code 2006 (IBC 2006). International Code Council, Falls Church, V.A. 
[25] Jain, S.K. and Jennings, P.C. 1985. Analytical models for low-rise buildings with flexible diaphragms. Earthquake Engineering and Structural Dynamics, 13: 225-241.

[26] Kim, S-C. and White, D.W. 2004a. Linear static analysis of low-rise buildings with flexible diaphragms using the structural separation method. Engineering Structures, 26: 83-93.

[27] Kim, S-C. and White, D.W. 2004b. Nonlinear analysis of a one-storey low-rise masonry building with a flexible diaphragm subjected to seismic excitation. Engineering Structures, 26: 2053-2067.

[28] Kunnath, S.K. Panahshahi, N., and Reinhorn, A.M. 1991. Seismic response of RC buildings with inelastic floor diaphragms. Journal of Structural Engineering, 117(4): 1218-1237.

[29] Lee, H.J., Kuchma, D., and Aschheim, M.A. 2007. Strength-based design of flexible diaphragms in low-rise structures subjected to earthquake loading. Engineering Structures, 29: 1277-1295

[30] Lee, K., and Bruneau, M. 2005. Energy dissipation of compressive members in concentrically braced frames: review of experimental data. Journal of Structural Engineering (C) ASCE, 131: 552-559.

[31] Lignos, D.G. 2008. Sidesway collapse of deteriorating structural systems under seismic excitations. Ph.D. Dissertation. Department of Civil and Environmental Engineering. Stanford University, Stanford, CA. 
[32] Lignos, D.G., and Krawinkler, H. 2011. Deterioration modelling of steel components in support of collapse prediction of steel moment frames under earthquake loading. Journal of Structural Engineering (C) ASCE, 137: 1291-1302.

[33] Lignos, D.G. and Krawinkler, H. 2012. Sidesway collapse of deteriorating structural systems under seismic excitations. Rep.No.TB 177. The John A. Blume Earthquake Engineering Research Center, Stanford University, Stanford, CA.

[34] Lignos, D.G., and Krawinkler, H. 2013. Development and utilization of structural component databases for performance-based earthquake engineering. Journal of Structural Engineering (C) ASCE, 139: 1382-1394.

[35] Massarelli, R., Franquet, J-E., Shrestha, K., Tremblay, R. and Rogers, C.A. 2012. Seismic testing and retrofit of steel-deck diaphragms for building structures. Thin-Walled Structures, 61: 239-247

[36] Mitchell, D., Tremblay, R., Karacabeyli, E., Paultre, P., Saatcioglu, M., and Anderson D.L. 2003. Seismic force modification factors for the proposed 2005 edition of the National Building Code of Canada. Canadian Journal of Civil Engineers, 30: 308327.

[37] Moncarz, P.D. and Krawinkler, H. 1981. Theory and application of experimental model analysis in earthquake engineering. Report no. 50, John A. Blume Earthquake Engineering Center, Stanford University, San Francisco, Ca. 
[38] NRCC. 2010. National Building Code of Canada 2010 (NBCC 2010). Canadian Commission on Building and Fire Code, National Research Council of Canada, Ottawa, Ont.

[39] NRCC. 2005. National Building Code of Canada 2005 (NBCC 2005). Canadian Commission on Building and Fire Code, National Research Council of Canada, Ottawa, Ont.

[40] NRCC. 1995. National Building Code of Canada 1995 (NBCC 1995). Canadian Commission on Building and Fire Code, National Research Council of Canada, Ottawa, Ont.

[41] OpenSees. 2014. Open system for earthquake engineering simulation. Pacific Earthquake Engineering Research Center (PEER), (http://opensees.berkeley.edu).

[42] Paquette, J. and Bruneau, M. 2006. Pseudo-dynamic testing of unreinforced masonry building with flexible diaphragm and comparison with existing procedures. Construction and Building Materials, 20: 220-228

[43] Rahnama, M., and Krawinkler, H. 1993. Effect of soft soil and hysteresis models on seismic design spectra. Rep. No. TB 108. The John A. Blume Earthquake Engineering Center, Stanford University, Stanford, CA.

[44] Rogers, C.A., and Tremblay, R. 2003a. Inelastic seismic response of side lap fasteners for steel roof deck diaphragms. Journal of Structural Engineering (C ASCE, 129: 1637-1646. 
[45] Rogers, C.A., and Tremblay, R. 2003b. Inelastic seismic response of frame fasteners for steel roof deck diaphragms. Journal of Structural Engineering (C) ASCE, 129: 16471657.

[46] SDI. 1981. Diaphragm design manual. 1st edition. Steel Deck Institute, Inc., Canton. Ohio.

[47] SDI. 1991. Diaphragm design manual. $2^{\text {nd }}$ edition. Steel Deck Institute, Inc., Canton. Ohio.

[48] SDI. 2011. ANSI/SDI C-2011 Standard for Composite Steel Floor Deck - Slabs. Steel Deck Institute, Inc., Canton. Ohio.

[49] Tena-Colunga, A. and Abrams, D.P. 1996. Seismic behaviour of structures with flexible diaphragms. Journal of Structural Engineering, 122(4): 439-445.

[50] Tokoro, K.T., Anderson, J.C., and Bertero, V.V. 2004. Uncertainties in determining diaphragm flexibility. $13^{\text {th }}$ World Conference on Earthquake Engineering. Vancouver, B.C. Paper No. 565.

[51] Tremblay, R. and Stiemer, S.F. 1996. Seismic behaviour of single-storey steel structures with a flexible roof diaphragm. Canadian Journal of Civil Engineering, 23(1): 49-62

[52] Tremblay, R., Rogers, C.A., Essa, H.S., and Martin, E. 2002. Dissipating seismic input energy in low-rise steel building through inelastic deformations in the metal roof diaphragm. $4^{\text {th }}$ Structural Speciality Conference. Montreal, Quebec. 
[53] Tremblay, R., Berair, T., and Filiatrault. 2000. Experimental behaviour of low-rise steel buildings with flexible roof diaphragms. $12^{\text {th }}$ World Conference on Earthquake Engineering. Auckland, NZ. Paper No. 2567.

[54] Tremblay, R., Archambault, M.-H., and Filiatrault, A. 2003. Seismic response of concentrically braced steel frames made with rectangular hollow bracing members. Journal of Structural Engineering $\odot$ ASCE, 129: 1626-1636.

[55] Trudel-Languedoc, S., Tremblay, R., Shrestha, K., and Rogers, C.A. 2012. Seismic force and ductility demand on the braced bents of single-storey buildings with flexible roof deck diaphragms. $15^{\text {th }}$ World Conference on Earthquake Engineering. Lisbon, Portugal. 